\title{
2016 Annual Merit Review, Vehicle Technologies Office
}

\author{
Results Report
}

December 2016

Prepared by Allegheny Science \& Technology for the

U.S. Department of Energy, Vehicle Technologies Office 
(This page intentionally left blank) 


\section{Table of Contents}

Introduction. 1

Evaluation Criteria - Research and Development Subprogram Projects.......................1

Evaluation Criteria - Technology Integration Projects........................................................ 3

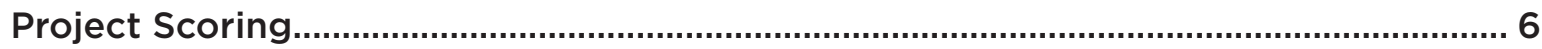

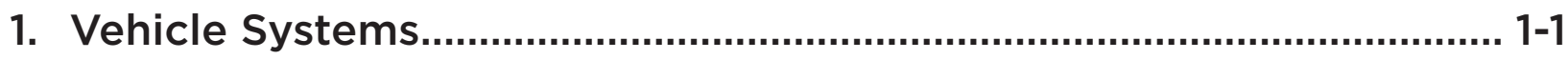

Overview of the VTO Vehicle Systems Program: Lee Slezak

(U.S. Department of Energy) - vsO00. $1-3$

Medium- and Heavy-Duty Vehicle Field Evaluations: Ken Kelly

(National Renewable Energy Laboratory) - vs001.

DOE's Effort to Improve Heavy Vehicle Fuel Efficiency through

Improved Aerodynamics: Kambiz Salari (Lawrence Livermore

National Laboratory) - vs006.

Idaho National Laboratory Testing of Advanced Technology Vehicles:

James Francfort (Idaho National Laboratory) - vs021.................................................... 1-22

Advanced Vehicle Testing and Evaluation: Richard Jacobson (Intertek) - vs029.......1-25

Advanced Technology Vehicle Lab Benchmarking (L1 and L2): Kevin Stutenberg (Argonne National Laboratory) - vs030.

SuperTruck - Development and Demonstration of a Fuel-Efficient Class 8

Tractor and Trailer Vehicle: Russ Zukouski (Navistar International Corporation)

- vs064.

Commercial Vehicle Thermal Load Reduction and VTCab-Rapid

HVAC Load Estimation Tool: Jason Lustbader (National Renewable

Energy Laboratory) - vs075.

Volvo SuperTruck: Pascal Amar (Volvo Trucks) - vs081

System for Automatically Maintaining Pressure in a Commercial Truck Tire:

Norm Anderson (The Goodyear Tire and Rubber Company) - vs085.

EV - Smart Grid Research and Interoperability Activities: Keith Hardy

(Argonne National Laboratory) - vs095.

Wireless and Conductive Charging Testing to Support Code and Standards:

Barney Carlson (Idaho National Laboratory) - vs096.

High-Efficiency, Low EMI and Positioning Tolerant Wireless Charging of EVs:

Rakan Chabaan (Hyundai) - vs102.

Wireless Charging of Electric Vehicles: Omer Onar

(Oak Ridge National Laboratory) - vs103.

Zero Emission Drayage Truck Demonstration (ZECT I): Matt Miyasato (SCAQMD) - vs115. 
Hydrogen Fuel-Cell Electric Hybrid Truck and Zero Emission Delivery Vehicle Deployment: Andrew DeCandis (Houston-Galveston Area Council) - vs116.

Combined Aero and Underhood Thermal Analysis for Heavy-Duty Trucks:

Tanju Sofu (Argonne National Laboratory) - vs132.

Cummins Medium-Duty and Heavy-Duty Accessory Hybridization CRADA:

Dean Deter (Oak Ridge National Laboratory) - vs133.

Vehicle Thermal System Modeling in Simulink: Jason Lustbader

(National Renewable Energy Laboratory) - vs134.

Advanced Climate Systems for EV Extended Range (ACSforEVER): John Meyer (Hanon Systems) - vs135.

ePATHS - electrical PCM Assisted Thermal Heating System: Mingyu Wang

(Mahle Behr USA, LLC) - vs136.

SAE J2907 Motor Power Ratings Standards Support: John Miller

(Oak Ridge National Laboratory) - vs144

Analyzing Real-World Light Duty Vehicle Efficiency Benefits: Jeff Gonder (National Renewable Energy Laboratory) - vs155.

UTEMPRA - Unitary Thermal Energy Management for Propulsion Range Augmentation: Sourav Chowdhury (Mahle Behr USA LLC) - vs157.

Zero Emission Cargo Transport II: San Pedro Bay Ports Hybrid and Fuel Cell Electric Vehicle Project: Joseph Impullitti (SCAQMD) - vs158

Multi-Speed Transmission for Commercial Delivery Medium-Duty Plug-In Electric Drive Vehicles: Bulent Chavdar (Eaton Corporation) - vs161.

Integrated Boosting and Hybridization for Extreme Fuel Economy and Downsizing: Vasilios Tsourapas (Eaton Corporation) - vs162

Advanced Bus and Truck Radial Materials for Fuel Efficiency:

Lucas Dos Santos Freire (PPG Industries) - vs163.

Design and Implementation of a Thermal Load Reduction System in a Hyundai

PHEV: Cory Kreutzer (National Renewable Energy Laboratory) - vs165.

EV Everywhere Charging Infrastructure Roadmap: Donald Karner

(Electric Applications Incorporated) - vs172

Energy Impact of Connected and Automated Vehicles: Huei Peng

(University of Michigan) - vs173.

Methods to Measure, Predict, and Relate Friction, Wear, and Fuel Economy:

Steve Gravante (Ricardo) - vs175.

Improved Tire Efficiency through Elastomeric Polymers Enhanced with Carbon-Based Nanostructured Materials: Georgios Polyzos (Oak Ridge National Laboratory) - vs176. $1-123$

VTO Vehicle to Building Integration Pathway: Richard Pratt (Pacific Northwest National Laboratory) - vs181 
VTO Systems Research Supporting Standards and Interoperability: John Smart (Idaho National Laboratory) - vs182.

VTO Modeling and Controls Software Tools to Support V2G Integration:

Samveg Saxena (Lawrence Berkeley National Laboratory) - vs183.

VTO Diagnostic Security Modules for Electric Vehicle to Building Integration:

Ken Rohde (Idaho National Laboratory) - vs184.

Evaluation of Vehicle Technology Benefits on Real World Driving Cycles

using Regional Transportation System Model: Aymeric Rousseau

(Argonne National Laboratory) - vs185.

Evaluation of Dynamic Wireless Charging Demand: James Li

(Oak Ridge National Laboratory) - vs186.

2. Electrochemical Energy Storage.

Overview of the DOE Advanced Battery R\&D Program: David Howell

(U.S. Department of Energy) - es000.

Materials Benchmarking Activities for CAMP Facility: Wenquan Lu

(Argonne National Laboratory) - es028.

Cell Analysis, Modeling, and Prototyping (CAMP) Facility Research Activities:

Andrew Jansen (Argonne National Laboratory) - es030.

Overview and Progress of United States Advanced Battery Consortium (USABC)

Activity: Ron Elder (United States Advanced Battery Consortium) - es097.

Thick Low-Cost, High-Power Lithium-Ion Electrodes via Aqueous Processing:

Jianlin Li (Oak Ridge National Laboratory) - es164.

Performance Effects of Electrode Coating Defects and IR Thermography

NDE for High-Energy Lithium-Ion Batteries: David Wood

(Oak Ridge National Laboratory) - es165.

Post-Test Analysis of Lithium-Ion Battery Materials at Argonne National

Laboratory: Ira Bloom (Argonne National Laboratory) - es166.

Process Development and Scale-Up of Advanced Active Battery Materials:

Greg Krumdick (Argonne National Laboratory) - es167..

Process Development and Scale-Up of Critical Battery Materials:

Greg Krumdick (Argonne National Laboratory) - es168.

Electrochemical Performance Testing: Ira Bloom

(Argonne National Laboratory) - es201..

INL Electrochemical Performance Testing: Matt Shirk

(Idaho National Laboratory) - es202.

Battery Safety Testing: Leigh Anna Steele

(Sandia National Laboratories) - es203..

Battery Thermal Characterization: Matthew Keyser

(National Renewable Energy Laboratory) - es204. 
Towards Solventless Processing of Thick Electron-Beam (EB) Cured LIB

Cathodes: David Wood (Oak Ridge National Laboratory) - es207.

New High-Energy Electrochemical Couple for Automotive Applications:

Khalil Amine (Argonne National Laboratory) - es208.

High-Energy High-Power Battery Exceeding PHEV-40 Requirements:

Jane Rempel (TIAX LLC) - es209.

Advanced High-Energy Li-Ion Cell for PHEV and EV Applications:

Jagat Singh (3M) - es210.

High-Energy Lithium Batteries for PHEV Applications:

Subramanian Venkatachala (Envia Systems) - es211.

High-Energy, Long Cycle Life Lithium-Ion Batteries for EV Applications:

Donghai Wang (Pennsylvania State University) - es212

High-Energy Density Li-ion Cells for EVs Based on Novel, High Voltage

Cathode Material Systems: Keith Kepler (Farasis) - es213.

Fundamental Studies of Lithium-Sulfur Cell Chemistry: Nitash Balsara

(Lawrence Berkeley National Laboratory) - es224..

BatPaC Model Development: Shabbir Ahmed (Argonne National Laboratory)

- es228.

Design of Sulfur Cathodes for High-Energy Lithium-Sulfur Batteries: Yi Cui (Stanford University) - es230

Efficient Rechargeable $\mathrm{Li}-\mathrm{O}_{2}$ Batteries Utilizing Stable Inorganic Molten Salt Electrolytes: Vincent Giordani (Liox) - es233.

Low-Cost, High-Energy Si-Graphene Anodes for Li-Ion Batteries: John Colwell (XG Sciences) - es237.

Low-Cost, High-Capacity Lithium Ion Batteries through Modified Surface and Microstructure: Pu Zhang (Navitas Systems) - es238.

Scale-Up of Low-Cost Encapsulation Technologies for High Capacity and High Voltage Electrode Powders: David King (Pneumaticoat Technologies) - es239.

High-Energy Anode Material Development for Li-Ion Batteries: Cary Hayner (Sinode Systems) - es240.

Advanced High-Performance Batteries for Electric Vehicle (EV) Applications: Ionel Stefan (Amprius) - es241.

A Disruptive Concept for a Whole Family of New Battery Systems: Farshid Roumi (Parthian Energy) - es242.

Dramatically Improve the Safety Performance of Li-Ion Battery Separators and Reduce the Manufacturing Cost Using Ultraviolet Curing and High-Precision Coating Technologies: John Arnold (Miltec UV International) - es243....

Low-Cost, High-Capacity Non-Intercalation Chemistry Automotive Cells:

Alex Jacobs (Sila Nanotechnologies) - es244. 
Low-Cost, Structurally Advanced Novel Electrode and Cell Manufacturing:

Billy Woodford (24M Technologies) - es245.

Advanced Drying Process for Lower Manufacturing Cost of Electrodes:

Iftikhar Ahmad (Lambda Technologies) - es246....

High-Energy Lithium Batteries for Electric Vehicles: Herman Lopez

(Envia Systems) - es247.

A 12V Start-Stop Li Polymer Battery Pack: Mohamed Alamgir

(LG Chem Power) - es249..

A Commercially Scalable Process for Silicon Anode Pre-Lithiation:

Ionel Stefan (Amprius) - es250.

Development of Advanced High-Performance Batteries for 12V Start-Stop

Vehicle Applications: Jeff Kim (Maxwell) - es251.

Enabling High-Energy, High-Voltage Li-Ion Cells for Transportation Applications:

Modeling and Analysis: Dennis Dees (Argonne National Laboratory) - es252.

Enabling High-Energy, High-Voltage Li-Ion Cells for Transportation Applications:

Project Overview: Dennis Dees (Argonne National Laboratory) - es253.

Enabling High-Energy, High-Voltage Li-Ion Cells for Transportation Applications:

Materials Characterization: Dennis Dees (Argonne National Laboratory) - es254..... 2-134

Next-Generation Anodes for Lithium-Ion Batteries: Overview: Dennis Dees

(Argonne National Laboratory) - es261.

Next-Generation Anodes for Li-Ion Batteries: Fundamental Studies of Si-C Model

Systems: Robert Kostecki (Lawrence Berkeley National Laboratory) - es262.............2-138

Electrodeposition for Low-Cost, Water-Based Electrode Manufacturing:

Stuart Hellring (PPG Industries) - es263.

Li-Ion Battery Anodes from Electrospun Nanoparticle/Conducting Polymer

Nanofibers: Peter Pintauro (Vanderbilt University) - es264.

UV Curable Binder Technology to Reduce Manufacturing Cost and Improve

Performance of LIB Electrodes: John Arnold (Miltec UV International) - es265.

Co-Extrusion (CoEx) for Cost Reduction of Advanced High-Energy-and-Power

Battery Electrode Manufacturing: Corie Cobb (Palo Alto Research Center)

- es266.

Commercially Scalable Process to Fabricate Porous Silicon: Peter Aurora

(Navitas Systems) - es267..

Low-Cost Manufacturing of Advanced Silicon-Based Anode Materials:

Aaron Feaver (Group14) - es268.

An Integrated Flame Spray Process for Low-Cost Production of

Battery Materials: Chad Xing (University of Missouri) - es269.

New Advanced Stable Electrolytes for High-Voltage Electrochemical

Energy Storage: Peng Du (Silatronix) - es271.

Pre-Lithiation of Battery Electrodes: Yi Cui (Stanford University) - es272. 
New Lamination and Doping Concepts for Enhanced Li-S Battery Performance: Prashant Kumta (University of Pittsburgh) - es279.

Novel Chemistry: Lithium Selenium and Selenium Sulfur Couple:

Khalil Amine (Argonne National Laboratory) - es280

Multi-Functional Cathode Additives for Li-S Battery Technology: Hong Gan

(Brookhaven National Laboratory) - es281.

Development of High-Energy Lithium-Sulfur Batteries: Jun Liu

(Pacific Northwest National Laboratory) - es282.

Addressing Internal "Shuttle" Effect: Electrolyte Design

and Cathode Morphology Evolution in Li-S Batteries:

Perla Balbuena (Texas A\&M University) - es283.

Statically and Dynamically Stable Lithium-Sulfur Batteries:

Arumugam Manthiram (University of Texas at Austin) - es284

Mechanistic Investigation for the Rechargeable Li-Sulfur Batteries:

Deyang Qu (University of Wisconsin, Milwaukee) - es285.

Development of Novel Electrolytes and Catalysts for Li-Air Batteries:

Khalil Amine (Argonne National Laboratory) - es286.

Exploratory Studies of Novel Sodium-Ion Battery Systems:

Xiao-Qing Yang (Brookhaven National Laboratory) - es287.

Construction of High Energy Density Batteries: Christopher Lang

(Physical Sciences, Inc.) - es288.

Advanced Polyolefin Separators for Li-Ion Batteries Used in

Vehicle Applications: Weston Wood (Entek) - es289.

Hybrid Electrolytes for PHEV Applications: Surya Moganty

(NOHMs Technologies) - es290.

SAFT-USABC 12V Start-Stop Phase II: Ian O’Connor (Saft) - es291.

Development of Advanced High-Performance Electrolytes for Lithium-Ion

Used in Vehicle Applications: Kristin Meyers (soulbrain) - es292.

A Closed Loop Process for the End-of-Life Electric Vehicle Li-Ion Batteries:

Yan Wang (Worcester Polytechnic Institute) - es293.

Computer Aided Battery Engineering Consortium: Ahmad Pesaran

(National Renewable Energy Laboratory) - es294.

Consortium for Advanced Battery Simulation: John Turner

(Oak Ridge National Laboratory) - es295.

Development and Validation of a Simulation Tool to Predict the Combined Structural, Electrical, Electrochemical, and Thermal Responses of Automotive Batteries: James Marcicki (Ford Motor Company) - es296

3. Electric Drive Technologies

Overview of the VTO Electric Drive Technologies Program: Susan Rogers

(U.S. Department of Energy) - edt000. 
Benchmarking EV and HEV Technologies: Tim Burress

(Oak Ridge National Laboratory) - edt006.

Development of Radically Enhanced alnico Magnets (DREaM) for

Traction Drive Motors: Iver Anderson (Ames Laboratory) - edt015.

North American Supply Chain for Traction Motors and PE:

Christopher Whaling (Synthesis Partners) - edt032.

Advanced Packaging Technologies and Designs: Zhenxian Liang

(Oak Ridge National Laboratory) - edt049.

Electric Drive Inverter R\&D: Madhu Chinthavali

(Oak Ridge National Laboratory) - edt053.

Innovative Technologies for Converters and Chargers: Gui-Jia Su

(Oak Ridge National Laboratory) - edt054

Advanced Low-Cost SiC and GaN Wide Bandgap Inverters for Under-the-Hood Electric Vehicle Traction Drives: Kraig Olejniczak (Wolfspeed) - edt058.

High Temperature DC-Bus Capacitor Cost Reduction

and Performance Improvements: Angelo Yializis

(Sigma Technologies International) - edt059.

High-Performance DC Bus Film Capacitor: Dan Tan (General Electric) - edt060.......3-38

Advanced Electric Motor Research: Tim Burress

(Oak Ridge National Laboratory) - edt062.

Performance and Reliability of Bonded Interfaces for High-Temperature

Packaging: Doug DeVoto (National Renewable Energy Laboratory) - edt063...........3-45

Electric Motor Thermal Management R\&D: Kevin Bennion

(National Renewable Energy Laboratory) - edt064

High-Efficiency High-Density GaN-Based 6.6 kW Bidirectional On-Board

Charger for PEVs: Charles Zhu (Delta Products Corporation) - edt067.

Gate Driver Optimization for WBG Applications: Nance Ericson

(Oak Ridge National Laboratory) - edt068.

Power Electronics Thermal Management R\&D: Gilbert Moreno

(National Renewable Energy Laboratory) - edt069.

Thermal Performance Benchmarking: Xuhui Feng

(National Renewable Energy Laboratory) - edt070.

Electric Motor Performance Improvement Techniques: Lixin Tang

(Oak Ridge National Laboratory) - edt071.

88 Kilowatt Automotive Inverter with New 900 Volt Silicon Carbide MOSFET Technology: Jeffrey Casady (Cree) - edt073.

4. Advanced Combustion Engines.

Overview of the VTO Advanced Combustion Engine R\&D Program:

Gurpreet Singh (U.S. Department of Energy) - ace000. 
Heavy-Duty Low-Temperature and Diesel Combustion and Heavy-Duty Combustion Modeling: Mark Musculus

(Sandia National Laboratories) - ace001.

Light-Duty Diesel Combustion: Stephen Busch

(Sandia National Laboratories) - ace002.

Low-Temperature Gasoline Combustion (LTGC) Engine Research:

John Dec (Sandia National Laboratories) - ace004

Spray Combustion Cross-Cut Engine Research: Lyle Pickett

(Sandia National Laboratories) - ace005.

Gasoline Combustion Fundamentals: Isaac Ekoto

(Sandia National Laboratories) - ace006.

Large Eddy Simulation (LES) Applied to Advanced Engine Combustion

Research: Joe Oefelein (Sandia National Laboratories) - ace007.

Fuel Injection and Spray Research Using X-Ray Diagnostics:

Christopher Powell (Argonne National Laboratory) - ace010

Advances in High-Efficiency Gasoline Compression Ignition:

Steve Ciatti (Argonne National Laboratory) - ace011.

Model Development and Analysis of Clean and Efficient Engine Combustion:

Russell Whitesides (Lawrence Livermore National Laboratory) - ace012.

Chemical Kinetic Models for Advanced Engine Combustion: Bill Pitz

(Lawrence Livermore National Laboratory) - ace013.

2016 KIVA-hpFE Development: A Robust and Accurate Engine Modeling

Software: David Carrington (Los Alamos National Laboratory) - ace014.

Stretch Efficiency for Combustion Engines: Exploiting New Combustion

Regimes: Stuart Daw (Oak Ridge National Laboratory) - ace015.

High-Efficiency Clean Combustion in Multi-Cylinder Light-Duty Engines:

Scott Curran (Oak Ridge National Laboratory) - ace016.

Accelerating Predictive Simulation of IC Engines with High Performance Computing: Kevin Edwards (Oak Ridge National Laboratory) - ace017..

Joint Development and Coordination of Emissions Control Data

and Models (CLEERS Analysis and Coordination): Stuart Daw

(Oak Ridge National Laboratory) - ace022.

CLEERS: Aftertreatment Modeling and Analysis: Yong Wang

(Pacific Northwest National Laboratory) - ace023.

Ash-Durable Catalyzed Filters for Gasoline Direct Injection (GDI)

Engines: Hee Je Seong (Argonne National Laboratory) - ace024.

Enhanced High- and Low-Temperature Performance of $\mathrm{NO}_{\mathrm{x}}$ Reduction Materials: Feng Gao (Pacific Northwest National Laboratory) - ace026.

Next Generation SCR-Dosing System Investigation: Abhijeet Karkamkar

(Pacific Northwest National Laboratory) - ace027.. 
Cummins-ORNL FEERC Emissions CRADA: $\mathrm{NO}_{\mathrm{x}}$ Control and Measurement Technology for Heavy-Duty Diesel Engines, Self-Diagnosing Smart

Catalyst Systems: Bill Partridge (Oak Ridge National Laboratory) - ace032

Emissions Control for Lean Gasoline Engines: Jim Parks

(Oak Ridge National Laboratory) - ace033.

Neutron Imaging of Advanced Transportation Technologies:

Todd Toops (Oak Ridge National Laboratory) - ace052.

RCM Studies to Enable Gasoline-Relevant Low-Temperature Combustion:

Scott Goldsborough (Argonne National Laboratory) - ace054.

Fuel-Neutral Studies of Particulate Matter Transport Emissions:

Mark Stewart (Pacific Northwest National Laboratory) - ace056

SuperTruck - Development and Demonstration of a Fuel-Efficient

Class 8 Tractor and Trailer, Engine Systems: Russ Zukouski

(Navistar International Corporation) - ace059.

Volvo SuperTruck - Powertrain Technologies for Efficiency

Improvement: Pascal Amar (Volvo Trucks) - ace060.

Advancements in Fuel Spray and Combustion Modeling with High-Performance

Computing Resources: Sibendu Som (Argonne National Laboratory) - ace075.

Improved Solvers for Advanced Engine Combustion Simulation:

Matthew McNenly (Lawrence Livermore National Laboratory) - ace076.

Cummins-ORNL \FEERC Combustion CRADA: Characterization

and Reduction of Combustion Variations: Bill Partridge

(Oak Ridge National Laboratory) - ace077.

Thermally Stable Ultra-Low Temperature Oxidation Catalysts:

Janos Szanyi (Pacific Northwest National Laboratory) - ace078.

High-Efficiency GDI Engine Research, with Emphasis on Ignition Systems:

Thomas Wallner (Argonne National Laboratory) - ace084.

Low-Temperature Emission Control to Enable Fuel-Efficient Engine

Commercialization: Todd Toops (Oak Ridge National Laboratory) - ace085.

High-Dilution Stoichiometric Gasoline Direct-Injection (SGDI) Combustion

Control Development: Brian Kaul (Oak Ridge National Laboratory) - ace090.

High-Efficiency VCR Engine with Variable Valve Actuation and

New Supercharging Technology: Charles Mendler (Envera LLC) - ace092.

Lean Miller Cycle System Development for Light-Duty Vehicles:

David Sczomak (General Motors) - ace093..

Ultra-Efficient Light-Duty Powertrain with Gasoline Low-Temperature

Combustion: Keith Confer (Delphi Advanced Powertrain) - ace094.

Metal Oxide Nano-Array Catalysts for Low-Temperature Diesel Oxidation:

Pu-Xian Gao (University of Connecticut) - ace095. 
Micro-Jet Enhanced Ignition with a Variable Orifice Fuel Injector for High-

Efficiency Lean-burn Combustion: Chia-Fon Lee (University of Illinois) - ace096.....4-154

Affordable Rankine Cycle (ARC) Waste Heat Recovery for Heavy-Duty

Trucks: Swami Subramanian (Eaton Corporation) - ace097.

Cummins 55\% BTE Project: Lyle Kocher (Cummins) - ace098.

Improved Fuel Efficiency through Adaptive Radio Frequency

Controls and Diagnostics for Advanced Catalyst Systems:

Alexander Sappok (Filter Sensing Technologies, Inc.) - ace099.

5. Fuel and Lubricant Technologies

Overview of the VTO Fuel and Lubricant Technologies R\&D: Kevin Stork

(U.S. Department of Energy) - ft000.

Engine Friction Reduction Technologies: George Fenske

(Argonne National Laboratory) - ft012.

Ionic Liquids as Engine Lubricant Additives, Impact on Emission

Control Catalysts, and Compatibility with Coatings: Jun Qu

(Oak Ridge National Laboratory) - ft014

Integrated Friction Reduction Technology to Improve Fuel Economy without

Sacrificing Durability: Stephen Hsu (George Washington University) - ft033.

Hybrid Ionic-Nano-Additives for Engine Lubrication to Improve

Fuel Efficiency: Bin Zhao (University of Tennessee) - ft034.

Hyperbranched Alkanes for Lubes: Lelia Cosimbescu

(Pacific Northwest National Laboratory) - ft035.

Lubricant Effects on Combustion, Emissions, and Efficiency:

Robert Wagner (Oak Ridge National Laboratory) - ft036..

Co-Optimization of Fuels and Engines Overview: John Farrell

(National Renewable Energy Laboratory) - ft037.

Co-Optimization of Fuels and Engines (Co-Optima) -- Fuel Properties and

Thrust I Engine Research: Jim Szybist (Oak Ridge National Laboratory) - ft038.......5-35

Co-Optimization of Fuels and Engines (Co-Optima) -- Thrust II Engine

Research, Sprays Research, and Emissions Control Research: Paul Miles

(Sandia National Laboratories) - ft039.

Co-Optimization of Fuels and Engines (Co-Optima) -- Simulation Toolkit

Team: Matt McNenly (Lawrence Livermore National Laboratory) - ft040..

Utilizing Alternative Fuel Ignition Properties to Improve

Spark-Ignited and Compression-Ignited Engine Efficiency:

Margaret Wooldridge (University of Michigan) - ft042

E85/Diesel Premixed Compression Ignition: Lyle Kocher (Cummins) - ft043. 5-56

GEFORCE: Gasoline Engine and Fuels Offering Reduced Fuel Consumption and Emissions: Scott Sluder (Oak Ridge National Laboratory) - ft044... $5-60$

GDI Metrics: Scott Goldsborough (Argonne National Laboratory) - ft045. $5-64$ 
Efficiency-Optimized Dual Fuel Engine with In-Cylinder Gasoline/CNG

Blending: Thomas Wallner (Argonne National Laboratory) - ft046.

6. Lightweight Materials.

Subprogram Overview Comments: Felix Wu

(U.S. Department of Energy) - Im000.

Scale-Up of Magnesium Production by Fully Stabilized Zirconia Electrolysis:

Adam Powell (INFINIUM, Inc.) - Im035.

Integrated Computational Materials Engineering Approach to

Development of Lightweight 3GAHSS Vehicle Assembly: Lou Hector

(United States Automotive Materials Partnership LLC) - Im080.

Validation of Material Models for Crash Simulation of Automotive Carbon

Fiber Composite Structures (VMM): Libby Berger (General Motors) - Im084

Collision Welding of Dissimilar Materials by Vaporizing Foil Actuator:

A Breakthrough Technology for Dissimilar Materials Joining:

Glenn Daehn (Ohio State University) - Im086.

Active, Tailorable Adhesives for Dissimilar Material Bonding, Repair

and Assembly: Mahmood Haq (Michigan State University) - Im087.

High-Strength Electroformed Nanostructured Aluminum for Lightweight

Automotive Applications: Robert Hilty (Xtalic Corporation) - Im089.

Vehicle Lightweighting: Mass Reduction Spectrum Analysis and

Process Cost Modeling: Tony Mascarin (IBIS Associates) - Im090.

Laser-Assisted Joining Process of Aluminum and Carbon Fiber Components:

Adrian Sabau (Oak Ridge National Laboratory) - Im097..

Brazing Dissimilar Metals with a Novel Composite Foil: Tim Weihs

(Johns Hopkins University) - Im098.

High-Strength, Dissimilar Alloy Aluminum Tailor-Welded Blanks:

Yuri Hovanski (Pacific Northwest National Laboratory) - Im099.

Upset Protrusion Joining Techniques For Joining Dissimilar Metals:

Steve Logan (Fiat Chrysler Automobiles US LLC) - Im100..

Integrated Computational Materials Engineering (ICME)

Development of Carbon Fiber Composites for Lightweight

Vehicles: Xuming Su (Ford Motor Company) - Im101.

Predictive Models for Integrated Manufacturing and Structural

Performance of Carbon Fiber Composites for Automotive

Applications: Venkat Aitharaju (General Motors) - Im102.

E. Coli Derived Spider Silk MaSp1 and MaSp2 Proteins as Carbon

Fiber Precursors: Randy Lewis (Utah State University) - Im103.

Solid-State Body-in-White Spot Joining of Al to AHSS at Prototype

Scale: Zhili Feng (Oak Ridge National Laboratory) - Im104.

Friction Stir Scribe Joining of Al to Steel: Yuri Hovanski

(Pacific Northwest National Laboratory) - Im105. 
Enhanced Sheared Edge Stretchability of AHSS/UHSS: Xin Sun

(Pacific Northwest National Laboratory) - Im106.

Optimizing Heat Treatment Parameters for $3^{\text {rd }}$ Generation AHSS Using

an Integrated Experimental-Computational Framework: Xin Sun

(Pacific Northwest National Laboratory) - Im107.

Development of Low-Cost, High-Strength Automotive Aluminum Sheet:

Russell Long (Alcoa) - Im108.

7. Propulsion Materials................................................................................. 7-1

Overview of VTO Material Technologies: Jerry Gibbs

(U.S. Department of Energy) - pmo00.

Novel Manufacturing Technologies for High-Power Induction and Permanent Magnet Electric Motors: Glenn Grant

(Pacific Northwest National Laboratory) - pm004.

Materials Issues Associated with EGR Systems: Michael Lance

(Oak Ridge National Laboratory) - pm009.

High-Temperature Materials for High-Efficiency Engines:

Govindarajan Muralidharan (Oak Ridge National Laboratory) - pm053

Enabling Materials for High-Temperature Power Electronics:

Andrew Wereszczak (Oak Ridge National Laboratory) - pm054

Biofuel Impacts on Aftertreatment Devices: Michael Lance

(Oak Ridge National Laboratory) - pm055.

Applied Computational Methods for New Propulsion Materials:

Charles Finney (Oak Ridge National Laboratory) - pm057.

Development of Advanced High-Strength Cast Alloys for Heavy-Duty

Engines: Rich Huff (Caterpillar) - pm059.

ICME Guided Development of Advanced Cast Aluminum Alloys for

Automotive Engine Applications: Mei Li (Ford Motor Company) - pm060

Computational Design and Development of a New, Lightweight

Cast Alloy for Advanced Cylinder Heads in High-Efficiency,

Light-Duty Engines: Mike Walker (General Motors) - pm061.

High-Performance Cast Aluminum Alloys for Next Generation Passenger

Vehicle Engines: Amit Shyam (Oak Ridge National Laboratory) - pm062.

Development of High-Performance Cast Crankshafts: Rich Huff

(Caterpillar) - pm065.

Innovative SCR Materials and Systems for Low-Temperature Aftertreatment:

Yong Wang (Pacific Northwest National Laboratory) - pm066.

Next Generation Three-Way Catalysts for Future, Highly Efficient

Gasoline Engines: Christine Lambert (Ford Motor Company) - pm067.

Sustained Low-Temperature $\mathrm{NO}_{\mathrm{x}}$ Reduction (SLTNR):

Yuhui Zha (Cummins) - pm068. 
8. Technology Integration.

Subprogram Overview Comments: Linda Bluestein

(U.S. Department of Energy) - tiooo..

Plug-In Hybrid Electric Vehicle Demonstration Program and

Social Media Campaign: Lizabeth Ardisana (ASG Renaissance) - ti064.

Drive Electric Orlando: April Combs (Florida Department of Agriculture and Consumer Services/Office of Energy) - ti065.

Alternative Fuel Vehicle Curriculum Development and Outreach Initiative:

Judy Moore (West Virginia University Research Corporation) - ti066.

Nationwide AFV Emergency Responder, Recovery,

Reconstruction and Investigation Training: Andrew Klock

(National Fire Protection Association) - ti067..

Safe Alternative Fuels Deployment in Mid-America (The SAF-D Project):

Kelly Gilbert (Metropolitan Energy Center, Inc.) - ti068.

Initiative for Resiliency in Energy through Vehicles (IREV): Cassie Powers

(National Association of State Energy Officials) - ti069.

EcoCAR 3: Kristen Wahl (Argonne National Laboratory) - ti070.................................8-38

9. Vehicle Analysis......................................................................................... 9-1

Subprogram Overview Comments: Jake Ward

(U.S. Department of Energy) - van999.

Transportation Data Program: A Multi-Lab Coordinated Project:

Stacy Davis (Oak Ridge National Laboratory) - van016.

ANL Vehicle Technologies Analysis Modeling Program: Michael Wang

(Argonne National Laboratory) - van017..

VTO Program Benefits Analysis: Tom Stephens

(Argonne National Laboratory) - van018.

Assessing Energy and Cost Impact of Advanced Technologies through Model-Based Design: Aymeric Rousseau

(Argonne National Laboratory) - van023.

10. Acronyms................................................................................... 10-1

Appendix A: 2016 Annual Merit Review Attendees..............................A-1 


\section{Introduction}

The 2016 U.S. Department of Energy (DOE) Hydrogen and Fuel Cells Program and Vehicle Technologies Office (VTO) Annual Merit Review and Peer Evaluation Meeting (AMR) was held June 6-9, 2016, in Washington, DC. The review encompassed work done by the Hydrogen and Fuel Cells Program and VTO: 226 individual activities were reviewed for VTO, by 171 reviewers. A total of 1,044 individual review responses were received for the VTO technical reviews.

The objective of the meeting was to review the accomplishments and plans for VTO over the previous 12 months, and provide an opportunity for industry, government, and academia to give inputs to DOE with a structured and formal methodology. The meeting also provided attendees with a forum for interaction and technology information transfer.

The peer review process followed the guidelines of the Peer Review Guide developed by the Office of Energy Efficiency and Renewable Energy (EERE). Each activity is reviewed every three years, at a minimum. However, VTO strives to have every activity reviewed every other year. The reviewers for the technical sessions were drawn from a wide variety of backgrounds, including current and former vehicle industry members, academia, government, and other expertise areas. Each reviewer was screened for conflicts of interest as prescribed by the Peer Review Guide. A complete list of the meeting participants is presented as Appendix A.

\section{Evaluation Criteria - Research and Development Subprogram Projects}

In the technical research and development $(R \& D)$ subprogram sessions, reviewers were asked to respond to a series of specific questions regarding the breadth, depth, and appropriateness of the VTO R\&D activities. The technical questions are listed below, along with appropriate scoring metrics. These questions were used for all formal VTO R\&D project reviews.

Question 1. Approach to performing the work-the degree to which technical barriers are addressed, the project is well-designed, feasible, and integrated with other efforts. (Scoring weight for overall average $=20 \%$ )

- $\quad 4.0=$ Outstanding (sharply focused on critical barriers; difficult to improve approach significantly).

- $\quad 3.5$ = Excellent (effective; contributes to overcoming most barriers).

- $\quad 3.0$ = Good (generally effective but could be improved; contributes to overcoming some barriers).

- $2.5=$ Satisfactory (has some weaknesses; contributes to overcoming some barriers).

- $\quad 2.0$ = Fair (has significant weaknesses; may have some impact on overcoming barriers).

- $\quad 1.5$ = Poor (minimally responsive to project objectives; unlikely to contribute to overcoming the barriers).

- $\quad 1.0=$ Unsatisfactory (not responsive to project objectives; unlikely to contribute to overcoming the barriers).

Question 2. Technical accomplishments and progress toward overall project and DOE goals-the degree to which progress has been made, measured against performance indicators and demonstrated progress toward DOE goals. (Scoring weight for overall average $=40 \%$ )

- $\quad 4.0=$ Outstanding (sharply focused on critical barriers; difficult to improve significantly).

- $\quad 3.5=$ Excellent (effective; contributes to overcoming most barriers).

- $\quad 3.0$ = Good (generally effective but could be improved; contributes to overcoming some barriers). 
- $2.5=$ Satisfactory (has some weaknesses; contributes to overcoming some barriers).

- 2.0 = Fair (has significant weaknesses; may have some impact on overcoming barriers).

- 1.5 = Poor (minimally responsive to project objectives; unlikely to contribute to overcoming the barriers).

- $1.0=$ Unsatisfactory (not responsive to project objectives; unlikely to contribute to overcoming the barriers).

Question 3. Collaboration and coordination with other institutions. (Scoring weight for overall average $=10 \%$ )

- $\quad 4.0=$ Outstanding (close, appropriate collaboration with other institutions; partners are full participants and well-coordinated).

- $\quad 3.5=$ Excellent (good collaboration; partners participate and are well-coordinated).

- $\quad 3.0=$ Good (collaboration exists; partners are fairly well-coordinated).

- 2.5 = Satisfactory (some collaboration exists; coordination between partners could be significantly improved).

- 2.0 = Fair (a little collaboration exists; coordination between partners could be significantly improved).

- $1.5=$ Poor (most work is done at the sponsoring organization with little outside collaboration; little or no apparent coordination with partners).

- $1.0=$ Unsatisfactory (no apparent coordination with partners).

Question 4. Proposed future research-the degree to which the project has effectively planned its future work in a logical manner by incorporating appropriate decision points, considering barriers to the realization of the proposed technology and, when sensible, mitigating risk by providing alternate development pathways. Note:

if the project has ended, please leave blank. (Scoring weight for overall average $=10 \%$ )

- $\quad 4.0=$ Outstanding (sharply focused on critical barriers; difficult to improve significantly).

- $\quad 3.5=$ Excellent (effective; contributes to overcoming most barriers).

- $\quad 3.0=$ Good (generally effective but could be improved; contributes to overcoming some barriers).

- $\quad 2.5$ = Satisfactory (has some weaknesses; contributes to overcoming some barriers).

- $\quad 2.0$ = Fair (has significant weaknesses; may have some impact on overcoming barriers).

- $\quad 1.5$ = Poor (minimally responsive to project objectives; unlikely to contribute to overcoming the barriers).

- $1.0=$ Unsatisfactory (not responsive to project objectives; unlikely to contribute to overcoming the barriers).

Question 5. Relevance-does this project support the overall DOE objectives of petroleum displacement? (Scoring weight, not included with overall average $=20 \%$ )

- Yes.

- No. 
Question 6. Resources: How sufficient are the resources for the project to achieve the stated milestones in a timely fashion?

- Excessive.

- Sufficient.

- Insufficient.

\section{Evaluation Criteria - Technology Integration Projects}

Reviewers for the Technology Integration (TI) technical session answered questions tailored to TI's 2016 AMR focus on petroleum reduction technologies and practices, alternative fuels, infrastructure, and related efforts. These technical questions are listed below, along with appropriate scoring metrics.

\section{Question 1. Project objectives-the degree to which the project objectives support the DOE/VTO objectives of reducing reliance on petroleum based fuels and reducing emissions. This includes the impact the project has on addressing the technical barriers from the Vehicle Technologies Office. (Scoring weight for overall average $=\mathbf{2 0} \%$ )}

- $\quad 4.0=$ Outstanding (project Objectives are sharply focused on supporting DOE/VTO goals of reducing reliance on petroleum based fuels and reducing emissions; project has a direct and substantial impact upon addressing technical barriers; difficult to improve project objectives significantly).

- $\quad 3.5=$ Excellent (project objectives are effective; project addresses a significant number of technical barriers; effectively contributes to reducing reliance on petroleum based fuels and reducing emissions).

- $\quad 3.0=$ Good (project objectives are generally effective, but could be improved; project addresses some technical barriers; contributes to reducing reliance on petroleum based fuels and reducing emissions).

- 2.5 = Satisfactory (project objectives have some weaknesses; project addresses some technical barriers; project may have some impact contributing to reducing reliance on petroleum based fuels and reducing emissions).

- $2.0=$ Fair (project objectives have significant weaknesses; project addresses few technical barriers; project may have a small impact contributing to reducing reliance on petroleum based fuels and reducing emissions).

- $\quad 1.5=$ Poor (project objectives are minimally responsive to DOE/VTO objectives; project does not address technical barriers; project is unlikely to contribute to reducing reliance on petroleum based fuels and reducing emissions).

- $1.0=$ Unsatisfactory (project objectives are not responsive to DOE/VTO objectives; project fails to address any technical barriers; project is highly unlikely to contribute to reducing reliance on petroleum based fuels or reducing emissions).

Question 2. Project approach to supporting deployment of petroleum reduction technologies and practices, alternative fuel vehicles, infrastructure, emissions reductions and related efforts-the degree to which the project is well-designed, feasible, and integrated with other efforts. (Scoring weight for overall average $=20 \%$ )

- $4.0=$ Outstanding (project approach is sharply focused on achieving project objectives; difficult to improve project approach significantly). 
- $\quad 3.5=$ Excellent (effective; project approach contributes to achieving the majority of project objectives).

- $\quad 3.0=$ Good (generally effective but project approach could be improved; contributes to achieving some of the project objectives).

- $2.5=$ Satisfactory (has some weaknesses; project approach contributes to achieving some project objectives).

- $\quad 2.0$ = Fair (has significant weaknesses; project approach may have some impact on achieving project objectives).

- $\quad 1.5=$ Poor (minimally responsive to project objectives; project approach is unlikely to contribute to achieving project objectives).

- $\quad 1.0$ = Unsatisfactory (not responsive to project objectives; project approach is highly unlikely to contribute to achieving project objectives).

Question 3. Project accomplishments and progress toward overall project and DOE goals-the degree to which progress/significant accomplishments have been achieved, measured against performance indicators and demonstrated progress toward project and DOE goals. (Scoring weight for Project Accomplishments $=40 \%$ )

- $\quad 4.0=$ Outstanding (project demonstrates significant accomplishments; strong progress toward achieving both project and DOE objectives; difficult to improve progress significantly).

- $\quad 3.5=$ Excellent (project demonstrates many accomplishments; very effective progress toward achieving overall project objectives and DOE goals).

- $\quad 3.0=$ Good (project accomplishments are generally effective; progress is on schedule to contribute to some project objectives and DOE goals).

- 2.5 = Satisfactory (project has some accomplishments, but also displays some weaknesses; progress could be improved; contributes to some project objectives and DOE goals).

- $2.0=$ Fair (project has few accomplishments and demonstrates significant weaknesses; rate of progress is slow; minimal contribution to project objectives or DOE goals).

- 1.5 = Poor (minimal demonstration of accomplishments; progress is significantly behind schedule; unlikely to contribute to project objectives or DOE goals).

- $\quad 1.0$ = Unsatisfactory (project demonstrates no accomplishments; limited or no demonstrated progress; not responsive to project objectives).

Question 4. Collaboration and coordination among project team-the degree to which the appropriate team members and partners are involved in the project work and the effectiveness of the collaboration between and among partners. (Scoring weight for Collaboration and Coordination $=10 \%$ )

- $\quad 4.0=$ Outstanding (sharply focused on collaboration among project team members; team is well-suited to effectively carry out the work of the project and have strong working relationships; no notable weaknesses).

- $3.5=$ Excellent (effective; team members meaningfully contribute to carrying out the work of the project, are well-suited to perform the work and have excellent working relationships).

- $\quad 3.0$ = Good (generally effective but could be improved; collaboration exists; team members are fairly wellsuited to project work and have good working relationships). 
- 2.5 = Satisfactory (has some weaknesses; collaboration among team members is satisfactory for carrying out the work of the project; project partnerships, team members and working relationships could be improved).

- $2.0=$ Fair (has significant weaknesses; little collaboration exists and team could be improved).

- $\quad 1.5=$ Poor (minimally responsive; little collaboration exists and team lacks effective working relationships).

- $\quad 1.0=$ Unsatisfactory (little or no apparent collaboration between team members; project team is lacking critical expertise to effectively carry out the work of the project).

Question 5. Market impact and sustainability-the degree to which the project has already contributed, as well as the potential to contribute in the future, to a sustainable alternative fuel vehicle market, alternative market expansion, and reduced petroleum dependence/greenhouse gas emissions in the transportation sector. This would include the potential to reduce barriers to large scale alternative fuel vehicle market penetration, making information about alternative fuels and petroleum reduction opportunities widely available to target audiences, and ability for the project to be replicated in other geographic areas or with other technologies. (Scoring Weight for Market Impact=10\%).

- $\quad 4.0=$ Outstanding (sharply focused on critical barriers and effective information products; clearly contributes to alternative fuel vehicle market expansion and/or petroleum/greenhouse gas reduction; difficult to improve significantly).

- $\quad 3.5=$ Excellent (effective; contributes to overcoming most barriers and informing appropriate audiences; contributes to alternative fuel vehicle market expansion and/or petroleum/greenhouse gas reduction).

- $\quad 3.0=$ Good (generally effective in overcoming barriers and providing information; has the potential to contribute to alternative fuel vehicle market expansion and/or petroleum/greenhouse gas reduction).

- 2.5 = Satisfactory (has some weaknesses; may contribute to market improvements and/or petroleum/ greenhouse gas reduction but needs better focus on overcoming some barriers and targeting appropriate audiences).

- $\quad 2.0$ = Fair (has significant weaknesses; may have some impact on overcoming barriers and reducing petroleum consumption/greenhouse gas emissions).

- 1.5 = Poor (minimally responsive; unlikely to advance an alternative fuel vehicle market or contribute to petroleum. reduction/greenhouse gas efforts).

- $1.0=$ Unsatisfactory (not responsive to eliminating barriers or providing information that will advance an alternative fuel vehicle market or lead to petroleum/greenhouse gas reductions).

Question 6. Use of resources-are DOE funds being used wisely? Should DOE fund similar efforts in the future? If not, what would be a better use of DOE resources to achieve alternative fuel vehicle and infrastructure expansion to support the broader goal of petroleum displacement and greenhouse gas reductions?

- Yes.

- Maybe.

- No. 


\section{Project Scoring}

\section{R\&D Subprogram Projects}

For R\&D subprogram sessions, reviewers were asked to provide numeric scores (on a scale of 1.0-4.0 in one-half point increments, as indicated above) for Question 1 through Question 4 of each formally reviewed activity. For each reviewed project, the individual reviewer scores for Question 1 through Question 4 were averaged to provide information on the project's question-by-question scoring. Scores for each of these four criteria were weighted using the formula below to create a Weighted Average for each project. This allows a project's question-byquestion and final overall scores to be meaningfully compared against another project:

\section{Weighted Average $=[$ Question 1 Score x 0.20] + [Question 2 Score x 0.40] + \\ [Question 3 Score x 0.10] + [Question 4 Score x 0.10]}

Each reviewed activity has a corresponding bar chart representing that project's average scores for each of the four designated criteria. As demonstrated in Figure 1, a bullet and error line are included within the green bars representing the corresponding average and standard deviation of criteria scores for all of the reviewed projects in the same subprogram.

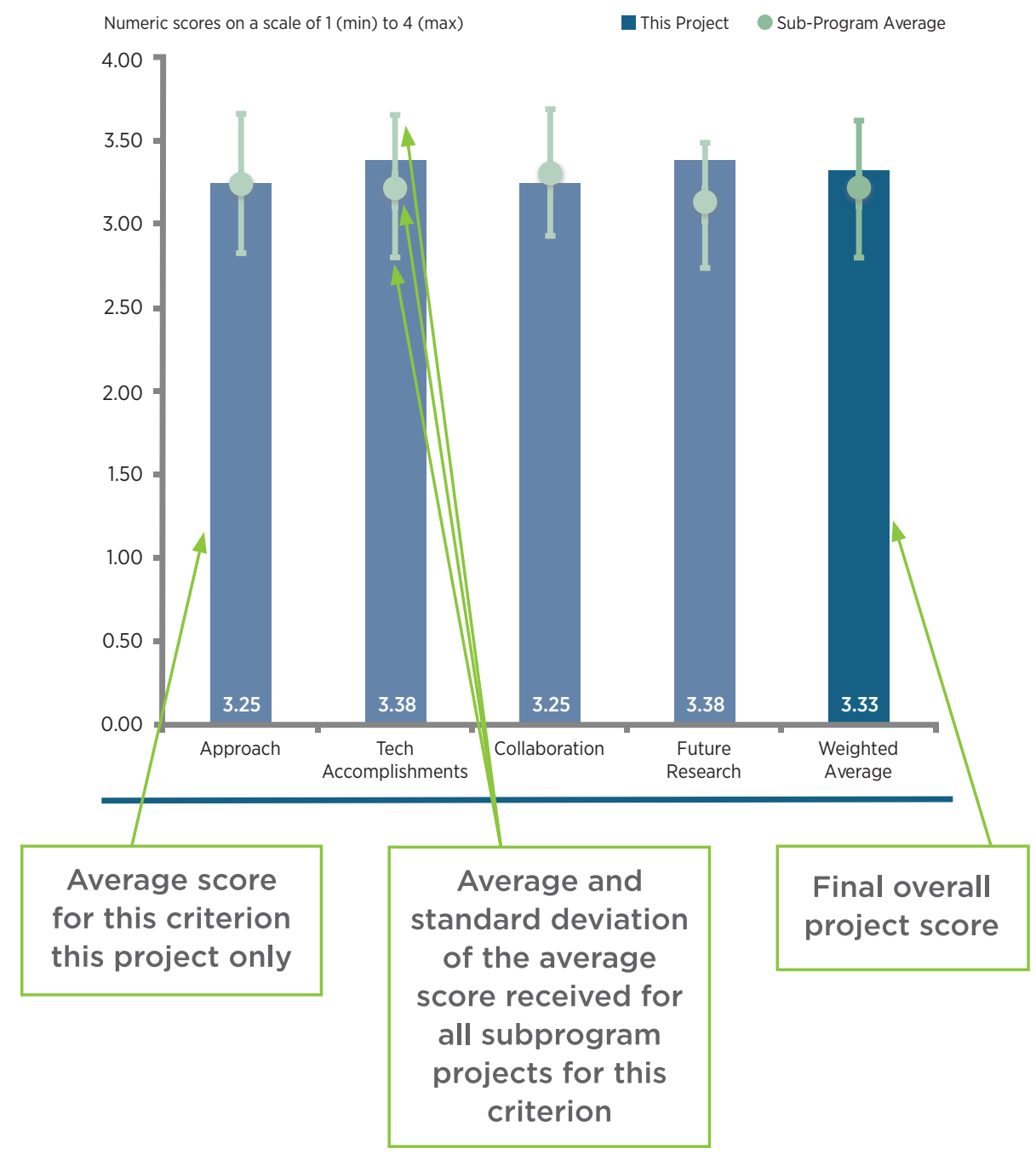

Figure 1. Sample Question 1 through Question 4 score averages, standard deviations, and overall Weighted Average for an R\&D project. 
Reviewers were also asked to evaluate a given project's relevance and funding through Question 5 and Question 6, which were each scored on a different scale than Question 1 through Question 4. For the R\&D subprogram sessions, while Question 1 through Question 4 were rated on a 1.0 to 4.0 scale in one-half point increments, Question 5 was rated on a yes or no scale, and Question 6 was rated on an excessive, sufficient, or insufficient scale. Consequently, Question 5 and Question 6 results were excluded from the Weighted Average calculation because the scoring scales are incompatible. As demonstrated in Figure 2, each reviewed activity has pie charts representing that project's population distributions for each reviewer rating associated with Question 5 and Question 6.
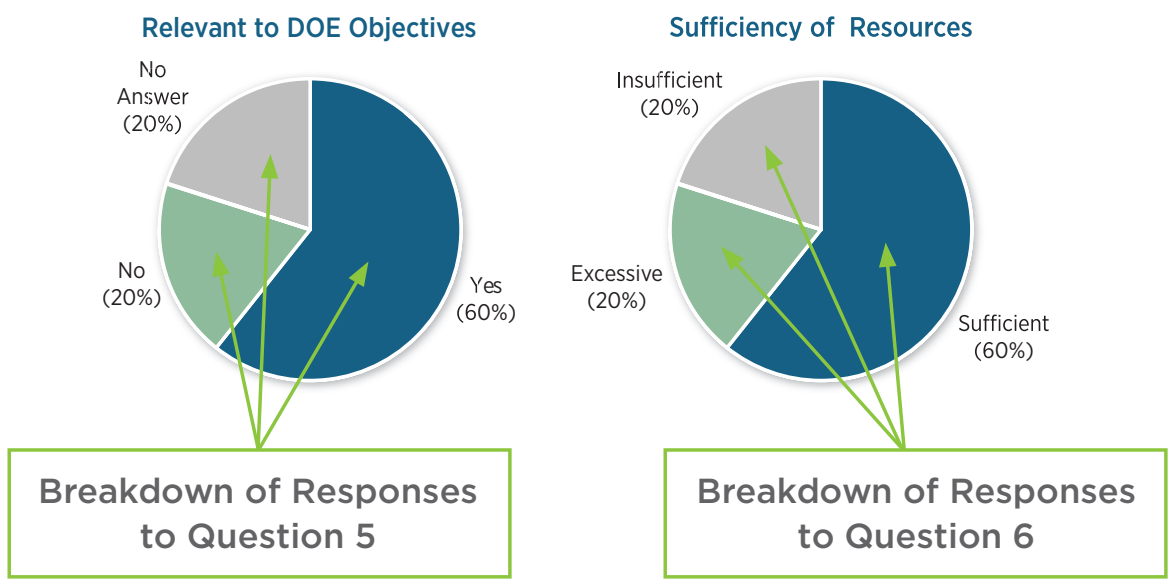

Figure 2. Sample Question 5 and Question 6 population distributions for R\&D subprogram project.

\section{TI Subprogram Projects}

For the TI subprogram session, reviewers were asked to provide numeric scores (on a scale of 1.0-4.0 in one-half point increments, as indicated above) for Question 1 through Question 5 of each formally reviewed activity. For each reviewed project, the individual reviewer scores for Question 1 through Question 5 were averaged to provide information on the project's question-by-question scoring. Scores for each of these five criteria were weighted using the formula below to create a Weighted Average for each project. This allows a project's question-byquestion and final overall scores to be meaningfully compared against another project:

$$
\begin{aligned}
& \text { Weighted Average }=[\text { Question } 1 \text { Score x 0.20] + [Question } 2 \text { Score x 0.20] + } \\
& {[\text { Question } 3 \text { Score x 0.40] + [Question 4 Score x 0.10] + [Question } 5 \text { Score x 0.10] }}
\end{aligned}
$$

Each reviewed TI activity has a corresponding bar chart representing that project's average scores for each of the five designated criteria. As demonstrated in Figure 3, a bullet and error line are included within the green bars representing the corresponding average and standard deviation of criteria scores for all of the reviewed projects in the same subprogram. 


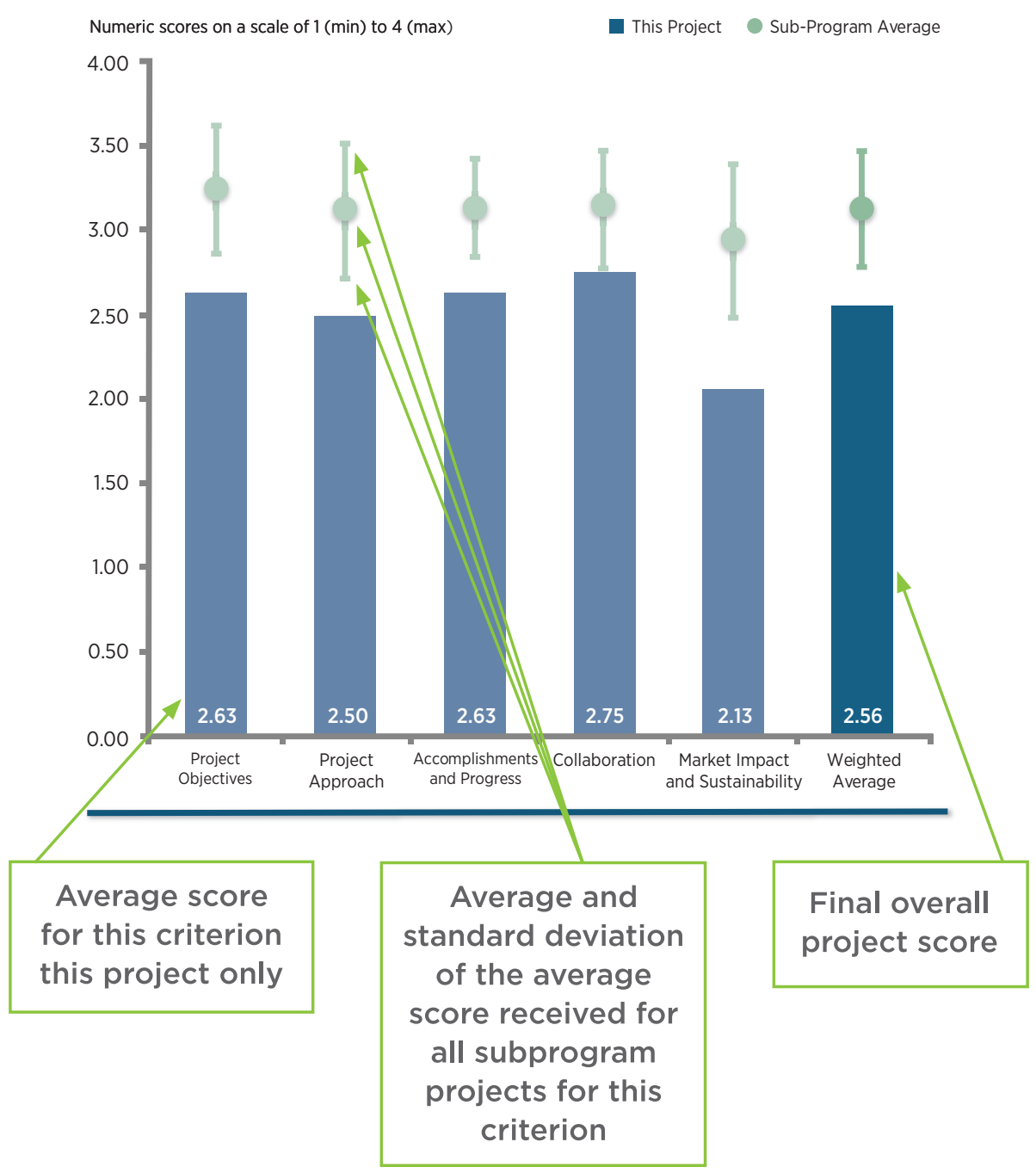

Figure 3. Sample Question 1 through Question 5 score averages, standard deviations, and overall Weighted Average for a TI subprogram project.

For TI projects, Question 1 through Question 5 were rated on a 1.0 to 4.0 scale in one-point increments, whereas Question 6 was rated on a yes, maybe, or no scale. Consequently, Question 6 results were excluded from the Weighted Average calculation because the scoring scales are incompatible. As demonstrated in Figure 4, similar to the R\&D subprograms, each reviewed activity for TI projects has a pie chart representing that project's population distributions for each reviewer rating associated with Question 6.

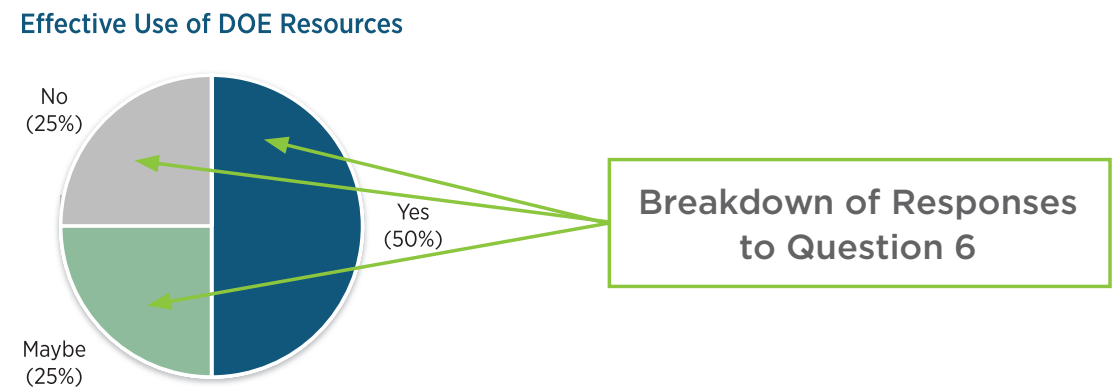

Figure 4. Sample Question 6 population distributions for TI Resources question. 


\section{Reviewer Responses}

Text responses and numeric scores to the questions were submitted electronically through a web-based software application, PeerNet, operated by Oak Ridge Associated Universities (ORAU). Database outputs from this software application were analyzed and summarized to collate the multiple-choice, text comments, and numeric scoring responses and produce the summary report.

Responses to the questions are summarized in this report, with summaries of numeric scores for each technical session, as well as text and graphical summaries of the responses for each individual technical activity. For each project, the reviewer sample size is identified.

Each reviewed activity is identified by the project title, followed by the Principal Investigator (PI), the PI's organization, and the project identification (ID) number. For each subprogram area, reviewed activities are ordered numerically by project number. Figure 5, below, provides an example project title.

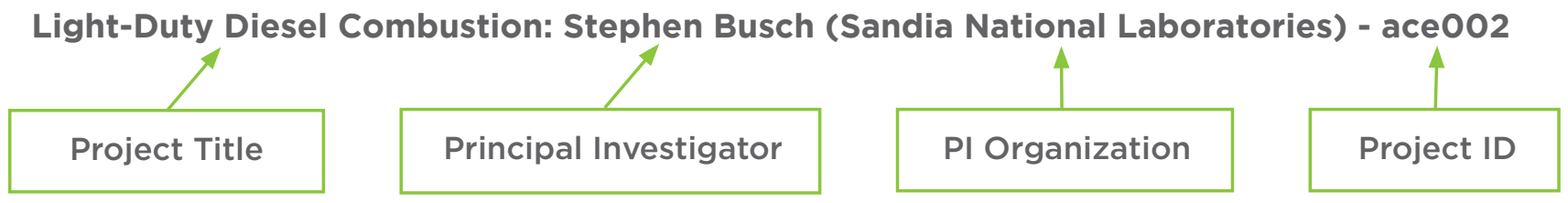

Figure 5. Sample project title with project title, PI, PI organization, and project ID.

For each project, in addition to the PI, the presenter at the AMR is identified, along with the reviewer sample size. For some projects, the presenter at the AMR was a project team member rather than the PI.

Individual reviewer comments for each question are identified under the heading Reviewer 1, Reviewer 2, etc. Note that for each question the order of reviewer comments may be different; for example, for each specific project the reviewer identified as Reviewer 1 in the first question may not be Reviewer 1 in the second question, etc. Not all reviewers provided a response to each question for a given project.

The report is organized by technical subprogram area. Each technical area section includes a summary of that subprogram, reviewer feedback received specific to the subprogram overview presentation(s) given by DOE, a subprogram activities score summary table (and page numbers), and project-specific reviewer evaluation comments with corresponding bar and pie charts. 


\title{
1. Vehicle Systems
}

\author{
Vehicle Systems (VS) is concerned with advancing light (LD)-, medium (MD)-, and heavy-duty (HD) \\ vehicle systems to support the U.S. Department of Energy's (DOE) goals of reducing petroleum \\ consumption, and reducing greenhouse gas (GHG) emissions in the U.S. transportation sector.
}

To help reach these goals, VTO conducts research and development (R\&D) programs implementing strategies to help maximize the number of electric vehicle (EV) miles driven, and increase the energy efficiency of transportation vehicles. VS's mission is to accelerate the market introduction and penetration of advanced vehicles and systems with R\&D that have a significant impact on petroleum displacement, GHG reduction, and DOE electrification goals.

The following outlines the outcome objectives that VS had identified as important to fulfilling its mission.

- Enable superior outcomes for VTO R\&D programs by evaluating technology targets.

- Accelerate the design, development, and market introduction through advanced design tools, analysis, and procedures.

- Provide stakeholders with data and analysis to support decision making.

- Accelerate codes \& standards development for EVs.

- Develop technology for auxiliary systems that improve vehicle efficiency and promote market acceptance.

Primary processes include the following.

- Develop, distribute, and use advanced modeling and simulation tools to evaluate efficiency potential of technologies.

- Conduct vehicle evaluations to guide future R\&D and validate component and system models.

- Support development and adoption of codes and standards for EVs.

- Support industry development, demonstration, and market introduction of advanced vehicle efficiency technologies.

- Investigate systems optimization strategies and enabling technologies to enhance vehicle efficiency, robustness, and effectiveness.

The following details sample project objectives.

- Provide updated Autonomie simulation tool to original equipment manufacturers (OEMs).

- Develop algorithms for proper transmission selection and shift parameter optimization.

- Benchmark the BMW i3 EV battery on a laboratory dynamometer.

- Organize and chair a Society of Automotive Engineers (SAE) task force to define methods for testing and validating the powertrain power rating for hybrid electric vehicles.

- Improve freight efficiency of HD trucks by 50\% compared with a model year 2009 highway truck.

- Integrate $6.5 \mathrm{~kW}$ wireless power transfer (WPT) technology into demonstration vehicles and validate in an independent testing laboratory.

- Increase electric range by $20 \%$ during operation of the climate control system through improved thermal management while maintaining or improving occupant thermal comfort.

The following details outcome objectives.

- Enable superior outcomes for VTO R\&D programs by evaluating technology targets.

- Accelerate design, development, and market introduction through advanced design tools, analysis, and procedures.

- Provide stakeholders with data and analysis to support decision making.

- Accelerate codes and standards development for EVs.

- Develop technology for auxiliary systems that improve vehicle efficiency and promote market acceptance. 


\section{Subprogram Feedback}

The U.S. Department of Energy (DOE) received feedback on the overall technical subprogram areas presented during the 2016 Annual Merit Review (AMR). Each subprogram technical session was introduced with a presentation that provided an overview of subprogram goals and recent progress, followed by a series of detailed topic area project presentations.

The reviewers for a given subprogram area responded to a series of specific questions regarding the breadth, depth, and appropriateness of that DOE VTO subprogram's activities. The subprogram overview questions are listed below, and it should be noted that no scoring metrics were applied. These questions were used for all VTO subprogram overviews.

Question 1: Was the program area, including overall strategy, adequately covered?

Question 2: Is there an appropriate balance between near- mid- and long-term research and development?

Question 3: Were important issues and challenges identified?

Question 4: Are plans identified for addressing issues and challenges?

Question 5: Was progress clearly benchmarked against the previous year?

Question 6: Are the projects in this technology area addressing the broad problems and barriers that the Vehicle Technologies Office (VTO) is trying to solve?

Question 7: Does the program area appear to be focused, well-managed, and effective in addressing VTO's needs?

Question 8: What are the key strengths and weaknesses of the projects in this program area? Do any of the projects stand out on either end of the spectrum?

Question 9: Do these projects represent novel and/or innovative ways to approach these barriers as appropriate?

Question 10: Has the program area engaged appropriate partners?

Question 11: Is the program area collaborating with them effectively?

Question 12: Are there any gaps in the portfolio for this technology area?

Question 13: Are there topics that are not being adequately addressed?

Question 14: Are there other areas that this program area should consider funding to meet overall programmatic goals?

Question 15: Can you recommend new ways to approach the barriers addressed by this program area?

Question 16: Are there any other suggestions to improve the effectiveness of this program area?

Responses to the subprogram overview questions are summarized in the following pages. Individual reviewer comments for each question are identified under the heading Reviewer 1, Reviewer 2, etc. Note that reviewer comments may be ordered differently; for example, for each specific subprogram overview presentation, the reviewer identified as Reviewer 1 in the first question may not be Reviewer 1 in the second question, etc 


\section{Overview of the VTO Vehicle Systems Program: Lee Slezak (U.S. Department of Energy) - vs000}

\section{Question 1: Was the program area, including overall strategy, adequately covered?}

\section{Reviewer 1:}

The reviewer stated that the VS Program area was adequately described, noting that the goals and objectives including technical guidance, vehicle systems R\&D, integration, optimization and inoperability, tech-to-market, and transformational transportation systems were all discussed thoroughly. In addition, the reviewer commented that the VS focus areas were described very well and shown to be very well integrated.

\section{Reviewer 2:}

The reviewer replied yes, a good job on fully covering objectives and strategy.

\section{Reviewer 3:}

The reviewer replied yes to this question.

\section{Reviewer 4:}

The reviewer said for the most part, yes, and that strategy was quite clear. The reviewer observed that because of the breadth of VS' reach, it is hard to boil everything going on into a relatively short presentation, but from the presentation, the focus on several primary areas is quite clear. The reviewer also noted that VS' value is that it also includes a number of issue-specific smaller areas of activity (which nearly fill the entire AMR time slots) that were not really highlighted much in this presentation, adding that these smaller areas, however, often provide critical insight and solutions to the challenges facing VS and the VTO in general.

\section{Question 2: Is there an appropriate balance between near- mid- and long-term research and development?}

\section{Reviewer 1:}

The reviewer replied yes, elaborating that by its nature, there is a greater emphasis toward nearer-term developments, but that is appropriate given VS goals and approach. The reviewer remarked that most of the targets for VS efforts are really to address issues being seen in prototype or early production vehicles, or for the next generation of vehicles, so that it really is a near-term/mid-term focus by necessity. There are appropriately a few longer-term areas of effort.

\section{Reviewer 2:}

The reviewer said yes, there are realistic timelines.

\section{Reviewer 3:}

The reviewer observed that it looks like most R\&D is focused on mid-term.

\section{Reviewer 4:}

The reviewer remarked that there was not any information provided about near-, mid-, and long-term research and development.

\section{Question 3: Were important issues and challenges identified?}

\section{Reviewer 1:}

The reviewer replied yes, elaborating that issues and challenges of reducing market barriers, technology validation, risk reduction, cost reduction, and performance improvement were discussed, and it was shown the program area helps support the Office of Energy Efficiency and Renewable Energy (EERE) Strategic Plan. The program area also identified the five EERE Core Questions.

\section{Reviewer 2:}

The reviewer answered yes, pointing out that VS is aimed at addressing a very broad range of challenges, so explaining this takes a special effort. The reviewer added that this effort was clearly taken to ensure that all major issues and challenges were identified and explained in a list that focused on cost reduction and performance 
improvement, technology validation and risk reduction, and reducing market barriers. There was also a specific tie presented to the five EERE Core Questions.

\section{Reviewer 3:}

The reviewer answered yes, adding that cybersecurity is a big issue.

\section{Reviewer 4:}

The reviewer replied yes to this question.

\section{Question 4: Are plans identified for addressing issues and challenges?}

\section{Reviewer 1:}

The reviewer said yes, adding that it was clearly explained how the various pieces within VS work together to ensure that overall goals are achieved. Thus, the reviewer concluded, this program was presented as much more than the sum of its parts, a key concept to grasp when looking at VS.

\section{Reviewer 2:}

The reviewer replied yes, adding that for each of the five EERE Core Questions the program area provided information as to how it will help to answer the questions.

\section{Reviewer 3:}

The reviewer answered yes, all the technical issues, but cautioned that cost and return on investment (ROI) issues need more attention

\section{Reviewer 4:}

The reviewer remarked that plans are not comprehensively outlined but that they are very much top level. The reviewer added that being a little bit specific with target specifications would be better.

\section{Question 5: Was progress clearly benchmarked against the previous year?}

\section{Reviewer 1:}

The reviewer said yes.

\section{Reviewer 2:}

The reviewer said that given the breadth of activities on-going within VS and the broad range of challenges, there was not a significant focus on comparing this year's successes against the previous year. Again, given this breadth, something needed to be eliminated for this presentation, and it appears that this specific comparison was likely an appropriate element to forego to ensure that the important elements of the VS Program were adequately addressed. The reviewer noted that key accomplishments over the past year were provided, and even divided out by activity area.

\section{Reviewer 3:}

The reviewer said that progress was identified in several areas of the program, including modeling and simulation, codes and standards, vehicle systems efficiency improvements and technology evaluations. However, the progress was not really benchmarked against the previous year.

\section{Reviewer 4:}

This reviewer acknowledged not being a reviewer last year for this specific program.

\section{Question 6: Are the projects in this technology area addressing the broad problems and barriers that the Vehicle Technologies Office (VTO) is trying to solve?}

\section{Reviewer 1:}

The reviewer declared absolutely, clarifying that these problems were clearly addressed in the presentation, focusing not only on its relationship with VTO overall but also in how VS is aimed at providing results tied to objectives ranging from technical guidance through transformational transportation systems. Specifically, the reviewer noted, the program identified how it is addressing the five EERE Core Questions. 


\section{Reviewer 2:}

Yes, the reviewer replied, elaborating that the overall goal of petroleum displacement, energy security, U.S. competitiveness, and emissions reduction are being addressed by using systems engineering R\&D to accelerate commercialization of integrated, highly efficient vehicles by reducing development risk, cost, and time.

\section{Reviewer 3:}

The reviewer replied yes to this question.

\section{Reviewer 4:}

The reviewer said somehow.

\section{Question 7: Does the program area appear to be focused, well-managed, and effective in addressing VTO's needs?}

\section{Reviewer 1:}

The reviewer responded yes, clarifying that by its very nature, the program is aimed at a very broad range of challenges so that its focus has to be very broad, but its efforts are focused upon the challenges that VTO needs solved. The reviewer noted that some are efficiency, some are technologies that need to enable new vehicle technologies (such as infrastructure), and some are verification of technology/vehicle performance. The reviewer concluded that VS is tasked with accomplishing a lot, yet it is where the "rubber hits the road" and performance of new technologies is really proven.

\section{Reviewer 2:}

The reviewer said yes, the presenter did a very good job in explaining the program and showing that it is quite effective in addressing the needs of VTO.

\section{Reviewer 3:}

The reviewer replied yes to this question.

\section{Reviewer 4:}

The reviewer said mostly.

\section{Question 8: What are the key strengths and weaknesses of the projects in this program area? Do any of the projects stand out on either end of the spectrum?}

\section{Reviewer 1:}

The reviewer remarked that modeling and simulation work under the VS Program area has been a key strength over the years. The reviewer specified Autonomie as having been used worldwide by companies and research organizations and as a true success story of the program.

\section{Reviewer 2:}

The reviewer praised as a true strength of the program its mandate - enabling and proving the performance of advanced/new technologies. Thus, the reviewer observed, the program is developing the tools, data, standards, etc., necessary for success, while also testing technologies and full vehicle designs (such as through involvement in SuperTruck II) to show progress toward overall goals. The capabilities of the program (within DOE, the national laboratories, and industry) have been developed to address this broad mandate.

The reviewer cited as a weakness of this program that it is also tied to this broad aim, commenting that there are a lot of moving parts in it and thus its structure can be complicated, and it can be difficult to explain to outside organizations (and even internal ones) the importance of these activities. It also appears to this reviewer that this complexity may have impacted the budgetary situation for the program, which has seen significant drops over the past few years.

More glaring, the reviewer remarked, is perhaps a weakness based upon its strength. Because of its systems-level focus and success, the program is increasingly asked to participate in VTO-level or even EERE-level initiatives, such as Grid Modernization. The reviewer stipulated that while such efforts provide significant opportunities, they 
cannot be at the expense of basic VS activities, and ultimately, capabilities, adding that additional funding must be provided with additional responsibilities.

\section{Reviewer 3:}

The reviewer cited as strengths the technical efficiencies of propulsion and charging while observing as a weakness cost not being fully vetted.

\section{Question 9: Do these projects represent novel and/or innovative ways to approach these barriers as appropriate?}

\section{Reviewer 1:}

The reviewer affirmed that the projects comprising the VS Program intended to provide vehicle system efficiency improvements, modeling and simulation, technology evaluation and codes and standards are innovative, well integrated, and successful in providing ways to eliminate barriers.

\section{Reviewer 2:}

The reviewer observed that many do represent novel applications, such as wireless charging technologies while other activities may appear to be simply bringing together a number of existing technologies to maximize impacts. However, the reviewer elaborated that these actually are innovative because they bring together technologies that have not necessarily been brought together before, and may require balancing among individual technologies that may have significant interactions complicating implementation. In addition, the reviewer stated, the VS Program also relies heavily upon innovative capabilities that have been developed at the national laboratories and that these capabilities have already resulted in significant progress such as in advanced aerodynamics.

\section{Reviewer 3:}

The reviewer replied yes, from a technical aspect, but added that cost needs more attention.

\section{Question 10: Has the program area engaged appropriate partners?}

\section{Reviewer 1:}

The reviewer declared absolutely, elaborating that efforts have been coordinated with both industry manufacturing partners and also with appropriate standards organizations such as SAE. In general, the reviewer observed that VS is collaborating on a number of sustainable transportation technologies with other government agencies, within other parts of DOE and EERE, with industry, and with a number of other institutions.

\section{Reviewer 2:}

The reviewer affirmed that the VS Program area has a wide variety of partners that make the program much stronger with their involvement, adding that the partners definitely seem to be very appropriate.

\section{Reviewer 3:}

The reviewer said yes, noting that many government, academia, and industry partners are involved.

\section{Reviewer 4:}

The reviewer indicated that it looks like most collaborations are between DOE research laboratories and some companies without enough attention from universities.

\section{Question 11: Is the program area collaborating with them effectively?}

\section{Reviewer 1:}

The reviewer replied yes, explaining that the organizations being collaborated with are exactly the types of companies or institutions that are necessary for technologies to move forward, and VS appears to focus heavily on making these successful relationships. The reviewer pointed out that a number of specific activities have been identified to ensure that efforts draw in the appropriate organizations and ensure completion of needed developments. In addition, there have been strong relationships built and maintained, both by DOE and National Laboratory personnel. The reviewer concluded that it is this more personal touch that clearly comes through when DOE management and individual researchers speak about VS projects. 


\section{Reviewer 2:}

Yes, the reviewer replied, stating that through the presentation it was clear that the program area is effectively collaborating with the partners.

\section{Reviewer 3:}

The reviewer answered yes, effective relationships are established.

\section{Reviewer 4:}

The reviewer stated that it is not clear.

\section{Question 12: Are there any gaps in the portfolio for this technology area?}

\section{Reviewer 1:}

The reviewer replied no, the program area does not seem to have any gaps in this area.

\section{Reviewer 2:}

The reviewer observed that it is not so much of a gap, as such, at least yet, but based upon recent interest within VTO and EERE in Systems and Modeling for Accelerated Research in Transportation (SMART) Mobility, it would seem that VS would be an important place for greater involvement. Other than a few specific pieces that may be included within SMART Mobility, the reviewer noted it does not appear that there has been a planning effort yet to take full advantage of the capabilities within VS to move SMART Mobility forward.

The reviewer recounted that in the past years, VS often included a mix of relatively large projects, along with a few smaller specifically-targeted ones, and that it appears reductions in funding (and greater impact of EERE-level initiatives such as Grid Modernization) have resulted in dropping some of these types of projects. The reviewer cautioned that the fear is more reductions will occur as greater interest is focused on office-level initiatives.

The reviewer concluded that while Vehicle Systems is a natural place for strong participation in many of these initiatives, such additional opportunities must be coupled with additional funding, or else core Vehicle Systems efforts (and capabilities) will continue to be cut. If this happens, VS will lose much of the expertise and learning that has made it successful.

\section{Reviewer 3:}

The reviewer stated that cost effectiveness needs to be a focus for successful deployments.

\section{Reviewer 4:}

The reviewer offered that there has been some focus on chargers, particularly wireless, which is fine, but it appears as though power electronics and electric machines are not part of this program.

\section{Question 13: Are there topics that are not being adequately addressed?}

\section{Reviewer 1:}

The reviewer stated that topics in the program area are being adequately addressed.

\section{Reviewer 2:}

The reviewer commented that there is always a greater need for performance testing and data from new technologies either ready to enter the market, or which have recently initiated market penetrations, such as under the test and evaluation activities. The reviewer observed that this is a particular place of coordination with VTO's deployment efforts, especially through Clean Cities, adding that fleets or individuals considering new technologies need data upon which to make informed decisions. The reviewer cautioned that VS has worked hard to maintain this capability but that recent program directions toward efforts such as Grid Modernization appear to have simply reduced the available funding for this and other important areas.

\section{Reviewer 3:}

The reviewer remarked economy of scale to obtain cost objectives. 


\section{Reviewer 4:}

The reviewer offered that there has been some focus on chargers, particularly wireless, which is fine, but it appears as though power electronics and electric machines are not part of this program.

\section{Question 14: Are there other areas that this program area should consider funding to meet overall programmatic goals?}

\section{Reviewer 1:}

The reviewer replied that this is very well thought out.

\section{Reviewer 2:}

No, the reviewer responded, adding that the programmatic goals should be able to be met with the current projects being studied.

\section{Reviewer 3:}

The reviewer praised the focus on software/code development as wonderful and asked if there is there a plan to unify controller area network protocols for EVs.

\section{Reviewer 4:}

The reviewer stated that if SMART Mobility is to become a focus for the EERE/VTO program, VS is a natural place to include targeted efforts focused upon transportation system-level efficiencies. However, the reviewer stipulated, adding this new area cannot be at the cost of existing VS activities, which are already struggling under significant budget reductions over the past few years.

\section{Question 15: Can you recommend new ways to approach the barriers addressed by this program area?}

\section{Reviewer 1:}

The reviewer replied no, the program is well structured to address the barriers in this program area.

\section{Reviewer 2:}

The reviewer responded that the program is on track, and added that cost and incentives need to be part of EV Everywhere for the charging infrastructure and vehicle incremental cost.

\section{Reviewer 3:}

The reviewer suggested that it could be useful to include some specific analysis of various electrification architectures in a range of applications in order to develop information upon which adopters could make decisions. In addition, the reviewer commented that it could also be useful if such data could be compared to various alternative fuel technologies and applications, focused upon performance against overall VTO goals (e.g., petroleum, GHG, and criteria emission reductions).

\section{Reviewer 4:}

The reviewer judged that additional university engagement should be mandatory, elaborating that the program should not let only one particular university - due to proximity to a specific laboratory or company — be listed on all the proposals. The reviewer added that each university should be limited to be partnered with only one submission or only one grant, offering that this diversifies university engagement.

\section{Question 16: Are there any other suggestions to improve the effectiveness of this program area?}

\section{Reviewer 1:}

The reviewer replied no, the program area continues to be effective in evaluating the VTO areas of research and development in a vehicle systems context.

\section{Reviewer 2:}

The reviewer said a good team is working on a very important issue, and added that this is just the first step to autonomous vehicles. 


\section{Reviewer 3:}

The reviewer recommended allowing the program the budgetary flexibility to maintain a mix between larger and smaller projects to ensure that the true breadth of VS can continue to be addressed successfully. The reviewer added that this program continues to accomplish a great deal, in spite of its reducing budget and increasing demand from EERE-level initiatives.

Reviewer 4:

The reviewer suggested enhanced engagement of universities, remarking that with little bit of money universities can contribute much more than the program expects. 


\section{Project Feedback}

In this merit review activity, each reviewer was asked to respond to a series of questions, involving multiple-choice responses, expository responses where text comments were requested, and numeric score responses (on a scale of 1.0 to 4.0). In the pages that follow, the reviewer responses to each question for each project will be summarized: the multiple choice and numeric score questions will be presented in graph form for each project, and the expository text responses will be summarized in paragraph form for each question. A table presenting the average numeric score for each question for each project is presented below.

\section{Table 1-1 - Project Feedback}

\begin{tabular}{|c|c|c|c|c|c|c|c|}
\hline Presentation Title & $\begin{array}{c}\text { Principal } \\
\text { Investigator } \\
\text { and } \\
\text { Organization }\end{array}$ & $\begin{array}{c}\text { Page } \\
\text { Number }\end{array}$ & Approach & $\begin{array}{c}\text { Technical } \\
\text { Accomplishments }\end{array}$ & Collaborations & $\begin{array}{c}\text { Future } \\
\text { Research }\end{array}$ & $\begin{array}{l}\text { Weighted } \\
\text { Average }\end{array}$ \\
\hline $\begin{array}{l}\text { Medium- and } \\
\text { Heavy-Duty } \\
\text { Vehicle Field } \\
\text { Evaluations }\end{array}$ & $\begin{array}{l}\text { Kelly, Ken } \\
\text { (NREL) }\end{array}$ & $1-15$ & 3.63 & 3.50 & 3.75 & 3.25 & 3.53 \\
\hline $\begin{array}{l}\text { DOE's Effort } \\
\text { to Improve } \\
\text { Heavy Vehicle } \\
\text { Fuel Efficiency } \\
\text { through Improved } \\
\text { Aerodynamics }\end{array}$ & $\begin{array}{l}\text { Salari, Kambiz } \\
\quad \text { (LLNL) }\end{array}$ & $1-18$ & 3.50 & 3.75 & 3.25 & 3.50 & 3.59 \\
\hline $\begin{array}{l}\text { Idaho National } \\
\text { Laboratory } \\
\text { Testing of } \\
\text { Advanced } \\
\text { Technology } \\
\text { Vehicles }\end{array}$ & $\begin{array}{l}\text { Francfort, } \\
\text { James (INL) }\end{array}$ & $1-22$ & 3.25 & 3.00 & 3.25 & 3.00 & 3.09 \\
\hline $\begin{array}{l}\text { Advanced Vehicle } \\
\text { Testing and } \\
\text { Evaluation }\end{array}$ & $\begin{array}{l}\text { Jacobson, } \\
\text { Richard } \\
\text { (Intertek) }\end{array}$ & $1-25$ & 3.10 & 3.10 & 3.20 & 2.80 & 3.08 \\
\hline $\begin{array}{l}\text { Advanced } \\
\text { Technology } \\
\text { Vehicle Lab } \\
\text { Benchmarking } \\
\text { (L1 and L2) }\end{array}$ & $\begin{array}{l}\text { Stutenberg, } \\
\text { Kevin (ANL) }\end{array}$ & $1-28$ & 3.60 & 3.60 & 3.40 & 3.40 & 3.55 \\
\hline $\begin{array}{l}\text { SuperTruck - } \\
\text { Development and } \\
\text { Demonstration of } \\
\text { a Fuel-Efficient } \\
\text { Class } 8 \text { Tractor } \\
\text { and Trailer, } \\
\text { Vehicle }\end{array}$ & $\begin{array}{l}\text { Zukouski, Russ } \\
\text { (Navistar) }\end{array}$ & $1-32$ & 3.50 & 3.50 & 3.60 & 3.40 & 3.50 \\
\hline
\end{tabular}




\begin{tabular}{|c|c|c|c|c|c|c|c|}
\hline Presentation Title & $\begin{array}{c}\text { Principal } \\
\text { Investigator } \\
\text { and } \\
\text { Organization }\end{array}$ & $\begin{array}{l}\text { Page } \\
\text { Number }\end{array}$ & Approach & $\begin{array}{c}\text { Technical } \\
\text { Accomplishments }\end{array}$ & Collaborations & $\begin{array}{l}\text { Future } \\
\text { Research }\end{array}$ & $\begin{array}{l}\text { Weighted } \\
\text { Average }\end{array}$ \\
\hline $\begin{array}{l}\text { Commercial } \\
\text { Vehicle Thermal } \\
\text { Load Reduction } \\
\text { and VTCab-Rapid } \\
\text { HVAC Load } \\
\text { Estimation Tool }\end{array}$ & $\begin{array}{l}\text { Lustbader, } \\
\text { Jason (NREL) }\end{array}$ & $1-36$ & 3.63 & 3.38 & 3.38 & 3.38 & 3.44 \\
\hline Volvo SuperTruck & $\begin{array}{l}\text { Amar, Pascal } \\
\text { (Volvo Trucks) }\end{array}$ & $1-39$ & 3.75 & 3.83 & 3.75 & 3.40 & 3.75 \\
\hline $\begin{array}{c}\text { System for } \\
\text { Automatically } \\
\text { Maintaining } \\
\text { Pressure in a } \\
\text { Commercial Truck } \\
\text { Tire }\end{array}$ & $\begin{array}{l}\text { Anderson, } \\
\text { Norm (The } \\
\text { Goodyear Tire } \\
\text { and Rubber } \\
\text { Company) }\end{array}$ & $1-43$ & 3.25 & 3.38 & 3.25 & 3.38 & 3.33 \\
\hline $\begin{array}{l}\text { EV - Smart Grid } \\
\text { Research and } \\
\text { Interoperability } \\
\text { Activities }\end{array}$ & $\begin{array}{l}\text { Hardy, Keith } \\
\text { (ANL) }\end{array}$ & $1-47$ & 3.50 & 3.63 & 3.88 & 3.00 & 3.55 \\
\hline $\begin{array}{l}\text { Wireless and } \\
\text { Conductive } \\
\text { Charging Testing } \\
\text { to Support Code } \\
\text { and Standards }\end{array}$ & $\begin{array}{c}\text { Carlson, } \\
\text { Barney (INL) }\end{array}$ & $1-50$ & 3.70 & 3.50 & 3.80 & 3.40 & 3.58 \\
\hline $\begin{array}{l}\text { High-Efficiency, } \\
\text { Low-EMI and } \\
\text { Positioning } \\
\text { Tolerant Wireless } \\
\text { Charging of EVs }\end{array}$ & $\begin{array}{l}\text { Chabaan, } \\
\text { Rakan } \\
\text { (Hyundai) }\end{array}$ & $1-53$ & 3.25 & 3.25 & 2.75 & 3.13 & 3.17 \\
\hline $\begin{array}{l}\text { Wireless Charging } \\
\text { of Electric } \\
\text { Vehicles }\end{array}$ & $\begin{array}{l}\text { Onar, Omer } \\
\text { (ORNL) }\end{array}$ & $1-56$ & 3.50 & 3.50 & 3.50 & 3.33 & 3.48 \\
\hline $\begin{array}{c}\text { Zero Emission } \\
\text { Drayage Truck } \\
\text { Demonstration } \\
\text { (ZECT I) }\end{array}$ & $\begin{array}{l}\text { Miyasato, Matt } \\
\text { (SCAQMD) }\end{array}$ & $1-58$ & 2.90 & 2.90 & 3.30 & 2.90 & 2.95 \\
\hline $\begin{array}{l}\text { Hydrogen Fuel- } \\
\text { Cell Electric } \\
\text { Hybrid Truck and } \\
\text { Zero Emission } \\
\text { Delivery Vehicle } \\
\text { Deployment }\end{array}$ & $\begin{array}{l}\text { DeCandis, } \\
\text { Andrew } \\
\text { (Houston- } \\
\text { Galvelston } \\
\text { Area Council) }\end{array}$ & $1-61$ & 2.38 & 2.00 & 2.75 & 2.13 & 2.20 \\
\hline
\end{tabular}




\begin{tabular}{|c|c|c|c|c|c|c|c|}
\hline Presentation Title & $\begin{array}{c}\text { Principal } \\
\text { Investigator } \\
\text { and } \\
\text { Organization }\end{array}$ & $\begin{array}{l}\text { Page } \\
\text { Number }\end{array}$ & Approach & $\begin{array}{c}\text { Technical } \\
\text { Accomplishments }\end{array}$ & Collaborations & $\begin{array}{c}\text { Future } \\
\text { Research }\end{array}$ & $\begin{array}{l}\text { Weighted } \\
\text { Average }\end{array}$ \\
\hline $\begin{array}{c}\text { Combined Aero } \\
\text { and Underhood } \\
\text { Thermal Analysis } \\
\text { for Heavy-Duty } \\
\text { Trucks }\end{array}$ & $\begin{array}{l}\text { Sofu, Tanju } \\
\text { (ANL) }\end{array}$ & $1-64$ & 2.50 & 2.90 & 2.70 & 2.40 & 2.71 \\
\hline $\begin{array}{l}\text { Cummins Medium- } \\
\text { Duty and Heavy- } \\
\text { Duty Accessory } \\
\text { Hybridization } \\
\text { CRADA }\end{array}$ & $\begin{array}{l}\text { Deter, Dean } \\
\text { (ORNL) }\end{array}$ & $1-69$ & 3.10 & 2.80 & 3.60 & 3.13 & 3.02 \\
\hline $\begin{array}{l}\text { Vehicle Thermal } \\
\text { System Modeling } \\
\text { in Simulink }\end{array}$ & $\begin{array}{l}\text { Lustbader, } \\
\text { Jason (NREL) }\end{array}$ & $1-73$ & 3.83 & 3.50 & 3.67 & 3.25 & 3.57 \\
\hline $\begin{array}{l}\text { Advanced Climate } \\
\text { Systems for EV } \\
\text { Extended Range } \\
\text { (ACSforEVER) }\end{array}$ & $\begin{array}{l}\text { Meyer, John } \\
\text { (Hanon } \\
\text { Systems) }\end{array}$ & $1-76$ & 3.38 & 3.38 & 3.25 & 3.38 & 3.36 \\
\hline $\begin{array}{c}\text { ePATHS - } \\
\text { electrical PCM } \\
\text { Assisted Thermal } \\
\text { Heating System }\end{array}$ & $\begin{array}{l}\text { Wang, Mingyu } \\
\text { (Mahle Behr } \\
\text { USA, LLC) }\end{array}$ & $1-79$ & 3.63 & 3.50 & 3.63 & 3.13 & 3.50 \\
\hline $\begin{array}{l}\text { SAE J2907 Motor } \\
\text { Power Ratings } \\
\text { Standards } \\
\text { Support }\end{array}$ & $\begin{array}{l}\text { Miller, John } \\
\text { (ORNL) }\end{array}$ & $1-82$ & 3.50 & 3.50 & 3.40 & 3.60 & 3.50 \\
\hline $\begin{array}{l}\text { Analyzing Real- } \\
\text { World Light-Duty } \\
\text { Vehicle Efficiency } \\
\text { Benefits }\end{array}$ & $\begin{array}{l}\text { Gonder, Jeff } \\
\text { (NREL) }\end{array}$ & $1-85$ & 3.13 & 3.25 & 3.25 & 3.00 & 3.19 \\
\hline $\begin{array}{c}\text { UTEMPRA } \\
\text { - Unitary } \\
\text { Thermal Energy } \\
\text { Management for } \\
\text { Propulsion Range } \\
\text { Augmentation }\end{array}$ & $\begin{array}{l}\text { Chowdhury, } \\
\text { Sourav (Mahle } \\
\text { Behr USA, } \\
\text { LLC) }\end{array}$ & $1-88$ & 3.00 & 3.25 & 3.38 & 3.13 & 3.19 \\
\hline
\end{tabular}




\begin{tabular}{|c|c|c|c|c|c|c|c|}
\hline Presentation Title & $\begin{array}{c}\text { Principal } \\
\text { Investigator } \\
\text { and } \\
\text { Organization }\end{array}$ & $\begin{array}{l}\text { Page } \\
\text { Number }\end{array}$ & Approach & $\begin{array}{c}\text { Technical } \\
\text { Accomplishments }\end{array}$ & Collaborations & $\begin{array}{l}\text { Future } \\
\text { Research }\end{array}$ & $\begin{array}{l}\text { Weighted } \\
\text { Average }\end{array}$ \\
\hline $\begin{array}{c}\text { Zero Emission } \\
\text { Cargo Transport } \\
\text { II: San Pedro Bay } \\
\text { Ports Hybrid and } \\
\text { Fuel Cell Electric } \\
\text { Vehicle Project }\end{array}$ & $\begin{array}{l}\text { Impullitti, } \\
\text { Joseph } \\
\text { (SCAQMD) }\end{array}$ & $1-92$ & 2.30 & 2.20 & 3.00 & 2.40 & 2.35 \\
\hline $\begin{array}{l}\text { Multi-Speed } \\
\text { Transmission } \\
\text { for Commercial } \\
\text { Delivery Medium- } \\
\text { Duty Plug-In } \\
\text { Electric Drive } \\
\text { Vehicles }\end{array}$ & $\begin{array}{l}\text { Chavdar, } \\
\text { Bulent (Eaton) }\end{array}$ & $1-96$ & 3.20 & 3.40 & 3.00 & 3.40 & 3.30 \\
\hline $\begin{array}{l}\text { Integrated } \\
\text { Boosting and } \\
\text { Hybridization } \\
\text { for Extreme Fuel } \\
\text { Economy and } \\
\text { Downsizing }\end{array}$ & $\begin{array}{c}\text { Tsourapas, } \\
\text { Vasilios } \\
\text { (Eaton) }\end{array}$ & $1-100$ & 3.50 & 3.30 & 3.10 & 3.20 & 3.31 \\
\hline $\begin{array}{c}\text { Advanced Bus } \\
\text { and Truck Radial } \\
\text { Materials for Fuel } \\
\text { Efficiency }\end{array}$ & $\begin{array}{l}\text { Dos Santos } \\
\text { Freire, Lucas } \\
\text { (PPG) }\end{array}$ & $1-103$ & 3.10 & 3.10 & 3.00 & 3.20 & 3.10 \\
\hline $\begin{array}{l}\text { Design and } \\
\text { Implementation } \\
\text { of a Thermal Load } \\
\text { Reduction System } \\
\text { in a Hyundai PHEV }\end{array}$ & $\begin{array}{l}\text { Kreutzer, Cory } \\
\text { (NREL) }\end{array}$ & $1-107$ & 3.25 & 3.13 & 3.63 & 3.25 & 3.23 \\
\hline $\begin{array}{c}\text { EV Everywhere } \\
\text { Charging } \\
\text { Infrastructure } \\
\text { Roadmap }\end{array}$ & $\begin{array}{c}\text { Karner, Donald } \\
\text { (EAI) }\end{array}$ & 1-111 & 2.25 & 2.25 & 2.00 & 2.00 & 2.19 \\
\hline $\begin{array}{c}\text { Energy Impact } \\
\text { of Connected } \\
\text { and Automated } \\
\text { Vehicles }\end{array}$ & $\begin{array}{l}\text { Peng, Huei } \\
\text { (University of } \\
\text { Michigan) }\end{array}$ & $1-115$ & 3.40 & 3.30 & 3.30 & 3.20 & 3.31 \\
\hline $\begin{array}{c}\text { Methods to } \\
\text { Measure, Predict, } \\
\text { and Relate } \\
\text { Friction, Wear, } \\
\text { and Fuel Economy }\end{array}$ & $\begin{array}{l}\text { Gravante, } \\
\text { Steve } \\
\text { (Ricardo) }\end{array}$ & $1-119$ & 3.42 & 3.17 & 3.42 & 3.25 & 3.27 \\
\hline
\end{tabular}




\begin{tabular}{|c|c|c|c|c|c|c|c|}
\hline Presentation Title & $\begin{array}{c}\text { Principal } \\
\text { Investigator } \\
\text { and } \\
\text { Organization }\end{array}$ & $\begin{array}{l}\text { Page } \\
\text { Number }\end{array}$ & Approach & $\begin{array}{c}\text { Technical } \\
\text { Accomplishments }\end{array}$ & Collaborations & $\begin{array}{l}\text { Future } \\
\text { Research }\end{array}$ & $\begin{array}{l}\text { Weighted } \\
\text { Average }\end{array}$ \\
\hline $\begin{array}{c}\text { Improved Tire } \\
\text { Efficiency through } \\
\text { Elastomeric } \\
\text { Polymers } \\
\text { Enhanced with } \\
\text { Carbon-Based } \\
\text { Nanostructured } \\
\text { Materials }\end{array}$ & $\begin{array}{l}\text { Polyzos, } \\
\text { Georgios } \\
\text { (ORNL) }\end{array}$ & $1-123$ & 2.83 & 3.33 & 2.67 & 2.67 & 3.04 \\
\hline $\begin{array}{l}\text { VTO Vehicle } \\
\text { to Building } \\
\text { Integration } \\
\text { Pathway }^{+}\end{array}$ & $\begin{array}{l}\text { Pratt, Richard } \\
\text { (PNNL) }\end{array}$ & $1-126$ & 3.33 & 3.50 & 3.67 & 3.33 & 3.46 \\
\hline $\begin{array}{l}\text { VTO Systems } \\
\text { Research } \\
\text { Supporting } \\
\text { Standards and } \\
\text { Interoperability }{ }^{\dagger}\end{array}$ & $\begin{array}{l}\text { Smart, John } \\
\text { (INL) }\end{array}$ & $1-128$ & 3.38 & 3.25 & 3.13 & 3.13 & 3.25 \\
\hline $\begin{array}{l}\text { VTO Modeling \& } \\
\text { Controls Software } \\
\text { Tools to Support } \\
\text { V2G Integration) }\end{array}$ & $\begin{array}{l}\text { Saxena, } \\
\text { Samveg } \\
\text { (LBNL) }\end{array}$ & $1-131$ & 3.40 & 3.20 & 3.30 & 3.30 & 3.28 \\
\hline $\begin{array}{l}\text { VTO Diagnostic } \\
\text { Security } \\
\text { Modules for } \\
\text { Electric Vehicle } \\
\text { to Building } \\
\text { Integration } \\
\end{array}$ & $\begin{array}{l}\text { Rohde, Ken } \\
\text { (INL) }\end{array}$ & $1-134$ & 3.50 & 3.50 & 3.50 & 3.50 & 3.50 \\
\hline $\begin{array}{l}\text { Evaluation } \\
\text { of Vehicle } \\
\text { Technology } \\
\text { Benefits on } \\
\text { Real World } \\
\text { Driving Cycles } \\
\text { using Regional } \\
\text { Transportation } \\
\text { System Model }\end{array}$ & $\begin{array}{c}\text { Rousseau, } \\
\text { Aymeric (ANL) }\end{array}$ & $1-136$ & 3.00 & 3.30 & 2.90 & 3.10 & 3.15 \\
\hline $\begin{array}{l}\text { Evaluation of } \\
\text { Dynamic Wireless } \\
\text { Charging Demand }\end{array}$ & $\begin{array}{l}\text { Li, James } \\
\text { (ORNL) }\end{array}$ & $1-139$ & 2.83 & 2.67 & 3.33 & 3.17 & 2.85 \\
\hline Overall Average & & & 3.24 & 3.22 & 3.28 & 3.12 & 3.22 \\
\hline
\end{tabular}

${ }^{\dagger}$ Denotes a poster presentation 


\section{Medium- and Heavy-Duty Vehicle Field Evaluations: Ken Kelly (National Renewable Energy Laboratory) - vs001}

\section{Presenter}

Ken Kelly, National Renewable Energy Laboratory

\section{Reviewer Sample Size}

A total of four reviewers evaluated this project.

\section{Question 1: Approach to performing the work-the degree to which technical barriers are addressed, the project is well-designed, feasible, and integrated with other efforts.}

\section{Reviewer 1:}

The reviewer found that the work with fleets and OEMs was excellent. Gathering these participants and getting their cost share was a critical barrier to success that was vigorously attacked on this project.

\section{Reviewer 2:}

The reviewer stated that the approach of this project was excellent. The field data collected, and the testing and analysis tools that were used provided very valuable information regarding MD and $\mathrm{HD}$ vehicles. This information was exchanged with a very large set of groups, including research organizations, other agencies, and within DOE programs.

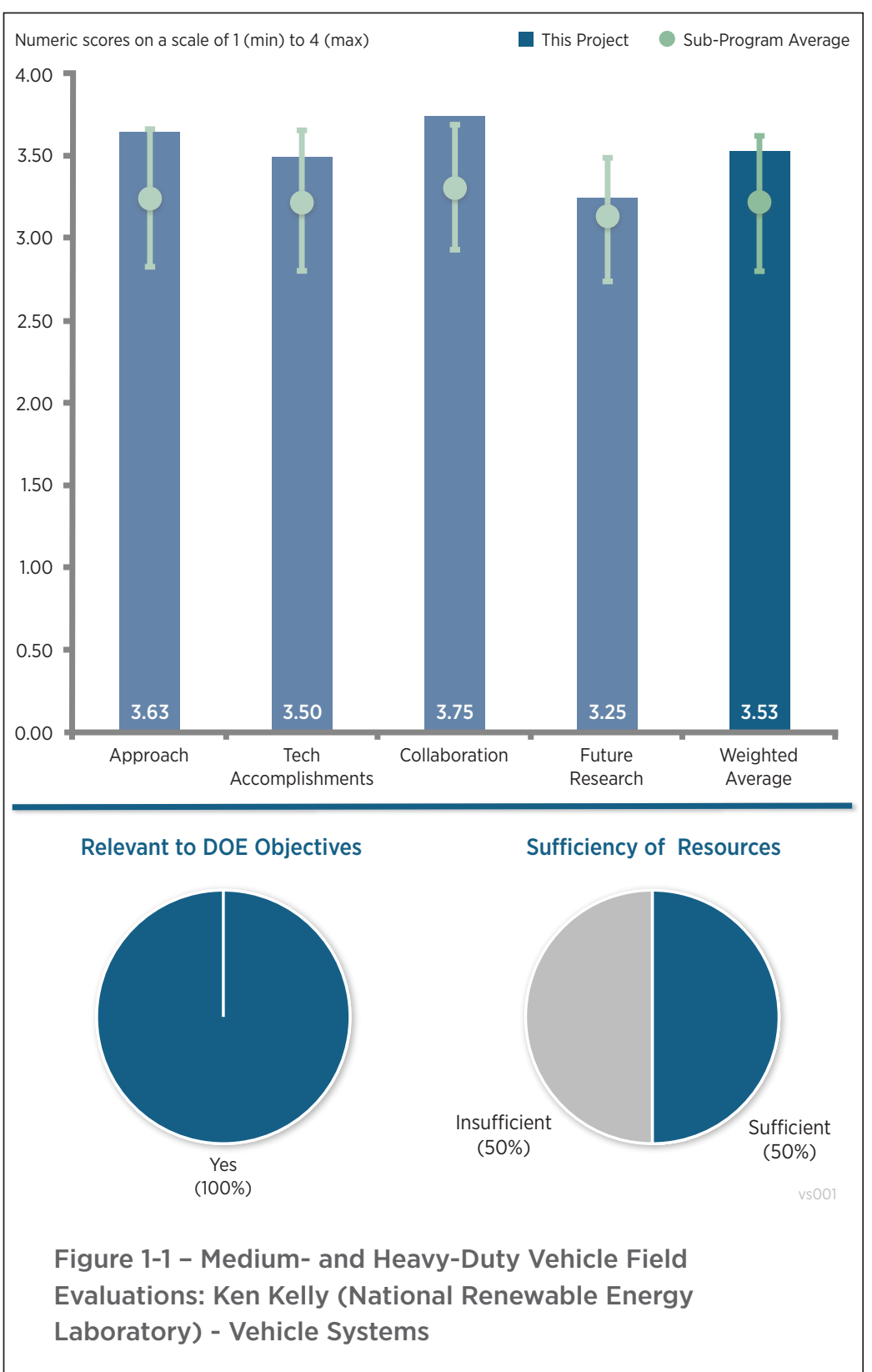

\section{Reviewer 3:}

The reviewer believed that analysis derived from the MD and HD data collection would have increased accuracy if the data collection included frequent direct measurements of the total mass of the vehicles. Several of the fleet data collection involved vehicles that frequently change the mass of their payloads.

\section{Reviewer 4:}

The reviewer stated that the project had very good fleet partners and that this real-world data were essential. The reviewer noted that the way in which fleets/applications were targeted could possibly be more methodical. The reviewer questioned where the holes were in terms of possible applications. Still, the reviewer commented that it was a good approach chosen of characterizing the data and then testing on the dynamometer. Also using a validated vehicle model to do what if scenarios was a very good idea. However, the reviewer commented that power characteristics would be helpful. 
Question 2: Technical accomplishments and progress toward overall project and DOE goals-the degree to which progress has been made, measured against performance indicators and demonstrated progress towards DOE goals.

\section{Reviewer 1:}

The reviewer stated that excellent progress had been made since the kick off in January 2015 on the Miami-Dade project and anticipated the final report will be submitted in fiscal year (FY) 2016. The Foothill Transit project had in use performance results presented to the public and will also have a final report completed in FY 2016. The United Parcel Service (UPS) project was complete and reported fuel economy and excellent emissions results.

\section{Reviewer 2:}

The reviewer thought that excellent progress was made with data collection across a wide range of fleet applications.

\section{Reviewer 3:}

The reviewer found that the amount of data collected was very impressive. The characterization of the fuel economy improvement in the Miami-Dade data will be very helpful in encouraging hybrid usage. The reviewer inquired about the point at which the project team can start recommending the technologies to be examined in future tests.

\section{Reviewer 4:}

The reviewer commented that the presenter provided evidence to support the idea that the data collection benefited the fleet owners, but did not support the idea that it significantly benefits R\&D planning, and strategy for DOE and the national laboratories.

\section{Question 3: Collaboration and coordination with other institutions.}

\section{Reviewer 1:}

The reviewer found that participation by fleets and vehicle OEMs was excellent and a key to the success of this project.

\section{Reviewer 2:}

The reviewer stated that collaboration and coordination in this project has been outstanding. The list of industry partners and other government organizations helped to make this project extremely successful.

\section{Reviewer 3:}

The reviewer stated that the fleets providing the data was good collaboration. Also fleets sharing their maintenance records showed other cost savings. The reviewer thought that when the killer applications are found, such as the Miami-Dade, some kind of gathering could be held with major fleets/OEMs in the same space to inform them of the results and influence their offering and selling positions.

Question 4: Proposed future research-the degree to which the project has effectively planned its future work in a logical manner by incorporating appropriate decision points, considering barriers to the realization of the proposed technology and, when sensible, mitigating risk by providing alternate development pathways.

\section{Reviewer 1:}

The reviewer stated that the proposed future work in collaboration with Idaho National Laboratory (INL) and Argonne National Lab (ANL) should prove to be an excellent project.

\section{Reviewer 2:}

The reviewer stated that there was an excellent focus on future work for existing fleets, but identification of future fleets was lacking.

\section{Reviewer 3:}

The reviewer liked the idea of collaborating with the other DOE institutions. However, the reviewer believed that more effort should have been made to target possible other killer applications by modeling in advance. The 
reviewer asked what power takeoff (PTO) applications are out there that could provide fuel savings and if there are certain cities with terrain, traffic, or other conditions that make hybrids pay off more quickly. The reviewer wondered how the next killer application could be found. Finally, if the project team would be able to make targets for technologies, routes, types of fleets, etc. based on results so far and modeling.

\section{Question 5: Does this project support the overall DOE objectives of petroleum displacement? Why or why not?}

\section{Reviewer 1:}

The reviewer stated that the data collected and analyzed throughout this project provided information to support the overall DOE objective of petroleum displacement.

\section{Reviewer 2:}

The reviewer observed significant petroleum use by MD and HD vehicles.

\section{Reviewer 3:}

The reviewer thought that the project provided objective third party operational test data to fleet operators. These data can help decision makers to make investments in technologies that are effective and economically viable for displacing petroleum consumption.

\section{Reviewer 4:}

The reviewer stated that this was the empirical way to confirm the best applications for hybrids and alternative fuels.

\section{Question 6: Resources: How sufficient are the resources for the project to achieve the stated milestones in a timely fashion?}

\section{Reviewer 1:}

The reviewer thought that the resources have been sufficient to fund the project to completion.

\section{Reviewer 2:}

The reviewer thought it was great that this program got more money this year, but mentioned it would be even more useful to find other applications where fuel saving technologies pay off. 


\section{DOE's Effort to Improve Heavy Vehicle Fuel Efficiency through Improved Aerodynamics: Kambiz Salari (Lawrence Livermore National Laboratory) - vs006}

\section{Presenter}

Kambiz Salari, Lawrence Livermore National Laboratory

\section{Reviewer Sample Size}

A total of four reviewers evaluated this project.

\section{Question 1: Approach to performing the work-the degree to which technical barriers are addressed, the project is well-designed, feasible, and integrated with other efforts.}

\section{Reviewer 1:}

The reviewer was impressed with the progress in the last year on this project. Good work on aerodynamics in general, platooning in particular, and with the generic speed form.

\section{Reviewer 2:}

The reviewer stated that the Principle Investigator (PI) and his team had a well-defined and logical approach to this aerodynamics research. A good scientific approach combined computational fluid dynamics (CFD) and wind tunnel experimentation, supplemented with on-road demonstration. The reviewer found the tanker trailer work intriguing: the fuel savings were not trivial, and the challenges for aerodynamic drag reduction were large, so this was worthwhile for government R\&D. The integrated tractor and trailer work gave the industry a new way to think about the aerodynamics of these vehicles, and demonstrated the potential improvements for looking at the tractor and trailer as a system.

\section{Reviewer 3:}

The reviewer found that the approach was a good mix of analysis, model testing, and real world testing. Testing the geometries in a wind tunnel was a great way to prove out a concept without incurring high costs of a field test. The reviewer stated that the PI leveraged the national laboratory and resources appropriately to fill in where the manufacturer's expertise lacked.

\section{Reviewer 4:}

The reviewer found that the approach to model, improve, then validate the results was a good approach. The presenter implied that all shape modifications are valid including dramatic changes to the trailer. The reviewer

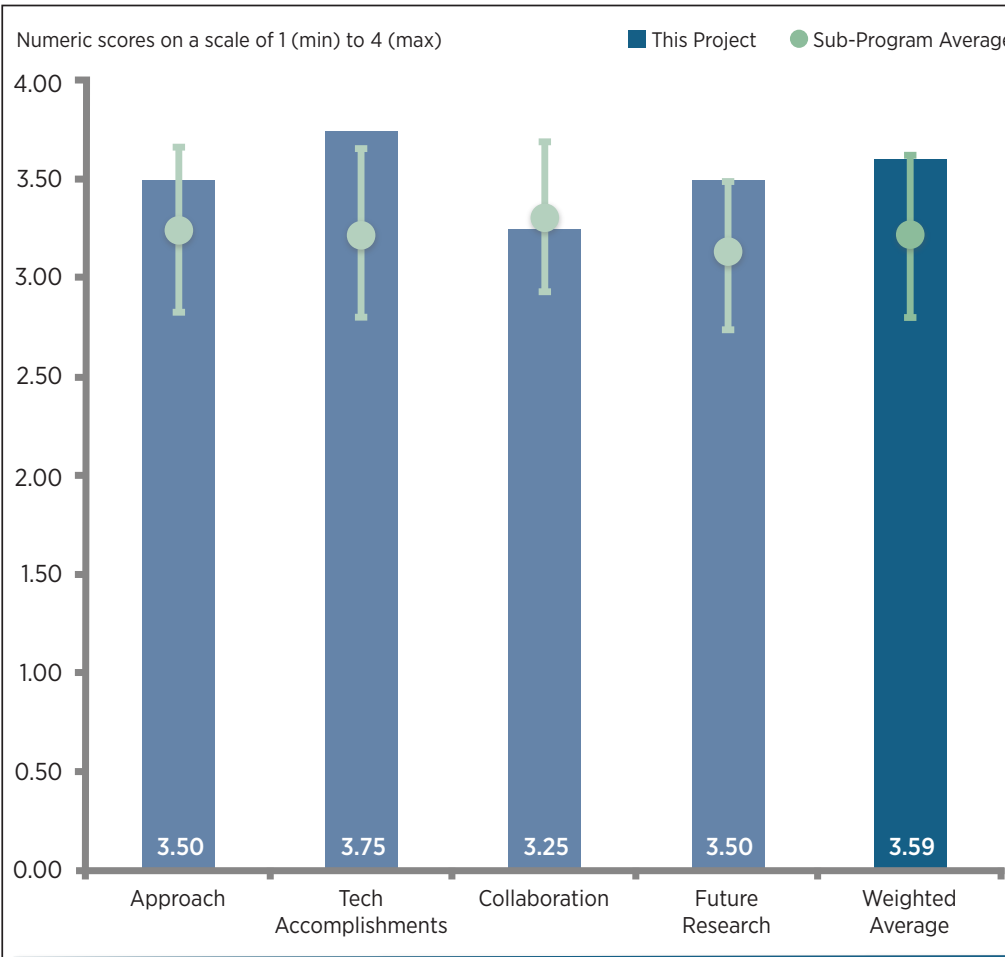

Sufficiency of Resources
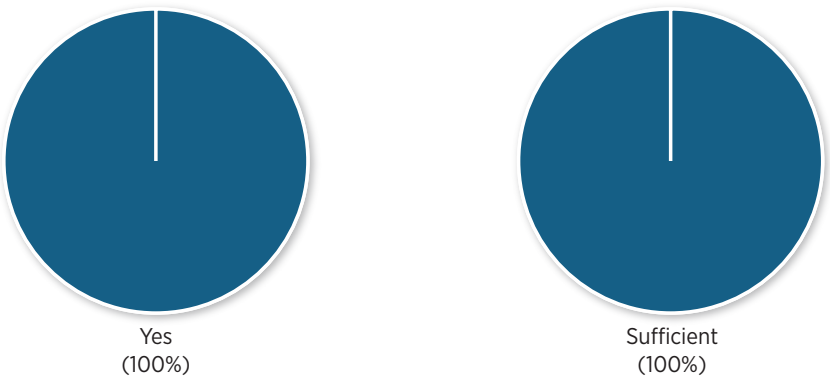

Figure 1-2 - DOE's Effort to Improve Heavy Vehicle Fuel Efficiency through Improved Aerodynamics: Kambiz Salari (Lawrence Livermore National Laboratory) - Vehicle Systems
Relevant to DOE Objectives 
thought that the project should have some industry feedback and certain constraints for logistics. For example, adding appendages to trailers and trucks to accomplish the goals is feasible. Outright changing shapes without considering things like inter modal transportation (very slow speed, but needs to stack) may be theoretically possible, but would not get DOE to its goals. The reviewer said that the project should bind itself to the state of the art that is possible.

\section{Question 2: Technical accomplishments and progress toward overall project and DOE goals - the degree to which progress has been made, measured against performance indicators and demonstrated progress towards DOE goals.}

\section{Reviewer 1:}

The reviewer stated that the Generic Speed Form 2 (GSF2) discoveries represented groundbreaking aerodynamics work, and it will be interesting to see how industrial partners use these results in their future work (perhaps elements of this can find their way to SuperTruck II eventually). The reviewer added that the drag characteristics of the GSF2 are completely different than baseline trucks; increased yaw angle drag reductions could be important in the real world as no truck operates in a zero-yaw condition in real life. The reviewer also stated that drag reductions were significant: $60-80 \%$ reduction or more. Hopefully this will change the discussion in the industry about truck shapes.

The reviewer continued saying that the truck platooning work addressed some previous concerns that the National Renewable Energy Laboratory (NREL) discovered in their first platooning tests. It will be important to quantify how the aerodynamic drag of the system of trucks improves with platooning, and how individual trucks may be affected. Finally, the reviewer stated that the cooling air work was important as increased fan power could negate aero drag reductions with platooning.

\section{Reviewer 2:}

The reviewer thought that the progress made on this project was great. There were several milestones that were achieved in this time period. The PI's willingness to share findings has led to more awareness of the breakthroughs, which will hopefully lead to more adoption of the GSF2. The comparison in drag coefficients on Slide 16 show the tremendous accomplishments made with this design.

\section{Reviewer 3:}

The reviewer stated that the accomplishments were completed and well described in this presentation. The reviewer added that it is important to share this work in a strong way to help industry deliver on the potential the project team is uncovering. For instance, sharing how the wind averaged drag is calculated was a good use of two minutes in the review. Finally, the reviewer commented that the platooning accomplishments were particularly noteworthy, very helpful in support of industry and NREL activities.

\section{Reviewer 4:}

The reviewer thought that progress appeared to be very good. The presenter verbally indicated that one of the designs was being used by Navistar (outstanding transition). The reviewer thought that the reporting aspect of the project was lacking, even though the technical was performing extremely well. The reviewer stated that the project should do the calculations and simulations (based on published drive cycles), and report on how much fuel is saved by the reduction of drag, so it is clear what impact there is and how close the project is to meeting the DOE goals.

\section{Question 3: Collaboration and coordination with other institutions.}

\section{Reviewer 1:}

The reviewer stated that the project has a very good mix of research, OEMs, manufacturers, and end users.

\section{Reviewer 2:}

The reviewer noted that the team is collaborating with a number of the right organizations, and added that expansion would always be helpful, but not necessary. Navistar is using the GSF2 geometry now, which is excellent to hear. The work on platooning aerodynamics shows a good inter-lab relationship with NREL, as they had identified the cooling challenge in a real-world testing effort last year that has now translated to lab research work at Lawrence Livermore National Laboratory (LLNL). 


\section{Reviewer 3:}

The reviewer noted that the project is using NREL and the National Aeronautics and Space Administration for their model validation and testing. NREL is also collecting on-the-road data from the industry. The reviewer thought that the team has good coordination and each member is playing to their strengths.

\section{Reviewer 4:}

The reviewer stated that these discoveries are very important for industries. The reviewer suggested that the project team continue to find opportunities to openly share these findings.

\section{Question 4: Proposed future research-the degree to which the project has effectively planned its future work in a logical manner by incorporating appropriate decision points, considering barriers to the realization of the proposed technology and, when sensible, mitigating risk by providing alternate development pathways.}

\section{Reviewer 1:}

The reviewer stated that it is very exciting to continue this work, particularly in this area of zero drag.

\section{Reviewer 2:}

The reviewer found that the team's future work plans are reasonable, logical, and are appropriate next steps based on the accomplishments of the project to date. The plans to continue coordination with industry on designing next-generation aerodynamic vehicles is extremely important, as it will move this groundbreaking lab work into production.

\section{Reviewer 3:}

The reviewer stated that the proposed future work is well thought out. The project is trying to further its efforts in reducing drag and improving fuel economy. The reviewer thought that the project team's efforts to coordinate industry participants makes it highly valuable.

\section{Reviewer 4:}

The reviewer stated that the additional iteration is good future research, but added that the project should add quantifiable goals of what the future research will be. For example: achieving a certain percentage of improvement over the current iteration. The reviewer noted that the presenter indicated greater spacing and possibly analyzing more trucks for the platooning is also a plan. This should all be captured as part of the program plan.

\section{Question 5: Does this project support the overall DOE objectives of petroleum displacement? Why or why not?}

\section{Reviewer 1:}

The reviewer stated very much so.

\section{Reviewer 2:}

The reviewer stated that reduction of aerodynamic drag of commercial trucks is very relevant to DOE petroleum displacement goals, due to the significant and necessary fuel use of these trucks. The potential drag reductions from this project can result in a large decrease in transportation petroleum use. The reviewer commented that research work is helping guide the discussions about the state-of-the-art in aerodynamics within the research and industrial communities, and as such moves the production vehicles forward in their aerodynamic performance.

\section{Reviewer 3:}

The reviewer noted that on Slides 3 and 4, the presenter made a great case for why the project was highly relevant to DOE's objective of reductions in petroleum consumption. The project informs DOE so they can in turn inform industry. Results of the project were also relevant to the industry as the speaker noted that Navistar is using the geometry developed in the project.

\section{Reviewer 4:}

The reviewer commented that reducing aerodynamic drag directly supports DOE's objectives of reduced fuel consumption. There is definitely an improvement in fuel consumption, but it is hard to quantify the actual savings as the presentation left the calculations up to the reader. 


\section{Question 6: Resources: How sufficient are the resources for the project to achieve the}

stated milestones in a timely fashion?

Reviewer 1:

The reviewer stated that the funds are sufficient to achieve the milestones set forth, but added that additional funds for groundbreaking work like this would always be helpful.

Reviewer 2:

The reviewer stated that based on what the project has been able to accomplish, the reviewer believed the resources are sufficient.

Reviewer 3:

The reviewer noted that progress is occurring and exciting.

Reviewer 4:

The reviewer thought that the project resources seem to be sufficient. 


\section{Idaho National Laboratory Testing of Advanced Technology Vehicles: James Francfort (Idaho National Laboratory) - vs021}

\section{Presenter}

Shawn Salisbury, Idaho National Laboratory

\section{Reviewer Sample Size}

A total of five reviewers evaluated this project.

\section{Question 1: Approach to performing the work-the degree to which technical barriers are addressed, the project is well-designed, feasible, and integrated with other efforts.}

\section{Reviewer 1:}

The reviewer found that this project is well designed and provides supportive data that can be utilized by consumers for choice and adaptation decisions. It collects needed data which will inform consumers and government agencies as to the life cycle and utility of the vehicles, and further program needs.

\section{Reviewer 2:}

The reviewer stated that there were comments by reviewers (this year, and apparently in last year's AMR as well) regarding the lack of standard drive cycles. In this particular case, the reviewer stated support for the approach that INL has taken, by not relying solely on standard drive cycles. The end goal of these projects is petroleum displacement, and while standard drive cycles provide a (rather unreliable) means to compare fuel consumption, they do not represent real world benefits. The testing that INL is performing goes a long way in providing the average customer more reliable information.

\section{Reviewer 3:}

The reviewer stated that the project tests a wide range of vehicles and lots of miles of miles on individual vehicles, which is helpful information to have. The tests are developed using standards, as well as experts on new tech that might not have standards. That is a good approach.

The reviewer thought that acquiring knowledge on battery degradation is important for resale value, second leases, etc. The reviewer questioned if that knowledge is getting to the car buying public, banks, or car selling networks to make use of that knowledge. The presentation says that results are presented and published, but the reviewer wanted to know how that is occurring to the widest possible audience. The reviewer questioned if the press is a possibility as well, for example the Wall Street Journal for business impact, car magazines, etc. Finally, the 
reviewer stated that getting the data on the public taxis is a great idea. It should point to how hybrid designs and the charging system can be improved.

\section{Question 2: Technical accomplishments and progress toward overall project and DOE goals-the degree to which progress has been made, measured against performance indicators and demonstrated progress towards DOE goals.}

\section{Reviewer 1:}

The reviewer stated that steady progress appears to be made.

\section{Reviewer 2:}

The reviewer noted that the testing of 95 vehicles within this program in the past couple years is statistically significant to derive the comparable performance data. By using large fleet operators (such as the New York City taxi cabs) some real-world data, such as efficiency compared to ambient temperature, become increasingly valuable for adoption decisions.

\section{Reviewer 3:}

The reviewer stated that the charger results are helpful and battery testing results are good. The project generated lots of reports with the 12 Volt (V) results given to OEMs. The reviewer noted that it looks like a fair amount of miles remain to be driven on some of the cars.

\section{Question 3: Collaboration and coordination with other institutions.}

\section{Reviewer 1:}

The reviewer stated that there were no issues here.

\section{Reviewer 2:}

The reviewer stated that INL and Intertek are well suited partners for these type of activities, each bringing complementary skills and expertise to the project. INL demonstrates exceptional leadership to organize the project with focused deliverables.

\section{Reviewer 3:}

The reviewer stated that taxis and Via would have good data, especially with taxis in New York City. The reviewer added that as much sharing as possible with OEMs would help leverage work. Follow up questions after the presentation indicated OEMs are involved.

Question 4: Proposed future research-the degree to which the project has effectively planned its future work in a logical manner by incorporating appropriate decision points, considering barriers to the realization of the proposed technology and, when sensible, mitigating risk by providing alternate development pathways.

\section{Reviewer 1:}

The reviewer stated that looking forward to the effect of electric propulsion to such areas as autonomous vehicle is paramount to the greater goals of EV acceptance and petroleum displacement. This project presentation brings that clarity to the future work.

\section{Reviewer 2:}

The reviewer stated that increased and enhanced data collection and exchange with national laboratories to support modeling and analysis for possible DOE VTO projects should help inform DOE of what projects to run. The reviewer questioned if the project team can get a hold of new vehicles before they are released, to help OEMs test and influence the tweaking of designs, or possibly the project team and DOE prefer testing to be done on production vehicles.

\section{Reviewer 3:}

The reviewer believed that Advanced Vehicle Test fleet can be leveraged to provide even more benefits by including a limited level of emissions testing during real world driving. This can provide an independent evaluation 
of the emissions reduction capability of these vehicles, similar to the work done by the International Council on Clean Transportation in uncovering the Volkswagen diesel issue.

\section{Reviewer 4:}

The reviewer thought that the project team needs more diverse users and must test in cold climates.

\section{Question 5: Does this project support the overall DOE objectives of petroleum displacement? Why or why not?}

\section{Reviewer 1:}

The reviewer stated that the data collected will help the EV industry optimize and gain some scale.

\section{Reviewer 2:}

The reviewer commented that this project brings needed data for consumer choice and modeling validation, which is necessary for adoption and greater goals of petroleum displacement.

\section{Reviewer 3:}

The reviewer stated that the test data are necessary to show how hybrids are saving fuel. Demonstrating how the vehicles age should increase trust of the car buying public in hybrids.

\section{Reviewer 4:}

The reviewer stated yes, by providing more real-world data to the consumer, and helping the consumer make a more informed selection. On the other hand, the reviewer believed much more benefit can be had by publicizing the website and its contents more. The reviewer acknowledged familiarity with the advanced vehicle testing activity (AVTA) testing for over seven years now, and knows where to look for it. However, the reviewer was not entirely sure that the average consumer knows that such a resource is available. A Google search for AVTA yields Antelope Valley Transit Authority. A Wikipedia page on fuel economy does not appear to have any reference to the AVTA site either. There appears to be no links to the AVTA website on www.fueleconomy.gov, which the reviewer thought was inexcusable. As a taxpayer, the reviewer believed that tax dollars are being put to very good use when they are spent on these kinds of testing activity, but also believed that there could be much more bang for the buck if the site was publicized more. More publicity could get more people within the United States to utilize the information that the AVTA website contains.

\section{Question 6: Resources: How sufficient are the resources for the project to achieve the stated milestones in a timely fashion?}

\section{Reviewer 1:}

The reviewer stated that it is not $100 \%$ clear that sufficient testers or people for the time remaining exist to complete the project.

\section{Reviewer 2:}

The reviewer stated that though resources are always spread thin for this type of expensive project, the project team appeared to have leveraged some excellent partners.

\section{Reviewer 3:}

The reviewer said that more of this kind of work can be done, but given the budget pressures, believed this project has the right level of funding. 
Advanced Vehicle Testing and Evaluation: Richard Jacobson (Intertek) - vs029

Presenter

Jeremy Diez, Intertek

\section{Reviewer Sample Size}

A total of five reviewers evaluated this project.

\section{Question 1: Approach to performing the work-the degree to which technical barriers are addressed, the project is well-designed, feasible, and integrated with other efforts.}

\section{Reviewer 1:}

The reviewer thought that the approach is good. However, questioned if the technical barrier is really "risk aversion from OEMs." The main barrier the reviewer saw was public resistance/ reluctance to buying advanced technology vehicles. This is where the program has value, by demonstrating long-term benefits of these vehicles.

\section{Reviewer 2:}

The reviewer commented that this project demonstrates a well-organized and well-managed program with clear objectives and goals.

\section{Reviewer 3:}

The reviewer stated that the approach of this project is adequate. By providing baseline, interim component and end of life testing a good set of data will be provided. A strong area of the project's approach is that it includes a very good set of vehicles being evaluated in areas of climate diversity.

\section{Reviewer 4:}

The reviewer stated that it is a good approach to monitor $12 \mathrm{~V}$ loads, but questioned how the components are picked. The reviewer added that it is great to check the components throughout the life of the vehicle, but wanted to know what the results were, besides the two vehicles that failed the transmissions. It would be helpful to get someone involved, possibly the OEM or a lab, to do root cause on the failures of the transmissions. This information could be used to improve components and perhaps system design. The reviewer commented that a good range of vehicles of consumer type hybrids was chosen. The MD trucks will be an important add in the future. The suggested direction of some cold climate testing is a good one; Colorado can get a little cold, but not like Minnesota or Alaska.

Question 2: Technical accomplishments and progress toward overall project and DOE goals-the degree to which progress has been made, measured against performance indicators and demonstrated progress towards DOE goals. 


\section{Reviewer 1:}

The reviewer thought that the data from this project is well intended to inform the DOE for future research and consumer information necessary to support widespread acceptance of plug-in/hybrid/electric (xEV) technology.

\section{Reviewer 2:}

The reviewer stated that the data collected is valuable and provides information that might be otherwise unavailable. It is useful for analysis supporting DOE goals of investing in technology for petroleum displacement and clarifying technology benefits for regulatory agencies (e.g., California Air Resources Board questions on plugin hybrid electric vehicles).

\section{Reviewer 3:}

The reviewer noted that many miles and components were tested for the money spent. The reviewer questioned if the $12 \mathrm{~V}$ testing gave an idea of the breakdown of auxiliary loads.

\section{Reviewer 4:}

The reviewer noted that there appears to be good technical accomplishments in the project, but it would be useful if more data could be provided on the vehicles that have been tested.

\section{Question 3: Collaboration and coordination with other institutions.}

\section{Reviewer 1:}

The reviewer noted that the array of national laboratories for technical support and fleet partnerships for road data are an outstanding example of quality coordination.

\section{Reviewer 2:}

The reviewer stated that the testing program is logistically challenging and requires a good deal of collaboration between various government and industry entities. The project team did very good coordination work.

\section{Reviewer 3:}

The reviewer found that the project has good collaboration with a variety of national laboratories. In the future it would be good if the project could also include OEMs as collaborators.

\section{Reviewer 4:}

The reviewer stated that there was good collaboration and breakdown in duties between INL, ANL, and NREL. Also, good fleet partners were selected. The reviewer was hopeful that the OEMs would join the project. Their involvement would decrease the amount of money needed to be spent, as the reviewer would imagine they could make testing of the vehicles and their components much easier. Agreements would have to be drawn up with them so that they feel they cannot be hurt in any way with the data obtained. Finally, the reviewer thought that a MD fleet that wants to test fuel saving technology needs to be found.

\section{Reviewer 5:}

The reviewer stated that there should be a diversified end user group providing data instead of the two fleets. Also, varied climates need to be incorporated.

\section{Question 4: Proposed future research-the degree to which the project has effectively planned its future work in a logical manner by incorporating appropriate decision points, considering barriers to the realization of the proposed technology and, when sensible, mitigating risk by providing alternate development pathways.}

\section{Reviewer 1:}

The reviewer noted that varied climates, including extreme cold and poor weather areas are included, like Chicago.

\section{Reviewer 2:}

The reviewer stated that the future work indicated for the project will continue to provide the necessary data needed to evaluate the advanced vehicle technologies and should provide information to help eliminate barriers. 


\section{Reviewer 3:}

The reviewer noted that as this project scope is quite long term, necessary for acquiring statistically relevant data, and that continuation with consistent data gathering is necessary.

\section{Reviewer 4:}

The reviewer liked the idea of investigating how the secondary-use market will be impacted by the battery condition. Also, finding out how battery condition at the end of the first life might influence the secondary lease/ sale auto market. The reviewer suggested a colder location than Colorado that also is close enough/convenient enough to get to.

\section{Reviewer 5:}

The reviewer found that the project was generally excellent, but possible reliance on future participation by OEMs seems questionable. OEMs would need to be convinced of more specific benefits. Also, the study loses some aura of independence if OEMs are providing vehicles.

\section{Question 5: Does this project support the overall DOE objectives of petroleum displacement? Why or why not?}

\section{Reviewer 1:}

The reviewer stated that this testing will validate electric propulsion vehicles.

\section{Reviewer 2:}

The reviewer believed that this demonstrates real-world benefits of advanced technology vehicles to the general public and provides data on benefits of specific technologies.

\section{Reviewer 3:}

The reviewer found that the project is definitely relevant and supports the overall DOE objectives of petroleum displacement. Testing and evaluating advanced vehicle technologies in fleet applications will provide good information to DOE about the state-of-the-art of these vehicles.

\section{Reviewer 4:}

The reviewer noted that the project provides real-world data to support consumer decisions and widespread acceptance of $x E V$ technology.

\section{Reviewer 5:}

The reviewer stated that the test data are key to lead the direction for future products which save fuel.

\section{Question 6: Resources: How sufficient are the resources for the project to achieve the stated milestones in a timely fashion?}

\section{Reviewer 1:}

The reviewer did not think it was clear that enough money has been, or will be, allocated to support the future research.

\section{Reviewer 2:}

The reviewer had the impression that budgetary constraints are limiting the test program more than in prior years.

\section{Reviewer 3:}

The resources stated that resources for this type of vehicle data can be endless, but the project appears to be meeting goals with present funding.

\section{Reviewer 4:}

The reviewer noted that the resources for this project seem to be adequate to accomplish the effort.

\section{Reviewer 5:}

The reviewer thought the project teams needs more diverse users and must go to cold places as heater, wipers, and defrosters place much more demand on the complete system. 


\section{Advanced Technology Vehicle Lab Benchmarking (L1 and L2): Kevin Stutenberg (Argonne National Laboratory) - vs030}

Presenter

Kevin Stutenberg, Argonne National Laboratory

\section{Reviewer Sample Size}

A total of five reviewers evaluated this project.

\section{Question 1: Approach to performing the work-the degree to which technical barriers are addressed, the project is well-designed, feasible, and integrated with other efforts.}

\section{Reviewer 1:}

The reviewer found that the testing work is sharply focused on obtaining vehicle data that are used in support of DOE projects and goals.

\section{Reviewer 2:}

The reviewer stated that testing under real-world conditions significantly enhances the value of the data, allowing it to inform off-cycle benefits of technology.

\section{Reviewer 3:}

The reviewer though it was a good idea to look at the beginning and end of life, as this project is doing. ANL fits into the partnership by doing the dyno testing. The in-vehicle component and standardized cycle testing on the vehicle level are needed. The reviewer understood that Level 2 testing is more invasive. If OEMs get the results and can use them, the purchase price seems like something they would be willing to donate. Finally, the reviewer questioned how the technology to be examined is determined.

\section{Reviewer 4:}

The reviewer stated that ANL has done great work with both the Level 1 and Level 2 testing. The reviewer was concerned with some of the issues faced with developing models for simulating the 5-cycle test; it is hard to obtain efficiency data for driveline components for low-temperature operation. Testing the components on a stand using a large reservoir of the lubricant maintained at the specified temperature is rather hard, and not quite representative. The reviewer wondered if ANL could add some extra instrumentation (torque sensors) and measure efficiencies in-situ, perhaps under steady state conditions to avoid driveline dynamic behavior, and use that to characterize component efficiencies.

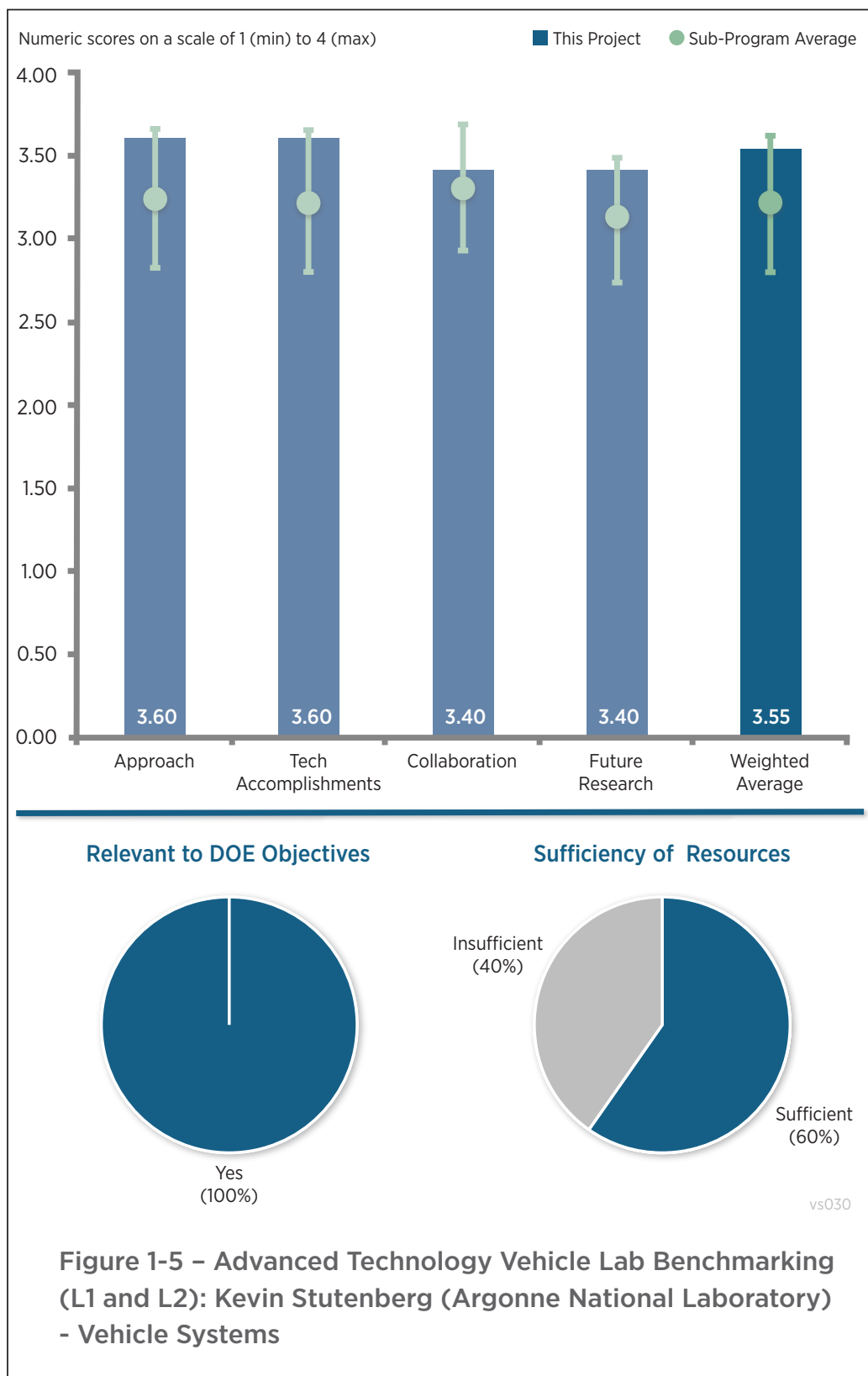




\section{Reviewer 5:}

The reviewer suggested some visible barrier flow-down from industry to perhaps guide the deep-dive testing program and provide this industry collaborative strategy in these AMR reviews. In other words, the reviewer questioned if the tough problems that OEMs or suppliers face today are getting enough attention in using the target vehicles and data from the benchmarking project most strategically to move the needle in the commercial world. The reviewer added that the slides say this is happening as part of the planning effort, but examples of this direct linkage would be useful. Overall the testing program is impressive, seems solid and well-refined over years of honing.

\section{Question 2: Technical accomplishments and progress toward overall project and DOE goals-the degree to which progress has been made, measured against performance indicators and demonstrated progress towards DOE goals.}

\section{Reviewer 1:}

The reviewer thought that this was excellent work, as always.

\section{Reviewer 2}

The reviewer stated that progress and technical accomplishments continue to be very good, with excellent reporting and analysis of the test data and good distribution of info to various partners. The public-facing data storehouse is a good way to disseminate data to the general public.

\section{Reviewer 3:}

The reviewer commented that there was good progress overall, but there are significant gaps in the range of vehicles that were tested due to budgetary and infrastructure constraints. Additional care needs to be taken to ensure that the most relevant and rapidly penetrating technologies are assessed.

\section{Reviewer 4:}

The reviewer found the result that heat pump/heater usage at cold temperatures is a big hit to the battery was an important result. That is a good comparison of energy needed for each vehicle for heat by the uses. The reviewer wanted to know who will look into why the Golf is lower, and what about their approach requires less energy.

\section{Reviewer 5:}

The reviewer stated that the project directly aligned with DOE missions/goals by continuously providing controlled data acquisition and providing a valuable service to the industry.

\section{Question 3: Collaboration and coordination with other institutions.}

\section{Reviewer 1:}

The reviewer stated that collaborations with appropriate laboratories and industry partners is thorough.

\section{Reviewer 2:}

The reviewer stated that there was good representation across industry and laboratories, and noted the comments in the approach section in terms of alignment to industry's strategic needs (for benchmarking data).

\section{Reviewer 3:}

Overall, the reviewer thought that the collaboration between the various labs is excellent. The extent of OEM involvement is quite good as well. The recent proposal by ANL to get the OEMs to have skin in the game by paying for the vehicle purchase, while the testing would be funded by DOE is a very interesting one, and should be followed with more vigor. The reviewer also commented about one of the observations made in last year's review regarding the exclusion of the U.S. Environmental Protection Agency (EPA). While the reviewer tended to agree with that observation, and felt that ANL could involve EPA more extensively, it could also be said that by working separately, ANL and EPA provide two neutral viewpoints.

\section{Reviewer 4:}

The reviewer noted that additional efforts to engage OEMs and Tier 1 suppliers would allow for more meaningful testing. The reviewer added that the engagement on the codes/standards efforts is good. 


\section{Reviewer 5:}

The reviewer found that the codes and standards, and modeling support are good areas for this project in which to collaborate. Some data are available for download, hopefully most of it, and U.S. Driving Research and Innovation for Vehicle Efficiency and Energy sustainability (U.S. DRIVE) also has some of the data available. The reviewer noted that there are many different collaborations, but asked if there are any OEMs. The reviewer stated that the project team and OEMs should be in this effort to characterize their vehicles together. Both organizations want to see more hybrids and battery electric vehicles (BEVs) sold.

\section{Question 4: Proposed future research-the degree to which the project has effectively planned its future work in a logical manner by incorporating appropriate decision points, considering barriers to the realization of the proposed technology and, when sensible, mitigating risk by providing alternate development pathways.}

\section{Reviewer 1:}

The reviewer thought that the budget is too constrained to allow for future work to be fully completed.

\section{Reviewer 2:}

The reviewer stated that by necessity, future work is dependent on what new advanced tech vehicles are available.

\section{Reviewer 3:}

The reviewer asked if the project team can tie the results to changes in hardware or software used by the OEMs. If so, it might make it easier to keep the budget. The reviewer added that getting the results analyzed and reported is very important.

\section{Reviewer 4:}

The reviewer stated that the inclusion of end-of-life assessments is a good supplement for future work. Of course, benchmarking data acquisition is a never-ending process so future work is generally similar work with new products and measurement technology advancements. The reviewer noted that any comments about how the measurement/analysis landscape has changed over time would be welcome.

\section{Reviewer 5:}

The reviewer suggested that ANL should look at measuring the emissions on some of some of the non-standard cycles that they run as well, to get a better understanding of how good the vehicle emissions are in real world driving.

\section{Question 5: Does this project support the overall DOE objectives of petroleum displacement? Why or why not?}

\section{Reviewer 1:}

The reviewer stated that this project provides hard data on benefits of advanced technology vehicles, and explains the mechanisms for fuel savings. This provides guidance on which technologies are most effective in achieving petroleum displacement.

\section{Reviewer 2:}

The reviewer commented that assessing the benefit of new technologies seems to be informing DOE on future areas to target for funding.

\section{Reviewer 3:}

The reviewer thought that this project is very relevant, especially if OEMs are using it to drive improvements in their design.

\section{Reviewer 4:}

The reviewer thought that supporting activity to the front lines is relevant to the DOE objectives.

\section{Reviewer 5:}

The reviewer stated that this project feeds into all the modeling activity as well as supports much of the AVTA. 


\section{Question 6: Resources: How sufficient are the resources for the project to achieve the stated milestones in a timely fashion?}

Reviewer 1:

The reviewer thought that further funding is needed to better assess rapidly evolving technologies in the marketplace.

\section{Reviewer 2:}

The reviewer asked how the project team would determine that start-stop may not make the cut. That indicates this area needs more money, asserted the reviewer.

\section{Reviewer 3:}

The reviewer believed that ANL is obtaining a wealth of data, especially from the Level 2 testing, and there could be significant knowledge gained if there were more resources dedicated to understanding the data better.

\section{Reviewer 4:}

The reviewer stated that the milestones are designed with budgetary constraints in mind, so by definition they are sufficient. However, if the desire is to accelerate the dissemination of data, then resources are insufficient.

\section{Reviewer 5:}

The reviewer noted that activity will simply fill the budget available. The numbers of samples per year appears appropriate for the pace the technology is fundamentally advancing. 


\section{SuperTruck - Development and Demonstration of a Fuel- Efficient Class 8 Tractor and Trailer Vehicle: Russ Zukouski (Navistar International Corporation) - vs064}

\section{Presenter}

Russ Zukouski, Navistar International Corporation

\section{Reviewer Sample Size}

A total of five reviewers evaluated this project.

\section{Question 1: Approach to performing the work-the degree to which technical barriers are addressed, the project is well-designed, feasible, and integrated with other efforts.}

\section{Reviewer 1}

The reviewer stated that all of the technical barriers appear to have been addressed in a well-designed manner and the project is producing meaningful results. The project team is doing a good job of approaching the efficiency challenge with a wholesystems approach.

\section{Reviewer 2:}

The reviewer stated that the approach of dividing the project into four focus areas was highly successful.

\section{Reviewer 3:}

The reviewer noted that the approach to restart is very strong. T3 testing and $\mathrm{T} 4$ plans are exhaustive and the project team should learn much from these two builds and test opportunities.

\section{Reviewer 4:}

The reviewer stated that the approach contains all the key enabling technologies that can help the program to achieve the goal. The reviewer noted it is unclear how the hybrid portion of technologies is contributing to overall improvement.

\section{Reviewer 5:}

The reviewer noted that the presentation described the team's approach to pursue fuel savings in four areas: lightweighting; rolling resistance; aerodynamic improvement; and powertrain technologies. The original approach called for use of an e-Turbo, but that was dropped due to problems. The reviewer stated that it seems like a good strategy was used to focus on waste heat recovery (WHR) and other technologies to adapt to this issue. The planned approach for testing includes multiple different drive cycles weighted together. This approach to consider multiple cycle effects is good, but it would have been nice to understand how the cycles and weights were chosen.

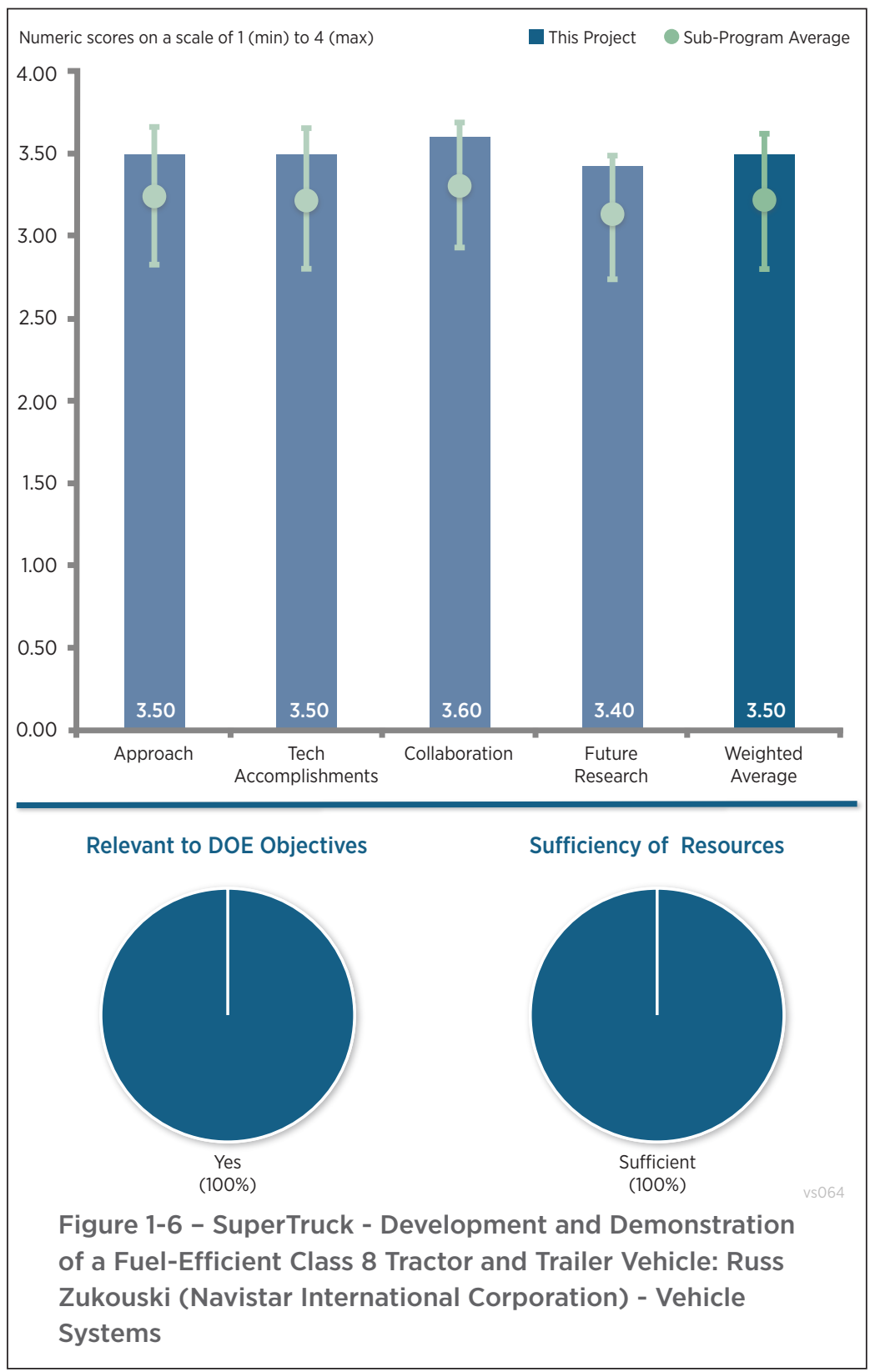

Vehicle Systems 
Furthermore, the reviewer asked why there is not a test component to capture the relative impact of overnight idling in the conventional baseline compared with running off the $48 \mathrm{~V}$ battery then recharging while driving during the next day.

\section{Question 2: Technical accomplishments and progress toward overall project and DOE goals - the degree to which progress has been made, measured against performance indicators and demonstrated progress towards DOE goals.}

\section{Reviewer 1:}

The reviewer stated that the project team is very close to the $50 \%$ brake thermal efficiency (BTE) goal and the $82.3 \%$ freight efficiency improved passed the goal. In spite of the period pause, their schedule has produced significant accomplishments.

\section{Reviewer 2:}

The reviewer noted that Navistar achieved the project goals with room to spare. According to the reviewer, the list of improvements is very long, and it was unfortunate that there was only 20 minutes to learn about them.

\section{Reviewer 3:}

The reviewer stated that following a pause on the project a couple of years ago it seems like the team has made good accomplishments in all four areas of fuel efficiency improvement: both for the T3 truck generation and so far on components incorporated into the T4 generation. The team seems on track to achieve the goals of the program with the upcoming demonstration and testing of the $\mathrm{T} 4$ vehicle. The reviewer thought it was great to hear that the electrified air conditioning system not only saves fuel, but also saves cost by enabling one electric compressor to replace what had previously been two compressors, one to cool the cab and another for the sleeper area. The reviewer also appreciated that the majority of the features in the T4 vehicle will get carried forward into new production vehicles.

\section{Reviewer 4:}

The reviewer was impressed with the technical accomplishments after the restart. The reviewer exclaimed that it can be very difficult to restore momentum on a project such as this and the team has, which was well done. The fuel economy performance of 10.45 after 10,000 miles is in line with expectations and should improve given the plans with T4. The reviewer also observed some shifts made with new learnings (e.g., WHR and e-Turbo).

\section{Reviewer 5:}

The reviewer stated that the $64 \%$ improvement is excellent considering that this is not the final package. However, the schedule is so much behind their competitors, that it would not look too good when SuperTruck II is coming. The reviewer questioned how many miles do the trucks run in the driving route for vehicle tests. Furthermore, the reviewer asked what the road grade is and the baseline miles per gallon (MPG).

\section{Question 3: Collaboration and coordination with other institutions.}

\section{Reviewer 1:}

The reviewer thought that this project has an outstanding set of partners.

\section{Reviewer 2:}

The reviewer stated that all of their partners appear to be fully engaged in the project.

\section{Reviewer 3:}

The reviewer stated that the project seemed to have a good list of partners and coordination/contribution from the partners. These included Bosch, Wabash, ANL, LLNL, Eaton, Dana, and Hendrickson.

\section{Reviewer 4:}

The reviewer stated that working with all key partners is excellent, as proposed.

\section{Reviewer 5:}

The reviewer did not see as much evidence of collaboration as expected. 
Question 4: Proposed future research-the degree to which the project has effectively planned its future work in a logical manner by incorporating appropriate decision points, considering barriers to the realization of the proposed technology and, when sensible, mitigating risk by providing alternate development pathways.

\section{Reviewer 1:}

The reviewer found the plan to be solid and comprehensive.

\section{Reviewer 2:}

The reviewer noted that the project's future work will focus on completing the build, integration/calibration, and testing of the T4 truck. Again, most promising is the future plans beyond the project to incorporate most of the efficiency features from the program into future production vehicles.

\section{Reviewer 3:}

The reviewer acknowledged being very interested to see the progress and results of T4, new cab shape, and other planned improvements.

\section{Reviewer 4:}

The reviewer noted that this project is nearing completion and is therefore short on future plans.

\section{Reviewer 5:}

The reviewer stated that the project team is done, too, and will include many of the features into their commercial product.

\section{Question 5: Does this project support the overall DOE objectives of petroleum displacement? Why or why not?}

\section{Reviewer 1:}

The reviewer thought that the returns on investment in long-haul fuel economy will be huge.

\section{Reviewer 2:}

The reviewer stated that the project has demonstrated significant petroleum displacement with its efficiency improvements.

\section{Reviewer 3:}

The reviewer noted that the program goals of increasing freight efficiency by over $50 \%$ and showing a path to $55 \%$ BTE are highly relevant to achieving national petroleum displacement, and this project appears on track to meet these goals.

\section{Reviewer 4:}

The reviewer stated that freight efficiency improvement is always in line with DOE objectives.

\section{Question 6: Resources: How sufficient are the resources for the project to achieve the stated milestones in a timely fashion?}

\section{Reviewer 1:}

The reviewer stated that, while $\$ 76$ million is significant funding, the upside petroleum reduction potential to the trucking industry is significant.

\section{Reviewer 2:}

The reviewer stated that progress is good and proves sufficient resources have been attained even after the restart.

\section{Reviewer 3:}

The reviewer commented that this was a huge job, requiring big bucks.

\section{Reviewer 4:}

The reviewer stated that this project represented a major funding effort, including over $\$ 35$ million in DOE funds over the course of the project and roughly $\$ 5$ million in FY 2016. This level of funding warrants more scrutiny than 
the reviewer can provide from a 20 minute presentation, and hoped that the DOE program managers have provided this scrutiny through the life of the project. It does seem like the team is realizing good results from the project funding.

Reviewer 5:

The reviewer stated that there seems to be sufficient to complete the program, but timing is a big concern when all other competitors all completed their goals. 


\section{Commercial Vehicle Thermal Load Reduction and VTCab-Rapid HVAC Load Estimation Tool: Jason Lustbader (National Renewable Energy Laboratory) - vs075}

\section{Presenter}

Jason Lustbader, National Renewable Energy Laboratory

\section{Reviewer Sample Size}

A total of four reviewers evaluated this project.

\section{Question 1: Approach to performing the work-the degree to which technical barriers are addressed, the project is well-designed, feasible, and integrated with other efforts.}

\section{Reviewer 1:}

The reviewer thought that the project has an appropriate scope and approach to meet the objectives, and that it was well presented and complete.

\section{Reviewer 2:}

The reviewer noted that the presentation graphics suggest that analysis possibly omits the effects of engine and pavement as a heat source.

\section{Reviewer 3:}

The reviewer stated that the project is very focused on reducing the load to benefit the plethora of options existing to help improve their efficiency. Expansion into other sectors is good, but should stay equally focused on those that need it. The reviewer commented that without sleeping in the trucks, the payback may be very limiting. Buses, yes, others maybe not.

\section{Reviewer 4:}

The reviewer stated that the project aims to increase the fuel efficiency of commercial vehicles by reduce the thermal load of the vehicles. A numerical tool is being developed for fast quantification of thermal load reduction solutions. Experimental testing and numerical modeling methods, which is typical, were adopted to evaluate the impact of the solution. The solution is a combination of ultra-white paint, advanced curtains, and insulations. The reviewer wondered why these three methods were adopted and whether there were any other methods being considered before coming to the final solution. 
Question 2: Technical accomplishments and progress toward overall project and DOE goals - the degree to which progress has been made, measured against performance indicators and demonstrated progress towards DOE goals.

\section{Reviewer 1:}

The reviewer found that the accomplishments of the project are impressive, taking three base technology areas and finding quite a bit of performance for minor improvements.

\section{Reviewer 2:}

The reviewer stated that the technical accomplishments and progress that have been made so far are impressive. The predicted payback time is three years, although this reviewer wondered about the collaborating OEMs' opinion on this and their future plan.

\section{Reviewer 3:}

The reviewer observed excellent progress. Acknowledging that it may be irrelevant, this reviewer had a little trouble following whether the accomplishments were a result of the 2015/2016 work or from the prior project.

\section{Question 3: Collaboration and coordination with other institutions.}

\section{Reviewer 1:}

The reviewer stated that the collaboration with the trucking industry is excellent!

\section{Reviewer 2:}

The reviewer stated that these features will be offered by the truck OEMs, so it is good to work with the truck builders. The reviewer did think better promotion of this with fleets will help pull the changes into more trucks

\section{Reviewer 3:}

The reviewer noted that OEMs of long-haul trucks and suppliers of the insulation solutions are engaged in this project. If the presentation can provide OEMs' opinion and future plan as the project moving forward, that would be great.

\section{Question 4: Proposed future research-the degree to which the project has effectively planned its future work in a logical manner by incorporating appropriate decision points, considering barriers to the realization of the proposed technology and, when sensible, mitigating risk by providing alternate development pathways.}

\section{Reviewer 1:}

The reviewer commented that the future work is well planned and will cover some other applications, e.g., day cab, buses, etc.

\section{Reviewer 2:}

The reviewer was sure the value exists in other applications. Keeping a high requirement for engagement and analysis is suggested.

\section{Reviewer 3:}

The reviewer stated that the future research proposal for calendar year (CY) 2016 and 2017 is a natural follow on. It would be good to see this work be included or brought into collaboration with SuperTruck at some level.

\section{Question 5: Does this project support the overall DOE objectives of petroleum displacement? Why or why not?}

\section{Reviewer 1:}

The reviewer stated that significant amounts of petroleum are consumed to satisfy HD auxiliary loads. This analytic capability offers OEMs and fleet owners the capability to quantitatively estimate ROI for investments in technologies that reduce petroleum consumption. 


\section{Reviewer 2:}

The reviewer thought that this is very relevant to the goals of the DOE, $21^{\text {st }}$ Century Truck Project, and the GHG regulations of EPA and the National Highway Traffic Safety Administration (NHTSA). The reviewer stated that the team did a good job.

\section{Reviewer 3:}

The reviewer noted that the project investigates the energy lost during the truck downtime, a substantial use of energy without transporting goods. The results, with some additions, could apply to both active as well as downtime. The reviewer added that this definitely applies to the DOE objective of petroleum displacement through efficiency.

\section{Reviewer 4:}

The reviewer stated that fuel efficiency improvement of commercial trucks is an important part of DOE's overall objective of energy saving and petroleum displacement.

\section{Question 6: Resources: How sufficient are the resources for the project to achieve the stated milestones in a timely fashion?}

\section{Reviewer 1:}

The reviewer stated that it seems sufficient for the great progress

\section{Reviewer 2:}

The reviewer found that the project appears to be adequately resourced.

\section{Reviewer 3:}

The reviewer stated that NREL has been collaborating with Volvo Trucks and Daimler Trucks for a while and they have the ability and sufficient resources to finish the project in time. The reviewer is looking forward to extensive participation and input from Volvo Trucks and Daimler Trucks. 
Volvo SuperTruck: Pascal Amar (Volvo Trucks) - vs081

Presenter

Pascal Amar, Volvo Trucks

Reviewer Sample Size

A total of six reviewers evaluated this project.

\section{Question 1: Approach to performing the work-the degree to which technical barriers are addressed, the project is well-designed, feasible, and integrated with other efforts.}

\section{Reviewer 1:}

The reviewer stated that no criticism of this project is possible given the outstanding freight efficiency improvements.

\section{Reviewer 2:}

The reviewer commented that the combination of simulation and testing yielded excellent results.

\section{Reviewer 3:}

The reviewer stated that Volvo's approach for the entire fouryear project has been robust and disciplined, and it delivered.

\section{Reviewer 4:}

The reviewer stated that this five-year effort has employed and successfully implemented a classical technical/ project approach. Phase One (Concept Selection) consisted of baseline test, technology development, and concepts evaluation through model development, engine and mule truck testing, and validation. Phase Two (Development and Integration) consisted of technology refinement and validation through engine bench testing and demonstrator truck development, and finally integration, optimization, and demonstration. The approach focused on achieving program goals through three pathways: fuel savings through various tractor and trailer technologies, hotel load savings measures, and overall vehicle weight reductions. This three-way approach provided flexibility to achieve project goals with each pathway playing a significant role. Finally, the approach employed a variety of robust simulation and testing mechanisms to guide technology selection and component sizing, develop algorithms, and ultimately verify freight efficiency improvements under controlled tests for repeatability and real world conditions. The reviewer believed that the early project focus (in Phase One) on mid-term freight efficiency impacts and customer ROI was a key element. Overall, a very strong approach which has led to project success.

\section{Reviewer 5:}

The reviewer stated that the approach includes all key enabling technologies, which is comprehensive.

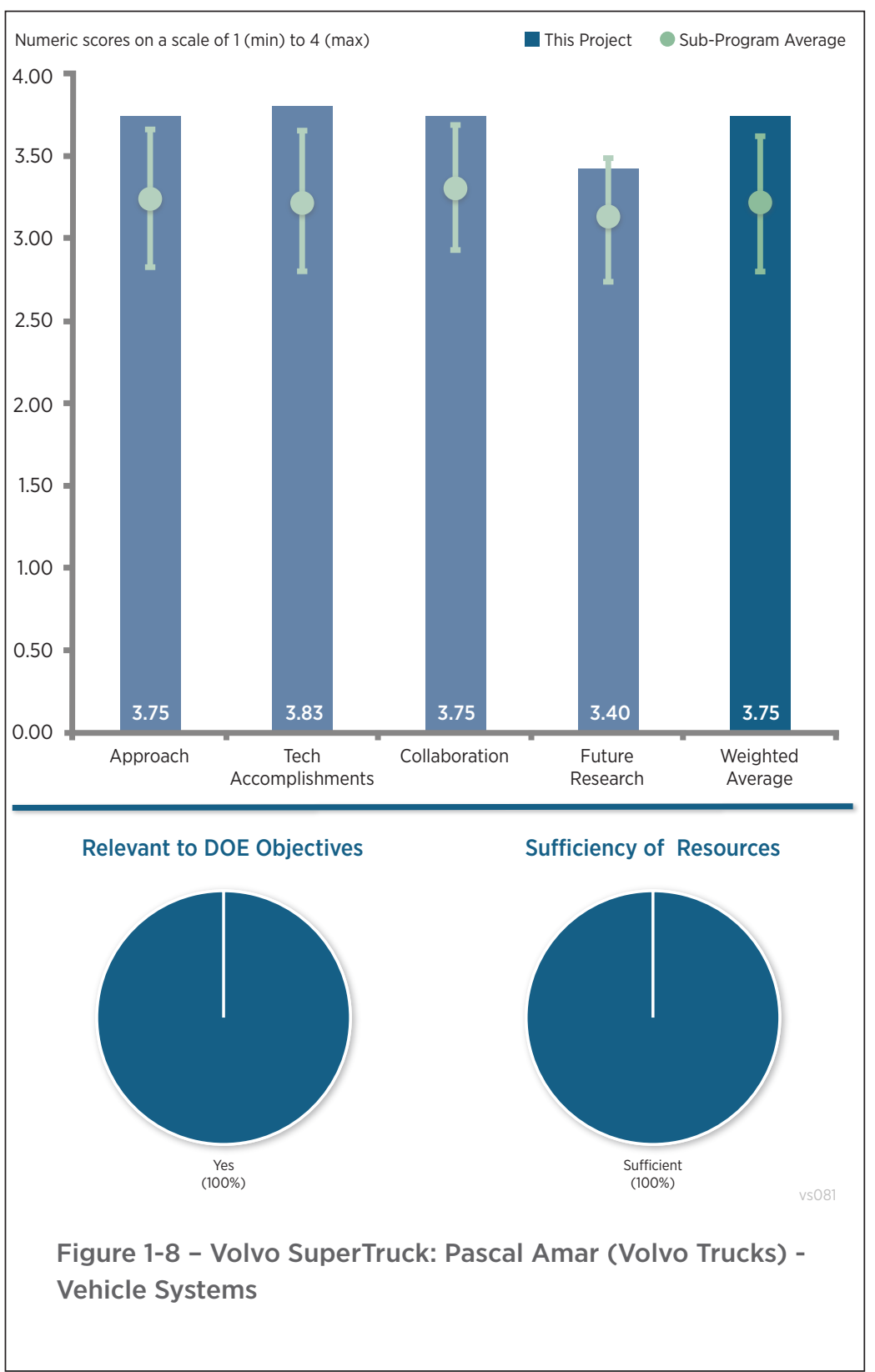




\section{Reviewer 6:}

The reviewer thought that the project had a good approach.

\section{Question 2: Comments on technical accomplishments and progress:}

\section{Reviewer 1:}

The reviewer exclaimed that with a goal of 50\% MPG improvement, the project team achieved $70 \%$ (and even higher on freight efficiency), which is pretty outstanding.

\section{Reviewer 2:}

The reviewer stated that the project far exceeded the goals, and received an award because of it. The improvements were sound and kept to what was viable and implementable. Outstanding!

\section{Reviewer 3:}

The reviewer found the freight efficiency strong and in line with features delivered. The project team developed some stretch goals, big and aggressive, for instance, all-aluminum frame rails.

\section{Reviewer 4:}

The reviewer stated that the fuel economy and freight efficiency improvement far exceeded the project goals, while also reducing vehicle weight and improving the payload capacity.

\section{Reviewer 5:}

The reviewer stated that the project is now completed and has demonstrated and verified a long list of successes, including surpassing the program goal for fuel economy (69\% as opposed to target of 50\%) and achieving $88 \%$ freight efficiency. Equally as important is the fact that several team members have already commercialized a number of technologies resulting from this project. Specifically, in 2015 improved trailer aero devices were implemented, in 2016 tractor aero improvements have been commercialized, and in 2017 powertrain improvements are being commercially implemented in 11 liter and 13 liter engines. Specifically, in 2016 chassis fairing and roof and bumper aero improvements have been launched in all Volvo vehicles. As a result, a lot of technologies are already making it to the customer.

The reviewer also noted that an interesting slide was presented outlining the technology readiness levels and customer pay back of a variety of the technologies examined. The ROI on the different technologies varies significantly depending upon the specific duty cycle and customer needs.

\section{Reviewer 6:}

The reviewer was very impressed with the achievement of $88 \%$ improvement. Specifically completed the goal one year shorter than their major competitors. Slide 6 is an excellent slide to show the individual key components and technology contribution to total improvement. However, the reviewer stated that it is not clear how the truck was tested for that $88 \%$ fuel economy improvement. The reviewer questioned how many miles were really running, at what road grade, and the baseline MPG.

\section{Question 3: Collaboration and coordination with other institutions.}

\section{Reviewer 1:}

The reviewer stated that partner coordination and participation appeared to be very strong, with each contributing in their area of expertise.

\section{Reviewer 2:}

The reviewer stated that the project team had a long list of collaborators, and together they got the job done.

\section{Reviewer 3:}

The reviewer noted that good collaboration was evident, and wants to see it continue with features going to production

\section{Reviewer 4:}

The reviewer stated that the project has incorporated a strong and diverse project team from the beginning, 
covering all the requisite areas. This team has helped bring the project to successful fruition and currently serves to promote commercialization.

\section{Reviewer 5:}

The reviewer stated that the project team utilized all partners to complete the program goal.

\section{Reviewer 6:}

The reviewer thought the project had a good team.

Question 4: Proposed future research-the degree to which the project has effectively planned its future work in a logical manner by incorporating appropriate decision points, considering barriers to the realization of the proposed technology and, when sensible, mitigating risk by providing alternate development pathways.

\section{Reviewer 1:}

The reviewer noted that the project team is done. The only suggestion of future work was coming up with lessexpensive alternatives for the small amount of carbon fiber used.

\section{Reviewer 2:}

The reviewer stated that the team wants to continue optimizations after the end of the project.

\section{Reviewer 3:}

The reviewer stated that the program is completed, and there is no need to detail the future work.

\section{Reviewer 4:}

The reviewer commented that as the project has ended, the proposed future research is limited. But, the PI does indicate efforts will be made to continue to evaluate the demonstrator vehicle to identify further areas of improvement, as well as possible additional candidate solutions for commercialization. Additionally, if not already planned, it may be beneficial to conduct a thorough project debrief to surface all the lessons learned over the past five years from this project and to use them to inform and guide similar future activities.

\section{Reviewer 5:}

The reviewer noted that none are really planned.

\section{Question 5: Does this project support the overall DOE objectives of petroleum displacement? Why or why not?}

\section{Reviewer 1:}

The reviewer stated that the project team is actually going to use much of what they did in their production vehicles, the reduction in petroleum use could be huge.

\section{Reviewer 2:}

The reviewer stated that the project exceeded the goals and directly supports the DOE objectives.

\section{Reviewer 3:}

The reviewer noted that the project team exceeded DOE's goals.

\section{Reviewer 4:}

The reviewer noted that HD transport is a major contributor to the nation's petroleum use and dependence, and added that it is actually the fastest growing component overall. Thereby, developing advanced technologies that improve the efficiency of the nation's trucking fleet and successfully implementing them will significantly contribute to meeting DOE's and the nation's petroleum displacement goals.

\section{Reviewer 5:}

The reviewer stated that freight efficiency is always in line with the DOE objectives.

Question 6: Resources: How sufficient are the resources for the project to achieve the stated milestones in a timely fashion? 


\section{Reviewer 1:}

The reviewer stated that it is amazing that Volvo is able to complete the program with the half of the funding compared to their competitors. The job was well done!

Reviewer 2:

The reviewer believed that there will be a big payback on the R\&D money spent.

Reviewer 3:

The reviewer stated that given the success, funding must have been sufficient.

Reviewer 4:

The reviewer stated that the resources (both financially and from a corporate capability perspective) have been sufficient, as demonstrated by the project's successful conclusion.

Reviewer 5:

The reviewer stated that this project is ending and has sufficient resources to close out. 


\section{System for Automatically Maintaining Pressure in a Commercial Truck Tire: Norm Anderson (The Goodyear Tire and Rubber Company) - vs085}

\section{Presenter}

Norm Anderson, Goodyear

\section{Reviewer Sample Size}

A total of four reviewers evaluated this project.

\section{Question 1: Approach to performing the work-the degree to which technical barriers are addressed, the project is well-designed, feasible, and integrated with other efforts.}

\section{Reviewer 1:}

The reviewer stated that the project's approach was specifically targeted to address issues arising from improperly inflated tires. The technology developed can be used on all vehicles, including in-use vehicles. The project also included surveying potential customers for interest in the technology. The project team also provided detailed analyses to support the rationale for the project. The reviewer found it interesting that despite providing a rationale focused on significant potential pressure losses without this technology (such as talk of the impact of a $20 \%$ pressure loss), the team's own testing appeared to indicate that their control tire lost 2.6 pounds per square inch (psi)/month compared to the technology under this project keeping this to a 0.3 psi/month drop, a much smaller difference. As fleet testing expands, it will be interesting to see how the technology-equipped and non-technology tires fare in comparison.

The reviewer added that the approach developed for the project was also interesting in that it originally included both internally- and externally-regulated systems. It was unfortunate that the internally-regulated system did not meet performance requirements, as that system may have been more interesting.

\section{Reviewer 2:}

The reviewer stated that the approach to develop a self-contained tire pressure maintenance system is reasonable and offers the potential for success. The switch from an internal pressure regulator (self-contained within the tire itself) versus an external regulator (which must be attached to the valve stem of the tire) is understandable, but there are implications in how complex the installation of the Air Maintenance Technology (AMT) tire is. The former design required no additional steps relative to a conventional tire, but this external regulator design 
requires an extra step to install the regulator on the stem. Perhaps this is a minor challenge, but fleets often look for operational reasons to avoid adopting a technology.

The reviewer continued, saying the approach to conduct real-world testing is very important, as it will demonstrate the benefits and challenges for the tires in operation. It is very good to talk directly to fleets about whether they would buy the system, as Goodyear is doing. This ensures that Goodyear understands how the technology will be received, increasing the chances for successful deployment. Goodyear is looking at multiple vocations (both long haul and regional haul), and looking at multiple tire positions on the truck, to increase the potential for market uptake.

\section{Reviewer 3:}

The reviewer stated that the overall approach is good: customer needs and interest were studied, some design requirements were specified, project management proved nimble enough to handle a major design change, and considerations were made for design for manufacturing and assembly. The approach to design verification is good. The first round of fleet testing resulted in variation in inflation loss. The reviewer noted that the project team determined that a leaking issue caused this variation. The product design was improved prior to the start of the second round of fleet testing (delayed to June). Time-history data collection made this possible. However, the reviewer believed that the design verification process could be better. New potential failure modes that may be introduced by the AMT system should be identified. The design verification plan should center on verifying those failure modes. Some of those failure modes have been exposed through standard lab and on-road testing, still some non-standard tests may be required to excite new failure modes.

The reviewer found that the combination of lab, track, fleet testing is good. A large amount of test miles have been accumulated. However, the author does not specify what constitutes a successful test. The reviewer commented that ideally, a statistically significant sample of tires should be tested to failure and cycles to failure compared against tires with AMT to verify superior life and that no new failure modes were introduced.

\section{Question 2: Technical accomplishments and progress toward overall project and DOE goals-the degree to which progress has been made, measured against performance indicators and demonstrated progress towards DOE goals.}

\section{Reviewer 1:}

The reviewer found that the team has successfully demonstrated the ability of the system to exceed the performance goals set for pumping rate, ensuring the system can maintain tire pressure appropriately. The performance of the external regulator (Goodyear's contingency plan) was acceptable, and solved issues seen with the internal regulator. This kept the basic DOE-funded concept of a tire with self-maintaining pressure viable. The reviewer noted that Goodyear has done extensive testing of the completed tires, in laboratory and real world testing with multiple trucks, demonstrating that the changes to the tires do not compromise their reliability and durability.

\section{Reviewer 2:}

The reviewer stated that during this year, the project made a no-go decision concerning the internal regulator, choosing the external regulator as the preferred solution. The devices went out for installation into test fleets this past February. Initial in-use tests appear to show monthly losses of $0.3 \mathrm{psi}$ (compared to $2.6 \mathrm{psi}$ for un-equipped tires). The original goal was to reduce psi loss to $1 \mathrm{psi} / 100$ miles. There were some leaking issues with the initial prototype on the first fleet, so there were some efforts to redesign slightly before application to additional fleets. The reviewer noted that the redesign did result in some delay moving out to the next fleets.

\section{Reviewer 3:}

The reviewer thought that the successful change in suppliers and design (internal compared to external regulator) is admirable. Size and weight were reduced, although it was not specified how much. The author does not address the approach to minimizing overall cost of pumping system including assembly, which was listed as a barrier to be overcome. The reviewer noted that two performance indicators are given. First, pumping rate exceeded bogey of greater than $1 \mathrm{psi} / 100$ miles. Lab and on-road testing demonstrated that the chosen design met the pumping rate 
requirement. Second, mean inflation loss was superior to tires without AMT. However, variation in inflation loss was significantly higher for tires with AMT.

The reviewer commented that while progress has been made with testing, it is unclear what the goals of testing are. Likewise, the manufacturing process was improved, but progress was not specified in terms of key metrics (production rate, process capability, scrap rate, process cost, etc.). The reviewer commented that at this stage in the project, the project team has not measured progress toward DOE goals. Fuel economy and life improvement targets must be validated. This is slated to be done as part of future work, but it behooves the project team to establish and communicate the validation plan.

\section{Question 3: Collaboration and coordination with other institutions.}

\section{Reviewer 1:}

The reviewer found that the collaborations were acceptable: most of this project is done by Goodyear with support from an (unnamed) set of suppliers for the pressure regulators. Goodyear has effectively collaborated with fleets to some extent by discussing the potential system with them to get feedback.

\section{Reviewer 2:}

The reviewer noted that suppliers provided prototype components and seemed to provide required support. Focus fleet testing is being performed by external customers. The author does not describe the terms of their participation, but apparently that interaction is contributing toward project goals.

\section{Reviewer 3:}

The reviewer stated that Goodyear has been the primary and largely lone member of the project team, working with several vendors for specific components/testing/assembly. Goodyear is also working with several fleets to conduct in-use testing. The reviewer commented that it is surprising to see this tight a circle of a team under a VS project, where there typically tends to be more collaborators. However, it seems largely appropriate for this project.

\section{Question 4: Proposed future research-the degree to which the project has effectively planned its future work in a logical manner by incorporating appropriate decision points, considering barriers to the realization of the proposed technology and, when sensible, mitigating risk by providing alternate development pathways.}

\section{Reviewer 1:}

The reviewer found that the future work appears acceptable and contains logical steps to wrap this project up in September. This reviewer would like to hear more about Goodyear's general plans and timetables for commercialization.

\section{Reviewer 2:}

The reviewer stated that the long-term trials planned, including retread testing and fuel economy evaluation, are important. The specific goals for testing should be communicated, including reaction plans if failures are experienced. Plans for product validation of tires with AMT produced using the production-intent assembly process should be established and communicated.

\section{Reviewer 3:}

The reviewer noted that the project is currently $90 \%$ complete, and scheduled to be finished in September 2016. This person indicated reported that there are still a number of important activities left to take place, which were clearly identified. Still, activities are significant enough that it could raise the question as to whether the project will be completed on time.

\section{Question 5: Does this project support the overall DOE objectives of petroleum displacement? Why or why not?}

\section{Reviewer 1:}

The reviewer stated that the project is focused upon increased efficiency through ensuring proper inflation of tires. This project has strong potential for relatively immediate impact upon in-use vehicles, resulting in near-term 
petroleum reduction, emissions reduction, and safety benefits. The reviewer commented that while the project's specific focus is on truck tires, the technology could be applied to LD vehicles too, increasing its impact.

Reviewer 2:

The reviewer stated that the technology is very promising. This technology could also have applications in LD fleets, further reducing petroleum losses and increasing safety.

\section{Reviewer 3:}

The reviewer commented that maintaining accurate and correct tire pressure is very important to maintaining fuel economy in commercial vehicles by reducing rolling resistance, and this system enables this to be done without user inputs. Although it was developed for commercial truck tires, its possible application to LD tires could be even more impactful for petroleum displacement. The reviewer commented that as Goodyear noted, the avoidance of premature tire wear and resulting replacement tire production is another petroleum displacement opportunity that can be significant.

\section{Reviewer 4:}

The reviewer stated that the project is highly relevant to DOE's overall objective of reducing petroleum consumption.

\section{Question 6: Resources: How sufficient are the resources for the project to achieve the stated milestones in a timely fashion?}

\section{Reviewer 1:}

The reviewer noted that this project highly leverages recipient cost share and represents a good value to DOE. The overall budget seems reasonable to accomplish the goals of this project and result in direct technology commercialization.

\section{Reviewer 2:}

The reviewer stated that a no-cost extension was received in FY 2015, with additional funding provided in FY 2016. With the end of the project approaching soon, it appears as though there are no issues, or at least none were identified.

\section{Reviewer 3:}

The reviewer stated that resources are sufficient to achieve the stated work, with more than adequate cost share. 


\section{EV - Smart Grid Research and Interoperability Activities: \\ Keith Hardy (Argonne National Laboratory) - vs095}

\section{Presenter}

Keith Hardy, Argonne National

Laboratory

\section{Reviewer Sample Size}

A total of four reviewers evaluated this project.

\section{Question 1: Approach to performing the work-the degree to which technical barriers are addressed, the project is well-designed, feasible, and integrated with other efforts.}

\section{Reviewer 1:}

The reviewer noted that the project provides a wide range of support across multiple industries and interfaces through interoperability committees. The focus on carrying interoperability from standards through implementation and validation makes good use of limited resources.

\section{Reviewer 2:}

The reviewer stated that collaboration with standards organizations seems to be the key approach to activities in which the ANL Grid Interoperability team participates. This approach can be effective, but can also hinder measurable progress. There also seems to be a large number of activities supported by this group, and they are spread across a number of grid-related standards and technologies. This breadth of involvement appears to be good, depth was difficult to determine in the short time allotted for presentation.

\section{Reviewer 3:}

The reviewer stated that the approach slide for this initiative seemed a little like a list of what is being done, compared to the approach of how it was selected. Understanding that this is a difficult (and diverse) collection of initiatives, it would be helpful to have a top-down viewpoint slide that sets the stage for the actions carried out. The reviewer asked whether there is an interoperability and standards development strategy that is guiding the selection of these specific sub-projects. The reviewer also asked what else remains unanswered that would be next in queue and why.

Question 2: Technical accomplishments and progress toward overall project and DOE goals-the degree to which progress has been made, measured against performance indicators and demonstrated progress towards DOE goals.

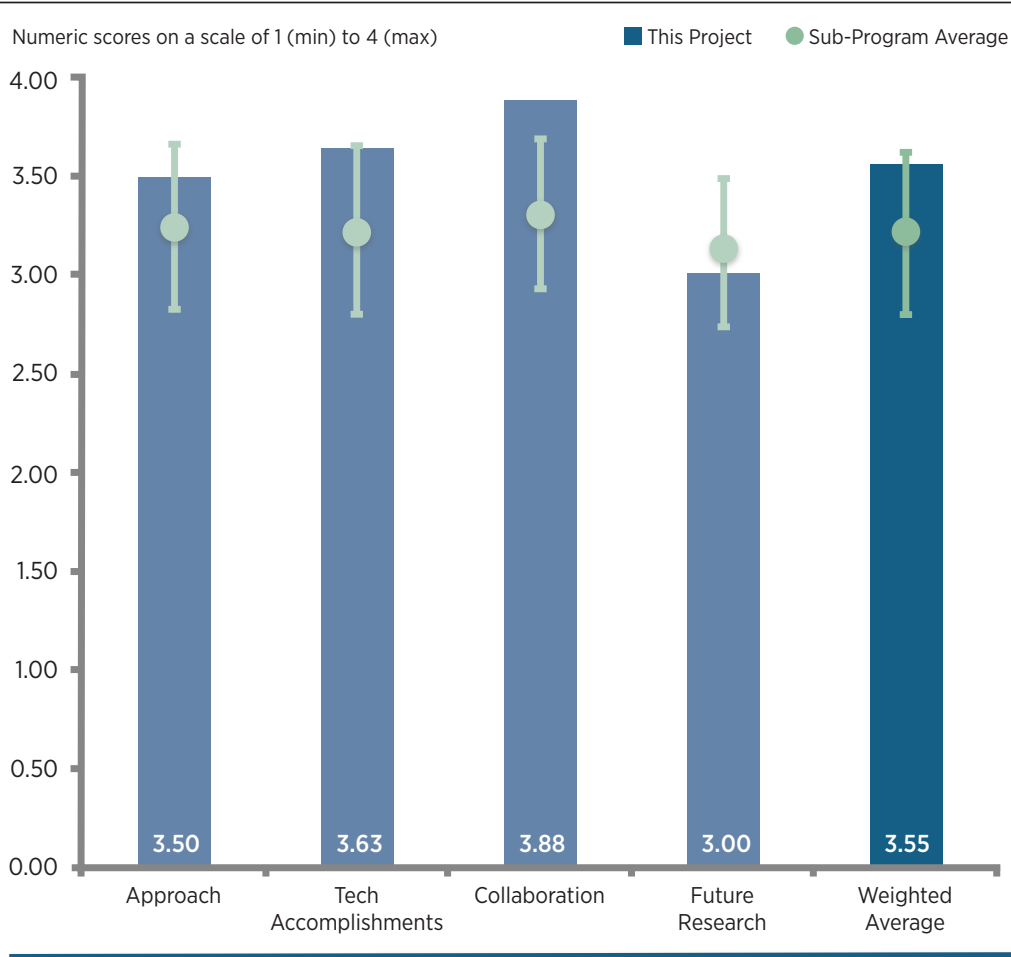

Relevant to DOE Objectives

Sufficiency of Resources

Figure 1-10 - EV - Smart Grid Research and Interoperability Activities: Keith Hardy (Argonne National Laboratory) Vehicle Systems 


\section{Reviewer 1:}

The reviewer commented that the project has pushed forward across a wide range of activities and the technical accomplishments are significant. Coordination across organizations and continents through participation in multiple interoperability committees (e.g., European Interoperability Center, Grid Integration Technical Team (GITT), etc.) provides extremely valuable coordination of national and international work.

\section{Reviewer 2:}

The reviewer stated that notwithstanding the approach comments, the technical accomplishments of selected initiatives seem substantial and reasonable to advance the multi-party work between labs, standards organizations, various government departments, and industry.

\section{Reviewer 3:}

The reviewer stated that the presentation shows the expected variation in progress when working on standards, which progresses more slowly than the technology they are setting standards for.

\section{Question 3: Collaboration and coordination with other institutions.}

\section{Reviewer 1:}

The reviewer stated that it was great work coordinating with various international bodies, OEMs, and other national laboratories.

\section{Reviewer 2:}

The reviewer thought that the coordination across organizations and continents through participation in multiple interoperability committees (e.g., European Interoperability Center, GITT, etc.) provides extremely valuable coordination of national and international work.

\section{Reviewer 3:}

The reviewer noted that the project is (by definition) required to have close coordination and collaboration, and appears to be quite successfully achieving the same.

\section{Reviewer 4:}

The reviewer commented that the key aspect of this groups activities is support and collaboration.

\section{Question 4: Proposed future research-the degree to which the project has effectively planned its future work in a logical manner by incorporating appropriate decision points, considering barriers to the realization of the proposed technology and, when sensible, mitigating risk by providing alternate development pathways.}

\section{Reviewer 1:}

The reviewer stated that there is ongoing activity with clearly presented timelines for future work in grid-related technologies.

\section{Reviewer 2:}

The reviewer commented that the continued efforts to develop tools and validate equipment provides significant value. Some effort should be spent on moving hardware and software developed into industry.

\section{Reviewer 3:}

The reviewer noted that the chart for future work needs to be grounded with qualitative comments on what the new frontiers are and why. The reviewer found it very difficult to navigate this chart without intimate knowledge of acronyms and connected project phasing logic. In other words, the roadmap is too complex for the audience to appreciate the sufficiency of detailed subjects and steps laid out.

\section{Question 5: Does this project support the overall DOE objectives of petroleum displacement? Why or why not?}

\section{Reviewer 1:}

The reviewer thought there was a clear alignment to advance standardization and in the public interest. 


\section{Reviewer 2:}

The reviewer acknowledged that, having sat on a SAE committee for standardization of some test procedures, the reviewer understood how hard it is to have all the OEMs agree with each other on a topic. The national laboratories provide a valuable service as a neutral third party. The reviewer did not think that the need for codes and standards can be exaggerated. It would not be very convenient to have each OEM with its own electric vehicle supply equipment (EVSE), as this would be a huge barrier for acceptability by the average consumer.

\section{Reviewer 3:}

The reviewer stated that DOE supporting industry codes and standards for grid-related technologies is very relevant to the objectives of the department.

\section{Reviewer 4:}

The reviewer found that the tools developed will be of significant assistance to industry in both facilitating connectivity and assuring interoperability.

\section{Question 6: Resources: How sufficient are the resources for the project to achieve the stated milestones in a timely fashion?}

\section{Reviewer 1:}

The reviewer stated that given the budget situation, this project is adequately resourced and funded. The reviewer believed that the work being done here is very important and the engineers at the national laboratories are in a unique position to perform this task, and perform it well. So, it is necessary that this activity is adequately funded.

\section{Reviewer 2:}

The reviewer stated that the team is doing well with current resources. Consideration should be given to developing and implementing a market transformation plan.

\section{Reviewer 3:}

The reviewer noted no exceptions flagged. As typical, the reviewer assumed that the project would make progress faster with additional funding.

\section{Reviewer 4:}

The reviewer stated that a large variety of activities supported requiring a lot of resources. Although doing more with less, the support of these technologies could involve less product development, which could make even better use of funds available. 


\section{Wireless and Conductive Charging Testing to Support Code and Standards: Barney Carlson (Idaho National Laboratory) - vs096}

\section{Presenter}

Barney Carlson, Idaho National Laboratory

\section{Reviewer Sample Size}

A total of five reviewers evaluated this project.

\section{Question 1: Approach to performing the work-the degree to which technical barriers are addressed, the project is well-designed, feasible, and integrated with other efforts.}

\section{Reviewer 1:}

The reviewer thought that the approach to the standards development process is logical and thorough. The proposed reporting methodology is excellent, covering both performance in a variety of possible charging connections as well as the health effects.

\section{Reviewer 2:}

The reviewer stated that the project has specific goals and methods to achieve those goals. Codes and standards support is critical for this emerging technology.

\section{Reviewer 3:}

The reviewer noted that as with any project that was well described up front and executed well, this project is very straight forward to addressing the wireless charging technology.

\section{Reviewer 4:}

The reviewer thought that testing support for standards development is a key component and has been planned to properly support the codes being developed.

\section{Reviewer 5:}

The reviewer stated that the project appears very focused and well-framed. Cybersecurity was mentioned on at least two slides (barriers and laboratory), but the reviewer assumed this was not in scope for this project as no other mention of work or results were presented.

Question 2: Technical accomplishments and progress toward overall project and DOE goals-the degree to which progress has been made, measured against performance indicators and demonstrated progress towards DOE goals.

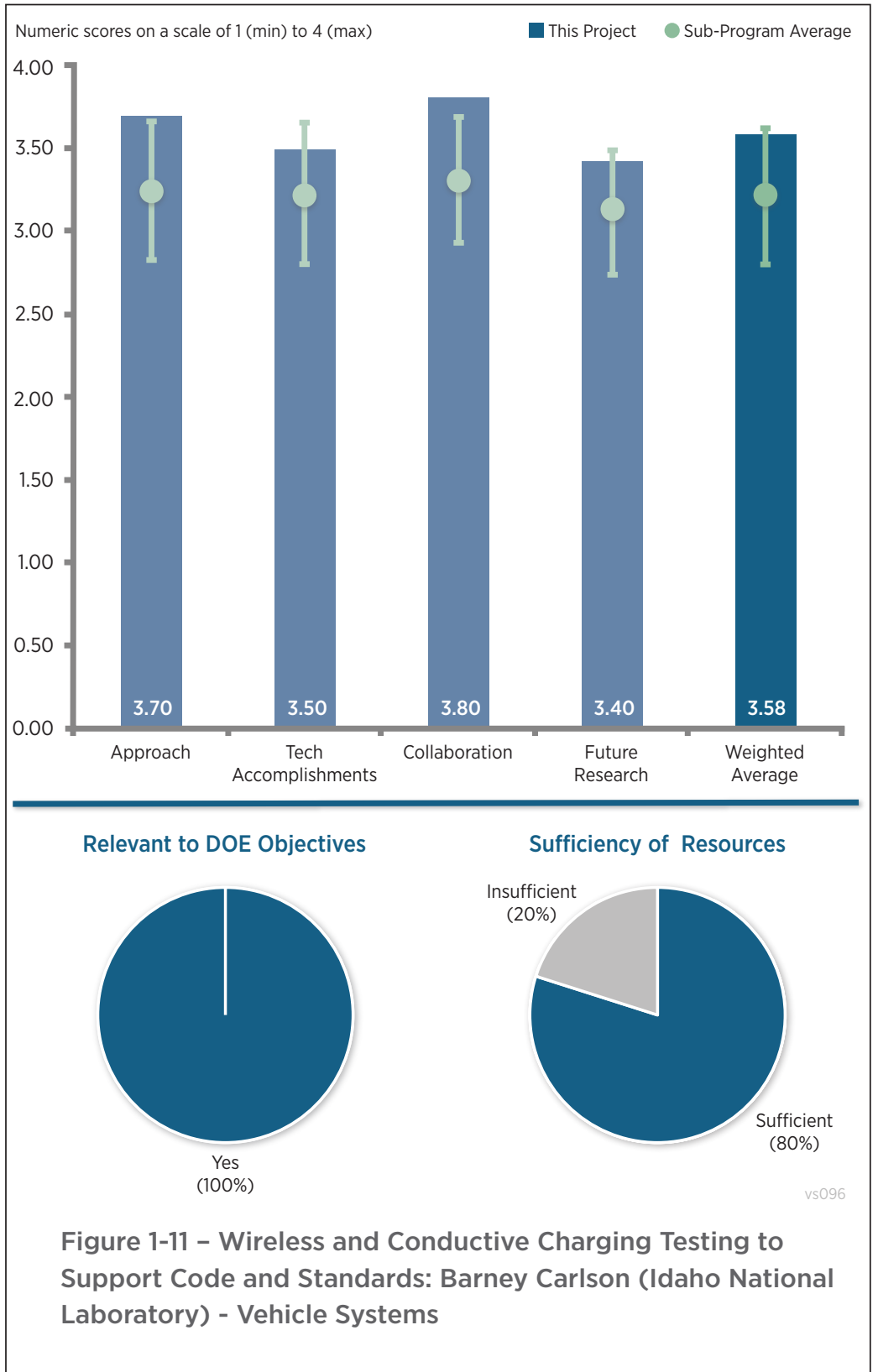




\section{Reviewer 1:}

The reviewer stated that there was excellent progress on objectives. The test facility is well designed to execute the research plan and the technical data acquired on the systems tested to date is valuable.

\section{Reviewer 2:}

The reviewer noted that beyond the testing and characterizations of the field intensity, this project has spawned an excellent support of the standards needed to advance this technology. The wireless technology success is critical in achieving market adoption and consumer acceptance.

\section{Reviewer 3:}

The reviewer stated that the plan is on track to support the codes and standards development.

\section{Reviewer 4:}

The reviewer found that the results to date are impressive and show the potential benefits, as well as the possible hazards of wireless charging.

\section{Reviewer 5:}

The reviewer stated that the work was presented in a very understandable way with apparent achievement of the stated objectives. Perhaps a little over-emphasis on details of the wireless charging work at the expense of some data results from the ENERGY STAR ${ }^{\circledR}$ evaluations of EVSEs. The reviewer would like to see a sampling of what kind of efficiency variations were observed in this testing and asked if there were any other qualitative insights.

\section{Question 3: Collaboration and coordination with other institutions.}

\section{Reviewer 1:}

The reviewer stated that the project has made the proper connections with SAE, other labs, and industry. The results from the project are reaching the appropriate audience.

\section{Reviewer 2:}

The reviewer stated that the choice of an OEM and standards organization created an exceptional partnership team that brings all of the diverse expectations of the stakeholders into one program; this is very likely to support a greater synergy to all of industry, and more robust standards.

\section{Reviewer 3:}

The reviewer stated that the project does not appear at a loss for appropriate collaborators.

\section{Reviewer 4:}

The reviewer stated that the right partners and coordination, through testing of the other DOE wireless charging projects, gives a wealth of data and analysis in support of the standards development activity.

\section{Reviewer 5:}

The reviewer stated that there was excellent coordination with interested vehicle manufacturers and charger suppliers. The reviewer would like to see SAE on the list if possible.

Question 4: Proposed future research-the degree to which the project has effectively planned its future work in a logical manner by incorporating appropriate decision points, considering barriers to the realization of the proposed technology and, when sensible, mitigating risk by providing alternate development pathways.

\section{Reviewer 1:}

The reviewer stated that future works supports the J2954 standards evolution and should provide good information on interoperability requirements.

\section{Reviewer 2:}

The reviewer stated that the proposed interoperability work somewhat rounds out the project scope and completing the standards will conclude the study. Without compelling issues, the work may be considered complete at that time. 


\section{Reviewer 3:}

The reviewer found that the work is all applicable to the intended outcome and well planned.

\section{Reviewer 4:}

The reviewer stated that the proposed future work is good and should be expanded to include additional wireless charging systems including HD if possible.

\section{Reviewer 5:}

The reviewer would have like a discussion of current and near-term continued work. The reviewer questioned what the longer-term outlook for R\&D is in this space.

\section{Question 5: Does this project support the overall DOE objectives of petroleum displacement? Why or why not?}

\section{Reviewer 1:}

The reviewer stated that providing testing for wireless charging technology enables introduction of the technology, which makes EV usage a bit more palatable to some audiences. EV usage will promote petroleum displacement.

\section{Reviewer 2:}

The reviewer noted that wireless charging is a technology enabler needed for widespread acceptance of $\mathrm{xEV}$ technology. Understanding the boundaries and limitations and bringing them into standards is supportive of that mission.

\section{Reviewer 3:}

The reviewer stated that this supports commercialization of wireless charging, which may increase adoption of EVs and can displace petroleum.

\section{Reviewer 4:}

The reviewer stated that DOE and the industry must have a picture of this technology and how it effects the use of electricity as a fuel for on road vehicles.

\section{Reviewer 5:}

The reviewer noted that this project assists in evaluating key parts of the DOE vision infrastructure for plug-in electric vehicles (PEVs).

\section{Question 6: Resources: How sufficient are the resources for the project to achieve the stated milestones in a timely fashion?}

\section{Reviewer 1:}

The reviewer thought that the resources appear to be barely adequate. Some work appears to have been deferred due to lack of budget.

\section{Reviewer 2:}

The reviewer stated that the resources appear to be sufficient at the moment, but the need could increase if the project takes on the testing of more vehicle wireless charging systems.

\section{Reviewer 3:}

The reviewer stated that without particular issues being expressed, it appears that the correct quantity were deployed into this project.

\section{Reviewer 4:}

The reviewer stated that the accomplishments were not specifically presented but seem to indicate sufficient funding.

\section{Reviewer 5:}

The reviewer stated that funding appears to have matched the work required to date. No mention of shortage or concern in material provided. 


\section{High Efficiency, Low EMI and Positioning Tolerant Wireless Charging of EVs: Rakan Chabaan (Hyundai) - vs102}

\section{Presenter}

Rakan Chabaan, Hyundai

\section{Reviewer Sample Size}

A total of four reviewers evaluated this project.

\section{Question 1: Approach to performing the work-the degree to which technical barriers are addressed, the project is well-designed, feasible, and integrated with other efforts.}

\section{Reviewer 1:}

The reviewer commented that this project is following a normal automotive company approach to $\mathrm{R} \& \mathrm{D}$ projects. It is an iterative approach to establish whether certain system configuration(s) can meet a set of predetermined threshold values for consideration for future production.

\section{Reviewer 2:}

The reviewer stated that the project looked at many alternatives, started large and worked towards small. It looks to be investigating the appropriate bandwidth of dimensions and their impact to efficiency.

\section{Reviewer 3:}

The reviewer stated that based on the original set of slides, the periods of time for each phase is not defined as there is no timeline provided for each phase. The technical barriers are explained well on Slide 2, and the power level and charging efficiency goals are clear.

\section{Question 2: Technical accomplishments and progress toward overall project and DOE goals - the degree to which progress has been made, measured against performance indicators and demonstrated progress towards DOE goals.}

\section{Reviewer 1:}

The reviewer stated that based on the system exceeding the $6.6 \mathrm{~kW}$ and $85 \%$ efficiency goals, the technical accomplishments are good.

\section{Reviewer 2:}

The reviewer thought that the evaluation of selected configuration was quite adequate for the purpose. There was no attempt to bring alternative configurations into the project. The project seems to be based on a pre-determined single configuration.

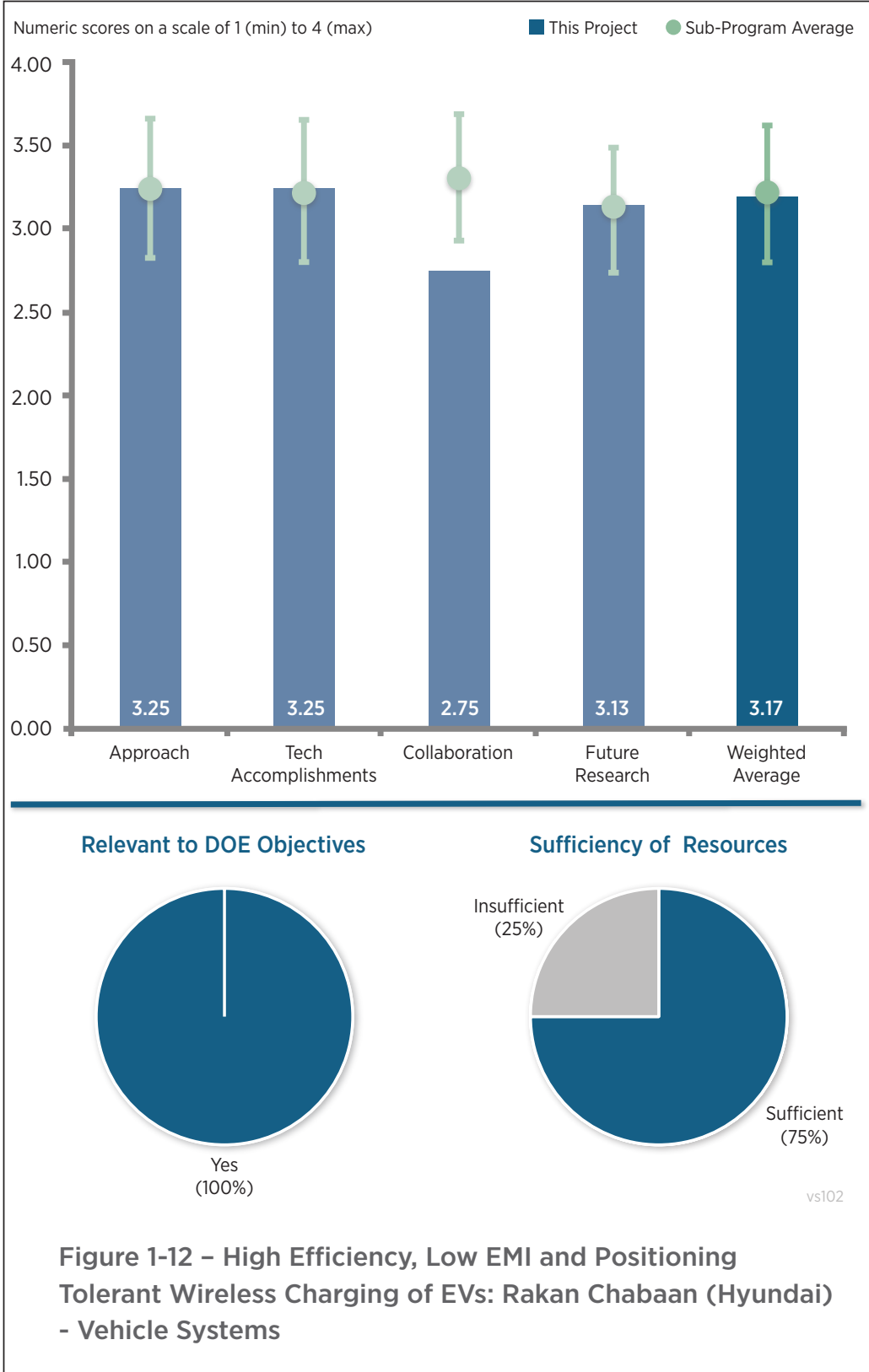




\section{Reviewer 3:}

The reviewer commented that the effects on efficiency and the study of energy transfer into unintended objects are good. The reviewer did not see a list of other issues that remain for commercialization. The reviewer further noted environmental concerns about installation spaces, how to help the user align with the charger for maximum efficiency and use, and working with SAE in the charging recommendation.

\section{Question 3: Collaboration and coordination with other institutions.}

\section{Reviewer 1:}

The reviewer stated that it seems adequate with Mojo, SAE, and others who have a serious stake in the outcome as collaborators.

\section{Reviewer 2:}

The reviewer would have liked to have seen a reference to SAE. Because there is variability in the charge efficiency, the reviewer questioned how EPA will align the charger during certification testing, and if there will be a recommendation in the SAE procedure. A $1 \%$ point difference in charge efficiency could affect MPG equivalent (MPGe) on the vehicle label by two MPGe.

\section{Reviewer 3:}

The reviewer noted that the Slide 22 collaboration and coordination information make it seem that Mojo is doing all the work. There is no detail as to how Mojo and Hyundai are interacting, nor is there any information as to what work Hyundai is doing. In addition, the presenter submitted his presentation at the start of the annual review instead of in April like he was required to do by DOE. The reviewer said that this suggests little coordination with DOE.

\section{Question 4: Proposed future research-the degree to which the project has effectively planned its future work in a logical manner by incorporating appropriate decision points, considering barriers to the realization of the proposed technology and, when sensible, mitigating risk by providing alternate development pathways.}

\section{Reviewer 1:}

The reviewer noted that the plan to continue refining the system is positive, as is the objective to add the system to five vehicles. Still, there is no indication that the future research will include a pathway to commercialization.

\section{Reviewer 2:}

The reviewer stated that the outcome of the last phase of the project is to have a proof of concept to present to management for decisions on commercialization. No comment was provided by Mojo who may be a prime commercialization partner. It would be good to have their input to the question of whether this technology is ready for introduction to the commercial sector.

\section{Reviewer 3:}

The reviewer thought that having the OEM partner will be helpful in overcoming the barriers to commercialization. This project presentation does not list the barriers of cost compared to current systems that charge the vehicle, so it is unclear if this will be hindrance to adoption of the technology.

\section{Question 5: Does this project support the overall DOE objectives of petroleum displacement? Why or why not?}

\section{Reviewer 1:}

The reviewer noted that one goal of wireless charging is to make recharging PEVs easier, which should result in more EV miles traveled. In turn, this results in achieving DOE's objective of petroleum displacement.

\section{Reviewer 2:}

The reviewer commented that yes, because if it is successful it would assist with barriers to charging and increase the transfer rate and efficiency of a wireless charge system. This can increase adoption of EVs thereby displacing petroleum use. 


\section{Reviewer 3:}

The reviewer stated that automatic charging of EVs will allow for more consistent charging of EVs which will result in less petroleum use.

Reviewer 4:

The reviewer stated that wireless charging must efficiently take place for EVs to charge at scale.

Question 6: Resources: How sufficient are the resources for the project to achieve the stated milestones in a timely fashion?

Reviewer 1:

The reviewer noted that based on the remaining funds of $\$ 427,000$ and the spend rate the last three fiscal years, there may be a funding issue looming.

Reviewer 2:

The reviewer stated that funding seems sufficient based on outcomes presented. Nothing was presented regarding resources or how partners have participated. 


\section{Wireless Charging of Electric Vehicles: Omer Onar (Oak Ridge National Laboratory) - vs103}

\section{Presenter}

Omer Onar, Oak Ridge National Laboratory

\section{Reviewer Sample Size}

A total of three reviewers evaluated this project.

\section{Question 1: Approach to performing the work-the degree to which technical barriers are addressed, the project is well-designed, feasible, and integrated with other efforts.}

\section{Reviewer 1:}

The reviewer noted that this is a multi-year project that is intended to define a set of parameters that may be used to develop and commercialize a high transfer rate wireless charging system. The work has been logical in its sequence of tasks in the development of the wireless system. The intended outcome of achieving a system definition for development of a commercial system seems to be on track.

\section{Reviewer 2:}

The reviewer questioned if there has been any cross-talk with the HyundaiMojo Mobility project, to pool together lessons learned.

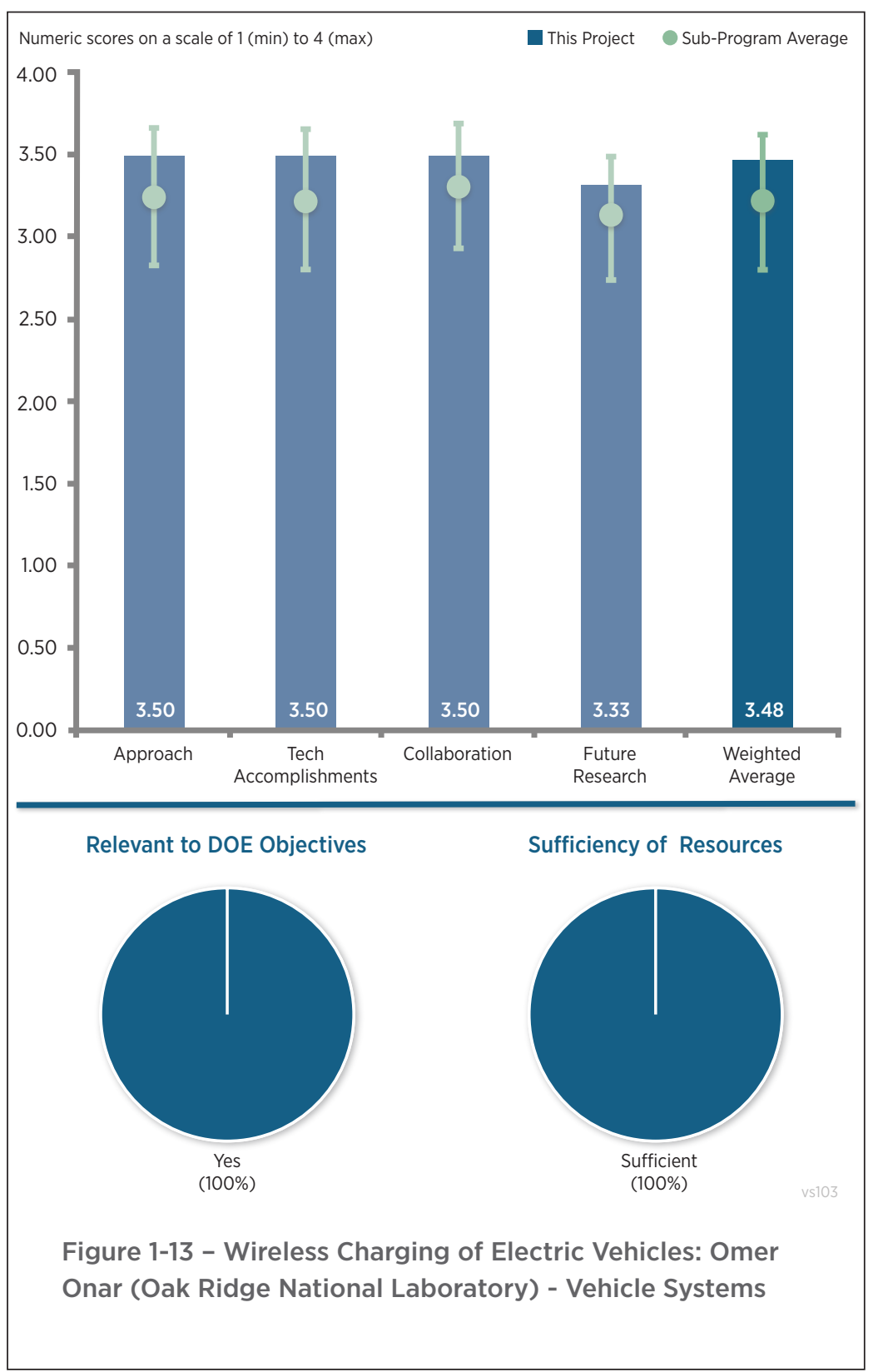

Question 2: Technical accomplishments and progress toward overall project and DOE goals-the degree to which progress has been made, measured against performance indicators and demonstrated progress towards DOE goals.

\section{Reviewer 1:}

The reviewer noted that there was excellent progress towards the $20 \mathrm{~kW}$ static and dynamic WPT.

\section{Reviewer 2:}

The reviewer stated that the project team is following the schedule according to plan.

\section{Question 3: Collaboration and coordination with other institutions.}

\section{Reviewer 1:}

The reviewer stated that the project team has the right partners to achieve the objectives of the project. More partners would seem to be a deterrent to progress at this level of R\&D. 


\section{Reviewer 2:}

The reviewer noted that the 2015 AMR review showed a partner funding level of $\$ 3.3$ million, while the 2016 AMR presentation showed \$2.6 million.

Question 4: Proposed future research-the degree to which the project has effectively planned its future work in a logical manner by incorporating appropriate decision points, considering barriers to the realization of the proposed technology and, when sensible, mitigating risk by providing alternate development pathways.

\section{Reviewer 1:}

The reviewer noted that the project is $98 \%$ complete. This person referenced comments regarding project resources and explained that this is an academic question. However, certain questions did come to mind for this reviewer. The impact of misaligned coils on loss of efficiency was described by the reviewer as significant. Thus, the reviewer inquired about the possibility of having a self-aligning mechanism in the stationary coil, perhaps even a ferromagnetic core, which would result in a significant enough energy saving that it would pay back the extra cost in a reasonable period of time.

\section{Reviewer 2:}

The reviewer stated that the work is designed to meet objectives and overcome barriers through testing of systems. This project, and what has been learned regarding high transfer rates, should be consolidated and a follow-on FOA should be initiated to take this work further and to get it dispersed into the commercial sector. The reviewer would like to see a larger test community in a real world operative environment included.

\section{Question 5: Does this project support the overall DOE objectives of petroleum displacement? Why or why not?}

\section{Reviewer 1:}

The reviewer stated that wireless charging is absolutely needed to run BEVs in large scale.

\section{Reviewer 2:}

The reviewer stated that wireless charging can support increased adoption of EVs by making charging much easier. It will contribute based on the economic realities of deploying these systems widely. Another project should evaluate the economics of wireless charging systems for vehicle populations of ever increasing volume.

\section{Reviewer 3:}

The reviewer could buy the argument that WPT results in customers less likely to have to rely on petroleum as an energy source because of forgetting to charge their vehicles, but only if the WPT system inefficiency does not swamp the benefits obtained by switching to electricity as a source of motive power. It would help if there are studies showing what the minimum efficiency of a WPT system needs to be (in comparison to a physically plugged in system) to ensure that, overall, there is no increase in petroleum consumption. The J2954 standard has a minimum efficiency target of $85 \%$. The reviewer noted that while this is not within the scope of this project, and the reviewer was not privy to all the discussions that went on in the J2954 committee that set this target, studies/ simulations backing the choice of this apparently arbitrary target would be helpful.

\section{Question 6: Resources: How sufficient are the resources for the project to achieve the stated milestones in a timely fashion?}

\section{Reviewer 1:}

The reviewer stated that at this phase of the project, with the project being listed as $98 \%$ complete, this is perhaps academic.

\section{Reviewer 2:}

The reviewer noted that no comment on resources was provided but this project seems to be going more slowly than it may have gone in a different environment. This could indicate either low efficiency of hours employed or a strain based on resource constraints. 


\section{Zero Emission Drayage Truck Demonstration (ZECT I): Matt Miyasato (SCAQMD) - vs115}

\section{Presenter}

Brian Choe, SCAQMD

\section{Reviewer Sample Size}

A total of five reviewers evaluated this project.

\section{Question 1: Approach to performing the work-the degree to which technical barriers are addressed, the project is well-designed, feasible, and integrated with other efforts.}

\section{Reviewer 1:}

The reviewer thought that this was a great project.

\section{Reviewer 2:}

The reviewer stated that the approach of developing Class 8 drayage trucks that will be used in real world service conditions, and compare the use data against baseline diesel trucks will address the barriers and objectives of the project to evaluate market viability and promote market acceptance.

\section{Reviewer 3:}

The reviewer thought it is admirable that the researchers chose to include both BEVs and plug-in hybrid electric trucks (PHETs) in the scope. This will provide very useful information about the comparative costs and benefits of the two types. Then the next step can be optimizing the designs for cost and efficacy in various types of use. The reviewer would be interested in seeing how the compressed natural gas part works out and if it is cost effective.

\section{Reviewer 4:}

The reviewer stated that the additional system weight and battery management system issues required a different and larger system is required, further delaying data collection and analysis. Prior modeling and systems analysis should have highlighted and predicted much of this.

\section{Reviewer 5:}

The reviewer stated that the project focuses on building trucks, but it is not clear that the vehicle requirements were considered before building them. Route and performance analysis should have been performed earlier. It is not clear if anything was learned about the technologies during the project, or if the technology is viable. There has been no discussions on the technical and financial viability of those options.

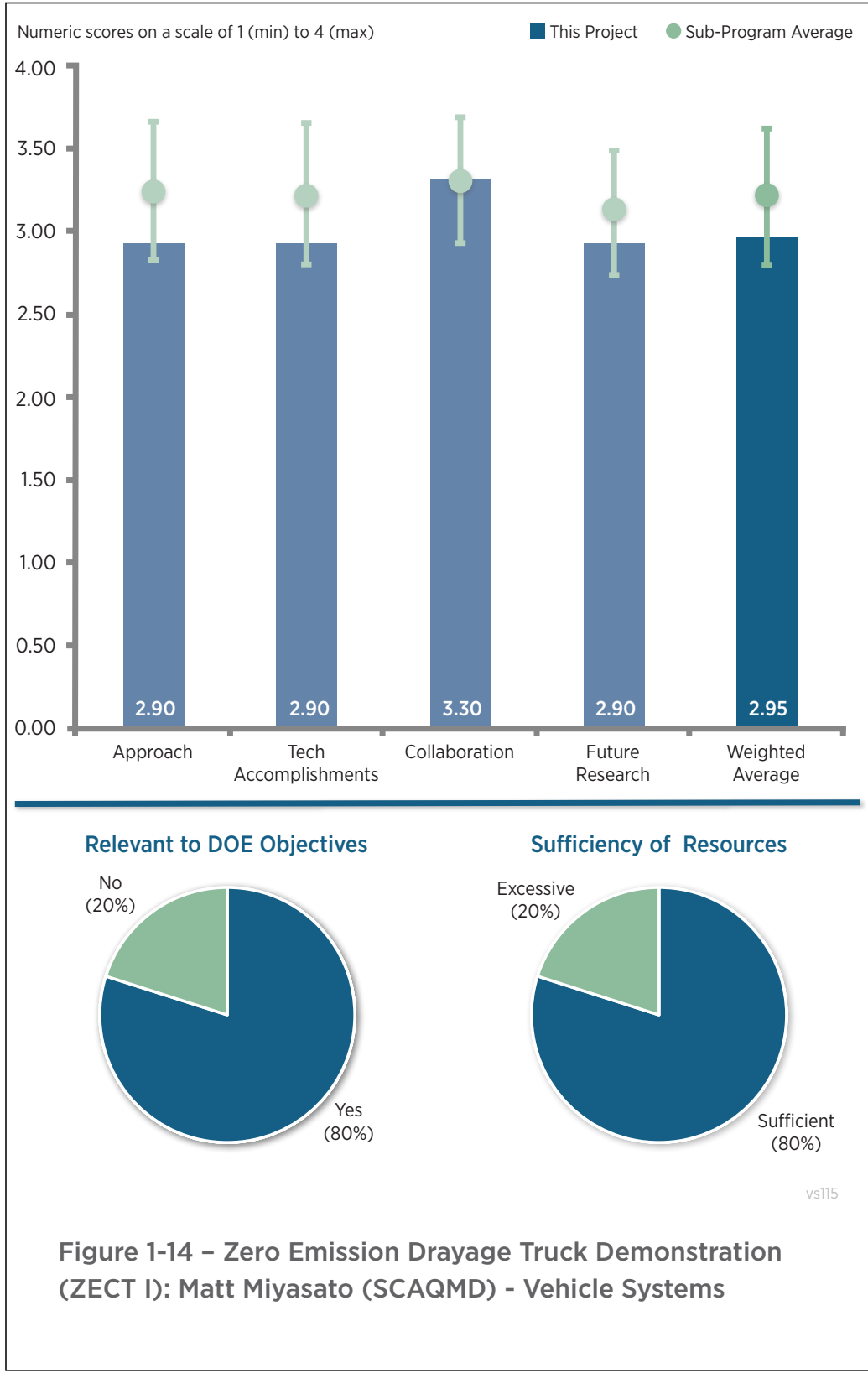


Question 2: Technical accomplishments and progress toward overall project and DOE goals-the degree to which progress has been made, measured against performance indicators and demonstrated progress towards DOE goals.

\section{Reviewer 1:}

The reviewer noted that the project team has trucks up and running, and have identified shortcomings and strengths so that the next set will work better. That is good progress!

\section{Reviewer 2:}

The reviewer noted that the technical accomplishments and progress has been satisfactory this year. Data has been collected and analyzed on baseline trucks and Transpower battery electric trucks (BETs). The contracts have been executed in 2015 and there is a schedule to have more vehicles put into service this year. The reviewer said that given the delays in finally getting the contracts executed in previous years of this project, it will be difficult to accomplish all of the data collection needed by September 2017.

\section{Reviewer 3:}

The reviewer stated that the trucks were built and tested, but it appears that little to no analysis was done regarding the viability of the technologies and remaining challenges. The project appears to have no R\&D or analysis.

\section{Question 3: Collaboration and coordination with other institutions.}

\section{Reviewer 1:}

The reviewer stated that this is a broad and obviously effective collaboration.

\section{Reviewer 2:}

The reviewer stated that the team assembled by the South Coast Air Quality Management District, of Transpower and U.S. Hybrid to develop the Class 8 trucks, as well as Total Transportation Services, Inc. and NREL to collect and analyze data, seem to be adequate and has good coordination.

\section{Reviewer 3:}

The reviewer stated that multiple partners are involved, including fleets, manufacturers, OEM, and universities.

Question 4: Proposed future research-the degree to which the project has effectively planned its future work in a logical manner by incorporating appropriate decision points, considering barriers to the realization of the proposed technology and, when sensible, mitigating risk by providing alternate development pathways.

\section{Reviewer 1:}

The reviewer is anxiously awaiting results that compare the BEVs to the PHETs. For drayage use, the smaller range and cheaper battery may enable most of the petroleum savings at much lower cost. The reviewer requested that some cost information be included in the future reports!

\section{Reviewer 2:}

The reviewer stated that the plans to deploy the remainder of the drayage trucks into service is very good. However, because many of the trucks will not be deployed until later this year, it will not be possible to collect the two years' worth of data while the trucks are in drayage service by September 2017 as required by the agreement. The reviewer asked if there is a possibility to have a no-cost extension to allow time for the data collection.

\section{Reviewer 3:}

The reviewer noted that considering the large amount of funding, some analysis should have been done ahead of time to assess the viability of the vehicle technologies considered and the vehicle requirements. In addition, it does not seem that any analysis was performed or is planned in the future. The reviewer questioned what metrics will be used to assess the success of the technologies.

Question 5: Does this project support the overall DOE objectives of petroleum displacement? Why or why not? 


\section{Reviewer 1:}

The reviewer stated that yes, it displaces large volumes of fuel in Class 8 operations.

\section{Reviewer 2:}

The reviewer stated that the project is relevant to the DOE objectives of reducing petroleum use by introducing BET and PHEV trucks into service and promoting acceptance to replace conventionally powered trucks.

\section{Reviewer 3:}

The reviewer stated that these vehicles reduce petroleum use, but noted that the cost is significant.

\section{Reviewer 4:}

The reviewer stated that this is a niche market with a very localized impact.

\section{Question 6: Resources: How sufficient are the resources for the project to achieve the stated milestones in a timely fashion?}

\section{Reviewer 1:}

The reviewer thought it is a lot of money, but the project team is buying unique trucks and operating them for years.

\section{Reviewer 2:}

The reviewer stated that there appears to be adequate funding to complete the project as outlined; however, given when the trucks will be deployed, there will not be enough time (two years) to collect the data required without a no-cost extension.

\section{Reviewer 3:}

The reviewer stated that the project is not focused on any R\&D activities, and so far no analysis has been performed. The reviewer acknowledged being puzzled as to what will be learned at the end of the project other than truck liability. 


\section{Hydrogen Fuel-Cell Electric Hybrid Truck and Zero Emission Delivery Vehicle Deployment: Andrew DeCandis (Houston-Galveston Area Council) - vs116}

\section{Presenter}

Andrew DeCandis, Houston-

Galveston Area Council

\section{Reviewer Sample Size}

A total of four reviewers evaluated this project.

\section{Question 1: Approach to performing the work-the degree to which technical barriers are addressed, the project is well-designed, feasible, and integrated with other efforts.}

\section{Reviewer 1:}

The reviewer found that the approach to put 30 all-electric delivery vehicles and three hydrogen $\left(\mathrm{H}_{2}\right)$ fuel cell Class 8 trucks into service and show them to be available, cost effective, and meeting performance expectations for operation and emissions is very good, and if successful will certainly help to address the barriers to be overcome in the project.

\section{Reviewer 2:}

The reviewer stated that the approach is to buy vehicles based on end user requirements and test the vehicles with a fleet operator. The fleet operator, UPS, verbally indicated from the audience that requirements were provided to the team. It would have been helpful if the specifications were provided as part of the reporting presentation. There should be some way to verify the requirements will be met, and a go/no-go decision point if the specifications do not meet the requirements.

\section{Reviewer 3:}

The reviewer stated that a preliminary analysis should have been performed before the start of the project to understand the vehicle requirements. The presenter mentioned several times the range anxiety in Houston area. This should have been understood and quantified before any vehicle was built.

\section{Reviewer 4:}

The reviewer thought that this project would have benefitted from some cursory modeling and analysis to understand what vehicles would be appropriate relative to drive cycle, requirements, etc. Without this, the vehicles can be poorly matched and underutilized due to range anxiety, cost, etc. The focus needs to be on demonstrating not only the capability but the feasibility (on a cost basis) of these technologies.

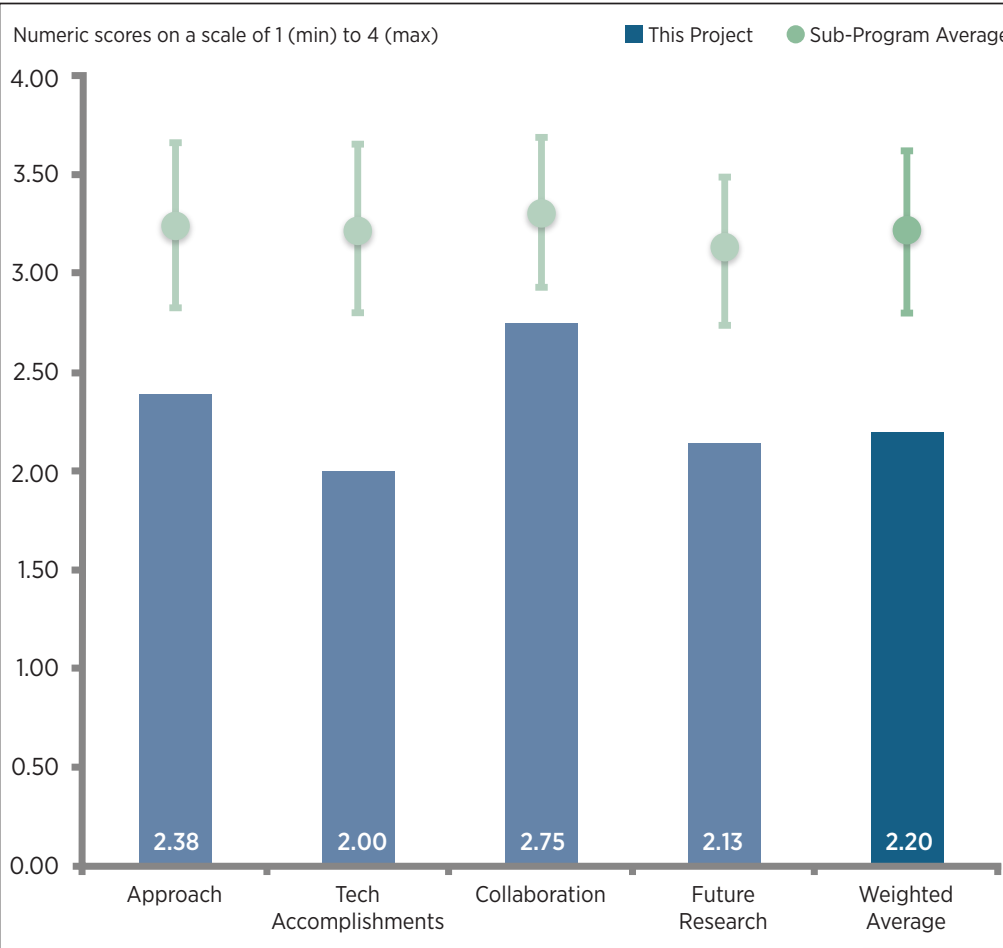

Relevant to DOE Objectives

Sufficiency of Resources
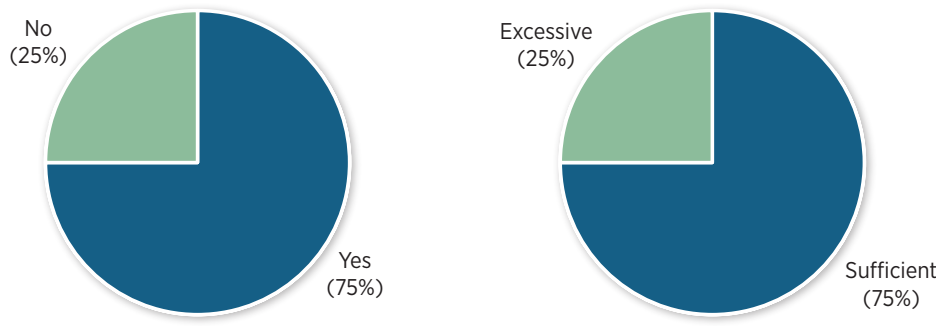

Figure 1-15 - Hydrogen Fuel-Cell Electric Hybrid Truck and Zero Emission Delivery Vehicle Deployment: Andrew DeCandis (Houston-Galveston Area Council) - Vehicle Systems 
Question 2: Technical accomplishments and progress toward overall project and DOE goals-the degree to which progress has been made, measured against performance indicators and demonstrated progress towards DOE goals.

\section{Reviewer 1:}

The reviewer noted that it was reported during the presentation that 10 of the UPS delivery trucks have now been delivered and that all 18 will be available in July of this year. It was good to see that the project has started to purchase parts and equipment for the hydrogen fuel cell electric hybrid trucks.

\section{Reviewer 2:}

The reviewer noted that the project has been running late and starting to purchase equipment at that point in time highlights the issues with the project and the technology.

\section{Reviewer 3:}

The reviewer stated that the project indicated that to be successful the technologies need to be cost effective, meet operational requirements, and emissions requirements. These metrics should be quantified up front and tracked to see if the project met its goals. The presenter indicated metrics would be figured out later, which is the wrong way to approach a project, as design choices need to be made up front to meet the metrics.

\section{Reviewer 4:}

The reviewer stated that work has largely been centered on revising scope, surveys, and subcontracts, yet it appears little has been done to develop or procure vehicles. Analysis needed to be done up front to understand the vehicle requirements relative to the market to make intelligent decisions on technology. Also, understanding the complexity in vehicle development, the reviewer believed that it will be difficult to have the fuel cell vehicle operating by this time next year.

\section{Question 3: Collaboration and coordination with other institutions.}

\section{Reviewer 1:}

The reviewer stated that the addition of UPS as a fleet partner that will be operating 18 of the zero emission delivery vehicles is excellent and will provide good information regarding the use of these trucks.

\section{Reviewer 2:}

The reviewer stated that the project has an experienced OEM as well as a large fleet operator as collaborators. There is a very high likelihood the technology will be adopted by UPS if it proves to be cost effective.

\section{Reviewer 3:}

The reviewer stated that fleets and OEM partners are involved in the project, but they appear to have little to no experience in the area of fuel cell vehicles and their specific safety.

\section{Reviewer 4:}

The reviewer was concerned that some suppliers have dropped and the project has required some re-scope to find other partners for the vehicles.

\section{Question 4: Proposed future research-the degree to which the project has effectively planned its future work in a logical manner by incorporating appropriate decision points, considering barriers to the realization of the proposed technology and, when sensible, mitigating risk by providing alternate development pathways.}

\section{Reviewer 1:}

The reviewer stated that to complete the project as planned with 30 zero emission delivery trucks, it is critical that a partner for the 12 trucks is decided upon quickly. Because the project ends in September 2017, it is not clear how two years' worth of data will be collected on all of the UPS trucks, and it is even more of a question regarding the 12 additional trucks for which a partner is not selected yet. 
There should be more detail provided in the presentation regarding the type of data that will be collected in this project. It was discussed in the question and answer (Q\&A) period, but in the future it should be specifically addressed in the presentation document.

\section{Reviewer 2:}

The reviewer noted that the plan is to buy vehicles and test them. The project does not define what the goal of testing the vehicles is other than to gather data. The testing goals should be defined up front. The reviewer questioned if the goal is to validate the goals of the project are being met and what the goals are to be tested. This will drive the data acquisition and the instrumentation preparation that is easier to design into the vehicle up front (saves time and money) rather than as a modification.

\section{Reviewer 3:}

The reviewer stated that the project appears to be focused on deployment with little to no R\&D focus. The reviewer was very unclear about what will be learned from this project. The reviewer questioned why DOE funding is needed if OEMs are producing similar vehicles.

\section{Question 5: Does this project support the overall DOE objectives of petroleum displacement? Why or why not?}

\section{Reviewer 1:}

The reviewer stated yes, by promoting hydrogen fuel cell Class 8 trucks and all-electric delivery vehicles, the project definitely supports the objective of petroleum displacement.

\section{Reviewer 2:}

The reviewer commented that by definition, an alternative fuel vehicle (i.e., fuel cell or EV) displaces petroleum.

\section{Reviewer 3:}

The reviewer stated that the project is now trying to find fleets to use vehicles rather than develop vehicles that need fleets. That statement highlights all the issues with the project.

\section{Question 6: Resources: How sufficient are the resources for the project to achieve the stated milestones in a timely fashion?}

\section{Reviewer 1:}

The reviewer stated that the resources for the project appear to be sufficient to complete the milestones.

\section{Reviewer 2:}

The reviewer thought that the project appears to currently have sufficient resources.

\section{Reviewer 3:}

The reviewer stated that the project does not appear to be focused on R\&D. It is very unclear why DOE would fund such activities. 


\section{Combined Aero and Underhood Thermal Analysis for Heavy-Duty Trucks: Tanju Sofu (Argonne National Laboratory) - vs132}

Presenter

Tanju Sofu, Argonne National Laboratory

\section{Reviewer Sample Size}

A total of five reviewers evaluated this project.

\section{Question 1: Approach to performing the work-the degree to which technical barriers are addressed, the project is well-designed, feasible, and integrated with other efforts.}

\section{Reviewer 1:}

The approach described on Slide 4 provides a well thought out process for accomplishing the project objectives.

\section{Reviewer 2:}

The reviewer expressed a little confusion about how this study could be beneficial to OEMs in designing a more fuel efficient HD truck. The distance between the leading truck and trailing truck and driving in single lane or two lanes do have some effect on the aerodynamics of the trailing vehicle and thus underhood heat transfer. However, the reviewer was not sure how OEMs can utilize the results of this study to guide truck design. The results might have some value to automated vehicles, though, because the speed and distance between vehicles can be fully controlled by computer.

\section{Reviewer 3:}

The reviewer commented that this project seems to be a routine CFD application study. The reviewer then stated that the first goal on Slide 2, "development of a computational framework," is not only unnecessary as it already exists, but is also not evidently being pursued from the presented progress. The reviewer noted that the third goal on Slide 2 seemed vague ("emission control issues") and somewhat disjointed from the other two goals; it should be dropped, particularly in view of the schedule delays encountered. Similarly, the emphasis on multi-vehicle platooning, which emerged suddenly on Slide 4 and seemed to dominate the rest of the presentation, seemed to have little direct connection to any of the stated goals.

On Slide 3, the reviewer asked for quantification of the amount of "useful mechanical work" that is parasitic to the cooling system and subject to reduction through design optimization. For heavy vehicles, it is typically a relatively small fraction of total fuel energy relative to the thirds discussed here. 
The reviewer stated that the value of this work would be significantly strengthened with recourse to some experimental data for model validation. The reviewer saw no indication of any effort or plan in that area.

\section{Reviewer 4:}

The reviewer said that the overall objectives of this project are basically to analyze heavy vehicle optimization through analysis of the interdependent phenomena of vehicle external aerodynamics, cooling system performance, and underhood thermal analysis. Predicting the engine and component temperatures under the hood can speed up the design cycle and help achieve greater fuel efficiencies through coolant system optimizations with impacts from aerodynamics.

The reviewer summarized the accomplishments in this project, which has been ongoing since 2012 and started with the development of extensive computer-aided design (CAD) and CFD models looking at aerodynamic drag and underhood thermal simulations in heavy and medium duty trucks. In FY 2015, vehicle platooning simulations commenced for different configurations, and in FY 2016 cooling package optimization in HD vehicles is underway as well as additional platooning underhood thermal simulations for two different configurations.

The reviewer found an area of some lack of clarity in the third bullet under Goals on Slide 2, which identified addressing emission control issues to meet the new diesel engine requirements and increased electrification of the engine system. It is not entirely clear to the reviewer how this directly fits into the balance of the primary scope of the project. Additionally, it is really not clear what the overall end point or targeted conclusion is of the project. The project appears to have followed a somewhat circuitous path to date, although this may be a result of funding limitations in more recent years. It may be beneficial to provide some additional clarity with a narrower focus and a clearer end point moving forward.

\section{Reviewer 5:}

The reviewer's view of the presentation material was that the project did not look like any optimization of fuel efficiency from aerodynamics had been done nor a trade-off of aerodynamics and the ability to cool the powertrain. This presentation came across as a study of vehicle positioning while driving down the road while the powertrain could remain at an appropriate temperature with less airflow due to shielding from a lead blocking vehicle.

\section{Question 2: Technical accomplishments and progress toward overall project and DOE goals-the degree to which progress has been made, measured against performance indicators and demonstrated progress towards DOE goals.}

\section{Reviewer 1:}

The reviewer acknowledged that a lot of analysis has been accomplished with this project, and the project team has done a good job of distilling the information down to metrics that matter (e.g., fuel savings). It seemed to the reviewer that the analysis was more than originally intended and the project team did allude to some scope creep in the project that allowed the team to do more than what was promised over the last three years.

\section{Reviewer 2:}

The reviewer was unclear as to why allowing or not allowing these vehicles to drive in the patterns described in the material is a barrier. The reviewer thought that it is well known that running vehicles close together improves fuel economy due to the changes in aerodynamic loads on the vehicles. This material is good in that it does discuss the magnitude of that advantage, but the reviewer did not see any clear results to what allows or does not allow operation in the vehicle on road formations described.

There were data presented about $4^{\circ}$ Celsius $(\mathrm{C})$ and $1{ }^{\circ} \mathrm{C}$ rises in the coolant temperatures from these conditions, but no indication if these rises in temperature are acceptable or not. The reviewer wanted to know if the single vehicle condition controlling to the limit and $+4^{\circ} \mathrm{C}$ is not acceptable, or is the $+4^{\circ} \mathrm{C}$ still beneath the allowable temperature limit.

\section{Reviewer 3:}

The reviewer remarked that there has been a steady list of accomplishments starting in FY 2013 until the present, including aerodynamic drag analysis of $\mathrm{HD}$ and MD vehicles, vehicle platooning and underhood thermal analysis under single and double lane scenarios with multiple vehicles, and fan shroud optimization. These analyses have 
shown significant potential platooning team fuel savings for two vehicles in a single lane configuration (24\%) and less advantages in two lane configurations (7\%), including optimal separation distances. The platooning cooling air flow rates are compared to that with no traffic for various scenarios.

It was not entirely clear to the reviewer as to the drive cycle used for the aerodynamic studies for the MD vehicles that exhibited a maximum $11 \%$ improvement in fuel economy. If the cycle were merely a steady state highway test speed, it is probably not very representative of actual use (and unlikely to be commercially viable from a ROI standpoint) as MD vehicles are not typically going to be traveling at highway speeds. Additionally, the reviewer was unclear about the value of the two-lane platooning studies as it is unlikely this configuration would be implemented in practice due to the resulting congested travel lanes for other vehicles.

\section{Reviewer 4:}

The reviewer said that the project team summarized the accomplishments in previous years before the current year. The reviewer found the slide of FY 2013 accomplishments hard to read and asked the project team to please increase the font size.

For the current year, the team analyzed the aerodynamic effect of the leading truck on the trailing truck and thus the air flow into the trailing truck. Based on the study, an optimized fan shroud was designed that has a $1.4 \%$ raise in cooling air flow. The aerodynamic modeling over the big truck is very computationally expensive, and the reviewer did not see too much value of it.

\section{Reviewer 5:}

In selecting vehicle changes, the reviewer suggested that some consideration should be given - and documented here - as to how to realize the cost of retrofit, weight, and other pragmatic fleet-usage considerations. The variables chosen for attention lacked realism or justification, such as a 30 foot following distance at 55 miles per hour (without any indication of adaptive or collaborative cruise control), multi-lane platooning, fan on/off scenarios, etc. Upon questioning in the session, the project team acknowledged some of these things being of academic interest only. The conclusions to date should be presented in less detail, at a higher level, looking beyond the raw CFD results or the fillet radius to a more synthesized basis, such as ranking of design improvements and implications for energy savings.

\section{Question 3: Collaboration and coordination with other institutions.}

\section{Reviewer 1:}

As mentioned by the presenters, the reviewer stated that there seemed to be difficulty in obtaining all the useful data to proceed. There also seemed to be a disconnection with the vs 006 project presented prior to this project.

\section{Reviewer 2:}

The reviewer indicated that this project originated as a cooperative research and development agreement (CRADA) with Cummins at a high 50/50 cost share. As such, the limited project partners are acceptable and understood although it might be good to consider an additional one now given the somewhat changing scope in particular, the new perspectives on platooning, and overall transportation as a system.

\section{Reviewer 3:}

The reviewer noted that the only collaborator on this project is Cummins. The reviewer commented that it would be great if the team can have discussions with other truck OEMs and get their opinions on this study.

\section{Reviewer 4:}

The reviewer observed that Cummins has its own modeling and simulation, but it was mostly limited to system integration. The project team acknowledged a stall in the project in order to find the collaborative partner. It would have been good to have more than one collaborator on this effort.

\section{Reviewer 5:}

The reviewer stated that the lack of a vehicle OEM or even a trailer manufacturer clearly limited and handicapped the study. 
Question 4: Proposed future research-the degree to which the project has effectively planned its future work in a logical manner by incorporating appropriate decision points, considering barriers to the realization of the proposed technology and, when sensible, mitigating risk by providing alternate development pathways.

\section{Reviewer 1:}

The reviewer stated that the project team listed other analyses that would be done to complete the project objective of an optimal design of vehicle thermal system.

\section{Reviewer 2:}

The reviewer commented that an expansion of more vehicle speeds will be useful in seeing the full value to the proposals.

\section{Reviewer 3:}

The reviewer found the very brief path forward material to be vague and insufficient for justifying the remaining $\$ 400,000$ DOE budget.

\section{Reviewer 4:}

The reviewer observed that only a brief synopsis of future proposed work is provided with regards to optimization of the cooling air mass flow rate of the heat exchanger and vehicle platooning underhood thermal transient analysis of varying heat rejection rates with fan on and off conditions. It would be beneficial to have a more focused and detailed pathway of planned future activities and where they are leading. As it stands, it is somewhat difficult to see a clear future project path and end point.

\section{Reviewer 5:}

The reviewer remarked that the project team has two paths forward. The first is heat exchanger optimization, which is necessary no matter what, although the reviewer did not expect much novel finding or breakthrough in this route because this work has been done for decades. The second is the transient thermal analysis of the trailing truck. This work would be even more computationally expensive. However, the reviewer had the same doubts as in the Approach section; namely, whether this is really necessary or beneficial to the design of a more fuel efficient truck.

\section{Question 5: Does this project support the overall DOE objectives of petroleum displacement? Why or why not?}

\section{Reviewer 1:}

The reviewer stated that successfully synergizing external aerodynamics, cooling system performance, and underhood thermal analysis can lead to a beneficial cascading effect with regards to achieving greater vehicle system efficiency. For example, optimizing these elements and their resultant cooling rates can lead to smaller and differently oriented radiators, potential application of other cooling strategies/fluids, and increased flexibility in external aerodynamic designs of truck tractors. There is an overall combinatorial effect that can be leveraged. As such, this analysis is relevant to the overall DOE objective of achieving greater vehicle fuel efficiency and increased petroleum displacement.

\section{Reviewer 2:}

While the reviewer did not see barriers to implementing this vehicle driving technique, or if these techniques are already in practice by Class 8 operators, the reviewer stated that this is clearly a method to reduce petroleum usage.

\section{Reviewer 3:}

The reviewer said that understanding how platooning impacts fuel efficiency essentially quantifies how driving behavior could help in lowering petroleum use even further after component design is optimized. The two lane platooning is viable perhaps on a four- or five- lane highway like I-495, but perhaps not so viable on a two-lane highway.

\section{Reviewer 4:}

The reviewer pointed out that the overall goal of this project is truck energy savings, which are relative to DOE's overall petroleum displacement objective. However, the reviewer did not think the research method is efficient enough to approach the goal. 


\section{Question 6: Resources: How sufficient are the resources for the project to achieve the stated milestones in a timely fashion?}

Reviewer 1:

The reviewer commented that the project completed quite a bit of analysis given the resources provided.

Reviewer 2:

The reviewer said that the project requires computer resource and manpower, and the budget is sufficient to support the project.

Reviewer 3:

The reviewer remarked that funding for this project has been limited in the last several years due to budgetary constraints. If a clearer project pathway with set milestones and end point is established moving forward, the project could justifiably be considered for additional funding. 


\section{Cummins Medium-Duty and Heavy-Duty Accessory Hybridization CRADA: Dean Deter (Oak Ridge National Laboratory) - vs133}

Presenter

Dean Deter, Oak Ridge National Laboratory

\section{Reviewer Sample Size}

A total of five reviewers evaluated this project.

\section{Question 1: Approach to performing the work-the degree to which technical barriers are addressed, the project is well-designed, feasible, and integrated with other efforts.}

\section{Reviewer 1:}

The reviewer stated that this is a two-phase project. In Phase One (modeling), the project team got accessory data to put into model. In Phase Two (testing and validation), the team put the system modeled into a test vehicle at Cummins. This approach was not only logical but cost effective, doing lower cost modeling earlier on and saving more costly field testing until the end of the project.

\section{Reviewer 2:}

The reviewer said that the project team modeled, then tested to validate the model. It is a valid approach.

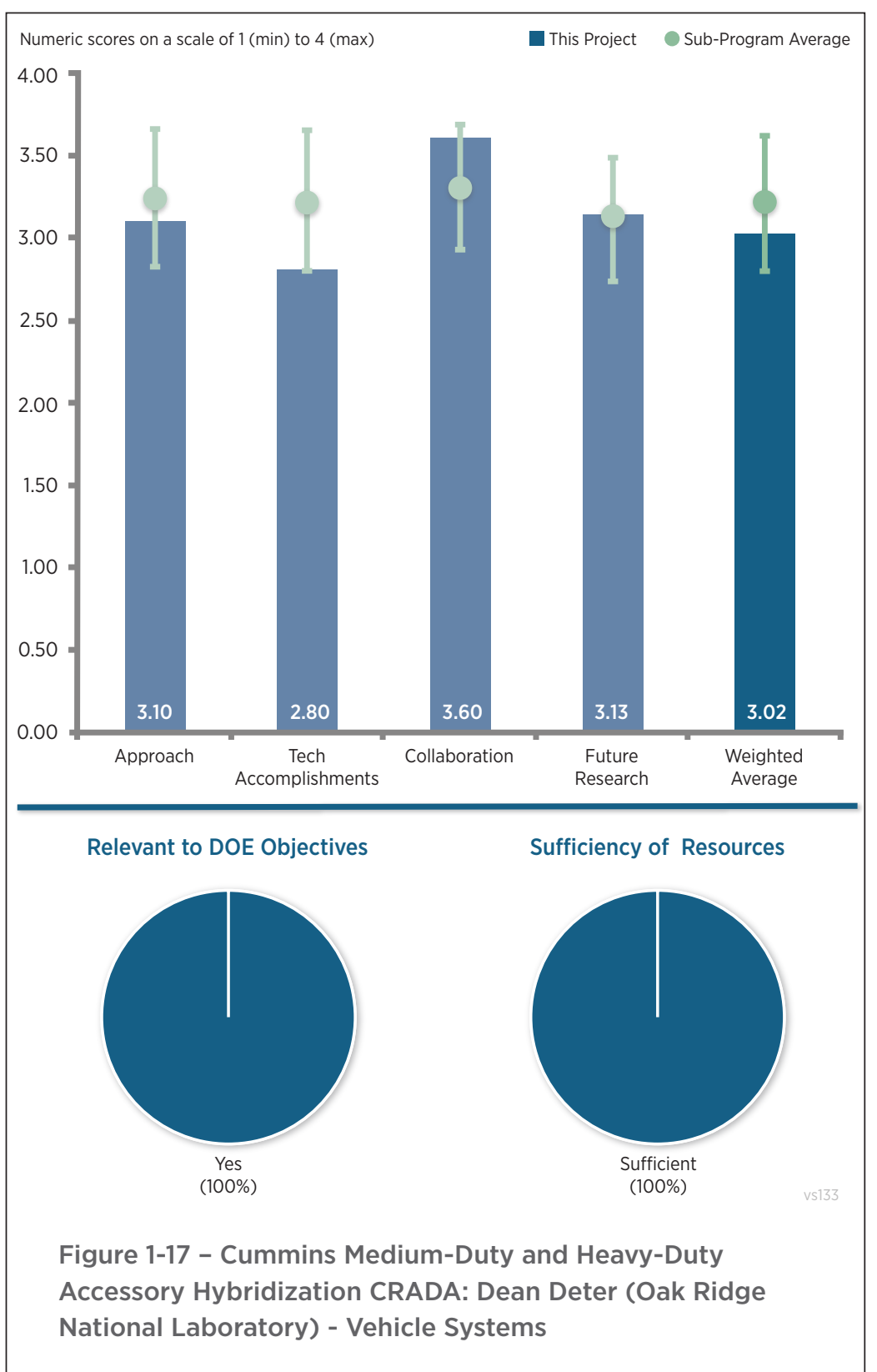

\section{Reviewer 3:}

The reviewer found the approach to be solid as it moved from analysis to prototype testing to full vehicle, and it was completed in a relatively short period of time.

\section{Reviewer 4:}

The reviewer stated that this project directly focuses on the real-world losses experienced by long-haul trucks while idling and in hotel mode. The use of a CRADA is sometimes necessary to gain more than pre-commercial results by working with industry; according to the reviewer, the project was unfairly criticized during the review as not showing results. In the context of the project approach, the project is outstanding for having the cooperation of a major OEM. The project did expose room for improvement on accessory loads that are optimized for in-use (which is obviously necessary) and lack of validated modeling tools for the load cases being studied.

\section{Reviewer 5:}

The reviewer remarked that these kinds of projects present a problem. The approach on using modeling and simulation to find more efficient solutions is well known and common practice. Because no results were presented 
due to a proprietary relationship with Cummins, it is impossible to evaluate whether the modeling was broad enough and complete.

The reviewer commented that a more appropriate way to present projects that have proprietary data should be identified. The suggestion on this particular project would be to discuss the technical approach, the modeling systems being used, and how they were applied. This reviewer emphasized that those items are not proprietary and, if presented in earnest, would allow evaluators to have a sensible discussion of the project without violating the agreement with the CRADA partner.

The reviewer said that it seemed that the project team takes this as an opportunity to, in effect, take a free pass on doing a serious presentation. Projects like this should be reviewed in the poster session in the future.

\section{Question 2: Technical accomplishments and progress toward overall project and DOE goals-the degree to which progress has been made, measured against performance indicators and demonstrated progress towards DOE goals.}

\section{Reviewer 1:}

The reviewer found that this project completed all five of the project milestones. The project team showed good alignment between model and field testing with about a 5\% error. The team considered all the accessories including refrigeration but believed that the ones focused on in this project were the best ones to attack.

\section{Reviewer 2:}

The reviewer noted that the project team achieved and completed its technical goals. The team focused on the long haul truck segment, and new model development occurred. Accessories are already designed well for steady state operation. Idling reduction is low hanging fruit. The air conditioning $(\mathrm{A} / \mathrm{C})$ test cell seems helpful to industry.

\section{Reviewer 3:}

The reviewer noted that, based on the boundaries in Question 1, the technical accomplishments to the engineering tools are very good. Of course, the CRADA does not allow elaboration to other results.

\section{Reviewer 4:}

The reviewer said that no technical accomplishments were presented. Because a statement was made as to the desire of Cummins to gain proprietary rights to some of the resulting systems configurations, it can only be surmised that there were valuable accomplishments but there was no way to evaluate that.

\section{Reviewer 5:}

The reviewer stated that the project is complete with a model and did well with the resources and time available. The major outstanding issue is the model, which is still not releasable due to proprietary threads. In order to really benefit other projects, this final closeout item needs to be worked out; otherwise, DOE did not gain from executing the CRADA (the reason for the reviewer's rating). It would have been helpful if there were more components modeled.

\section{Question 3: Collaboration and coordination with other institutions.}

\section{Reviewer 1:}

The reviewer said that collaboration with Cummins has the greatest potential of future product impact than any other engine system development company could have.

\section{Reviewer 2:}

The reviewer remarked that there were a variety of players involved including ORNL, Cummins, NREL, EMP, and MasterFlux. The collaboration and the role each member took on was well thought out.

\section{Reviewer 3:}

The reviewer stated that there was a nice example of collaboration with EPA/Southwest Research Institute (SwRI) on the available model and test. The reviewer asked how this project team can educate the suppliers in this space better. 


\section{Reviewer 4:}

The reviewer noted that this project was a CRADA with one specific manufacturing company. There was collaboration with NREL as well. The reviewer noted that CRADAs by definition are cooperative agreements with one company. This question really does not apply to this project.

\section{Reviewer 5:}

The reviewer asserted that this was a closed CRADA and Cummins led the decisions on who was involved. There were some suppliers involved, so it seemed that proper collaborations were included.

Question 4: Proposed future research-the degree to which the project has effectively planned its future work in a logical manner by incorporating appropriate decision points, considering barriers to the realization of the proposed technology and, when sensible, mitigating risk by providing alternate development pathways.

\section{Reviewer 1:}

The reviewer stated that if the initiatives of this program come to fruition, they could have an enormous effect on petroleum displacement.

\section{Reviewer 2:}

The reviewer picked up on a statement that Cummins will proceed with further R\&D based on the work completed. This is an excellent outcome of the program.

\section{Reviewer 3:}

The reviewer proposed that the models from this completed project be used to feed other projects. It would have been helpful if more components could be modeled, but this project ran out of time and funding to do so.

\section{Reviewer 4:}

The reviewer said that the project has ended.

\section{Reviewer 5:}

The reviewer noted that the program is basically completed.

\section{Question 5: Does this project support the overall DOE objectives of petroleum displacement? Why or why not?}

\section{Reviewer 1:}

The reviewer commented that if the initiatives of this program come to fruition, they could have an enormous effect on petroleum displacement.

\section{Reviewer 2:}

The reviewer stated that this was an enabling project that can be used to evaluate technologies that directly support DOE's goal.

\section{Reviewer 3:}

The reviewer said that reducing energy losses due to accessory loads will reduce fuel consumption, which reduces petroleum use.

\section{Reviewer 4:}

The reviewer commented that the project focuses on reducing or optimizing accessory loads of HD line haul trucks. Overnight drivers are down for 10 hours consuming 4-7 gallons of diesel while idling to support accessory loads needed during down time. Optimizing the loads help to reduce petroleum consumption.

\section{Reviewer 5:}

The reviewer thought that this area has real opportunity, but so many players and concepts makes it tough. The reviewer very much encouraged DOE to find some way to share the highest level learnings from projects like this one and asked how that can that be done. It sort of falls into how DOE promotes learnings to the industry. 
Question 6: Resources: How sufficient are the resources for the project to achieve the stated milestones in a timely fashion?

Reviewer 1:

The reviewer believed that there was no indication that project funding was an issue in this program.

Reviewer 2:

The reviewer stated that the project is complete and there are enough resources.

Reviewer 3:

The reviewer noted that because the project is finished and Cummins is proceeding with using the work, resources must have been sufficient.

Reviewer 4:

The reviewer remarked that the project was able to complete all its milestones with the funds provided. 
Vehicle Thermal System

Modeling in Simulink:

Jason Lustbader (National

Renewable Energy

Laboratory) - vs134

\section{Presenter}

Jason Lustbader, National Renewable

Energy Laboratory

\section{Reviewer Sample Size}

A total of six reviewers evaluated this project.

\section{Question 1: Approach to performing the work-the degree to which technical barriers are addressed, the project is well-designed, feasible, and integrated with other efforts.}

\section{Reviewer 1:}

The reviewer asserted that making a first principles model for heating, ventilation, and $\mathrm{AC}$ (HVAC)/thermal system is an excellent approach. Having it integrated into the rest of the vehicle/powertrain model is great.

\section{Reviewer 2:}

The reviewer remarked that the project objectives and approach are commendable. They are realistic, logical, and offer a solid foundation for future work by NREL and the industry.

\section{Reviewer 3:}

The reviewer stated that this simulation has appropriate fidelity to make meaningful contributions to the design of advanced HVAC systems for EVs as it uses the Matlab/Simulink platform, a simulation platform that is widely used by industry and academia. This simulation can co-simulate with Autonomie.

\section{Reviewer 4:}

The reviewer commented that the proposed approach to handle the stiff network by using an artificially low bulk modulus would also introduce lower frequency modes in the system. The reviewer asked if this could result in the system behaving oddly. If there is no interest in the fast transients at all, the reviewer proposed that the obvious approach would be to ignore compressibility of the fluid (at least, where it exists as a single phase) as this would not give rise to any of the spurious modes.

\section{Reviewer 5:}

The reviewer remarked that the emphasis on freely available prototype code based on the popular Matlab/Simulink

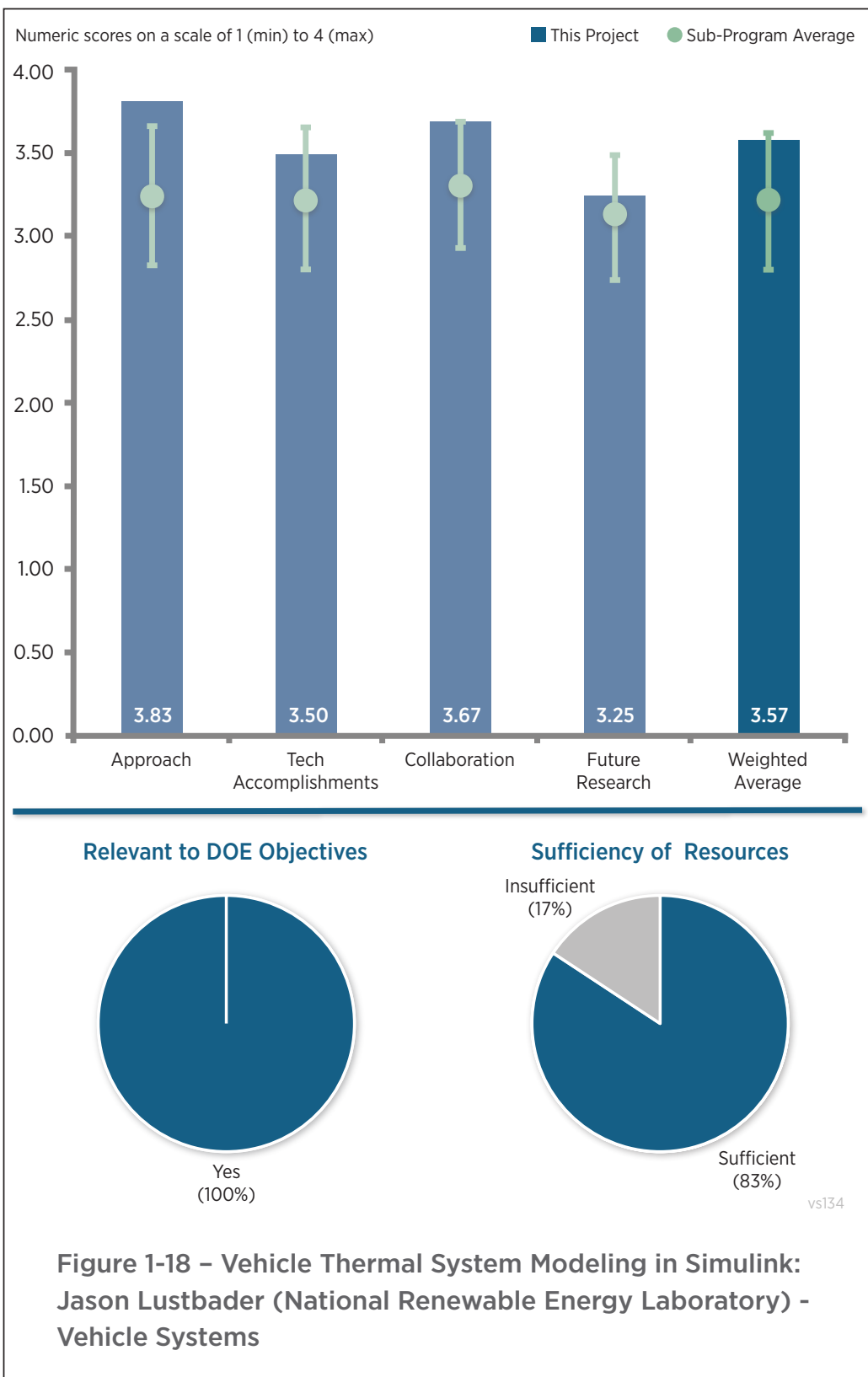


framework, CRADAs for application studies, and the consideration of integration with Autonomie and similar tools seems smart and appropriate.

Improvements for milestone four should ideally be at least partly directed by the end-user. This could be facilitated by a small beta-test group outside of NREL to try out the available capabilities, supported by an intermediate milestone three deliverable from the documentation task.

\section{Question 2: Technical accomplishments and progress toward overall project and DOE goals-the degree to which progress has been made, measured against performance indicators and demonstrated progress towards DOE goals.}

\section{Reviewer 1:}

The reviewer enthused that the project accomplishments to date are impressive. The approach to fill in the holes in exiting vehicle environmental controls analysis is working.

\section{Reviewer 2:}

The reviewer commented that the simulation is being applied to multiple advanced HVAC design projects that vehicle component OEMs are conducting. It appears to be a successful enabler for innovating and transitioning HVAC technology for EVs.

\section{Reviewer 3:}

The reviewer indicated that the project shows flexibility of operation and integrated control.

\section{Reviewer 4:}

The reviewer commented that the project is encountering the typical bootstrapping challenge of balancing capabilities development with application studies to verify and demonstrate the capabilities. The presentation made the latter part seem a little too heavy and detailed for the current status of the tool. In other words, more numerous and diverse yet simpler test cases at this stage may lead to a more robust and versatile software.

\section{Reviewer 5:}

The reviewer noted that, while significant progress has been made, it appears that the FY 2017 deliverables are identical to the FY 2016 deliverables. According the 2015 AMR presentation, milestone three should have been completed sometime in the second quarter of FY 2016, but it has been moved back to end of FY 2016 in this year's AMR presentation.

\section{Question 3: Collaboration and coordination with other institutions.}

\section{Reviewer 1:}

The reviewer observed that presumably the changes seen due to mergers and acquisitions in the industry have not had much impact on the collaboration.

\section{Reviewer 2:}

The reviewer mentioned that there are multiple industry partners that are actively using this simulation on their R\&D projects.

\section{Reviewer 3:}

The reviewer said that the project has an excellent set of partners.

Question 4: Proposed future research-the degree to which the project has effectively planned its future work in a logical manner by incorporating appropriate decision points, considering barriers to the realization of the proposed technology and, when sensible, mitigating risk by providing alternate development pathways.

\section{Reviewer 1:}

The reviewer enthused about not wanting to wait to see this project integrated with drive cycles to refine controls and understand consumption benefits. The reviewer suggested that the project team also needs to make the component models parametric and validate this as well. 
The next phase of the project should be showing real-world benefits of these technologies on advanced powertrain vehicles.

\section{Reviewer 2:}

The reviewer commented that the future project work is an absolute must. Improving the simulation environment in this area is desperately needed.

\section{Reviewer 3:}

This reviewer highlighted the FY 2015 reviewer question 2 on Slide 30, and strongly pointed out that the response failed to answer the question. Assuming the answer is "no, not as of today," two recommendations arise for devoting a portion of the remaining project resources. The first recommendation is, as application cases continue (Slide 31, bullet 2), to strongly prioritize those that are non-proprietary and work to develop detailed, hands-on tutorials based on them. The reviewer's second recommendation is, before end of project, to issue a competitive Request for Information for licensing CoolCalc and CoolSim and work out terms so that an interested party other than NREL can be positioned to provide the essential, substantial training, maintenance, and support services needed by vehicle system engineers in industry.

\section{Reviewer 4:}

The reviewer remarked that developing a flexible, publicly available Matlab/Simulink-based framework for vehicle thermal management system is a perfectly reasonable goal. However, it would be a stretch to expect that this tool would be used by the OEMs and the suppliers. The reviewer said that this is not to say that the tool is lacking in capability - it is just that most of the companies that have been involved in this kind of work for some time have their own established processes that are very reliant on specific advantages that their software of choice offers. Many man-years of effort would have gone into developing these processes, and it would it be hard for the OEMs to abandon all of that in a favor of new software, even if it were more capable. That said, as someone from the industry, the reviewer would have been more than happy to give it a try and see if it can do a better job than the current set of tools in use!

\section{Question 5: Does this project support the overall DOE objectives of petroleum displacement? Why or why not?}

\section{Reviewer 1:}

The reviewer stated that this is an excellent project and very worthwhile.

\section{Reviewer 2:}

The reviewer said this was great modeling work and will be very useful.

\section{Reviewer 3:}

The reviewer commented that, yes, this simulation provides useful capability to increase EV range and displace petroleum.

\section{Reviewer 4:}

Besides motive power, the reviewer noted that climate control is the second most energy expensive service. Further development is needed to mature the technology portfolio.

\section{Question 6: Resources: How sufficient are the resources for the project to achieve the stated milestones in a timely fashion?}

\section{Reviewer 1:}

The reviewer stated that this project deserves more funding because it is a useful tool for industry and academia to design more efficient and effective HVAC solutions for EVs. The current funding level is insufficient to add new capabilities and successfully address relevant domain challenges.

\section{Reviewer 2:}

The reviewer commented that the project appears to be appropriately resourced. 


\section{Advanced Climate Systems for EV Extended Range (ACSforEVER): John Meyer (Hanon Systems) - vs135}

\section{Presenter}

Nicos Agathocleous, Hanon Systems

\section{Reviewer Sample Size}

A total of four reviewers evaluated this project.

\section{Question 1: Approach to performing the work-the degree to which technical barriers are addressed, the project is well-designed, feasible, and integrated with other efforts.}

\section{Reviewer 1:}

The reviewer noted that the work plan and goals are well thought out. The approach addresses the questions surrounding the implementation of the technology. The notion of utilizing the thermal inertia of the components is a practical way to maximize benefit at minimum cost.

\section{Reviewer 2:}

The reviewer found the objective to achieve passenger comfort with reduced auxiliary loads to be a very significant hurdle to overcome in efforts to achieving widespread acceptance of xEV technology. This project does attempt a logical and direct approach in achieving the stated objectives of the program.

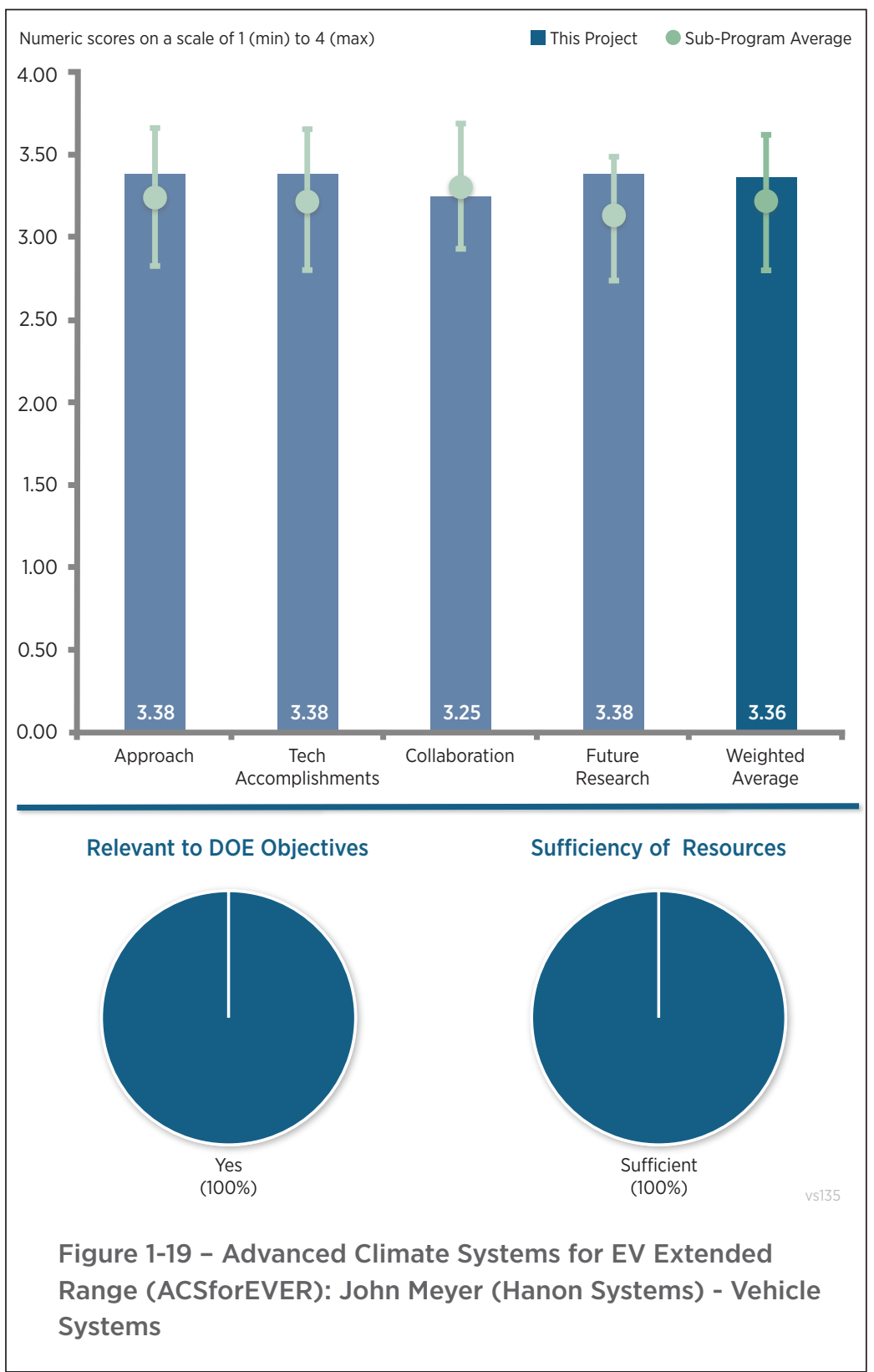

\section{Reviewer 3:}

The reviewer stated that this is a very comprehensive technical approach. However, cost should be a factor in technology selection.

\section{Reviewer 4:}

The reviewer wanted to see the results of the thermal comfort simulation being presented in terms of a statistical confidence interval, given that in real life the fact that one person may find the thermal comfort acceptable by no means implies that another person would. Alternatively, it would also help if it were made clear that because the purpose is to improve EV range, the results of the simulations would imply an improvement provided we have ceteris paribus. And from that perspective, the reviewer wanted to clarify whether a simpler measure of comfort could not be used to quantify the benefits of the new systems.

\section{Question 2: Technical accomplishments and progress toward overall project and DOE goals-the degree to which progress has been made, measured against performance indicators and demonstrated progress towards DOE goals.}




\section{Reviewer 1:}

The reviewer noted that the project team appears to have been able to provide greater understanding of the limitations of existing modeling tools and hopefully has improved on their limitations. The challenges of adding or taking away heat in rapid volume will be arduous for this team to overcome; hopefully, a human factors or psychology-based metric will be added to correlate the physical data to human comfort.

\section{Reviewer 2:}

The reviewer commented that the project appears to be behind by a couple of months, which is not a big deal. Separately, on Slide 7, the project team stated that "neither the Berkeley nor the Fiala models adequately predicted the effects of cabin temperature changes on thermal sensation." While the reviewer did not claim to be an expert on human physiological models, the reviewer presumed that the Berkeley and the Fiala models were developed to predict human comfort and had been peer-reviewed. The reviewer then asked on what basis the team concluded that they are not adequate and that some weighted average version of it was actually preferable. Presumably, much research has gone into developing the Berkeley and Fiala models, and it seems that the dismissal of these models is almost casual, and neither is any basis presented for the acceptability of the new thermal sensation metric. If this were based on feedback from one or more test subjects, then this calls into question the statistical validity of the conclusion.

\section{Reviewer 3:}

The reviewer pronounced the progress to be very good. For future reviews, the reviewer suggested some explanation or other notation on Slide 15 as the graphs are hard to interpret. The wall-to-wheels efficiency of the system should also be indicated somewhere.

\section{Reviewer 4:}

The reviewer commented that there was a good approach using several technologies simultaneously to achieve objective. There seemed to be no consideration apparent on $\$ / \mathrm{mi}$ of range extension. The project team, according to the reviewer, has not addressed the issue of starting trips without prior charging (trips not starting after residential or workplace charging).

\section{Question 3: Collaboration and coordination with other institutions.}

\section{Reviewer 1:}

The reviewer commented that there was very good OEM participation.

\section{Reviewer 2:}

The reviewer noted that the appropriate partners are involved (OEM, supplier, national laboratories) to properly execute the project.

\section{Reviewer 3:}

The reviewer did not understand the coordination very well, but as the project does appear to be focused in the correct direction, the results are sufficient to state an acceptable effort.

\section{Reviewer 4:}

The reviewer stated that it appears that NREL (which has had experience in the area) was not involved in the thermal comfort modeling. The different set of experiences that the NREL personnel would have had could have provided valuable feedback and ensured the best possible outcome.

Question 4: Proposed future research-the degree to which the project has effectively planned its future work in a logical manner by incorporating appropriate decision points, considering barriers to the realization of the proposed technology and, when sensible, mitigating risk by providing alternate development pathways.

\section{Reviewer 1:}

The reviewer believed the objectives are well understood and support future work in this area.

\section{Reviewer 2:}

The reviewer commented that future work is to complete the remaining deliverables. 


\section{Reviewer 3:}

The reviewer would like to have seen more justification for some of the decisions that were made and asked the project team to take a look at the reviewer's previous comments.

\section{Reviewer 4:}

The reviewer said that there was insufficient time allowed for vehicle testing and proposed that the project team may want to do a no-cost time extension.

\section{Question 5: Does this project support the overall DOE objectives of petroleum displacement? Why or why not?}

\section{Reviewer 1:}

The reviewer stated that research informs technology choices that can be made to improve EV range under realworld driving conditions. The impact of the environment on EV range is a barrier to more widespread EV adoption.

\section{Reviewer 2:}

The reviewer commented that, absolutely, the ability to maintain passenger compartment comfort with minimal energy used (lost) is critical to widespread acceptance of xEV technology and therefore petroleum displacement.

\section{Reviewer 3:}

The reviewer praised the technical accomplishments as excellent; however, cost must be a consideration.

\section{Question 6: Resources: How sufficient are the resources for the project to achieve the} stated milestones in a timely fashion?

\section{Reviewer 1:}

The reviewer said that the project appears to be adequately funded.

\section{Reviewer 2:}

The reviewer found that no information to the contrary was presented on resources. 


\section{ePATHS - electrical PCM Assisted Thermal Heating System: Mingyu Wang (Mahle Behr USA, LLC) - vs136}

\section{Presenter}

Mingyu Wang, Mahle Behr USA, LLC

\section{Reviewer Sample Size}

A total of four reviewers evaluated this project.

\section{Question 1: Approach to performing the work-the degree to which technical barriers are addressed, the project is well-designed, feasible, and integrated with other efforts.}

\section{Reviewer 1:}

The reviewer commented that the project team has been very thorough in attacking all aspects of the problem and was impressed by the team's going the extra mile to find a new phase change material (PCM).

\section{Reviewer 2:}

The reviewer noted that PCMs have been discussed for a long time for this type of application. It was, therefore, an advantageous study to promote the development of PCMs for thermal storage in vehicle space.

\section{Reviewer 3:}

The reviewer said that this is an ambitious, challenging project, and the approach being taken is excellent and increases the likelihood of success.

The project directly addresses multiple barriers to meeting DOE goals, and the technical approach is sufficiently rigorous for a systems engineering project of this level.

\section{Reviewer 4:}

The reviewer found that the technical approach, with testing and validation, was well laid out.

\section{Question 2: Technical accomplishments and progress toward overall project and DOE} goals-the degree to which progress has been made, measured against performance indicators and demonstrated progress towards DOE goals.

\section{Reviewer 1:}

The reviewer commented that the development of unique if not novel PCMs for this application is arduous and complex. The analysis of production feasibility was insightful and demonstrated a barrier of high quantity (technology widespread acceptance in terms of applications). 
The reviewer asked what the vehicle mass and packaging volume tradeoffs are for this application and how these will affect commercial feasibility.

\section{Reviewer 2:}

The reviewer pronounced the execution of the work to be very good. Yet, the PCM packaging issue is a bit of a setback because it limits practical application of the technology. As suggested during the review, perhaps downsizing the thermal storage may be an available avenue. The reviewer proposed that the overall energy efficiency of the system from a wall-to-wheels perspective should be reported.

\section{Reviewer 3:}

The reviewer found a need to evaluate energy efficiency of the overall system and specifically compare cost against adding a battery to recover range. The reviewer said that the assumption of a 1 million $\mathrm{kg} / \mathrm{year}$ PCM manufacturing facility may be an overreach when trying to meet cost objectives.

\section{Reviewer 4:}

The reviewer stated that numerous accomplishments were identified and the project appears to be on schedule. Performance metrics were met in bench testing and prototype builds.

The results of the manufacturing cost study showed that a dedicated plant must be used to achieve sufficient scale to meet cost targets for the PCM material. This decreases the likelihood that this product will make it to production, but this determination is an accomplishment, nonetheless. The project team indicated that the grid-to-wheels efficiency of the system is better than using the battery to run the positive temperature coefficient heater. This should be quantified and communicated.

The reviewer stated that the system must be superior to lithium-ion batteries on a cost basis. The project team indicated that the system is expected to achieve this. Cost targets and status to target should be specified.

\section{Question 3: Collaboration and coordination with other institutions.}

\section{Reviewer 1:}

The reviewer said that the project had an excellent team working well together.

\section{Reviewer 2:}

The reviewer found outstanding collaboration and coordination, with partners taking active roles in all major areas.

\section{Reviewer 3:}

The right partners are involved in the project to make it a success, according to the reviewer.

\section{Reviewer 4:}

It was unclear to the reviewer what the partnership responsibilities actually were, but the companies involved are high quality stakeholders.

\section{Question 4: Proposed future research-the degree to which the project has effectively planned its future work in a logical manner by incorporating appropriate decision points, considering barriers to the realization of the proposed technology and, when sensible, mitigating risk by providing alternate development pathways.}

\section{Reviewer 1:}

The reviewer noted that the project team is focused on completing the project goals.

\section{Reviewer 2:}

The reviewer believed that the best evaluation will done at vehicle levels and the commercial salability will be accurately assessed there in the final months of this project.

\section{Reviewer 3:}

The reviewer stated that vehicle-level testing is important and will be conducted, but timing is tight. The remaining 
work must be accomplished flawlessly to be successfully completed. Volume (and presumably weight) must be reduced in order to be viable for production. Packaging the unit in the trunk can have a negative impact on customer satisfaction. The reviewer exclaimed that customers may care more about trunk space than EV range.

\section{Reviewer 4:}

The reviewer commented that the schedule to complete testing unrealistically assumes no issues revealed during tests.

\section{Question 5: Does this project support the overall DOE objectives of petroleum displacement? Why or why not?}

\section{Reviewer 1:}

The reviewer said that this project addresses the issue of reduced EV range due to occupant comfort considerations under real world driving conditions. Success of this technology will improve the chances of EV adoption by the average driver.

\section{Reviewer 2:}

The reviewer commented that any technology that improves efficiency and market acceptance of xEVs in transportation is directionally correct in the goals of petroleum displacement.

\section{Reviewer 3:}

The reviewer said that this project is highly relevant to DOE's goals of improving PEV performance and appeals to promoting PEV adoption and reducing petroleum consumption.

\section{Reviewer 4:}

The reviewer noted that a goal has been set to restore the equivalent of $3 \mathrm{kWh}$ of battery at a cost of $\$ 200 / \mathrm{kWh}$. The project presenter indicated that the current overall cost is $\$ 270-\$ 300$, or half of the per-kWh cost objective. If accurate, this is an excellent result and is very relevant.

\section{Question 6: Resources: How sufficient are the resources for the project to achieve the stated milestones in a timely fashion?}

\section{Reviewer 1:}

The reviewer said that the funds and personnel needed to complete the remaining milestones appear to be sufficient.

\section{Reviewer 2:}

The reviewer noticed no obvious financial barriers in the discussion.

\section{Reviewer 3:}

The reviewer stated that the funding level is reasonable, considering the scope of work, number of partners involved, and the technology readiness level of the technology at the start of the project. Additional time will likely be required for successful completion of remaining work. 


\section{SAE J2907 Motor Power Ratings Standards Support: John Miller (Oak Ridge National Laboratory) - vs144}

Presenter

John Miller, Oak Ridge National Laboratory

\section{Reviewer Sample Size}

A total of five reviewers evaluated this project.

\section{Question 1: Approach to performing the work-the degree to which technical barriers are addressed, the project is well-designed, feasible, and integrated with other efforts.}

\section{Reviewer 1:}

The reviewer noted that it is amazing that this has not been done before. The project is well structured and certainty needed in the industry.

\section{Reviewer 2:}

The reviewer commented that the project helps to promote a common language around the way we discuss electric traction drives, especially at the consumer level. This should help demystify technology for consumers.

\section{Reviewer 3:}

The reviewer remarked that the project approach conforms to a wellestablished SAE method of developing standards: the technical community builds a committee, which then works with the user stakeholders to find the technical solutions that fit the intent of the standard being established. It is a tried and proven method.

\section{Reviewer 4:}

The reviewer said that it seems that all the steps needed to develop the standard for ballot have been achieved.

\section{Reviewer 5:}

The reviewer stated that the project and its presentation did not effectively show why DOE is involved. The industry needs may be clear, but the need for DOE involvement is less clear. Also arguable is that this figure is important to vehicle buyers, who are unlikely to be very concerned with motor output levels when selecting which electrically propelled vehicle to purchase.

Question 2: Technical accomplishments and progress toward overall project and DOE goals - the degree to which progress has been made, measured against performance indicators and demonstrated progress towards DOE goals. 


\section{Reviewer 1:}

Given the difficulty of reaching consensus among the many stakeholders, the reviewer commented that the project is progressing well and achieving basic accepted definitions around electric traction drive system characterization.

\section{Reviewer 2:}

The reviewer noted the project is aligning with the normal progress of SAE standards, and parallel work on SAE J2908 is an important accomplishment as both standards are needed because of the way the drive motors are used in the market.

\section{Reviewer 3:}

The reviewer said that the project accomplishments have been reached through good technical work as well as a consensus with the target community for the standards, which is very tough to do.

\section{Reviewer 4:}

The reviewer stated that the project has a tight objective, and the result appears to be on track to achieve the consensus procedure for component-level peak and continuous power ratings.

\section{Reviewer 5:}

With the goal being a consensus based standard that meets the technical needs described, the reviewer remarked that the progression to a technical information report (TIR) is the accomplishment that matters the most. The subsurface technical issues that were debated have a lesser level of importance.

\section{Question 3: Collaboration and coordination with other institutions.}

\section{Reviewer 1:}

The reviewer noted that all appropriate institutions are involved in the effort.

\section{Reviewer 2:}

The reviewer stated that this project is clearly a collaborative effort as shown in the presentation.

\section{Reviewer 3:}

The reviewer pointed out that the partners are the members of the committee, and DOE is properly represented.

\section{Reviewer 4:}

The reviewer said that the project is collaborating with the appropriate organizations.

\section{Reviewer 5:}

The reviewer was not clear about what happens outside the United States. The reviewer asked about the breadth of global OEM participation, whether any other key markets are participating in this standard development, or if the European Union, Korea, and China continue independently.

Question 4: Proposed future research-the degree to which the project has effectively planned its future work in a logical manner by incorporating appropriate decision points, considering barriers to the realization of the proposed technology and, when sensible, mitigating risk by providing alternate development pathways.

\section{Reviewer 1:}

The reviewer noted that the proposed future work involves the standard process of creating an SAE standard.

\section{Reviewer 2:}

The reviewer commented that future work shown and the schedule presented made clear that this project should be completed in 2016.

\section{Reviewer 3:}

The reviewer said that the future is to get the TIR out and the standard finalized. This is the original intent of the program. 


\section{Reviewer 4:}

The reviewer found the proposed future work to be appropriate to reach the standards completion objective.

\section{Reviewer 5:}

The reviewer observed that the project appears to hit its objectives and the process will be concluded.

\section{Question 5: Does this project support the overall DOE objectives of petroleum displacement? Why or why not?}

\section{Reviewer 1:}

The reviewer supported this standard as it is certainly relevant to the objective of reducing petroleum consumption.

\section{Reviewer 2:}

The reviewer believed that a standardized measurement for power in the electric propulsion system arena is needed and that would give a reliable value to consumers to make a decision regarding the purchase of an EV. To the extent this comforts consumers as to the validity of marketing claims, it can foster adoption of electrified powertrains.

\section{Reviewer 3:}

The reviewer stated that this project supports the continued use of EVs through a better understanding of the product by those outside these technical fields.

\section{Reviewer 4:}

The reviewer found this project to be an industry enabler to measure EV drive systems appropriately and consistently.

\section{Reviewer 5:}

The reviewer agreed about supporting future research, if only weakly. Clarifying EV power requirements for consumers should promote better understanding of what is being offered and should provide a better basis for assessing products.

\section{Question 6: Resources: How sufficient are the resources for the project to achieve the stated milestones in a timely fashion?}

\section{Reviewer 1:}

The reviewer remarked that the resources appear to be adequate.

\section{Reviewer 2:}

The reviewer commented that this is a support activity with clearly qualified resources.

\section{Reviewer 3:}

The reviewer noted that the current level of resources has kept the program on time so the resources are assumed to be sufficient.

\section{Reviewer 4:}

The reviewer said that the resources appear to be sufficient.

\section{Reviewer 5:}

The reviewer indicated that the project appears to be on track for conclusion with its current resource level. There is no mention of any exception to that. 


\section{Analyzing Real-World Light Duty Vehicle Efficiency Benefits: Jeff Gonder (National Renewable Energy Laboratory) - vs155}

\section{Presenter}

Jeff Gonder, National Renewable Energy Laboratory

\section{Reviewer Sample Size}

A total of four reviewers evaluated this project.

\section{Question 1: Approach to performing the work-the degree to which technical barriers are addressed, the project is well-designed, feasible, and integrated with other efforts.}

\section{Reviewer 1:}

The reviewer stated that the technical barrier is that the existing standard certification cycles cannot give credit to some new fuel saving technologies (e.g., start-stop, engine encapsulation, and connected and automated vehicles). A new model is being developed. The model is then calibrated using dynamometer data and validated by on-road testing. This is a standard and efficient approach to develop a new model and fuel economy evaluation tool.

\section{Reviewer 2:}

The reviewer commented that this is a great framework for studying the real-world benefits of efficiency improvement technologies. It would be useful to add the impact of air density with temperature and elevation as well as effective draft coefficient $(\mathrm{Cd})$ changes with yaw-rate of cross-wind. This may be harder to get from the manufacturer, but cross-wind should be added as the project team suggested.

The reviewer asked that the project team please add the rate dependence of short versus long trips (based on 30 or 40 mile demarcation from NHTSA data) and alternative powertrain technology vehicles.

\section{Reviewer 3:}

The reviewer stated that the approach does a good job of being all encompassing, but now needs to focus on making the results practical and useful. As opposed to driving for more accuracy, the reviewer suggested focusing on why the current accuracy is good enough by showing that the effects on the output are not significant when singular points are improved or reduced in accuracy.

\section{Reviewer 4:}

The reviewer found the approach of this project to be confusing to a short term observer. The objectives say the

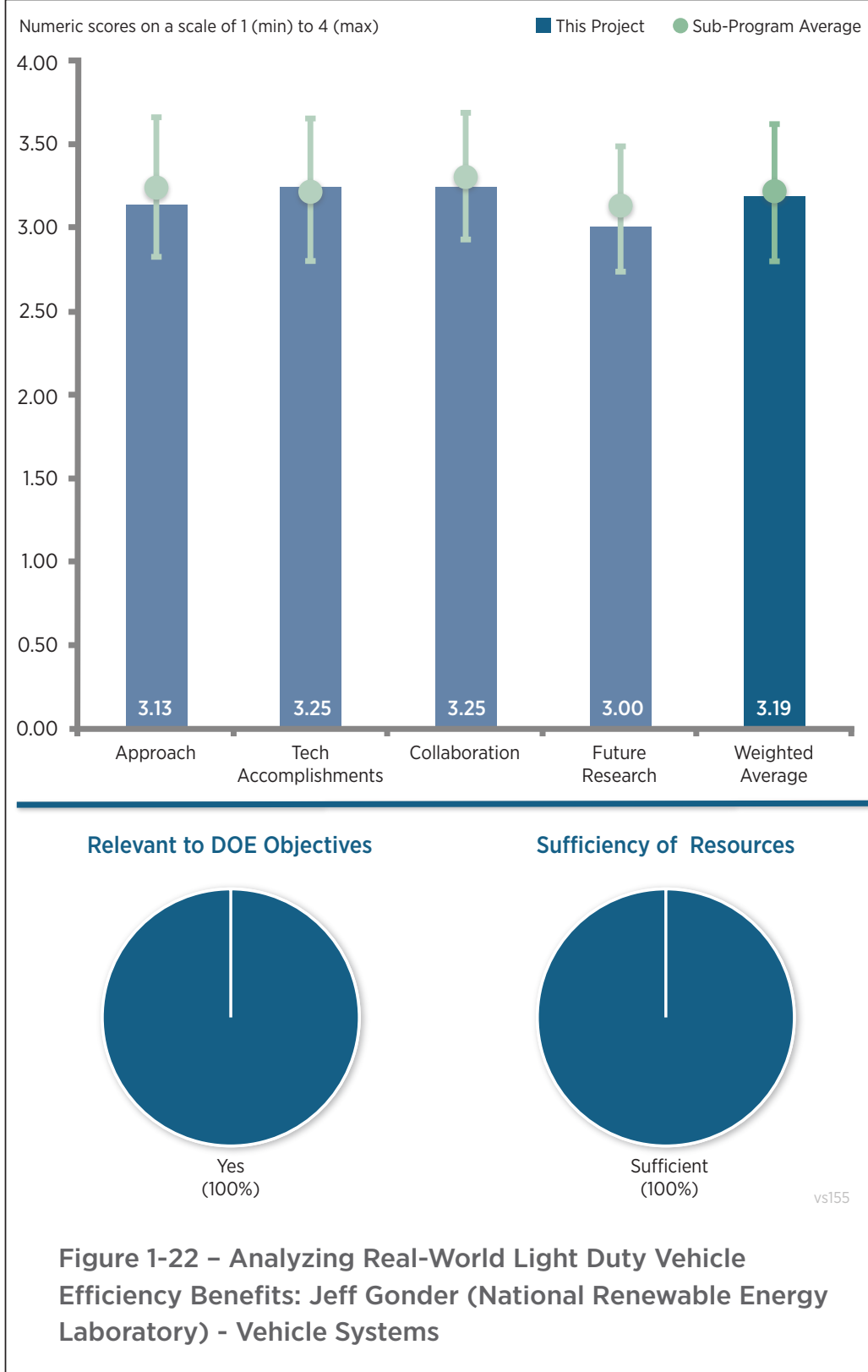


purpose is to evaluate nonstandard technologies for efficiency quantification, but the dynamometer approach is quite traditional. The project also appears to be spending quite a bit of time validating the modeling methods. It would be good to see a focus on the objectives.

\section{Question 2: Technical accomplishments and progress toward overall project and DOE goals-the degree to which progress has been made, measured against performance indicators and demonstrated progress towards DOE goals.}

\section{Reviewer 1:}

The reviewer stated that the data seem very good and useful. The reviewer appreciated the data on the spread of customer in-use fuel economies and the effect of $\mathrm{A} / \mathrm{C}$ and where the step change occurs. The project team mentioned solar loading skewing the $\mathrm{A} / \mathrm{C}$ data, but variability in humidity is also a factor.

\section{Reviewer 2:}

The reviewer complimented the project team on its great technical accomplishments and progress made so far. From the comparison of model prediction and real-world on-road tests, it can be seen that the model can predict the mpg within $\pm 10 \%$ with a root mean square error of $6.4 \%$. It is good.

On Slide 15, when the real-world estimate is compared with other calculation approach, the reviewer suggested that it would be great to put the on-road test data in the table for comparison.

\section{Reviewer 3:}

The reviewer noted that the technical accomplishments for the first year are mostly results validating the models and dynamometer testing, which appear to be standard tests. The reviewer was not really sure where this is going.

\section{Question 3: Collaboration and coordination with other institutions.}

\section{Reviewer 1:}

The reviewer stated that it can be seen that many institutes are involved in the project. This project cannot be done without extensive collaboration among different parties.

\section{Reviewer 2:}

The reviewer urged the project team to keep up the connection with EPA and try to create a workable alternative to using five cycle for off-cycle credit determination. Tens of thousands of simulations depending on set-up may not be a workable solution for all technology cases.

\section{Reviewer 3:}

If the U.S. auto industry wants this evaluation to get the credit, then the reviewer pointed out that a more in-depth collaboration should be in order. At the moment it is only for project reviews. The EPA connection should be stronger as well. The reviewer said that a connection to NHTSA would be helpful as it is setting the standards for future vehicles.

\section{Question 4: Proposed future research-the degree to which the project has effectively planned its future work in a logical manner by incorporating appropriate decision points, considering barriers to the realization of the proposed technology and, when sensible, mitigating risk by providing alternate development pathways.}

\section{Reviewer 1:}

The reviewer remarked that the project is near its completion and asked the project team to please focus on the arguments against the use of this technique. The reviewer's point of view was that adding accuracy to the modeling might not be needed to make the data useful. The reviewer encouraged the team to look to simplify the technique as much as possible while maintaining the usefulness of the output.

\section{Reviewer 2:}

The reviewer commented that the project is approaching its deadline of September 2016. The remaining three or four months may not enough for the proposed future work. 


\section{Reviewer 3:}

The reviewer strongly and enthusiastically wanted to see this framework implemented for EVs. The reviewer asked the project team to please plot the energy consumption versus trip distance as EPA is hungry for this plot for both EVs and conventional vehicles.

\section{Reviewer 4:}

The reviewer observed that the proposed research is much like that of the past year as it appears unorganized and distant from the objectives.

\section{Question 5: Does this project support the overall DOE objectives of petroleum displacement? Why or why not?}

\section{Reviewer 1:}

The reviewer stated that the project is helping to describe the value of technologies in a way that has not been previously examined in depth.

\section{Reviewer 2:}

The reviewer believed that the project is on the border here, but with some better structuring, or clarification of the current structure, it could be strongly relevant to the DOE mission.

\section{Reviewer 3:}

The reviewer noted that many new fuel saving technologies are emerging while the existing standard certification cycles cannot assess them correctly. Giving credit to these new technologies will promote the adoption of these technologies.

\section{Question 6: Resources: How sufficient are the resources for the project to achieve the stated milestones in a timely fashion?}

\section{Reviewer 1:}

The reviewer stated that the project appears to be sufficiently resourced.

\section{Reviewer 2:}

The reviewer said that the project has many collaborators and testing facilities to get the work done in time. 


\section{UTEMPRA - Unitary Thermal Energy Management for Propulsion Range Augmentation: Sourav Chowdhury (Mahle Behr USA LLC) - vs157}

\section{Presenter}

Sourav Chowdhury, Mahle Behr USA, LLC

\section{Reviewer Sample Size}

A total of four reviewers evaluated this project.

\section{Question 1: Approach to performing the work-the degree to which technical barriers are addressed, the project is well-designed, feasible, and integrated with other efforts.}

\section{Reviewer 1:}

The reviewer stated that much effort has been expended in preparing for future production of the multi-mode flow controller and flux-less brazing hardware.

\section{Reviewer 2:}

The reviewer commented that the design concept appears promising. Although the system complexity is high, it consolidates all thermal management into one unit to provide an overall vehicle system benefit. The reviewer commended the team for using a contemporary systems engineering approach, following the define/design/verify process that leverages state-of-the-art systems modeling capabilities. Project management appears adequate and capable of managing multiple concurrent efforts by multiple partners.

The reviewer noted that it will be challenging to meet the aggressive (but appropriate) cost target of zero delta cost while also ensuring adequate performance. The project team should establish a design verification plan with objective performance targets based on comparators (baseline Fiat 500e and/or conventional internal combustion engine [ICE] vehicle) to ensure targets are met at the component and systems levels. Performance targets should include energy consumption at the component and systems levels to ensure that the end goal of $15 \%$ vehicle range improvement at $-10^{\circ} \mathrm{C}$ is met. Performance targets and verification must include operation below $-10^{\circ} \mathrm{C}$ ambient temperatures in order to be commercially viable for the entire North American market.

\section{Reviewer 3:}

The reviewer stated that the Unitary Thermal Energy Management for Propulsion Range Augmentation (UTEMPRA) project follows a fairly classic project approach to establishing vehicle requirements and conducting

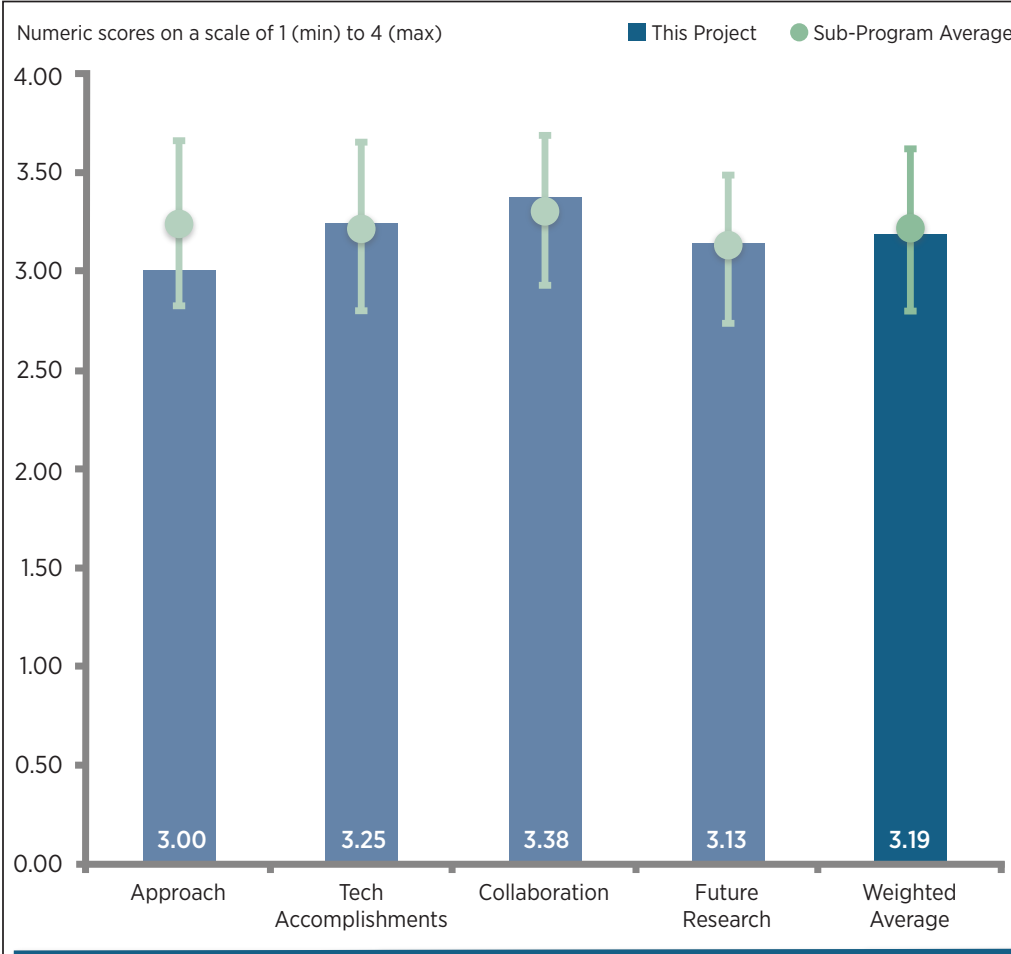

Relevant to DOE Objectives

Sufficiency of Resources
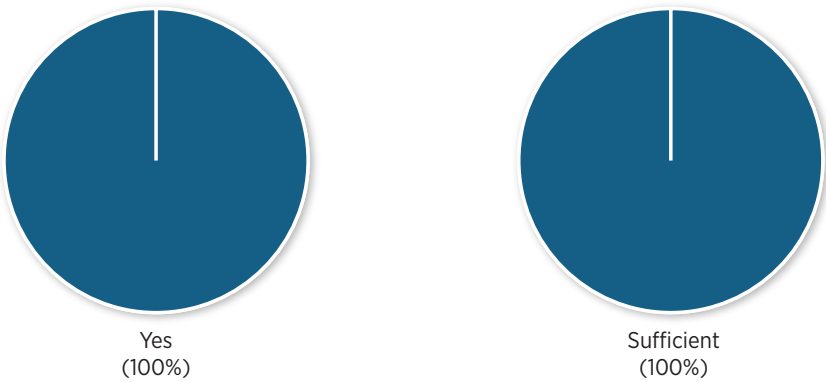

Figure 1-23 - UTEMPRA - Unitary Thermal Energy Management for Propulsion Range Augmentation: Sourav Chowdhury (Mahle Behr USA LLC) - Vehicle Systems 
baseline testing, designing systems and component requirements, conducting modeling and testing, designing in durability and performing validation activities, conducting manufacturing and cost estimates, and finalizing vehicle build and testing. This approach addresses the traditional barriers to new systems design, integration, optimization, and validation. A lower temperature limit of $-10^{\circ} \mathrm{C}$ has been targeted for the HVAC system as this is widely accepted as the lower limit of coolant capabilities.

A Fiat 500e BEV was chosen as the baseline vehicle for design and incorporation of the UTEMPRA system. This vehicle was chosen because the UTEMPRA system is compatible with Fiat's philosophy and Mahle Behr has a working history with Fiat. What was not clear to the reviewer is whether the choice of this OEM and vehicle type would in any way limit the potential broader commercial applicability of the UTEMPRA system with other larger vehicle OEMs. However, the project team did indicate that a vehicle battery that is cooled by coolant is the best approach for future industry flexibility and that other OEMs have expressed interest.

\section{Reviewer 4:}

The reviewer stated that the schematic of the UTEMPRA system is not clear enough for understanding the concept.

The reviewer remarked that at mild ambient temperature, e.g., spring and fall, there is no need for cabin heating or cooling and only thermal management of power electronics and battery is needed. The reviewer wondered, under this condition, how the UTEMPRA system would help with the driving range extension.

In some places like Minnesota and Michigan, there are a few months when the ambient temperature is lower than $-10^{\circ} \mathrm{C}$. The reported driving range comparison is only to $-10^{\circ} \mathrm{C}$. The reviewer wondered how the UTEMPRA system outperforms the traditional system when the heat pump system is reaching its low end temperature limit.

When the ambient temperature is higher than $25^{\circ} \mathrm{C}$, which is usually when $\mathrm{A} / \mathrm{C}$ is needed in summer, it seemed to the reviewer that the UTEMPRA system performs worse than traditional systems. This reviewer observed the presenter argue that the project team's system can approach the traditional system by using PCMs. However, using PCMs will increase the vehicle weight, and the reviewer was unsure how efficiently PCMs will help.

It seems to this reviewer that, over the whole year, the UTEMPRA system outperforms or underperforms the traditional system at different times at different locations. When driving range is compared between different systems, the reviewer recommended averaging over the whole year at different locations. The reviewer thought that the project target - "to increase $15 \% \mathrm{BEV}$ drive range at $-10^{\circ} \mathrm{C}$ with equivalent cabin comfort"- should be changed. It would not make any sense if the driving range is increased by $15 \%$ at $-10^{\circ} \mathrm{C}$ ambient temperature but decreases the driving range at lower or high temperature. The reviewer asserted that the driving range extension should be looked into over the whole year.

\section{Question 2: Technical accomplishments and progress toward overall project and DOE goals-the degree to which progress has been made, measured against performance indicators and demonstrated progress towards DOE goals.}

\section{Reviewer 1:}

The reviewer enthused about the excellent accomplishments across the full range of project areas: baseline testing and target setting, systems model development, prototype build, component sizing and design, vehicle integration, and manufacturing process and equipment development.

\section{Reviewer 2:}

The reviewer noted that the project is on schedule and on budget. With $30 \%$ of the budget spent on brazing equipment, the percentage of budget completion is exaggerated at approximately $75 \%$. The project seems to have sufficient funds to complete the scope.

\section{Reviewer 3:}

The reviewer recounted a solid list of technical accomplishments that includes packaging studies, systems and component specifications and design, proof-of-concept of components and brazing equipment, and heat exchanger and compressor builds. Starting in late 2015 through the present, all milestones have been completed on schedule 
with only milestone six (braze equipment installation and qualification) being slightly behind. There has been an extensive amount of vehicle baseline testing and system/component modeling and evaluation.

An area of potential concern to the reviewer was that while the UTEMPRA system shows respectable efficiency improvements at low and mild temperatures, it does show worsening performance at higher temperatures compared to conventional direct $\mathrm{A} / \mathrm{C}$ systems currently in use. This could potentially limit the commercial attractiveness of the UTEMPRA system in warmer climates. The project team thought that this deficiency could be reduced/bridged by additionally implementing PCMs and other technologies in warmer climates. However, this would come with an additional cost barrier.

An additional area of concern to the reviewer was that the cost viability of the UTEMPRA system is really not discussed. There are no details as to the potential cost premium although the target is apparently to achieve cost neutrality with today's conventional HVAC systems. The presentation mentions towards the end the need for an update of the projected system cost to ensure its commercial viability. The reviewer remarked that it would be good to prioritize this effort sooner rather than later and to clearly define the parameters guiding this effort.

\section{Reviewer 4:}

A lot of work has been done and the project is making progress as planned. The reviewer had a concern similar to comments on Question 1 about whether the proposed system has advantages over the traditional system in different seasons and at different locations. If the advantage is limited from $-10^{\circ} \mathrm{C}$ to $+10^{\circ} \mathrm{C}$, then the OEMs may not consider adopting the new system.

\section{Question 3: Collaboration and coordination with other institutions.}

\section{Reviewer 1:}

The reviewer found the collaboration to be outstanding, with no noticeable shortcoming.

\section{Reviewer 2:}

The reviewer complimented the project team on its excellent partnership with Fiat Chrysler Automobiles (FCA).

\section{Reviewer 3:}

The reviewer remarked that this project has collaboration with an OEM and a national laboratory. Getting more OEMs involved might help.

\section{Reviewer 4:}

The reviewer acknowledged that the project has a respectable list of partners including FCA (OEM partner), Norgren (component design), and NREL (modeling). Several suppliers of flux-less materials are being considered and furnace specification and design are being reviewed by a large team of braze experts. However, the reviewer suggested that it would have been beneficial to have additional OEMs on board to increase confidence in the broad applicability of the technology at the consumer end.

\section{Question 4: Proposed future research-the degree to which the project has effectively planned its future work in a logical manner by incorporating appropriate decision points, considering barriers to the realization of the proposed technology and, when sensible, mitigating risk by providing alternate development pathways.}

\section{Reviewer 1:}

The reviewer wanted to see future work include a cost and performance comparison with increased battery size.

\section{Reviewer 2:}

The reviewer stated that the presentation lacked specifics about future work in the remainder of budget period two and budget period three. However, based on current progress and the general plan communicated, future research is properly focused and the team is in position to overcome most barriers. A plan for recovering from the one-month delay is needed.

\section{Reviewer 3:}

The reviewer observed that the proposed future research and project plan for the balance of FY 2016 and all of 
FY 2017 is relatively well detailed and focused on brazing technology development, further component design and build, testing, and vehicle build and validation. No major omissions appear evident. However, as mentioned above, a primary emphasis should be placed in the near-term on in-depth cost studies to assess the true commercial viability of the UTEMPRA system for vehicle OEMs.

\section{Reviewer 4:}

The reviewer reported that it seems the next step would be prototyping and testing. Before jumping into that, the reviewer recommended that the team go back to look into the yearly averaged driving range increase at different locations and with the cabin heating/cooling on and off.

\section{Question 5: Does this project support the overall DOE objectives of petroleum displacement? Why or why not?}

\section{Reviewer 1:}

The reviewer noted that, with cost evaluation, this project can set priorities for cold weather range recovery.

\section{Reviewer 2:}

The reviewer found the project to be completely relevant and in line with DOE VTO goals and objectives.

\section{Reviewer 3:}

The reviewer remarked that, yes, reduced EV driving range as a result of extreme temperatures is a significant challenge to mainstream consumer market viability of these vehicles. Overcoming, or significantly mitigating, substantial range reductions at extreme temperatures is critical.

\section{Reviewer 4:}

The reviewer stated that looking for a replacement for the traditional cabin heating and cooling system is very critical considering that the current system draws a lot of juice from the battery, which limits the driving range of EV.

\section{Question 6: Resources: How sufficient are the resources for the project to achieve the stated milestones in a timely fashion?}

\section{Reviewer 1:}

The reviewed stated that there are sufficient financial and company resources to conduct the scheduled project activities.

\section{Reviewer 2:}

The reviewer commented that the first phase of the project seemed to use only $6 \%$ of the budget. The next phase would cost much more money, but the total budget should be sufficient. The team can get more OEMs involved and get their opinion on the system. 


\section{Zero Emission Cargo \\ Transport II: San Pedro Bay Ports Hybrid and Fuel Cell Electric Vehicle Project: Joseph Impullitti (SCAQMD) - vs158}

Presenter

Joseph Impullitti, SCAQMD

\section{Reviewer Sample Size}

A total of five reviewers evaluated this project.

\section{Question 1: Approach to performing the work-the degree to which technical barriers are addressed, the project is well-designed, feasible, and integrated with other efforts.}

\section{Reviewer 1:}

The reviewer noted that the project has a reasonable and effective approach to achieving its stated goals, and it is technically sound. The reviewer would have liked for the project team to have included an analysis of where the $\mathrm{H}_{2}$ will come from in the future. If it comes from $\mathrm{CH} 4$, there is not much $\mathrm{GHG}$ benefit. The basic idea of adding on something expensive to another expensive technology is not likely to be very favorable on economic grounds, either. The reviewer would have added a comparative economic assessment to the scope of the project.

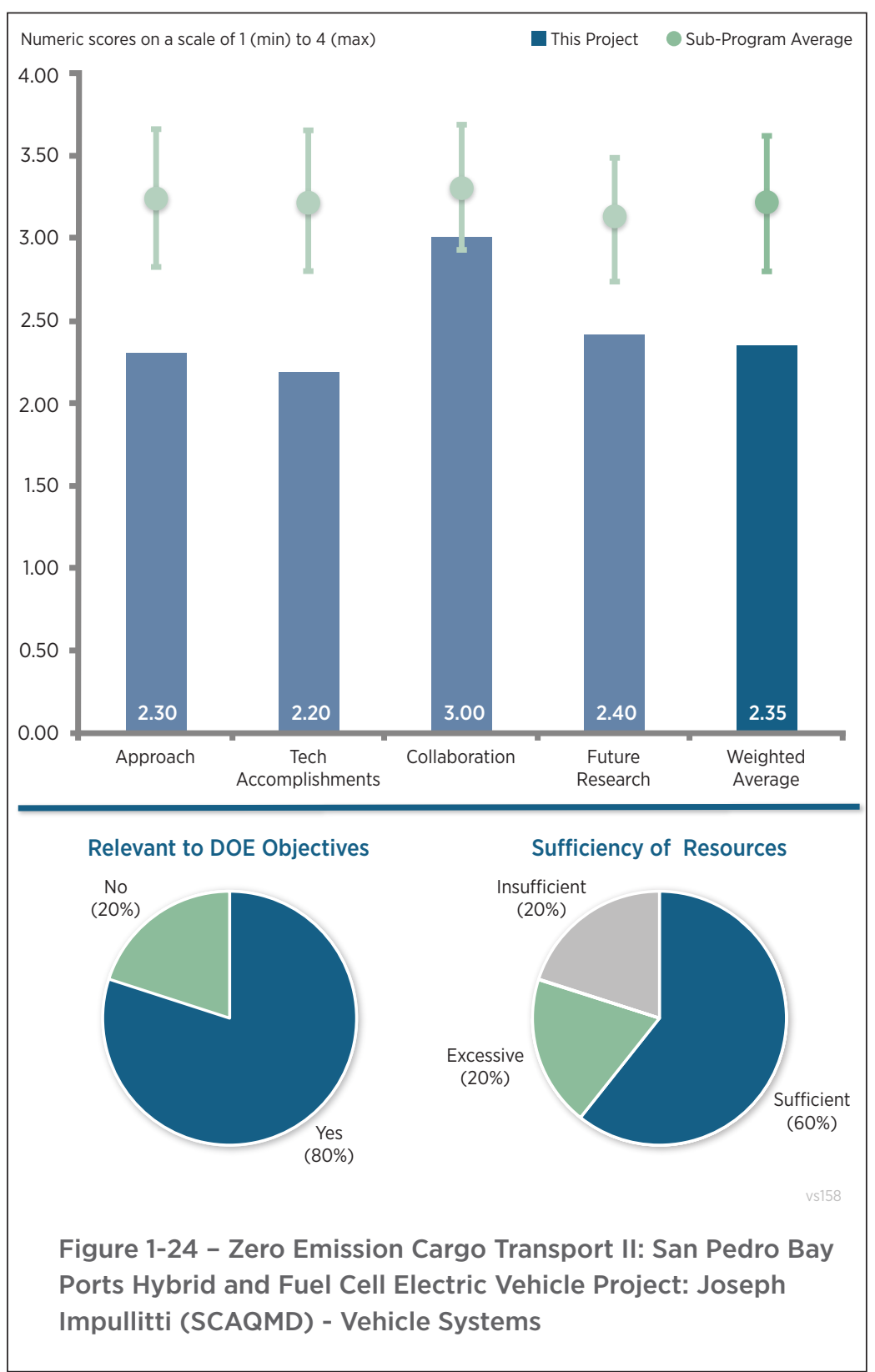

\section{Reviewer 2:}

The goal of the project is to help zero/near zero emission vehicles penetrate the fleet. The approach appears to be primarily building prototypes that extend the range of previous electric/fuel cell vehicles. The project team indicated the vehicles will be heavier than the incumbents, which affects load hauling capability. In order to penetrate the fleet long term, the reviewer encouraged the project team to benchmark against the range and load carrying capability of the incumbent fleet. The reviewer stated that the load hauling capacity is what should be compared. If the load capacity is the same as a Class 7 truck, use the Class 7 truck as the comparison because that is how a user would need to use it.

\section{Reviewer 3:}

The reviewer commented that the team's approach to draw on the results and experience from previous projects is good in order to leverage experience from one to another and avoid reinventing technologies. The requirement to have an OEM for this work is absolutely critical - the technologies are unlikely to progress beyond a science project level if this is not the case. The system designs for the various truck projects appear to be technically sound. The reviewer said that the team members are using proven components in their designs (but as these are not always 
production systems, proven is a relative thing). The project team has done some initial work to address the $\mathrm{H}_{2}$ infrastrcture question for both the short- and long-term: the team will have tube trailer refueling for $\mathrm{H}_{2}$ in the short term and wants a permanent $\mathrm{H}_{2}$ station later in the long term. The approach to test all trucks on the same duty cycle is important to enable accurate comparison of performance among trucks.

\section{Reviewer 4:}

The reviewer remarked that the vehicle technical specification should have been based on a previous analysis of requirements. Instead, it looks like one of the main accomplishments of the project was to have found a contractor to build a truck. There was no consideration of the infrastructure as the $\mathrm{H}_{2}$ is expected to be delivered by trailers.

\section{Reviewer 5:}

The proposed size of batteries and fuel cell (FC) systems seems extremely costly and unrealistic relative to what the market could bear. The reviewer asked whether there is any analysis regarding the cost feasibility of such an endeavor. The reviewer noted that batteries of more than $300 \mathrm{kWh}$ would cost well in excess of $\$ 100,000$ in the best case, cell cost scenarios. Additionally, the comment at the end of the presentation discussing the use of tools to analyze the vehicles needed to be done up front. The reviewer said that understanding the drive cycles, energy requirements, etc., would lead to a rational engineered approach to the system that could be more cost effective and optimized relative to the needs.

\section{Question 2: Technical accomplishments and progress toward overall project and DOE goals-the degree to which progress has been made, measured against performance indicators and demonstrated progress towards DOE goals.}

\section{Reviewer 1:}

The project has made good progress towards its technical goals. The reviewer questioned how anybody can seriously evaluate a $\$ 20$ million project in 20 minutes, and expressed being very uncomfortable with the whole review process [DOE Program Clarification: DOE will take under consideration in planning future AMR presentations.].

\section{Reviewer 2:}

Work seems to be progressing slowly given that the project award was at the end of 2014, but the project has now begun to move (and truck designs are now started). Kenworth is taking a larger role in vehicle builds/integration, and the reviewer hoped this means this OEM is interested in the technology.

The reviewer said it was good to see these are all range extended vehicles, as these might be more practical and appeal to a larger group of potential buyers than an electric-only truck. Despite this, the range limitations of the designs may still prevent wider interest beyond the port/drayage application. The TransPower truck will be able to be used beyond the ports, extending to warehouses, because of its somewhat longer range.

This reviewer explained that incorporating FC dominant and battery dominant designs will demonstrate which design may work better for the drayage/port application. The compressed natural gas ICE hybrid is interesting as it may be closer to a possible production design than the FC concepts. The reviewer noted that the team is looking at another FC concept to replace the International Rectifier project; since the team has several FC projects already, it may be an opportunity to consider another architecture to expand the experience base of the project.

The reviewer stated that alignment of architectures with duty cycles is important. Team members have looked at drayage duty cycles, plus TIAX drayage cycles, to use as a basis for their designs, and this should align the architectures appropriately with the intended use. However, this person asserted that weight is still an issue, both with batteries and $\mathrm{H}_{2} \mathrm{FC} / \mathrm{H}_{2}$ storage.

\section{Reviewer 3:}

One of the manufacturers was lost, due to corporate restructuring. The project is working to find a replacement, and recover the schedule. It is unclear to the reviewer from the presentation what the overall project schedule really is other than to buy items before the two-year presentation period is over. A project schedule should be developed, tracked, and presented as part of the reporting, rather than just a history or an individual company projection. It was helpful to the reviewer to see the project reporting usable energy storage numbers for a valid comparison of 
options. Quantifiable benchmarks and targets should be added to track the goals of the project (i.e., energy savings, cost savings, equivalent fuel savings, etc.).

\section{Reviewer 4:}

The reviewer said that it appears a year has passed with little more than some meetings. The reviewer asked whether anything is being done to manage the development of the relatively complex systems for FC range extender, which can take a significant amount of time and resources.

\section{Reviewer 5:}

The reviewer commented that having a contract signed should not be considered as an accomplishment.

\section{Question 3: Collaboration and coordination with other institutions.}

\section{Reviewer 1:}

The reviewer noted that the project includes financial partners besides DOE, such as OEMs and national laboratories. It is a good mix.

\section{Reviewer 2:}

The reviewer stated that the set of collaborators is excellent.

\section{Reviewer 3:}

The reviewer said that two OEMs are involved (i.e., Kenworth and Navistar) as well as several electrified systems companies. It is important to engage the truck OEMs to ensure the technologies will move forward-a good collaboration activity would be to consider how the project will encourage the OEMs to include these trucks in future product plans. California financial partners are supporting this project, which shows commitment. The project team stated that the team will be looking at commercialization later, and hoped the trucks will be wellaccepted and then pushed to commercialization.

\section{Reviewer 4:}

The reviewer remarked that several institutions are involved; however, it appears that there were no vehicle requirements defined before the start of the project. Prior studies to develop those requirements would certainly have led to different vehicles being designed.

\section{Question 4: Proposed future research-the degree to which the project has effectively planned its future work in a logical manner by incorporating appropriate decision points, considering barriers to the realization of the proposed technology and, when sensible, mitigating risk by providing alternate development pathways.}

\section{Reviewer 1:}

Given the scope as defined, the reviewer stated that the project plan should be effective in carrying out the work as expected.

\section{Reviewer 2:}

Future activities are logical (build and test the trucks, collect information from a 24-month demonstration, and develop a project to replace the team that withdrew). All of this work makes sense to complete the project as described.

\section{Reviewer 3:}

The reviewer commented that the project plans to continue to acquire things and to test them, but did not address challenges. The project team indicated that the vehicle was heavier than the incumbents, which affects the load capability. The reviewer suggested that the project team should look at what the risks and challenges are and document a mitigation strategy.

\section{Reviewer 4:}

In the reviewer's opinion, the presenters do not appear to have any clear plan to quantify the impact or the potential of the technology behind building a couple of prototypes. 


\section{Question 5: Does this project support the overall DOE objectives of petroleum displacement? Why or why not?}

\section{Reviewer 1:}

The reviewer pointed out that, by definition, an alternative fuel/EV displaces petroleum.

\section{Reviewer 2:}

The project could certainly be relevant to DOE petroleum reduction objectives, to some degree, depending on how well these trucks will appeal to a market outside of the port and drayage application. The project appears to be focused more on local criteria emissions reduction at ports, which is a major concern in Southern California.

\section{Reviewer 3:}

The reviewer commented that there would of course be reductions in petroleum use, but the project's main purpose is emissions reduction in a highly polluted area. The technology combinations chosen are expensive and unlikely to find large markets; therefore, the long-term potential for petroleum replacement is small. The reviewer found it unfortunate that the project scope does not include a preliminary estimate of cost-effectiveness of the technology combinations. If it did, the reviewer opined that it would probably lead to the conclusion that the project made no sense, other than as a demonstration. The reviewer recommended that the project team return the uncommitted $\$ 800,000$ [DOE Program Clarification: DOE will take this under advisement when looking at establishing similar projects in the future.].

\section{Reviewer 4:}

This is a lot of budget to assess the potential of a technology without any prior analysis.

\section{Question 6: Resources: How sufficient are the resources for the project to achieve the stated milestones in a timely fashion?}

\section{Reviewer 1:}

If the question is whether the sum is appropriate for the project as scoped, then the amount is reasonable. But, given the low long-term potential, the reviewer thought too much money is being allocated.

\section{Reviewer 2:}

The reviewer enumerated that the project lost one of the manufacturers, the project team is working to finding a replacement, and the resultant delay in the schedule still needs remediation.

\section{Reviewer 3:}

The reviewer's comment was that the resources appear to be more than adequate to achieve the goals of the project. 


\section{Multi-Speed Transmission for Commercial Delivery Medium- Duty Plug-In Electric Drive Vehicles: Bulent Chavdar (Eaton Corporation) - vs161}

\section{Presenter}

Bulent Chavdar, Eaton Corporation

\section{Reviewer Sample Size}

A total of five reviewers evaluated this project.

\section{Question 1: Approach to performing the work-the degree to which technical barriers are addressed, the project is well-designed, feasible, and integrated with other efforts.}

\section{Reviewer 1:}

The reviewer stated that this project is an excellent idea, elaborating that it a relatively inexpensive and straightforward way to improve the performance of electric trucks. The reviewer added that the project as described seems to have developed an effective approach that will lead to a real product. The reviewer remarked being impressed by the re-thinking that led to an improved concept.

\section{Reviewer 2:}

The reviewer stated that the overall approach is good and observed that the scope for optimization of design for efficiency, cost, weight, and performance is limited due to constraints of the program. The reviewer concluded that some lack of total investment and implementation cost details need further refinement.

\section{Reviewer 3:}

The reviewer commented that the timeline is not well defined with dates in a sufficiently detailed enough manner for the reviewers to understand how this re-scoping will be successful and that this does not provide enough confidence.

\section{Reviewer 4:}

The reviewer warned that while the approach has been satisfactory at this project stage, not currently having an EV OEM partner going forward, and unidentified motor, inverter and battery manufacturers, puts the job of integrating the entire system at risk.
Figure 1-25 - Multi-Speed Transmission for Commercial Delivery Medium-Duty Plug-In Electric Drive Vehicles: Bulent Chavdar (Eaton Corporation) - Vehicle Systems 
Question 2: Technical accomplishments and progress toward overall project and DOE goals - the degree to which progress has been made, measured against performance indicators and demonstrated progress towards DOE goals.

\section{Reviewer 1:}

The reviewer affirmed that the technical accomplishments are proceeding, adding that there is a strong team that appears to have excellent resources for success. The reviewer said tools are being utilized well and that the team has used a solid process to select the 3-speed automated manual transmission over other possible solutions and then matching gear ratios with this setup.

\section{Reviewer 2:}

The reviewer agreed that the modeling and design efforts appear to be right on target in addressing the technical difficulties. The reviewer hoped to see something built next year.

\section{Reviewer 3:}

The reviewer offered that the technical development of the solution and verification of modeled results are very good.

\section{Reviewer 4:}

The reviewer stated that analysis and modeling has resulted in the proposed design and projected benefits and observed that $\mathrm{CAD}$ is complete for a modular multi-speed transmission and manufacturing to begin.

\section{Reviewer 5:}

The reviewer remarked that modeled results to-date suggest improvements in drivability and efficiency.

\section{Question 3: Collaboration and coordination with other institutions.}

\section{Reviewer 1:}

The reviewer praised as excellent the collaboration with DOE laboratories, but cautioned that the challenge of not having Smith Electric Vehicles intimately involved is significant.

\section{Reviewer 2:}

The reviewer stated there is sufficient modeling and design group presence but that the supplier for the EV delivery truck is questionable and said there is a need to look into other partnerships.

\section{Reviewer 3:}

The reviewer concluded that there will obviously be more activity by the collaborators after something is actually built but that finding a new EV OEM will be somewhat of a challenge.

\section{Reviewer 4:}

The reviewer commented that not having an EV OEM onboard significantly impacts the project's ability collaborate. However, the reviewer also remarked that ongoing collaboration with ORNL and NREL with these laboratories performing vehicle-level simulations based on historic duty cycles at this stage of the project is appropriate.

\section{Reviewer 5:}

The reviewer cautioned that lack of a vehicle partner leads to some uncertainty in whether the design will be optimized for a future OEM, or whether the work will need to be redone once partner requirements are provided.

Question 4: Proposed future research-the degree to which the project has effectively planned its future work in a logical manner by incorporating appropriate decision points, considering barriers to the realization of the proposed technology and, when sensible, mitigating risk by providing alternate development pathways.

\section{Reviewer 1:}

The reviewer exclaimed that lightweighting is a big win by saving 240 pounds. The reviewer is excited for the project to move on to prototyping. 


\section{Reviewer 2:}

The reviewer observed that the real test is building something that works. The reviewer is looking forward to very positive results.

\section{Reviewer 3:}

The reviewer judged that the decision points for future work are good.

\section{Reviewer 4:}

The reviewer stipulated that finding an appropriate EV partner will be critical for this work. The reviewer cautioned that current pressures on EV development due to low gasoline cost could be problematic on the short term, adding that this is outside the control of the collaboration. That said, the reviewer concluded the multi-speed development is promising for performance and energy improvements in EVs.

\section{Reviewer 5:}

The reviewer warned that lack of an EV OEM places all future research at risk, adding that even if one can be identified, much of the modeling to-date may need to be repeated, significantly reducing the potential to succeed within the designated timeframe. The reviewer also remarked that future presentations must spell out all the acronyms.

\section{Question 5: Does this project support the overall DOE objectives of petroleum displacement? Why or why not?}

\section{Reviewer 1:}

The reviewer stated that modeling results to-date suggest improved efficiency improved, which support DOE's petroleum reduction goals. In addition, the reviewer commented that the increased drivability, as measured by acceleration and top speed, may make the potential EV truck a more attractive choice, which would in turn support the administration's goal of getting more EVs deployed.

\section{Reviewer 2:}

The reviewer offered that successful completion of this project should do much to increase market penetration of EVs into the delivery truck market, which now relies heavily on gasoline and diesel.

\section{Reviewer 3:}

The reviewer commented that this technology is important to help reduce the barrier of entry MD EV fleets.

\section{Reviewer 4:}

The reviewer remarked that EVs for personal use and work applications are a direct petroleum-displacing disruptive technology.

\section{Reviewer 5:}

The reviewer said that this is a key integration to make MD EVs more efficient and to provide a better overall payback.

\section{Question 6: Resources: How sufficient are the resources for the project to achieve the stated milestones in a timely fashion?}

\section{Reviewer 1:}

The reviewer concluded that the budget of this project is quite reasonable, especially given that the project team will need to actually build a product and test it on operating vehicles.

\section{Reviewer 2:}

The reviewer stated that it seems right, although the project needs an OEM for success, or at a minimum, an engineering firm who can perform like an OEM.

\section{Reviewer 3:}

The reviewer indicated that resources seem to be appropriate, but added that it is unclear if further funding will be needed if scope changes after an EV OEM partner is on board. 
Reviewer 4:

The reviewer remarked that it is difficult to rate the level of resources needed going forward without knowing the future battery, EV OEM, converter and motor integrator, and manufacturers, as well as the resources that will be needed. The reviewer concluded that given the unknowns, the risk is always that funding will be insufficient as excessive funding rarely occurs. 


\section{Integrated Boosting and Hybridization for Extreme Fuel Economy and Downsizing: Vasilios Tsourapas (Eaton Corporation) - vs162}

\section{Presenter}

Vasilios Tsourapas, Eaton Corporation

\section{Reviewer Sample Size}

A total of five reviewers evaluated this project.

\section{Question 1: Approach to performing the work-the degree to which technical barriers are addressed, the project is well-designed, feasible, and integrated with other efforts.}

\section{Reviewer 1:}

The reviewer praised an excellent project and presentation, elaborating that the hybridization of waste heat recovery and electrification of a supercharger demonstrates a more complete solution set of technology. The reviewer looks forward to seeing the final fuel economy optimization results next year.

The reviewer inquired if one only looks at the closed system of these two components, by what percent of efficiency does the electricity recovered compare to the energy needed to utilize the supercharger.

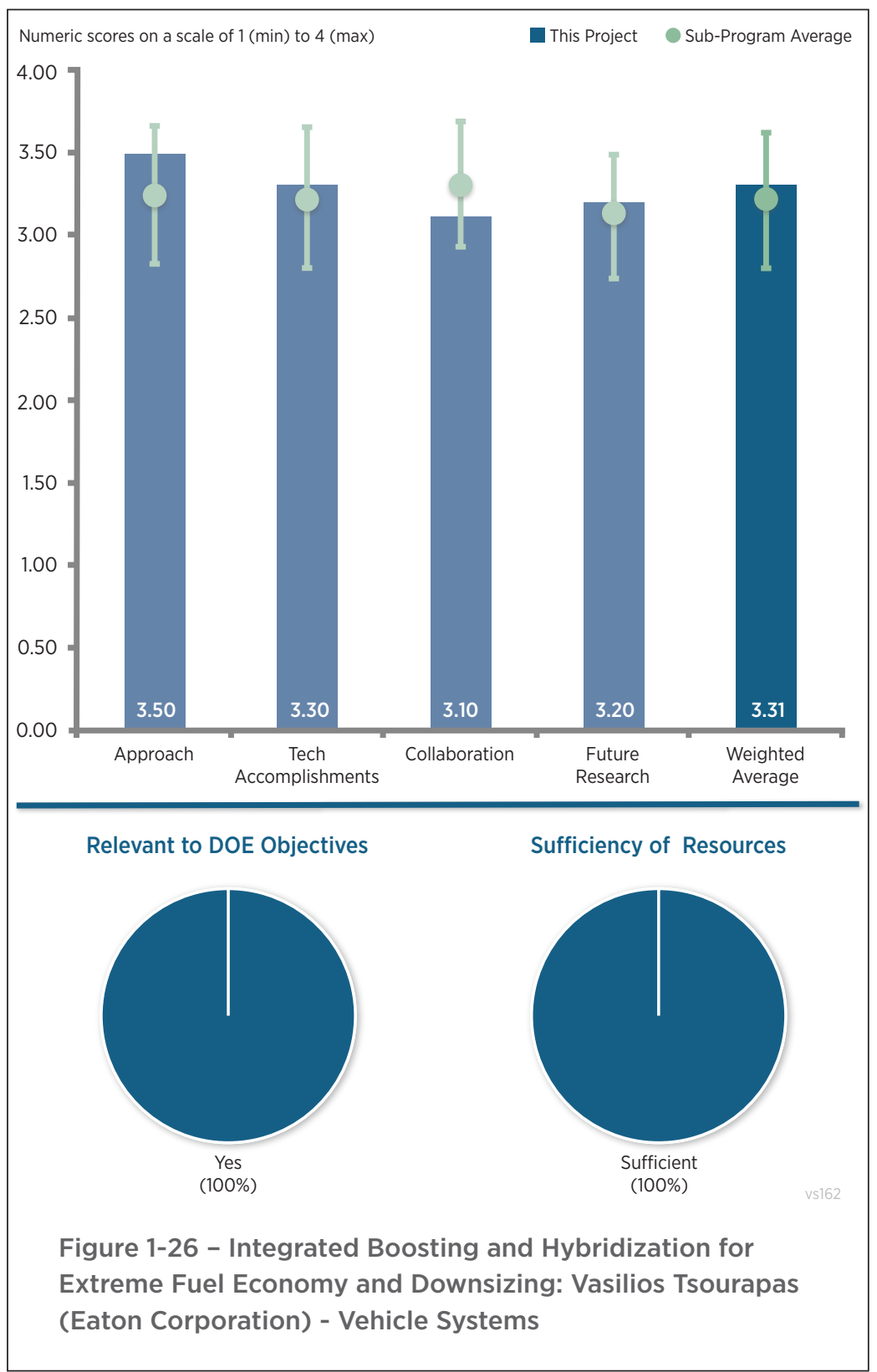

\section{Reviewer 2:}

The reviewer observed that the project takes the approach of designing the new components for integration into the engine and then, once that is complete, of integrating them into the vehicle. The reviewer remarked that this incremental approach is good for the introduction of new components and trying to evaluate their impact on subsystems (the engine) and the overall system (vehicle).

\section{Reviewer 3:}

The reviewer stated that the plan is solid and progress is occurring.

\section{Reviewer 4:}

The reviewer affirmed that the approach described is sound and that the reference technology and the different steps are well described.

Question 2: Technical accomplishments and progress toward overall project and DOE goals-the degree to which progress has been made, measured against performance indicators and demonstrated progress towards DOE goals. 


\section{Reviewer 1:}

The reviewer observed that the project made good progress over the last year with the models at the component level. The reviewer also noted that this second year is an important one for the project and that milestone slide (Slide 4) indicates the design of the electrically assisted variable speed supercharger and WHR were delayed. The reviewer remarked that because these are the primary components in the project strategy, it is important that they stay on schedule. However, the PI, when asked about his progress, felt that the project could meet the fuel economy targets.

\section{Reviewer 2:}

The reviewer stated that the project team should clearly find the best application and solidify for next steps to best demonstrate this disruptive technology, adding that it is good to tradeoff items to exploit the fuel economy opportunity.

\section{Reviewer 3:}

The reviewer recounted that Delphi used the model-based systems engineering approach by utilizing first modeling and simulation with GT-POWER and using higher fidelity models to design the different components.

\section{Reviewer 4:}

The reviewer referenced prior comments.

\section{Question 3: Collaboration and coordination with other institutions.}

\section{Reviewer 1:}

The reviewer stated that having both Isuzu and SwRI on board as partners is very helpful in order to follow the project approach, adding that the support required from each is well thought out.

\section{Reviewer 2:}

The reviewer urged the project team to stay focused with the few collaborators to complete this project on time and on budget and accomplish goals.

\section{Reviewer 3:}

The reviewer remarked that the addition of Isuzu would be beneficial to the project.

\section{Reviewer 4:}

The reviewer regretted that Isuzu was the only partner willing to invest in this project, as opposed to a Ford or GM, which the reviewer suggested might have realized a more direct gain to the U.S. market, and in the case of Ford started with a baseline more-optimized system.

Question 4: Proposed future research-the degree to which the project has effectively planned its future work in a logical manner by incorporating appropriate decision points, considering barriers to the realization of the proposed technology and, when sensible, mitigating risk by providing alternate development pathways.

\section{Reviewer 1:}

The reviewer remarked that while several issues still need to be addressed, the team appears to have a good understanding of the remaining challenges.

\section{Reviewer 2:}

This reviewer acknowledged looking forward to seeing the final results.

\section{Reviewer 3:}

The reviewer stated that the proposed future work is to continue forward with the project approach, noting that this coming year the PI will be focused on durability, cost, and system control of the design and adding that these are all important activities prior to vehicle integration.

Question 5: Does this project support the overall DOE objectives of petroleum displacement? Why or why not? 


\section{Reviewer 1:}

The reviewer observed that if the project team is able to demonstration newly designed components that take advantage of waste heat and variable speed supercharger, the design will allow for a smaller more efficient engine that results in less petroleum consumption.

\section{Reviewer 2:}

The reviewer commented that optimization and integration of this technology could show marked fuel economy improvement.

\section{Reviewer 3:}

The reviewer noted the following: big opportunity; big risk; and like to swing for the fences sometime. The reviewer expressed looking forward to next year's review.

\section{Reviewer 4:}

The reviewer remarked that an obvious question as it applies to this project, but major downsizing and optimization projects are, by definition, directly proportional to petroleum displacement.

\section{Question 6: Resources: How sufficient are the resources for the project to achieve the stated milestones in a timely fashion?}

\section{Reviewer 1:}

The reviewer observed that the project is heavily funded in year two and that this should be sufficient to get the team through subsystem integration.

\section{Reviewer 2:}

The reviewer said there are no apparent issues noted in this project. 


\section{Advanced Bus and Truck Radial Materials for Fuel Efficiency: Lucas Dos Santos Freire (PPG Industries) \\ - vs163}

\section{Presenter}

Lucas Dos Santos Freire, PPG

Industries

\section{Reviewer Sample Size}

A total of five reviewers evaluated this project.

\section{Question 1: Approach to performing the work-the degree to which technical barriers are addressed, the project is well-designed, feasible, and integrated with other efforts.}

\section{Reviewer 1:}

The reviewer characterized the approach as reasonable and addresses a very important area for reducing fuel use in the commercial truck sector. The reviewer observed that the team is addressing the use of silica for commercial truck tires, which have different material characteristics because of the use of natural rubber. The reviewer elaborated that incorporation of silica should produce the desired rolling resistance effects, as the ability of silica to reduce rolling resistance has been proven in the $\mathrm{LD}$ market. The team is targeting highervolume tire applications (Class 7-8) to improve market uptake. The reviewer concluded that the team's processes appear to be reasonable and analysis is thorough, adding that verification of the fuel economy will be done on realworld trucks at Bridgestone, which is an important step.

\section{Reviewer 2:}

The reviewer observed that the project appeared to have an approach tightly focused upon achieving the very specific Budget Period One requirement. The reviewer remarked that the project team relied upon historical analyses to provide the tie between tire compound, then rolling resistance, and ultimately vehicle efficiency, all of which appeared to be clearly based upon extensive experience in the technology area.

\section{Reviewer 3:}

The reviewer commented that this project builds on previous work with Silica and offered that sooner, rather than later, the tires are needed to prove the results and see how they measure to the goals.

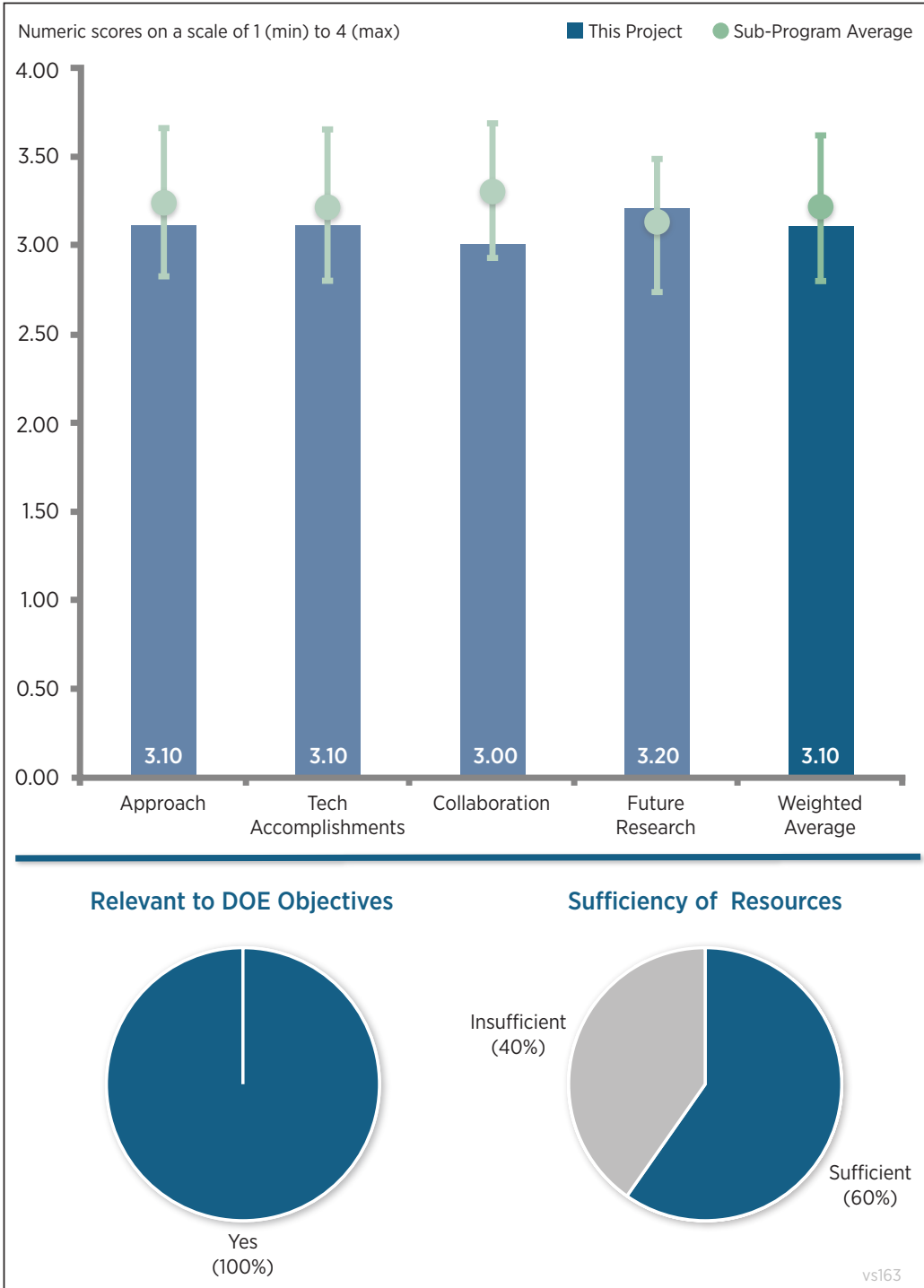

Figure 1-27 - Advanced Bus and Truck Radial Materials for Fuel Efficiency: Lucas Dos Santos Freire (PPG Industries) Vehicle Systems 


\section{Reviewer 4:}

The reviewer stated that the project is focused on developing a novel approach to tire manufacturing to significantly reduce rolling resistance and improve vehicle fuel economy. The reviewer praised as excellent the approach to tire compound design and process development, elaborating that elements of this work includes treatment of silica for increased dispersion, quantification of new compound properties, and comparison to properties of tires produced using existing state-of-the-art properties. However, the reviewer cautioned that the approach lacks the necessary rigor to predict and verify the relationship between tire material properties and on-vehicle performance.

The reviewer remarked that the presenter asserts there is an understood relationship between tire material properties tangent $(\tan )$ delta and rolling resistance and characterized this institutional knowledge as valuable, but that it seems insufficient to establish a link to on-vehicle tire performance and resulting vehicle fuel economy. The reviewer further explained that multiple targets for rolling resistance reduction were established for laboratory test of samples and on-tire measurements, and that it appears tan delta is being used as a measure of rolling resistance, but no transfer function between tan delta and rolling resistance is given.

The reviewer suggested the team should more clearly define metrics, test methods, and transfer functions to show the relationship between samples in lab, full-tire laboratory testing, on-road tire performance testing (if any), and vehicle fuel economy testing.

\section{Question 2: Technical accomplishments and progress toward overall project and DOE goals-the degree to which progress has been made, measured against performance indicators and demonstrated progress towards DOE goals.}

\section{Reviewer 1:}

The reviewer observed that multiple silica prototypes have been developed and excellent test data has been gathered, which show that the compounds meet many of the material targets and manufacturability. The reviewer summarized that the project appears to be on track for timing and budget and the go/no-go decision was successfully passed in March.

\section{Reviewer 2:}

The reviewer agreed that the team has achieved its initial goal of gaining a scientific understanding of silica dispersion performance and increasing the dispersion of silica into the natural rubber material. The reviewer noted that the team has identified silica prototype systems that achieved the required $10 \%$ increase in dispersion, and are thus able to move forward with subsequent project phases. Furthermore, the team has demonstrated with both testing and microscopy that its prototype silica will be dispersed into the natural rubber. The reviewer concluded that initial results of the prototype silica system are promising for both rolling resistance and durability.

\section{Reviewer 3:}

The reviewer judged that overall, the silicas appeared to have significantly better dispersion that the baseline compound (carbon black), which seems to be the major focus at this point. The PI indicated that the project achieved the dispersion level required for the Budget Period One go/no-go decision, although it might not have been the full $10 \%$ and instead perhaps was closer to $9 \%$. The reviewer concluded that this appeared to be the culmination of the efforts under this first phase of the project, along with greater understanding of the impact of surface area and chemistries on ultimate achievement of project goals.

\section{Reviewer 4:}

The reviewer remarked that the project looks to be behind schedule and asked what is being done to make up the time.

The reviewer said that improved dispersion with lower surface energy treatment for silica is a good result, but then added that tan delta of the LD0380-2 does not appear to be that much different from the Control 1 . The reviewer wondered if more than one sample used. Finally, the reviewer indicated that the connection between dispersion and tan delta to rolling resistance is unclear and asked if this been experimentally correlated.

\section{Question 3: Collaboration and coordination with other institutions.}




\section{Reviewer 1:}

The reviewer observed the team has made strides in expanding (or clarifying the extent of) collaboration with a critical partner (i.e., the tire manufacturer). The tire OEM will provide the appropriate feedback on the benefits of the new compound for its tires and intended tire customers. The reviewer commented that a trucking fleet could add perspective, as noted, but Bridgestone Americas Tire Operations (BATO) should cover this, as the tire company will be the one to decide how to use the new silica in its tire products. Critical measurements at the molecular level are provided by a scientific laboratory.

\section{Reviewer 2:}

The reviewer stated that the project partners seem to be working well together and have the required capabilities for the bulk of the work. However, the reviewer cautioned that it is not clear whether BATO is capable of performing the requisite on-road testing to verify the expected relationship between tire material properties, including tan delta, rolling resistance, other tire performance, and vehicle fuel economy. The reviewer suggested it may be necessary to bring in another partner with on-road testing expertise (auto OEM, test laboratory, or national laboratory), adding that perhaps this has already been defined with DOE and will be done as follow-on work with tires that will be provided to DOE for independent testing.

\section{Reviewer 3:}

The reviewer remarked that any OEM or large fleet interaction would have been helpful, commenting that they might differ in their opinions sometimes from what Bridgestone thinks and says.

\section{Reviewer 4:}

The reviewer observed that the project highlighted collaboration was with Bridgestone (advisor and ultimate manufacturer) and Augustine Scientific (measurement laboratory). The reviewer noted that the project team had considered including a user (fleet) as an advisor too, but it sounds as though Bridgestone may have convinced the team that was not necessary. An alternative may have been to include at least one knowledgeable bus and truck fleet to provide a check on Bridgestone's advice. The reviewer indicated that the PI felt Bridgestone had put together a sufficient argument that Bridgestone knew the market, and had actually gathered letters of support from fleets for this project to take place. The reviewer concluded that it still would appear that the project could have benefited from greater collaboration, and may want to revisit this as the project moves along.

\section{Question 4: Proposed future research-the degree to which the project has effectively planned its future work in a logical manner by incorporating appropriate decision points, considering barriers to the realization of the proposed technology and, when sensible, mitigating risk by providing alternate development pathways.}

\section{Reviewer 1:}

The reviewer observed that it appears that Phase Two will include a focus on additional tire properties, such as rolling resistance. The reviewer elaborated that additional compound work is planned, and then no more than two formulations will be selected for an experimental tire build (followed by a down-select to a single compound in Phase Three), adding that there were several interesting suggestions for research beyond the project. The reviewer stated that, in general, the proposed future research appears to be a rational approach to completing the project, and the project team has clearly given this subject a great deal of thought with regard to future directions.

\section{Reviewer 2:}

The reviewer described as excellent future work proposed for silica materials, rubber compounds, and manufacturing process development. Again, the reviewer cautioned, the project plan lacks a sufficiently robust design verification plan to connect material properties to on-road performance. However, the reviewer concluded that the identification of research beyond the end of this project is admirable, in that it demonstrates how the prime contractor will continue to strive for a return on DOE's funding investment.

\section{Reviewer 3:}

The reviewer judged that proposed future work, as described, appears reasonable to complete the project and achieve the desired goals, adding that the team members are distributing the future work appropriately based on their respective areas of expertise. 


\section{Reviewer 4:}

The reviewer stated that tires are needed and asked if the schedule and resources still allow the team to get them when needed. The reviewer agreed with a past reviewer that it would be helpful to have a truck OEM providing input. The reviewer pointed out that Bridgestone will get rolling resistance numbers but not fuel economy numbers which would be helpful. If the fuel economy cannot be tested, the reviewer assumes the rolling resistance numbers will be used to calculate the fuel economy.

\section{Question 5: Does this project support the overall DOE objectives of petroleum displacement? Why or why not?}

\section{Reviewer 1:}

The reviewer stated that the project is focused upon improving bus and truck efficiency through better tire technology, judging that this is clearly the type of project that is highly appropriate for inclusion in the VS R\&D portfolio, in order to address overall VTO goals of greater heavy vehicle efficiency.

\section{Reviewer 2:}

The reviewer observed that the project offers the potential for increasing fuel efficiency for truck and bus tires, which are typically used and replaced more frequently than LD tires, giving the opportunity for faster market uptake. The reviewer also noted the team is looking at tires for the largest truck fuel users - Class 7-8 trucks-and that improvements in this market will have a large impact on petroleum displacement.

\section{Reviewer 3:}

The reviewer stated the goals are very relevant, adding that improvement in rolling resistance would decrease fuel use and at a relatively inexpensive cost.

\section{Reviewer 4:}

The reviewer said this project is highly relevant to DOE goals.

\section{Question 6: Resources: How sufficient are the resources for the project to achieve the stated milestones in a timely fashion?}

\section{Reviewer 1:}

The reviewer stated that resources for this project appear to be sufficient to complete the work as described.

\section{Reviewer 2:}

The reviewer observed that there was no indication made that the funding was either high or low, so the assumption is that it is sufficient. The reviewer added that the only question here might be that it could have appeared to perhaps warrant more than a $25 \%$ cost-share on the part of industry.

\section{Reviewer 3:}

The reviewer offered that it is not clear that enough resources exist to accomplish all the goals of this project.

\section{Reviewer 4:}

The reviewer cautioned that there do not seem to be sufficient funds to perform sufficiently rigorous verification/ validation testing, adding that no details are given as to how full-tire laboratory and road testing will be carried out. It is the reviewer's opinion that significant resources will be needed to design and implement a test program necessary to understand the relationship between prototype silica dispersion in multiple compounds, rolling resistance, and vehicle fuel economy; and verify that the chosen tire design(s) meet the rolling resistance and fuel economy targets. 
Design and Implementation of a Thermal Load Reduction System in a Hyundai PHEV: Cory Kreutzer (National Renewable Energy Laboratory) - vs165

\section{Presenter}

John Rugh, National Renewable Energy Laboratory

\section{Reviewer Sample Size}

A total of four reviewers evaluated this project.

\section{Question 1: Approach to performing the work-the degree to which technical barriers are addressed, the project is well-designed, feasible, and integrated with other efforts.}

\section{Reviewer 1:}

The reviewer praised the approach being used to estimate the range across the nation as very good. The reviewer then noted that since the project team is using Future Automotive Systems Technology Simulator to perform simulations, it should not be having a resource issue regarding computation power. That being the case, the reviewer asked if it is necessary to reduce the number of weather stations considered from 839 to 204 . The reviewer would like to know what the basis is for choosing the most important weather environments.

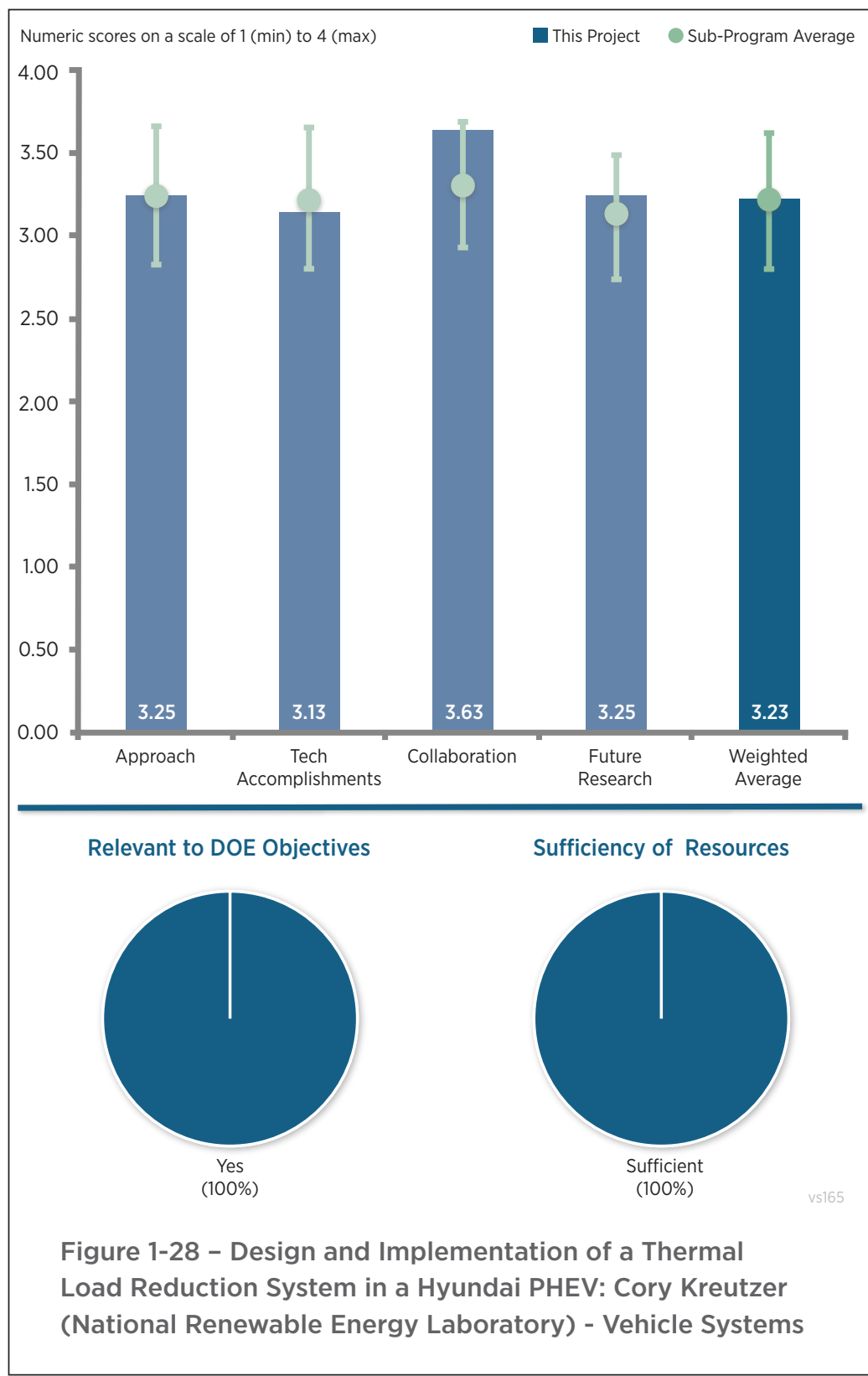

\section{Reviewer 2:}

The reviewer remarked that this is interesting work, but added that the old barrier that does not appear to be addressed or referenced is a cost benefit to the customer. In the reviewer's experience, the fuel savings-to-insulation and heated surfaces initial cost does have a payback to the customer.

\section{Reviewer 3:}

The reviewer remarked that this project has a significantly compelling value proposition in seeking to increase grid-connected electric drive vehicle (EDV) range by $20 \%$ during operation of the climate control system over the standard vehicle configuration. The reviewer added that it follows a classic project approach with Phase One consisting of technology down-selection, specification, and development supported by modelling and analysis and evaluation. The reviewer explained that a broad range of technologies are considered including insulation, climate control seating, grid-connected preconditioning, and advanced vehicle shell approaches. Phase Two consists of technology/vehicle integration, testing, and validation. The reviewer concluded that there does not seem to be any significant omissions nor deficiencies in the project approach. 
However, the reviewer suggested that it is worth considering whether future projects similar to this should consider a somewhat different or more truncated approach. For example, there is a very strong tendency for advanced thermal control technologies (as well as other vehicular technologies) to be successfully demonstrated in prototype or production vehicles but frequently never reach the production line. This can be due to a myriad of commercial/ business reasons from cost, to lack of compatibility with a changing vehicle system environment, consumer acceptability, and others.

The reviewer offered that it may be worth considering an approach that focuses much harder on extensive cost analyses for technology down-selection in the early phases of the project with heavy OEM input. Additionally, the project could be truncated to end after Phase One with a portfolio of potentially viable technologies merely tested and validated via simulation and bench top testing. In this way, the reviewer summarized, OEMs would have a broad portfolio of viable technologies from which to choose and further investigate if so desired. This would potentially lower project costs and offer more flexibility on the OEM side to account for inevitable vehicle system and commercialization vagaries as time progresses.

\section{Reviewer 4:}

The reviewer summarized that the project aims to evaluate the impact of different thermal load reduction technologies on the energy consumption of HVAC systems. The general research strategy is gathering available thermal load reduction technologies and down-selecting them by evaluating their individual impact. In the meantime, a predictive model is developed and validated by test data. The reviewer characterized this approach as typical and appropriate. The reviewer offered that one of the biggest barriers is OEMs' concern of the increased cost, and suggested that some analysis on the increased cost and payback period could be added to the project.

\section{Question 2: Technical accomplishments and progress toward overall project and DOE goals-the degree to which progress has been made, measured against performance indicators and demonstrated progress towards DOE goals.}

\section{Reviewer 1:}

The reviewer cited a solid list of accomplishments that have been achieved including successful winter baseline testing demonstrating a strong correlation between vehicles at varying environmental conditions; insulation which demonstrates a $9.6 \%$ improvement at steady state (10.6\% penalty in warm-up); and heated surfaces which show a $29-59 \%$ improvement in steady state heating with only a $2 \%$ penalty during warm-up. The reviewer pointed out that CoolCalc cabin modeling has been developed and validated for the Hyundai Sonata showing correlation within $3.6 \%$ to experimental data and that additional HVAC and detailed cabin modeling is in progress. The accomplishments the reviewer found most appealing, though, is the down-selection process established for meteorological data based on vehicle registrations because this helps simplify and focus the process in support of the nation-wide analysis.

The reviewer suggested it would be beneficial if a better understanding was provided of the level of efficiency improvement over the typical driving cycle (as just opposed to at-warm-up and steady state) and identification of the not so obvious customer/commercial barriers to potential implementation of these technologies.

\section{Reviewer 2:}

The reviewer noted that the presenter referenced delivering an "OEM Quality System" in his initial verbal framing of the project, but the project material never really discussed any barriers to a production quality system.

The reviewer commented that the energy savings look to be substantial and measurable and this reviewer looks forward to see that balance of energy saved from these driving conditions balanced against all driving.

\section{Reviewer 3:}

The reviewer observed that on Slide 12, the results for the transient warm-up showing a $10.6 \%$ penalty needs to be clarified. The reviewer explained that because any insulation is better than no insulation, the expectation is that there would be a decrease in energy input with added insulation, unless of course, the energy is coming in from the outside. 


\section{Reviewer 4:}

The reviewer commented that the project started in FY 2015 and 40\% of the work has been done, but from the presentation, it seems only the technologies of cabin insulation and heated surface have been tested. The reviewer asked if this means all the other technologies have reached a no-go decision.

The reviewer claimed that the impact of the insulation to the transient warm-up has some doubt, adding that the thermal capacity of the added insulation material should not have such a big effect. As the presenter mentioned, this part needs further investigation.

\section{Question 3: Collaboration and coordination with other institutions.}

\section{Reviewer 1:}

The reviewer characterized the NREL team has having a strong list of partners and seemingly well positioned to collaborate and coordinate appropriately. The reviewer observed that all the major areas of the project have another sub-tier industry partner, including at the OEM level and with regards to specific technology area development. The reviewer concluded there are no significant gaps with regards to project partners.

\section{Reviewer 2:}

The reviewer remarked that there appears to be synergy between this project and two other projects in particular VS155 and VS134. The reviewer presumes this synergy is being exploited, but because there was no mention of it, the reviewer wanted to mention it here just to be sure.

\section{Reviewer 3:}

The reviewer stated that OEM partnership will help drive research to production use and that the tie-in with the Vehicle Systems Analysis Technical Team seem to have the program framed properly. The suppliers of the surface heated parts and insulation appear to be properly engaged.

\section{Reviewer 4:}

The reviewer indicated that this project has OEM and suppliers involved and the contribution of these institutions are clearly listed.

\section{Question 4: Proposed future research-the degree to which the project has effectively planned its future work in a logical manner by incorporating appropriate decision points, considering barriers to the realization of the proposed technology and, when sensible, mitigating risk by providing alternate development pathways.}

\section{Reviewer 1:}

The reviewer praised the proposed future work for Phase One and Phase Two as seemingly comprehensive and logically sequenced, adding that there do not appear to be any major deficiencies. The reviewer remarked it would be useful though to have a clear definition of what criteria are being used to determine the go/ no-go technology milestone evaluation at the end of Phase One. Additionally, the reviewer suggested it may be worth considering a very minimal national-level analysis as this is predicated on a specific set of technologies penetrating a set number of vehicles over a given time frame, which is highly subjective and largely predicated on conjecture.

\section{Reviewer 2:}

The reviewer noted that the presenter mentioned during questioning that the tradeoff to mass and parasitic electrical loading for $100 \%$ of driving is balanced against the large savings in this subset of driving, but the current material did not show or discuss that overall balance. The reviewer said the presenter mentioned that it will be part of the final conclusions and the reviewer looks forward to seeing those results. Additionally, the reviewer remarked it would also be interesting to see if, as part of this testing, the project team identifies any production barriers to the surface heating of the new proposed surfaces not currently in production.

\section{Reviewer 3:}

The reviewer indicated a national level analyses that needs to be performed have a large degree of uncertainty associated with them, and that it is not clear that the chosen approach can provide the required level of confidence. 


\section{Reviewer 4:}

The reviewer cautioned that based on the listed future work, it seems there are still a lot to do. The reviewer is also unsure whether any other thermal load reduction technologies will be evaluated or only the two presented at this meeting will be considered for integration.

\section{Question 5: Does this project support the overall DOE objectives of petroleum displacement? Why or why not?}

\section{Reviewer 1:}

The reviewer noted that climate uses a measurable amount of fuel energy and that reducing the need to use energy to heat and cool the vehicle occupants will reduce petroleum use.

\section{Reviewer 2:}

The reviewer observed that significantly reduced driving ranges of EDVs in adverse weather (cold and hot) conditions is a major barrier to the widespread marketability of EDVs. The reviewer explained that if thermal load/ climate control technologies achieving a $20 \%$ increase in vehicle range can be implemented commercially, it will go a long way to increasing the broad consumer acceptance of these vehicles.

\section{Reviewer 3:}

The reviewer stated that thermal load reduction is important for energy saving of HVAC systems and that this is one way to extend the EV driving range.

\section{Question 6: Resources: How sufficient are the resources for the project to achieve the stated milestones in a timely fashion?}

\section{Reviewer 1:}

There reviewer indicated there are sufficient resources both financially and from a corporate qualifications and facilities standpoint to conduct the project within the defined timeframe.

\section{Reviewer 2:}

The reviewer said that it seems the budget is sufficient for the project. 
EV Everywhere Charging Infrastructure Roadmap: Donald Karner (Electric Applications Incorporated) - vs172

\section{Presenter}

Tom Garetson, Electric Applications Incorporated

\section{Reviewer Sample Size}

A total of five reviewers evaluated this project.

\section{Question 1: Approach to performing the work-the degree to which technical barriers are addressed, the project is well-designed, feasible, and integrated with other efforts.}

\section{Reviewer 1:}

The reviewer characterized the project approach as a reasoned, rational one based upon evaluation of available data/studies, as well as input from stakeholders (i.e., users, manufacturers, etc.) and concluded that the approach could be seen as solid, if not necessarily innovative or novel. However, the reviewer added that the interesting part of the approach was really the input from stakeholders, and that it was unclear how much of this information was used for this analysis. The reviewer indicated the project used some interesting definitions to address specific elements of the analysis, including intra-urban charging, interurban charging, and different phases (early, transitional, and mature). These allowed the project to coalesce some ideas that may have been less than clear in some other studies.

\section{Reviewer 2:}

The reviewer stated that the goal of the current project is to lay out the EV Everywhere charging infrastructure roadmap but noted that there are no technical barriers to complete the roadmap. The reviewer explained that the project team takes four approaches to identify and prioritize the next steps for PEV charging infrastructure: analyze DOE infrastructure studies; apply experience of the authors; use EV project data; and gather input from PEV industry leaders from OEMs, electric vehicle service providers, electric utilities, government, and PEV drivers. The reviewer concluded that the approach is generally effective considering it is only a one-year project, then commenting that the team could gather input not only from PEV drivers but also gasoline vehicle drivers who may have concerns and viewpoints that PEV drivers do not have.

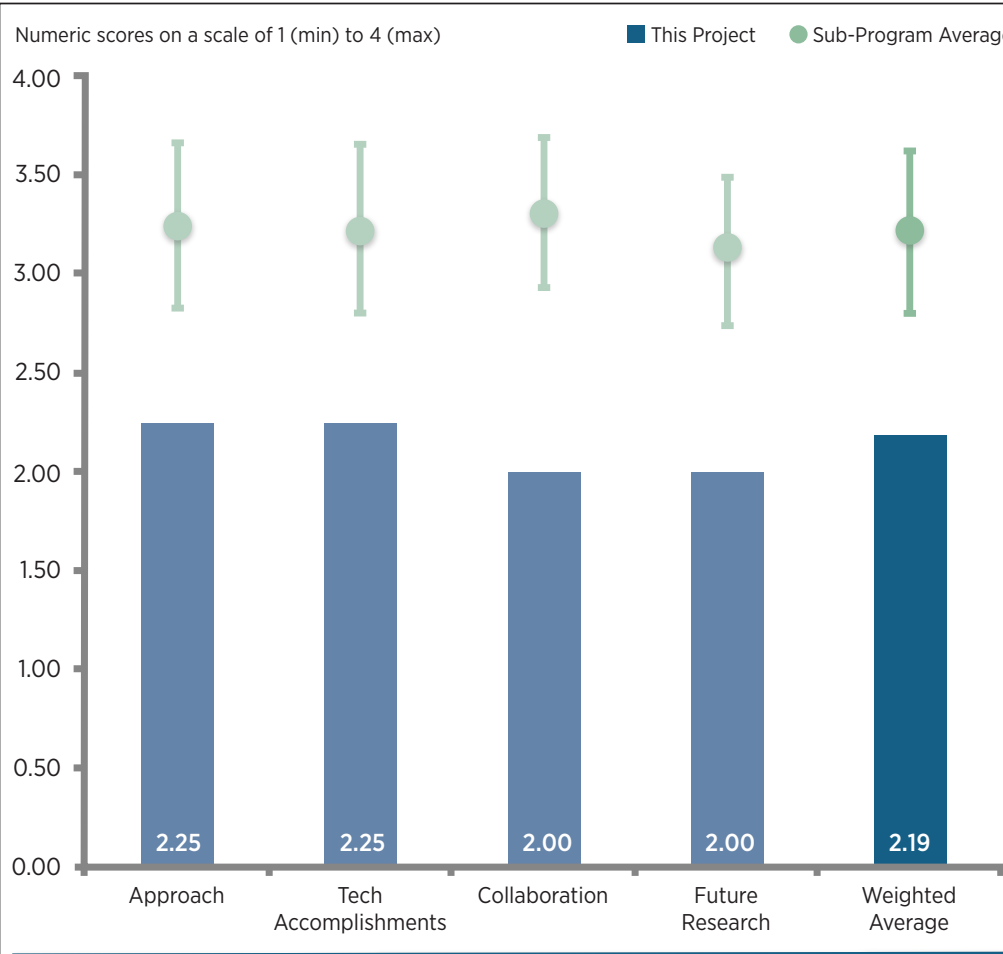

Relevant to DOE Objectives

No
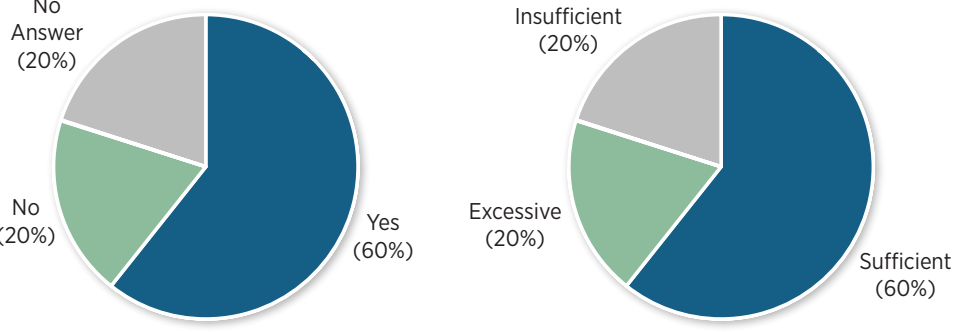

Figure 1-29 - EV Everywhere Charging Infrastructure Roadmap: Donald Karner (Electric Applications Incorporated) - Vehicle Systems 


\section{Reviewer 3:}

The reviewer indicated that it is not clear that this work can significantly inform any future decisions about buildout of charging infrastructure or the best regional deployment of PEVs or all-electric vehicles.

\section{Reviewer 4:}

The reviewer noted that the approach was stated to be specified but this is not a roadmap in any way that this reviewer understands a roadmap, explaining that the concept of a roadmap in a technical realm needs to include projections of outcomes based on different scenarios and be based on the future that is not predetermined. What was reported here was anecdotal information that relates to past scenarios. The reviewer pointed out that the presenters stated that the approach was prescribed by those that sponsored the project, and if that was the case, the mistake was with them rather than the project operatives. Nonetheless, the reviewer concluded, the project does not result in a roadmap to any future set of conditions, which is quite unfortunate because such a roadmap is needed to support and promote adoption of EVs [DOE Program Clarification: To clarify the project's objective and scope, the investigators are identifying and prioritizing EV charging infrastructure actions that support an EV Everywhere objective, specifically, that charging infrastructure should promote PEV adoption and increase electric miles driven without compromising the reliability or performance of the electric grid. The approach is to identify and prioritize the next steps for PEV charging infrastructure, including analyzing DOE infrastructure studies; applying the investigators' own experience; evaluating EV Project data; and seeking input from PEV industry leaders from OEMs, EV service providers, utilities, government, and PEV drivers.].

\section{Question 2: Technical accomplishments and progress toward overall project and DOE goals-the degree to which progress has been made, measured against performance indicators and demonstrated progress towards DOE goals.}

\section{Reviewer 1:}

The reviewer pointed out that the project appears to have largely confirmed the expectations that have been identified many other places, namely, that the vast majority of PEV charging is done at home, with most of the remainder done at workplaces. Therefore, only a small amount of charging would need to be done publicly. The reviewer noted that the team's findings were likely a bit higher than previously postulated $(85 \%$ home, $13 \%$ workplace), but likely not substantially different. Nevertheless, the reviewer characterized the project as providing some additional, perhaps more formal, analysis supporting these conclusions.

The reviewer concluded that the results presented appeared rational, although not that much detail was provided. The reviewer expressed the hope that the final report will provide more, noting in particular that very little of the input directly from stakeholders was clearly identified and presented. As indicated above, this was the part that would have set this project apart perhaps from a simple literature search. Without this information presented, the reviewer described the accomplishments as less than impressive.

\section{Reviewer 2:}

The reviewer commented that details of laying out the roadmap may have been technically met, but there is a lack of details needed for implementation.

\section{Reviewer 3:}

The reviewer observed that it seems the project is almost done at more than $90 \%$ complete, and that there were not many important findings based on the presentation. The reviewer is looking forward to seeing a more detailed report.

\section{Reviewer 4:}

The reviewer remarked that because this is a listing of anecdotal information rather than a roadmap, there was little, if any, technical progress. The reviewer noted that in questioning, the project participants have agreed to send the draft final report for review to some of the reviewers. The reviewer looked forward to seeing it and hoped that it includes some data or scenario-based roadmapping methodologies.

\section{Question 3: Collaboration and coordination with other institutions.}




\section{Reviewer 1:}

The reviewer stated that it is great to see the program has collaboration between a national laboratory and industry with input from PEV drivers.

\section{Reviewer 2:}

The reviewer remarked that there seems to be very little collaboration on this project to assess the significant data and research going on in this field, and that the presenter instead seems to rely significantly on personal experience. Little data was presented to justify recommendations.

\section{Reviewer 3:}

The reviewer noted that the project team worked with INL as well as representatives from industry, utilities, service providers, and users, adding that at least the presentation indicated that the project team contacted stakeholders. However, with none of the stakeholders' input clearly identified, the reviewer stated that it is hard to say what the level of collaboration on this was.

The reviewer highlighted as a glaring hole was that there was no mention of the project explicitly coordinating with Clean Cities. The reviewer pointed out that only a few years ago, Clean Cities awarded 16 EV Community Readiness Projects around the country, and the results of what these groups found, and the documents and solutions they developed, were very close ties to the future research needs identified in the presentation. The reviewer further observed that the Center for Climate and Energy Solutions prepared a lessons learned report for Clean Cities bringing together the results of the 16 projects and stipulated that this should have been a mandatory inclusion for evaluation within this project, but no mention of it was made. The reviewer summarized that what the project was left with was something that appeared to solely be a literature search done somewhat in a vacuum.

\section{Reviewer 4:}

The reviewer commented that there were many inputs that would have to have been investigated to determine the infrastructure needs of a future high level adoption of EVs, but that because none of those were investigated, not much collaboration existed. Hopefully, the reviewer said, something different will be seen when the report is drafted for evaluation.

\section{Question 4: Proposed future research-the degree to which the project has effectively planned its future work in a logical manner by incorporating appropriate decision points, considering barriers to the realization of the proposed technology and, when sensible, mitigating risk by providing alternate development pathways.}

\section{Reviewer 1:}

The reviewer stated that almost all the investigation has been done and that the remaining future work is final editing and incorporating reviewer comments into the roadmap document.

\section{Reviewer 2:}

The reviewer indicated that no future efforts for detailing a roadmap were presented but only high-level findings of the study.

\section{Reviewer 3:}

The reviewer said that because project is $90 \%$ complete and ending at the end of the FY, with largely only final editing and input compilation remaining, therefore the score was left blank.

\section{Reviewer 4:}

The reviewer noted that the base work has been completed and a report is in draft, but that because what was presented was not what was described in the objectives, the lack of future work is a major shortcoming.

\section{Question 5: Does this project support the overall DOE objectives of petroleum displacement? Why or why not?}

\section{Reviewer 1:}

The reviewer remarked that limited charging infrastructure is one of the barriers to increase the number of electric 
vehicle drivers and that development of a charging infrastructure roadmap is helpful to replace petroleum vehicles with PEV/EVs.

\section{Reviewer 2:}

The reviewer replied yes, this project ties to (and obviously supports) EV Everywhere activities and that the project has been focused on identifying and prioritizing EVSE actions to support greater use of EV technologies. However, the reviewer said the concern is with its approach and implementation.

\section{Reviewer 3:}

The reviewer stated that it is an obvious DOE priority to develop a roadmap for EV charging infrastructure build out, but that execution of this study lacked detailed data to back the conclusions.

\section{Reviewer 4:}

The reviewer commented that a well-conceived roadmap for EV infrastructure does need to be developed; however, this work does not satisfy that need.

\section{Question 6: Resources: How sufficient are the resources for the project to achieve the stated milestones in a timely fashion?}

\section{Reviewer 1:}

The reviewer stated that it seems that $\$ 150,000$ is sufficient to conduct an investigation of and lay out a roadmap for the charging infrastructure.

\section{Reviewer 2:}

The reviewer said it is unclear if the lack of funding prevented the development of a more extensive roadmapping process.

\section{Reviewer 3:}

The reviewer stipulated that the project needed to have more and different resources from a variety of stakeholders to answer the question of what types of EV infrastructure were needed for a future of high volume EV penetration and concluding that a true roadmapping effort needs to be conducted.

\section{Reviewer 4:}

The reviewer remarked that to be honest, based upon what was presented, the funding appears excessive. As a caveat, the reviewer said assuming that a number of interviews with stakeholders took place, and that this input will be clearly identified and incorporated into the final report, then it may be sufficient, but based upon what was presented, this seems high. 


\section{Energy Impact of Connected and Automated Vehicles: Huei Peng (University of Michigan) - vs173}

\section{Presenter}

Huei Peng, University of Michigan

\section{Reviewer Sample Size}

A total of five reviewers evaluated this project.

\section{Question 1: Approach to performing the work-the degree to which technical barriers are addressed, the project is well-designed, feasible, and integrated with other efforts.}

\section{Reviewer 1:}

The reviewer described the project approach as crisply presented and significantly detailed with extensive milestones and go/no-go decision points identified over a defined three-year project schedule. The reviewer said that there are five major tasks distributed amongst the three principal project participants: University of Michigan (UM), ANL, and INL. The reviewer explained that the approach leverages a significant amount of existing expertise, fleet operations (and drivers), and data sets to extend the funding mileage for the project, adding that there is a clear final outcome, specifically, the establishment of a portfolio of tools and test platforms for the evaluation of the energy impact of clean air vehicles (CAVs).

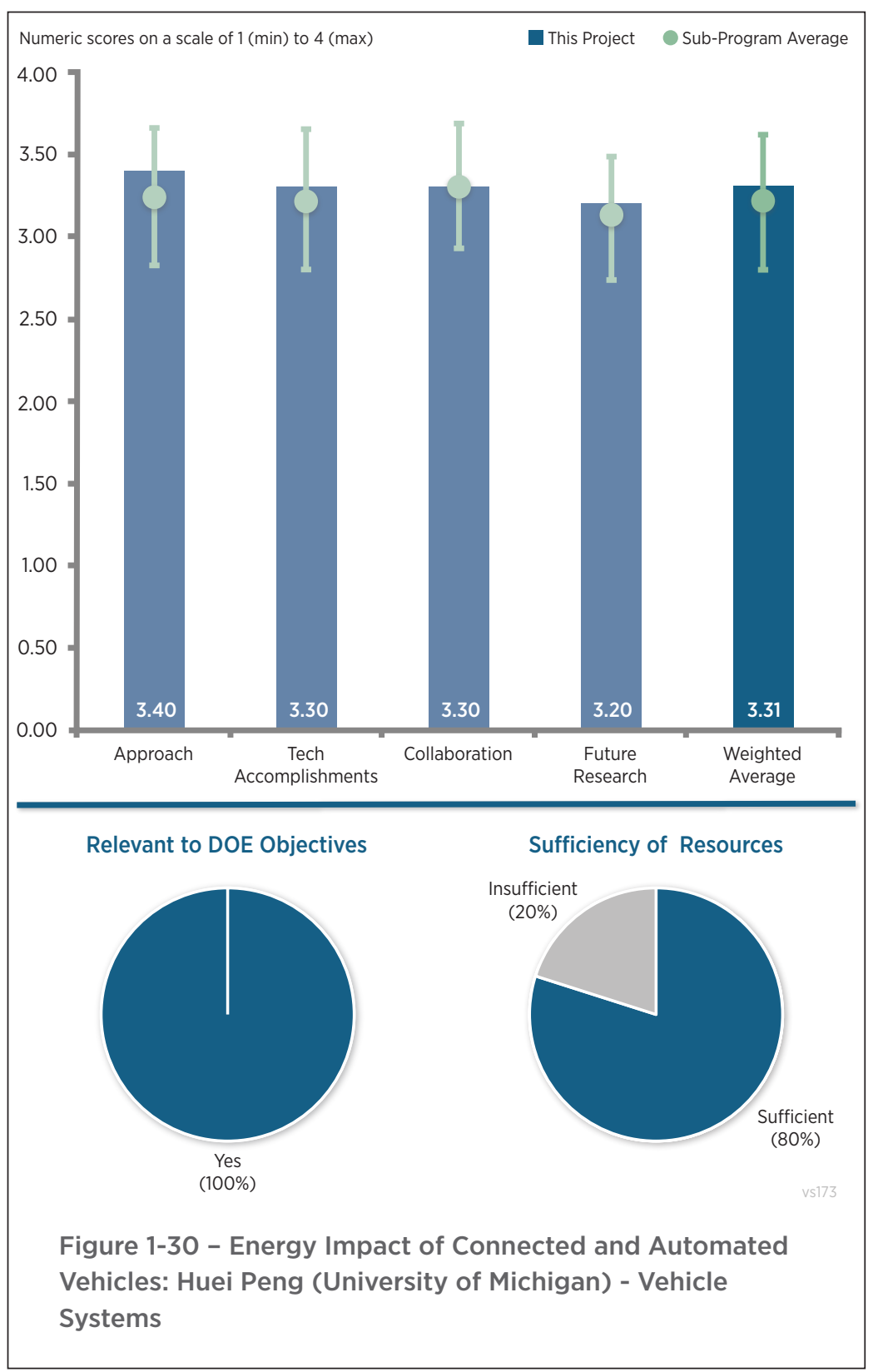

The reviewer cautioned that a continuing challenge will be to appropriately frame and bound the overall project effort given it is breaking significant new ground and contains a lot of previously undefined or amorphous elements which are likely to evolve over time.

\section{Reviewer 2:}

The reviewer stated that this project has a completely new research topic that aims to develop a model to study the energy impact of connected and automated vehicles at a large scale. The reviewer characterized the approach and strategy as sound but added that the work looks challenging because many human factors are involved, e.g., recruiting and training volunteer drivers, and effect of user behavior.

\section{Reviewer 3:}

The reviewer remarked that based on the presenter's reference to signal phase and timing data, the approach 
appears to leverage extensive previous work by the U.S. Department of Transportation and numerous standards organizations that have established physical, functional, and data models as part of the connected vehicle reference implementation architecture. The reviewer characterized the project as having a good plan to collect real-world data to inform a baseline representation of vehicle energy usage in Ann Arbor, Michigan.

The reviewer suggested one idea that may improve the project approach would be to add a stakeholder review panel that would review the plans for simulation experiments, clarifying that this stakeholder review panel should include technical experts from industry, government, and standards organizations. The guidance that would be provided by the stakeholder review panel has the potential to increase the relevancy and value of the project's results.

\section{Reviewer 4:}

The reviewer described a generally sound approach on a challenging set of problems involving modeling of multiple fuzzy (behavioral) factors. The reviewer added that the choice of Ann Arbor is a convenient and appropriate starting-point, but that the team will need to take care to avoid sweeping national energy-use conclusions until more diverse demographic data-points can be added.

\section{Reviewer 5:}

The reviewer observed very interesting and relevant research. This person further noted that the project is to remain focused on small tasks as this could become so large in scope that it becomes an unmanageable amount of information and tries to answer too many questions that it is not designed to answer. The reviewer indicated that the presentation material did not clarify "driver behaviors."

\section{Question 2: Technical accomplishments and progress toward overall project and DOE goals-the degree to which progress has been made, measured against performance indicators and demonstrated progress towards DOE goals.}

\section{Reviewer 1:}

The reviewer said that given the recent start of the project (Fall 2015), there has been in general steady progress on all fronts. The reviewer described as an especially attractive approach for the presentation the clear identification of the specific challenge(s) under each accomplishment area followed by assumptions and proposed solution pathways, adding that this is an effective way to address project challenges and present the approach to reviewers. The reviewer described ongoing activities and accomplishments to include a comprehensive variety of modeling/ simulation, testing, device and software development, field experiments and data collection, and human behavior studies. The reviewer remarked that there is a significant element of uniqueness to many of these activities that cover a lot of ground.

\section{Reviewer 2:}

The reviewer stated there has been impressive progress and milestone accomplishment for less than a year into the project.

\section{Reviewer 3:}

The reviewer noted that the project has been going for less a year and is making progress as planned.

\section{Reviewer 4:}

The reviewer concluded that at $10 \%$ in to the project, it is too difficult to judge accomplishments and process, but added that it looks like the set-up to obtain the necessary data are in place and the scope is under control.

\section{Question 3: Collaboration and coordination with other institutions.}

\section{Reviewer 1:}

The reviewer remarked that there is a strong and appropriate project team that includes UM, ANL, and INL, and other collaborators such as the University of Michigan Transportation Research Institute (UMTRI)/Michigan Mobility Transformation Center (MTC) (leveraging their connected fleet), Danlaw and Cohda Wireless (data loggers and dedicated short range communications, and the EPA for broad consultation on key signals to collect, model inputs, and CAV functions. The reviewer observed that the team has already met twice with EPA and added 
that this seems to be significantly broad team well-positioned to address the project challenges, with no glaring partner deficiencies identified.

\section{Reviewer 2:}

The reviewer observed that a lot of collaboration is necessary for this project to move forward and that it seems there have been many parties involved (e.g., national laboratories, universities, suppliers, government, volunteer drivers, etc.).

\section{Reviewer 3:}

The reviewer stated that involvement on INL and ANL with UM is appropriate. The reviewer added that at some point in time there may be a need to involve an OEM to help discuss technical barriers that may be overlooked as a research project as it pertains to implementation of an end product.

\section{Reviewer 4:}

The reviewer noted that in the Q\&A session, the ANL collaborator spoke about the new DOE SMART Mobility initiative, which was helpful and should be elaborated in future reviews. The reviewer also recommended reaching out to the Unifying Control and Verification of Cyber-Physical Systems (UnCoVerCPS) project sponsored by the European Commission with its ongoing, overlapping research with emphasis on autonomous multi-vehicle cooperation, and provided the following address: http://cps-vo.org/group/UnCoVerCPS.

\section{Question 4: Proposed future research-the degree to which the project has effectively planned its future work in a logical manner by incorporating appropriate decision points, considering barriers to the realization of the proposed technology and, when sensible, mitigating risk by providing alternate development pathways.}

\section{Reviewer 1:}

The reviewer commented that the proposed future work is sufficiently well outlined and detailed to understand future activities. Additionally, the reviewer said that broad barriers/challenges to the project are identified, including design of data logging equipment, recruiting of volunteer drivers, and mutual dependency of progress on test design and model development. This transparency helps provide reassurance that the project is being thoughtfully considered over the long term.

\section{Reviewer 2:}

The reviewer stated that as mentioned in the discussion, connected and automated vehicles have both the potential to save fuel and to overuse fuel. The reviewer suggested that this research should be able to describe those boundary conditions and help set a framework to begin algorithm development towards an end product. As the project progresses, the reviewer suggested that it would be good to see the steps of how to take or apply Ann Arbor to other regions. While Ann Arbor has a good variety of traffic conditions, the footprint is small and population density in relationship is large population basis. The reviewer wondered if those larger cities can reduce gridlock and benefit from this connected and automation, or whether their density is past a critical mass to have significant impact. Some description of barriers to production will be appropriate as the project gets closer to completion.

\section{Reviewer 3:}

The reviewer described it as a long list in the project's future plan. The reviewer is looking forward to seeing future accomplishments.

\section{Reviewer 4:}

The reviewer indicated the chief concern is that the plans seem somewhat rudimentary, academic, early stage, and possibly missing the bigger-picture context. For example, the presentation was unclear whether the research includes inter-vehicle cooperation, or drive-by-wire, other than simulated.

\section{Question 5: Does this project support the overall DOE objectives of petroleum displacement? Why or why not?}

\section{Reviewer 1:}

The reviewer remarked that this is a very relevant project given the potential energy and environmental benefits 
of advances in the area of transportation as a system and CAVs/advanced vehicles While the potential magnitude of energy benefits (or possibly energy losses) is presently unclear, the reviewer surmised that there is a significant untapped opportunity here which needs to be explored in detail, adding that if successful, this project as outlined will enormously benefit the knowledge base in this regard and lay the foundation for further analyses and assessments.

\section{Reviewer 2:}

The reviewer stated that automated vehicles have a great impact on energy consumption looking from big picture.

\section{Reviewer 3:}

The reviewer observed the project looks to develop opportunities to reduce running losses for vehicles in operation, which would reduce petroleum consumption.

\section{Reviewer 4:}

The project will evaluate the potential for connected vehicles to reduce energy consumption which will result in displacement of petroleum consumption.

\section{Question 6: Resources: How sufficient are the resources for the project to achieve the stated milestones in a timely fashion?}

\section{Reviewer 1:}

The reviewer indicated that there are sufficient financial resources and project partner qualifications and facilities to successfully achieve the project milestones and schedule as presented.

\section{Reviewer 2:}

The reviewer stated that so far it seems the budget and resource is sufficient to support the project.

\section{Reviewer 3:}

The reviewer concluded that this project looks like it has a significantly large budget for a significantly large task.

\section{Reviewer 4:}

The reviewer cautioned that the main objective, if interpreted broadly as written in bullet two of Slide 4, is very ambitious and will require extensive leverage of time, budget, and effort beyond what is allocated here. The reviewer offered that writing this project's objective more tightly and specifically would be helpful. 


\section{Methods to Measure, Predict, and Relate Friction, Wear, and Fuel Economy: Steve Gravante (Ricardo) - vs175}

\section{Presenter}

Steve Gravante, Ricardo

\section{Reviewer Sample Size}

A total of six reviewers evaluated this project.

\section{Question 1: Approach to performing the work-the degree to which technical barriers are addressed, the project is well-designed, feasible, and integrated with other efforts.}

\section{Reviewer 1:}

The reviewer praised the excellent overall work that has broad relevance to improving vehicle models.

\section{Reviewer 2:}

The reviewer explained that this project varies lubricants and coatings within an Isuzu $4 \mathrm{H}$ engine to achieve friction reduction, elaborating that the approach is an iterative one (model, test, revise model) to arrive at a best practice. The reviewer praised this as a truly scientific approach to meet the project objectives combining both modeling and empirical test data. The reviewer thinks this was a well thought out approach and, if successful, there will be a way to more accurately measure the friction between piston, skirt, and lining.

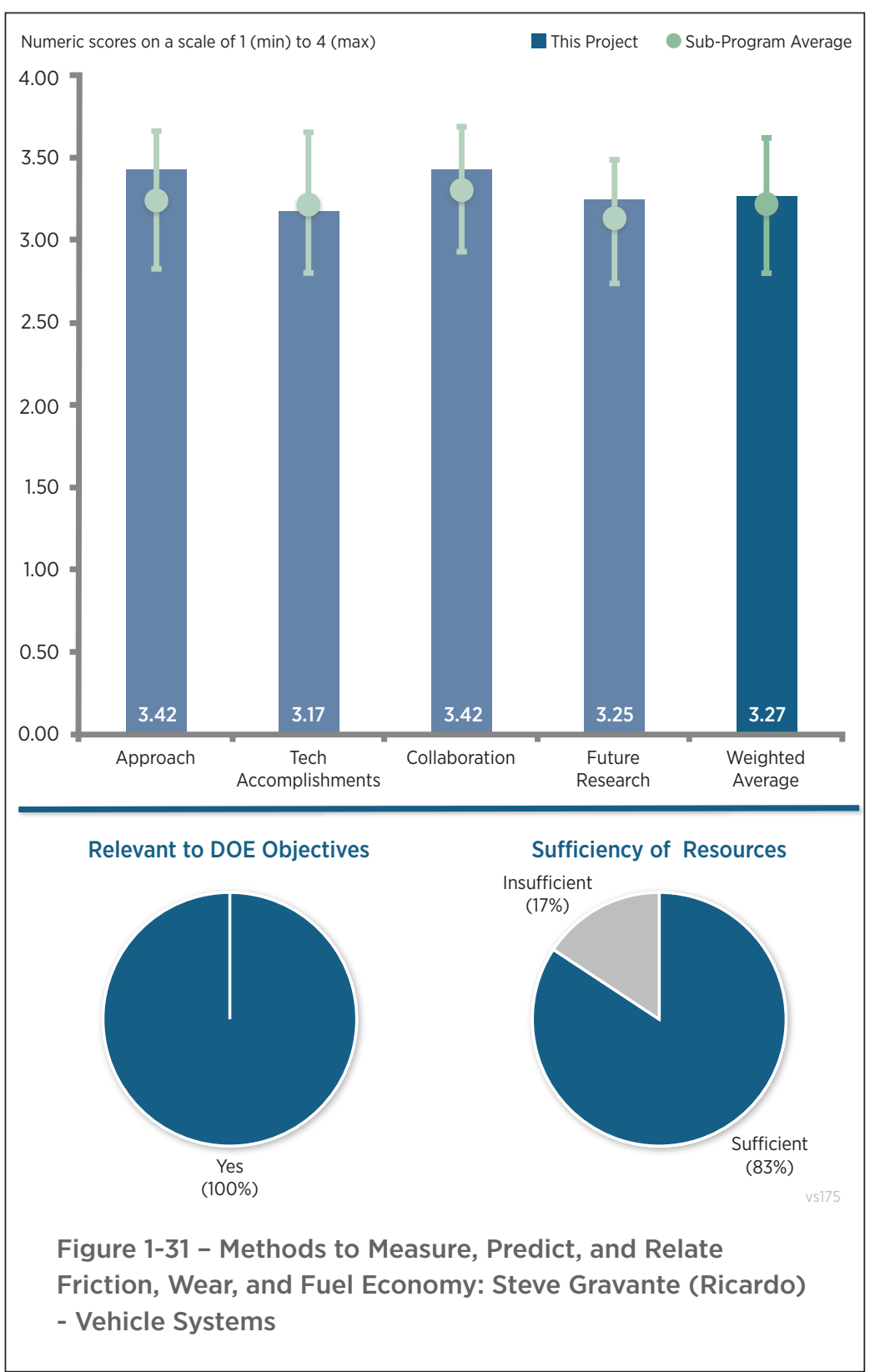

\section{Reviewer 3:}

The reviewer described the approach as good for developing an alternative test method and then validating it against the incumbent method.

\section{Reviewer 4:}

The reviewer characterized the objective as important and suitably ambitious. The reviewer noted the necessary focus on ring/skirt is a major source of risk of failure, because of the need to develop "an appropriate means to separate out the impact of lubricant changes on engine friction and fuel consumption realized through other components, e.g., main bearings, valve train, etc." However, the reviewer characterized as concerning that the approach to doing that was mentioned only vaguely and briefly, and even an understanding of the typical breakdown of the magnitudes of these components was not evident from the presentation. 
A second major concern that the reviewer pointed out is the dependence of modeling on surface roughness. While the careful and methodical approach is good and was well summarized, the concern is that findings from one specific (Isuzu) engine and its peculiar surface manufacturing processes may not be broadly applicable; in other words, a more diverse experimental sample may be needed for robust conclusions.

\section{Reviewer 5:}

The reviewer explained that due to many factors, it has proven extremely difficult over the years to develop a means of predicting the impact of friction reduction technologies on engine fuel economy and wear. As a result, the progression and implementation of advanced lubricants into commercialized HD engines has proven extremely evolutionary. Nonetheless, the reviewer noted, the compelling reason to do so is strong because if successful, advanced tribological solutions could be applied to millions of vehicles, saving large amounts of fuel.

The reviewer further explained that due to the high cost of running dyno and fired engine tests, the project is looking to determine ways to understand key tribological factors that can be measured at the laboratory scale and accurately translate them to the engine-scale via simulation in order to predict the ultimate effect on fuel economy and engine wear and life. The reviewer said this project is incorporating a variety of approaches including extensive laboratory-scale testing, modeling and simulation, and dyno and fired engine tests in the hopes of achieving a means to accurately predict the impact of advanced lubricants, surface treatments, and materials.

The reviewer concluded that a detailed accounting of the approach along with comprehensive milestones for 2015/2016 is presented, providing a good understanding of the project scope and challenges. Additionally, a detailed listing of the critical assumptions and issues is provided which helps ground the audience to the realities of the task moving forward.

\section{Question 2: Technical accomplishments and progress toward overall project and DOE goals-the degree to which progress has been made, measured against performance indicators and demonstrated progress towards DOE goals.}

\section{Reviewer 1:}

The reviewer remarked that overall, the progress is quite good on this effort. The reviewer added that there is some uncertainty in how to account for friction losses in bottom end system that could confound results and correlations and that more effort may be needed to assess these noise factors.

\section{Reviewer 2:}

The reviewer observed that the project appears to be progressing technically and that the deep dive into understanding the physics behind what the alternative test methods are saying is a good accomplishment. The reviewer indicated that programmatically, the project should report actual dates and percentage complete for milestones instead of just reporting to be on track. It is difficult to track progress without more information.

\section{Reviewer 3:}

The reviewer characterized the project as having made good progress, noting it has picked 2 oils from 22 variants and tried them on the piston components. The reviewer noted that the RINGPAK modeling shows moderate correlation, clarifying that the project team can get the peaks but not the velocities. The reviewer surmised that the change in partners probably impacted the progress made.

\section{Reviewer 4:}

The reviewer stated that the project is somewhat behind schedule but is achieving a steady list of accomplishments, including successful down-selection of oil candidates which fulfills the first go/no-go requirement. The reviewer also noted that extensive surface roughness characterization studies are being conducted to help find means to filter out or mitigate the effects of noise and curvature to allow a reasonably accurate measurement of surface roughness. In addition, a RINGPAK model is being developed and sensitivity studies conducted looking at surface roughness, ring tension, oil film thickness, honing parameters asperity function, and so forth, and that resulting trends/observations from these studies are being assessed. The reviewer concluded that the presentation provides a reasonably modest assessment of some of the challenges and barriers moving forward.

\section{Question 3: Collaboration and coordination with other institutions.}




\section{Reviewer 1:}

The reviewer praised the project team as broad and deep with a major engine manufacturer, vehicle OEM, ANL, and two companies specializing in advanced lubricants and laboratory-scale testing, concluding that the team appears to be appropriately structured covering all the major requirements.

\section{Reviewer 2:}

The reviewer noted that this project is using ANL for the modeling and laboratory-scale testing and characterized that as great. The reviewer also thinks the project team was able to make a great recovery bringing on Isuzu when the main OEM pulled out of the project.

\section{Reviewer 3:}

The reviewer stated that this is a good team for modeling and testing, but added that there may be some benefit if academia were included in the effort as well.

\section{Reviewer 4:}

The reviewer replied there is good collaborations that supports the technical areas of this project.

\section{Reviewer 5:}

The reviewer replied it is an overall solid future plan for work.

\section{Reviewer 6:}

The reviewer referenced prior comments.

Question 4: Proposed future research-the degree to which the project has effectively planned its future work in a logical manner by incorporating appropriate decision points, considering barriers to the realization of the proposed technology and, when sensible, mitigating risk by providing alternate development pathways.

\section{Reviewer 1:}

The reviewer stated that the PI showed a slide with the milestones for the project and the status of each and that they all appear on track, so the future work is a continuation of the effort funded. The reviewer concluded that this makes sense given project is in the second year of a three-year project and nothing in the findings indicates a reason to change course. The reviewer observed that some of the specifics are accelerated wear tests, test plans for motored, and fired engine friction tests.

\section{Reviewer 2:}

The reviewer characterized as good the future research of validating the model through physical testing. The reviewer suggested the project would benefit by expanding on the model to include non-power cylinder components (indicated as a challenge/barrier).

\section{Reviewer 3:}

The reviewer agreed that a fairly good accounting is made of next steps/future research, noting that these largely will focus on accelerated wear tests and development and execution of test plans for motored and fired engine friction tests. The reviewer suggested it would have been beneficial to have presented detailed milestones for 2017 as was done for $2015 / 2016$.

\section{Reviewer 4:}

The reviewer replied that other components should have been included in the coating process to complete the test.

\section{Reviewer 5:}

The reviewer remarked that there were many unanswered questions, some of which were touched on in the live Q\&A session including: constant-speed engine applications versus passenger vehicles with frequent cold starts; trade-offs between efficiency and durability; whether the oil-film hydrodynamics are important and, if so, why these are not being studied; history effects and warm-up transient; and getting film temperature correct, noting that there was little discussion of hot versus cold day ambient effects. 


\section{Question 5: Does this project support the overall DOE objectives of petroleum displacement? Why or why not?}

\section{Reviewer 1:}

The reviewer characterized this project as very relevant because the results could be applied to millions of vehicles in the HD sector and also potentially to the medium- and LD sectors as well.'

\section{Reviewer 2:}

The reviewer described this project as an enabler for quickly evaluating other technologies that may directly reduce fuel consumption and that the test methods being developed are expected to be a much quicker evaluation method then traditional engine testing.

\section{Reviewer 3:}

The reviewer noted that friction reduction is crucial to improving powertrain design for fuel economy improvement.

\section{Reviewer 4:}

The reviewer stated that this project works on reducing friction which equates to less losses and less fuel consumption.

\section{Reviewer 5:}

The reviewer replied that lower friction tools will help improve mpg.

\section{Question 6: Resources: How sufficient are the resources for the project to achieve the stated milestones in a timely fashion?}

\section{Reviewer 1:}

The reviewer thinks this project could have benefitted from more funds. There were several times during the presentation when the PI mentioned that work was out of scope, which led the reviewer to believe that the project team was unable to do the full suite of testing and modeling with the budget provided. The reviewer observed that the number of oils tested were down-selected and the number of components with coatings was fewer.

\section{Reviewer 2:}

The reviewer noted that the project is $20 \%+$ cost shared and has strong industry participants that should have the expertise and facilities to potentially carry it out successfully. The reviewer concluded the project is sufficiently funded over its three-year duration.

\section{Reviewer 3:}

The reviewer stated that the budget is sufficient for this scope of work.

\section{Reviewer 4:}

The reviewer replied that the project appears to have sufficient resources. 


\section{Improved Tire Efficiency through Elastomeric Polymers Enhanced with Carbon-Based Nanostructured Materials: Georgios Polyzos (Oak Ridge National Laboratory) - vs176}

\section{Presenter}

Georgios Polyzos, Oak Ridge National Laboratory

\section{Reviewer Sample Size}

A total of three reviewers evaluated this project.

\section{Question 1: Approach to performing the work-the degree to which technical barriers are addressed, the project is well-designed, feasible, and integrated with other efforts.}

\section{Reviewer 1:}

The reviewer stated that the project's overall approach seems rational. The reviewer noted that the project is focused upon developing new materials with tailored properties, going down to almost a very elemental stage of tire design. The reviewer also noted the project team identified parallel paths to increase the chances of success, reducing risks associated with the limitations of restricting the approach to a single formulation. A key criterion for the project is cost-competitiveness, relying upon realistic manufacturing techniques.

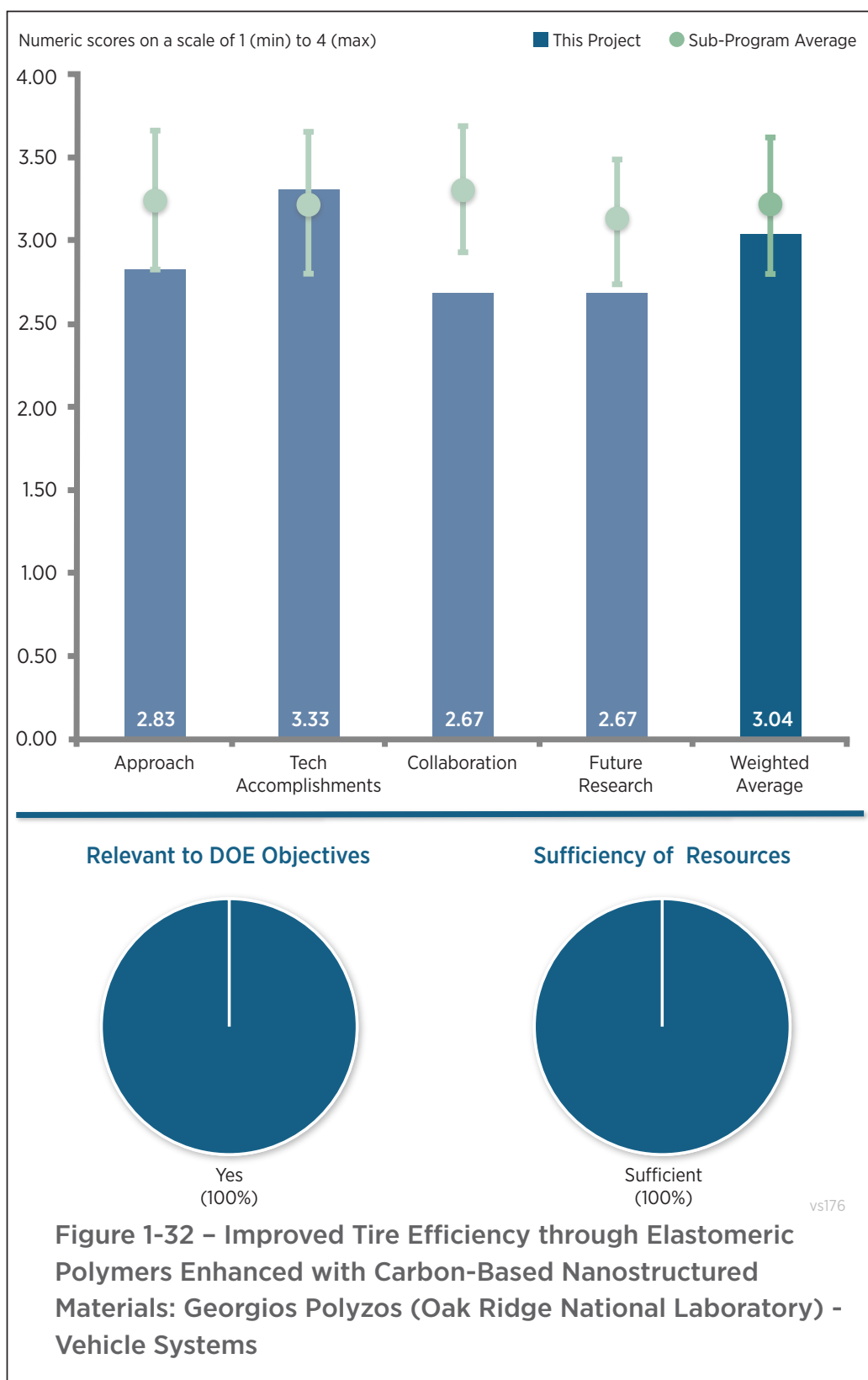

\section{Reviewer 2:}

The reviewer observed that no preliminary data or modeling work was presented to show that the approach taken would result in the claimed benefit of lower rolling resistance. The reviewer added, though, that it seems quite hopeful that scale up costs and needed durability and road grip targets could meet many OEM requirements.

\section{Reviewer 3:}

The reviewer indicated that the approach was not very well defined and that a description of the approach should be enhanced.

\section{Question 2: Technical accomplishments and progress toward overall project and DOE goals-the degree to which progress has been made, measured against performance indicators and demonstrated progress towards DOE goals.}

\section{Reviewer 1:}

The reviewer noted that the project began in January 2016, so this presentation only includes the initial results of approximately $25 \%$ of the project but that it has already completed both of its scheduled milestones for this 
period (first quarter of the project). The reviewer remarked that the results for exfoliated graphene do appear to be relatively dramatic. As for synthesizing silica nanofibers with diameters smaller than 100 nanometers $(\mathrm{nm})$, the reviewer observed that the project achieved fibers with diameters in the $85-110 \mathrm{~nm}$ size range and thus the average likely met the milestone, although there were some in the range above this level.

The reviewer concluded that given the recent start to this project however, these accomplishments have shown very rapid work and added that three milestones are scheduled for the second quarter of the project (during June 2016).

\section{Reviewer 2:}

The reviewer stated that the project appears on target to meet its agreed Phase One deliverables.

\section{Reviewer 3:}

The reviewer observed that the technical accomplishments milestones have been met already this year even though the project only started in January 2016.

\section{Question 3: Collaboration and coordination with other institutions.}

\section{Reviewer 1:}

The reviewer stated that project team is coordinating closely with the industrial partner, but the agreements necessary are not yet in place to allow partner identification. Given the stage of activity of this project (materials formulation), the reviewer acknowledged it may be appropriate that there is not a large team of collaborators. However, the reviewer noted it is a bit surprising that there are no additional collaborators at all, such as perhaps a key fleet user or other organization to provide some level of additional/independent input. The reviewer also noted it was assumed that the industrial partner is a tire manufacturer. If it is not, and is instead another organization in the production/development chain (such as a materials supplier), the reviewer suggested then it may be appropriate to eventually move toward additional collaborators.

\section{Reviewer 2:}

The reviewer replied that the project indicates there is an industrial partner but the organization's name is not provided.

\section{Reviewer 3:}

The reviewer stated there is a lack of coordination to gain insight into the many OEM requirements and instead the focus seems to be fully on compounding and characterization of materials. The reviewer offered that a larger view could be taken by including industry.

\section{Question 4: Proposed future research-the degree to which the project has effectively planned its future work in a logical manner by incorporating appropriate decision points, considering barriers to the realization of the proposed technology and, when sensible, mitigating risk by providing alternate development pathways.}

\section{Reviewer 1:}

The reviewer indicated that the project seems on track to meet its objectives as agreed by DOE.

\section{Reviewer 2:}

The reviewer remarked that ongoing work has been identified by listing the milestones to be met but that no discussion of future work in FY 2017 was provided.

\section{Reviewer 3:}

The reviewer commented that only a few elements of future research were identified, mostly those for completion this month. The reviewer concluded that while overall plan appears to be focused on moving toward testing rolling resistance by the end of the $\mathrm{CY}$, the future plans appear a bit vague, at least according to what was presented.

\section{Question 5: Does this project support the overall DOE objectives of petroleum displacement? Why or why not?}




\section{Reviewer 1:}

The reviewer described the project's objective as improving tire efficiency to meet vehicle fuel consumption goals and that this is fully in line with DOE and VTO objectives. The reviewer explained that the project is using wellreferenced estimates that a reduction of $25-30 \%$ in rolling resistance equates to a $4 \%$ improvement in fuel mileage, as well as California's estimate that $1.5-4.5 \%$ of gasoline use could be reduced if all replacement tires were low rolling resistance models. The reviewer also noted that an additional benefit of improving tire tear resistance is cited as resulting from this project.

\section{Reviewer 2:}

The reviewer stated that the project supports the DOE objective of petroleum displacement through improving fuel economy by reducing rolling resistance of tires.

\section{Reviewer 3:}

The reviewer observed that reducing rolling resistance will support improvements in vehicle fuel economy.

\section{Question 6: Resources: How sufficient are the resources for the project to achieve the stated milestones in a timely fashion?}

\section{Reviewer 1:}

The reviewer commented that the funding appears to be sufficient for the scope of the project.

\section{Reviewer 2:}

The reviewer replied the project is on budget.

\section{Reviewer 3:}

The reviewer pointed out there was no indication given as to the sufficiency of the current level of funding, so it was assumed to be sufficient. 


\section{VTO Vehicle to Building Integration Pathway: Richard Pratt (Pacific Northwest National Laboratory) - vs181}

\section{Presenter}

Richard Pratt, Pacific Northwest National Laboratory

\section{Reviewer Sample Size}

A total of three reviewers evaluated this project.

\section{Question 1: approach to} performing the work-the degree to which technical barriers are addressed, the project is well-designed, feasible, and integrated with other efforts.

\section{Reviewer 1:}

The reviewer praised the preparation of Use Cases and support of project VS183 as providing an excellent structured and integrated approach to this work.

\section{Reviewer 2:}

The reviewer remarked that the project is a worthy effort but seems somewhat broadly defined in terms of goals.

\section{Question 2: Technical} accomplishments and progress toward overall project and DOE goals-the degree to which progress has been made, measured against performance indicators and demonstrated progress towards DOE goals.

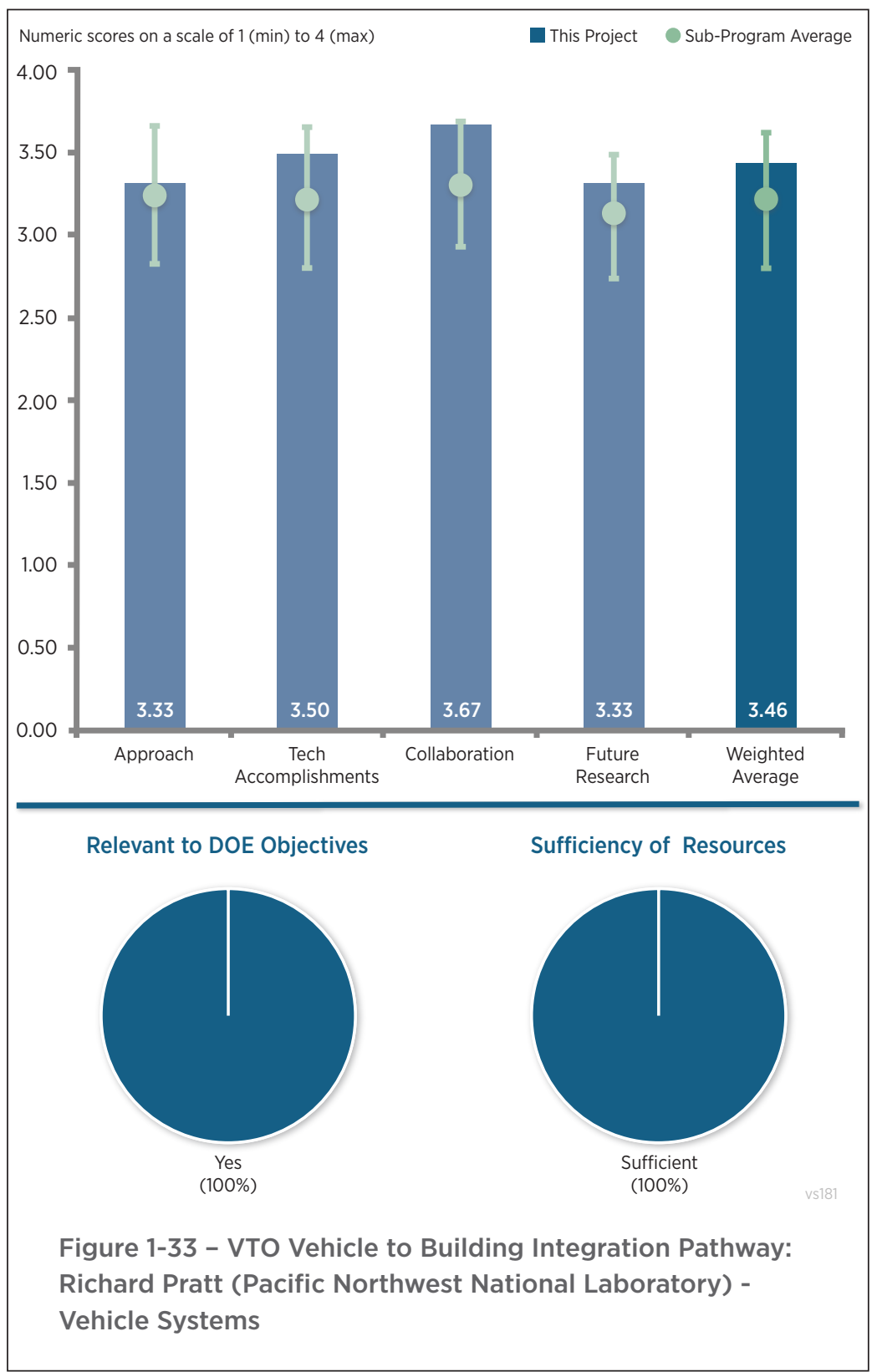

\section{Reviewer 1:}

The reviewer replied there is a new start and that the anticipated October objective would be excellent progress.

\section{Reviewer 2:}

The reviewer commented it is early into the project; integration with the project VS183 has occurred; and an advisory group is being established.

\section{Question 3: Collaboration and coordination with other institutions.}

\section{Reviewer 1:}

The reviewer applauded the great integration with project VS183 and the cooperation across multiple laboratories. The reviewer also commented that the advisory group will provide excellent input from a wide variety of backgrounds. 


\section{Reviewer 2:}

The reviewer observed that the primary stakeholders on the grid side are in place and confirmed that there is good communication with OEMs through the U.S. DRIVE GITT. The reviewer noted that the project is looking for additional stakeholders in EVSE and possibly additional OEM connections.

Question 4: Proposed future research-the degree to which the project has effectively planned its future work in a logical manner by incorporating appropriate decision points, considering barriers to the realization of the proposed technology and, when sensible, mitigating risk by providing alternate development pathways.

\section{Reviewer 1:}

The reviewer evaluated as appropriate the proposed future work and the approach to executing, allowing for feedback from stakeholders.

\section{Reviewer 2:}

The reviewer indicated that the future work will quantify the cost and benefits of various vehicle-to-grid (V2G) Use Cases, thus providing a clear focus on where to apply resources in this area.

\section{Question 5: Does this project support the overall DOE objectives of petroleum displacement? Why or why not?}

\section{Reviewer 1:}

The reviewer replied that this work will satisfy both DOE EERE and DOE Office of Electricity Delivery and Energy Reliability (OE) objectives.

\section{Reviewer 2:}

The reviewer remarked that it promotes adoption of EV usage by better management of electrical energy supply and demand from various sources and sinks.

\section{Question 6: Resources: How sufficient are the resources for the project to achieve the} stated milestones in a timely fashion?

\section{Reviewer 1:}

The reviewer replied that there was no explicit detail but that the reviewers were told the project has strong backing. 


\section{VTO Systems Research Supporting Standards and Interoperability: John Smart (Idaho National Laboratory) - vs182}

\section{Presenter}

John Smart, Idaho National

Laboratory

\section{Reviewer Sample Size}

A total of four reviewers evaluated this project.

\section{Question 1: approach to performing the work-the degree to which technical barriers are addressed, the project is well-designed, feasible, and integrated with other efforts.}

\section{Reviewer 1:}

The reviewer praised the excellent approach to this project, noting that while it is just beginning, the initial objectives are sound.

\section{Reviewer 2:}

The reviewer concurred that the approach and strategy identified by the project will be effective in overcoming barriers. The reviewer observed that this project is one of three projects that will demonstrate PEV charging as an integral part of the renewable electricity grid of the future. In addition, the reviewer stated that this project overlaps with other grid modernization laboratory consortium projects, which will aid in meeting DOE goals.

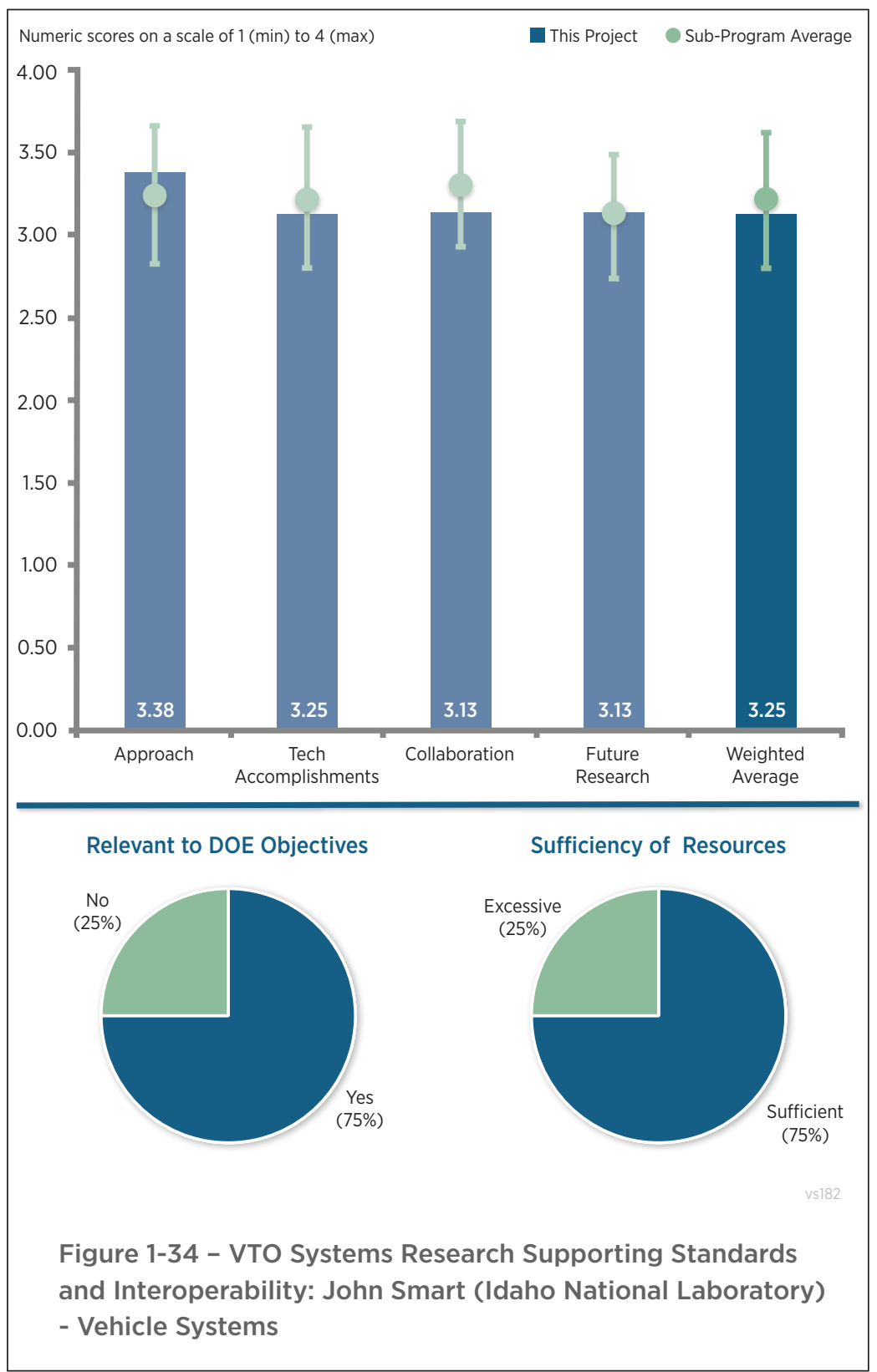

\section{Reviewer 3:}

The reviewer remarked that emulating communications using hardware in the loop and performing dynamic real-time simulation seems like the best way to model these complex system interactions and help predict how integration of PEVs with the grid may take place.

\section{Reviewer 4:}

The reviewer criticized the approach of building hardware first to solve a standards issue in this area with many standards and configurations as an ineffective and expensive approach to the issue. The reviewer noted that the hardware-in-the-loop (HIL) system will come up with a single solution, and asked if it will apply to all of the potential variations. The reviewer said that the approach should have been preceded with a simulation that could be varied to cover widely varying applications followed by validation of one or two with the HIL route. 
Question 2: Technical accomplishments and progress toward overall project and DOE goals-the degree to which progress has been made, measured against performance indicators and demonstrated progress towards DOE goals.

\section{Reviewer 1:}

The reviewer thinks given the short time period since the start of the project that the progress on this effort is excellent.

\section{Reviewer 2:}

The reviewer remarked that even though the project only recently started in April 2016, there have been several accomplishments identified which indicates good progress thus far in the project.

\section{Reviewer 3:}

The reviewer agreed that initial infrastructure and alignment efforts seem to be on track for a successful Phase One go/no-go decision.

\section{Reviewer 4:}

The reviewer stated that technically, the accomplishments are good if only looking at the project.

\section{Question 3: Collaboration and coordination with other institutions.}

\section{Reviewer 1:}

The reviewer applauded the project lead as having assembled an excellent set of partners including four additional national laboratories each with its own specific area of expertise. The reviewer also commented that the use of an Advisory Board consisting of energy companies, a U.S. DRIVE technical team and federal and state organizations will provide good guidance and review of the project.

\section{Reviewer 2:}

The reviewer characterized as excellent the collaboration between the laboratories, and added that it would be helpful to add more insight and feedback in a structured manner from utilities and grid operators.

\section{Reviewer 3:}

The reviewer stated that this is a highly collaborative project and the PI has the right people involved, adding that it is clear that the cooperation from the different laboratories will be there. However, the reviewer commented that it is not clear if sufficient incentive has been provided to the utility partners to have them as fully-engaged collaborators and suggesting it would be good to understand more about how decisions are going to be made in such a collaborative environment. The reviewer further remarked that the project is also linked to other laboratory projects, and thus it is important to align timelines and define success even if other laboratories projects fall behind.

\section{Reviewer 4:}

The reviewer concluded that much more work could have been done here, adding that the project is much bigger than this is planned for.

Question 4: Proposed future research-the degree to which the project has effectively planned its future work in a logical manner by incorporating appropriate decision points, considering barriers to the realization of the proposed technology and, when sensible, mitigating risk by providing alternate development pathways.

\section{Reviewer 1:}

The reviewer commented that the proposed future work in FY 2016, as well as the milestones identified to be accomplished in future years, will be effective in providing solutions to overcoming the specified barriers.

\section{Reviewer 2:}

The reviewer replied that the initial plan is sound. 


\section{Reviewer 3:}

The reviewer stated that this section is planned and well thought out but cautioned that it is still too soon in the project to understand if the planned research is appropriate.

\section{Reviewer 4:}

The reviewer said that the project should have a much bigger vision than it does, commenting that standards work for the utility industry is not effective with a single hardware set demonstration.

\section{Question 5: Does this project support the overall DOE objectives of petroleum displacement? Why or why not?}

\section{Reviewer 1:}

The reviewer characterized the project as relevant to the DOE objective of petroleum displacement through the overall objective of showing the feasibility of PEVs providing grid services and renewable energy integration at the electric utility distribution without negatively impacting PEV customer experience.

\section{Reviewer 2:}

The reviewer stated that the assessment of grid interoperability is a key piece to EV deployment.

\section{Reviewer 3:}

The reviewer remarked that understanding whether EVs can play in utility markets can assist with the market penetration of EVs. The challenge here is if the results will be useful to the grid community once they are available.

\section{Reviewer 4:}

The reviewer described the project as not well founded to reach the stated objectives, and suggested the approach should be reconsidered for a much bigger vision.

\section{Question 6: Resources: How sufficient are the resources for the project to achieve the stated milestones in a timely fashion?}

\section{Reviewer 1:}

The reviewer stated that funding seems to be sufficient to meet current objectives.

\section{Reviewer 2:}

The reviewer indicated that resources appear to be adequate for the overall project but that it would be useful for the project lead to indicate what the funding allocation will be for each laboratory.

\section{Reviewer 3:}

The reviewer thinks the resources are good-to-high. The reviewer would have liked to have seen some of the funds going to the utilities if possible to give them more reason to be active participants in the project.

\section{Reviewer 4:}

The reviewer replied that this project should be reconstructed. 
VTO Modeling and Controls

Software Tools to Support

V2G Integration: Samveg

Saxena (Lawrence Berkeley

National Laboratory) - vs183

\section{Presenter}

Samveg Saxena, Lawrence Berkeley

National Laboratory

\section{Reviewer Sample Size}

A total of five reviewers evaluated this project.

Question 1: approach to performing the work-the degree to which technical barriers are addressed, the project is well-designed, feasible, and integrated with other efforts.

\section{Reviewer 1:}

The reviewer praised the holistic approach to determining value as unique and will provide great insight into what makes sense to implement with V2G.

\section{Reviewer 2:}

The reviewer stated there are ambitious objectives with a detailed plan, adding that as a new start, it is very comprehensive.

\section{Reviewer 3:}

The reviewer said it is a welldocumented approach and that the presentation shows the team is aware of significant challenges to the implementation of this approach. The reviewer remarked that the intent to effectively create a tool for use in evaluating feasibility seems a sensible approach for this simple concept with a complex application.

\section{Reviewer 4:}

The reviewer replied that the proposed modeling and control tool will support $\mathrm{V} 2 \mathrm{G}$ integration through considering various components such as battery calendar and life degradation, time of use, etc.

\section{Question 2: Technical accomplishments and progress toward overall project and DOE goals-the degree to which progress has been made, measured against performance indicators and demonstrated progress towards DOE goals.}

\section{Reviewer 1:}

The reviewer noted the new start, and remarked that progress seems very good in terms of prior work to be leveraged and an explicit roadmap of deliverables.

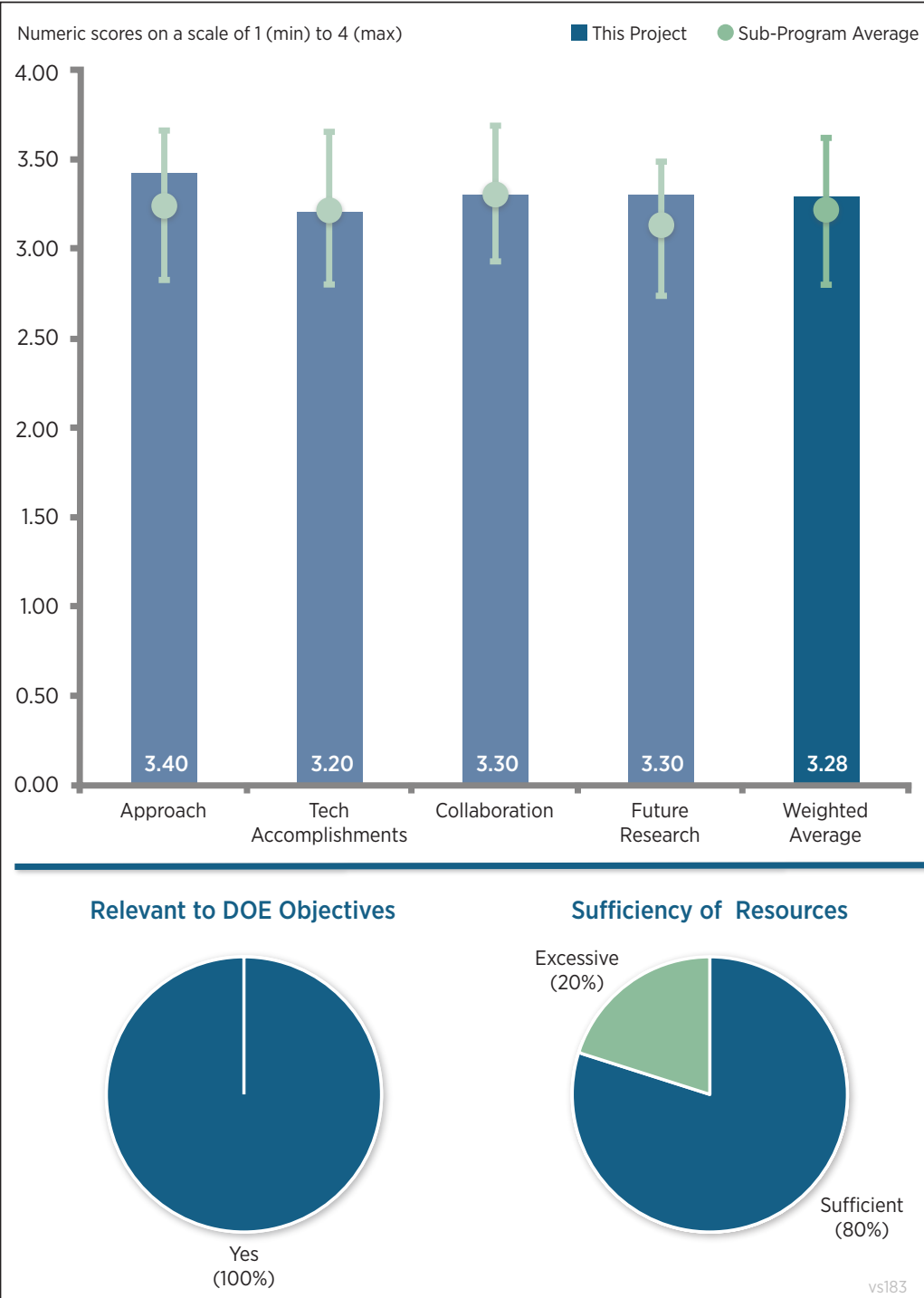

Figure 1-35 - VTO Modeling \& Controls Software Tools to Support V2G Integration: Samveg Saxena (Lawrence Berkeley National Laboratory) - Vehicle Systems 


\section{Reviewer 2:}

The reviewer stated that progress to-date has been to quantify possibilities, but implementation of the rest will provide the means to realize value from the modelling.

\section{Reviewer 3:}

The reviewer commented that while it is early in the project, a significant accomplishment already is the integration of multiple efforts to bring as much granularity to decision making as possible.

\section{Reviewer 4:}

The reviewer replied that it is just two months into the project and too early to confidently review progress.

\section{Question 3: Collaboration and coordination with other institutions.}

\section{Reviewer 1:}

The reviewer remarked that pulling multiple laboratories together with affiliated projects demonstrates excellent cooperation rather than competition for resources.

\section{Reviewer 2:}

The reviewer stated there is good collaboration between the California Independent System Operator (CAISO) and the California Energy Commission and noted that there are stakeholder advisors from automotive OEMs and EVSE suppliers.

\section{Reviewer 3:}

The reviewer commented that the project leverages other laboratories' work and is well connected with other projects.

\section{Reviewer 4:}

The reviewer replied that the multi-laboratory approach seems to be well supported. No named collaboration with electric utility(ies) might have been useful, but it may be unnecessary because this is a model. The reviewer expressed concern for effectively herding cats (when working with so many laboratories) that the objective is met in a timely manner.

\section{Reviewer 5:}

The reviewer recommended careful coordination with the large body of grid-side research by the DOE grid technical team and the results of the DOE Advanced Modeling Grid Research program for the period 2012-2016. The reviewer indicated that awareness of this foundation was not evident from the poster and cautioned that collaboration outside the VTO-managed EV working group seemed in need of strengthening.

\section{Question 4: Proposed future research-the degree to which the project has effectively planned its future work in a logical manner by incorporating appropriate decision points, considering barriers to the realization of the proposed technology and, when sensible, mitigating risk by providing alternate development pathways.}

\section{Reviewer 1:}

The reviewer described the proposed work as ambitious and added that the tools being built should help various stakeholders to better answer the pressing questions around viability of V2G.

\section{Reviewer 2:}

The reviewer agreed that the future work will quantify the cost and benefits of various V2G Use Cases, providing a clear focus on where to apply resources in this area.

\section{Reviewer 3:}

The reviewer stated that it is early in the process, so that future research work is really work to be done on this new project. The reviewer added that results from this project may lead to future work being defined. 


\section{Question 5: Does this project support the overall DOE objectives of petroleum displacement? Why or why not?}

Reviewer 1:

The reviewer replied that the project provides options for better understanding of $\mathrm{EV}$ impact on the electrical grid and vice versa.

\section{Reviewer 2:}

The reviewer stated that this supports objectives of both EERE and OE.

\section{Reviewer 3:}

The reviewer commented that the relevance is good, but that it is not a direct petroleum displacement benefit. Rather, the reviewer remarked, much of the benefit identified is in managing renewables and peak loads.

Question 6: Resources: How sufficient are the resources for the project to achieve the stated milestones in a timely fashion?

\section{Reviewer 1:}

The reviewer replied that the resources appear adequate.

\section{Reviewer 2:}

The reviewer stated that there appears to be significant effort and sufficient funding available to support six laboratories but remarked that it could be clearer how coordination will be managed because an effective organization of the team and their respective responsibilities will be necessary to keep this project on track to deliver.

\section{Reviewer 3:}

The reviewer suggested that many compounding uncertainties involved in V2G integration are so large that a more cautious, incremental approach and budget may be worth considering. The reviewer added that a rush to optimize should be avoided as the EV market, battery, vehicle, internet of things, and grid capabilities continue to significantly evolve under commercial and regulatory pressures. 


\section{VTO Diagnostic Security Modules for Electric Vehicle to Building Integration: Ken Rohde (Idaho National Laboratory) - vs184}

\section{Presenter}

Barney Carlson, Idaho National Laboratory

\section{Reviewer Sample Size}

A total of two reviewers evaluated this project.

\section{Question 1: approach to performing the work-the degree to which technical barriers are addressed, the project is well-designed, feasible, and integrated with other efforts.}

\section{Reviewer 1:}

The reviewer replied that the approach appears to be a logical stepby-step one that builds on previous steps and has appropriate go/no-go decision points.

\section{Reviewer 2:}

The reviewer stated that the approach to the project is good but added that the lack of OEM participation may be problematic in later phases.

\section{Question 2: Technical} accomplishments and progress toward overall project and DOE goals-the degree to which progress has been made, measured against performance indicators and demonstrated progress towards DOE goals.

\section{Reviewer 1:}

The reviewer identified no issues in early phase of this project.

\section{Reviewer 2:}

The reviewer stated it is very early in the project and progress is based on a sound plan and successful application for funding.

\section{Question 3: Collaboration and coordination with other institutions.}

\section{Reviewer 1:}

The reviewer characterized this as a project clearly based on collaboration, adding it should be especially productive by having partners from national laboratories, industry and academia.

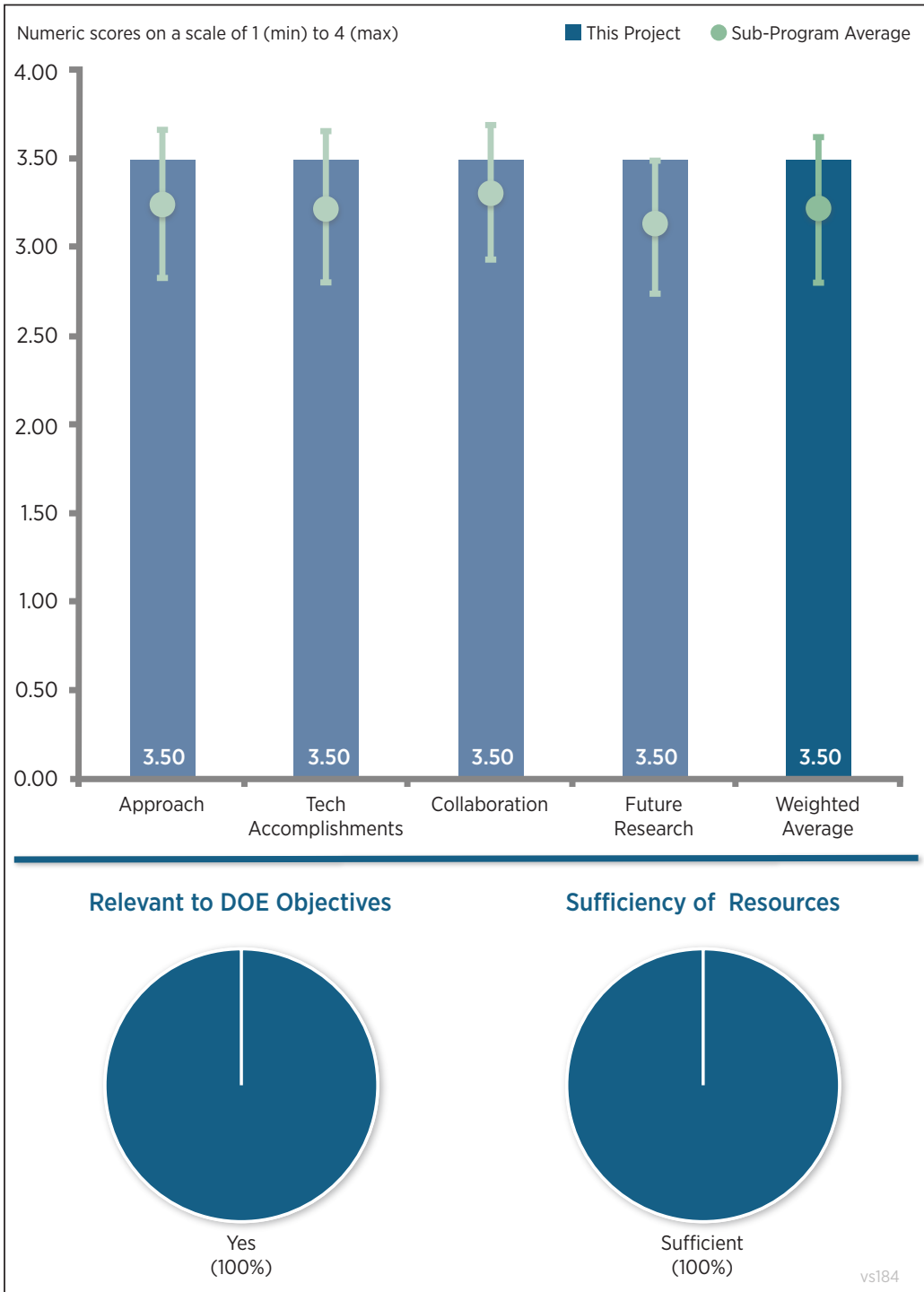

Figure 1-36 - VTO Diagnostic Security Modules for Electric Vehicle to Building Integration: Ken Rohde (Idaho National Laboratory) - Vehicle Systems 


\section{Reviewer 2:}

The reviewer remarked that what will be needed to ensure that the findings and results can be acted upon is broad industry collaboration through standards committees or other approaches for involvement of the ultimate implementers.

Question 4: Proposed future research-the degree to which the project has effectively planned its future work in a logical manner by incorporating appropriate decision points, considering barriers to the realization of the proposed technology and, when sensible, mitigating risk by providing alternate development pathways.

\section{Reviewer 1:}

The reviewer replied there is a solid proposal for future work, and added that more industry involvement at later stages may be useful to inform findings and expected deployment of security countermeasures.

\section{Reviewer 2:}

The reviewer stated that proposed future work is identified in approach.

\section{Question 5: Does this project support the overall DOE objectives of petroleum displacement? Why or why not?}

\section{Reviewer 1:}

The reviewer commented that security is a potential barrier to EV deployment and therefore this project is relevant to DOE objectives.

\section{Reviewer 2:}

The reviewer remarked that cybersecurity is an extraordinarily relevant area of study and a device is an unusual and interesting approach to addressing the concern.

\section{Question 6: Resources: How sufficient are the resources for the project to achieve the stated milestones in a timely fashion?}

\section{Reviewer 1:}

The reviewer responded that the initial requested budget seems to be consistent with meeting project goals.

\section{Reviewer 2:}

The reviewer stated that it is too early to know for sure, but there certainly does not appear to be a shortage of resources base on the list of collaborators. 


\section{Evaluation of Vehicle Technology Benefits on Real World Driving Cycles using Regional Transportation System Model: Aymeric Rousseau (Argonne National Laboratory) - vs185}

\section{Presenter}

Ram Vijayagopal, Argonne National Laboratory

\section{Reviewer Sample Size}

A total of five reviewers evaluated this project.

\section{Question 1: approach to performing the work-the degree to which technical barriers are addressed, the project is well-designed, feasible, and integrated with other efforts.}

\section{Reviewer 1}

The reviewer praised the overall great approach for evaluating the benefits, but added that it would be good to see how the real-world cycles differ from standard cycles with some highlevel metrics such as average speed, velocity ratio, and characteristic acceleration (reviewer suggested looking up the Ph.D. work of Andrew Simpson). The reviewer also added that it would be useful to see what technology benefits 2020 has over 2010 and asking what is causing consumption to improve.

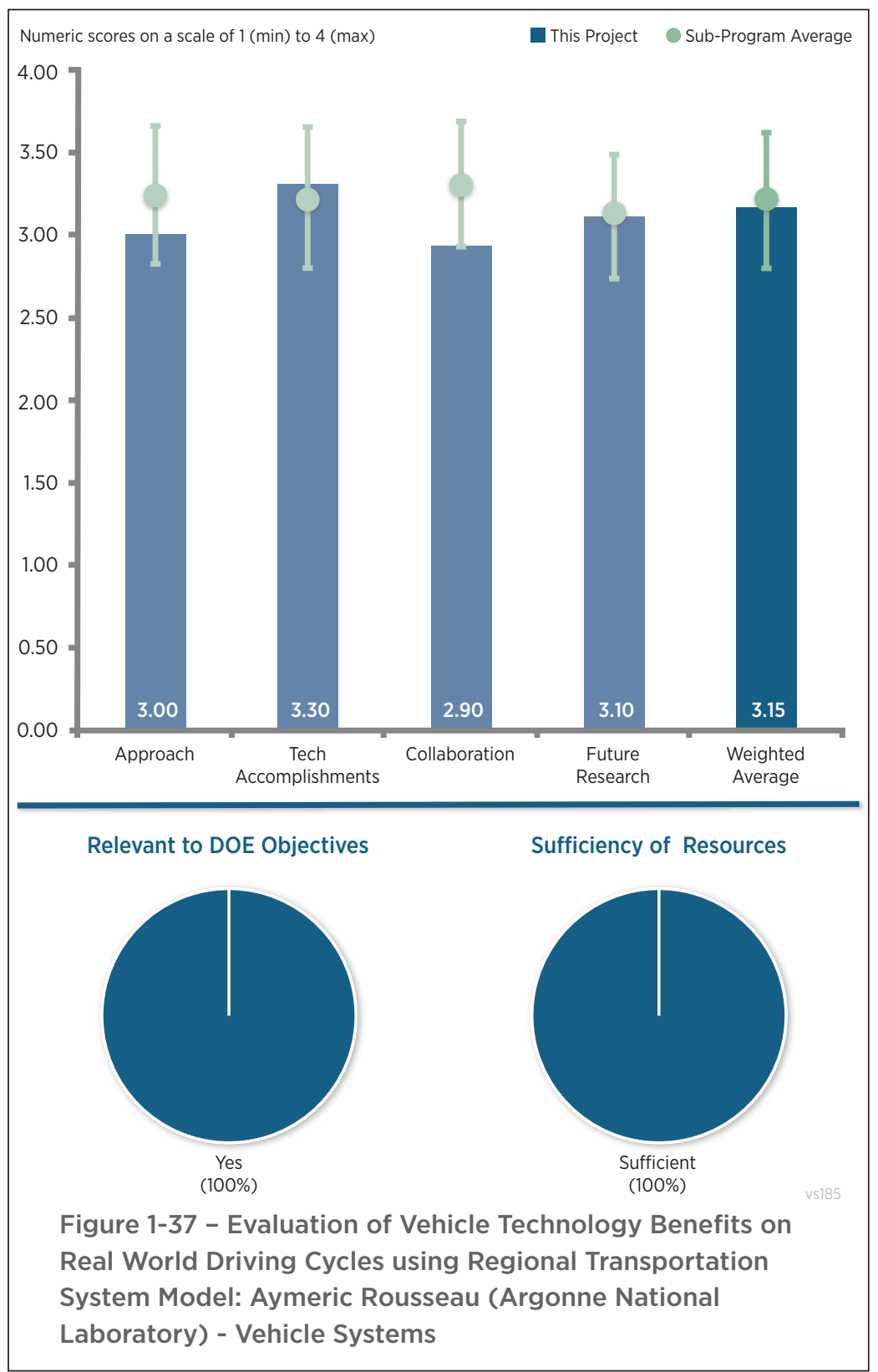

\section{Reviewer 2:}

The reviewer characterized the project approach as a step forward for producing fuel efficiency estimates for specific regional transportation scenarios because it includes real world information, such as road networks and elevation data in the fuel consumption calculations. The reviewer added that the approach focuses on quick production of aggregate fuel consumption estimates for sets of vehicles that employ a mix of technologies. However, the reviewer suggested the approach could be improved through more emphasis on providing quick insights into what are driving differences in the results. The reviewer stated that this additional capability would increase the value of the model to impart information and also provide mechanisms for sanity checking the simulation results.

\section{Reviewer 3:}

The reviewer stated that the approach taken in this project is aligned with meeting the key barriers; however, the approach has some challenges in that its assumption for future fleet mix and technology integration may not match current projections. 


\section{Reviewer 4:}

The reviewer offered that by its very nature, this project has significant uncertainty associated with it (market penetration predictions, etc.). Given these uncertainties, the reviewer believed it would be worthwhile to consider lower fidelity vehicle driveline models to study the outcomes. However, the reviewer acknowledged that using the higher fidelity models along with the large amount of data that it generates does have its advantages, adding that new processes and methods have to be developed to handle these data sets with minimal interaction and to generate consistent and accurate results. Nevertheless, the reviewer said, even if simpler approaches are not used, it may still be worthwhile to compare the results of lower and higher fidelity models to understand what the limitations are of each approach.

\section{Reviewer 5:}

The reviewer replied that the technical approach for this work is very good; however, the overall approach is to validate an ANL model in a vacuum.

\section{Question 2: Technical accomplishments and progress toward overall project and DOE goals-the degree to which progress has been made, measured against performance indicators and demonstrated progress towards DOE goals.}

\section{Reviewer 1:}

The reviewer replied that the technical accomplishments in terms of validating the model are good.

\section{Reviewer 2:}

The reviewer stated that the project appears to be on track to meet the technical objectives laid out for this reporting period.

\section{Reviewer 3:}

The reviewer noted great progress but added that it would help if the labels and titles on the graphs were a little more descriptive. The reviewer pointed out that with the understanding that MA3T predictions are just thatpredictions - the predicted 2025 figures (as was pointed out by another reviewer as well) appear to be unrealistic.

The reviewer concluded it is good that assessing the impact of multiple market penetration scenarios will be addressed in the future.

\section{Reviewer 4:}

The reviewer emphasized that total energy consumption (for fleet level) needs to be shown as energy/mile (not as total energy) and that benefits of 2025 over 2015 vehicles need to be broken down by powertrain type. The reviewer wondered whether electric Watt-hour per mile $(\mathrm{Wh} / \mathrm{mi})$ was referring to only EVs and, if so, what technological benefits were considered for 2025 vehicles for this class.

\section{Question 3: Collaboration and coordination with other institutions.}

\section{Reviewer 1:}

The reviewer replied that the coordination with other organizations is good but added that more engagement from other agencies to improve forecasting could be beneficial.

\section{Reviewer 2:}

The reviewer stated that there appears to be considerable synergy between this project and the work done by Jeff Gonder, Eric Wood, and others at NREL on evaluating the real-world benefits of various technologies such as thermal encapsulation, etc., with respect to fuel consumption. However, the reviewer noted, NREL does not appear to be a partner in this project.

\section{Reviewer 3:}

The reviewer indicated that it is not clear how this methodology will be used and commented that there needs to be a concrete example and use case, adding that a few suggestions were made for potential use cases, but one of these needs to be pursued in depth. 


\section{Reviewer 4:}

The reviewer said that the collaboration on this project as very shallow, adding that the importance of its message should be getting out to other organizations such as EPA and NHTSA.

Question 4: Proposed future research-the degree to which the project has effectively planned its future work in a logical manner by incorporating appropriate decision points, considering barriers to the realization of the proposed technology and, when sensible, mitigating risk by providing alternate development pathways.

\section{Reviewer 1:}

The reviewer stated that future modeling procedures to support off-cycle credits is a good proposed future step to support OEM incorporation of new technologies.

\section{Reviewer 2:}

The reviewer answered that the objective of providing a tool that can be used in assessment of off-cycle credits is worthy of pursuit.

\section{Reviewer 3:}

The reviewer referenced prior comments found in "Technical Accomplishments." The reviewer also remarked, if it were possible, that it would also be interesting to know whether the drive cycles generated by Polaris have statistical properties similar to the drive cycles available at the Transportation Secure Data Center website hosted by NREL.

\section{Reviewer 4:}

The reviewer replied that the proposed future work for this project is good but should be supplement with a much broader coordination.

\section{Question 5: Does this project support the overall DOE objectives of petroleum displacement? Why or why not?}

\section{Reviewer 1:}

The reviewer stated that besides being relevant in terms of evaluating the future petroleum displacement potential, this entire process will also likely develop procedures for handling large amounts of data and reducing them with minimal input to easily comprehensible packets.

\section{Reviewer 2:}

The reviewer judged that the relevance is weak here if the project continues to remain within ANL. The reviewer offered that DOE should be coordinating broadly with the other two agencies responsible for setting vehicle mileage standards.

\section{Question 6: Resources: How sufficient are the resources for the project to achieve the stated milestones in a timely fashion?}

\section{Reviewer 1:}

The reviewer stated that current project efforts are adequate to complete the current tasks.

\section{Reviewer 2:}

The reviewer replied that resources are sufficient. 


\section{Evaluation of Dynamic Wireless Charging Demand: James Li (Oak Ridge National Laboratory) - vs186}

\section{Presenter}

James Li, Oak Ridge National Laboratory

\section{Reviewer Sample Size}

A total of three reviewers evaluated this project.

\section{Question 1: approach to performing the work-the degree to which technical barriers are addressed, the project is well-designed, feasible, and integrated with other efforts.}

\section{Reviewer 1:}

The reviewer affirmed that the presenter clearly listed out the technical barriers of dynamic wireless charging (e.g., lack of effective decision support tool for investment of charging infrastructure; lack of knowledge about en route EV performance regimes; etc.). The presenter also addressed the methods the project team adopted to approach the barriers (e.g., developing and validating representing traffic models, etc.).

\section{Reviewer 2:}

The reviewer commented it is interesting research, and added that while it is early in the program, it is not the best course to ignore the economic factors, and noting this concept seems like a very large infrastructure cost. The reviewer pointed out that many public transportation systems need to subsidized to continue to function and cautioned that if the cost of dynamic charging of the vehicle is significantly more per mile than to drive on gasoline per mile, it will be difficult to gain enough customer usage to warrant such a large investment.

The reviewer also wondered how many more EV miles are created by dynamic charging, and observed that at 40 miles of EV range, the Chevrolet Volt is already displacing $60-70 \%$ of gasoline miles with EV miles while the Nissan LEAF displaces approximately $80 \%$, but still requires a second vehicle for those remaining $20 \%$.

The reviewer asked how many more sales are possible; what percent of roads need to be electrified to reduce range anxiety; and if high-voltage batteries hit their cost targets, what is the cost difference between electrified roads and smaller batteries.

\section{Reviewer 3:}

The reviewer remarked that a lot of time was spent during the presentation just setting up the problem and the 
approach and that it left no time to review the work progress and technical accomplishments or progress. The reviewer also pointed out that the slides indicate the approach taken, the models used, and so forth, but that very little data were shown. The reviewer urged the project team to share some of the modeling results to assure that work is being done. The reviewer exclaimed enough about the potential impact and the milestones.

\section{Question 2: Technical accomplishments and progress toward overall project and DOE goals-the degree to which progress has been made, measured against performance indicators and demonstrated progress towards DOE goals.}

\section{Reviewer 1:}

The reviewer indicated that it is too early in the project to make a strong evaluation of progress and accomplishments. The reviewer thinks there are so many questions to the adoption of this technology that it will be difficult to prove out the value.

\section{Reviewer 2:}

The reviewer replied it was difficult to gauge this from the presentation given.

\section{Question 3: Collaboration and coordination with other institutions.}

\section{Reviewer 1:}

The reviewer affirmed that it is great to see that the program has collaboration between other national laboratories (i.e., ANL and NREL) utilizing their vehicle simulator and testing model to help with the study.

\section{Reviewer 2:}

The reviewer applauded the involvement of ANL and NREL as appropriate to this project at this stage. The reviewer suggested INL may have some useful data on plug-in behavior that could be leveraged to help describe need to electrify road ways.

\section{Reviewer 3:}

The reviewer replied that there seems like a lot of collaboration from the slides shown, but it is hard to see tangible progress because no results were shown.

\section{Question 4: Proposed future research-the degree to which the project has effectively planned its future work in a logical manner by incorporating appropriate decision points, considering barriers to the realization of the proposed technology and, when sensible, mitigating risk by providing alternate development pathways.}

\section{Reviewer 1:}

The reviewer replied that the project is going well so far and the future work is well planned. The reviewer is looking forward to seeing future results.

\section{Reviewer 2:}

The reviewer suggested that to determine how the vehicle will be controlled to please consider use EPA's data (namely, the draft MOVES2004) for speed-acceleration histograms for national driving. The reviewer also offered future questions to consider. The reviewer asked how this is advantageous over fixed point charging infrastructure. The reviewer pointed out that there are many stopping points for intercity trips and that this perhaps only makes sense for urban areas and, more specifically, intersections. The reviewer queried whether automakers would allow their products to be compatible with dynamic wireless charging, and how much that would add to the OEM system cost.

\section{Reviewer 3:}

The reviewer reiterated that the biggest barrier for adopting this technology will be with customer value, and said that this project does not address this barrier.

\section{Question 5: Does this project support the overall DOE objectives of petroleum displacement? Why or why not?}




\section{Reviewer 1:}

The reviewer referenced the presenter's remark that dynamic wireless charging is a promising technology to reduce electric car drivers' range anxiety and size of battery by providing capability of en route charging, which will increase the percentage of EV drivers, and that electrification of the nation's vehicle fleet offers large potential reductions in energy consumption, criteria emissions, and greenhouse gases.

Reviewer 2:

The reviewer stated that this project does have an opportunity to provide additional EV miles displacing fueled miles.

\section{Question 6: Resources: How sufficient are the resources for the project to achieve the} stated milestones in a timely fashion?

\section{Reviewer 1:}

The reviewer remarked that at $\$ 180,000$, it looks to be a low-cost research project to DOE that could identify some opportunity to displace petroleum and that this funding level seems appropriate at this time.

\section{Reviewer 2:}

The reviewer thinks that $\$ 180,000$ is sufficient for this project considering there is no need for prototyping or experiment. 


\section{Acronyms and Abbreviations}

$\mathrm{A} / \mathrm{C}$

AMR

AMT

ANL

AVTA

BATO

BET

BEV

BTE

${ }^{\circ} \mathrm{C}$

CAD

CAISO

CAV

$\mathrm{Cd}$

CFD

CRADA

CY

DOE

EDV

EERE

EPA

EV

EVSE

FC

FCA

FY

GDI

GHG

GITT

GSF2
Air-Conditioning

Annual Merit Review

Air Maintenance Technology

Argonne National Laboratory

Advanced Vehicle Testing Activity

Bridgestone Americas Tire Operations

Battery Electric Truck

Battery Electric Vehicle

Brake thermal efficiency

Degrees Celsius

Computer-Aided Design

California Independent System Operator

Clean Air Vehicles

Drag Coefficient

Computational Fluid Dynamics

Cooperative research and development agreement

Calendar Year

U.S. Department of Energy

Electric Drive Vehicle

Energy Efficiency and Renewable Energy

U.S. Environmental Protection Agency

Electric Vehicle

Electric Vehicle Supplemental (Supply) Equipment

Fuel Cell

Fiat Chrysler Automobiles

Fiscal Year

Gasoline direct injection

Greenhouse Gas

Grid Integration Technical Team

Generic Speed Form 2 


\begin{tabular}{|c|c|}
\hline $\mathrm{H}_{2}$ & Hydrogen \\
\hline $\mathrm{HC}$ & Hydrocarbons \\
\hline HD & Heavy-Duty \\
\hline HIL & Hardware in the Loop \\
\hline HVAC & Heating, Ventilating and Air Conditioning \\
\hline ICE & Internal Combustion Engine \\
\hline INL & Idaho National Laboratory \\
\hline $\mathrm{kW}$ & Kilowatt \\
\hline $\mathrm{kWh}$ & Kilowatt Hour \\
\hline LD & Light-Duty \\
\hline LLNL & Lawrence Livermore National Laboratory \\
\hline MD & Medium-Duty \\
\hline MPG & Miles per gallon \\
\hline MPGe & Miles per gallon equivalent \\
\hline MTC & Michigan Mobility Transformation Center \\
\hline NHTSA & National Highway Traffic Safety Administration \\
\hline NM & Nanometer \\
\hline NREL & National Renewable Energy Laboratory \\
\hline $\mathrm{OE}$ & Office of Electricity Delivery and Energy Reliability \\
\hline OEM & Original Equipment Manufacturer \\
\hline ORNL & Oak Ridge National Laboratory \\
\hline PCM & Phase change material \\
\hline PEV & Plug-in Electric Vehicle \\
\hline PHET & Plug-In Hybrid Electric Truck \\
\hline PI & Principal Investigator \\
\hline PSI & Pounds per Square Inch \\
\hline PTO & Power Takeoff \\
\hline Q\&A & Question and Answer \\
\hline $\mathrm{R} \& \mathrm{D}$ & Research and Development \\
\hline ROI & Return on Investment \\
\hline SAE & Society of Automotive Engineers \\
\hline
\end{tabular}


SMART Systems and Modeling for Accelerated Research in Transportation

SwRI Southwest Research Institute

TIR Technical Information Report

UM University of Michigan

UMTRI University of Michigan Transportation Research Institute

UnCoVerCPS Unifying Control and Verification of Cyber-Physical Systems

U.S. DRIVE U.S. Driving Research and Innovation for Vehicle efficiency and Energy sustainability

UTEMPRA Unitary Thermal Energy Management for Propulsion Range Augmentation

V2G Vehicle-to-Grid

V Volt

VS Vehicle Systems

VTO Vehicle Technologies Office

$\mathrm{Wh} / \mathrm{mi} \quad$ Watt-hour per Mile

WHR Waste Heat Recovery

WPT Wireless Power Transfer 


\section{Electrochemical Energy Storage}

The Vehicle Technologies Office (VTO) focuses on reducing the cost, volume, and weight of batteries, while simultaneously improving the vehicle batteries' performance (power, energy, and durability) and ability to tolerate abuse conditions. Reaching the Office's goals in these areas and commercializing advanced energy storage technologies will allow more people to purchase and use electric drive vehicles. It will also help the U.S. Department of Energy (DOE) meet the EV Everywhere Grand Challenge goal to enable plug-in electric vehicles (PEVs) that are as affordable and convenient for the American family as gasoline-powered vehicles by 2022.

VTO pursues three major areas of research in batteries:

- Exploratory Battery Materials Research: Addresses fundamental issues of materials and electrochemical interactions associated with lithium ( $\mathrm{Li}$ ) and beyond-Li batteries. This research attempts to develop new and promising materials, use advanced material models to predict the modes in which batteries fail, and employ scientific diagnostic tools and techniques to gain insight into why materials and systems fail. Building on these findings, it works to develop ways to mitigate those failures.

- Applied Battery Research: Focuses on optimizing next generation, high-energy Li-ion electrochemistries that incorporate new battery materials. The activity emphasizes identifying, diagnosing, and mitigating issues that negatively impact the performance and life of cells using advanced materials.

- Advanced Battery Development, System Analysis, and Testing: Focuses on the development of robust battery cells and modules to significantly reduce battery cost, increase life, and improve performance. This research aims to ensure these systems meet specific goals for particular vehicle applications.

This research builds upon decades of work that DOE has conducted in batteries and energy storage. Research supported by VTO led to today's modern nickel (Ni) metal hydride batteries, which nearly all first-generation hybrid electric vehicles (HEV) used. Similarly, the Office's research also helped develop the Li-ion battery (LIB) technology used in the Chevrolet Volt, the first commercially available plug-in hybrid electric vehicle (PHEV). This technology is now being used in a variety of HEVs and PEVs coming on the market now and in the next few years, including the Ford Focus electric vehicle (EV).

The batteries subprogram works extensively with a number of different organizations, including national laboratories and universities. Within the Department, the office collaborates with the Office of Science and Advanced Research Projects Agency-Energy (ARPA-e). Across the federal government, the subprogram collaborates with:

- The Interagency Advanced Power Group;

- The U.S. Environmental Protection Agency;

- The National Aeronautics and Space Administration;

- The National Science Foundation;

- The National Highway Traffic Safety Administration (U.S. Department of Transportation); and

- The U.S. Army Tank, Automotive Research and Development and Engineering Center (U.S. Department of Defense).

The subprogram collaborates on international research with:

- International Energy Agency's Implementing Agreement on Hybrid Electric Vehicles;

- The Clean Energy Ministerial's Electric Vehicle Initiative; and

- The Clean Energy Research Center bilateral agreement between the United States and China.

Much of the subprogram's research is conducted in sync with industry partners through:

- The U.S. DRIVE Partnership focusing on light-duty vehicles;

- The $21^{\text {st }}$ Century Truck Partnership, focusing on heavy-duty vehicles; and

- The United States Advanced Battery Consortium (USABC), a partnership between DOE, Fiat Chrysler Au- 
tomobiles, Ford, and General Motors to develop and demonstrate advanced battery technologies for hybrid and electric vehicles (EVs), as well as benchmark test emerging technologies.

As described in the EV Everywhere Blueprint, the major goals of the Batteries and Energy Storage subprogram are by 2022 to:

- Reduce the production cost of an EV battery to a quarter of its current cost;

- Halve the size of an EV battery; and

- Halve the weight of an EV battery.

Achieving these goals would result in:

- Lowering battery cost from $\$ 500 / \mathrm{kwh}$ to $\$ 125 / \mathrm{kWh}$; and

- Increasing density from $100 \mathrm{Wh} / \mathrm{kg}$ to $250 \mathrm{Wh} / \mathrm{kg}, 200 \mathrm{Wh} / 1$ to $400 \mathrm{Wh} / 1$, and $400 \mathrm{~W} / \mathrm{kg}$ to $2,000 \mathrm{~W} / \mathrm{kg}$

\section{Subprogram Feedback}

The U.S. Department of Energy (DOE) received feedback on the overall technical subprogram areas presented during the 2016 Annual Merit Review (AMR). Each subprogram technical session was introduced with a presentation that provided an overview of subprogram goals and recent progress, followed by a series of detailed topic area project presentations.

The reviewers for a given subprogram area responded to a series of specific questions regarding the breadth, depth, and appropriateness of that DOE VTO subprogram's activities. The subprogram overview questions are listed below, and it should be noted that no scoring metrics were applied. These questions were used for all VTO subprogram overviews.

Question 1: Was the program area, including overall strategy, adequately covered?

Question 2: Is there an appropriate balance between near- mid- and long-term research and development?

Question 3: Were important issues and challenges identified?

Question 4: Are plans identified for addressing issues and challenges?

Question 5: Was progress clearly benchmarked against the previous year?

Question 6: Are the projects in this technology area addressing the broad problems and barriers that the Vehicle Technologies Office (VTO) is trying to solve?

Question 7: Does the program area appear to be focused, well-managed, and effective in addressing VTO's needs?

Question 8: What are the key strengths and weaknesses of the projects in this program area? Do any of the projects stand out on either end of the spectrum?

Question 9: Do these projects represent novel and/or innovative ways to approach these barriers as appropriate?

Question 10: Has the program area engaged appropriate partners?

Question 11: Is the program area collaborating with them effectively?

Question 12: Are there any gaps in the portfolio for this technology area?

Question 13: Are there topics that are not being adequately addressed?

Question 14: Are there other areas that this program area should consider funding to meet overall programmatic goals? 
Question 15: Can you recommend new ways to approach the barriers addressed by this program area?

Question 16: Are there any other suggestions to improve the effectiveness of this program area?

Responses to the subprogram overview questions are summarized in the following pages. Individual reviewer comments for each question are identified under the heading Reviewer 1, Reviewer 2, etc. Note that reviewer comments may be ordered differently; for example, for each specific subprogram overview presentation, the reviewer identified as Reviewer 1 in the first question may not be Reviewer 1 in the second question, etc. 


\section{Overview of the DOE Advanced Battery R\&D Program: David Howell (U.S. Department of Energy) - es000}

\section{Question 1: Was the program area, including overall strategy, adequately covered?}

\section{Reviewer 1:}

The reviewer replied yes, and stated that the program's background, prediction of battery cost, funding distribution, research roadmap, and strategy are adequately covered and easy to understand.

\section{Reviewer 2:}

The reviewer replied yes to this question.

\section{Reviewer 3:}

The reviewer replied yes to this question.

\section{Reviewer 4:}

The reviewer praised the presentation as having clearly outlined the different projects and focus areas of the program and provided an indication of how awards are decided. Nevertheless, the reviewer cautioned, it is not $100 \%$ clear why these areas are chosen and what requirements were used. Overall, though, the reviewer stated that from an original equipment manufacturer (OEM) perspective, a majority of the projects make sense and might ease the path for the implementation of electric vehicles.

\section{Question 2: Is there an appropriate balance between near- mid- and long-term research and development?}

\section{Reviewer 1:}

The reviewer replied yes, the balance of the projects is good, remarking that the focus on silicon ( $\mathrm{Si}$ )/intermetallic anodes and metallic Li covers very well the trend in industry and results can be implement quickly into already existing industry roadmaps. The reviewer added also the approach to develop diagnostic tools that support the material developments and decipher root causes of electrochemical energy storage degradation is useful as this can prove to be invaluable for the development of new materials. The reviewer concluded that the framework of funding agencies, this program covers a very important range of topics and besides the occasional overlap has his own footprint.

\section{Reviewer 2:}

The reviewer said yes, adding that there is an appropriate balance between near- and long-term research and development (R\&D). The reviewer observed that the timeline chart clearly shows that Si anode coupled with a high capacity cathode which presents moderate risk pathway is one of the current emphasis, while Li-metal, Li-sulfur (Li-S), and Li-air would be the long-term research.

\section{Reviewer 3:}

The reviewer offered that there seems to have been at least some movement towards more near-term R\&D and manufacturing issues as well as towards more advanced fundamental science, with reduced focus on mid-term R\&D. This reviewer suggested this is favorable and should provide better value and benefit to U.S. industry in this technology area.

\section{Reviewer 4:}

The reviewer replied yes to this question.

\section{Question 3: Were important issues and challenges identified?}

\section{Reviewer 1:}

The reviewer said yes, adding that most of the challenges are well known in the community and a majority of them are addressed in this program.

\section{Reviewer 2:}

The reviewer replied yes. 


\section{Reviewer 3:}

The reviewer said.

\section{Reviewer 4:}

The reviewer said yes and expressed approval of the slide that shows the breakdown of the battery cost and the breakthroughs required at different process steps. The reviewer cautioned, however, that not all the research areas have challenges identified and present.

\section{Question 4: Are plans identified for addressing issues and challenges?}

\section{Reviewer 1:}

The reviewer replied yes.

\section{Reviewer 2:}

The reviewer said yes, generally.

\section{Reviewer 3:}

The reviewer praised the plan on the anode side as very clearly and outlined. However, the reviewer stated that in other areas, it is not as accurate for the case of a high-level overview but added that this could be caused by the time constraint for the projects.

\section{Reviewer 4:}

The reviewer remarked that most focus areas have plans laid out to address issues and challenges while some of them do not and noted as an example that advanced battery material has today's technology but a next generation target listed. The reviewer said that except for anode material, issues not addressed include how to reach the goal, what are the challenges, and what are any solutions.

\section{Question 5: Was progress clearly benchmarked against the previous year?}

\section{Reviewer 1:}

The reviewer replied yes.

\section{Reviewer 2:}

The reviewer said yes, stating that progress was clearly benchmarked against the previous year and the year of 2012, and that it seems that a continuous progress is being made to approach the 2022 target. The reviewer asked, however, about whether the $\$ 125 / \mathrm{kWh}$ for 2020 as listed on Slide two is the goal or a typo and it should be in 2022 .

\section{Reviewer 3:}

The reviewer remarked that certain areas such as the licensing and commercialization of the components/ technologies from the Materials Engineering Research Facility (MERF) have clearly progressed since last year while other highlights were the conclusion of multi-year projects. The reviewer concluded that, in general, the progress was outlined well on a high level versus the general roadmap, which is adequate for the purpose of this presentation.

\section{Reviewer 4:}

The reviewer said yes in some areas and no in some areas. The reviewer elaborated that in some cases, it may be implied that progress which might have occurred independently within the global industry in this technology is explicitly due to DOE-funded activity. The reviewer observed an enormous amount of DOE-funded activity underway for which progress has been made in some areas that can be directly noted.

\section{Question 6: Are the projects in this technology area addressing the broad problems and barriers that the Vehicle Technologies Office (VTO) is trying to solve?}

\section{Reviewer 1:}

The reviewer said yes, adding that increasing the usage of renewable energy source and electrifying the vehicles is obviously one of the focus of VTO. 


\section{Reviewer 2:}

The reviewer replied yes to this question.

\section{Reviewer 3:}

The reviewer said yes, in almost all cases.

\section{Reviewer 4:}

The reviewer replied that in general yes, but cautioned that sometimes the projects seem to be too specifically focused on a single problem and forget to address other barriers needed to be overcome in order to implement this technology in commercial products and/or use the software in industry.

\section{Question 7: Does the program area appear to be focused, well-managed, and effective in addressing VTO's needs?}

\section{Reviewer 1:}

The reviewer noted that program breaks down the battery research into many focus areas and that those research areas progress hand-by-hand to approach the advanced low-cost battery system. The reviewer concluded therefore, yes, the program is focused, well-managed and effective.

\section{Reviewer 2:}

The reviewer said yes, in general, remarking that in particular there seems to have been improved focus on some key topics which impact time-sensitive decisions for future direction in the last few years and that this indicates an improved focus on potential payoff versus continued effort. The reviewer assessed this as a positive trend.

\section{Reviewer 3:}

The reviewer replied yes to this question.

\section{Reviewer 4:}

The reviewer stated that the majority of the projects are well-managed and aligned with the VTO's needs and roadmaps, although the reviewer added that some of the projects seem a bit out of the scope and cover topics that should be addressed by the OEMs themselves if there is need for this particular technology. However, this is only true for a minority of the modelling and battery pack projects.

\section{Question 8: What are the key strengths and weaknesses of the projects in this program area? Do any of the projects stand out on either end of the spectrum?}

\section{Reviewer 1:}

The reviewer stated that a key strength is the strong focus of the program towards materials and a focus on characterization and that one of the key solutions to improve Li-ion is to find new high-performance materials and coatings to protect and support existing materials. On the other hand, the reviewer noted, it is important to find the root causes of the degradation of energy storage systems and identify these interactions and mechanisms. A combination of these projects could be a powerful conclusion to enable better batteries.

A suggestion from the reviewer to further strengthen the program would be to widen the landscape of funding recipients, adding that although several new companies and institutes have received awards recently, the group could be extended to represent the changing structure of the battery research.

\section{Reviewer 2:}

The reviewer stated that Computer-Aided Engineering for Electric-Drive Vehicle Batteries (CAEBAT) should be very helpful for continuous battery R\&D.

\section{Reviewer 3:}

The reviewer described as one of the key strengths that have developed more recently with some of the projects is the creation of large groups focused on key technical issues that must either must be resolved or which must be determined to be unresolvable, and upon which some reasonable time limit of effort has been placed. The reviewer noted another key strength is focused on battery manufacturing methods and related manufacturing innovation. 
Efforts in this area may provide a pathway for the United States to show some leadership at some level in the general global battery industry.

The reviewer characterized as one of the weaknesses of some of the projects is in putting particular focus on scaleup, optimization of scale-up and optimization of processes for battery materials, particularly for ultra-specialty battery materials and chemicals. This does not seem to be an area in which the United States would be expected to be significant in the global battery industry, when other areas may still have potential (like battery manufacturing methods, etc.).

\section{Reviewer 4:}

The reviewer said the requirements and time line to meet requirements.

\section{Question 9: Do these projects represent novel and/or innovative ways to approach these barriers as appropriate?}

\section{Reviewer 1:}

The reviewer replied yes.

\section{Reviewer 2:}

The reviewer said yes, generally.

\section{Reviewer 3:}

The reviewer stated that the majority of the projects are novel and innovative, which is also driven by the feedback loop in the award process with industry. However, the reviewer suggested that some of the projects however could use more input from the OEM's perspective, e.g., considering the volumetric capacity of novel Si compounds or the costs basis of manufacturing new materials.

\section{Reviewer 4:}

The reviewer indicated that the program does identify the critical research areas of battery technology and linked them together to meet the program target but pointed out that is difficult to comment whether each project adopts innovative ways to approach the barriers due to limited information shared at the meeting.

\section{Question 10: Has the program area engaged appropriate partners?}

\section{Reviewer 1:}

The reviewer answered yes to this question, noting it is possible to see that many universities, industrial members, and national laboratories are engaged in this program.

\section{Reviewer 2:}

The reviewer replied yes.

\section{Reviewer 3:}

The reviewer replied more or less, noting that the overall team includes the broad majority of key players in North America and is well-based to establish a supply-chain network for EVs in the United States. The reviewer suggested to increase the broadness of recipients towards start-ups and shorten the duration of the awards, remarking that this would increase the number of recipients and provide a faster reality check to new companies and provide early guidance.

\section{Reviewer 4:}

The reviewer stated that the program projects generally provide good collaboration with useful partners within the United States. However, the reviewer observed that almost completely, the program projects fail to involve any of the significant global battery manufacturers. While this is most generally due to limitations imposed by DOE and U.S. government, the reviewer warned that this limits the impact that the program can have on the industry in general and on the impact the program can have on U.S. industry specifically

\section{Question 11: Is the program area collaborating with them effectively?}




\section{Reviewer 1:}

The reviewer said yes, commenting that once you are in the program the interaction with the partners and the funding agencies is good.

\section{Reviewer 2:}

The reviewer replied yes.

\section{Reviewer 3:}

This reviewer thinks and hopes so.

\section{Reviewer 4:}

The reviewer voiced that the program area is very effective in collaboration within the United States, but that it is perhaps by design generally ineffective in collaboration with global partners of importance.

\section{Question 12: Are there any gaps in the portfolio for this technology area?}

\section{Reviewer 1:}

The reviewer stated that there are no major gaps in the program portfolio.

\section{Reviewer 2:}

The reviewer said no.

\section{Reviewer 3:}

The reviewer indicated a desire to see some battery performance test data while making progress at different research sub-levels.

\section{Reviewer 4:}

The reviewer remarked that some limited but clear effort in technologies beyond Li would be useful within VTO, even if this is already covered at some level within DOE external to VTO.

\section{Question 13: Are there topics that are not being adequately addressed?}

\section{Reviewer 1:}

The reviewer replied yes.

\section{Reviewer 2:}

The reviewer remarked that as an overview of the program, it is difficult to cover every aspect of each topic but that, in general, the overview is very well organized and presented.

\section{Reviewer 3:}

The reviewer wondered if VTO is maybe too focused on the core topics and whether some projects should also address potential mid-term candidates which are not present in the current landscape. Additionally, the reviewer observed, the question of the viability of Li-metal-sulfur batteries for EV applications is not solved. To mitigate the risk here, the reviewer suggested that the program could fund specific projects that advance particular key technologies which could enable Li-metal-sulfur batteries later (e.g., a stronger focus on protecting Li-metal or strengthen the efforts in the development of solid-state electrolytes [SSEs]).

\section{Question 14: Are there other areas that this program area should consider funding to meet overall programmatic goals?}

\section{Reviewer 1:}

The reviewer praised the program as very comprehensive and covering almost all critical aspects of the battery technology.

Reviewer 2:

The reviewer said no 


\section{Reviewer 3:}

The reviewer commented that the requirements and challenges described by the VTO already cover most of the interesting areas but suggested potential additions could include fast charging and cold temperature performance or pack architectures enabling cold climate operation. The reviewer noted that most of the projects are strongly focused on the VTO roadmap and that perhaps a certain percentage of the projects should be awarded to potential competitors, i.e., to have a good overview in the project and to strengthen the completion between the technologies. The reviewer remarked that a stronger connection between cost calculation and materials project would also help to reach the program's goal.

\section{Question 15: Can you recommend new ways to approach the barriers addressed by this program area?}

\section{Reviewer 1:}

The reviewer characterized the program as going well and making progress continuously and had no new ways to recommend.

\section{Reviewer 2:}

The reviewer replied no to this question.

\section{Reviewer 3:}

The reviewer praised the overall project landscape and approach as very good. The reviewer offered one way to improve on it would be to implement a very competitive sub-program with strict go/no-go decisions in which award recipients have only a short amount of time to prove their technology, but then receive a full project after a strict elimination process.

\section{Question 16: Are there any other suggestions to improve the effectiveness of this program area?}

\section{Reviewer 1:}

The reviewer judged that overall, the VTO is in a good position and covers its topics very well. On the task to establish a supply-chain for EVs in the United States, the reviewer suggested that the program might have to find a way to work and strengthen their interaction with partners who are not headquartered in the United States, but have major activities in North America (i.e., cell manufactures and component suppliers).

\section{Reviewer 2:}

The reviewer recommended some level of direct engagement with the global battery industry.

\section{Reviewer 3:}

The reviewer claimed that only future needs of fast charging will be needed to make battery electric vehicles more acceptable.

\section{Reviewer 4:}

The reviewer suggested that for each project, listing the main contribution of each team member might help to improve the effectiveness of the program. 


\section{Project Feedback}

In this merit review activity, each reviewer was asked to respond to a series of questions, involving multiple-choice responses, expository responses where text comments were requested, and numeric score responses (on a scale of 1.0 to 4.0). In the pages that follow, the reviewer responses to each question for each project will be summarized: the multiple choice and numeric score questions will be presented in graph form for each project, and the expository text responses will be summarized in paragraph form for each question. A table presenting the average numeric score for each question for each project is presented below.

Table 2-1 - Project Feedback

\begin{tabular}{|c|c|c|c|c|c|c|c|}
\hline Presentation Title & $\begin{array}{c}\text { Principal } \\
\text { Investigator } \\
\text { and } \\
\text { Organization }\end{array}$ & $\begin{array}{l}\text { Page } \\
\text { Number }\end{array}$ & Approach & $\begin{array}{c}\text { Technical } \\
\text { Accomplishments }\end{array}$ & Collaborations & $\begin{array}{l}\text { Future } \\
\text { Research }\end{array}$ & $\begin{array}{l}\text { Weighted } \\
\text { Average }\end{array}$ \\
\hline $\begin{array}{c}\text { Materials } \\
\text { Benchmarking } \\
\text { Activities For } \\
\text { CAMP Facility }\end{array}$ & $\begin{array}{l}\text { Lu, Wenquan } \\
\text { (ANL) }\end{array}$ & 2-19 & 3.20 & 3.40 & 3.60 & 3.30 & 3.36 \\
\hline $\begin{array}{l}\text { Cell Analysis, } \\
\text { Modeling, and } \\
\text { Prototyping } \\
\text { (CAMP) Facility } \\
\text { Research } \\
\text { Activities }\end{array}$ & $\begin{array}{c}\text { Jansen, } \\
\text { Andrew (ANL) }\end{array}$ & $2-22$ & 3.60 & 3.30 & 3.50 & 3.40 & 3.41 \\
\hline $\begin{array}{l}\text { Overview and } \\
\text { Progress of } \\
\text { United States } \\
\text { Advanced Battery } \\
\text { Consortium } \\
\text { (USABC) Activity }\end{array}$ & $\begin{array}{l}\text { Elder, Ron } \\
\text { (USABC) }\end{array}$ & $2-25$ & 3.33 & 3.17 & 3.42 & 3.08 & 3.23 \\
\hline $\begin{array}{l}\text { Thick Low-Cost, } \\
\text { High-Power } \\
\text { Lithium-Ion } \\
\text { Electrodes } \\
\text { via Aqueous } \\
\text { Processing }\end{array}$ & $\begin{array}{l}\text { Li, Jianlin } \\
\text { (ORNL) }\end{array}$ & $2-29$ & 3.25 & 3.13 & 3.75 & 3.25 & 3.25 \\
\hline $\begin{array}{l}\text { Performance } \\
\text { Effects of } \\
\text { Electrode Coating } \\
\text { Defects and IR } \\
\text { Thermography } \\
\text { NDE for High- } \\
\text { Energy Lithium- } \\
\text { Ion Batteries }\end{array}$ & $\begin{array}{l}\text { Wood, David } \\
\text { (ORNL) }\end{array}$ & $2-31$ & 3.17 & 3.00 & 3.33 & 3.17 & 3.10 \\
\hline $\begin{array}{l}\text { Post-Test Analysis } \\
\text { of Lithium- } \\
\text { Ion Battery } \\
\text { Materials at } \\
\text { Argonne National } \\
\text { Laboratory }\end{array}$ & $\begin{array}{l}\text { Bloom, Ira } \\
\text { (ANL) }\end{array}$ & $2-33$ & 3.00 & 3.13 & 3.38 & 3.25 & 3.14 \\
\hline
\end{tabular}




\begin{tabular}{|c|c|c|c|c|c|c|c|}
\hline Presentation Title & $\begin{array}{c}\text { Principal } \\
\text { Investigator } \\
\text { and } \\
\text { Organization }\end{array}$ & $\begin{array}{c}\text { Page } \\
\text { Number }\end{array}$ & Approach & $\begin{array}{c}\text { Technical } \\
\text { Accomplishments }\end{array}$ & Collaborations & $\begin{array}{c}\text { Future } \\
\text { Research }\end{array}$ & $\begin{array}{c}\text { Weighted } \\
\text { Average }\end{array}$ \\
\hline $\begin{array}{c}\text { Process } \\
\text { Development } \\
\text { and Scale-Up of } \\
\text { Advanced Active } \\
\text { Battery Materials }\end{array}$ & $\begin{array}{l}\text { Krumdick, } \\
\text { Greg (ANL) }\end{array}$ & $2-36$ & 3.50 & 3.13 & 3.50 & 3.25 & 3.28 \\
\hline $\begin{array}{c}\text { Process } \\
\text { Development } \\
\text { and Scale-Up of } \\
\text { Critical Battery } \\
\text { Materials }\end{array}$ & $\begin{array}{l}\text { Krumdick, } \\
\text { Greg (ANL) }\end{array}$ & $2-38$ & 3.50 & 3.50 & 3.63 & 3.25 & 3.48 \\
\hline $\begin{array}{l}\text { Electrochemical } \\
\text { Performance } \\
\text { Testing }^{+}\end{array}$ & $\begin{array}{l}\text { Bloom, Ira } \\
\text { (ANL) }\end{array}$ & $2-40$ & 3.50 & 3.33 & 3.83 & 3.17 & 3.42 \\
\hline $\begin{array}{c}\text { INL } \\
\text { Electrochemical } \\
\text { Performance } \\
\text { Testing }^{+}\end{array}$ & $\begin{array}{l}\text { Shirk, Matt } \\
\text { (INL) }\end{array}$ & $2-42$ & 3.33 & 3.33 & 3.50 & 3.33 & 3.35 \\
\hline $\begin{array}{c}\text { Battery Safety } \\
\text { Testing }^{+}\end{array}$ & $\begin{array}{c}\text { Steele, Leigh } \\
\text { Anna (SNL) }\end{array}$ & $2-44$ & 3.50 & 3.38 & 3.63 & 3.38 & 3.44 \\
\hline $\begin{array}{l}\text { Battery Thermal } \\
\text { Characterization }\end{array}$ & $\begin{array}{l}\text { Keyser, } \\
\text { Matthew } \\
\text { (NREL) }\end{array}$ & $2-47$ & 3.38 & 3.38 & 3.25 & 3.38 & 3.36 \\
\hline $\begin{array}{l}\text { Towards } \\
\text { Solventless } \\
\text { Processing of } \\
\text { Thick Electron- } \\
\text { Beam (EB) Cured } \\
\text { LIB Cathodes }\end{array}$ & $\begin{array}{l}\text { Wood, David } \\
\text { (ORNL) }\end{array}$ & $2-50$ & 3.38 & 3.38 & 3.25 & 3.25 & 3.34 \\
\hline $\begin{array}{l}\text { New High-Energy } \\
\text { Electrochemical } \\
\text { Couple for } \\
\text { Automotive } \\
\text { Applications }\end{array}$ & $\begin{array}{l}\text { Amine, Khalil } \\
\text { (ANL) }\end{array}$ & 2-52 & 3.50 & 3.20 & 3.30 & 2.83 & 3.24 \\
\hline $\begin{array}{c}\text { High-Energy } \\
\text { High-Power } \\
\text { Battery Exceeding } \\
\text { PHEV-40 } \\
\text { Requirements }\end{array}$ & $\begin{array}{l}\text { Rempel, Jane } \\
\text { (TIAX) }\end{array}$ & $2-55$ & 2.88 & 2.75 & 2.75 & 2.50 & 2.75 \\
\hline $\begin{array}{c}\text { Advanced } \\
\text { High-Energy } \\
\text { Lithium-Ion Cell } \\
\text { for PHEV and EV } \\
\text { Applications }\end{array}$ & $\begin{array}{l}\text { Singh, Jagat } \\
\text { (3M) }\end{array}$ & $2-58$ & 3.25 & 2.75 & 3.50 & 2.88 & 2.98 \\
\hline
\end{tabular}




\begin{tabular}{|c|c|c|c|c|c|c|c|}
\hline Presentation Title & $\begin{array}{c}\text { Principal } \\
\text { Investigator } \\
\text { and } \\
\text { Organization }\end{array}$ & $\begin{array}{l}\text { Page } \\
\text { Number }\end{array}$ & Approach & $\begin{array}{c}\text { Technical } \\
\text { Accomplishments }\end{array}$ & Collaborations & $\begin{array}{l}\text { Future } \\
\text { Research }\end{array}$ & $\begin{array}{l}\text { Weighted } \\
\text { Average }\end{array}$ \\
\hline $\begin{array}{c}\text { High-Energy } \\
\text { Lithium Batteries } \\
\text { for PHEV } \\
\text { Applications }\end{array}$ & $\begin{array}{c}\text { Venkatachala, } \\
\text { Subramanian } \\
\text { (Envia } \\
\text { Systems) }\end{array}$ & $2-61$ & 3.30 & 2.90 & 3.40 & 2.33 & 2.99 \\
\hline $\begin{array}{l}\text { High-Energy, } \\
\text { Long Cycle Life } \\
\text { Lithium-Ion } \\
\text { Batteries for EV } \\
\text { Applications }\end{array}$ & $\begin{array}{c}\text { Wang, } \\
\text { Donghai (Penn } \\
\text { State) }\end{array}$ & $2-64$ & 3.50 & 3.25 & 3.25 & 3.08 & 3.29 \\
\hline $\begin{array}{c}\text { High-Energy } \\
\text { Density Lithium- } \\
\text { Ion Cells for EVs } \\
\text { Based on Novel, } \\
\text { High Voltage } \\
\text { Cathode Material } \\
\text { Systems }\end{array}$ & $\begin{array}{l}\text { Kepler, Keith } \\
\text { (Farasis) }\end{array}$ & $2-68$ & 3.08 & 2.75 & 3.42 & 2.50 & 2.89 \\
\hline $\begin{array}{l}\text { Fundamental } \\
\text { Studies of } \\
\text { Lithium-Sulfur } \\
\text { Cell Chemistry }{ }^{+}\end{array}$ & $\begin{array}{l}\text { Balsara, Nitash } \\
\text { (LBNL) }\end{array}$ & $2-71$ & 3.33 & 3.33 & 3.17 & 3.00 & 3.27 \\
\hline $\begin{array}{l}\text { BatPaC Model } \\
\text { Development }\end{array}$ & $\begin{array}{c}\text { Ahmed, } \\
\text { Shabbir (ANL) }\end{array}$ & $2-73$ & 3.70 & 3.50 & 3.40 & 3.40 & 3.53 \\
\hline $\begin{array}{l}\text { Design of Sulfur } \\
\text { Cathodes for } \\
\text { High-Energy } \\
\text { Lithium-Sulfur } \\
\text { Batteries }{ }^{+}\end{array}$ & $\begin{array}{c}\text { Cui, Yi } \\
\text { (Stanford } \\
\text { University) }\end{array}$ & $2-75$ & 3.63 & 3.63 & 3.38 & 3.63 & 3.59 \\
\hline $\begin{array}{c}\text { Efficient } \\
\text { Rechargeable } \\
\mathrm{Li}^{-} \mathrm{O}_{2} \text { Batteries } \\
\text { Utilizing Stable } \\
\text { Inorganic Molten } \\
\text { Salt Electrolytes }{ }^{\dagger}\end{array}$ & $\begin{array}{c}\text { Giordani, } \\
\text { Vincent (Liox) }\end{array}$ & $2-78$ & 3.13 & 3.25 & 3.13 & 3.13 & 3.19 \\
\hline $\begin{array}{l}\text { Low-Cost, } \\
\text { High-Energy } \\
\text { Si/Graphene } \\
\text { Anodes for Li-Ion } \\
\text { Batteries }^{+}\end{array}$ & $\begin{array}{l}\text { Colwell, John } \\
\text { (XG Sciences) }\end{array}$ & $2-81$ & 3.50 & 3.40 & 3.40 & 3.40 & 3.43 \\
\hline $\begin{array}{l}\text { Low-Cost, High- } \\
\text { Capacity Lithium- } \\
\text { lon Batteries } \\
\text { through Modified } \\
\text { Surface and } \\
\text { Microstructure }^{+}\end{array}$ & $\begin{array}{l}\text { Zhang, Pu } \\
\text { (Navitas } \\
\text { Systems) }\end{array}$ & $2-84$ & 3.50 & 3.10 & 3.20 & 3.20 & 3.23 \\
\hline
\end{tabular}




\begin{tabular}{|c|c|c|c|c|c|c|c|}
\hline Presentation Title & $\begin{array}{c}\text { Principal } \\
\text { Investigator } \\
\text { and } \\
\text { Organization }\end{array}$ & $\begin{array}{l}\text { Page } \\
\text { Number }\end{array}$ & Approach & $\begin{array}{c}\text { Technical } \\
\text { Accomplishments }\end{array}$ & Collaborations & $\begin{array}{l}\text { Future } \\
\text { Research }\end{array}$ & $\begin{array}{l}\text { Weighted } \\
\text { Average }\end{array}$ \\
\hline $\begin{array}{c}\text { Scale-Up of } \\
\text { Low-Cost } \\
\text { Encapsulation } \\
\text { Technologies for } \\
\text { High-Capacity } \\
\text { and High-Voltage } \\
\text { Electrode } \\
\text { Powders }\end{array}$ & $\begin{array}{l}\text { King, David } \\
\text { (Pneumaticoat } \\
\text { Technologies) }\end{array}$ & $2-86$ & 3.80 & 3.50 & 3.20 & 3.00 & 3.48 \\
\hline $\begin{array}{l}\text { High-Energy } \\
\text { Anode Material } \\
\text { Development for } \\
\text { Li-Ion Batteries }^{\dagger}\end{array}$ & $\begin{array}{l}\text { Hayner, Cary } \\
\text { (Sinode } \\
\text { Systems) }\end{array}$ & $2-89$ & 3.00 & 3.00 & 3.00 & 3.00 & 3.00 \\
\hline $\begin{array}{l}\text { Advanced High- } \\
\text { Performance } \\
\text { Batteries for } \\
\text { Electric Vehicle } \\
\text { (EV) Applications }{ }^{\dagger}\end{array}$ & $\begin{array}{l}\text { Stefan, lonel } \\
\text { (Amprius) }\end{array}$ & $2-92$ & 3.25 & 3.38 & 2.38 & 2.50 & 3.11 \\
\hline $\begin{array}{c}\text { A Disruptive } \\
\text { Concept for a } \\
\text { Whole Family } \\
\text { of New Battery } \\
\text { Systems }\end{array}$ & $\begin{array}{l}\text { Roumi, Farshid } \\
\text { (Parthian } \\
\text { Energy) }\end{array}$ & $2-95$ & 2.20 & 2.30 & 2.60 & 2.40 & 2.33 \\
\hline $\begin{array}{l}\text { Dramatically } \\
\text { Improve } \\
\text { the Safety } \\
\text { Performance } \\
\text { of Lithium- } \\
\text { Ion Battery } \\
\text { Separators and } \\
\text { Reduce the } \\
\text { Manufacturing } \\
\text { Cost Using } \\
\text { Ultraviolet } \\
\text { Curing and High- } \\
\text { Precision Coating } \\
\text { Technologies }\end{array}$ & $\begin{array}{l}\text { Arnold, John } \\
\text { (Miltec UV } \\
\text { International) }\end{array}$ & 2-99 & 2.50 & 2.30 & 2.60 & 2.20 & 2.38 \\
\hline $\begin{array}{l}\text { Low-Cost, High- } \\
\text { Capacity Non- } \\
\text { Intercalation } \\
\text { Chemistry } \\
\text { Automotive Cells }\end{array}$ & $\begin{array}{l}\text { Jacobs, Alex } \\
\text { (Sila Nano- } \\
\text { Technologies) }\end{array}$ & $2-103$ & 3.08 & 3.33 & 3.25 & 3.08 & 3.23 \\
\hline $\begin{array}{l}\text { Low-Cost, } \\
\text { Structurally } \\
\text { Advanced Novel } \\
\text { Electrode and Cell } \\
\text { Manufacturing }\end{array}$ & $\begin{array}{c}\text { Woodford, } \\
\text { Billy (24M } \\
\text { Technologies) }\end{array}$ & $2-107$ & 2.70 & 2.80 & 2.20 & 2.60 & 2.68 \\
\hline
\end{tabular}




\begin{tabular}{|c|c|c|c|c|c|c|c|}
\hline Presentation Title & $\begin{array}{c}\text { Principal } \\
\text { Investigator } \\
\text { and } \\
\text { Organization }\end{array}$ & $\begin{array}{l}\text { Page } \\
\text { Number }\end{array}$ & Approach & $\begin{array}{c}\text { Technical } \\
\text { Accomplishments }\end{array}$ & Collaborations & $\begin{array}{l}\text { Future } \\
\text { Research }\end{array}$ & $\begin{array}{c}\text { Weighted } \\
\text { Average }\end{array}$ \\
\hline $\begin{array}{l}\text { Advanced Drying } \\
\text { Process for Lower } \\
\text { Manufacturing } \\
\text { Cost of Electrodes }\end{array}$ & $\begin{array}{c}\text { Ahmad, } \\
\text { Iftikhar } \\
\text { (Lambda } \\
\text { Technologies) }\end{array}$ & $2-111$ & 3.30 & 3.20 & 3.20 & 3.20 & 3.23 \\
\hline $\begin{array}{l}\text { High-Energy } \\
\text { Lithium Batteries } \\
\text { for Electric } \\
\text { Vehicles }^{\dagger}\end{array}$ & $\begin{array}{c}\text { Lopez, } \\
\text { Herman (Envia } \\
\text { Systems) }\end{array}$ & 2-115 & 3.20 & 3.30 & 3.40 & 2.50 & 3.19 \\
\hline $\begin{array}{l}\text { A 12V Start-Stop } \\
\text { Li Polymer Battery } \\
\text { Pack }^{+}\end{array}$ & $\begin{array}{c}\text { Alamgir, } \\
\text { Mohamed (LG } \\
\text { Chem Power) }\end{array}$ & 2-119 & 3.00 & 2.25 & 2.75 & 2.13 & 2.48 \\
\hline $\begin{array}{l}\text { A Commercially } \\
\text { Scalable Process } \\
\text { for Silicon Anode } \\
\text { Pre-lithiation }\end{array}$ & $\begin{array}{l}\text { Stefan, lonel } \\
\text { (Amprius) }\end{array}$ & $2-123$ & 3.42 & 3.33 & 1.92 & 3.00 & 3.14 \\
\hline $\begin{array}{l}\text { Development of } \\
\text { Advanced High- } \\
\text { Performance } \\
\text { Batteries for } 12 \mathrm{~V} \\
\text { Start-Stop Vehicle } \\
\text { Applications }^{\dagger}\end{array}$ & $\begin{array}{l}\text { Kim, Jeff } \\
\text { (Maxwell) }\end{array}$ & $2-127$ & 3.25 & 3.25 & 2.88 & 3.50 & 3.23 \\
\hline $\begin{array}{l}\text { Enabling } \\
\text { High-Energy, } \\
\text { High-Voltage } \\
\text { Li-Ion Cells for } \\
\text { Transportation } \\
\text { Applications: } \\
\text { Modeling and } \\
\text { Analysis }\end{array}$ & $\begin{array}{l}\text { Dees, Dennis } \\
\text { (ANL) }\end{array}$ & $2-130$ & 3.50 & 3.50 & 3.33 & 3.00 & 3.42 \\
\hline $\begin{array}{l}\text { Enabling } \\
\text { High-Energy, } \\
\text { High-Voltage } \\
\text { Li-Ion Cells for } \\
\text { Transportation } \\
\text { Applications: } \\
\text { Project Overview }\end{array}$ & $\begin{array}{l}\text { Dees, Dennis } \\
\text { (ANL) }\end{array}$ & $2-132$ & 3.63 & 3.25 & 3.38 & 3.25 & 3.36 \\
\hline $\begin{array}{c}\text { Enabling } \\
\text { High-Energy, } \\
\text { High-Voltage } \\
\text { Li-lon Cells for } \\
\text { Transportation } \\
\text { Applications: } \\
\text { Materials } \\
\text { Characterization }\end{array}$ & $\begin{array}{l}\text { Dees, Dennis } \\
\text { (ANL) }\end{array}$ & $2-134$ & 3.38 & 3.38 & 3.50 & 3.25 & 3.38 \\
\hline $\begin{array}{c}\text { Next-Generation } \\
\text { Anodes for } \\
\text { Lithium-Ion } \\
\text { Batteries: } \\
\text { Overview }\end{array}$ & $\begin{array}{l}\text { Dees, Dennis } \\
\text { (ANL) }\end{array}$ & $2-136$ & 3.50 & 3.38 & 3.50 & 3.38 & 3.42 \\
\hline
\end{tabular}




\begin{tabular}{|c|c|c|c|c|c|c|c|}
\hline Presentation Title & $\begin{array}{c}\text { Principal } \\
\text { Investigator } \\
\text { and } \\
\text { Organization }\end{array}$ & $\begin{array}{l}\text { Page } \\
\text { Number }\end{array}$ & Approach & $\begin{array}{c}\text { Technical } \\
\text { Accomplishments }\end{array}$ & Collaborations & $\begin{array}{l}\text { Future } \\
\text { Research }\end{array}$ & $\begin{array}{l}\text { Weighted } \\
\text { Average }\end{array}$ \\
\hline $\begin{array}{l}\text { Next-Generation } \\
\text { Anodes for Li- } \\
\text { Ion Batteries: } \\
\text { Fundamental } \\
\text { Studies of Si-C } \\
\text { Model Systems }\end{array}$ & $\begin{array}{c}\text { Kostecki, } \\
\text { Robert (LBNL) }\end{array}$ & $2-138$ & 3.50 & 3.13 & 3.25 & 3.38 & 3.27 \\
\hline $\begin{array}{l}\text { Electrodeposition } \\
\text { for Low-Cost, } \\
\text { Water-Based } \\
\text { Electrode } \\
\text { Manufacturing }\end{array}$ & $\begin{array}{c}\text { Hellring, } \\
\text { Stuart (PPG) }\end{array}$ & $2-140$ & 3.10 & 2.90 & 2.80 & 3.10 & 2.96 \\
\hline $\begin{array}{l}\text { Li-Ion Battery } \\
\text { Anodes from } \\
\text { Electrospun } \\
\text { Nanoparticle/ } \\
\text { Conducting } \\
\text { Polymer } \\
\text { Nanofibers }\end{array}$ & $\begin{array}{l}\text { Pintauro, Peter } \\
\text { (Vanderbilt) }\end{array}$ & $2-143$ & 3.00 & 3.00 & 3.25 & 3.00 & 3.03 \\
\hline $\begin{array}{l}\text { UV Curable Binder } \\
\text { Technology } \\
\text { to Reduce } \\
\text { Manufacturing } \\
\text { Cost and Improve } \\
\text { Performance of } \\
\text { LIB Electrodes }\end{array}$ & $\begin{array}{l}\text { Arnold, John } \\
\text { (Miltec UV } \\
\text { International) }\end{array}$ & $2-147$ & 3.25 & 2.83 & 2.83 & 2.75 & 2.93 \\
\hline $\begin{array}{l}\text { Co-Extrusion } \\
\text { (CoEx) for } \\
\text { Cost Reduction } \\
\text { of Advanced } \\
\text { High-Energy- } \\
\text { and-Power } \\
\text { Battery Electrode } \\
\text { Manufacturing }\end{array}$ & $\begin{array}{l}\text { Cobb, Corie } \\
\text { (PARC) }\end{array}$ & $2-151$ & 2.70 & 3.00 & 3.10 & 3.10 & 2.95 \\
\hline $\begin{array}{l}\text { Commercially } \\
\text { Scalable Process } \\
\text { to Fabricate } \\
\text { Porous Silicon }\end{array}$ & $\begin{array}{c}\text { Aurora, Peter } \\
\text { (Navitas } \\
\text { Systems) }\end{array}$ & $2-155$ & 3.29 & 3.14 & 3.29 & 3.21 & 3.21 \\
\hline $\begin{array}{l}\text { Low-Cost } \\
\text { Manufacturing of } \\
\text { Advanced Silicon- } \\
\text { Based Anode } \\
\text { Materials }\end{array}$ & $\begin{array}{l}\text { Feaver, Aaron } \\
\text { (Group14) }\end{array}$ & $2-160$ & 2.64 & 2.79 & 2.93 & 2.86 & 2.78 \\
\hline $\begin{array}{l}\text { An Integrated } \\
\text { Flame Spray } \\
\text { Process for Low- } \\
\text { Cost Production of } \\
\text { Battery Materials }\end{array}$ & $\begin{array}{l}\text { Xing, Chad } \\
\text { (University of } \\
\text { Missouri) }\end{array}$ & $2-165$ & 2.42 & 2.75 & 2.75 & 2.92 & 2.69 \\
\hline
\end{tabular}




\begin{tabular}{|c|c|c|c|c|c|c|c|}
\hline Presentation Title & $\begin{array}{c}\text { Principal } \\
\text { Investigator } \\
\text { and } \\
\text { Organization }\end{array}$ & $\begin{array}{l}\text { Page } \\
\text { Number }\end{array}$ & Approach & $\begin{array}{c}\text { Technical } \\
\text { Accomplishments }\end{array}$ & Collaborations & $\begin{array}{l}\text { Future } \\
\text { Research }\end{array}$ & $\begin{array}{c}\text { Weighted } \\
\text { Average }\end{array}$ \\
\hline $\begin{array}{l}\text { New Advanced } \\
\text { Stable } \\
\text { Electrolytes for } \\
\text { High-Voltage } \\
\text { Electrochemical } \\
\text { Energy Storage }\end{array}$ & $\begin{array}{l}\text { Du, Peng } \\
\text { (Silatronix) }\end{array}$ & 2-169 & 3.20 & 3.20 & 3.20 & 3.20 & 3.20 \\
\hline $\begin{array}{l}\text { Pre-Lithiation } \\
\text { of Battery } \\
\text { Electrodes }^{\dagger}\end{array}$ & $\begin{array}{c}\text { Cui, Yi } \\
\text { (Stanford } \\
\text { University) }\end{array}$ & $2-173$ & 3.33 & 3.33 & 2.83 & 3.17 & 3.25 \\
\hline $\begin{array}{l}\text { New Lamination } \\
\text { and Doping } \\
\text { Concepts for } \\
\text { Enhanced } \\
\text { Li-S Battery } \\
\text { Performance }^{+}\end{array}$ & $\begin{array}{c}\text { Kumta, } \\
\text { Prashant (U. of } \\
\text { Pittsburgh) }\end{array}$ & 2-175 & 3.50 & 3.38 & 3.13 & 3.38 & 3.38 \\
\hline $\begin{array}{l}\text { Novel Chemistry: } \\
\text { Lithium Selenium } \\
\text { and Selenium } \\
\text { Sulfur Couple }^{\dagger}\end{array}$ & $\begin{array}{l}\text { Amine, Khalil } \\
\text { (ANL) }\end{array}$ & $2-178$ & 3.38 & 3.13 & 3.38 & 3.13 & 3.22 \\
\hline $\begin{array}{l}\text { Multi-Functional } \\
\text { Cathode Additives } \\
\text { for Li-S Battery } \\
\text { Technology }\end{array}$ & $\begin{array}{l}\text { Gan, Hong } \\
\text { (BNL) }\end{array}$ & 2-180 & 3.17 & 3.00 & 3.33 & 3.50 & 3.15 \\
\hline $\begin{array}{l}\text { Development } \\
\text { of High-Energy } \\
\text { Lithium-Sulfur } \\
\text { Batteries }^{\dagger}\end{array}$ & $\begin{array}{l}\text { Liu, Jun } \\
\text { (PNNL) }\end{array}$ & $2-183$ & 3.50 & 3.38 & 3.63 & 3.38 & 3.44 \\
\hline $\begin{array}{l}\text { Addressing } \\
\text { Internal } \\
\text { "Shuttle" Effect: } \\
\text { Electrolyte Design } \\
\text { and Cathode } \\
\text { Morphology } \\
\text { Evolution in Li-S } \\
\text { Batteries }^{\dagger}\end{array}$ & $\begin{array}{c}\text { Balbuena, } \\
\text { Perla (Texas } \\
\text { A\&M) }\end{array}$ & $2-186$ & 3.38 & 3.25 & 2.88 & 3.00 & 3.20 \\
\hline $\begin{array}{l}\text { Statically and } \\
\text { Dynamically } \\
\text { Stable Lithium- } \\
\text { Sulfur Batteries }\end{array}$ & $\begin{array}{l}\text { Manthiram, } \\
\text { Arumugam } \\
\text { (U of Texas at } \\
\text { Austin) }\end{array}$ & $2-188$ & 3.25 & 3.38 & 3.25 & 3.13 & 3.30 \\
\hline $\begin{array}{c}\text { Mechanistic } \\
\text { Investigation for } \\
\text { the Rechargeable } \\
\text { Li-Sulfur } \\
\text { Batteries }^{\dagger}\end{array}$ & $\begin{array}{c}\text { Qu, Deyang (U. } \\
\text { of Wisconsin - } \\
\text { Madison) }\end{array}$ & 2-191 & 3.63 & 3.50 & 3.63 & 3.25 & 3.52 \\
\hline
\end{tabular}




\begin{tabular}{|c|c|c|c|c|c|c|c|}
\hline Presentation Title & $\begin{array}{c}\text { Principal } \\
\text { Investigator } \\
\text { and } \\
\text { Organization }\end{array}$ & $\begin{array}{l}\text { Page } \\
\text { Number }\end{array}$ & Approach & $\begin{array}{c}\text { Technical } \\
\text { Accomplishments }\end{array}$ & Collaborations & $\begin{array}{l}\text { Future } \\
\text { Research }\end{array}$ & $\begin{array}{c}\text { Weighted } \\
\text { Average }\end{array}$ \\
\hline $\begin{array}{l}\text { Development of } \\
\text { Novel Electrolytes } \\
\text { and Catalysts } \\
\text { for Lithium-Air } \\
\text { Batteries }^{+}\end{array}$ & $\begin{array}{l}\text { Amine, Khalil } \\
\text { (ANL) }\end{array}$ & $2-193$ & 3.38 & 3.50 & 3.00 & 3.00 & 3.34 \\
\hline $\begin{array}{c}\text { Exploratory } \\
\text { Studies of Novel } \\
\text { Sodium-Ion } \\
\text { Battery Systems }\end{array}$ & $\begin{array}{l}\text { Yang, Xiao- } \\
\text { Qing (BNL) }\end{array}$ & 2-195 & 3.33 & 3.33 & 3.33 & 3.00 & 3.29 \\
\hline $\begin{array}{c}\text { Construction } \\
\text { of High Energy } \\
\text { Density Batteries }\end{array}$ & $\begin{array}{c}\text { Lang, } \\
\text { Christopher } \\
\text { (Physical } \\
\text { Sciences Inc.) }\end{array}$ & $2-197$ & 2.80 & 2.80 & 3.10 & 2.90 & 2.85 \\
\hline $\begin{array}{c}\text { Advanced } \\
\text { Polyolefin } \\
\text { Separators for } \\
\text { Li-Ion Batteries } \\
\text { Used in Vehicle } \\
\text { Applications }^{\dagger}\end{array}$ & $\begin{array}{l}\text { Wood, Weston } \\
\text { (Entek) }\end{array}$ & $2-200$ & 3.33 & 3.00 & 3.00 & 3.17 & 3.10 \\
\hline $\begin{array}{c}\text { Hybrid } \\
\text { Electrolytes } \\
\text { for PHEV } \\
\text { Applications }\end{array}$ & $\begin{array}{c}\text { Moganty, } \\
\text { Surya (NOHMs } \\
\text { Technologies) }\end{array}$ & $2-202$ & 3.63 & 3.38 & 3.25 & 3.50 & 3.44 \\
\hline $\begin{array}{c}\text { SAFT-USABC 12V } \\
\text { Start-Stop Phase } \\
\mathrm{II}^{+}\end{array}$ & $\begin{array}{l}\text { O'Connor, Ian } \\
\text { (Saft) }\end{array}$ & $2-205$ & 3.25 & 3.13 & 3.13 & 2.88 & 3.13 \\
\hline $\begin{array}{l}\text { Development of } \\
\text { Advanced High- } \\
\text { Performance } \\
\text { Electrolytes } \\
\text { for Lithium-lon } \\
\text { Used in Vehicle } \\
\text { Applications }^{+}\end{array}$ & $\begin{array}{l}\text { Meyers, Kristin } \\
\text { (soulbrain) }\end{array}$ & $2-209$ & 2.40 & 2.50 & 3.20 & 2.60 & 2.58 \\
\hline $\begin{array}{l}\text { A Closed Loop } \\
\text { Process for the } \\
\text { End-of-Life } \\
\text { Electric Vehicle } \\
\text { Li-Ion Batteries }^{\dagger}\end{array}$ & $\begin{array}{l}\text { Wang, Yan } \\
\text { (WPI) }\end{array}$ & $2-212$ & 3.63 & 3.38 & 3.25 & 3.25 & 3.41 \\
\hline $\begin{array}{l}\text { Computer } \\
\text { Aided Battery } \\
\text { Engineering } \\
\text { Consortium }^{+}\end{array}$ & $\begin{array}{c}\text { Pesaran, } \\
\text { Ahmad (NREL) }\end{array}$ & $2-215$ & $3 . .67$ & 4.00 & 3.33 & 3.33 & 3.75 \\
\hline
\end{tabular}

+ Denotes a poster presentation 


\begin{tabular}{|c|c|c|c|c|c|c|c|}
\hline Presentation Title & $\begin{array}{c}\text { Principal } \\
\text { Investigator } \\
\text { and } \\
\text { Organization }\end{array}$ & $\begin{array}{l}\text { Page } \\
\text { Number }\end{array}$ & Approach & $\begin{array}{c}\text { Technical } \\
\text { Accomplishments }\end{array}$ & Collaborations & $\begin{array}{l}\text { Future } \\
\text { Research }\end{array}$ & $\begin{array}{l}\text { Weighted } \\
\text { Average }\end{array}$ \\
\hline $\begin{array}{l}\text { Consortium for } \\
\text { Advanced Battery } \\
\text { Simulation }^{+}\end{array}$ & $\begin{array}{l}\text { Turner, John } \\
\text { (ORNL) }\end{array}$ & $2-218$ & 3.33 & 3.50 & 3.50 & 3.50 & 3.46 \\
\hline $\begin{array}{l}\text { Development } \\
\text { and Validation } \\
\text { of a Simulation } \\
\text { Tool to Predict } \\
\text { the Combined } \\
\text { Structural, } \\
\text { Electrical, } \\
\text { Electrochemical, } \\
\text { and Thermal } \\
\text { Responses of } \\
\text { Automotive } \\
\text { Batteries }^{+}\end{array}$ & $\begin{array}{c}\text { Marcicki, } \\
\text { James (Ford) }\end{array}$ & $2-221$ & 3.67 & 3.50 & 3.33 & 3.17 & 3.48 \\
\hline Overall Average & & & 3.27 & 3.17 & 3.20 & 3.07 & 3.19 \\
\hline
\end{tabular}

+ Denotes a poster presentation 


\section{Materials Benchmarking Activities for CAMP Facility: Wenquan Lu (Argonne National Laboratory) - es028}

\section{Presenter}

Wenquan Lu, Argonne National Laboratory

\section{Reviewer Sample Size}

A total of five reviewers evaluated this project.

\section{Question 1: Approach to performing the work-the degree to which technical barriers are addressed, the project is well-designed, feasible, and integrated with other efforts.}

\section{Reviewer 1:}

The reviewer found that the Cell Analysis, Modeling, and Prototyping (CAMP) facility provides an excellent service to the community. The facility's organized information, consistent electrodes, and cycling protocols allow for others to enter the field with an expert's guidance.

\section{Reviewer 2:}

The reviewer said that the team's aim is to collaborate with material developers and leverage Argonne National Laboratory's (ANL) expertise in electrode design and cell testing to develop next-generation battery technology. The team has established standardized material testing protocols for evaluating samples from various developer for use in batteries for vehicles.

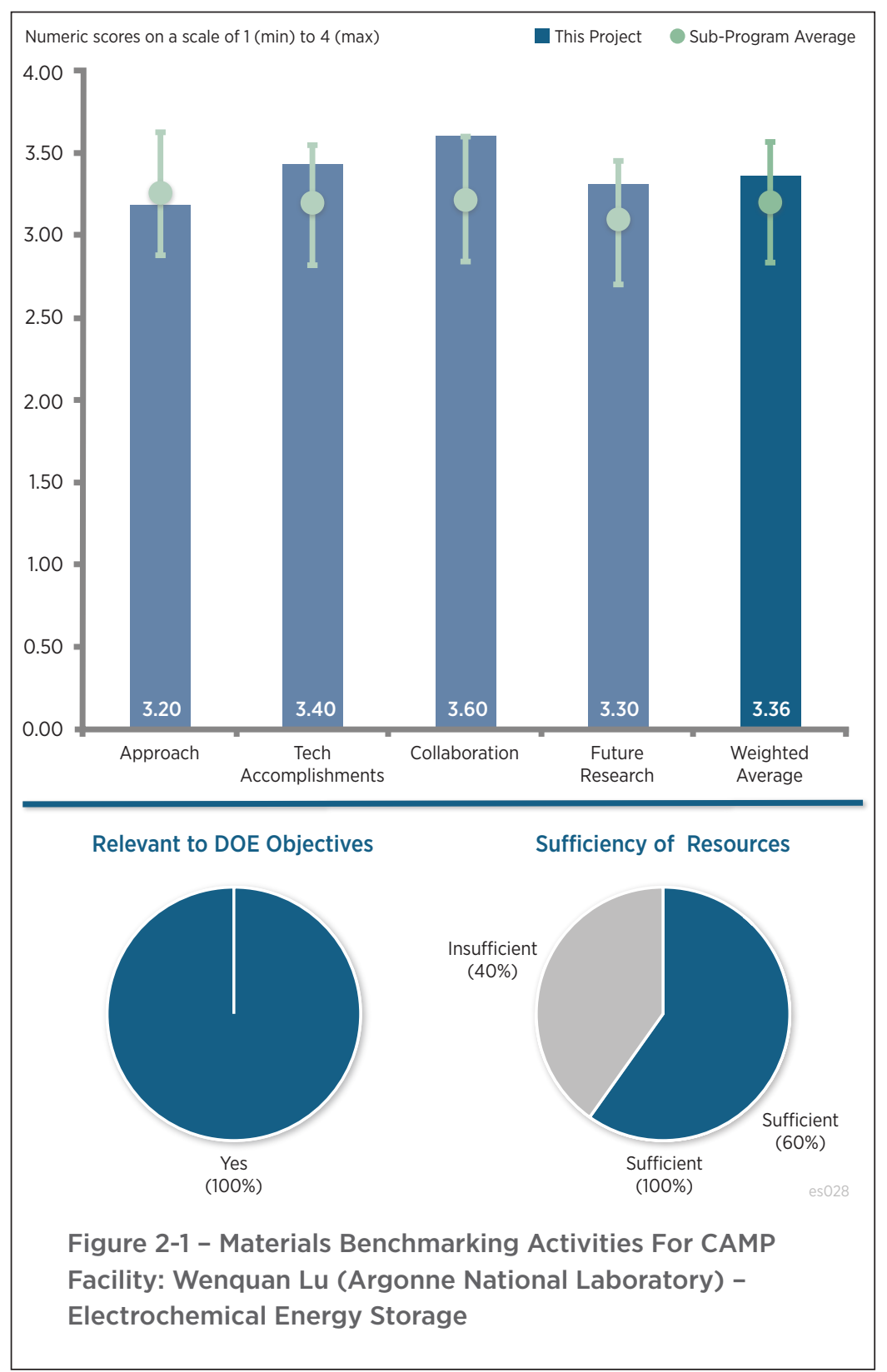

\section{Reviewer 3:}

The reviewer remarked that the presented work covered pre-lithiation, carbon additive, and nickel manganese cobalt oxide (NMC) material. All these are important issues in Li batteries. The reviewer suggested that the presentation next year further explain the relationship between this project and the general project on the CAMP facility. The project is apparently doing original battery work rather than benchmarking.

\section{Reviewer 4:}

The reviewer remarked that CAMP serves an important function in validating materials in a consistent protocol for fair evaluation and comparison. The evaluation of methods of pre-lithiation are good, we need this. However, the reviewer would not recommend putting a low-density Li source into a high-density cathode. The reviewer suggested putting it in the anode, which is already low density. If researchers are going to put it in the anode, the reviewer suggested considering the volume fraction of the Li source and its overall effect on volumetric energy density. 


\section{Reviewer 5:}

The reviewer said CAMP could be a bit tighter focus, particularly given the modest budget and duration. According to the reviewer, it was not as clear as it could be whether CAMP is a service role or discovery program.

\section{Question 2: Technical accomplishments and progress toward overall project and DOE goals-the degree to which progress has been made, measured against performance indicators and demonstrated progress towards DOE goals.}

\section{Reviewer 1:}

The team worked on a broad area of interest such as reduction of initial capacity loss (ICL), effect of conductive additive on power and life, as well as studies on nickel-cobalt-manganese (NCM). Some of the data are preliminary and we need additional data to assess the true potential of these ideas. The reviewer said that among all the projects, the use of $\mathrm{Li}$ iron oxide (LFO) to reduce ICL is quite interesting and could be promising. The reviewer remarked that given the adverse effect of iron $(\mathrm{Fe})$ in Li-ion batteries, it will be interesting to see full cell data especially at high temperature as well as storage data to examine its efficacy as a lithiation agent.

\section{Reviewer 2:}

The reviewer remarked that many materials have been made available for the community that show excellent performance in cycling.

\section{Reviewer 3:}

The reviewer said that despite a concern about focus, this program has been very productive for the level of support. The reviewer found that the pre-lithiation technologies, though early, are very compelling. The reviewer would direct more resources there and hence indicated that resources on this program were not sufficient. The reviewer suggested defining go/no-go criteria for these approaches, or for pre-lithiation in general—-this may fit with the stated benchmark mission.

\section{Reviewer 4:}

The reviewer commented that using hard carbon as a surrogate to study the issue of pre-lithiation through irreversible Li source in the cathode is interesting; it avoids the complications of using Si. The reviewer wondered that given that oxygen is generated during initial activation, what happens to the oxygen, does it oxidize the electrolyte. The reviewer also wondered about the microstructure of the cathode because the volume formerly occupied by the Li-rich oxides will now disappear. The reviewer suggested that the energy density of the cell made with hard carbon anode needs to be better described to include all cell components.

\section{Reviewer 5:}

The reviewer acknowledged having some difficulty judging this one. The reviewer knows that CAMP does a lot of work, but it is not well reflected in this set of slides. The reviewer noted that the focus was on a pre-lithiation source that has fundamental limitations, at the expense of all the other work the project team does. The reviewer acknowledged that the project included Cabot as a partner, but there are a lot of highly structured carbon blacks out there. The reviewer asked if the project team benchmark against other structured carbons, or just super P.

\section{Question 3: Collaboration and coordination with other institutions.}

\section{Reviewer 1:}

The reviewer remarked that the collaboration was extensive between the team and many internal and external labs.

\section{Reviewer 2:}

The reviewer said that the project has extensive interactions with both industrial and research institutions.

\section{Reviewer 3:}

The reviewer was glad to see that the project has an industrial collaborator. The reviewer asked if this collaborator does more than supply material.

\section{Reviewer 4:}

The reviewer was unclear how materials are selected for evaluation at CAMP. The reviewer inquired how decisions are made, are potential collaborators ever turned away, and if so, what percent and why. The reviewer inquired 
that if not, does this mean that the facility needs more advertisement for those outside of the core energy storage community.

Question 4: Proposed future research-the degree to which the project has effectively planned its future work in a logical manner by incorporating appropriate decision points, considering barriers to the realization of the proposed technology and, when sensible, mitigating risk by providing alternate development pathways.

\section{Reviewer 1:}

The reviewer said that CAMP will continue to be a gateway to next-generation battery materials and the work proposed fully reflects that.

\section{Reviewer 2:}

The reviewer commented that proposed work is a continuation of presented work and includes both benchmarking and original research on materials. The reviewer thinks that it would be help if the project mandate is better articulated in terms of the ratio between these two classes of activities.

\section{Reviewer 3:}

The reviewer suggested trying to be a bit clearer on the hypothesis (or hypotheses) and how opportunities are selected and prioritized.

\section{Question 5: Does this project support the overall DOE objectives of petroleum displacement? Why or why not?}

\section{Reviewer 1:}

The reviewer commented that this project supports DOE's overall objective of petroleum displacement by aiding in the development of low-cost and durable materials for vehicle applications.

\section{Reviewer 2:}

The reviewer remarked that this facility contributes to practical evaluation of materials for researchers not otherwise able to do so and is important for validation of new materials.

\section{Reviewer 3:}

The reviewer said yes, the project is to help with the transition of laboratory materials discovery into higher levels of readiness for commercial deployment. The reviewer commented that thorough understanding of the state of the art is also important for projecting performance improvement and cost reduction.

\section{Question 6: Resources: How sufficient are the resources for the project to achieve the stated milestones in a timely fashion?}

\section{Reviewer 1:}

The reviewer suggested placing more resources into a program like this, but changing the focus to evaluate more materials coming from outside ANL. This would solve all those press releases where companies claim to have solved all battery problems. The reviewer also equated CAMP to mythbusters.

\section{Reviewer 2:}

The reviewer hoped to see the effort and focus pick up on pre-lithiation. 


\section{Cell Analysis, Modeling, and Prototyping (CAMP) Facility Research Activities: Andrew Jansen (Argonne National Laboratory) - es030}

\section{Presenter}

Andrew Jansen, Argonne National Laboratory

\section{Reviewer Sample Size}

A total of five reviewers evaluated this project.

\section{Question 1: Approach to performing the work-the degree to which technical barriers are addressed, the project is well-designed, feasible, and integrated with other efforts.}

\section{Reviewer 1:}

The reviewer said that CAMP is certainly providing a platform to many material researchers who lack stateof-the-art electrode fabrication and testing facilities. The work is focused and well-planned, taking advantage of the ANL team's long experience in battery research.

\section{Reviewer 2:}

The reviewer commented that the project serves an important function for the research community to provide the early stage scale-up.

\section{Reviewer 3:}

The reviewer remarked that this facility is a great service to the energy storage community, including academic, industrial, and government institutions. The team is well qualified to run the facility.

\section{Reviewer 4:}

The reviewer thanked the project team because this was one of the few presentations the entire day where error bars were included on graphs. The reviewer appreciated the analysis of volumetric energy density fade, this is an interesting way to look at the results that the reviewer believes add value when thinking of a solution to the problem. The reviewer observed a careful, thoughtful approach to experimentation and data analysis. The comparison of pouch to coin cells is very important. The reviewer pointed out that researchers need to be able to use small format/easy to construct cells for early R\&D and be confident it will translate.

\section{Reviewer 5:}

The reviewer hoped that one or more pre-lithiation concepts graduates to CAMP and is not bogged down by intellectual property concerns.

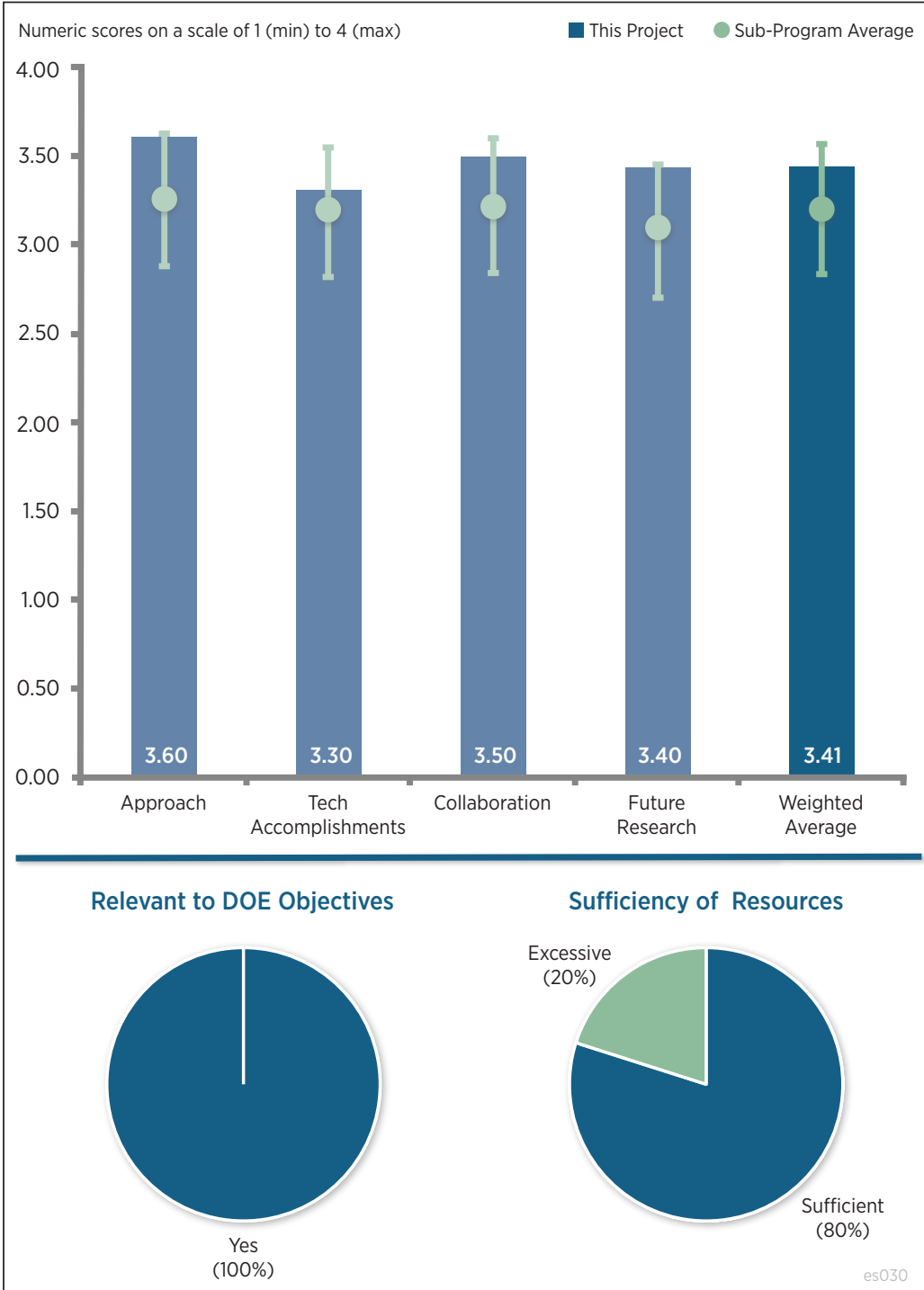

Figure 2-2 - Cell Analysis, Modeling, and Prototyping (CAMP) Facility Research Activities: Andrew Jansen (Argonne National Laboratory) - Electrochemical Energy Storage 
Question 2: Technical accomplishments and progress toward overall project and DOE goals-the degree to which progress has been made, measured against performance indicators and demonstrated progress towards DOE goals.

\section{Reviewer 1:}

The reviewer commented that the fabrication of matched electrodes for distribution to other laboratories is enabling, both for materials being available and including protocols for cycling performance and analysis.

\section{Reviewer 2:}

The reviewer said that the team has carried out high quality work, from designing electrodes to studying Si anodes extensively for use in EVs as well as providing electrodes to various partnering organizations who themselves are trying to develop next generation cell technologies. The work on Si has been significant and thorough. The reviewer noted that of course there has not been any breakthrough result as yet, but this is a challenging task around the globe and studies carried out at CAMP will certainly help in better understanding the factors that will help us in developing a robust $\mathrm{Si}$ electrode.

\section{Reviewer 3:}

The reviewer commented clearly a lot of work by a very competent team, and guessed that each separate effort could have filled a presentation.

\section{Reviewer 4:}

The reviewer noted that the facility has supplied materials and electrodes to many projects at ANL and beyond. The reviewer expressed concern that little was discussed with respect to quality control of the fabrication process. For these scale-up to be impactful, the performance of the tapes and cells have to be truly state of the art. This is very hard to do given the rapidly maturing manufacturing technologies. The reviewer asked how much benchmarking has the project done as compared to commercial products, and would like to know what the details of the quality control are.

\section{Question 3: Collaboration and coordination with other institutions.}

\section{Reviewer 1:}

The reviewer said that collaboration with other institutions is impressive. Many groups are involved and benefit from the work done at the CAMP facility.

\section{Reviewer 2:}

The reviewer said that the list of collaborating teams is quite extensive and impressive, spanning new and wellknown material suppliers and coating companies, some of whom are entering the battery field for the first time. The reviewer was reassured to learn that these companies have access to very standardized fabrication and test protocols.

\section{Reviewer 3:}

The reviewer remarked that the facility is well integrated with the research community in academia and national laboratories. The reviewer pointed out that the difficulty in working with industrial leaders is understandable, but the team needs to be creative to address the issue.

\section{Reviewer 4:}

The reviewer remarked pretty impressive, and would be interested on guidance based on successes and failures with regard to what problems/projects are likely to have impact and be well received under the open source constraints.

\section{Question 4: Proposed future research-the degree to which the project has effectively planned its future work in a logical manner by incorporating appropriate decision points, considering barriers to the realization of the proposed technology and, when sensible, mitigating risk by providing alternate development pathways.}

\section{Reviewer 1:}

The reviewer remarked that the list of proposed work is quite comprehensive, and the work on Si and other 
next-generation anode and high energy cathodes with the max impact will be most interesting to follow in the future.

\section{Reviewer 2:}

The reviewer wondered if this may be the right facility to look at formation process optimization and whether all the fuss over electrolyte additives and formation conditions is making a difference.

\section{Reviewer 3:}

The reviewer encouraged the team to come up with creative solutions to access technologies from industrial leaders. The reviewer said that it is very challenging but has to be done.

\section{Question 5: Does this project support the overall DOE objectives of petroleum displacement? Why or why not?}

\section{Reviewer 1:}

The reviewer said that enabling intermediate-format testing of materials in non-flooded cell environments allows for a more realistic evaluation of new materials for many groups who would not otherwise have the resources to do so. This project, in this reviewer's opinion, is of the utmost importance for continued support.

\section{Reviewer 2:}

The reviewer said that the project supports DOE objectives of petroleum displacement. The CAMP offers a platform to many researchers for scaling up new battery materials and independently testing at the cell level.

\section{Reviewer 3:}

The reviewer said that the facility is an integral part of maturing technologies in the battery space.

\section{Reviewer 4:}

The reviewer commented that this program includes material development and techniques that could span many other efforts in the development of new materials for high-energy density batteries

\section{Question 6: Resources: How sufficient are the resources for the project to achieve the stated milestones in a timely fashion?}

\section{Reviewer 1:}

The reviewer said that the funds seems to on the high side considering the fact that CAMP had done a lot of work for outside partners who the reviewer is sure paid for their own work. 
Overview and Progress of

United States Advanced

Battery Consortium (USABC)

Activity: Ron Elder (United

States Advanced Battery

Consortium) - es097

\section{Presenter}

Ron Elder, United States Advanced

Battery Consortium

\section{Reviewer Sample Size}

A total of six reviewers evaluated this project.

Question 1: Approach to performing the work-the degree to which technical barriers are addressed, the project is well-designed, feasible, and integrated with other efforts.

\section{Reviewer 1:}

The reviewer said that the United States Advanced Battery Consortium (USABC) with DOE's full support is undoubtedly taking the leading role in advancing the state-of-the-art in automotive battery technology.

\section{Reviewer 2:}

The reviewer commented that USABC has been very productive in fostering domestic battery technology development. The reviewer would like to know more about its plan on working differently with startups versus established businesses. Startups face significant financial and schedule challenges and could use every bit of help they can get from USABC.

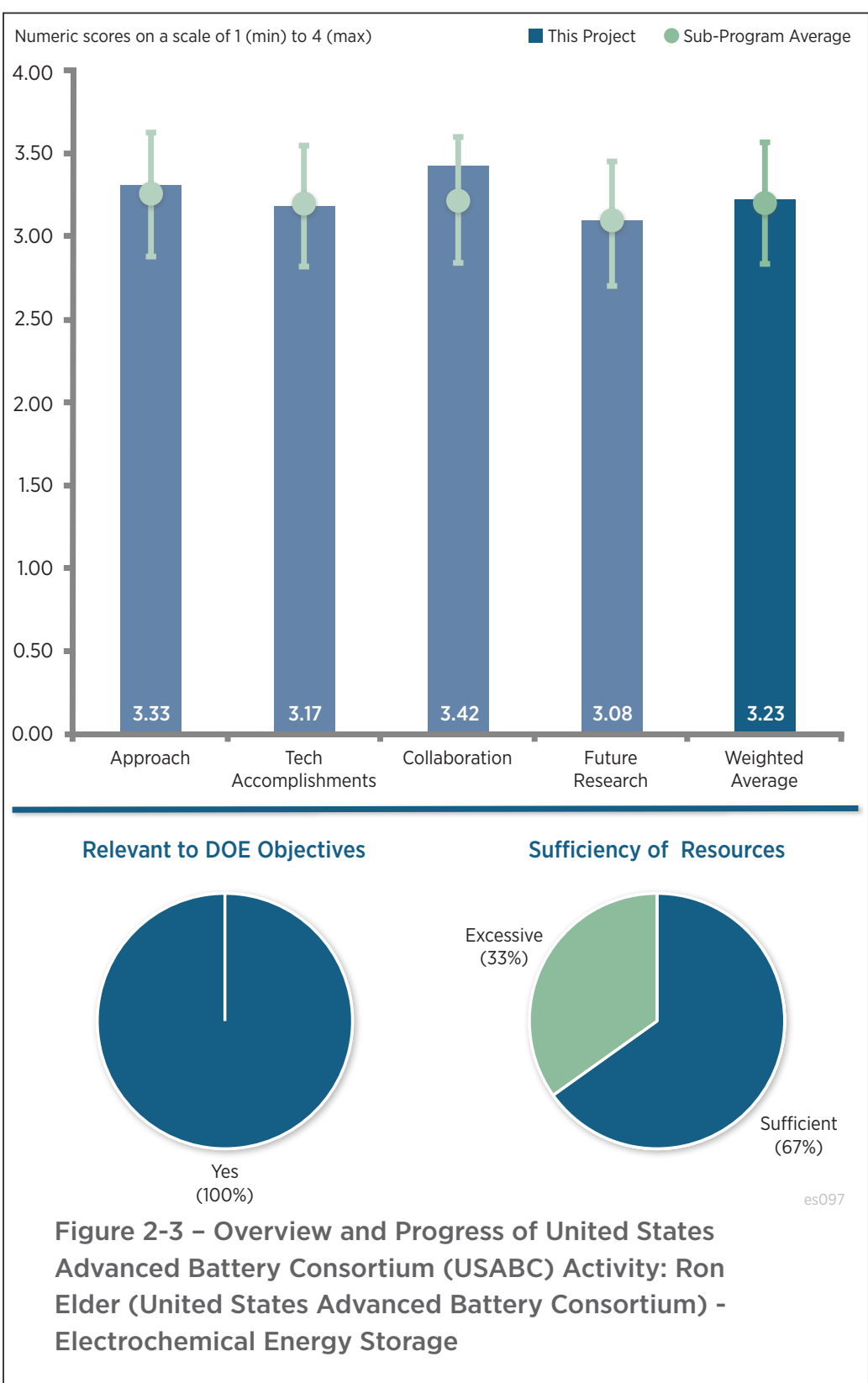

\section{Reviewer 3:}

The reviewer said that USABC has provided a significant amount of funding for the industrial partners to develop new energy storage technologies for EVs. The funding mechanism has been proven critical for the development and introduction of the new vehicle technologies, which would otherwise take a long time.

\section{Reviewer 4:}

The reviewer appreciated the scope of projects for EVs, PHEVs, start-stop, etc. The reviewer said that we need these efforts to ensure development across applications. The reviewer expressed a concern about the high level of administrative/review to project work, which USABC seems to be addressing

\section{Reviewer 5:}

The reviewer noted that the objective is to develop advanced electrochemical energy technologies for EVs, PHEVs and 12 Volt (V) start-stop. The approach is to leverage U.S. Council for Automotive Research (USCAR) OEMs, 
national laboratories, and industry to develop the components and materials. The reviewer noted that performance goals were summarized in tables. The reviewer said that the RFPI schedule, along with the RFPI task descriptions, were clear. Five years is reasonable to develop mitigations for cost, calendar life and cycle life barriers. The reviewer said that the RFPIs cover a wide scope, ranging from materials, testing and system components. This USABC program appeared to have a lot of overlap with the other DOE vehicle energy technology programs. The reviewer recommended that USABC should focus on accelerating the maturity of technologies at the system level while DOE programs focus on the basic and applied R\&D level.

\section{Reviewer 6:}

The reviewer noted that the process for application and review is excessive. Decisions on funding should be made earlier in the process. The reviewer said that the use of DOE targets for short-term projects is unrealistic, and a realistic set of performance targets should be used.

\section{Question 2: Technical accomplishments and progress toward overall project and DOE goals-the degree to which progress has been made, measured against performance indicators and demonstrated progress towards DOE goals.}

\section{Reviewer 1:}

The reviewer said that USABC is selecting all highly pertinent topics from an automotive applications points of view. The reviewer commented that unlike before, it is good to see that USABC is funding projects on all types of energy sources and key components.

\section{Reviewer 2:}

The reviewer noted that USABC selectively funded a few industrial development programs. The programs covered the major area of vehicle applications. The reviewer suggested that as a DOE program, USABC should focus more on U.S.-owned and U.S.-based industries.

\section{Reviewer 3:}

The reviewer said that the metrics for evaluations of applications were provided, but more information is needed to allow for an appropriate review. The reviewer asked, out of the initial inquiries, what percent of applicants continued to the full application, and of applicants who finished the application, what percent were funded. The reviewer asked what the range of scores in evaluation was, and what the cutoff for funding was. The reviewer asked when companies did not meet requirements, where did they fail. Additionally, the reviewer noted that other qualitative descriptions were provided instead of numerical data. The reviewer inquired what accomplishments have truly been made by the funded researchers. The reviewer said that data should be provided for improvements, for example, if higher voltages have been accessed through improvements in electrolytes, how was this determined, and provided as an example cycle life with one electrolyte versus another in the same condition.

\section{Reviewer 4:}

The reviewer would like to hear about the project accomplishments, not just the fact that USABC kicked off a specific number of them. The reviewer said that it is difficult to judge the value of USABC funding without hearing about the specific progress made within the technical development projects.

\section{Reviewer 5:}

The reviewer said that USABC updated test manuals and funded companies to develop the materials needed to mitigate the aforementioned barriers. The reviewer pointed out that although the goals were presented, current status (two years into a five-year program) against those goals was not presented, thus it was difficult to gauge progress on the mitigation of barriers.

\section{Question 3: Collaboration and coordination with other institutions.}

\section{Reviewer 1:}

The reviewer observed good collaboration between car OEMs, DOE, national laboratories, and industry. 


\section{Reviewer 2:}

The reviewer said that by its very nature, USABC requires collaboration, and that the team appears to engage well with a variety of collaborators.

\section{Reviewer 3:}

The reviewer said that USABC-sponsored programs are diversified, which involved the collaboration of government labs and industry.

\section{Reviewer 4:}

The reviewer said that while benchmarking and development efforts are well selected, it will be good to see more engagement from overseas suppliers such as the Japanese. However, the process needs to be made friendlier to attract more participation. The reviewer said that financial due diligence is a serious burden on many companies, as is the $50 / 50$ cost-share.

\section{Reviewer 5:}

The reviewer remarked that a limited set of companies was included. The reviewer asked about the major players, like Tesla, and wondered if this application process is too onerous for them to participate. If not, the reviewer would like to know why Tesla is not included.

The reviewer pointed out that eliminating smaller companies as a result of the cost share requirement may reduce unique ideas. The reviewer would like to know what validation USABC has that cost share requirements lead to better projects, participation, and results.

Question 4: Proposed future research-the degree to which the project has effectively planned its future work in a logical manner by incorporating appropriate decision points, considering barriers to the realization of the proposed technology and, when sensible, mitigating risk by providing alternate development pathways.

\section{Reviewer 1:}

The reviewer said that there is a good list of topics.

\section{Reviewer 2:}

The reviewer said that it is a good idea to encourage collaboration teams to address cost, calendar life, and cycle life barriers. The reviewer remarked that it is also a good use of USABC resources to increase scale-up of promising technologies.

\section{Reviewer 3:}

The reviewer commented that the USABC proposal to update the manuals for test methods and cost models are very useful, and the development of cost reduction manufacture process is critical for American manufacture industry to remain competitive. The reviewer remarked that more detailed plans should be presented.

\section{Reviewer 4:}

The reviewer remarked that the plan for modifying the application process was unclear. The reviewer said that no numerical values or specific targets were provided, so it will be difficult to know if improvements were met. The reviewer commented that the goal of improving the process is too vague for this reviewer to have confidence in knowing that effective changes will be made.

\section{Reviewer 5:}

The reviewer acknowledged not seeing reference to what (from a project portfolio perspective) USABC would be looking for in the future. The reviewer knows that USABC has a roadmap, but according to the reviewer it was not emphasized in this presentation and there was not a link presented between projects being funded to this roadmap

\section{Question 5: Does this project support the overall DOE objectives of petroleum} displacement? Why or why not? 


\section{Reviewer 1:}

The reviewer remarked highly relevant for the DOE objective of petroleum displacement by developing advanced electrochemical energy technologies.

\section{Reviewer 2:}

The reviewer commented that by enabling funding of research at companies involved in battery research, it is likely that some improvements will be made in LIB performance.

\section{Reviewer 3:}

The reviewer said that projects were mainly focused on improvement in energy density or cost reduction of alternatives to petroleum

\section{Question 6: Resources: How sufficient are the resources for the project to achieve the stated milestones in a timely fashion?}

\section{Reviewer 1:}

The $\$ 125$ million/5 years of effort was appropriate given the amount of work and teams involved.

\section{Reviewer 2:}

The reviewer thought that these funds would be better allocated by DOE directly, instead of through the USABC.

\section{Reviewer 3:}

The reviewer said that without knowing more details on the technical projects, this is difficult to assess. The reviewer said that it does seem like there are an excessive amount of resources directed to reviewing and rereviewing proposals. 
Thick Low-Cost, High-Power Lithium-Ion Electrodes via Aqueous Processing: Jianlin Li (Oak Ridge National Laboratory) - es164

Presenter

Jianlin Li, Oak Ridge National Laboratory

\section{Reviewer Sample Size}

A total of four reviewers evaluated this project.

Question 1: Approach to performing the work-the degree to which technical barriers are addressed, the project is well-designed, feasible, and integrated with other efforts.

\section{Reviewer 1:}

The reviewer said that the program appears to cover all of the challenges associated with moving to an aqueousbased electrode coating process.

\section{Reviewer 2:}

The reviewer said that at this stage of the project, it will be helpful to incorporate statistical analyses tools and compare results for different cathode families. In addition to using internal baseline, it is important to do benchmarking. The reviewer said that it is strange to see the data on Slide 9 where the industrially processed electrode sheet is so inferior.

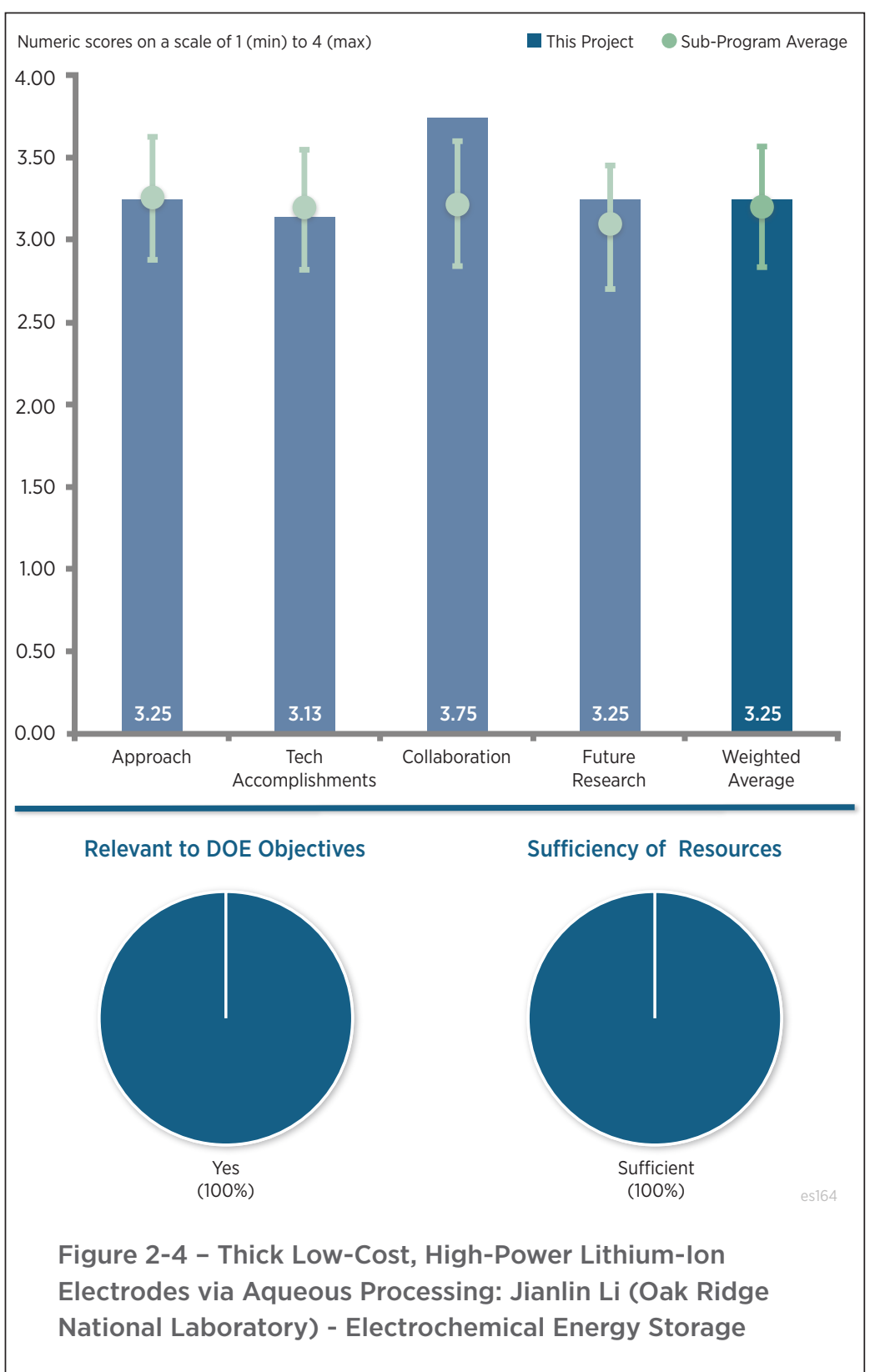

\section{Question 2: Technical}

accomplishments and progress toward overall project and DOE goals-the degree to which progress has been made, measured against performance indicators and demonstrated progress towards DOE goals.

\section{Reviewer 1:}

The reviewer found that overall, progress is impressive. The benefit for the industry will be enhanced through the use of the experimental design tools where interactions between the parameters studied could be assessed and quantified. The reviewer said that this will also improve focus on the program objectives.

\section{Reviewer 2:}

The reviewer remarked that establishing the theoretical improvement of heat load for drying on N-Methylpyrrolidone (NMP) versus water calls into question the potential of the process improvement targets. There may be other benefits, including the environmental ease of handling water versus NMP, however the cost savings may not be part of those benefits. The reviewer said that it does not appear that any lack of progress 
has contributed to the questionable target, but rather some initial errors in the initial evaluation of the potential improvement such a system could offer.

\section{Reviewer 3:}

The reviewer asked if the $15 \%$ cost reduction is really relevant and does it justify changes to the process.

\section{Question 3: Collaboration and coordination with other institutions.}

\section{Reviewer 1:}

The reviewer gave kudos for the great team assembled.

\section{Reviewer 2:}

The reviewer said that there appears to be a comprehensive list of appropriate collaborators across the spectrum of process, materials and end users.

Question 4: Proposed future research-the degree to which the project has effectively planned its future work in a logical manner by incorporating appropriate decision points, considering barriers to the realization of the proposed technology and, when sensible, mitigating risk by providing alternate development pathways.

\section{Reviewer 1:}

The reviewer said that proposed future research is well aligned with program objectives.

\section{Question 5: Does this project support the overall DOE objectives of petroleum displacement? Why or why not?}

\section{Reviewer 1:}

The reviewer said that it is necessary to address and justify the significantly lower cost reduction expectations to move further.

\section{Question 6: Resources: How sufficient are the resources for the project to achieve the} stated milestones in a timely fashion?

No comments were received in response to this question. 


\section{Performance Effects of Electrode Coating Defects and IR Thermography NDE for High-Energy Lithium-Ion Batteries: David Wood (Oak Ridge National Laboratory) - es165}

\section{Presenter}

David Wood, Oak Ridge National Laboratory

\section{Reviewer Sample Size}

A total of three reviewers evaluated this project.

\section{Question 1: Approach to performing the work-the degree to which technical barriers are addressed, the project is well-designed, feasible, and integrated with other efforts.}

\section{Reviewer 1:}

The reviewer described that quantifying the types and effect of coating defects can have a large impact on the quality and cost of electrode manufacturing. The reviewer said that it seems critical in this program that the results are relevant and transferable to commercial manufactures as they are the location that will ultimately be able to translate the finding to real cost advantage.

\section{Question 2: Technical} accomplishments and progress toward overall project and DOE goals-the degree to which progress has been made, measured against performance indicators and demonstrated progress towards DOE goals.

\section{Reviewer 1:}

The reviewer stated that the target defects appear to be identifiable with the approaches developed. The reviewer assumed that these are the correct target defects.

\section{Question 3: Collaboration and coordination with other institutions.}

\section{Reviewer 1:}

The reviewer detailed that the fact there is real collaboration with commercial entities is good. It is critical for this program to make sure that it is working on a capability that does not currently exist in the industry as well as working within production parameters (line speed, coating process etc.) that are commercially relevant. The reviewer concluded that with that in mind, the list of commercial end users (those actually coating electrodes in commercial environments) could be stronger.

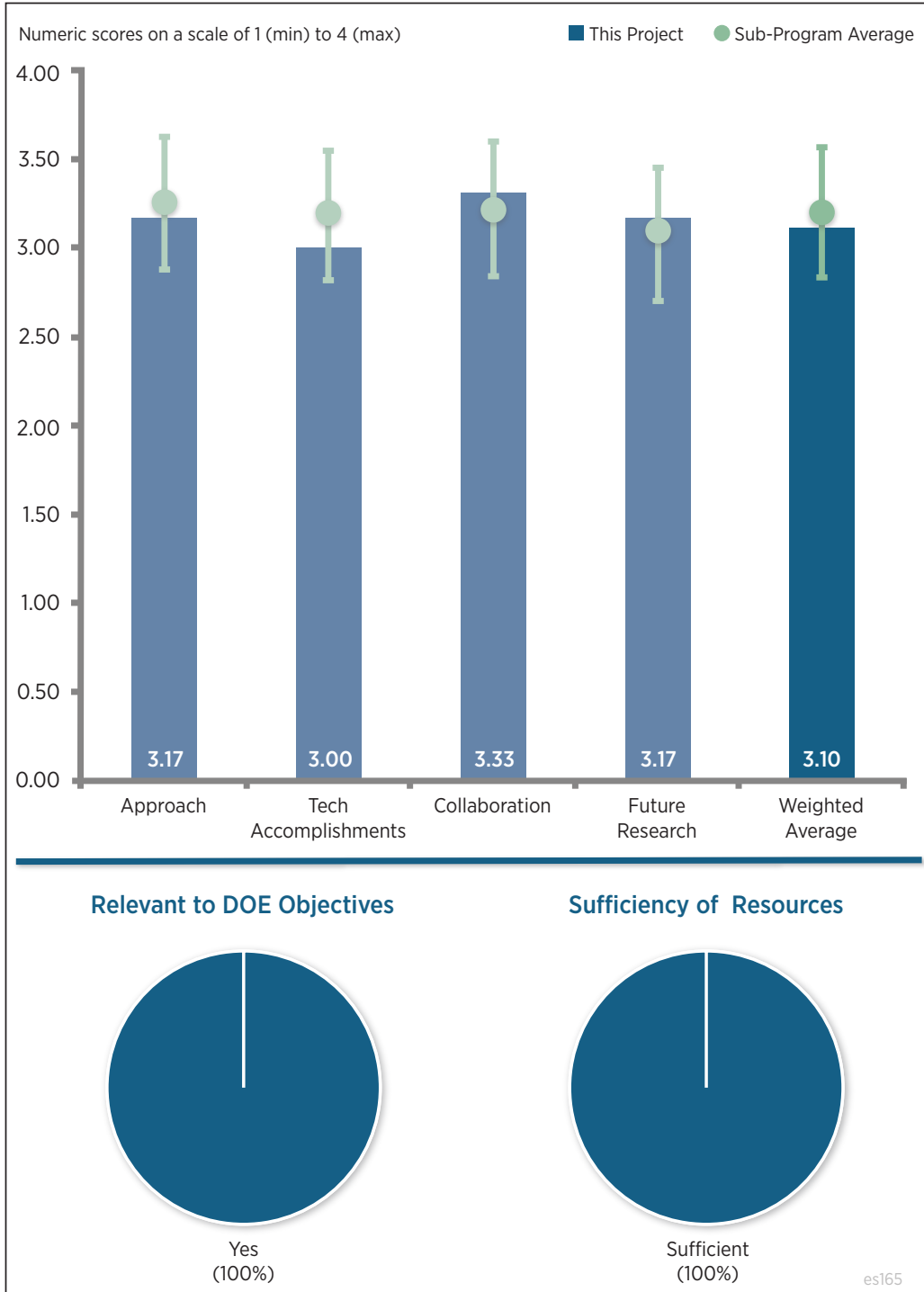

Figure 2-5 - Performance Effects of Electrode Coating Defects and IR Thermography NDE for High-Energy LithiumIon Batteries: David Wood (Oak Ridge National Laboratory) - Electrochemical Energy Storage 
Question 4: Proposed future research-the degree to which the project has effectively planned its future work in a logical manner by incorporating appropriate decision points, considering barriers to the realization of the proposed technology and, when sensible, mitigating risk by providing alternate development pathways.

Reviewer 1:

The reviewer reiterated that it is critical to address areas that are not currently available in the commercial world, as well as to address areas on where the industry is going rather than where it has been.

Question 5: Does this project support the overall DOE objectives of petroleum displacement? Why or why not?

No comments were received in response to this question.

Question 6: Resources: How sufficient are the resources for the project to achieve the stated milestones in a timely fashion?

No comments were received in response to this question. 


\section{Post-Test Analysis of Lithium- Ion Battery Materials at Argonne National Laboratory: Ira Bloom (Argonne National Laboratory) - es166}

\section{Presenter}

Ira Bloom, Argonne National Laboratory

\section{Reviewer Sample Size}

A total of four reviewers evaluated this project.

\section{Question 1: Approach to performing the work-the degree to which technical barriers are addressed, the project is well-designed, feasible, and integrated with other efforts.}

\section{Reviewer 1:}

The reviewer was very glad to see this being studied in detail, and commented that the project plays to ANL's strengths. The reviewer said that it would be better to use commercial electrodes, but the reviewer understands the challenges in doing that.

\section{Reviewer 2:}

The reviewer commented that the consortium is much needed to understand the effect of Li-ion materials after abusive conditions and to understand the impact of processing methods on cell performance. Three years is reasonable schedule for this effort.

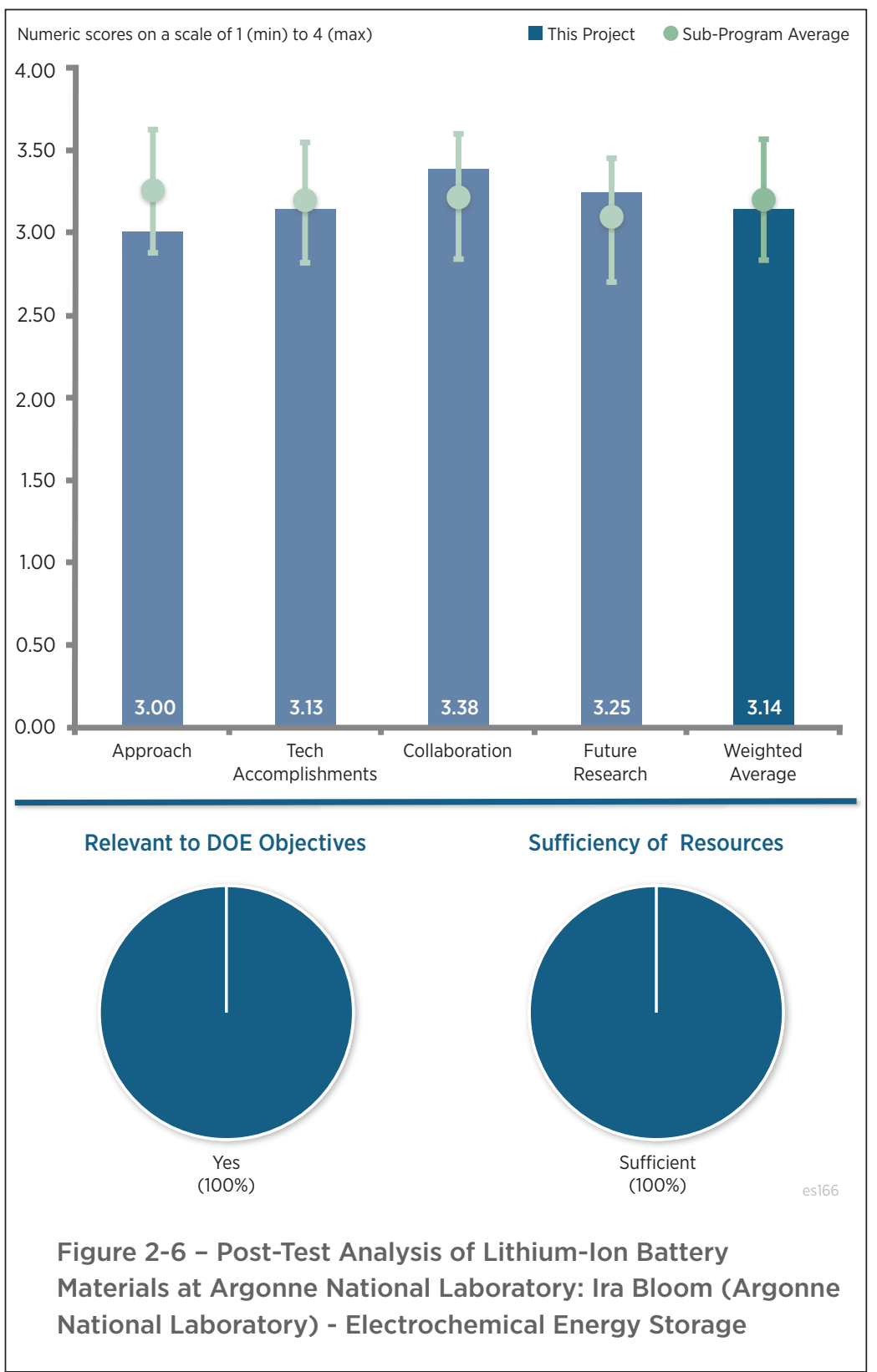

The reviewer remarked that quantifiable milestones and go/no-go decision points were not provided. This program appears to have some overlap with Wood's effort at Oak Ridge National Laboratory (ORNL) to examine effect of processing conditions (e.g., electrode coating) on performance.

\section{Reviewer 3:}

The reviewer said that this program shades a bit toward a testing capability looking for a mission, and asked who the gatekeeper is for what to evaluate, and what criteria/decision process is used to initiate an evaluation. The reviewer suggested that the project team consider looking at formation and/or other places OEMs may be paying for quality or process control that we may not need, and cited robust engineering principles. The reviewer asked what the meaningful changes are in cell design or chemistry that should warrant requalification by customers, or by regulators. The reviewer asked what limitations there are on this front if restricted to open source bill of materials $(\mathrm{BOM})$, processing, and fabrication equipment/tooling. 


\section{Reviewer 4:}

The reviewer expressed uncertainty (at least from the presentation) how participants are planning to prepare samples for testing, and which processing parameters, beyond two types of chemistries and two types of solvent, will be evaluated. The reviewer asked if there is a detailed experimental plan in place.

\section{Question 2: Technical accomplishments and progress toward overall project and DOE goals-the degree to which progress has been made, measured against performance indicators and demonstrated progress towards DOE goals.}

\section{Reviewer 1:}

The reviewer said that the work done on the baseline cells looks good and in line with project objectives.

\section{Reviewer 2:}

The reviewer noted that the project is still early, so it is not fair to judge. The reviewer suggested that the project team not proceed until the team gets the electrode quality issues figured out. The reviewer said that the team should be able to make high quality anodes with NMP processing.

\section{Reviewer 3:}

The reviewer said that the team established effective experimental methods (e.g., Fourier transform infrared spectroscopy and impedance) for post-mortem analysis. The reviewer expressed uncertainty if some of the electrode issues (rippling, pin holes) were specific to ANL's coating process or were they caused by materials. The reviewer cited that ANL's study showed that aqueous process produced anodes had higher impedance than that of NMP. However, aqueous process has been used to produce good anode in commercial cells. Thus, according to the reviewer, it was not clear how applicable the insights learned from ANL's study were.

\section{Reviewer 4:}

The reviewer said that the project is still early, and was not clear on how copper $(\mathrm{Cu})$ corrosion is tied to failure mode, whether through delamination, impedance gain, or electrochemical activity. The reviewer asked what the role of residual moisture and electrode drying operation is.

\section{Question 3: Collaboration and coordination with other institutions.}

\section{Reviewer 1:}

The reviewer remarked excellent team with synergetic capabilities.

\section{Reviewer 2:}

The reviewer observed a great team with the perfect skill sets to tackle this project.

\section{Reviewer 3:}

The reviewer noted good collaboration between ANL, ORNL, and Sandia National Laboratories (SNL). The roles of each team member were clearly identified to justify their participation in the project.

\section{Reviewer 4:}

The reviewer remarked that there seems to be good lab coordination, but these presentations tended to just post long lists of contributors and not defined roles or RASIC (Responsible, Approving, Supporting, Informed, and Consulted). The reviewer said that it starts to look like every national laboratory investigator throws in on every program to avoid being cut out. The reviewer remarked that it might help to have some internal competition/ incentives and more defined roles. The reviewer also noted that the SNL abuse test role seemed downplayed.

Question 4: Proposed future research-the degree to which the project has effectively planned its future work in a logical manner by incorporating appropriate decision points, considering barriers to the realization of the proposed technology and, when sensible, mitigating risk by providing alternate development pathways.

\section{Reviewer 1:}

The reviewer commented that the proposed effort to understand the effect of aging and abusive conditions, e.g., overcharging, on Li-ion materials is much needed. 


\section{Reviewer 2:}

The reviewer said that proposed future research has good focus and understanding, and that it is important to start including statistical tools like design of the experiments to understand the interaction between parameters studied.

\section{Reviewer 3:}

The reviewer said that the project reflects thoughtful experimentation to ensure final results will be meaningful, e.g., getting good electrode quality. The reviewer noted that it is important to develop and validate all procedures. The reviewer asked if the project team could use commercial materials for the validation.

\section{Reviewer 4:}

The reviewer said that proposed future research could be better focused.

\section{Question 5: Does this project support the overall DOE objectives of petroleum displacement? Why or why not?}

\section{Reviewer 1:}

The reviewer said that we need to address safety as we move towards higher energy materials and cells.

\section{Reviewer 2:}

The reviewer remarked that understanding the effect of aging and abusive conditions on Li-ion materials is very relevant to achieve reliable cell performance.

\section{Reviewer 3:}

The reviewer commented that this program is very important in aiding the fundamental understanding of the complex events in the cell causing its failure.

\section{Question 6: Resources: How sufficient are the resources for the project to achieve the stated milestones in a timely fashion?}

\section{Reviewer 1:}

The reviewer commented that it is great to see young scientists being trained by the best in this field.

\section{Reviewer 2:}

The reviewer said that the $\$ 1.1$ million/year for three years should be sufficient for this effort.

\section{Reviewer 3:}

The reviewer said that once electrode issues are solved, this team has the skills to do the safety testing and analytical work required. The reviewer remarked that it looks like this project evaluates combinations of existing materials, and is not new material development.

\section{Reviewer 4:}

The reviewer would advocate for more emphasis on abuse testing and root cause failure, but acknowledged that this can be resource intensive if doing multiple large format cells and extended cycling on channels that can handle high capacity. 


\section{Process Development and Scale-Up of Advanced Active Battery Materials: Greg Krumdick (Argonne National Laboratory) - es167}

\section{Presenter}

Youngho Shin, Argonne National Laboratory

\section{Reviewer Sample Size}

A total of four reviewers evaluated this project.

\section{Question 1: Approach to performing the work-the degree to which technical barriers are addressed, the project is well-designed, feasible, and integrated with other efforts.}

\section{Reviewer 1:}

The reviewer remarked outstanding performance meeting the needs of the industry and research community while adding value to ANL's thorough licensing strategies.

\section{Reviewer 2:}

The reviewer said that providing consistent program scale volumes of advanced materials is a worthy goal to support ongoing development within the community. The reviewer said that it would be interesting to have more visibility into the process of how the target materials are determined in the first place. The development of any single material is an enormous commitment, so choosing the target materials seems as if it should be a highly critical step in the process.

\section{Question 2: Technical accomplishments and progress toward overall project and DOE goals-the degree to which progress has been made, measured against performance indicators and demonstrated progress towards DOE goals.}

\section{Reviewer 1:}

The reviewer remarked excellent use of the statistical tools for the optimization work. It is important to conduct comparison/benchmarking studies versus the industrially produced materials.

\section{Reviewer 2:}

The reviewer commented that the identified target materials appear to have been successfully developed.

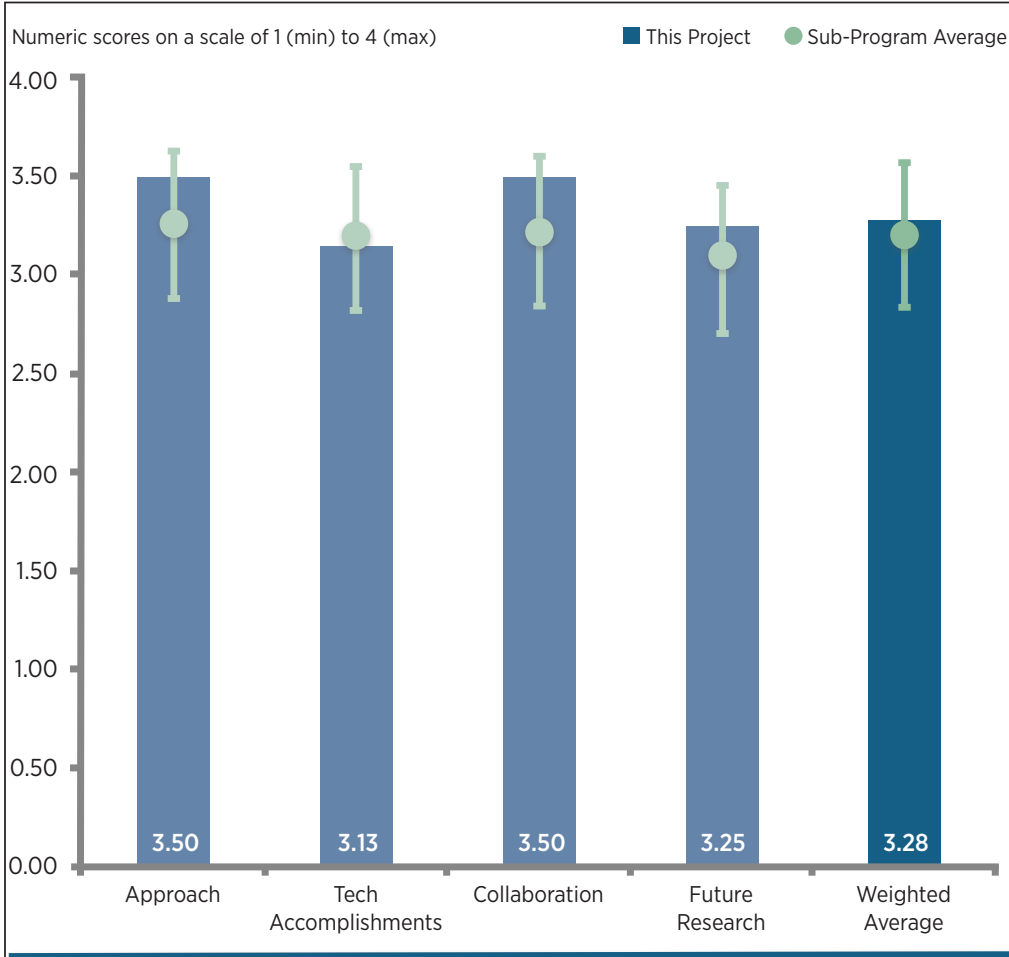

Relevant to DOE Objectives

Sufficiency of Resources
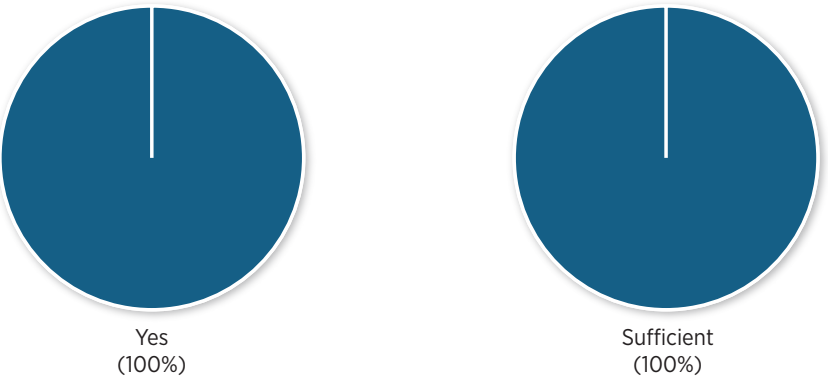

Figure 2-7 - Process Development and Scale-Up of Advanced Active Battery Materials: Greg Krumdick (Argonne National Laboratory) - Electrochemical Energy Storage

Question 3: Collaboration and coordination with other institutions. 


\section{Reviewer 1:}

The reviewer said that the list of the collaborators demonstrate the trust this group has earned, well done. It will be interesting to see the performance of the commercialized products.

\section{Reviewer 2:}

The reviewer commented that there appears to be reasonable collaboration with various process and equipment partners, and there could be better collaboration with the target material audience, particularly in the commercial arena.

Question 4: Proposed future research-the degree to which the project has effectively planned its future work in a logical manner by incorporating appropriate decision points, considering barriers to the realization of the proposed technology and, when sensible, mitigating risk by providing alternate development pathways.

\section{Reviewer 1:}

The reviewer said that proposed future research is well-balanced to meet the program objectives.

\section{Question 5: Does this project support the overall DOE objectives of petroleum} displacement? Why or why not?

\section{Reviewer 1:}

The reviewer commented that this program plays a supporting role in supplying critical materials for advanced development activities.

\section{Reviewer 2:}

The reviewer said that the project addressed the gap between research, development, and commercialization.

Question 6: Resources: How sufficient are the resources for the project to achieve the stated milestones in a timely fashion-

\section{Reviewer 1:}

The reviewer said highly qualified, hard-working team. 


\section{Process Development and Scale-Up of Critical Battery Materials: Greg Krumdick (Argonne National Laboratory) - es168}

\section{Presenter}

Krzysztof Pupek, Argonne National Laboratory

\section{Reviewer Sample Size}

A total of four reviewers evaluated this project.

\section{Question 1: Approach to performing the work-the degree to which technical barriers are addressed, the project is well-designed, feasible, and integrated with other efforts.}

\section{Reviewer 1:}

The reviewer remarked impressive systematic engineering approach, sharp focus on the objectives.

\section{Reviewer 2:}

The reviewer pointed out that in order to provide critical advanced materials to the community, critical process developments must occur in areas where common material will be made. The focus on processes for fluorinated solvents and salts, and for advanced binders, appears to be a good choice as an area of development. The reviewer commented that as always in this type of program, the choice of target is critical as it is a long-term commitment to develop even pilot level processes,

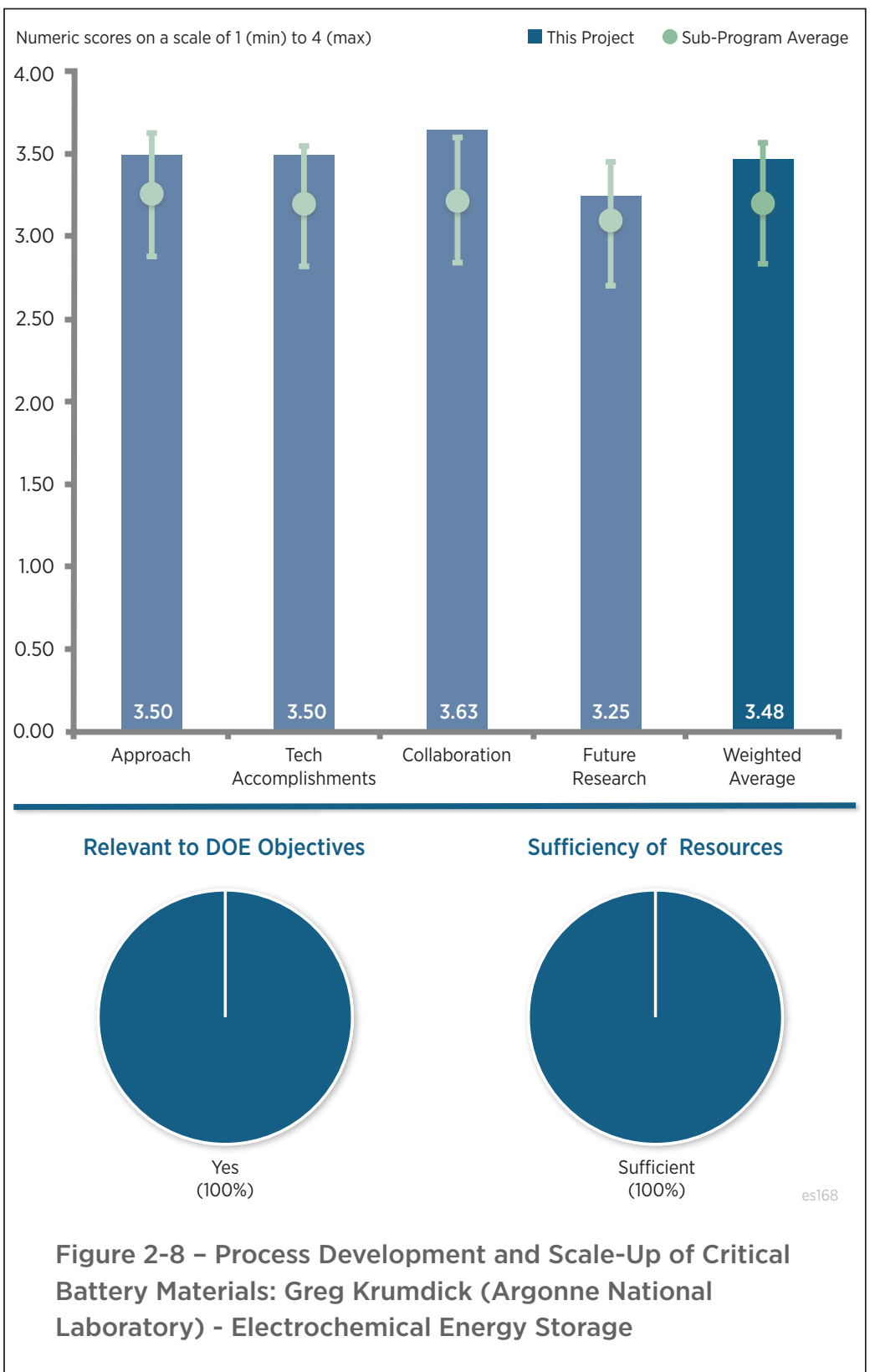

so the choice of areas must be relevant to the industry both today and tomorrow.

Question 2: Technical accomplishments and progress toward overall project and DOE goals-the degree to which progress has been made, measured against performance indicators and demonstrated progress towards DOE goals.

\section{Reviewer 1:}

The reviewer said that a number of unique materials were developed that can be applied to the advanced development efforts.

\section{Reviewer 2:}

The reviewer commented that the program meets DOE's needs and fills in the gaps between R\&D and commercial efforts.

\section{Question 3: Collaboration and coordination with other institutions.}




\section{Reviewer 1:}

The reviewer remarked that bringing on board a large-scale manufacturer will further increase the project value, and that establishing licensing revenue is impressive.

Question 4: Proposed future research-the degree to which the project has effectively planned its future work in a logical manner by incorporating appropriate decision points, considering barriers to the realization of the proposed technology and, when sensible, mitigating risk by providing alternate development pathways.

\section{Reviewer 1:}

The reviewer said that the pipeline is well balanced.

Question 5: Does this project support the overall DOE objectives of petroleum displacement? Why or why not?

\section{Reviewer 1:}

The reviewer commented that the project is filling the gaps and reducing the risks.

Question 6: Resources: How sufficient are the resources for the project to achieve the stated milestones in a timely fashion?

No comments were received in response to this question. 


\section{Electrochemical Performance Testing: Ira Bloom (Argonne National Laboratory) - es201}

\section{Presenter}

Ira Bloom, Argonne National

Laboratory

\section{Reviewer Sample Size}

A total of three reviewers evaluated this project.

\section{Question 1: Approach to performing the work-the degree to which technical barriers are addressed, the project is well-designed, feasible, and integrated with other efforts.}

\section{Reviewer 1:}

The reviewer stated that the electrochemical cycle testing is the best way to verify that money spent on battery material and especially cell invention and development does in fact do what is claimed, and to authoritatively compare those results to commercial, state of the art performance and cell or material goals. The reviewer concluded that this work thus helps approach many of the barriers.

\section{Reviewer 2:}

The reviewer said that the study of the different international test standards is very interesting. It would be nice to see this this done on more standards beyond the United States and China. The reviewer said that for the fast charging test, the data should be plotted as a function of ampere hour (Ah) throughput. This may help make more sense of the idea that the fast-charge test profile seems to cause an increase in aging over the constant-current profile. Also, the use of another chemistry type such as NMC/carbon for the standard comparison study would be more relevant to the United States.

\section{Question 2: Technical accomplishments and progress toward overall project and DOE goals-the degree to which progress has been made, measured against performance indicators and demonstrated progress towards DOE goals.}

\section{Reviewer 1:}

The reviewer said that as mentioned above, it would be nice to see more a few more standards included in the study. Other than that, the reviewer characterized the benchmarking work was excellent. The testing done at the national laboratories is an important non-biased source of data for these technologies.

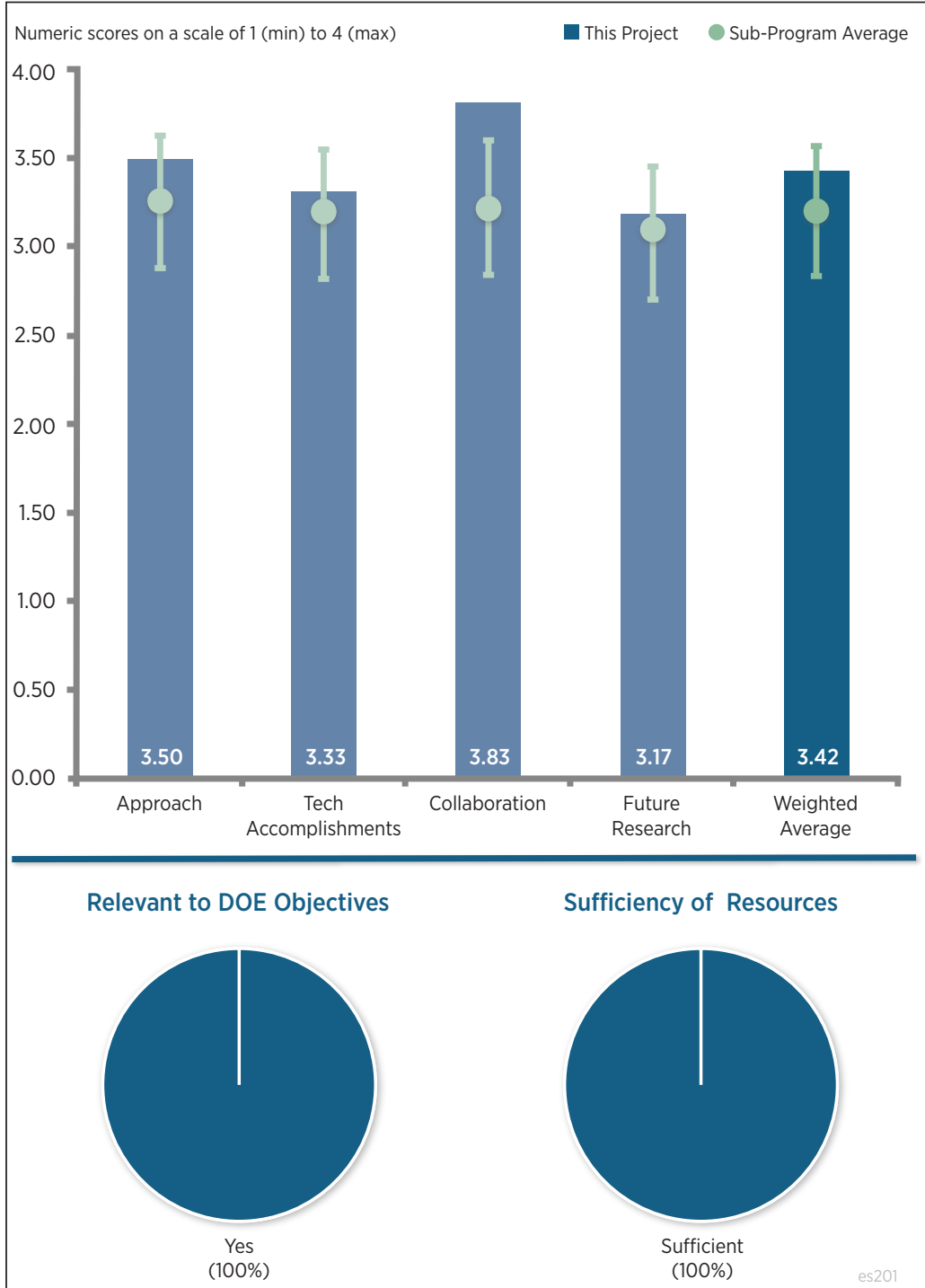

Figure 2-9 - Electrochemical Performance Testing: Ira Bloom (Argonne National Laboratory) - Electrochemical Energy Storage 


\section{Reviewer 2:}

The reviewer said that ANL is conducting ongoing and new testing on many cells. The comparison of U.S. and China protocols is interesting, but would be more useful if it were then used to harmonize regulation or methods. The reviewer commented that assistance in fast-charge protocol is important and of increasing importance in the future.

\section{Question 3: Collaboration and coordination with other institutions.}

\section{Reviewer 1:}

The reviewer pointed out that ANL has a wide range of partners with several partners in each of the areas.

\section{Reviewer 2:}

The reviewer said that this project collaborates with many different organizations including cell makers, vehicle makers, the USABC, and others. ANL creates an important independent source of data to compare technologies from many different developers.

Question 4: Proposed future research-the degree to which the project has effectively planned its future work in a logical manner by incorporating appropriate decision points, considering barriers to the realization of the proposed technology and, when sensible, mitigating risk by providing alternate development pathways.

\section{Reviewer 1:}

The reviewer said that continued electrochemical testing is appropriate and well planned.

China test work would benefit from a partner who can help use it to harmonize testing. The reviewer understands that is the goal, but ANL would benefit from the help of say the Society of Automotive Engineers (SAE) or some other organization that can help navigate the international harmonization waters.

\section{Reviewer 2:}

The reviewer remarked that the national laboratories will continue to be an important source of independent data for the industry. While the collaboration with the Chinese labs is interesting, the reviewer expressed uncertainty if more testing with lithium-iron phosphate (LFP) is very interesting for the U.S. market. That chemistry is not often used here.

\section{Question 5: Does this project support the overall DOE objectives of petroleum displacement? Why or why not?}

\section{Reviewer 1:}

The reviewer said that this testing basically determines which chemistry is ready for marketing and thus participates in petroleum displacement.

\section{Reviewer 2:}

The reviewer stated yes and reiterated that the independent test data produced by the national laboratories is of a very high quality and an important source of information for the industry.

\section{Question 6: Resources: How sufficient are the resources for the project to achieve the stated milestones in a timely fashion?}

No comments were received in response to this question. 


\section{INL Electrochemical Performance Testing: Matt Shirk (Idaho National Laboratory) - es202}

\section{Presenter}

Matt Shirk, Idaho National Laboratory

\section{Reviewer Sample Size}

A total of three reviewers evaluated this project.

\section{Question 1: Approach to performing the work-the degree to which technical barriers are addressed, the project is well-designed, feasible, and integrated with other efforts.}

\section{Reviewer 1:}

The reviewer said that electrochemical testing supports almost all the barriersurmounting efforts by validating the results, so this supports all the cell and material work. The manuals the project team support help and guide research internationally and so effectively leverage other country's efforts toward DOE goals.

\section{Reviewer 2:}

The reviewer stated that the national laboratories are an important source of independent test data for the battery development programs conducted through the various DOE funding opportunities. To the extent that it is possible, due to restrictions on the data etc., the reviewer said that it would be nice to see comparison work done between the different cells tested. If the cells tested are all tested to the same test manuals, Idaho National Laboratory (INL) would be in the best position to understand what the state of the art is, and how each technology performs relative to that standard.

\section{Question 2: Technical accomplishments and progress toward overall project and DOE goals - the degree to which progress has been made, measured against performance indicators and demonstrated progress towards DOE goals.}

\section{Reviewer 1:}

The reviewer said that the project is probably the best (if by only a small margin) electrical test work at DOE, and the team has a large number of cells on the test, and are developing new tests such as vibration impacts on cells. At the same time, INL holds the lion's share of the test manuals, many of which were recently updated.

\section{Reviewer 2:}

The reviewer said that the slide/study on memoryless aging was very interesting. The reviewer would like to

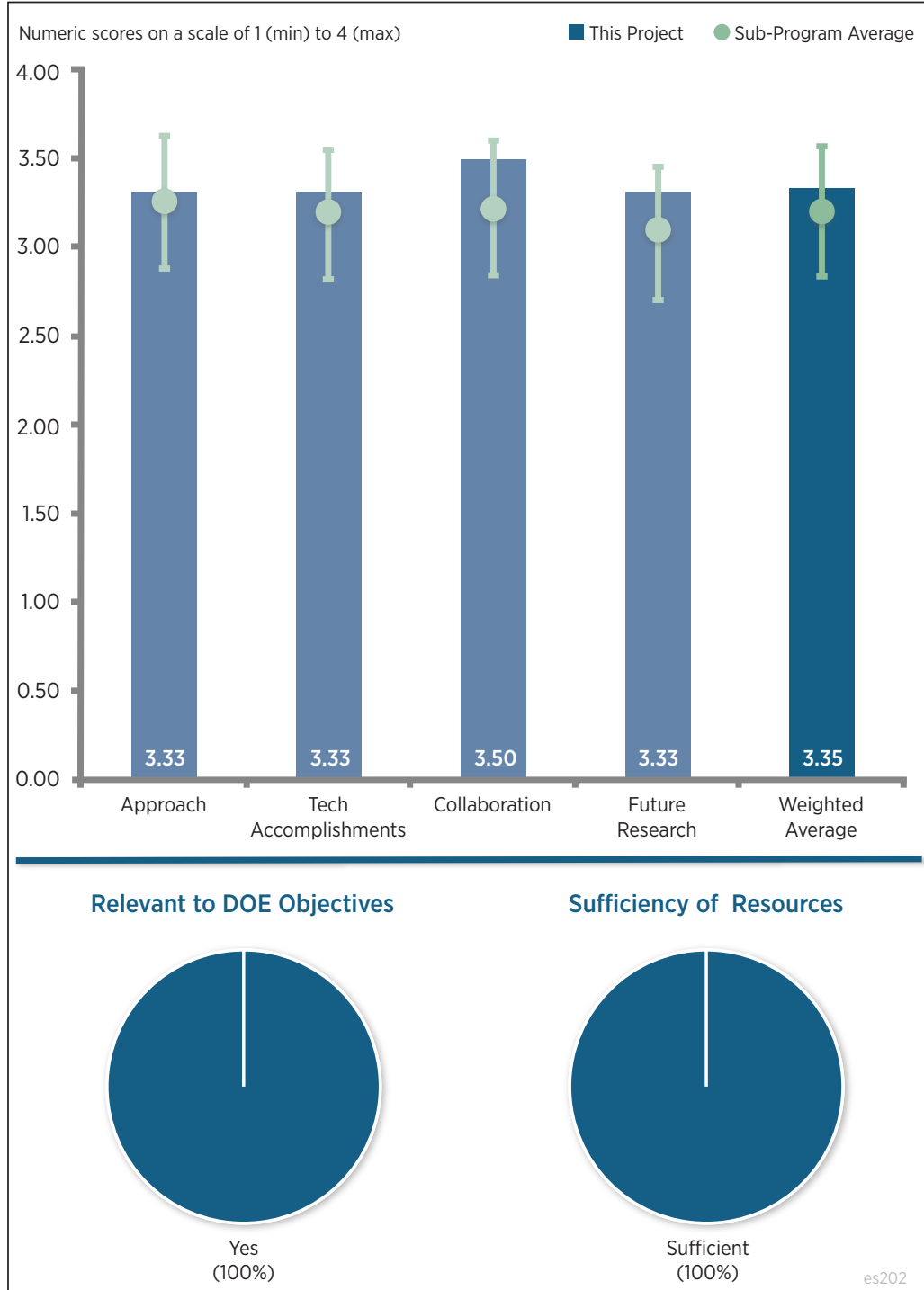

Figure 2-10 - INL Electrochemical Performance Testing: Matt Shirk (Idaho National Laboratory) - Electrochemical Energy Storage 
see a similar study with NMC only cathodes, as LMO has known calendar aging issues that may affect the results. The reviewer also expressed an interest to see a similar study with combinations of cycling and calendar aging. The reviewer also thinks that it is important to include the capacity fade information as well as the resistance rise, even though the memoryless aging is unlikely to exist when it comes to capacity fade.

\section{Question 3: Collaboration and coordination with other institutions.}

\section{Reviewer 1:}

The reviewer remarked that coordination with all the other research programs, laboratories, USABC, DOE, etc., is outstanding. As mentioned before, the independent testing at the national laboratories is a great contribution to the battery R\&D community.

\section{Reviewer 2:}

The reviewer said that collaboration is great with government and industry, and decent with universities.

Question 4: Proposed future research-the degree to which the project has effectively planned its future work in a logical manner by incorporating appropriate decision points, considering barriers to the realization of the proposed technology and, when sensible, mitigating risk by providing alternate development pathways.

\section{Reviewer 1:}

The reviewer said that proposed future research is appropriate for their mission.

\section{Reviewer 2:}

The reviewer remarked that INL maintains a state-of-the-art test facility, and that it is a good idea to think ahead about what new equipment will be needed to test future technologies. The reviewer also pointed out methods for measuring the swelling of Si containing cells under cycling, high current channels for the next generation of $12 \mathrm{~V}$ cell testing, and combination vibration and cycling testing, possibly in a temperature controlled environment.

\section{Question 5: Does this project support the overall DOE objectives of petroleum displacement? Why or why not?}

\section{Reviewer 1:}

The reviewer remarked that by helping USABC bring improved batteries to market, INL is definitely helping displace petroleum.

\section{Reviewer 2:}

The reviewer commented yes, the data produced from this program is critical to the success of the DOE battery programs.

\section{Question 6: Resources: How sufficient are the resources for the project to achieve the stated milestones in a timely fashion?}

\section{Reviewer 1:}

The reviewer pointed out that INL is on the edge of needing more facilities and staff as the load of new materials and cells to test grows. 


\section{Battery Safety Testing: Leigh Anna Steele (Sandia National Laboratories) - es203}

\section{Presenter}

Leigh Anna Steele, Sandia National Laboratories

\section{Reviewer Sample Size}

A total of four reviewers evaluated this project.

\section{Question 1: Approach to performing the work-the degree to which technical barriers are addressed, the project is well-designed, feasible, and integrated with other efforts.}

\section{Reviewer 1 :}

The reviewer affirmed that this group is the gold standard for safety and abuse testing. The project team plays a dramatically powerful role in getting batteries in vehicles and consumer times, without their data the risk would outweigh the possible benefits. The reviewer said that the team directly attacks the abuse response but also contribute to the durability evaluation and thus support the progress of all the other work done on new materials by ensuring they can focus on acceptable chemistry.

\section{Reviewer 2:}

The reviewer said that the approach seems solid. The reviewer expressed one concern related to the propagation experiments, specifically that the nail penetration (mechanical initiation) and the laser penetration methods are both very abrupt in the initiation. This provides an extremely fast temperature ramp-up that is rarely seen in real-life incidents. The reviewer noted that in particular, a common failure mode in real life is that a weak cell (low capacity) in an array can go into overcharge if a voltage monitoring failure occurs and that can precipitate a Li deposition-shorting mechanism and a slower temperature ramp-up as Li bridges are made and broken due to localized heating. The reviewer believed that this could be approached by a deliberate overcharging of a single cell in center or edge location and observe propagation.

\section{Reviewer 3:}

The reviewer commented that SNL continues to be the leader in the field of battery abuse testing. The work done there, specifically in developing and studying battery abuse test procedures, is extremely valuable to the field of battery R\&D and commercialization. The reviewer said that it would be nice to see the work move beyond cell level testing and into larger module level testing in order to help the industry create best practices when dealing with larger test items.

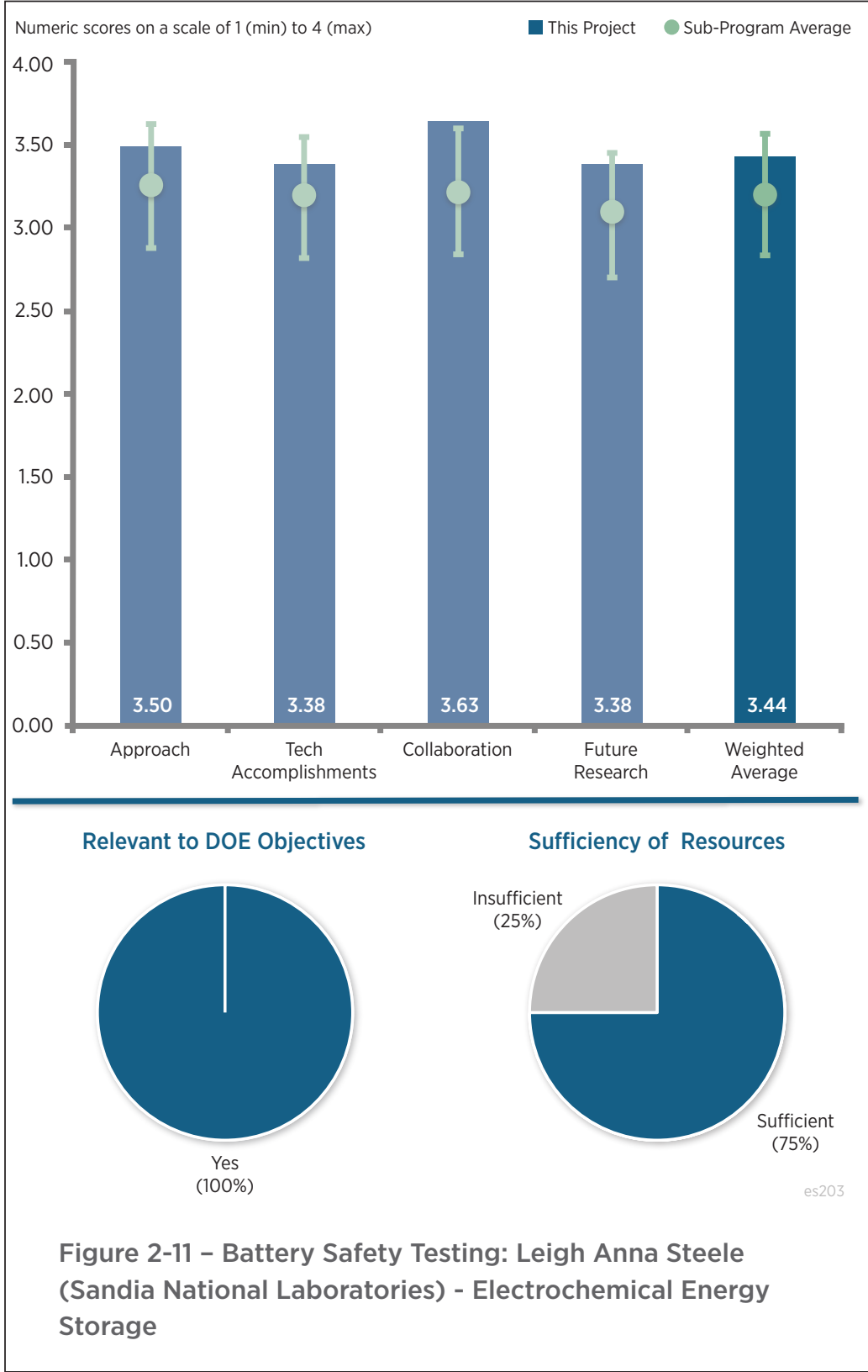


Question 2: Technical accomplishments and progress toward overall project and DOE goals-the degree to which progress has been made, measured against performance indicators and demonstrated progress towards DOE goals.

Reviewer 1:

The reviewer observed very solid results.

\section{Reviewer 2:}

The reviewer commented that the progress in the testing of DOE-sponsored cells is well done, critical, and voluminous. The team also supported modeling work and are exploring an emerging problem of failure propagation as well. The reviewer remarked there is not much more that could be asked of the team available.

\section{Reviewer 3:}

The reviewer remarked that the short circuit/propagation work is very interesting. The reviewer would like to see more cell formats tested, particularly larger format pouch and prismatic can cells. The reviewer thought that the choice of cell cathodes is not optimal. NMC or nickel cobalt aluminum oxide (NCA) or blends containing NMC/ $\mathrm{NCA} / \mathrm{LMO}$ would be more relevant to the transportation industry.

\section{Question 3: Collaboration and coordination with other institutions.}

\section{Reviewer 1:}

The reviewer pointed out that in particular, the collaboration with the National Renewable Energy Laboratory (NREL) and the modeling program is very valuable. The reviewer asked if it would be possible to see some appraisals from customers of their satisfaction with test protocols and results.

\section{Reviewer 2:}

The reviewer commented that SNL does a great job of collaborating with other institutes, and the reviewer is looking forward to seeing the outcome of the continued collaboration between the experimental work at SNL and the modeling work at other national laboratories.

\section{Reviewer 3:}

The reviewer remarked that the team works with government, academic, and industry groups too numerous to mention in this box.

Question 4: Proposed future research-the degree to which the project has effectively planned its future work in a logical manner by incorporating appropriate decision points, considering barriers to the realization of the proposed technology and, when sensible, mitigating risk by providing alternate development pathways.

\section{Reviewer 1:}

The reviewer said that the proposed future research is not sexy but it is what the project team should be doing. The reviewer noted that staying in your swim lane and doing it well is important.

\section{Reviewer 2:}

The reviewer would like to see some studies on liquid coolant effects with regards to runaway propagation. The reviewer asked if the ten-pin array could be modified to include coolant in the air gap space.

\section{Reviewer 3:}

The reviewer reiterated comments that it would be nice to see larger cells and more automotive relevant chemistries included in the future work.

\section{Question 5: Does this project support the overall DOE objectives of petroleum displacement? Why or why not?}

\section{Reviewer 1:}

The reviewer commented that this is one of the most important DOE programs to support the objectives as safety is paramount to achieving public acceptance of electric powered vehicles. 


\section{Reviewer 2:}

The reviewer said that without question the project supports the goal. Without understanding the response to abuse conditions, much more engineering structure would be needed to ensure safety in vehicles and would probably raise the cost, mass and volume past a saleable point. The reviewer emphasized that this is key support.

\section{Question 6: Resources: How sufficient are the resources for the project to achieve the stated milestones in a timely fashion?}

\section{Reviewer 1:}

The reviewer would like to see the abuse program at SNL expanded. These data are very important to the development of battery technologies for transportation and the reviewer believed the funding level is low relative to the importance of the work to the industry.

\section{Reviewer 2:}

The reviewer qualified that the backlog the project team has points to the lab being on the edge of what is needed to provide appropriate investigation of enough batteries to hit project milestones, and more to the point to support the work that needs to be done outside the contract shown in this poster. The reviewer thought that added facilities and staff would be money well spent. 
Battery Thermal

Characterization: Matthew

Keyser (National Renewable

Energy Laboratory) - es204

\section{Presenter}

Matthew Keyser, National Renewable

Energy Laboratory

\section{Reviewer Sample Size}

A total of four reviewers evaluated this project.

\section{Question 1: Approach to performing the work-the degree to which technical barriers are addressed, the project is well-designed, feasible, and integrated with other efforts.}

\section{Reviewer 1:}

The reviewer described NREL as the world's best thermal characterization lab and group, most likely. The reviewer certainly trusts their work over all others. The project team is helping get batteries into commerce by overcoming all the barriers that internal and asymmetric heat generation cause.

\section{Reviewer 2:}

The reviewer said that the experimental approach is good based on the use of calorimetry as a key technique in evaluating battery function. The reviewer would like to see a comparison with other methods such as impedance analysis to separate the various components such as Joule heating and entropic effects to sort out the causes of efficiency loss and the growth in time and cycling on efficiency loss. The emphasis is on only part of the story.

\section{Reviewer 3:}

The reviewer remarked that the thermal analysis data NREL provides is an important tool in the study of Li-ion batteries. The information on entropic heating is particularly interesting. The reviewer said that it would be nice to see more collaboration between the experimental data produced by NREL and the battery models produced by the other national laboratories. The reviewer pointed out that there were no slides showing the comparison between experimental and modeling results.

\section{Question 2: Technical accomplishments and progress toward overall project and DOE goals-the degree to which progress has been made, measured against performance indicators and demonstrated progress towards DOE goals.}

\section{Reviewer 1:}

The reviewer noted good progress on important questions. The project tested a nice variety of cells and in

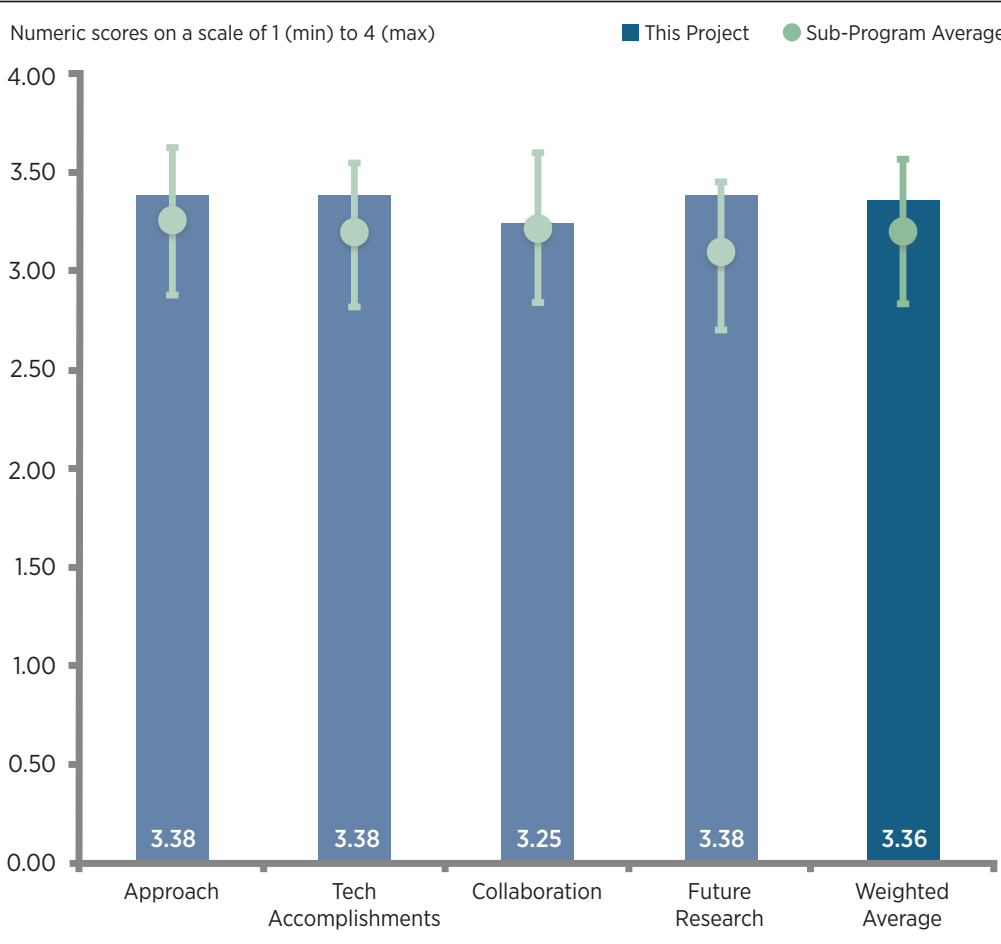

Relevant to DOE Objectives

Sufficiency of Resources

Figure 2-12 - Battery Thermal Characterization: Matthew Keyser (National Renewable Energy Laboratory) Electrochemical Energy Storage 
discussion it was apparent the project team helped suppliers save money in their designs and improve function, helping advance the market toward use by consumers.

\section{Reviewer 2:}

The reviewer found that the technical accomplishments are good as far as the presentation is concerned. However, the reviewer would like to see more conclusions regarding the effects of active and passive cooling and the various approaches to this important aspect of battery design. The calorimetry studies should be able to shed considerable light on these aspects.

\section{Reviewer 3:}

The reviewer remarked that the term efficiency used on a few slides is a little vague. The reviewer expressed that it would be a good idea to include a definition about how that is calculated. The reviewer remarked that the plots of heat rate during discharge of various cell chemistries was interesting, but rather than using a constant $\mathrm{C}$ discharge rates, it would be nice to see the dynamic stress test (DST) cycle used with multipliers, for example, or possibly a comparison of charge versus discharge heating rates. The reviewer pointed out that it is not easy to size a thermal system based on constant current (CC) discharges alone.

\section{Question 3: Collaboration and coordination with other institutions.}

\section{Reviewer 1:}

The reviewer remarked that collaboration was very good with government and industry, less so with university, but this is okay.

\section{Reviewer 2:}

The reviewer said that there does not seem to be a close collaboration with battery/vehicle designers, at least from the presentation. There is no mention of other U.S. producers such as Tesla Motors, which is the largest producer of EVs in the United States. The reviewer noted that cost of battery systems and cell or module replacement issues interact strongly with degradation issues, and the reviewer would like to see some interaction with DOE and USABC partners to make some policy recommendations in this arena.

\section{Reviewer 3:}

The reviewer commented that the data on the multiple cell chemistries is interesting; however, there seems to be a lack of collaboration with the modeling groups within the national laboratories.

\section{Question 4: Proposed future research-the degree to which the project has effectively planned its future work in a logical manner by incorporating appropriate decision points, considering barriers to the realization of the proposed technology and, when sensible, mitigating risk by providing alternate development pathways.}

\section{Reviewer 1:}

The reviewer commented good plans, the modeling work will extend the capabilities. The reviewer found that the planned coordination is well chosen and helpful.

\section{Reviewer 2:}

The reviewer acknowledged being happy to see collaboration with the modeling teams listed under future work. This seems to be the big missing piece in an otherwise good body of work.

\section{Reviewer 3:}

The reviewer would like to see an emphasis on applications to specific systems in use today to offer guidance to designers of active and passive cooling systems.

\section{Question 5: Does this project support the overall DOE objectives of petroleum displacement? Why or why not?}

\section{Reviewer 1:}

The reviewer said that these studies are highly relevant to the U.S. EV industry in order to improve petroleum 
displacement measures. The effects of temperature are profound in both calendar life and cycle life limitations of batteries in present use and the interaction of cost measures.

\section{Reviewer 2:}

The reviewer pointed out that high and/or uneven heating can destroy cells due to the change in how it then charges or discharges making the cell exist at various states of charge (SOC) in different places so that it can easily over- or under-charge and thus destroy those areas. This work helps avoid that situation so batteries will last a long time and be acceptable to the public.

\section{Reviewer 3:}

The reviewer said yes, these thermal studies are an important source of data for the USABC and other battery research projects. The reviewer also said that the capabilities NREL has to do these studies is not easily found elsewhere.

\section{Question 6: Resources: How sufficient are the resources for the project to achieve the stated milestones in a timely fashion?}

\section{Reviewer 1:}

The reviewer said that the backlog is acceptable. 


\section{Towards Solventless \\ Processing of Thick Electron- Beam (EB) Cured LIB Cathodes: David Wood (Oak Ridge National Laboratory) - es207}

\section{Presenter}

David Wood, Oak Ridge National Laboratory

\section{Reviewer Sample Size}

A total of four reviewers evaluated this project.

\section{Question 1: Approach to performing the work-the degree to which technical barriers are addressed, the project is well-designed, feasible, and integrated with other efforts.}

\section{Reviewer 1 :}

The reviewer said that this is a subtly complicated program. The development of a high-speed curing process begets challenges in the development of a high-speed coating process for the very viscous starting material. The reviewer commented that these are all interesting components; however, it can be a bit of a rabbit hole to develop one technique that demands the development of another in order to be effective.

\section{Reviewer 2:}

The reviewer said it is not very clear what the contribution is of the individual barriers identified to the cost savings. If established, it would be easier to prioritize and maintain focus.

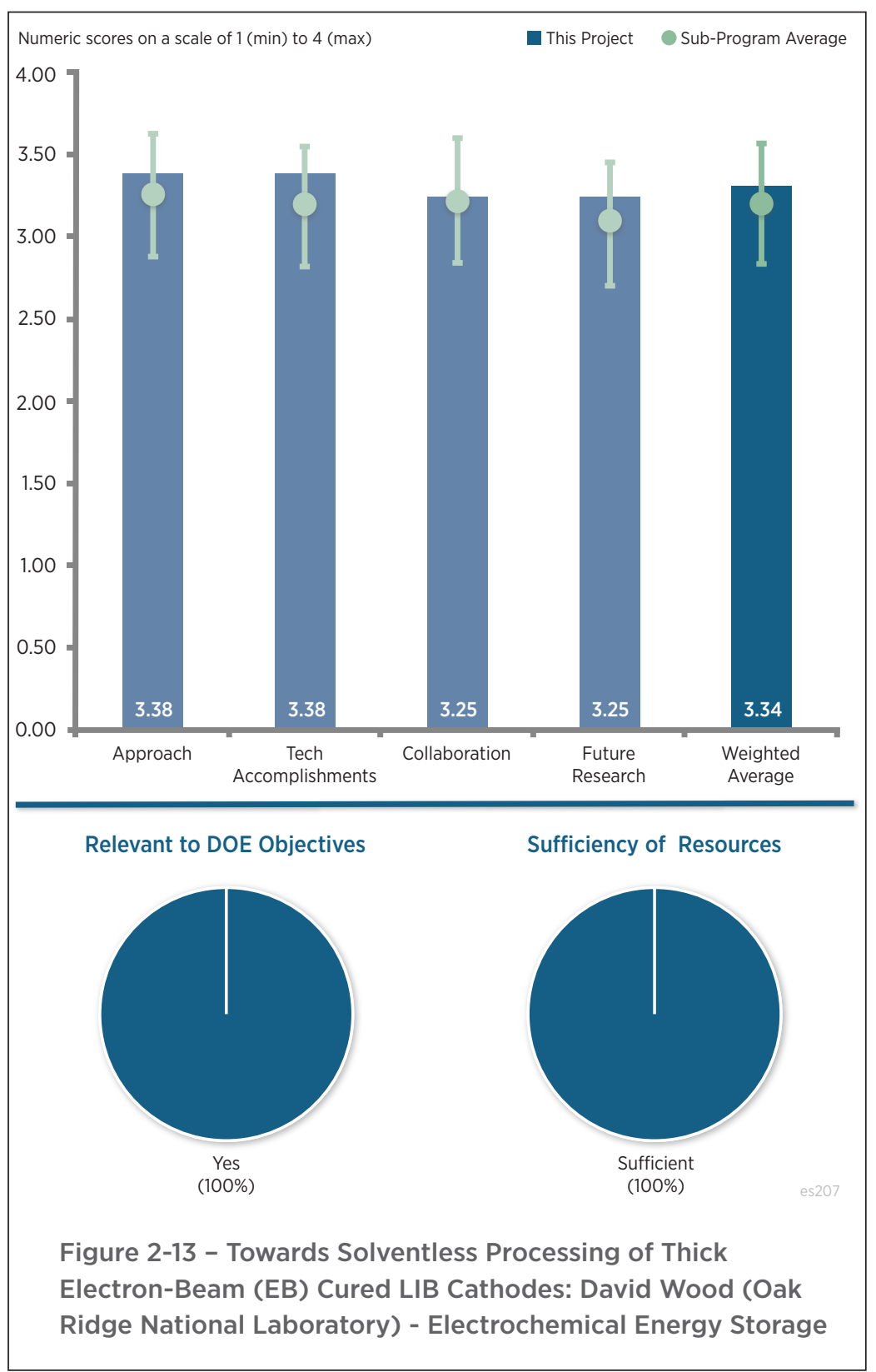

\section{Question 2: Technical accomplishments and progress toward overall project and DOE goals-the degree to which progress has been made, measured against performance indicators and demonstrated progress towards DOE goals.}

\section{Reviewer 1:}

The reviewer commented that the initial results look very promising and need to be quantified (cost/benefit analyses).

\section{Reviewer 2:}

The reviewer found that the interaction of a high-speed curing line with a high-speed coating line using curable polymers appears to be a greater challenge than initially considered. 


\section{Reviewer 3:}

The reviewer remarked that it would be useful to understand if choice of electrode formulation impacts the coating quality.

\section{Question 3: Collaboration and coordination with other institutions.}

\section{Reviewer 1:}

The reviewer commented that as with all programs of this type, it is critical that this technology has relevance within the manufacturing direction of commercial entities for it to be relevant. The reviewer said that it would have been good to hear about specific comparisons to any other competing techniques that may or may not be in development.

\section{Reviewer 2:}

The reviewer said that guidance from or the independent project evaluation of a major high-volume global cell supplier would be a tremendous benefit to the project.

\section{Reviewer 3:}

The reviewer remarked it will be helpful to identify the larger cell producer to validate the approach.

Question 4: Proposed future research-the degree to which the project has effectively planned its future work in a logical manner by incorporating appropriate decision points, considering barriers to the realization of the proposed technology and, when sensible, mitigating risk by providing alternate development pathways.

\section{Reviewer 1:}

The reviewer remarked that the project should include cost assessment/validation as more data are collected.

\section{Question 5: Does this project support the overall DOE objectives of petroleum} displacement? Why or why not?

\section{Reviewer 1:}

The reviewer said that the program addressed the need for the battery cost reduction.

\section{Question 6: Resources: How sufficient are the resources for the project to achieve the} stated milestones in a timely fashion?

\section{Reviewer 1:}

The reviewer said that resources were sufficient for perhaps developing the drying/curing portion of the program, and perhaps under-resourced if expanded into high-speed deposition of high-viscosity coatings. 


\section{New High-Energy \\ Electrochemical Couple for Automotive Applications: Khalil Amine (Argonne National Laboratory) - es208}

\section{Presenter}

Khalil Amine, Argonne National Laboratory

\section{Reviewer Sample Size}

A total of five reviewers evaluated this project.

\section{Question 1: Approach to performing the work-the degree to which technical barriers are addressed, the project is well-designed, feasible, and integrated with other efforts.}

\section{Reviewer 1:}

The reviewer said that the authors have focused on key barriers such as cathode and anode capacities to develop the next generation PHEV battery.

\section{Reviewer 2:}

The reviewer commented that the project is reducing the cost of the LIB through the energy density enhancement, and $\$ / \mathrm{kWh}$ reduction is one of the most relevant approaches for the automotive application.

\section{Reviewer 3:}

The reviewer detailed that the project's objective is to increase the specific energy to over $200 \mathrm{Wh} / \mathrm{kg}$ using Si-Sn anode and full gradient concentration cathode (FGC). The project team identified the first cycle irreversible loss issue of the $\mathrm{Si}-\mathrm{Sn}$ anode and mitigated it with a novel pre-lithiation technique that can be incorporated during the first charge. Milestones were clear and quantifiable. The reviewer found that the two-year schedule was reasonable to demonstrate specific energy improvement. The reviewer said that $200 \mathrm{Wh} / \mathrm{kg}$ should be achievable using graphite anode and FGC, and commented that the Si-Sn alloy anode seemed to introduce unnecessary risks.

\section{Reviewer 4:}

The reviewer remarked that this particular cathode/anode couple potentially improves energy density, while maintaining safety. The alloys do not take such a hit on electrode density, which is a good thing. The reviewer said that the pre-lithiation process is problematic due to the high first charge voltage required to access the Li, yet this was stated nowhere in the slides or the presentation in spite of repeated questions about downsides to the proposed approach.

\section{Question 2: Technical accomplishments and progress toward overall project and DOE goals-the degree to which progress has been made, measured against performance}




\section{indicators and demonstrated progress towards DOE goals.}

\section{Reviewer 1:}

The reviewer said that pre-lithiation may represent a significant advance.

\section{Reviewer 2:}

The reviewer said that this is a highly focused, well-organized innovative research project that led to several key results, such as the FCG cathode, as well as the discovery of the lithia for pre-lithiation. The authors deserve credit for their work.

The reviewer remarked that while the analytical and cell data show strong potential for the FCG cathode to be a viable material for PHEV applications, comprehensive data for durability and cost guidance would have been useful. A Mn-rich outer layer naturally raises the question of Mn-dissolution at high temperatures and high-temperature durability of the cells. The reviewer noted that only room temperature data for full cells were presented, and the author referenced other data using a lithium salt additive, but the reviewer was unsure this salt is a panacea for Mn dissolution. Additionally, the reviewer said that guidance for cost (because FCG does involve separate processing steps) would be useful. The reviewer wondered how cost-efficient the manufacturing is and how difficult it is to control the morphology in a cstr synthesis. The use of lithia as a pre-lithiationn agent is definitely a neat idea that will enable researchers to circumvent other alternative and cumbersome techniques. The reviewer said it was not clear how tedious it will be to incorporate lithia during the electrode manufacturing steps. The reviewer asked if one would need to work in a moisture-controlled atmosphere. The reviewer identified this as a major and costly challenge.

\section{Reviewer 3:}

The reviewer said that researchers demonstrated the feasibility of the high-capacity Si-Sn composite anode and the FGC cathode with limited cycle life, and also demonstrated feasibility of the Si-Sn + MAG composite with limited cycle life. To mitigate the high first cycle irreversible loss of the Si-Sn anode, researchers demonstrated a novel in situ pre-lithiation technique activating lithium oxide $\left(\mathrm{Li}_{2} \mathrm{O}\right)$ at high voltage at the cathode.

The reviewer said that the Si-Sn anode had high operating voltage versus Li and will require low discharge cutoff voltage (less than $2 \mathrm{~V}$ ) in a full cell to achieve full utilization. The approximately $2.5 \mathrm{~V}$ voltage swing at the cell level might pose a challenge for electronics at the battery pack level. The reviewer said that the researchers demonstrated the feasibility of cycle life and calendar life using the Si-Sn anode and FGC cathode. However, in order to claim that researchers mitigated the cycle life and calendar life barriers, the reviewer said that the team needed to have more than 50 cycles. According to the reviewer, the researchers projected specific energy greater than $200 \mathrm{Wh} / \mathrm{kg}$, but have yet to demonstrate achieving much greater than $200 \mathrm{Wh} / \mathrm{kg}$ using the Si-Sn anode and the FGC cathode in a full cell. To achieve the high capacity using the FGC cathode at high voltages, such as greater than $4.3 \mathrm{~V}$, the reviewer said that researchers need to address long-term stability of their electrolyte. The reviewer pointed out that $\mathrm{Li}_{2} \mathrm{O}$ is extremely hygroscopic and tends to form lithium carbonate $\left(\mathrm{Li}_{2} \mathrm{CO}_{3}\right)$ in air. Researchers need to provide some data on the processability of $\mathrm{Li}_{2} \mathrm{O}$ in a manufacturing environment, such as dry room.

\section{Reviewer 4:}

The reviewer remarked excellent progress on the cathode development, and the reviewer asked what the barriers are preventing its large adoption. The reviewer was not clear what is new in using Li salts for the pre-lithiation, and referenced G. Amatucci's work on MeF. The reviewer asked how it is different from the Li-excess in the cathode, and how will the physical mixing effect the cathode structures on charge. The reviewer inquired if there are results on the cycleability. The reviewer asked, in general, how will adding $\mathrm{Li}_{2} \mathrm{O}$ impact the slurry $\mathrm{pH}$, and asked about slurry gelling. The reviewer inquired how the high voltage of the activation will affect the electrolyte stability, and where will the oxygen go.

\section{Reviewer 5:}

The reviewer said that the project is complete, yet there was no cycle life on cells containing the Si anode. The reviewer said that the gradient material scale-up to $1 \mathrm{~kg}$ is significant, and while the pre-lithiation was explored, researchers needed to show the downsides of the approach.

\section{Question 3: Collaboration and coordination with other institutions.}




\section{Reviewer 1:}

The reviewer remarked excellent team for collaborative work.

\section{Reviewer 2:}

The reviewer commented excellent team of experts and institutional capabilities.

\section{Reviewer 3:}

The reviewer said that the team demonstrated good collaboration within ANL and with external ANL members. The roles of each team member was clearly identified to justify their participation in the project.

\section{Reviewer 4:}

The reviewer suggested that collaboration with a cell partner might have been good as a check on the overall materials that the team ended up with; otherwise, team members appeared to have each contributed

\section{Reviewer 5:}

The reviewer remarked there was limited collaboration beyond ANL, and the team should have reached out to OEM coaters when the CAMP lab was struggling.

Question 4: Proposed future research-the degree to which the project has effectively planned its future work in a logical manner by incorporating appropriate decision points, considering barriers to the realization of the proposed technology and, when sensible, mitigating risk by providing alternate development pathways.

\section{Reviewer 1:}

The reviewer said that the project has ended.

\section{Reviewer 2:}

The reviewer said that the project is complete, so not relevant

\section{Reviewer 3:}

The reviewer remarked project completed.

\section{Question 5: Does this project support the overall DOE objectives of petroleum displacement? Why or why not?}

\section{Reviewer 1:}

The reviewer said that the project definitely supports the DOE objectives of petroleum displacement.

\section{Reviewer 2:}

The reviewer said that the project is highly relevant for DOE objective of petroleum displacement by increasing the specific energy of the battery.

\section{Reviewer 3:}

The reviewer pointed out that high energy density electrochemical couple are necessary for the e-mobility success.

\section{Question 6: Resources: How sufficient are the resources for the project to achieve the stated milestones in a timely fashion?}

\section{Reviewer 1:}

The reviewer noted that the project is complete.

\section{Reviewer 2:}

The reviewer commented that the $\$ 2.5$ million over 2 years of funding was more than sufficient to achieve the greater than $200 \mathrm{Wh} / \mathrm{kg}$ goal. The reviewer commented that the funding should be sufficient to demonstrate greater than $200 \mathrm{Wh} / \mathrm{kg}$ using the Si-Sn and FGC cathode in a full cell, and to demonstrate cycle life and calendar life beyond feasibility using the high-capacity Si-Sn and FGC cathode. 


\section{High-Energy High-Power Battery Exceeding PHEV-40 Requirements: Jane Rempel (TIAX LLC) - es209}

\section{Presenter}

Jane Rempel, TIAX LCC

\section{Reviewer Sample Size}

A total of four reviewers evaluated this project.

\section{Question 1: Approach to performing the work-the degree to which technical barriers are addressed, the project is well-designed, feasible, and integrated with other efforts.}

\section{Reviewer 1:}

The reviewer said that using the project team's in-house developed cathode in combination with Sianode, the team had the objective of developing a PHEV-40 mile pack technology.

\section{Reviewer 2:}

The reviewer characterized the project as a try everything approach, which is unlikely to lead to large improvement, given all the work that has been done along these lines. Still, someone has to do this.

\section{Reviewer 3:}

The reviewer said the project seemed like an anode survey and applied development of an active material that was relatively optimized at the outset.

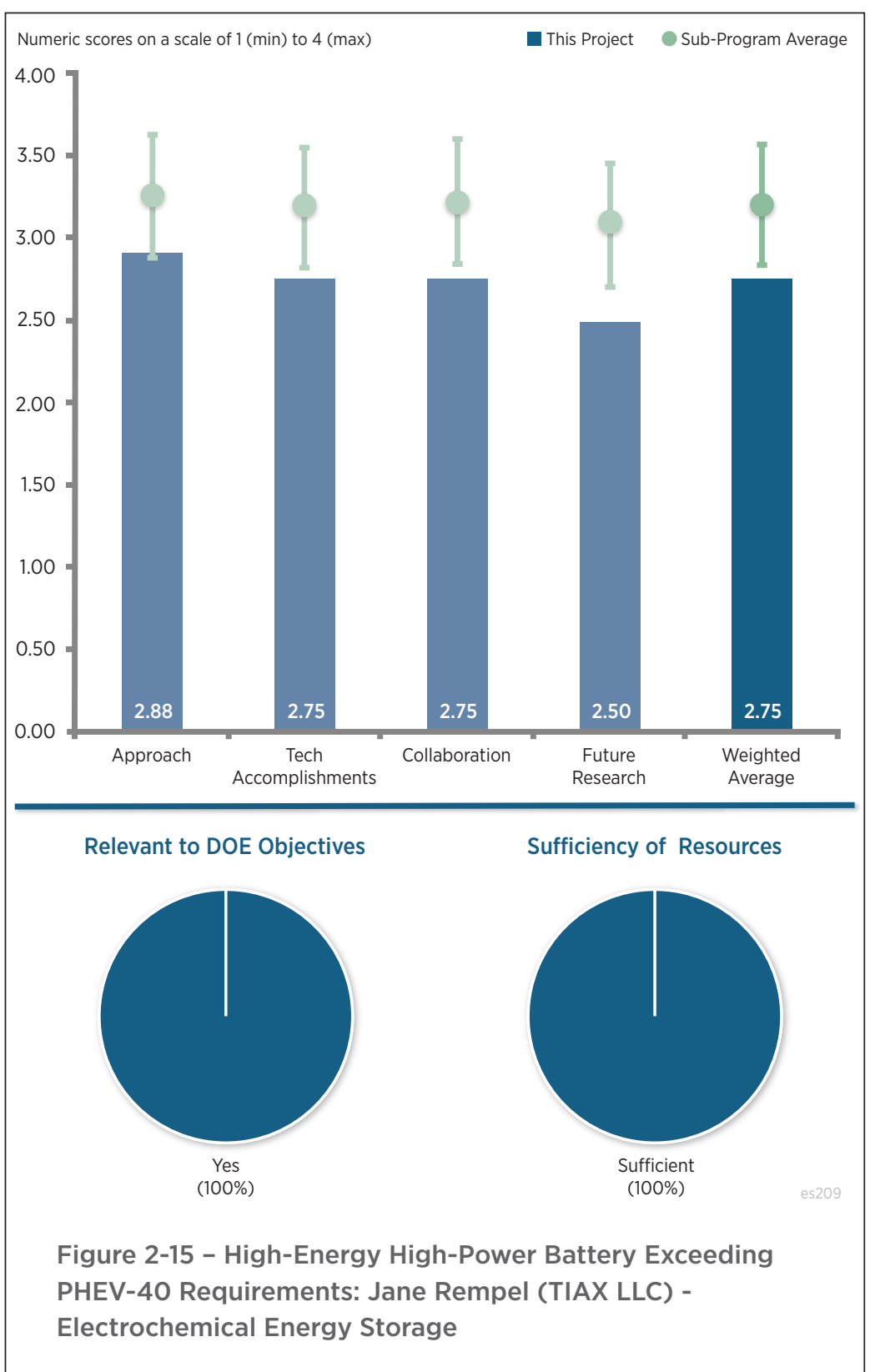

\section{Reviewer 4:}

The reviewer expressed uncertainty about what is novel here. CAM-7 has been around for a long time, and the reviewer said that rather standard Si anodes were used.

The reviewer would like to see more effects studied as other components were incorporated/varied (highperformance separators, binders, electrolytes, etc.). The reviewer asked if the team learned anything from the different combinations. The reviewer could not tell if there was development of the CAM-7 in this project, or if this is the same as in the past. If so, the reviewer would have liked to have seen the progression in performance.

Question 2: Technical accomplishments and progress toward overall project and DOE goals-the degree to which progress has been made, measured against performance indicators and demonstrated progress towards DOE goals. 


\section{Reviewer 1:}

The reviewer said that the CAM-7 data look quite impressive from performance and durability points of view. While the full cell with graphite show attractive life data, that with the Si-anode appears to be limited by the Si-anode performance, which is still an emerging technology (only limited cycle-life data shown and only at room temperature). The reviewer said that data on calendar-life and abuse would have been welcome.

\section{Reviewer 2:}

The reviewer observed decent cycle life against graphite, and poor cycle life against $\mathrm{Si}$. The reviewer also noted poor first cycle efficiency, and moderate to poor coulombic efficiency (CE). The reviewer said that it would have been nice to see a comparison with other state of the art Si systems.

\section{Reviewer 3:}

The reviewer said that many can show high energy density using a high-energy cathode and a Si anode, as did this project, but researchers have to couple that with long cycle life, which this project did not show. Even if researchers have poor cycle life, it is better to show the data at the same voltage for which you calculate the energy density than to show cycle life at a lower voltage. The reviewer acknowledged appreciating the work on electrode design that is too often overlooked.

\section{Reviewer 4:}

The reviewer said that the concept of a silicon oxide $\left(\mathrm{SiO}_{\mathrm{x}}\right)$ anode with high $\mathrm{Ni}$ cathode is already commercial, and the reviewer acknowledged missing what the accomplishment is here. The reviewer asked how this advanced the state of the art. The reviewer pointed out that abuse tolerance is a concern with this chemistry and needed to be addressed in a more relevant EV format than an 18650 lagging state of the art capacity and design.

\section{Question 3: Collaboration and coordination with other institutions.}

\section{Reviewer 1:}

The reviewer said that there were other partners, so that is good. However, the reviewer suggested using ANL's diagnostic capabilities more extensively to refine knowledge on failure mechanisms. Although Si material suppliers were acknowledged, it is not clear how much work was done with them or what their contribution was other than supply powder.

\section{Reviewer 2:}

The reviewer said that collaborations with teams who could carry out diagnostic work would have been a nice complement

\section{Reviewer 3:}

The reviewer said that the program was not collaborative.

\section{Question 4: Proposed future research-the degree to which the project has effectively planned its future work in a logical manner by incorporating appropriate decision points, considering barriers to the realization of the proposed technology and, when sensible, mitigating risk by providing alternate development pathways.}

\section{Reviewer 1:}

The reviewer said that the project has ended.

\section{Reviewer 2:}

The reviewer said that the program is complete.

\section{Reviewer 3:}

The reviewer said that the project is complete, so not a relevant question.

\section{Reviewer 4:}

The reviewer said that the project is completed. 
Question 5: Does this project support the overall DOE objectives of petroleum displacement? Why or why not?

Reviewer 1:

The reviewer said that the development of a durable, high-energy density cathode/battery supports the DOE objectives of petroleum displacement.

Question 6: Resources: How sufficient are the resources for the project to achieve the stated milestones in a timely fashion?

Reviewer 1:

The reviewer said that the project is complete, so not a relevant question. 


\section{Advanced High-Energy Li- Ion Cell for PHEV and EV Applications: Jagat Singh (3M) - es210}

\section{Presenter}

Jagat Singh, 3M

\section{Reviewer Sample Size}

A total of six reviewers evaluated this project.

\section{Question 1: Approach to performing the work-the degree to which technical barriers are addressed, the project is well-designed, feasible, and integrated with other efforts.}

\section{Reviewer 1 :}

The reviewer stated that this was a well-planned and well-coordinated effort to resolve key barriers to develop advanced battery technology.

\section{Reviewer 2:}

The reviewer stated that this is a good set of collaborators where each brings strength in specific cell components. The material set is very relevant and both the anode and cathode have significant challenges to be addressed.

\section{Reviewer 3:}

The reviewer stated that the approach is conventional.

\section{Reviewer 4:}

The reviewer stated that it was a good approach to use $3 \mathrm{M} \mathrm{Si}$ anode coupled with $3 \mathrm{M}$ high-V coated NMC to achieve the high specific energy. Excellent modeling projections of the specific energy and cycle life as a function of the charge cut-off voltages. The reviewer stated that two and a half years is a reasonable schedule for this project. The reviewer noted that milestones were not quantifiable and that descriptions in the milestone table were task descriptions, not milestones.

\section{Reviewer 5:}

The reviewer stated that in general it was a well-structured approach. A clearer definition of targets to be demonstrated would have helped in this project. It was not always clear as to how choices were made between the various cases studied.

Question 2: Technical accomplishments and progress toward overall project and DOE goals-the degree to which progress has been made, measured against performance indicators and demonstrated progress towards DOE goals.

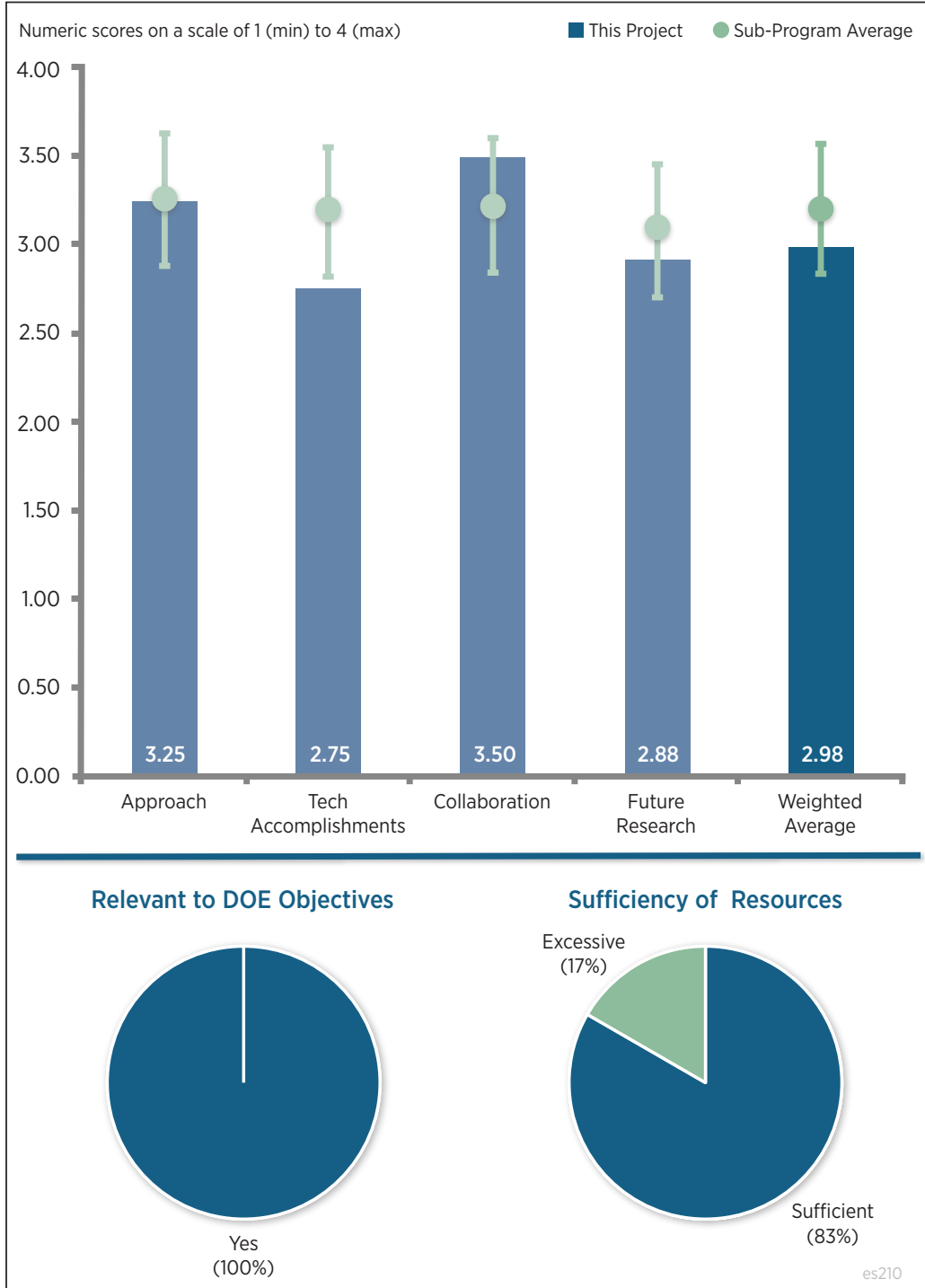

Figure 2-16 - Advanced High-Energy Li-Ion Cell for PHEV and EV Applications: Jagat Singh (3M) - Electrochemical Energy Storage 


\section{Reviewer 1:}

The reviewer stated that the team demonstrated over $250 \mathrm{Wh} / \mathrm{kg}$ at $1 \mathrm{C}$ rate and good cycle life over 100 cycles. Unlike other teams which only presented room temperature performance data, this team actually provided performance data at other temperatures. The reviewer stated that the project team needs a high charge cut-off voltage for the high specific energy achieved, long-term stability of the high-energy cells are unclear due to instability of electrolyte at high voltages. It was unclear if the Army Research Lab (ARL) high voltage electrolyte was used in their cell that demonstrated over $250 \mathrm{Wh} / \mathrm{kg}$.

\section{Reviewer 2:}

The reviewer referenced the same issue as indicated above. Cycle life development was unconvincing, as data were lacking at this point in the project. The reviewer stated that low-temperature performance was unimpressive, for an EV-based goal. Also, that a lack of reporting on root cause analysis leads to questions, regarding some of the design selections made.

\section{Reviewer 3:}

The reviewer stated that the technical challenges here are very difficult, but considering the strength of the team, the progress is disappointing. This reviewer noted improvement in Si capacity and cycle efficiency, but pointed out that cycle life is only out to 100 cycles. There were no significant advancements in electrolytes.

\section{Reviewer 4:}

The reviewer noted that energy density is not very high. Cycling life is only 100 cycles and power capability is also low. It should also be noted the project team did C-rate test using 100\% SOC but cycling using 5-95\% SOC (maybe to help extend cycle life), and use $35 \%$ capacity loss as threshold for cycling end of life (20\% capacity loss is well-accepted for end-of-life).

The reviewer noted that state-of-the-art 18650 cells (Panasonic, LG, or Sony) can now reach around 3.4 to 3.5 Ah. Tesla has used 3.2 to $3.3 \mathrm{Ah} 18650$ cells for many years. Both Tesla 18650 cells and iPhone pouch cells can reach $260 \mathrm{Wh} / \mathrm{kg}$ at low rate and have better power than those in the slides; and they can be used for 1,000 cycles. The reviewer commented that current Tesla and iPhone cells are only charged to $4.2 \mathrm{~V}$ or $4.3 \mathrm{~V}$. Increasing $4.2 \mathrm{~V}$ to 4.5 $\mathrm{V}$ or even $4.6 \mathrm{~V}$, one can obtain $20 \%$ more energy density. The reviewer stated that the use of the word, "excellent," on page six is pretty far out there and that Slide 7 is hardly something of which to be proud.

\section{Question 3: Collaboration and coordination with other institutions.}

\section{Reviewer 1:}

The reviewer stated that this was an excellent team for collaboration encompassing key cell components with OEM, labs, a university, as well as developers.

\section{Reviewer 2:}

The reviewer thought that it was a well-picked team. Responsibilities were well-assigned and the project appeared to have been very well-managed.

\section{Reviewer 3:}

The reviewer stated that there was good collaboration between industry, national laboratories, and ARL. The roles and responsibilities for each team member were clearly specified, however, it was not clear the contribution of each team member in the demonstrated high energy cell.

\section{Reviewer 4:}

The reviewer noted that there was a good breadth of skills and technologies. This is the kind of team that really could make a breakthrough, which is why the reviewer was disappointed.

\section{Reviewer 5:}

The reviewer stated that it was not clear what ARL or Dr. Jow's roles were.

Question 4: Proposed future research-the degree to which the project has effectively planned its future work in a logical manner by incorporating appropriate decision points, 


\section{considering barriers to the realization of the proposed technology and, when sensible, mitigating risk by providing alternate development pathways.}

\section{Reviewer 1:}

The reviewer stated that this question was not scored, as project has ended. It was indicated that additional characterization testing will be performed by the national laboratories. This will be very important in evaluating the merits of the approaches taken within this program.

\section{Reviewer 2:}

The reviewer noted that the project is complete.

\section{Reviewer 3:}

The reviewer stated that the project is complete, so this question is not so relevant

\section{Reviewer 4:}

The reviewer stated that the future work is sharply focused on key aspects of the work ( $\mathrm{Si}$ alloy anode, high energy NMC, and electrolyte additives). The reviewer recommended that for future presentations, include all test conditions on slides and any guidance on mechanisms/cost of new materials that improve the performance such as electrolyte additives.

\section{Reviewer 5:}

The reviewer stated that it is an unsuccessful project.

\section{Question 5: Does this project support the overall DOE objectives of petroleum displacement? Why or why not?}

\section{Reviewer 1:}

The reviewer stated that if the goal is to lower EV battery costs and improve energy, then yes.

\section{Reviewer 2:}

The reviewer stated that this was highly relevant because high specific energy is critical to achieving petroleum displacement.

\section{Reviewer 3:}

The reviewer stated that the focus on higher voltage and reducing anode ICL are key targets to improve EV battery performance.

\section{Question 6: Resources: How sufficient are the resources for the project to achieve the stated milestones in a timely fashion?}

\section{Reviewer 1:}

The reviewer stated that the project ran slightly longer than planned, but found no apparent issues.

\section{Reviewer 2:}

The reviewer stated that there was sufficient resources for energy improvement over two and a half years.

\section{Reviewer 3:}

The reviewer stated that the scope seemed a bit broad.

\section{Reviewer 4:}

The reviewer reiterated that the project is complete, so this does not really matter

\section{Reviewer 5:}

The reviewer noted that Slide 2 says that the program end date is September 30, 2015, but is $15 \%$ complete. The reviewer questioned what happened.

\section{Reviewer 6:}

The reviewer stated that way too many resources were put into this project, considering that there were so many known problems with this material. 


\section{High-Energy Lithium Batteries for PHEV Applications: Subramanian Venkatachala (Envia Systems) - es211}

\section{Presenter}

Subramanian Venkatachala, Envia Systems

\section{Reviewer Sample Size}

A total of five reviewers evaluated this project.

\section{Question 1: Approach to performing the work-the degree to which technical barriers are addressed, the project is well-designed, feasible, and integrated with other efforts.}

\section{Reviewer 1:}

The reviewer thought that the team has done outstanding work in trying to improve upon the properties of this high energy cathode material.

\section{Reviewer 2:}

The reviewer stated that the initial approach to use coatings to eliminate the $\mathrm{DC}$ resistance of $\mathrm{Li}$-rich $\mathrm{NMC}$ at low SOC was a good one (unfortunately, it did not work). The reviewer appreciated the flexibility of the team to switch to an alternative cathode when the first approach did not work. There was good fundamental work by this team but marginal improvements demonstrated.

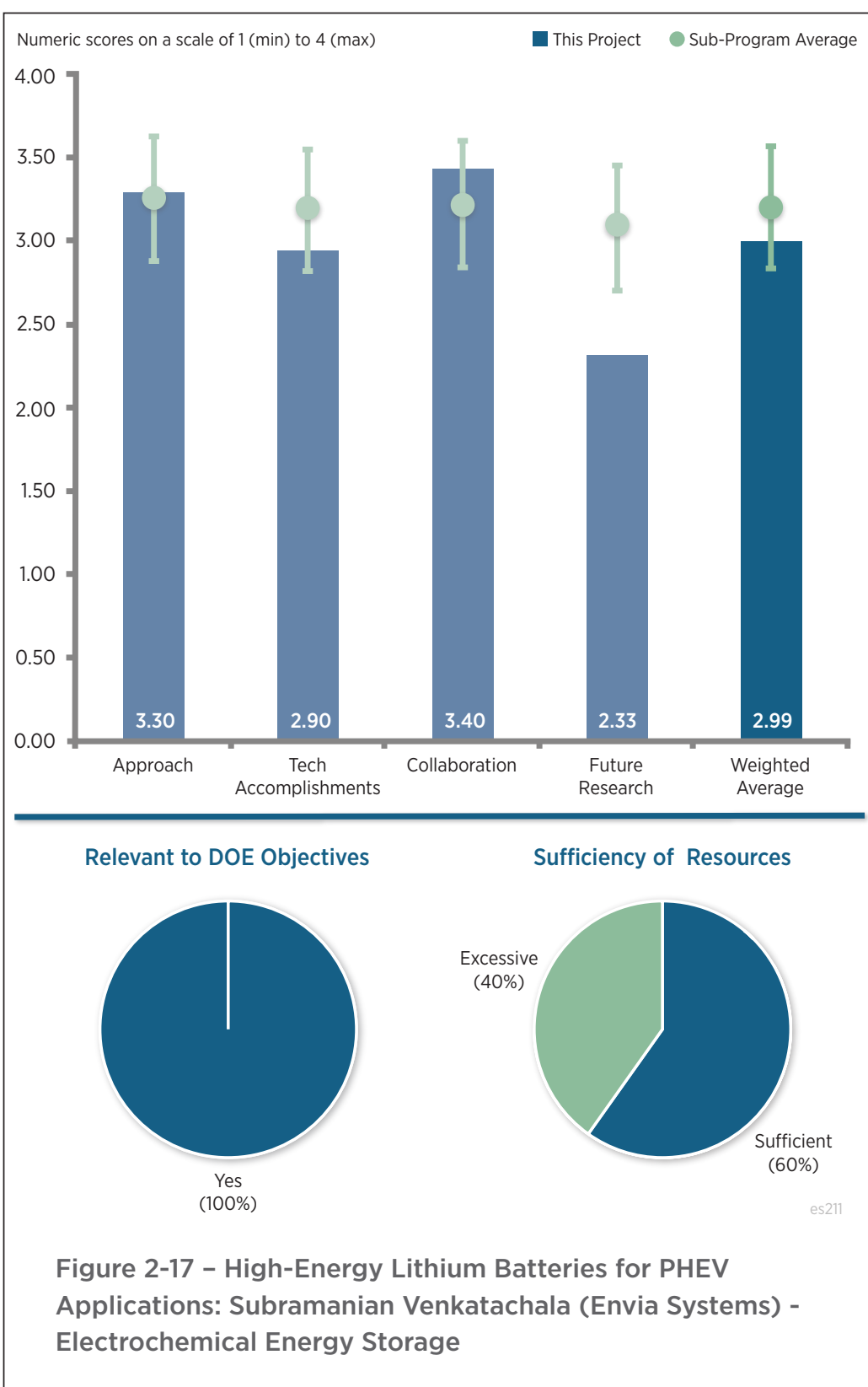

\section{Reviewer 3:}

The reviewer noted that the objective is to increase specific energy to over $200 \mathrm{Wh} / \mathrm{kg}$ using Si/C composite anode and high-capacity Mg-rich (HCMR) Li-rich cathode material. The project team identified and mitigated the power fade issue with the HCMR material at low SOC, and with cycling. The team showed the tasks, subtasks and timeline clearly in a table. The area specific impedance (ASI) milestones were clear and quantifiable. The presentation described the coating and composition efforts succinctly to show the effectiveness of composition optimization in mitigating the power fade issue. Two and a half years was a reasonable schedule to demonstrate specific capacity improvement.

The modeling data were not clear, it would have been more useful to model the ASI as a function of SOC. Si-based anode coupled with NMC should be sufficient to demonstrate over $200 \mathrm{Wh} / \mathrm{kg}$.

\section{Reviewer 4:}

The reviewer stated that it seems like everything but the kitchen sink was thrown into this project, rather than focusing on a few areas that were most likely to have a payoff. 


\section{Question 2: Technical accomplishments and progress toward overall project and DOE goals-the degree to which progress has been made, measured against performance indicators and demonstrated progress towards DOE goals.}

\section{Reviewer 1:}

The reviewer stated that while the issues of HCMR are well-known, the team has carried out highly focused and comprehensive studies to improve upon the vexing issues of voltage fade and durability. The results on materials manipulation, diagnostic and modeling are extensive and very useful even though the HCMR has turned out to be a hard nut to crack.

While the compositional changes brought about some significant improvement in the resistance of the materials, it is unfortunate that a lot of effort was aimed at surface modification, which turned out to be a futile attempt. However, the diagnostic studies were really well-planned and thorough. These results build the core of these studies and the authors have done an excellent job in analyzing the significant amount of especially spectroscopic and electrochemical data. Making the HCMR or a blend of HCMR and NMC to cycle to a charge voltage lower than that that does not give the expected energy boost might only have an appeal if there is a significant cost and safety advantages are there. It is not clear from the data that it is the case. The reviewer really loved the slides.

\section{Reviewer 2:}

The reviewer stated that the team clearly understood the issues of power fade and capacity fade with the HCMR cathode. The team demonstrated the effectiveness of composition modification in mitigating the power fade issue. The team demonstrated $200 \mathrm{Wh} / \mathrm{kg}$ at $1 \mathrm{C}$, without using the high $\mathrm{V}$ cathodes that requires over $4.3 \mathrm{~V}$. The team also demonstrated good cycle, greater than 300 cycles to $80 \%$ capacity retention. The high specific energy was not achieved by using very high cathode cut-off of over $4.3 \mathrm{~V}$, thus should be less prone to stability issue. No data were presented regarding the voltage fade with cycling issue.

\section{Reviewer 3:}

The reviewer stated that there were marginal improvements (this was a difficult task), but more work should be/ have been done on the blends.

\section{Reviewer 4:}

The reviewer stated that there was limited anode work or progress. Payoff in cathode progress for amount invested seems somewhat incremental.

\section{Reviewer 5:}

The reviewer stated that $200 \mathrm{Wh} / \mathrm{kg}$ at $1 \mathrm{C}$, plus 300-500 cycles should not have been the team's initial target. Under such a power requirement, one can easily get cells with over $220 \mathrm{Wh} / \mathrm{kg}$ at $1 \mathrm{C}$ and over 1,500 cycles from any of the major suppliers. In fact, Envia has better cells (greater than $320 \mathrm{Wh} / \mathrm{kg}$ ) with a different cathode and anode, which perhaps are not involved in the DOE/General Motors (GM) projects.

\section{Question 3: Collaboration and coordination with other institutions.}

\section{Reviewer 1:}

The reviewer stated that this was an enormous multi-institution effort.

\section{Reviewer 2:}

The reviewer thought it was a good team that tried a lot of different things. The team gained fundamental understanding due to team members with different skill sets

\section{Reviewer 3:}

The reviewer stated there was excellent collaboration among teams that really complement each other's expertise such as ORNL, Lawrence Berkeley National Laboratory (LBNL), etc.

\section{Reviewer 4:}

The reviewer thought that the team demonstrated good collaboration within Envia and with external Envia members. The roles of each team member was clearly identified to justify their participation in the project. 


\section{Reviewer 5:}

The reviewer stated that it seems to be a heavily Envia effort and no sign that decisions were driven by partner input despite apparent \$1.6 million effort at partners. The surface stabilization efforts looked similar to work done elsewhere, notably ANL, and seems like there is potential overlap or need for more collaboration.

Question 4: Proposed future research-the degree to which the project has effectively planned its future work in a logical manner by incorporating appropriate decision points, considering barriers to the realization of the proposed technology and, when sensible, mitigating risk by providing alternate development pathways.

\section{Reviewer 1:}

The reviewer stated that the project has ended.

\section{Reviewer 2:}

The reviewer noted that the project is complete.

\section{Reviewer 3:}

The reviewer stated that the project is complete, so not relevant.

\section{Reviewer 4:}

The reviewer thought that this was a failed project.

\section{Question 5: Does this project support the overall DOE objectives of petroleum} displacement? Why or why not?

\section{Reviewer 1:}

The reviewer stated definitely.

\section{Reviewer 2:}

The reviewer stated that this was highly relevant for the DOE objective of petroleum displacement by increasing the specific energy of the battery.

\section{Question 6: Resources: How sufficient are the resources for the project to achieve the stated milestones}

\section{Reviewer 1:}

The reviewer commented that the $\$ 3.8$ million effort was appropriate given the amount of work and the demonstrated results.

\section{Reviewer 2:}

The reviewer was not sure why project was continued absent progress on high-V stability.

\section{Reviewer 3:}

The reviewer thought that way too many resources were put into this project, considering that there were so many known problems with this material. 


\section{High-Energy, Long Cycle Life Lithium-Ion Batteries for EV Applications: Donghai Wang (Pennsylvania State University) - es212}

\section{Presenter}

Donghai Wang, Pennsylvania State University

\section{Reviewer Sample Size}

A total of six reviewers evaluated this project.

\section{Question 1: Approach to performing the work-the degree to which technical barriers are addressed, the project is well-designed, feasible, and integrated with other efforts.}

\section{Reviewer 1 :}

The reviewer found that the project is focused, well-organized, and addresses all key barriers using proven approaches and powerful diagnostic tools.

\section{Reviewer 2:}

The reviewer commented that this was a well-organized, carefully thought out set of coordinated experiments.

\section{Reviewer 3:}

The reviewer thought that it was a good approach to use Ni-rich layered oxide coupled with $\mathrm{Si}$-alloy/C anode to achieve greater than $330 \mathrm{Wh} / \mathrm{kg}$. To achieve greater than 500 cycles, their approach was also reasonable, to use functional conductive binder, fluorinated electrolytes and additives, and pre-lithiation. Quantifiable milestones were provided, but a schedule was not provided. The period of performance was three years, which might be sufficient to demonstrate technologies feasible of achieving the specific energy and cycle life.

\section{Reviewer 4:}

The reviewer appreciated the attention to electrode loading and abuse tolerance.

\section{Reviewer 5:}

The reviewer stated that the approaches basically addressed most of the critical issues associated with high energy density electrode materials and are well laid-out.

\section{Reviewer 6:}

The reviewer stated that it was an excellent choice to include scale up of the material in this project. Good fundamental work with the secondary ion mass spectrometry (SIMS) analysis and good performance of the cathode material in graphite cells. However, disappointing results on the anode (this project was not alone in that regard). 


\section{Question 2: Technical accomplishments and progress toward overall project and DOE goals-the degree to which progress has been made, measured against performance indicators and demonstrated progress towards DOE goals.}

\section{Reviewer 1:}

The reviewer stated that the team has made significant progress on different components. The integrity of the optimized electrodes, electrolyte, and binder, including the pre-lithiation, is well done.

\section{Reviewer 2:}

The reviewer stated that there was good progress on the cathode, especially synthesis. However, less good progress on anode. Results on analytical are very interesting and can form the basis of new projects

\section{Reviewer 3:}

The reviewer stated that the authors have investigated several well-established techniques to enhance the durability of Ni-rich cathodes which initially deliver a capacity of about 200 millampere-hour (mAh)/g but unfortunately show considerable fade. The approaches show somewhat similar improvement in their durability at room temperature, but the full cell performance needs to be improved, as the authors recognize. The diagnostic data, especially those derived using time of flight (TOF), are highly insightful. The Si-anode results show promise but considerable work remains to be done.

\section{Reviewer 4:}

The reviewer stated that high quality cathodes were developed. Si anodes have poor cycle life, as demonstrated in Slides 12 and 13. There was also good electrolyte development. The reviewer commended the authors for the CE graph on Slide 15, with efficiency going from $90 \%$ to $100 \%$, instead of hiding the results going from $0 \%$ to $100 \%$. Finally the reviewer stated that first cycle efficiency needs to improve.

\section{Reviewer 5:}

The reviewer stated that the team leveraged TOF-SIMS to quantify the decomposition products, which resulted from Al-doped FGC cathode, on anode and cathode. The project team demonstrated pre-lithiation using stable Li-metal powder to improve coulombic efficiency from $80 \%$ to close to $99 \%$. Fluorinated electrolyte seemed to improve cycle life over 50 limited cycles.

The reviewer said that surface coating seemed to improve the cycle life of the Ni-rich layered oxide, but it was not clear if the team mitigated the cycle life voltage sag issue with surface modification. Even though the approach was to use the Ni-rich layered oxide to achieve the high specific energy, electrolyte optimization, electrode optimization, and pre-lithiation in a pouch-type full cell was based on NMC523, not the Ni-rich layered oxide. Because NMC71515 is less stable than NMC523, the team would have to requalify the NMC71515 with the fluorinated electrolyte, the pre-lithiation, and the electrode optimization. The project team demonstrated limited cycle life using the functional conductive binder at a Si-graphite loading of $4.9 \mathrm{mAh} / \mathrm{cm}^{2}$ in a half cell, but no full cell data based on this binder. The team has yet to demonstrate a full cell using the Ni-rich layered oxide coupled with the Si-graphite anode, as was described in their approaches.

\section{Question 3: Collaboration and coordination with other institutions.}

\section{Reviewer 1:}

The reviewer stated that there was good collaboration among the teams.

\section{Reviewer 2:}

The reviewer thought that the co-presentation showed good collaborative participation from two participants. There are a variety of efforts here, ranging from cathode to anode to scale-up to analytical. It showed a good comprehensive team and skill sets.

\section{Reviewer 3:}

The reviewer stated that there was good team composition with the roles and responsibilities of each team member clearly specified. 


\section{Reviewer 4:}

The reviewer stated that the team and the collaborators are well known experts in the field from different aspects.

\section{Reviewer 5:}

The reviewer stated that the project may have benefited from a manufacturing partner on the team.

\section{Question 4: Proposed future research-the degree to which the project has effectively planned its future work in a logical manner by incorporating appropriate decision points, considering barriers to the realization of the proposed technology and, when sensible, mitigating risk by providing alternate development pathways.}

\section{Reviewer 1:}

The reviewer stated that there were promising results on many fronts. This work should be encouraged.

\section{Reviewer 2:}

The reviewer stated that the future work addressed the remaining challenges. The plans are realistic and achievable in the remaining project period.

\section{Reviewer 3:}

The reviewer stated that there are about three months left before the project completion in September 2016. The team's main focus should be to integrate the improved components (Ni-rich layered cathode, Si-graphite anode, functional conductive binder, fluorinated electrolyte, and pre-lithiation) in a full cell and demonstrate specific energy and cycle life.

\section{Reviewer 4:}

The reviewer stated that the team is well qualified to advance understanding and suggest solutions on thermal runaway mitigation. The reviewer would like to see remaining resources continue to address that.

\section{Reviewer 5:}

The reviewer noted that while the team has focused more on cathode material (bulk/surface) properties, it is possible that the use of suitable electrolytes might improve further the performance, especially at high temperatures. Thus the reviewer recommended expanding the work on electrolyte. As is well-known, additives are known to considerably improve the durability of high Ni content cathode performance.

\section{Reviewer 6:}

The reviewer suggested the team focus more on improving the cycle life of the $\mathrm{Si}$ at current capacity, rather than improving capacity. Pre-lithiation is being worked on by others, let them do it; get your cycle life improved without pre-lithiation (that is just a band aid). The reviewer believed that this is the right team to address these challenges

\section{Question 5: Does this project support the overall DOE objectives of petroleum displacement? Why or why not?}

\section{Reviewer 1:}

The reviewer stated definitely.

\section{Reviewer 2:}

The reviewer stated that the project was highly relevant because high cycle life at high specific energy are critical to achieving petroleum displacement.

\section{Reviewer 3:}

The reviewer stated that the project addressed the barriers for the high energy battery well. If successful, it can meet the DOE objectives.

Question 6: Resources: How sufficient are the resources for the project to achieve the stated milestones in a timely fashion? 


\section{Reviewer 1:}

The reviewer stated that it was a good team with lots of results. With focus, this team can make progress on remaining challenges

Reviewer 2:

The reviewer stated that there were sufficient resources for energy improvement over three years.

Reviewer 3:

The reviewer commented that the resources are sufficient. The team members and collaborators have all the necessary expertise and sources to address the barriers. 


\section{High-Energy Density Li- ion Cells for EVs Based on Novel, High Voltage Cathode Material Systems: Keith Kepler (Farasis) - es213}

\section{Presenter}

Michael Slater, Farasis

\section{Reviewer Sample Size}

A total of six reviewers evaluated this project.

\section{Question 1: Approach to performing the work-the degree to which technical barriers are addressed, the project is well-designed, feasible, and integrated with other efforts.}

\section{Reviewer 1:}

The reviewer stated that the multifaceted cathode approach was very good. Sufficient work on the anode was demonstrated.

\section{Reviewer 2:}

The reviewer noted that the research team's claim that nanowires, with less surface area than spheres, should have better first cycle efficiency. This is reasonable, but has been tried before without much success. Also, the reviewer did not see where the project team reported their values. Finally, the ion exchange synthesis has some potential.

\section{Reviewer 3:}

The reviewer was glad to see someone looking further into Chris Johnson's excellent work on the ion exchange approach. It was also good to see the project team working on nanowires, a different approach from most of the other projects. Same for the titanium-modified (Ti) NMC (even though it did not work out). The reviewer would like to see more projects like this that explore different approaches.

\section{Reviewer 4:}

The reviewer stated that the approach was to leverage high capacity layered-layered metal oxide coupled with Sibased anode to achieve the specific energy goal. Two and a half years is reasonable to demonstrate the feasibility of achieving the specific energy goal. However, the milestones were not quantified, and the team should provide some modeling data on how they plan to achieve greater than $350 \mathrm{Wh} / \mathrm{kg}$ using their Si anode that only had two times higher specific capacity than that of graphite.

\section{Question 2: Technical accomplishments and progress toward overall project and DOE goals-the degree to which progress has been made, measured against performance indicators and demonstrated progress towards DOE goals.}




\section{Reviewer 1:}

The reviewer stated that the progress seemed moderate on anode and electrolyte.

\section{Reviewer 2:}

The reviewer stated that the program would have benefited from compiling results in a gap chart. It was not clear as to how great the remaining gaps were.

\section{Reviewer 3:}

The reviewer stated that the results were okay for the novelty of the approach. The reviewer appreciated the team's point on the voltage window of 4.4-3 V, however the good cycle life cannot really be compared to others cycling over a wider voltage range. It would have been good to show the cycle life over a wider voltage range for the $\mathrm{NMC}$ /graphite. Also there was not much progress on the Si anode. Furthermore, the presenter said that there was extensive electrolyte screening, but the reviewer saw no evidence of that and questioned the number of different electrolytes. The reviewer also asked the team if it could learn anything based on what worked and what did not work.

\section{Reviewer 4:}

The reviewer noted that the team demonstrated several methods to mitigate the voltage stability and impedance issues of the high-capacity layered NMC materials. The team also demonstrated the potential cycle life benefit of fluorinated solvents while highlighting the gassing issue due to fluorinated solvents. Specific capacity of Generation 2 cells was not provided, only cycle life compared to capacity retention was presented. The team modified lithium manganese rich-NCM (LMR) via ion-x to improve the rate capability and showed decrease in impedance, but there was no data at the full cell level. The reviewer pointed out that there was also no data demonstrating the mitigation of the voltage sag issue.

\section{Reviewer 5:}

The reviewer thought that overall, the project team's Si results are poor. The statement in Slide 11 that their material is cost-effective is not supported. Finally, Ti substitution does not improve things very much, according to Slide 15 , up to only 25 cycles.

\section{Question 3: Collaboration and coordination with other institutions.}

\section{Reviewer 1:}

The reviewer found that there was a good set of partners and balance of contribution and capabilities, which set the standard for this group of awards.

\section{Reviewer 2:}

The reviewer thought that the collaboration appeared to be comprehensive and effective.

\section{Reviewer 3:}

The reviewer stated that there was a good team and a diverse set of materials. Materials developers (e.g., OneD, DuPont) can have trouble in optimizing a single component as they do not have the whole system. This project is a good example of how they can be integrated into a larger effort with other component developers.

\section{Reviewer 4:}

The reviewer noted that there was good team composition with the roles and responsibilities of each team member clearly specified.

Question 4: Proposed future research-the degree to which the project has effectively planned its future work in a logical manner by incorporating appropriate decision points, considering barriers to the realization of the proposed technology and, when sensible, mitigating risk by providing alternate development pathways.

\section{Reviewer 1:}

The reviewer stated that the project has ended. 


\section{Reviewer 2:}

The reviewer stated that the program is complete, so this question was left blank. It will be essential to review the final test results from the labs once testing is complete, in order to verify that this technical approach was successful.

\section{Reviewer 3:}

The reviewer stated that the project is complete, so this question is not relevant.

\section{Reviewer 4:}

The reviewer stated that the project is completed.

\section{Reviewer 5:}

The reviewer stated that the project should be ended.

\section{Question 5: Does this project support the overall DOE objectives of petroleum displacement? Why or why not?}

\section{Reviewer 1:}

The reviewer stated that the specific goals to improve energy density, and lower overall energy storage costs, appear to have been kept central to the program.

\section{Reviewer 2:}

The reviewer stated that the project was highly relevant because high specific energy is critical to achieving petro displacement.

\section{Question 6: Resources: How sufficient are the resources for the project to achieve the stated milestones in a timely fashion?}

\section{Reviewer 1:}

The reviewer noted that Farasis did respond to the concern on the anode research. The no-cost extension applied made sense, given the scope of the project.

\section{Reviewer 2:}

The reviewer stated that the project is complete, so not relevant.

\section{Reviewer 3:}

The reviewer stated that $\$ 3.5$ million over two and a half years should be sufficient to demonstrate the specific energy goals.

\section{Reviewer 4:}

The reviewer commented that funds were possibly excessive, but funding splits were not provided. 


\section{Fundamental Studies of Lithium-Sulfur Cell Chemistry: Nitash Balsara (Lawrence Berkeley National Laboratory) - es224}

\section{Presenter}

Nitash Balsara, Lawrence Berkeley National Laboratory

\section{Reviewer Sample Size}

A total of three reviewers evaluated this project.

\section{Question 1: Approach to performing the work-the degree to which technical barriers are addressed, the project is well-designed, feasible, and integrated with other efforts.}

\section{Reviewer 1:}

The reviewer thought that the project team's approach is relatively unique and should add light to what remains a challenging problem and technology.

\section{Reviewer 2:}

The reviewer stated that the project combines multiple tools to probe the speciation of the polysulfide solutions in different solvents. The project also designed in situ cells for X-ray based techniques. The study promises to improve our understanding of the solution chemistry in order to improve Li-S battery performance.

\section{Reviewer 3:}

The reviewer noted that the approach is to use calculations and in situ diagnostics to understand the reaction products in $\mathrm{Li} / \mathrm{S}$ cells during cycling. The approach seems good, although the reviewer wondered about the use of symmetric polystyrene-block-poly (ethylene oxide) (SEO)-based electrolytes for some of these studies as those are not used in actual cells.

\section{Question 2: Technical accomplishments and progress toward overall project and DOE goals-the degree to which progress has been made, measured against performance indicators and demonstrated progress towards DOE goals.}

\section{Reviewer 1:}

The reviewer stated that there was good progress and all milestones planned to be met, have been met, or are on track. Team has succeeded in building cells to permit in situ X-ray absorption spectroscopy (XAS) studies through the anode and cathode side of the cell.

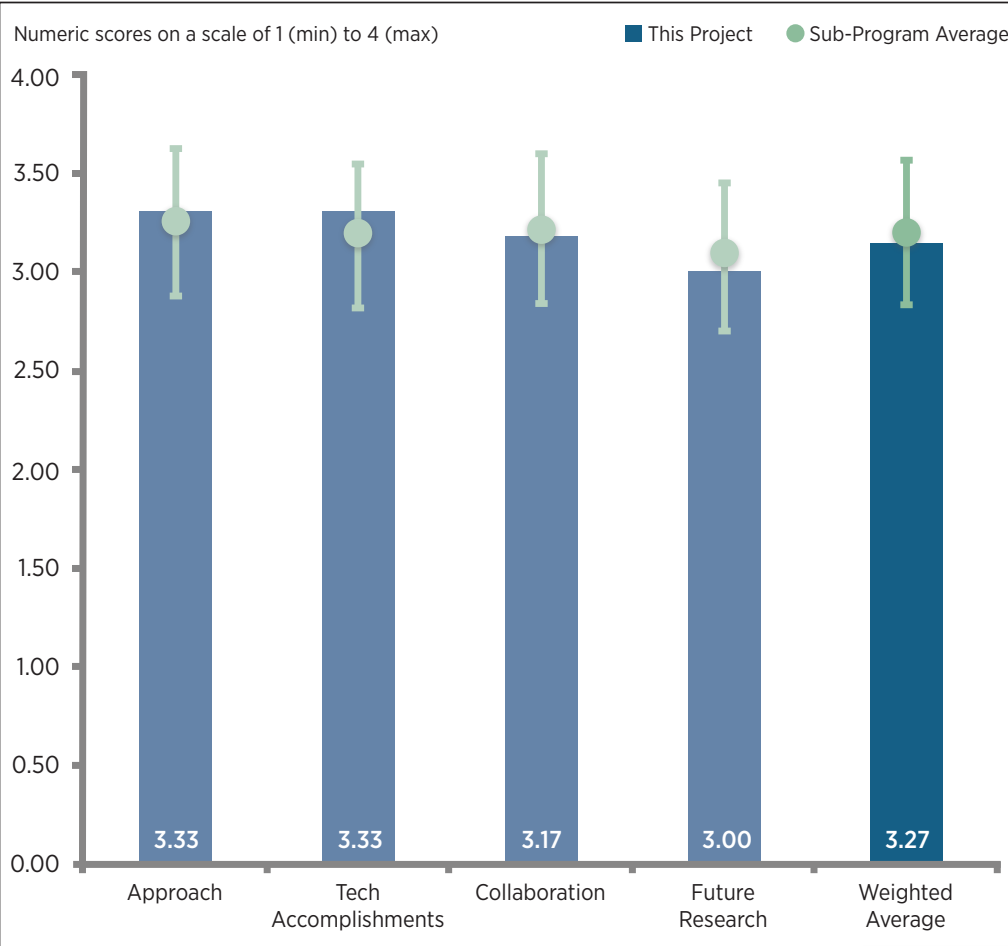

Relevant to DOE Objectives

Sufficiency of Resources

Figure 2-20 - Fundamental Studies of Lithium-Sulfur Cell Chemistry: Nitash Balsara (Lawrence Berkeley National Laboratory) - Electrochemical Energy Storage 


\section{Reviewer 2:}

The reviewer stated that the work on free radical detection is comprehensive. Computational work on sulfur species also shows promise.

\section{Reviewer 3:}

The reviewer stated that the project team made good progress on understanding the speciation of the polysulfides. The reviewer did not understand why the team is not focusing on polyethylene oxide (PEO) electrolytes if that is the team's plan for the future.

\section{Question 3: Collaboration and coordination with other institutions.}

\section{Reviewer 1:}

The reviewer stated that the collaboration was good and reasonable.

\section{Reviewer 2:}

The reviewer stated that the project team has a number of collaborators, most of them are from University of California at Berkeley.

Question 4: Proposed future research-the degree to which the project has effectively planned its future work in a logical manner by incorporating appropriate decision points, considering barriers to the realization of the proposed technology and, when sensible, mitigating risk by providing alternate development pathways.

\section{Reviewer 1:}

The reviewer stated that further in situ studies promises to provide insight on limiting issues in Li-S; initial indication seems to be related to $\mathrm{Li}$ anode.

\section{Reviewer 2:}

The reviewer stated that the project team is continuing their work. The reviewer expects them to make significant progress on understanding the polysulfides, but it is not clear the team has a plan for improvement of the present state of the technology.

\section{Reviewer 3:}

The reviewer stated that given that many of the basic goals of the project have been met, and encouraged the PI to solicit input from companies working on Li-S battery technology to determine the best next steps.

\section{Question 5: Does this project support the overall DOE objectives of petroleum displacement? Why or why not?}

\section{Reviewer 1:}

The reviewer stated that yes, Li-S is a low-cost couple that needs fundamental studies, although the reviewer wondered what the differences are when using the solid polymer electrolyte compared to the typical liquid ones.

\section{Question 6: Resources: How sufficient are the resources for the project to achieve the stated milestones in a timely fashion?}

No comments were received in response to this question. 


\section{BatPaC Model Development: Shabbir Ahmed (Argonne National Laboratory) - es228}

\section{Presenter}

Shabbir Ahmed, Argonne National Laboratory

\section{Reviewer Sample Size}

A total of five reviewers evaluated this project.

\section{Question 1: Approach to performing the work-the degree to which technical barriers are addressed, the project is well-designed, feasible, and integrated with other efforts.}

\section{Reviewer 1:}

The reviewer stated that the effort is well focused to improve upon and expand the capability of the BatPac model.

\section{Reviewer 2:}

The reviewer stated that the BatPac has continued to evolve and serve as a resource for the battery community.

\section{Question 2: Technical} accomplishments and progress toward overall project and DOE goals-the degree to which progress has been made, measured against performance indicators and demonstrated progress towards DOE goals.

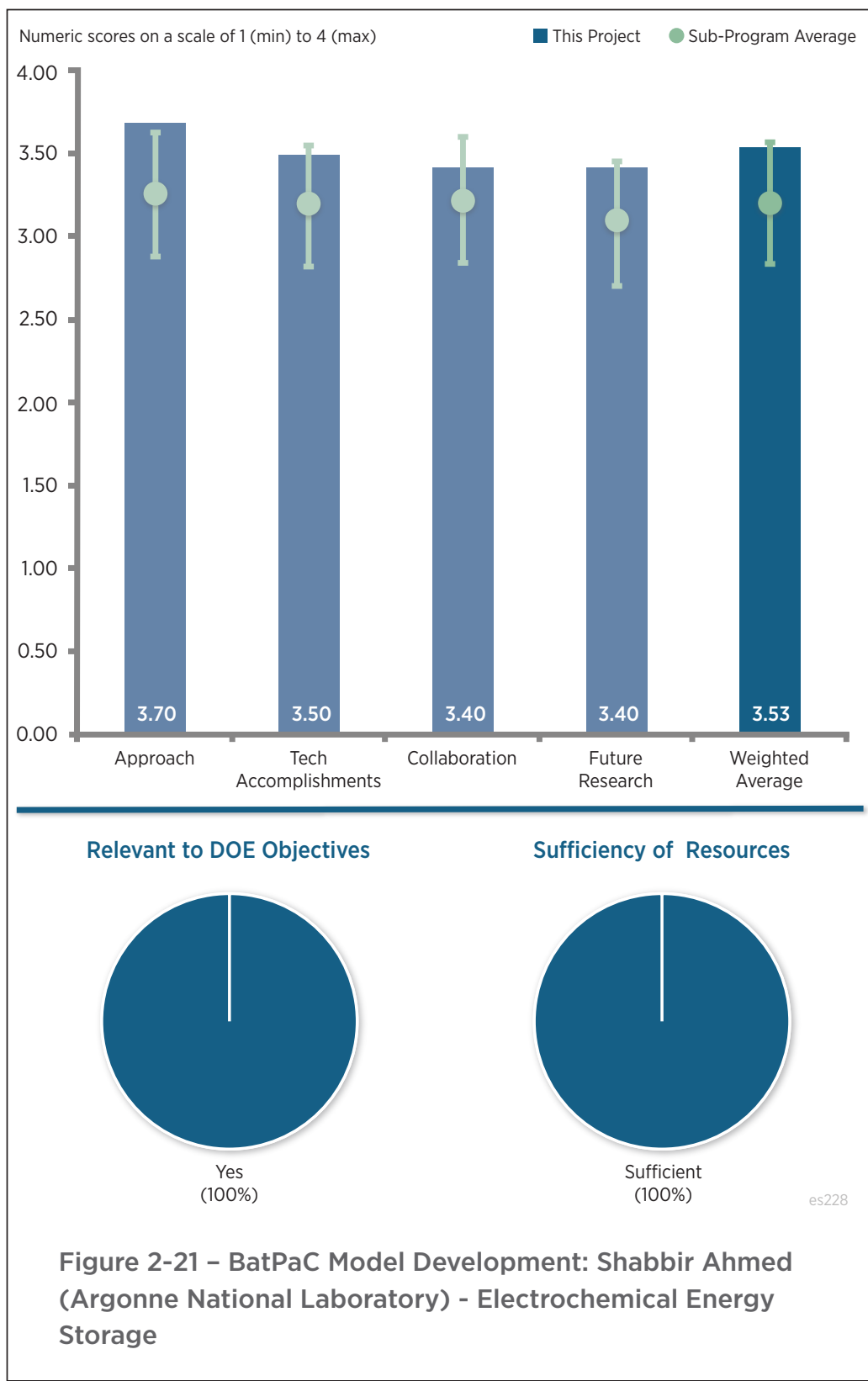

\section{Reviewer 1:}

The reviewer thought the authors have done a great job in establishing important parameters such as optimal electrode loading (current density for Li plating) and the impact of key processes such as NMC production and cathode drying, dry room operation, and formation steps on the cost of battery pack model. These are key steps that contribute to the cost of the battery manufacturing.

\section{Reviewer 2:}

The reviewer stated that the fidelity of the model continues to improve by incorporating increased level of details, particularly on the processing aspects. The challenge appears to be model validation.

\section{Question 3: Collaboration and coordination with other institutions.}

\section{Reviewer 1:}

The reviewer noted that there was excellent collaboration with many key material suppliers, equipment manufacturers, and researchers. 


\section{Reviewer 2:}

The reviewer commented that the team has served the research community well and the model appears to be popular. There is concern that the model is not as well communicated to industry. The team is encouraged to think creatively about how to do model validation: it is difficult to get direct input from industry so it is necessary to develop various metadata sets so that concerns of propriety can be alleviated.

\section{Reviewer 3:}

The reviewer stated that the presenter was not able to justify how collaborators' contributions affected the model. The efforts in reaching out to industrial partners is weak, especially in light of the criticisms last year.

\section{Question 4: Proposed future research-the degree to which the project has effectively planned its future work in a logical manner by incorporating appropriate decision points, considering barriers to the realization of the proposed technology and, when sensible, mitigating risk by providing alternate development pathways.}

\section{Reviewer 1:}

The reviewer thought that the project has identified valuable additions (mainly manufacturing) to consider. However, increasing validation from leading industrial players is the key.

\section{Reviewer 2:}

The reviewer thought that the proposed research areas look good, and made three additional suggestions. First, the reviewer questioned if there is any work done on processing cost for anodes, such natural or artificial graphite, Si-based materials, as well as titanate anode. Even though their cost impact is less significant than that of cathodes, it will be good to complement the database already generated. Second, the study on the impact of plant automations on cost looks interesting. It will be instructive to compare this to the announced cost reduction by Tesla in their gigafactory. Of course NCA becomes then a part of the study. Third, the reviewer noted that $24 \mathrm{M}$ has predicted $\$ 50 / \mathrm{kWh}$ battery cost, but asked if the model can validate that.

\section{Question 5: Does this project support the overall DOE objectives of petroleum displacement? Why or why not?}

\section{Reviewer 1:}

The reviewer stated that a reliable battery pack model is essential and useful for tracking the goal and progress of battery pack technology development.

\section{Reviewer 2:}

The reviewer commented that this model is useful for industries to make well-informed decisions on materials and battery designs.

\section{Reviewer 3:}

The reviewer stated that the model calculates the expected cost of the batteries, which helps the battery customers and suppliers to make decisions on business strategies and provides the batteries for electric vehicle applications.

\section{Question 6: Resources: How sufficient are the resources for the project to achieve the stated milestones in a timely fashion?}

\section{Reviewer 1:}

The reviewer stated that additional resources could be beneficial to support greater marketing/public relations of BatPac in general and specifically internationally. 


\section{Design of Sulfur Cathodes for High-Energy Lithium-Sulfur Batteries: Yi Cui (Stanford University) - es230}

\section{Presenter}

Yi Cui, Stanford University

\section{Reviewer Sample Size}

A total of four reviewers evaluated this project

\section{Question 1: Approach to performing the work-the degree to which technical barriers are addressed, the project is well-designed, feasible, and integrated with other efforts.}

\section{Reviewer 1:}

The reviewer found that the approach the PI is taking is excellent. Novel sulfur nanostructures and multifunctional coatings are being designed and fabricated to overcome the issues related to volume expansion, polysulfide dissolution, and insulating nature of sulfur. This is complimented by structure and property characterization and electrochemical testing.

\section{Reviewer 2:}

The reviewer stated that the use of nanostructured cathode materials to confine polysulfides, or shield them from electrolyte interaction, could be the key to Li-S cell cycling. Very good use of multiple approaches to managing this issue.

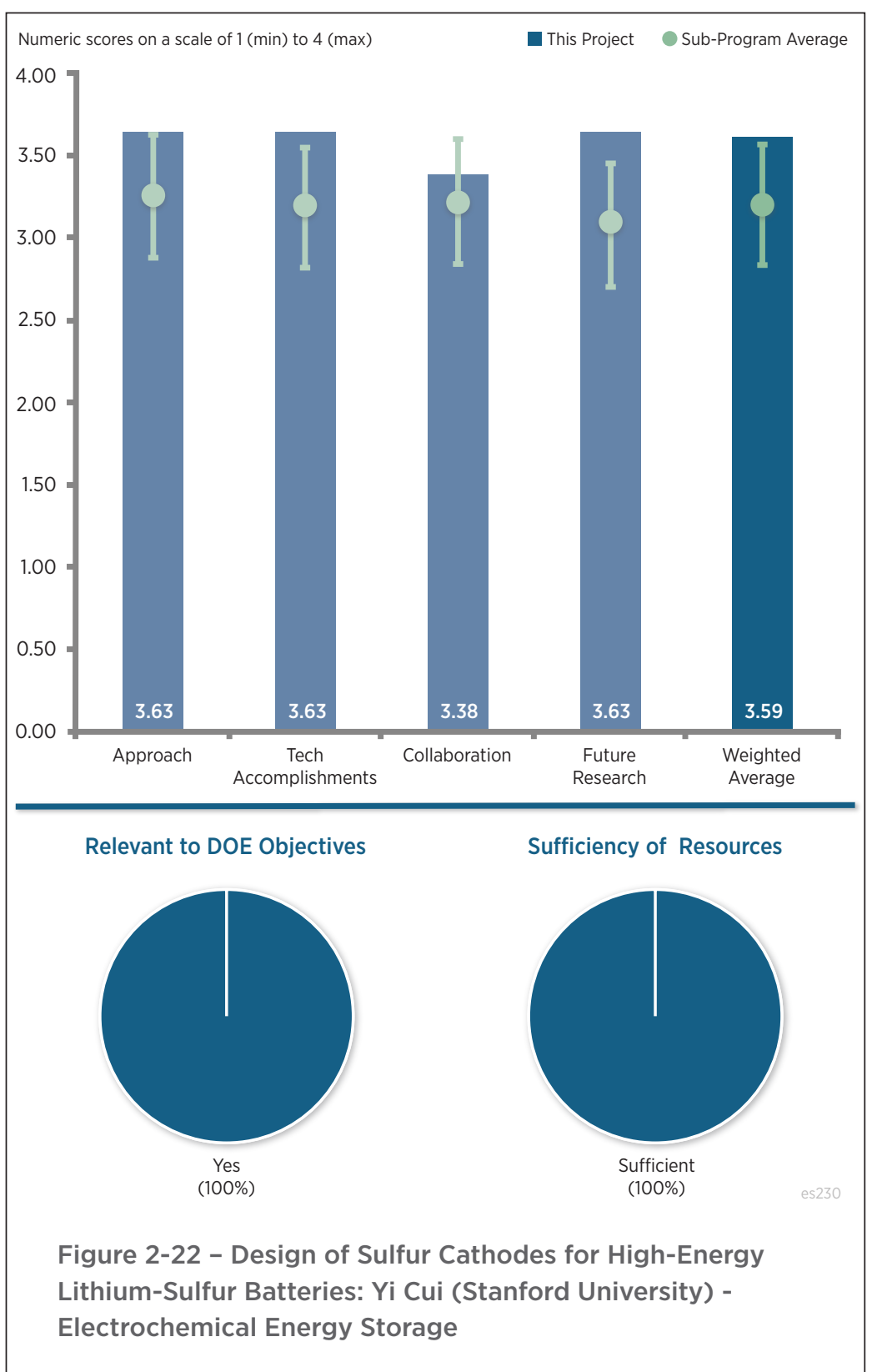

\section{Reviewer 3:}

The reviewer thought that a better fundamental understanding of polysulfide adsorption on metal oxides and other approaches in the scope of this project are important contributions to achieving effective Li-S electrode designs that are goal of the projects. The systematic screening is excellent, valuable work.

\section{Question 2: Technical accomplishments and progress toward overall project and DOE goals - the degree to which progress has been made, measured against performance indicators and demonstrated progress towards DOE goals.}

\section{Reviewer 1:}

The reviewer noted that the project will be completed at the end of July. Outstanding progress was achieved this past year. Milestones were met in a timely manner. The project team identified the interaction mechanism between sulfur species and different types of sulfides/oxides/metals, and is on a path to find the optimal material to 
improve the capacity and cycling of S cathode. There were numerous peer-reviewed journals describing the novel approaches that were undertaken.

\section{Reviewer 2:}

The reviewer noted that apparently a very nice cycling stability has been achieved, with $3000.2 \mathrm{C}$ cycles shown, with a cathode capacity of $1,000 \mathrm{mAh} / \mathrm{g}$, using conductive polymer coated hollow $\mathrm{S}$ cathodes. Another approach was to use $\mathrm{TiS}_{2}$ coated $\mathrm{S}$, those showed 400 very stable cycles near $600 \mathrm{mAh} / \mathrm{g}$. Finally, the project team evaluated polysulfide capture on the surface of metal oxides.

\section{Reviewer 3:}

The reviewer stated that the results demonstrate good progress and are convincing in technical accomplishment; however, cost and energy density are main goals and the presentation should quantify such parameters, even if the goal is not in reach due to the difficulty of the challenges. To convey the result, it is not ideal to scale coulombic efficiency plots from $0 \%$ to $100 \%$.

\section{Reviewer 4:}

The reviewer thought that the reliance of using lithium sulfide $\left(\mathrm{Li}_{2} \mathrm{~S}\right)$ as the initial electrode materials could pose a financial burden to the adoption of this technology. The reviewer asked if the researchers of this project can come up with a synthesis route that can lower the cost of $\mathrm{Li}_{2} \mathrm{~S}$.

\section{Question 3: Collaboration and coordination with other institutions.}

\section{Reviewer 1:}

The PI had a number of highly effective collaborations (Ab initio simulations: Prof. Qianfan Zhang, and Amprius).

\section{Reviewer 2:}

The reviewer stated that it makes sense to have simulation work and other tasks that can be completed with little interaction at partners in China and in situ X-ray work right in the area.

\section{Reviewer 3:}

The reviewer thought that it looks okay, but would appreciate more collaborations with domestic institutions, including other $\mathrm{S}$ focused battery companies as this project is sharply focused on solutions to enable Li-S cells as opposed to a more fundamental study.

\section{Question 4: Proposed future research-the degree to which the project has effectively planned its future work in a logical manner by incorporating appropriate decision points, considering barriers to the realization of the proposed technology and, when sensible, mitigating risk by providing alternate development pathways.}

\section{Reviewer 1:}

The reviewer noted that the project will be completed this year.

\section{Reviewer 2:}

The reviewer stated that future research is reasonable, but wondered if the electrolyte volume issue is under control yet. Traditionally, Li-S cells have operated with an effective S catholyte, meaning the cell $\mathrm{Wh} / \mathrm{kg}$ and $\mathrm{Wh} / 1$ is severely restrained by the amount of electrolyte in the cell.

\section{Reviewer 3:}

The reviewer noted that the researcher pointed out that the dendritic growth of $\mathrm{Li}$ on the anode could be a problem in Li-S battery. This is a point that has been omitted/evaded by the Li-S research field due to the view that the crossover of polysulfides can reduce this catastrophic effect. It would be interesting to know more about this fundamental process specifically in the Li-S system.

\section{Question 5: Does this project support the overall DOE objectives of petroleum} displacement? Why or why not? 


\section{Reviewer 1:}

The reviewer stated that the project is highly relevant. Efforts like this will aid in the development of Li-S batteries. This technology is expected to decrease the high cost of batteries.

Reviewer 2:

The reviewer stated yes, Li-S is a low-cost couple and the $\mathrm{S}$ cathode needs novel approaches to manage the polysulfides.

Reviewer 3:

The reviewer thought that the findings can lead to commercial solutions for high energy density, low-cost batteries.

Question 6: Resources: How sufficient are the resources for the project to achieve the stated milestones in a timely fashion?

\section{Reviewer 1:}

The reviewer thought that there was excellent value for the investment. 


\section{Efficient Rechargeable $\mathrm{Li}-\mathrm{O}_{2}$ Batteries Utilizing Stable Inorganic Molten Salt Electrolytes: Vincent Giordani (Liox) - es233}

Presenter

Vincent Giordani, Liox

\section{Reviewer Sample Size}

A total of four reviewers evaluated this project

\section{Question 1: Approach to performing the work-the degree to which technical barriers are addressed, the project is well-designed, feasible, and integrated with other efforts.}

\section{Reviewer 1:}

The reviewer stated that there are many commercial and/or lower cost methods of making micro, meso, and nano porous $\mathrm{Ni}$. It is unclear why the three-dimensional (3D) Ni truss nanolattice structure was chosen for investigation and whether this structure is superior to existing and commercially available porous $\mathrm{Ni}$.

\section{Reviewer 2:}

The reviewer stated that it was a generally good approach to overcome some issues. The materials and electrodes used are very fundamental. The PIs need to address the motive behind choosing the catalysts and electrode structures. Aspects about operating at high temperature need to be also addressed (from a system perspective). Rate capability tests need to be performed, as well as operation at low temperatures and system start-up. The reviewer also thought that safety needs to be discussed.

\section{Reviewer 3:}

The reviewer stated that the approach to find a tolerant Li/oxygen electrolyte at elevated temperature seems good. It seems incredibly hopeful that this electrolyte, if found, will also solve the issues of $\mathrm{V}$ hysteresis, low rate, and ability to cycle only low $\mathrm{mAh} / \mathrm{cm}^{2}$. It also seems very aggressive to hope that it will permit the cell to operate in air compared to pure oxygen.

\section{Reviewer 4:}

The reviewer had a comment regarding the topic $\mathrm{Ni}$ catalyzes the formation of $\mathrm{Li}_{2} \mathrm{O}$ discharge product. It is unclear to what the real catalyst is here and if it is $\mathrm{NiO}$. The investigators showed $\mathrm{Ni}_{3}+$ in the post mortem study of the Ni catalysts. It would be interesting to know if these $\mathrm{Ni}_{3}$ are relevant to the catalytic process and what crystal form they are in.

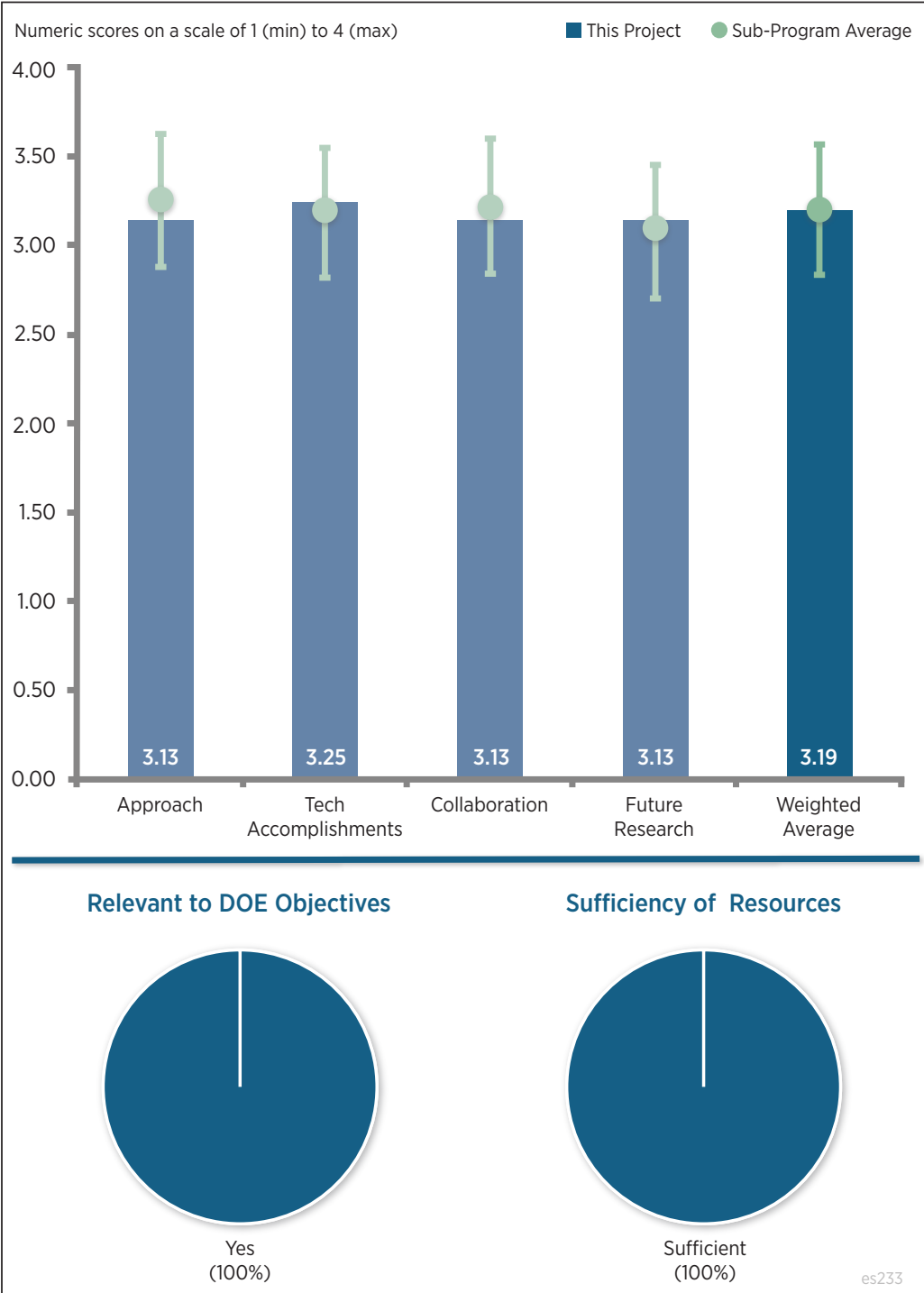

Figure 2-23 - Efficient Rechargeable $\mathrm{Li}-\mathrm{O}_{2}$ Batteries Utilizing Stable Inorganic Molten Salt Electrolytes: Vincent Giordani (Liox) - Electrochemical Energy Storage 
Question 2: Technical accomplishments and progress toward overall project and DOE goals - the degree to which progress has been made, measured against performance indicators and demonstrated progress towards DOE goals.

\section{Reviewer 1:}

The reviewer thought that up to now results have shown use materials and electrodes that are challenging to be scaled up and used. Iridium oxide $\left(\mathrm{IrO}_{2}\right)$ that has been identified as the most promising catalyst but the cost and availability need to be addressed.

\section{Reviewer 2:}

$\mathrm{IrO}_{2}$ was identified as promising non-carbonaceous electrode candidate for oxygen reduction reaction (ORR) electrochemistry. However, Ir is expensive and may not be economically viable.

\section{Reviewer 3:}

The reviewer stated that it was good finding that cells made with $\mathrm{Ni}$ - and $\mathrm{Li}$-doped $\mathrm{NiO}$ permits high coulombic and round trip efficiencies. However, cathode materials screened, from carbon through to $\mathrm{TiC}$ showed relatively poor oxygen evolution reaction ORR ratios

\section{Question 3: Collaboration and coordination with other institutions.}

\section{Reviewer 1:}

The reviewer stated that materials and electrodes used from the California Institute of Technology need to be designed for the project. LBNL work contributed well to the project.

Question 4: Proposed future research-the degree to which the project has effectively planned its future work in a logical manner by incorporating appropriate decision points, considering barriers to the realization of the proposed technology and, when sensible, mitigating risk by providing alternate development pathways.

\section{Reviewer 1:}

The reviewer stated that because Ir is expensive, the PI should consider low-cost alternatives.

\section{Reviewer 2:}

The reviewer stated that there is a long way to go, lots of challenges remaining. The major issue here of course is that even if this cell can be made to cycle reversibly, it is not clear that automotive customers would be willing to commercialize a $100-150^{\circ}$ Celsius (C) battery. Nevertheless, this is a good study for proof in principal.

\section{Reviewer 3:}

The reviewer stated that future research points mentioned need to be expanded and more topics need to be added. The technology has potential but needs to be aligned with DOE timeline and targets. Even if the problems mentioned are addressed and/or solved there are still open points that need to be overcome.

\section{Reviewer 4:}

The reviewer stated that it is unclear to how the researchers would address the goal to provide a cell and system that can operate robustly in ambient air without $\mathrm{O}_{2}$ purification. The researchers of this project have not even tangentially studied any effects of water vapor and carbon dioxide $\left(\mathrm{CO}_{2}\right)$

\section{Question 5: Does this project support the overall DOE objectives of petroleum displacement? Why or why not?}

\section{Reviewer 1:}

The reviewer stated that this molten salt battery could potentially scale up for stationary applications.

\section{Reviewer 2:}

The reviewer thought it was reasonable, not a home run however due to the temperature range of the cell. 
Reviewer 3:

The reviewer stated yes, but Li-air technology is in general challenging to address the targets of DOE.

Question 6: Resources: How sufficient are the resources for the project to achieve the stated milestones in a timely fashion?

No comments were received in response to this question. 


\section{Low-Cost, High-Energy Si/ Graphene Anodes for Li-Ion Batteries: John Colwell (XG Sciences) - es237}

\section{Presenter}

Robert Privette, XG Sciences

\section{Reviewer Sample Size}

A total of five reviewers evaluated this project.

\section{Question 1: Approach to performing the work-the degree to which technical barriers are addressed, the project is well-designed, feasible, and integrated with other efforts.}

\section{Reviewer 1:}

The reviewer stated that XG Sciences is developing $\mathrm{SiG}^{\mathrm{TM}}$ anode with improved performance, and thus addressing the three barriers: specific energy, life, and cost.

\section{Reviewer 2:}

The reviewer noted that this was material-centric, and the developer did a good job of defining the key program metrics, then seeking to meet them.

\section{Reviewer 3:}

The reviewer stated that this is possible way to stabilize Si negative electrodes. Seem to be hitting the goal of $600 \mathrm{Ah} /$ $\mathrm{kg}$ for the anode. However, it would be nice to have independent verification, and know what this enables in full cells at the consumer or large format cell level. The reviewer thought that the project team needs to understand better if the Si is truly sealed inside or if the graphene is loosely wrapped around the particle.

\section{Question 2: Technical accomplishments and progress toward overall project and DOE goals - the degree to which progress has been made, measured against performance indicators and demonstrated progress towards DOE goals.}

\section{Reviewer 1:}

The reviewer stated that the $\mathrm{SiG}^{\mathrm{TM}}$ anode materials shows good consistency in first cycle capacity (FCC), first cycle efficiency (FCE) and capacity retention. The best $2 \mathrm{Ah}$ full cell built by XG with SiGTM anode has a cycle life of 1,176 at $86.9 \%$ retention, which is better than other anode projects. However, the good performance is limited in the voltage window of 4.2-3.25.

\section{Reviewer 2:}

The reviewer stated that it seems appropriate for the level of funding. Seems on track vis-a-vis the plan. The initial

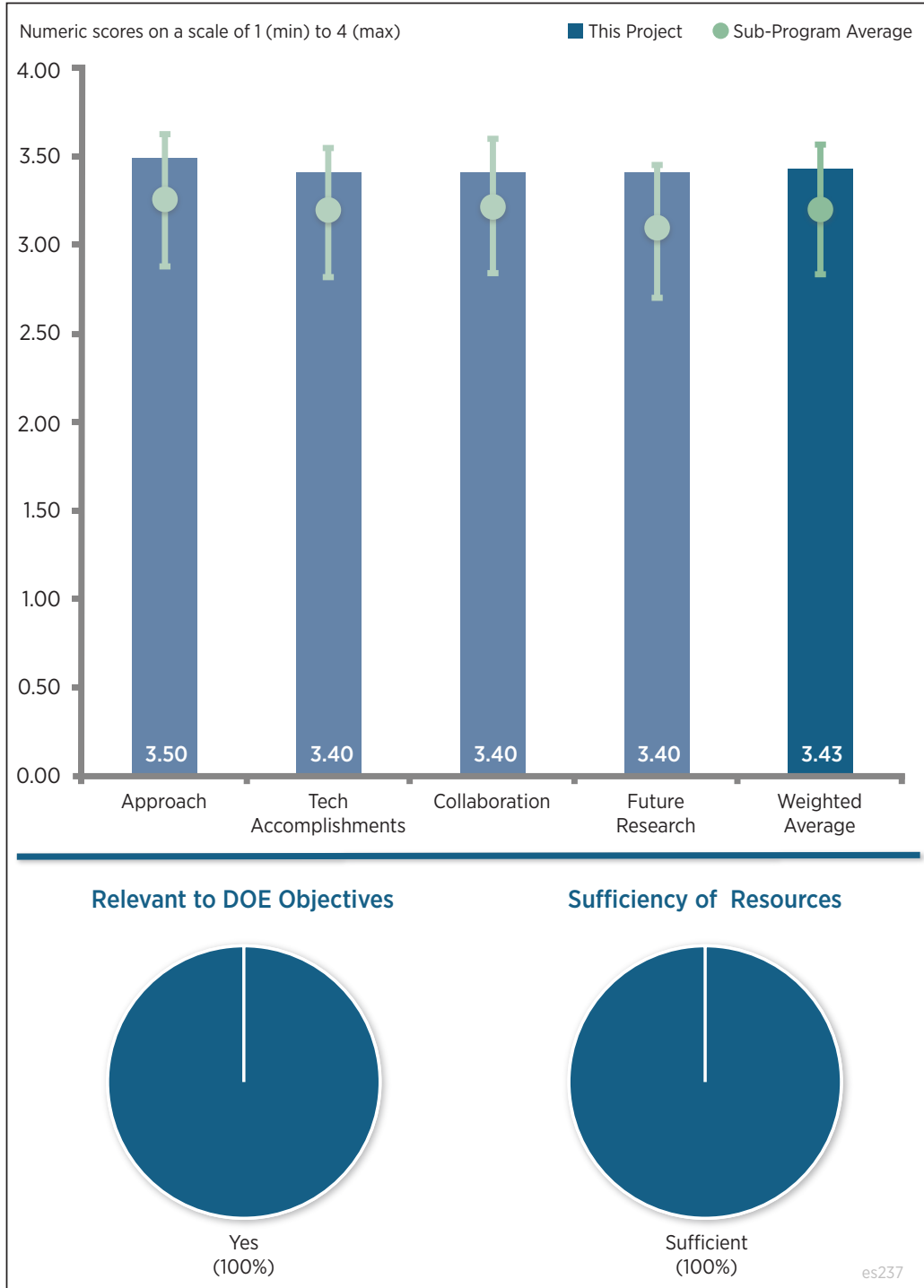

Figure 2-24 - Low-Cost, High-Energy Si/Graphene Anodes for Li-Ion Batteries: John Colwell (XG Sciences) - Electrochemical Energy Storage 
durability goal was hit. The reviewer would have liked to see more energy and indeed reporting of energy as well as Ah capacity.

\section{Reviewer 3:}

The reviewer stated that more information on cost model approach would have been beneficial. A target of 600 $\mathrm{mAh} / \mathrm{g}$ was set for the anode, but the modelling had shown that $800 \mathrm{mAh} / \mathrm{g}$ was really required, in order to achieve $350 \mathrm{Wh} / \mathrm{kg}$. Some additional modeling, predicting an EV-sized cell (with their collaborators) would have been beneficial.

\section{Question 3: Collaboration and coordination with other institutions.}

\section{Reviewer 1:}

The reviewer stated that XG Sciences collaborates with several national laboratories and industrial partners.

\section{Reviewer 2:}

The reviewer said it appeared that there had been good collaboration, but the exact roles of each institution could have been made more clear.

\section{Reviewer 3:}

The reviewer stated that the addition of more collaborators and nature of additional collaborators is excellent; however, achieving the involvement of global high-volume cell producer(s) would have been one remaining area for improvement.

\section{Reviewer 4:}

The reviewer noted that many of the partners are more like suppliers, but still sufficient to get outside ideas to help the project team do good work.

\section{Question 4: Proposed future research-the degree to which the project has effectively planned its future work in a logical manner by incorporating appropriate decision points, considering barriers to the realization of the proposed technology and, when sensible, mitigating risk by providing alternate development pathways.}

\section{Reviewer 1:}

The reviewer stated that the project is nearly over, but there are good plans for future.

\section{Reviewer 2:}

The reviewer noted that the program is closing out, but the developer is making good use of the data generated during the program. It will be important to evaluate the final articles against the program goals, to verify that this project approach was effective.

\section{Reviewer 3:}

The reviewer stated that XG clearly indicated the next step task: to improve voltage window performance with higher energy specific energy $(850 \mathrm{mAh} / \mathrm{g})$. The reviewer added that XG needs data to support the claim that XG $\mathrm{SiG}^{\mathrm{TM}}$ achieves competitive price as compared to graphite, and how far it is to the EV Everywhere target \$125/ $\mathrm{kWh}$ for full battery.

\section{Reviewer 4:}

The reviewer stated that given funding and progress the future plans seem right. Again, it would be nice if the project team could give some sort of idea how this would enable improvements in commercial cells. The reviewer would like to see more emphasis on durability, and suitable or compatible positive electrodes and electrolytes.

\section{Question 5: Does this project support the overall DOE objectives of petroleum displacement? Why or why not?}

\section{Reviewer 1:}

The reviewer stated yes, the work is in right direction. 


\section{Reviewer 2:}

The reviewer stated that this moderate cost (hopefully) method to make higher energy cells, which will help with penetration of electrified vehicles.

Reviewer 3:

The reviewer stated that this low-cost high-energy Si anode will promote the use of batteries and EVs, which will help with the petroleum displacement.

Reviewer 4:

The reviewer noted local advanced material supply, allowing for an appreciable energy density increase.

Question 6: Resources: How sufficient are the resources for the project to achieve the stated milestones in a timely fashion?

\section{Reviewer 1:}

The reviewer stated that the current achievements demonstrate that XG Sciences has sufficient resources to achieve the milestones.

\section{Reviewer 2:}

The reviewer stated that there were no issues observed. 


\section{Low-Cost, High-Capacity Lithium Ion Batteries through Modified Surface and Microstructure: Pu Zhang (Navitas Systems) - es238}

\section{Presenter}

$\mathrm{Pu}$ Zhang, Navitas Systems

\section{Reviewer Sample Size}

A total of five reviewers evaluated this project.

\section{Question 1: Approach to performing the work-the degree to which technical barriers are addressed, the project is well-designed, feasible, and integrated with other efforts.}

\section{Reviewer 1 :}

The reviewer stated that 2016 works concentrated on improving tap density scale up process.

\section{Reviewer 2:}

The reviewer noted that as shown, the approach is clearly focused on addressing specific and relevant material metrics.

\section{Reviewer 3:}

The reviewer stated that the low-cost and standard manufacturing method to make stable Si electrodes attacks major barriers and seems well planned.

\section{Question 2: Technical} accomplishments and progress toward overall project and DOE goals-the degree to which progress has been made, measured against performance indicators and demonstrated progress towards DOE goals.

\section{Reviewer 1:}

The reviewer stated that progress has been good. Cycle life improvement requires an explanation as to why pouch cells have performed better than coin cells. The reviewer encouraged the developer to track progress via a gap chart, as a summary.

\section{Reviewer 2:}

The reviewer stated that the project team seems to have made good progress with the scale-up complete. Not clear that the team will reach cycling goals that are relevant to customer use.

\section{Reviewer 3:}

The reviewer noted that the pilot scale products match or exceed the capacity retention of lab scale product, but
Figure 2-25 - Low-Cost, High-Capacity Lithium Ion Batteries through Modified Surface and Microstructure: Pu Zhang (Navitas Systems) - Electrochemical Energy Storage

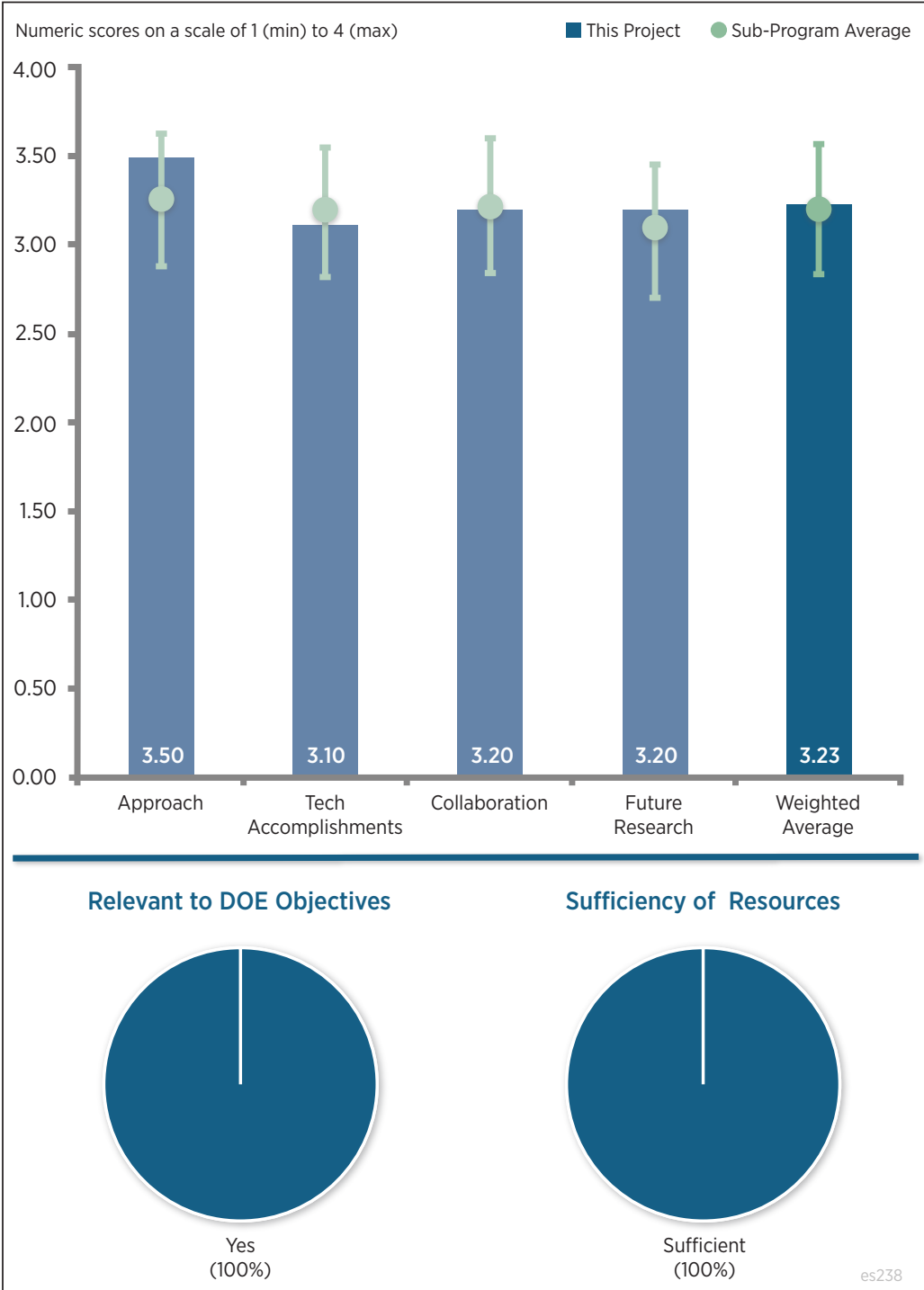


both are about only 200 cycles with less than $80 \%$ retention. The half cell (anode) test shows only 450 cycles at a capacity of $550 \mathrm{mAh} / \mathrm{g}$. Object was greater than $800 \mathrm{mAh} / \mathrm{g}$ and greater than 1000 cycle at $80 \%$ depth of discharge (DOD). 2 Ah full cell delivered but cycle life test shows less than 200 cycles at $90 \%$ retention.

\section{Question 3: Collaboration and coordination with other institutions.}

\section{Reviewer 1:}

The reviewer noted that ANL and A123 Systems, LLC joined the project in 2016.

\section{Reviewer 2:}

The reviewer did not find a large variety of collaborators, but enough.

\section{Reviewer 3:}

The reviewer thought that more clarification of partners' roles throughout the presentation would help.

Question 4: Proposed future research-the degree to which the project has effectively planned its future work in a logical manner by incorporating appropriate decision points, considering barriers to the realization of the proposed technology and, when sensible, mitigating risk by providing alternate development pathways.

\section{Reviewer 1:}

The reviewer found the future work well defined.

\section{Reviewer 2:}

The reviewer thought it seems appropriate and matches the plan that the team showed as the DOE agreement.

\section{Reviewer 3:}

The reviewer was concerned that a path to 1,000 cycles is not clear.

\section{Question 5: Does this project support the overall DOE objectives of petroleum} displacement? Why or why not?

\section{Reviewer 1:}

The reviewer stated that the works are in the right direction.

\section{Reviewer 2:}

The reviewer said this could lower cost of cells and thereby get them in commerce.

\section{Reviewer 3:}

The reviewer stated that this project enhances the energy density and improves cost of the batteries, which will help EVs to displace petroleum.

\section{Reviewer 4:}

The reviewer commented yes, insofar as this is a domestic material, which at a competitive cost could enhance the manufacture and supply of energy storage devices.

\section{Question 6: Resources: How sufficient are the resources for the project to achieve the stated milestones in a timely fashion?}

\section{Reviewer 1:}

The reviewer stated that Navitas, in collaboration with ANL and A123 Systems, LLC, has the potential to do the job (low-cost long cycle Si anodes).

\section{Reviewer 2:}

The reviewer stated that this is a good partnership, although the exact contributions of each throughout the project could be made clearer. 


\section{Scale-Up of Low-Cost Encapsulation Technologies for High Capacity and High Voltage Electrode Powders: David King (Pneumaticoat Technologies) - es239}

\section{Presenter}

David King, Pneumaticoat Technologies

\section{Reviewer Sample Size}

A total of five reviewers evaluated this project.

\section{Question 1: Approach to} performing the work-the degree to which technical barriers are addressed, the project is well-designed, feasible, and integrated with other efforts.

\section{Reviewer 1:}

The reviewer stated that the PneumatiCoat Small Business Innovation Research (SBIR) project is a successful project that helps improving cycling life of a variety of cathode and anode materials with economic viability. The presentation is clear and convincing.

\section{Reviewer 2:}

The reviewer stated that there was an excellent structure of the program.

\section{Reviewer 3:}

The reviewer commented that the coating will improve the stability of the active material and scale up will reduce the cost of coating.

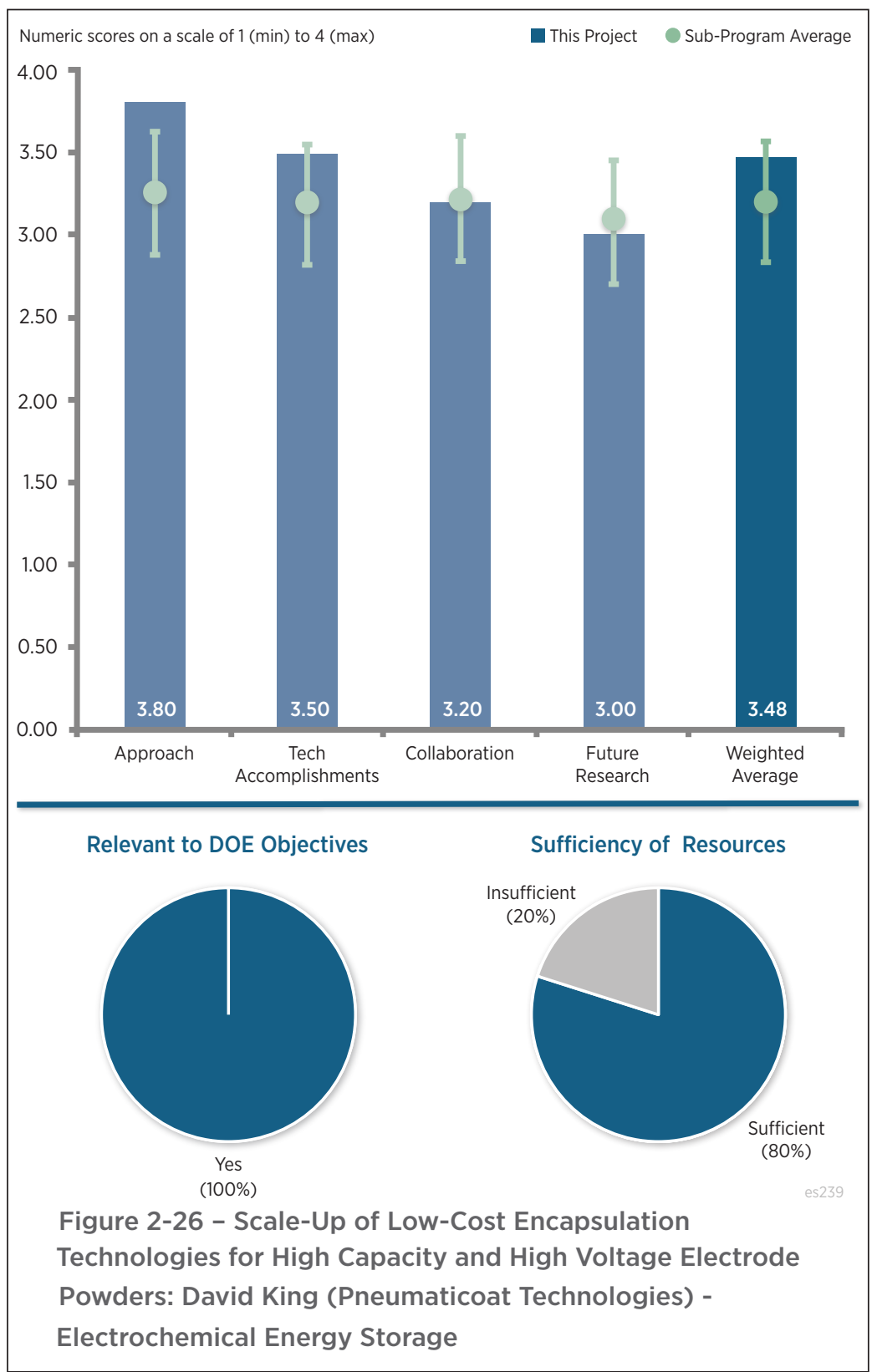

\section{Reviewer 4:}

The reviewer though that the approach is excellent based on SBIR limitations of effort. It might have been nice to see more comparisons of other coating methods, there was at least a good effort to compare the chemical precipitation method in detailed performance studies. The decision to go with atomic layer deposition (ALD) on powders rather than electrodes led to an excellent implementation of scale-up to pilot plant operation of an ALD methodology.

\section{Question 2: Technical accomplishments and progress toward overall project and DOE goals-the degree to which progress has been made, measured against performance indicators and demonstrated progress towards DOE goals.}

\section{Reviewer 1:}

The reviewer thought that the results were very impressive. The one area of concern for the developer should be 
understanding the limitations of aluminum oxide-based ALD coatings for some high voltage systems. The reviewer encouraged the investigations in the phosphates.

\section{Reviewer 2:}

The reviewer stated that the ALD particle semi-continuous coating process has the advantages of low-cost and high-throughput, and is effective in improving electrode performance.

\section{Reviewer 3:}

The presence of pilot plant equipment sets the stage for in detail heavy implementation in particular systems. The reviewer would like to see such implementation extended to other more promising systems such as low cobalt $\mathrm{NMC}$ positive and $\mathrm{Si}$ or $\mathrm{Si} / \mathrm{C}$ negative electrode materials.

\section{Reviewer 4:}

The reviewer noted that although progress previously reported at the last AMR was excellent, the nature of actual accomplishments, progress, or new outcomes since then either is not as clearly reported or simply has been of less significance.

\section{Question 3: Collaboration and coordination with other institutions.}

\section{Reviewer 1:}

The reviewer enthused that PneumatiCoat can actually collaborate will all players in the battery field by providing their coating technologies.

\section{Reviewer 2:}

The reviewer stated that the team is well structured, and all contributions from partners were shown.

\section{Reviewer 3:}

The reviewer found that the collaboration is limited so far and no information is given about industrial partners. Material suppliers should definitely be included although the reviewer understands the need for protection of proprietary issues. This could be a breakthrough technology, and needs to be fast tracked to get best results.

\section{Reviewer 4:}

The reviewer noted that while collaboration and coordination with main partners appears to have been productive, the nature or possible utility of collaboration with unidentified corporate partnerships is completely opaque.

\section{Question 4: Proposed future research-the degree to which the project has effectively planned its future work in a logical manner by incorporating appropriate decision points, considering barriers to the realization of the proposed technology and, when sensible, mitigating risk by providing alternate development pathways.}

\section{Reviewer 1:}

The reviewer stated that it seems PneumatiCoat has done everything and is ready for phase III.

\section{Reviewer 2:}

The reviewer thought that future work is correctly directed. The reviewer also referenced prior comments regarding balancing of focus of work. Anode-based coating studies may be an area of future work as well.

\section{Reviewer 3:}

The reviewer stated that while the project is nearly done, no future work or plans are noted.

\section{Reviewer 4:}

The reviewer commented that future research is really not documented in report.

Question 5: Does this project support the overall DOE objectives of petroleum displacement? Why or why not? 


\section{Reviewer 1:}

The reviewer stated that the good results reported need to be fully validated and put on fast track because of the high relevance of the techniques. The improved cycle life should also result in improved calendar life of the batteries to increase the level of relevance to DOE programs.

\section{Reviewer 2:}

The reviewer stated that yes, the work is on right track

\section{Reviewer 3:}

The reviewer thought that the low-cost coating technology will provide long lasting batteries to encourage petroleum displacement in automotive applications.

\section{Reviewer 4:}

The reviewer stated yes, insofar that this process may enable significant improvement in cathode material durability. The big question on advanced anode durability improvement remains.

\section{Question 6: Resources: How sufficient are the resources for the project to achieve the stated milestones in a timely fashion?}

\section{Reviewer 1:}

The reviewer thought that the pilot plant can produce a lot of material, but it is not clear that cell building and testing resources are adequate for fast track evaluations.

\section{Reviewer 2:}

The reviewer stated that the initial progress earlier in project seemed to be excellent in light of budget size and apparent resources.

\section{Reviewer 3:}

The reviewer stated that the current achievements demonstrate that PneumatiCoat has sufficient resource to achieve the milestones.

\section{Reviewer 4:}

The reviewer commented that the project appeared to be well-balanced. 


\section{High-Energy Anode Material Development for Li-Ion Batteries: Cary Hayner (Sinode Systems) - es 240}

\section{Presenter}

Cary Hayner, Sinode Systems

\section{Reviewer Sample Size}

A total of four reviewers evaluated this project.

\section{Question 1: Approach to performing the work-the degree to which technical barriers are addressed, the project is well-designed, feasible, and integrated with other efforts.}

\section{Reviewer 1:}

The reviewer commented that the efforts of development and commercialization of silicon-carbon (Si-C)-based anode by SiNode Systems are in line with DOE's targets on battery materials: performance, life and cost.

\section{Reviewer 2:}

The reviewer thought that the approach of using a graphene-Si anode has definite advantages in making a high energy anode with good cycling properties and low cost. These properties can be optimized with a good selection of materials with an emphasis on low-cost and high-performance. The approach of using a staged marketing approach runs the risk of floundering on an early marketing stage without ever reaching the desired EV market, however.

\section{Reviewer 3:}

The reviewer commented that the main issue is calling out USABC battery system cost target, without clearly identifying the anode material properties necessary to achieve that and the performance goals. A more comprehensive set of material targets (e.g., include tap density, sheet conductivity, etc.) should be considered.

\section{Reviewer 4:}

The reviewer stated that the objectives do not target the final cost DOE battery targets of $\$ 125 / \mathrm{kWh}$ with the detail of cost of producing cells. Also the business case is not well defined on being the anode material supplier to the battery industry. The cost versus production rate is not included.

Question 2: Technical accomplishments and progress toward overall project and DOE goals - the degree to which progress has been made, measured against performance indicators and demonstrated progress towards DOE goals. 


\section{Reviewer 1:}

The reviewer stated that the 1,000 times scale-up was achieved with comparable performance. However, cycle life data are only up to 140, far from the end performance targets: 750-1,500 mAh/g anode, 1,000 cycles.

\section{Reviewer 2:}

The reviewer commented that the progress to date is promising, but still far from acceptable in terms of cycle life. The use of additives and surface treatments are hard to evaluate because the types of additives and surface treatments are not discussed at all. At least some generic explanations would be helpful in attempting evaluations of these crucial steps.

\section{Reviewer 3:}

The reviewer commented that this is a very interesting approach, and encouraging improvement in cycle life. More discussion of key material parameters influencing cycle life, and associated identified failure modes would be worthwhile. The reviewer suggested that the team continue with the binder investigations, as this could have a considerable impact on this work.

\section{Reviewer 4:}

The reviewer stated that the status of the cost of the material and improvement from last year is missing in the accomplishments.

\section{Question 3: Collaboration and coordination with other institutions.}

\section{Reviewer 1:}

The reviewer stated that there were well-defined partnerships, and good evidence of the effective collaboration.

\section{Reviewer 2:}

The reviewer stated that the collaboration with the state-of-the-art cell supplier will help to validate the cost benefit of the advanced anode material.

\section{Reviewer 3:}

The reviewer stated that SiNode Systems should seek collaboration with national laboratories for advanced characterization techniques.

\section{Reviewer 4:}

The reviewer stated that collaborations to date have only involved pragmatic evaluation and material supply questions. The group needs to develop more forward looking collaborations if they are to move in the direction of intermediate product development and ultimately EV products. In particular, the group needs to develop collaborators to make cathodes, to coat their anode materials to a high standard, and to manufacture finished cells. The team also needs collaboration to enter the above business areas.

Question 4: Proposed future research-the degree to which the project has effectively planned its future work in a logical manner by incorporating appropriate decision points, considering barriers to the realization of the proposed technology and, when sensible, mitigating risk by providing alternate development pathways.

\section{Reviewer 1:}

The reviewer stated that the remaining challenges and barriers are clearly recognized in the presentation.

\section{Reviewer 2:}

The reviewer noted that this project is nearly finished, so a strong finish is necessary to obtain funding for the next step of development.

\section{Reviewer 3:}

The reviewer stated that the future research should include the cost analysis to show the gap between DOE cost goals and SiNode Systems business strategy. 


\section{Reviewer 4:}

The reviewer commented that without identifying the failure modes, it is difficult to see how the cycling goals shall be met. This is the open work however, so the developer should focus on this. The reviewer also suggested considering calendar life in the evaluation.

\section{Question 5: Does this project support the overall DOE objectives of petroleum displacement? Why or why not?}

\section{Reviewer 1:}

The reviewer thought that the work is in right direction.

\section{Reviewer 2:}

The reviewer stated yes, insofar the development of a low-cost Si-containing anode material (and supplier) furthers industrialization of large format and durable energy storage systems.

\section{Reviewer 3:}

The reviewer stated that the R\&D provides low-cost, high-energy density solutions to encourage petroleum displacement.

\section{Reviewer 4:}

The reviewer commented that the relevance is somewhat downgraded because of the staged marketing approach, which may take many years before the desired DOE objectives can be addressed.

\section{Question 6: Resources: How sufficient are the resources for the project to achieve the stated milestones in a timely fashion?}

\section{Reviewer 1:}

The reviewer stated that there were no issues.

\section{Reviewer 2:}

The reviewer stated that at present levels of resources, the chance of entering even the first stage of cell manufacture and market entry is unlikely. The project team needs a strong partner in these areas. 


\section{Advanced High-Performance Batteries for Electric Vehicle (EV) Applications: Ionel Stefan (Amprius) - es241}

\section{Presenter}

Ionel Stefan, Ampirus

\section{Reviewer Sample Size}

A total of four reviewers evaluated this project.

\section{Question 1: Approach to performing the work-the degree to which technical barriers are addressed, the project is well-designed, feasible, and integrated with other efforts.}

\section{Reviewer 1:}

The reviewer stated that Amprius is starting with well-performing $\mathrm{Si}$ anodes and is trying to improve them.

\section{Reviewer 2:}

The reviewer noted that the objective is to develop high specific energy Li-ion cells utilizing Amprius Si nanorod anodes for EV batteries. There were two specific objectives. First, to design and fabricate Si nanowire anodes matching with advanced (high-capacity and high-energy density) cathodes and state-of-the-art cell components. Second, to design, fabricate, test, and deliver $2 \mathrm{Ah}, 10 \mathrm{Ah}$, and $40 \mathrm{Ah} \mathrm{Li}$-ion cells with Si nanowire anodes that meet the USABC 2020 goals: $350 \mathrm{Wh} /$ $\mathrm{kg}$ and $750 \mathrm{Wh} / 1$ at end-of-life (EOL), a 12:1 power:energy ratio, and 1,000 anode for higher energy density, specific energy, and lower costs, and to improve the cycle life by optimizing the nanowire structure.

The reviewer thought that the approach looks good and is consistent with the project/DOE goals. The use of Si anode can result in moderate gains in specific energy and energy density, especially after proper pre-lithiation. However, with the Si anode, there is a huge penalty in the cycle life even with the nanorods here and it is not clear to what extent further optimization can mitigate this. Yet, this is one of the viable approaches. The specific approach involves matching Si nanowire anodes with advanced (high capacity and high energy density) cathodes and state-of-the-art cell components, and the development of an anode and other cell components in a $2 \mathrm{Ah}$ cell form factor and later scale it up to an intermediate $10 \mathrm{Ah}$ cell and alter to $40 \mathrm{Ah}$ cells for performance demonstration. Overall, the project is well designed, and integrated with other efforts.

\section{Reviewer 3:}

The reviewer thought that the approach to anode development rates much higher than the cathode development 
or the full cell approach. The emphasis on cobalt (especially that charged to high voltage) presents a severe safety hazard and should be discontinued. Other cathode materials should be considered that can meet the requirements for full cell energy and power.

\section{Reviewer 4:}

The reviewer thought that the PI is putting too much emphasis on cobalt-based cathode materials. This is definitely wrong for transportation applications, on which the project is focused. High-nickel NMCs are able to attain similar capacities at high voltages and more in line with the transportation sector. Indeed, cost is the overriding issue for this project. It is hard to see how the anode fabrication method could ever meet USABC and DOE cost targets.

\section{Question 2: Technical accomplishments and progress toward overall project and DOE goals-the degree to which progress has been made, measured against performance indicators and demonstrated progress towards DOE goals.}

\section{Reviewer 1:}

The reviewer thought that the accomplishments for the negative electrode development are excellent. However, the use of cobalt oxide is not acceptable for EV usage and no acceptable alternatives are presented. The limiting electrode for cycle life seems to be the negative electrode and the results to date do not meet the goals of DOE, but the progress is encouraging, especially when compared with other Si attempts by other contractors.

\section{Reviewer 2:}

The reviewer noted that there has been good progress made on the cell fabrication and scale up. Specifically, $\mathrm{Si}$ nanowire cells were developed with capacities of 2.6 to 3.1 Ah depending on the cathode, which met USABC requirements expect the cycle life. Also, 30 of the Si nanowire-NMC 2.0 Ah cells were delivered to INL and SNL for performance and safety evaluation.

Furthermore, the reviewer stated that the design and tooling has been completed for $10 \mathrm{Ah}$ cells and the testing of first $10 \mathrm{Ah}$ prototype cell. These cells performed well in the short-terms tests and met the energy and power goals. However, the performance relative the cycle life and calendar life is less than desired. It is not obvious or stated clearly what the strategies would be for improving the life characteristics, such as modifying the electrolyte or reducing the Si content. Nevertheless, the overall progress is good and is consistent with the scheduled milestones and DOE goals.

\section{Reviewer 3:}

The reviewer stated that it is near the beginning of the program. Gap analysis shows substantial progress, with decent energy density and life, although not yet at the USABC goals, especially to meet EOL goals. A discussion of pre-lithiation ended with an admission (during questions) that pre-lithiation is not really viable.

\section{Reviewer 4:}

The reviewer stated that in general for a Si anode Li-ion cell, these are pretty good results. Unfortunately, they have a long way to go for transportation applications.

\section{Question 3: Collaboration and coordination with other institutions.}

\section{Reviewer 1:}

The reviewer stated that there are no formal partners, but most potential partners are way behind what Amprius has already accomplished. Amprius does collaborate on an ad hoc basis

\section{Reviewer 2:}

The reviewer stated that there are no collaborators listed and this is a deficiency of the project. The workers should be spending their full time on anode development, but could benefit by collaborating with cathode and full cell experts to enhance the anode work.

\section{Reviewer 3:}

The reviewer commented that there are no collaborations on this project, but will be established (with the DOE laboratories) for testing after the cells are delivered. 


\section{Reviewer 4:}

The reviewer reiterated that the researchers stated that there is no team in the team overview.

Question 4: Proposed future research-the degree to which the project has effectively planned its future work in a logical manner by incorporating appropriate decision points, considering barriers to the realization of the proposed technology and, when sensible, mitigating risk by providing alternate development pathways.

\section{Reviewer 1:}

The reviewer stated that there are three goals going forward. First, to continue studies to increase the cycle life by optimizing the electrolyte formulation, anode structure, cathode materials and coating, and separator type. Second, to verify and/or demonstrate that the EOL energy density and specific energy will exceed the USABC requirements. Finally, to continue studies to source the appropriate cathode material with high capacity and cycle life. It is imperative that a systematic failure mode analysis (DPA) will be performed to identify the failure modes and develop strategies to mitigate them. This is indeed a challenge for the Si-anode based $\mathrm{Li}$-ion cells. Another intricate characteristic is the abuse tolerance, which is reportedly worse compared to the conventional Li-ion batteries. The future work planned is logical with appropriate decision points in the materials selection and cell fabrication processes.

\section{Reviewer 2:}

The reviewer stated that it is too early to make much of a judgment on whether their ideas will lead to big improvements.

\section{Reviewer 3:}

The reviewer found that the proposed future work is ambitious, but the path to advancing the state of cell development is not well delineated. The cathode and electrolyte developments are only stated as goals, but no details regarding the approach are given.

\section{Reviewer 4:}

The reviewer pointed out that there is little to no detail concerning how the team is going to attack the cycle/ calendar life issue, which should be its highest priority.

\section{Question 5: Does this project support the overall DOE objectives of petroleum displacement? Why or why not?}

\section{Reviewer 1:}

The reviewer stated that the energy requirements are close, but the cycling life work needs improvement to meet the DOE goals.

\section{Reviewer 2:}

The reviewer commented that the low specific energies and high costs of $\mathrm{Li}$-ion batteries are serious impediments to their widespread adoption in vehicles. High capacity anode materials are required to improve the specific energy of Li-ion cells. Si anode has the potential to offer twice the capacity of graphitic anodes, and Amprius has developed a fairly robust Si anode based on Si nanorods. There are early results on small laboratory cells and it would be timely to demonstrate the performance benefits (higher specific energy and energy density) in large format cells against the USABC requirements, which is the objective in this project.

\section{Question 6: Resources: How sufficient are the resources for the project to achieve the stated milestones in a timely fashion?}

\section{Reviewer 1:}

The reviewer stated that the group needs to collaborate with others, as discussed above, to have a chance to meet the goals. This would require additional resources.

\section{Reviewer 2:}

The reviewer commented that the resources seem to be excessive, but make sense based on the cost share from the sub-contractor. 


\section{A Disruptive Concept for a Whole Family of New Battery Systems: Farshid Roumi (Parthian Energy) - es242}

\section{Presenter}

Farshid Roumi, Parthian Energy

\section{Reviewer Sample Size}

A total of five reviewers evaluated this project.

\section{Question 1: Approach to performing the work-the degree to which technical barriers are addressed, the project is well-designed, feasible, and integrated with other efforts.}

\section{Reviewer 1:}

The reviewer observed a nontraditional cell design that appears to be at the very early stages of development, and also described the project as quite novel. The objectives were clearly stated, but the discussion of the approach was incomplete and focused more on the results that approach. Slide five is incomplete in the discussion of the battery architecture and Slide 6 is confusing in terms of an approach. From discussions with the presenter, a better approach is believed to exist than was presented.

\section{Reviewer 2:}

The reviewer stated that this project approach is very unconventional and ambitious relative to most other battery designs. Due to this novel approach, the performers should present technical results showing the progress of their technology since their start in February of 2015. The performer did not present any new experimental data regarding their novel cell design concept, and the only data presented on Slide 5 were identical to what was presented last year. Based on this lack of technical concept presentation, the validation of this unusually approach is not supported by any technical data.

\section{Reviewer 3:}

The reviewer pointed that this is alternative cell architecture, which is chemistry agnostic, to achieve high energy density. This is much needed and will complement advanced materials to achieving high energy density. This approach was based on a multi-layer, Supper Cell (Scell ${ }^{\mathrm{TM}}$ ) 3D architecture to achieve high energy density and high rate goals. Two and a half years is sufficient to demonstrate feasibility of this technology.

The reviewer commented that the project team needs to provide assumptions for modeling in order to assess modeling validity. Milestones were not quantifiable, and they were task descriptions, not milestones. Given the 
small size of the components, the team needs to provide a schematic on how they plan to integrate the components in the ScellTM.

\section{Reviewer 4:}

The reviewer stated that the ScellTM design was not innovative, and as a matter of fact, similar designs can be found in the U.S. patents in the 1990's for alkaline batteries. The ideal was to increase the interfacial surface area, but the interfacial area in the ScellTM would not be larger than the traditional cell. Many problems were encountered in the engineering of a primary alkaline battery. The likelihood for the design to work in rechargeable batteries is low.

\section{Question 2: Technical accomplishments and progress toward overall project and DOE goals-the degree to which progress has been made, measured against performance indicators and demonstrated progress towards DOE goals.}

\section{Reviewer 1:}

The reviewer noted that good technical progress was shown, but the discussion on methods for low-cost fabrication of the various battery elements were overstated in that there was no clear path to low-cost production at scale. At best, an approach to producing battery elements to allow prototype cells was demonstrated. The reviewer added that, to be fair, it is clearly very early in the development process.

\section{Reviewer 2:}

The reviewer stated that the team presented very limited technical accomplishments during the performance period. The majority of the new experimental data shared were based on coin cells and spacer height variation, data which used conventional materials and delivered no performance improvement over what is commonly achievable with Li-ion technology.

\section{Reviewer 3:}

The reviewer acknowledged that the PI has demonstrated the feasibility of the design and tested prototypes, but exclaimed that the PI needed to show some real electrode capacity data from a prototype, especially with this type of project. The work presented focused on how the key parameters were determined. However, the team is still at the beginning of the development phase and a long way from deciding on optimizing process steps.

\section{Reviewer 4:}

The reviewer stated that the project team demonstrated the concept of an in situ, conformal separator via electrophoretic deposition (EPD). If successful, this conformal separator technique can be an enabler for 3D cells. The project team also measured the effect of ionic diffusion distance on capacity utilization. Even though the 3D design should be capable of achieving full capacity at high rates, all the experimental results so far showed that full capacity was only achieved at low rates. Thus, even though full capacity was achieved at low rate, the team has not demonstrated the high rate feasibility enabled by the 3D architecture at the component level.

\section{Reviewer 5:}

The reviewer did not understand why the half coin cells were made and tested. The faults of the design were not identified: both anode and cathode were thick, unsure if the cell can sustain practical rate of charge and discharge, and unsure if the Li-ion become uniformly distributed in the cathode. The reviewer found that the conclusion was totally unsupported.

\section{Question 3: Collaboration and coordination with other institutions.}

\section{Reviewer 1:}

The reviewer stated that the PI has a slide listing contributions from partners. This is good and clear.

\section{Reviewer 2:}

The reviewer stated that this is a good collaboration with national laboratories, academia, and industry. The roles of each team member was clearly identified to justify their participation in the project.

\section{Reviewer 3:}

The reviewer noted that the developer identifies many partner organizations with significant battery skills. The 
collaborators' contribution should extend to guiding the performance of basic electrochemical tests to provide the cycle life, specific energy, energy density, power, etc. of this technology.

\section{Reviewer 4:}

The reviewer opined that collaborations with a potential manufacturer are lacking. While it is obvious that a partnership with a cell manufacturer is probably not viable, HydroQuebec does not appear to be the commercialization partner that will be required. It was not obvious until Slide 20 who was doing what.

\section{Question 4: Proposed future research-the degree to which the project has effectively planned its future work in a logical manner by incorporating appropriate decision points, considering barriers to the realization of the proposed technology and, when sensible, mitigating risk by providing alternate development pathways.}

\section{Reviewer 1:}

The reviewer thought that the discussion of the future work does not appear to support the identified challenges and barriers.

\section{Reviewer 2:}

The reviewer stated that the project team needs to characterize the performance of the in situ conformal separator via EPD. The team should also demonstrate full integration using the in situ conformal separator.

\section{Reviewer 3:}

The reviewer stated that efforts to study high risk/reward anode systems, like $\mathrm{Si}$, seem inappropriate given the extremely limited technical data presented with more conventional materials like graphite. This approach uses novel cell geometries and it is highly probable that the high volume change of $\mathrm{Si}$ will compound the challenge in proving the technical viability of this performer's base approach. Future work should center on experimentally proving the advantages of the performer's proposed cell geometry system using conventional materials.

\section{Reviewer 4:}

The reviewer warned that no solid future research plan was proposed to address the major design problem. The half-cell work has nothing different as those reported in the literature.

\section{Reviewer 5:}

The reviewer noted that the PI realizes the daunting task ahead and has already developed alternative approaches for moving ahead. The PI will have to make significant progress with working prototypes and results. The reviewer expressed concerns regarding whether expansion of Si anode rods will deteriorate causing increased impedance with cycling.

\section{Question 5: Does this project support the overall DOE objectives of petroleum displacement? Why or why not?}

\section{Reviewer 1:}

The reviewer stated that this is highly relevant, because high energy density is critical to achieving petro displacement.

\section{Reviewer 2:}

The reviewer stated that while it is high risk, the concept is quite novel and worthy of funding.

\section{Reviewer 3:}

The reviewer noted that DOE objectives would be supported if the Scell ${ }^{\mathrm{TM}}$ design works.

\section{Reviewer 4:}

The reviewer warned that currently this project does not support the mission of DOE for petroleum replacement. No new technical performance level has been demonstrated and the proposed cell geometry is very high risk with an unclear promise of reward. 


\section{Question 6: Resources: How sufficient are the resources for the project to achieve the stated milestones in a timely fashion?}

\section{Reviewer 1:}

The reviewer stated that $\$ 750,000$ for two and a half years might not be sufficient to demonstrate feasibility of the Scell ${ }^{\mathrm{TM}}$ concept.

\section{Reviewer 2:}

The reviewer stated that the PI will probably need additional funding to achieve milestones. However, the project team should demonstrate proof of concept sufficiently to get additional funding.

\section{Reviewer 3:}

The reviewer noted that this project is in a very early stage and has many challenges. The level of funding requested to complete the proof of concept is a minimum of three times what is available.

Reviewer 4:

The reviewer stated that project resources are appropriate. 


\section{Dramatically Improve the Safety Performance of Li- Ion Battery Separators and Reduce the Manufacturing Cost Using Ultraviolet Curing and High-Precision Coating Technologies: John Arnold (Miltec UV International) - es243}

\section{Presenter}

John Arnold, Miltec UV International

\section{Reviewer Sample Size}

A total of five reviewers evaluated this project.

\section{Question 1: Approach to performing the work-the degree to which technical barriers are addressed, the project is well-designed, feasible, and integrated with other efforts.}

\section{Reviewer 1:}

The reviewer said that the work's objective is very relevant-to improve separator performance at elevated temperatures; however, there are not enough technical details discussed.

\section{Reviewer 2:}

The reviewer said that the objective here is to improve the safety of LIBs by improving the shutdown characteristics of Li-ion cells and reduce the risk of thermal runaway and fire, without impacting the conductivity of the separators and the high rate discharge rates. Another specific objective is to reduce the manufacturing costs for the ceramic coated separators by $50 \%$. The reviewer stated that ceramic-coated separators reduce the risk from Li dendrites, minimize the separator shrinkage during thermal runaway, and also improve the interfacial stability at the electrodes. The approach here involves ultraviolet (UV)-cured binders to coat the separators with ceramic materials. Methods will be developed at the bench-top level to identify suitable binder chemistry with good adhesion and to prove its viability to coat separator rolls and to validate these separators before commercialization. The reviewer said that the studies address the technical barriers of safety and cost Li-ion batteries, though it is not clear if the proposed coating method will result in significant cost benefits over the conventional ceramic coating. The project is well designed, and integrated with other efforts, but according to the reviewer the relevance and justification of this project are to be substantiated with a proper cost assessment.

\section{Reviewer 3:}

The reviewer said that the technical advantages of UV processing compared to conventional ceramic separators are not clear. Through the addition of a new step, the promise of a cost reduction is not supported by the data

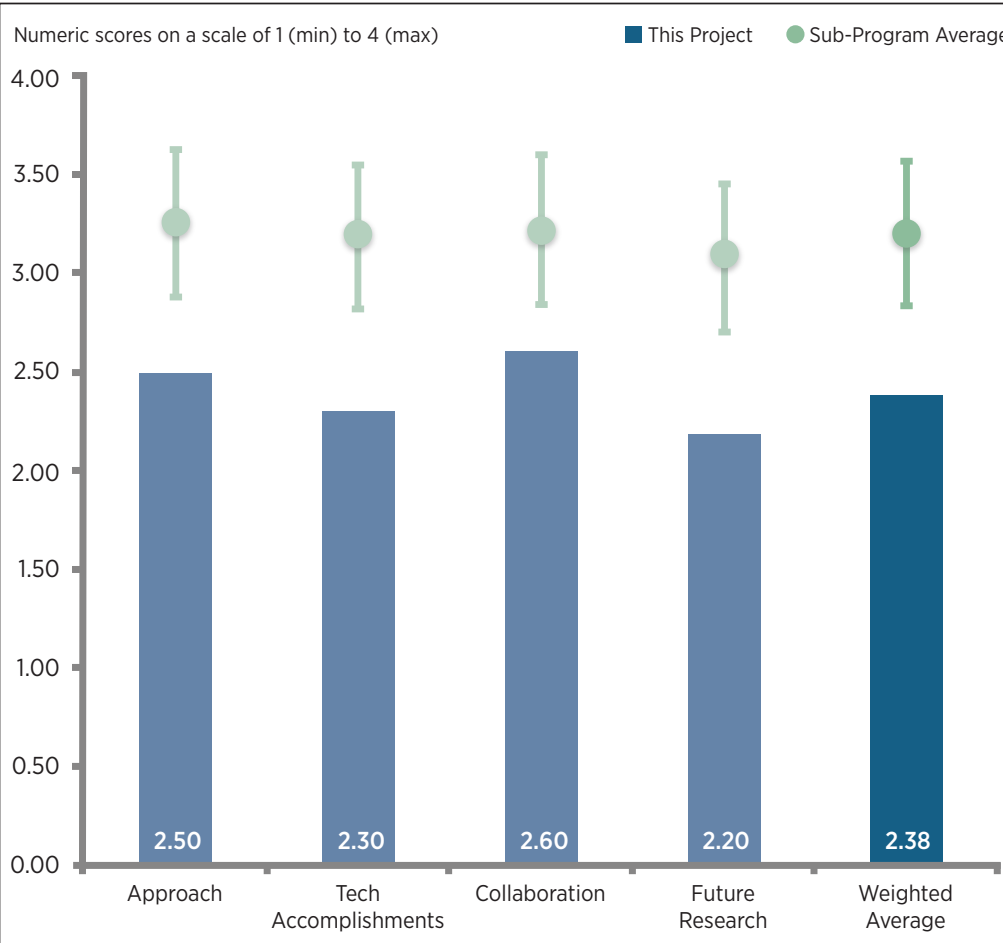

Relevant to DOE Objectives
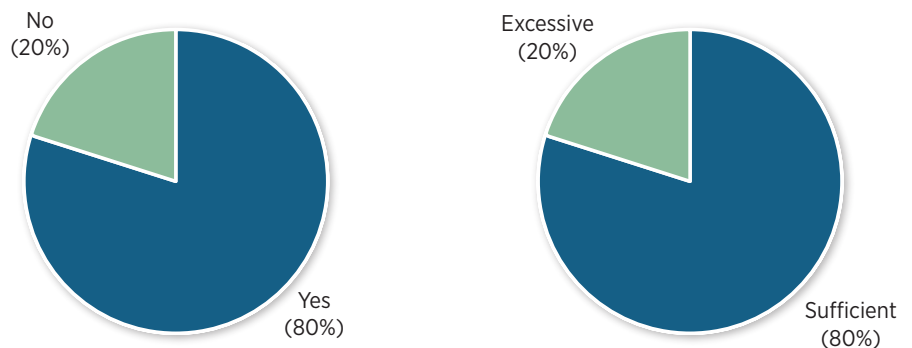

Figure 2-30 - Dramatically Improve the Safety Performance of Li-Ion Battery Separators and Reduce the Manufacturing Cost Using Ultraviolet Curing and High-Precision Coating Technologies: John Arnold (Miltec UV International) Electrochemical Energy Storage 
shown and the technical performance improvements are either the same as the incumbent (mechanical/temperature stability) or unproven (voltage stability).

\section{Reviewer 4:}

The reviewer remarked that the project involves utilizing UV curing to create ceramic-coated separators. The emphasis is on making the materials and comparing to uncoated versions. The reviewer said that this area of research is worthwhile for investigation, but this team is too inexperienced to know what tests need to be performed to analyze its materials for LIB applications. The reviewer remarked that no cost analysis was presented, and questions about commercial viability due to cost increases in fabrication were inadequately answered.

\section{Reviewer 5:}

The reviewer said that it would be great to see cost economic analysis and technical target specifications based on costs. The reviewer said that the work plan also needs to include full cell testing using carbon anodes; characterize permeability; uniformity; loss in pore volume; and chemical stability in electrolyte

\section{Question 2: Technical accomplishments and progress toward overall project and DOE goals-the degree to which progress has been made, measured against performance indicators and demonstrated progress towards DOE goals.}

\section{Reviewer 1:}

The reviewer said that the project team made good progress towards meeting the proposed objectives with UV curing and coatings.

\section{Reviewer 2:}

The reviewer remarked that good progress has been made on the UV-cured polymer-bonded ceramic coatings on the separators and their assessment in a Li-ion cell. Specifically, the team developed UV-curing coatings for various separators coatings for polyethylene (PE), polypropylene (PP), and trilayer separators that show low hightemperature shrinkage and good functionality in a $5 \mathrm{~V} \mathrm{Li-ion} \mathrm{chemistry.} \mathrm{Additionally,} \mathrm{patterned} \mathrm{coatings} \mathrm{have} \mathrm{been}$ developed on these separators, though the effect of these patterns is yet to be determined. The reviewer commented that the assessment, however is incompatible at the material level. The effects of the coating on the porosity, conductivity and electrochemical compatibility have not been quantitatively determined. The reviewer said that it is essential to establish the chemical/electrochemical compatibility of the UV-curable pre-cursors with the electrode materials (before and after curing). The assessment in the cell in also in complete without proper comparison with baseline, discharge rate. Above all, according to the reviewer, proper cost assessment is to be made to provide a convincing argument that this UV-cure method is beneficial. Overall, progress is good and is consistent with DOE's goals.

\section{Reviewer 3:}

The reviewer said that some of the test data look promising, but there really should be more technical details released to allow a proper evaluation. Without those, it is hard to judge whether uniformity, cost, etc. would be commercially competitive.

\section{Reviewer 4:}

According to the reviewer, the performer stated that the mechanical/thermal performance shown on Slide nine is comparable to a conventional ceramic separator. Additionally, the performer has very limited data to show electrochemical stability. The reviewer cited that Slide 11 shows limited cycling without disclosing voltage window, current rates, temperature or many other relevant technical details. The stated reason for using a lithium manganese, nickel oxide (LMNO) versus a lithium titanium oxide (LTO) system was to achieve high voltage on the cathode and safety on the anode. In a $1.5 \mathrm{mAh}$ cell, the rational of safety for using LTO instead of carbon is not well supported, this would also raise questions of reductive stability of the material.

The reviewer said that a basic cyclic voltammetry experiment should be performed to determine the stability of this new material. The project team mentioned that they are not electrochemists, but some of their collaborators are and should assist them. Barring that help, the reviewer said that a possible, experimental setup for the team to perform 
is a coin cell-based stainless steel (SUS) blocking cell setup with cyclic voltammetry at 1 to $10 \mathrm{mV} / \mathrm{second} \mathrm{scan}$ rate sweeping 0 to $5 \mathrm{~V}$ versus $\mathrm{Li}$ using $5 \mathrm{uA} / \mathrm{cm}^{2}$ as the threshold for stability.

\section{Reviewer 5:}

The reviewer pointed out that demonstrating a coating is possible was already performed (see 2015 slides). The reviewer asked what has really been accomplished to address whether these materials are practical for LIB operation and if they are commercially competitive. The reviewer said that this study lacks any wetting investigation, analysis of performance of separators in environments that would create Li dendrites that lead to cell failure, and cycling with more reducing anodes. The PI did not understand high-voltage battery operations with the current cathode and LTO anode versus using a graphitic anode; the reviewer said that it is worrisome that the PI is not aware and that the PI's collaborator has not given enough guidance to understand. Moreover, the comparisons are unfair; comparisons should not be made to uncoated materials but instead to competitive coated separators.

\section{Question 3: Collaboration and coordination with other institutions.}

\section{Reviewer 1:}

The reviewer said that there are good collaborations with a national laboratory (ANL) for the product validation and with the separator manufacturer (Celgard) for subsequent scale up and commercialization.

\section{Reviewer 2:}

The reviewer suggested that the collaborators should more strongly support the prime performer in electrochemical testing.

\section{Reviewer 3:}

The reviewer remarked that at ANL, Amine and coworkers performed charge/discharge experiments. This collaboration is minimal and has not resulted in the PI understanding the necessary performance metrics needed to evaluate the material. The reviewer said that simple charge/discharge tests are inadequate to evaluate the material; Amine should have facilitated further testing.

\section{Reviewer 4:}

The reviewer said that it is not clear how the collaboration worked, and gave as an example how there are no target specifications resulting from the collaborative activities.

Question 4: Proposed future research-the degree to which the project has effectively planned its future work in a logical manner by incorporating appropriate decision points, considering barriers to the realization of the proposed technology and, when sensible, mitigating risk by providing alternate development pathways.

\section{Reviewer 1:}

The reviewer detailed that the proposed future research is to demonstrate the benefits of printed coating, develop coatings with additional shut-down temperatures, and design customized commercial prototype press and verify the ability for high-speed coating with superior coating uniformity, tension, and consistency. The reviewer pointed out that it is important to augment these efforts with a proper cost assessment. The future work planned is logical and consistent with project objectives.

\section{Reviewer 2:}

The reviewer remarked that the first bullet on Slide 16, "Confirm advantages of printed coating," should be performed before any other work is done. Exploring additional shutdown temperatures, scale-up and coating speed improvements are not relevant if the performer's base chemistry is not electrochemically stable or provides a clear technical advantage over conventional ceramic separators.

\section{Reviewer 3:}

The reviewer said that future research directions for the project team should include testing membranes in real cells in their partners' laboratories. The details do not include the challenges associated with such testing (on what conditions with respect to accelerated testing, etc.). The reviewer was unclear how one can measure success with this project, i.e., in terms of applicability of their coated membranes for LIB cells. 


\section{Reviewer 4:}

The reviewer stated that the PI's focus has been on creating separators, and that the PI's plans to validate materials are lacking.

\section{Question 5: Does this project support the overall DOE objectives of petroleum displacement? Why or why not?}

\section{Reviewer 1:}

The reviewer said that in addition to improving performance characteristics, such as specific energy, energy density and specific power of the EV batteries, it is also important to improve their safety characteristics to enable their widespread adoption. Large-capacity Li-ion cells and batteries can release substantial amounts of energy during thermal runaway, which can propagate to adjacent cells in a multi-cell module. Advanced separators with less thermal shrinkage, e.g., a ceramic-coated separator, are currently being used, which partly prevent such a runaway, but the ceramic-coating add to process costs for the separators. The reviewer detailed that this project is aimed at developing new low-cost methods for ceramic-coated separators.

\section{Reviewer 2:}

The reviewer commented that the project helps in developing safer LIB technology and energy storage industry.

\section{Reviewer 3:}

The reviewer said that if this project could demonstrate some improvements over the incumbent ceramic coated separator technology, this could aid DOE's mission of petroleum reduction.

\section{Reviewer 4:}

The reviewer remarked that this project's accomplishments and plans are not sufficient to determine if this material is competitive with others on the market. Fabrication is not enough. The reviewer said that a thorough series of tests is needed, and the project team seems unaware of these tests in many cases.

\section{Question 6: Resources: How sufficient are the resources for the project to achieve the stated milestones in a timely fashion?}

\section{Reviewer 1:}

The reviewer said sufficient resources

\section{Reviewer 2:}

The reviewer said that the resources seem to be adequate for the scope of the project.

\section{Reviewer 3:}

The reviewer remarked that the resources are sufficient, but they are not being used. The reviewer said that with the experts at ANL, this company should be able to do much more for product validation.

\section{Reviewer 4:}

The reviewer commented that the project resources are large compared to the relatively small amount of technical data performed and presented. 


\section{Low-Cost, High-Capacity Non-Intercalation Chemistry Automotive Cells: Alex Jacobs (Sila Nanotechnologies) \\ - es244}

Presenter

Gleb Yushin, Georgia Tech

\section{Reviewer Sample Size}

A total of six reviewers evaluated this project.

\section{Question 1: Approach to performing the work-the degree to which technical barriers are addressed, the project is well-designed, feasible, and integrated with other efforts.}

\section{Reviewer 1:}

The reviewer said that the anode portion of this project seems to be an excellent approach. The decision to couple this with iron(III) fluoride $\left(\mathrm{FeF}_{3}\right)$ also offers a fresh approach to dealing with the shortcoming of these materials. The reviewer said that whether this will be low-cost needs to be addressed.

\section{Reviewer 2:}

The reviewer said that the objective here is to develop next-generation battery chemistries that can provide a two-fold improvement in performance. The approach involves the development of electrochemically stable ultra-high specific capacity metal fluoride (MFx)-based cathodes based on novel core-shell MFx nanocomposite powders. Such core-shell designs are expected to help mitigate the challenges, including dissolution, low electrical conductivity, volume changes, etc., that otherwise lead to large voltage hysteresis and rapid capacity fading. The reviewer remarked that later, the synthetic methods will be scaled up, which could be a drop-in-replacement to the conventional material synthesis. These metal fluoride cathodes will be combined with the $\mathrm{Si}$ anodes with a similar core-shell design being developed by Sila Nanotechnologies Inc. The reviewer remarked that the project is well designed, and integrated with other efforts.

\section{Reviewer 3:}

The reviewer observed that good progress was made on the iron(II) fluoride $\mathrm{FeF}_{2}$ system, particularly relative to previous attempts. The reviewer indicated that questions remain regarding the temperature sensitivity of the 4.6M Lithium bis(fluorosulfonyl)imide (LiFSI)/dimethoxyethane (DME) electrolyte, the performer should report performance figures at a span of relevant temperatures (i.e. $-40^{\circ}$ to $60^{\circ} \mathrm{C}$ ). Additionally, the reviewer pointed out that no information was presented towards the original target of a low-cost solution.

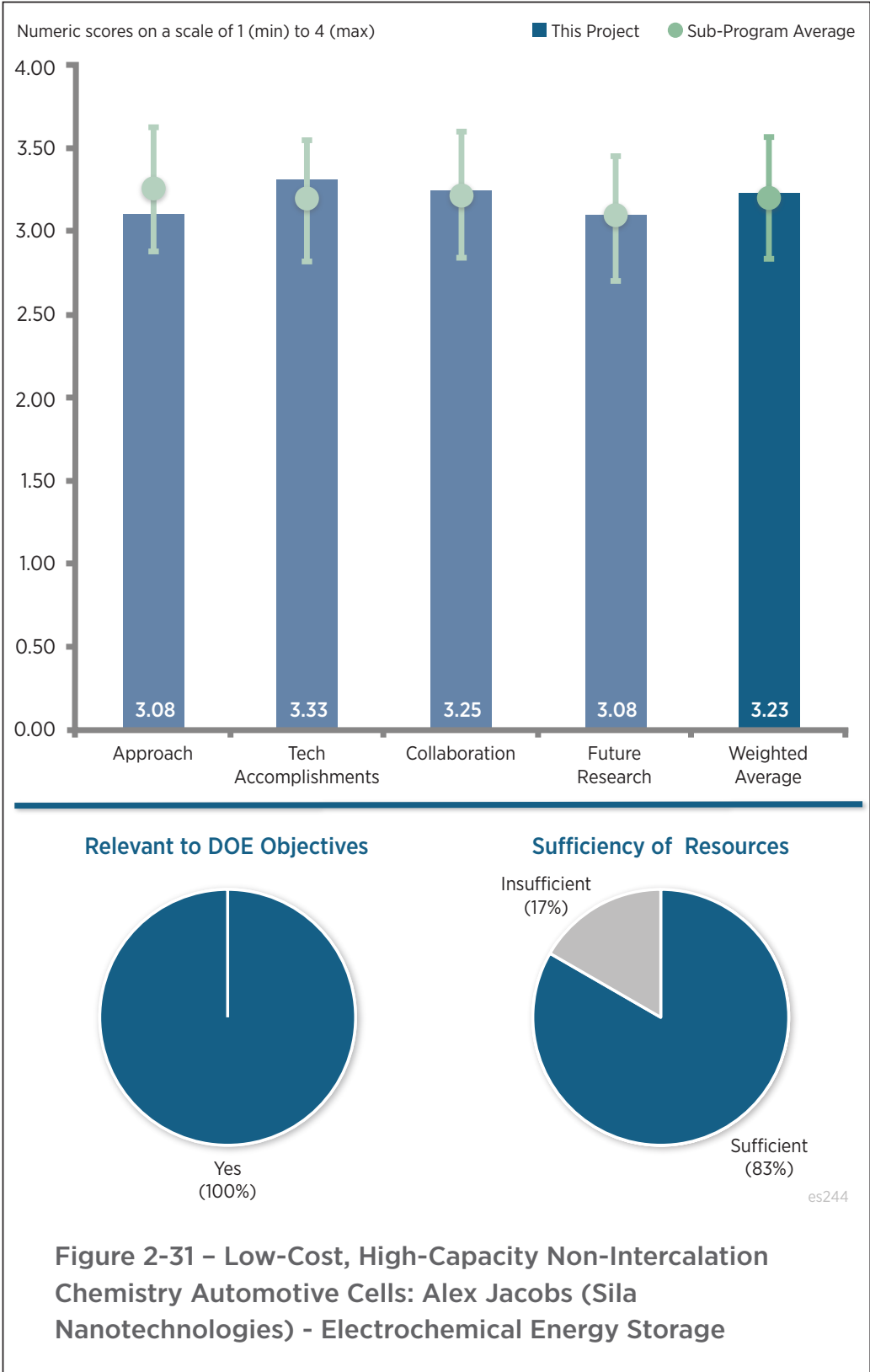




\section{Reviewer 4:}

The reviewer noted that the objectives state cost reduction and battery lifetime increase. The reviewer commented that while the Sila Si anode was shown to increase lifetime, the discussion of an approach to cost reduction was incomplete and merely based on the increased energy density.

\section{Reviewer 5:}

The reviewer said that the project aims to develop a conversion-based cathode material in couple with a Si anode with a shell. The approach of confining the MFx in the porous carbon material has been reported elsewhere, but the PI reported decent cycle life.

However, conversion materials have tested very extensively. The reviewer said that the distinction between this work and those reported elsewhere was not clear.

\section{Reviewer 6:}

The reviewer said that this project is extremely challenging, and recommended that the developer first define the cell design the project team intends to target, and from there derive the respective anode and cathode material properties to be addressed. These properties can then become the target of the research, through prioritization. The reviewer agreed that reversibility of $\mathrm{FeF}_{2}$ and copper(II) fluoride $\left(\mathrm{CuF}_{2}\right)$ would remain highest priority.

\section{Question 2: Technical accomplishments and progress toward overall project and DOE goals-the degree to which progress has been made, measured against performance indicators and demonstrated progress towards DOE goals.}

\section{Reviewer 1:}

The reviewer said that the PI demonstrated that the conversion cathode $\left(\mathrm{FeF}_{2}\right)$ was synthesized and good cycle life can be achieved. The solubility of Fe was eliminated, and the technical milestones were met.

\section{Reviewer 2:}

The reviewer said that good progress has been made on the Si anode and to a lesser extent on the metal fluoride cathodes, using core-shell design. For the $\mathrm{Si}$ anode, the core consists of porous nano-structured $\mathrm{Si}$, covered by a solvent impermeable and mechanically robust shell with fast Li transport. The anode shows a high capacity of $\sim 800-100 \mathrm{mAh} / \mathrm{g}$ with a low-volume change of less than $12 \%$ and as a result good cycle life in half-cells and laboratory full cells with NCM and lithium iron phosphate (LFP) cathodes. However, according to the reviewer, the anode loading is noticeably small with $2-5 \mathrm{mAh} / \mathrm{cm}^{2}$, which is not much better than graphite anodes. For the metal fluoride cathode, the MFx is confined in carbon nanopores, and is also coated with a protective outer shell. The electrolyte is a highly concentrated solution of LiFSI in DME, which shows good cycle life for Li but might be too viscous for high rate discharges and is also not cost-effective. The reviewer said that even though the cycle life demonstrated here is fairly good, the cathode loading is not mentioned here (thin electrode generally cycle well) and the hysteresis is still noticeable. The reviewer identified as another shortcoming how the Si-MFx cell would need some pre-lithiation, which is a difficult proposition. The data shown here are only in half cells. The reviewer concluded that the overall progress is good and is consistent with the DOE goals.

\section{Reviewer 3:}

The reviewer remarked that the PI has progressed well and has demonstrated capacity and cycle life of the anode. The project team has gone on to make a working system from FeFx producing the longest cycle life cell to date. The reviewer said that the PI has collaborated well with partners.

\section{Reviewer 4:}

The reviewer said that the developer was not clear on the cycle rate applied to achieve current cycle life. Anode progress appears to be excellent, but progress on the cathode has not been as much. The reviewer recommended that the PI continue to build a foundational structure around the cathode active material cycling mechanistics, and resultant failure modes.

\section{Reviewer 5:}

The reviewer said that the hysteresis seen in the $\mathrm{FeF}_{2}$ system is perhaps less than other approaches with this chemistry, but far larger than conventional cathodes. If the long-term goal is to pair this cathode with a Si anode, 
the already large problem of SOC determination with Si systems owing to hysteresis maybe exacerbated. The reviewer said that the PI should present a strategy/approach to deal with this potential implementation barrier.

Reviewer 6:

The reviewer said that the PI was evasive when asked about how Li was introduced into the cell.

\section{Question 3: Collaboration and coordination with other institutions.}

\section{Reviewer 1:}

The reviewer said there are a small number of collaborators, but the collaboration is great.

\section{Reviewer 2:}

The reviewer said that the PI has collaborated well with the ARL. Together the team resolved the solid electrode interface (SEI) component identification, leading to the use of electrolyte formulation that reduced Fe dissolution, making the FeFx system workable. Without this collaboration, the project would be dead.

\section{Reviewer 3:}

The reviewer commented that there is an on-going collaboration with the ARL in the development of suitable electrolytes for $\mathrm{Li}-\mathrm{FeF}_{2}$ cells and with the university partner for the anode/cathode development.

\section{Reviewer 4:}

The reviewer found that the level of collaboration seems appropriate.

\section{Reviewer 5:}

The reviewer said that the PI only has limited collaborators.

\section{Reviewer 6:}

The reviewer said that additional collaborations would help.

Question 4: Proposed future research-the degree to which the project has effectively planned its future work in a logical manner by incorporating appropriate decision points, considering barriers to the realization of the proposed technology and, when sensible, mitigating risk by providing alternate development pathways.

\section{Reviewer 1:}

The reviewer said that the proposed future research is reasonable.

\section{Reviewer 2:}

The reviewer said that the proposed future research is aimed at developing $\mathrm{Li}$-ion permeable (solvent impermeable) shells on $\mathrm{CuF}_{2}-\mathrm{C}$ composite powders prior to forming them into electrodes, exploring doping of MFx in order to enhance rate performance and stability, developing high-capacity loading cathodes for matching with Si anodes, and finally optimizing electrode construction and electrolyte composition to achieve high rate performance in full cells. The reviewer said that these studies are well aligned with the project objectives and DOE's program goals.

\section{Reviewer 3:}

The reviewer remarked that the progress towards a $\mathrm{CuF}_{2}$ systems seems promising; however, the previously raised questions of cost, hysteresis and temperature sensitivity should also be addressed for that system.

\section{Reviewer 4:}

The reviewer requested that the project team please focus on a single material class, and understand the key failure modes, before pursuing another class of material. While the desire to move to $\mathrm{CuF}_{2}$ is understood, it may be premature.

\section{Reviewer 5:}

The reviewer was unsure that it is wise to attempt to perfect the $\mathrm{CuFx}$ system at this point. More resources should be focused on improving the FeFx system and then getting a handle on developing a low-cost process. The 
reviewer commented that more information is needed to compare expected cost reduction. The project team will need to develop a process for pre-lithiating either the Si or the FeFx for the cell to work.

\section{Reviewer 6:}

The reviewer said that the future work appears incomplete when compared to the remaining challenges and barriers.

\section{Question 5: Does this project support the overall DOE objectives of petroleum displacement? Why or why not?}

\section{Reviewer 1:}

The reviewer said that this is a highly innovative, and potentially disruptive development. It is worth further investigation.

\section{Reviewer 2:}

The reviewer remarked that this project is relevant to the DOE mission of improved batteries to decrease petroleum consumption.

\section{Reviewer 3:}

The reviewer said that the cathode materials being considered have direct relevance to DOE's objectives.

\section{Reviewer 4:}

The reviewer pointed out that low specific energies and high costs of $\mathrm{Li}$-ion batteries are serious impediments to their widespread adoption in vehicles. The conventional cathodes based on intercalation of Li, typically less than one Li per metal, and have low specific capacities and thus limit the specific energy and energy density of the Liion cells. It is imperative that new cathodes that involve multi-lithiums are to be developed to address this technical barrier. The reviewer commented that conversion cathodes have the ability to involve multiple lithiums and metal fluorides (e.g., $\mathrm{FeF}_{3}$ ) involve two-three reversible lithiums and thus have high specific capacity and can lead to improved specific energy and energy densities but require further developments to overcome the challenges of poor conductivity poor reversibility and large hysteresis during cycling.

\section{Reviewer 5:}

The reviewer remarked that if this project is successful, it will exceed many of DOE's targets making EVs a commercialization a reality.

\section{Question 6: Resources: How sufficient are the resources for the project to achieve the stated milestones in a timely fashion?}

\section{Reviewer 1:}

The reviewer found that the resources seem to be adequate for the scope of the project.

\section{Reviewer 2:}

The reviewer commented that the budget appears commensurate to the work plan.

\section{Reviewer 3:}

The reviewer said that the project funding seems appropriate for scope.

\section{Reviewer 4:}

The reviewer expressed concern that, if some promise is shown with the cathode work, this project may be too small to address the complexities associated with this class of cathode active material (CAM). The developer should watch their resource needs closely.

\section{Reviewer 5:}

The reviewer believed that the financial resources are sufficient at this stage of the R\&D. 


\section{Low-Cost, Structurally Advanced Novel Electrode and Cell Manufacturing: Billy Woodford (24M Technologies) - es245}

\section{Presenter}

Billy Woodford, 24M Technologies

\section{Reviewer Sample Size}

A total of five reviewers evaluated this project.

\section{Question 1: Approach to performing the work-the degree to which technical barriers are addressed, the project is well-designed, feasible, and integrated with other efforts.}

\section{Reviewer 1:}

The reviewer commented that the idea is unique and methods of evaluation are sufficient.

\section{Reviewer 2:}

The reviewer summarized that the objective here is to modify the electrode design that allow better utilization of thick electrodes, which results in a reduction of inactive materials (separators and current collectors) that translates to higher specific energy and energy density. To enable proper utilization of the active material in these dense electrodes, the porosity/tortuosity need to be sufficiently high, as is being done here using magnetic methods. With fewer unit operations for the electrode fabrication and low capital equipment costs compared to the conventional Li-ion cells, this modified design is expected to be easier to scale up. The reviewer commented that this architecture is anticipated to be amenable for high volume manufacturing, especially for stationary applications. The reviewer said that it is not clear, however, how this modified design of electrodes will enable an abuse tolerant battery system. The reviewer wondered if this is possibly by the mechanical flexibility of the cells. The project is well designed, and integrated with other efforts.

\section{Reviewer 3:}

The reviewer said that this is a potentially disruptive idea for battery technology in general. However, not enough information was provided to assess the impact on EVs.

\section{Reviewer 4:}

The reviewer commented that the performer is targeting the barriers of cost, performance, and abuse, but did not provide technical data to support progress despite being $75 \%$ complete with their project's timeline. The reviewer 
said that the project disclosed very little technical detail regarding the technical details of the semisolid electrodes at the center of this project.

\section{Reviewer 5:}

The reviewer said that there are many concerns with the approach. The proposed work has several barriers, including manufacturing cost, energy density, and reliability. In terms of manufacturing cost, this is something that is being partially addressed. Even though a number of manufacturing steps are reduced, it is not clear on the costs of specialty materials used for making gels/slurries for enhanced conductivity. The reviewer said that it would be good to see the cost analysis and comparison of costs associated with new electrode chemistries. In terms of energy density, the reviewer said that these calculations need to be more transparent at both cell level and pack level. In terms of reliability, the company is delivering cells to DOE labs for testing.

\section{Question 2: Technical accomplishments and progress toward overall project and DOE goals-the degree to which progress has been made, measured against performance indicators and demonstrated progress towards DOE goals.}

\section{Reviewer 1:}

The reviewer said that technical and investment accomplishments so far are fairly good.

\section{Reviewer 2:}

The reviewer said that good progress has been made in demonstrating the scaling-up capability of this technology, and in fabricating 4-6 Ah cells with these thick electrodes to demonstrate good cycle life and calendar life characteristics. However, there were a few issues that were not quite clear. The reviewer cited analysis showing that high areal capacity can be realized at high current densities in these electrodes, but the actual current densities in these (thick) electrodes may be higher than in the conventional designs. It was not clear how well these cells behave at high rates (data not provided), but the round trip efficiency of $90 \%$ is lower than normal (95\%). Further, the reviewer commented that no information was provided on the specific energy and energy density of the $6 \mathrm{Ah}$ cells, nor on the cost benefits associated with this design. As may be expected, the volumetric energy density is considerably low; the reviewer believed $200 \mathrm{Wh} / 1$ was mentioned. The reviewer said that it is not clear how the electrode design can be optimized to improve cycle life. The reviewer was encouraged to see that the $6 \mathrm{Ah}$ cells have passed all the United Nations (UN), UL and USABC safety tests. Overall, the progress is good and consistent with DOE's goals.

\section{Reviewer 1:}

The reviewer said that there was really not enough technical information presented to provide a meaningful assessment, e.g., mixing rate, formation cycle, etc.

\section{Reviewer 2:}

The reviewer commented that regarding cost, only broad statements of process simplification were stated without any technical detail to assess the feasibility of this performer's approach. Regarding performance, the performer did not answer basic questions regarding cells, such as specific energy and energy density, despite receiving these specific questions in the 2015 review feedback, and during the 2016 presentation. Regarding abuse, the reviewer said that Slide 12 has blanket statements of pass of a mixture of test procedures (UN, UL and USABC) without any technical detail (European Council for Automotive R\&D [EUCAR] score, temperature, voltage, current, time, etc.) shared. Additionally, during the review the question of temperature came up and the performer stated that low-temperature $\left(40^{\circ} \mathrm{C}\right)$ survivability was possible, but did not comment on performance. The reviewer commented that given the high viscosity nature of this performer's semisolid electrodes, a range of temperatures $\left(-40^{\circ}\right.$ to $\left.60^{\circ} \mathrm{C}\right)$ should be studied and the technology's energy, power and cycle life reported.

\section{Reviewer 3:}

The reviewer said that it is difficult to evaluate the progress because of missing (needed) information in the presentation slides and in response to reviewer questions. The reviewer remarked that it was amazing was how difficult it was to extract $\mathrm{mAh} / \mathrm{kg}$ and $\mathrm{mAh} / \mathrm{L}$ values for any of the cells presented. The reviewer said that it was unclear why testing at low temperatures has never been attempted given the simplicity and low-cost of acquiring a 
cooling chamber. Low-temperature operation is important for stationary storage; if batteries are not cold tolerant, then insulation should be considered in cost analysis.

The reviewer said that values for conductivity should be reported for representative suspensions. If conductivities can be tuned, the range of accessible conductivities should be provided. The effect of SEI formation on individual particles was unclear, as was the consequence on ionic and electronic conductivity. The reviewer remarked that numerical values for conductivity should have been provided for the reviewers, and were not given in response to reviewer questions.

\section{Question 3: Collaboration and coordination with other institutions.}

\section{Reviewer 1:}

The reviewer said that there were no external partners mentioned, but the cells ( $4 \mathrm{Ah}$ ) were sent to ANL for an independent assessment.

\section{Reviewer 2:}

The reviewer remarked that some mention of cells being cycled at ANL was given during the presentation, but no results were shared. The reviewer would like to know what has been done for cell validation.

\section{Reviewer 3:}

The reviewer commented that no collaborations were described.

\section{Reviewer 4:}

The reviewer said there were no data on collaborations.

Question 4: Proposed future research-the degree to which the project has effectively planned its future work in a logical manner by incorporating appropriate decision points, considering barriers to the realization of the proposed technology and, when sensible, mitigating risk by providing alternate development pathways.

\section{Reviewer 1:}

The reviewer remarked that despite the lack of information provided for review, it appears that plans to meet UL requirements will be completed.

\section{Reviewer 2:}

The reviewer said that the proposed future research is aimed at demonstrating the final cathode and anode volume loading, electrode quality metrics at $260+\mathrm{cm}^{2} \mathrm{f}$ ootprint and target electrode yield, target cell manufacturing yield, and delivering final program deliverables: cells with $260+\mathrm{cm}^{2}$ footprint exceeding target yield. The reviewer said that these studies are well aligned with the project objectives and DOE's program goals. However, it would be helpful if there are numbers associated with the demonstration of enhanced performance or reduced cost, which are the technical barriers for the DOE VTO program.

\section{Reviewer 3:}

The reviewer said that the plan is to deliver on the project milestones. One issue the reviewer identified is that very few things are quantitative in the presented milestones, so it is very difficult to gauge how much remains to be done.

\section{Reviewer 4:}

The reviewer said that the appropriate future direction is not possible to determine given the lack of technical content presented by the performer.

\section{Reviewer 5:}

The reviewer commented that the company claims to have raised a lot of venture capital. The reviewer said that it is simply puzzling to see the company relying on DOE and Advanced Research Projects Agency - Energy (ARPA-E) grants. The reviewer said that it is not clear what is being accomplished with the DOE support. The future goals include meeting target anode and cathode loadings. In the progress, the project team already showed delivery of cells and packs of cells. So, according to the reviewer, it is not clear why the company has delivered 
the cells without meeting the target loadings for anode and cathode. The reviewer commented that the actual challenges with increasing loadings are also not detailed enough to see whether the team has the proper plan to overcome them.

\section{Question 5: Does this project support the overall DOE objectives of petroleum displacement? Why or why not?}

\section{Reviewer 1:}

The reviewer said that these cells may lead to lower-cost, longer-lasting cells that could be used in electrified vehicles and in stationary storage.

\section{Reviewer 2:}

The reviewer commented improved energy storage technology with low manufacturing cost.

\section{Reviewer 3:}

The reviewer noted that low specific energies and high costs of Li-ion batteries are serious impediments to their widespread adoption in vehicles. Fabrication of conventional Li-ion cells involves complex, wet/dry/wet operations with an expensive infrastructure and with a high proposition of inactive materials resulting in lower specific energy/energy density and higher costs. The reviewer said that new methods of electrode fabrication are desired that would lead to improved energy densities, reduced cost and increased ease of scale up, which are being addressed by the project.

\section{Reviewer 4:}

The reviewer said that given the lack of technical content shared, it is not possible to determine if this project will contribute to the DOE mission of petroleum reduction.

\section{Question 6: Resources: How sufficient are the resources for the project to achieve the stated milestones in a timely fashion?}

\section{Reviewer 1:}

The reviewer said that the resources seem to be slightly excessive for a two-year project with this scope.

\section{Reviewer 2:}

The reviewer remarked that the project resources are large relative to the small amounts of technical content presented.

\section{Reviewer 3:}

The reviewer commented that the company raised a large amount of venture capital monies and it is unclear why the project team needs support from DOE and ARPA-E to make technical progress. The reviewer said this does not seem right [DOE Program Clarification: 24M has support for a particular high risk/high reward activity, namely a detailed quantitative analysis of its unique battery architecture/manufacturing to vehicle electrification needs. DOE's view is that this is not an activity that venture capital funding entities would support at this time in 24M's life cycle.]. 


\section{Advanced Drying Process for Lower Manufacturing Cost of Electrodes: Iftikhar Ahmad (Lambda Technologies) - es246}

\section{Presenter}

Iftihkar Ahmad, Lambda

Technologies

\section{Reviewer Sample Size}

A total of five reviewers evaluated this project.

\section{Question 1: Approach to performing the work-the degree to which technical barriers are addressed, the project is well-designed, feasible, and integrated with other efforts.}

\section{Reviewer 1:}

The reviewer remarked that the objective here is to develop an alternate electrode fabrication process that consists of an advanced rapid drying process that will allow increased electrode loading for high energy density batteries and also reduced fabrication costs. The conventional method of electrode drying is a slow and high-cost operation needing a large footprint. In contrast, the advanced drying process (ADP) with variable frequency microwaves (VFM) is shorter $(1 / 5$ in length) and quicker, with microwaves penetrating the electrode slurry and rapidly driving the solvent molecules out of thick coatings. The reviewer commented that the VFM provides more uniform heating than fixed frequency microwaves processes and also allows processing on metal foil without any damage. It is possible this process will result in a reduction in the electrode manufacturing cost by $30 \%$, but the cost benefit at the battery level will be much less. The reviewer noted that the approach involves developing an ADP/VFM processing chamber that is designed to fit in the pilot coating line at Navitas. Prototype cells will be built to demonstrate the efficacy of the ADP/VFM over the conventional (infrared) heating. The milestones look reasonable to verify and validate this new drying process. The reviewer concluded that overall, the project is well designed, and integrated with other efforts.

\section{Reviewer 2:}

The reviewer remarked that the approach seems interesting, i.e., using microwave to dry the electrodes.

\section{Reviewer 3:}

The reviewer said that the approach of a new drying method for electrode manufacturing appears sound and to have been achieved. The true impact of manufacturing cost and any unintended performance degradations still

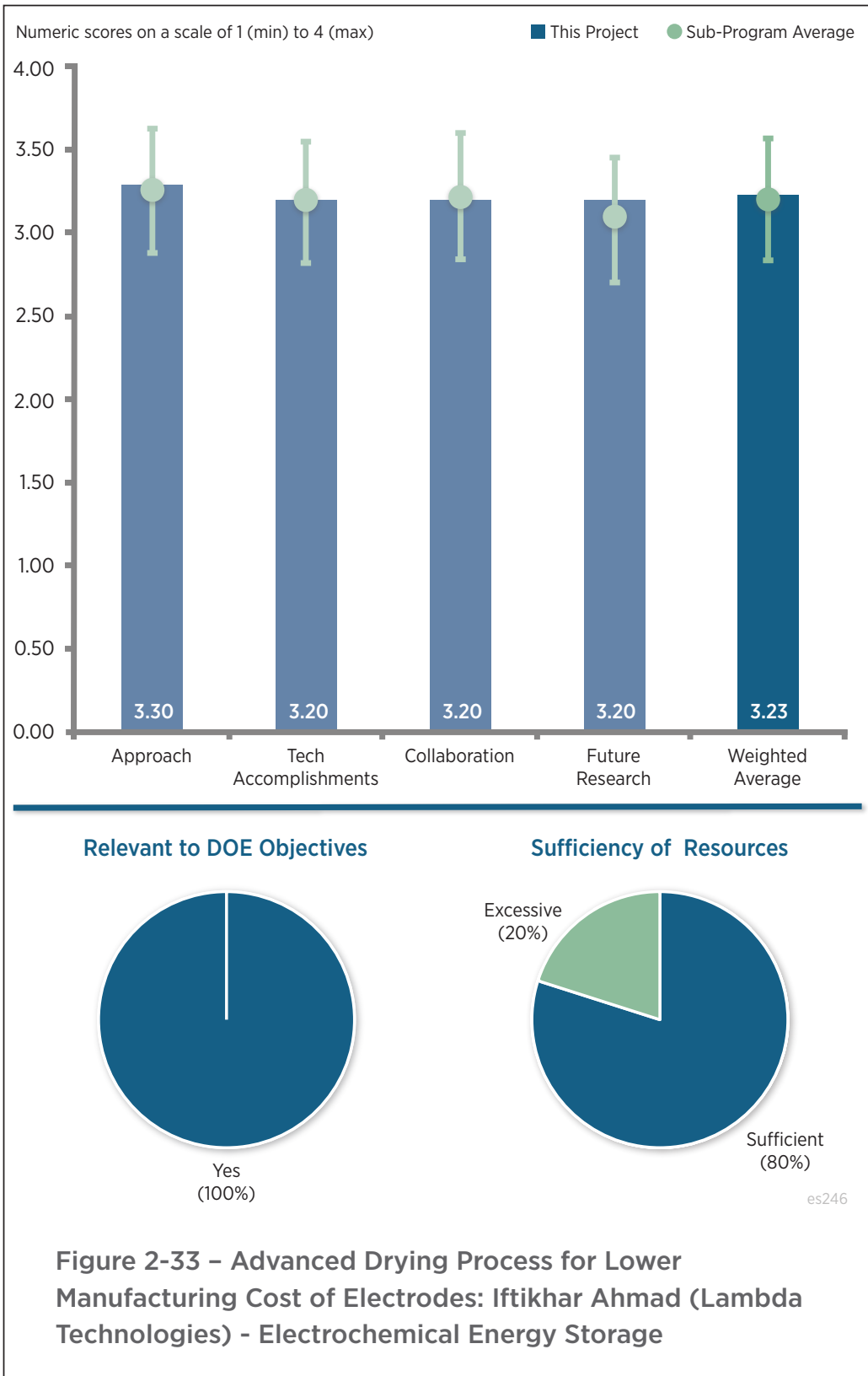


need to be studied. The reviewer commented that the advantage of this approach is often described in terms of equipment dimensions (Slide 10 and 14), which is not a priority, unlike the project goals of cost or energy density improvement.

\section{Reviewer 4:}

The reviewer remarked that using a VFM to dry the electrode web will accelerate the production speed, which would reduce production cost. The rapid variable frequency will ensure the uniformity of microwave distribution and the metal exposure. The reviewer said that the PI should provide a detailed cost analysis to forecast the potential impact on the cost.

\section{Reviewer 5:}

The reviewer said this approach provides a unique way — at least for the battery community — to more effectively dry coatings.

\section{Question 2: Technical accomplishments and progress toward overall project and DOE goals-the degree to which progress has been made, measured against performance indicators and demonstrated progress towards DOE goals.}

\section{Reviewer 1:}

The reviewer remarked that the project team seemed to make great progress in demonstrating a workable microwave apparatus for drying a continuous roll-roll process. The progress in preventing microwave power leak for a metal foil going through the oven is interesting.

\section{Reviewer 2:}

The reviewer said that good progress has been made in the first year towards designing the ADP/VFM processing chamber that will be integrated with the pilot plant cell fabrication at Navitas. This chamber will allow $170 \mathrm{~mm}$ foil (without much microwave leakage) and has a sub-chamber to contain hot-air for surface drying and solvent removal, in addition to internal heating with microwaves. The reviewer detailed that after fabricating this device, roll-to-roll dried electrodes have been fabricated and are being tested for cycle life. Full cell testing shows no difference in rate performance between standard and ADP dried electrodes. The reviewer said that even though this process looks simpler, faster and supposedly cheaper, there are a couple of issues, including the following: First, rapid drying can lead to non-uniform drying (especially with thick electrodes) and cracks in the electrode surface. No data were presented (e.g., scanning electrode microscopes [SEMs] showing the uniformity of the dried electrodes. Second, it is not clear how much optimizing (frequency range) is needed, if there is a change in the slurry composition (e.g., organic solvent or water). Third, it is helpful to have a preliminary cost model, with the savings in the process time, footprint etc. and project the overall cost savings at the battery level. Overall, according to the reviewer, the progress is good and consistent with the project objectives and DOE's goals.

\section{Reviewer 3:}

The reviewer said that the oven development and electrode/cell build work by Navitas shows good technical progress. The reviewer noted that open questions remain, including if the increased drying speed distorts the creation of pore volumes of active material and/or impacts the current collector (i.e., in the case of aluminum by changing the exfoilating paths whereby $\mathrm{LiPF}_{6}$-based electrolytes create $\mathrm{AlF}_{3}$ and passivate the layer). The reviewer said that this should be investigated by power/resistance studies with Navitas-made electrodes (electrochemical impedance spectroscopy [EIS], hybrid pulse-power capability, current/voltage [i-V] pulse, etc.).

\section{Reviewer 4:}

The reviewer stated that the project progressed well. The prototype VFM dryer was built and tested as planned. The traditional drying and VFM drying were compared and the advantages were demonstrated. The reviewer suggested that the PI should focus more on the impact on the electrode physical properties, e.g., density, conductivity, etc.

\section{Question 3: Collaboration and coordination with other institutions.}

\section{Reviewer 1:}

The reviewer noted that there is an on-going collaboration with a cell manufacturer (Navitas). 


\section{Reviewer 2:}

The reviewer said that collaborations seemed appropriate.

\section{Reviewer 3:}

The reviewer said that collaboration seems fine.

\section{Reviewer 4:}

The reviewer encouraged the PI to collaborate with both material and cell manufactures.

Question 4: Proposed future research-the degree to which the project has effectively planned its future work in a logical manner by incorporating appropriate decision points, considering barriers to the realization of the proposed technology and, when sensible, mitigating risk by providing alternate development pathways.

\section{Reviewer 1:}

The reviewer said that the proposed work in the remaining few months of the project is directed at demonstrating the viability of ADP for electrode fabrication in prototype Li-ion cells. Specific relevant activities being planned are cell fabrication (at Navitas) and continuing the cycle life testing on the ADP-processed electrodes, verifying the process with electrodes containing higher active material loadings, and completing energy related cost analysis for the ADP drying processes and assess the overall cost benefits.

\section{Reviewer 2:}

The reviewer commented that future work should focus on initial project targets of cost reduction (supported by technical data now that the project team has a working system) and prospect for increasing electrode energy density. Additionally, the reviewer recommended that any impact on electrode/power from the different drying method should also be studied.

\section{Reviewer 3:}

The reviewer stated that the proposed future research covers the method development. Both cost and impacts on electrode properties should be included.

\section{Reviewer 4:}

The reviewer recommended that analysis of recovered NMP through $1 \mathrm{H}$ and $13 \mathrm{C}$ nuclear magnetic resonance (NMR) spectroscopy should be performed in comparison to analysis of NMP recovered using more common heating methods for NMP removal to determine if (partial) decomposition is occurring, and if so, are the byproducts the same. The reviewer said that amides are thermally sensitive, and even with average temperatures of $120^{\circ} \mathrm{C}$, or possibly higher in localized areas, decomposition is a concern as far as the impact on the recyclability of NMP. The reviewer pointed out that determining if $\mathrm{Cu}$ corrosion occurs and its effect on cell cycling is an important area to explore.

\section{Reviewer 5:}

The reviewer suggested that the project needs more attention on the electrode characterization in terms of porosity, mechanism of drying, effect of drying with thickness, etc. In addition, the reviewer recommended that a task should be added for monitoring the microwave leakage and ensuring personnel safety. For example, under what conditions could the microwaves leak from this system and how will this be detected.

\section{Question 5: Does this project support the overall DOE objectives of petroleum displacement? Why or why not?}

\section{Reviewer 1:}

The reviewer detailed that low specific energies and high costs of Li-ion batteries are serious impediments to their widespread adoption in vehicles. The high costs are partly attributed to the complex time-intensive fabrication processes for the electrodes with the conventional coaters/dryer. New methods of coating the active materials are desired with the aim of increasing electrode loading and/or reducing the cost of electrode fabrication, which are being addressed here. 


\section{Reviewer 2:}

The reviewer commented that this process may lead to lowered costs of electrode fabrication through faster processing.

\section{Reviewer 3:}

The reviewer stated improved manufacturing practice and potentially low-cost for electrode manufacturing for Li-ion batteries. The reviewer said that the project potentially applies to other applications as well.

\section{Reviewer 4:}

The reviewer said that if the benefits of this novel drying method can be quantified (such as cost reduction and energy density improvement), this could serve DOE's mission to reduce petroleum reduction.

\section{Question 6: Resources: How sufficient are the resources for the project to achieve the stated milestones in a timely fashion?}

Reviewer 1:

The reviewer pointed out that the PI's background is ideal for addressing the challenges of this project, and the pairing with Navitas appears to be quite effective in the production and testing of materials.

\section{Reviewer 2:}

The reviewer said that the resources are adequate for the scope of the project.

\section{Reviewer 3:}

The reviewer commented that resources seem appropriate. 


\section{High-Energy Lithium Batteries for Electric Vehicles: Herman Lopez (Envia Systems) \\ - es247}

\section{Presenter}

Herman Lopez, Envia Systems

\section{Reviewer Sample Size}

A total of five reviewers evaluated this project.

\section{Question 1: Approach to performing the work-the degree to which technical barriers are addressed, the project is well-designed, feasible, and integrated with other efforts.}

\section{Reviewer 1:}

The reviewer said that the approach used in this effort is very effective at addressing the technical barriers identified for this project. For this reviewer, areas of concern for this effort would be at this point not much work has been done in the area of power capability, and at this point there is limited testing at high/low temperatures. The reviewer said these data would significantly strengthen this effort.

\section{Reviewer 2:}

The reviewer opined that the flow diagram is not very informative, but that one can get a flavor of the project team's approach from the rest of the presentation.

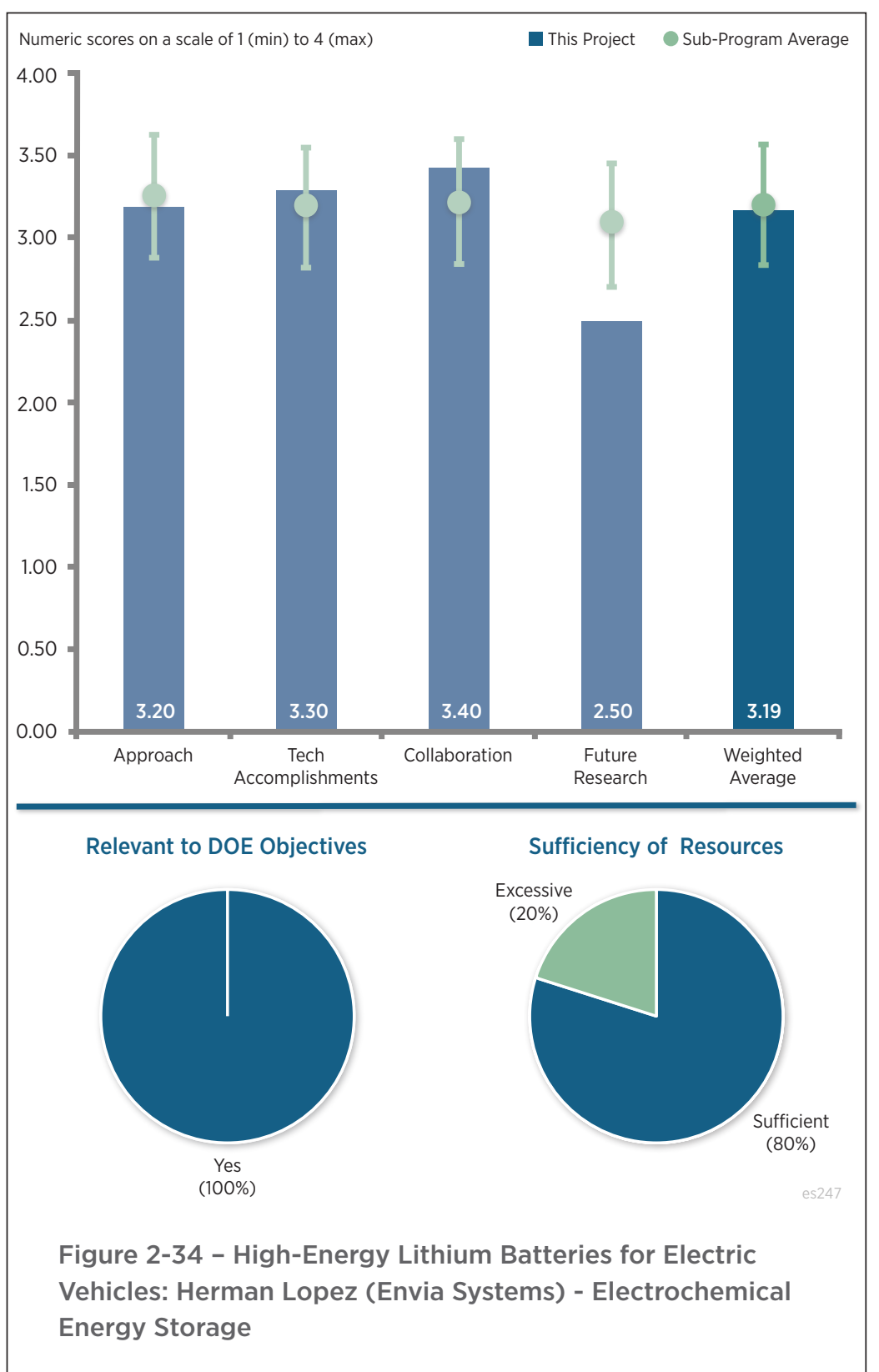

\section{Reviewer 3:}

The reviewer commented that the approach to cell development is good and seems to be achieving the specific energy goals, but the testing protocol as reported is quite incomplete. No pulse testing during cycling is reported, and the reviewer is concerned that the optimization based solely on specific energy and energy density will result in severe problems with pulse capability later in life. This can cause a back to the drawing board effect on the cell development protocols. The reviewer suggested that if there are data on pulsing, these should be reported so that a valid assessment of status can be done.

\section{Reviewer 4:}

The reviewer said that not a lot of testing on the power capability of cell for cathode/anode with different surface area/porosity and electrolytes have been conducted. It was unclear to this reviewer what the target is of cell resistance for the contractor to meet the power target for a $12 \mathrm{~V}$ battery. Furthermore, it was unclear to this reviewer if the contractor can meet the cost target without other factors being considered, such as the materials cost and manufacturing cost reduction. 


\section{Reviewer 5:}

The reviewer detailed that the objective here is to develop high capacity cathode and anode materials, screen commercial electrolytes and separators, optimize pre-lithiation processes and integrate them into high-capacity pouch cells that meet the USABC EV battery goals. The previous efforts were with Li-rich Mn-rich layeredlayered cathode materials, which have not shown the desired performance (poor cycle life and voltage fade). The current approach is to develop cathodes based on Mn-rich and Ni-rich cathodes that seem to have good cycle life characteristics, but with slightly lower specific capacities. The reviewer said that the anode development is however more intricate and is based on n-type Si and polymer composite with pre-lithiation affected electrochemically. Additionally, the electrolyte and separator are also being optimized, especially to stabilize the SEI on the Si anode. The reviewer pointed out that these strategies are similar to those being evaluated in various laboratories, with the difference being the electrochemical-pre-lithiation of Si. The reviewer said that the issues in handling prelithiated Si during fabrication have not been listed, nor have the benefits of such pre-lithiation been demonstrated. The reviewer remarked that these studies address the technical barriers for high-energy Li-ion batteries, though the targeted specific energy and energy densities are already realized in commercial cells with graphitic anodes and the state of art cathodes. The reviewer said that the project is well designed, and integrated with other efforts, but the feasibility of $\mathrm{Si}$ anode with the projected performance enhancements will be a challenge for this project.

\section{Question 2: Technical accomplishments and progress toward overall project and DOE goals-the degree to which progress has been made, measured against performance indicators and demonstrated progress towards DOE goals.}

\section{Reviewer 1:}

The reviewer noted that there was a wide range of technical accomplishments on several fronts.

\section{Reviewer 2:}

The reviewer pointed out that having demonstrated over 500 cycles in a full 21 Ahr pouch cell with specific energy of approximately $260 \mathrm{Whr} / \mathrm{kg}$, this effort is demonstrating outstanding progress. Furthermore, demonstrating this performance using greater than $50 \% \mathrm{Si}$ show significant promise for this material.

\section{Reviewer 3:}

The reviewer said that the results to date are promising for the $21 \mathrm{Ah}$ cells using the $\mathrm{C} / 3$ test regime. There seems to be an effort to include some statistical evaluations as well, which the reviewer feels is mandatory in this kind of development project. The lack of pulse data are a concern because of the possibility of making decisions based only on CC cycling and having a severe deficiency in pulse characteristics. Thus, the decisions to go on to larger cells have been taken without DST cycling data to determine DST cycle life and without periodic 30s pulse tests to determine power density. The previous cells were quite deficient with respect to both of these quantities. The reviewer is also concerned about the downward curvature of the cycle life data as to the impact on other types of cycling, e.g., DST or other current rates, and C/1.

\section{Reviewer 4:}

The reviewer said that good progress had been made with various cell components and cell designs. A cycle life of 500 cycles was realized in $21 \mathrm{Ah}$, (approximately $260 \mathrm{Wh} / \mathrm{kg}$ ) pouch cells with NMC cathode, Si anode (greater than 50\%) and with selected electrolyte and surface modification. At the component-level, the cathode performance looks good, but the reviewer was not sure what the loading is, if it is comparable to that in the full cell. In contrast, the $\mathrm{Si}$ anode could be a problem to meet the requirements of cycle life (of 1,000 cycles) and specific energy. The reviewer said that anode development is still ongoing with respect to composition, coatings and electrode morphology. Surprisingly, the cell specific energy is not high even with $50 \%$ of Si, possibly due to the irreversible capacity loss. The reviewer found that roll to roll pre-lithition development is encouraging, but no data were presented on the pre-lithiated anodes. It would be interesting to see if the irreversible capability loss is totally compensated by this pre-lithiation. The reviewer said that proper cost analysis needs to be made to assess from the pre-lithiation and the associated electrode handling needs versus its benefits. There is substantial anode delamination even in the baseline cells; the reviewer speculated it was with a graphite anode. The reviewer said that the prototype cells $(21 \mathrm{Ah})$ showed decent performance, but the specific energy and cycle life are well short of the goals, and need to be improved in in the third year to meet the project (or USABC) targets. The reviewer concluded that overall, progress is good and is consistent with DOE's goals. 


\section{Reviewer 5:}

The reviewer remarked that it was unclear if the resistance data provided consists of polarization resistance, and on-cell power performance data were provided to show cells' power capability. With limited time remaining for this project to be finished, the reviewer expressed concern if the project can meet the on time. The battery management system (BMS) progress was not detailed, and a detailed testing of the BMS design by the end of 2016 is a concern.

\section{Question 3: Collaboration and coordination with other institutions.}

\section{Reviewer 1:}

The reviewer enthused that this program is a great example of the value of a strong collaborative partnership to significantly accelerate the advancement of an R\&D effort.

\section{Reviewer 2}

The reviewer commented that there are excellent collaborations with several researchers from different organizations, specialized in different components and manufacturing processes.

\section{Reviewer 3:}

The reviewer remarked that there seems to be a good level of collaboration on the materials and cell making sides. There does not seem to be oversight on the cell testing aspects, as noted above.

\section{Reviewer 4:}

The reviewer said that the project team is reaching out to partners in many different areas.

\section{Reviewer 5:}

The reviewer suggested that the contractor leverage efforts by academia such as ANL and/or LBNL or facility to see if the project team can reduce the time in testing and trying with different size and porosity cathodes/anodes, etc.

Question 4: Proposed future research-the degree to which the project has effectively planned its future work in a logical manner by incorporating appropriate decision points, considering barriers to the realization of the proposed technology and, when sensible, mitigating risk by providing alternate development pathways.

\section{Reviewer 1:}

The reviewer remarked that proposed future research is to continue to develop the Si anode with pre-lithiation, $\mathrm{Mn} / \mathrm{Ni}$ rich cathodes, and electrolytes and incorporate the down-selected materials into large capacity cells. Further, the studies will focus on understanding and mitigating the cells through destructive physical analysis (DPA) studies. The reviewer found that the future work planned is logical with appropriate decision points in the materials selection and cell fabrication processes.

\section{Reviewer 2:}

The reviewer believed that there should be a lot more test data before launching into a new cell vehicle (41 Ah cells). This could be a real problem depending on pulse and DST results.

\section{Reviewer 3:}

The reviewer said that there is little to no detail concerning how the project team is going to attack the cycle/ calendar life issue, which should be their highest priority.

\section{Reviewer 4:}

The reviewer said that it appears extremely risky to accomplish the cell and $12 \mathrm{~V}$ pack design, while finishing the testing of the $12 \mathrm{~V}$ pack to demonstrate the design meeting goals (life and low-temperature performance) may be extremely challenging.

\section{Reviewer 5:}

The reviewer said that the PI provided very limited information on the future work for this effort. In part this is due to the excellent results that the team has been able to achieve to date; however, much work remains as the team is not tracking to meet all of the EV targets. 


\section{Question 5: Does this project support the overall DOE objectives of petroleum displacement? Why or why not?}

\section{Reviewer 1:}

The reviewer commented that this effort is a highly relevant as it addresses key challenges to reaching the aggressive EV energy storage targets. This is one of the efforts that shows the most promise of pushing toward the specific energy goals without completely sacrificing cycle life. The reviewer said that having demonstrated 500 cycles with a very high Si content anode is encouraging.

\section{Reviewer 2:}

The reviewer remarked that this is highly relevant as the specific energy gains are substantial compared to present production cells.

\section{Reviewer 3:}

The reviewer said that low specific energies and high costs of Li-ion batteries are serious impediments to their widespread adoption in vehicles. High-capacity cathode and anode materials are required to improve the specific energy of Li-ion cells. Blends of Ni/Mn rich cathodes and Si composite anodes are promising both from energy and cost perspectives, and are being addressed in this project.

\section{Reviewer 4:}

The reviewer remarked that the work conducted to fulfill USABC's $12 \mathrm{~V}$ battery pack requirement support DOE's goals in pushing for vehicle electrification.

\section{Question 6: Resources: How sufficient are the resources for the project to achieve the stated milestones in a timely fashion?}

\section{Reviewer 1:}

The reviewer remarked that the contractor appears to need some help or collaboration from academia or industry.

\section{Reviewer 2:}

The reviewer commented that if the lack of pulse test data are due to lack of resources, the researchers should seek ways to improve the test positions.

\section{Reviewer 3:}

The reviewer said that it appears that the PI has sufficient resources to complete this effort.

\section{Reviewer 4:}

The reviewer said that resources seem to be slightly high for the scope of the project, but may be reasonable based on the large number of team members. 
A 12 V Start-Stop Li Polymer Battery Pack: Mohamed Alamgir (LG Chem Power)

- es249

\section{Presenter}

Mohamed Alamgir, LG Chem Power

\section{Reviewer Sample Size}

A total of four reviewers evaluated this project.

\section{Question 1: Approach to performing the work-the degree to which technical barriers are addressed, the project is well-designed, feasible, and integrated with other efforts.}

\section{Reviewer 1:}

The reviewer remarked that the objective here is to develop a highpower Li-ion cell suitable for use in the $12 \mathrm{~V}$ start-stop battery by optimizing the cell chemistry for cold-cranking requirements, design a low-cost, simplified BMS and finally battery with a cost target of less than \$220. The approach involves optimizing various cell components, including cathode and anode and their structure, electrolyte and separator for enhanced power, especially at low temperatures. The reviewer noted that the approach also includes the development of low-cost battery pack designs (mechanical, thermal and electrical) to meet USABC targets. The reviewer said that the approach addresses the technical barriers, and the project is well-designed, feasible and integrated with other Vehicle Technologies projects.

\section{Reviewer 2:}

The reviewer said that battery life and power performance are still a challenge, and there is no clear path provided to overcome the technical barriers.

\section{Reviewer 3:}

The reviewer commented that in general, the approach is effective and contributes to overcoming some of the identified barriers. One area of concern is that there has been limited progress in addressing one of the key challenges, which is cold crank at lower states of charge. The reviewer remarked that there is very little info on the approach to address this deficiency.

\section{Reviewer 4:}

The reviewer said that this project was very difficult to analyze for approach because the cell specifics were not

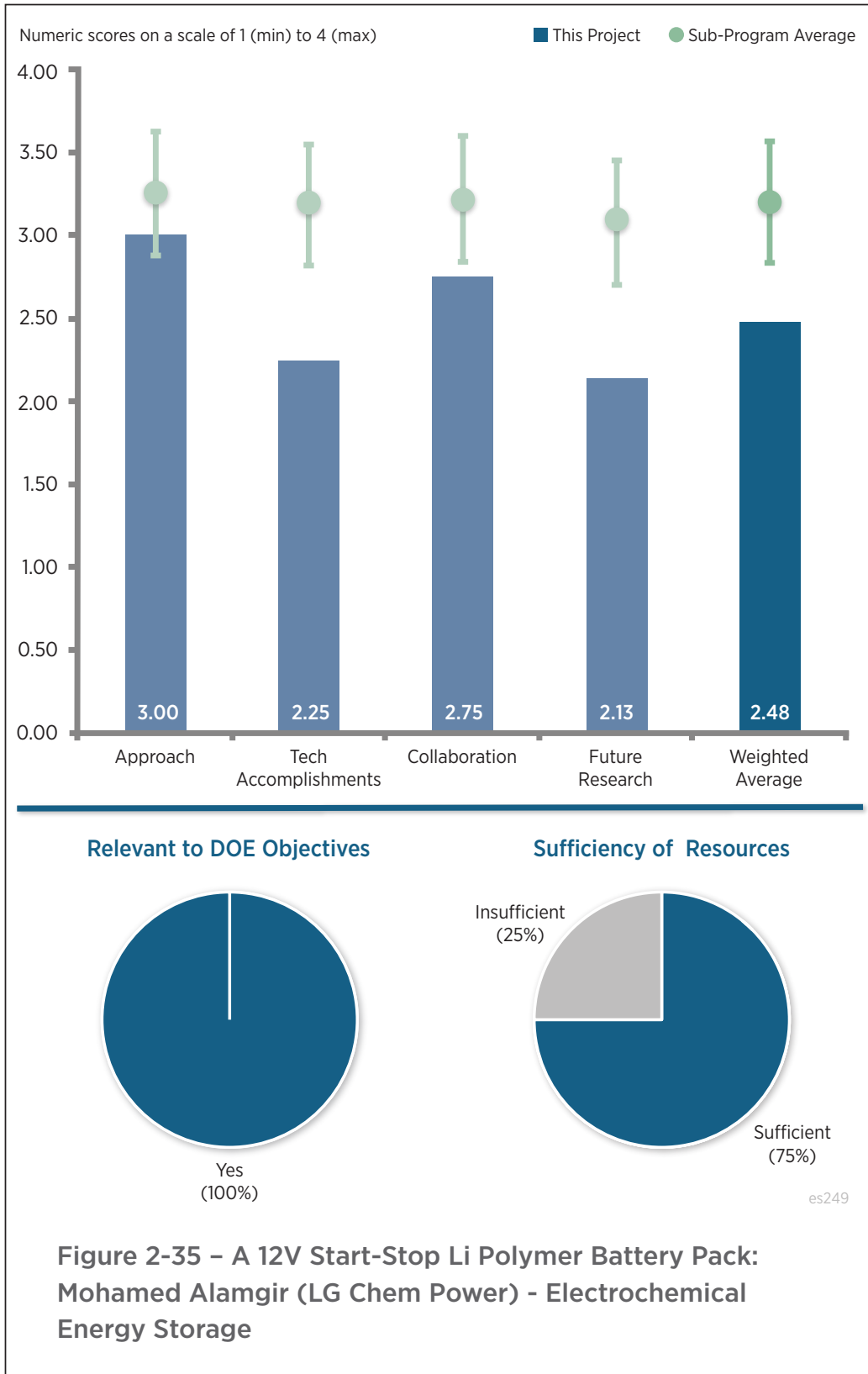


presented. Furthermore, there were inconsistencies, such as the porosity study in 2015 in which the cell resistance at $10 \mathrm{~s}$ after charge and discharge decreased as the porosity decreased through three trials. The reviewer would expect this variable to reverse as the porosity continued to decrease and the electrolyte available volume decreases, and as a result, the ion flux in the electrolyte will now introduce substantial resistance. The reviewer pointed out that on other studies such as cathode material surface area in 2016, the resistance was lowered as the surface area increased, but again the reviewer would expect a reversal when the particles get sufficiently small and the surface area increases. Also, if was not clear if the porosity was held constant through this study.

\section{Question 2: Technical accomplishments and progress toward overall project and DOE goals-the degree to which progress has been made, measured against performance indicators and demonstrated progress towards DOE goals.}

\section{Reviewer 1:}

The reviewer remarked that the project studied various cathodes and anodes with different compositions and morphologies and different electrolyte formulations to enhance cold-cranking power and high-temperature durability. Also, the effect of dopant, surface area and porosities were studied for the cathode material. There was a progressive development in the ASI and EIS impedance and in the high-temperature resilience at $60^{\circ} \mathrm{C}$, with the recent cathode, anode and electrolyte materials. In addition, the team designed a battery pack fabricated and delivered with a low-cost BMS and thermal system. Even though it is convincing that good progress has been made relative to the material development and cell/pack design towards the project goals, not enough data have been presented to assess the compliance of this progresses with the requirement (e.g., cell and battery discharge curves at low temperatures and after storage and pack design details to the extent permitted by intellectual property). The reviewer said that only relative data on the ASI, EIS and high temperatures was shown, and it is not clear as to how the battery temperature could be pegged to $52^{\circ} \mathrm{C}$ even after four hours of exposure to $75^{\circ} \mathrm{C}$; the reviewer wondered what happens after four hours or, say, 12 hours.

\section{Reviewer 2:}

The reviewer said that limited progress have been achieved to meet project and DOE goals in terms of life, specific energy and power density, etc. The reviewer suggested adding more electrolyte screening work in to help realize the goals.

\section{Reviewer 3:}

The reviewer remarked that this project is near completion (November 2016), yet is only $50 \%$ complete with multiple challenges remaining, in particular in the area of cold crank. Furthermore, the program was only able to achieve $25 \%$ progress during the year. The reviewer said that this effort will not meet the stated goals.

\section{Reviewer 4:}

The reviewer commented that because an insufficient range of variables was studied (particularly in porosity and surface area), a true optimization was not found. Therefore, the cold cranking was unsatisfactory, according to discussion with the authors. Furthermore, the use of arbitrary units for the resistance at $10 \mathrm{~s}$, it was not possible to assess the sensitivity of the resistance to the different variables. The reviewer would like to see a valid statistical analysis including absolute values in order to evaluate progress in the program.

\section{Question 3: Collaboration and coordination with other institutions.}

\section{Reviewer 1:}

The reviewer noted that there is formal collaboration and partnership with the DOE laboratories for testing the cells and batteries from this project (deliverables).

\section{Reviewer 2:}

The reviewer said that the only collaborations are with national laboratories and that seems to be mainly based on submitting battery packs for evaluation at the end of the program. Thus, according to this reviewer there does not seem to be any real collaboration or even discussion of the work in progress. 


\section{Reviewer 3:}

The reviewer suggested that the contractor include an academic partner in helping to attack key barriers to DOE goals.

\section{Reviewer 4:}

The reviewer said that there exists some collaboration within the LG team to develop the technology. However, the reviewer would like to have seen the PI explore additional collaboration to address some of the key technical barriers that will not likely be addressed with this effort.

Question 4: Proposed future research-the degree to which the project has effectively planned its future work in a logical manner by incorporating appropriate decision points, considering barriers to the realization of the proposed technology and, when sensible, mitigating risk by providing alternate development pathways.

\section{Reviewer 1:}

The reviewer commented that the proposed future research is to continue the development of cell components to improve the cold-cranking power, by evaluating various electrolyte compositions and separators. Also, there will be further optimization of the pack and the BMS designs to reduce the overall cost of the $12 \mathrm{~V}$ battery. The reviewer said that these activities are well aligned with the project goals; however, details are not provided on the decision points and risk mitigation strategies.

\section{Reviewer 2:}

The reviewer said that because pack and BMS design remain to be optimized and the program ends in November 2016, there does not seem to be enough time to improve the cell basis for the project, although it is stated that further optimization will be done on cells.

\section{Reviewer 3:}

The reviewer said that there insufficient information provided to prove that the proposed future research can achieve the battery life, specific energy goals, etc. for EV batteries.

\section{Reviewer 4:}

The reviewer commented that this program is reaching completion and in all likelihood will fail to overcome the remaining technical barriers. It is unlikely that the proposed future work will successfully enable the cold crank requirements to be met.

\section{Question 5: Does this project support the overall DOE objectives of petroleum displacement? Why or why not?}

\section{Reviewer 1:}

The reviewer pointed out that the project is important in improving stop/start systems to improve auto fuel efficiency.

\section{Reviewer 2:}

The reviewer remarked that this effort is relevant and addresses a key enabling energy storage solution needed to facilitate $12 \mathrm{~V}$ start/stop vehicle platforms.

\section{Reviewer 3:}

The reviewer detailed that the replacement for standard $12 \mathrm{~V}$ lead-acid batteries with Li-ion batteries will result in lower battery mass and volume of over $60 \%$, enhanced cycle and service life and reduced maintenance due to lower self-discharge. In addition to these benefits, $\mathrm{Li}$-ion batteries reduce the load on the alternator as they retain more power and are able to handle the charge faster than lead-acid batteries. The reviewer said that this results in reduced fuel consumption and thus reduced $\mathrm{CO}_{2}$ emissions. Current active materials have low specific power to support cranking, especially at low temperatures. The reviewer also said that new active materials and cell components and/or designs are required to meet the demands for high power density, and also pack costs, which are being addressed here. 


\section{Reviewer 4:}

The reviewer commented that EV battery development may help the vehicle electrification efforts promoted by DOE.

\section{Question 6: Resources: How sufficient are the resources for the project to achieve the stated milestones in a timely fashion?}

Reviewer 1:

The reviewer commented that the resources are adequate for the project scope.

\section{Reviewer 2:}

The reviewer said that the contractor appears to have sufficient funds to conduct the work, but collaboration with academia such as a national laboratory is recommended to help speed the process.

\section{Reviewer 3:}

The reviewer remarked that this effort will run out of time before addressing the stated milestones. 


\section{A Commercially Scalable Process for Silicon Anode Pre-Lithiation: Ionel Stefan (Amprius) - es250}

\section{Presenter}

Ionel Stefan, Ampirus

\section{Reviewer Sample Size}

A total of six reviewers evaluated this project.

\section{Question 1: Approach to performing the work-the degree to which technical barriers are addressed, the project is well-designed, feasible, and integrated with other efforts.}

\section{Reviewer 1:}

The reviewer remarked that the team performed an outstanding work to exhaustively explore different ways of prelithiating $\mathrm{Si}$ anodes and increasing its energy density. The project team systematically downselected a series of chemical, physical and electrochemical approaches and summarized their feasibility. The reviewer remarked that the knowledge gained would be of value for the Sibased chemistry.

\section{Reviewer 2:}

The reviewer said that pre-lithiation is an effective way to compensate the capacity loss due to SEI formation. The PI pretty much covered all the possible ways for pre-lithiation.

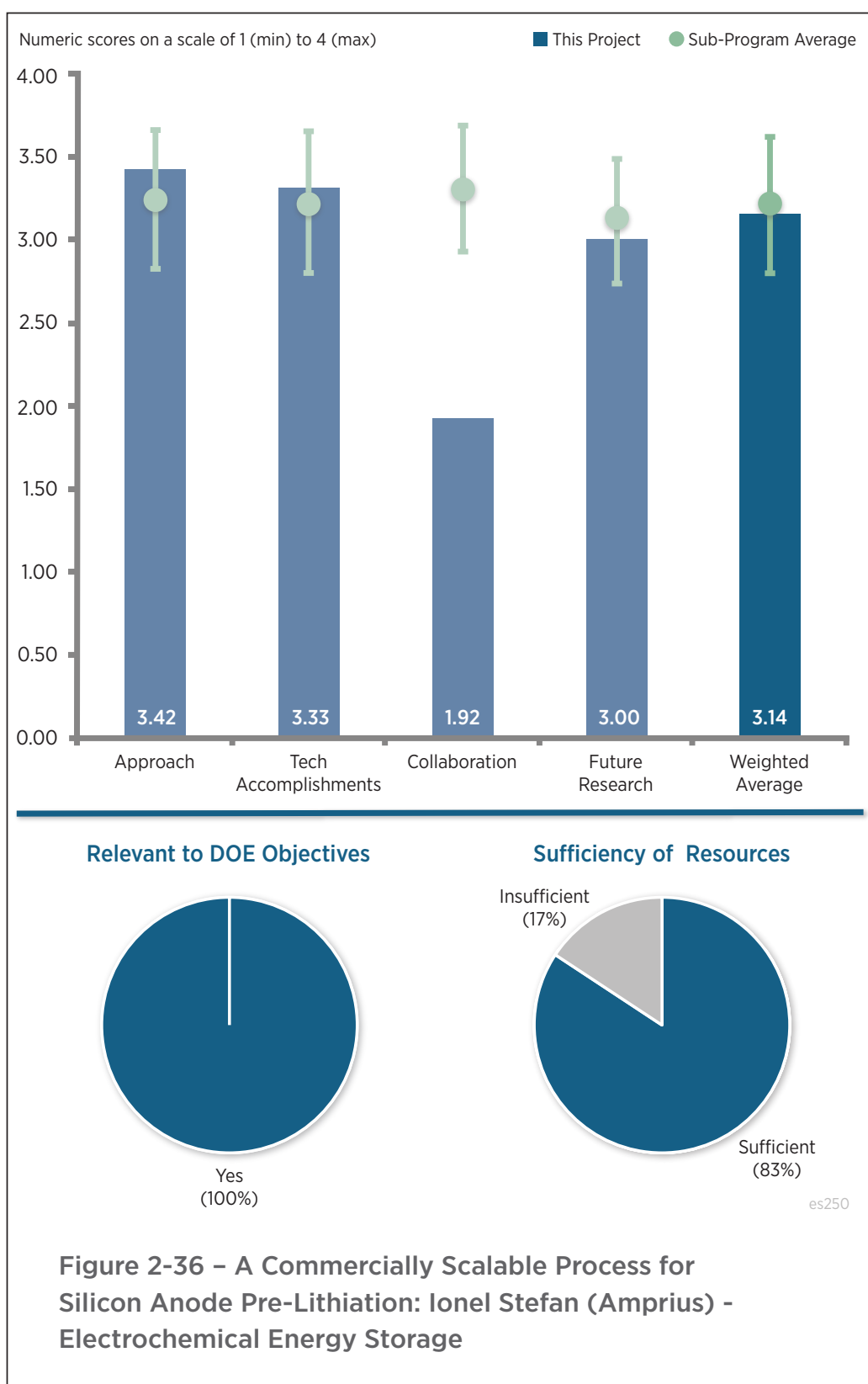

The reviewer suggested that the cost estimate should include the potential compromise of the manufacture throughput.

\section{Reviewer 3:}

The reviewer remarked that the prelithation approach outlined seems reasonable. The overall cost target is described as $\$ 0.10 /$ Ah based on a comparison to the equivalent cost of adding $\$ 0.18 /$ Ah of lithium cobalt oxide $\left(\mathrm{LiCoO}_{2}\right)$. The reviewer suggested that the cost impact of each preliathion method should be explicitly quantified to assess the progress of the overall approach.

\section{Reviewer 4:}

The reviewer found that the approaches are feasible. The reviewer suggested that the team should also compare the additional cost from the prelithation process and the saving from reducing the loading of cathode materials.

\section{Reviewer 5:}

The reviewer remarked that the approach was well thought out and indeed it seemed that a lot of the pre-lithiation selection methodology was done as a paper exercise. The reviewer would have liked to have seen some additional 
trails. This project is near completion and it seems that the PI has already concluded that pre-lithiation is not going to have much of an effect on their system and any commercial pre-lithiation station will be expensive and perhaps uneconomical (for their system).

\section{Reviewer 6:}

The reviewer remarked that the objective is to develop and demonstrate a commercially scalable process for Si anode pre-lithiation that will add no more than $10 \%$ to the cost of producing Si nanowires, facilitating production of $\mathrm{Si}$ anodes that cost significantly less than today's premium graphite anodes. The eventual goals are to demonstrate the pre-lithiation strategy at pilot level pre-lithiation capacity (greater than 100 cells/day) with a cost of less than $\$ 0.1 / \mathrm{Ah}$ and providing a cathode utilization of greater than $5 \%$. This would address the barriers of poor utilization of the cathode and lower capacity/energy of Li-ion cell with the Si anode. The reviewer said that the project team investigated various pre-lithiation schemes, including electrochemical (in situ and ex situ), chemical and physical methods, in terms of effectiveness and feasibility, i.e., in terms of improving the coulombic efficiency, cell capacity and cycle life of the (full) Li-ion cells. The reviewer detailed that after down-selecting two pre-lithiation methods, plans are to set up pilot tool/set up to deliver prelithiated Si nanorod anodes and deliver 10 2-Ah-cells for demonstrating the benefits of this pre-lithiation. The reviewer found that this project is well designed, and integrated with other efforts, but the feasibility of the Si anode with the projected performance enhancements will be a challenge.

\section{Question 2: Technical accomplishments and progress toward overall project and DOE goals-the degree to which progress has been made, measured against performance indicators and demonstrated progress towards DOE goals.}

\section{Reviewer 1:}

The reviewer remarked that the team evaluated different possible approaches systematically, and identified the best one based on cost and technical feasibility.

\section{Reviewer 2:}

The reviewer found that good progress had been made on the assessment of various pre-lithiation strategies and down-selecting a couple of approaches for the pilot scale production and demonstration. After identifying the optimum pre-lithiation charge level required, four methods (two electrochemical, one physical and one chemical) have been selected for further evaluation of technical feasibility. Guidelines were established for the cost and process implications, including pre-lithiation in cell assembly. Among the methods evaluated, the electrochemical method with a sacrificial Li salt seems to be adaptable and tested further in proof-of concept cells to show the improvement in the cell capacity and cycle life. However, the improvement in the cell capacity is marginal only $5-7 \%$, which leads to the question if the pre-lithiation is worth the additional cost/effort. The reviewer commented that the improvement in cycle life, though less significant, implies that the pre-lithiation does not entirely eliminate the irreversible capacity loss continuing to happen in the first few cycles. It would be useful to show some more data on the benefits of pre-lithiation, for example, cell coulombic efficiency during cycling and the initial formation of the cells with and without pre-lithiation. The reviewer asked is the solvent for pre-lithiation different from that in the battery electrolyte, and if so, how is its stability versus $\mathrm{Si}$ and cathode. The reviewer queried what happens if there is residual sacrificial salt after pre-lithiation.

The reviewer noted that, finally, the cycle life is not substantially better with the Si nanorods compared with the other Si anodes reported in the literature, suggesting that further developments are warranted for their use in EVs. The reviewer said that a proper cost analysis needs to be made to assess the benefits from the pre-lithiation and the associated electrode handling needs versus its benefits. The reviewer commented that overall progress is good and is consistent with the scheduled milestones and DOE goals.

\section{Reviewer 3:}

The reviewer applauded the systematic way to approach the pre-lithiation issues (e.g., determination of the impacts of the loading capacity on cycleability and discharge capacity). Using Student's t-test is a big plus.

\section{Reviewer 4:}

The reviewer pointed out that during the technical review, two of the project objectives (greater than 100 cells/ day and greater than $95 \%$ cathode utilization) were not addressed. The technical progress towards these two other 
objectives (in addition to the $\$ 0.10 /$ Ah goal) should also be described. The ideal process condition is described as gas formation during pre-lithiation. The reviewer asked does this new step add to the formation related cost of manufacturer. Slide 16 describes a pre-lithiation protocol and cell response, but without further technical detail the statements made of "anode voltage and pre-lithiation charge reached target values" is not possible to assess independently. The reviewer said that Slide 17 quantifies the impact of pre-lithiation on cell capacity as 5-7\%, Si fade/volume expansion is often tied to the utilization of Si active material (i.e., 800 versus $1,500 \mathrm{mAh} / \mathrm{g}$ will lead to very different crystal structures of the LiSi alloy and as a result life). The reviewer asked if the decreased cycle life shown on Slide 18 is a result of such a move. The reviewer asked if the performer has tested the new system using half-cell and/or three-cell electrode setups to explicitly quantify the enhancement of anode capacity.

\section{Reviewer 5:}

The reviewer said that progress continues to be made on this project and the PI has demonstrated that pre-lithiation is possible. However, more progress might have been made if collaboration had been sorted rather than going at it alone.

\section{Reviewer 6:}

The reviewer said that the final results using sacrificial Li salt approach showed 5-7\% increase in overall energy density. This outcome is less than what is expected from a project of such size.

\section{Question 3: Collaboration and coordination with other institutions.}

\section{Reviewer 1:}

The reviewer said that there are no collaborations on this project, but these will be established (with the DOE laboratories) for testing after the cells are delivered.

\section{Reviewer 2:}

The reviewer said that no collaborators are identified, but it does not seem obvious that the work remaining would benefit from the addition of new partners at this late stage of the project.

\section{Reviewer 3:}

The reviewer remarked no collaborators.

\section{Reviewer 4:}

The reviewer stated that Ampirus conducted most if not all of the work themselves.

\section{Reviewer 5:}

The reviewer said that it looks as though the project does not involve any collaborations from national laboratories or universities. The reviewer suggested that the PI should reach out and get more expertise from institutions in order to accelerate the utilization of this process into practical applications.

\section{Reviewer 6:}

The reviewer observed no collaboration as Amprius is the only performer in this project. Because there is no "N/A" category, the reviewer selected "Unsatisfactory," which it is unfair to the project [DOE Program Clarification: DOE will take this into consideration for future Annual Merit Reviews].

Question 4: Proposed future research-the degree to which the project has effectively planned its future work in a logical manner by incorporating appropriate decision points, considering barriers to the realization of the proposed technology and, when sensible, mitigating risk by providing alternate development pathways.

\section{Reviewer 1:}

The reviewer detailed that plans are to continue optimizing the process conditions; design, test and optimize a pilot set up and equipment compatible with the current cell assembly process; and produce and deliver cells with a prelithiated Si anode. There are corresponding challenges, however, in sourcing the materials for prelithiation, scaling up to the pilot production and long-term feasibility and process reproducibility. The reviewer said that the future work planned is logical with appropriate decision points in the materials selection and cell fabrication processes. 


\section{Reviewer 2:}

The reviewer commented that the proposed research for the remaining three-four months is solid, but it is hard to be accomplished within the timeframe.

\section{Reviewer 3:}

The reviewer remarked that future efforts should focus on the cost evaluation and feasibility of the chosen method before scale-up is planned.

\section{Reviewer 4:}

The reviewer suggested that perhaps the PI should focus more on just further reducing the irreversible capacity loss to the system.

\section{Reviewer 5:}

The reviewer remarked that the future plan looks ambitious considering the project will end in September.

\section{Reviewer 6:}

The reviewer said that the project is ending in September 2016. The reviewer was unclear if the PI would continue the pilot effort if there is no continuous funding from DOE.

\section{Question 5: Does this project support the overall DOE objectives of petroleum displacement? Why or why not?}

\section{Reviewer 1:}

The reviewer said that incorporating $\mathrm{Si}$ anodes into Li-ion batteries will support DOE's goal

\section{Reviewer 2:}

The reviewer commented that if successfully commercialized, the development of this prelithation work would contribute to DOE's mission to reduce petroleum consumption.

\section{Reviewer 3:}

The reviewer remarked that pre-lithiation is critical for the high energy batteries, particularly when nanostructured active materials are used. Improving the first cycle efficiency and reducing the cathode loading is the right way to achieve the high energy density of next generation of Li-ion batteries.

\section{Reviewer 4:}

The reviewer said that low specific energies and high costs of Li-ion batteries are serious impediments to their widespread adoption in vehicles. High capacity anode materials are required to improve the specific energy of Li-ion cells. Si anode has the potential to offer twice the capacity of graphitic anodes, and Amprius has developed a fairly robust SI anode based on Si nanorods. The reviewer said that despite the high specific capacity, these anodes pose the problem of high irreversible capacity, which consumes the reversible $\mathrm{Li}$ in the cells and lowers the cell capacity/energy. New strategies of prelithiating Si would be useful to mitigate this problem, which is being addressed in this project.

\section{Question 6: Resources: How sufficient are the resources for the project to achieve the stated milestones in a timely fashion?}

\section{Reviewer 1:}

The reviewer said that the resources seem to be adequate for the scope of the project, but the initial schedule of one year is not sufficient. It has now been extended to another year.

\section{Reviewer 2:}

The reviewer commented that project resources seem appropriate.

\section{Reviewer 3:}

The reviewer said yes.

\section{Reviewer 4:}

The reviewer emphasized that the team should collaborate with national laboratories and universities. 
Development of Advanced High-Performance Batteries for 12V Start-Stop Vehicle Applications: Jeff Kim (Maxwell) - es251

\section{Presenter}

Jeff Kim, Maxwell

\section{Reviewer Sample Size}

A total of four reviewers evaluated this project.

\section{Question 1: Approach to performing the work-the degree to which technical barriers are addressed, the project is well-designed, feasible, and integrated with other efforts.}

\section{Reviewer 1:}

The reviewer said that the general approach is good, but details are significantly lacking. The reviewer was unable to assess key aspects of the approach because of the lack of details, e.g. the type of electrolyte, the types of additives to reduce gassing, etc.

\section{Reviewer 2:}

The reviewer described that the objectives here are to develop a robust low-cost ultra-capacitor pouch cell that can operate over a wide temperature range; hybrid power system comprising the ultracap pouch cells and commercial Li-iron phosphate $\left(\mathrm{LiFePO}_{4}\right)$-based Li-ion cells for the $12 \mathrm{~V}$ start stop system, and demonstrate the technical and economic feasibility; battery/capacitor management system (BCMS); CAD model of the $12 \mathrm{~V}$ module; and a system cost model. Specific challenges addressed in the development of ultra-capacitors are the electrolytes with suitable additives, separators and low-cost robust cell designs for the pouch cells that would enable low-temperature performance and yet minimize gassing at warm temperatures. The reviewer commented that the approach addresses the technical barriers of the $12 \mathrm{~V}$ start-stop battery system. The project is well-designed towards the program objectives, feasible and integrated with other Vehicle Technologies projects.

\section{Reviewer 3:}

The reviewer said that the approach used in this effort is very effective at addressing the technical barriers identified for this project. One area of concern for this effort would be the potential toxicity of the final electrolyte solvent for the capacitor; this is something that has not been addressed in the approach. The reviewer would also like to see quantitative metrics that demonstrate that the approach of a hybridized ultracapacitor/LIB solution is better that high-power Li-ion batteries alone.

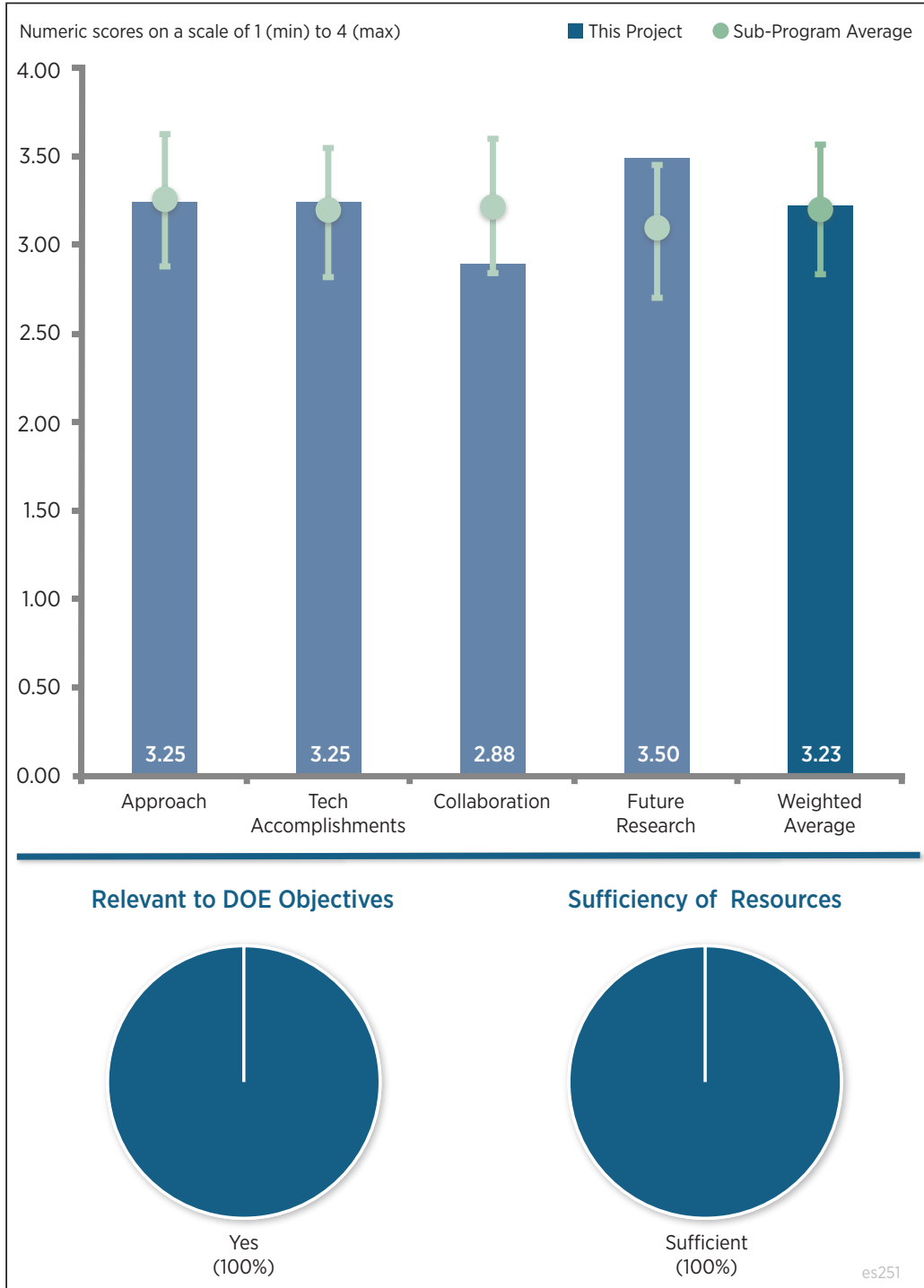

Figure 2-37 - Development of Advanced High-Performance Batteries for 12V Start-Stop Vehicle Applications: Jeff Kim (Maxwell) - Electrochemical Energy Storage 


\section{Reviewer 4:}

The reviewer said that the developed system appears to have sufficient energy at $-30^{\circ} \mathrm{C}$, but can only provide power to limited cranking numbers. The reviewer inquired if there were any efforts conducted to study how to increase battery utilization if there is energy that has not been used.

\section{Question 2: Technical accomplishments and progress toward overall project and DOE goals-the degree to which progress has been made, measured against performance indicators and demonstrated progress towards DOE goals.}

\section{Reviewer 1:}

The reviewer detailed that in an effort to identify cell components that minimizes high-temperature gas generation while having no negative impact on other key cell operating characteristics, several electrolyte combinations including acetonitrile, carbonate and ionic liquids and gas-suppression additives and separators were evaluated and suitable components were selected for the ultracapacitor pouch cell. The pouch cells were designed, fabricated and demonstrated to performance requirements. These ultracapacitor pouch cells are being tested by the national laboratories. The reviewer remarked that a proof-of concept system was built with a battery/capacitor management system, which was demonstrated to meet USABC energy and cold crank requirements. The cost model is being developed, which projects a cost closer to the targeted $\$ 180$. The reviewer found that these accomplishments are in agreement with the plans, and the progress is consistent with the project objectives and DOE's goals.

\section{Reviewer 2:}

The reviewer said that a tremendous amount of progress has been achieved so far, but it remains a challenge to meet the goal for the module lifecycle.

\section{Reviewer 3:}

The reviewer said that the technical accomplishments and progress towards the DOE goals were highly effective. However, the PI did not provide any supporting data to substantiate the progress that was made - supporting data and information would strengthen this criteria.

\section{Reviewer 4:}

The reviewer commented that technical accomplishments were evaluated on the final results in a gap analysis; however, the path to the results was not clear because of a lack of details. The problem with the report is that only the DOE requirements are evaluated and no results are shown beyond these parameters. Therefore, according to the reviewer it is not possible to even guess what the long-term value and usage of a capacitor-battery hybrid system for $12 \mathrm{~V}$ stop/start system may be.

\section{Question 3: Collaboration and coordination with other institutions.}

\section{Reviewer 1:}

The reviewer said that the collaborating partners that will be leveraged for this program are primarily in the area of verification testing. There appears to be effective, well-coordinated collaboration.

\section{Reviewer 2:}

The reviewer commented that the only collaborations are the confidential testing done at national laboratories, so no access to collaboration status can be seen.

\section{Reviewer 3:}

The reviewer said that there is an on-going collaboration with the DOE laboratories, specifically with ANL in the testing of the ultracapacitors, with NREL for understanding the thermal aspects of the hybrid system, and with SNL for abuse tolerance.

\section{Reviewer 4:}

The reviewer commented none noted.

Question 4: Proposed future research-the degree to which the project has effectively planned its future work in a logical manner by incorporating appropriate decision points, 
considering barriers to the realization of the proposed technology and, when sensible, mitigating risk by providing alternate development pathways.

\section{Reviewer 1:}

This project has demonstrated the benefits of utilizing a hybrid power system combining Li-ion cells and ultracapacitors, which may be useful for other applications as well. The remaining challenges are to finalize the ultracap pouch cell configuration, build the hybrid power system based on the CAD model and a digital version of the power management system, test the system against the USABC specifications and demonstrate its viability for the start-stop application. It is not clear, however, if the problem of gas generation has been completely solved and the ultracap provides adequate survivability at warm temperatures.

\section{Reviewer 2:}

The reviewer stated that this effort ends in June 2016 with a two-month extension. The next steps include conducting verification testing to evaluate the performance and abuse data.

\section{Reviewer 3:}

The reviewer commented that a lack of detail on specifics makes assessment difficult.

\section{Reviewer 4:}

The reviewer remarked none noted.

\section{Question 5: Does this project support the overall DOE objectives of petroleum displacement? Why or why not?}

\section{Reviewer 1:}

The reviewer said that it appears that most DOE criteria are met, which gives a good state of relevance to the project.

\section{Reviewer 2:}

The reviewer commented that replacing the conventional $12 \mathrm{~V}$ lead-acid batteries with Li-ion batteries for startup applications will reduce the lower battery mass, volume (by 60\%), improve the service life and reduce the maintenance. Their rapid recharge reduces the load on the alternator as they retain more power and are able to handle the charge faster than lead-acid batteries. All these characteristics will result in reduced fuel consumption and thus reduced $\mathrm{CO}_{2}$ emissions. The reviewer stated that the technical challenges for the $\mathrm{Li}$-ion batteries for this application are high power densities at low temperature for cold cranking, long cycle life and a wide range of operating temperatures. A hybrid system comprising commercial high-power Li-ion cells and low-temperature ultracapacitors may be an interesting combination to meet these performance and cost challenges. The reviewer stated that this project is aimed at developing such a power system.

\section{Reviewer 3:}

The reviewer remarked development of a hybrid energy storage may help the vehicle start and stop strategy to increase fuel economy.

\section{Reviewer 4:}

The reviewer said that this effort is relevant and addresses a key enabling energy storage solution needed to facilitate $12 \mathrm{~V}$ start/stop vehicle platforms.

\section{Question 6: Resources: How sufficient are the resources for the project to achieve the stated milestones in a timely fashion?}

\section{Reviewer 1:}

The reviewer commented that the resources are adequate for the scope of the project to achieve the stated milestones per the schedule.

\section{Reviewer 2:}

The reviewer pointed out that this program is near completion; final deliverables are being made for verification testing. 


\section{Enabling High-Energy, High- Voltage Li-Ion Cells for Transportation Applications: Modeling and Analysis: Dennis Dees (Argonne National Laboratory) - es252}

\section{Presenter}

Daniel Abraham, Argonne National Laboratory

\section{Reviewer Sample Size}

A total of three reviewers evaluated this project.

\section{Question 1: Approach to performing the work-the degree to which technical barriers are addressed, the project is well-designed, feasible, and integrated with other efforts.}

\section{Reviewer 1:}

The reviewer commented that this is part of the broader program, in this case focusing on the fundamental and performance analysis of the system. Overall, the program approach is ambitious and well laid out.

\section{Question 2: Technical} accomplishments and progress toward overall project and DOE goals-the degree to which progress has been made, measured against performance indicators and demonstrated progress towards DOE goals.

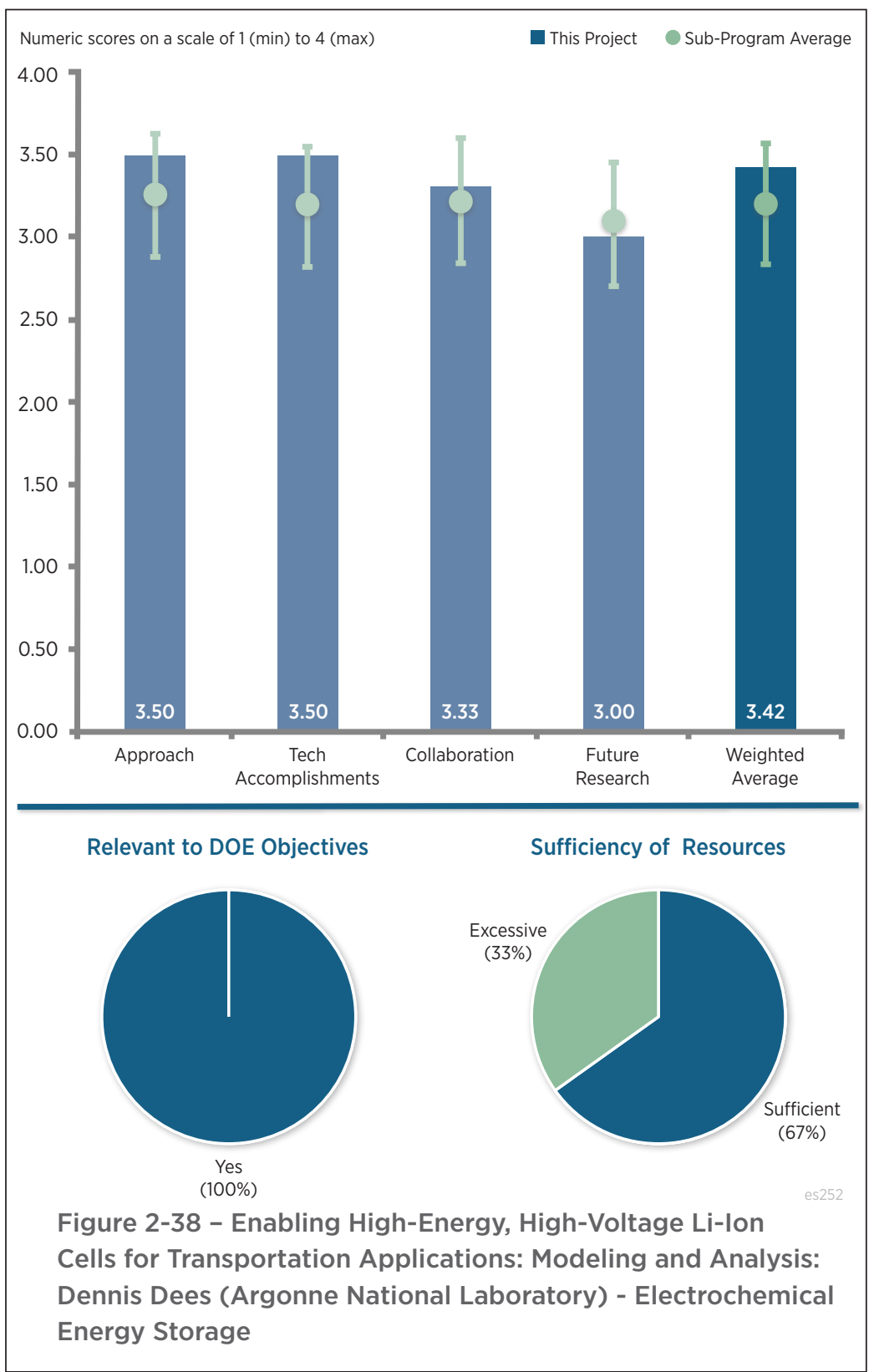

Reviewer 1:

The reviewer stated that it is rather early in the program to make a significant comment on progress. If the material standardization and the protocol standardization have been completed and are operable, that would be a good start. The reviewer said that laying out the known challenges of the system in this format was also a good start.

\section{Question 3: Collaboration and coordination with other institutions.}

\section{Reviewer 1:}

The reviewer reiterated that this is an ambitious program that is dependent on effective coordination and collaboration. The project appears to be successfully accomplished in terms of the internal group collaboration. There was no mention of external industry collaborations. The reviewer was unclear if this was sought or considered so the reviewer is not clear if it is a negative or not.

Question 4: Proposed future research-the degree to which the project has effectively planned its future work in a logical manner by incorporating appropriate decision points, 
considering barriers to the realization of the proposed technology and, when sensible, mitigating risk by providing alternate development pathways.

Reviewer 1:

The reviewer said that while this is a coordinated deep dive effort, this is an area where considerable work has occurred in the past. Hopefully, there will be a critical focus on key identified mechanism issues rather than a rehash of work to date.

Reviewer 2:

The reviewer commented that it would be useful to understand if Li-trapping on the anode side is a continuous process throughout the cycle life of the battery.

Reviewer 3:

The reviewer suggested slightly less focus on the modeling-only portion of future work, and more focus on the additional electrolyte study portion.

Question 5: Does this project support the overall DOE objectives of petroleum displacement? Why or why not?

No comments were received in response to this question.

Question 6: Resources: How sufficient are the resources for the project to achieve the stated milestones in a timely fashion?

No comments were received in response to this question. 


\section{Enabling High-Energy, High- Voltage Li-Ion Cells for Transportation Applications: Project Overview: Dennis Dees (Argonne National Laboratory) - es253}

\section{Presenter}

Jason Croy, Argonne National Laboratory

\section{Reviewer Sample Size}

A total of four reviewers evaluated this project.

\section{Question 1: Approach to} performing the work-the degree to which technical barriers are addressed, the project is well-designed, feasible, and integrated with other efforts.

\section{Reviewer 1:}

The reviewer remarked excellent progress on demonstrating a systematic approach to problem solving and utilizing talent across the DOE community.

\section{Reviewer 2:}

The reviewer said it is a worthy goal to provide standardized material sets and analysis protocols across a large multi-group program.

\section{Question 2: Technical} accomplishments and progress toward overall project and DOE goals-the degree to which progress has been made, measured against performance indicators and demonstrated progress towards DOE goals.

\section{Reviewer 1:}

The reviewer remarked a lot of good and trusted data due to the use of the statistical tools.

\section{Reviewer 2:}

The reviewer commented he specific technical progress of this program will be evaluated in the technical sections of the presentation.

\section{Question 3: Collaboration and coordination with other institutions.}

\section{Reviewer 1:}

The reviewer commented well-coordinated effort.

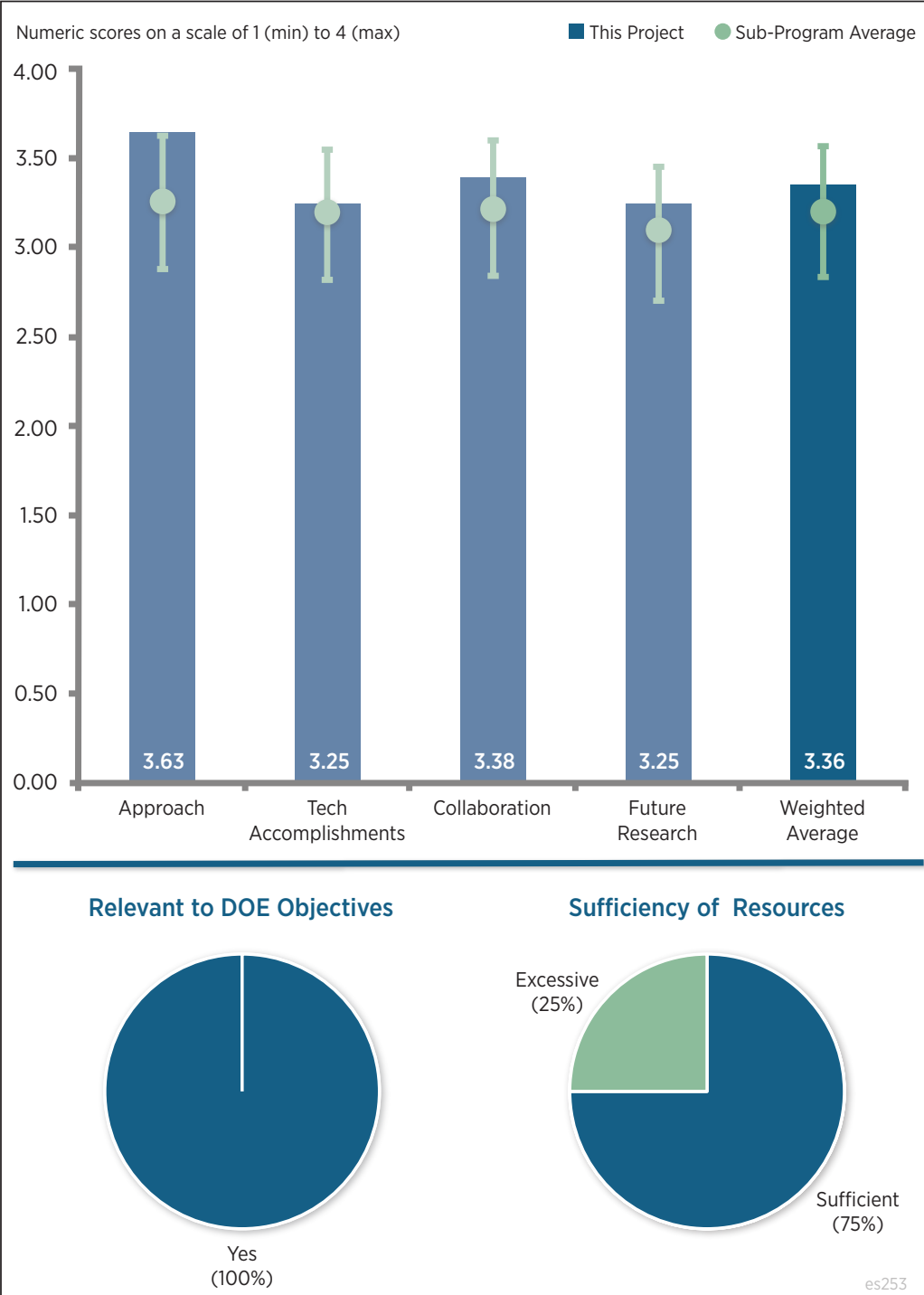

Figure 2-39 - Enabling High-Energy, High-Voltage Li-Ion Cells for Transportation Applications: Project Overview: Dennis Dees (Argonne National Laboratory) Electrochemical Energy Storage 


\section{Reviewer 2:}

The reviewer remarked that in particular, this portion of the multiple presentations is focused on the collaboration and coordinating aspects of the program. It appears to be going quite well and will hopefully be a model for programs going forward. It was not clear to this reviewer if commercial collaboration is anticipated or sought.

Question 4: Proposed future research-the degree to which the project has effectively planned its future work in a logical manner by incorporating appropriate decision points, considering barriers to the realization of the proposed technology and, when sensible, mitigating risk by providing alternate development pathways.

\section{Reviewer 1:}

The reviewer commented very high expectation for this program in the research and commercial community.

\section{Reviewer 2:}

The reviewer said that future research was not discussed specifically in this presentation; however, we can assume that the standardization of materials and protocols will aid future work.

\section{Question 5: Does this project support the overall DOE objectives of petroleum displacement? Why or why not?}

\section{Reviewer 1:}

The reviewer commented one of the promising approaches to higher energy density cells.

Question 6: Resources: How sufficient are the resources for the project to achieve the stated milestones in a timely fashion?

No comments were received in response to this question. 


\section{Enabling High-Energy, High- Voltage Li-Ion Cells for Transportation Applications: Materials Characterization: Dennis Dees (Argonne National Laboratory) - es254}

\section{Presenter}

John Vaughey, Argonne National Laboratory

\section{Reviewer Sample Size}

A total of four reviewers evaluated this project.

\section{Question 1: Approach to performing the work-the degree to which technical barriers are addressed, the project is well-designed, feasible, and integrated with other efforts.}

\section{Reviewer 1 :}

The reviewer said that this portion of the program is aimed at evaluating the role of surface coatings for improvement of cycle life behavior of Ni-rich cathode materials. The reviewer said the approach appears to be well laid out with baseline system analysis, model system development, and analytical tool development.

\section{Reviewer 2:}

The reviewer remarked that based on the question and answer session, it is apparent that surface stability is a function of the Ni-content and affinity to certain coating techniques is a function of the Mn content in the studied cathode compositions. Thus, the reviewer suggested that it might be helpful to re-think the approach and to better design experiments for easier results interpretation.

\section{Question 2: Technical accomplishments and progress toward overall project and DOE goals-the degree to which progress has been made, measured against performance indicators and demonstrated progress towards DOE goals.}

\section{Reviewer 1:}

The reviewer commented some good understanding of some of the basic characteristics of coated cathode materials has been developed.

\section{Reviewer 2:}

The reviewer believed that results indicating that alumina coating negatively impacts the performance warrant deeper analysis. Industry is currently using this approach so it would be nice to understand which benefits industry sees.

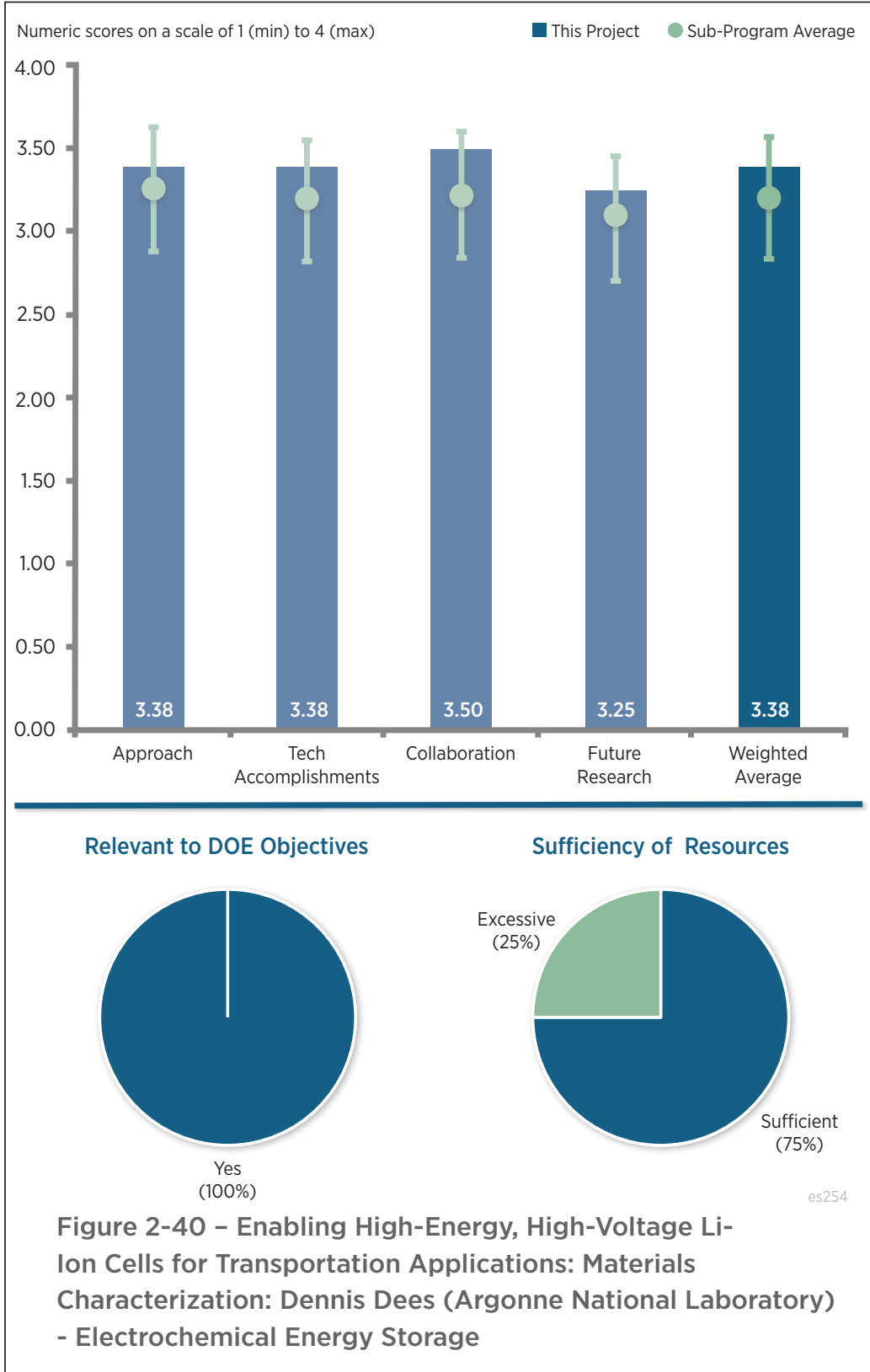




\section{Reviewer 3:}

The reviewer remarked a lot of good work and a lot of data, but it was difficult to draw conclusions; a more systematic experimental approach will be helpful.

\section{Question 3: Collaboration and coordination with other institutions.}

\section{Reviewer 1:}

The reviewer commented seasoned team of professionals.

\section{Reviewer 2:}

The reviewer said that the large team appears to be functioning well. It was unclear to this reviewer what level of commercial collaboration is anticipated or needed.

Question 4: Proposed future research-the degree to which the project has effectively planned its future work in a logical manner by incorporating appropriate decision points, considering barriers to the realization of the proposed technology and, when sensible, mitigating risk by providing alternate development pathways.

\section{Reviewer 1:}

The reviewer commented that future research continues to focus on cathode coating development. It is not clear if any other approaches have been considered.

\section{Reviewer 2:}

The reviewer remarked that it would be interesting to see the comparison of the coating versus bulk doping as a function of the $\mathrm{Ni} / \mathrm{Mn}$ content. This might lead to the different strategies for different families of materials. In addition, high $\mathrm{Ni}$ content materials are made using $\mathrm{Li}$ hydroxide, not $\mathrm{Li}_{2} \mathrm{CO}_{3}$ at lower sintering temperatures. The reviewer asked what effect on surface stability that might have.

\section{Question 5: Does this project support the overall DOE objectives of petroleum displacement? Why or why not?}

\section{Reviewer 1:}

The reviewer said that the project addresses energy density/safety needs.

Question 6: Resources: How sufficient are the resources for the project to achieve the stated milestones in a timely fashion?

No comments were received in response to this question. 


\section{Next-Generation Anodes for Lithium-Ion Batteries: Overview: Dennis Dees (Argonne National Laboratory) - es261}

\section{Presenter}

Dennis Dees, Argonne National Laboratory

\section{Reviewer Sample Size}

A total of four reviewers evaluated this project.

\section{Question 1: Approach to performing the work-the degree to which technical barriers are addressed, the project is well-designed, feasible, and integrated with other efforts.}

\section{Reviewer 1:}

The reviewer remarked outstanding effort to systematically assess advanced anode systems.

\section{Reviewer 2:}

The reviewer said that the project uses the same program structure as the High Voltage cell development program, which in theory has promise. This is a potentially large scope technical challenge that has been studied intensely for many years. The reviewer said that finding new approaches and developing useful new knowledges will be a challenge. The reviewer said that it is too early to tell how the program shape will roll out so the reviewer believed it is a little early to review quantitatively.

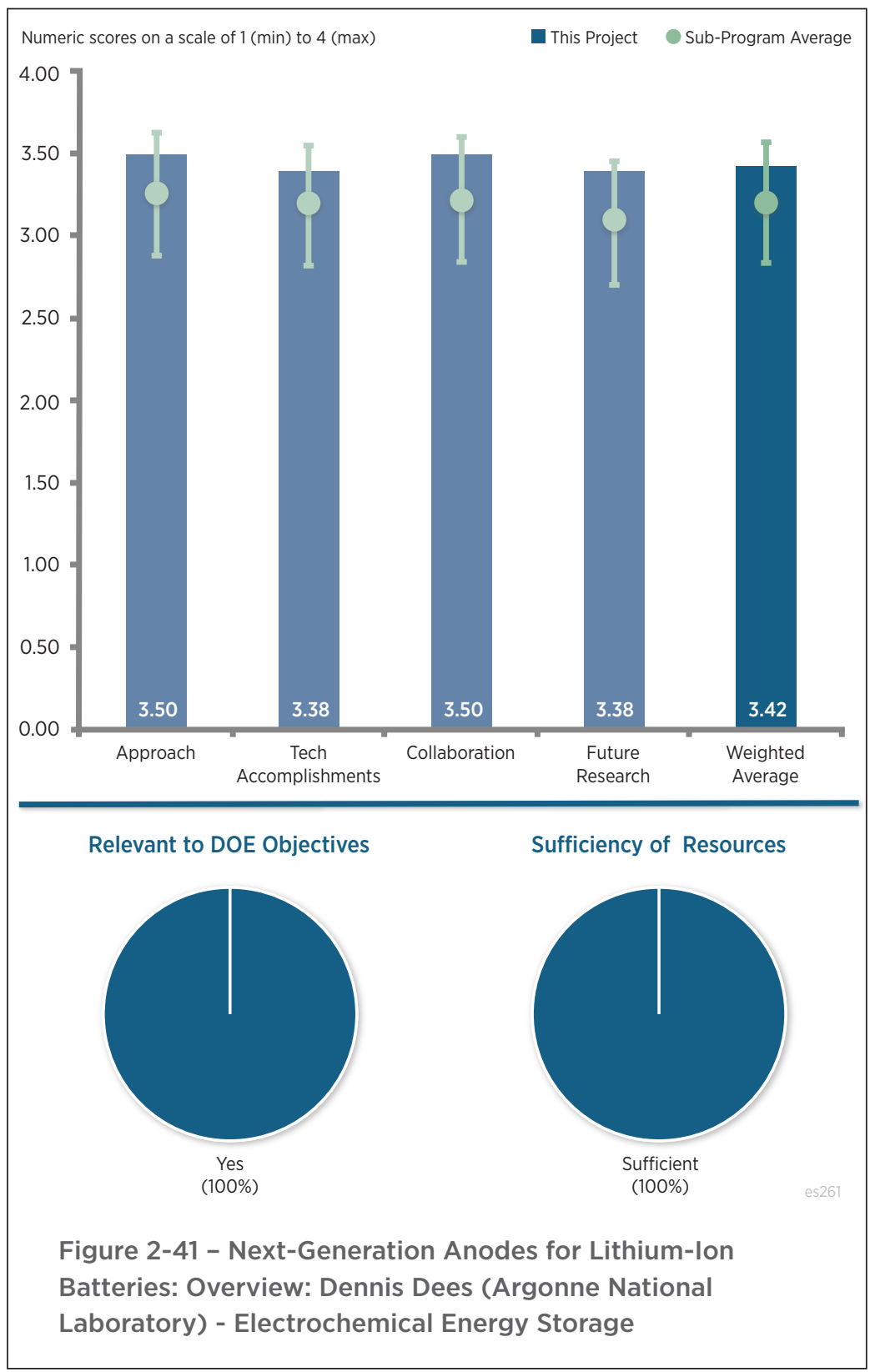

Question 2: Technical accomplishments and progress toward overall project and DOE goals-the degree to which progress has been made, measured against performance indicators and demonstrated progress towards DOE goals.

\section{Reviewer 1:}

The reviewer said excellent use of the characterization tools/capabilities of the collaborating entities, and good effort on addressing Li inventory in the cell.

\section{Reviewer 2:}

The reviewer commented that preliminary work on program structure, material standards and protocol standards appears to be moving along well. 


\section{Question 3: Collaboration and coordination with other institutions.}

\section{Reviewer 1:}

The reviewer commented that the assembled team is excellent.

\section{Reviewer 2:}

The reviewer observed a good use of resources/synergies of the national laboratories.

\section{Reviewer 3:}

The reviewer said that collaboration is currently with internal contributors, and the reviewer was unclear if industrial partners are sought after or anticipated.

Question 4: Proposed future research-the degree to which the project has effectively planned its future work in a logical manner by incorporating appropriate decision points, considering barriers to the realization of the proposed technology and, when sensible, mitigating risk by providing alternate development pathways.

\section{Reviewer 1:}

The reviewer believed the direction of the proposed work should provide many insights into the issues associated with Si anode development.

\section{Reviewer 2:}

The reviewer commented a good plan for continued work on a reasonable set of key focus areas of importance.

\section{Reviewer 3:}

The reviewer said that the need for adding Li inventory was established decades ago. It is important to utilize national laboratory modeling capabilities to identify the most effective pre-lithiation route. The LFO concept was developed by ANL at least five years ago and it is important to understand its utility versus just being used as a research tool. Questions to consider include, for example, how LFO hygroscopicity will effect slurry quality, slurry $\mathrm{pH}$ and possible gelling. The reviewer asked what the effect is of the dead weight of the LFO on the gravimetric/ volumetric energy density. Another approach the reviewer detailed could be fundamental understanding of the irreversible capacity in the $\mathrm{Si}$ anode and mitigating it through the new material architectures design

\section{Question 5: Does this project support the overall DOE objectives of petroleum displacement? Why or why not?}

\section{Reviewer 1:}

The reviewer said that it is important to validate the hopes for the commercial use of the advanced high-capacity anodes.

\section{Question 6: Resources: How sufficient are the resources for the project to achieve the stated milestones in a timely fashion?}

\section{Reviewer 1:}

The reviewer said that the resource structure may have to change over time with new understanding. 


\section{Next-Generation Anodes for Li-Ion Batteries: Fundamental Studies of Si-C Model Systems: Robert Kostecki (Lawrence Berkeley National Laboratory) - es262}

Presenter

Robert Kostecki, Lawrence Berkeley National Laborator

\section{Reviewer Sample Size}

A total of four reviewers evaluated this project.

\section{Question 1: Approach to performing the work-the degree to which technical barriers are addressed, the project is well-designed, feasible, and integrated with other efforts.}

\section{Reviewer 1 :}

The reviewer acknowledged that the $\mathrm{PI}$ is an intelligent, critical thinker and he lays out the challenges in $\mathrm{Si}$ development in a very compelling manner. The PI laid out the history that there has been 40 years of development on the Si system and progress still is not where it needs to be. So this program will be challenged to bring new insight to the table, but the energy storage community can hope that researchers like Kostecki are up to the task.

\section{Reviewer 2:}

The reviewer said that it was a good approach to have an integrated electrochemical and diagnostic study to study the two key issues with Si-based anodes, namely the large irreversible loss and the large volume change. The reviewer noted that a schedule and quantifiable milestones were not provided.

\section{Question 2: Technical accomplishments and progress toward overall project and DOE goals - the degree to which progress has been made, measured against performance indicators and demonstrated progress towards DOE goals.}

\section{Reviewer 1:}

The reviewer said that the program is too new to provide any significant technical progress beyond laying out the challenges and approach.

\section{Reviewer 2:}

The reviewer noted that the project was just kicked off in January 2016. The PI formed the project team and established the experimental procedures for diagnosis. In addition to the polypyrrole (PPy) binder, the reviewer 
suggested that the project team should also examine the validity of the self-healing binder for use in Si-based anode to mitigate the volume expansion issue.

\section{Question 3: Collaboration and coordination with other institutions.}

\section{Reviewer 1:}

The reviewer said that this is another deep dive program where the internal collaboration appears to be quite extensive and well supported, and external collaboration has not been developed yet, if indeed it is going to be.

\section{Reviewer 2:}

The reviewer commented good team composition but the project needs to specify the roles and responsibilities of each team member.

\section{Question 4: Proposed future research-the degree to which the project has effectively planned its future work in a logical manner by incorporating appropriate decision points, considering barriers to the realization of the proposed technology and, when sensible, mitigating risk by providing alternate development pathways.}

\section{Reviewer 1:}

The reviewer said that the areas laid out for study appear to be the correct areas to provide insight into Si anode performance.

\section{Reviewer 2:}

The reviewer suggested that future research should focus on the role of additives. For example, fluorinated ethylene carbonate (EC) additive were shown to improve the cycle life of Si-based anode. The reviewer remarked that understanding the role of the additives in the SEI should provide guidance in search of even better additives for Si-based anode.

\section{Question 5: Does this project support the overall DOE objectives of petroleum displacement? Why or why not?}

\section{Reviewer 1:}

The reviewer said that the project is highly relevant because Si-based anodes are an enabler to achieve DOEspecific energy goals, but we still lack fundamental understanding that correlates performance with Si-based materials.

\section{Question 6: Resources: How sufficient are the resources for the project to achieve the stated milestones in a timely fashion?}

\section{Reviewer 1:}

The reviewer remarked that the issues brought here could significantly change the landscape of critical research for $\mathrm{Si}$ anode development. The ability to respond to new information with new research directions and perhaps new funded programs will be a challenge for DOE to respond to.

\section{Reviewer 2:}

The reviewer commented sufficient resources for this diagnostic effort over 2.5 years. 


\section{Electrodeposition for Low- Cost, Water-Based Electrode Manufacturing: Stuart Hellring (PPG Industries) \\ - es263}

\section{Presenter}

Stuart Hellring, PPG Industries

\section{Reviewer Sample Size}

A total of five reviewers evaluated this project.

\section{Question 1: Approach to performing the work-the degree to which technical barriers are addressed, the project is well-designed, feasible, and integrated with other efforts.}

\section{Reviewer 1:}

The reviewer said that the technical approach seems novel and is premised on viewing the electrode coating as a more common automotive e-coat system. The battery electrode is significantly more technically complex than a typical e-coat system, so this approach appears to be very high risk if also high reward.

\section{Reviewer 2:}

The reviewer said that the objective here is to develop a new low-cost water-based electrodeposition method to manufacture battery electrodes for Li-ion cells. In contrast to the conventional NMP-based slot-die coating method, the proposed electrodeposition method has the advantages of being low-cost, easier to scale up with high throughput and has low emissions. Dense and uniform coatings are possible in a shorter time, with both the sides coated simultaneously and thus reducing the overall cost. The reviewer described that the approach involves identifying suitable resins with charged groups and developing cathode materials of a suitable particle size to facilitate rapid mobility and deposition of the cathode material using electrophoretic method. The reviewer said that this project is well designed, and integrated with other efforts.

\section{Reviewer 3:}

The reviewer commented that the aqueous electro deposition to replace slot dye coating seems promising, especially for the two-side simultaneous coating. As any electrochemical process, diffusion limitation will come to play, which will limit the deposition rate.

\section{Reviewer 4:}

The reviewer expressed concern about the deposition of multiple materials to create working electrodes ever

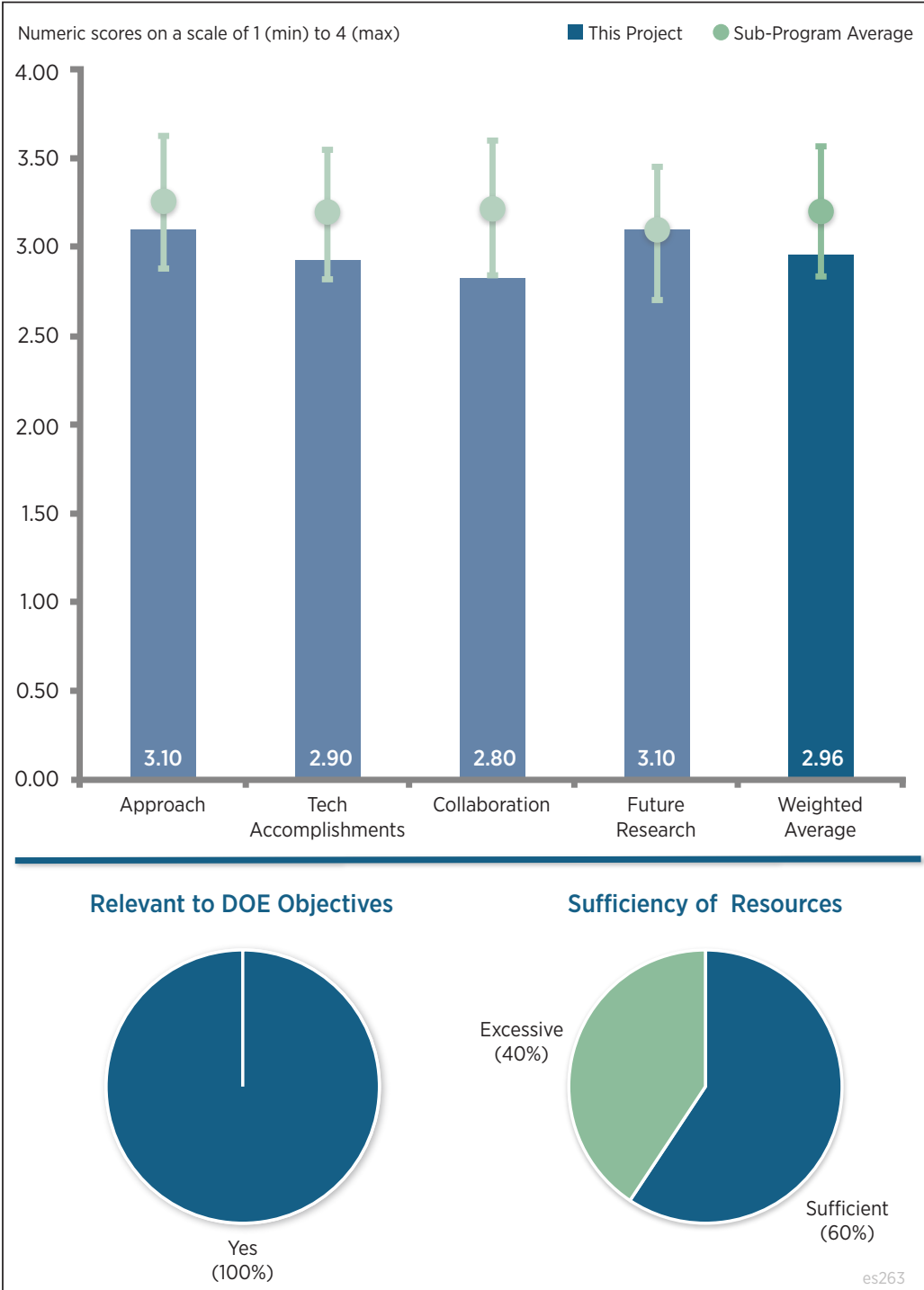

Figure 2-43 - Electrodeposition for Low-Cost, Water-Based Electrode Manufacturing: Stuart Hellring (PPG Industries) Electrochemical Energy Storage 
working well. However, the reviewer thinks the idea should be explored and that the team is appropriate for this study.

\section{Reviewer 5:}

The reviewer was not entirely clear on the motivation for this approach, using an electrophoretic method to deposit powders to make electrodes. The reviewer was unclear how well the project team competes with speed of other manufacturing processes.

\section{Question 2: Technical accomplishments and progress toward overall project and DOE goals-the degree to which progress has been made, measured against performance indicators and demonstrated progress towards DOE goals.}

\section{Reviewer 1:}

The reviewer said that the project is at its onset, but the initial data on materials properties seems reasonable.

\section{Reviewer 2:}

The reviewer remarked that within the short period of the project, substantial results were obtained.

\section{Reviewer 3:}

The reviewer said that being a new project, reasonably good progress has been made in the first quarter with the electrodeposition process for the cathodes. A cathode material (NMC-523) of desired particle size has been synthesized and its Li dissolution characteristics during water soak have been determined. The reviewer remarked that several binders (eight) have been synthesized. This progress is consistent with the schedule. However, the reviewer said that it is important to assess the chemical/electrochemical stability of the resins (binders) in the battery electrolytes; the electrochemical stability of the substrate (the reviewer wondered if this is $\mathrm{Al}$ ) at these high deposition potentials (and in aqueous medium); the effects of gas evolution during deposition; the effects of residual water, if any; and demonstrate the feasibility of coating both sides simultaneously. The reviewer also commented that a preliminary cost estimate needs to be made to make the argument that this is indeed economical and will lead to a noticeable reduction in battery cost, which is one of DOE's goals.

\section{Reviewer 4:}

The reviewer said that it is hard to evaluate at this stage. It is difficult to give a higher rating than that assigned, but based on further accomplishments, this value could raise.

\section{Reviewer 5:}

The reviewer commented that the project just started.

\section{Question 3: Collaboration and coordination with other institutions.}

\section{Reviewer 1:}

The reviewer pointed out that there are useful collaborations with DOE's laboratories (ANL and ORNL) to provide suitable cathode materials and develop aqueous deposition methods, respectively. Also, there will be a collaboration with the cell manufacturer, Navitas, to assist in the manufacturing and commercialization efforts.

\section{Reviewer 2:}

The reviewer remarked that the PI has established adequate collaborations.

\section{Reviewer 3:}

The reviewer commented that the performer cited many collaborators with strong battery groups. The reviewer suggested that partners should support the prime strongly in establishing an early proof of concept for the novel approach so that the prime does not invest time and resources in developing an e-coat system that they by themselves cannot determine is functional.

Question 4: Proposed future research-the degree to which the project has effectively planned its future work in a logical manner by incorporating appropriate decision points, considering barriers to the realization of the proposed technology and, when sensible, mitigating risk by providing alternate development pathways. 


\section{Reviewer 1:}

The reviewer said that the future work described in Slide 16 seems appropriate. The performer should work strongly with the collaborators to the confirm battery performance of resulting coatings early in their development process.

\section{Reviewer 2:}

The reviewer remarked that the future research plan is adequate. The PI should put more attention to the feasibility evaluation, e.g., can the uniform coating be achieved at a practically adequate rate.

\section{Reviewer 3:}

The reviewer pointed out that the project just started, and remarked that the proposed research strategy seems fine even though the overall motivation for this approach is not clear.

\section{Reviewer 4:}

The reviewer remarked that the proposed future research is to address the remaining challenges of synthesizing the active materials in the desired particle (small) size; optimizing the formulation of the electrodeposition bath, verifying its stability towards electrodes, and identifying the electrodeposition parameters; assessing the performance of the cathode films; and estimating the performance characteristics in Li-ion batteries (with these cathodes). It is also important to have a preliminary cost model to support the claims of reduced costs. In short, according to this reviewer a lot has to be done to prove the viability of this concept.

\section{Question 5: Does this project support the overall DOE objectives of petroleum displacement? Why or why not?}

\section{Reviewer 1:}

The reviewer pointed out that low specific energies and high costs of Li-ion batteries are serious impediments to their widespread adoption in vehicles. The high costs are partly attributed to the complex fabrication processes for cathodes and anodes. The reviewer commented that new methods of coating the active materials are desired that would lead to a reduction of overall costs for the cell fabrication and for the batteries, which is being addressed here.

\section{Reviewer 2:}

The reviewer remarked that lowered costs of electrode fabrication and eliminating NMP from the deposition method are both helpful toward creating electrodes for EVs at more competitive costs.

\section{Reviewer 3:}

The reviewer commented that this project is high risk and high reward with the potential to support DOE's mission to reduce petroleum consumption.

\section{Reviewer 4:}

The reviewer said that the project will potentially lead to improved battery electrodes and the process for making them.

\section{Question 6: Resources: How sufficient are the resources for the project to achieve the stated milestones in a timely fashion?}

\section{Reviewer 1:}

The reviewer said that this group is ideal for performing the study.

\section{Reviewer 2:}

The reviewer said that project resources seem appropriate for the scope.

\section{Reviewer 3:}

The reviewer said that resources seem to be slightly excessive for the scope of the project, possibly due to the participation of multiple organizations (including national laboratories). 


\section{Li-Ion Battery Anodes from Electrospun Nanoparticle/ Conducting Polymer Nanofibers: Peter Pintauro (Vanderbilt University) - es264}

\section{Presenter}

Peter Pintauro, Vanderbilt University

\section{Reviewer Sample Size}

A total of six reviewers evaluated this project.

\section{Question 1: Approach to performing the work-the degree to which technical barriers are addressed, the project is well-designed, feasible, and integrated with other efforts.}

\section{Reviewer 1:}

The reviewer remarked that the approach of using electrospinning to make fibers containing active materials for making thick electrodes is interesting.

\section{Reviewer 2:}

The reviewer said that the objective here is to develop high specific and volumetric capacity of nanofiber anode mats with Si nanoparticles and an electronically conductive binder for Li-ion batteries. The targeted performance is greater than $750 \mathrm{mAh} / \mathrm{g}$ and $800 \mathrm{Ah} / \mathrm{cc}$ after 50 cycles and with a high anode loading corresponding to an areal capacity of $3 \mathrm{mAh} / \mathrm{cm}^{2}$.

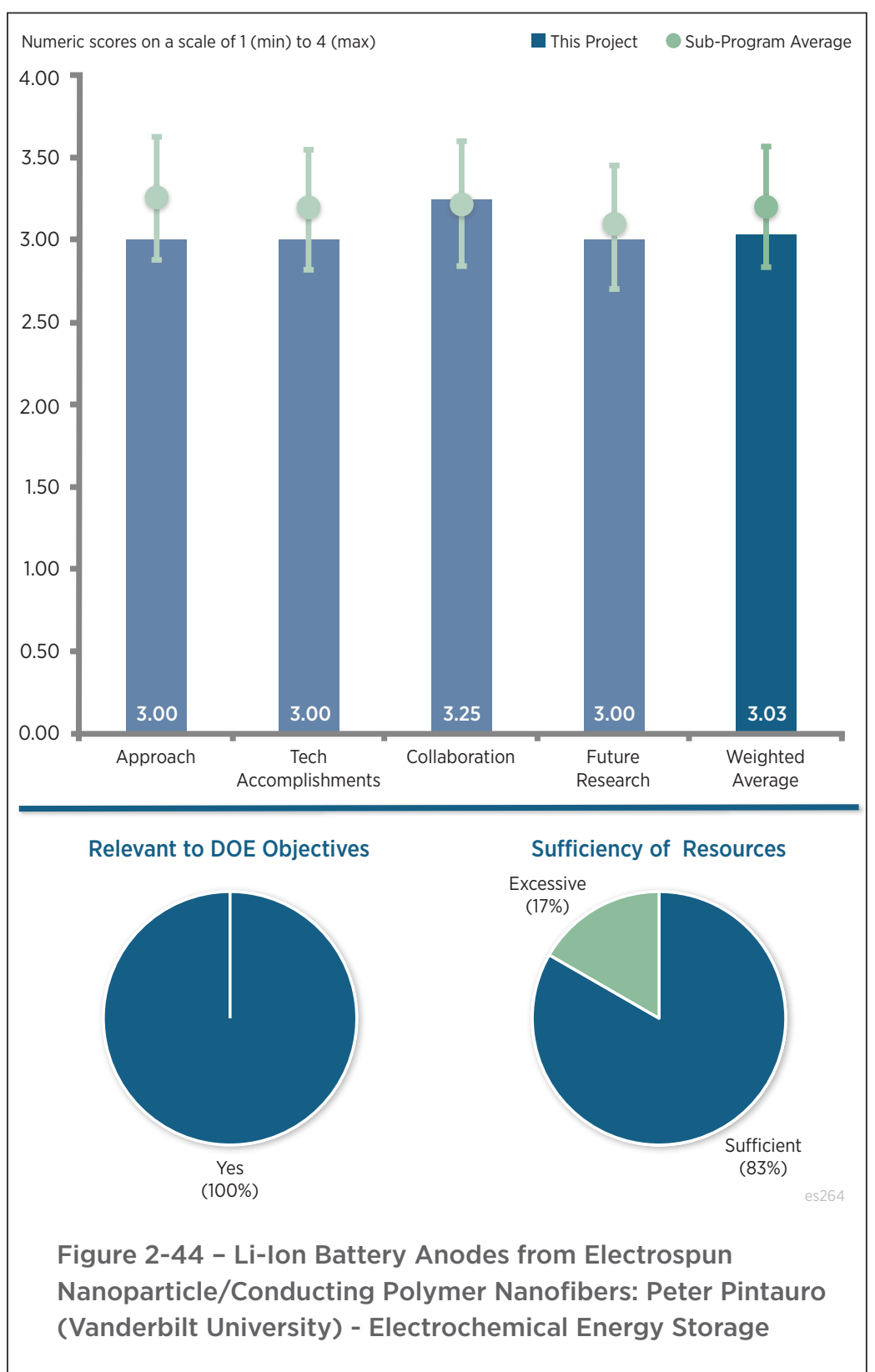

The reviewer said that the technical

that will result in high energy densities for Li-ion cells. The approach is to synthesize electro-spun Si-C nanofiber (with at least $50 \%$ of Si nanoparticles) with a conductive binder (initially the binder developed at LBNL earlier and later another binder), and fabricate and test Si-based nanofiber anodes in Li-ion half cells. The reviewer said that the approach looks feasible with proper milestones to demonstrate the feasibility. The reviewer identified that one problem, however, has to do with the evaluation of Si nanoanodes in half-cells, which is not as complete and even misleading. The performance in a full cell is more challenging, with considerably reduced cycle life and the irreversibility and SEI build on Si determine the overall gains in specific energy and energy density at the cell level. The reviewer said that the project is well designed, and integrated with other efforts.

\section{Reviewer 3:}

The reviewer said that this approach appears to have volumetric energy density issues, with the target performance value (Slide three) of 1.07 and what was achieved (Slide 6) of 0.56. Moreover, the referenced conventional cast $\mathrm{C} /$ poly(vinylidene fluoride) (PVDF) value of $25 \mathrm{mAh} / \mathrm{cm}^{3}$ shown on Slide 9 is extremely low and unrealistic 
(example calculations of $1 \mathrm{~g} / \mathrm{cm}^{3}$ in a finished electrode assuming $2.27 \mathrm{~g} / \mathrm{cm}^{3}$ starting density and losses for binder and $50 \%$ porosity lead to an effective density of $1 \mathrm{~g} / \mathrm{cm}^{3}$ which leads to $25 \mathrm{mAh} / \mathrm{g}$ which at $2 \mathrm{C}$ for any graphite electrode is highly suspect) as a comparison basis. The reviewer said that care should be taken in the future to cite more relevant comparison values for the incumbent technology and to clarify the volumetric issues of this approach.

\section{Reviewer 4:}

The reviewer liked the novelty of the approach, but was concerned about cost. The reviewer wondered if a cost evaluation can be done early in the project (like now).

The reviewer suggested that the project team be very clear in calculations on areal and volumetric energy densities. The PI spoke a lot about porosity, between fibers and within fibers. This caused the reviewer to think about low energy density as the project fills up the electrodes with air.

\section{Reviewer 5:}

The reviewer pointed out that large volume expansion caused mechanical fracture, unstabilized SEI, and poor electrode integrity are the main challenges for applying Si based materials in Li-ion batteries. The project does not address how to solve that problem sufficiently. The reviewer said that electrospun Si nanoparticles in a fiber shape has been investigated by other researchers for many years. Although the conductive polymer binder has been introduced into this project, however, the thickness of the electrode is still a challenge.

\section{Reviewer 6:}

The reviewer said that the PI pursues an integrated Si-based electrode using electrospinning technique. It will create a unique electrode mat with porosity structure and well-connected matrix. However, this approach will have to use high polymer contact in order to achieve spun-fiber. The reviewer said that this will inevitably impact the energy density by volume. The reviewer said that an electrospinning technique is also unfit for large scale production considering its inefficiency.

\section{Question 2: Technical accomplishments and progress toward overall project and DOE goals-the degree to which progress has been made, measured against performance indicators and demonstrated progress towards DOE goals.}

\section{Reviewer 1:}

The reviewer said that the team made significant progress in making Si containing electrospun fibers for making thick anode.

\section{Reviewer 2:}

The reviewer said that good progress has been made in the last few months of the project on the electro-spun Si-C fibrous mats with a conducting polymer. Specifically, using an electronically conductive polymer binder synthesized at LBNL, Si-C fibers were electrospun with 20-50 weight percentage Si nanoparticles. Thick electrodes of approximately $62 \mu \mathrm{m}$ have been fabricated using Si-C fibers, and polyacrylic acid (PAA) binder and tested in half-cells. The reviewer said that high gravimetric $(1,000 \mathrm{mAh} / \mathrm{g})$, areal $\left(3.5 \mathrm{mAh} / \mathrm{cm}^{2}\right)$, and volumetric $(560 \mathrm{mAh} /$ $\mathrm{cm}^{3}$ ) capacities were achieved with the Si-C/PAA mat anodes, especially after compaction. The reviewer detailed that fiber mat strength appears to be important for achieving high initial capacity and to minimize capacity fade with cycling. Even though these results look promising, the reviewer pointed out a couple of issues. Details are not provided on the irreversible capacity (or Coulombic efficiency), which is an important design parameter. Also, cyclic performance in a half-cell gives little indication of how well the electrode cycles in a full cell. The reviewer concluded that overall, the progress is good and is consistent with DOE's goals.

\section{Reviewer 3:}

The reviewer said that the slurry cast values shown on Slide 10 fade approximately $1 \%$ /cycle through 25 cycles and although the Vanderbilt results may be comparable to the LBNL results, the absolute performance is not excellent as stated. Slide 15 mentions welding electrodes but does not give a clear description of process used. The reviewer suggested that the conditions of testing (voltage swept, current, temperature, electrode thickness, etc.) should be added to Slides 9, 10,16 and 17 for a better understanding of work performed. 


\section{Reviewer 4:}

The reviewer said that the project is rather early to judge; however, the reviewer cited good progress on getting the electrospinning process working with the $\mathrm{PFM} / \mathrm{Si}$.

\section{Reviewer 5:}

The reviewer remarked that the project just started last year, and the team has made some progresses to optimize the process and control the electrode structure. These are all necessary initial steps towards addressing the barriers listed at the beginning.

\section{Reviewer 6:}

The reviewer said that in the short period since the project was awarded, the PI tested various polymers as fibermaking agent, including the electronically-conductive binder and PAA. The preliminary results demonstrated that this approach is viable for making Si-composite electrodes, but it will take more time to prove that the electrode materials are indeed superior to state-of-the-art Si electrode materials processed via bulk production, and the process is indeed viable to scale-up for $\mathrm{Kg}$-scale production.

\section{Question 3: Collaboration and coordination with other institutions.}

\section{Reviewer 1:}

The reviewer said that this is a good multi-intuitional project involving a university (Vanderbilt University), two DOE laboratories (LBNL and ORNL), and an industrial partner (e-Spin Technologies).

\section{Reviewer 2:}

The reviewer said that collaborators seem appropriate.

\section{Reviewer 3:}

The reviewer said that the collaborators in this project have sufficient expertise in this field, which can address the barriers effectively.

\section{Reviewer 4:}

The reviewer commented that partners have all the skill sets technically, but asked who the commercialization partner is, and who can provide feedback on manufacturability, cost, etc.

\section{Reviewer 5:}

The reviewer commented that there is apparent close collaboration between the PI and the team members.

Question 4: Proposed future research-the degree to which the project has effectively planned its future work in a logical manner by incorporating appropriate decision points, considering barriers to the realization of the proposed technology and, when sensible, mitigating risk by providing alternate development pathways.

\section{Reviewer 1:}

The reviewer remarked that future work seems appropriate.

\section{Reviewer 2:}

The reviewer said that the proposed future efforts seem to be fine with the milestones and goals.

\section{Reviewer 3:}

The reviewer detailed that the proposed future research is to continue electrospinning studies for Si/PFM/PEO fibers of less than $1 \mu \mathrm{m}$ diameter and demonstrate stable capacities of greater than $500 \mathrm{mAh} / \mathrm{g}$ at $0.1 \mathrm{C}$; and develop dual fiber electrospun nanofiber mats (40-100 $\mu \mathrm{m}$ with an areal capacity of $\left.3 \mathrm{mAh} / \mathrm{cm}^{2}\right)$ containing separate Si/PFM/PEO fibers (for Li storage) and C/PVDF fibers (for electrical conduction). These targets are reasonable provided the irreversible capacity is low (less than 10\%), Coulombic efficiency is high (greater than $99 \%$ ) and capacity fade is low (less than $0.4 \%$ ) during cycling in a full cell. The reviewer said that future work planned is logical with appropriate decision points in the materials selection and demonstration. 


\section{Reviewer 4:}

The reviewer said that the addition of carbon fibers to improve conductivity after pressing is a good idea. The reviewer asked what the surface area is of the polymer fibers, and are these Si or carbon fibers. It seemed to the reviewer as though there could be a lot of parasitic reactions/SEI formation that would result in poor CE. The reviewer asked if the project team makes an SEI on the polymer. If it is electronically conducting enough, the electrolyte will be exposed to a reducing potential

\section{Reviewer 5:}

The reviewer expressed a concern. As this process needs Si in the form of nanoparticles, the cost of this process will certainly depend upon the cost of Si nanoparticles. The reviewer said that it would be interesting to try to make a composite electrode using a Si containing precursor.

\section{Question 5: Does this project support the overall DOE objectives of petroleum displacement? Why or why not?}

\section{Reviewer 1:}

The reviewer said that Si-based materials are the desirable candidate for achieve the DOE objectives.

\section{Reviewer 2:}

The reviewer said that low specific energies and high costs of Li-ion batteries are serious impediments to their widespread adoption in vehicles. High capacity cathode and anode materials are required to improve the specific energy of Li-ion cells. Si anodes are promising to provide high specific capacity $(\mathrm{mAh} / \mathrm{g})$ and volumetric capacity $(\mathrm{mAh} / \mathrm{cc})$ compared to graphitic anodes, which will result in high gravimetric and volumetric energy densities for Li-ion cells. However, there is an inherent issue of volume expansion with the Si anode that affects its cyclic stability. The reviewer said that in order to mitigate this issue, new anode nanostructure architectures are being explored worldwide, which is also the aim for this project.

\section{Reviewer 3:}

The reviewer said that if successful, this project could help contribute to DOE's mission to reduce petroleum consumption.

\section{Reviewer 4:}

The reviewer commented an improved electrode manufacturing process and potentially a high energy density $\mathrm{Si}$ anode.

\section{Reviewer 5:}

The reviewer affirmed that the project is relevant to DOE objectives.

\section{Question 6: Resources: How sufficient are the resources for the project to achieve the stated milestones in a timely fashion?}

\section{Reviewer 1:}

The reviewer said that resources seem to be quite appropriate for the scope of the project.

\section{Reviewer 2:}

The reviewer commented that project resources seem appropriate.

\section{Reviewer 3:}

The reviewer said that resources are sufficient. 


\section{UV Curable Binder \\ Technology to Reduce \\ Manufacturing Cost and \\ Improve Performance of \\ LIB Electrodes: John Arnold \\ (Miltec UV International) \\ - es265}

Presenter

John Arnold, Miltec UV International

\section{Reviewer Sample Size}

A total of six reviewers evaluated this project.

\section{Question 1: Approach to performing the work-the degree to which technical barriers are addressed, the project is well-designed, feasible, and integrated with other efforts.}

\section{Reviewer 1:}

The reviewer stated that the objective is to develop UV curable binders for LIB electrodes to reduce manufacturing cost and also to improve performance of Li-ion batteries. The reviewer noted that in contrast to the conventional electrode fabrication with polyvinylidene difluoride (PVDF) and NMP solvent, this UV-cured method provides faster curing and rapid drying, possibly with simultaneous two-sided coating, resulting in considerable reduction in capital costs $(80 \%)$ and some savings in the operational costs for Li-ion cells. The reviewer added that it will also allow a reduction in the binder content, improved binder stability during cycling. The reviewer explained that UV light instantly polymerizes photo-reactive mixture into a solid plastic, and the coating is fully cured and ready to use or test immediately after light exposure. After identifying suitable binders, the reviewer added, high-speed coating and curing with slot die and printing technology will be explored. Such UV-cured polymers were used to form polymer electrolytes successfully. The reviewer remarked that the project is well designed and integrated with other efforts, especially with a similar project from this organization on using UV-cured resin for coating ceramic layers on separators.

\section{Reviewer 2:}

The reviewer stated that using UV-curable technique to process the electrode binder is an innovative approach as it will rid of many heavy equipment for drying and solvent-recovery, as well as saving time and energy.

\section{Reviewer 3:}

The reviewer pointed out that UV-cured coating has substantial advantages (e.g., high speed, two-side simultaneous curing, no solvent, etc.).

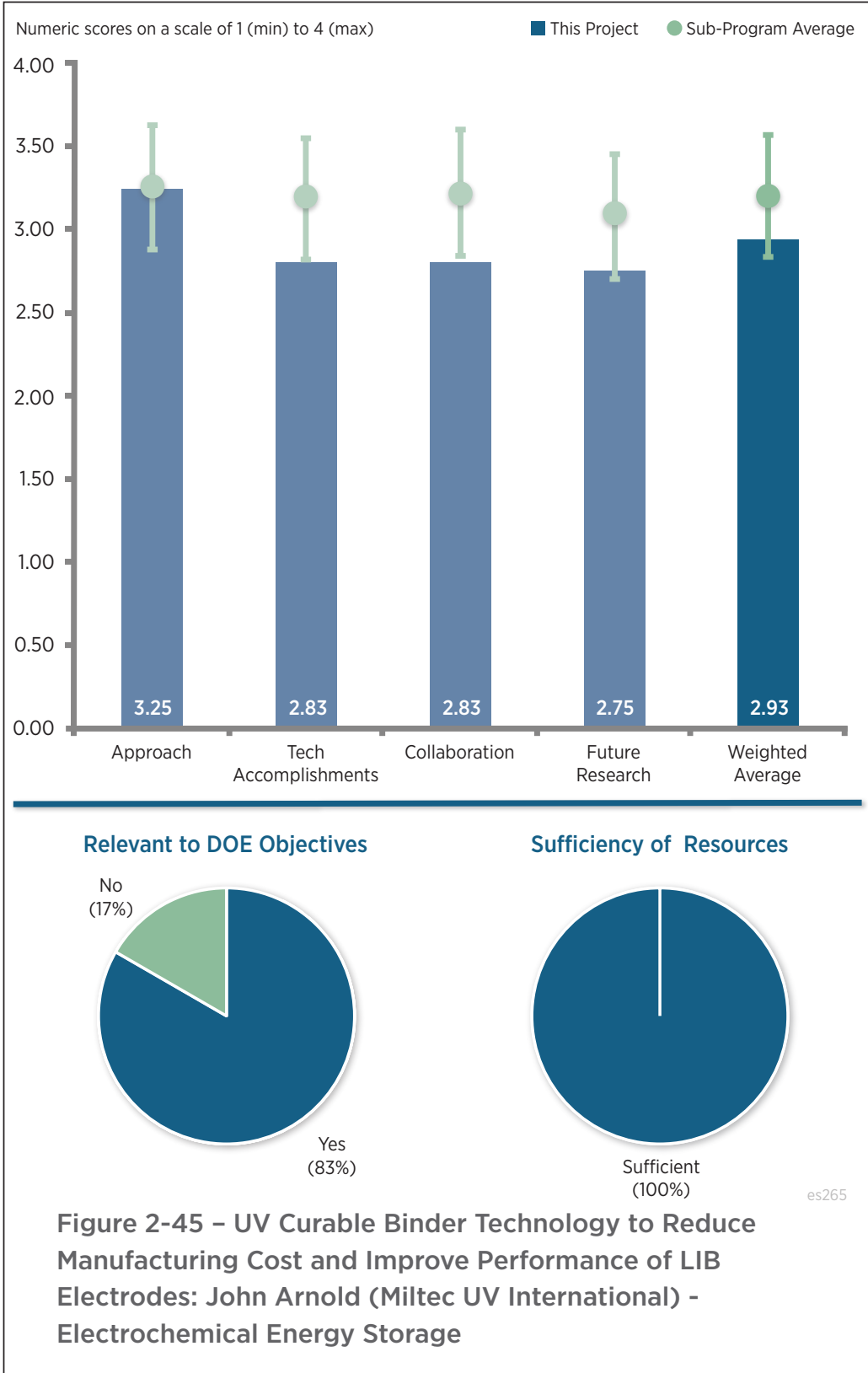




\section{Reviewer 4:}

The reviewer remarked that the approach is generally effective in terms of saving the cost. The reviewer added that the trade-off on performance needs to be further studied. The large particle of active materials might block UV and, as a result, the reviewer explained, curing might not be uniform.

\section{Reviewer 5:}

The reviewer commented that manufacturing cost reductions of $80-95 \%$ are claimed for this approach, but are not substantiated by any technical data provided.

\section{Question 2: Technical accomplishments and progress toward overall project and DOE goals-the degree to which progress has been made, measured against performance indicators and demonstrated progress towards DOE goals.}

\section{Reviewer 1:}

The reviewer stated that good progress has been made in the first quarter on the UV-cured binders for fabricating electrodes for Li-ion cells. Specifically, suitable binders have been identified and electrodes have been fabricated using UV-curing. These electrodes demonstrated performance comparable to the standard electrodes with PVDF binder (different amounts), even showing lower impedance growth and better capacity retention during cycling both at high and low temperatures. However, the reviewer questioned that it has not been mentioned what the electrode loadings are in these studies. Further, the process has been demonstrated to be faster $(100 \mathrm{~m} / \mathrm{min})$. Finally, the reviewer added, modeling studies have been performed which show that the manufacturing costs will be reduced by $80-90 \%$, and $24 \%$ of the cost reduction included materials for $\mathrm{Li}$-ion cells. There will also be additional benefits in the capital and operational costs. The reviewer remarked that even though the initial results are encouraging, it is not clear how good the electrode uniformity will be with higher electrode loadings. The reviewer concluded that overall progress has been good and consistent with DOE goals.

\section{Reviewer 2:}

The reviewer remarked that, given the short period since the project was awarded, the PI was able to show that the new electrode processed by their UV approach can indeed rival the semiconductor optical amplifier (SOA) electrode.

\section{Reviewer 3:}

The reviewer stated that electrodes were made using a UV-curing method in comparison with traditional PVDF coating, and that cells were made from the electrode and tested electrochemically. The reviewer noted that preliminary data show the parity performance, and that more cycle data should be included.

\section{Reviewer 4:}

The reviewer noted that the accomplishment meets the planned milestone. However, the reviewer added, PI claimed that the major challenges of capacity, impedance, and long-term cycling have been overcome, but no convincing data were shown in the presentation.

\section{Reviewer 5:}

The reviewer noted that very few technical details were provided on Slide 8 regarding impedance, such as the alternating current (AC) impedance test pattern (e.g., frequency swept, amplitude). The reviewer questioned why only the $-10^{\circ} \mathrm{C}$ data were shown, and whether impedance means the real axis intercept, or the radius of curve 1,2 , or other feature. Similarly, the reviewer commented that very few technical experimental details were provided, such as details of cycles run (e.g., voltage, current, temperature, etc.). The reviewer questioned whether the data plotted belonged to one cell or the average of many. The reviewer pointed out that Slide 9 shows 200 cycles of $2 \mathrm{C} / 2 \mathrm{C}$ testing, which would take approximately 10 days to reach depending on further details of the test pattern not shared (such as voltage swept, rest periods, cell type/construction, electrode details, etc.). The reviewer remarked that further testing with a variety of temperatures, cycle voltages, rates, etc. should be shown if similar performance relative to the PVDF system is to be believed.

\section{Question 3: Collaboration and coordination with other institutions.}




\section{Reviewer 1:}

The reviewer praised the collaborations with DOE laboratories (ANL ORNL) for technical guidance and product validation. ANL is assisting in the testing and analysis of UV-cured cathodes in pouch cells and comparing performance to PVDF baseline, while ORNL is assisting in the coating and testing of a multilayered UV cathode with goals of higher energy density and higher voltage cells.

\section{Reviewer 2:}

The reviewer remarked that the collaborators are the experts in the battery field, and that the collaboration is expected to be effective.

\section{Reviewer 3:}

The reviewer commented that there is close collaboration between the PI and the team members.

\section{Reviewer 4:}

The reviewer noted that collaborations seem appropriate, but should contribute more strongly to help the prime perform basic electrochemical characterization to determine if this material change is workable in a battery system.

\section{Reviewer 5:}

The reviewer indicated that the PI should find more collaborators.

Question 4: Proposed future research-the degree to which the project has effectively planned its future work in a logical manner by incorporating appropriate decision points, considering barriers to the realization of the proposed technology and, when sensible, mitigating risk by providing alternate development pathways.

\section{Reviewer 1:}

The reviewer stated that the proposed future research is adequate.

\section{Reviewer 2:}

The reviewer stated that the proposed future efforts are reasonable.

\section{Reviewer 3:}

The reviewer noted that the proposed future research focuses on the installation and operation of high-speed coating with slot die and printer technology to demonstrate high-speed coating and curing, both single- and doublesided. The reviewer expressed that it needs to be confirmed if this method is applicable to high-energy density multi-layered cathode coatings. The reviewer added that planned future work is logical and the milestones are reasonable and consistent with project objectives.

\section{Reviewer 4:}

The reviewer indicated that future efforts should focus on supporting the very large cost-reduction potential claimed by the performer. The reviewer observed that the first bullet of Slide 11 states that challenges of capacity, impedance, and cycling have been overcome, but that little technical data to support these conclusions have been shown. The reviewer said that the performer needs to provide much more electrochemical testing data at the electrode proof-of-concept stage before focusing on any equipment and speed scale ups.

\section{Reviewer 5:}

The reviewer pointed out that chemistry and electrode structures still need further optimization, which are not included in the future plan.

\section{Question 5: Does this project support the overall DOE objectives of petroleum displacement? Why or why not?}

\section{Reviewer 1:}

The reviewer remarked that in addition to improving the specific energy, energy density, and specific power of Li-ion batteries, it is also important to reduce their costs for a widespread adoption in the EV market. The conventional electrodes, or at least cathodes, are fabricated using fluorinated binders dissolved in NMP, which requires elaborate drying to remove the solvent. Newer binders and with simpler drying (i.e., curing) processes are 
required to reduce the cost of electrode fabrication and thus the cost of $\mathrm{Li}$-ion batteries. The reviewer stated that the project is aimed at developing new such binders cured by low-cost methods, i.e., UV irradiation.

\section{Reviewer 2:}

The reviewer agreed that reducing manufacturing costs is an effective way to reduce the overall cost of a battery pack.

Reviewer 3:

The reviewer stated that the project is relevant to DOE goals.

\section{Reviewer 4:}

The reviewer commented that, based on the low amounts of technical data shown, it is not possible to determine if this work would be able to serve the DOE mission to reduce petroleum usage.

\section{Question 6: Resources: How sufficient are the resources for the project to achieve the stated milestones in a timely fashion?}

\section{Reviewer 1:}

The reviewer said the resources seem to be adequate for the scope of the project and the number of participants.

\section{Reviewer 2:}

The reviewer stated that, based on the low amounts of technical data shown, the resources seem large. 


\section{Co-Extrusion (CoEx) for Cost Reduction of Advanced High- Energy-and-Power Battery Electrode Manufacturing: Corie Cobb (Palo Alto Research Center) - es266}

\section{Presenter}

Corie Cobb, Palo Alto Research Center

\section{Reviewer Sample Size}

A total of five reviewers evaluated this project.

\section{Question 1: Approach to performing the work-the degree to which technical barriers are addressed, the project is well-designed, feasible, and integrated with other efforts.}

\section{Reviewer 1:}

The reviewer observed that the objective of the project is to develop pilot-scale fabrication of highcapacity pouch cells through coextrusion. The use of thick-structured cathodes and matching-thick anode is expected to result in improved performance ( $20 \%$ gain in $\mathrm{Wh} / \mathrm{kg}$ ) and reduced cost (greater than $30 \%$ ). The reviewer indicated that near-term objectives include: demonstrating the CoEx cathodes in coin cell with over $20 \%$ gain in $\mathrm{Wh} / \mathrm{kg}$ over a baseline cell (the reviewer questioned how such a quantitative comparison can be made in a coin cell where the ratio of active material to cell casing is not favorable); optimizing the thick CoEx cathode design; developing matching-thick graphite anode; and performing an assessment of its scale up and commercialization. The reviewer added that even though the CoEx concept looks appealing, especially with the structured electrodes, such structural pattern may not be retained after calendering. Without calendaring and compaction, the performance will be poor for a thick electrode due to low electronic conductivity, and result in low volumetric energy density. The reviewer expressed that the milestones look reasonable, except that for the demonstration of the cathode, its thickness is not specified. The reviewer noted that the design of print head and the slurry consistency are critical for the co-extrusion of the cathode. The anode will be slot-die coated, i.e., in the conventional manner, but with different conductive diluent and binder for thicker electrodes $(125-200 \mu \mathrm{m})$. Overall, the reviewer concluded, the project is well designed and integrated with other efforts.

\section{Reviewer 2:}

The reviewer noted that the approach addressed the barriers effectively, and that the cost should dramatically be reduced by increasing the loading of active materials in the batteries.
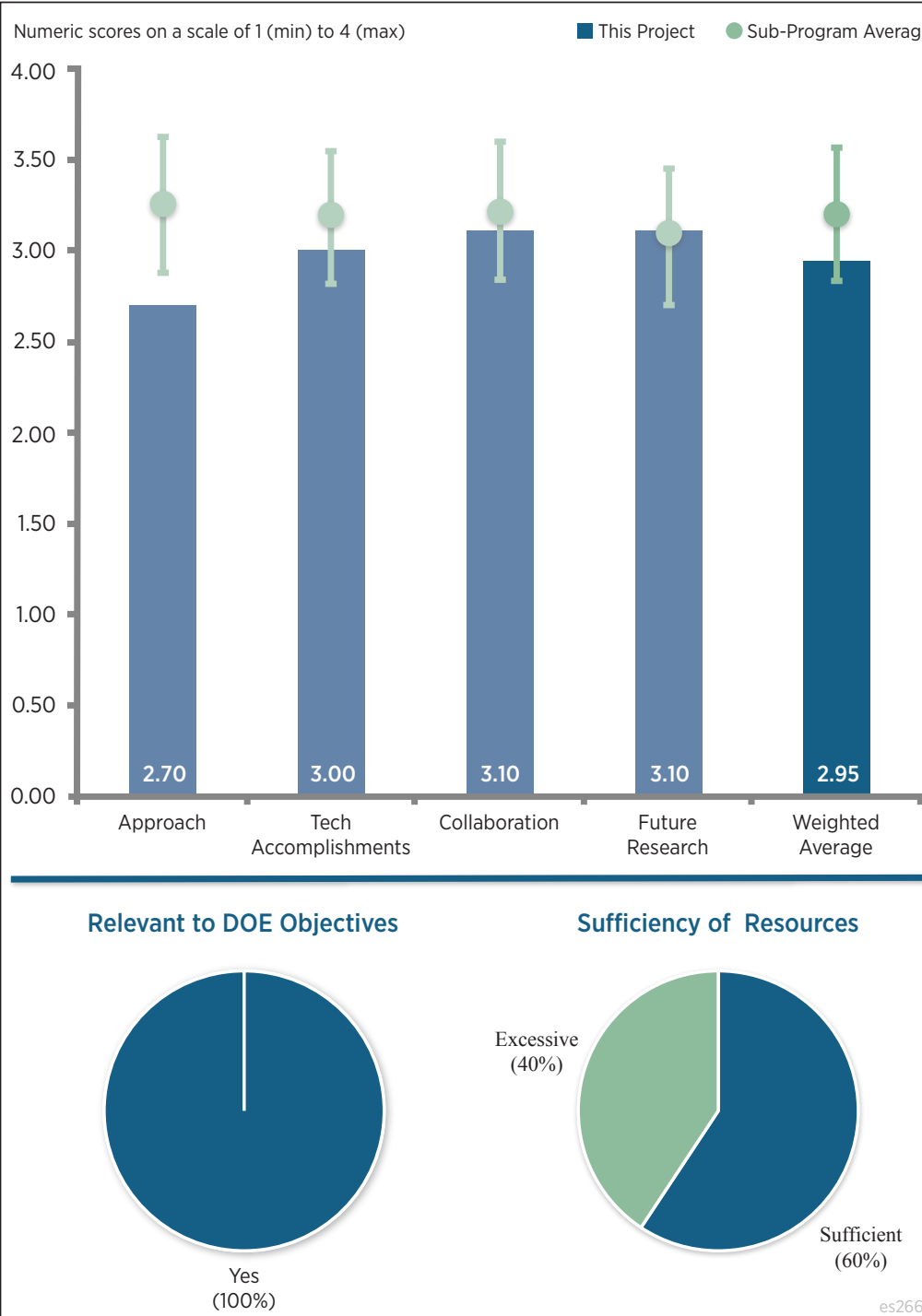

Figure 2-46 - Co-Extrusion (CoEx) for Cost Reduction of Advanced High-Energy-and-Power Battery Electrode Manufacturing: Corie Cobb (Palo Alto Research Center) Electrochemical Energy Storage
Relevant to DOE Objectives 


\section{Reviewer 3:}

The reviewer stated that the advantages of the proposed approach as opposed to making mesoporous composite electrodes are not clear. In fact, the reviewer elaborated, the proposed approach will be limited in the volume fraction for active material loading due to the lateral gap limitation between gaps. The reviewer expressed that the mechanical integrity of the cathode layer near hollow groves is also questionable. Similarly, the reviewer added, the chemical stability through dissolution will also be enhanced in the hollow groves filled with electrolytes.

\section{Reviewer 4:}

The reviewer observed that the PI proposed to use a printing technique to create a structure electrode matrix so that requirements for both energy density and power density can be satisfied. The reviewer commented that it seems unnecessary to use a printing technique, at least for the single-material processing, and that a mechanical patterning will probably create the same effect at a much lower cost.

\section{Reviewer 5:}

The reviewer expressed difficulty in evaluating this question with the information given in the presentation. The reviewer acknowledged that the program has just started, but indicated a lack of technical detail in the slides. The reviewer commented that goals for the cells need to be disclosed, and that it is impossible to determine the merit of the technology without an idea of the power density targets. The reviewer pointed out that USABC publishes its targets, and claimed that DOE funding should not be used to fund projects without clear targets for the cell performance [DOE Program Clarification: This is an exploratory Advanced Processing R\&D project; it is significantly early in the project to build a reasonable cost model. Cost models will be deliverables in the final year of the project.]. The reviewer warned that cost claims are dubious, and added that more specific cost modeling needs to be shown to give merit to cost claims. The reviewer did not see how a $30 \%$ decrease of cost is possible on a $\mathrm{kWh}$ basis. The reviewer pointed out that, as far as the reviewer understands, the program is not decreasing the amount of active material, only the number of layers in a cell. Because the current collectors and separator do not add up to $30 \%$ of the cell cost, the reviewer added, even removing them completely will not result in a 30\% reduction in cost. Similarly, manufacturing accounts for only around $30 \%$ of the cost in the worst case, thus the same issues exist. The reviewer expressed that energy density claims are similarly dubious, and that electrochemical modeling data should be shown to support the claims.

\section{Question 2: Technical accomplishments and progress toward overall project and DOE goals-the degree to which progress has been made, measured against performance indicators and demonstrated progress towards DOE goals.}

\section{Reviewer 1:}

Although the project started late, the reviewer commented, the team has made significant progress.

\section{Reviewer 2:}

The reviewer noted that progress seems okay in demonstrating the fabrication of striped electrodes.

\section{Reviewer 3:}

The reviewer commented that good progress has been made in the first quarter towards the CoEx and thickstructured cathodes in Li-ion cells for improved specific energy and reduced cost. The reviewer pointed out that work is in progress to design the cell targets for the NMC cathode graphite materials; to perform modeling for the optimal geometries of the CoEx cathode for a 10-30\% energy improvement; to demonstrate single-layer CoEx cathode films with over $142 \mathrm{mAh} / \mathrm{g}$ at C/2 discharge rate; and to verify that baseline anode matches well with CoEX thick cathode with suitable binder and conductive diluent. The reviewer questioned what the thickness of the single-layer CoEx cathode films was. The reviewer also noted that preliminary experiments were completed to demonstrate initial print feasibility for CoEx cathode structures. The reviewer said that, even though the process looks simpler, there are a couple of issues: First, the post-extrusion calendering will damage the structural aspects (with gradient porosities) of the cathode. Second, it is not clear if thick anode will function as well, without any additional channels for electrolyte; even though cathode is limiting at room temperature, at low temperatures anode is known to be limiting. Third, with only 10-30\% gain at the cathode level, the gains at the cell and battery level will be less attractive. Finally, because cost is one of the presumptive benefits here, it requires substantiation with a preliminary cost model. Overall, the reviewer concluded, the progress is good and consistent with the DOE goals. 


\section{Reviewer 4:}

The reviewer stated that even though it is early in the program, at this point the reviewer would expect to see more detailed cost modeling and electrochemical modeling to give merit to the cost reduction and energy density improvement claims.

\section{Reviewer 5:}

The reviewer observed that results shown are preliminary and not convincing that the electrodes created are superior to the state of the art. The reviewer added that more time is needed to make that judgment.

\section{Question 3: Collaboration and coordination with other institutions.}

\section{Reviewer 1:}

The reviewer pointed out that there are useful collaborations with external partners that include DOE laboratories (ANL and ORNL) and industry (Navitas).

\section{Reviewer 2:}

The reviewer expressed that future collaboration with ORNL is good, but strongly suggested collaboration with the national laboratories to work on cost modeling. The cell performance targets for the program need to be disclosed.

\section{Reviewer 3:}

The reviewer stated that the team is strong, involving national laboratories and the automotive industry, and that it would be better to also involve battery manufactures at some point in the future.

\section{Reviewer 4:}

The reviewer commented that collaboration is close.

Question 4: Proposed future research-the degree to which the project has effectively planned its future work in a logical manner by incorporating appropriate decision points, considering barriers to the realization of the proposed technology and, when sensible, mitigating risk by providing alternate development pathways.

\section{Reviewer 1:}

The reviewer observed that the proposed future research is aimed at the following tasks: develop CoEx cathode films with high utilization; develop suitable matching thick graphite anode; perform a scale up analysis; and modify the print heads for 1 Ampere-hour $(\mathrm{Ah})$ pouch cells. In addition, alternate drying methods will be established to avoid electrode cracks, multiple CoEx structures will be explored to achieve the desired structural properties, and multiple graphite anodes will be assessed for the matching thick anode. The reviewer suggested a preliminary cost versus performance model based on the material properties and coin cell data to support the claims of benefits over the devices with conventional electrodes. The reviewer concluded that the future work is logical and addresses the risks in the proposed electrode architecture.

\section{Reviewer 2:}

The reviewer stated that the future plan is trying to address all the barriers and was laid out in detail.

\section{Reviewer 3:}

The reviewer commented that the proposed research strategy is okay with respect to the funded project objectives.

\section{Reviewer 4:}

The reviewer noted the proposed future efforts are reasonable.

\section{Reviewer 5:}

The reviewer said that notes from first section apply to this as well.

Question 5: Does this project support the overall DOE objectives of petroleum displacement? Why or why not? 


\section{Reviewer 1:}

The reviewer stated that low specific energies and high costs of Li-ion batteries are serious impediments to their widespread adoption in vehicles, and that reducing the inactive materials (current collectors and separators) by utilizing thick electrodes will have benefits in both performance (higher energy density) and cost. However, the reviewer added, the current electrode designs yield poor results if used in thick configuration. New electrode designs and fabrication methods are desired that would allow the use of thicker electrodes and improve both performance and cost of Li-ion batteries, which are being addressed by the project.

\section{Reviewer 2:}

The reviewer agreed that the idea of reducing cost and improving energy density support DOE objectives.

\section{Reviewer 3:}

The reviewer indicated improved manufacturing processes for LIB electrodes.

\section{Reviewer 4:}

The reviewer observed that improving the loading of the active materials is an effective way to reduce the cost of the high energy batteries in terms of dollars-per-kWh.

\section{Reviewer 5:}

The reviewer stated that the project is relevant to DOE goals.

\section{Question 6: Resources: How sufficient are the resources for the project to achieve the stated milestones in a timely fashion?}

\section{Reviewer 1:}

The reviewer indicated that resources seem a little excessive for the scope of the project, even with multiple partners.

\section{Reviewer 2:}

The reviewer stated that the amount of funding seems high given that no new electrochemistry or equipment will be developed during the program. The project is using existing chemistries and coating technologies for the anode and existing printing technologies for the cathode. 


\section{Commercially Scalable Process to Fabricate Porous Silicon: Peter Aurora (Navitas Systems) - es267}

\section{Presenter}

Peter Aurora, Navitas Systems

\section{Reviewer Sample Size}

A total of seven reviewers evaluated this project.

\section{Question 1: Approach to performing the work-the degree to which technical barriers are addressed, the project is well-designed, feasible, and integrated with other efforts.}

\section{Reviewer 1 :}

The reviewer observed that the objective is to develop a novel, commercially-scalable approach to produce microporous Si with a targeted specific capacity of $800 \mathrm{mAh} / \mathrm{g}$, in the desired powder morphology, and at low process cost. In contrast to the conventional methods that uses hazardous materials, such as silane and hydrofluoric acid, this method has three steps: solid-state milling, reduction and thermal treatment, followed by etching. The estimated cost of the microporous Si thus produced is about $\$ 10 / \mathrm{kg}$, as opposed to $\$ 24 / \mathrm{kg}$ for the conventional process. The reviewer noted that specific objectives include a bench-scale optimization of the three processes, demonstrating the performance of the materials in baseline prototype cells, establishing a pilot-scale production plant (over 10kg per batch), and assessing its economic feasibility. Suitable tasks with appropriate milestones and performance targets have been identified. The reviewer concluded that the project is well designed and integrated with other efforts.

\section{Reviewer 2:}

The reviewer noted that the development of a production method to make Si anode material in which the hydrofluoric acid (HF) etching is eliminated is a step toward a feasible industrial process.

\section{Reviewer 3:}

The reviewer remarked that the approach proposed by the PI to make microporous Si through green etching without using HF is novel.

\section{Reviewer 4:}

The reviewer observed that the project is still in its early stages, but that the approach seems technically sound. The

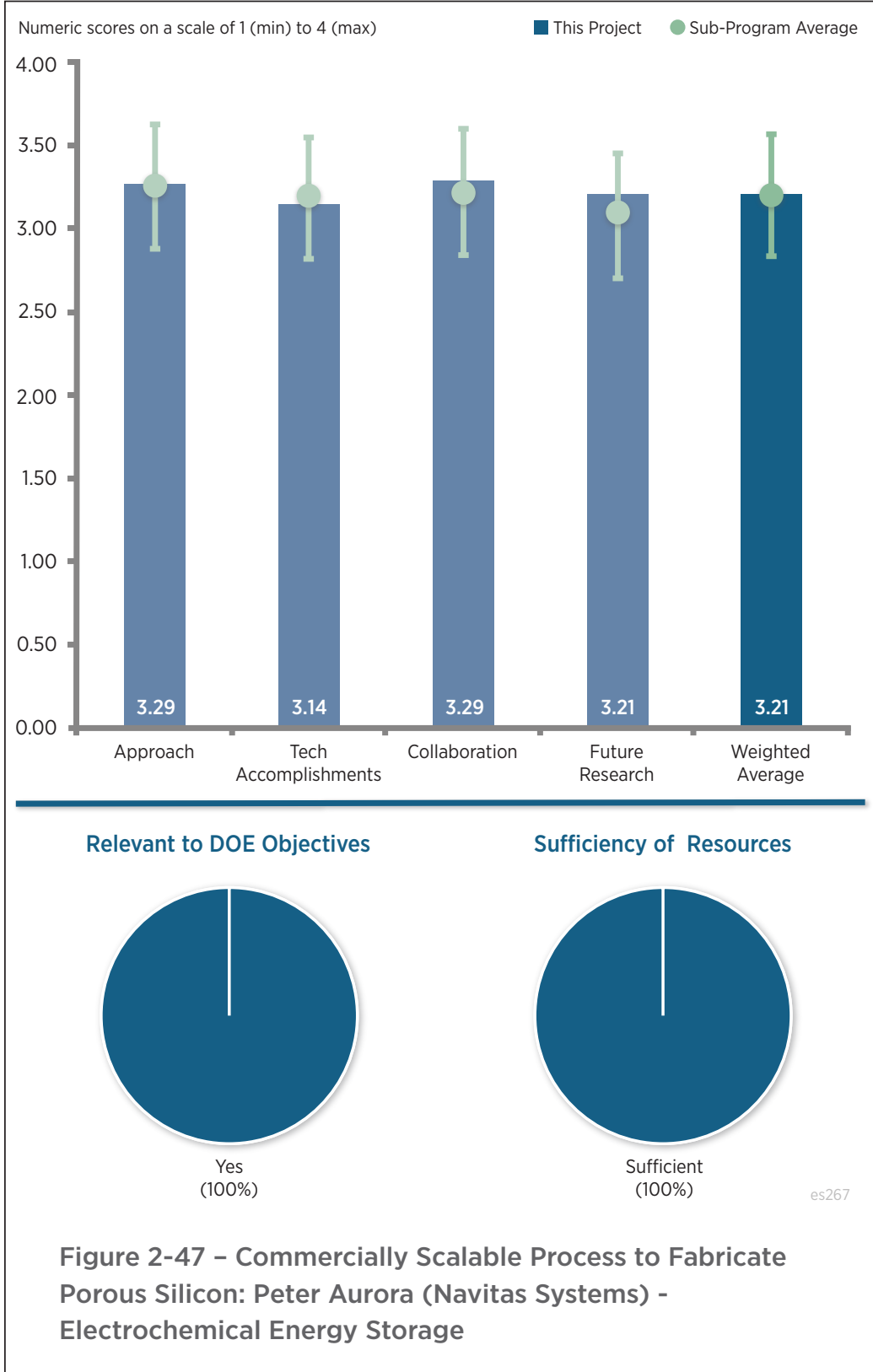


reviewer commented that it is not clear from the slides where the cost savings come from. The reviewer added that Navitas Systems' approach to making the microporous Si seems more involved than the standard process, and that next year it is important to show more details from the in progress cost modeling step. The reviewer recommended avoiding the use of the term green etching being that it is overly vague for a scientific presentation.

\section{Reviewer 5:}

The reviewer commented that the strategy and approach were fine. Commercial use of HF for etching can be expensive; additional safety precautions are required, and waste disposal also leads to added cost. The reviewer added that additional information on cost benefits should be broken down to the various process steps, and that a second way to express cost reduction, such as dollars-per-kWh savings, may be helpful.

\section{Reviewer 6:}

The reviewer stated that although the team proposed a new approach to scale up the process to make porous $\mathrm{Si}$ electrodes, it has not been demonstrated that porous Si would meet the requirement of the anode materials on cycle life, volumetric energy density, and cost. The reviewer added that a lot of potential issues associated with porous $\mathrm{Si}$ have not yet been solved, including low cycle efficiency and tap density.

\section{Reviewer 7:}

The reviewer commented that the approach to change microporous Si processing steps for cost reduction seems appropriate. However, an inherent assumption is made that conventional microporous Si is desirable as an anode, and that if only the price was cheaper it would be commercialized. The reviewer expressed that this case is not made convincingly by this project and is at the center of its motivation. More technical comparisons against the conventional materials should be made in this approach to help clearly differentiate the impact of this project's approach.

\section{Question 2: Technical accomplishments and progress toward overall project and DOE goals-the degree to which progress has been made, measured against performance indicators and demonstrated progress towards DOE goals.}

\section{Reviewer 1:}

The reviewer praised that good progress has been made in the last few months of the project on developing a lowcost process for manufacturing microporous Si powder for Li-ion cells. Laboratory-scale process optimization has been initiated that includes three steps: mechanical milling, thermal reduction to achieve desirable particle structure and composition, and etching to fully remove metal oxide. Mechanical milling allows the reduction temperature to be lowered by $200^{\circ} \mathrm{C}$. The microporous Si produced here compares well with the commercial microporous material in physical characteristics as well as electrochemical performance. However, the reviewer observed, the electrochemical performance seems to be quite preliminary with a rapid capacity fade even in half-cell, though the microporous material is slightly superior to the commercial baseline. The reviewer added that apart from the technical performance, the critical step here is to perform an economic assessment of the process to verify its economic advantages over current battery material manufacturing processes. Overall, the reviewer concluded, the progress is good and is consistent with the DOE goals.

\section{Reviewer 2:}

The reviewer noted that the team has made a lot of progress. For the half cell data, the reviewer suggested the team also show the ratio between porous $\mathrm{Si}$ and graphite in the $\mathrm{Si} /$ graphite blend anode.

\section{Reviewer 3:}

The reviewer expressed that while the program is in the very early stages, the initial data show the approach has promise. The reviewer would have liked to see more information to support the cost claims.

\section{Reviewer 4:}

The reviewer stated that a $\mathrm{Si}$ anode with high porosity was synthesized, characterized, and tested in electrochemical cells, and decent cycle life was obtained against benchmark material.

\section{Reviewer 5:}

The reviewer commented that the technical targets on Slide 5 seem reasonable, but that all the cycling data shown 
on Slides 13 and 14 are based on percentage of capacity retention and thus it is not possible to determine progress towards goals. Slide 11 highlights that impurities in the new material are less 3\%; however, the reviewer suggested that the existing value in the commercial microporous Si be reported for comparison. On Slide 13, the reviewer observed that the microporous approach appears to have no impact on cycling. The reviewer suggested that these data be shown in absolute terms, and that cell details (e.g., size, construction type) be shared and commented on.

\section{Reviewer 6:}

The reviewer observed that given the short period since the project was awarded, the preliminary results are far from being convincing that the current approach will produce superior Si materials.

\section{Reviewer 7:}

The reviewer strongly suggested that the PI find the best commercial reference source for all the baseline studies. The reviewer recommended that the PI report CE and cycle life next year. The reviewer expressed that the PI has a good handle on the process parameters to investigate in Tasks 1 and 2 with the metals removal step. The reviewer also suggested the PI investigate the degree of final purity needed by carrying out performance tests at different etching efficiencies.

\section{Question 3: Collaboration and coordination with other institutions.}

\section{Reviewer 1:}

The reviewer observed that there are good ongoing collaborations with several partners: Nexceris, LLC for scaleup; ANL for material characterization and cost modeling; Navitas Systems for assessment in prototype Li-ion cells; XG Sciences for anode evaluation; and the University of Utah for scaling up the powder milling process.

\section{Reviewer 2:}

The reviewer stated that the collaborations seem appropriate.

\section{Reviewer 3:}

The reviewer expressed that the list of partners in the project looks well thought-out and likely to contribute to the project's success.

\section{Reviewer 4:}

The reviewer noted that collaborators are quite strong and the expertise are complementary to one another.

\section{Reviewer 5:}

The reviewer commented that the PI seems to have arranged for sufficient collaboration and external expertise on project.

\section{Reviewer 6:}

The reviewer suggested that cell manufacturers be included.

\section{Reviewer 7:}

The reviewer said that collaboration is close between PI and team members.

Question 4: Proposed future research-the degree to which the project has effectively planned its future work in a logical manner by incorporating appropriate decision points, considering barriers to the realization of the proposed technology and, when sensible, mitigating risk by providing alternate development pathways.

\section{Reviewer 1:}

The reviewer stated that reasonable future efforts were proposed.

\section{Reviewer 2:}

The reviewer pointed out that the proposed future research is to demonstrate the benefits of the microporous $\mathrm{Si}$ being developed, optimize the synthesis further, and scale it up. Specific activities include completing the labscale process optimization (mechanical milling, thermal treatment, and oxide removal; identifying alternative etching chemistries to reduce cost with reduced environmental footprint; validating electrochemical properties; 
and identifying opportunities for cost reduction associated with scaling up the processes. The reviewer noted it is crucial to demonstrate that this process has technical and economic advantages over a conventional method, which is a go/no-go milestone. The reviewer concluded that the future work planned is logical, with appropriate decision points in the process development.

\section{Reviewer 3:}

The reviewer expressed the plan was reasonable.

\section{Reviewer 4:}

The reviewer appreciated the inclusion of the go/no-go date based on process cost.

\section{Reviewer 5:}

The reviewer observed that monitoring coulombic efficiency and cycle life against a good reference will be important measurement tools in progress.

\section{Reviewer 6:}

The reviewer suggested that future work prioritize the focus on cost estimation to reduce the cost of microporous $\mathrm{Si}$, as that is stated as the main purpose of this project.

\section{Reviewer 7:}

The reviewer recommends the team consider both improving the performance and reducing the cost.

\section{Question 5: Does this project support the overall DOE objectives of petroleum displacement? Why or why not?}

\section{Reviewer 1:}

The reviewer said that, if successful, this project may support the DOE objective of petroleum displacement.

\section{Reviewer 2:}

The reviewer observed that the use of microporous $\mathrm{Si}$ in anodes shows promise, and having a cost effective source of the material will contribute to the DOE goals of petroleum displacement.

\section{Reviewer 3:}

The reviewer noted that the project supports DOE's overall objective.

\section{Reviewer 4:}

The reviewer commented that reducing the cost and improving the energy density/cycle life are closely related with DOE objectives.

\section{Reviewer 5:}

The reviewer stated that the project is relevant to DOE goals.

\section{Reviewer 6:}

The reviewer pointed out that low specific energies and high costs of Li-ion batteries are serious impediments to their widespread adoption in vehicles. High capacity cathode and anode materials are required to improve the specific energy of Li-ion cells. Si anodes are promising to provide high specific capacity $(\mathrm{mAh} / \mathrm{g})$ and volumetric capacity $(\mathrm{mAh} / \mathrm{cc})$ compared to graphitic anodes, which will result in high gravimetric and volumetric energy densities for Li-ion cells. However, the reviewer expressed that there is an inherent issue of volume expansion with the $\mathrm{Si}$ anode that affect its cyclic stability. In order to be resilient to the volume expansion and the resulting cracking, microporous Si material is used, which this project is aiming to develop.

\section{Question 6: Resources: How sufficient are the resources for the project to achieve the stated milestones in a timely fashion?}

\section{Reviewer 1:}

The reviewer expressed that the resources seem appropriate for the scope of the project. 
Reviewer 2:

The reviewer noted that resources seem appropriate.

\section{Reviewer 3:}

The reviewer stated that resources are sufficient for now. 


\section{Low-Cost Manufacturing of Advanced Silicon-Based Anode Materials: Aaron Feaver (Group14) - es268}

\section{Presenter}

Aaron Feaver, Group14

\section{Reviewer Sample Size}

A total of seven reviewers evaluated this project.

\section{Question 1: Approach to performing the work-the degree to which technical barriers are addressed, the project is well-designed, feasible, and integrated with other efforts.}

\section{Reviewer 1 :}

The reviewer stated that the objective is to develop a high-capacity, low-cost and long-life silicon-carbon (Si-C) composite anode material for $\mathrm{Li}$-ion batteries, based on EnerG2's carbon technology works. Specific targets are a specific capacity of $1,000 \mathrm{mAh} / \mathrm{g}$ (or 1,000 cycles) and with lower cost than the current graphite based on dollars-per-Ah. However, there is no quantification on the cost. The reviewer suggested that a better cost target be based on dollars-per-Wh rather than dollars-per-Ah (because Si lowers the cell voltage) that also takes into account initial irreversible capacity. The reviewer pointed out that the strategy is based on the EnerG2 expertise in carbon materials manufacturing to create an ideal Sisupport matrix material, using feedstock materials from suitable suppliers. Upon a successful demonstration in full cells, the subsequent efforts will focus on manufacturing a pilot scale plant for the $\mathrm{Si}-\mathrm{C}$ material production. The reviewer concluded that the task plan and milestone look reasonable, with a cost target of \$0.034/Ah for the anode alone, and that, overall, the project is well designed and integrated with other efforts.

\section{Reviewer 2:}

The reviewer said that, although not disclosed, the approach taken by the PI to make $\mathrm{Si}-\mathrm{C}$ composite seems to be innovative. The trade-off correlation between capacity and volume expansion follows a much less sloppy trend.

\section{Reviewer 3:}

The reviewer commented that little information regarding the approach taken in this project was shared, except to cite using a carbon developed by the EnerG2 parent company. The reviewer added that more technical information regarding the approach is needed to judge its chance of reaching the ambitious cost and energy barriers targeted. 


\section{Reviewer 4:}

The reviewer expressed that there is not enough baseline data on the material to judge if investing in a scaleup process is a good use of DOE funds. Cycle data out to 300-500 cycles would be more consistent with other published Si anode technologies, and would give more confidence in the scale-up process. The reviewer pointed out that Slide nine, Cycle Life Comparison, is not very meaningful, and that it is an apples-to-oranges comparison. The reviewer noted that because the two materials are cycled at different capacities, it is hard to say that one is better than the other. For example, at least for the first graph, the pure Si is cycling at a higher capacity than the $\mathrm{Si}-\mathrm{C}$ material at the end of cycling. The reviewer said that, if the goal was 30 cycles, the reviewer would take the pure Si material. Likewise, if pure Si was cycled to the same capacity as the Si-C material, it may show better cycle life and it would then be the preferred material again. Regarding Slide 10, the reviewer suggested that the figure be plotted as a function of volumetric rather than gravimetric energy density. The reviewer explained that it would be easy to achieve a result like this by just leaving a significant amount of open space in the material, but that, unless there is also an improvement in gravimetric energy density, the strategy is meaningless.

\section{Reviewer 5:}

The reviewer pointed out that the detailed approach is unclear and not fully revealed. Based on the information provided, it seems that carbon is being mixed with $\mathrm{Si}$, which is not unique.

\section{Reviewer 6:}

The reviewer observed that Si-C composite electrodes have apparently been explored extensively. The reviewer expressed that the approach does not show any novelty and how to address the critical issues related with $\mathrm{Si}$, such as mechanical fracture and low cycle efficiency.

\section{Reviewer 7:}

The reviewer recommended Slide 4 be modified from " $3-4 x$ increase in energy density" to " $30-40 \%$ increase." The reviewer commented that utilization of EnerG2 expertise in carbon materials manufacturing to create an ideal $\mathrm{Si}$ support matrix material has good basis.

\section{Question 2: Technical accomplishments and progress toward overall project and DOE goals-the degree to which progress has been made, measured against performance indicators and demonstrated progress towards DOE goals.}

\section{Reviewer 1:}

The reviewer praised that good progress has been made in the first quarter towards developing the Si-C composite material based on EnerG2's carbon expertise. Suppliers have been identified for feedstock for Si-C composites, matching the targeted anode costs (less than $\$ 0.034 / \mathrm{Ah}$ ), and preliminary studies have been carried out on the Si-C composite materials synthesized in-house. The reviewer observed that the materials show good cycle life in half cells, with high coulombic efficiencies. However, the irreversible capacity in the first cycle is missing, which has a bearing on the overall cell energy densities. The reviewer pointed out that there is no information on the anode loading, and that it is more challenging to achieve good cycle life in a thick electrode and in full cells, where the Li loss from the irreversible capacity and continued solid electrolyte interface (SEI) build up is crucial. The reviewer added that mechanistic studies have been also made, which suggest reduced volume expansion with the Si-C materials. The reviewer commented that it would be useful to have a preliminary cost/performance model for the Si-C materials that takes into account the irreversible capacity and loss of reversible $\mathrm{Li}$, lower operating voltage versus graphite, etc. in order to revise the project targets. Overall, the reviewer concluded, progress is good and consistent with the project objectives and DOE goals.

\section{Reviewer 2:}

The reviewer expressed that, given the short period, encouraging results are shown.

\section{Reviewer 3:}

The reviewer said that the initial work looks very promising. The reviewer pointed out that low-expansion composites are a definite plus, and added that the PI understands the relationship between this and high coulombic efficiency/cycle life. 


\section{Reviewer 4:}

The reviewer commented that, because the program is just in its beginning stages, there is not much data to judge the merit of the approach. However, the reviewer would have liked to see more preliminary cycle data, adding that other Si-C anode makers are showing data out to 500-800 cycles with similar capacities. The reviewer pointed out that the $2.0 \mathrm{~V}$ cutoff shown in Slide 13 is a very low voltage cutoff, and questioned what the reason was for choosing such a harsh condition.

\section{Reviewer 5:}

The reviewer observed that the two achievements claimed were low expansion rate and stable SEI layer. However, the presentation of percentage expansion did not fully reveal the actual expansion in comparison with benchmarks. The reviewer added that there is no evidence showing the SEI layer is stable.

\section{Reviewer 6:}

The reviewer stated that the technical comparison against pure Si in Slide nine is not relevant; pure material is known to behave extremely poorly, regardless of supplier source, and developed material is only shown for up to 80 cycles, which is a very short period. Slides 11 and 12, the reviewer continued, cite improvements made to coulombic efficiency without any description of what was changed or the test conditions used to demonstrate this improvement. Improved coulombic efficiency is typically cited as evidence of improved capacity stability. However, the reviewer explained, this trend is not clearly shown in the dataset provided, and would only be applicable where the mechanism was dominated by irreversible capacity loss.

\section{Reviewer 7:}

The reviewer noted that the presentation included some preliminary electrochemical performance. However, the reviewer said, baseline performance is poor.

\section{Question 3: Collaboration and coordination with other institutions.}

\section{Reviewer 1:}

The reviewer observed that there is an ongoing collaboration with a university partner, University of Washington, and a DOE laboratory, Pacific Northwest National Laboratory (PNNL).

\section{Reviewer 2:}

The reviewer expressed that collaboration partners seem to have the necessary tools to help on the project.

\section{Reviewer 3:}

The reviewer stated that there is a collaboration with University of Washington and PNNL.

\section{Reviewer 4:}

The reviewer described the team as strong, and pointed out that the collaboration might start in the near future because the project just began this year.

\section{Reviewer 5:}

The reviewer recommended getting a cell manufacturer to collaborate and verify full cell results.

\section{Reviewer 6:}

The reviewer commented that the existing collaborations seem appropriate, and that the project should consider adding a partner with extensive electrode/cell making/testing experience. If the prime or the partners already have this skill, the reviewer suggested more technical data be provided at future reviews.

\section{Reviewer 7:}

The reviewer noted that collaboration is close.

Question 4: Proposed future research-the degree to which the project has effectively planned its future work in a logical manner by incorporating appropriate decision points, considering barriers to the realization of the proposed technology and, when sensible, mitigating risk by providing alternate development pathways. 


\section{Reviewer 1:}

The reviewer stated that the proposed work is directed at demonstrating the benefits (cost and performance) of Si-C composite anode with EnerG2's carbon. Specific relevant activities being planned are: developing Si-C commensurate with the technical and cost targets and demonstrating in full cells; optimizing the manufacturing process; and setting up a pilot manufacturing plant for the material. The reviewer expressed that these studies are well-aligned with targeted objectives and milestones.

\section{Reviewer 2:}

The reviewer noted that future work seems appropriate.

\section{Reviewer 3:}

The reviewer commented that the proposed future research looks reasonable at this stage.

\section{Reviewer 4:}

The reviewer said that the future plan is realistically reasonable.

\section{Reviewer 5:}

The reviewer stated that the proposed future research seems to be feasible.

\section{Reviewer 6:}

The reviewer expressed that the goals seem to be more about materials scale-up than improving the cycle life performance of the material, and that there is no information given on the approach to improving performance. The reviewer pointed out a big jump between 500 cycles in Year 1 and 1,000 cycles in Year 2 with no details on how this will be accomplished. The reviewer added that cell level goals are also missing, and without those investing in process scale-up is not reasonable.

\section{Reviewer 7:}

The reviewer observed that the proposed future research was very brief and hard to evaluate.

\section{Question 5: Does this project support the overall DOE objectives of petroleum displacement? Why or why not?}

\section{Reviewer 1:}

The reviewer commented that low specific energies and high costs of $\mathrm{Li}$-ion batteries are serious impediments to their widespread adoption in vehicles, and that high specific-capacity materials, especially with low-cost synthesis, are desired to improve the specific energy and energy density of Li-ion batteries to make the EV batteries more acceptable. The reviewer stated that Si-based anode materials are promising to provide three times the improvement in specific capacity compared to graphitic materials, but are hampered by poor cycle life due to considerable volume expansion. One approach to mitigate this, the reviewer pointed out, is to form composite materials with carbons, which is being pursued here.

\section{Reviewer 1:}

The reviewer agreed that increasing the energy density and reducing the cost of anode materials supports the DOE objectives of petroleum displacement.

\section{Reviewer 2:}

The reviewer said that the project supports the overall DOE objectives given that $\mathrm{Si}-\mathrm{C}$ composites are a potential candidate to improving the energy density of next-generation Li-ion batteries.

\section{Reviewer 3:}

The reviewer called the project relevant.

\section{Reviewer 4:}

The reviewer expressed that the specific approach of this project is not clearly delineated from the broad class of $\mathrm{Si}$ anode work, and therefore it is difficult to judge their probability of contributing to the mission of DOE to reduce petroleum consumption. 


\section{Question 6: Resources: How sufficient are the resources for the project to achieve the} stated milestones in a timely fashion?

Reviewer 1:

The reviewer commented that the resources seem appropriate.

Reviewer 2:

The reviewer said that the funding is sufficient, but that more emphasis should be placed on improving the electrochemical characteristics (cycle life) of the material before scale-up.

Reviewer 3:

The reviewer noted that the resources are sufficient for now.

Reviewer 4:

The reviewer expected a lot more progress with the funding level.

Reviewer 5:

The reviewer stated that the resources seem to be slightly excessive for the scope and novelty of the project. 


\section{An Integrated Flame Spray \\ Process for Low-Cost \\ Production of Battery \\ Materials: Chad Xing \\ (University of Missouri) \\ - es269}

\section{Presenter}

Chad Xing, University of Missouri

\section{Reviewer Sample Size}

A total of six reviewers evaluated this project.

\section{Question 1: Approach to performing the work-the degree to which technical barriers are addressed, the project is well-designed, feasible, and integrated with other efforts.}

\section{Reviewer 1:}

The reviewer pointed out that the objective here is to develop an integrated manufacturing technology for producing battery materials, based on a water-based flame spray process. Cathode precursors (metal acetates) are dissolved in deep eutectic solvents (e.g., glycerol) and the metal oxides are deposited by a flame spray process and converted into battery material powders in a single process. Both cathode and anode materials will be produced in this manner and tested in laboratory cells similar to the standard materials. Upon verifying the performance of these materials, a pilot plant production line will be developed. This process is expected to result in a $25 \%$ reduction in the cost of the material, which needs to be substantiated with a cost model. However, the reviewer explained, the overall benefit in the battery costs will be lower and needs to be properly estimated. The reviewer concluded that the project is well designed and integrated with other efforts.

\section{Reviewer 2:}

The reviewer noted that the goal of reducing the cost of cathode material production is very relevant, adding that a process that removes the intensive calcining step in traditional material production would be good for the industry. The reviewer suggested avoiding using the term green manufacturing process in a scientific presentation because the term is vague and largely without meaning. The reviewer recommended elaborating on what is meant by green. The reviewer expressed lack of clarity on the coating and processing steps of the process shown in Slide 5. The reviewer questioned if those steps are included in the project; if that is not the case, the reviewer recommended they be left off the process flow.

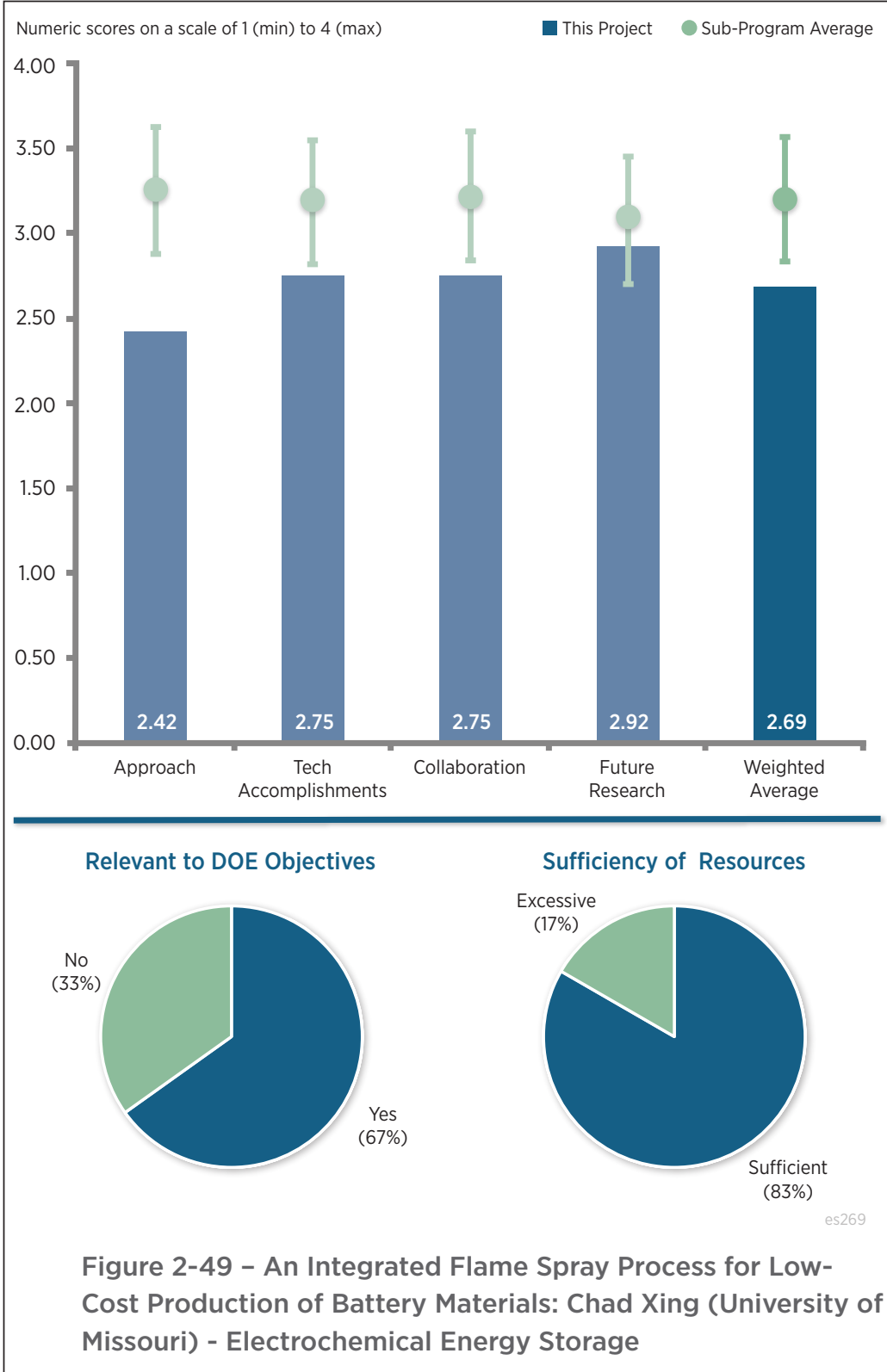




\section{Reviewer 3:}

The reviewer stated that the green manufacturing objective is clear based on the approach described. However, the reviewer pointed out that other objectives of $25 \%$ cost reduction and a $250 \mathrm{Wh} / \mathrm{kg}$ target are less clearly related. The reviewer recommended a quantitative cost comparison be shared early on in this project. Additionally, the $250 \mathrm{Wh} / \mathrm{kg}$ target at the cell level is not so relevant. The reviewer added that given the unclear background in cell making of the prime and the technical details shared, targets in terms of cathode material performance would make more sense.

\section{Reviewer 4:}

The reviewer indicated that although the reviewer is in favor of additional studies into cathode production via flame pyrolysis, the reviewer is not a great fan of this project. The reviewer expressed that the PI seems to have wound the project around the use of green deep eutectic solvents (DESs) perhaps in order to get funded. The reviewer's concern is that the PI has no intention at present to compare their future findings to what could be produced using water with the same raw materials. The reviewer added that it may well be that this approach produces a better final material, but we will never know. The reviewer also expressed concerns about the fact that the PI intends to use this material in cells directly from the reactor.

\section{Reviewer 5:}

The reviewer said that while the team claims the process can significantly reduce the cost, there is no cost analysis to show how much the cost reduction might be.

\section{Reviewer 6:}

The reviewer commented that the proposed approach of flame spray does not seem to support the objective of lowcost and green process. The reviewer added that, considering the large volume of precursors and the low efficiency of production as compared with solid state or other solution techniques, the energy consumption versus conversion efficiency reflect a high production cost. Additionally, the production parameters have to be explored in order for the materials produced to be well-controlled in terms of both morphology and performances. The reviewer expressed that this project should not be in the Applied Battery Research (ABR) program, and that perhaps moving to Battery Materials Research (BMR) is a better option.

\section{Question 2: Technical accomplishments and progress toward overall project and DOE goals-the degree to which progress has been made, measured against performance indicators and demonstrated progress towards DOE goals.}

\section{Reviewer 1:}

The reviewer said that, being a new project, fairly good progress has been made in the first quarter with identification of DESs and precursor formulations. The initial design as well as preliminary testing of the flame spray process has been completed, and NMC cathode powders were produced. The distribution of the transition metals $(\mathrm{Co}, \mathrm{Ni}$, and $\mathrm{Mn}$ ) is uniform within these powders. The reviewer pointed out that even though this process seems to simplify the synthesis of lithiated metal oxides, some details are missing. For example, it is not clear how well $\mathrm{Li}$ is distributed in the cathode powders. The reviewer questioned what the tap density of these powders would be, whether it will be high enough as conventional solid-state methods, especially with the acetate precursors. The reviewer also asked how this method is adopted for graphitic anode materials. The reviewer added that, now that the preliminary process has been developed, it is important to verify if the cost benefits will be at the expected level, i.e., 2\% reduction. Overall, the reviewer concluded, the progress is good and consistent with the DOE goals.

\section{Reviewer 2:}

The reviewer noted that much progress has been made within three months, and that the process apparently needs further optimization.

\section{Reviewer 3:}

The reviewer commented that given the short period since the project was awarded, the PI already delivers a lot of results. However, it is still too early to tell whether the materials produced is indeed superior to those produced via state-of-the-art approaches. 


\section{Reviewer 4:}

The reviewer pointed out that Slide 12 lists some particle diameters of NMC materials, but that more material properties (e.g., D10, D50, D99, tap density, BET, impurities, X-ray diffraction, etc.) should be shown to determine the quality of cathode material made.

\section{Reviewer 5:}

The reviewer observed that the presence of $\mathrm{Ni}, \mathrm{Mn}$, and Co does not necessarily indicate that the material is a viable cathode material, and that the presence of $\mathrm{Li}$ is still unknown, as is the structure of the material. The reviewer added that because powders have been produced by the process, there should be some coin half-cell data to show they are actually capable of functioning as cathode materials, and the tap density should have been determined.

\section{Reviewer 6:}

The reviewer noted that this is just the first quarter and not a lot has actually been accomplished. The reviewer claimed that the PI mentioned the tap density of the product was $\sim 1 \mathrm{~g} / \mathrm{cc}$, and that this is very low given that $\mathrm{NMC}$ is typically $2-2.8 \mathrm{~g} / \mathrm{cc}$, adding that this is a challenge regarding energy density. The reviewer suggested that before doing too much work with half or full cells, the team should do a few post-calcine treatments and report how the EC performance changes or does not change. The reviewer said that the PI is already seeing a variety of morphology in its early days, but should closely monitor for continuous improvement.

\section{Question 3: Collaboration and coordination with other institutions.}

\section{Reviewer 1:}

The reviewer noted that a strong contribution from EaglePicher Technologies as a battery maker/user seems very important to this project, and it is hoped that their contribution is shown as the project progresses.

\section{Reviewer 2:}

The reviewer observed that there will be a formal collaboration with EaglePicher Technologies and also possibly with ANL to assess these electrode powders in comparison with conventional materials.

\section{Reviewer 3:}

The reviewer said that collaboration with EaglePicher Technologies is a good step.

\section{Reviewer 4:}

The reviewer commented that it seems the collaboration has not started yet because the project has just begun. The reviewer suggested the PI talk to the experts on cathode materials so the barriers can be addressed appropriately.

\section{Reviewer 5:}

The reviewer expressed that there is very little evidence of collaboration with partners. The reviewer suggested finding a partner with experience producing and testing coin cells, and added that a partner with knowledge of using the process in an industrial setting is important.

\section{Reviewer 6:}

The reviewer stated that collaboration is unclear at the current stage.

Question 4: Proposed future research-the degree to which the project has effectively planned its future work in a logical manner by incorporating appropriate decision points, considering barriers to the realization of the proposed technology and, when sensible, mitigating risk by providing alternate development pathways.

\section{Reviewer 1:}

The reviewer observed that the proposed future research is to continue the optimization of the DESs precursors for making stoichiometric compositions of the NMC powders, to improve the nozzle design and operational conditions for spraying the DES, to optimize the flame reactor design, to understand the factors affecting the powder morphologies, and to begin the assessment of these powders in Li-ion test cells in comparison with the baseline materials. The reviewer said these studies are well aligned with the project objectives and with the DOE program goals of lowering battery costs. 


\section{Reviewer 2:}

The reviewer stated that future work seems appropriate and should also be compared against an existing commercially-available material for reference.

\section{Reviewer 3:}

The reviewer noted that proposed future efforts are reasonable.

\section{Reviewer 4:}

The reviewer was happy to see cell testing included in the future work, but that cost modeling is missing and should absolutely be included as part of the future work.

\section{Reviewer 5:}

The reviewer suggested the PI compare to products from aqueous system.

\section{Reviewer 6:}

The reviewer pointed out that the team did not emphasize the chemistry.

\section{Question 5: Does this project support the overall DOE objectives of petroleum displacement? Why or why not?}

\section{Reviewer 1:}

The reviewer commented that low specific energies and high costs of Li-ion batteries are serious impediments to their widespread adoption in vehicles. The high costs are partly attributed to the complex and multi-step synthesis of cathode materials. The reviewer stated that new low-cost and simpler methods are desired that would lead to reduced costs for the batteries, which is being addressed here.

\section{Reviewer 2:}

The reviewer noted that high cost of the cathode materials is a large barrier for the applications in electrical vehicles. The PI is targeting to significantly reduce the cost by developing a novel and green approach. The reviewer expressed that the project fits overall DOE objectives quite well.

\section{Reviewer 3:}

The reviewer commented that if it turns out there is a viable cathode material produced by the process, then yes, the project does support DOE goals.

\section{Reviewer 4:}

The reviewer indicated that it is unclear how this manufacturing approach improves specific energy or reduces cost, and thus its contribution to the DOE mission to reduce petroleum consumption is difficult to determine.

\section{Reviewer 5:}

The reviewer stated that it is not yet a proven technology.

\section{Question 6: Resources: How sufficient are the resources for the project to achieve the stated milestones in a timely fashion?}

\section{Reviewer 1:}

The reviewer stated that project resources seem appropriate.

\section{Reviewer 2:}

The reviewer noted that project resources are sufficient for now.

\section{Reviewer 3:}

The reviewer indicated that the resources seem to be slightly excessive for a project with this scope, especially for an academic institution. 


\section{New Advanced Stable Electrolytes for High-Voltage Electrochemical Energy Storage: Peng Du (Silatronix) - es271}

\section{Presenter}

Peng Du, Silatronix

\section{Reviewer Sample Size}

A total of five reviewers evaluated this project.

\section{Question 1: Approach to performing the work-the degree to which technical barriers are addressed, the project is well-designed, feasible, and integrated with other efforts.}

\section{Reviewer 1:}

The reviewer stated that the objective of this project is to develop an electrolyte system stable at high voltage (greater than $5 \mathrm{~V}$ ) to enable the development of high energy density Li-ion batteries required by the automotive industry. Such electrolytes will allow the use of recently-emerging high specific energy cathode materials. Specific targets for these electrolytes are good oxidative stability up to $6 \mathrm{~V}$ with very low oxidative rates (less than $0.02 \mathrm{~mA} / \mathrm{cm}^{2}$ ). These electrolytes will be evaluated against the $5 \mathrm{~V}$ lithium nickel manganese oxide (LNMO) cathode anticipating an improved performance versus the carbonate-based electrolytes, with a cycle life greater than 300 at $50^{\circ} \mathrm{C}$ and a capacity retention greater than $80 \%$. These new electrolytes are based on organosilicon (OS) solvents with selective functional and bulky groups for achieving the desired electrochemical stability. Similar OS solvents systems were studied earlier in the literature with some marginal success. Also, these solvents, when added in small proportions, provide improved thermal stability for $\mathrm{LiPF}_{6}$ salt. The reviewer concluded that the approach looks logical, addressing the key technical barriers of electrolyte stability, and the project is well integrated with the other cathode developmental efforts.

\section{Reviewer 2 :}

The reviewer noted that the PI has developed a series of fluorinated OS compounds that are stable at high voltages. This provides an opportunity to investigate whether they are suitable for Li-ion electrolytes for high voltage cathodes.

\section{Reviewer 3:}

The reviewer commented that targeting the polysiloxane-based chemistry to yield a high voltage stability material

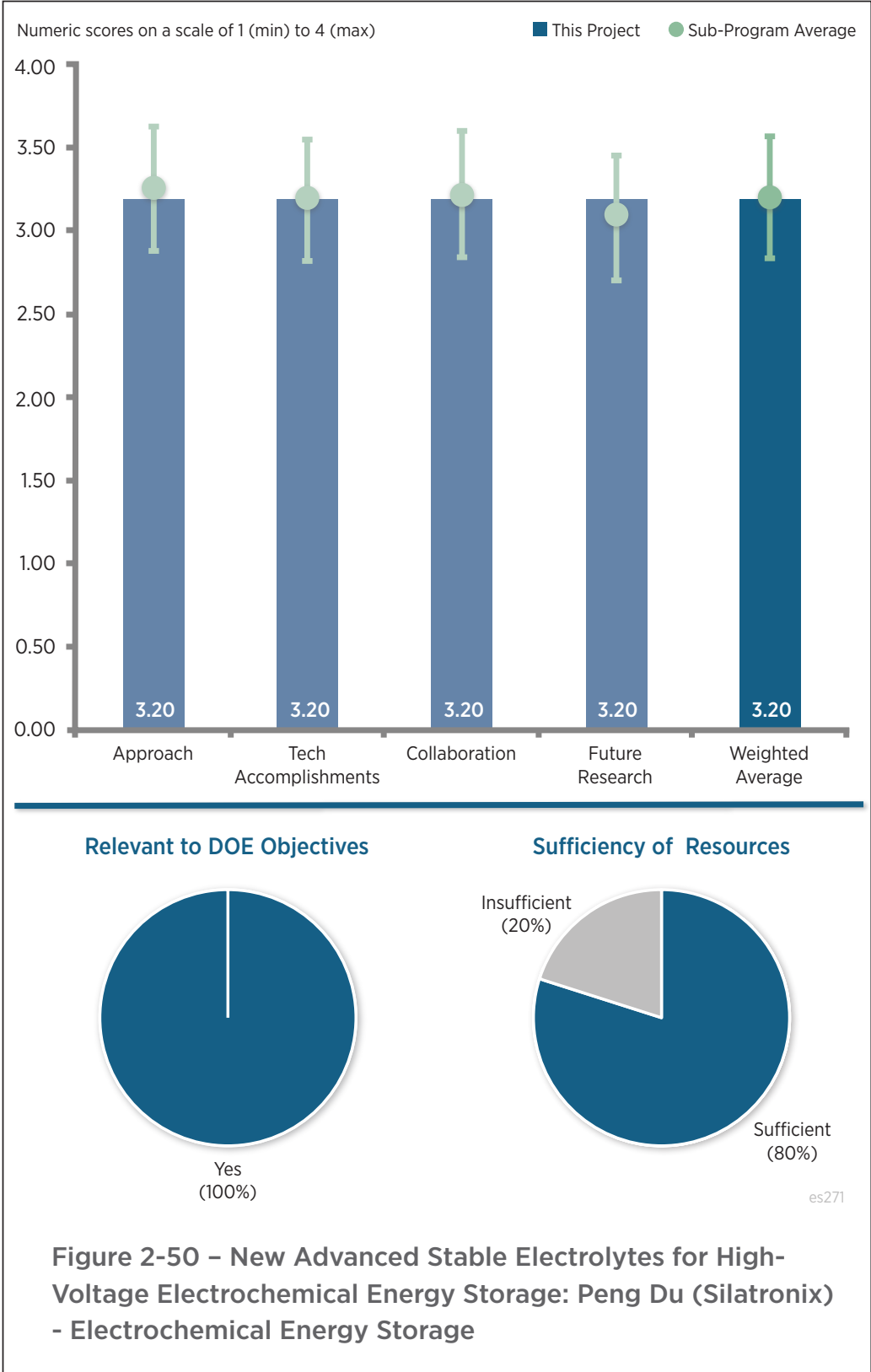


is appropriate, but that very little is described regarding the specific technical approach (e.g., polymer engineering for high voltage).

\section{Reviewer 4:}

The reviewer pointed out that OS-based electrolytes have proven to be safe for elevated temperatures. Making these electrolytes sustain high voltages is a good approach for developing high energy Li-ion electrolytes. The reviewer added that more attention should be paid to low-temperature performance.

\section{Reviewer 5:}

The reviewer expressed that the approach addressed the issue related with the decomposition of $\mathrm{LiPF}_{6}$ at high voltages. However, it does not cover the oxidation of electrolyte solvent.

\section{Question 2: Technical accomplishments and progress toward overall project and DOE goals-the degree to which progress has been made, measured against performance indicators and demonstrated progress towards DOE goals.}

\section{Reviewer 1:}

The reviewer praised the team for making impressive progress in such a short period after the project started last October.

\section{Reviewer 2:}

The reviewer stated that reasonably good progress has been made in the last six months in developing the new $5 \mathrm{~V}$ electrolytes. Four OS solvents have been successfully synthesized and characterized, and some of them show less oxidative floating current at high voltage of 5-6 V, even at high temperatures, compared to the baseline electrolytes. In parallel, several new additives with bifunctional groups have been identified for protecting the anode/cathode surface at these high (and low corresponding to graphite) voltages. Another $5 \mathrm{~V}$ cathode, lithium cobalt phosphate (LCP), has been shown to perform well with improved discharge profile and cycling stability in the baseline electrolyte containing one of these additives. Also, against the $5 \mathrm{~V}$ LMNO cathode, some OS-rich formulations show lower oxidative current and expectedly better cycle life. The reviewer commented that these studies look encouraging. However, it should be noted that the additive impacts both anode and cathode in different ways. Some of the additives (e.g., lithium bis(oxalate)borate [LiBOB]) seem to be protecting the cathode, but add to the SEI build up the in anode. The reviewer added that it is therefore important to select and optimize the solvents/additives in full cells rather than half cells. The reviewer concluded that, overall, progress is consistent with the objectives of the project and DOE goals.

\section{Reviewer 3:}

The reviewer stated that the technical metric of $20 \mu \mathrm{A} / \mathrm{cm}^{2}$ for electrochemical stability introduced in Slide three is too high and should be more like 5 or $10 \mu \mathrm{A} / \mathrm{cm}^{2}$ to align better with requirements and published literature. The reviewer also pointed out that the volume growth over time plot shown in Slide six is not so informative without knowing the test details (e.g., original volume size, pressure of constraint). Additionally, four different OS variants are introduced in Slide nine without any explanation of their structure, design goals/purpose, or information describing why they were made or tested. On Slides 10 and 11, the reviewer explained, OS3 and OS3a decompose before the reference, and OS3b/c show comparable current flow in the 5.0-5.7 V range. The reviewer asked whether there are purity issues with these materials or if they are inherently unstable. The reviewer stated that the rate of cyclic voltammetry (CV) in millivolts-per-second for these and all experiments should be shared, and asked if this behavior is sensitive to the scan rate. The reviewer pointed out that Slide 18 shows LCP with a low CE, and asked if this is a result of electrolyte decomposition or if the material unstable. The reviewer also asked what the voltage of cycling is. The reviewer said that Slides 19-23 should describe the cell used (e.g., type, dimensions, electrode features, design capacities), and the voltage stand plots on Slides 19 and 22-23 should be normalized for surface area $\left(\mu \mathrm{A} / \mathrm{cm}^{2}\right)$ instead of current $(\mathrm{mA})$. Description of the design of experiments for 21-23 and the future ARL species is necessary as the addition of additives is introduced in a haphazard, non-rigorous way. The reviewer also pointed out that the carbonate reference is superior to OS3 at 20\% in the voltage stand tests on Slides 19 and 21 , and questioned if this result is reproducible and whether any of the other OS3 variants (i.e., a, b, c) were tested in a similar way. 


\section{Reviewer 4:}

The reviewer commented that multiple solvents were synthesized and tested, and that decent performance was demonstrated against benchmark electrolyte.

\section{Reviewer 5:}

The reviewer observed good technical results in half cells, and added that work needs to be done in full cells as well. The reviewer suggested trying to improve conductivity.

\section{Question 3: Collaboration and coordination with other institutions.}

\section{Reviewer 1:}

The reviewer praised good and useful collaborations with ARL and ANL.

\section{Reviewer 2:}

The reviewer stated that the collaborators identified seem appropriate. The reviewer added that coordination between the parties should ensure that the effort is not divided between too many cathodes (LCP versus LNMO) and sources of additives (prime, ARL and ANL), and that complexity is managed.

\section{Reviewer 3:}

The reviewer said collaboration is good.

\section{Reviewer 4:}

The reviewer noted that the team is very strong in the field of electrolytes.

\section{Reviewer 5:}

The reviewer suggested the PI seek more collaborators in both chemical and battery companies.

Question 4: Proposed future research-the degree to which the project has effectively planned its future work in a logical manner by incorporating appropriate decision points, considering barriers to the realization of the proposed technology and, when sensible, mitigating risk by providing alternate development pathways.

\section{Reviewer 1:}

The reviewer observed that the proposed future research is to continue developing new OS solvents/additives based on modeling and experiments in cells with the $5 \mathrm{~V}$ cathodes. Successful electrolytes will be tested in full cells at different temperatures, which will be augmented by post-cycling analysis, with the eventual goal of demonstrating the performance in $300 \mathrm{mAh}$ pouch cell with the $5 \mathrm{~V}$ LNMO cathode. With the multiple options on solvents and additives, the reviewer said that the risk is adequately mitigated. The reviewer stated that these studies are well aligned with project goals, with appropriate decision points.

\section{Reviewer 2:}

The reviewer noted that future work seems appropriate.

\section{Reviewer 3:}

The reviewer commented that the proposed future research is solid, and that more attention should be paid to lowtemperature performance.

\section{Reviewer 4:}

The reviewer said that the planned future research is well defined. The reviewer recommended testing more in full cells and adding $-30^{\circ} \mathrm{C}$ rate testing.

\section{Reviewer 5:}

The reviewer suggested the future plan include how to stabilize the electrolyte solvent at high voltage as well.

Question 5: Does this project support the overall DOE objectives of petroleum displacement? Why or why not? 


\section{Reviewer 1:}

The reviewer stated that low specific energies and energy densities and high costs are serious impediments for Li-ion batteries to be widely adopted in vehicles. New materials, such as high specific energy cathode and anode materials, are required to improve the gravimetric and volumetric energy densities of Li-ion cells. The cathode specific energy can be improved by increasing its specific capacity and/or increasing its operating voltage. New cathode materials have emerged recently that can function at higher voltages 4.3-5.0 V. However, their applicability is being limited by the absence of suitable electrolytes that are stable at these high voltages. The reviewer concluded that new types of electrolytes stable at $5 \mathrm{~V}$ are, therefore, desired, which is being addressed by this project.

\section{Reviewer 2:}

The reviewer commented that, if successful, this project could contribute to DOE's mission of reducing petroleum consumption.

\section{Reviewer 3:}

The reviewer expressed that if these materials can deliver on high voltage expectations, the project will help to provide high energy density cells.

\section{Reviewer 4:}

The reviewer noted that the project closely supports the overall DOE objectives, adding that it is a straightforward approach to increasing the energy density by expanding the cell voltage.

\section{Question 6: Resources: How sufficient are the resources for the project to achieve the stated milestones in a timely fashion?}

\section{Reviewer 1:}

The reviewer observed that if deliverables are met, the PI may need additional support to lower production costs.

\section{Reviewer 2:}

The reviewer commented that the resources seem to be appropriate for the scope of the project.

\section{Reviewer 3:}

The reviewer stated that project resources seem appropriate. However, the reviewer pointed out inconsistencies in the funding values shown on Side 2 (i.e., Total: $\$ 1.665$ million, DOE: $\$ 897,000$, Contractor \$333,000). 
Pre-Lithiation of Battery Electrodes: Yi Cui (Stanford University) - es272

\section{Presenter}

Yi Cui, Stanford University

\section{Reviewer Sample Size}

A total of three reviewers evaluated this project

\section{Question 1: Approach to performing the work-the degree to which technical barriers are addressed, the project is well-designed, feasible, and integrated with other efforts.}

\section{Reviewer 1:}

The reviewer observed that multiple approaches attempted to achieve pre-lithiationpre-lithiation, and that all provided Li to the anode material to compensate for the ICL. Given the huge number of people developing pre-lithiationpre-lithiation technologies, the reviewer encouraged this team to tackle both the technical aspects and the economic ones. The reviewer added that there is no point in developing a pre-lithiation technique if it costs more than, say, just adding an extra cathode to the cell.

\section{Reviewer 2:}

The reviewer noted that the work utilized a systematic approach to the issue from many possible angles.

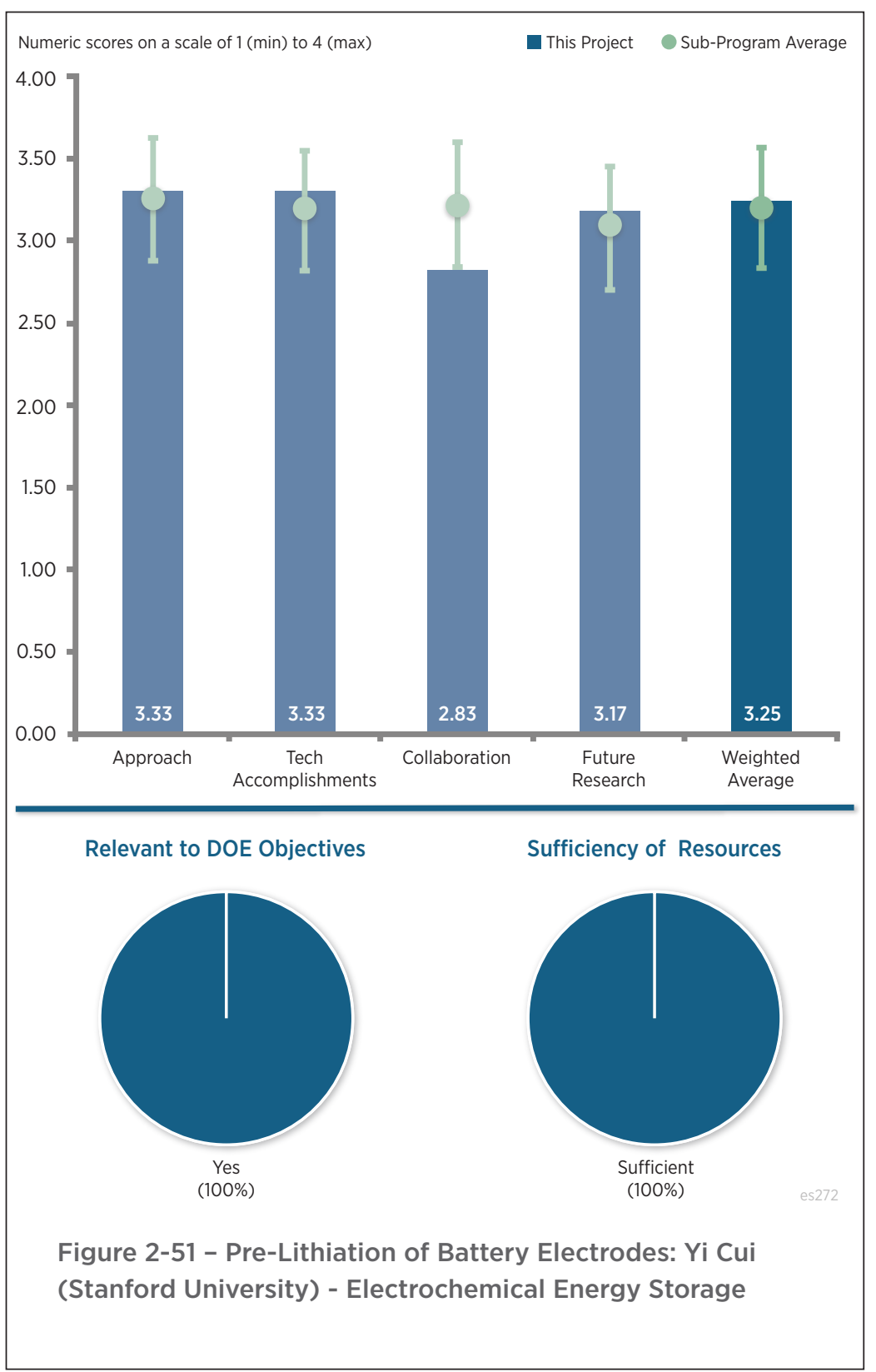

\section{Reviewer 3:}

The reviewer stated that this effort employs a sound approach to prelithiate Si. The PI pursues two main directions: increasing first-cycle coulombic efficiency via anode pre-lithiationpre-lithiation, and increasing first-cycle coulombic efficiency via cathode pre-lithiation.

\section{Question 2: Technical accomplishments and progress toward overall project and DOE goals-the degree to which progress has been made, measured against performance indicators and demonstrated progress towards DOE goals.}

\section{Reviewer 1:}

The reviewer observed good progress in the first year.

\section{Reviewer 2:}

The reviewer stated that good progress was made this last year, but that challenges still remain for the team. The reviewer pointed out that the results have been published in top peer-reviewed scientific journals and provided the following citation: Nature Energy 1, 15008(2016) JACS, 137, 8372(2015), Nano Letters16, 1497(2016). 


\section{Question 3: Collaboration and coordination with other institutions.}

\section{Reviewer 1:}

Given that Amprius, Inc. already has a funded project from DOE to develop pre-lithiation technology, the reviewer would like to see other developers and research institutions here as partners.

\section{Reviewer 2:}

The reviewer noted that the PI has established collaborations with SLAC and Amprius, Inc., and that it does not appear that the team reached out to other investigators outside their immediate circle.

\section{Question 4: Proposed future research-the degree to which the project has effectively planned its future work in a logical manner by incorporating appropriate decision points, considering barriers to the realization of the proposed technology and, when sensible, mitigating risk by providing alternate development pathways.}

\section{Reviewer 1:}

The reviewer noted that the proposed future research is good, but that the cost of these various approaches should be evaluated.

\section{Reviewer 2:}

The reviewer expressed for the team's consideration that exploring other materials with high pre-lithiation capacity may not be the best use of resources late in the project.

\section{Reviewer 3:}

The reviewer stated that future activities are appropriate. The team will improve the stability of pre-lithiation reagents in the slurry process by developing new solvent-binder combination, and plans to improve the stability of pre-lithiation reagents in the dry and ambient air condition by exploring different kinds of coatings and nanostructures.

\section{Question 5: Does this project support the overall DOE objectives of petroleum displacement? Why or why not?}

\section{Reviewer 1:}

The reviewer commented that the project is extremely relevant to many advanced anode and cathode cell designs, but pointed out that there are many people trying techniques like this.

\section{Reviewer 2:}

The reviewer noted that pre-lithiation of high capacity electrode materials such as $\mathrm{Si}$ is an important means to enabling those materials in high-energy batteries.

\section{Question 6: Resources: How sufficient are the resources for the project to achieve the stated milestones in a timely fashion?}

\section{Reviewer 1:}

The reviewer commented that there is good amount of work and progress for the money. 


\section{New Lamination and Doping Concepts for Enhanced Li-S Battery Performance: Prashant Kumta (University of Pittsburgh) - es279}

\section{Presenter}

Prashant Kumta, University of Pittsburgh

\section{Reviewer Sample Size}

A total of four reviewers evaluated this project.

\section{Question 1: Approach to performing the work-the degree to which technical barriers are addressed, the project is well-designed, feasible, and integrated with other efforts.}

\section{Reviewer 1:}

The reviewer said that the approach to solving the issues of polysulfide dissolution and poor conductivity in Li-S cells in intriguing. The team is attempting to coat the nano sulfur particles with Li conducting material, to dope the $\mathrm{S}$ to improve its conductivity, and using $\mathrm{LiC} / \mathrm{S}$ nanoparticles to prevent polysulfide dissolution.

\section{Reviewer 2:}

The reviewer commented systematic development of potential solutions to address the known hurdles that keep Li-S from achieving specific targets.

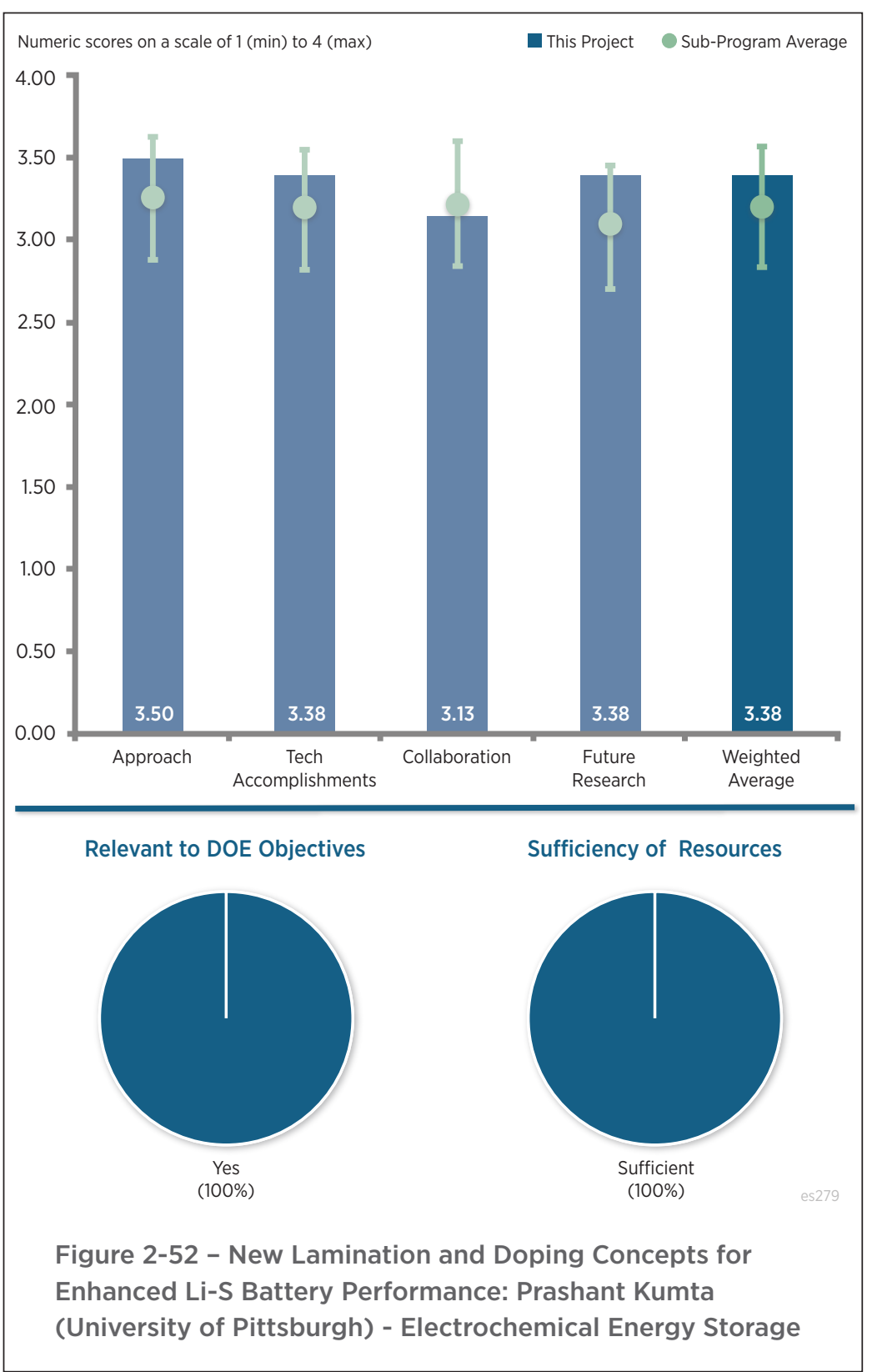

\section{Reviewer 3:}

The reviewer said that the investigation is logical and is expected to move the science of Li-S battery technology forward. The team recognizes the difficulty and are using several approaches to increase their chances of success. For example, the team will synthesis and characterize Li-ion conductor (LIC) coating materials to prevent polysulfide dissolution. The reviewer pointed out that the team will develop LIC coated sulfur nanoparticles and doping strategies to improve the electronic conductivity of sulfur and use framework materials to ensure polysulfide retention.

\section{Question 2: Technical accomplishments and progress toward overall project and DOE goals-the degree to which progress has been made, measured against performance indicators and demonstrated progress towards DOE goals.}

\section{Reviewer 1:}

The reviewer said that excellent progress has been achieved this past year. The team successfully identified an effective LIC membrane and demonstrated its ability to shield polysulfide from dissolving into the electrolyte. The reviewer pointed out that the PI had numerous publications and presentations. 


\section{Reviewer 2:}

The reviewer said excellent, all milestones to date have been met. However, cycling results using nano sulfur coated with LIC are modest at best, with approximately $25 \%$ fade in only 60 cycles. The reviewer found it laudable, however, that the team is making sulfur electrodes with loadings of 3-6 mAh/cm ${ }^{2}$, which is needed for EV cells. Flexible sulfur wires appear to do better, with minimal capacity lose but again over only 60 cycles.

\section{Reviewer 3:}

The reviewer commented that as to be expected, some promising solutions toward achieving the final goal have been demonstrated.

\section{Reviewer 4:}

The reviewer commented that the multilayer composite cathode certainly is a viable concept. It would be worth knowing how possible it is to scale this up.

\section{Question 3: Collaboration and coordination with other institutions.}

\section{Reviewer 1:}

The reviewer pointed out that collaborations exist with Drs. Maiti and Achary at the University of Pittsburgh and with Dr. Manivannan at the National Energy Technology Laboratory.

\section{Reviewer 2:}

The reviewer remarked good partners for a relatively small university effort. However, as this project is specifically focused on solving practical issues with sulfur cathodes (as opposed to a more fundamental study), involving companies trying to commercialize Li-S technology might be valuable.

\section{Question 4: Proposed future research-the degree to which the project has effectively planned its future work in a logical manner by incorporating appropriate decision points, considering barriers to the realization of the proposed technology and, when sensible, mitigating risk by providing alternate development pathways.}

\section{Reviewer 1:}

The reviewer commented that the doping of sulfur is particularly interesting.

\section{Reviewer 2:}

The reviewer said that the proposed efforts are excellent and are focused on the remaining technical challenges, such as high electronic conductivity, reduce weight and increase energy density.

\section{Reviewer 3:}

The reviewer observed some excellent ideas, e.g., first-principle alteration of sulfur to improve electronic conductivity and reaction kinetics; the reviewer also noted some ideas that might not be as innovative. The reviewer remarked good thoughts have been put into the continuation, maybe some more prioritization for the future work would increase the chance for achieving the best result.

\section{Question 5: Does this project support the overall DOE objectives of petroleum displacement? Why or why not?}

\section{Reviewer 1:}

The reviewer commented that the project could enable alternative lightweight low-cost batteries.

\section{Reviewer 2:}

The reviewer said that the project is highly relevant. The team seeks to identify new sulfur cathode materials displaying higher gravimetric and volumetric energy densities than present materials in conventional Li-ion batteries. The reviewer commented that if successful, it will result in a new battery that is capable of delivering better energy and power densities and will be more lightweight than current Li-ion battery packs. 
Reviewer 3:

The reviewer said extremely relevant, high-loading sulfur cathodes are an excellent way to achieve the DOE EV Everywhere energy and cost goals.

Question 6: Resources: How sufficient are the resources for the project to achieve the stated milestones in a timely fashion?

No comments were received in response to this question. 


\section{Novel Chemistry: Lithium Selenium and Selenium Sulfur Couple: Khalil Amine (Argonne National Laboratory) - es280}

\section{Presenter}

Khalil Amine, Argonne National Laboratory

\section{Reviewer Sample Size}

A total of four reviewers evaluated this project.

\section{Question 1: Approach to performing the work-the degree to which technical barriers are addressed, the project is well-designed, feasible, and integrated with other efforts.}

\section{Reviewer 1 :}

The reviewer praised a good approach to add selenium (Se) to $\mathrm{S}$ to improve conductivity, which is similar to what other groups are doing but with the added benefit that $\mathrm{Se}$ is electroactive. The reviewer questioned whether there is a clear path to manage polysulfide dissolution and migration to the Li-metal anode.

\section{Reviewer 2:}

The reviewer noted that the work is a methodical investigation of the applicability of the Se-based chemistry with helpful parallels to the somewhat similar Li-S system. The reviewer added that the approach to addressing the barriers is excellent.

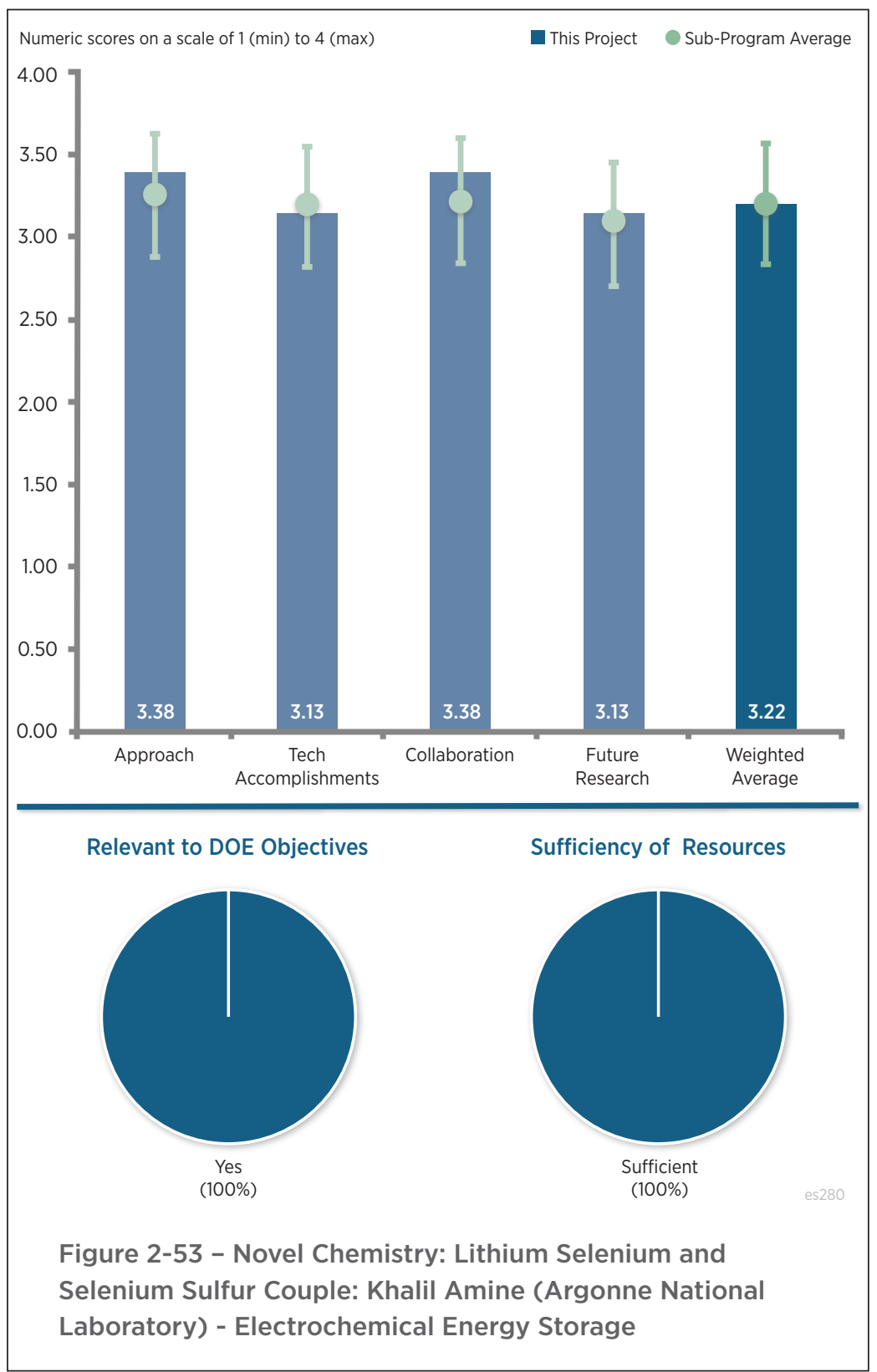

\section{Reviewer 3:}

The reviewer stated that the approach is unique. The team plans to solve the problems associated with the redox shuttle effect of dissolved Li polysulfides and higher loading of active material by nano-confining the SxSey in a nanoporous conductive matrix, and partially replacing $\mathrm{S}$ with Se.

\section{Question 2: Technical accomplishments and progress toward overall project and DOE goals-the degree to which progress has been made, measured against performance indicators and demonstrated progress towards DOE goals.}

\section{Reviewer 1:}

The reviewer observed that a goal of 500 cycles was set, but only 50 were shown. The reviewer suggested that battery cycling plots be shown until failure is observed. The reviewer added that, given the number of years devoted to the project and only having 50 cycles reported, reaching 500 cycles seems unrealistic. 


\section{Reviewer 2:}

The reviewer noted that good progress was achieved this past year. The electrical conductivity of S cathode was significantly improved by adding Se, and better compatibility between the Se cathode and the carbonate-based electrolyte was validated with no dissolution of polyselenide phases. The reviewer pointed out that an article describing the project's results was accepted in Nano Letters.

\section{Reviewer 3:}

The reviewer commented that nearly all of the results to date are on $\mathrm{Li} / \mathrm{Se}$ couples, which is not the focus of this project, adding that work on $\mathrm{S}-\mathrm{Se}$ alloys is just beginning. The reviewer pointed out that the project overview mentions cycling of S5Se2, but that results are not shown. The reviewer also mentioned that the one set of cycling data show about $300 \mathrm{mAh} / \mathrm{g}$, but at only $2.5 \mathrm{~V}$, meaning the energy is barely better than today's Li-ion cathodes. The reviewer concluded that the barriers to $\mathrm{S}$ utilization are well known, and that this approach seems to only address the $\mathrm{S}$ conductivity issue, which is not a deal breaker right now.

\section{Question 3: Collaboration and coordination with other institutions.}

\section{Reviewer 1:}

The reviewer said that good collaborations exist with Prof. Chunsheng Wang of University of Maryland, Dr. X. Chen and Dr. L Curtis of the Materials Science Division at ANL, and Dr. Yang Ren and Dr. Chengjun Sun of the Advanced Photon Source Office at ANL.

\section{Reviewer 2:}

The reviewer stated that it is unclear how the computational co-PIs have contributed to the work reported in this presentation.

Question 4: Proposed future research-the degree to which the project has effectively planned its future work in a logical manner by incorporating appropriate decision points, considering barriers to the realization of the proposed technology and, when sensible, mitigating risk by providing alternate development pathways.

\section{Reviewer 1:}

The reviewer said that the proposed future research is perfect and very strategic.

\section{Reviewer 2:}

The reviewer stated that the proposed work is good and should allow the team to advance the technology.

\section{Question 5: Does this project support the overall DOE objectives of petroleum displacement? Why or why not?}

\section{Reviewer 1:}

The reviewer commented that the project is relevant. The objective is to develop a novel SxSey cathode material for rechargeable Li batteries with high energy density and long life as well as low-cost and high safety. The reviewer stated that improved battery chemistries will enable the DOE to reach the PHEV and EV target goals.

\section{Reviewer 2:}

The reviewer pointed out that Li-S cells are clearly relevant as they offer the possibility of a much lower cost cell. It is not clear to this reviewer if this approach will address the multiple issues with Li-S.

\section{Reviewer 3:}

The reviewer noted that high capacity Li-Se/S batteries could be used in energy storage applications if performance is improved.

\section{Question 6: Resources: How sufficient are the resources for the project to achieve the stated milestones in a timely fashion?}

\section{Reviewer 1:}

The reviewer stated that $\$ 500,000$ per year is a good amount of money for relatively few new results. 


\section{Multi-Functional Cathode Additives for Li-S Battery Technology: Hong Gan (Brookhaven National Laboratory) - es281}

\section{Presenter}

Hong Gan, Brookhaven National Laboratory

\section{Reviewer Sample Size}

A total of three reviewers evaluated this project.

\section{Question 1: Approach to performing the work-the degree to which technical barriers are addressed, the project is well-designed, feasible, and integrated with other efforts.}

\section{Reviewer 1 :}

The reviewer commented that, in general, the approach is systematic and the topic important for addressing Li-S barriers. The reviewer observed that the baseline system may not be ideal because it comes with a number of problems unrelated to the area the project is addressing.

\section{Reviewer 2:}

The reviewer said that the approach is well thought out, and is contributing to overcoming the technology barriers. The team will focus on improving the cathode energy density, power capability, and cycling stability of a Li-S battery by introducing multifunctional cathode additives

(MFCA). The team will investigate transition metal sulfides due to their high electronic conductivity and chemical compatibility to the sulfur cell system. The reviewer added that this approach is sound.

\section{Reviewer 3:}

The reviewer stated that the approach to improve $\mathrm{S}$ utilization through improved conductivity using additives is reasonable. The reviewer added that there are several issues that this approach will likely not address, like polysulfide dissolution and reaction at the Li-metal anode, and the need for large volumes of electrolytes that reduce $\mathrm{Wh} / \mathrm{kg}$ values.

\section{Question 2: Technical accomplishments and progress toward overall project and DOE goals-the degree to which progress has been made, measured against performance indicators and demonstrated progress towards DOE goals.}

\section{Reviewer 1:}

The reviewer praised that excellent progress has been made and that several MFCAs were investigated. Transition 
metal sulfides (e.g., CuS, titanium disulfide [ $\left.\mathrm{TiS}_{2}\right], \mathrm{FeS}_{2}$, and $\mathrm{CoS}_{2}$ ) in S:MFCA hybrid cathodes were found to promote initial sulfur cell discharge power capability at $1 \mathrm{C}$ rate. The reviewer added that the team filed patents and published the project findings in the Journal of the Electrochemical Society.

\section{Reviewer 2:}

The reviewer noted that a good amount of work was reported, but that most of the results are not of interest due to the low energies reported. For example, $300-500 \mathrm{mAh} / \mathrm{g}$ at $2 \mathrm{~V}$ is barely better than $\mathrm{NMC}$, which cycles thousands of times.

\section{Reviewer 3:}

The reviewer stated that the project contributes to the understanding of benefits of transition metal sulfide additives, but could not identify a sulfide that adds significant value.

\section{Question 3: Collaboration and coordination with other institutions.}

\section{Reviewer 1:}

The reviewer stated that collaboration is very effective.

\section{Reviewer 2:}

The reviewer indicated that excellent collaborations exist. They include investigators from Brookhaven National Laboratory (BNL), Stony Brook University, and Columbia University.

\section{Reviewer 3:}

The reviewer observed that there is a relatively small amount of collaboration, which may be appropriate at this stage.

Question 4: Proposed future research-the degree to which the project has effectively planned its future work in a logical manner by incorporating appropriate decision points, considering barriers to the realization of the proposed technology and, when sensible, mitigating risk by providing alternate development pathways.

\section{Reviewer 1:}

The reviewer noted that the team is concentrating on the most promising additive, $\mathrm{TiS}_{2}$, adding that it will be interesting to see if there are any new approaches to mitigating/reducing polysulfide dissolution.

\section{Reviewer 2:}

The reviewer pointed out that required system-level attention was recognized to efficiently investigate the beneficial sulfur/TiS 2 interaction.

\section{Reviewer 3:}

The reviewer commented that future activities are focused on overcoming technical barriers, noting that the investigators understand the many challenges facing the development of a Li-S battery. The team will focus on the leading MFCA candidate $\left(\mathrm{S} / \mathrm{TiS}_{2}\right)$ and advance the fundamental understanding of the system. This will be followed by hybrid electrode processing where the team will optimize the energy density. After obtaining a highperformance electrode, cells will be built and undergo electrochemical performance testing. The reviewer agreed that the plan is an effective method to advance and then to assess the technology.

\section{Question 5: Does this project support the overall DOE objectives of petroleum displacement? Why or why not?}

\section{Reviewer 1:}

The reviewer agreed that Li-S cells hold promise for very low costs.

\section{Reviewer 2:}

The reviewer described the investigation as highly relevant. The Li-S battery system has gained significant interest due to its low material cost potential and its attractive volumetric and gravimetric energy density, which is theoretically higher than conventional Li-ion batteries. 
Question 6: Resources: How sufficient are the resources for the project to achieve the stated milestones in a timely fashion?

No comments were received in response to this question. 
Development of High-Energy Lithium-Sulfur Batteries: Jun Liu (Pacific Northwest National Laboratory) - es282

\section{Presenter}

Jun Liu, Pacific Northwest National Laboratory

\section{Reviewer Sample Size}

A total of four reviewers evaluated this project.

\section{Question 1: Approach to performing the work-the degree to which technical barriers are addressed, the project is well-designed, feasible, and integrated with other efforts.}

\section{Reviewer 1:}

The reviewer declared that the focus on thick sulfur cathodes is excellent, and that separating the Li-metal and its issues from the sulfur cathode is a very nice idea. The reviewer expressed that good progress on making 2-8 $\mathrm{mg} / \mathrm{cm}^{2}$ sulfur cathodes has been made. The reviewer also liked that the team plans to directly address the electrolyte amount by reducing the pore volume.

\section{Reviewer 2:}

The reviewer called the approach a very well prepared strategy that enables the focused investigation of multiple issues in Li-S batteries.

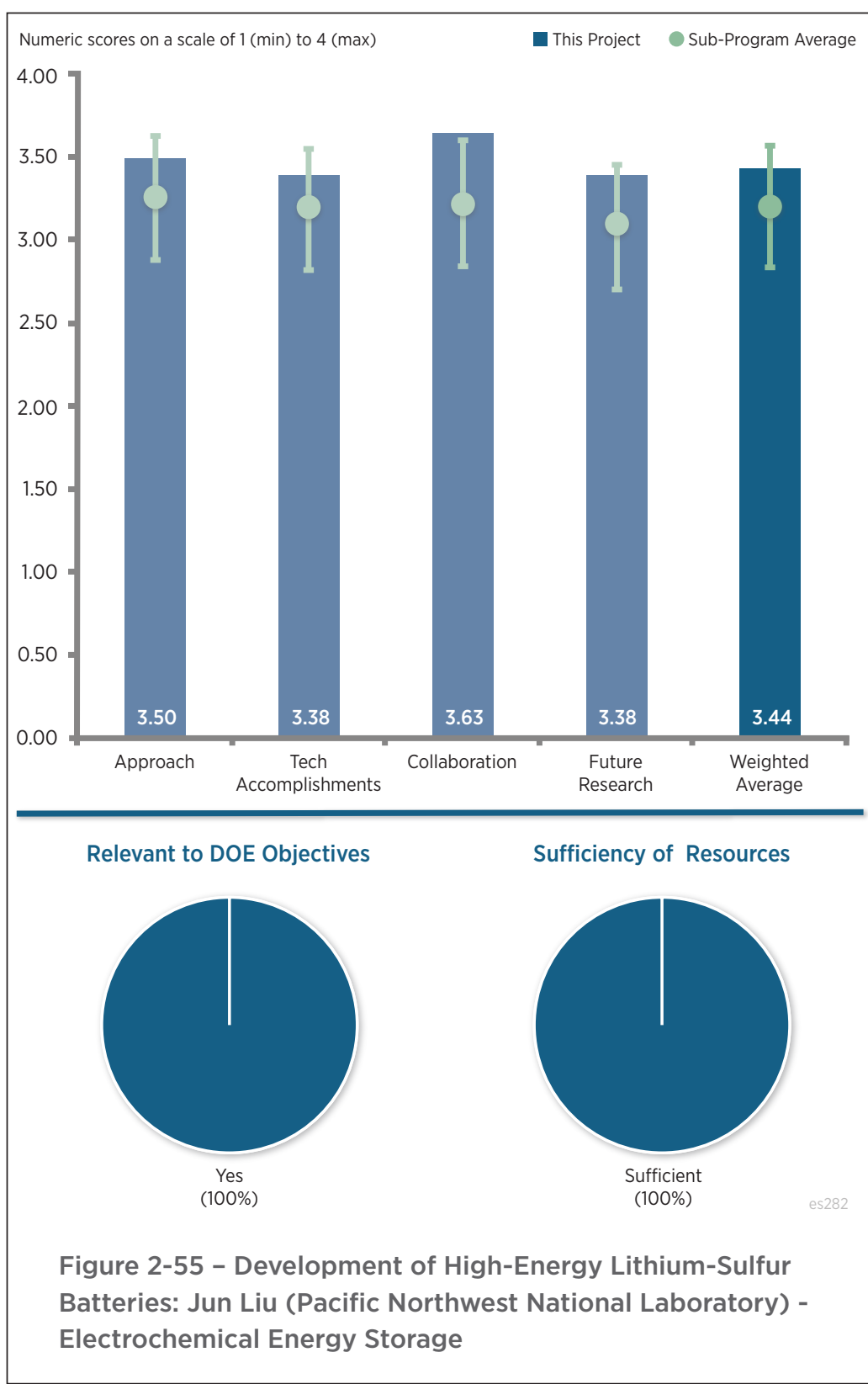

\section{Reviewer 3:}

The reviewer indicated that this work has more thorough characterization than many other Li-S projects, which is needed to understand mechanisms.

\section{Reviewer 4:}

The reviewer commented that the approach is sound. Previous studies indicated that the corrosion of Li anode is one of the key degradation mechanisms for Li-S batteries, and it is thus difficult to critically evaluate the performance of the cathode. The team is therefore decoupling the influences from the Li anode side by using a lithiated graphite anode in the Li bis(trifluoromethanesulfonyl)imide dioxolane (LiTFSI-DOL) electrolyte. The reviewer stated that this should allow the team to successfully investigate the intrinsic properties of cathode.

Question 2: Technical accomplishments and progress toward overall project and DOE goals - the degree to which progress has been made, measured against performance indicators and demonstrated progress towards DOE goals. 


\section{Reviewer 1:}

The reviewer indicated that very good progress has been made in building and cycling a graphite/sulfur $(\mathrm{Gr} / \mathrm{S})$ cell. The reviewer pointed out that the fade rate was still high at about $25 \%$ over 100 cycles, but that it is a very good first step. The reviewer added that it will be interesting to see if the team can identify causes of fade.

\section{Reviewer 2:}

The reviewer stated that the work lays a good foundation for further investigations. The reviewer added that findings confirming established knowledge are good but could go further. The reviewer also commented that thick electrode fabrication provides helpful data.

\section{Reviewer 3:}

The reviewer declared that good progress was made this year. High reversibility and rate capability were achieved on graphite electrodes with the LiTFSI/DOL electrolyte. The team also showed Gr/S cells displaying high capacity retention and coulombic efficiency with sulfur loadings greater than $2 \mathrm{mg} / \mathrm{cm}^{2}$.

\section{Reviewer 4:}

The reviewer expressed that it does not appear that the group has met the performance metric for March 2016. A goal of $80 \%$ capacity retention over 100 cycles was stated, and it can be seen from the plot of Capacity versus Cycle Number that more than $20 \%$ of the capacity was lost.

\section{Question 3: Collaboration and coordination with other institutions.}

\section{Reviewer 1:}

The reviewer said the team of national laboratories, industry, and universities is excellent.

\section{Reviewer 2:}

The reviewer indicated that many institutions are listed as collaborators, and their scope of work is described.

\section{Reviewer 3:}

The reviewer observed that the team is collaborating with ANL, BNL, General Motors, and University of Western Ontario, and that their roles were clearly defined.

\section{Question 4: Proposed future research-the degree to which the project has effectively planned its future work in a logical manner by incorporating appropriate decision points, considering barriers to the realization of the proposed technology and, when sensible, mitigating risk by providing alternate development pathways.}

\section{Reviewer 1:}

The reviewer expressed that the future work is well defined and consistent with the results to date and the project objectives.

\section{Reviewer 2:}

The reviewer stated that it is unclear how barriers that have led to capacity fade will be addressed. For example, the reviewer called the comment that reads "Address electrolyte amount and penetration issues in high loading sulfur electrode" unspecific, and therefore not possible to evaluate.

\section{Question 5: Does this project support the overall DOE objectives of petroleum displacement? Why or why not?}

\section{Reviewer 1:}

The reviewer particularly liked the concentration of thick S cathodes of 3-4 $\mathrm{mAh} / \mathrm{cm}^{2}$, which will be needed for high energy automotive cells.

\section{Reviewer 2:}

The reviewer said the Li-S system is a potential system for low-cost and high-capacity batteries. The reviewer indicated that this project increases the understanding of strategies toward high loading cathodes, and electrolyte 
and cathode reactions. The reviewer noted that this knowledge is critically important for the successful implementation of Li-S batteries.

\section{Reviewer 3:}

The reviewer stated that this effort supports DOE objectives. The theoretical specific energy of Li-S batteries is approximately three times higher than Li-ion batteries. However, the reviewer commented that the major challenge for Li-S batteries is polysulfide shuttle reactions, and that this effort seeks to stop those reactions and to enable its transition into the market place.

\section{Question 6: Resources: How sufficient are the resources for the project to achieve the stated milestones in a timely fashion?}

\section{Reviewer 1:}

The reviewer called the resources adequate, and noted that there has been good progress for the investment to date.

Reviewer 2:

The reviewer pointed out that the total amount of funding for this work was not provided on Slide 2. 


\section{Addressing Internal "Shuttle" Effect: Electrolyte Design and Cathode Morphology Evolution in Li-S Batteries: Perla Balbuena (Texas A\&M University) - es283}

\section{Presenter}

Perla Balbuena, Texas A\&M

University

\section{Reviewer Sample Size}

A total of four reviewers evaluated this project

\section{Question 1: Approach to performing the work-the degree to which technical barriers are addressed, the project is well-designed, feasible, and integrated with other efforts.}

\section{Reviewer 1 :}

The reviewer noted that the project combines theoretical simulation with cathode synthesis and electrochemical testing to tackle the major problems with developing Li-S batteries.

\section{Reviewer 2:}

This reviewer observed a mostly fundamental model approach to understanding potential effects of complex structures on polysulfide cathode structures in Li-S systems. As with all complex molecular level models, explained the reviewer, the concern will be whether it represents to real-world systems in a meaningful way. Despite this concern, the reviewer acknowledged that the approach adds to the field.

\section{Reviewer 3:}

The reviewer opined that the project team probably promises a bit too much, but has an aggressive plan to meet its expectations.

\section{Question 2: Technical accomplishments and progress toward overall project and DOE goals-the degree to which progress has been made, measured against performance indicators and demonstrated progress towards DOE goals.}

\section{Reviewer 1:}

This reviewer particularly liked the project team's examination of interactions between the polysulfides and cathode matrix, which could certainly lead to long term improvements of the technology.

\section{Reviewer 2:}

The reviewer noted that work has progressed as planned and further observed a modest amount of commendable, 
experimental work. This reviewer added that it is difficult to judge the relative merits of the results because there does not appear to be any control variations.

\section{Reviewer 3:}

This reviewer asserted that the project team made some significant progresses in simulation, and suggested the need for more efforts in improving electrochemical performance of the carbon sulfur composites $(\mathrm{CCs} / \mathrm{S})$. Further, the reviewer observed that not much synergy between the simulation and synthesis/testing works so far.

\section{Question 3: Collaboration and coordination with other institutions.}

\section{Reviewer 1:}

This reviewer indicated that collaboration appears appropriate for the work.

\section{Reviewer 2:}

The reviewer noted that the project team started collaboration with PNNL and ANL, and recommended that the project team adopt the advanced characterization methods available in national laboratories to link the simulation with the materials structure and morphology.

\section{Reviewer 3:}

The reviewer asserted that the project team has limited collaborations.

Question 4: Proposed future research-the degree to which the project has effectively planned its future work in a logical manner by incorporating appropriate decision points, considering barriers to the realization of the proposed technology and, when sensible, mitigating risk by providing alternate development pathways

\section{Reviewer 1:}

The reviewer identified a need for more specific plans regarding future work, such as how to identify reasons for failures and successes of specific electrolyte compositions.

\section{Reviewer 2:}

The reviewer pointed out that here is not a lot of detail here.

\section{Reviewer 3:}

This reviewer commented that the future research list appears very ambitious, and it was not clear whether the focus will be on modeling or experimental approaches.

\section{Question 5: Does this project support the overall DOE objectives of petroleum displacement? Why or why not?}

\section{Reviewer 1:}

This reviewer recognized that Li-S batteries have great potential and any progress in this direction will help support DOE objectives.

\section{Reviewer 2:}

The reviewer opined that the Li-S system is a system of interest that has very complex issues associated with its potential improvement.

\section{Question 6: Resources: How sufficient are the resources for the project to achieve the stated milestones in a timely fashion?}

\section{Reviewer 1:}

This reviewer noted that the project team has both theoretical and experimental expertise, and started collaborating with national laboratories.

\section{Reviewer 2:}

The reviewer stated pending clarification of future work. 


\section{Statically and Dynamically Stable Lithium-Sulfur Batteries: Arumugam Manthiram (University of Texas at Austin) - es284}

\section{Presenter}

Arumugam Manthiram, University of Texas at Austin

\section{Reviewer Sample Size}

A total of four reviewers evaluated this project.

\section{Question 1: Approach to performing the work-the degree to which technical barriers are addressed, the project is well-designed, feasible, and integrated with other efforts.}

\section{Reviewer 1:}

This reviewer asserted that the project is sound and focused. The investigators will develop statically and dynamically stable Li-S batteries by developing polysulfide (PS)filter-coated separators that will protect the Li-metal anode. This will be accomplished by establishing a materials chemistry database for coating materials and for PS-filtered coated separators. The reviewer further noted that investigators from other facilities may be able to benefit from the database, too.

\section{Reviewer 2:}

The reviewer observed a good approach to filtering polysulfides, which keeps them from reaching the Li-metal anode, and increases S loading to commercially relevant values. However, explained this reviewer, at some point the huge volume of electrolyte needed to enable $\mathrm{S}$ cathodes to work has to be addressed. This reduces $\mathrm{Wh} / \mathrm{kg}$ by quite a bit.

\section{Reviewer 3:}

Trapping polysulfides closer to their place of origin is better than filtering at the separator, opined this reviewer. Nevertheless, the reviewer acknowledged that experience gained and knowledge about PS-filter separators will contribute to solving the PS issue.

\section{Question 2: Technical accomplishments and progress toward overall project and DOE goals-the degree to which progress has been made, measured against performance indicators and demonstrated progress towards DOE goals.}

\section{Reviewer 1:}

This reviewer commented that good progress has been achieved thus far: the databases were established; various 
coating materials with different morphologies and microstructures were analyzed and categorized; and four different carbon materials for PS-filter coatings are undergoing a thorough investigation.

\section{Reviewer 2:}

For cells that have not failed after 200 cycles, the reviewer recommended continued cycling to determine how long they will last.

\section{Reviewer 3:}

This reviewer reported that a very large number of possible filtering materials (applied to the cell separator) have been screened. However, the reviewer opined that results to date are not particularly promising. The reviewer commented that cells with spherical C materials suffer $25 \%$ capacity fade in 75 cycles, while those with CNF suffer about the same fade in 200 cycles. Although the reviewer observed good improvement, there is still a long way to go and work on thick electrodes is just starting.

\section{Question 3: Collaboration and coordination with other institutions.}

\section{Reviewer 1:}

The reviewer noted good collaboration with ORNL.

\section{Reviewer 2:}

This reviewer described collaborations with Veith group on thin films as appropriate for this study.

\section{Reviewer 3:}

The reviewer reported that there is collaboration with Dr. Gabriel Veith, ORNL.

\section{Reviewer 4:}

This reviewer observed that collaboration is used for a specific task only and suggested that it might be useful to have other perspectives contribute to the project on a regular basis.

Question 4: Proposed future research-the degree to which the project has effectively planned its future work in a logical manner by incorporating appropriate decision points, considering barriers to the realization of the proposed technology and, when sensible, mitigating risk by providing alternate development pathways.

\section{Reviewer 1:}

The reviewer described this thorough project with extensive characterization as an excellent approach to understanding Li-S batteries.

\section{Reviewer 2:}

This reviewer asserted that the proposed work is well thought out and includes clear go/no-go decision points.

\section{Reviewer 3:}

The reviewer observed an ambitious goal to test metal sulfides and address high-loading cathodes in FY 2017. Less may be more, suggested this reviewer.

\section{Reviewer 4:}

It was unclear to this reviewer whether the team understands the cause of the observed capacity fade: loss of S through reactions of polysulfides at the Li-metal interface; loss of access to $\mathrm{S}$ due to electrical isolation; or buildup of Li-metal SEI.

\section{Question 5: Does this project support the overall DOE objectives of petroleum displacement? Why or why not?}

\section{Reviewer 1:}

This reviewer opined that $\mathrm{Li} / \mathrm{S}$ research is very relevant due to the potential low-cost of the technology. 


\section{Reviewer 2:}

The reviewer affirmed that the project is relevant to DOE. A basic science understanding gained during this effort will be used to develop the Li-S technology as the next-generation power source for electric vehicles.

\section{Reviewer 3:}

The reviewer commented that realizing commercial Li-S cells would allow for increased reliance on renewable energy sources in connection to the electrical grid.

Reviewer 4:

This reviewer stated that the results contribute to the goal of low-cost, high-capacity Li-S batteries.

Question 6: Resources: How sufficient are the resources for the project to achieve the stated milestones in a timely fashion?

Reviewer 1:

The reviewer observed excellent value for the investment. 


\section{Mechanistic Investigation for the Rechargeable Li- Sulfur Batteries: Deyang Qu (University of Wisconsin, Milwaukee) - es285}

\section{Presenter}

Deyang Qu, University of Wisconsin

\section{Reviewer Sample Size}

A total of four reviewers evaluated this project.

\section{Question 1: Approach to performing the work-the degree to which technical barriers are addressed, the project is well-designed, feasible, and integrated with other efforts.}

\section{Reviewer 1:}

The reviewer observed an excellent approach and explained that the investigators will develop an analytical method for the quantitative and qualitative assessment of dissolved elemental sulfur and polysulfide ions in non-aqueous electrolytes. These kinds of studies are essential in order to investigate the kinetics of the sulfur redox reaction.

\section{Reviewer 2:}

The reviewer emphasized that setup of an in situ electrochemical HPLCMS cell is an outstanding approach.

\section{Reviewer 3:}

This reviewer indicated that it is definitely interesting to try and understand the specific polysulfides formed during S lithiation. Although this is a worthwhile study, it is not immediately clear to the reviewer how this will lead to solutions to the polysulfide anode reaction issues or the need for so much excess electrolyte.

\section{Question 2: Technical accomplishments and progress toward overall project and DOE goals - the degree to which progress has been made, measured against performance indicators and demonstrated progress towards DOE goals.}

\section{Reviewer 1:}

The reviewer stated that the polysulfide dissolution details achieved in this project are unprecedented and make important corrections to the existing view in the field.

\section{Reviewer 2:}

This reviewer observed very good progress in less than one work year.

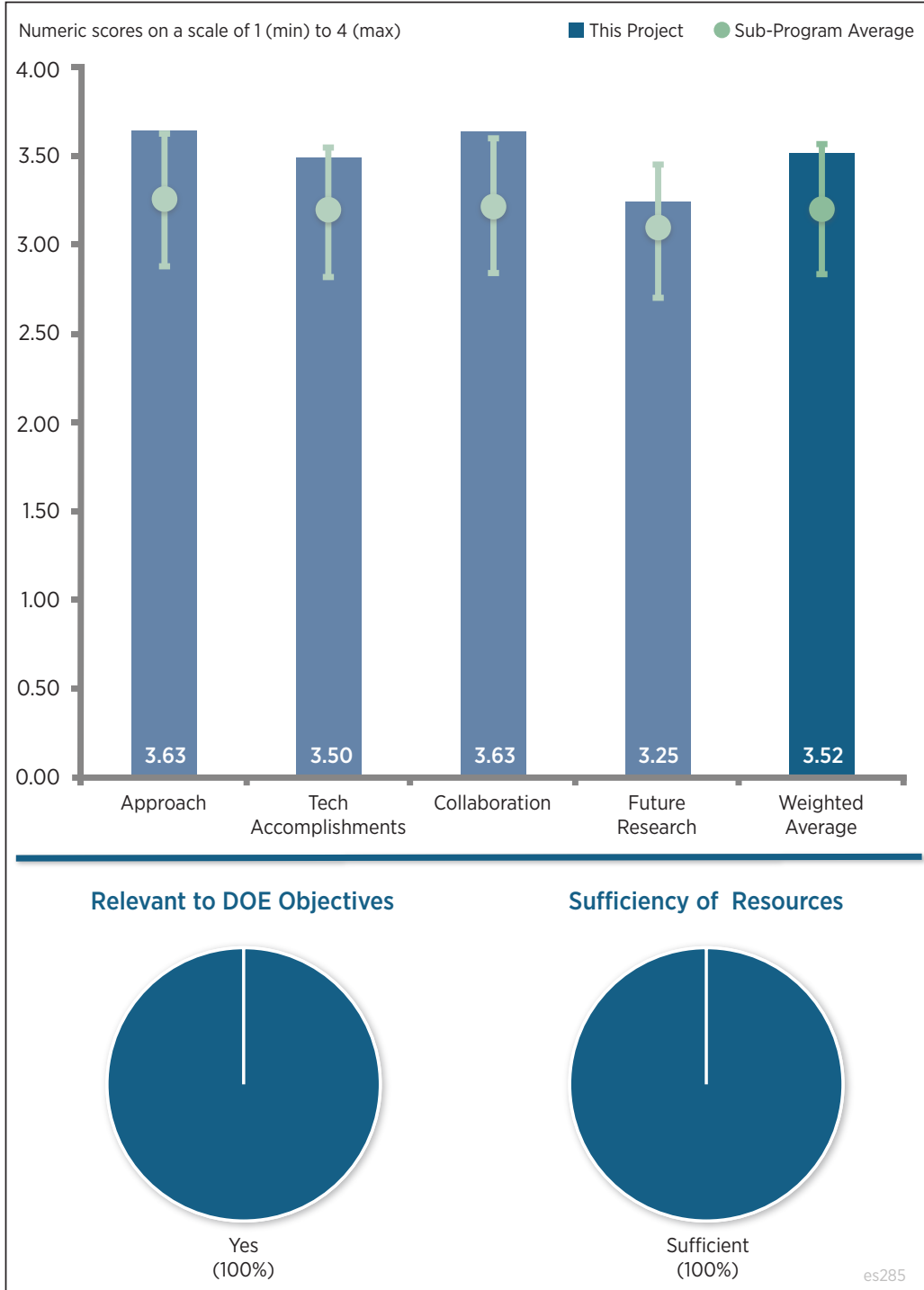

Figure 2-58 - Mechanistic Investigation for the Rechargeable Li-Sulfur Batteries: Deyang Qu (University of Wisconsin, Milwaukee) - Electrochemical Energy Storage 


\section{Reviewer 3:}

This reviewer commented that good progress was achieved and reported that several polysulfides were separated and qualitatively analyzed for the first time. Further, the reviewer explained that there were numerous publications and presentations.

\section{Question 3: Collaboration and coordination with other institutions.}

\section{Reviewer 1:}

The reviewer observed an excellent use of industry, national laboratory, and university resources.

\section{Reviewer 2:}

The reviewer indicated that there are excellent collaborations with other institutions and industry. The reviewer added that the PI has close collaborations with Johnson Controls' scientists and engineers, and that this collaboration may be beneficial to the project as it would allow the validation of fundamental research findings in pilot-scale cells.

\section{Question 4: Proposed future research-the degree to which the project has effectively planned its future work in a logical manner by incorporating appropriate decision points, considering barriers to the realization of the proposed technology and, when sensible, mitigating risk by providing alternate development pathways}

\section{Reviewer 1:}

The reviewer opined that in situ confocal microscopy setup would deliver very promising results.

\section{Reviewer 2:}

Future plans were outlined and look appropriate according to this reviewer.

\section{Reviewer 3:}

This reviewer expressed confusion, and specified that there is a large amount of future work listed, which is odd because the project is scheduled to end September 2016. The reviewer believed the project concluded that polysulfide concentrations can only be measured during the first electron transfer due to subsequent reactions in the electrolyte that form multiple polysulfide species. This issue has been known in the literature for some time, explained the reviewer.

\section{Question 5: Does this project support the overall DOE objectives of petroleum displacement? Why or why not?}

\section{Reviewer 1:}

According to the reviewer, this work presents a major contribution to understanding mechanisms in Li-S batteries, which are a candidate for low-cost, high-energy batteries.

\section{Reviewer 2:}

The reviewer asserted that the project is relevant to the DOE. Li-S batteries could enable a competitive market entry of electric vehicles by reducing the cost and extending the driving distance per charge.

\section{Reviewer 3:}

A Li-S battery is probably the only practical route for breaking the $300 \mathrm{Wh} / \mathrm{kg}$ barrier for VT applications, opined this reviewer. The reviewer further explained that fundamental understanding of the failure modality of the electrode is critical for improving the cycle life of the Li-S battery.

\section{Reviewer 4:}

This reviewer stated that the project is relevant because Li-S offers a clear path to low-cost cells.

\section{Question 6: Resources: How sufficient are the resources for the project to achieve the stated milestones in a timely fashion?}

\section{Reviewer 1:}

Although this project seems to have reached a natural ending point, the reviewer observed good value for the investment. 
Development of Novel Electrolytes and Catalysts for Li-Air Batteries: Khalil Amine (Argonne National Laboratory) - es286

\section{Presenter}

Khalil Amine, Argonne National Laboratory

\section{Reviewer Sample Size}

A total of four reviewers evaluated this project.

\section{Question 1: Approach to performing the work-the degree to which technical barriers are addressed, the project is well-designed, feasible, and integrated with other efforts.}

\section{Reviewer 1:}

The reviewer stated that this is very much a science project and the team's combined modeling and experimental approaches are very adequate. The focus on catalyst development has proven productive, according to this reviewer, who also explained that electrolyte work has been largely on the modeling side.

\section{Reviewer 2:}

This reviewer described the challenges to a practical lithium-air $\left(\mathrm{Li}-\mathrm{O}_{2}\right)$ system as enormous. While this is one piece of this complex system, the study of new options for cathode catalyst systems is of some interest to the reviewer.

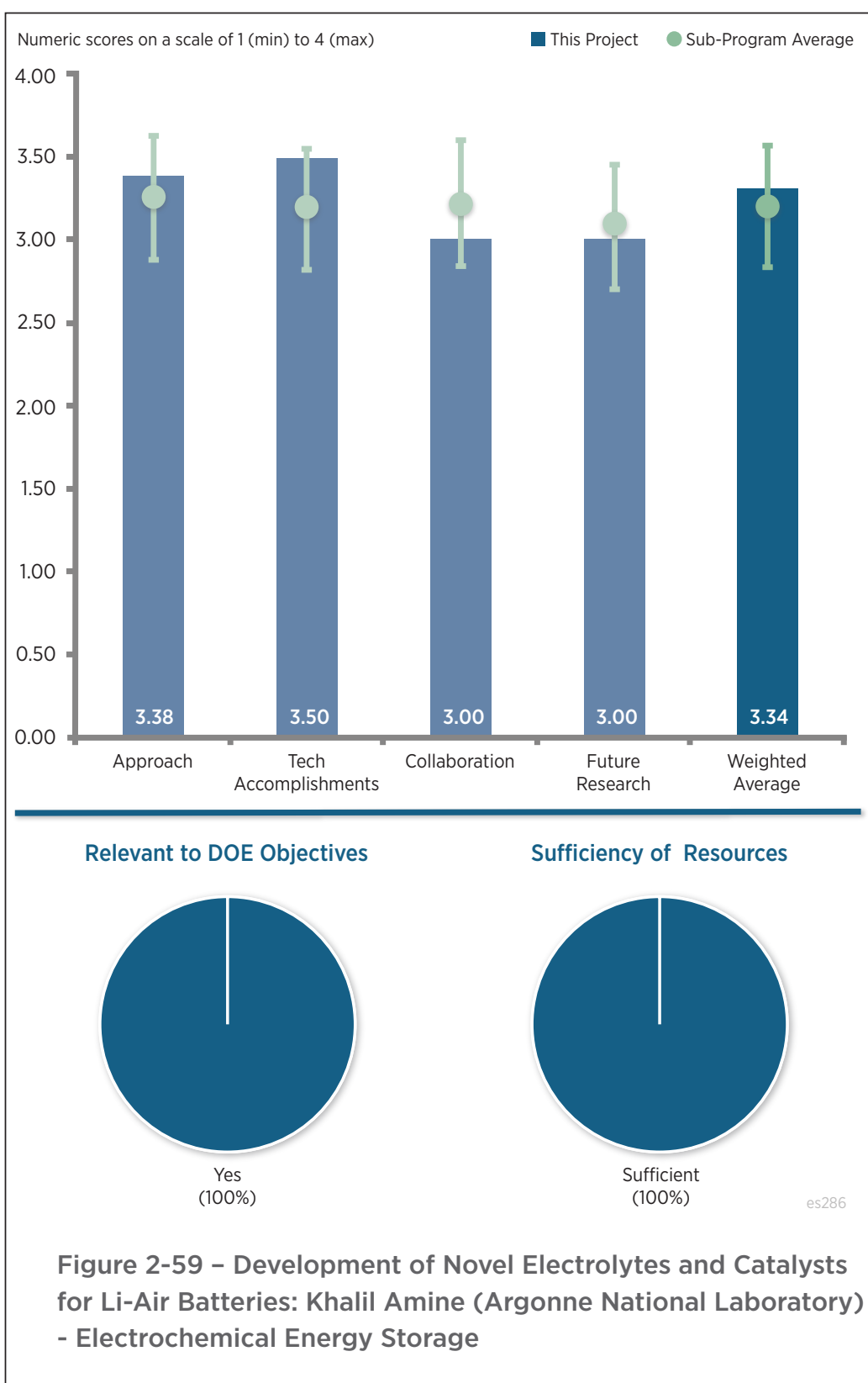

\section{Reviewer 3:}

The reviewer was unclear what experiments are planned for "Electrolyte Development," in particular, "Test new Li-air battery electrolytes."

\section{Question 2: Technical accomplishments and progress toward overall project and DOE goals-the degree to which progress has been made, measured against performance indicators and demonstrated progress towards DOE goals.}

\section{Reviewer 1:}

This reviewer opined that the project has made tremendous progress in identifying the formation of peroxide versus superoxide under different conditions and how that affects overpotential.

\section{Reviewer 2:}

This reviewer commented that the work resulted in materials producing noticeably different electrochemical 
performance of unique catalyst structures, adding to the knowledge base against the system. While this is not placed in context with all of the overall challenges faced by the system, the reviewer asserted that it is a contribution to the field.

\section{Reviewer 3:}

This reviewer highlighted that there is no experimental work on "Electrolyte Development," particularly with regard to experiments on "Test new Li-air battery electrolytes."

\section{Question 3: Collaboration and coordination with other institutions.}

\section{Reviewer 1:}

The reviewer stated that collaboration appears appropriate for the project.

\section{Question 4: Proposed future research-the degree to which the project has effectively planned its future work in a logical manner by incorporating appropriate decision points, considering barriers to the realization of the proposed technology and, when sensible, mitigating risk by providing alternate development pathways}

\section{Reviewer 1:}

The reviewer recommended a strong experimental component to test modeling predictions under the topic of "Electrolyte Development," and specifically, "Test new Li-air battery electrolytes."

\section{Reviewer 2:}

The proposed list of work is rather expansive and generic, according to this reviewer, who also pointed out that the potential work plan needs more detail.

\section{Question 5: Does this project support the overall DOE objectives of petroleum displacement? Why or why not?}

\section{Reviewer 1:}

This reviewer commented that further developing this system is important in the context of diversifying choices of battery chemistries for EV. It would be helpful, suggested the reviewer, if the project can provide an analysis on the system performance (i.e., if a closed system is designed) and its expected energy density.

\section{Reviewer 2:}

The reviewer opined that the ultimate practicality of $\mathrm{Li}-\mathrm{O}_{2}$ is highly debatable, though some fundamental work in the field can help establish just how practical it may or may not be.

\section{Question 6: Resources: How sufficient are the resources for the project to achieve the stated milestones in a timely fashion?}

No comments were received in response to this question. 


\section{Exploratory Studies of Novel Sodium-Ion Battery Systems: Xiao-Qing Yang (Brookhaven National Laboratory) - es287}

\section{Presenter}

Xiao-Qing Yang, Brookhaven

National Laboratory

\section{Reviewer Sample Size}

A total of three reviewers evaluated this project.

\section{Question 1: Approach to performing the work-the degree to which technical barriers are addressed, the project is well-designed, feasible, and integrated with other efforts.}

\section{Reviewer 1:}

This reviewer observed some really good work that not only gives insight into the sodium $(\mathrm{Na})$-ion cathode system, but all intercalation systems. Quite complete and well grounded, continued the reviewer, who also said good job.

\section{Reviewer 2:}

The reviewer described the work on new materials for $\mathrm{Na}$-ion batteries as very good, carefully planned, and well documented. More focus is needed on the cell level though, and the reviewer further suggested that linking the choice of active materials to the capacities at the cell level be beneficial.

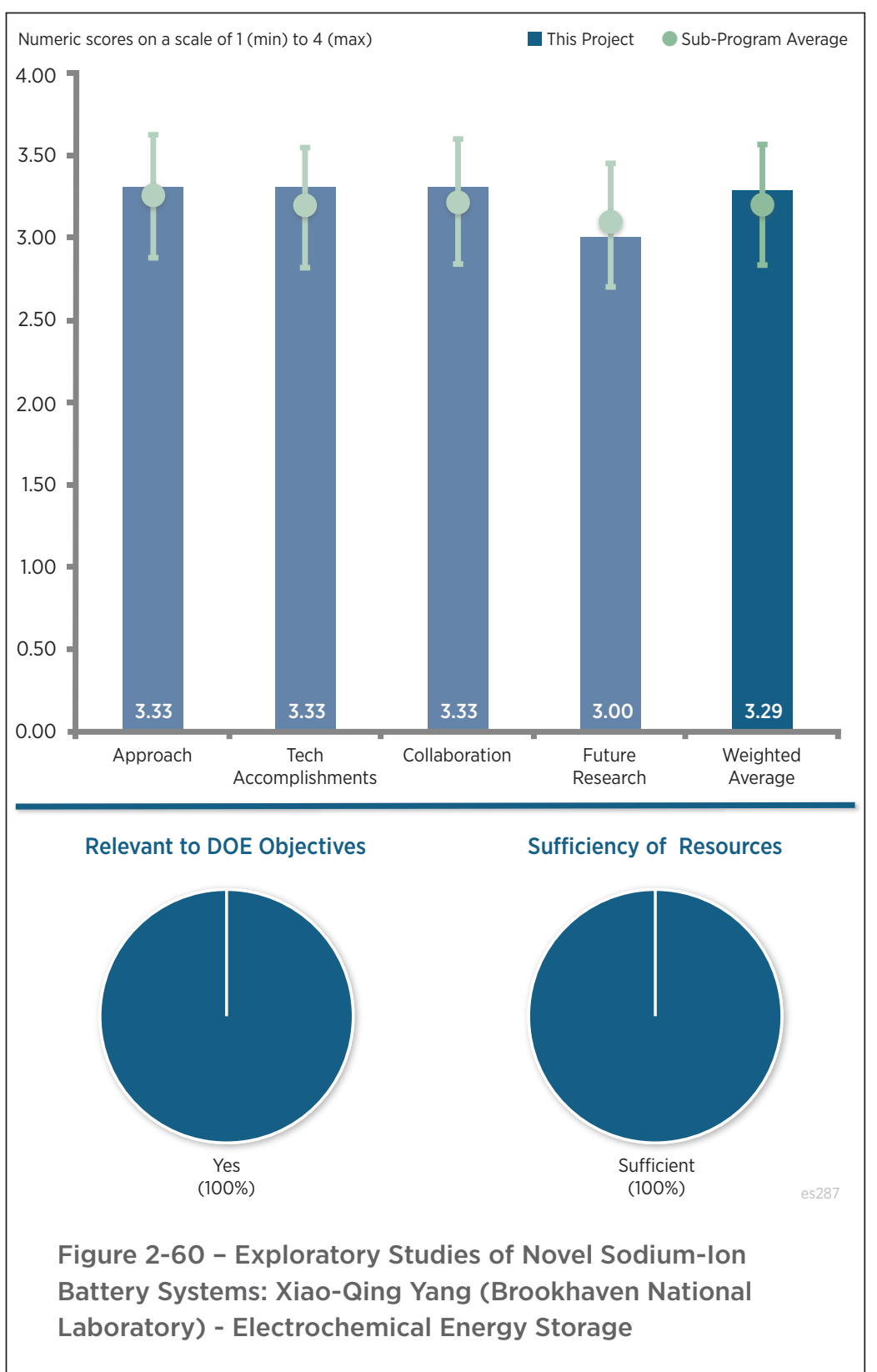

Question 2: Technical accomplishments and progress toward overall project and DOE goals-the degree to which progress has been made, measured against performance indicators and demonstrated progress towards DOE goals.

\section{Reviewer 1:}

The reviewer remarked that mechanisms of Na-ion batteries are identified with well-planned and documented experiments. This reviewer also observed very useful insights on Na-ion battery technology.

\section{Reviewer 2:}

This reviewer discerned a very nice set of experimental results that allow good insight into intercalation dynamics. The techniques appear sound and the results are reasonable. The reviewer described the work as really good, and suggested that there could be a little more focus on summarizing the relevance of results.

Question 3: Collaboration and coordination with other institutions. 


\section{Reviewer 1:}

The reviewer commented that collaboration appears appropriate for this work. This reviewer further reported a lot of development, then application of advanced techniques, and appropriate expertise in them.

\section{Reviewer 2:}

The reviewer stated that even though the external partner network is large, the contribution is well organized and all partners seem to participate in the work.

\section{Question 4: Proposed future research-the degree to which the project has effectively planned its future work in a logical manner by incorporating appropriate decision points, considering barriers to the realization of the proposed technology and, when sensible, mitigating risk by providing alternate development pathways}

\section{Reviewer 1:}

The reviewer reported that focus is largely on mechanisms and fundamental work, and that this is well explained given the maturity of technology.

\section{Reviewer 2:}

This reviewer indicated that the focus of future work appears to be on expanded technique development. Hopefully, added the reviewer, this will applied across a number of system studies.

\section{Question 5: Does this project support the overall DOE objectives of petroleum displacement? Why or why not?}

\section{Reviewer 1:}

The reviewer opined that this is the type of fundamental work that can be used to develop new insight into important chemical processes.

\section{Reviewer 2:}

The reviewer stated yes, and explained that the work is aimed towards an understanding of sodium-ion batteries, which is needed to further develop these systems.

\section{Question 6: Resources: How sufficient are the resources for the project to achieve the stated milestones in a timely fashion?}

\section{Reviewer 1:}

This reviewer reported that there seems to be a good level of collaboration and ability to apply a number of advanced techniques to the study. 


\section{Construction of High Energy Density Batteries: Christopher Lang (Physical Sciences, Inc.) - es288}

\section{Presenter}

Christopher Lang, Physical Sciences Inc.

\section{Reviewer Sample Size}

A total of five reviewers evaluated this project.

\section{Question 1: Approach to performing the work-the degree to which technical barriers are addressed, the project is well-designed, feasible, and integrated with other efforts.}

\section{Reviewer 1:}

PSI's efforts, as reported by this reviewer, are concentrated in increasing energy density of batteries by reducing inactive mass. The target is $25 \%$ increase in cell energy density.

\section{Reviewer 2:}

The reviewer commented that this is an incremental improvement in electrode design, higher active loading content, and improved volumetric efficiency. More evidence as to how the $25 \%$ energy density improvement is to be achieved should be provided. The reviewer further recommended that the developer would benefit from organizing the deliverable goals and performance objectives into a gap chart.

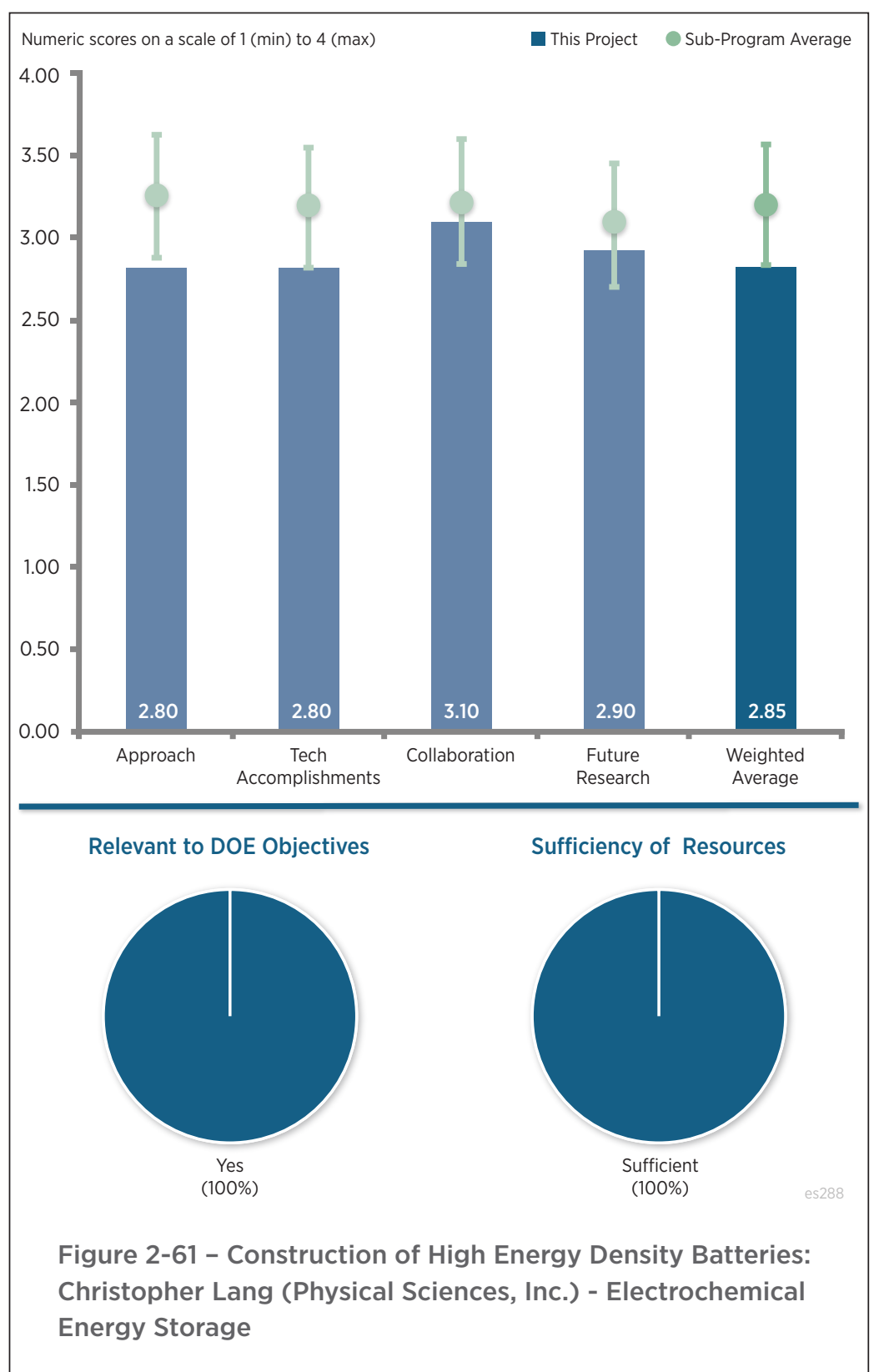

\section{Reviewer 3:}

This reviewer stated that the approach to make lower weight electrodes with equivalent performance to conventional electrodes is an interesting one. The composite material is not defined, but it apparently has a lower weight than $\mathrm{Cu}$ or aluminum conventionally used as negative or positive electrode substrate. The reviewer added that no idea of the energy density $(\mathrm{Wh} / \mathrm{L})$ can be assessed because nothing was said about the thickness or density of the test substrate. The reviewer explained that what the project team calls energy density is actually the specific energy $(\mathrm{Wh} / \mathrm{kg})$, and the possible improvement in this property may be accompanied by a decrease in energy density if the composite density is too low. This reviewer also highlighted two other aspects that are not considered: conductance of the materials may be insufficient for many higher rate discharge tests (cells seem to be tested only at $\mathrm{C} / 3$ rate); and the specification of the coating (which contains less carbon and binder than conventional coatings) may also lead to poorer performance under higher rate continuous or pulse testing. The reviewer suggested that these tests be conducted as part of the approach to fully evaluate the project. 


\section{Reviewer 4:}

Although the objectives are with respect to the state of the art technologies, this reviewer noted that the state of the art is not well defined. Furthermore, the reviewer reported that cost justification of the innovative process is not provided.

\section{Reviewer 5:}

The reviewer found it difficult to evaluate the technology or approach due to a lack of any useful details regarding basic benefit mechanisms or technology content.

\section{Question 2: Technical accomplishments and progress toward overall project and DOE goals-the degree to which progress has been made, measured against performance indicators and demonstrated progress towards DOE goals.}

\section{Reviewer 1:}

The reviewer described progress to date as good, and suggested that the developer needs to provide more information on the composite current collector so that its benefits and potential failure modes can be better understood. It was also recommended by this reviewer that anode-side work be discussed within the program, such that partner development may better support the cell designs for deliverables, and take advantage of potential anode innovations.

\section{Reviewer 2:}

This reviewer commented that progress to date is interesting because apparently better stability at high voltage is achieved than with conventional cells. The reviewer reiterated that there is a lack of rate date presented; so, the down side of this approach cannot be assessed. The reviewer further noted that using a test with only partial discharge has little meaning if comparative tests are not performed, as is the case here.

\section{Reviewer 3:}

The reviewer highlighted that the progress reported (i.e., lower mass of composite current collector, 50\% less solvent, and $98.5 \%$ active materials in electrode) does not convert to a benchmark showing the progress in increasing the overall energy density. This reviewer inquired about current achievement to the targeted 25\% energy density increase. The reviewer explained that rate performance of high active (HA) NCM-622 in 3+ Ah cells is basically the same as the baseline NCM-622, but the key information about the energy density improvement of HA cell is missing. The reviewer further recommended that the cycling test of the HA cells charged to different voltages should include results of a baseline cell.

\section{Reviewer 4:}

The reviewer acknowledged that understanding 80-100\% SOC cycling performance can be useful. However, avoiding something closer to full DOD cycling seemed troubling to this reviewer, even if notably less cycling can be accomplished.

\section{Reviewer 5:}

This reviewer remarked that cost reduction due to high active material loading, energy density improvement due to higher voltage, and effect on cycle life are not addressed.

\section{Question 3: Collaboration and coordination with other institutions.}

\section{Reviewer 1:}

The reviewer observed a good team, and added that it will be interesting to see the progress once more advanced active materials are incorporated.

\section{Reviewer 2:}

According to this reviewer, the collaboration with the state of the art cell developers is necessary to evaluate success of the innovative coating technology.

\section{Reviewer 3:}

This reviewer reported that PSI collaborates with both national laboratory and industry partners. 


\section{Reviewer 4:}

The reviewer opined that it is valuable to have a scale-up partner because the background of the PI is not strong in this area.

Question 4: Proposed future research-the degree to which the project has effectively planned its future work in a logical manner by incorporating appropriate decision points, considering barriers to the realization of the proposed technology and, when sensible, mitigating risk by providing alternate development pathways

\section{Reviewer 1:}

This reviewer reported that remaining challenges and future works are identified.

\section{Reviewer 2:}

The reviewer commented that the investigator seems to recognize the need for rate evaluation in future work, and suggested that the project team also investigate the true energy density in this work $(\mathrm{Wh} / \mathrm{L})$.

\section{Reviewer 3:}

This reviewer recommended that the developer include a contrast of cell design with an advanced anode.

\section{Reviewer 4:}

The reviewer recommended that cycle life using the automotive cycling profile and calendar life to last 15 years should be planned for the future research.

\section{Question 5: Does this project support the overall DOE objectives of petroleum displacement? Why or why not?}

\section{Reviewer 1:}

The reviewer explained that the need for higher specific energy is an important DOE goal.

\section{Reviewer 2:}

This reviewer asserted that the work is in the right direction.

\section{Reviewer 3:}

The reviewer remarked that the research, if successful, should improve the domestic cell assembly cost and will meet or beat DOE targets.

\section{Reviewer 4:}

While incremental, this reviewer indicated that the technical approach could benefit industry quickly.

\section{Question 6: Resources: How sufficient are the resources for the project to achieve the} stated milestones in a timely fashion?

\section{Reviewer 1:}

The reviewer observed no issues at this time.

\section{Reviewer 2:}

This reviewer opined that Physical Sciences Inc. is capable of providing the resources to optimize the coating technologies. 


\section{Advanced Polyolefin \\ Separators for Li-Ion Batteries Used in Vehicle Applications: Weston Wood (Entek) - es289}

\section{Presenter}

Weston Wood, Entek

\section{Reviewer Sample Size}

A total of three reviewers evaluated this project.

\section{Question 1: Approach to performing the work-the degree to which technical barriers are addressed, the project is well-designed, feasible, and integrated with other efforts.}

\section{Reviewer 1:}

The reviewer commented that the technical approach is up-to-date and detail led in order to reach the USABC final deliverables. This reviewer further reported that all standard test and metrics were followed for separator testing. If successful, opined the reviewer, the manufacturing approach will reduce cost and improve performance as described in the target.

\section{Reviewer 2:}

This reviewer noted that the project addresses some of the points and identified the following gaps: there is no work on high voltage stability; cost is only addressed by linking it to the fill time; there are no details on process for the coating of the multilayer separator; and there are no cell measurements.

\section{Question 2: Technical accomplishments and progress toward overall project and DOE goals-the degree to which progress has been made, measured against performance indicators and demonstrated progress towards DOE goals.}

\section{Reviewer 1:}

The reviewer remarked that the project team made good technical progress towards the silica and alumina filled separator. Optimization of the conductivity and wetting was also noted by this reviewer. The reviewer further acknowledged good improvement over the baseline separator.

\section{Reviewer 2:}

This reviewer reported the lack of electrochemical tests, compatibility of coated separators to cathode materials, and high voltage stability.

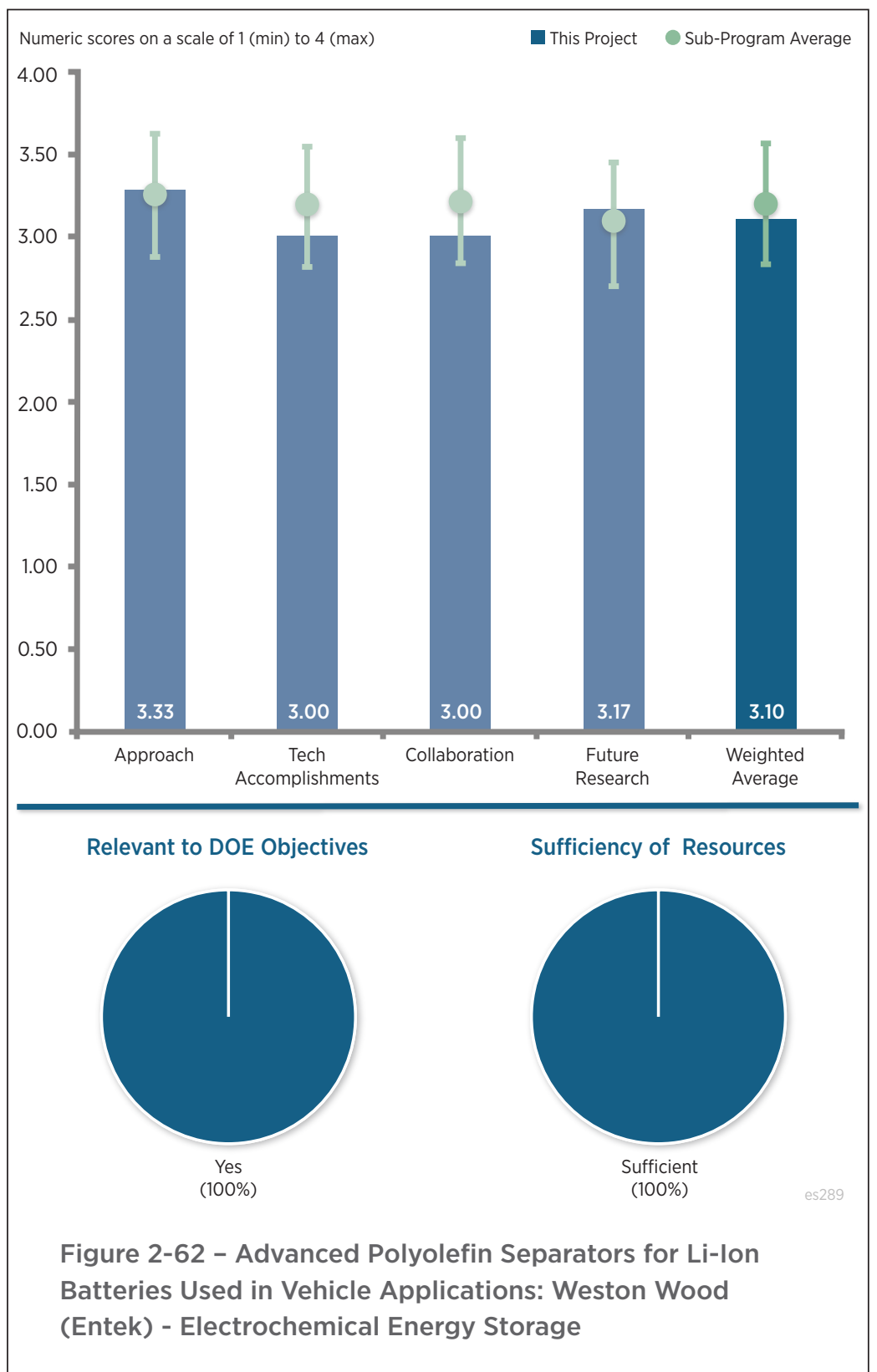




\section{Question 3: Collaboration and coordination with other institutions.}

\section{Reviewer 1:}

This reviewer observed good teaming with Farasis on cell testing and development and Portland State University for characterization.

\section{Reviewer 2:}

The reviewer commented that Farasis' role was not active at the moment, and the role of the other partner except from SEM imaging is unclear.

Question 4: Proposed future research-the degree to which the project has effectively planned its future work in a logical manner by incorporating appropriate decision points, considering barriers to the realization of the proposed technology and, when sensible, mitigating risk by providing alternate development pathways

\section{Reviewer 1:}

The reviewer praised that improvement in energy density is a plus, especially designing separators for $5 \mathrm{~V}$ stability.

\section{Reviewer 2:}

According to the reviewer, this project has the potential to improve over the current status, if next year's work is completed. However, continued the reviewer, a link of production methods cost needs to be discussed and the cost cannot be addressed by only linking it to cell fill time.

\section{Reviewer 3:}

This reviewer explained that the proposed future work addresses what is to be done, but not how it will be done. The reviewer provided the following examples: "Drying methods and ceramic coating formulation optimization will be evaluated," was stated without specifying what drying methods will be attempted; and "Evaluate the feasibility of continuously coating separator using immersion, spray coating, and powder coating systems" was stated without specifying coating methods. The reviewer also recommended that coating adhesion strength should be measured and quantified.

\section{Question 5: Does this project support the overall DOE objectives of petroleum displacement? Why or why not?}

\section{Reviewer 1:}

The reviewer summarized that this project aims at developing advanced Li-ion batteries with improved safety and energy density. It also aims at improving the manufacturing process for separators that could potentially reduce cost and energy consumption.

\section{Reviewer 2:}

This reviewer asserted that enabling better batteries is a clear DOE target.

\section{Question 6: Resources: How sufficient are the resources for the project to achieve the stated milestones in a timely fashion?}

\section{Reviewer 1:}

This reviewer described resources as adequate. 


\section{Hybrid Electrolytes for PHEV Applications: Surya Moganty (NOHMs Technologies) \\ - es290}

\section{Presenter}

Surya Moganty, NOHMs Technologies

\section{Reviewer Sample Size}

A total of four reviewers evaluated this project.

\section{Question 1: Approach to performing the work-the degree to which technical barriers are addressed, the project is well-designed, feasible, and integrated with other efforts.}

\section{Reviewer 1:}

This reviewer commented that the project is very well designed with quantifiable milestones.

\section{Reviewer 2:}

This reviewer stated that the PI proposed to synthesize and formulate ionic liquid based electrolyte for high voltage electrolytes. Considering the intrinsic problem with carbonatebased solvents, which release $\mathrm{CO}_{2}$ at high voltage and high-temperature, it is necessary to explore non-carbonatebased solvents. The reviewer opined that this project started a meaningful direction.

\section{Reviewer 3:}

The reviewer indicated that targets and the project pathway are very well defined and organized. However, the reviewer noted that the project does not include safety tests, even though this is critical for electrolytes.

\section{Reviewer 4:}

The reviewer highlighted several strong points: good baseline comparison with EC-DMC/EMC electrolyte with NOHMS ionic liquids (IL) and additives; the team has adequate tools and characterization methods to test the performance of ILs for high voltage applications; and first testing on $2 \mathrm{Ah}$ cell is a good idea even before on a small single layer pouch cell is preferable as listed in the project team's milestones. This reviewer identified one major issue and emphasized that, as the team knows, even if $5 \mathrm{~V}$ electrolytes are designed, the cathodes and cathode surfaces may not be stable above $4.4 \mathrm{~V}$ or so. This calls for surface modifications and other treatments to stabilize, advised the reviewer. The capacity loss at higher voltage could be due to multiple reasons as the cathodes like NMC may not be stable as well.

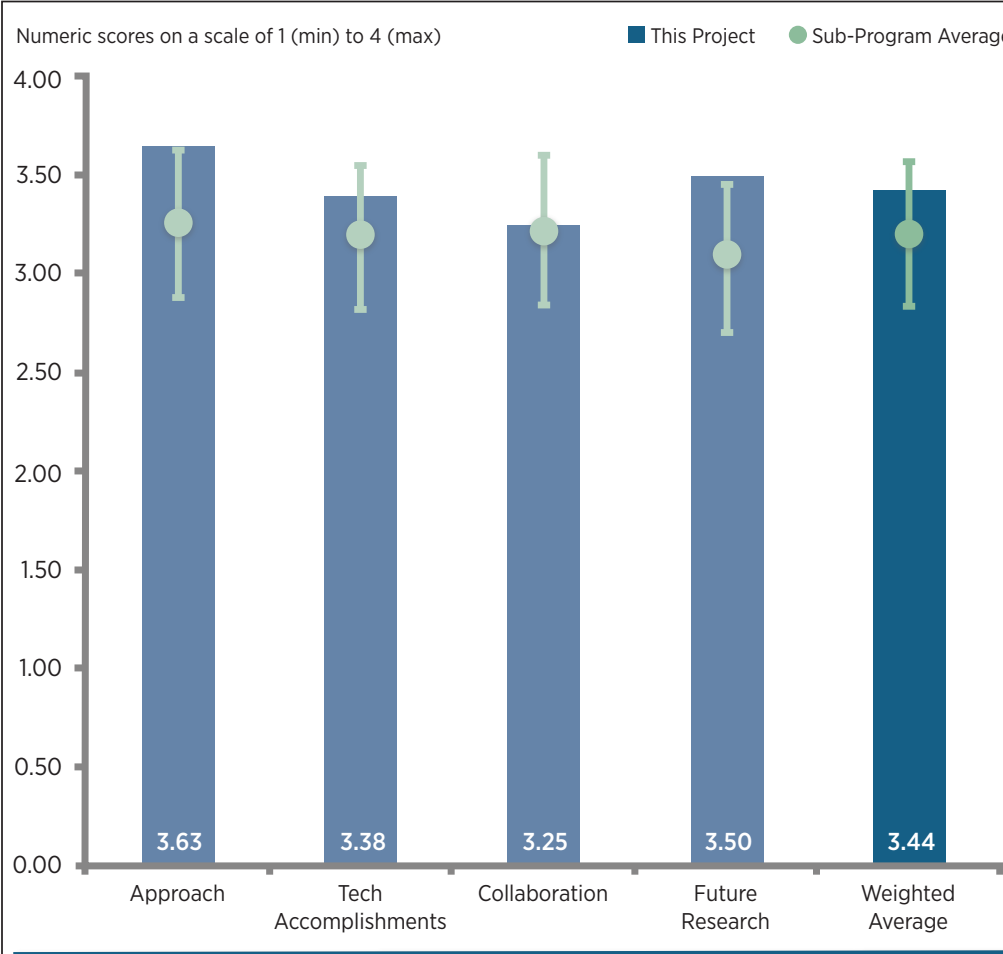

Relevant to DOE Objectives

Sufficiency of Resources
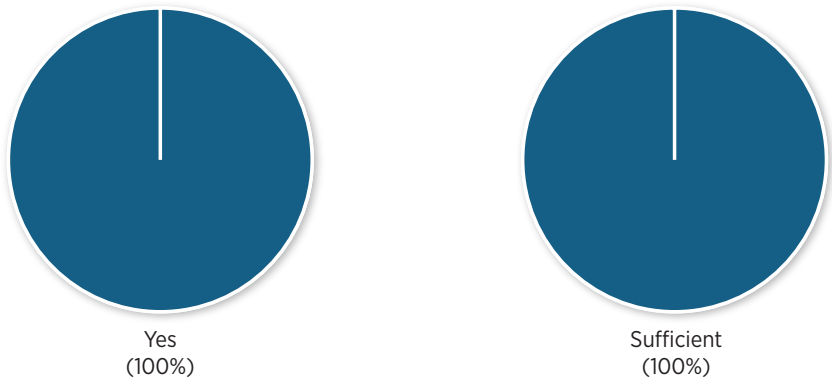

Figure 2-63 - Hybrid Electrolytes for PHEV Applications: Surya Moganty (NOHMs Technologies) - Electrochemical Energy Storage 
Question 2: Technical accomplishments and progress toward overall project and DOE goals - the degree to which progress has been made, measured against performance indicators and demonstrated progress towards DOE goals.

\section{Reviewer 1:}

The reviewer listed impressive technical accomplishments: higher temperature stability than EC; correlation between ring-size and temperature stability; improved low-temperature conductivity; lower vapor pressure at elevated temperatures than conventional electrolytes; improved stability against the graphite, NMC, and nickel manganese oxide (NMO) electrodes; and improved capacity retention.

\section{Reviewer 2:}

Given the short period since the project award, the reviewer asserted that the PI has already made significant progress in synthesis and characterization.

\section{Reviewer 3:}

This reviewer described the technical accomplishments and progress as good, but stated that there is no information about the electrode loading in the presentation. Further, the reviewer inquired whether there is a plan to get higher loading cathodes.

\section{Reviewer 4:}

The reviewer observed that the cycling data are presented between 4.95 V-3.5 V for NMO-graphite, and asked what the justification was for the lower voltage cut-off. The reviewer stated that should be clear, and wondered if it is reactivity with the negative electrode. Further, this reviewer expected that all of the cathodes used for comparison have similar loading and test metrics for a meaningful comparison. The reviewer further asked what the typical electrode loading was for graphite and NMC/NMO, and stated that this should have been listed.

\section{Question 3: Collaboration and coordination with other institutions.}

\section{Reviewer 1:}

This reviewer observed close collaboration between the PI and team members.

\section{Reviewer 2:}

CoorsTek's contribution in the project was not fully clear to this reviewer.

\section{Reviewer 3:}

Referencing Slide 17 ("New Partner TBD"), the reviewer commented that it was unclear why a new partner is needed.

Question 4: Proposed future research-the degree to which the project has effectively planned its future work in a logical manner by incorporating appropriate decision points, considering barriers to the realization of the proposed technology and, when sensible, mitigating risk by providing alternate development pathways

\section{Reviewer 1:}

The reviewer noted that proposed efforts are innovative and highly meaningful.

\section{Reviewer 2:}

Proposed future work is well defined and clearly stated, as stated by this reviewer.

\section{Reviewer 3:}

The reviewer observed a pretty ambitious goal as illustrated in the challenges and barriers section. Partnering with A123 Venture and CoorsTek is a positive. This reviewer looked forward to constructive feedback about the cost and scale up, and added that it is a good idea to provide some fundamental or mechanistic picture about why ILs are stable at higher oxidative voltage. Details are often hidden in literature, but this reviewer indicated that a clear picture needs to be provided for the cell and engineering community. 


\section{Reviewer 4:}

This reviewer asserted that several issues have to be addressed to finish the project in a good way: mass production of the ionic liquids and electrolyte; safety tests have to be performed; electrolyte formulation recommendations for specific cathodes (i.e., NMC and NCA) have to be published.

\section{Question 5: Does this project support the overall DOE objectives of petroleum displacement? Why or why not?}

\section{Reviewer 1:}

This reviewer asserted that advances and mass penetration of EVs are one of the approaches to displace fossil fuels.

\section{Question 6: Resources: How sufficient are the resources for the project to achieve the stated milestones in a timely fashion?}

\section{Reviewer 1:}

The reviewer described resources as adequate. Overall, opined this reviewer, USABC milestones are very ambitious given the very nature and status of IL R\&D, but the team has the right approach and tools to push the envelope.

\section{Reviewer 2:}

This reviewer recommended use of a proof-reader because there are many typos in the es290_moganty_2016_p presentation. 
SAFT-USABC 12V Start-Stop Phase II: Ian O'Connor (Saft) - es291

\section{Presenter}

Ian O'Connor, Saft

\section{Reviewer Sample Size}

A total of four reviewers evaluated this project.

\section{Question 1: Approach to performing the work-the degree to which technical barriers are addressed, the project is well-designed, feasible, and integrated with other efforts.}

\section{Reviewer 1:}

The reviewer reported that the objective is to develop an advanced, high-performance battery module for $12 \mathrm{~V}$ start-stop (SS) vehicle applications, in compliance with the USABC performance requirements, based on SAFT's proprietary allaluminum LTO LIB technology. The goal is to deliver to USABC $12 \mathrm{~V}$ SS module assemblies with cells in a prismatic format placed in thermoplastic module along with battery management electronics, for a cost of less than $\$ 220$. This reviewer further explained that the approach involves the use of a LTO anode, which has the advantages of high power capability, long life, and free Li plating and lithium manganese oxide (LMO) cathode. Different electrolyte blends (i.e., binary and ternary) are being examined for improved low-temperature conductivity and high-temperature stability (i.e., gassing). In parallel, continued this reviewer, a simple battery pack design is being developed with SAFT's BMS to meet the USABC targets. The reviewer concluded that the approach addresses the technical barriers, and added the project is well-designed, feasible, and integrated with other VTO projects.

\section{Reviewer 2:}

This reviewer commented that the roadmap of the work is good and covers most of the critical targets. The reviewer suggested that the only aspect missing is lifetime testing of LIB for start-stop applications, and inquired whether this is supposed to be the same as for the sealed lead acid battery of three years. This reviewer further expressed interest in whether the use of LMO as the cathode with the Mn dissolution would effect this lifetime.

\section{Reviewer 3:}

The reviewer explained that the presentation was only available to this reviewer in a poster format rather than the traditional AMR format. Thus, the reviewer could not rank this as some of the mandatory slides were missing. The

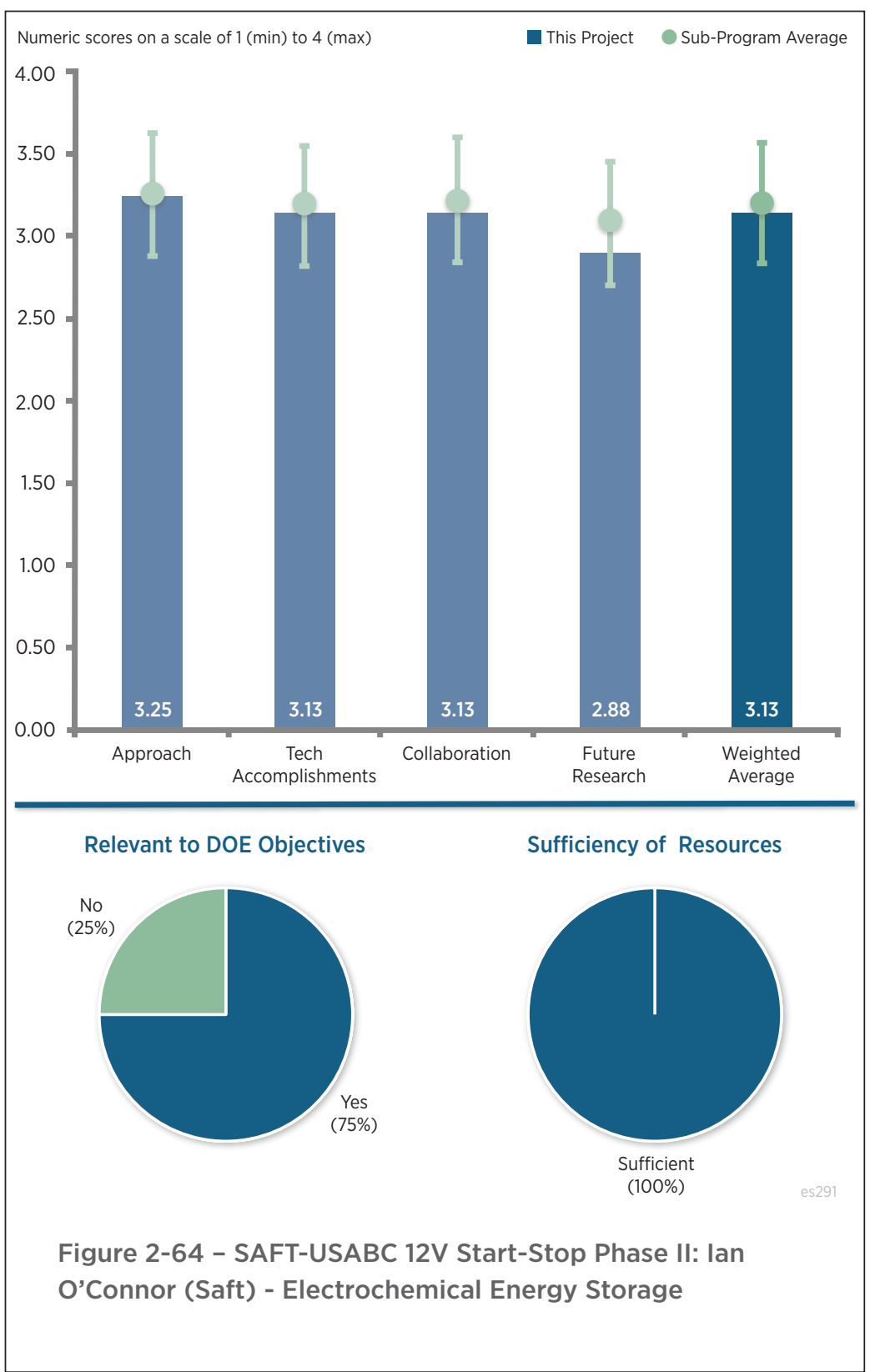

traditional AMR format. Thus, the reviewer could not rank this as some of the mandatory slides were missing. The 
reviewer checked with the project team during the poster session to determine if there was a formal template to read and evaluate.

The reviewer observed a technically sound and mature project that should be ready as a deliverable baring some issues. LTO/LMO full cell should work for this kind of start-stop application. The only issue identified by the reviewer, and also pointed out by the project team, is the electrolyte performance at $-30^{\circ} \mathrm{C}$. This could affect the cold cranking. This reviewer would have liked to have seen more data and/or results about the project team's electrolyte test results and other relevant data.

\section{Question 2: Technical accomplishments and progress toward overall project and DOE goals-the degree to which progress has been made, measured against performance indicators and demonstrated progress towards DOE goals.}

\section{Reviewer 1:}

Because the key requirement for the USABC program is the cold cranking, the reviewer indicated that much of the recent effort was focused on identifying electrolytes with good low-temperature conductivity and also high-temperature stability. Some formulations with binary solvents show improved conductivity and also low-temperature cranking, but the high-temperature gassing continued to be an issue. This reviewer stated that the addition of some additives reduced gassing, but affected the low-temperature performance. Undoubtedly, according to the reviewer, it is a challenge to find suitable electrolyte systems or low-temperature power and high-temperature stability. The reviewer asserted that SAFT's approach of electrolyte optimization is appropriate. In addition, a battery pack was designed with five prismatic cells and also contains a system control in the battery to ensure optimum performance, as well as longevity. The final module is intended to be a drop in replacement for lead acid batteries to eliminate the need for a dual battery in a start-stop system. The reviewer concluded that these accomplishments are encouraging and progress is consistent with the project objectives and DOE goals.

\section{Reviewer 2:}

This reviewer stated that measurable progress has been made towards high-power and low-temperature (e.g., $-30^{\circ} \mathrm{C}$ ) operations of LTO LIB cells.

\section{Reviewer 3:}

Although progress is good, the reviewer noted that the project timeline in terms of years is not mentioned. Therefore, the reviewer could not provide an evaluation in terms of progress towards the ultimate goals.

\section{Reviewer 4:}

This reviewer reported that the team has identified or down selected a few electrolyte compositions that are in the range of approximately $10 \mathrm{mS} / \mathrm{cm}$ at $-30^{\circ} \mathrm{C}$. That should augur well. The reviewer expressed interest in seeing some cell-level performance using those electrolytes, especially at low temperatures. Additionally, the reviewer pointed out that no technical information was provided for the project team's choice of binary and ternary solvent systems for its electrolytes.

\section{Question 3: Collaboration and coordination with other institutions.}

\section{Reviewer 1:}

This reviewer indicated that no external collaboration was specified. The reviewer added that the project seemed self-contained and did not need collaboration and coordination with other institutions.

\section{Reviewer 2:}

The reviewer reported that there were no other project partners listed.

\section{Reviewer 3:}

This reviewer assumed collaboration and coordination with other institutions were satisfactory and indicated that an evaluation could not be made because of a lack of information presented in this regard.

\section{Reviewer 4:}

The reviewer commented that there is no collaboration explicitly mentioned, but that it is possible that the project deliverables will be tested by the DOE laboratories. 
Question 4: Proposed future research-the degree to which the project has effectively planned its future work in a logical manner by incorporating appropriate decision points, considering barriers to the realization of the proposed technology and, when sensible, mitigating risk by providing alternate development pathways

\section{Reviewer 1:}

This reviewer summarized that proposed future research includes determining the root cause for gas generation in an LMO/LTO cell at high temperatures, especially in electrolytes that provide good low-temperature performance. The goal of the future studies is to prevent and/or diminish gas generation in the LMO/LTO cell design while concurrently identifying an alternative electrolyte that achieves the cold cranking requirements. The reviewer added that the eventual goal is to develop and manufacture more than 20 fully operational batteries with an integrated electronic system contained in a novel architecture, and identify a path to full commercialization.

\section{Reviewer 2:}

The reviewer commented that future research is well defined, and listed the following statements: "Moving forward, SAFT's goal is to prevent and/or diminish gas generation in the LMO/LTO cell design whilst concurrently identifying an alternative electrolyte that achieves the cold cranking requirements;" and "... develop and manufacture over 20 fully operational batteries with an integrated electronic system contained in a novel architecture, and identify a path to full commercialization."

\section{Reviewer 3:}

This reviewer noted that there was no project timeline on the poster.

\section{Reviewer 4:}

This reviewer was unable to evaluate the future work proposed because no information was presented. An assumption was made by this reviewer that proposed future work is satisfactory.

\section{Question 5: Does this project support the overall DOE objectives of petroleum displacement? Why or why not?}

\section{Reviewer 1:}

The reviewer explained that replacing conventional $12 \mathrm{~V}$ lead-acid batteries with LIBs for start-up applications will reduce battery mass and volume (by $60 \%$ ), improve service life, and reduce maintenance. The rapid recharge reduces load on the alternator because more power is retained and the charge is handled faster than lead-acid batteries. The reviewer asserted that all of these characteristics will result in reduced fuel consumption and, thus, reduced $\mathrm{CO}_{2}$ emissions. Current active materials have low specific power to support cranking, especially at low temperatures. New active materials, in conjunction with advanced electrolytes, are needed to provide lowtemperature cranking and high-temperature resilience. Also, continued this reviewer, simpler pack designs and battery management systems are essential to make the LIB a viable replacement. The reviewer stated that this project is aimed at addressing these aforementioned challenges.

\section{Reviewer 2:}

This reviewer offered that start-stop application can increase fuel efficiency by turning off the engine operation during traffic stops and/or signals.

\section{Reviewer 3:}

The reviewer indicated that displacement of sealed lead acid (SLA) batteries for start-stop applications would have minimal impacts on the use of internal combustion engines for propulsion. Additionally, this reviewer has not seen a carbon lifecycle analysis that has shown that LIBs use less carbon to produce than SLA batteries. This would be good to include, suggested the reviewer, and would allow for a project partner.

\section{Question 6: Resources: How sufficient are the resources for the project to achieve the stated milestones in a timely fashion?}

\section{Reviewer 1:}

This reviewer described resources as adequate. 
Reviewer 2:

The reviewer commented that resources are adequate for the scope of the project to meet the stated milestones in the scheduled time.

Reviewer 3:

Although funding amount was not mentioned, the reviewer explained that Saft is a large company and has multiple efforts on increasing the durability and performance of LIBs that can be leveraged for this work. 


\section{Development of Advanced High-Performance Electrolytes for Lithium-Ion Used in Vehicle Applications: Kristin Meyers (soulbrain) - es292}

\section{Presenter}

Kristin Meyers, soulbrain

\section{Reviewer Sample Size}

A total of five reviewers evaluated this project.

\section{Question 1: Approach to performing the work-the degree to which technical barriers are addressed, the project is well-designed, feasible, and integrated with other efforts.}

\section{Reviewer 1:}

The reviewer acknowledged that this is a new project and commented that there is insufficient information provided in terms of the PI's precise approach to develop high voltage electrolytes.

\section{Reviewer 2:}

Referencing the introduction, this reviewer reported that the barriers to technology adoption mention abuse tolerance, but the planned scope of work only includes overcharge testing. The reviewer noted that the pathway towards cost reduction is not addressed, though this may be addressed later in this new project.

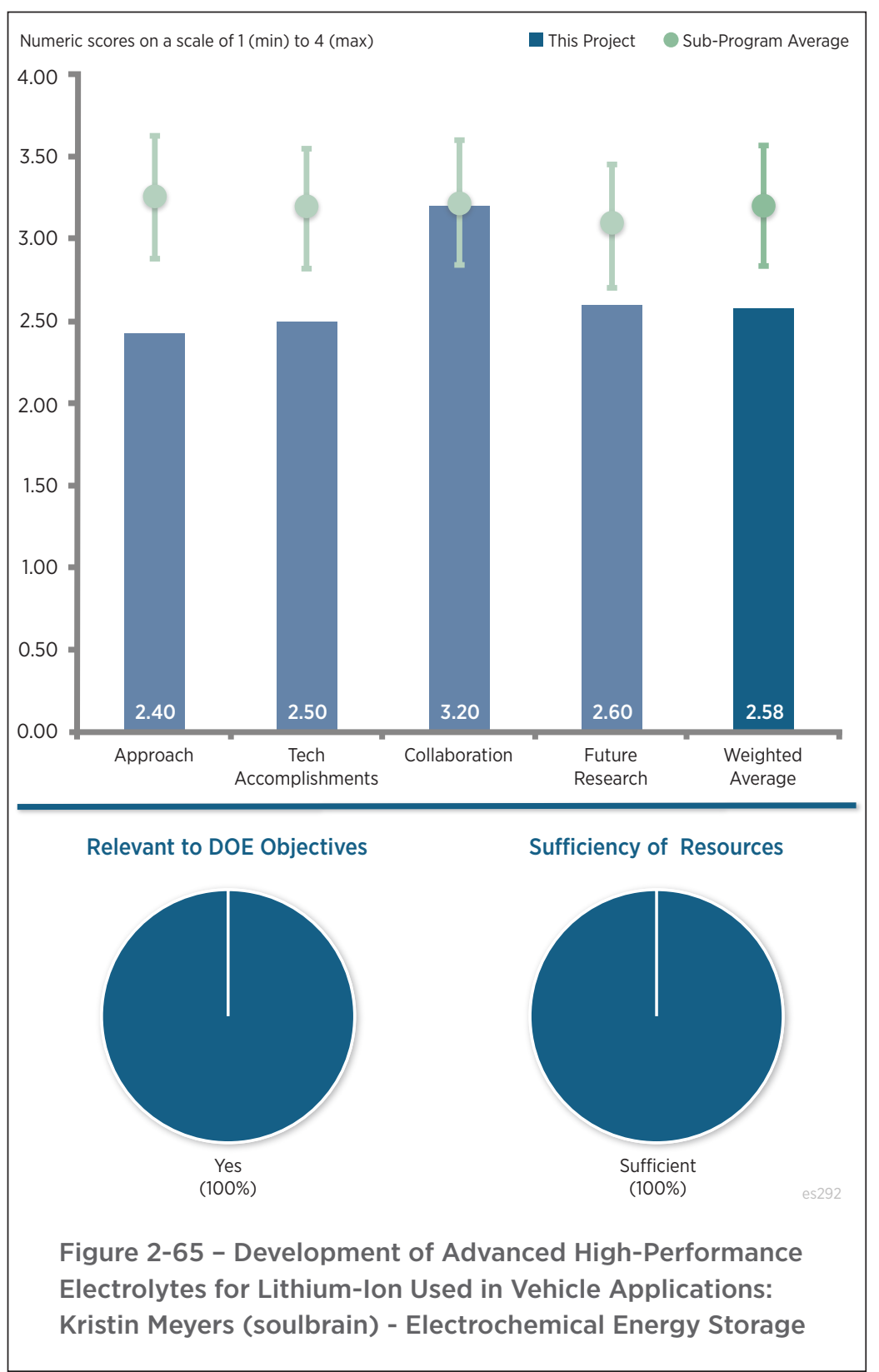

\section{Reviewer 3:}

The reviewer noted that the current cost of LIB packs is listed as $\$ 800-\$ 1,000 / \mathrm{kWh}$ in the "Barriers" section of Slide 2. The reviewer strongly expressed certainty that the price has reduced by at least a factor of 2.5 in 2016 , and recommended that this be reevaluated. It was difficult for this reviewer to appreciate the approach due to the lack of technical information. The reviewer further reported that the project team is working on various high voltage electrolyte formulation and cathodes form suppliers.

\section{Reviewer 4:}

The eventual success of the project depends on too many parameters, according to this reviewer. While the title of the project is "Development of Advanced High-Performance Electrolytes..," the reviewer observed that much of the effort is on testing NMC (from three vendors) and $\mathrm{LiNi}_{0.5} \mathrm{Mn}_{1.5} \mathrm{O}_{4}$ (from two vendors) in several electrolytes. The reviewer offered the following recommendations: the project should use one or two readily available and well-characterized high voltage cathodes and focus on developing electrolytes; the PI should disclose the scientific principle, if not the chemical composition, underlying the electrolyte development; conduct experiments on 
the effect of single wall carbon nanotube (SWCNT) conductive additives after developing a better electrolyte formulation; and the PI should justify the cost reduction chart on Slide 5 by providing the relevant data.

\section{Question 2: Technical accomplishments and progress toward overall project and DOE goals-the degree to which progress has been made, measured against performance indicators and demonstrated progress towards DOE goals.}

\section{Reviewer 1:}

Understanding that the project started less than a year ago, this reviewer indicated that the only technical progress presented was optimizing the electrode performance using conductive additives, Super $\mathrm{P}{ }^{\circledR}$ versus SWCNT. All others were observed by this reviewer to be in progress with no data yet.

\section{Reviewer 2:}

The reviewer acknowledged that the project started this year and the technical accomplishments to date would be small. Additionally, the reviewer offered that soulbrain mentioned that the reduction of conductive additive surface would reduce electrolyte oxidation, but did not show a pathway to measure the conductive additive surface area.

\section{Reviewer 3:}

This reviewer reiterated that the results provided are inadequate to judge.

\section{Reviewer 4:}

The project only started in January, and this reviewer commented that scarce data and information were given to evaluate project progress.

\section{Reviewer 5:}

Based on the scant data presented, the reviewer found that the various formulations with additives designated as "A" to "E" do not change the density and conductivity very much. Therefore, it seemed to this reviewer that an improved electrolyte has not been found. This reviewer advised that the PI should provide convincing data on improved performance before moving the project to the "Optimization of the electrolyte..." stage under "Remaining Challenges and Barriers."

\section{Question 3: Collaboration and coordination with other institutions.}

\section{Reviewer 1:}

The reviewer opined that the following partnership is good and should conclude in successful conclusion of this project: USABC for project management expertise; soulbrain for electrolyte expertise; and Iontensity for cell building and testing expertise.

\section{Reviewer 2:}

The reviewer observed that the collaboration seems to be close.

\section{Reviewer 3:}

This reviewer highlighted that no slide was presented. Therefore, this reviewer could not provide a ranking, and an assumption was made that collaboration and coordination with other institutions is satisfactory.

\section{Question 4: Proposed future research-the degree to which the project has effectively planned its future work in a logical manner by incorporating appropriate decision points, considering barriers to the realization of the proposed technology and, when sensible, mitigating risk by providing alternate development pathways}

\section{Reviewer 1:}

This reviewer looked forward to seeing the progress in the project team's high voltage electrolyte testing using various surface-modified cathodes from various suppliers.

\section{Reviewer 2:}

The reviewer described identification of the high-voltage capable cathodes as good. The project team identified the need to reduce the surface area of the conductive binder, but have not identified a way to test this. This reviewer 
suggested that a dynamic gas generation test (cell swelling, etc.) should be identified, because differential scanning calorimetry (DSC) testing is done simply on a component level, and overcharging and gassing will react on a cell level. The reviewer further suggested that a level of acceptable amount of swelling (due to gassing) should be identified.

\section{Reviewer 3:}

This reviewer commented that it was unclear what future efforts would be made, and opined that more time is needed because there is not much information to judge in the poster and slides provided.

\section{Reviewer 4:}

The reviewer strongly advised that experiments on the effect of SWCNT conductive additives should come after developing a better electrolyte formulation. It was further suggested by this reviewer that the project should use one or two readily available and well-characterized high voltage cathodes and focus on developing electrolytes.

\section{Question 5: Does this project support the overall DOE objectives of petroleum displacement? Why or why not?}

\section{Reviewer 1:}

The reviewer stated that this research topic is targeted towards electric drive train vehicles that will reduce dependence on fossil fuels.

\section{Reviewer 2:}

This reviewer explained that increasing the abuse tolerance of Li-ion batteries will help with the technology adoption, especially in vehicle technologies, which will help displace petroleum based technologies.

\section{Reviewer 3:}

The cost and performance of electrolyte is an essential part of the LIB industry, asserted this reviewer.

\section{Question 6: Resources: How sufficient are the resources for the project to achieve the stated milestones in a timely fashion?}

\section{Reviewer 1:}

Resources are adequate as observed by this reviewer.

\section{Reviewer 2:}

The reviewer commented that there should be enough technical resources from soulbrain and Iontensity to complete the project. 


\section{A Closed Loop Process for the End-of-Life Electric Vehicle Li-Ion Batteries: Yan Wang (Worcester Polytechnic Institute) - es293}

\section{Presenter}

Yan Wang, Worcester Polytechnic Institute

\section{Reviewer Sample Size}

A total of four reviewers evaluated this project.

\section{Question 1: Approach to performing the work-the degree to which technical barriers are addressed, the project is well-designed, feasible, and integrated with other efforts.}

\section{Reviewer 1 :}

The reviewer observed a unique, innovative, technical approach that appears to be promising for battery recycle.

\section{Reviewer 2:}

This reviewer asserted that this is a very cost effective approach for recycling LIBs.

\section{Reviewer 3:}

The reviewer commented that the approach used in this effort is very effective at addressing the technical barriers identified for this project. One area of concern for this effort will be the quality of the materials that are generated as part of this recycling process. The reviewer advised that this is an area that needs to be more thoroughly addressed as the program proceeds.

\section{Reviewer 4:}

The approach mentioned the need for recovery of $\mathrm{Cu}$ and aluminum, but the reviewer noted that there did not seem to be any specific work to accomplish this activity. The reviewer added that the approach to NCM recovery seemed valid.

\section{Question 2: Technical accomplishments and progress toward overall project and DOE goals - the degree to which progress has been made, measured against performance indicators and demonstrated progress towards DOE goals.}

\section{Reviewer 1:}

The reviewer stated that this effort just started and that it is somewhat premature to comment on the project team's progress. However, even at this early stage, the project team has effectively demonstrated feasibility of 
this approach at generating a highly flexible process for converting recycled batteries into usable NMC cathode material. Clearly, noted this reviewer, the next steps of improving the quality of the cathode materials generated from this process will be key.

\section{Reviewer 2:}

This reviewer indicated that, although the recovery efficiency of NCM is less than the target (greater than $80 \%$ ), the actual value was not given. Subsequently, it was not possible to evaluate progress. The reviewer further commented that the extracted product seems to be of good, but not excellent, quality.

\section{Reviewer 3:}

The reviewer reported that some preliminary progress has been achieved in less than five months and expressed interest in seeing more progress at the next AMR meeting.

\section{Reviewer 4:}

This reviewer shared that the recovery efficiency is currently 50\%, which has room for improvement. Additionally, the reviewer inquired about the recovery efficiency from the shredded pack to recovered powders, as well as how to improve the sieving process.

\section{Question 3: Collaboration and coordination with other institutions.}

\section{Reviewer 1:}

Excellent collaborations to provide cells and packs were observed by this reviewer.

\section{Reviewer 2:}

The reviewer indicated that good collaboration has been identified and partners are well coordinated.

\section{Reviewer 3:}

It was unclear to this reviewer whether the group has the expertise for synthesizing state-of-the-art NMC.

\section{Reviewer 4:}

The reviewer opined that collaboration and coordination with other institutions is one of the only weaknesses in this effort. The reviewer recommended that the program would significantly benefit from a broader collaborative team to include battery manufacturers that have a NMC or mixed metal oxide cathode as part of their base offering. This would provide the team with valuable input regarding the quality of the recycled materials that are being generated from their process and be key at identifying potential issues early in the development process.

Question 4: Proposed future research-the degree to which the project has effectively planned its future work in a logical manner by incorporating appropriate decision points, considering barriers to the realization of the proposed technology and, when sensible, mitigating risk by providing alternate development pathways

\section{Reviewer 1:}

The reviewer praised the effective future work plan and highlighted that the PI has properly identified the key challenges and areas of concern for this effort.

\section{Reviewer 2:}

This reviewer reported that the proposed future research focused on key barriers.

\section{Reviewer 3:}

The reviewer advised that actual recovery results should be plotted in future research, and pointed out that no details are given as to how the recovery process will be optimized, nor how the metals may be recovered.

\section{Question 5: Does this project support the overall DOE objectives of petroleum} displacement? Why or why not? 


\section{Reviewer 1:}

This reviewer commented that development and demonstration of LIB recycling technique is the key to fulfill DOE goals on vehicle electrification.

\section{Reviewer 2:}

This reviewer observed a highly relevant effort that addresses one of the next major issues associated with the electrification of vehicles (i.e., how one can recycle materials from spent LIBs).

Reviewer 3:

Recycling will be crucial for EV acceptance and economics, opined this reviewer.

\section{Question 6: Resources: How sufficient are the resources for the project to achieve the stated milestones in a timely fashion?}

\section{Reviewer 1:}

It seemed to this reviewer that the budget may be tight to fulfill the proposed research goals and recommended more funds in the future, if required.

\section{Reviewer 2:}

The reviewer that this effort appears to have sufficient resources, and suggested it would be strengthened with additional collaborative partners. 


\section{Computer Aided Battery Engineering Consortium: Ahmad Pesaran (National Renewable Energy Laboratory) - es294}

\section{Presenter}

Ahmad Pesaran, National Renewable Energy Laboratory

\section{Reviewer Sample Size}

A total of three reviewers evaluated this project.

\section{Question 1: Approach to performing the work-the degree to which technical barriers are addressed, the project is well-designed, feasible, and integrated with other efforts.}

\section{Reviewer 1:}

The reviewer commented that the PI has developed an outstanding approach to address the technical barriers to this program. The project is building on years of demonstrated success and is leveraging/well integrated with other efforts. This reviewer concluded that it would be very difficult to improve on the project team's plan.

\section{Reviewer 2:}

This reviewer explained that the approach to increase computational efficiency is based upon the multi-scale multi-domain (GH-MSMD) developed in CAEBAT II, which seems effective.

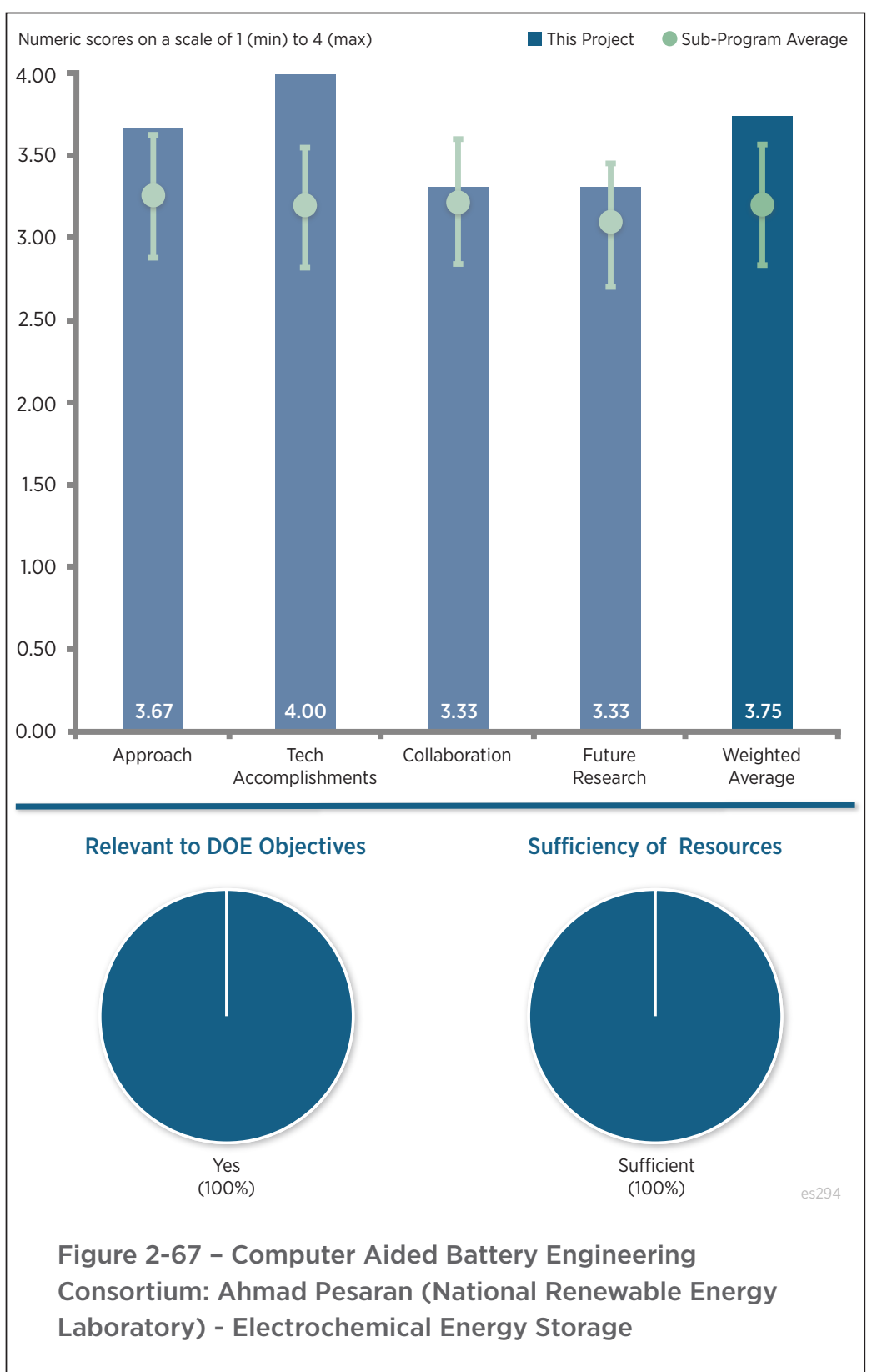

\section{Reviewer 3:}

The reviewer noted the approach involves building a front-end onto commercial solvers (e.g., Fluent) to allow for battery pack analysis. This will be very useful for automotive and battery companies, opined this reviewer, who also added that earlier versions were already incorporated into ANSYS. The reviewer asked whether it will be useful to academic researchers without access to these large commercial codes.

Question 2: Technical accomplishments and progress toward overall project and DOE goals-the degree to which progress has been made, measured against performance indicators and demonstrated progress towards DOE goals.

\section{Reviewer 1:}

Although very early, this reviewer observed considerable accomplishments and referenced Slide 12 and Slide 13. The reviewer added that the project team is in touch with major solver companies to incorporate their work. 


\section{Reviewer 2:}

This reviewer pointed out that the team has already demonstrated very good progress for each of the three tasks during the relative short duration of the program. Based on this work, it appeared very likely to the reviewer that the team will be able to achieve the stated goals for this effort.

\section{Reviewer 3:}

It appeared to this reviewer that the research has been conducted smoothly and progress has been achieved as planned so far.

\section{Question 3: Collaboration and coordination with other institutions.}

\section{Reviewer 1:}

The reviewer strongly praised the PI for having assembled an outstanding collaborative partnership with world leading institutions, which serves as a template for how to assemble a team. This reviewer particularly commended how the PI breaks out roles and responsibilities for each of the supporting organizations.

\section{Reviewer 2:}

This reviewer observed good research collaboration between the contract and the academia/industry partners. The reviewer added that the contractor has collaborated with the GM/ANSYS team on CAEBAT III, co-funded by DOE and the Army to increase the pack level combined mechanical/electrochemical/thermal modeling computational efficiency. This reviewer asked whether this CAEBAT III project can leverage that GM project.

\section{Reviewer 3:}

This person expressed concern about overlap among three groups who are doing more or less the same thing, led by ORNL, NREL, and Ford. The reviewer understood that DOE may feel it wants a competition in order to get the best results. The reviewer warned of a danger that the three groups will produce three different program sets that will be very difficult to compare, in case they do not happen to compute the same quantities. In that case, the reviewer cautioned that the community may start making incompatible predictions, which could lead to confusion and slow progress.

Of the three groups, ORNL seemed best positioned to this reviewer because ORNL is collaborating with both Ford and NREL. Ford, which wants to create a proprietary system, seemed least connected according to this reviewer. Without some overall coordination, this reviewer expressed concern that the result will be messy.

Question 4: Proposed future research-the degree to which the project has effectively planned its future work in a logical manner by incorporating appropriate decision points, considering barriers to the realization of the proposed technology and, when sensible, mitigating risk by providing alternate development pathways

\section{Reviewer 1:}

The proposed research was sound and reasonable to this reviewer.

\section{Reviewer 2:}

The reviewer conveyed that each of the tasks has a very robust and detailed future work plan. This person further suggested that an assessment of risks and corresponding mitigation strategies would be helpful to determine the viability of the approach.

\section{Reviewer 3:}

It was unclear to this reviewer that the tomographic work has been carefully planned. Referencing Slide 15 , the reviewer pointed out that the problem with measuring diffusivity is unresolved. This reviewer also noted that the problem of identifying the minimum representative volume element size has not been addressed. The reviewer added that mechanical failure is a statistical, not deterministic, process in which the presence and intensity of local flaws and/or inhomogeneities may control failure rate. Texas A\&M University, with its stochastic reconstruction 
and meso-scale physics may address this issue, but the reviewer stated that there is no detail. The reviewer agreed that crush, rather than nail-tests, are considered important.

\section{Question 5: Does this project support the overall DOE objectives of petroleum displacement? Why or why not?}

\section{Reviewer 1:}

The reviewer affirmed that this project provides a critically needed capability for the auto industry.

\section{Reviewer 2:}

The reviewer asserted that this effort is highly relevant to DOE objectives. This person added that advanced modeling and simulation $(M \& S)$ tools will accelerate the development of cost effective, advanced energy storage solutions with improved safety and performance characteristics.

\section{Reviewer 3:}

If successful, this reviewer commented that the developed tolls may help reduce battery cell development time to speed vehicle electrification.

\section{Question 6: Resources: How sufficient are the resources for the project to achieve the stated milestones in a timely fashion?}

\section{Reviewer 1:}

The reviewer indicated that sufficient funding has been provided and/or budgeted to support the success of this project.

Reviewer 2:

This effort has sufficient resources to achieve the stated milestones, asserted the reviewer. 


\section{Consortium for Advanced Battery Simulation: John Turner (Oak Ridge National Laboratory) - es295}

Presenter

John Turner, Oak Ridge National Laboratory

\section{Reviewer Sample Size}

A total of three reviewers evaluated this project.

\section{Question 1: Approach to performing the work-the degree to which technical barriers are addressed, the project is well-designed, feasible, and integrated with other efforts.}

\section{Reviewer 1:}

The approach seemed reasonable to this reviewer, who also inquired whether there is any possibility for this project to leverage other, on-going CAEBAT III.

\section{Reviewer 2:}

The reviewer observed an approach that involves building a front-end onto commercial solvers (e.g., Fluent) that allows for battery pack analysis. This will be very useful for auto and battery companies. The reviewer reported greater than 150 downloads via the website, http://batterysim.org/. The following inquiries were also made by this reviewer: whether it will be useful to academic researchers without access to these large commercial codes; the level of necessary detail to reproduce results in baseline performance modeling; and whether the project team really needs to go to DualFoil, or whether a lumped model would work, because both have adjustable parameters.

\section{Reviewer 3:}

The reviewer declared that the approach used in this effort is very effective at addressing the technical barriers identified for this project. One particular advantage to this effort is that it is building on the Open Architecture Software (OAS) and components of the Virtual Integrated Battery Environment (VIBE), developed as part of CAEBAT 1. This reviewer offered that one area of concern is the project team needs to have a more robust methodology to validate the completed models.

Question 2: Technical accomplishments and progress toward overall project and DOE goals-the degree to which progress has been made, measured against performance indicators and demonstrated progress towards DOE goals.

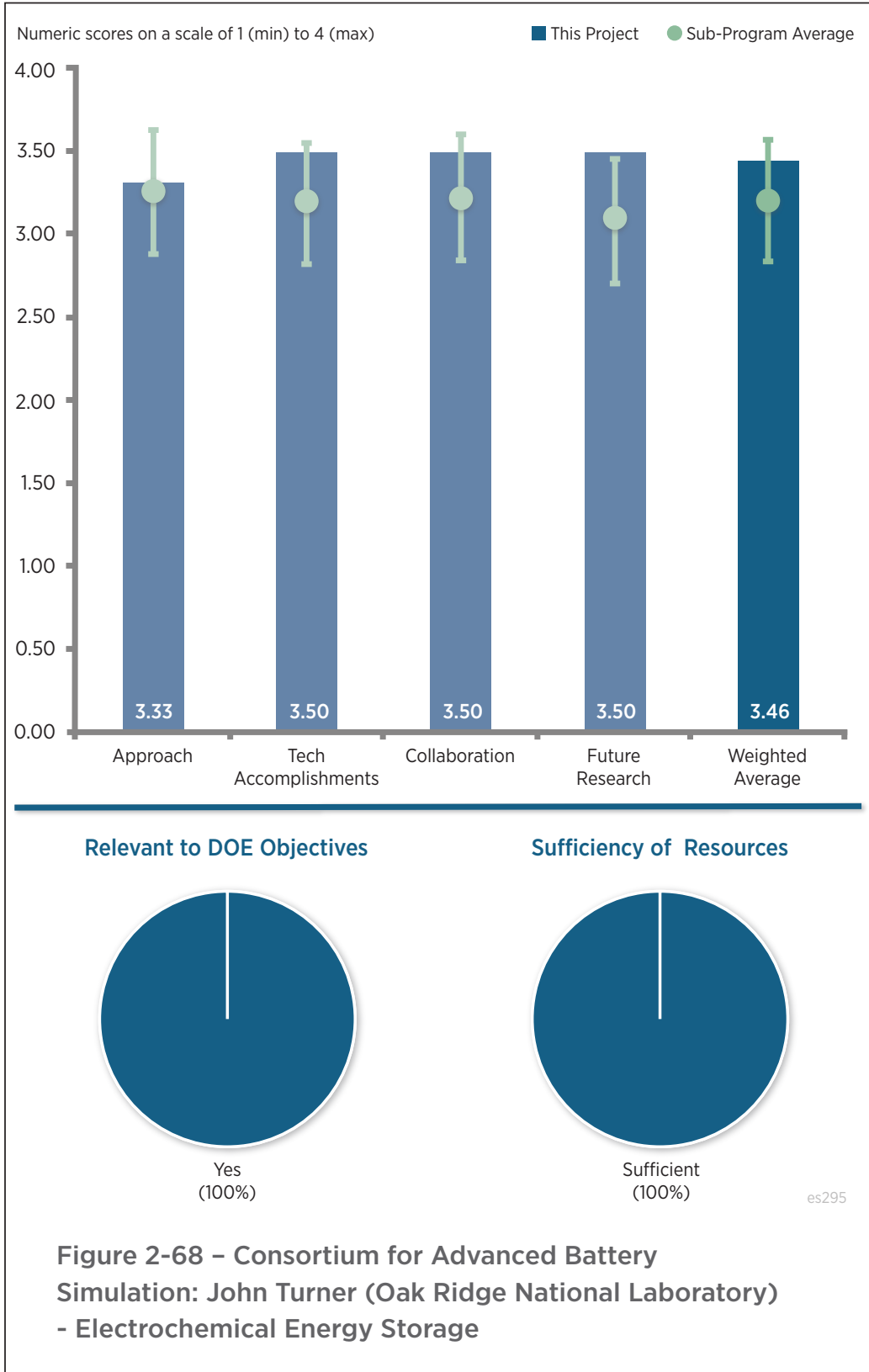




\section{Reviewer 1:}

This reviewer commented that the team appears to be making excellent progress addressing the technical barriers to this program.

\section{Reviewer 2:}

So far, this reviewer found that the research progress achieved in this project is as planned.

\section{Reviewer 3:}

The reviewer reported that the program has just begun, and opined that it is not enough to mention the modulus under "Effective properties with binder." This person inquired as to where the project team was going to get binder failure data, and whether this failure is ductile, brittle, or fatigue, for example.

\section{Question 3: Collaboration and coordination with other institutions.}

\section{Reviewer 1:}

This reviewer noted good collaboration with partners has been conducted as planned.

\section{Reviewer 2:}

The reviewer commended the PI for having done a very nice job developing collaborative relationships to meet this effort's goals. This person stated that there is a significant amount of work being conducted at Naval Surface Warfare Center (NSWC) Carderock that will be particularly helpful to validate abuse simulations.

\section{Reviewer 3:}

This person expressed concern about overlap among three groups who are doing more or less the same thing, led by ORNL, NREL, and Ford. The reviewer understood that DOE may feel it wants a competition in order to get the best results. The reviewer warned of a danger that the three groups will produce three different program sets that will be very difficult to compare, in case they do not happen to compute the same quantities. In that case, the reviewer cautioned that the community may start making incompatible predictions, which could lead to confusion and slow progress.

Of the three groups, ORNL seemed best positioned to this reviewer because ORNL is collaborating with both Ford and NREL. Without some overall coordination, this reviewer expressed concern that the result will be messy.

Question 4: Proposed future research-the degree to which the project has effectively planned its future work in a logical manner by incorporating appropriate decision points, considering barriers to the realization of the proposed technology and, when sensible, mitigating risk by providing alternate development pathways

\section{Reviewer 1:}

This reviewer indicated that future research outlined by the contractor is effective and it targets on the key barriers identified in the project team's proposal.

\section{Reviewer 2:}

The reviewer commented that a lot of work remains, particularly with this being a new start program. The reviewer added that the PI has laid out a very comprehensive plan complete with risks and mitigation strategies to address the remaining technical barriers.

\section{Reviewer 3:}

It was unclear to the reviewer that the tomographic work has been carefully planned, and noted that the problem of identifying minimum representative volume element size has not been addressed. The reviewer pointed out that mechanical failure is a statistical, not deterministic, process in which the presence and intensity of local flaws/ inhomogeneities may control failure rate. This person inquired about how this will be addressed. The reviewer opined that NMR is probably not a good way to measure diffusivity for a system where the diffusion coefficient depends strongly on concentration, which is the case with $\mathrm{Li}+$. The reviewer further explained that this is because 
the concentration of species (e.g., contact ion pairs) depends strongly on concentration, and these objects diffuse at much different rates than solvent-separated ion pairs. See work by Oleg Borodin, suggested the reviewer, who added that this issue is recognized in FY 2017 Q2, but no solution is presented.

\section{Question 5: Does this project support the overall DOE objectives of petroleum displacement? Why or why not?}

\section{Reviewer 1:}

The reviewer opined that this supports DOE's EV Everywhere goals by enabling increases in energy density, specific energy, and power while maintaining safety.

\section{Reviewer 2:}

The reviewer offered that this effort is highly relevant to DOE objectives, and that advanced M\&S tools will accelerate the development of cost effective advanced energy storage solutions with improved safety and performance characteristics.

\section{Reviewer 3:}

The reviewer asserted that this project provides a critically needed capability for the auto industry.

\section{Question 6: Resources: How sufficient are the resources for the project to achieve the stated milestones in a timely fashion?}

\section{Reviewer 1:}

It appeared to this reviewer that there are sufficient resources to address the technical barriers for this program.

\section{Reviewer 2:}

None were noted by this reviewer. 
Development and Validation

of a Simulation Tool to Predict

the Combined Structural, Electrical, Electrochemical, and Thermal Responses

of Automotive Batteries: James Marcicki (Ford Motor Company) - es296

\section{Presenter}

James Marcicki, Ford Motor Company

\section{Reviewer Sample Size}

A total of three reviewers evaluated this project.

\section{Question 1: Approach to} performing the work-the degree to which technical barriers are addressed, the project is well-designed, feasible, and integrated with other efforts.

\section{Reviewer 1:}

This reviewer commended the approach used in this effort as very effective at addressing the technical barriers identified for this project. The reviewer further highlighted that one area of particular strength for this approach is the robust validation plan that has been put together.

\section{Reviewer 2:}

The reviewer reported that the approach involves building a front-end onto LS-DYNA that allows for battery pack analysis, and added that this is a very useful thing to do.

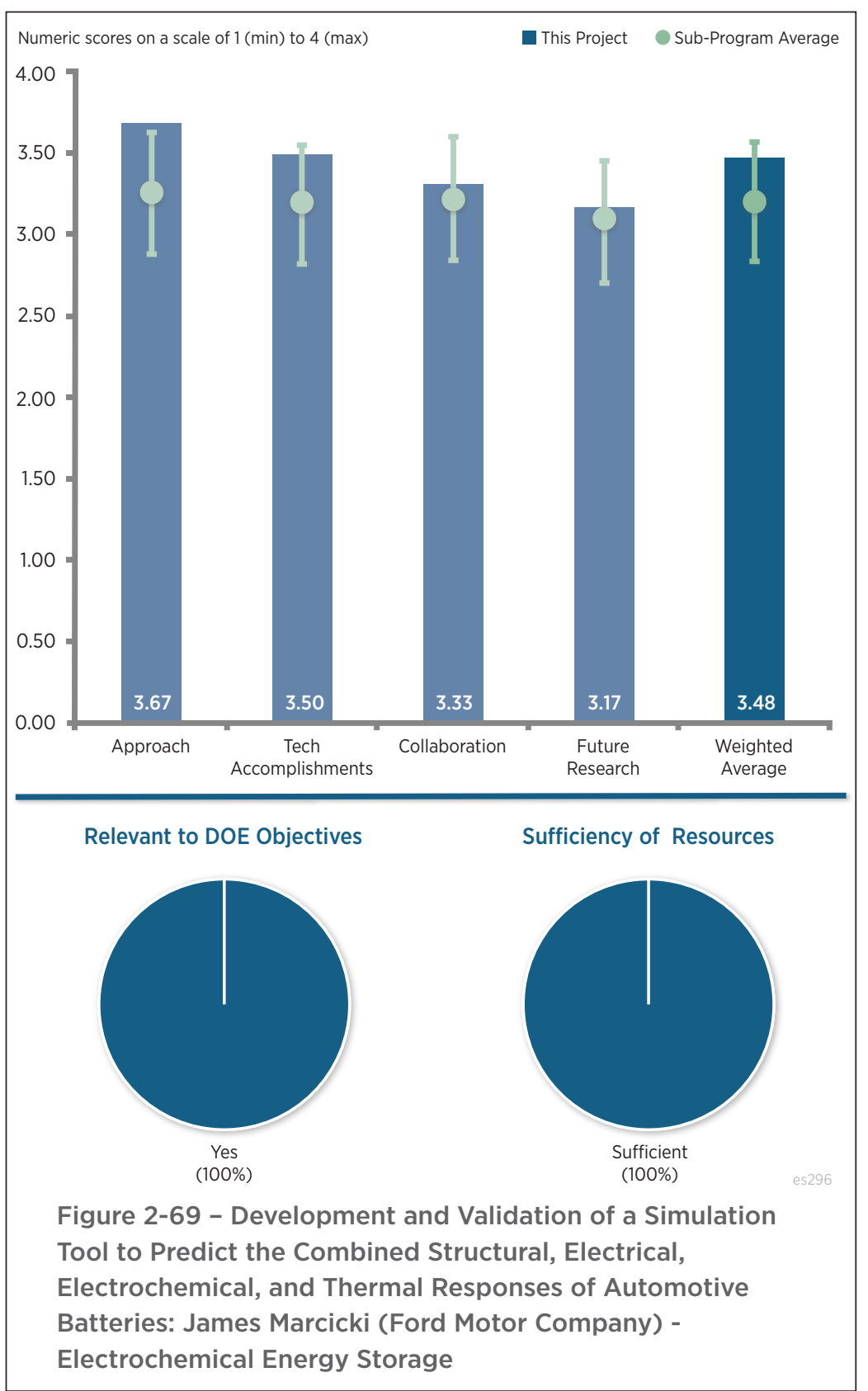

\section{Reviewer 3:}

This reviewer stated that the approach addressed the key barriers to understanding battery tolerance under mechanical abuse.

Question 2: Technical accomplishments and progress toward overall project and DOE goals-the degree to which progress has been made, measured against performance indicators and demonstrated progress towards DOE goals.

\section{Reviewer 1:}

This reviewer observed impressive technical achievement given that this project was started about four months ago. 


\section{Reviewer 2:}

The reviewer pointed out that this is a new start program that began January 2016, and the team is already making very good progress developing and validating the initial models needed to complete this effort.

\section{Reviewer 3:}

This reviewer noted that the project is new, and few solid accomplishments are described compared to those in the NREL and ORNL projects.

\section{Question 3: Collaboration and coordination with other institutions.}

\section{Reviewer 1:}

The reviewer stated that good collaboration appeared to be occurring and asked whether the modes to be developed in this project can fit into OAS.

\section{Reviewer 2:}

This reviewer commented that the PI has assembled an effective collaborative team addressing the challenges associated with this effort. The reviewer suggested that collaboration with NSWC Carderock may be useful because it collects a significant amount of abuse data.

\section{Reviewer 3:}

This person expressed concern about overlap among three groups who are doing more or less the same thing, led by ORNL, NREL, and Ford. The reviewer understood that DOE may feel it wants a competition in order to get the best results. The reviewer warned of a danger that the three groups will produce three different program sets that will be very difficult to compare, in case they do not happen to compute the same quantities. In that case, the reviewer cautioned that the community may start making incompatible predictions, which could lead to confusion and slow progress.

Of the three groups, ORNL seemed best positioned to this reviewer because ORNL is collaborating with both Ford and NREL. Without some overall coordination, this reviewer expressed concern that the result will be messy.

\section{Question 4: Proposed future research-the degree to which the project has effectively planned its future work in a logical manner by incorporating appropriate decision points, considering barriers to the realization of the proposed technology and, when sensible, mitigating risk by providing alternate development pathways}

\section{Reviewer 1:}

The reviewer commented that a lot of work remains given that this is a new start program. This person further observed that the PI has laid out a very comprehensive plan to complete the work, with a particular strength being in the validation plan. The reviewer suggested that providing more info on the risks and approaches to mitigate the risks would be one area that may strengthen future work. This person found the critical issues and risks discussion to be very useful, and also recommended that additional discussion/consideration about how abuse testing is very dependent on the cell chemistry and cell design, as well as on the test condition, would be very useful.

\section{Reviewer 2:}

This reviewer suggested having battery validation in several different temperatures (such as one at low temperature) to represent vehicle environment.

\section{Reviewer 3:}

The reviewer noted that the problem of identifying minimum representative volume element size has not been addressed. The reviewer explained that mechanical failure is a statistical, not deterministic, process in which the presence and intensity of local flaws/inhomogeneities may control failure rate, and inquired about how this will be addressed. 
Question 5: Does this project support the overall DOE objectives of petroleum displacement? Why or why not?

Reviewer 1:

This reviewer found this effort to be highly relevant to DOE objectives. Advanced M\&S tools, especially tools to increase abuse tolerance during crash systems will accelerate the development of cost effective advanced energy storage solutions for electrified vehicle platforms.

\section{Reviewer 2:}

The goals of this project support the overall DOE objective for vehicle electrification, observed this reviewer.

\section{Reviewer 3:}

The reviewer asserted that this project provides a critically needed capability for the auto industry.

Question 6: Resources: How sufficient are the resources for the project to achieve the stated milestones in a timely fashion?

\section{Reviewer 1:}

It appeared to this reviewer that there are sufficient resources allocated to this project in order to achieve its goals.

\section{Reviewer 2:}

The reviewer perceived that the team has sufficient resources to achieve the stated milestones. 


\section{Acronyms and Abbreviations}

3D

$\mathrm{ABR}$

$\mathrm{AC}$

ADP

$\mathrm{Ah}$

ALD

$\mathrm{AlF}_{3}$

AMR

ANL

ARK

ARL

ARPA-E

ASI

ATR

BATT

BCMS

BES

BMR

BMS

BNL

$\mathrm{BOM}$

$\mathrm{C}$

CAD

CAEBAT

CAM

CAM-7

CAMP

$\mathrm{CC}$

$\mathrm{CC} / \mathrm{S}$

$\mathrm{CE}$

Co

$\mathrm{CO}_{2}$

$\mathrm{CoS}_{2}$

$\mathrm{Cu}$

$\mathrm{CuF}_{2}$

$\mathrm{CV}$

DME

DoD

DOE

DPP

DSC

DST
Three Dimensional

Advanced Battery Research

Alternating current

Advanced drying process

Ampere-hour

Atomic Layer Deposition

Aluminum fluoride

Annual Merit Review

Argonne National Laboratory

Abuse Reaction Kinetics

Army Research Lab

Advanced Research Projects Agency - Energy

Area Specific Impedance

Attenuated Total Reflectance

Batteries for Advanced Transportation Technologies

Battery/Capacitor Management System

Office of Basic Energy Sciences

Battery Materials Research

Battery Management System

Brookhaven National Laboratory

Bill of Materials

Carbon

Computer-aided design

Computer-aided engineering of batteries

Cathode Active Material

Proprietary cathode material for Li-ion batteries

Cell Analysis, Modeling, and Prototyping

Constant Current

Carbon Sulfur Composite

Coulombic Efficiency

Cobalt

Carbon Dioxide

A Cobalt-Sulfide Cattierite

Copper

Copper (II) Fluoride

Cyclic Voltammetry

Dimethoxyethane

Depth of Discharge

U.S. Department of Energy

Dynamic particle-packing

Differential Scanning Calorimetry

Dynamic Stress Test 


\begin{tabular}{|c|c|}
\hline $\mathrm{EC}$ & Ethylene Carbonate \\
\hline EIS & Electrochemical Impedance Spectroscopy \\
\hline EOL & End-of-Life \\
\hline EPD & Electrophoretic Deposition \\
\hline EUCAR & European Council for Automotive R\&D \\
\hline $\mathrm{EV}$ & Electric Vehicle \\
\hline FCC & First Cycle Capacity \\
\hline FCE & First Cycle Efficiency \\
\hline $\mathrm{Fe}$ & Iron \\
\hline $\mathrm{FeF}_{3}$ & Iron Fluoride \\
\hline $\mathrm{FeS}_{2}$ & Iron Sulfide \\
\hline FGC & Full Gradient Cathode \\
\hline FY & Fiscal Year \\
\hline GM & General Motors \\
\hline $\mathrm{Gr} / \mathrm{S}$ & Graphite/Sulfur \\
\hline HA & High-Active \\
\hline HCMR & High Capacity Manganese Rich \\
\hline $\mathrm{HEV}$ & Hybrid Electric Vehicle \\
\hline ICL & Initial capacity loss \\
\hline IL & Ionic Liquid \\
\hline INL & Idaho National Laboratory \\
\hline IR & Infrared \\
\hline $\mathrm{IrO}_{2}$ & Iridium Oxide \\
\hline $\mathrm{kg}$ & Kilogram \\
\hline LBNL & Lawrence Berkeley National Laboratory \\
\hline LCP & Lithium Cobalt Phosphate \\
\hline LFO & Lithium Iron Oxide \\
\hline LFP & Lithium Iron Phosphate \\
\hline LFP & Iron Phosphate \\
\hline $\mathrm{Li}$ & Lithium \\
\hline $\mathrm{Li}_{2} \mathrm{CO}_{3}$ & Lithium Carbonate \\
\hline $\mathrm{Li}_{2} \mathrm{O}$ & Lithium Oxide \\
\hline $\mathrm{Li}_{2} \mathrm{~S}$ & Lithium Sulfide \\
\hline LIB & Lithium Ion Battery \\
\hline LiBOB & Lithium bis(oxalate)borate \\
\hline LIC & Lithium-ion Conductor \\
\hline $\mathrm{LiCoO}_{2}$ & Lithium Cobalt Oxide \\
\hline $\mathrm{LiFePO}_{4}$ & Lithium-Iron Phosphate \\
\hline LiFSI & Lithium Bis(flurosulfonyl)mide \\
\hline Li-ion & Lithium Ion \\
\hline $\mathrm{LiO}_{2}$ & Lithium Oxygen \\
\hline $\mathrm{LiPF}_{6}$ & Effective electrolyte salt for lithium-ion battery \\
\hline Li-S & Lithium-Sulfur \\
\hline
\end{tabular}




\begin{tabular}{|c|c|}
\hline LiTFSI & Lithium Bis(Trifluoromethanesulfonyl)Imide \\
\hline LMNO & Lithium-Manganese Nickel Oxide \\
\hline $\mathrm{LMO}$ & Lithium Manganese Oxide \\
\hline LMR & Lithium Manganese Rich \\
\hline LNMO & Lithium Nickel Manganese Oxide \\
\hline LS-DYNA & Non-linear finite element analysis software program \\
\hline LTO & Lithium Titanium Oxide \\
\hline $\mathrm{M} \& \mathrm{~S}$ & Modeling and Simulation \\
\hline MERF & Materials Engineering Research Facility \\
\hline MFCA & Multifunctional Cathode Additives \\
\hline MFx & Metal Fluoride \\
\hline $\mathrm{Mg}$ & Magnesium \\
\hline $\mathrm{Mn}$ & Manganese \\
\hline MSMD & Multi-Scale Multi-Domain \\
\hline $\mathrm{Na}$ & Sodium \\
\hline $\mathrm{NaOH}$ & Sodium hydroxide \\
\hline NCA & Battery cathode material (nickel cobalt aluminum oxide) \\
\hline NCA & Nickel Cobalt Aluminum \\
\hline NCM & Nickel Cobalt Manganese \\
\hline ND & Neutron diffraction \\
\hline NDE & Non-Destructive Evaluation \\
\hline $\mathrm{Ni}$ & Nickel \\
\hline NMC & Nickel Manganese Cobalt oxide \\
\hline NMO & Nickel Manganese Oxide \\
\hline NMP & N-Methylpyrrolidone \\
\hline NMR & Nuclear Magnetic Resonance \\
\hline NREL & National Renewable Energy Laboratory \\
\hline NSWC & Naval Surface Warfare Center \\
\hline $\mathrm{O}_{2}$ & Oxygen \\
\hline OAS & Open Architecture Software \\
\hline OEM & Original equipment manufacturer \\
\hline ORNL & Oak Ridge National Laboratory \\
\hline ORR & Oxygen Reduction Reaction \\
\hline OS & Organosilicon \\
\hline PAA & Polyacrylic Acid \\
\hline $\mathrm{PE}$ & Polyethylene \\
\hline PEO & Polyethylene oxide \\
\hline PEV & Plug-in Electric Vehicle \\
\hline PFM & Poly(9,9-dioctylfuorene-co-9-fluorenone-co-methybenzoic ester \\
\hline PHEV & Plug-In Hybrid Electric Vehicle \\
\hline PI & Principal Investigator \\
\hline PNNL & Pacific Northwest National Laboratory \\
\hline $\mathrm{PP}$ & Polypropylene \\
\hline
\end{tabular}




\begin{tabular}{|c|c|}
\hline PPy & Polypyrrole \\
\hline PS & Polysulfide \\
\hline PVDF & Polyvinylidene Difluoride \\
\hline RASIC & Responsible, Approving, Supporting, Informed, and Consulted \\
\hline $\mathrm{R} \& \mathrm{D}$ & Research and Development \\
\hline $\mathrm{S}$ & Sulfur \\
\hline SAE & Society of Automotive Engineers \\
\hline SBIR & Small Business Innovation Research \\
\hline SE & Selenium \\
\hline SEI & Solid Electrolyte Interface \\
\hline SEI & Solid Electrolyte Interface \\
\hline SEM & Scanning Electron Microscope \\
\hline SEO & Symmetric Polystyrene-block-poly (ethylene oxide) \\
\hline $\mathrm{Si}$ & Silicon \\
\hline $\mathrm{Si}-\mathrm{C}$ & Silicon Carbon \\
\hline SIMS & Secondary ion mass spectrometry \\
\hline SLA & Sealed Lead Acid \\
\hline SNL & Sandia National Laboratories \\
\hline SOA & Semiconductor Optical Amplifier \\
\hline $\mathrm{SOC}$ & State of Charge \\
\hline SS & Start/Stop \\
\hline SSEs & Solid State Electrolytes \\
\hline SUS & Stainless Steel \\
\hline SWCNT & Single Wall Carbon Nanotube \\
\hline $\mathrm{Ti}$ & Titanium \\
\hline $\mathrm{TiS}_{2}$ & Titanium Disulfide \\
\hline TOF & Time of flight \\
\hline U.S. DRIVE & $\begin{array}{l}\text { United States Driving Research and Innovation for Vehicle efficiency and Energy } \\
\text { sustainability }\end{array}$ \\
\hline UN & United Nations \\
\hline USABC & U.S. Advanced Battery Consortium \\
\hline USCAR & U.S. Council for Automotive Research \\
\hline UV & Ultraviolet \\
\hline V & Vanadium \\
\hline V & Volt \\
\hline VFM & Variable Frequency Microwaves \\
\hline VIBE & Virtual Integrated Battery Environment \\
\hline VTO & Vehicle Technologies Office \\
\hline Wh & Watt hour \\
\hline XAS & X-ray Absorption Spectroscopy \\
\hline
\end{tabular}




\title{
3. Electric Drive Technologies
}

\author{
Electric drive technologies (EDT), including the electric motor, inverter, boost converter, and on- \\ board charger, are essential components of hybrid and plug-in electric vehicle (PEV) propulsion \\ systems. The Vehicle Technologies Office (VTO) supports research and development (R\&D) to \\ reduce the cost and improve the performance of innovative electric drive devices, components, and \\ systems.
}

Reducing the cost of electric drive vehicles (EDVs) is essential for increasing consumer adoption and meeting the EV Everywhere Grand Challenge goal. EV Everywhere is a Clean Energy Grand Challenge for PEVs that are as affordable and convenient for the American family as gasoline-powered vehicles by 2022 .

VTO funds research to advance EDT in two key areas:

- Power electronics (PE); and

- Electric motors.

VTO funds research on EDT to:

- Reduce cost, weight, and volume;

- Improve performance, efficiency, and reliability;

- Develop innovative modular and scalable designs; and

- Improve manufacturability and accelerate commercialization.

Within these areas, VTO focuses on:

- Wide bandgap (WBG) devices for power electronics;

- Advanced motor designs to reduce or eliminate rare earth (RE) materials;

- Novel packaging for power electronics and electric motors;

- Improvements in thermal management and reliability; and

- Integration of power electronics functions.

VTO works extensively with a number of different organizations. The EDT subprogram supports a number of unique user facilities at the national laboratories. Within the U.S. Department of Energy (DOE), the office collaborates with the Office of Science, Advanced Research Projects Agency-Energy (ARPA-e), and the Clean Energy Manufacturing Initiative Across the federal government, the EDT subprogram works with:

- The National Network for Manufacturing Innovation;

- The Interagency Advanced Power Group; and

- The U.S. Army Tank, Automotive Research and Development and Engineering Center (TARDEC) in the U.S. Department of Defense.

Much of the subprogram's research is conducted in sync with industry partners through:

- The United States Driving Research and Innovation for Vehicle efficiency and Energy sustainability (U.S. DRIVE) Partnership focusing on light-duty vehicles; and

- The $21^{\text {st }}$ Century Truck Partnership, focusing on heavy-duty vehicles.

As described in the EV Everywhere Blueprint, the major goals of the subprogram are to reduce the cost of electric drive systems by half and decrease their volume by one-third by 2022 . 


\section{Subprogram Feedback}

The U.S. Department of Energy (DOE) received feedback on the overall technical subprogram areas presented during the 2016 Annual Merit Review (AMR). Each subprogram technical session was introduced with a presentation that provided an overview of subprogram goals and recent progress, followed by a series of detailed topic area project presentations.

The reviewers for a given subprogram area responded to a series of specific questions regarding the breadth, depth, and appropriateness of that DOE VTO subprogram's activities. The subprogram overview questions are listed below, and it should be noted that no scoring metrics were applied. These questions were used for all VTO subprogram overviews.

Question 1: Was the program area, including overall strategy, adequately covered?

Question 2: Is there an appropriate balance between near- mid- and long-term research and development?

Question 3: Were important issues and challenges identified?

Question 4: Are plans identified for addressing issues and challenges?

Question 5: Was progress clearly benchmarked against the previous year?

Question 6: Are the projects in this technology area addressing the broad problems and barriers that the Vehicle Technologies Office (VTO) is trying to solve?

Question 7: Does the program area appear to be focused, well-managed, and effective in addressing VTO's needs?

Question 8: What are the key strengths and weaknesses of the projects in this program area? Do any of the projects stand out on either end of the spectrum?

Question 9: Do these projects represent novel and/or innovative ways to approach these barriers as appropriate?

Question 10: Has the program area engaged appropriate partners?

Question 11: Is the program area collaborating with them effectively?

Question 12: Are there any gaps in the portfolio for this technology area?

Question 13: Are there topics that are not being adequately addressed?

Question 14: Are there other areas that this program area should consider funding to meet overall programmatic goals?

Question 15: Can you recommend new ways to approach the barriers addressed by this program area?

Question 16: Are there any other suggestions to improve the effectiveness of this program area?

Responses to the subprogram overview questions are summarized in the following pages. Individual reviewer comments for each question are identified under the heading Reviewer 1, Reviewer 2, etc. Note that reviewer comments may be ordered differently; for example, for each specific subprogram overview presentation, the reviewer identified as Reviewer 1 in the first question may not be Reviewer 1 in the second question, etc. 


\title{
Overview of the VTO Electric Drive Technologies Program: Susan Rogers (U.S. Department of Energy) - edt000
}

\author{
Question 1: Was the program area, including overall strategy, adequately covered? \\ Reviewer 1: \\ The reviewer agreed that all of the activities of the program were adequately covered. The reviewer also \\ commented that the strategic goals of the program were very clearly presented and that industry engagement was \\ mentioned. One recommendation made by the reviewer is to more explicitly describe how the value chain of \\ advanced EDT components is being developed.
}

\section{Reviewer 2:}

The reviewer stated that it was a good overview that covered the area adequately.

\section{Reviewer 3:}

The reviewer replied yes to this question.

\section{Reviewer 4:}

The reviewer observed that the vehicle program covers a comprehensive set of areas including the traction machines, traction inverters, and battery charger, and that the traction machine area covers new materials, new machine topologies, and packaging. The reviewer also noted that the power converter area covers the application of next generation WBG semiconductors, thermal management, and additive manufacturing of power converter heat sinks.

\section{Question 2: Is there an appropriate balance between near- mid- and long-term research and development?}

\section{Reviewer 1:}

The reviewer agreed that the program has a balanced near-, mid-, and long-term R\&D plan to achieve the goals in cost reduction, power density, and energy saving. This reviewer also noted greater adoption of hybrid electric vehicle (HEV), plug-in hybrid electric vehicle (PHEV), and battery electric vehicle (BEV) technology.

\section{Reviewer 2:}

The reviewer replied yes to this question.

\section{Reviewer 3:}

The reviewer commented that the research portfolio is centered at a point between mid- and long-term research. The reviewer also observed that near-term research is represented, particularly in the advanced manufacturing of capacitors and power electronics, and that the mid-term focus on advanced packaging for WBG components is appropriate. The reviewer recommended some focus on long-term WBG components, for example, the impact of the next generation of semiconductors beyond silicon carbide $(\mathrm{SiC})$ and gallium nitride $(\mathrm{GaN})$ on EDT. This would be a good opportunity to integrate the results of advanced WBG work funded by ARPA-E.

\section{Reviewer 4:}

The reviewer remarked that the near- and-mid-term research goals up to 2022 were well identified and that the balanced approach to research topic areas and funding is an effective approach and creates a good balance of technologies. However, the reviewer stated that long term (i.e., far out, hard to achieve) research was not identified, adding that while general statements were made for looking forward statements, there was not a path mapped or specific research identified to get there.

\section{Question 3: Were important issues and challenges identified?}

\section{Reviewer 1:}

The reviewer stated that the program identified the following major challenges: cost and size reduction of traction 
motor (using non-rare earth [NREL]) materials, new materials for laminations, and thermal management), packaging, and thermal management of power converters using WBG devices and their reliability.

\section{Reviewer 2:}

The reviewer agreed that important issues and challenges were identified, adding that cost was the largest concern area identified.

\section{Reviewer 3:}

The reviewer replied yes to this question.

\section{Reviewer 4:}

The reviewer remarked that the focus on reducing system cost was clear and appropriate with improved manufacturability a complementary goal. However, the reviewer commented that less emphasis was explicitly placed on system and component reliability although it was mentioned. The reviewer's recommendation would be to identify system end-of-life management (recycling and/or remanufacturing) as an important challenge.

\section{Question 4: Are plans identified for addressing issues and challenges?}

\section{Reviewer 1:}

The reviewer commented that the roadmap for achieving the 2022 targets was clear and very well presented, and that it provided an appropriately-graded strategy that segmented the development needs for component technologies into an appropriate timeline. The reviewer offered that the rates of improvement in the system performance metrics that are needed to meet the 2022 targets are aggressive.

\section{Reviewer 2:}

The reviewer replied yes to this question.

\section{Reviewer 3:}

The reviewer observed that the program plans to engage national laboratories, universities, original equipment manufacturers (OEMs), and suppliers to tackle the above-mentioned challenges through benchmarking and new technology development and verification.

\section{Reviewer 4:}

The reviewer stated that the plan to address the issues and challenges was to engage the right team focusing including research and manufacturing, in conjunction with educating engineers at OEMs who are making design choices. The team includes national laboratories, universities, and manufacturers.

\section{Question 5: Was progress clearly benchmarked against the previous year?}

\section{Reviewer 1:}

The reviewer stated that progress was clearly stated against the previous year including the cost reduction and power density improvement.

\section{Reviewer 2:}

The reviewer replied yes to this question.

\section{Reviewer 3:}

The reviewer commented that progress was benchmarked against the overall system development activities that have been undertaken since 1995 and added that a specific benchmark of progress made against the 2014 achievements was not observed.

\section{Reviewer 4:}

The reviewer indicated that benchmarking at the low level (individual year by year) was not presented, although a history of past achievements was presented at a macro level to show that progress is being made longer term. 


\section{Question 6: Are the projects in this technology area addressing the broad problems and barriers that the Vehicle Technologies Office (VTO) is trying to solve?}

\section{Reviewer 1:}

The reviewer affirmed that there is good progress being made to solve broad problems and barriers with the goal to have affordable, higher efficiency vehicles for the mass market through electrification.

\section{Reviewer 2:}

The reviewer stated that this program is centered on meeting part of the technological challenges identified in the EV Everywhere Grand Challenge and agreed that the scope of EDT is appropriately focused on electric machine and power electronics systems and component technologies.

\section{Reviewer 3:}

The reviewer replied yes to this question.

\section{Reviewer 4:}

The reviewer remarked that the projects in this technology area contribute to the VTO from the aspect of electric drive train technology including the traction electric machines, traction power converters, and battery chargers.

\section{Question 7: Does the program area appear to be focused, well-managed, and effective in addressing VTO's needs?}

\section{Reviewer 1:}

The reviewer agreed that the project's focus is appropriate for VTO goals and objectives, adding that the management team is experienced and has been effective in managing the research portfolio over the past several years.

\section{Reviewer 2:}

The reviewer indicated that the program area is effective in addressing VTO's needs in vehicle electric drivetrain technology.

\section{Reviewer 3:}

The reviewer replied yes to this question.

\section{Reviewer 4:}

The reviewer stated that the program appears to be well managed and effectively advancing the agenda.

\section{Question 8: What are the key strengths and weaknesses of the projects in this program area? Do any of the projects stand out on either end of the spectrum?}

\section{Reviewer 1:}

The reviewer identified involvement with OEMs as one of the key strengths, adding that the technology is being adopted by industry and really stands out going into production (i.e., 2016 Chevy Volt). The reviewer stated that three-dimensional (3D) printing of cold plates also stands out, as it is a low risk, high potential gain item. Thermal management is one of the challenges identified and historically has been a challenge for power electronics. The reviewer further specified that new approaches like 3D printing to optimize the heat flow are needed and stand out.

\section{Reviewer 2:}

The reviewer described as a key strength the program's effective integration of research projects at national laboratories with those demonstration activities done at companies. This close interaction would help speed up technology commercialization. 


\section{Reviewer 3:}

The reviewer emphasized two key strengths in this program area as the demonstration of advanced power electronic systems that incorporate the latest in advanced manufacturing as well as the latest in WBG devices. The reviewer also identified the development and demonstration of advanced motor designs that use less RE elements as another notable success. The reviewer suggested revisiting the focus on RE reduction as a program goal, pointing out that the rare-earth supply crisis has abated, due in no small part to programs such as this one that have produced system- and component-level substitutes. The reviewer stated that it might be appropriate to expand the focus to include more critical elements beyond REs that could possibly be used in EDT systems. The benefit might be to anticipate and avoid future critical material supply shocks.

\section{Reviewer 4:}

The reviewer listed the following two key strengths: existing projects cover many important challenges (e.g., cost and size reduction of motors and power converters); and good collaboration is going on between collaborators). The key weakness observed by the reviewer is that little effort is given to the control of machines and power converters.

\section{Question 9: Do these projects represent novel and/or innovative ways to approach these barriers as appropriate?}

\section{Reviewer 1:}

The reviewer praised these projects as having had a proven track record of investigating and supporting novel approaches to a level where reasonable extrapolations of their system level performance can be made. The reviewer described the projects as having shown a disciplined approach towards redirecting program focus to the most promising areas and demonstrating them at the benchtop or test-stand level.

\section{Reviewer 2:}

The reviewer offered that the projects represent innovative approaches to the barriers, such as 3D printing components to optimize weight and thermal management.

\section{Reviewer 3:}

The reviewer agreed that most of the projects are innovative.

\section{Question 10: Has the program area engaged appropriate partners?}

\section{Reviewer 1:}

The reviewer stated the program area has a good mix of national laboratories, academia, and industry.

\section{Reviewer 2:}

The reviewer characterized the program area as having engaged appropriate partners including industry, national laboratories, and universities.

\section{Reviewer 3:}

The reviewer replied yes to this question.

\section{Reviewer 4:}

The reviewer agreed that the program area has identified and engaged the appropriate partners among the national laboratories, academia, OEMs, and Tier 1, 2, and 3 suppliers. The reviewer recommended an increased focus on promoting the formation of supply chains at the component to sub-system level and remarked that the focus on WBG packaging is a good example that could be emulated in other parts of the EDT portfolio.

\section{Question 11: Is the program area collaborating with them effectively?}




\section{Reviewer 1:}

The reviewer affirmed that the program area is collaborating effectively with its partners, describing the technologies being developed as proven to be effective (both from a technical and cost standpoint) and transitioned to production (e.g., the Chevy Volt).

\section{Reviewer 2:}

The reviewer agreed that the program area is effectively collaborating with all of the partner organizations, adding that the list of research highlights was encouraging and shows the progress the program area is making across several fronts.

\section{Reviewer 3:}

The reviewer replied yes to this question.

\section{Reviewer 4:}

The reviewer stated that collaborating partners have adequate communication going on with each other.

\section{Question 12: Are there any gaps in the portfolio for this technology area?}

\section{Reviewer 1:}

The reviewer stated that the portfolio seems to broadly cover the technology sufficiently.

\section{Reviewer 2:}

The reviewer replied yes to this question.

\section{Reviewer 3:}

The reviewer remarked that, as noted, there may need to be more focus on developing the supply chain at the component to sub-system level.

\section{Reviewer 4:}

The reviewer cautioned that the electromagnetic interference (EMI) and electromagnetic compatibility (EMC) issues do not seem to be given enough attention in this program.

\section{Question 13: Are there topics that are not being adequately addressed?}

\section{Reviewer 1:}

The reviewer replied no to this question.

\section{Reviewer 2:}

The reviewer cautioned that system end-of-life considerations such as recycling and/or remanufacturing of EDT components may warrant more investigation. The reviewer also indicated that the impact of the evolving electric vehicle (EV) market may require more investigation in the future. In particular, the possibility of self-driving vehicles and the future growth of car-sharing services may change the balance of system performance priorities.

\section{Reviewer 3:}

The reviewer remarked that the high temperature range and power density metrics are a little weak. The reviewer said that the individual DOE projects and Society of Automotive Engineers (SAE) design practices suggest engine compartment components should be designed for 145 Celsius $\left({ }^{\circ} \mathrm{C}\right)$ to $150^{\circ} \mathrm{C}$ ambient temperatures. The reviewer recommended helping to identify the operating conditions for the system rather than just the junction temperatures of discrete components. The reviewer noted that junction temperatures are an important enabling technology to get to the end goals (especially integrated power electronics on motors), but that the real design requirements are having the larger operating environment defined (coolant temperatures and ambient). The reviewer stated that it was not readily obvious in the presentation, but in conjunction with other presentations, the power density metrics are unclear. Other presentations refer to these power density metrics as being relatively short duration peak 
requirements. The reviewer explained that power density goals should be stated for continuous operation (hours, not a couple of seconds), or at a minimum for both continuous and peak, adding that these would be really good goals if they are for continuous operation.

\section{Reviewer 4:}

The reviewer did not see anything related to the controls of machines and power converters.

\section{Question 14: Are there other areas that this program area should consider funding to meet overall programmatic goals?}

\section{Reviewer 1:}

The reviewer judged the program as currently reasonably funded to achieve the proposed work and goals.

\section{Reviewer 2:}

The reviewer replied no to this question.

\section{Reviewer 3:}

The reviewer stated that the program should also consider adding low temperatures $\left(-40^{\circ} \mathrm{C}\right.$ to $\left.-50^{\circ} \mathrm{C}\right)$ requirements in conjunction with high temperatures because some of the design choices chosen to address high temperatures affect low temperature operation. The reviewer pointed out that SAE temperature ranges have a relatively large range to address the Upper Midwest snowbelt states and Alaska.

\section{Reviewer 4:}

The reviewer suggested that the program area may wish to evaluate the risk posed by other critical materials in the supply chain for EDT systems and component technology beyond RE ones. The reviewer stated that coordination with the Critical Materials Institute may identify future supply chain risks that may be avoided if prudent investments are made in substitute technologies.

\section{Question 15: Can you recommend new ways to approach the barriers addressed by this program area?}

\section{Reviewer 1:}

The reviewer observed that there may be older technologies that could be used for conventional low-cost electronics that are being overlooked because they were hard to control previously. The reviewer noted new ways to cascade, control, and cool conventional electronics and to reach the cost goals quicker than with WBG. The reviewer said that micro machines integrated in the heat sink used to create closed loop air conditioning (hot side going to an engine temperature cooling loop) may be able to cool conventional electronics more effectively than thermal electric and other methods.

\section{Reviewer 2:}

The reviewer stated that coordination with the Vehicles Systems (VS) subprogram can uncover challenges that the evolving EV market may place on EDT systems and components. In particular, the development of self-driving vehicles and car-sharing services may place unforeseen challenges on EDT systems and components.

\section{Reviewer 3:}

The reviewer said no.

Question 16: Are there any other suggestions to improve the effectiveness of this program area?

\section{Reviewer 1:}

The reviewer stated that this comment is for the effectiveness of the presentation rather than the program area. The 
reviewer urged that for the presentation, review the questions that reviewers will be reviewing, make sure they are all covered, and make it blatantly obvious.

\section{Reviewer 2:}

The reviewer had no other suggestions.

Reviewer 3:

The reviewer replied no to this question. 


\section{Project Feedback}

In this merit review activity, each reviewer was asked to respond to a series of questions, involving multiple-choice responses, expository responses where text comments were requested, and numeric score responses (on a scale of 1.0 to 4.0). In the pages that follow, the reviewer responses to each question for each project will be summarized: the multiple choice and numeric score questions will be presented in graph form for each project, and the expository text responses will be summarized in paragraph form for each question. A table presenting the average numeric score for each question for each project is presented below.

\section{Table 3-1 - Project Feedback}

\begin{tabular}{|c|c|c|c|c|c|c|c|}
\hline Presentation Title & $\begin{array}{c}\text { Principal } \\
\text { Investigator } \\
\text { and } \\
\text { Organization }\end{array}$ & $\begin{array}{l}\text { Page } \\
\text { Number }\end{array}$ & Approach & $\begin{array}{c}\text { Technical } \\
\text { Accomplishments }\end{array}$ & Collaborations & $\begin{array}{l}\text { Future } \\
\text { Research }\end{array}$ & $\begin{array}{l}\text { Weighted } \\
\text { Average }\end{array}$ \\
\hline $\begin{array}{l}\text { Benchmarking } \\
\text { EV and HEV } \\
\text { Technologies }\end{array}$ & $\begin{array}{l}\text { Burress, Tim } \\
\text { (ORNL) }\end{array}$ & $3-13$ & 3.64 & 3.79 & 3.57 & 3.50 & 3.69 \\
\hline $\begin{array}{c}\text { Development } \\
\text { of Radically } \\
\text { Enhanced alnico } \\
\text { Magnets (DREaM) } \\
\text { for Traction Drive } \\
\text { Motors }\end{array}$ & $\begin{array}{c}\text { Anderson, Iver } \\
\text { (Ames) }\end{array}$ & $3-17$ & 3.58 & 3.42 & 3.50 & 3.42 & 3.47 \\
\hline $\begin{array}{l}\text { North American } \\
\text { Supply Chain for } \\
\text { Traction Motors } \\
\text { and PE }\end{array}$ & $\begin{array}{l}\text { Whaling, } \\
\text { Christopher } \\
\text { (Synthesis } \\
\text { Partners) }\end{array}$ & $3-21$ & 3.00 & 2.67 & 3.17 & 2.50 & 2.79 \\
\hline $\begin{array}{c}\text { Advanced } \\
\text { Packaging } \\
\text { Technologies and } \\
\text { Designs } \dagger\end{array}$ & $\begin{array}{l}\text { Liang, } \\
\text { Zhenxian } \\
\text { (ORNL) }\end{array}$ & $3-23$ & 3.13 & 3.25 & 3.00 & 3.13 & 3.17 \\
\hline $\begin{array}{l}\text { Electric Drive } \\
\text { Inverter Research } \\
\text { and Development }\end{array}$ & $\begin{array}{l}\text { Chinthavali, } \\
\text { Madhu (ORNL) }\end{array}$ & $3-26$ & 3.33 & 3.08 & 3.25 & 3.08 & 3.17 \\
\hline $\begin{array}{c}\text { Innovative } \\
\text { Technologies for } \\
\text { Converters and } \\
\text { Chargers } \dagger\end{array}$ & $\begin{array}{l}\text { Su, Gui-Jia } \\
\text { (ORNL) }\end{array}$ & $3-29$ & 3.36 & 3.36 & 3.21 & 3.21 & 3.32 \\
\hline
\end{tabular}




\begin{tabular}{|c|c|c|c|c|c|c|c|}
\hline Presentation Title & $\begin{array}{c}\text { Principal } \\
\text { Investigator } \\
\text { and } \\
\text { Organization }\end{array}$ & $\begin{array}{l}\text { Page } \\
\text { Number }\end{array}$ & Approach & $\begin{array}{c}\text { Technical } \\
\text { Accomplishments }\end{array}$ & Collaborations & $\begin{array}{c}\text { Future } \\
\text { Research }\end{array}$ & $\begin{array}{c}\text { Weighted } \\
\text { Average }\end{array}$ \\
\hline $\begin{array}{l}\text { Advanced Low- } \\
\text { Cost SiC and GaN } \\
\text { Wide Bandgap } \\
\text { Inverters for } \\
\text { Under-the-Hood } \\
\text { Electric Vehicle } \\
\text { Traction Drives }\end{array}$ & $\begin{array}{c}\text { Olejniczak, } \\
\text { Kraig (APEI } \\
\text { Inc.) }\end{array}$ & $3-32$ & 3.83 & 3.83 & 3.67 & 3.50 & 3.77 \\
\hline $\begin{array}{l}\text { High Temperature } \\
\text { DC-Bus Capacitor } \\
\text { Cost Reduction } \\
\text { and Performance } \\
\text { Improvements }\end{array}$ & $\begin{array}{c}\text { Yializis, } \\
\text { Angelo (Sigma } \\
\text { Technologies } \\
\text { International) }\end{array}$ & $3-35$ & 3.83 & 3.83 & 3.33 & 3.33 & 3.71 \\
\hline $\begin{array}{l}\text { High-Performance } \\
\text { DC Bus Film } \\
\text { Capacitor }\end{array}$ & $\begin{array}{l}\text { Tan, Dan } \\
\text { (General } \\
\text { Electric) }\end{array}$ & 3-38 & 3.33 & 3.33 & 3.50 & 3.17 & 3.33 \\
\hline $\begin{array}{l}\text { Advanced Electric } \\
\text { Motor Research }\end{array}$ & $\begin{array}{l}\text { Burress, Tim } \\
\text { (ORNL) }\end{array}$ & $3-41$ & 3.25 & 3.25 & 3.38 & 3.00 & 3.23 \\
\hline $\begin{array}{c}\text { Performance } \\
\text { and Reliability } \\
\text { of Bonded } \\
\text { Interfaces for } \\
\text { High-Temperature } \\
\text { Packaging }\end{array}$ & $\begin{array}{l}\text { DeVoto, Doug } \\
\text { (NREL) }\end{array}$ & $3-45$ & 3.30 & 3.20 & 3.50 & 3.00 & 3.24 \\
\hline $\begin{array}{l}\text { Electric Motor } \\
\text { Thermal } \\
\text { Management } \\
\text { Research and } \\
\text { Development }\end{array}$ & $\begin{array}{l}\text { Bennion, Kevin } \\
\text { (NREL) }\end{array}$ & $3-48$ & 3.50 & 3.67 & 3.50 & 3.50 & 3.58 \\
\hline $\begin{array}{c}\text { High-Efficiency } \\
\text { High-Density } \\
\text { GaN-Based } 6.6 \\
\text { kW Bidirectional } \\
\text { On-Board Charger } \\
\text { for PEVs }\end{array}$ & $\begin{array}{l}\text { Zhu, Charles } \\
\text { (Delta } \\
\text { Products } \\
\text { Corporation) }\end{array}$ & 3-51 & 3.25 & 3.50 & 3.25 & 3.25 & 3.38 \\
\hline $\begin{array}{c}\text { Gate Driver } \\
\text { Optimization for } \\
\text { WBG Applications }\end{array}$ & $\begin{array}{c}\text { Ericson, Nance } \\
\text { (ORNL) }\end{array}$ & 3-55 & 3.20 & 3.50 & 3.40 & 3.30 & 3.39 \\
\hline
\end{tabular}




\begin{tabular}{|c|c|c|c|c|c|c|c|}
\hline Presentation Title & $\begin{array}{c}\text { Principal } \\
\text { Investigator } \\
\text { and } \\
\text { Organization }\end{array}$ & $\begin{array}{l}\text { Page } \\
\text { Number }\end{array}$ & Approach & $\begin{array}{c}\text { Technical } \\
\text { Accomplishments }\end{array}$ & Collaborations & $\begin{array}{l}\text { Future } \\
\text { Research }\end{array}$ & $\begin{array}{l}\text { Weighted } \\
\text { Average }\end{array}$ \\
\hline $\begin{array}{c}\text { Power Electronics } \\
\text { Thermal } \\
\text { Managment } \\
\text { Research and } \\
\text { Development }\end{array}$ & $\begin{array}{c}\text { Moreno, } \\
\text { Gilbert (NREL) }\end{array}$ & $3-58$ & 3.33 & 3.00 & 3.00 & 3.00 & 3.08 \\
\hline $\begin{array}{c}\text { Thermal } \\
\text { Performance } \\
\text { Benchmarking } \dagger\end{array}$ & $\begin{array}{l}\text { Feng, Xuhui } \\
\text { (NREL) }\end{array}$ & $3-60$ & 3.38 & 3.25 & 3.13 & 3.13 & 3.25 \\
\hline $\begin{array}{l}\text { Electric Motor } \\
\text { Performance } \\
\text { Improvement } \\
\text { Techniques } \dagger\end{array}$ & $\begin{array}{l}\text { Tang, Lixin } \\
\text { (ORNL) }\end{array}$ & $3-63$ & 3.50 & 3.25 & 3.25 & 3.25 & 3.31 \\
\hline $\begin{array}{c}88 \text { Kilowatt } \\
\text { Automotive } \\
\text { Inverter with New } \\
900 \text { Volt Silicon } \\
\text { Carbide MOSFET } \\
\text { Technology }\end{array}$ & $\begin{array}{c}\text { Casady, } \\
\text { Jeffrey (Cree) }\end{array}$ & $3-67$ & 3.67 & 3.83 & 3.67 & 3.33 & 3.71 \\
\hline Overall Average & & & 3.41 & 3.39 & 3.35 & 3.20 & 3.37 \\
\hline
\end{tabular}

† denotes poster presentation. 


\section{Benchmarking EV and HEV Technologies: Tim Burress (Oak Ridge National Laboratory) - edt006}

\section{Presenter}

Tim Burress, Oak Ridge National Laboratory

\section{Reviewer Sample Size}

A total of seven reviewers evaluated this project.

\section{Question 1: Approach to performing the work-the degree to which technical barriers are addressed, the project is well-designed, feasible, and integrated with other efforts.}

\section{Reviewer 1:}

The reviewer recognized that this project provides valuable information about state-of-the-art EDT that can be used to improve EV and HEV systems in the United States. This person commented that the approach has proven effective on several other systems.

\section{Reviewer 2:}

This reviewer noted it was good that both the motor dyno tests and chassis dyno tests were used to benchmarking the new vehicle products.

\section{Reviewer 3:}

The reviewer acknowledged that this project provides a resource to U.S. researchers to help them understand the state of the commercial art.

\section{Reviewer 4:}

This reviewer stated that the approach to baseline the system with the same controls allows some standardized comparisons between systems. This person noted there should be some standard tests/metrics to try and level the systems such as long term continuous power capability (i.e., two hours plus), instead of just manufacturer's specifications. The reviewer expressed it would also be very relevant and useful to check the devices out as a system using the manufacturer's controls. This person pointed out the manufacturer may be trading off efficiency on one component (i.e., the motor), but getting better efficiency with the combination of motor, inverter, and controls. At a minimum this reviewer remarked the project should spot check a few data points with the manufacturer's controls, even if it is not a full duplication of the characterization.

\section{Reviewer 5:}

The reviewer praised the approach as a logical method that should lead to detailed knowledge of the selected

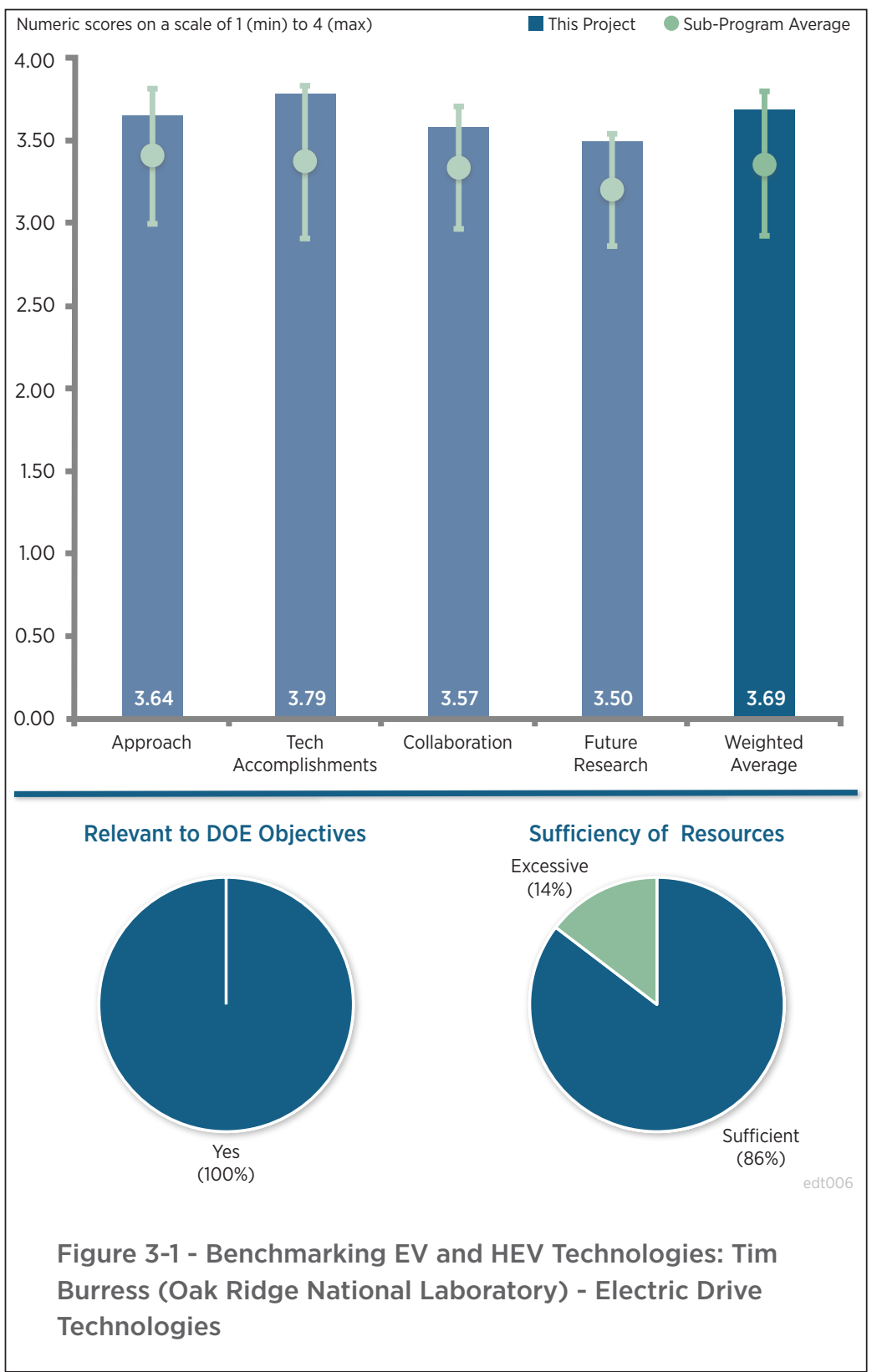


system. This person noted the high level description of the selection process was fine; however, how the final decision was made as to which two vehicles were selected in this case lacks detail. The reviewer remarked the vehicle testing followed by the tear down analysis was excellent; however, the reviewer suggested it would be beneficial to have a ruler or some other method of providing a size guide in the photos as well as some correlation between the vehicle performance testing and what is seen on the dyno testing. This person acknowledged that the motor controls used for the dyno testing may not be the same as those used in the vehicle.

\section{Question 2: Technical accomplishments and progress toward overall project and DOE goals-the degree to which progress has been made, measured against performance indicators and demonstrated progress towards DOE goals.}

\section{Reviewer 1:}

The reviewer confirmed the benchmarking work is detailed and impressive as was finding the new hardware designs and performance of the two vehicle models under benchmark.

\section{Reviewer 2:}

This reviewer stated the program is effectively validating and testing manufacturer's known specs and characterizing the unknown performance.

\section{Reviewer 3:}

The reviewer remarked that results from the remaining tests on the 2014 Honda Accord were presented, along with initial results on the $2016 \mathrm{BMW}$ i3. This person found the efficiency plots and details on motor and inverter construction methods very helpful.

\section{Reviewer 4:}

This reviewer confirmed the Honda Accord was nearly done, and the BMW i3 was in progress, which appeared on track. For the Honda Accord, this person suggested that the report include data on the boost direct current (DC/DC) such as inductor size, core, and DC-DC converter efficiency measurements.

\section{Reviewer 5:}

The reviewer claimed the progress was very good, noting that trying to re-engineer a control/communication system is not a simple task nor is adapting a motor from a transmission into a housing suitable for dyno use. This person observed that the comparison chart continues to grow as new vehicles are tested and added. The reviewer suggested it might be interesting to provide a chart comparing and showing the changes to the Prius system/ performance from the earliest to the latest as well as a quick comparison to the other Toyota vehicles. While the task appears concentrated on the performance of the electrical propulsion system and the various technologies used in it, the reviewer pondered other aspects of the system/vehicle performance, such as EMC performance of the vehicle, and asked whether it meets Federal Motor Vehicle Safety Standards (FMVSS) standards or are performed as part of the vehicle testing. The reviewer also had questions about the analysis and whether any of it has been performed showing the benefits of the various cooling system designs in terms of reliability or junction temperatures over the various drive cycles tested.

\section{Question 3: Collaboration and coordination with other institutions.}

\section{Reviewer 1:}

The reviewer stated there is good collaboration with Argonne National Laboratory (ANL), National Renewable Energy Laboratory (NREL), Electrical and Electronics Tech Team (EETT), and the U.S. DRIVE Vehicle Systems Analysis Tech Team (VSATT).

\section{Reviewer 2:}

The reviewer noted the degree of collaboration seems appropriate for this project. 


\section{Reviewer 3:}

This reviewer acknowledged the team has improved its workflow in response to reviewer comments.

\section{Reviewer 4:}

This reviewer recognized that the results indicate that the collaboration between the team members/organization seems to be working very well. The person offered a comparison of the Oak Ridge National Laboratory (ORNL) component performance data to that taken at the vehicle level would help show the level of collaboration as well as the impact of the components on the overall vehicle's performance. This person wondered if data are available related to the types of materials used in the construction of the chassis and how it performs in the field, in terms of corrosion resistance.

\section{Question 4: Proposed future research-the degree to which the project has effectively planned its future work in a logical manner by incorporating appropriate decision points, considering barriers to the realization of the proposed technology and, when sensible, mitigating risk by providing alternate development pathways.}

\section{Reviewer 1:}

This reviewer declared the BMW i3, the LEAF, and new Prius are good choices.

\section{Reviewer 2:}

The reviewer ascertained there is a plan to test additional systems such as the next-generation Prius. This person confirmed the specific vehicles have not been narrowed down, but there is a plan.

\section{Reviewer 3:}

The reviewer indicated the BMW i3 will be the focus for the remainder of Fiscal Year (FY) 2016 while specific systems for FY 2017 and FY 2018 have not yet been determined.

\section{Reviewer 4:}

This reviewer confirmed the proposed future research continues the process of testing leading vehicles in the market place. This person pondered whether there are any plans for investigating start-stop systems or EVs or if this is project limited to just hybrids. The reviewer wondered if the interface methods/components are being investigated such as high-voltage (HV)/high current connectors.

\section{Reviewer 5:}

Continuing the question about the implementation of double-side cooled power modules, it will be useful to this reviewer to have quantifiable data or metrics showing the benefits and impacts of the packaging technology on the electric drive system.

\section{Question 5: Does this project support the overall DOE objectives of petroleum displacement? Why or why not?}

\section{Reviewer 1:}

The reviewer confirmed this work evaluates the performance and manufacturing methods of state-of-the-art EV and HEV systems. This person acknowledged that this project allows DOE to gauge how close the systems are to the DOE targets and provides insight into how manufacturers can reach those targets.

\section{Reviewer 2:}

This reviewer said that characterizing the technologies that exist helps to gauge the effectiveness of fuel reduction technologies. The person commented that characterization also helps to understand how much room there is left for improvement on these technologies or for example whether the fundamental limit has been approached.

\section{Reviewer 3:}

The reviewer confirmed this project is very relevant to what the competition is doing to solve basically the same issue. By comparing different model years of the same vehicle, this person suggested it should be possible to determine growth in the technology used as well as future trends when comparing the various vehicles. This 
reviewer pointed out the work should be useful for planning future products and the technology required to successfully compete in the market place.

\section{Question 6: Resources: How sufficient are the resources for the project to achieve the stated milestones in a timely fashion?}

\section{Reviewer 1:}

This reviewer claimed the team definitely has the best facility in the United States to conduct the proposed work.

\section{Reviewer 2:}

The reviewer confirmed the project seems to have sufficient resources to execute.

\section{Reviewer 3:}

This reviewer expressed the project appears to have sufficient resources.

\section{Reviewer 4:}

The reviewer concluded the resources on this project are sufficient based on the results achieved. This person cautioned whether any additional resources would significantly reduce the time required to perform the testing and analysis, but it might reduce the time required to prepare the units for testing in the ORNL lab by some small amount; however, it is probably not worth the cost.

\section{Reviewer 5:}

This reviewer suggested more resources might allow more and quicker teardowns. total of seven reviewers evaluated this project. 


\section{Development of Radically Enhanced alnico Magnets (DREaM) for Traction Drive Motors: Iver Anderson (Ames Laboratory) - edt015}

\section{Presenter}

Matt Kramer, Ames Laboratory

\section{Reviewer Sample Size}

A total of six reviewers evaluated this project.

\section{Question 1: Approach to performing the work-the degree to which technical barriers are addressed, the project is well-designed, feasible, and integrated with other efforts.}

\section{Reviewer 1:}

This reviewer confirmed the emphasis on simulation and theoretical analysis was the right approach to address the technical barriers. The sample test results proved this approach effective.

\section{Reviewer 2:}

The reviewer indicated the project is being very systematic and doing the right things to understand the physics of the barriers. The work is well focused on what is needed to be done to advance the technology.

\section{Reviewer 3:}

The reviewer claimed the approach was well thought out and reasonable.

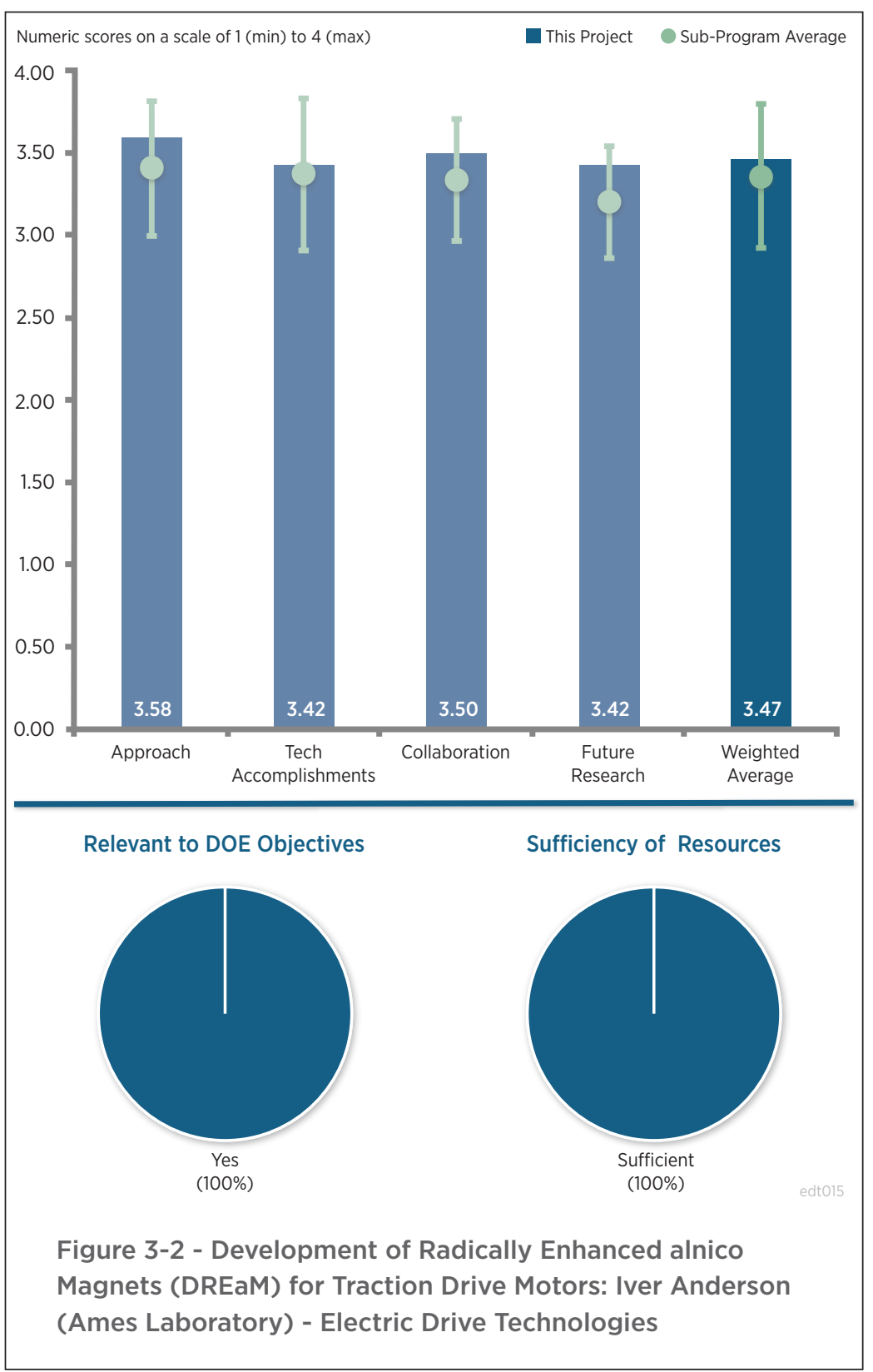

\section{Reviewer 4:}

The reviewer observed great details in understanding magnet chemistry and their impact on the magnet physical properties.

\section{Reviewer 5:}

The reviewer said the team is taking a good approach focused on improved aluminum-nickel-cobalt (AlNiCo) as a replacement for RE permanent magnets (PMs) in traction motors. This reviewer wondered, though, if the goals are narrowly focused on the specific requirements of the UQM Technologies, Inc., motor. It appears, from industry comments at the review, that higher coercivity (4,000-4,500 Oersteds [Oe]) with high energy density may be required for practical applications. The reviewer suggested it will be good to show a line of sight to getting there, even if the current project does not attain these values.

Question 2: Technical accomplishments and progress toward overall project and DOE goals-the degree to which progress has been made, measured against performance indicators and demonstrated progress towards DOE goals. 


\section{Reviewer 1:}

The reviewer commended the comprehensive multi-scale modeling in optimization of magnets as the major accomplishment, and noted it definitely serves the DOE goals by speeding up the magnetics improvements process.

\section{Reviewer 2:}

The reviewer applauded model development to speed design as a welcomed accomplishment, providing fundamental insights. The synthesis of magnetic material demonstrates process understanding and control.

\section{Reviewer 3:}

The reviewer expressed the project is steadily improving the characteristics of AlNiCo magnets. There is a lot of great research being performed, and the progress made was at an appropriate level of technical detail. This person agreed the historical progression was well presented. Programmatically (that is reporting), the project indicated there were intermediate goals, but did not present what the intermediate goals were. The reviewer voiced that it appears there are multiple iterations being performed for optimization, but it is unclear how many iterations are expected and what timeline is expected for the intermediate steps. Verbally, the presenter indicated the end goal was an intrinsic coercivity $\left(\mathrm{H}_{\mathrm{ci}}\right)$ of around 4,000-4,500, but it was unclear how long the project was expecting to get there (hard to track on a future timeline).

\section{Reviewer 4:}

The reviewer reinforced the team shared good progress, but it was not clear if the projected energy density with high coercivity has been obtained. It sounded like UQM will fabricate their motor with commercially available AlNiCo magnets. This reviewer cautioned this sounds like a lost opportunity to demonstrate the new magnets within an actual motor.

\section{Reviewer 5:}

This reviewer declared it is good that the team has been able to increase the coercivity of the proposed AlNiCobased magnet materials for the past year. It would be good for the team to give a theoretical prediction on the maximum coercivity that the materials can eventually achieve. This person warned if the coercivity of the material cannot meet the requirements of automotive grade magnets, the risk of magnet demagnetization can be a major barrier to use these materials.

\section{Reviewer 6:}

This reviewer brought to light the marginal improvement in AlNiCo magnet's coercivity in the latest grade presented with respect to the last year's presentation. The target of greater than 2,500 Oe is still not enough to meet some of the less thermally challenged automotive applications.

\section{Question 3: Collaboration and coordination with other institutions.}

\section{Reviewer 1:}

The reviewer expressed there is excellent collaboration on this project that includes motor manufacturers, a magnet manufacturer (supplier), universities, ORNL, and NREL.

\section{Reviewer 2:}

This reviewer confirmed there was a comprehensive team with significant engagement from all parties.

\section{Reviewer 3:}

This reviewer indicated good collaboration took place, although not much was addressed in the presentation, probably due to the time constraints.

\section{Reviewer 4:}

The reviewer mentioned the team appears to be collaborating broadly. One addition this reviewer recommended is to also consider partnering with motor developers that can take advantage of AlNiCo's high-temperature performance to reduce overall system level cost.

Question 4: Proposed future research-the degree to which the project has effectively planned its future work in a logical manner by incorporating appropriate decision points, 


\section{considering barriers to the realization of the proposed technology and, when sensible, mitigating risk by providing alternate development pathways.}

\section{Reviewer 1:}

This reviewer commented the future path to advancing the technology is well thought out and very systematic. It is very likely the barrier issues will be overcome given enough time.

\section{Reviewer 2:}

This reviewer ascertained the team is clearly focused on the stated goals of achieving about a two times increase in energy density of AlNiCo at $180^{\circ} \mathrm{C}$. The team feels confident this can be achieved by improving grain alignment and reducing the iron-cobalt $(\mathrm{Fe}-\mathrm{Co})$ needle diameters.

\section{Reviewer 3:}

The reviewer noted the remaining tasks are formulated well and the approach to overcoming the remaining barriers is clear.

\section{Reviewer 4:}

This reviewer declared Ames National Laboratory has a clear understanding of the need for improvements in coercivity of AlNiCo magnet. It will be very helpful in the future presentation if the magnetic hysteresis curve (B$\mathrm{H})$ of their latest grades are included and compared for various grade and Neo magnets. The reviewer suggested the tradeoffs between lower magnetic remanence $\left(\mathrm{B}_{\mathrm{r}}\right)$ and higher coercivity should also be studied

\section{Reviewer 5:}

The reviewer expressed concern over how this work results in a solution for automotive traction application with AlNiCo still so inferior to heavy RE magnets.

\section{Question 5: Does this project support the overall DOE objectives of petroleum displacement? Why or why not?}

\section{Reviewer 1:}

The reviewer claimed absolutely yes, as high-temperature and high-durability magnetic materials not requiring a special cooling is one of the greatest challenges in the electrical machine design.

\section{Reviewer 2:}

The reviewer explained this project is directly attacking a key barrier to cost effective traction motors by developing NRE magnets with competitive properties.

\section{Reviewer 3:}

The reviewer confirmed heavy RE material is the most expensive part of the motor. If AlNiCo grades have sufficient coercivity, then it may provide an alternate magnet solution to the motor designer to save cost in future designs.

\section{Reviewer 4:}

The reviewer confirmed this project focuses on an enabling technology (the material) that can help meet the performance objectives of DOE cost effectively.

\section{Reviewer 5:}

This reviewer stated this project tries to develop new AlNiCo-based magnet materials to be used on traction machines to reduce the dependency of foreign RE magnet materials.

\section{Question 6: Resources: How sufficient are the resources for the project to achieve the stated milestones in a timely fashion?}




\section{Reviewer 1:}

This reviewer observed the project has the right people assigned, and appears to be moving at a pace to achieve the goals.

\section{Reviewer 2:}

The reviewer noted there were no comments on the resources shortages; they seemed to be sufficient to accomplish the project goals. 


\section{North American Supply Chain for Traction Motors and PE: Christopher Whaling (Synthesis Partners) - edt032}

\section{Presenter}

Christopher Whaling, Synthesis Partners

\section{Reviewer Sample Size}

A total of three reviewers evaluated this project.

\section{Question 1: Approach to performing the work-the degree to which technical barriers are addressed, the project is well-designed, feasible, and integrated with other efforts.}

\section{Reviewer 1:}

This reviewer characterized the efforts in conducting interviews with North American (NA) suppliers and OEMs to understand the gaps in the supply chain as good.

\section{Reviewer 2:}

The reviewer found the approach to collect information reasonable, but the project lacks a clear method of analyzing the data.

\section{Reviewer 3:}

The reviewer asserted this project is unlike the other projects on the deck with respect to technical content. While the reviewer understood the goals and the importance of investigating potential challenges with the NA supply chain for traction motors and PE, this person found it hard to assess the validity of the approach. Synthesis Partners likely has a tried and tested method to gather and analyze market intelligence in a rigorous manner, but it is not clear to this reviewer how the collected information can be validated. The reviewer questioned how sampling bias is avoided.

\section{Question 2: Technical accomplishments and progress toward overall project and DOE goals - the degree to which progress has been made, measured against performance indicators and demonstrated progress towards DOE goals.}

\section{Reviewer 1:}

This reviewer agreed the study showed clear technological gaps in the NA supply chain for motors and PE.

\section{Reviewer 2:}

The reviewer noted the team gathered information that shed light on important questions. This person would recommend probing more deeply into some of the points raised by vendors. For example, lack of strategic investment planning/situational awareness has been highlighted as a major barrier. The reviewer suggested it will 
be good to understand how much of this can be attributed to a lack of vision from business leaders and corporate averseness to risk, versus a reaction to a lack of strategic plan by the nation.

Also, this reviewer asserted it will be good to see areas where a lack of core competencies and/or technology are the bottlenecks, in addition to economics driven challenges.

\section{Reviewer 3:}

The reviewer observed the information presented was too general and there was a lack of actionable data provided. This person suggested a need to be more specific of how or what should be done.

\section{Question 3: Collaboration and coordination with other institutions.}

\section{Reviewer 1:}

The reviewer commented the work was done with a wide variety of industry participants.

\section{Reviewer 2:}

This reviewer cautioned some notable vendors were not highlighted in the example lists shared. The person suggested it will be good to review how the vendors being interviewed are selected and are representative of the breadth of the industry.

\section{Question 4: Proposed future research-the degree to which the project has effectively planned its future work in a logical manner by incorporating appropriate decision points, considering barriers to the realization of the proposed technology and, when sensible, mitigating risk by providing alternate development pathways.}

\section{Reviewer 1:}

The reviewer concluded continuing the interviews, understanding the gaps, and informing VTO officials will be helpful in directing attention where needed to bridge the gaps.

\section{Reviewer 2:}

This reviewer suggested the project explore the possibility of proactively reaching out to a broader set of vendors that are representative of the industry.

\section{Reviewer 3:}

This reviewer expressed that analysis needs to be performed on the information already collected to derive conclusions that can be acted on. Asking further questions as described in future work would not provide value at this time.

\section{Question 5: Does this project support the overall DOE objectives of petroleum displacement? Why or why not?}

\section{Reviewer 1:}

The reviewer expressed having strong and robust supply base in NA provides cost savings for manufacturing motors and PE components for NA applications.

\section{Reviewer 2:}

The reviewer summarized that this is a good area of work, but the project currently is not providing tangible and actionable information. Actionable information should show what needs to be done and provides a recommendation of what action should be carried out to address needs.

\section{Reviewer 3:}

This reviewer reported that while this is an important activity, it has more do to with having manufacturing capability and jobs in the United States rather than on petroleum displacement.

\section{Question 6: Resources: How sufficient are the resources for the project to achieve the stated milestones in a timely fashion?}

No comments were received in response to this question. 


\section{Advanced Packaging Technologies and Designs: Zhenxian Liang (Oak Ridge National Laboratory) - edt049}

\section{Presenter}

Zhenxian Liang, Oak Ridge National Laboratory

\section{Reviewer Sample Size}

A total of four reviewers evaluated this project.

\section{Question 1: Approach to performing the work-the degree to which technical barriers are addressed, the project is well-designed, feasible, and integrated with other efforts.}

\section{Reviewer 1:}

This reviewer summarized the approach as prototyping of a power module to demonstrate that WBG devices and chipsets can indeed be packaged in the form of a half-bridge power module.

This person stated $3 \mathrm{D}$ printing to manufacture heat-sink and power module case is utilized.

The reviewer recounted the method for WBG power device package optimization is described and benefits of optimized package is documented in the project report.

\section{Reviewer 2:}

The reviewer observed that the approach seems to be; package a half bridge between two substrates; Planar-Bond-All (PBA) components within the bridge with attached heat sink coolers; use modeling, prototyping and evaluation to guide and validate the approach over time. This person suggested it would be useful to see a listing of the challenges related to the PBA packaging approach.

\section{Reviewer 3:}

The reviewer said there was relevant direction, but the approach appears to be somewhat behind what industry is doing and from some other presentations at this AMR.

Question 2: Technical accomplishments and progress toward overall project and DOE goals - the degree to which progress has been made, measured against performance indicators and demonstrated progress towards DOE goals.

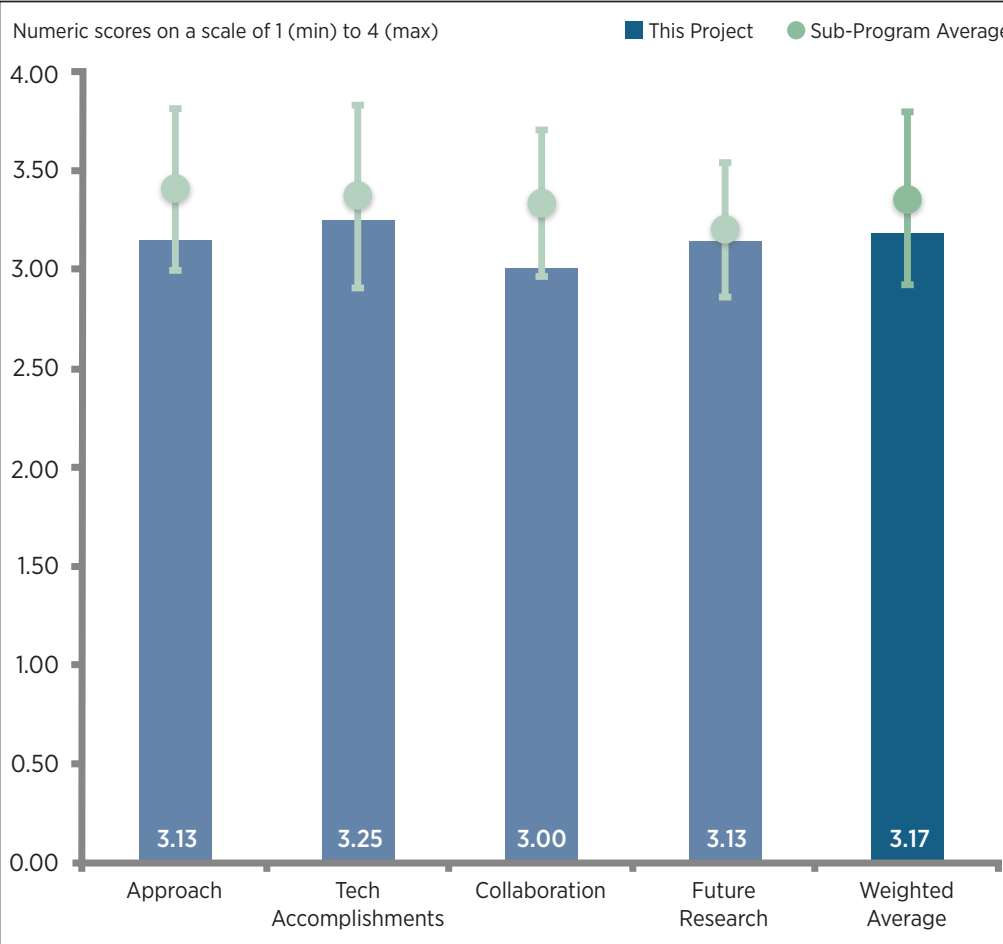

Relevant to DOE Objectives

Sufficiency of Resources
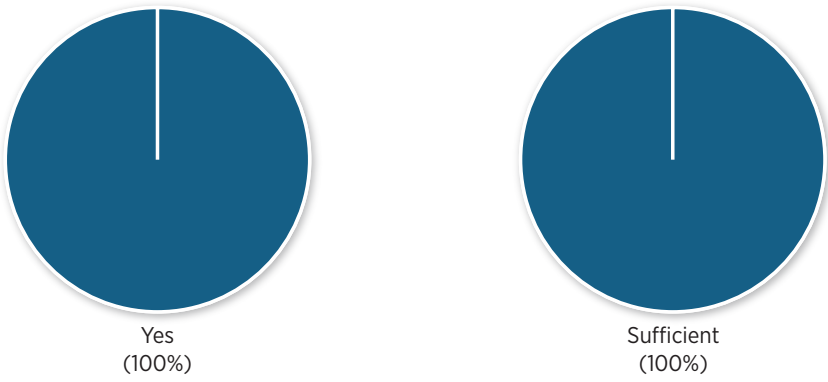

Figure 3-4 - Advanced Packaging Technologies and Designs: Zhenxian Liang (Oak Ridge National Laboratory) - Electric Drive Technologies 


\section{Reviewer 1:}

This reviewer recounted the accomplishments: packaging process for power module is developed; 300 amperes (A)/1,200 Volt (V) power module packaged and successfully used in air-cooled inverter; parasitic inductance of power module is reduced by $75 \%$ by layout optimization for WBG chipsets; benefits of laminated and planar structure of inverter power stage demonstrated; and solver sintering is used in module packaging.

\section{Reviewer 2:}

The reviewer stipulated some progress was made in silver (Ag) die attach and in reduction of loop inductances, but cautioned the inductances need to be further reduced.

\section{Reviewer 3:}

The reviewer applauded the modeling of the electrical parasitics as excellent, and noted this helps with the electrical layouts. This person questioned whether it would be possible to share more model results for the overall mechanical package including a thermo-mechanical model for the overall package that gives an indication that the PBA package will hold together under thermal stress. This will help to support the goal of a highly reliable integrated $\mathrm{SiC}$ module prototype. The reviewer wondered, with a system that is called PBA, why there are wire bond interconnects.

\section{Question 3: Collaboration and coordination with other institutions.}

\section{Reviewer 1:}

The reviewer reinforced the project has the right team of people working. It would be helpful to know what reliability tests the partners are asking for in order to verify the design. This person questioned if the PBA approach considered a power module, and if so, if it is tested as a power module using Automotive Electronics Council (AEC) Q101 tests. Additionally, the reviewer wondered if it is not a power module what tests are being used to validate the design and predict its high reliability.

\section{Reviewer 2:}

This reviewer offered that the team would benefit from industrial collaborators who would actually use the results.

\section{Reviewer 3:}

This reviewer warned that the Principal Investigator (PI) is collaborating with part suppliers while collaboration with end user is absent. It is recommended that an industry partner is identified and the PI should work closely with industry toward objectives such as U.S. manufacturing and technology commercialization pathway.

This person cautioned the technology to market and commercialization pathway is absent.

\section{Question 4: Proposed future research-the degree to which the project has effectively planned its future work in a logical manner by incorporating appropriate decision points, considering barriers to the realization of the proposed technology and, when sensible, mitigating risk by providing alternate development pathways.}

\section{Reviewer 1:}

The reviewer expressed that the development of high-temperature power modules and their integration in inverter fed motor control system is stated as future research work, which could somehow provide some sort of pathway towards commercialization.

\section{Reviewer 2:}

The reviewer wondered when the thermo-mechanical analysis comes into play and would like to see life test results for the overall package, not just die attach after the PBA module has undergone life testing.

\section{Question 5: Does this project support the overall DOE objectives of petroleum} displacement? Why or why not? 


\section{Reviewer 1:}

The reviewer reinforced that WBG PE know-how produced at ORNL will indirectly benefit industry and make a case for adoption by automotive industries.

\section{Reviewer 2:}

The reviewer indicated if the project can show it meets the DOE 2022 targets, it is an enabler for lower cost PE, which will expand the market for EDVs and reduce our dependence on foreign oil.

Question 6: Resources: How sufficient are the resources for the project to achieve the stated milestones in a timely fashion?

\section{Reviewer 1:}

This reviewer concluded the project has the right team of industry and labs. 


\section{Electric Drive Inverter R\&D: Madhu Chinthavali (Oak Ridge National Laboratory) - edt053}

\section{Presenter}

Madhu Chinthavali, Oak Ridge

National Laboratory

\section{Reviewer Sample Size}

A total of six reviewers evaluated this project.

\section{Question 1: Approach to performing the work-the degree to which technical barriers are addressed, the project is well-designed, feasible, and integrated with other efforts.}

\section{Reviewer 1:}

This reviewer mentioned multiple activities are being performed on the project and there is excellent dissemination of data for the benefit of the technical community.

\section{Reviewer 2:}

The reviewer expressed the approach to bench line existing converters, build one with the same components, build an improved one, then modify to find better methods or optimize as a sound one.

\section{Reviewer 3:}

The reviewer considered that this project aids the $R \& D$ of the electric drive inverter in the United States.

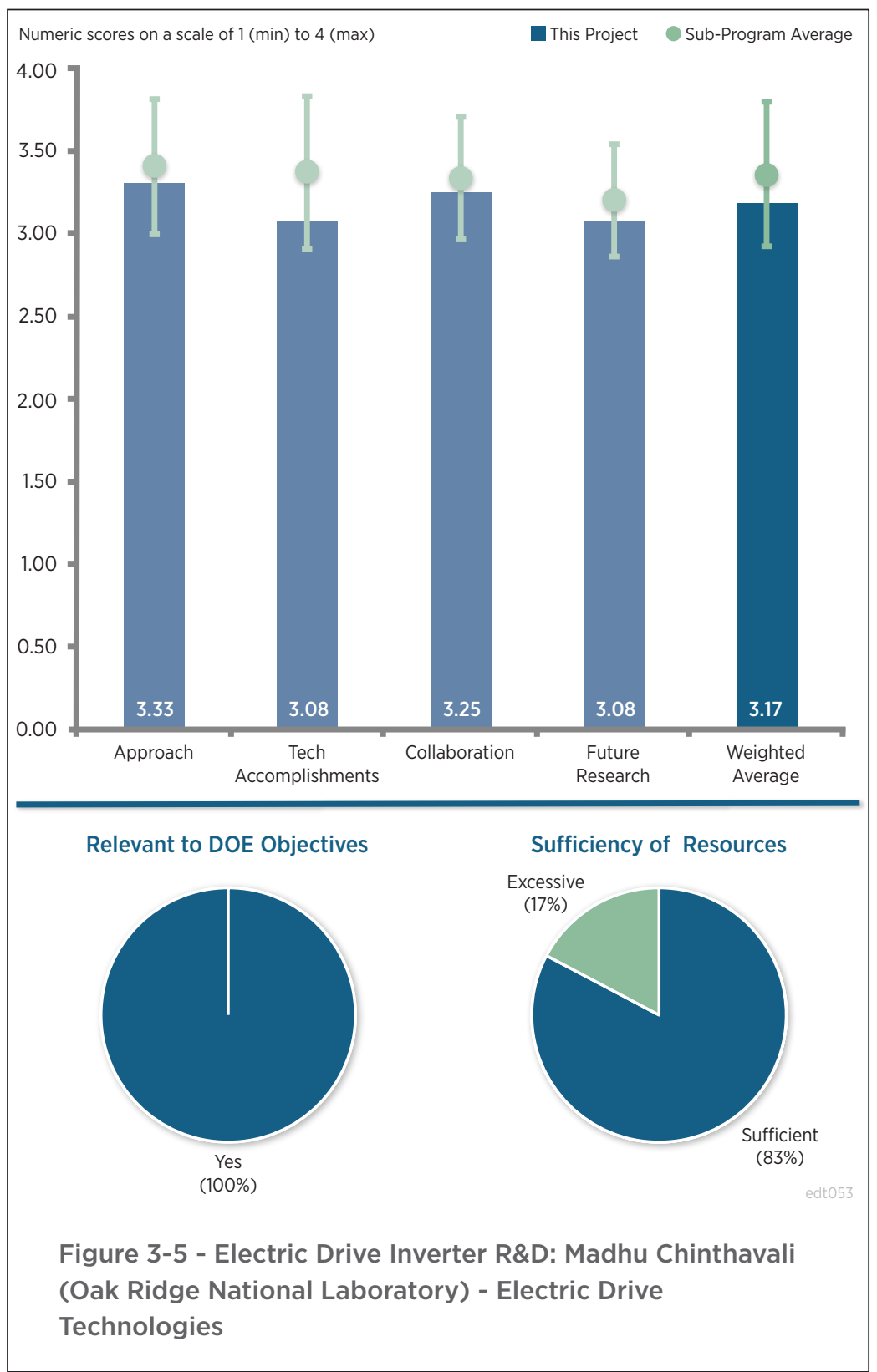

\section{Reviewer 4:}

The reviewer recounted the approach as testing of various WBG devices supplied by manufacturers, apparently to verify data sheet parameters followed by a trench metal-oxide-semiconductor (MOS) this year. This approach appears more directed at performing double pulse tests, rather than demonstrating new approaches to WBG PE to meet APEEM targets.

\section{Question 2: Technical accomplishments and progress toward overall project and DOE goals-the degree to which progress has been made, measured against performance indicators and demonstrated progress towards DOE goals.}

\section{Reviewer 1:}

The reviewer observed that the testing and simulation results look very promising and it appears there is good progress being made. Some of the test results had temperatures (test conditions) listed, but it would be helpful if all reported tests (and simulations) had the ambient and coolant temperatures clearly stated. 


\section{Reviewer 2:}

This reviewer declared that the team has performed significant work on gate drive design, module layout, and overall system layout. The person suggested the overall system layout also incorporate structural analysis to accommodate typical vibration seen in automotive inverters.

\section{Reviewer 3:}

The reviewer judged that the technical accomplishments and progress were on track, but more innovations are necessary to meet the 2022 power density requirement.

\section{Reviewer 4:}

This reviewer concluded that compared with other projects, this one appears to be substantially behind. It is true that WBG gate drivers can be challenging, but there are many examples in the literature, industry, and other AMR projects that work. The person noted that efforts to achieve 100 kilohertz $(\mathrm{kHz})$ experimental switching frequency, power density, etc., have been unsuccessful so far because of layout and noise issues. The reviewer concluded if the experimental setup does not work, then testing of devices is suspect. Perhaps the team could benefit from using evaluation kits from GE, Cree, Transphorm, etc.

\section{Question 3: Collaboration and coordination with other institutions.}

\section{Reviewer 1:}

This reviewer offered that the team has excellent connections with manufacturers for devices and passive elements.

\section{Reviewer 2:}

The reviewer considered all the partners have contributed to this work.

\section{Reviewer 3:}

This reviewer recounted that collaboration is summarized as a list of WBG device and capacitor vendors.

\section{Reviewer 4:}

The reviewer emphasized that this project is focused on methods, rather than specific hardware transition, so manufacturability does not fully come into play, but it may be helpful to have an OEM as a collaborator to give an end user/integrator/manufacture perspective.

\section{Question 4: Proposed future research-the degree to which the project has effectively planned its future work in a logical manner by incorporating appropriate decision points, considering barriers to the realization of the proposed technology and, when sensible, mitigating risk by providing alternate development pathways.}

\section{Reviewer 1:}

The reviewer specified that the design and testing of the inverter design would be a good accomplishment. The test results would enable the community to evaluate the benefits of WBG devices.

\section{Reviewer 2:}

This reviewer described the future research as good overall, but some of the barrier issues such as capacitor volume and lower parasitic inductance are not listed as research items. These appear to be needed to achieve the goals, so there should be some time dedicated to these barriers.

\section{Reviewer 3:}

This reviewer wondered what the optimization target was with the current simulated parasitic inductance below five nanohenries $(\mathrm{nH})$. The reviewer further questioned to what frequency range the reduced parasitic inductance will increase, if this target has been met.

\section{Reviewer 4:}

The reviewer highlighted that it would be good to pay attention in the upcoming tests if the use of WBG PE causes any concerns on reflected wave phenomenon. 


\section{Reviewer 5:}

To this reviewer, it is not clear how the investigators will overcome their gate driver problems so that the team can address APEEM targets.

\section{Question 5: Does this project support the overall DOE objectives of petroleum displacement? Why or why not?}

\section{Reviewer 1:}

This reviewer reinforced that this project helps provide approaches to meeting the DOE cost and power density goals. The research into a smarter gate control and incorporating 3D printing to optimize utilization of devices and space claim will help drive the end result.

\section{Reviewer 2:}

The reviewer agreed that the WBG inverter would support the objectives of petroleum displacement.

\section{Question 6: Resources: How sufficient are the resources for the project to achieve the} stated milestones in a timely fashion?

\section{Reviewer 1:}

The reviewer considered the project to be resourced and funded appropriately.

\section{Reviewer 2:}

This reviewer pointed out that the resources appear to be sufficient to accomplish the goals. 


\section{Innovative Technologies for Converters and Chargers: Gui- Jia Su (Oak Ridge National Laboratory) - edt054}

Presenter

Gui-Jia Su, Oak Ridge National

Laboratory

\section{Reviewer Sample Size}

A total of seven reviewers evaluated this project.

\section{Question 1: Approach to performing the work-the degree to which technical barriers are addressed, the project is well-designed, feasible, and integrated with other efforts.}

\section{Reviewer 1:}

This reviewer expressed this was a novel approach that incorporates WBG devices, planar magnetics, and up-todate converter topologies.

This reviewer affirmed combining or the sharing of components to increase the overall power density and targeting lower system cost is a good approach.

\section{Reviewer 2:}

The reviewer claimed that a high power density integrated charger will be required by the automotive industry and this project is addressing issues associated with this technology. This person recognized the proposed topology of the power converter used in integrated charger and inverter systems uses fewer components and takes advantages of WBG devices.

\section{Reviewer 3:}

The reviewer claimed the approach is to focus on high switching frequencies and 3D printing of components to reduce the space claim and weight. The project appears to be moving forward, but it would help if there were a more systematic approach defined to help keep things on track. The presentation indicated more work was needed on the 3D printing, but did not expand on what the plan forward was.

\section{Reviewer 4:}

This reviewer pointed out the proposed charging solutions are only single phase and need access to the neutral point of the motor. Even though bringing out the neutral might not be very costly, it would have potential reliability issues.

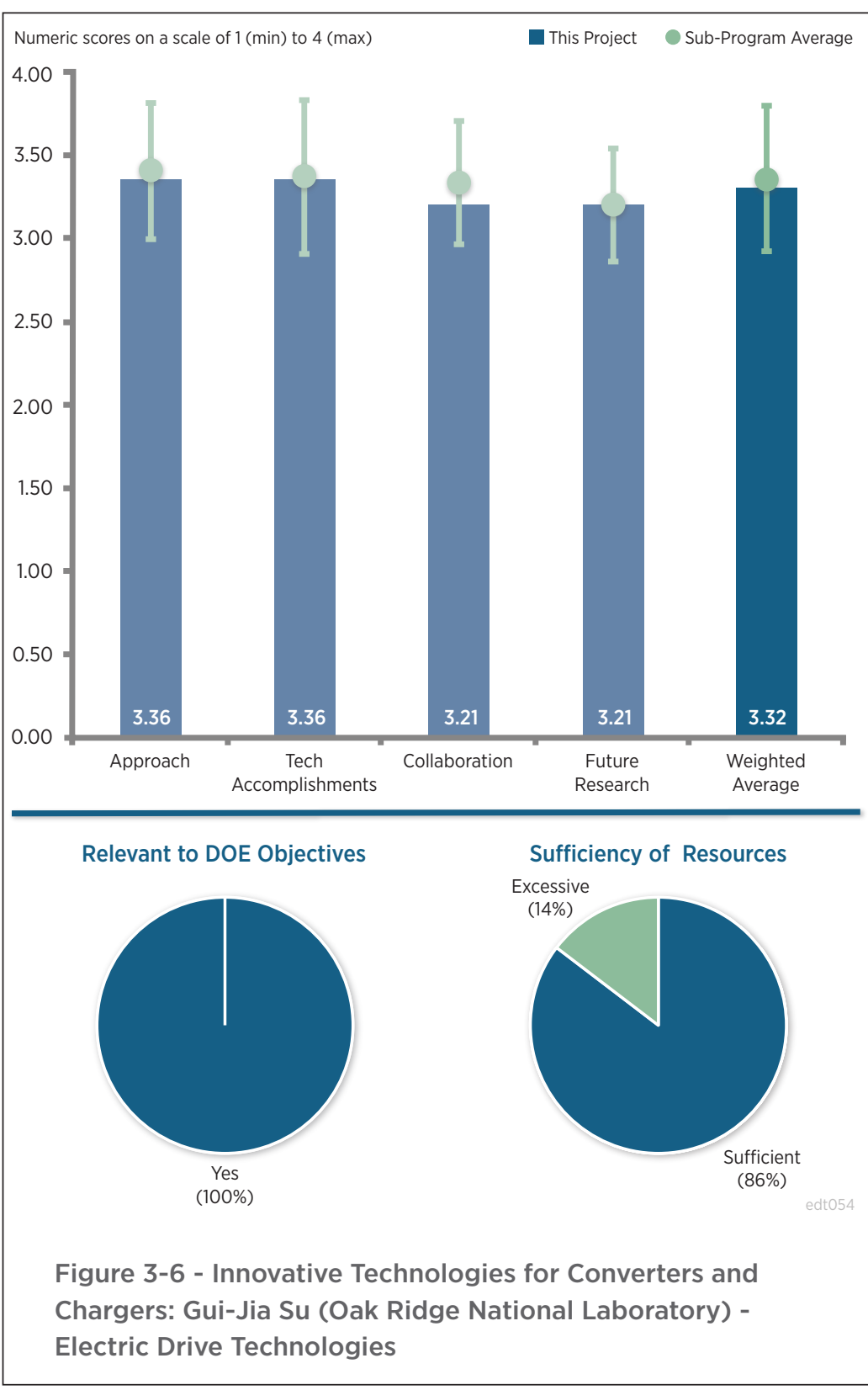

Chargers: Gui-Jia Su (Oak Ridge National Laboratory) -

54 


\section{Question 2: Technical accomplishments and progress toward overall project and DOE goals-the degree to which progress has been made, measured against performance indicators and demonstrated progress towards DOE goals.}

\section{Reviewer 1:}

This reviewer expressed that an interesting contribution of this work is the lightweight, low-loss nano-magnetic powder material and printed E-core manufacturing.

\section{Reviewer 2:}

This reviewer claimed the team's charger comparison to the Nissan LEAF charger is a good comparison as well as showing the project is on the right path. Use of the 3D printed cold plate and E-core is a good approach to advancing additive manufacturing. Now that the project can print magnetics, this person questioned why it was limited to a traditional E-core rather than something that is more space efficient. The reviewer concluded the progress appears to be on track.

\section{Reviewer 3:}

This reviewer declared the Dual Active Bridge design using GaN and planar magnetics has led to good experimental results in both efficiency and power density. The printed magnetic core was unsuccessful.

\section{Reviewer 4:}

The reviewer explained that $\mathrm{SiC}$ and silicon (Si) on-board chargers (OBC) are compared for efficiency over load in the range from 1 kilowatt $(\mathrm{kW})$ to $6 \mathrm{~kW}$. It is demonstrated that the Si-based charger runs into limit for load power greater than $6 \mathrm{~kW}$. This reviewer also noted the following: $6.6 \mathrm{~kW} \mathrm{SiC} \mathrm{OBC}$ built and tested; GaN devices are characterized, such as double pulse testing and GaN H-bridge circuit tested; GaN H-bridge circuit's efficiency over voltage and frequency range is measured; design of $\mathrm{GaN}$ based $6.6 \mathrm{~kW}$ OBC is completed, including planar magnetics needed on OBC; magnetic cores are printed at ORNL; and NREL did thermal analysis of OBC in computational fluid dynamics (CFD) analysis using pin-fin heat-sink.

\section{Reviewer 5:}

The reviewer agreed the 3D printed heat sink has great promise for targeted heat rejection with minimal materials. The 3D printed inductor slightly increases inductance over an open air inductor, but is a long way away from the inductance of a traditional iron core inductor. This person explained the presentation did not elaborate what benefit there was to using the printed core (other than an implied slight decrease in size). With some work still needing to be done on packaging, it is unclear to this reviewer why there is a push to integrate with a traction drive on schedule. It seems the GaN converter should be optimized a bit more prior to integration.

This person emphasized there are not clear milestones as compared to the DOE goals for power density. The chart that compares the LEAF to the ORNL charger is helpful, but does not directly show the path to get to the DOE goals. The reviewer offered that milestones and a timeline be added to help track the progress of the project. The project should report both coolant and ambient temperatures at which the devices are being tested. This person wondered whether temperature reported was room temperature or vehicle under-hood temperatures with engine temperature coolant. In order to be relevant to the DOE goals, the project should be focused on the end vehicle installation temperatures.

\section{Question 3: Collaboration and coordination with other institutions.}

\section{Reviewer 1:}

This reviewer concluded the project includes collaboration with vendors but not with OEMs or Tier 1 suppliers who would use the results.

\section{Reviewer 2:}

The reviewer claimed the project has good representation from the component supplier base, but would benefit from an OEM or end manufacturer. A manufacturer/OEM would help transition the product, bring insight into the manufacturability of the product, and provide a transition path. 


\section{Reviewer 3:}

This reviewer would like to see an auto industry partner or collaborator.

\section{Reviewer 4:}

This reviewer acknowledged the PI is collaborating with part and engineering service suppliers, such as NREL, GaN Systems, ROHM, Aegis Tech, and Ferroxcube USA. Collaboration with end user industry is absent, and so the path to commercialization and U.S. manufacturing is also absent.

Question 4: Proposed future research-the degree to which the project has effectively planned its future work in a logical manner by incorporating appropriate decision points, considering barriers to the realization of the proposed technology and, when sensible, mitigating risk by providing alternate development pathways.

\section{Reviewer 1:}

The reviewer declared the future research as an appropriate plan for completion of work.

\section{Reviewer 2:}

The reviewer recounted that GaN-based isolated converter and its integration with $\mathrm{SiC}$ traction drive are identified as future research work to demonstrate feasibility of all WBG OBC systems.

\section{Reviewer 3:}

The reviewer observed focus on meeting project objectives.

\section{Reviewer 4:}

This reviewer observed the future research jumps directly to integration of the converter without optimizing the converter. A level of integration is good to see what else needs to be done in the converter design, but it seems the research should focus on getting to the DOE power density goals prior to advancing to the next step.

\section{Question 5: Does this project support the overall DOE objectives of petroleum displacement? Why or why not?}

\section{Reviewer 1:}

This reviewer affirmed this project supports the overall DOE objectives. Having low cost, power dense electronics enables fuel savings through electrification.

\section{Reviewer 2:}

The reviewer acknowledged the team is trying to enhance efficiency and power density.

\section{Reviewer 3:}

This reviewer noted the WBG PE research aligns with DOE objectives.

\section{Reviewer 4:}

The reviewer observed that if the project can show it meets the DOE 2022 targets, it becomes an enabler for lower cost PE, which will expand the market for EDVs and reduce our dependence on foreign oil.

\section{Question 6: Resources: How sufficient are the resources for the project to achieve the stated milestones in a timely fashion?}

\section{Reviewer 1:}

The reviewer indicated the resources appear to be sufficient and appropriate for the project.

\section{Reviewer 2:}

The reviewer said the project has the right team of both industry and labs; however, this person would like to see an auto industry collaborator. 


\section{Advanced Low-Cost SiC and GaN Wide Bandgap Inverters for Under-the-Hood Electric Vehicle Traction Drives: Kraig Olejniczak (Wolfspeed) - edt058}

\section{Presenter}

Kraig Olejniczak, Wolfspeed

\section{Reviewer Sample Size}

A total of three reviewers evaluated this project.

\section{Question 1: Approach to performing the work-the degree to which technical barriers are addressed, the project is well-designed, feasible, and integrated with other efforts.}

\section{Reviewer 1:}

This reviewer mentioned multiple activities are being performed on the project and there is excellent dissemination of data for the benefit of the technical community.

\section{Reviewer 2:}

The reviewer expressed the approach to bench line existing converters, build one with the same components, build an improved one, then modify to find better methods or optimize as a sound one.

\section{Reviewer 3:}

The reviewer considered that this project aids the $R \& D$ of the electric drive inverter in the United States.

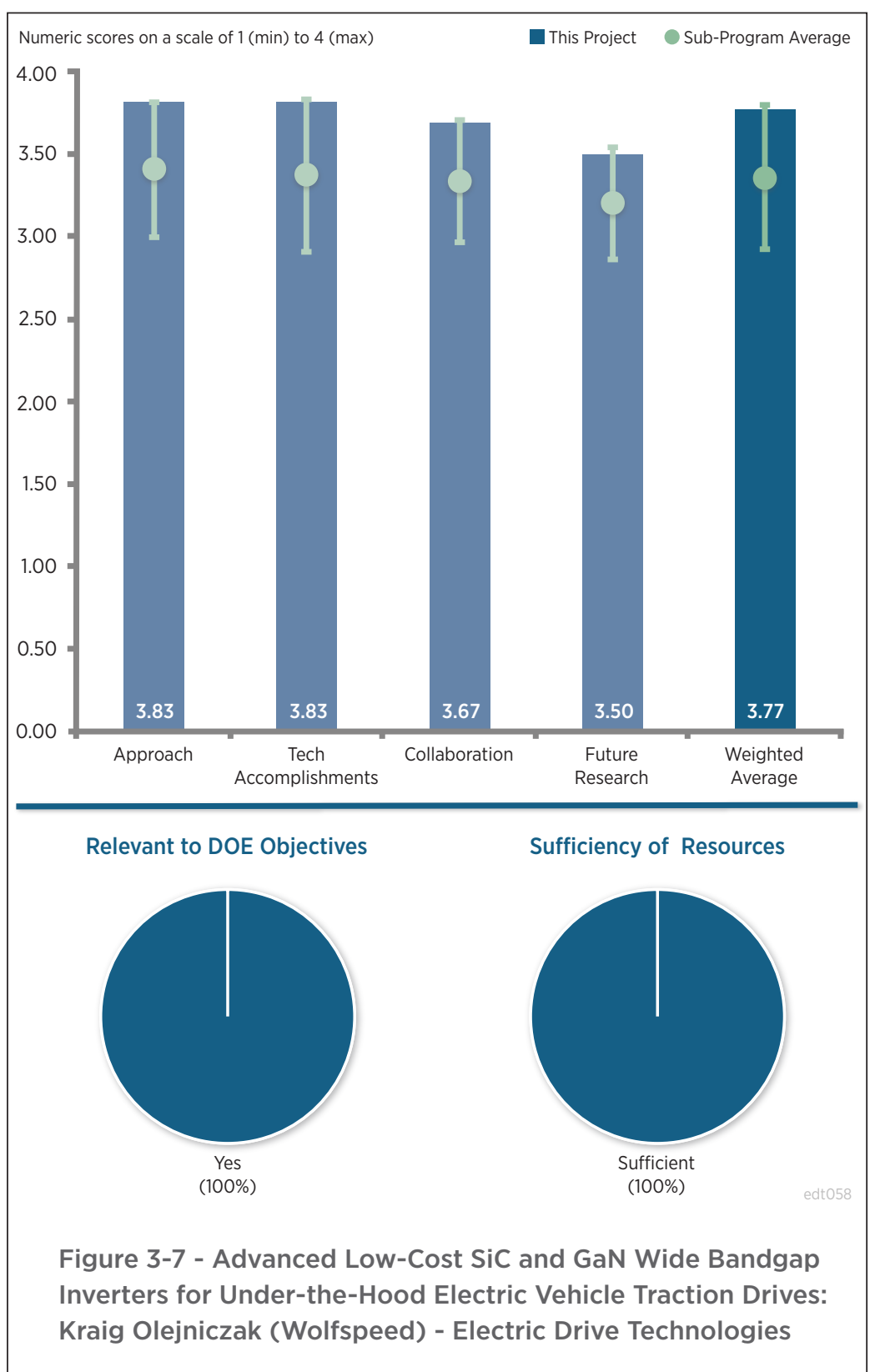

\section{Reviewer 4:}

The reviewer recounted the approach as testing of various WBG devices supplied by manufacturers, apparently to verify data sheet parameters followed by a trench metal-oxide-semiconductor (MOS) this year. This approach appears more directed at performing double pulse tests, rather than demonstrating new approaches to WBG PE to meet APEEM targets.

\section{Question 2: Technical accomplishments and progress toward overall project and DOE goals-the degree to which progress has been made, measured against performance indicators and demonstrated progress towards DOE goals.}

\section{Reviewer 1:}

The reviewer observed that the testing and simulation results look very promising and it appears there is good progress being made. Some of the test results had temperatures (test conditions) listed, but it would be helpful if all reported tests (and simulations) had the ambient and coolant temperatures clearly stated. 


\section{Reviewer 2:}

This reviewer declared that the team has performed significant work on gate drive design, module layout, and overall system layout. The person suggested the overall system layout also incorporate structural analysis to accommodate typical vibration seen in automotive inverters.

\section{Reviewer 3:}

The reviewer judged that the technical accomplishments and progress were on track, but more innovations are necessary to meet the 2022 power density requirement.

\section{Reviewer 4:}

This reviewer concluded that compared with other projects, this one appears to be substantially behind. It is true that WBG gate drivers can be challenging, but there are many examples in the literature, industry, and other AMR projects that work. The person noted that efforts to achieve 100 kilohertz $(\mathrm{kHz})$ experimental switching frequency, power density, etc., have been unsuccessful so far because of layout and noise issues. The reviewer concluded if the experimental setup does not work, then testing of devices is suspect. Perhaps the team could benefit from using evaluation kits from GE, Cree, Transphorm, etc.

\section{Question 3: Collaboration and coordination with other institutions.}

\section{Reviewer 1:}

This reviewer offered that the team has excellent connections with manufacturers for devices and passive elements.

\section{Reviewer 2:}

The reviewer considered all the partners have contributed to this work.

\section{Reviewer 3:}

This reviewer recounted that collaboration is summarized as a list of WBG device and capacitor vendors.

\section{Reviewer 4:}

The reviewer emphasized that this project is focused on methods, rather than specific hardware transition, so manufacturability does not fully come into play, but it may be helpful to have an OEM as a collaborator to give an end user/integrator/manufacture perspective.

\section{Question 4: Proposed future research-the degree to which the project has effectively planned its future work in a logical manner by incorporating appropriate decision points, considering barriers to the realization of the proposed technology and, when sensible, mitigating risk by providing alternate development pathways.}

\section{Reviewer 1:}

The reviewer specified that the design and testing of the inverter design would be a good accomplishment. The test results would enable the community to evaluate the benefits of WBG devices.

\section{Reviewer 2:}

This reviewer described the future research as good overall, but some of the barrier issues such as capacitor volume and lower parasitic inductance are not listed as research items. These appear to be needed to achieve the goals, so there should be some time dedicated to these barriers.

\section{Reviewer 3:}

This reviewer wondered what the optimization target was with the current simulated parasitic inductance below five nanohenries $(\mathrm{nH})$. The reviewer further questioned to what frequency range the reduced parasitic inductance will increase, if this target has been met.

\section{Reviewer 4:}

The reviewer highlighted that it would be good to pay attention in the upcoming tests if the use of WBG PE causes any concerns on reflected wave phenomenon. 


\section{Reviewer 5:}

To this reviewer, it is not clear how the investigators will overcome their gate driver problems so that the team can address APEEM targets.

\section{Question 5: Does this project support the overall DOE objectives of petroleum displacement? Why or why not?}

\section{Reviewer 1:}

This reviewer reinforced that this project helps provide approaches to meeting the DOE cost and power density goals. The research into a smarter gate control and incorporating 3D printing to optimize utilization of devices and space claim will help drive the end result.

\section{Reviewer 2:}

The reviewer agreed that the WBG inverter would support the objectives of petroleum displacement.

\section{Question 6: Resources: How sufficient are the resources for the project to achieve the} stated milestones in a timely fashion?

\section{Reviewer 1:}

The reviewer considered the project to be resourced and funded appropriately.

\section{Reviewer 2:}

This reviewer pointed out that the resources appear to be sufficient to accomplish the goals. 


\section{High Temperature DC-Bus Capacitor Cost Reduction and Performance Improvements: Angelo Yializis (Sigma Technologies International) - edt059}

\section{Presenter}

Angelo Yializis, Sigma Technologies International

\section{Reviewer Sample Size}

A total of three reviewers evaluated this project.

\section{Question 1: Approach to performing the work-the degree to which technical barriers are addressed, the project is well-designed, feasible, and integrated with other efforts.}

\section{Reviewer 1:}

The reviewer emphasized that this project has demonstrated a very novel approach to produce film capacitors and the results obtained are very impressive.

\section{Reviewer 2:}

The reviewer summarized the main approach of this work is to scale-up a liquid/vapor deposition process for polymer-metal multilayer capacitors. Sigma has established equipment and processes for synthesizing thin layers of high-temperature polymers, which are important for capacitors operating in an electric vehicle. This person noted the novel process allows for sub-micron films to be fabricated. Conventional extrusion processes are currently limited to above three microns in high-temperature polymer systems. The energy density will increase as thickness decreases for the $450 \mathrm{~V}$ electric vehicle application.

\section{Reviewer 3:}

This reviewer expressed the research of this project is to engineer a capacitor that could overcome limitation of polypropylene capacitor. As per Sigma Technologies, liquid monomer and aluminum wire are converted in a single step into Mother Capacitor material, which become building block polymer-multi-layer (PML) capacitor. This person noted there is a new electrode mask which doubles the number of capacitors produced in a single run. Elimination of specialized termination needed for electrical connection could help reduce cost and increase micro Farad/Liter. Passivation of aluminum electrodes prevents corrosion and hence could enhance life and reliability of

PML capacitors.

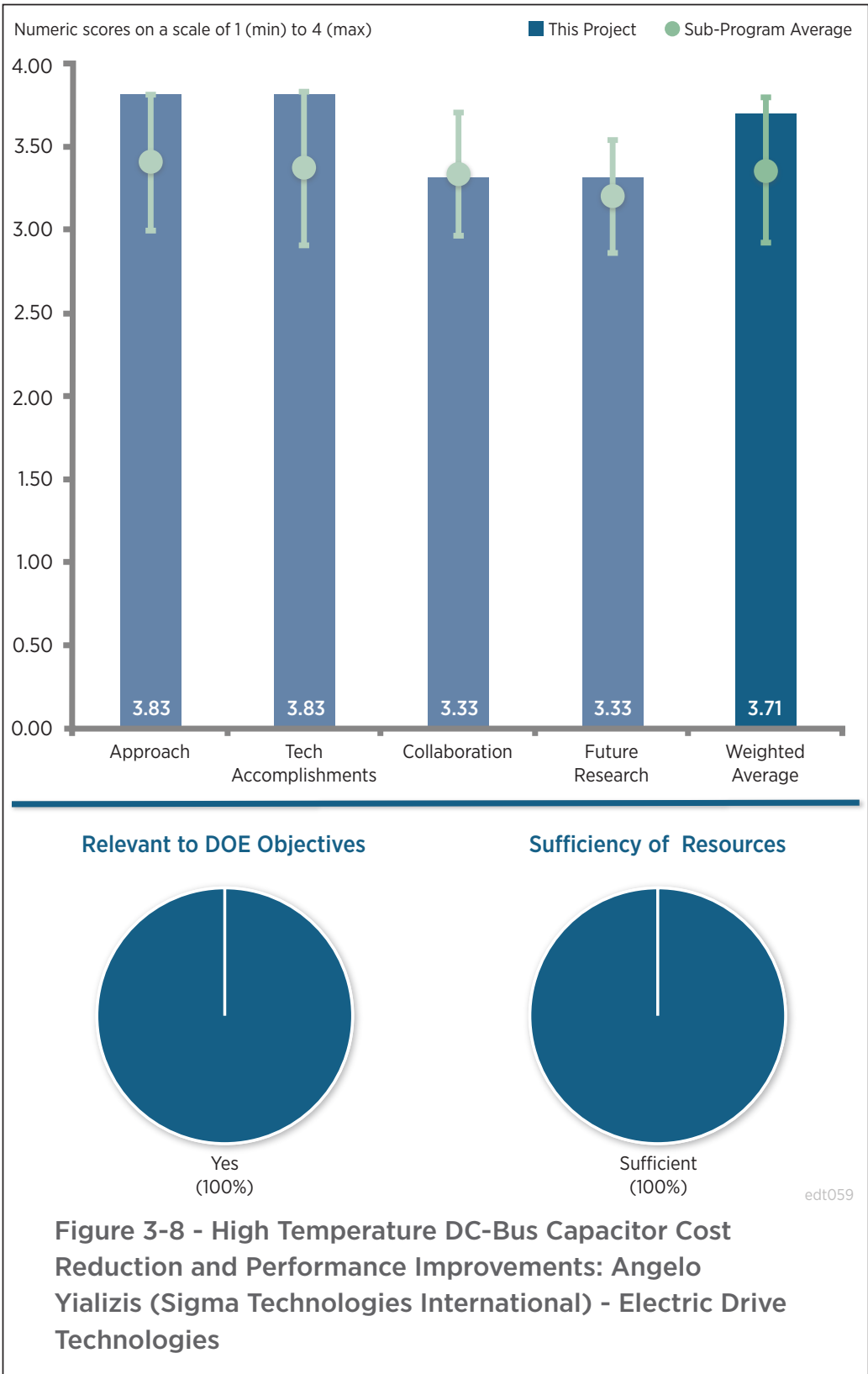


Question 2: Technical accomplishments and progress toward overall project and DOE goals-the degree to which progress has been made, measured against performance indicators and demonstrated progress towards DOE goals.

\section{Reviewer 1:}

This reviewer mentioned outstanding results and prototype hardware built so far has clearly exceeded DOE goals. Test results shown in the presentation meet the requirements and the team is requested to perform rigorous reliability testing.

\section{Reviewer 2:}

This reviewer expressed prototype parts produced and sent off to Delphi for characterization can be stated as a major success of this project. The U.S. manufacturing and commercialization pathway is not clear.

\section{Reviewer 3:}

This reviewer indicated the DOE targets for the DC bus capacitor are related to operation temperature and reliability and all these issues are being addressed by the work. Specifically, 700 microfarad capacitors have been fabricated with sizes that are significantly smaller than the capacitors that are currently used in electric vehicles. High energy densities have also been demonstrated in thin ( 0.5 micron $)$ materials. This person pointed out a typographical error on Slide 10 where units should be microns not millimeters $(\mathrm{mm})$ and a typographical error on Slide 19 that should be milliohms.

\section{Question 3: Collaboration and coordination with other institutions.}

\section{Reviewer 1:}

This reviewer declared very good collaboration with Delphi to evaluate the reliability of the parts. Depending on the remaining budget, the team is requested to provide samples to other key organizations in the vehicle technologies space to jumpstart development and foster incorporation of this capacitor technology into product designs.

\section{Reviewer 2:}

This reviewer stated the PI is collaborating with Delphi.

\section{Reviewer 3:}

This reviewer assessed that a majority of the work was done at Sigma. ORNL is collaborating on the thermal mechanical modeling but no results were shown in the presentation. Delphi is working on the potting process for capacitor arrays.

Question 4: Proposed future research-the degree to which the project has effectively planned its future work in a logical manner by incorporating appropriate decision points, considering barriers to the realization of the proposed technology and, when sensible, mitigating risk by providing alternate development pathways.

\section{Reviewer 1:}

This reviewer offered reliability evaluation is key in gaining confidence in this technology. The team is requested to share the results through presentations in conferences and papers. This would enable a stronger pull to adopt this capacitor concept into product designs.

\section{Reviewer 2:}

This reviewer recounted the focus is on cost and commercialization plan. The technical plan is to integrate these capacitors with an inverter.

\section{Reviewer 3:}

This reviewer expressed the AEC Q200 qualification is planned and it could help PML capacitor adoption 
in automotive applications. It would be nice if the developed technology is commercialized soon and finds applications in automotive and industrial applications.

Question 5: Does this project support the overall DOE objectives of petroleum displacement? Why or why not?

\section{Reviewer 1:}

This reviewer explained capacitor size reduction is key to increase the power density of PE for electric hybrid vehicles. This effort is clearly tied to DOE goals in this area.

\section{Reviewer 2:}

This reviewer offered that high-temperature capacitors are needed for WBG PE, which is essential for petroleum displacement due to efficiency gains possible.

\section{Reviewer 3:}

This reviewer indicated the project directly addresses the system cost and performance of EDVs. Higher temperature capacitors will result in lower cooling requirements for the power converters and hopefully the cooling systems can be eliminated.

\section{Question 6: Resources: How sufficient are the resources for the project to achieve the} stated milestones in a timely fashion?

\section{Reviewer 1:}

The reviewer judges the financial support is sufficient for the capacitor development, prototyping, and characterization efforts. 


\section{High-Performance DC Bus Film Capacitor: Dan Tan (General Electric) - edt060}

\section{Presenter}

Dan Tan, General Electric

\section{Reviewer Sample Size}

A total of three reviewers evaluated this project.

\section{Question 1: Approach to performing the work-the degree to which technical barriers are addressed, the project is well-designed, feasible, and integrated with other efforts.}

\section{Reviewer 1:}

The reviewer specified the main objective of this work is to develop high-temperature polymer films for DC bus capacitors. Specifically, polyetherimide (PEI) films are being explored because this polymer has excellent high-temperature dielectric performance. This person noted there are several important criteria that must be achieved before this material can be commercialized for electric vehicle applications. The DOE targets for the DC bus capacitor are related to operation temperature, cost, and reliability, and all these issues are being addressed by the work.

\section{Reviewer 2:}

This reviewer declared hightemperature extruded polymer film could result in higher packing density parts, particularly for application voltages greater than $800 \mathrm{~V}$, provided $\mathrm{V} /$ micro-meter is properly engineered. The inorganic coating of PEI films increased breakdown voltage. Therefore, the coating can be advantageous; however, the process needs to be scaled for PEI film for all possible thickness.

\section{Reviewer 3:}

This reviewer reported the team has overcome significant challenges to be able to put together the individual elements for the proposed capacitor. However, it appears as if the team still has multiple issues to deal with to meet the project milestones and deadlines. The reviewer expressed concern that the team will not be able to meet the milestones without further delays.

Question 2: Technical accomplishments and progress toward overall project and DOE goals-the degreeand DOE goals-the degree to which progress has been made, measured against performance indicators and demonstrated progress towards DOE goals. 


\section{Reviewer 1:}

This reviewer claimed the team has made significant progress; however, testing on the film dissipation factor (DF) and breakdown at elevated temperatures needs to be performed.

\section{Reviewer 2:}

This reviewer acknowledged there has been continued progress in decreasing the PEI film thickness over time, with over a $40 \%$ decrease in the last six years. This metric is very important for volumetric efficiency and cost. This person offered the process challenge is to create wrinkle-free films with a minimum number of surface defects. The process challenges become increasingly difficult for PEI films below four microns in thickness.

The roll-to-roll oxide deposition method shows promising results, but scale-up was shown to be difficult. The reviewer recounted the PI decided to stop development efforts on the coating. Self-healing was also explored under this program and a number of aluminum deposition parameters were surveyed. It was found that a specific sheet resistance range was required for self-healing.

The reviewer mentioned once the film is fabricated, there are many steps in capacitor manufacturing including winding, end terminations, and packaging. In addition to film resistance, higher equivalent series resistance (ESR) was also found after winding process. This person commented it was also interesting that the DF increased by an order of magnitude when the sheet resistance increased by two.

The reviewer ascertained there have been delays in delivering the large capacitor prototypes and pondered when these will be delivered for final testing.

\section{Reviewer 3:}

This reviewer recounted that the 3.5 micrometer $(\mu \mathrm{m})$ PEI film was produced by extrusion and the wrinkle-free PEI films scaled-up and are ready for capacitor prototyping. Film tested in oil and higher break-down voltage indicates that impregnation of PEI capacitor could be helpful for breakdown voltage and environment management, such as impregnation lending itself to prevent intrusion of humidity and moisture. This person noted roll-to-roll oxide coating was demonstrated on 5- $\mu \mathrm{m}$ PEI film. The U.S. manufacturing and commercialization pathway is not very clear.

\section{Question 3: Collaboration and coordination with other institutions.}

\section{Reviewer 1:}

This reviewer confirmed General Electric (GE) is collaborating with companies along the entire supply chain. At the materials level, GE is working with two film manufacturers that were unnamed. It appears that most of the GE effort is in the scale-up of the capacitor manufacturing and testing, so this is at the component level. Delphi provides the systems perspective.

\section{Reviewer 2:}

This reviewer highlighted the team appears to have reached out to multiple corporations to mitigate the schedule and technical challenges.

\section{Reviewer 3:}

This reviewer indicated that Ralph Taylor from Delphi is identified as collaborator in project report. PI did not put much emphasis upon any on-going collaboration for this project.

\section{Question 4: Proposed future research-the degree to which the project has effectively planned its future work in a logical manner by incorporating appropriate decision points, considering barriers to the realization of the proposed technology and, when sensible, mitigating risk by providing alternate development pathways.}

\section{Reviewer 1:}

The reviewer brought to light this is the last year of the program so finishing the prototype development is the most important future task. The 4-micron film will be used to fabricate the 700 microfarad capacitor bank. 


\section{Reviewer 2:}

This reviewer commented that some tasks are briefly stated and the PI did not put much emphasis upon future research.

\section{Reviewer 3:}

The reviewer declared the team has quite a few tasks remaining this year to complete the project. The person suggested the team ensure that the film quality and capacitor manufacturing process are carefully monitored to ensure no further delays.

\section{Question 5: Does this project support the overall DOE objectives of petroleum displacement? Why or why not?}

\section{Reviewer 1:}

This reviewer affirmed the project directly addresses the system cost and performance of EDVs, which will significantly decrease the overall fuel consumption. Higher temperature capacitors will result in lower cooling requirements for the power converters and hopefully the cooling systems can be eliminated.

\section{Reviewer 2:}

The reviewer explained high-temperature film capacitors could enable successful realization and commercialization of WBG PE, which have far higher efficiency versus Si-based PE.

\section{Question 6: Resources: How sufficient are the resources for the project to achieve the stated milestones in a timely fashion?}

\section{Reviewer 1:}

The reviewer offered the resources are sufficient and there is a significant cost match from the GE Aerospace Division, which will probably be the first market for these high-temperature capacitors. 


\section{Advanced Electric Motor Research: Tim Burress (Oak Ridge National Laboratory) - edt062}

\section{Presenter}

Tim Burress, Oak Ridge National Laboratory

\section{Reviewer Sample Size}

A total of four reviewers evaluated this project.

\section{Question 1: Approach to performing the work-the degree to which technical barriers are addressed, the project is well-designed, feasible, and integrated with other efforts.}

\section{Reviewer 1:}

This reviewer recognized the comprehensive approach to the motor design and control optimization as well as the winning strategy were clearly presented. The results proved this approach very effective.

\section{Reviewer 2:}

While the approach is reasonable, this reviewer recommended reviewing the progress made so far and trying to streamline and focus the strategy towards one or two key threads with the highest potential of making impact. The current scope looks like a collection of smaller projects that all have merit, but maybe limited synergy. This person recognized at least two distinct threads; development of advanced electrical steel and a novel motor topology. However, these activities could be on very different timelines. It can take 5-10 years to develop a new material so it is hard to imagine these impacting the motors being developed within this project.

\section{Reviewer 3:}

This reviewer confirmed the project has identified the key barriers impeding progress to the final goals. The parallel efforts of machine design and modeling, materials characterizations, and motor build and test are all appropriate ways to accelerate progress relative to a sequential task timeline. The tasks seem feasible for the amount of resources provided. The project seems well integrated with the efforts at NREL. This person expressed it was unclear, at least from this presentation, how much value the project is receiving from the interactions with UQM Technologies, Inc., Ames, and the University of Wisconsin.

\section{Reviewer 4:}

The reviewer confirmed heavy RE-free developments are presented in many academic research papers lately.

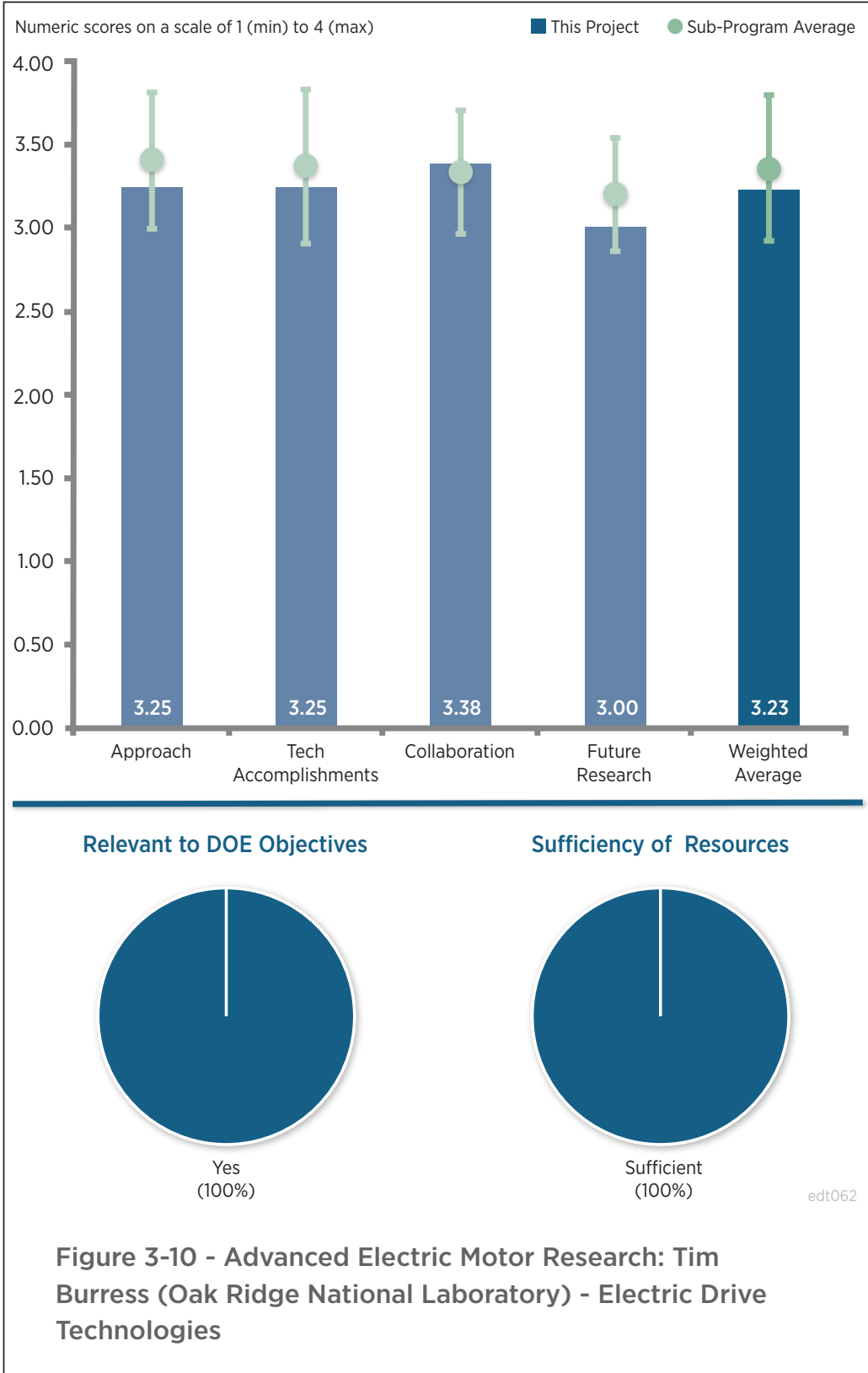


The success of these type of developments would depend on the specific motor design technology and successful material development. This person acknowledged that any success, however, would have significant impact for automotive application. Successful completion of this project would be quite useful; however, the project lacks clear definition.

\section{Question 2: Technical accomplishments and progress toward overall project and DOE goals-the degree to which progress has been made, measured against performance indicators and demonstrated progress towards DOE goals.}

\section{Reviewer 1:}

This reviewer concluded the combination of modeling, materials research, and motor design and testing delivered great practical results. The optimization of the ferrite motor topology and motor performance and efficiency are the major project accomplishments.

\section{Reviewer 2:}

This reviewer characterized the project as making excellent progress in developing an alternate NRE magnet-based ferrite motor design. Fundamental work is being performed on the modeling of high Si content, soft magnetic materials with the goal of improving manufacturability.

This person declared the ab-initio and micromagnetic modeling of the magnetic domain distribution in a soft magnetic material as groundbreaking, representing the state of the art in computational materials science. This part of the project should be emphasized and focused more on modeling real-life microstructures and textures observed in rolled Si steel sheet. Further, the domain wall dynamics should be placed on a more solid theoretical foundation. This may involve broadening the collaborations to groups that have experience in micromagnetic modeling of simpler thin film systems such as magnetic recording media.

The reviewer confirmed the projected performance of the ferrite motor design with distributed windings seems quite promising as compared to that in the 2010 Toyota Prius. However, it was noted that the ferrite motor performance was estimated at a magnet temperature of $100^{\circ} \mathrm{C}$. Ferrite magnets are unique in that their coercivity decreases with decreasing temperature, a behavior opposite to all other commercially available permanent magnets. This person indicated it was not clear if the project had accounted for the risk of demagnetizing the magnets at the initial startup transient at room temperature, before the magnets have a chance to equilibrate at their operating temperature.

\section{Reviewer 3:}

The reviewer mentioned the team appears to have developed a novel motor architecture that can make NRE motors competitive with current RE- based motors. This is exciting; however, details of the innovation were not shared at the review so this person was not able to assess technical accomplishment. This reviewer cautioned the potential for more accurate motor models significantly impacting cost and specific power of motors was not clear.

\section{Reviewer 4:}

This reviewer stated a detailed first principle material model is presented that can predict B-H curves of nonoriented electrical steel. The automotive industry works directly with the material manufacturer and relies on their expertise on high performing material development. This person noted spoke-type ferrite designs have been developed both with DOE funding as well as in the academic projects. These types of designs can provide some flux concertation but still falls short of conventional heavy RE-based designs. The reviewer judged that such construction would introduce many other mechanical issues so adoption of such designs for automotive productions is not very likely.

\section{Question 3: Collaboration and coordination with other institutions.}

\section{Reviewer 1:}

The reviewer applauded that the four partners' collaboration with well-defined role has been perfectly coordinated.

\section{Reviewer 2:}

This reviewer said the team appears to be collaborating well with other teams in the industry and in academia. It 
will be good to see how the team is incorporating lessons learned from other DOE funded efforts, such as the GE and UQM motor projects on integrated permanent magnet (IPM)/spoke rotor designs.

\section{Reviewer 3:}

This reviewer observed the collaboration with NREL appears to be tightly integrated with the efforts presented in this presentation. It was unclear from this presentation the degree to which collaboration is proceeding with Ames, UQM Technologies, Inc. and the University of Wisconsin. This person noted according to Slide 24, UQM Technologies, Inc., was to contribute injection molded potting compounds, but it was not clear where or when this occurred. Also, it was not clear to what degree the University of Wisconsin was involved in the motor design efforts.

\section{Question 4: Proposed future research-the degree to which the project has effectively planned its future work in a logical manner by incorporating appropriate decision points, considering barriers to the realization of the proposed technology and, when sensible, mitigating risk by providing alternate development pathways.}

\section{Reviewer 1:}

This reviewer cautioned that the future work plan is quite clear but the plan is lacking specificity.

\section{Reviewer 2:}

The reviewer suggested the stated timeline of completing the ferrite prototype by the end of September seems aggressive unless substantial portions of the hardware are already built. This person said it was also somewhat unclear how the various modeling results were going to be integrated into the advanced finite element analysis (FEA) modeling method, or how much component analysis remained to be done before that integration could take place. The reviewer wondered how are the domain wall dynamics that are being calculated in the micromagnetic modeling task being integrated into the advanced FEA modeling method and what software package is being used for the advanced FEA modeling method.

\section{Reviewer 3:}

This reviewer warned the objective and definition of this project is very general and broad. Model development with manufacturing issues such as stress, stamping damage, etc., could be useful. This person observed the project does not present any specific modeling methods that are practical and can be adopted by automotive industry.

\section{Reviewer 4:}

The reviewer said that the team should consider performing a risk assessment on proposed technologies and focus the demonstration activities on specific experiments to retire key risks, which may or may not include a full blown motor demonstration.

\section{Question 5: Does this project support the overall DOE objectives of petroleum displacement? Why or why not?}

\section{Reviewer 1:}

This reviewer acknowledged the project does support the DOE objectives by focusing on several promising motor technologies.

\section{Reviewer 2:}

The reviewers suggested a NRE motor that is competitive with RE-based machines will impact cost of EV/HEV's and help with DOE's objective of petroleum displacement.

\section{Reviewer 3:}

The reviewer emphasized the advanced motor designs and the soft magnetic material modeling efforts will provide considerable support to the DOE objectives of petroleum displacement. More efficient electric motor designs will reduce gallons of gasoline equivalent through direct substitution as well as increasing the marketability of electric machines. This person reinforced that improvements in the modeling and manufacturing of soft magnetic materials will have broad impact in the transportation sector beyond electric vehicles into any market segment seeing penetration of electric drive technology. This includes rail, off-highway vehicles (i.e., mining vehicles), marine, and electric aircraft propulsion. 
Question 6: Resources: How sufficient are the resources for the project to achieve the stated milestones in a timely fashion?

\section{Reviewer 1:}

This reviewer declared the resources seem sufficient to complete the proposed tasks; however, the budget and timeline are only specified for FY 2016, while the proposed future work has tasks listed for FY 2017.

Reviewer 2:

This reviewer stated the resources seemed to be sufficient although nothing was specifically stated in relation to that. 


\section{Performance and Reliability of Bonded Interfaces for High-Temperature Packaging: Doug DeVoto (National Renewable Energy Laboratory) - edt063}

\section{Presenter}

Sreekant Narumanchi, National Renewable Energy Laboratory

\section{Reviewer Sample Size}

A total of five reviewers evaluated this project.

\section{Question 1: Approach to performing the work-the degree to which technical barriers are addressed, the project is well-designed, feasible, and integrated with other efforts.}

\section{Reviewer 1:}

The reviewer considered the project to be well addressed with regards to the technical barriers.

\section{Reviewer 2:}

The reviewer described the approach as basic research on crack formation modeling and reliability of sintered Ag contacts.

\section{Reviewer 3:}

This reviewer expressed the approach of systematic characterization is for the most part good although higher temperature results should be captured. One large weakness is the strategy describes a lack of information at $200^{\circ} \mathrm{C}$, but the testing only shows thermal cycling going to $175^{\circ} \mathrm{C}$ (Slide 6). If the goal is $200^{\circ} \mathrm{C}$, the test should cover that range.

\section{Reviewer 4:}

This reviewer stated the reliability of bonded interfaces is key in determining the performance of the power module and the project is addressing this important topic. Reliability modeling and testing are inherently challenging tasks and the team has laid out a very systematic approach in evaluating the performance of Ag sinter based joints. The reviewer requested the team address the following comments. First, modeling the silver material in ANSYS is key to developing a useful model that users can utilize to evaluate the reliability of their designs. Typical Anand viscoplastic models may not be sufficient in accurately representing the failure mode of sintered Ag joints. The reviewer requested that the team evaluate if Anand's model is sufficient for the conditions being investigated and if not to refer to literature to determine other models that are more relevant. Second, the nature of the sintered $\mathrm{Ag}$ joint depends on a large number of process parameters, and the reviewer requested that the team see if porosity of

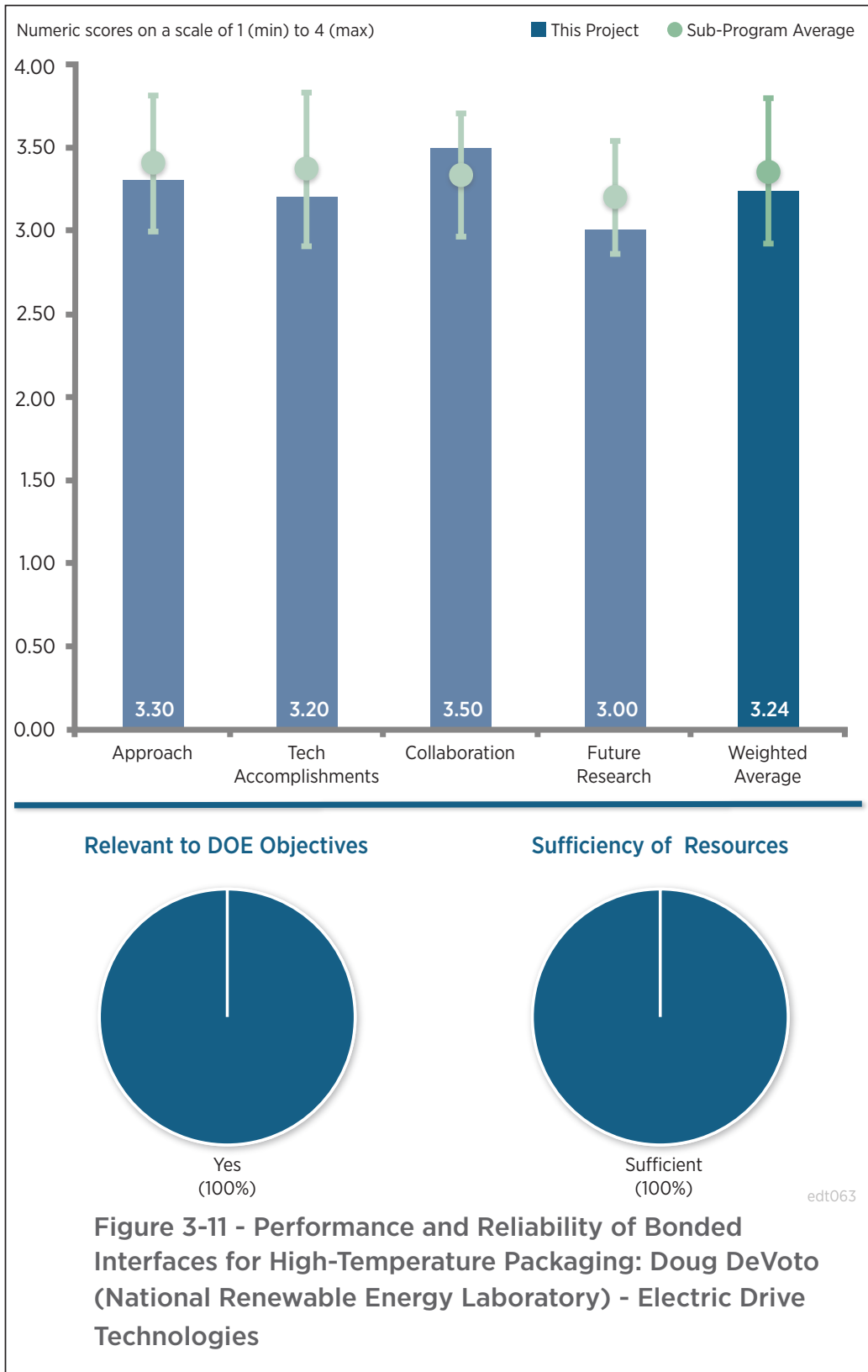


the joint is sufficient to represent these. This would greatly simplify the modeling approach and evaluation of test results.

\section{Question 2: Technical accomplishments and progress toward overall project and DOE goals-the degree to which progress has been made, measured against performance indicators and demonstrated progress towards DOE goals.}

\section{Reviewer 1:}

The reviewer declared this is important research that can inform the electronic packaging industry on sintered Ag.

\section{Reviewer 2:}

The reviewer pointed out the team has made excellent progress so far and has quite a few challenging tasks ahead of them. Results from this effort will clearly help in meeting DOE power electronic goals in the EDT area.

\section{Reviewer 3:}

The reviewer considered the project to be progressing well.

\section{Reviewer 4:}

This reviewer said that it is very important to understand the bonded interface crack propagation modes for high-temperature packaging. Using ANSYS to model the crack propagation may be extremely challenging. This person suggested the team think about how to verify the accuracy of the modeling work, i.e., how to compare the simulations and tests.

\section{Reviewer 5:}

This reviewer acknowledged the methods are good but do not appear to be covering the characterization at the $200^{\circ} \mathrm{C}$ goal. The $175^{\circ} \mathrm{C}$ data point is also useful, but does not reach the $200^{\circ} \mathrm{C}$ or greater goals. The project seems a little behind with the extra data point.

\section{Question 3: Collaboration and coordination with other institutions.}

\section{Reviewer 1:}

This reviewer reported there was very good collaboration between ORNL and NREL on this effort.

\section{Reviewer 2:}

The reviewer expressed that the ORNL and NREL researchers had established a very good collaboration.

\section{Reviewer 3:}

This reviewer considered that perhaps this project does not require a high degree of collaboration with other institutions.

\section{Reviewer 4:}

This reviewer stipulated that this is low level research, so it does not necessarily warrant a large collaboration team. It may be helpful if there were some sort of industry involvement, even if it were just as advisors on manufacturing techniques currently being used, or for feedback on new techniques.

Question 4: Proposed future research-the degree to which the project has effectively planned its future work in a logical manner by incorporating appropriate decision points, considering barriers to the realization of the proposed technology and, when sensible, mitigating risk by providing alternate development pathways.

\section{Reviewer 1:}

The reviewer stated the future work to be well defined, but it seemed the time was not sufficient. 


\section{Reviewer 2:}

The reviewer confirmed the continued testing is good, but should cover the full temperature $\left(200^{\circ} \mathrm{C}\right)$ stated as the project goal instead of stopping at $175^{\circ} \mathrm{C}$.

\section{Reviewer 3:}

The reviewer concluded the project has made good progress and has some challenging tasks ahead. Modeling crack propagation in simulation is interesting. This person suggested that the team check if this is necessary or if it would be sufficient to estimate strength reduction.

\section{Reviewer 4:}

This reviewer said that using ANSYS to measure the crack propagation may be extremely challenging. The team may want to evaluate the computational requirement and time to conduct this simulation.

\section{Reviewer 5:}

This reviewer warned the milestones are not very strong although this work appears to show good results.

\section{Question 5: Does this project support the overall DOE objectives of petroleum displacement? Why or why not?}

\section{Reviewer 1:}

The reviewer pointed out the project addresses reliability of bonded interfaces, which is a key failure mechanism in power converters. Power converters are a key component of electric hybrid technologies and hence this project is clearly in line with DOE goals.

\section{Reviewer 2:}

The reviewer confirmed the work of characterizing the sintered-Ag would be helpful and critical.

\section{Reviewer 3:}

The reviewer noted that the performance and reliability of higher temperature electronics is not well documented. In order for DOE to meet its power density goals, and for the electronics to be installed in areas like the engine compartment, higher temperatures need to be tolerated by the electronics. This person said this research is enabling information that will help electronic designers know the electronics' limitations and help with design choices/ methods for building the high-temperature electronics that will meet the DOE goals.

\section{Reviewer 4:}

The reviewer ascertained it is very important to understand the bonded interfaces failure mechanism as DOE is targeting to further increase the power density of the vehicle traction inverters.

\section{Question 6: Resources: How sufficient are the resources for the project to achieve the stated milestones in a timely fashion?}

\section{Reviewer 1:}

The reviewer noted the resources seem to be sufficient to accomplish the goals.

\section{Reviewer 2:}

The reviewer declared the resources to be sufficient. 


\section{Electric Motor Thermal Management R\&D: Kevin Bennion (National Renewable Energy Laboratory) - edt064}

\section{Presenter}

Kevin Bennion, National Renewable Energy Laboratory

\section{Reviewer Sample Size}

A total of three reviewers evaluated this project.

\section{Question 1: Approach to performing the work-the degree to which technical barriers are addressed, the project is well-designed, feasible, and integrated with other efforts.}

\section{Reviewer 1:}

The reviewer reported that the project is well positioned to contribute valuable information on the heat transfer performance of motor end windings. The experimental and data collection efforts are well designed and appropriate for collecting the required data. This person noted that the collaborations with the partners, particularly ORNL, appear to have led to cross-cutting impact.

\section{Reviewer 2:}

The reviewer indicated that the approach is great, with both good fundamental and rigorous experimental work. This person suggested that perhaps there is opportunity to broaden the impact of the project. The team may want to consider increasing the collaboration with one of the motor design efforts to integrate novel cooling schemes derived from the new understanding being obtained from bench tests. The reviewer said it will also be good to see the connection to complete machine models described in more detail. This person questioned if standardized salable models be can be generated and shared broadly.

\section{Question 2: Technical accomplishments and progress toward overall project and DOE goals-the degree to which progress has been made, measured against performance indicators and demonstrated progress towards DOE goals.}

\section{Reviewer 1:}

The reviewer observed, though this topic sounds simple and fundamental, there is no literature on the cooling effect of direct spraying of transmission fluids on surfaces representative of electrical machines, and certainly no publicly available empirical data that motor designers can use to optimize their machines. This person suggested that this project fills that gap.

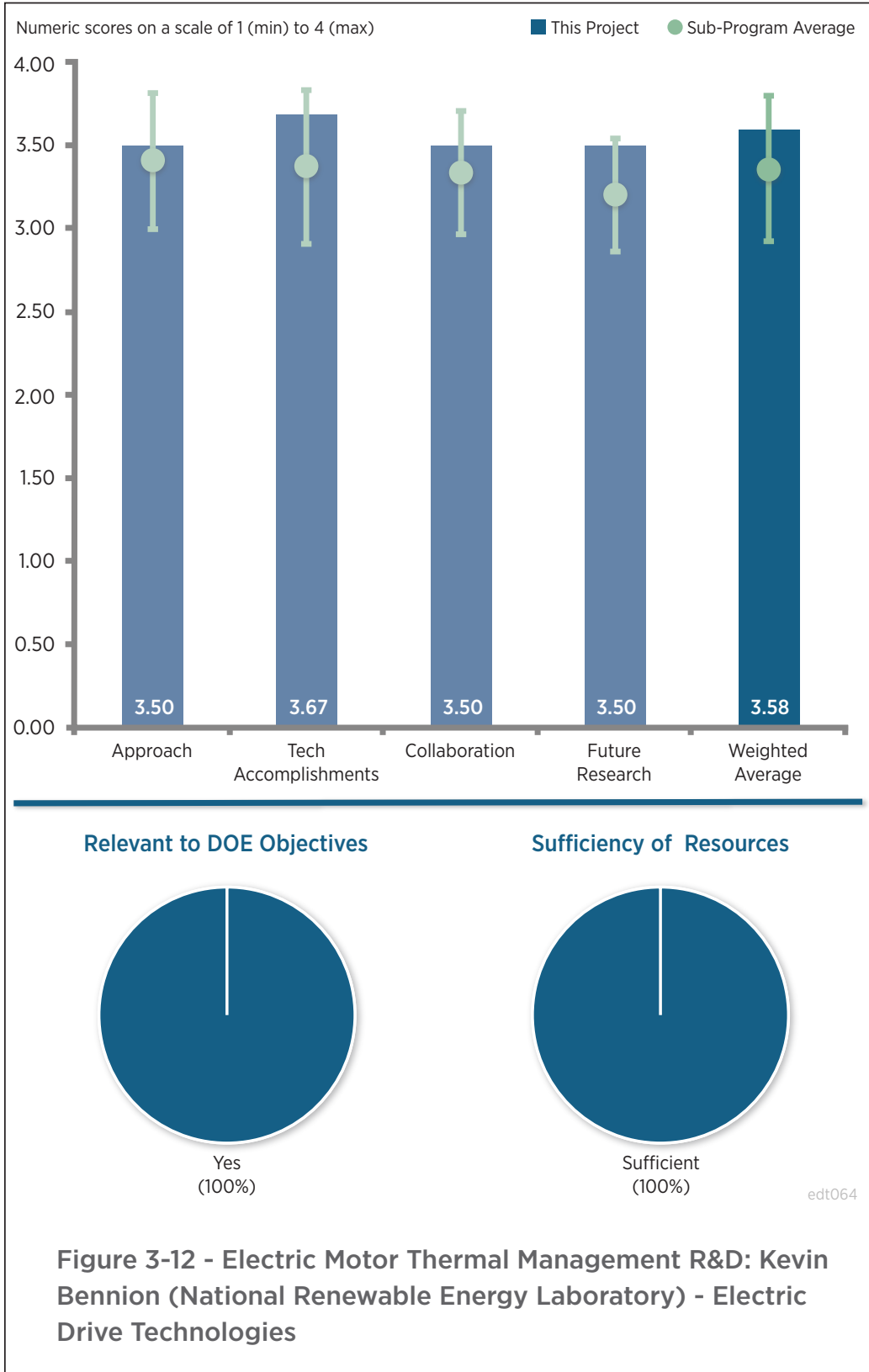




\section{Reviewer 2:}

The reviewer indicated that the jet impingement test rig represents a simple, effective method for measuring the heat transfer coefficients in realistic winding configurations. It is well suited for measuring the direction-dependent thermal conductivity of laminate stacks as well as the thermal contact resistances. The reviewer noted that the comparison between fan and orifice jets covers the range of impingement methods used in practical motor designs. The thermal contact resistance on passive materials also provides valuable data for motor designers, the reviewer stated.

\section{Reviewer 3:}

The reviewer mentioned that the measurement of the heat transfer coefficient with automatic transmission fluid (ATF) flow as a function of flow rate could be quite useful. In addition, proposing several orifice jets and subsequent estimation of the thermal coefficient and the measurement of heat transfer coefficient from the end winding could be very useful as well. The reviewer concluded that these data should be useful for automotive thermal estimation.

\section{Question 3: Collaboration and coordination with other institutions.}

\section{Reviewer 1:}

The reviewer reported that it appears this team is working closely with other motor focused projects and helping with their modeling efforts. The interaction will benefit both sides, the reviewer stated.

\section{Reviewer 2:}

The reviewer said that the collaboration with ORNL was well documented, but it was unclear if there were any collaboration with Ames directly relating towards the goals of this project.

Question 4: Proposed future research-the degree to which the project has effectively planned its future work in a logical manner by incorporating appropriate decision points, considering barriers to the realization of the proposed technology and, when sensible, mitigating risk by providing alternate development pathways.

\section{Reviewer 1:}

The reviewer indicated that the project team has a clear, focused plan for performing representative tests to better understand oil spray cooling. This person suggested the project team should consider being more ambitious in the test campaign and partner with others to investigate novel machine level cooling schemes that can impact motors being developed currently.

\section{Reviewer 2:}

The reviewer stated that the proposed future research should complete the remaining goals and objectives of the project.

\section{Reviewer 3:}

The reviewer remarked that the project concentrates on stranded design. This person suggested that it would be interesting to know how a bar wound motor would perform. Estimating these parameters for bar wound motors would be quite useful, the reviewer said.

\section{Question 5: Does this project support the overall DOE objectives of petroleum displacement? Why or why not?}

\section{Reviewer 1:}

The reviewer commented that this work indirectly supports the DOE objectives of petroleum displacement by providing much needed experimental data on heat transfer coefficients and thermal resistances to electric machine designers. This person stated that this will help the industry reach the 2022 system performance targets, thus making the domestic EV industry more competitive.

\section{Reviewer 2:}

The reviewer acknowledged that thermal constraints are clearly a key barrier to improved power density and cost of electric machines and drives. This person suggested that if efficiency considerations allow it, better 
understanding of oil impingement cooling and other advanced cooking schemes can enable more cost effective electric traction systems.

Question 6: Resources: How sufficient are the resources for the project to achieve the stated milestones in a timely fashion?

Reviewer 1:

The reviewer indicated that the resources and timeline as indicated are sufficient for the team to complete their stated milestones. 


\section{High-Efficiency High- \\ Density GaN-Based 6.6 kW \\ Bidirectional On-Board \\ Charger for PEVs: Charles Zhu \\ (Delta Products Corporation) \\ - edt067}

\section{Presenter}

Charles Zhu, Delta Products

Corporation

\section{Reviewer Sample Size}

A total of four reviewers evaluated this project.

\section{Question 1: Approach to performing the work-the degree to which technical barriers are addressed, the project is well-designed, feasible, and integrated with other efforts.}

\section{Reviewer 1:}

The reviewer considered the project to be well designed and feasible. In addition, the technical barriers have been well addressed.

\section{Reviewer 2:}

The reviewer explained that electric vehicles require battery chargers to fill the tank and it needs to be efficient due to the length of time that it will be charging, which is based on battery size and charger capability. A typical limit for a normal household without having to add special wiring or plugs is $6.6 \mathrm{~kW}$. With the EV market spreading to areas with less than perfect weather, the addition of a bi-directional charger allowing for the EV battery to be used for providing power to the home is a good addition to the vehicle. This person reported that Delta has chosen to use a high frequency switching design based on GaN devices, which allow a switching frequency much greater than the typical $50 \mathrm{kHz}$ found in Si-based chargers. The higher switching frequency allows the passive components such as capacitors and inductors to be significantly smaller thus allowing for a more compact design as well as an efficient design. The reviewer reported that the approach of building a Si-based charger to verify the concept and allow controls to be developed while the GaN devices were being improved allowed the team to concentrate on technical issues related to the new hardware rather than trying to determine if the problem had hardware, software, or control as the source. This person also pointed out that another good approach was carrying a second architecture designed by their partner, Virginia Tech, which had the same operational goals but different implementation which allowed them to select the better design.

\section{Reviewer 3:}

The reviewer remarked that reducing the number of switching devices will reduce cost of OBC and simplify packaging of $\mathrm{OBC}$ converter systems. In addition, increased switching frequency will reduce magnetics size and 
cost of magnetics used in OBC. The reviewer recommended that the PI should explore a possibility that the vehicle identifies if there is one $3.3 \mathrm{~kW}$ OBC or there are two $3.3 \mathrm{~kW} \mathrm{OBC}$ in parallel, and then configure the control system so that interleaving could be used to reduce EMI, EMC, and filtering capacitor requirements.

\section{Reviewer 4:}

The reviewer noted that the approach consists of two basic statements, which are to reduce the number of switching devices and increase the switching frequency. This person pointed out that the statements are not quite well coordinated with the project objectives and goals.

\section{Question 2: Technical accomplishments and progress toward overall project and DOE goals-the degree to which progress has been made, measured against performance indicators and demonstrated progress towards DOE goals.}

\section{Reviewer 1:}

The reviewer stated that the target efficiency achievement and excellent dynamic load responses are two major accomplishments. This person commented that the dependency of the system topology upon specifics of the GaN switches and associated challenges in driving and cooling them has not been addressed sufficiently.

\section{Reviewer 2:}

The reviewer indicated that the design, build, and testing of charger versions using three iterations of GaN switches is impressive. The performance and efficiency of the device as both a charger and an inverter is quite good. The reviewer prefers the two parallel $3.3 \mathrm{~kW}$ power stages as it provides options for use such as running a single stage if connected to a $120 \mathrm{~V}$ alternating current, 16 ampere service and still operate at a reasonable efficiency point. This approach can also be used to provide fault tolerance if desired. Performance of both the mainstream and back up charger are both very good with the backup charger being higher risk due to the power levels that the circuit card needs to handle with the planar magnetic approach which does save space and volume. The reviewer remarked that when the cost of GaN devices drops to competitive levels and the Virginia Tech University Center for Power Electronics Systems (CPES) in-board inductor method has been made into a product, these designs will be very competitive in the EV charger market place.

\section{Reviewer 3:}

The reviewer reported that three iterations of GaN MOS field-effect transistor (MOSFET) were evacuated and a GaN device supplier states that reliability of iterations of GaN MOSFET has improved reliability offered by iteration I and II GaN MOSFETs. The topology of concept design was selected. The prototype of $3.3 \mathrm{~kW} \mathrm{GaN}$ $\mathrm{OBC}$ was developed and tested for power-factor and efficiency. The reviewer further explained that the efficiency of $3.3 \mathrm{~kW} \mathrm{GaN} \mathrm{OBC}$ is also measured at variable voltage across the battery and it is identified that $350 \mathrm{~V}$ could be the preferred voltage for the on-board battery. Additionally, the reviewer noted the following: concept topology of $6.6 \mathrm{~kW} \mathrm{OBC}$ is proposed; project report includes a picture of the sample $6.6 \mathrm{~kW} \mathrm{OBC}$; and the project report includes a variety of test results.

\section{Reviewer 4:}

The reviewer considered the progress to be moderate. This person would like to see the justifications for developing a $6.6 \mathrm{~kW}$ module by paralleling two $3.3 \mathrm{~kW}$ modules. The reviewer questioned whether it would be feasible to parallel switch modules or do multiphase. Specific to the DC/DC and PFC stages, this reviewer asked whether the same challenges would be faced when scaling from $3.3 \mathrm{~kW}$ to $6.6 \mathrm{~kW}$. The reviewer also suggested a system architecture optimization procedure.

\section{Question 3: Collaboration and coordination with other institutions.}

\section{Reviewer 1:}

The reviewer stated that the role of each partner was well defined and the coordination of efforts appeared to be effective.

\section{Reviewer 2:}

The reviewer indicated that the PI has clear plan to work with academia (Virginia Tech), key part supplier (Transform), and end-user industry (Fiat Chrysler Automobiles [FCA]). 


\section{Reviewer 3:}

The reviewer did not see a clear contribution from FCA.

\section{Reviewer 4:}

The reviewer said that the team collaboration appears to be functioning well at least with Delta and Virginia Tech University CPES. This person observed that the vehicle integration task will need to rely on the FCA connection and has no reason to believe that it will not work well.

Question 4: Proposed future research-the degree to which the project has effectively planned its future work in a logical manner by incorporating appropriate decision points, considering barriers to the realization of the proposed technology and, when sensible, mitigating risk by providing alternate development pathways.

\section{Reviewer 1:}

The reviewer reported that the future planned tasks are logical and reasonable and should lead to a successful completion of this project. This person questioned what the control interface between the vehicle and the grid will be because this was not mentioned.

\section{Reviewer 2:}

The reviewer noted that the emphasis on the vehicle test and the creation of the commercialization plan are very important.

\section{Reviewer 3:}

The reviewer commented that deployment and testing of $\mathrm{OBC}$ in the vehicle is identified as future research.

\section{Reviewer 4:}

The reviewer remarked that the future work needs to be better defined.

\section{Question 5: Does this project support the overall DOE objectives of petroleum displacement? Why or why not?}

\section{Reviewer 1:}

The reviewer stated that using the vehicle charger as the emergency power to your house is the very important feature benefit.

\section{Reviewer 2:}

The reviewer acknowledged that WBG devices-based OBC research work underway at Delta falls within the objective of DOE due to higher efficiency possible with the GaN based OBC system.

\section{Reviewer 3:}

The reviewer considered this project supported the overall DOE objectives well.

\section{Reviewer 4:}

The reviewer explained that as EVs become a larger percentage of the vehicle usage in the world market, the amount of energy required to charge them will require more efficient chargers. The addition of bi-directionality will not only be an attractive option but may soon be seen as an imperative especially in areas prone to loss of power and as the smart grid becomes more common place which can take advantage of this capability. The reviewer pointed out that it might even pay for the additional cost of the circuitry.

\section{Question 6: Resources: How sufficient are the resources for the project to achieve the stated milestones in a timely fashion?}

\section{Reviewer 1:}

The reviewer said that the resources were definitely sufficient. This person observed that the amount of work that has been done considering the relatively low budget was quite impressive. 


\section{Reviewer 2:}

The reviewer considered the resources to be sufficient.

\section{Reviewer 3:}

The reviewer mentioned that the progress to date indicates that at the design level the resources are adequate. This person indicated that the device development may have under-estimated the number of turns to produce the desired level of performance, which is understandable as it is a complicated task. 


\section{Gate Driver Optimization for WBG Applications: Nance Ericson (Oak Ridge National Laboratory) - edt068}

\section{Presenter}

Nance Ericson, Oak Ridge National

Laboratory

\section{Reviewer Sample Size}

A total of five reviewers evaluated this project.

\section{Question 1: Approach to performing the work-the degree to which technical barriers are addressed, the project is well-designed, feasible, and integrated with other efforts.}

\section{Reviewer 1:}

The reviewer considered this project to be very helpful to the research and development of the WBG applications in the auto industry.

\section{Reviewer 2:}

The reviewer remarked that the approach is sound benchmarking against existing chips, followed by iterating and improving them with advanced methods and controls.

\section{Reviewer 3:}

The reviewer explained that the project focuses on optimizing the gate drive for WBG devices with an aim to control the instantaneous rates of change of voltage $(\mathrm{dv} / \mathrm{dt})$, and current (di/dt). This is a fairly challenging task but definitely has benefit to improve system level performance (decreased EMI and motor breakdown). However, there are quite a few challenges that the team is asked to respond to. First, typically in a module or a package, the manufacturers tend to reduce common source inductance to improve switching speed. This would negatively impact the measurement required for the proposed scheme. The reviewer requested that the team derive what the minimum common source inductance is required for this scheme to work. The second challenge is that in a module, multiple dies are placed in parallel and invariably these have slightly different parameters (transconductance, threshold voltage, etc.). The reviewer questioned how this can be addressed in the proposed scheme.

\section{Reviewer 4:}

The reviewer noted that previous attempts by commercial integrated circuit (IC) houses to produce analog feedback-based gate drivers for (relatively slower) Si MOSFETs have been failures because of the very high bandwidths needed and the accompanying problems with layout, grounding, etc. The reviewer is very skeptical that this project will succeed. This person commented that the 4 kilowatt per liter $(\mathrm{kW} / \mathrm{L})$ stated power density target is out of date.

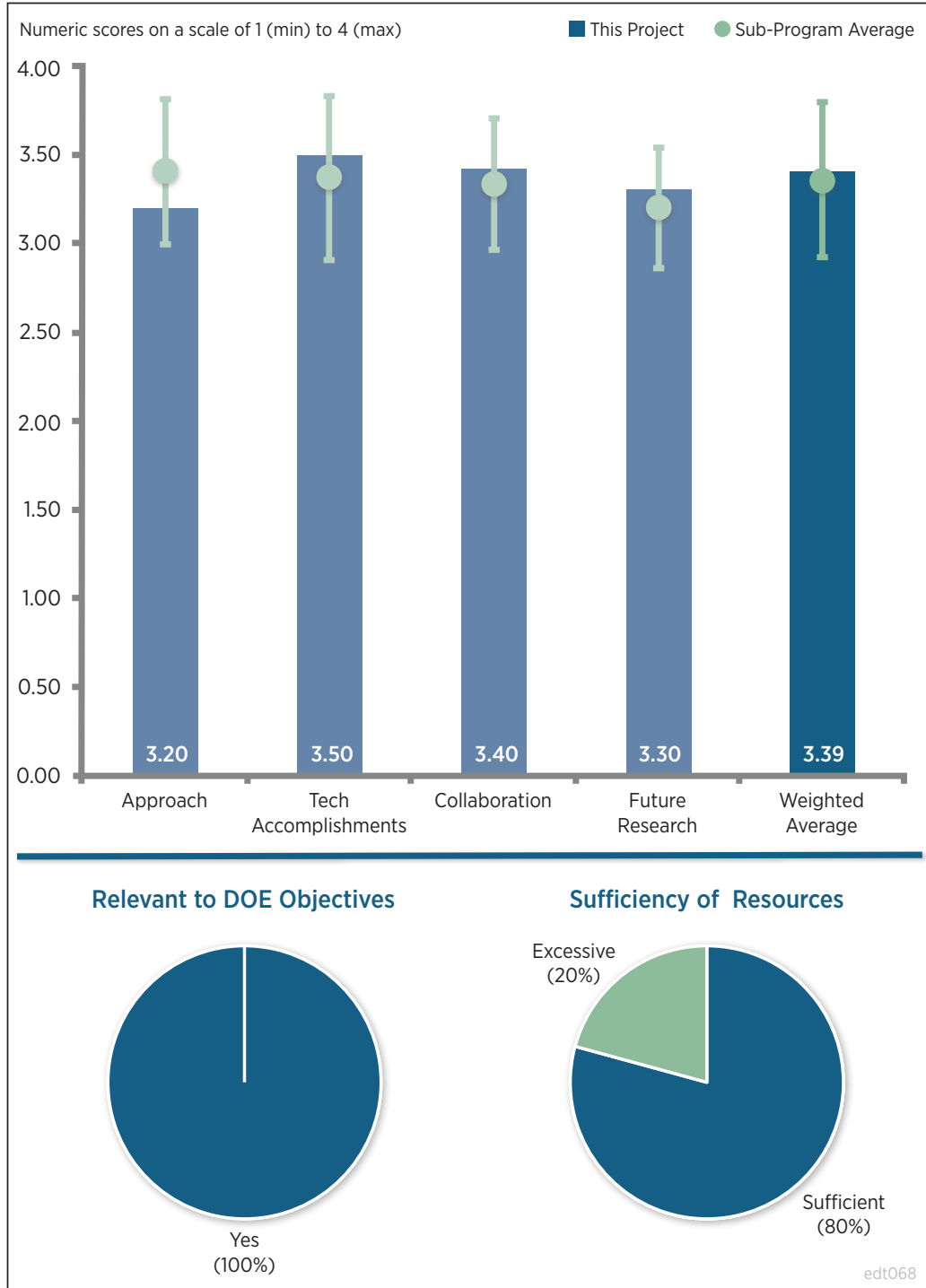

Figure 3-14 - Gate Driver Optimization for WBG Applications: Nance Ericson (Oak Ridge National Laboratory) - Electric Drive Technologies 
Question 2: Technical accomplishments and progress toward overall project and DOE goals-the degree to which progress has been made, measured against performance indicators and demonstrated progress towards DOE goals.

\section{Reviewer 1:}

The reviewer pointed out that optimizing the gate drive performance is very important to the WBG device based inverter performance and reliability and helps mitigate the voltage stress on the motor and bearing current. This person stated that there has been excellent progress made to simulate the performance of the proposed gate drive circuits. The reviewer acknowledged that the team has completed the hardware design and is looking forward to seeing comprehensive test results next year.

\section{Reviewer 2:}

The reviewer mentioned that the project is tracking well and appears to be on track to meet its goals. Simulations and baseline testing were done and the design improvements are in progress, the reviewer reported.

\section{Reviewer 3:}

The reviewer observed that the team has shown very good progress so far. Modeling and simulation work look thorough and experimental evaluation has a very good likelihood to be successful.

\section{Reviewer 4:}

The reviewer stated that simulation is fine, but the experiment will be a key to proving feasibility of this approach. This person warned that it appears that there is very little time left to meet the very difficult experimental milestones and go/no-go checkpoint. The plateau voltage control scheme appears to be novel, remarked the reviewer.

\section{Reviewer 5:}

The reviewer would like to know the measures to evaluate the stability of the integrated gate drive. This person also questioned if the specific target is efficiency or frequency.

\section{Question 3: Collaboration and coordination with other institutions.}

\section{Reviewer 1:}

The reviewer acknowledged that there is collaboration between ORNL and the University of Tennessee, Knoxville (UTK) researchers. This person suggested that this project would benefit from direction by IC producers with interest in the area of WBG gate drivers.

\section{Reviewer 2:}

The reviewer commented that the project has a good team with a manufacturing partner, Wolfspeed, a chip manufacturer and potential transition partner, and academia (UTK) represented.

\section{Reviewer 3:}

The reviewer noted that from the presentation it looks like the team is leveraging the competence of UTK in gate drive design and chip development. Device simulation appears to be in partnership with CoolCad.

\section{Reviewer 4:}

The reviewer agreed that all the partners have contributed to the project.

Question 4: Proposed future research-the degree to which the project has effectively planned its future work in a logical manner by incorporating appropriate decision points, considering barriers to the realization of the proposed technology and, when sensible, mitigating risk by providing alternate development pathways.

\section{Reviewer 1:}

The reviewer noted that the future work had been well defined and planned. 


\section{Reviewer 2:}

The reviewer pointed out that the upcoming milestones are appropriate although tight. This person is skeptical that the experimental milestones will be met.

\section{Reviewer 3:}

The reviewer commented that the future research for the gate driver is good. This person said that it is unclear how the solution will be implemented as the presentation indicated isolation is still needed. The researcher suggested that, at a minimum, whatever isolation is required should be tested and recommendations for various implementations should be included in the future research.

\section{Reviewer 4:}

The reviewer declared that the project is addressing quite a challenging task. The reviewer suggested that, in the proposed future work, the project team be asked to incorporate automatic tuning (from user) to achieve desired dv/ $\mathrm{dt}$ and/or di/dt instead of manual tuning and device parameter variation and impact of paralleling devices on the proposed scheme.

\section{Question 5: Does this project support the overall DOE objectives of petroleum displacement? Why or why not?}

\section{Reviewer 1:}

The reviewer indicated that an optimized gate driver circuit for WBG devices is an enabling technology to help DOE meet its goals.

\section{Reviewer 2:}

The reviewer stated that WBG device performance and system benefits depend on proper gate drive design. The project addresses a key risk in utilizing these devices and hence clearly supports the overall DOE objectives, commented the reviewer.

\section{Reviewer 3:}

The reviewer considered this project to be well supportive of the overall DOE objectives.

\section{Reviewer 4:}

The reviewer observed that the reliability of the inverter and motor system using the WBG devices will improve the performance and efficiency of the future HEV/ PHEV/BEV products.

\section{Question 6: Resources: How sufficient are the resources for the project to achieve the stated milestones in a timely fashion?}

\section{Reviewer 1:}

The reviewer commented that this topic is of high importance to the proper use of WBG devices. The project scope is quite challenging, and $\$ 200,000-\$ 250,000$ appears to be too small for addressing the proposed research and the additional concerns raised by the reviewer.

\section{Reviewer 2:}

The reviewer said that the resources appear to be sufficient to meet the goals

\section{Reviewer 3:}

The reviewer considered the resources to be sufficient. 


\section{Power Electronics Thermal Management R\&D: Gilbert Moreno (National Renewable Energy Laboratory) - edt069}

\section{Presenter}

Kevin Bennion, National Renewable Energy Laboratory

\section{Reviewer Sample Size}

A total of three reviewers evaluated this project.

\section{Question 1: Approach to performing the work-the degree to which technical barriers are addressed, the project is well-designed, feasible, and integrated with other efforts.}

\section{Reviewer 1:}

The reviewer acknowledged that the project focuses on the thermal bottlenecks to using WBG devices in under hood applications. Simulation results show the expected temperature rise in various components and the effect of increasing under hood temperature. The reviewer concluded that this is a key topic for successful incorporation of WBG devices.

\section{Reviewer 2:}

The reviewer considered this project to be interesting, but the applications of the thermal management techniques need to be better defined.

\section{Reviewer 3:}

The reviewer identified macroscale thermal modeling of PE systems. This person further noted that the project started with a 2012 LEAF (benchmarked by Burress), developed thermal system model, and then considered effects of modifications/ improvements.

\section{Question 2: Technical accomplishments and progress toward overall project and DOE goals-the degree to which progress has been made, measured against performance indicators and demonstrated progress towards DOE goals.}

\section{Reviewer 1:}

The reviewer reported good experimental validation and good insights. The reviewer questioned if this will be applied to a John Deere design. This person noted that the comments on $140^{\circ} \mathrm{C}$ capacitors are consistent with what others have observed and questioned if there is a proposed solution.

\section{Reviewer 2:}

The reviewer stated that the thermal modeling work carried out is comprehensive; however, validation of the
Figure 3-15 - Power Electronics Thermal Management R\&D: Gilbert Moreno (National Renewable Energy Laboratory) Electric Drive Technologieses

Electric Drive Technologies 
thermal model is limited to few components. This person requested that the team check if more experimental data are available or can be carried out to validate the model.

\section{Reviewer 3:}

The reviewer reported that the percentage of the work completed seemed to be insufficient. The reviewer would like to see a flowchart or other representation that summarizes the thermal management methodology which would be applicable for most inverter and converter designs. This person would also like to see a comparison of the issues or challenges of the thermal management techniques for Si-based and WBG devices.

\section{Question 3: Collaboration and coordination with other institutions.}

\section{Reviewer 1:}

The reviewer noted that the team appears to have good collaboration with other institutions.

\section{Reviewer 2:}

The reviewer commented that apparently there is collaboration with John Deere and Kyocera, but no details were given so this is difficult to judge.

Question 4: Proposed future research-the degree to which the project has effectively planned its future work in a logical manner by incorporating appropriate decision points, considering barriers to the realization of the proposed technology and, when sensible, mitigating risk by providing alternate development pathways.

\section{Reviewer 1:}

The reviewer pointed out that the project has reached a key point where new technologies need to be evaluated for thermal management. This person observed that jet impingement techniques appear to be unsuitable for some cases but no details on the other potential solutions are presented.

\section{Question 5: Does this project support the overall DOE objectives of petroleum displacement? Why or why not?}

\section{Reviewer 1:}

The reviewer explained that thermal management is a key challenge for hybrid electronics and the project is well aligned with DOE objectives.

Question 6: Resources: How sufficient are the resources for the project to achieve the stated milestones in a timely fashion?

No comments were received in response to this question. 


\section{Thermal Performance Benchmarking: Xuhui Feng (National Renewable Energy Laboratory) - edt070}

\section{Presenter}

Xuhui Feng, National Renewable Energy Laboratory

\section{Reviewer Sample Size}

A total of four reviewers evaluated this project.

\section{Question 1: Approach to performing the work-the degree to which technical barriers are addressed, the project is well-designed, feasible, and integrated with other efforts.}

\section{Reviewer 1:}

The reviewer reported that this approach to select a vehicle, measure the desired performance attributes, create models, and validate it using the data makes sense. Based on the data and validated modeling to determine what needs to be done to improve the performance is also a logical step. This person indicated that this project will include the electric motor and transmission as well as the PE but it was not clear if the PE includes a charger and $12 \mathrm{~V}$ power supply for the vehicle. The reviewer thought that just the drivetrain is fine.

\section{Reviewer 2:}

The reviewer observed that this project examines state of the art thermal management systems employed in EVs and HEVs. The results can be used to guide future R\&D in thermal management, which can lead to higher efficiency and reliability in the vehicles. This person indicated that the project is aligned with ORNL's benchmarking project.

\section{Reviewer 3:}

The reviewer mentioned that the Nissan LEAF and Honda Accord inverter's thermal performance have been analyzed. This person further noted that the Nissan LEAF is shown to possibly have cost and reliability advantages.

\section{Question 2: Technical accomplishments and progress toward overall project and DOE goals-the degree to which progress has been made, measured against performance indicators and demonstrated progress towards DOE goals.}

\section{Reviewer 1:}

The reviewer stated that the technical progress looks good. This person mentioned that thermal management 
systems for the 2012 Nissan LEAF and the 2014 Honda Accord were characterized and compared to finite element models and that several areas of improvement were identified.

\section{Reviewer 2:}

The reviewer reported that the information provided on the analysis and testing of the Nissan LEAF and Honda Accord provided good results for the cooling systems. The comparison charts are a good method to show the differences between the approaches. This person pointed out that the conclusions in the report were substantiated by the data indicating that the process is controlled. The reviewer noted that, as mentioned in the presentation, the test conditions do not match in vehicle conditions. This person asked if there is a plan to develop vehicle instrumentation procedures to provide an indication of what these might be. The reviewer realized that the vehicle environment is pretty harsh for instrumentation, but perhaps a few significant test points might be monitored during the routine vehicle testing being performed.

\section{Question 3: Collaboration and coordination with other institutions.}

\section{Reviewer 1:}

The reviewer stated the project team is collaborating with ORNL.

\section{Reviewer 2:}

The reviewer acknowledged that this project aligns well with ORNL's benchmarking project (edt006).

\section{Reviewer 3:}

The reviewer voiced that collaboration between the laboratories appears to be working well in this case.

Question 4: Proposed future research-the degree to which the project has effectively planned its future work in a logical manner by incorporating appropriate decision points, considering barriers to the realization of the proposed technology and, when sensible, mitigating risk by providing alternate development pathways.

\section{Reviewer 1:}

The reviewer pointed out that the project team will focus on thermal management characterization of the 2015 BMW i3 PE, inverter, and motor.

\section{Reviewer 2:}

The reviewer commented that the future research is focused on the BMW i3, which complements the work in EDT006.

\section{Reviewer 3:}

The reviewer observed that the plan to continue to characterize the performance is reasonable, but the reviewer is not sure how the project team will characterize and improve the thermal performance on a production vehicle. This person questioned if the plan is to modify the thermal stack up, heatsink, and thermal fluid path, or just modify the models and simulate the results. The reviewer asked, if so, how the project team will validate this process.

\section{Question 5: Does this project support the overall DOE objectives of petroleum displacement? Why or why not?}

\section{Reviewer 1:}

The reviewer stipulated that this work can guide future thermal management R\&D efforts and identify areas to improve efficiency, reliability, and cost.

\section{Reviewer 2:}

The reviewer remarked that the thermal system performance drives the number of switching devices required, has a significant impact on the size and mass of the PE, and to a large degree the amount of power that the electric machine can deliver within its cost target, which makes it relevant. 
Question 6: Resources: How sufficient are the resources for the project to achieve the stated milestones in a timely fashion?

Reviewer 1:

The reviewer said that the project appears to have sufficient resources.

\section{Reviewer 2:}

The reviewer explained that the data indicate that the resources are sufficient for the current project objectives. If on-vehicle testing and/or performing modifications to the hardware are added, then the resources may not be adequate but then that is a scope change, the reviewer noted. 


\section{Electric Motor Performance Improvement Techniques: Lixin Tang (Oak Ridge National Laboratory) - edt071}

Presenter

Lixin Tang, Oak Ridge National Laboratory

\section{Reviewer Sample Size}

A total of four reviewers evaluated this project.

Question 1: Approach to performing the work-the degree to which technical barriers are addressed, the project is well-designed, feasible, and integrated with other efforts.

\section{Reviewer 1:}

The reviewer indicated that the project clearly states the technical barriers towards realizing the goals of multispeed motor control schemes with series and parallel windings. The development program as outlined appears to have made substantial progress towards comparing and contrasting the benefits and drawbacks of this method. The reviewer pointed out that the increase in focus on understanding system cost and complexity, as well the outreach to external partners such as Borg Warner, was particularly noteworthy.

\section{Reviewer 2:}

The reviewer commented that it is good to see a motor plus drive system level approach that can help reduce motor size, weight, and cost. This person said it is especially interesting because solutions that require more active switching could become more attractive given the rapid advances being made in PE.

\section{Reviewer 3:}

The reviewer mentioned that the project seeks to increase the power density and efficiency of electric drives by using reconfigurable windings in the motor. The project team is investigating several methods and the high level approach seems good. The reviewer noted that the specific approach was not presented, but that is understandable because some of the design is under patent review.

\section{Reviewer 4:}

The reviewer remarked that the use of a multi-speed range (MSR) motor is one potential approach to achieving the desired performance goals. In theory, it will work but there are still issues that need to be addressed before it is acceptable for use in a vehicle. The reviewer suggested that these include issues with torque interruptions during

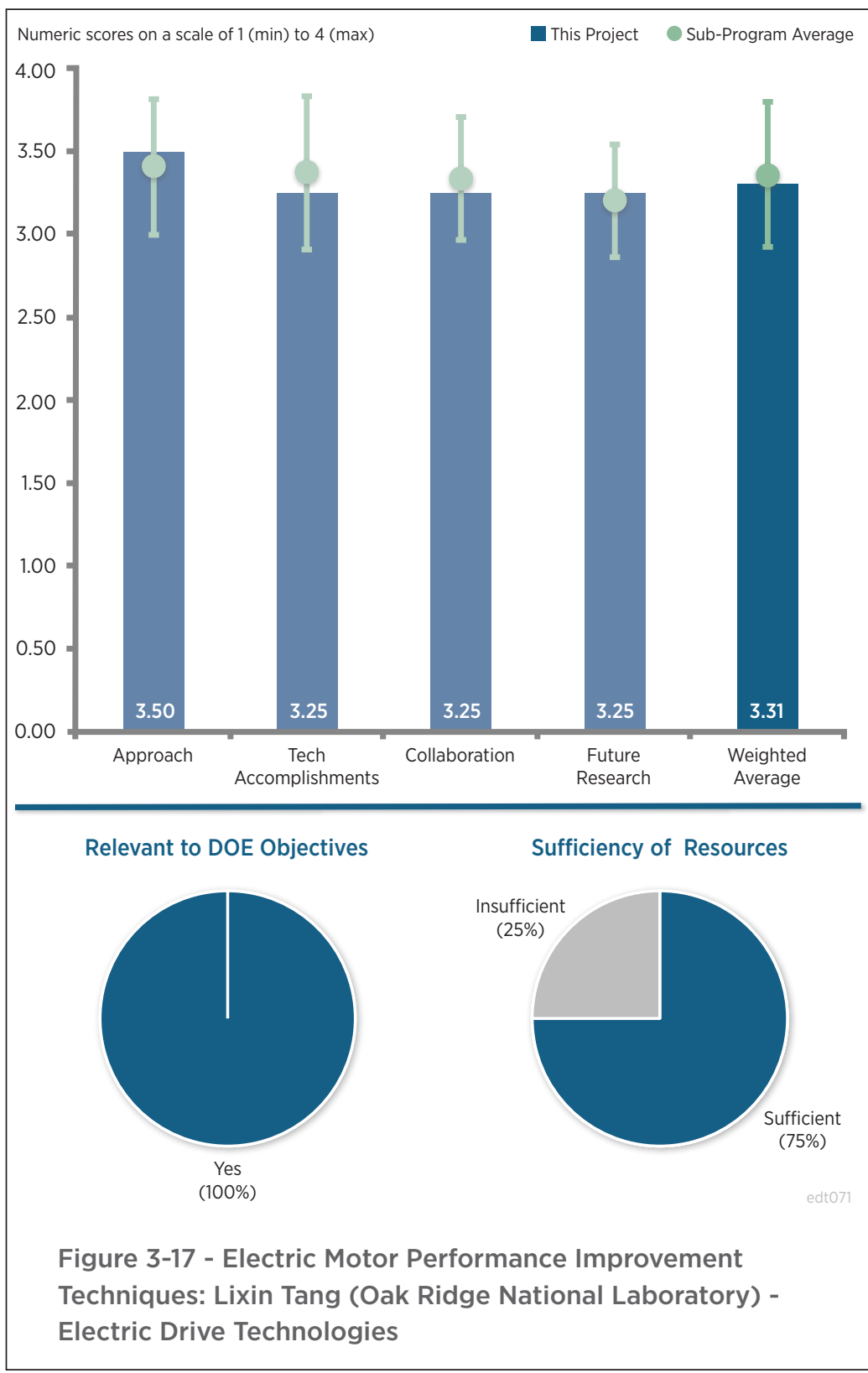


range switching, added cost of the switches, and torque issues in the event of a failure. This person emphasized that before the MSR can be judged successful, it must compete not only in terms of performance but also in the cost and packaging. This has not been addressed to date.

\section{Question 2: Technical accomplishments and progress toward overall project and DOE goals-the degree to which progress has been made, measured against performance indicators and demonstrated progress towards DOE goals.}

\section{Reviewer 1:}

The reviewer stated that the project team has made excellent progress on understanding a key risk of the multispeed method, namely the reconfiguration transient that occurs when switching from series to parallel mode and vice-versa. In addition, the potential reduction of permanent magnet content was noteworthy but should be verified in a tested prototype. The reviewer noted the FEA-generated efficiency maps were thoroughly developed.

\section{Reviewer 2:}

The reviewer said that the simulation results look very promising with increased power density and efficiency over traditional designs.

\section{Reviewer 3:}

The reviewer observed that the project team has made good progress by optimizing the design and quantifying system level value through analysis. The real challenges may be with reducing this to practice and understanding challenges that show up when an actual motor is integrated with the drive at scale. This person questioned what happens to transients as inductances scale-up, how stable are the controls as the motor operates continuously at a speed close to the transition, and are there any reliability considerations with a large number of repeated switching.

The reviewer mentioned that it would be good to understand why this promising approach has not been pursued seriously even though it has been proposed in the past (e.g., Panchien, L. I. N. "Adaptive winding system and control method for electric machines." U.S. Patent No. 7,977,842).

\section{Reviewer 4:}

The reviewer noted that the simulated results indicate that this approach has potential and have started to address some of the concerns, such as the torque interruptions. This person questioned if the cost comparisons on Slide 8 include the cost of the gate drive or just the switches. In addition, the reviewer wanted to know what the implications are in terms of the inverter packaging by adding additional switches and gate drives as well as the added complexity of routing the power to these switches. Furthermore, the reviewer was interested to find out if the project team can provide an efficiency map of the inverter for the two operating modes. Lastly, this person was interested in finding out what the impact is to the drive unit with the increase in stator outer radius increase to 141 $\mathrm{mm}$ from $100 \mathrm{~mm}$ and if this will cause issues with vehicle mounting.

The reviewer pointed out that, at this point in the project, the progress has been limited to simulations only, which indicate that the approach has merit along with issues that need to be addressed.

\section{Question 3: Collaboration and coordination with other institutions.}

\section{Reviewer 1:}

The reviewer observed that at this early stage the team is probably doing as well as they can in collaborating with others. As the technology readiness levels (TRL) increase, closer collaborations with industry may be required to understand and address practical issues with implementing this architecture in a product.

\section{Reviewer 2:}

The reviewer stated that the team is collaborating with Borg Warner on the design of their motor hairpin windings and cost evaluation of the system.

\section{Reviewer 3:}

The reviewer indicated that the team appears to be working well together performing the simulations. The proof will be in the building of a prototype system. 


\section{Reviewer 4:}

The reviewer reported that the project team responded well to a previous reviewer's suggestion to engage an outside collaborator to understand the system cost impact of multi-speed control. This person suggested that the project team should leverage this collaboration to the maximum extent possible to understand the cost increases due to additional parts and motor complexity.

Question 4: Proposed future research-the degree to which the project has effectively planned its future work in a logical manner by incorporating appropriate decision points, considering barriers to the realization of the proposed technology and, when sensible, mitigating risk by providing alternate development pathways.

\section{Reviewer 1:}

The reviewer indicated that future research is in line with the original plan and what is needed to provide the required data to determine if this is indeed a valid approach to meeting DOE's 2022 goals. This person suggested that the additional simulations should focus on identifying the source of the circulating current as well as control methods for minimizing torque transients while switching modes. The challenges identified in the presentation are still valid.

\section{Reviewer 2:}

The reviewer acknowledged that the team will optimize, build, and test the benchtop prototype in FY 2016 and work on the final design in FY 2017.

\section{Reviewer 3:}

The reviewer suggested that a failure mode and effects analysis (FMEA) and risk assessment could help guide future work by the team. It may make sense to consider demonstration of a motor and drive at close to full rating to understand scale-up issues. Also, it would be interesting to see how similar approaches would impact induction machines, maybe by including pole changing techniques.

\section{Reviewer 4:}

The reviewer commented that building the benchtop and final prototype is an appropriate method to verify the model predictions. However, the available budget may make this challenging unless a substantial amount of the hardware is already built.

\section{Question 5: Does this project support the overall DOE objectives of petroleum displacement? Why or why not?}

\section{Reviewer 1:}

The reviewer stated yes, this project supports the petroleum displacement objective by investigating the use of a potentially more efficient motor design.

\section{Reviewer 2:}

The reviewer indicated that the goals of this project include increasing the efficiency and power density of the electric drive system, both of which decrease energy use in the vehicle.

\section{Reviewer 3:}

The reviewer pointed out that with the design space opened up by the reconfigurable windings, more optimal machines that meet torque speed requirements at lower cost and lighter weight could be designed, which can in turn lead to improved viability of EV/HEVs.

\section{Reviewer 4:}

The reviewer commented that this project is one method to increase the efficiency at low torque and speed levels that needs to be investigated to determine if it is viable.

Question 6: Resources: How sufficient are the resources for the project to achieve the stated milestones in a timely fashion? 


\section{Reviewer 1:}

The reviewer explained that, if the team decides to adopt the above recommendation for demonstration of a motor and drive at scale, significantly more resources would be required.

\section{Reviewer 2:}

The reviewer agreed that the budget for the final build and test seems appropriate if existing hardware can be effectively leveraged.

\section{Reviewer 3:}

The reviewer noted that the resources for this project appear to be sufficient.

\section{Reviewer 4:}

The reviewer mentioned that, based on the simulations, resources appear to be sufficient to date. This person further described the resources as sufficient if the concerns are addressed in a timely manner. 


\section{Kilowatt Automotive Inverter with New 900 Volt Silicon Carbide MOSFET Technology: Jeffrey Casady (Cree) - edt073}

Presenter

Jeffrey Casady, Cree

\section{Reviewer Sample Size}

A total of three reviewers evaluated this project.

\section{Question 1: Approach to performing the work-the degree to which technical barriers are addressed, the project is well-designed, feasible, and integrated with other efforts.}

\section{Reviewer 1:}

The reviewer declared that the emphasis on achieving the lowest $\mathrm{R}_{\mathrm{DSON}}$ over operating temperature range and the reduction of the power losses in comparison with insulated-gate bipolar transistors (IGBT) and high-temperature operation address the critical barriers perfectly.

\section{Reviewer 2:}

The reviewer considered this to be a well-designed and structured project.

\section{Reviewer 3:}

The reviewer reported that this project is concentrating on the development and commercialization of an automotivequalified, high-power SiC MOSFET with plans to demonstrate it in an inverter. The approach shown is logical and realistic in timing assuming that the modifications from the previous version are successful which is reasonable since it is an improvement to a known chip. This person suggested that the process is by no means a simple one but appears to be well understood by the project team. The reviewer indicated that the project team is improving the basic building block of the power module. The module appears to have been designed on a different project but with the new device in mind.

Question 2: Technical accomplishments and progress toward overall project and DOE goals-the degree to which progress has been made, measured against performance indicators and demonstrated progress towards DOE goals.

\section{Reviewer 1:}

The reviewer acknowledged that the innovative 900V module design and the conduction and switching losses reduction (67\% total losses reduction) in comparison with the state-of-the-art automotive Infineon IGBT module are the major technical accomplishments of the project.

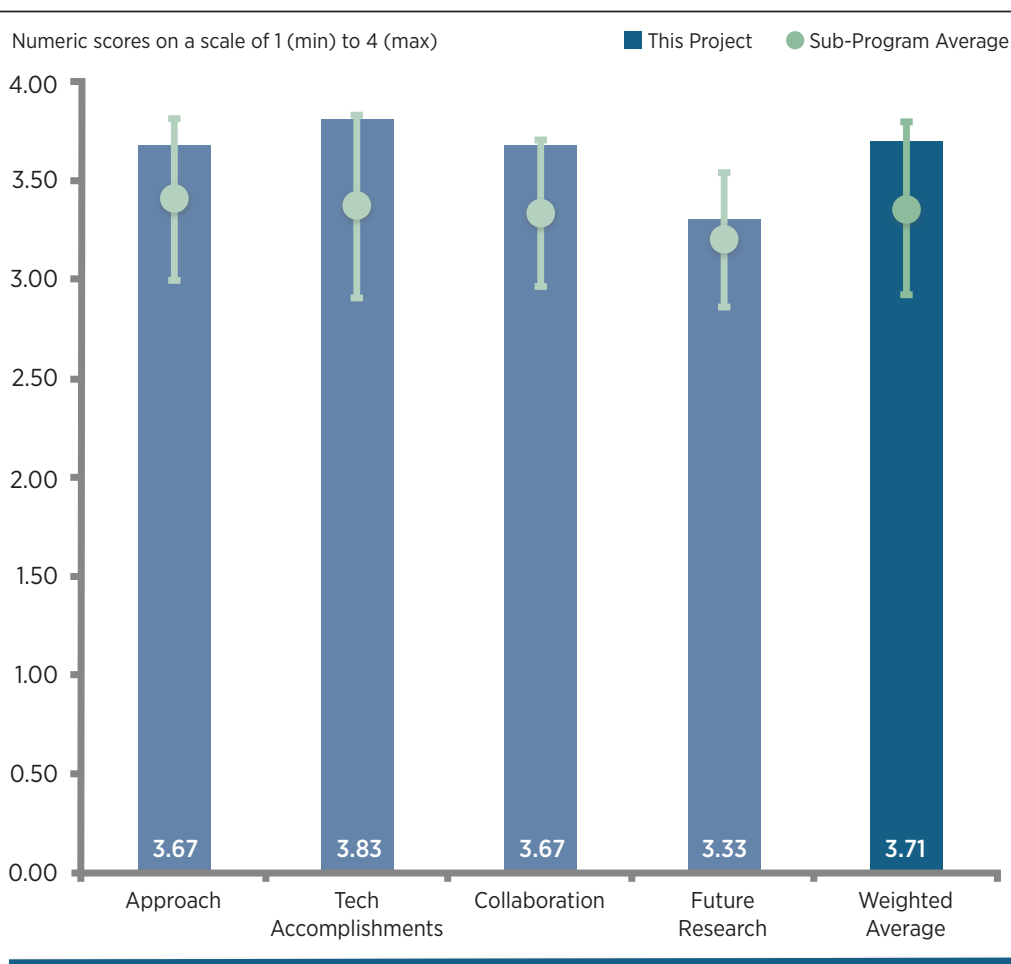

Sufficiency of Resources
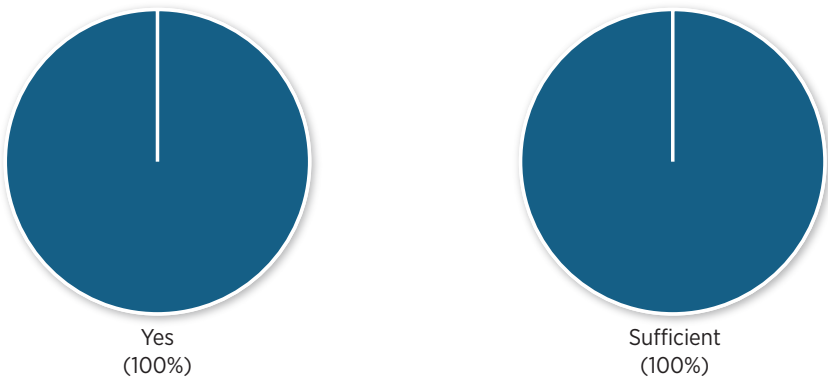

Figure 3-18 - 88 Kilowatt Automotive Inverter with New 900 Volt Silicon Carbide MOSFET Technology: Jeffrey Casady (Cree) - Electric Drive Technologies
Relevant to DOE Objectives 


\section{Reviewer 2:}

The reviewer stated that the test data presented indicates that the project team has been successful in building this improved device. This person noted that the modeling of vehicle performance using these devices shows a significant increase in efficiency at the vehicle level, which correlates with the data shown in the APEI inverter project efficiency maps. The project team has manufactured a significant number of wafers and dies for use during the qualification and the results to date are very good. The reviewer questioned if the TO247-4 maintains its performance advantage over the TO247-3 package over the full temperature range. The reviewer suggested that the real test will be what the actual inverter testing show as the performance increases and wondered if it will match the simulation.

\section{Question 3: Collaboration and coordination with other institutions.}

\section{Reviewer 1:}

The reviewer said collaboration with nine automotive OEMs demonstrates the undisputable practical value of the project's results.

\section{Reviewer 2:}

The reviewer explained the team members are appropriate for this project and seem to be working well together. This person is not sure how the creation of Wolfspeed will impact this project but the results to date indicate the team is working well together.

Question 4: Proposed future research-the degree to which the project has effectively planned its future work in a logical manner by incorporating appropriate decision points, considering barriers to the realization of the proposed technology and, when sensible, mitigating risk by providing alternate development pathways.

\section{Reviewer 1:}

The reviewer mentioned that the AEC-Q101 qualification plan at the chip level and the three-phase inverter demonstration is the great future plan and the path to the commercialization. The project has a very high real application potential in terms of the future production, said the reviewer.

\section{Reviewer 2:}

This person remarked that the proposed future work is the next step in readying the device for commercial use, which includes finishing the qualification and demonstrating the reliability of the devices and modules. The schedule for this was not shown nor was any significant concern mentioned during the presentation.

\section{Question 5: Does this project support the overall DOE objectives of petroleum displacement? Why or why not?}

\section{Reviewer 1:}

The reviewer stated that the DOE objectives were directly addressed by the project scope.

\section{Reviewer 2:}

The reviewer indicated that the switching device is the main determining factor in the performance of an EV and any improvement in that device is relevant.

\section{Question 6: Resources: How sufficient are the resources for the project to achieve the stated milestones in a timely fashion?}

\section{Reviewer 1:}

The reviewer reported that a lot was accomplished with the given resources and acknowledged it was really impressive. 


\section{Reviewer 2:}

The reviewer mentioned that the number of devices manufactured and tested indicate that the resources were sufficient for this project. This person pointed out that the test results indicate that the resources used in the design of the device were very capable in doing their job. 


\section{Acronyms and Abbreviations}

3D

A

$\mathrm{AC}$

AEC

Ag

Al

AlNiCo

AMR

ANL

APEEM

ARPA-E

ATF

B

B-H

BEV

$\mathrm{Br}$

${ }^{\circ} \mathrm{C}$

CEMI

CFD

Co

CPES

DC

DF

DOE

EDT
Three-dimensional

Ampere

Alternating current

Automotive Electronics Council

Silver

Aluminum

Aluminum-nickel-cobalt

Annual Merit Review

Argonne National Laboratory

Advanced Power Electronics and Electric Motors

Advanced Research Projects Agency - Energy

Automatic transmission fluid

Magnetic flux density

Magnetic hysteresis curve (magnetic flux density versus magnetic field strength)

Battery electric vehicle

Magnetic remanence

Degrees Celsius (Centigrade)

Clean Energy Manufacturing Initiative

Computational fluid dynamics

Cobalt

Center for Power Electronics Systems

Direct current

Dissipation factor

U.S. Department of Energy

Electric Drive Technologies 


\begin{tabular}{|c|c|}
\hline EDV & Electric drive vehicle \\
\hline EETT & Electrical and Electronics Technical Team \\
\hline EMC & Electromagnetic compatibility \\
\hline EMI & Electromagnetic interference \\
\hline ESR & Equivalent series resistance \\
\hline EV & Electric vehicle \\
\hline FMEA & Failure mode and effects analysis \\
\hline FCA & Fiat Chrysler Automobiles \\
\hline $\mathrm{Fe}$ & Iron \\
\hline FEA & Finite element analysis \\
\hline $\mathrm{Fe}-\mathrm{Co}$ & Iron-cobalt \\
\hline FMVSS & Federal Motor Vehicle Safety Standards \\
\hline FY & Fiscal year \\
\hline $\mathrm{GaN}$ & Gallium nitride \\
\hline GE & General Electric \\
\hline $\mathrm{H}$ & Henry (the unit of electrical inductance) \\
\hline $\mathrm{H}$ & Magnetic field strength \\
\hline Hci & Intrinsic coercivity \\
\hline $\mathrm{HEV}$ & Hybrid electric vehicle \\
\hline HV & High-voltage \\
\hline IAPG & Interagency Advanced Power Group \\
\hline IC & Integrated circuit \\
\hline IGBT & Insulated-gate bipolar transistors \\
\hline IPM & Integrated permanent magnet \\
\hline $\mathrm{kHz}$ & Kilohertz \\
\hline $\mathrm{kW}$ & Kilowatt \\
\hline
\end{tabular}


Liter

$\mu \mathrm{m} \quad$ Micrometer (micron)

millimeter

MOS Metal-oxide-semiconductor

MOSFET Metal-oxide-semiconductor field-effect transistor

MSR Multi-speed range

NA North American

nH Nanohenry

$\mathrm{Ni} \quad$ Nickel

NNMI National Network for Manufacturing Innovation

NRE Non-rare earth

NREL National Renewable Energy Laboratory

OBC On-board charger

Oe Oersteds

OEM Original equipment manufacturer

ORNL Oak Ridge National Laboratory

PBA Planar-Bond-All

PE Power electronics

PEI Polyetherimide

PEV Plug-in electric vehicle

PHEV Plug-in hybrid electric vehicle

PI Principal Investigator

PM Permanent magnet

PML Polymer-multi-layer

R\&D Research and development

RE Rare earth 
SAE Society of Automotive Engineers

$\mathrm{Si} \quad$ Silicon

SiC Silicon carbide

TARDEC U.S. Army Tank Automotive Research, Development and Engineering Center

TRL Technology readiness levels

UTK University of Tennessee, Knoxville

U.S. United States

U.S. DRIVE United States Driving Research and Innovation for Vehicle efficiency and Energy sustainability

V Volt

VAC Volt alternating current

VS Vehicle Systems

VSATT Vehicle Systems Analysis Tech Team

VTO Vehicle Technologies Office

WBG Wide bandgap 


\section{Advanced Combustion Engines}

Improving the efficiency of internal combustion engines (ICE) is one of the most promising and cost-effective near- to mid-term approaches to increasing highway vehicles' fuel economy. The Vehicle Technologies Office's (VTO) research and development (R\&D) activities address critical barriers to commercializing higher efficiency, very low emissions advanced internal combustion engines for passenger and commercial vehicles. This technology has great potential to reduce U.S. petroleum consumption, resulting in greater economic, environmental, and energy security.

Already offering outstanding drivability and reliability to over 230 million passenger vehicles, ICEs have the potential to become substantially more efficient. Initial results from laboratory engine tests indicate that passenger vehicle fuel economy can be improved by more than up to $50 \%$, and some vehicle simulation models estimate potential improvements of up to $75 \%$. Advanced combustion engines can utilize renewable fuels, and when combined with hybrid electric powertrains could have even further reductions in fuel consumption. As the U.S. Energy Information Administration reference case forecasts that, by 2035, more than 99\% of light-duty (LD) and heavy-duty (HD) vehicles sold will still have ICEs, the potential fuel savings is tremendous.

VTO undertakes R\&D activities to improve the efficiency of engines for both LD and HD highway vehicles, whether they run on petroleum-based (gasoline and diesel) or alternative fuels. We support every type of research in these areas, from fundamental science to prototype demonstration. VTO's research focuses on improving engine efficiency while meeting future federal and state emissions regulations through three main approaches.

- Developing advanced combustion strategies that maximize engine efficiency and minimize the formation of emissions within the engine cylinders.

- Developing cost-effective aftertreatment technologies that further reduce exhaust emissions.

- Reducing losses and recovering energy from engine waste heat.

The combustion engines subprogram also works with other subprograms in VTO to integrate and test advanced combustion engines in vehicles, such as the SuperTruck project. Commercialization of these advanced combustion engine technologies could allow the United States to cut its transportation fuel use and corresponding greenhouse gas (GHG) emissions by as much as $20 \%-40 \%$.

The combustion subprogram supports a number of unique user facilities at the national laboratories. In addition to the national laboratories, research and development is done in collaboration with industry, other federal agencies (such as the National Science Foundation) and universities, as well as through government/industry partnerships:

- The United States Driving Research and Innovation for Vehicle efficiency and Energy sustainability (U.S. DRIVE) Partnership focusing on LD vehicles and

- The 21st Century Truck Partnership, focusing on HD vehicles.

To enable further advances in combustion research and development, VTO also supports research on materials that can withstand high operating temperatures and pressures needed to capitalize on these engines' potential benefits, materials for energy recovery systems and controlling exhaust gases, and materials by design to solve specific issues.

The major goals of the Advanced Combustion Engine (ACE) R\&D subprogram are:

- By 2020, improve the fuel economy of gasoline vehicles by 35\% compared to 2009 model year baseline, and diesel vehicles by $30 \%$.

- By 2020, further improve HD engine efficiency to 55\% (a 30\% improvement) with demonstrations on commercial vehicle platforms.

- By 2020, improve $\mathrm{NO}_{\mathrm{x}}$ and PM emissions to EPA Tier 3 and California Low Emission Vehicles (LEV) III standards for LD engines and EPA standards for HD engines. 


\section{Subprogram Feedback}

The U.S. Department of Energy (DOE) received feedback on the overall technical subprogram areas presented during the 2016 Annual Merit Review (AMR). Each subprogram technical session was introduced with a presentation that provided an overview of subprogram goals and recent progress, followed by a series of detailed topic area project presentations.

The reviewers for a given subprogram area responded to a series of specific questions regarding the breadth, depth, and appropriateness of that DOE VTO subprogram's activities. The subprogram overview questions are listed below, and it should be noted that no scoring metrics were applied. These questions were used for all VTO subprogram overviews.

Question 1: Was the program area, including overall strategy, adequately covered?

Question 2: Is there an appropriate balance between near- mid- and long-term research and development?

Question 3: Were important issues and challenges identified?

Question 4: Are plans identified for addressing issues and challenges?

Question 5: Was progress clearly benchmarked against the previous year?

Question 6: Are the projects in this technology area addressing the broad problems and barriers that the Vehicle Technologies Office (VTO) is trying to solve?

Question 7: Does the program area appear to be focused, well-managed, and effective in addressing VTO's needs?

Question 8: What are the key strengths and weaknesses of the projects in this program area? Do any of the projects stand out on either end of the spectrum?

Question 9: Do these projects represent novel and/or innovative ways to approach these barriers as appropriate?

Question 10: Has the program area engaged appropriate partners?

Question 11: Is the program area collaborating with them effectively?

Question 12: Are there any gaps in the portfolio for this technology area?

Question 13: Are there topics that are not being adequately addressed?

Question 14: Are there other areas that this program area should consider funding to meet overall programmatic goals?

Question 15: Can you recommend new ways to approach the barriers addressed by this program area? 


\section{Question 16: Are there any other suggestions to improve the effectiveness of this program area?}

Responses to the subprogram overview questions are summarized in the following pages. Individual reviewer comments for each question are identified under the heading Reviewer 1, Reviewer 2, etc. Note that reviewer comments may be ordered differently; for example, for each specific subprogram overview presentation, the reviewer identified as Reviewer 1 in the first question may not be Reviewer 1 in the second question, etc. 


\section{Overview of the VTO Advanced Combustion Engine R\&D Program: Gurpreet Singh (U.S. Department of Energy) - ace000}

\section{Question 1: Was the program area, including overall strategy, adequately covered?}

\section{Reviewer 1:}

The reviewer stated that the strategic goal of reducing petroleum dependence by increasing efficiency of emissionscompliant ICE powertrains is clearly outlined, as are the primary directions for achieving this down to the goals in terms of percentage gains through advanced combustion strategies, aftertreatment technologies, and loss reduction and waste heat recovery (WHR) techniques. The reviewer further stated that the broad range of programs being pursued to support these approaches is also outlined at a reasonable level of detail.

\section{Reviewer 2:}

The reviewer agreed that the overall program was properly covered with many critical aspects of the program discussed. The reviewer noted that this program supports several national laboratories and each laboratory appears to have specific focuses. The reviewer commented that while the strategy and the synergy between the national laboratories are very good, the support to universities does not seem significant.

\section{Question 2: Is there an appropriate balance between near- mid- and long-term research and development?}

\section{Reviewer 1:}

The reviewer commented that there is a good balance between more academic, even basic research in terms of diagnostics, etc., through component technologies to all up engine demonstrations of new technologies.

\section{Reviewer 2:}

The reviewer stated that the balance is appropriate.

\section{Question 3: Were important issues and challenges identified?}

\section{Reviewer 1:}

The reviewer characterized the important issues and challenges as properly identified.

\section{Reviewer 2:}

The reviewer agreed that the challenges in each technology area are briefly outlined but cautioned that it is difficult to get into too much detail given the scope of the overall program. Nevertheless, the reviewer concluded that the individual project presentations cover these details well in any case. This is based on the reviewer having just finished reviewing 15 individual projects and thus having a relatively good idea of what's being done up and down the line of this research topic.

\section{Question 4: Are plans identified for addressing issues and challenges?}

\section{Reviewer 1:}

The reviewer affirmed that the near- and long-term plans are clearly outlined and describe how the program is tackling the various issues being faced to achieve the overall whole program's efficiency and emissions goals.

\section{Reviewer 2:}

The reviewer replied yes.

\section{Question 5: Was progress clearly benchmarked against the previous year?}

\section{Reviewer 1:}

The reviewer replied yes.

\section{Reviewer 2:}

The reviewer remarked that more could have been done to specifically address this in this presentation. 
Question 6: Are the projects in this technology area addressing the broad problems and barriers that the Vehicle Technologies Office (VTO) is trying to solve?

\section{Reviewer 1:}

The reviewer agreed that this is detailed very specifically.

\section{Reviewer 2:}

The reviewer answered yes.

Question 7: Does the program area appear to be focused, well-managed, and effective in addressing VTO's needs?

\section{Reviewer 1:}

The reviewer replied yes to all of these criteria.

\section{Reviewer 2:}

The reviewer replied yes.

\section{Question 8: What are the key strengths and weaknesses of the projects in this program area? Do any of the projects stand out on either end of the spectrum?}

\section{Reviewer 1:}

The reviewer declared as tremendous strengths the coordination and participation across academia, DOE laboratories, component and tool suppliers (such as computational fluid dynamics [CFD] vendors and others), energy companies, and engine manufacturers. The reviewer also described the Engine Combustion Network (ECN) (part of ACE005) as a standout example of this level of cooperative research to achieve a common goal. The reviewer concluded that no glaring weaknesses come to mind.

\section{Reviewer 2:}

The reviewer stated that the key strength is the experimental study and the fundamental modeling research in all of the national laboratories that are supported by this program. Conversely, the reviewer described the main weakness as the applied modeling research at Argonne National Laboratory (ANL), which uses commercial code (CONVERGE) to perform engine performance simulation. The reviewer offered that such modeling work using commercial codes does not appear to be appropriate for a national laboratory and that such modeling work should be left to the industry or academia. In the meantime, the numerical model development at universities need to be strengthened. The reviewer further commented that Los Alamos National Laboratory (LANL), which has been developing the new engine simulation code for quite a number of years, has never been able to release anything. The reviewer observed that nowadays the industry has its own code and models, and it is highly probably that the code developed at LANL will never get used.

\section{Question 9: Do these projects represent novel and/or innovative ways to approach these barriers as appropriate?}

\section{Reviewer 1:}

The reviewer agreed that all of the projects incorporate innovative and sometimes even novel approaches to overcoming the barriers.

\section{Reviewer 2:}

The reviewer replied yes.

\section{Question 10: Has the program area engaged appropriate partners?}

\section{Reviewer 1:}

The reviewer agreed, noting as in a previous question that a broad range of partners have been engaged, adding that about the only thing lacking perhaps is engaging government laboratories outside of DOE to a larger extent (such as those with the U.S. Department of Defense and others). 


\section{Reviewer 2:}

The reviewer encouraged more collaborations with universities.

\section{Question 11: Is the program area collaborating with them effectively?}

\section{Reviewer 1:}

The reviewer replied yes.

\section{Reviewer 2:}

The reviewer replied yes.

\section{Question 12: Are there any gaps in the portfolio for this technology area?}

\section{Reviewer 1:}

The reviewer said no.

\section{Reviewer 2:}

The reviewer observed that some reviewers of individual projects in previous years have complained about the balance between diesel and gasoline engine research, but there appears to be a greater emphasize on gasoline engine research in this year's program, which addresses this concern.

\section{Question 13: Are there topics that are not being adequately addressed?}

\section{Reviewer 1:}

The reviewer stated that while there is great focused research looking at spray combustion chemistry, emissions, etc., the interactions between them (combustion-turbulence, sprays-emissions, etc.) might themselves be topics of more focused research. The reviewer also suggested that some of the CFD research could be better tied into commercialization to make the progress seen in modeling codes KIVA FE and RAPTOR trickle down faster into the commercial tools used by industry.

\section{Question 14: Are there other areas that this program area should consider funding to meet overall programmatic goals?}

\section{Reviewer 1:}

The reviewer referenced previous comments made in question 13.

\section{Question 15: Can you recommend new ways to approach the barriers addressed by this program area?}

\section{Reviewer 1:}

The reviewer replied that the approaches being pursued here appear to address the barriers present in this area.

\section{Reviewer 2:}

The reviewer warned that regarding the new KIVA code development at LANL, it is not clear how successful this activity would be. The reviewer observed that many advancements in engine modeling have been made in the industry in the past 10 years, and that the code development does not incorporate the state-of-the-art models. Even if the new KIVA code is released, it is unlikely that industry will spend resources to move all the sub-models into the new KIVA code. Plus, there is no customer support for the new KIVA code, which would defeat the interest of using it by the industry. The reviewer also pointed out that nowadays, the industry does not use a code that does not have customer support, and concluded that this is why many industry players have abandoned their own version of KIVA-3V and use commercial codes.

The reviewer recommended that VTO form an ad-hoc committee to investigate the necessity of developing new KIVA code. Similarly, the reviewer suggested that the CONVERGE modeling work at ANL may need to be evaluated because this work possibly can be done by universities at a lower cost. The reviewer concluded that the numerical modeling at national laboratories should be focused on fundamental aspects rather than using a commercial code to investigate the engine performance optimization. 
Question 16: Are there any other suggestions to improve the effectiveness of this program area?

Reviewer 1:

The reviewer stated that ECN as a cooperative team approach to looking at problems is such a good concept that perhaps it can be applied to other areas (aftertreatment, simulation, emissions, etc.) as well. 


\section{Project Feedback}

In this merit review activity, each reviewer was asked to respond to a series of questions, involving multiple-choice responses, expository responses where text comments were requested, and numeric score responses (on a scale of 1.0 to 4.0). In the pages that follow, the reviewer responses to each question for each project will be summarized: the multiple choice and numeric score questions will be presented in graph form for each project, and the expository text responses will be summarized in paragraph form for each question. A table presenting the average numeric score for each question for each project is presented below.

\section{Table 4-1 - Project Feedback}

\begin{tabular}{|c|c|c|c|c|c|c|c|}
\hline Presentation Title & $\begin{array}{c}\text { Principal } \\
\text { Investigator } \\
\text { and } \\
\text { Organization }\end{array}$ & $\begin{array}{l}\text { Page } \\
\text { Number }\end{array}$ & Approach & $\begin{array}{c}\text { Technical } \\
\text { Accomplishments }\end{array}$ & Collaborations & $\begin{array}{c}\text { Future } \\
\text { Research }\end{array}$ & $\begin{array}{c}\text { Weighted } \\
\text { Average }\end{array}$ \\
\hline $\begin{array}{l}\text { Heavy-Duty } \\
\text { Low-Temperature } \\
\text { and Diesel } \\
\text { Combustion } \\
\text { and Heavy-Duty } \\
\text { Combustion } \\
\text { Modeling }\end{array}$ & $\begin{array}{l}\text { Musculus, } \\
\text { Mark (SNL) }\end{array}$ & 4-14 & 3.50 & 3.38 & 3.38 & 3.25 & 3.39 \\
\hline $\begin{array}{l}\text { Light-Duty Diesel } \\
\text { Combustion }\end{array}$ & $\begin{array}{l}\text { Busch, } \\
\text { Stephen } \\
\text { (SNL) }\end{array}$ & 4-17 & 3.30 & 3.00 & 3.30 & 3.00 & 3.11 \\
\hline $\begin{array}{l}\text { Low-Temperature } \\
\text { Gasoline } \\
\text { Combustion } \\
\text { (LTGC) Engine } \\
\text { Research }\end{array}$ & $\begin{array}{l}\text { Dec, John } \\
\text { (SNL) }\end{array}$ & 4-22 & 2.90 & 3.10 & 2.90 & 2.70 & 2.98 \\
\hline $\begin{array}{l}\text { Spray Combustion } \\
\text { Cross-Cut Engine } \\
\text { Research }\end{array}$ & $\begin{array}{l}\text { Pickett, Lyle } \\
\text { (SNL) }\end{array}$ & 4-26 & 3.38 & 3.38 & 3.25 & 3.38 & 3.36 \\
\hline $\begin{array}{c}\text { Gasoline } \\
\text { Combustion } \\
\text { Fundamentals }\end{array}$ & $\begin{array}{l}\text { Ekoto, Isaac } \\
\text { (SNL) }\end{array}$ & $4-30$ & 3.00 & 3.00 & 2.83 & 3.17 & 3.00 \\
\hline $\begin{array}{l}\text { Large Eddy } \\
\text { Simulation } \\
\text { (LES) Applied to } \\
\text { Advanced Engine } \\
\text { Combustion } \\
\text { Research }\end{array}$ & $\begin{array}{l}\text { Oefelein, Joe } \\
\text { (SNL) }\end{array}$ & $4-32$ & 3.60 & 3.50 & 3.30 & 3.20 & 3.46 \\
\hline $\begin{array}{l}\text { Fuel Injection and } \\
\text { Spray Research } \\
\text { Using X-Ray } \\
\text { Diagnostics }\end{array}$ & $\begin{array}{l}\text { Powell, } \\
\text { Christopher } \\
\text { (ANL) }\end{array}$ & 4-36 & 3.30 & 3.30 & 3.30 & 3.20 & 3.29 \\
\hline
\end{tabular}




\begin{tabular}{|c|c|c|c|c|c|c|c|}
\hline Presentation Title & $\begin{array}{c}\text { Principal } \\
\text { Investigator } \\
\text { and } \\
\text { Organization }\end{array}$ & $\begin{array}{l}\text { Page } \\
\text { Number }\end{array}$ & Approach & $\begin{array}{c}\text { Technical } \\
\text { Accomplishments }\end{array}$ & Collaborations & $\begin{array}{l}\text { Future } \\
\text { Research }\end{array}$ & $\begin{array}{l}\text { Weighted } \\
\text { Average }\end{array}$ \\
\hline $\begin{array}{l}\text { Advances in } \\
\text { High-Efficiency } \\
\text { Gasoline } \\
\text { Compression } \\
\text { Ignition }\end{array}$ & $\begin{array}{l}\text { Ciatti, Steve } \\
\text { (ANL) }\end{array}$ & $4-40$ & 2.83 & 2.67 & 3.17 & 2.67 & 2.77 \\
\hline $\begin{array}{l}\text { Model } \\
\text { Development } \\
\text { and Analysis } \\
\text { of Clean and } \\
\text { Efficient Engine } \\
\text { Combustion }\end{array}$ & $\begin{array}{l}\text { Whitesides, } \\
\text { Russell } \\
\text { (LLNL) }\end{array}$ & 4-42 & 3.33 & 3.17 & 2.92 & 2.92 & 3.15 \\
\hline $\begin{array}{l}\text { Chemical Kinetic } \\
\text { Models for } \\
\text { Advanced Engine } \\
\text { Combustion }\end{array}$ & $\begin{array}{l}\text { Pitz, Bill } \\
\text { (LLNL) }\end{array}$ & 4-46 & 3.80 & 3.60 & 3.40 & 3.60 & 3.63 \\
\hline $\begin{array}{l}2016 \text { KIV A-hpFE } \\
\text { Development: } \\
\text { A Robust and } \\
\text { Accurate Engine } \\
\text { Modeling } \\
\text { Software }\end{array}$ & $\begin{array}{c}\text { Carrington, } \\
\text { David } \\
\text { (LANL) }\end{array}$ & 4-49 & 2.50 & 2.63 & 2.00 & 2.38 & 2.48 \\
\hline $\begin{array}{l}\text { Stretch Efficiency } \\
\text { for Combustion } \\
\text { Engines: } \\
\text { Exploiting New } \\
\text { Combustion } \\
\text { Regimes }\end{array}$ & $\begin{array}{l}\text { Daw, Stuart } \\
\text { (ORNL) }\end{array}$ & 4-52 & 3.08 & 3.08 & 2.58 & 2.92 & 3.00 \\
\hline $\begin{array}{l}\text { High-Efficiency } \\
\text { Clean Combustion } \\
\text { in Multi-Cylinder } \\
\text { Light-Duty } \\
\text { Engines }\end{array}$ & $\begin{array}{l}\text { Curran, Scott } \\
\text { (ORNL) }\end{array}$ & 4-57 & 3.38 & 3.38 & 3.13 & 3.38 & 3.34 \\
\hline $\begin{array}{l}\text { Accelerating } \\
\text { Predictive } \\
\text { Simulation } \\
\text { of Internal } \\
\text { Combustion } \\
\text { Engines with High } \\
\text { Performance } \\
\text { Computing }\end{array}$ & $\begin{array}{l}\text { Ewards, Kevin } \\
\text { (ORNL) }\end{array}$ & $4-60$ & 2.86 & 3.07 & 3.21 & 3.07 & 3.04 \\
\hline
\end{tabular}




\begin{tabular}{|c|c|c|c|c|c|c|c|}
\hline Presentation Title & $\begin{array}{c}\text { Principal } \\
\text { Investigator } \\
\text { and } \\
\text { Organization }\end{array}$ & $\begin{array}{l}\text { Page } \\
\text { Number }\end{array}$ & Approach & $\begin{array}{c}\text { Technical } \\
\text { Accomplishments }\end{array}$ & Collaborations & $\begin{array}{l}\text { Future } \\
\text { Research }\end{array}$ & $\begin{array}{l}\text { Weighted } \\
\text { Average }\end{array}$ \\
\hline $\begin{array}{l}\text { Joint } \\
\text { Development and } \\
\text { Coordination of } \\
\text { Emissions Control } \\
\text { Data and Models } \\
\text { (CLEERS Analysis } \\
\text { and Coordination) }\end{array}$ & $\begin{array}{l}\text { Daw, Stuart } \\
\text { (ORNL) }\end{array}$ & 4-65 & 3.88 & 3.63 & 3.88 & 3.63 & 3.72 \\
\hline $\begin{array}{c}\text { CLEERS: } \\
\text { Aftertreatment } \\
\text { Modeling and } \\
\text { Analysis }\end{array}$ & $\begin{array}{l}\text { Wang, Yong } \\
\text { (PNNL) }\end{array}$ & $4-69$ & 3.13 & 3.25 & 3.38 & 3.25 & 3.23 \\
\hline $\begin{array}{l}\text { Ash-Durable } \\
\text { Catalyzed Filters } \\
\text { for Gasoline } \\
\text { Direct Injection } \\
\text { (GDI) Engines }\end{array}$ & $\begin{array}{l}\text { Seong, Hee Je } \\
\text { (ANL) }\end{array}$ & 4-73 & 3.30 & 3.00 & 3.40 & 3.10 & 3.14 \\
\hline $\begin{array}{l}\text { Enhanced } \\
\text { High- and Low- } \\
\text { Temperature } \\
\text { Performance of } \\
\text { NO }_{\mathrm{x}} \text { Reduction } \\
\text { Materials }\end{array}$ & $\begin{array}{l}\text { Gao, Feng } \\
\text { (PNNL) }\end{array}$ & 4-77 & 3.38 & 3.38 & 3.63 & 3.13 & 3.38 \\
\hline $\begin{array}{l}\text { Next Generation } \\
\text { SCR-Dosing } \\
\text { System } \\
\text { Investigation }\end{array}$ & $\begin{array}{l}\text { Karkamkar, } \\
\text { Abhijeet } \\
\text { (PNNL) }\end{array}$ & $4-80$ & 3.17 & 3.17 & 3.08 & 3.00 & 3.14 \\
\hline $\begin{array}{c}\text { Cummins-ORNL/ } \\
\text { FEERC Emissions } \\
\text { CRADA: } \mathrm{NO}_{\mathrm{x}} \\
\text { Control and } \\
\text { Measurement } \\
\text { Technology for } \\
\text { Heavy-Duty } \\
\text { Diesel Engines, } \\
\text { Self-Diagnosing } \\
\text { SmartCatalyst } \\
\text { Systems }\end{array}$ & $\begin{array}{l}\text { Partridge, Bill } \\
\text { (ORNL) }\end{array}$ & $4-85$ & 3.10 & 3.10 & 3.10 & 3.10 & 3.10 \\
\hline $\begin{array}{c}\text { Emissions Control } \\
\text { for Lean Gasoline } \\
\text { Engines }\end{array}$ & $\begin{array}{l}\text { Parks, Jim } \\
\text { (ORNL) }\end{array}$ & $4-90$ & 3.33 & 3.67 & 3.83 & 3.50 & 3.58 \\
\hline
\end{tabular}




\begin{tabular}{|c|c|c|c|c|c|c|c|}
\hline Presentation Title & $\begin{array}{c}\text { Principal } \\
\text { Investigator } \\
\text { and } \\
\text { Organization }\end{array}$ & $\begin{array}{l}\text { Page } \\
\text { Number }\end{array}$ & Approach & $\begin{array}{c}\text { Technical } \\
\text { Accomplishments }\end{array}$ & Collaborations & $\begin{array}{c}\text { Future } \\
\text { Research }\end{array}$ & $\begin{array}{l}\text { Weighted } \\
\text { Average }\end{array}$ \\
\hline $\begin{array}{l}\text { Neutron Imaging } \\
\text { of Advanced } \\
\text { Transportation } \\
\text { Technologies }\end{array}$ & $\begin{array}{l}\text { Toops, Todd } \\
\text { (ORNL) }\end{array}$ & 4-93 & 3.40 & 3.40 & 3.10 & 3.20 & 3.34 \\
\hline $\begin{array}{l}\text { RCM Studies to } \\
\text { Enable Gasoline- } \\
\text { Relevant Low- } \\
\text { Temperature } \\
\text { Combustion }\end{array}$ & $\begin{array}{l}\text { Goldsborough, } \\
\text { Scott (ANL) }\end{array}$ & 4-96 & 3.10 & 3.30 & 3.20 & 3.10 & 3.21 \\
\hline $\begin{array}{l}\text { Fuel-Neutral } \\
\text { Studies of } \\
\text { Particulate } \\
\text { Matter Transport } \\
\text { Emissions }\end{array}$ & $\begin{array}{l}\text { Stewart, Mark } \\
\text { (PNNL) }\end{array}$ & 4-100 & 3.50 & 3.38 & 3.50 & 3.38 & 3.42 \\
\hline $\begin{array}{c}\text { SuperTruck- } \\
\text { Development and } \\
\text { Demonstration of } \\
\text { a Fuel-Efficient } \\
\text { Class } 8 \text { Tractor } \\
\text { and Trailer, Engine } \\
\text { Systems }\end{array}$ & $\begin{array}{c}\text { Zukouski, } \\
\text { Russ (Navistar } \\
\text { International } \\
\text { Corp.) }\end{array}$ & 4-103 & 3.33 & 3.08 & 3.50 & 3.25 & 3.22 \\
\hline $\begin{array}{l}\text { Volvo SuperTruck } \\
\text { - Powertrain } \\
\text { Technologies } \\
\text { for Efficiency } \\
\text { Improvement }\end{array}$ & $\begin{array}{c}\text { Amar, Pascal } \\
\text { (Volvo) }\end{array}$ & 4-108 & 3.64 & 3.93 & 3.36 & 3.71 & 3.76 \\
\hline $\begin{array}{l}\text { Advancements } \\
\text { in Fuel Spray } \\
\text { and Combustion } \\
\text { Modeling with } \\
\text { High-Performance } \\
\text { Computing } \\
\text { Resources }\end{array}$ & $\begin{array}{l}\text { Som, Sibendu } \\
\text { (ANL) }\end{array}$ & 4-113 & 3.00 & 3.00 & 2.83 & 2.67 & 2.94 \\
\hline $\begin{array}{l}\text { Improved } \\
\text { Solvers for } \\
\text { Advanced Engine } \\
\text { Combustion } \\
\text { Simulation }\end{array}$ & $\begin{array}{l}\text { McNenly, } \\
\text { Matthew } \\
\text { (LLNL) }\end{array}$ & 4-116 & 3.50 & 3.42 & 3.42 & 3.08 & 3.40 \\
\hline
\end{tabular}




\begin{tabular}{|c|c|c|c|c|c|c|c|}
\hline Presentation Title & $\begin{array}{c}\text { Principal } \\
\text { Investigator } \\
\text { and } \\
\text { Organization }\end{array}$ & $\begin{array}{c}\text { Page } \\
\text { Number }\end{array}$ & Approach & $\begin{array}{c}\text { Technical } \\
\text { Accomplishments }\end{array}$ & Collaborations & $\begin{array}{l}\text { Future } \\
\text { Research }\end{array}$ & $\begin{array}{c}\text { Weighted } \\
\text { Average }\end{array}$ \\
\hline $\begin{array}{c}\text { Cummins/ORNL- } \\
\text { FEERC Combustion } \\
\text { CRADA: } \\
\text { Characterization } \\
\text { and Reduction } \\
\text { of Combustion } \\
\text { Variations }\end{array}$ & $\begin{array}{l}\text { Partridge, Bill } \\
\text { (ORNL) }\end{array}$ & 4-120 & 3.00 & 3.13 & 3.25 & 3.00 & 3.09 \\
\hline $\begin{array}{l}\text { Thermally Stable } \\
\text { Ultra Low- } \\
\text { Temperature } \\
\text { Oxidation } \\
\text { Catalysts }\end{array}$ & $\begin{array}{l}\text { Szanyi, Janos } \\
\text { (PNNL) }\end{array}$ & $4-123$ & 3.13 & 3.50 & 3.38 & 3.13 & 3.34 \\
\hline $\begin{array}{l}\text { High-Efficiency } \\
\text { GDI Engine } \\
\text { Research, with } \\
\text { Emphasis on } \\
\text { Ignition Systems }\end{array}$ & $\begin{array}{c}\text { Wallner, } \\
\text { Thomas (ANL) }\end{array}$ & 4-127 & 2.50 & 1.50 & 2.50 & 2.50 & 2.00 \\
\hline $\begin{array}{l}\text { Low-Temperature } \\
\text { Emission Control } \\
\text { to Enable Fuel } \\
\text { Efficient Engine } \\
\text { Commercialization }\end{array}$ & $\begin{array}{l}\text { Toops, Todd } \\
\text { (ORNL) }\end{array}$ & 4-129 & 3.80 & 3.70 & 3.60 & 3.40 & 3.68 \\
\hline $\begin{array}{l}\text { High-Dilution } \\
\text { Stoichiometric } \\
\text { Gasoline Direct- } \\
\text { Injection (SGDI) } \\
\text { Combustion } \\
\text { Control } \\
\text { Development }\end{array}$ & $\begin{array}{l}\text { Kaul, Brian } \\
\text { (ORNL) }\end{array}$ & 4-133 & 2.90 & 2.70 & 2.70 & 2.70 & 2.75 \\
\hline $\begin{array}{l}\text { High-Efficiency } \\
\text { VCR Engine with } \\
\text { Variable Valve } \\
\text { Actuation and New } \\
\text { Supercharging } \\
\text { Technology }\end{array}$ & $\begin{array}{c}\text { Mendler, } \\
\text { Charles } \\
\text { (Envera LLC) }\end{array}$ & 4-136 & 2.25 & 2.44 & 2.13 & 2.25 & 2.33 \\
\hline $\begin{array}{l}\text { Lean Miller } \\
\text { Cycle System } \\
\text { Development } \\
\text { for Light-Duty } \\
\text { Vehicles }\end{array}$ & $\begin{array}{l}\text { Sczomak, } \\
\text { David } \\
\text { (General } \\
\text { Motors) }\end{array}$ & 4-142 & 3.20 & 2.90 & 3.00 & 3.20 & 3.03 \\
\hline
\end{tabular}




\begin{tabular}{|c|c|c|c|c|c|c|c|}
\hline Presentation Title & $\begin{array}{c}\text { Principal } \\
\text { Investigator } \\
\text { and } \\
\text { Organization }\end{array}$ & $\begin{array}{l}\text { Page } \\
\text { Number }\end{array}$ & Approach & $\begin{array}{c}\text { Technical } \\
\text { Accomplishments }\end{array}$ & Collaborations & $\begin{array}{l}\text { Future } \\
\text { Research }\end{array}$ & $\begin{array}{c}\text { Weighted } \\
\text { Average }\end{array}$ \\
\hline $\begin{array}{l}\text { Ultra-Efficient } \\
\text { Light-Duty } \\
\text { Powertrain with } \\
\text { Gasoline Low- } \\
\text { Temperature } \\
\text { Combustion }\end{array}$ & $\begin{array}{l}\text { Confer, Keith } \\
\text { (Delphi } \\
\text { Powertrain) }\end{array}$ & 4-146 & 3.33 & 3.50 & 2.83 & 3.00 & 3.31 \\
\hline $\begin{array}{l}\text { Metal Oxide Nano- } \\
\text { Array Catalysts for } \\
\text { Low-Temperature } \\
\text { Diesel Oxidation }\end{array}$ & $\begin{array}{l}\text { Gao, Pu-Xian } \\
\text { (U. Conn) }\end{array}$ & 4-150 & 3.00 & 2.75 & 3.00 & 2.88 & 2.86 \\
\hline $\begin{array}{c}\text { Micro-Jet } \\
\text { Enhanced Ignition } \\
\text { with a Variable } \\
\text { Orifice Fuel } \\
\text { Injector for High- } \\
\text { Efficiency Lean- } \\
\text { Burn Combustion }\end{array}$ & $\begin{array}{l}\text { Lee, Chia-Fon } \\
\text { (U. of Illinois) }\end{array}$ & 4-154 & 2.50 & 2.13 & 1.88 & 2.75 & 2.27 \\
\hline $\begin{array}{l}\text { Affordable Rankine } \\
\text { Cycle (ARC) Waste } \\
\text { Heat Recovery for } \\
\text { Heavy-Duty Trucks }\end{array}$ & $\begin{array}{l}\text { Subramanian, } \\
\text { Swami (Eaton } \\
\text { Corp.) }\end{array}$ & 4-157 & 3.08 & 3.08 & 3.67 & 3.25 & 3.18 \\
\hline $\begin{array}{c}\text { Cummins 55\% BTE } \\
\text { Project }\end{array}$ & $\begin{array}{l}\text { Kocher, Lyle } \\
\text { (Cummins) }\end{array}$ & 4-161 & 3.50 & 3.38 & 2.63 & 3.31 & 3.30 \\
\hline $\begin{array}{l}\text { Improved Fuel } \\
\text { Efficiency through } \\
\text { Adaptive Radio } \\
\text { Frequency } \\
\text { Controls and } \\
\text { Diagnostics for } \\
\text { Advanced Catalyst } \\
\text { Systems }\end{array}$ & $\begin{array}{c}\text { Sappok, } \\
\text { Alexander } \\
\text { (Filter Sensing } \\
\text { Technologies, } \\
\text { Inc.) }\end{array}$ & 4-166 & 3.43 & 3.14 & 3.64 & 3.36 & 3.30 \\
\hline Overall Average & & & 3.20 & 3.14 & 3.12 & 3.09 & 3.15 \\
\hline
\end{tabular}




\section{Heavy-Duty Low- \\ Temperature and Diesel \\ Combustion and Heavy- \\ Duty Combustion Modeling: \\ Mark Musculus (Sandia \\ National Laboratories) - ace001}

\section{Presenter}

Mark Musculus, Sandia National Laboratories

\section{Reviewer Sample Size}

A total of four reviewers evaluated this project.

\section{Question 1: Approach to performing the work-the degree to which technical barriers are addressed, the project is well-designed, feasible, and integrated with other efforts.}

\section{Reviewer 1:}

The reviewer stated the project has shown a clear path to achieve the long-term objective, and the team has executed key milestones through optical imaging and CFD modeling of low-temperature combustion (LTC) combustion processes.

\section{Reviewer 2:}

The reviewer stated this remains an excellent program that combines state-of-the-art optical diagnostics with multidimensional engine modeling to examine some of the more challenging issues in engine development today. The innovation seemed very high to this reviewer, who highlighted said it is quite clever to take the beam steering effect, which at first glance appears to be a serious problem, and turning it around to become a diagnostic solution by using beam width as a measure for scalar dissipation. As is employing infrared (IR) thermometry via window coating and soot luminosity coupling with CFD to potentially develop a soot mass quantification technique.

\section{Reviewer 3:}

The reviewer commented that there is an unusually good balance of experimental and computational approaches within the project. It is a nice presentation of experimental imaging challenges and approaches to overcome them. The reviewer said on the other hand, additional bigger-picture materials pointing to the desired progress directions and connections would have helped. 


\section{Question 2: Technical accomplishments and progress toward overall project and DOE goals-the degree to which progress has been made, measured against performance indicators and demonstrated progress towards DOE goals.}

\section{Reviewer 1:}

The reviewer declared valuable engine in-cylinder spray data on ECN Spray B was obtained. As the project is for HD engine focus, the reviewer recommended using a larger nozzle with one to three holes if possible. The reviewer also commented that the new heat transfer diagnostic method using IR thermometry is very interesting. Additionally, measurement and CFD modeling on at least some engine conditions is highly anticipated in fiscal year (FY) 2016/2017.

\section{Reviewer 2:}

The reviewer said the progress and technical accomplishments are excellent. The Spray B measurements and analysis are very illuminating, and there seems to be a potential breakthrough in understanding ignition delay increase with injection duration as a scalar dissipation effect with the possibility that the beam steering problem could be turned into a diagnostic tool itself. There are challenges to be overcome with the IR thermometry technique owing to the issues of the metal coatings used initially, but the reviewer remains confident in the team's problem solving abilities; the same with the soot luminosity correlation approach for obtaining integrated soot mass estimates.

\section{Reviewer 3:}

The reviewer noted that experimental challenges and investigation of mechanistic questions seem to have limited the progress on two of the three barriers cited on Slide 2 (i.e., LTC aftertreatment integration and impact of future fuels on LTC). The reviewer pointed out that uncertainty analysis discussion focused on the error bars in the experimental data only. This person recommended considering uncertainty quantification (UQ) and sensitivities for the simulation models, including not just the model-form uncertainty inherent in the three models presented, but also the myriad input coefficients to those models.

\section{Question 3: Collaboration and coordination with other institutions.}

\section{Reviewer 1:}

There were good collaborations with various partners from industries and universities the reviewer noted. As stated in Critical Assumptions and Issues, the current optical engine testing is limited in engine speed and load for HD applications, especially off-road diesel engines where rated condition is the most important one. The reviewer highly recommends collaboration in this area.

\section{Reviewer 2:}

The reviewer commented collaboration with ECN is particularly noteworthy, but also with the various universities and industrial concerns. It might be interesting to work with code vendors such as Convergent Science, Inc. (CSI) to do comparative studies with commercial CFD software in addition to KIVA calculations.

\section{Reviewer 3:}

The reviewer stated the Advanced Engine Combustion collaboration is very effective.

Question 4: Proposed future research-the degree to which the project has effectively planned its future work in a logical manner by incorporating appropriate decision points, considering barriers to the realization of the proposed technology and, when sensible, mitigating risk by providing alternate development pathways. 


\section{Reviewer 1:}

The reviewer declared each milestone in 2016 was critically reviewed and future work is suggested. It would be good to see a detailed plan from University of Wisconsin (UW) on in-cylinder heat transfer modeling and validation. Wall boundary-layer resolution and heat transfer model are critical for accurate modeling, but the current approach in KIVA-ERC code is proven to be not very accurate.

\section{Reviewer 2:}

The reviewer stated future plans appear to be well thought out and will continue to provide valuable insights to the engine community.

\section{Reviewer 3:}

The reviewer noted that Slide 27 mentions a "range of ... in-cylinder geometries," but it was unclear how the single ECN engine could or would be modified or augmented to achieve this worthwhile expansion. As another reviewer noted during the live session, concern begins to arise that some details of the findings/conclusions are engine-specific.

\section{Question 5: Does this project support the overall DOE objectives of petroleum displacement? Why or why not?}

\section{Reviewer 1:}

The reviewer commented that multi-injection is a promising and innovative approach to improve efficiency and reduce emissions.

\section{Reviewer 2:}

The reviewer stated gains in engine efficiency and emissions reductions that the technology developed in this project will aid in developing, and will certainly lead to decreased petroleum usage.

\section{Reviewer 3:}

The reviewer declared this project can provide valuable understanding on LTC.

\section{Question 6: Resources: How sufficient are the resources for the project to achieve the stated milestones in a timely fashion?}

\section{Reviewer 1:}

The reviewer declared resources, including budget, seem appropriate.

\section{Reviewer 2:}

The reviewer stated resources appear sufficient. 


\section{Light-Duty Diesel \\ Combustion: Stephen \\ Busch (Sandia National \\ Laboratories) - ace002}

\section{Presenter}

Stephen Busch, Sandia National

Laboratories

Reviewer Sample Size

A total of five reviewers evaluated this project.

\section{Question 1: Approach to performing the work-the degree to which technical barriers are addressed, the project is well-designed, feasible, and integrated with other efforts.}

\section{Reviewer 1:}

The reviewer declared the project is a great combination of experimental, diagnostic, and simulation work.

\section{Reviewer 2:}

The reviewer commented the approach is well coordinated with Sandia National Laboratories' (SNL) optical engine experiments at the core and with supporting activities by UW, CSI, and other national laboratories. The team has an opportunity to further the understanding of LD diesel combustion. The reviewer remarked the scope of work focuses on piston geometry and pilot injections, and injection timing appears to be quite limited. The project team may want to consider the roadmap for a target higher efficiency based on optimized dilution, compression ratio (CR), heat transfer, etc., from predictive tools and then explore the required hardware. It appears the approach is the other way around: testing hardware and examining its effects.

\section{Reviewer 3:}

The reviewer expressed that the project has a good technical approach. There is a good balance between optical, simulation, and soon-to-be-metal engine work. But, the project needs to directly compare to DOE's engine efficiency goals. Indicated efficiency results show progress, but those results do not relate directly to DOE goals. Metal engine work at Oak Ridge National Laboratory (ORNL) is one way to accomplish this, the reviewer suggested. The reviewer is pleased to see a commercial software vendor as part of project; it is a quick and efficient way to take knowledge gained from this project and impact consumer products.

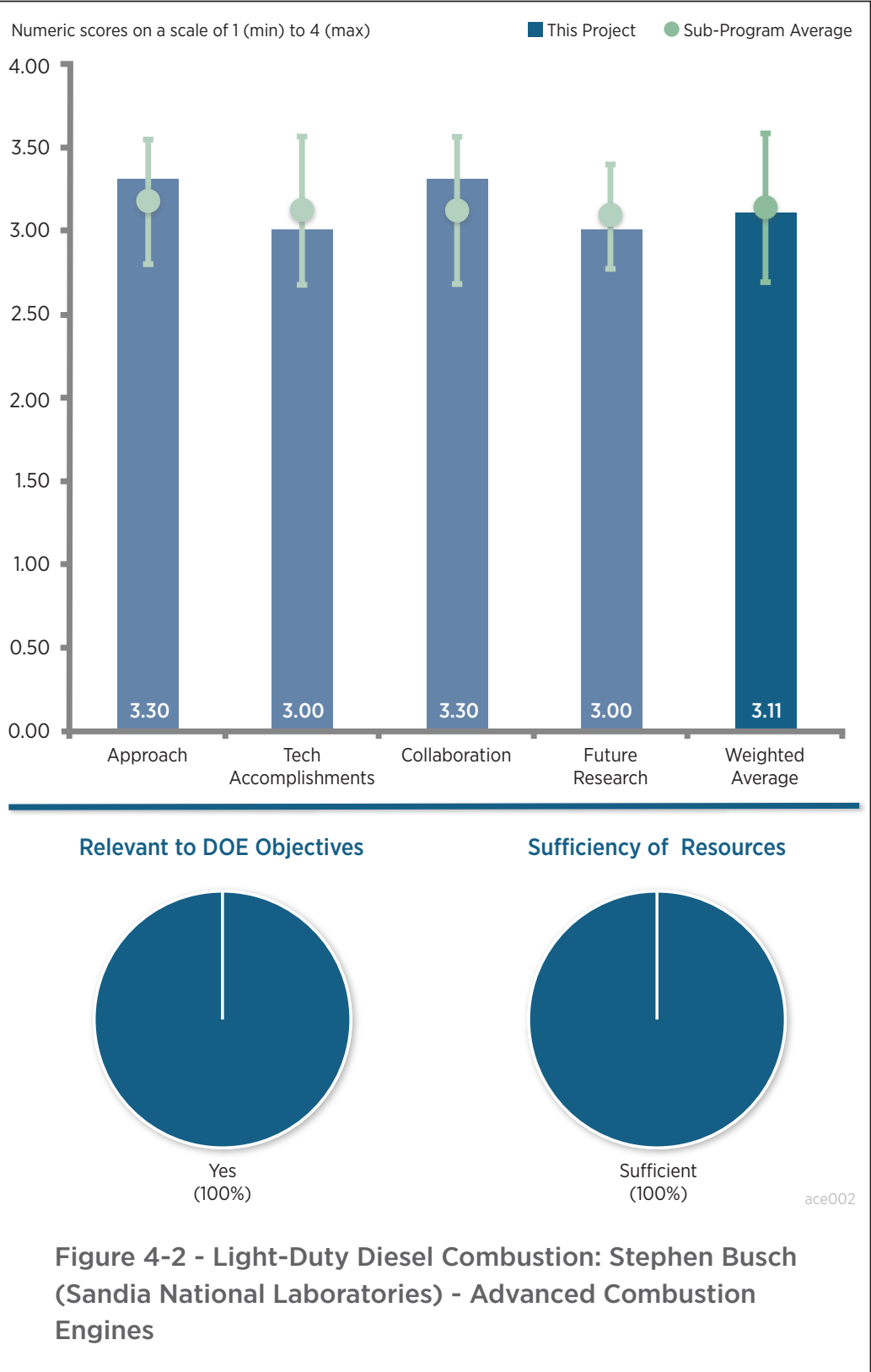




\section{Reviewer 4:}

The reviewer observed a good approach and that the fundamentals of diesel combustion are being attached. The project work is made up of primarily optical engine work and looking at injection, ignition, and combustion processes. The reviewer reported that the work is supported with simulations by UW with their Fast and Reliable Engine Simulation Code (FRESCO) code as well as CFD support from CSI.

\section{Reviewer 5:}

The reviewer stated the project uses a common engine platform (General Motors [GM] 1.9 liter [L] head) to provide data that ostensibly provides what the principal investigator (PI) terms a "fundamental understanding of advanced combustion processes." The data are also being used to improve computational modeling capabilities mainly using the commercial code CONVERGE, which is used in industry, as well as to test UW's RAN's simulation capabilities (FRESCO simulations). The reviewer also declared that SNL provides data from their optical engine related to measurements of flow patterns and emissions in their optical engine, and three-dimensional (3D) CFD simulations are being carried out using FRESCO. The SNL data are also being used to improve the simulations.

The reviewer remarked the project has been pursued for over 20 years; the most recent emphasis is on piston bowl geometry and the impact of pilot injections. The combination of detailed numerical modeling and experimental in-cylinder measurements is good and is providing information that should improve the predictions. The reviewer remarked some discussions should be included that show comparisons with modeling efforts and what about the model should be changed.

The reviewer asked how important knowing combustion chemistry is in FRESCO, and how sensitive the simulations are to alterations in specific reaction mechanisms. This question relates to surrogates and their chemistries and to using FRESCO in the potential validation of combustion chemistry.

\section{Question 2: Technical accomplishments and progress toward overall project and DOE goals-the degree to which progress has been made, measured against performance indicators and demonstrated progress towards DOE goals.}

\section{Reviewer 1:}

The reviewer commented there was great progress despite much time spent rebuilding the metal engine.

\section{Reviewer 2:}

The reviewer said the benefits of stepped-lip piston shown as up to $3 \%$ in thermal efficiency while simultaneously reducing smoke and oxides of nitrogen $\left(\mathrm{NO}_{\mathrm{x}}\right)$. This is a good result. In addition to this, the reviewer would like to have seen more direct comparisons between the optical engine and the two software platforms (UW and CSI). The reviewer remarked it would be even better to include spray results from ANL.

\section{Reviewer 3:}

The reviewer said that it is very encouraging to see that squish flow behavior is being understood by exercising the model. This will help understand to interpret engine data when injection timings are swept. The reviewer stated the role of pilot injections in modifying the radial squish flow will be important. However, it may be that heat transfer is primarily being reduced.

\section{Reviewer 4:}

The reviewer stated the experimental work appears to have had a significant setback with the need to rebuild the engine following a piston failure in June. There are, however, interesting data presented as from the stepped piston bowl. Nevertheless, the reviewer noted, the data give little insight to viability or success with respect to LD diesel requirements from the VTO program (Slide 1). Delphi is mentioned as providing advanced injection systems. The reviewer asked if the report could include what the supplier contributed to the program. 


\section{Reviewer 5:}

The reviewer said that a range of results were presented, including scoping studies to assess performance of a stepped-lip piston design. The PI provided no substantive discussion why this design was worthy of investigation; the design appeared to be just fabricated and tested. The reviewer stated that UW's CFD FRESCO code was shown to be able to simulate some of the piston bowl flow patterns. The PI reported on the development of a velocimetry technique to provide temporally and spatially resolved measurements in the piston bowl. The reviewer commented that this technique seems quite interesting and should be further developed and placed in the context of existing measurement capabilities.

The reviewer said that in response to an apparent request for a closer coupling of this project with thermomechanical material stress issues, the PI noted that folding in such an aspect was not within the scope of their efforts. This perspective should be revisited. The reviewer commented that it will certainly be an issue if an engine ultimately flies apart from material failures if operating at peak efficiencies for long periods of time. That is the project team's choice not to consider it. The reviewer suggested that perhaps as part of a more research oriented investigation, the PIs can afford to omit this consideration from their project. However, the reviewer noted, original equipment manufacturers (OEM) are part of this effort (GM/Ford) and they would most certainly need to consider the compatibility of achieving high engine efficiencies with material stresses. If the OEMs do not care about this problem, certainly the PIs need not as well; ultimately though, the consumer could pay the price.

The reviewer remarked that because piston bowl development is included in this project, the PIs should consider employing solid free-form fabrication techniques to cost-effectively fabricate a range of designs. This technology is also capable of fabricating parts in metal. The reviewer said the PIs may consider contacting DOE's Advanced Manufacturing Office, which has a vested interest in this technology, for insights on the optimal rapid prototyping (RP) technology for this application. Even in an acrylonitrile butadiene styrene plastic, much can be learned about flow patterns with rapid fabrication of different designs. The reviewer noted that RP piston bowl geometries will facilitate identifying an optimal bowl design, which apparently is not currently a part of the research plan. It is something the PIs should look into. Again, fabrication in metal is possible, and at the least can be accomplished in a plastic that could facilitate fluid flow patterns which the particle image velocimetry (PIV) capability could use for comparing with computations.

The reviewer noted that the codes considered, UW developed their own code (FRESCO). The reviewer asked if the code, as well as SNL's RAPTOR, is or will become open source. This person also commented that more evidence should be provided about precisely how the data reported in this study are informing the modeling. The reviewer asked where the discrepancies are and what the strategy is for closing the gap with modeling. The reviewer recommended to not just present comparisons, and to provide insights on what need to be changed in the modeling.

The reviewer noted the reference list included articles from earlier reporting periods; this is fine. However, it is more typical that references refer to articles/publications/presentations referenced only in the reporting period.

\section{Question 3: Collaboration and coordination with other institutions.}

\section{Reviewer 1:}

The reviewer expressed that excellent collaboration exists with GM, Ford, UW, and CSI, and the parties are playing their roles well. Additionally, new collaborations for spray measurements with ANL are a very good move.

\section{Reviewer 2:}

The reviewer stated the project has all the elements required from a collaboration perspective. It would be good to show more evidence of the degree to which the collaboration is occurring. For example, the 
reviewer asked what technical input the OEMs have offered.

\section{Reviewer 3:}

The reviewer remarked the project would merit a great deal with active participation from OEM that is committed to the LD diesel product in the United States. A committed OEM may be able to provide a more focused approach to the current work.

\section{Reviewer 4:}

The reviewer commented that the collaborations include close coupling with GM/Ford. The PI noted that the project team is interacting with all parties to provide technical input. The reviewer stated the PI should be more specific about the GM/Ford input and how it informs the work that evidences a substantive impact. As presented, it is vague.

\section{Question 4: Proposed future research-the degree to which the project has effectively planned its future work in a logical manner by incorporating appropriate decision points, considering barriers to the realization of the proposed technology and, when sensible, mitigating risk by providing alternate development pathways.}

\section{Reviewer 1:}

The reviewer said that it was good to see that the Advanced Combustion and Emissions Control Technical Team (ACEC) noise guidelines will be followed. The reviewer also commented that a First Law analysis should be conducted accounting for the combustion, work conversion, heat transfer, and internal energy portions of indicated efficiency for the re-entrant and stepped-lip piston bowls to get additional insight regarding from exactly where the efficiency benefit is coming.

Reviewer 2:

The reviewer remarked future work will include continuing study of bowl geometry and pilot injection effects. As noted previously, consideration should be considered to using capabilities of RP to fabricate piston bowl configurations. The reviewer asked if FRESCO has the capability to deal with multicomponent liquid effects, which will be important for surrogate fuels beyond simple single component surrogates and if not, how the PIs will handle this problem. The reviewer noted soot oxidation is mentioned and asked if the FRESCO model will be used in the CFD. If so, the reviewer inquired about what diagnostics will be employed in the experiments and what strategies are proposed if the model does not match the data.

\section{Reviewer 3:}

The reviewer commented the program does little to address the technical targets of $40 \%$ fuel economy improvements or Tier 2 Bin 2 emission targets, or cost effective combustion-emission solutions.

\section{Reviewer 4:}

The reviewer asked how the current piston geometry was determined and how the geometry can be improved. Additionally, the reviewer questioned whether the models are now of sufficient fidelity to start to explore an optimized geometry (e.g., applying a generic algorithm). The reviewer stated this has to be on the list of future work at some point. Additionally, this person would also like to see a direct comparison between the two software codes and the optical experiments.

\section{Question 5: Does this project support the overall DOE objectives of petroleum displacement? Why or why not?}

\section{Reviewer 1:}

The reviewer affirmed, yes, from a broad perspective. The project has been in development for a long time. The reviewer asked what timeline the PIs envision before work will be completed. 


\section{Reviewer 2:}

The reviewer said, yes, it is relevant. However, the project team can do a better job showing this by comparing back directly to the DOE goals (i.e., a projected brake thermal efficiency [BTE]), and showing how it improves every year.

\section{Reviewer 3:}

The reviewer stated the project scope is relevant. The work underway, however, is far from making any real impact on the merits of LD diesels in the United States. A question the team may ask themselves, the reviewer remarked, is whether the work the team is doing would invite manufacturers towards the introduction of LD diesels to the United States in the next 20 years.

Question 6: Resources: How sufficient are the resources for the project to achieve the stated milestones in a timely fashion?

\section{Reviewer 1:}

The reviewer stated that resources seem adequate although ultimate judgement would have to come from a cost/benefit analysis based on DOE's investment relative to the commercialization potential. 


\section{Low-Temperature Gasoline Combustion (LTGC) Engine Research: John Dec (Sandia National Laboratories) - ace004}

\section{Presenter}

John Dec, Sandia National

Laboratories

\section{Reviewer Sample Size}

A total of five reviewers evaluated this project.

\section{Question 1: Approach to performing the work-the degree to which technical barriers are addressed, the project is well-designed, feasible, and integrated with other efforts.}

\section{Reviewer 1:}

The reviewer stated the project had a good approach and liked that the project team has both an optical and metal engine. The reviewer was pleased to see the focus shift from chasing efficiency to demonstrating control robustness and minimizing boost demand. The reviewer would like to see a more thorough 3D simulation effort to show how well the current tools can predict the team's results.

\section{Reviewer 2:}

The reviewer remarked that as the project team moves the concept forward, it will need to begin to address, at least conceptually, how Tier 3 emissions levels would be achieved. This reviewer pointed out that the presenter commented on ultra-low $\mathrm{NO}_{\mathrm{x}}$ and soot, which implies minimal aftertreatment for these constituents, or at least minimal impact on fuel economy to regenerate such aftertreatment. The reviewer stated that hydrocarbons (HC) remains an issue, especially for cold starting of the engine. The reviewer questioned if there are any means to address how the project team's engine concept would be cold started and does it present an issue for Tier 3 Bin 30 emissions targets. OEM partners could help identify targets for this. The reviewer also asked how does the efficiency of the team's concept compare to a conventional hybrid electric vehicle (HEV) optimized stoichiometric engine concept using an Atkinson or Miller cycle approach.

\section{Reviewer 3:}

The reviewer remarked that understanding the potential of assisted auto-ignition (i.e., spark in this case) could be an important enabler for LTC approaches. 


\section{Reviewer 4:}

The reviewer stated that $\mathrm{NO}_{\mathrm{x}}$ and soot emissions below $2010 \mathrm{HD}$ requirements are not likely to satisfy future regulations because regulated emissions requirements will invariably decrease in the future. Engine-out $\mathrm{NO}_{\mathrm{x}}$ and soot should be reported along with exhaust temperature so that aftertreatment feasibility can be assessed in the context of tailpipe emissions requirements. The reviewer also commented that a realistic boosting system will likely erode the indicated efficiency benefits to the point that the brake efficiency is not very attractive.

\section{Question 2: Technical accomplishments and progress toward overall project and DOE goals-the degree to which progress has been made, measured against performance indicators and demonstrated progress towards DOE goals.}

\section{Reviewer 1:}

The reviewer remarked that cylinder head comparison between the two different heads is very interesting. The reviewer asked, given that there was such a large difference in low-temperature gasoline combustion (LTGC) with cylinder heads that were designed for diesel engines, what characteristics would be desirable for a cylinder head designed for LTGC. Additionally, the reviewer questioned what does $5 \mathrm{MW} / \mathrm{m}^{2}$ ringing intensity sound like. It would be useful for the audience to bring an audio recording of the engine running at this condition so that the audience can hear it. In order to implement this combustion approach in the marketplace, phi sensitive fuel will be required. The reviewer asked how could it be determined if all available gasoline in the market is phi sensitive. The reviewer also questioned what the impact on $\mathrm{NO}_{\mathrm{x}}$ is when spark ignition is introduced into the LTGC combustion approach.

\section{Reviewer 2:}

The reviewer expressed that the results are encouraging and that fuel timing and spark provide some level of combustion phasing control. It was unclear to this reviewer how much control authority is actually needed. The project team should engage their industrial partners and develop a method to quantify combustion robustness to control factors, including both slow-path (air, exhaust gas recirculation [EGR]) and fast-path (fuel timing, s/a) actuators. The reviewer stated this should then be compared to a goal and/or a relevant benchmark. It was disappointing to see the new head perform worse than the new one. The reviewer asked what level of analysis was performed before making the head. Additionally, the reviewer questioned whether the tools are incapable or was the work simply poorly executed. No exhaust gas temperatures were shown. The reviewer requested that they be included in the analysis next time.

\section{Reviewer 3:}

The reviewer commented that some of the project team's colleagues have employed an uncertainty analysis into the project. Given the control challenges of this combustion mode, an uncertainty analysis could help sort/rank control and noise factors and could be very valuable given the transition to CoOptimization of Fuels and Engines Initiative (Co-Optima) and the inclusion of fuel properties.

\section{Reviewer 4:}

The reviewer stated that careful base-lining of the new head and its comparison to the previous head will be important in understanding the results that will be forthcoming.

\section{Question 3: Collaboration and coordination with other institutions.}

\section{Reviewer 1:}

The reviewer stated there appears to be strong interaction with relevant stakeholders.

\section{Reviewer 2:}

The reviewer saw very little proof of collaboration. Yes, there was a fuel specification developed with 
GM and a head provided by Cummins, but there were no results from ANL/Lawrence Livermore National Laboratory (LLNL) shown. The reviewer stated that if GM is performing the 3D CFD analyses, please include a sample of the results. If the project team is unable to do so, please find a partner that can support the team's 3D CFD needs in an open way.

\section{Question 4: Proposed future research-the degree to which the project has effectively planned its future work in a logical manner by incorporating appropriate decision points, considering barriers to the realization of the proposed technology and, when sensible, mitigating risk by providing alternate development pathways.}

\section{Reviewer 1:}

The reviewer stated that integrating this work with the Co-Optima fuels program should involve efforts to quantify the range of relevant thermodynamic, in-cylinder time histories present relative to the physical and kinetic characteristics of the fuel being used. For example, to achieve the successful operation over the speed and load range being studied, certain temperature, pressures, and composition time histories are required. The reviewer stated these histories are needed because they match well with the physical characteristics and auto-ignition chemistry of the fuel being used. The interdependence of the in-cylinder thermodynamic time histories and the physical and kinetic characteristics of the fuel is the important fundamental understanding that is needed. The reviewer remarked there is debate as to whether $\mathrm{K}$, the empirical parameter proposed as an attempt to connect the engine operating characteristics with research octane number (RON) and motor octane number (MON) via the Octane Index, is an appropriate metric. Whether it is or is not the relevant metric is an important question that may deserve directed attention. The reviewer said it seems that this is an important issue for the Co-Optima program and to achieve optimization of fuels and engines for minimal GHG and emission impact it will be important to identify the range of in-cylinder conditions present in the current portfolio of combustion approaches and connect those conditions into the physical characteristics and autoignition chemistry of viable fuel mixtures. The reviewer said that this research could be a rich source for that database.

\section{Reviewer 2:}

The reviewer remarked future work should include a complete survey of market available gasoline to ensure that all gasoline is phi sensitive. If any gasoline is found that is not phi sensitive, then this combustion approach will never be more than a laboratory novelty.

\section{Reviewer 3:}

The reviewer expressed there needs to be more work performed to understand control robustness as previously mentioned - both experimentally and 3D (with results that can be shared).

\section{Question 5: Does this project support the overall DOE objectives of petroleum displacement? Why or why not?}

\section{Reviewer 1:}

The reviewer affirmed the work is relevant, but the project team should be doing more to show this. Indicated thermal efficiency and indicated mean effective pressure (IMEP) are good indicators, but they do not relate directly back to DOE goals, which are described in fuel economy or BTE terms. The reviewer stated there should be at least a directional one-dimensional (1D) model created to show what boosting system is required. The project team can also work with Cummins on a friction assumption. With this, the reviewer can estimate a BTE. The reviewer further noted all researchers should be doing this and comparing themselves to one another to show who is the most relevant. There should also be a forum to vet the assumptions that go into the model. Maybe this becomes an activity for the advanced combustion engine working group, the reviewer suggested. 
Question 6: Resources: How sufficient are the resources for the project to achieve the stated milestones in a timely fashion?

\section{Reviewer 1:}

The reviewer would encourage the project team to find a way to work within their available budget to perform the $1 \mathrm{D}$ and $3 \mathrm{D}$ work in a way that the results can be openly shared. 


\section{Spray Combustion Cross- Cut Engine Research: Lyle Pickett (Sandia National Laboratories) - ace005}

\section{Presenter}

Lyle Pickett, Sandia National Laboratories

\section{Reviewer Sample Size}

A total of four reviewers evaluated this project.

\section{Question 1: Approach to performing the work-the degree to which technical barriers are addressed, the project is well-designed, feasible, and integrated with other efforts.}

\section{Reviewer 1:}

The reviewer said that the approach here is outstanding. SNL focuses on the advanced spray optical diagnostics where it has the facilities and expertise and then combines this with a large external pool of CFD and experimental collaborators to leverage its results with theirs. The reviewer commented that there appears to be a better balance this year between diesel and gasoline work.

\section{Reviewer 2:}

The reviewer stated that a highquality resolved measurement dataset is an invaluable aid to the engine and CFD community in industrial practice.

\section{Reviewer 3:}

The reviewer noted the approach involves engaging multiple research organizations for experimental and computational spray research for diesel and gasoline engines.

\section{Reviewer 4:}

The reviewer remarked that this project is motivated by the need to develop a better understanding of sprays (gasoline/diesel) and to provide data for the ECN. The constant-volume chamber (CVC) with spray injection is being used to develop spray data. The CVC ostensibly provides well-defined conditions, allows assessing performance of several injectors, and provides better control of conditions than found in an engine. The reviewer stated that the presentation noted that the CVC is thought to provide data under engine-relevant spray conditions. However, the environment of a CVC is static (constant volume) while that of an engine is dynamic with time-dependent conditions. The reviewer 
noted that, in fact, there seems to be a lot in the CVC geometry that is not closely aligned with an engine, which is not necessarily an impediment. The reviewer stated that the situation is of no more concern than claims that a rapid compression machine (RCM) or shock tube provides engine-relevant condition (it does not). Rather, the CVC provides a means to provide fundamental information about spray processes, and that is what should be advertised rather than trying to claim relevancy to the environment of an engine. The reviewer recommended that some clarification of this view is needed. Interest in understanding flash boiling was noted from the presentation by the reviewer, who asked what is being done in this area by this project. This person explained that it is an interesting subject, but information about it was scarce.

\section{Question 2: Technical accomplishments and progress toward overall project and DOE goals-the degree to which progress has been made, measured against performance indicators and demonstrated progress towards DOE goals.}

\section{Reviewer 1:}

The reviewer said that the increased focus on gasoline fuel Spray G this year has been outstanding. The work with the new PIV diagnostic to understand collapse is excellent work and has thrown new light on the entrainment and velocity inside the spray cone as a function of time.

\section{Reviewer 2:}

The reviewer stated the PIV technique demonstrated this year looks very promising and there are significant findings in many areas, such as the ambient temperature and nozzle inlet passage effects on spray characteristics. The broader collaborative work with the various ECN members also shows quite a bit of progress and useful accomplishments in a wide range of areas: soot, mixing, dribble, etc.

\section{Reviewer 3:}

The reviewer said the development of the high-speed PIV diagnostic is impressive. The project demonstrated operation of this capability by scoping out its operation with iso-octane as the fuel. The reviewer noted that for PIV to work, the seed particles need to faithfully follow the flow and asked if this is the case here with the zirconium $(\mathrm{Zr})$ particles. For example, the reviewer asked, could the droplets be a sort of seed, or even the soot produced. The reviewer said that the project has shown a lot of results of the experiments. However, a better demonstration of how the CVC data are used in CFD development should be established. This reviewer reported that a lot of results were shown: demonstration of the high-speed PIV diagnostic; time evolution between plumes; axial temperature variations at different gas temperatures and its influence on droplet vaporization; nozzle shape effects on spray development; structure of the spray for different fuels; and influence of ambient temperature on ignition delay. The reviewer commented that because the data are ostensibly supposed to be coupled with CFD, it would be appropriate to show more clearly how the spray data and diagnostic capabilities for velocity are being used in the CFD simulations. Many CFD collaborators are listed; however, precisely how they are being incorporated in the project is unclear.

\section{Reviewer 4:}

The reviewer said it is understood that this type of fundamental understanding requires a sustained methodical approach and the fruition in terms of engine design may be slow to develop.

\section{Question 3: Collaboration and coordination with other institutions.}

\section{Reviewer 1:}

The reviewer commented that the ECN must be one of the best examples of collaborative engine research around. The broad inclusiveness of national laboratories, academia, engine makers, component suppliers, CFD vendors, etc., defines outstanding. 


\section{Reviewer 2:}

The reviewer commented that the breadth and scope of the ECN collaboration are impressive, but what seemed to be missing (at least in this brief presentation) is a sense of coordination. The reviewer questioned who is working on which sub-topics/questions and why. Notwithstanding the "very tight coordination" touted on Slide 20, the reviewer asked whether the ECN performers are free to define their own niches, and if not, how they are being steered in ways that will create a synergistic whole. The reviewer noted that this is an important aspect to summarize in the AMR.

\section{Reviewer 3:}

The reviewer noted that there is an extensive array of collaborators. In fact, the list is very extensive. However, the precise roles of the collaborators were not evident. The reviewer commented a stronger demonstration for how the CVC data are being used in the CFD modeling should be demonstrated. The reviewer asked what the project is providing to the ECN and what an example is for how the data developed here are being used.

\section{Question 4: Proposed future research-the degree to which the project has effectively planned its future work in a logical manner by incorporating appropriate decision points, considering barriers to the realization of the proposed technology and, when sensible, mitigating risk by providing alternate development pathways.}

\section{Reviewer 1:}

The reviewer noted the project appears to be focused on the topics of greatest interest and shows a continued responsiveness to the needs of the engine manufacturing community.

\section{Reviewer 2:}

The reviewer observed that the proposed work on gasoline particulate formation at the tip of the injectors is very relevant and timely.

\section{Reviewer 3:}

The reviewer commented that the anticipated speed-up with a heated chamber is suitably ambitious.

\section{Reviewer 4:}

The reviewer stated that future work notes improving model capabilities for the Spray G, with several codes being listed (CONVERGE, OpenFoam, etc.). The reviewer commented that better specificity of precisely what data will be developed that the codes will predict should be provided. For example, soot formation is noted to quantify its formation with Spray C, which is apparently a cavitating spray. The soot diagnostics should be specified and discussed.

\section{Question 5: Does this project support the overall DOE objectives of petroleum displacement? Why or why not?}

\section{Reviewer 1:}

The reviewer said the learning from this project will continue to facilitate building cleaner and more efficient engines, thus reducing petroleum usage.

\section{Reviewer 2:}

The reviewer noted that the overall importance of diagnostics to evaluate engine performance is high and in that regard the project is very relevant. It may be beneficial for the PI, and indeed all the national laboratories, to consider developing a diagnostic consortium of sorts to advertise experimental capabilities across the national laboratories. 


\section{Reviewer 3:}

The reviewer stated that the project is indirectly relevant because better spray understanding is essential to better petroleum-combustion efficiency and emissions reduction.

Question 6: Resources: How sufficient are the resources for the project to achieve the stated milestones in a timely fashion?

\section{Reviewer 1:}

The reviewer commented the resources appear adequate, especially considering the scope of outside collaborators contributing to the project.

\section{Reviewer 2:}

The reviewer stated that resources seem adequate although ultimate judgement would have to come from a cost/benefit analysis based on DOE's investment relative to the commercialization potential. 


\section{Gasoline Combustion Fundamentals: Isaac Ekoto (Sandia National Laboratories) - ace006}

\section{Presenter}

Isaac Ekoto, Sandia National

Laboratories

\section{Reviewer Sample Size}

A total of three reviewers evaluated this project.

\section{Question 1: Approach to performing the work-the degree to which technical barriers are addressed, the project is well-designed, feasible, and integrated with other efforts.}

\section{Reviewer 1:}

The reviewer remarked that spark and plasma ignition is more important than the negative valve overlap (NVO) work. The calorimeter is a good addition, and it might also be important to develop a bench test to investigate ignition system behavior in a flow field.

\section{Reviewer 2:}

The reviewer noted that the work is transitioning to focus on advanced ignition concepts for various combustion concepts.

\section{Reviewer 3:}

The reviewer stated that the purpose of the ignition work is not clear and asked if it is to establish conditions for auto-ignition or to actually initiate combustion.

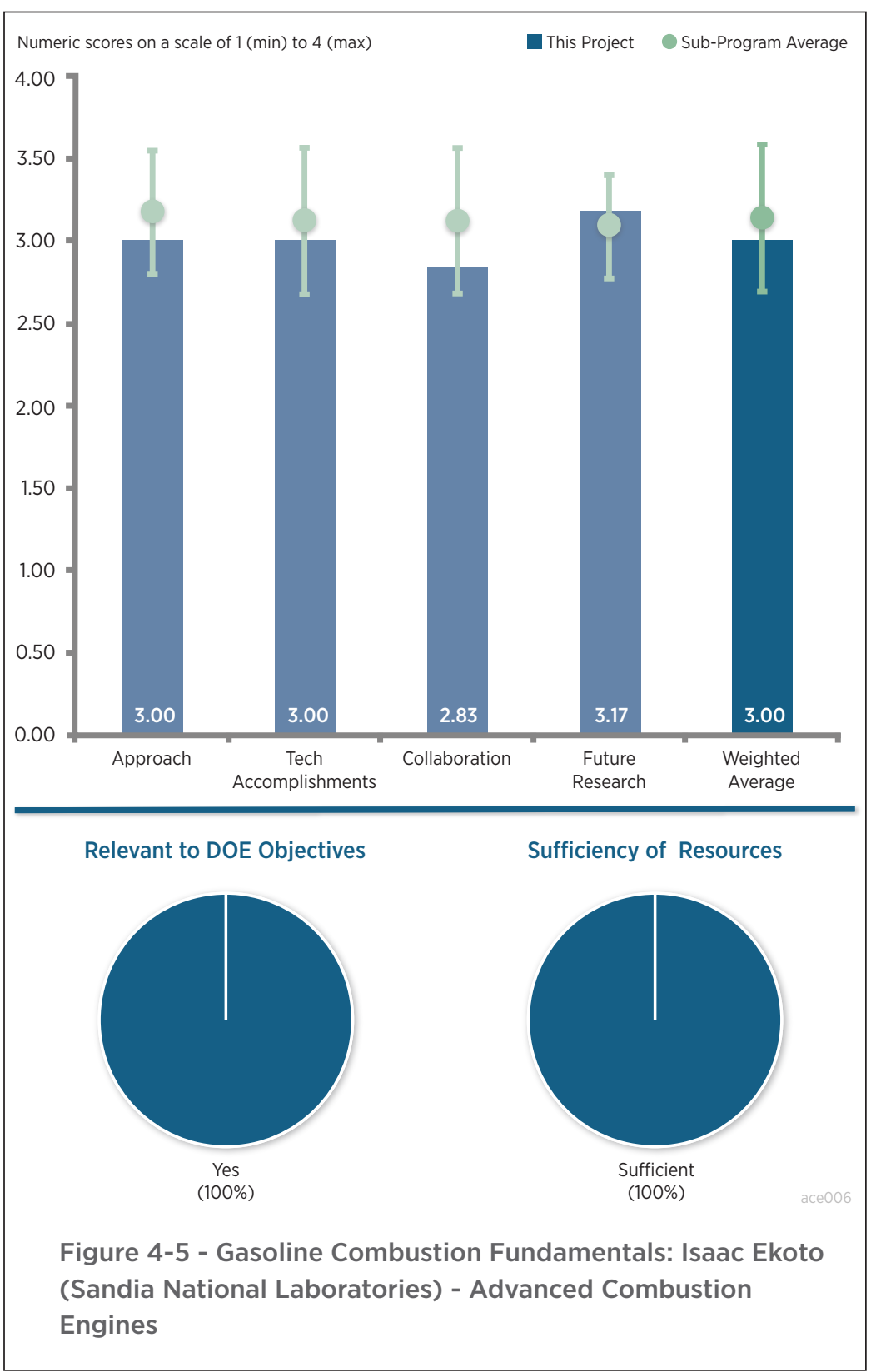

\section{Question 2: Technical accomplishments and progress toward overall project and DOE goals-the degree to which progress has been made, measured against performance indicators and demonstrated progress towards DOE goals.}

\section{Reviewer 1:}

The reviewer commented that the analysis was an insightful method to explain results from NVO reformate behavior. Furthermore, the calorimeter results were also good, and the reviewer expects more interesting results to come.

\section{Reviewer 2:}

The reviewer stated that there was a good explanation of observed effects of reformate addition. While the explanations offered are noted, project progress continues to be slow. 


\section{Question 3: Collaboration and coordination with other institutions.}

Reviewer 1:

The reviewer stated the response to industry needs is good.

Reviewer 2:

The reviewer commented that more collaboration with OEMs regarding the ignition system testing would be helpful to provide guidance for the project and feedback to industry.

Question 4: Proposed future research-the degree to which the project has effectively planned its future work in a logical manner by incorporating appropriate decision points, considering barriers to the realization of the proposed technology and, when sensible, mitigating risk by providing alternate development pathways.

\section{Reviewer 1:}

No comments were received in response to this question.

\section{Question 5: Does this project support the overall DOE objectives of petroleum} displacement? Why or why not?

\section{Reviewer 1:}

The reviewer noted that improved understanding of ignition systems and processes in gasoline engines is critical to improving engine efficiency.

Question 6: Resources: How sufficient are the resources for the project to achieve the stated milestones in a timely fashion?

\section{Reviewer 1:}

No comments were received in response to this question. 


\section{Large Eddy Simulation (LES) Applied to Advanced Engine Combustion Research: Joe Oefelein (Sandia National Laboratories) - ace007}

\section{Presenter}

Joe Oefelein, Sandia National Laboratories

\section{Reviewer Sample Size}

A total of five reviewers evaluated this project.

\section{Question 1: Approach to performing the work-the degree to which technical barriers are addressed, the project is well-designed, feasible, and integrated with other efforts.}

\section{Reviewer 1:}

The reviewer stated that this project provides invaluable information on combusting spray, which can be used for model development. However, it is not clear how complex engine geometry with moving valves and a piston can be handled in the framework of RAPTOR. The reviewer also noted that it is not clear if the end goal is to provide reference simulation data for model development or to provide a software tool for engine development. When considering computing time and a high-performance computing (HPC) resource for engineering purposes, the project should focus on the former.

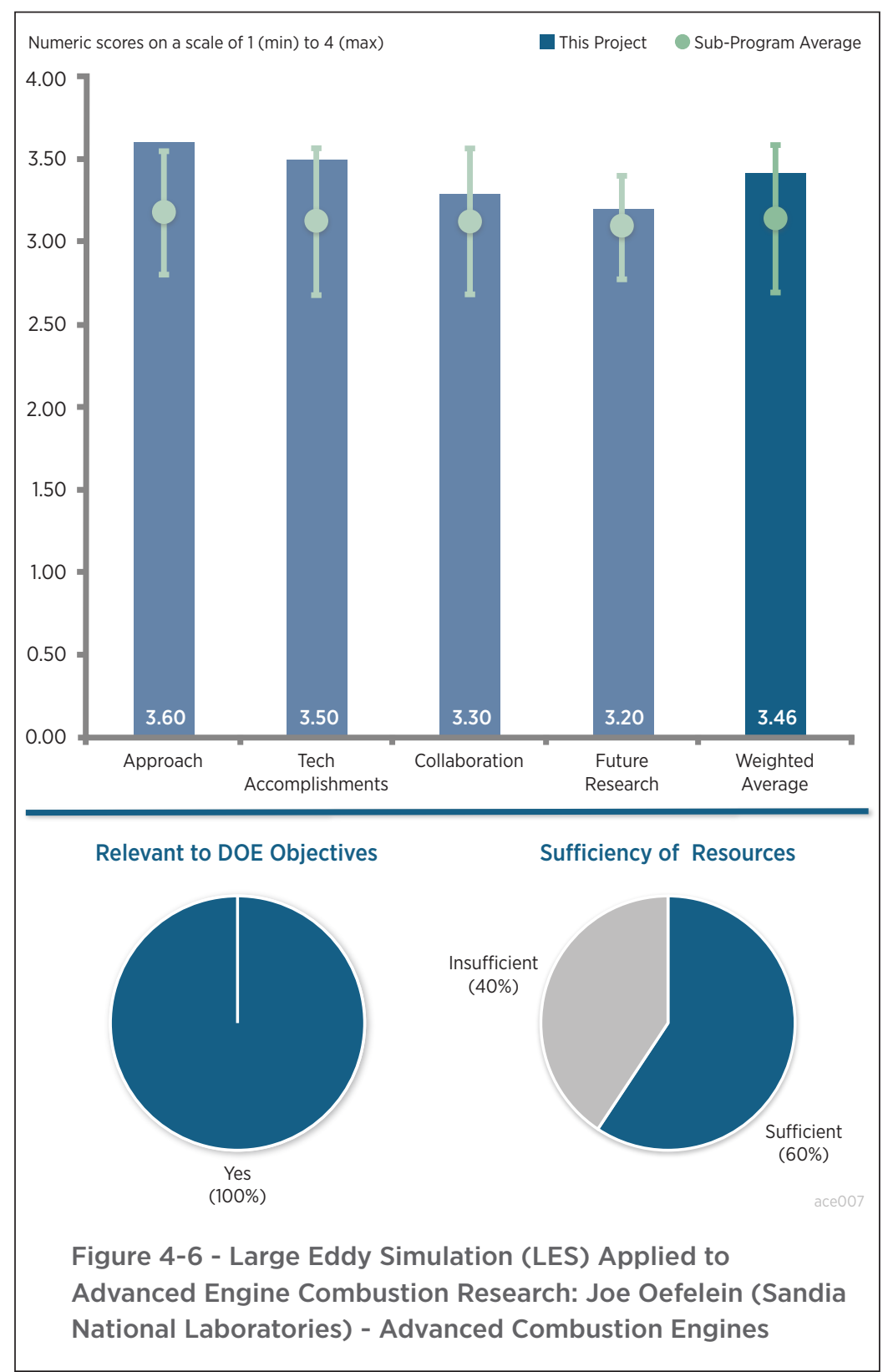

\section{Reviewer 2:}

The reviewer commented that this is a well-focused synthesis and presentation of the root-cause challenges faced by engine combustion simulation. The near-DNS approach is a worthy attack on the problem of too many uncertain input tuning coefficients in multiphase reacting CFD.

\section{Reviewer 3:}

The reviewer noted that this project is motivated by the need to develop a better understanding of spray combustion dynamics as related to gasoline direct injection (GDI) and diesel engines. The PI has made a convincing case that the current art on engine simulations is lacking for their abilities to provide high accuracy simulations of in-cylinder processes. The reviewer said the approach advanced in this project is to use large eddy simulation (LES) through the code RAPTOR, which is a first-principles solver optimized for LES. RAPTOR has significant potential for a high impact on engine design when completed, and the PI seems to be making a lot of progress. The reviewer expressed that one can only wish that the pace of development could be increased because the potential for RAPTOR is significant. The reviewer said that the presentation was clear and well developed. 


\section{Reviewer 4:}

The reviewer stated that the approach of applying detailed first principles models for the wide range of complex incylinder processes is excellent. However, progress to combine these into an all-up simulation of a diesel or gasoline direct-injected (GDI) engine has yet to be achieved (although progress is measurable and continuing towards that goal). The reviewer noted that the key to the ultimate success of this program will be how the knowledge gained can be transferred to commercial code vendors and ultimately used in a practical way by engine developers. It will be a challenge.

\section{Question 2: Technical accomplishments and progress toward overall project and DOE goals-the degree to which progress has been made, measured against performance indicators and demonstrated progress towards DOE goals.}

\section{Reviewer 1:}

The reviewer commented that the project is a good systematic use of UQ to help optimize accuracy per unit computational cost. Additionally, the interphase filtering and new combustion LES closure are highly innovative.

\section{Reviewer 2:}

The reviewer stated that project progress remains good and steady; much is being learned about turbulence, sprays, and combustion and the interactions between them. However, some of this is more along the lines of confirming the known limitations of current methodologies that have to also meet the constraint of speed. The real progress will be in finding ways to take these advanced approaches and producing better engineering tools combustion system designers can use on a daily basis to develop better engines.

\section{Reviewer 3:}

The reviewer stated that the accomplishments included an array of simulations on several combustion and nonreacting configurations. Calibration of the results through comparisons with the configurations selected is an essential part of proving the efficacy of RAPTOR to provide high accuracy results. The reviewer noted that the code appears to incorporate detailed transport and chemical kinetic mechanisms along with multiphase effects from sprays folded into the capabilities so it seems to be quite robust. The reviewer commented that configurations used to provide data for testing RAPTOR include the CVC, non-reacting fuel jet injection, auto-ignition of reacting diesel jets, and ignition delay time predictions from RCM and shock tubes. The work on GDI sprays is perhaps closer to the reality of spray injection in engines and seems to be in progress. The reviewer asked whether, in this simulation, the flame exists only in the region where the droplets have fully vaporized. The reviewer also questioned the conditions where liquid is present in the flaming region and liquid vaporization effects are present and how are they treated.

The reviewer said that in regards to combustion chemistry, a number of chemical mechanisms were used. If RAPTOR is a first-principles solver, the project team should comment on its ability to provide a stringent test of the combustion chemistry. The reviewer asked what strategies would be used to adjust the chemistry to bring measurement and theory into better alignment. The reviewer realized that the chemistry is provided by others and presumably they have strategies for adjusting reactions to improve predictions. To facilitate this effort, the PI might consider a simpler multiphase configuration to test RAPTOR's ability to deal with the complexities of liquid/ combustion/vaporization/transport that might better provide a platform to adjust the inputs to RAPTOR (chemistry, property database, etc.).

\section{Reviewer 4:}

The reviewer noted that although milestones and achievements in FY 2016 are not clearly described, several valuable tasks have been well performed (e.g., combusting spray on ECN Spray A with good agreement with experiment and optimization of chemical models using UQ).

\section{Question 3: Collaboration and coordination with other institutions.}

\section{Reviewer 1:}

The reviewer indicated that collaboration with other institutions looks very well done. This person recommended that the PI also talk to industry to determine current and future needs in engine combustion modeling. 


\section{Reviewer 2:}

The reviewer commented that collaboration perhaps along the lines of a combustion CFD equivalent to the ECN, which is more focused on spray diagnostics and some simulation but not at the high end for the most part, might be a way forward for the project. Engaging CFD practitioners in the engine industry with commercial code vendors as well as research code and model developers in academia and national laboratories might provide more of a dialog to transfer learning from the detailed simulations to the development and application of engineering tools to design and understand in-cylinder combustion systems.

\section{Reviewer 3:}

The reviewer noted that a range of collaborators are included in the project and some appear to be contributing actual data, which the PI is using to assess the efficacy of RAPTOR's capabilities (several examples were given in the presentation). Regarding the radiation modeling, the reviewer asked whether RAPTOR currently has this capability. Concerning wall impingement effects, the reviewer queried whether RAPTOR can simulate the impingement and spreading dynamics of a single droplet, or an aggregate of droplets, at a wall.

\section{Reviewer 4:}

The reviewer observed that the last summary sub-bullet mentions "working closer with industry" on simulation software development, and, on Slide 31, the second comment response cites an attempt to do that. It would have been helpful to hear the project team's specific ideas and plans. The reviewer noted there is zero industry participation evident in Slide 32. Deliberate avoidance of reinventing the wheel of commercial Reynolds-Averaged Navier-Stokes (RANS)/LES-centric CFD was applauded by the reviewer. However, this person recommended the earliest possible coordination with code developers supporting today's design community, and with products that do scale well to $\mathrm{O}(10,000)$ processor so that RAPTOR can have the maximum long-term impact.

\section{Question 4: Proposed future research-the degree to which the project has effectively planned its future work in a logical manner by incorporating appropriate decision points, considering barriers to the realization of the proposed technology and, when sensible, mitigating risk by providing alternate development pathways.}

\section{Reviewer 1:}

The reviewer commented that it is good to see that actual engine simulations are in the project plan for the coming couple of years. Hopefully, these will prove the concept of detailed modeling that has been followed in this program.

\section{Reviewer 2:}

The reviewer opined that there is a good forward look at milestones and several slides provided helpful historical context. However, a formal timeline is missing. The reviewer noted that the information in that section of the quad chart (Slide 2) is completely off topic and the reviewer was left with no idea of the project duration, past or future.

\section{Reviewer 3:}

The reviewer commented that because RAPTOR is so potentially robust, consideration should be given to using it as a tool to assess kinetic and property inputs required for simulation. For example, combustion chemistry can be uncertain. The project team should consider applying RAPTOR to a simplified multiphase configuration where transport is well defined to assess performance of the combustion chemistry. The reviewer stated this could also provide significant benefit to other projects using commercial codes where the combustion chemistry is needed but validation of it is limited. The reviewer also noted that future work should comment upon the specific computer platform used as well as the computational times involved when presenting results.

\section{Reviewer 4:}

The reviewer stated that it is directionally right to move toward realistic (optical) engine geometries, but the current codes cannot handle as is mentioned in Slide 34. The project team should develop a plan to address this situation.

\section{Question 5: Does this project support the overall DOE objectives of petroleum displacement? Why or why not?}




\section{Reviewer 1:}

The reviewer stated that the project can provide important simulation data for engineering model development.

\section{Reviewer 2:}

The reviewer stated this project is certainly relevant. Indeed, it seems to be the only simulation effort that is incorporating a first-principles approach to solving the multiphase dynamics associated with fuel spray injection and combustion. The reviewer said that when completed, RAPTOR should be the most advanced tool for simulating the complex multiphase processes found in engines and, thus, provide the means for high accuracy simulations for facilitating engine design.

\section{Reviewer 3:}

The reviewer noted that in the long run, better simulation tools lead to better, more efficient engines that will lead to reduced petroleum use.

\section{Question 6: Resources: How sufficient are the resources for the project to achieve the stated milestones in a timely fashion?}

\section{Reviewer 1:}

The reviewer said that the budget or manpower seems too low for progressing faster and producing more outcomes.

\section{Reviewer 2:}

The reviewer commented that hopefully the funding can be stabilized at the current level. In comparing all the different avenues of research being pursued by DOE, with a limited funding resources, sometimes cuts have to be made. The reviewer stated this project probably cannot make adequate progress if further cuts are imposed.

\section{Reviewer 3:}

The reviewer stated that resources seem adequate although ultimate judgement would have to come from a cost/ benefit analysis based on DOE's investment relative to the commercialization potential. 


\section{Fuel Injection and Spray Research Using X-Ray Diagnostics: Christopher Powell (Argonne National Laboratory) - ace010}

\section{Presenter}

Christopher Powell, Argonne

National Laboratories

\section{Reviewer Sample Size}

A total of five reviewers evaluated this project.

\section{Question 1: Approach to performing the work-the degree to which technical barriers are addressed, the project is well-designed, feasible, and integrated with other efforts.}

\section{Reviewer 1 :}

The reviewer said that the application of ANL's unique X-ray source for spray and injector internal flow diagnostics and studies is brilliant and an excellent example of repurposing DOE's technology to aid the engine industry. With this tool, the team has already performed many useful studies and continues to develop techniques and capabilities to extend their research to other important areas.

\section{Reviewer 2:}

The reviewer said that the ANL team has put together a very impressive visualization tool for spray diagnostics and that it has been well-documented over the last years. The reviewer said that it would be useful to understand if this tool is considered to be mature or it needs to be improved for successful integration to the modeling and predictive tools (e.g., whether the spatial or temporal resolutions are sufficient or whether the room temperature limitations are a significant impediment). The reviewer found that, overall, the impression given is that there is little integration of this work with actual modeling.

\section{Reviewer 3:}

The reviewer said that this project is motivated by a lack of an understanding of fuel injection processes. To fill this gap, the project is rather narrowly focused on using the ANL X-ray source to provide unique measurements related to fuel injection with an emphasis on or near the nozzle. The reviewer commented that data are taken that are indicated as being important for improved spray models. The X-ray source provides unique abilities to image both inside and outside an injector. In this way, it is possible to view inside the nozzle to assess optimal flow paths for atomization including droplet size and their trajectories 
downstream of injection.

The reviewer detailed that, thus far, the project has demonstrated considerably improved photographic resolution that show significant details nozzle geometry. The multi-hole (Spray G) nozzle images have been shared with the ECN, and industrial partners are showing interest. The approach of using a beryllium nozzle is good because of its ability to facilitate better imaging.

Comparisons with simulations at the University of Massachusetts were shown. More details should be provided. The reviewer asked if the simulation is from a commercial code or was it written in-house. The reviewer noted that the approach provides information under relevant conditions, and asked if this includes high-temperature gas environments.

\section{Question 2: Technical accomplishments and progress toward overall project and DOE goals - the degree to which progress has been made, measured against performance indicators and demonstrated progress towards DOE goals.}

\section{Reviewer 1:}

The reviewer praised the improvement to the beam line and the resulting improvement in resolution as staggering. The reviewer suggested that the team continue to pursue correlations between observed features or anomalies in the injector design and a measurable engine or combustion system-level attribute.

\section{Reviewer 2:}

The reviewer said that the effects of cavitation, needle motion, etc. have been observed with extremely high resolution in plastic and metal injector hardware. The results include qualitative and quantitative data, both of great import to the engine community. The reviewer noted that improvements in hardware and techniques are continuously sought to improve the quality and extend the domain of these measurements.

\section{Reviewer 3:}

The reviewer said that the presentation provides little update of technical work for this calendar year. It appears limited to submerged cavitation, and measurements of cavitation on a GDI injector. The reviewer noted that many of the slides touch on collaboration, but there is no content reported.

\section{Reviewer 4:}

The reviewer cited that listed accomplishments include working with the ECN to provide information on injector geometry, internal needle motion, near-nozzle spray density, and mean droplet diameter measurements. Regarding diameter measurements, the reviewer said that more information should be provided. The reviewer asked if such measurements are through the cylinder and what the time resolution is. The reviewer asked how the diameter measurements are made. Some examples should be provided. The reviewer inquired how the measurements compare with more conventional diagnostics (a Phase Doppler Particle Analyzer, etc.).

The reviewer commented that much of the presentation seemed more like an advertisement of the virtue of X-ray diagnostics than a demonstration of new results over the past year and that more results should be provided. The reviewer asked if there is a jumping off point when the diagnostic will be mature and a mainstream instrument or will it always be tied to ANL's facility. If the latter, the reviewer found that would be a limitation to its wider use in industry.

\section{Question 3: Collaboration and coordination with other institutions.}

\section{Reviewer 1:}

The reviewer said that the team has clearly worked closely with many groups and freely provided data 
to many. The coordination with the neutron imaging team at ORNL is particularly noteworthy as these two facilities are very complementary, but could also be competitive. The reviewer was glad to see the former appears to be winning.

\section{Reviewer 2:}

The reviewer said that many collaborations are reported, but there was no clear assessment as to the significance of these collaborations. The reviewer noted that there are extensive presentations given, 15 in total, including 7 international conferences. The reviewer asked if this is necessary or seen as distracting from the actual work.

\section{Reviewer 3:}

The reviewer commented that a range of collaborators was indicated that included groups from industry, academia, and national laboratories. However, the importance/necessity of the listed collaborators to the overall success of the project was unclear. Few results were included from these groups. The reviewer also noted that partners from industry were included, such as Delphi Diesel, which perhaps has an intense interest in the X-ray diagnostic. Nonetheless, the collaborators should be expanded to include the major manufactures of atomizers. The reviewer said that the X-ray diagnostic can be used as a design tool and nozzle designers should be part of the team as they stand to gain significantly from the success of this project.

The reviewer noted that the collaboration with ORNL is interesting because Oak Ridge Associated Universities (ORAU) is pursuing virtual injector design. Precisely what ORAU and ANL are sharing in this partnership is not clear. The reviewer said that this concern further indicates that the necessity of the listed partners, and what they brought to this project should be clearly evident.

\section{Question 4: Proposed future research-the degree to which the project has effectively planned its future work in a logical manner by incorporating appropriate decision points, considering barriers to the realization of the proposed technology and, when sensible, mitigating risk by providing alternate development pathways.}

\section{Reviewer 1:}

The reviewer said that moving into more of a combustion environment will provide even greater benefits to the engine community.

\section{Reviewer 2:}

The reviewer said that there is a good list of tasks listed, citing Slide 18. The authors may explain how these will be incorporated into the main path towards helping the predictive modeling efforts. One suggestion the reviewer offered may be participating in a specific simulation program where the information from the X-ray images proves to be the enabler to overcome a specific barrier, thus resulting in a significant breakthrough in the modeling capability.

\section{Reviewer 3:}

The reviewer commented that future work seems to be developed around measurements for the ECN, cavitation studies, measurement of fuel/air mixing inside a duct, nozzle geometry measurements, etc. The reviewer noted barriers included windows, broadband X-ray capabilities, and high-temperature conditions. While it was not evident from the list of the proposed future work, e.g., and measurements of GDI nozzle geometry, cavitation studies, spark and laser ignition studies and nozzle geometry measurements would contribute to the listed barriers. The reviewer said that more thought should be given to a more-focused effort with clear connections between articulated needs and tasks that will specifically address those needs.

The reviewer noted a study of flash boiling. The reviewer said that this is interesting, but it was not 
evident precisely how the X-ray diagnostic would be used on this problem, what data it would provide, and how they it will be used.

\section{Question 5: Does this project support the overall DOE objectives of petroleum displacement? Why or why not?}

\section{Reviewer 1:}

The reviewer commented that improved spray and combustion diagnostics aid the development of more efficient engines that will use less petroleum.

\section{Reviewer 2:}

The reviewer remarked that the project is relevant. The nature of the diagnostics needs to grow towards providing more useful, close to real-life conditions, and data to the research and engine manufacturers.

\section{Reviewer 3:}

The reviewer said that the project is relevant from a broad perspective. Greater relevance could be realized with a stronger connection with the atomizer/nozzle design industry.

\section{Resources: How sufficient are the resources for the project to achieve the stated milestones in a timely fashion?}

\section{Reviewer 1:}

The reviewer was sure that more money could be usefully spent here, but the resources seem to be adequate for the program presented.

\section{Reviewer 2:}

The reviewer stated that resources seem adequate although ultimate judgement would have to come from a cost/benefit analysis based on DOE's investment relative to the commercialization potential. All that said, the cost/benefit ratio at a level of $\$ 700,000$ per year seems high. 


\section{Advances in High-Efficiency Gasoline Compression Ignition: Steve Ciatti (Argonne National Laboratory) - ace011}

\section{Presenter}

Steve Ciatti, Argonne National Laboratories

\section{Reviewer Sample Size}

A total of three reviewers evaluated this project.

\section{Question 1: Approach to performing the work-the degree to which technical barriers are addressed, the project is well-designed, feasible, and integrated with other efforts.}

\section{Reviewer 1:}

The reviewer was glad to see some uncertainty analysis brought into the project. The reviewer would like to see a ranking of the most-important control and noise variables, which could help focus the project on leading barriers to control of this combustion mode.

\section{Reviewer 2:}

The reviewer said that the approach does not seem to be systematic and connected from one year to another and referenced comments in the next section.

\section{Question 2: Technical} accomplishments and progress toward overall project and DOE goals-the degree to which progress has been made, measured against performance indicators and demonstrated progress towards DOE goals.

\section{Reviewer 1:}

The reviewer said that it would be helpful to see a speed or load map showing efficiencies and highlighting your control approaches in differing areas and also the chief barriers.

The reviewer asked if the exhaust temperatures the team reported are pre- or post-turbo and noted that post-turbo is most relevant to aftertreatment performance. The reviewer said that reporting brake specific fuel consumption (BSFC) with supercharger parasitics included, when the ultimate concept will not include a supercharger, is not helpful. The reviewer asked if maybe indicated specific fuel consumption and BSFC are reported together.

\section{Reviewer 2:}

The reviewer said that it is difficult to relate the technical accomplishments presented this year with the progress made in the past. The benefits of gasoline compression ignition (GCI) are high efficiency, ultra-low $\mathrm{NO}_{\mathrm{x}}$ and low

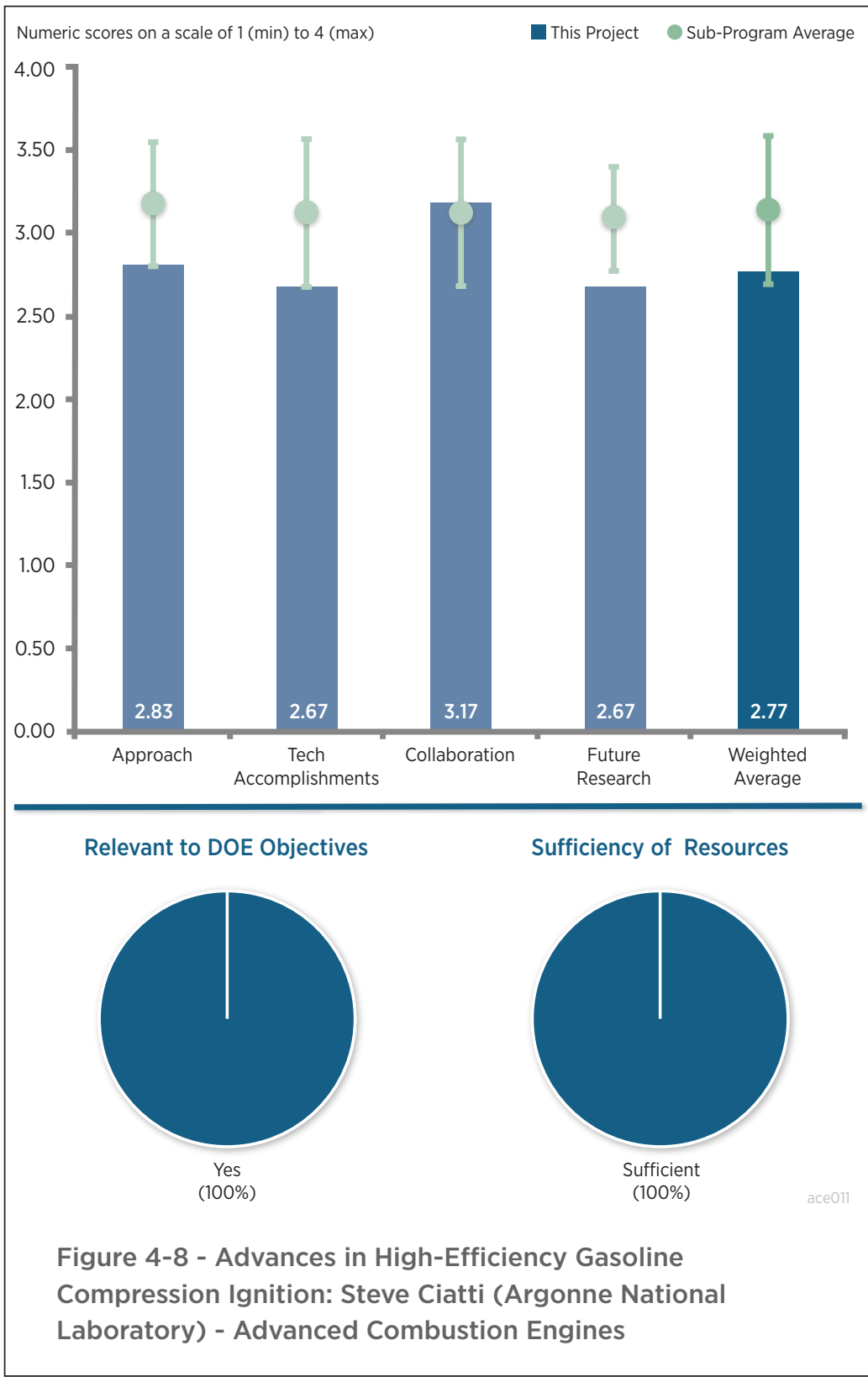

Avvanced Combustion Engines 
particulate matter (PM), but its shortcomings are high carbon monoxide (CO) and $\mathrm{HC}$ emissions. The reviewer wondered can each of these key metrics be tracked from year to year to facilitate gauging progress, or if there is a better way to gauge progress.

The reviewer noted that experiments with a supercharger reported for several loads of interest. However, only BSFC results (which naturally are not competitive) are shown. The reviewer asked what the indicated or net efficiency was, how it compares to the efficiency results shown in earlier years, and how it compares to a conventional diesel engine.

The reviewer said that good experiments were conducted this year on minimum injection quantity and start of injection (SOI) effects with E10. Experiments have also been conducted at higher engine speed, at constant boost, and at constant lambda. The reviewer asked how the learnings from this are going to be applied to moving the overall concept forward. The reviewer asked can these learnings be made profitably in the context of highefficiency, low- $\mathrm{NO}_{\mathrm{x}}$ and low PM. If so, the reviewer wondered if the reviewer and reader can be made aware of how these experiments and learnings enable moving the GCI concept forward. For example, if low-pressure (LP)-EGR along with late injection appears to have the leverage to increase exhaust temperature at low load, the reviewer asked how did those these two changes affect efficiency, $\mathrm{NO}_{\mathrm{x}}$, and $\mathrm{PM}$.

\section{Question 3: Collaboration and coordination with other institutions.}

No comments were received in response to this question.

Question 4: Proposed future research-the degree to which the project has effectively planned its future work in a logical manner by incorporating appropriate decision points, considering barriers to the realization of the proposed technology and, when sensible, mitigating risk by providing alternate development pathways.

\section{Reviewer 1:}

The reviewer said that it is proposed that indicated as well as $\mathrm{BTE}, \mathrm{NO}_{\mathrm{x}}$, and $\mathrm{PM}$ always be presented so as to be able to relate any new fuel, operating strategy, experiment, or learnings back to the original benefits of the concept. The reviewer also said that progress in reducing $\mathrm{CO}$ and $\mathrm{HC}$ emission should be systematically tracked.

\section{Question 5: Does this project support the overall DOE objectives of petroleum displacement? Why or why not?}

\section{Reviewer 2:}

No comments were received in response to this question.

\section{Question 6: Resources: How sufficient are the resources for the project to achieve the stated milestones in a timely fashion?}

\section{Reviewer 2:}

No comments were received in response to this question. 


\section{Model Development and Analysis of Clean and Efficient Engine Combustion: Russell Whitesides (Lawrence Livermore National Laboratory) - ace012}

\section{Presenter}

Russell Whitesides, Lawrence Livermore National Laboratory

\section{Reviewer Sample Size}

A total of six reviewers evaluated this project.

\section{Question 1: Approach to performing the work-the degree to which technical barriers are addressed, the project is well-designed, feasible, and integrated with other efforts.}

\section{Reviewer 1:}

The reviewer applauded the uncertainty analysis and said that too many simulations use nominal values. The reviewer commented that real-world application of advanced combustion regimes, both design and control, will need to deal with these noisy variables.

\section{Reviewer 2:}

The reviewer said that as a counterbalance to a 2015 reviewer's comment, it is refreshing to see a recognition that smaller, pragmatic kinetic mechanisms will continue to be the norm in industrial practice for some time to come and are deserving of careful study and emphasis in the quest to democratize engine simulation.

\section{Reviewer 3:}

The reviewer commented that the development of fast chemistry solver technologies and the application of them to new architectures that promise significant performance versus cost gains, such as a graphics processing unit (GPU), is of great import to combustion system designers striving to increase simulation accuracy while also reducing computational time. The reviewer said that getting this technology into the hands of commercial code vendors to make these gains of practical utility to the design community is, of course, just as important.

\section{Reviewer 4:}

The reviewer said that the PIs identify the time to perform simulations of in-cylinder simulations of combustion engines as a limitation for achieving detailed simulations of performance of combustion engines. The reviewer explained that GPU refers to NVIDIA's hardware specification for its general purpose GPU. GPU technology is not new and it is widely used in biomolecular simulations. The reviewer remarked that, here, the PIs are developing

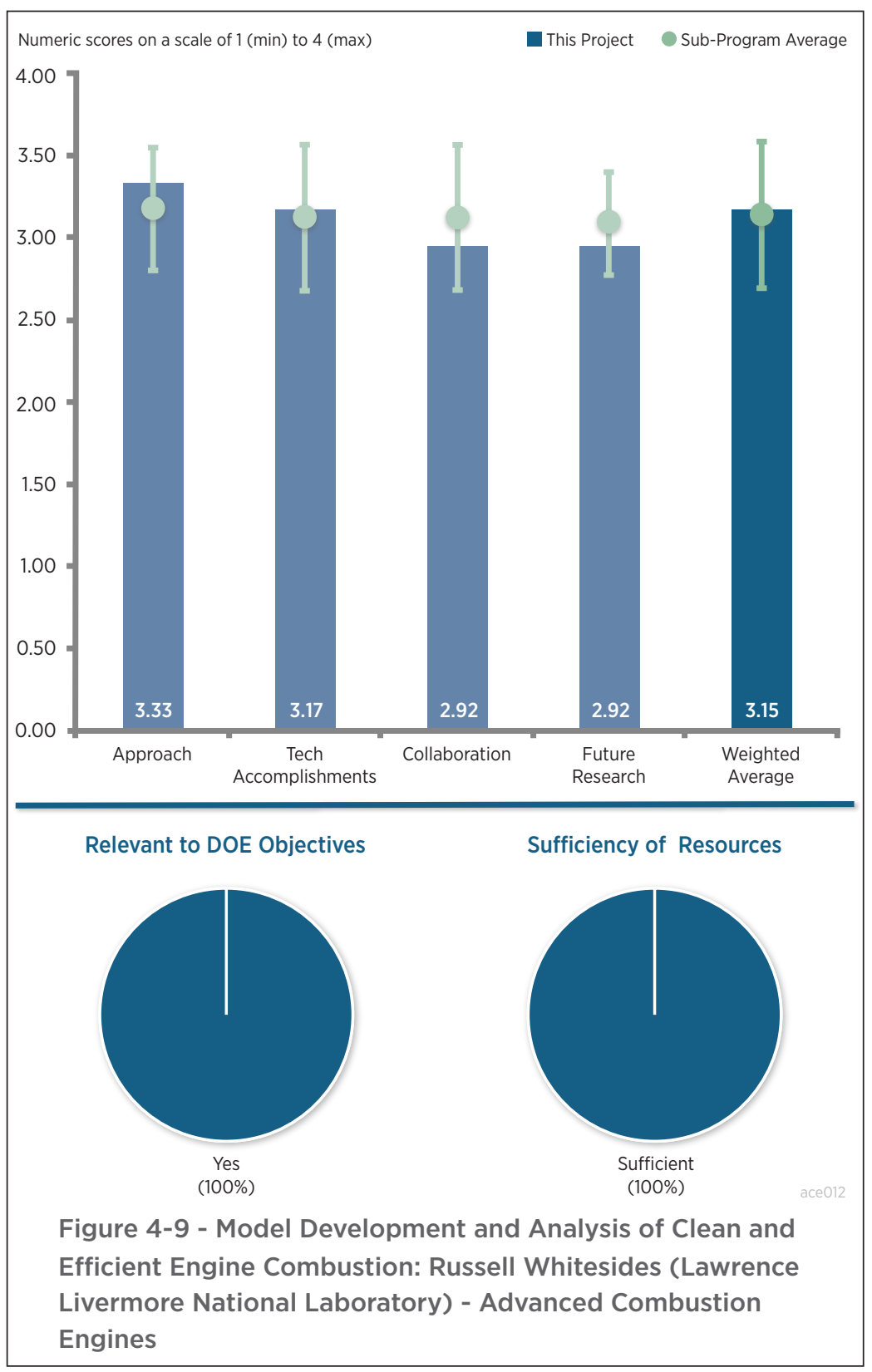


its capability for integrating complex chemistry in combustion engine simulations so that such simulations can be performed in a more time-efficient manner.

The reviewer stated that it is not clear why cetane chemistry is of interest, especially given the current emphasis on surrogates, beyond single component fuels, for real fuels and biofuels. The reviewer remarked that this project should move away from cetane and emphasize chemical mechanisms for multicomponent surrogates for gasoline or diesel.

The reviewer commented that the homogeneous charge compression ignition (HCCI) concept is rather mature, and the problems with it well-known. The reviewer questioned what this work brings to the HCCI problem and how will it alleviate the issues with HCCI. The reviewer asked if this project addresses the concerns that have limited wider use of HCCI. Similarly, high-efficiency clean combustion has been known for some time. The reviewer wondered how this project will strengthen its development and how long will it take. The reviewer also asked what the roadmap is for solving the problems with this engine technology and if the yearly work here is more incremental than transformative.

The reviewer commented that a lot of the work seems to be to run a code and evaluate the results, which seems a bit pedestrian. The project repeatedly refers to CFD simulations, but the actual code being used was unclear from the presentation. The reviewer requested that this be specified.

\section{Reviewer 5:}

The reviewer said that this project is to develop a fast interface method for engine simulation where a detailed mechanism is needed. The method is integrated with engine combustion solvers and detailed mechanisms being developed by other LLNL project teams (i.e., ACE076, Matt McNenly of LLNL; ACE013, Bill Pitz of LLNL). The reviewer remarked that the approach seems reasonable, but the project title seems too general and misleading. The reviewer wondered if the model includes physical models like spray and combustion, and commented that, if so, the focus seems incorrect and it should be done by other ACE teams. The reviewer said that if it is simply using a commercial code (e.g., CONVERGE), the project title should not use the term "model development."

\section{Question 2: Technical accomplishments and progress toward overall project and DOE goals-the degree to which progress has been made, measured against performance indicators and demonstrated progress towards DOE goals.}

\section{Reviewer 1:}

The reviewer said the accomplishments reported for the past year included an uncertainty analysis of SNL HCCI experiments (for example, data for engine speed, crank angle, CR, temperatures, and engine speed). The reviewer questioned what code was used to predict these things. The reviewer also asked if the SNL fuel used cetane. The reviewer said the presenter should clarify the uncertainty analysis. The reviewer commented that the presenter showed a flowchart that indicates how measured quantities related to derived quantities. The reviewer asked how this was used and what effort was involved with this activity. The reviewer also asked if it is just a flowchart of expected links.

The reviewer said that what the presenter reported was apparently the first engine simulations using all central processing units (CPUs) and GPUs on a computer cluster code. The reviewer asked what the base code was. The reviewer said that the PIs demonstrated results that showed GPUs producing the same results as CPUs but in much less time. The PI showed that they can handle big engine combustion problems.

\section{Reviewer 2:}

The reviewer said the milestone of this project in FY 2016 is to evaluate different load-balancing schemes for chemical kinetics and implement the most promising technique, and that collaboration work is going on with the ACE projects. The reviewer observed that development of the GPU accelerated chemistry solution was demonstrated on a gasoline spark ignited engine case on a workstation class computer. The reviewer remarked that it seems impractical to develop a method on a small-scale computer when a detailed mechanism is considered. It would not be that simple to port the method into a HPC environment. The reviewer said the GPU-enabled zeroorder reaction kinetics (Zero-RK) chemistry solver through ACE076 seems like a great achievement. 


\section{Reviewer 3:}

The reviewer said the smart-batching of reactions for GPU is innovative. The UQ work seems particularly important. It would be reassuring to see the simulation (kinetics and CFD parts) studied with the same UQ framework as another source of uncertainties alongside the measurement uncertainties, not only the myriad coefficients and grid-dependencies but also the more subtle and basic model-form uncertainty.

\section{Reviewer 4:}

The reviewer said that while the approach sounds good, test problems have shown that the performance gains have not been as good as anticipated. The reviewer commented that work has begun on smart batching, load balancing, and more in an effort to optimize performance. The reviewer said that preliminary tests look good. A lot of test work so far has focused on HCCI, and while there are some benefits of doing so, testing the capability for conventional diesel is also important and should be given more priority.

\section{Question 3: Collaboration and coordination with other institutions.}

\section{Reviewer 1:}

The reviewer noted Science and NVIDIA, and said the link with some of these groups was a bit vague. The SNL connection seemed clear (e.g., presumably data), but the link with the academic institutions and what they specifically bring to this project was not evident. The reviewer commented that a better connection needs to be established with the academic institutions listed to show the need for what they can provide. The reviewer wondered if the project could proceed without any of the academic partners and asked if they provide data or perform simulations.

As previously noted, the reviewer said the actual code used was unclear and wondered if it was the framework of CONVERGE. If so, then the CONVERGE PIs in other presentations at the AMR would presumably reference the combustion chemistry emphasized here and the efficient integration of GPU technology. The reviewer concluded that if that is being done, the researcher should note it in this project.

\section{Reviewer 2:}

The reviewer questioned how the methods developed through the project can be used for other software vendors than Convergent Science, Inc., and universities or industries using other software. The reviewer commented that the project team should consider this and make the methods available to others when needed.

\section{Reviewer 3:}

The reviewer suggested that the research team could adopt a deliberate multi-code approach in areas where R\&D conclusions or even software design decisions are historically proven to be shaped to varying degrees by the peculiar limitations, methods, or assumptions of an individual simulation tool. The reviewer said that in these situations, to robustly achieve the verbally stated goal of "bringing fast chemistry to CFD," code-redundancy can add value by not only avoiding such distortions but also exposing otherwise hidden lessons and uncertainty sources. The reviewer commented that this collaboration is too important to be left downstream to the Technology Licensing Office.

\section{Reviewer 4:}

The reviewer observed that some collaboration with other national laboratories, a software vendor, a hardware vendor, and an engine company are noted, but the researchers should seek and support broader collaborations.

\section{Question 4: Proposed future research-the degree to which the project has effectively planned its future work in a logical manner by incorporating appropriate decision points, considering barriers to the realization of the proposed technology and, when sensible, mitigating risk by providing alternate development pathways.}

\section{Reviewer 1:}

The reviewer said that the future work should move away from a single component fuel (cetane) and begin to incorporate combustion chemistries of multicomponent surrogate fuels. The reviewer stated that the results of this effort were disseminated through a range of presentations at various meetings and conferences. However, to have wider visibility, the PI should emphasize archival publications such as the articles in Proceedings of the 
Combustion Institute, Society of Automotive Engineers (SAE), and International Journal of Engines. The reviewer commented that the project could benefit from more publications in such journals.

\section{Reviewer 2:}

The reviewer expressed that it is not clear how the UW work helps this project. The reviewer recommended solving multiple engine cycles, or RANS, to avoid ambiguity on setting up the intake valve closing (IVC) conditions.

\section{Reviewer 3:}

The reviewer said that in addition to the two very worthy topics presented, see comments above under question number three.

\section{Reviewer 4:}

The reviewer said that a one-slide summary of future work planned is somewhat sketchy. The reviewer agreed that greater speed-up is needed if the effort put into this work is to truly pay off in faster, better engine designs. The reviewer commented that uncertainty work could possibly take lower priority to improving computational speed. The reviewer added that increased collaboration is definitely needed.

\section{Question 5: Does this project support the overall DOE objectives of petroleum displacement? Why or why not?}

\section{Reviewer 1:}

The reviewer said yes, from a broad perspective.

\section{Reviewer 2:}

The reviewer said this project can support faster simulation time for engine development CFD analysis using detailed mechanism.

\section{Reviewer 3:}

The reviewer said that faster and more accurate simulations will lead to better engine designs with greater efficiency, hence reduced petroleum use.

\section{Question 6: Resources: How sufficient are the resources for the project to achieve the stated milestones in a timely fashion?}

\section{Reviewer 1:}

The reviewer said that the FY 2016 budget seems reasonable.

\section{Reviewer 2:}

The reviewer stated that resources seem adequate although ultimate judgement would have to come from a cost/ benefit analysis based on DOE's investment relative to the commercialization potential.

\section{Reviewer 3:}

The reviewer commented that leveraging more collaborators inside and outside of DOE would be beneficial to effectively increase the project resources at no additional cost to the program. 


\section{Chemical Kinetic Models for Advanced Engine Combustion: Bill Pitz (Lawrence Livermore National Laboratory) - ace013}

\section{Presenter}

Bill Pitz, Lawrence Livermore National Laboratory

\section{Reviewer Sample Size}

A total of five reviewers evaluated this project.

\section{Question 1: Approach to performing the work-the degree to which technical barriers are addressed, the project is well-designed, feasible, and integrated with other efforts.}

\section{Reviewer 1:}

The reviewer stated that it is encouraging to see a recognition that pragmatic surrogate and reduced kinetic mechanisms will continue to be the norm in industrial practice for some time to come. The mechanisms are deserving of careful study and emphasis to support engine simulation. The reviewer encouraged the team to balance its methodical, one-componentat-a-time approach with holistic validation and calibration, to the extent practical, helping ensure that the largest error and uncertainty sources, such as high pressure effects, EGR, and realcycle effects, receive commensurate attention.

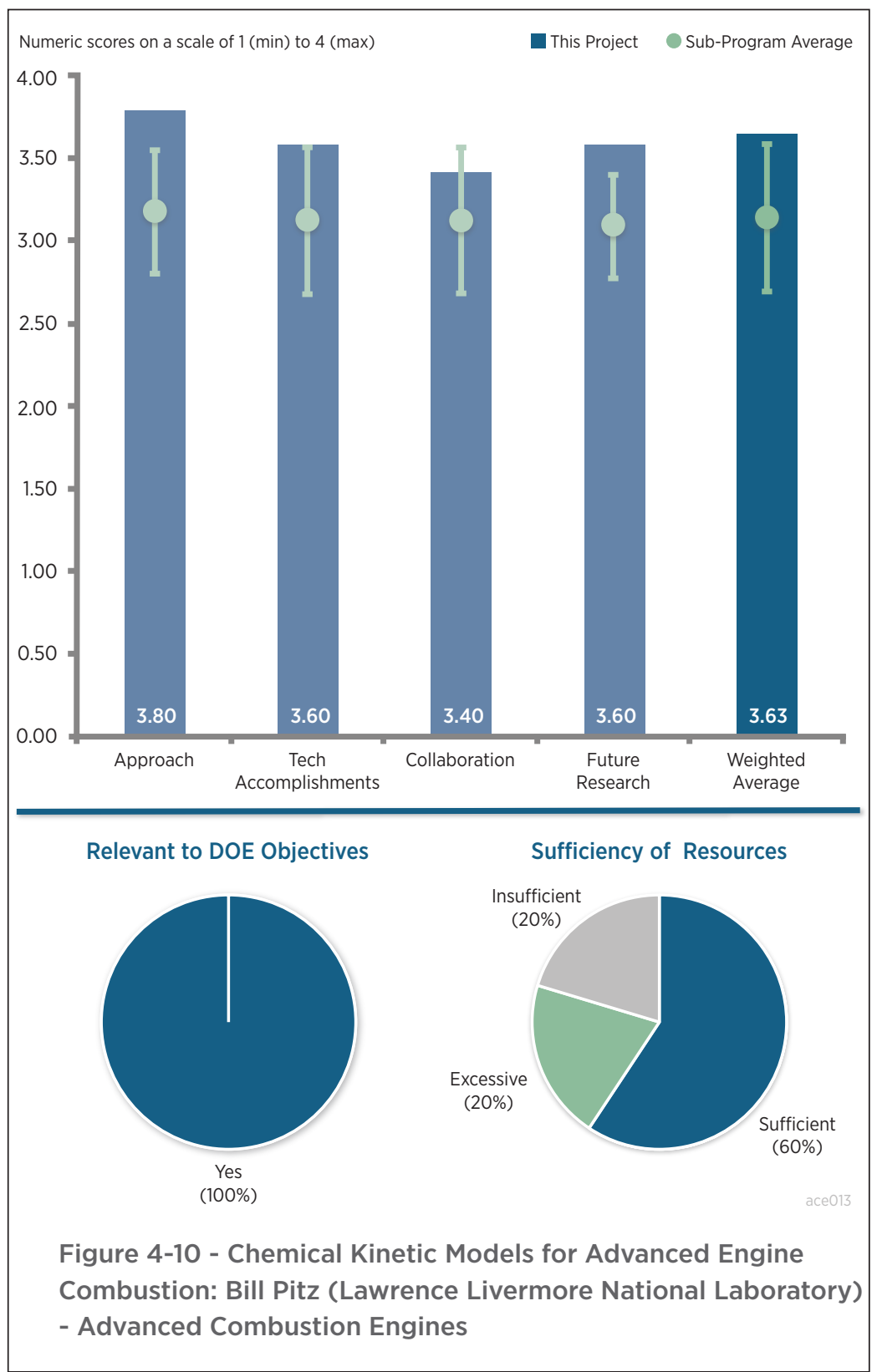

\section{Reviewer 2:}

The reviewer said that understanding and developing kinetic models for combustion chemistry is vital but difficult work. The reviewer commented that the LLNL team has demonstrated over the years that their methodology works. The researchers have produced some very accurate, detailed mechanisms that explain what is observed experimentally in laboratory devices and engines. The reviewer said that the researchers' approach works, adding that it may not be as fast as might be desirable, but experience shows the LLNL team's results are much better than faster, more automated methods.

\section{Reviewer 3:}

The reviewer commented that this is very important work to improve combustion simulation accuracy.

\section{Reviewer 4:}

The reviewer said that the approach is outstanding and has been used for many years. The reviewer expressed that it has proven successful in the development of accurate chemical reaction kinetics. 


\section{Reviewer 5:}

The reviewer commented that the approach is sound in that fundamental chemical kinetic models are generated for surrogate fuels for gasoline and diesel. The reviewer added that these models are validated by comparison to fundamental experimental data. The reviewer said such models have become more important in recent years with the growing interest in LTC.

\section{Question 2: Technical accomplishments and progress toward overall project and DOE goals-the degree to which progress has been made, measured against performance indicators and demonstrated progress towards DOE goals.}

\section{Reviewer 1:}

The reviewer said that the researchers continue to improve and refine their mechanisms for widely used fuels, such as gasoline and diesel, to better match data and extend to new operating regimes. The reviewer said the researchers also continue to expand their palette to new fuel species, adding that the team's process is relatively slow but methodical and deliberate. The reviewer concluded that the researchers' contributions are vital to the community.

\section{Reviewer 2:}

The reviewer commented that the researchers made great progress on improving reactions and developing new reactions.

\section{Reviewer 3:}

The reviewer said that the researchers' presented several worthy accomplishments on Slides 6 through 15. The reviewer said that work on gasoline should continue to be accelerated, including the effect of EGR and more equivalence ratios, pressures, and temperatures. The reviewer commented that the development of improved surrogate mechanisms for high-octane gasoline fuels and gasoline fuels with ethanol is a very critical need.

\section{Reviewer 4:}

The reviewer expressed that the researchers' progress is up to expectation. However, the reviewer requested that the researchers elaborate on the availability of the reaction mechanisms to the broader engine modeling community.

\section{Question 3: Collaboration and coordination with other institutions.}

\section{Reviewer 1:}

The reviewer applauded the direct exposure of results through the LLNL website as an effective, frictionless mode of dissemination that encourages open collaboration. The reviewer said that coordination with Co-Optima, mentioned and discussed in this year's slides, will be very valuable.

\section{Reviewer 2:}

The reviewer said that the team has a large number of partners and clients in industry, academia, and government institutions. The reviewer added that the team's results are the foundation of much of the engine modeling work done today.

\section{Reviewer 3:}

The reviewer said that while the PI has close interactions with other institutions, it would be really nice to show results of such collaborations. The reviewer noted that examples include how the reaction mechanisms are utilized for practical engine combustion simulations and the results as well as how the mechanisms are reduced and the final outcomes.

Question 4: Proposed future research-the degree to which the project has effectively planned its future work in a logical manner by incorporating appropriate decision points, considering barriers to the realization of the proposed technology and, when sensible, mitigating risk by providing alternate development pathways.

\section{Reviewer 1:}

The reviewer said that this is one of the best, most cogent summaries of reviewer comments and future plans seen at this year's review. 


\section{Reviewer 2:}

The reviewer said that the researchers' entry into improved soot modeling is welcomed as existing models fail to match engine behavior under various conditions. The reviewer also welcomed the continued diesel and gasoline surrogate development in conjunction with better RCM data from various collaborators.

\section{Reviewer 3:}

The reviewer said that improved gasoline surrogates are very important, and it is good to see that they are part of the plan.

\section{Reviewer 4:}

The reviewer stated that it is natural to keep developing reaction mechanisms for higher-order HCs and keep refining the mechanisms. However, there may be a limit above which the usefulness diminishes. The reviewer commented that engine spray combustion is complex and there are other determining factors in the modeling accuracy. The reviewer said that it is good to see that the PI plans to develop soot models, which are extremely important.

\section{Question 5: Does this project support the overall DOE objectives of petroleum displacement? Why or why not?}

\section{Reviewer 1:}

The reviewer said that in order to develop better, more efficient engines, developers need accurate kinetics to aid the design process.

\section{Reviewer 2:}

The reviewer expressed that this is crucial work to improve simulations of combustion chemistry that can be used by industry to improve engine efficiency.

\section{Reviewer 3:}

The reviewer noted that the work also includes the chemical kinetics of certain biofuels.

\section{Reviewer 4:}

The reviewer expressed that very important chemical kinetic mechanisms needed for LTC development result from this project.

\section{Question 6: Resources: How sufficient are the resources for the project to achieve the stated milestones in a timely fashion?}

\section{Reviewer 1:}

The reviewer commented that more money could be spent here, but the funding appears adequate for the program proposed.

\section{Reviewer 2:}

The reviewer expressed that more resources should be applied to accelerate progress.

\section{Reviewer 3:}

The reviewer stated that the PI appears to be able to utilize related resources. 
2016 KIVA-hpFE

Development: A Robust and

Accurate Engine Modeling

Software: David Carrington

(Los Alamos National

Laboratory) - ace014

\section{Presenter}

David Carrington, Los Alamos

National Laboratory

\section{Reviewer Sample Size}

A total of four reviewers evaluated this project.

Question 1: Approach to performing the work-the degree to which technical barriers are addressed, the project is well-designed, feasible, and integrated with other efforts.

\section{Reviewer 1:}

The reviewer said that it is not clear if this project can achieve the goal of software development for advanced ICE modeling satisfying industries. The reviewer said that mesh generation seems like old technology, and key physical and chemical models such as spray, combustion, and engine-out emissions, are not clearly directed.

\section{Reviewer 2:}

The reviewer said that turning to hp-adaptive finite element method technology is an innovative approach to attack the critical barriers. The reviewer commented that the new code appears likely to become an effective open-source tool for the research community studying combustion fundamentals. However, the reviewer said that to meet the more ambitious stated goal of "user-friendly (or industry-friendly) software" for engine design, much more investment will be required in the end-user environment, workflow aids, and integration with commercial computer-aided engineering (CAE). The reviewer added that success will require more integration and advanced planning than is presently evident.

\section{Reviewer 3:}

The reviewer said that the mathematical approach seems to be the right direction to improve accuracy. However, it is not clear what the plan is for including chemistry. The reviewer would like to see a comparison to engine simulations.

Question 2: Technical accomplishments and progress toward overall project and DOE goals-the degree to which progress has been made, measured against performance indicators and demonstrated progress towards DOE goals. 


\section{Reviewer 1:}

The reviewer commented that the researchers demonstrated an impressive rate of progress given the lean development team.

\section{Reviewer 2:}

The reviewer said that key milestones and progress in 2016 are not clearly mentioned or compared with those in 2015. The reviewer noted that the two grid generation methods were tried for a very simple port and valve geometry. The reviewer remarked that the researchers should have demonstrated on real engine geometries, such as a light-duty diesel engine from SNL that was used for CFD analysis by UW-Madison.

\section{Reviewer 3:}

The reviewer would prefer to see more progress and results regarding moving parts for engine simulations and noted that the researcher presented no results for engine-relevant air flow or chemistry.

\section{Reviewer 4:}

The reviewer said that comments below have been made in previous reviews and are still relevant. The reviewer said that it is encouraging to see efforts through the radio frequency interference (RFI) and ANSYS to commercialize the code and make it competitive with other codes so that industry can get interested in using it. It will be healthy to have more competitive CFD codes in the marketplace. The reviewer said that KIVA-3 and KIVA-4 are seeing less and less use within industry. KIVA has become more of a free resource to universities that want an open source-type format so they can do physical modeling, but even there, other competitors like OpenFOAM are taking over the market share.

The reviewer expressed that presenters need to seriously evaluate the business model. The reviewer said it would really be healthy to continue to have KIVA as a competitor to other commercial codes. The reviewer questioned what can be done to hasten the development and deployment of KIVA within industry. The reviewer stated that plenty of work has been done and numerous test cases are shown. However, overall technical progress over the last few years on KIVA-hpFE has been very slow. The reviewer said the key issue now is whether industry is really interested in KIVA-hpFE or not, and observed that it is a free code, yet industry prefers to use other commercial codes. The reviewer said there something wrong with this picture and questioned what can be done to make the usefulness and deployment of KIVA-hpFE within industry faster.

The reviewer stated that perhaps a new business model that increases the chances of KIVA not fading away in the next few years would demand different types of collaborations. The reviewer said that, overall, there needs to be faster progress on getting the remaining work done.

\section{Question 3: Collaboration and coordination with other institutions.}

\section{Reviewer 1:}

The reviewer said that it is hard to see any collaboration with other ACE project leaders but with the current project team members. The reviewer suggested the researchers should talk to ACE project teams on LES at SNL, chemistry solver (Zero-RK), and multi-point injection (MPI).

\section{Reviewer 2:}

The reviewer said that to broaden collaboration and increase its ultimate impact, this project might consider (in parallel with the present RFI, or as an alternative if that proves unsuccessful) the CAE for batteries software approach being pursued by the VTO for CFD-based battery simulation tools. The reviewer commented that it will reduce risk and improve odds for ultimate success to collaborate earlier and more strongly with the engine design, and, more broadly, with CAE software leaders, even if only as a hedge relative to a small or startup business attempting to commercialize the software through monolithic licensing. The reviewer said that if KIVA-hpFE is highly modular as advertised, then this approach might involve licensing novel individual nuggets while leveraging established and well-supported commercial software frameworks, infrastructures, and user communities.

\section{Reviewer 3:}

The reviewer said that some collaborations were mentioned, but it is not clear how they contributed to the success 
of the project. The reviewer questioned if there are any collaborative efforts to commercialize the code to promote usage in industry.

\section{Reviewer 4:}

The reviewer stated that there could be more direct collaboration and interaction with industry.

Question 4: Proposed future research-the degree to which the project has effectively planned its future work in a logical manner by incorporating appropriate decision points, considering barriers to the realization of the proposed technology and, when sensible, mitigating risk by providing alternate development pathways.

\section{Reviewer 1:}

The reviewer stated that further improvements in accuracy are good, but questioned who the customer for the software is. The reviewer asked what efforts are being made to commercialize the code and when engine simulation comparisons will be made to engine measurements.

\section{Reviewer 2:}

The reviewer said that incorporating CHEMKIN into the software seems a wrong direction unless user CHEMKIN license cost is resolved, and the same for mesh generation code and other solvers. The reviewer said the current software should not require additional user cost for third party licenses but be a standalone code like previous versions of KIVA code.

\section{Reviewer 3:}

The reviewer commented that many pragmatic software development, integration, deployment, and sustainment challenges lie ahead that were not explicitly acknowledged in this AMR briefing.

\section{Question 5: Does this project support the overall DOE objectives of petroleum displacement? Why or why not?}

\section{Reviewer 1:}

The reviewer said that the project outcome, if successful, will help industries and academia with developing fuelefficient ICEs.

\section{Question 6: Resources: How sufficient are the resources for the project to achieve the stated milestones in a timely fashion?}

\section{Reviewer 1:}

The reviewer said that $\$ 700,000$ is not a small amount of budget to perform this project.

\section{Reviewer 2:}

The reviewer remarked that the presentation provides zero forward-looking milestones, only a long list of efforts, some of which are inherently rather open-ended. The reviewer said that achieving the stated goals of the overall project will require many tens of additional person-years. Slide 2 only presented the budget in terms of "funding to date," but the concern from the 2018 end date is that the planned future funding may be insufficient to fully meet the objectives.

\section{Reviewer 3:}

The reviewer wondered if more resources should be provided to make the code more attractive to industry. 


\section{Stretch Efficiency for Combustion Engines: Exploiting New Combustion Regimes: Stuart Daw (Oak Ridge National Laboratory) - ace015}

\section{Presenter}

Jim Szybist, Oak Ridge National Laboratory

\section{Reviewer Sample Size}

A total of six reviewers evaluated this project.

\section{Question 1: Approach to performing the work-the degree to which technical barriers are addressed, the project is well-designed, feasible, and integrated with other efforts.}

\section{Reviewer 1:}

The reviewer appreciated that there are novel approaches under investigation. The reviewer stated that this high risk project is exactly what DOE should be supporting because it is something unlikely for industry to investigate due to the perceived risk.

\section{Reviewer 2:}

The reviewer stated that it was a very good approach and applauded the search for the high-reward solutions.

\section{Reviewer 3:}

The reviewer said the project focuses on the high EGR dilution and thermochemical recuperation as a form of waste heat recovery. It has focused on critical issues such as hydrogen $\left(\mathrm{H}_{2}\right)$ generation and catalyst formulations, with detailed experiments documenting the challenges experienced. The reviewer said the approach may be extended to consider (at least with some estimations) the impact of the various approaches (for example, dedicated cylinders for reformation) on load capabilities, or the impact of more sophisticated exhaust flow routes on back pressure and efficiency. The reviewer stated that the schematics seem to omit necessary details such as valves and required probes.

\section{Reviewer 4:}

The reviewer said that steam reforming seems to be the thermodynamic pathway of choice, but it seems that having the requisite exhaust enthalpy will be a challenge.

\section{Reviewer 5:}

The reviewer questioned if the researchers, if successful, are at a point where they can estimate the ultimate benefit. The reviewer also wondered if the researchers could leverage some of the EGR loop-reforming techniques to improve the catalyst light-off during cold start.

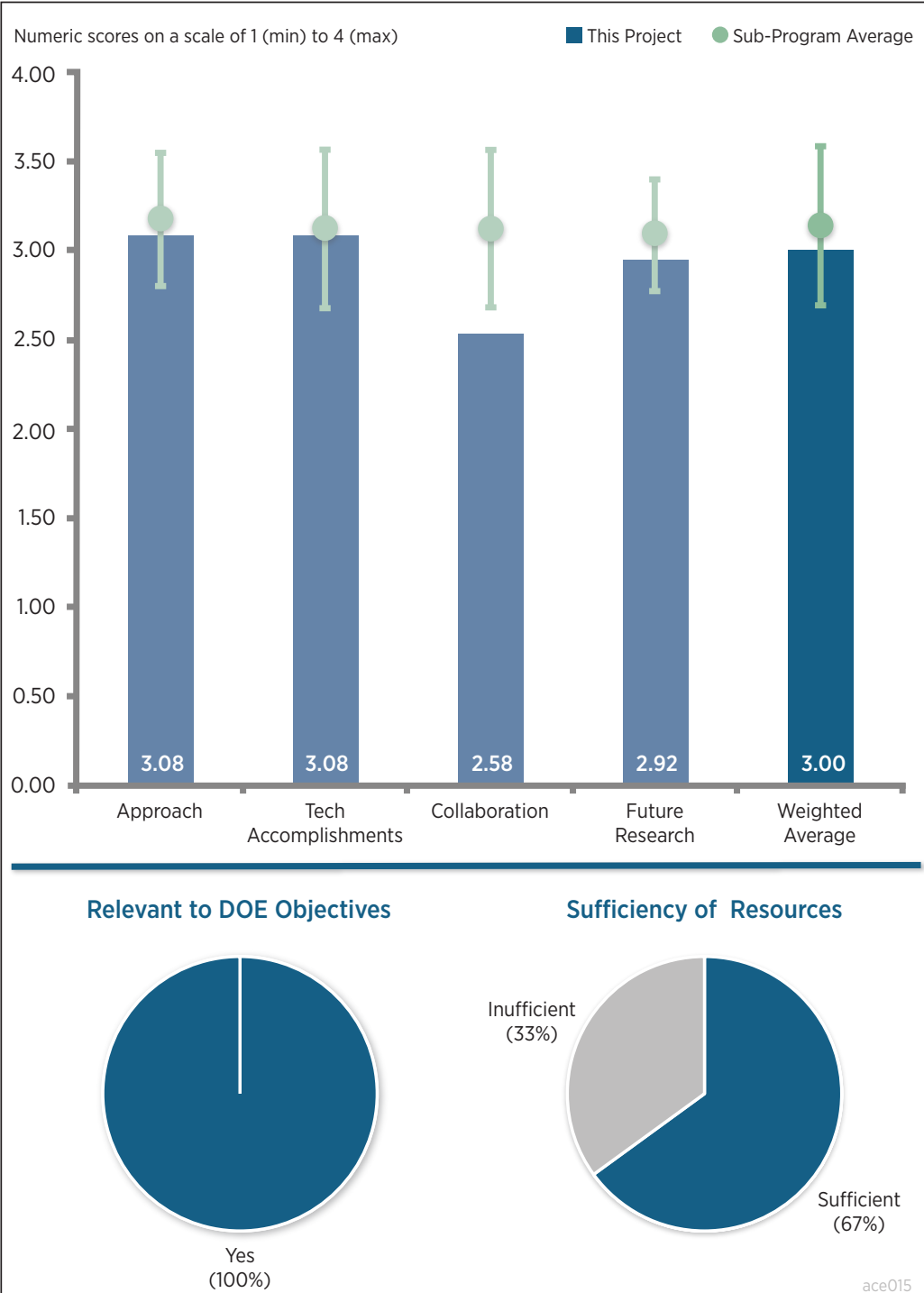

Figure 4-12 - Stretch Efficiency for Combustion Engines: Exploiting New Combustion Regimes: Stuart Daw (Oak Ridge National Laboratory) - Advanced Combustion Engines 


\section{Reviewer 6:}

The reviewer observed that this project is a continuation of an effort to reform gasoline into syngas during operation of an ICE. The reviewer said that the PI's approach is to essentially sacrifice a cylinder, for example, in a four-cylinder engine, and carry out the reforming within the cylinder, or using EGR loop-reforming in which the gases are passed through a catalytic device where the reforming would be carried out. The reviewer said the reporting period appears to be the last year of this project beyond its potential continuation through the combustion laboratory call.

The reviewer stated that the overall approach of reforming gasoline seems interesting, and there are a number of issues with the concept that the work of this project has investigated. The reviewer said that reformed fuel seems to be hydrogen and syngas production. The reviewer wondered if syngas is better than gasoline to warrant converting gasoline to it or hydrogen. The reviewer also asked if the engine would need to be retrofitted in any way with hardware to accommodate the reformed fuel.

\section{Question 2: Technical accomplishments and progress toward overall project and DOE goals-the degree to which progress has been made, measured against performance indicators and demonstrated progress towards DOE goals.}

\section{Reviewer 1:}

The reviewer observed that in the reporting period, the researchers approached three strategies: higher CR, partial oxidation, and exhaust manifold. A bench flow reactor was used to study catalyst reforming. The reviewer said that the PI states that the flow reactor replicates engine conditions. This seems highly unlikely because a flow reactor is largely a steady-state device and in-cylinder processes are highly transient.

The reviewer said the overall results pointed to EGR reforming as preferred over in-cylinder reforming where a range of conclusions were noted such as that $\mathrm{CR}$ seems to have no substantial benefit. The reviewer stated that a number of issues were addressed, such as the effect of endothermic reactions on catalyst temperature, which cause the temperature to decrease until reforming is not effective, and the effect of fuel addition, which was shown to not significantly increase $\mathrm{H}_{2}$ production.

The reviewer remarked that a rhodium $(\mathrm{Rh})$ catalyst was used though few details of the catalyst were provided. Future presentations should discuss more the catalyst properties that yield the highest $\mathrm{H}_{2}$ production as this will determine the efficacy of EGR loop-reforming. The reviewer also said that the researchers noted that "gasoline speciated as a mixture of iso-octane and toluene... results in lower energy balance than iso-octane;" however, this was unclear. The reviewer questioned if the PIs are stating that iso-octane and toluene mixtures are a surrogate for gasoline and asked the researchers to clarify.

The reviewer said that partial oxidation studies showed that $\mathrm{H}_{2}$ production is significantly increased compared to in-cylinder reforming (using iso-octane as the fuel) and yielded the best overall engine efficiency. The review stated that steam reforming of iso-octane provides the route for thermochemical recuperation. The reviewer commented that accomplishments also showed that the optimal reforming conditions were highly dependent on engine response. Results for ethanol reforming were presented that showed significant amounts of acetaldehyde and methane formation that were dependent on temperature and fuel flow rate. The reviewer said that though it may not fall under technical accomplishments, for the proposed EGR loop-reforming concept to be commercially viable, there should be some interest among OEMs to pursue the idea. The reviewer concluded that without such interest, the concept will remain only as a laboratory demonstration.

\section{Reviewer 2:}

The reviewer said that work has not yielded significant nor expected results. The level of reformate generated in the partial oxidation approach appears to be insufficient to change and enhance the combustion. The reviewer commented that the EGR loop-reforming approach did not provide the sought $\mathrm{H}_{2}$ concentrations except at extremely high temperatures. However, the iso-octane appears to be more promising. The reviewer said there is some discussion and justification to the work, such as the impact of EGR and combustion phasing on combustion duration, which is indicative of efficiency. Nevertheless, the data do not indicate the impact of $\mathrm{H}_{2}$ on extended EGR operation. 


\section{Reviewer 3:}

The reviewer said the work is technically sound; however, the reviewer would liked to have seen a more explicit roadmap of how the researchers plan to improve the efficiency of the engine via thermochemical exhaust heat recuperation. The reviewer questioned if the options included: increased energy, as shown in the energy balance and implied in the comments made on WHR during the presentation; the capability to run more dilute and pick up efficiency that way; or a combination of these two or others. The reviewer thought the researchers should be able to frame these potential improvements through their modeling capability.

\section{Reviewer 4:}

The reviewer said sometimes a negative result is a good result as it is in this case. The reviewer also commented that it is good that the researchers identified the issues with the in-cylinder reforming so that the approach can be abandoned to focus on the catalytic reforming, which might provide better results.

\section{Reviewer 5:}

The reviewer stated that though the in-cylinder reforming did not perform as desired, researchers performed a very thorough test and analysis campaign. The reviewer said the technology was given a fair chance; it just did not work.

\section{Question 3: Collaboration and coordination with other institutions.}

\section{Reviewer 1:}

The reviewer was pleased to see the addition of a 3D CFD collaborator.

\section{Reviewer 2:}

The reviewer said that collaborations were noted between ORNL and industry (Umicore, Aramco), academia (University of Michigan, University of Minnesota), and other laboratories (SNL). However, the reviewer said some of the indicated collaborations were a bit unclear; for example, a 2015 SAE paper is indicated as being in manuscript form. The reviewer wondered if it has been finalized yet. The reviewer said researchers refer to a 2014 SAE paper as evidence of collaborations with SNL, which seems dated. Regarding "kinetic simulations," it was not clear how this information was used in the project. The reviewer commented that a clearer need for the partnerships listed with the overall project goals should be established in future presentations. For example, the reviewer questioned what the collaborator brings to the project that the PI is unable to provide from within the PI's own organization.

The reviewer also said the collaborators do not appear to include an engine OEM and questioned if that was correct. The reviewer remarked that to establish commercial viability, at least of the concept, an OEM should be included as part of the team with a commitment to pursue this concept if the EGR loop-reforming concept is attractive.

\section{Reviewer 3:}

The reviewer remarked that the project should look for active participation from OEMs that are supportive of these advanced concepts.

\section{Reviewer 4:}

The reviewer stated that the activity would benefit from collaboration with an automotive OEM and that there are many practical issues that the researcher needs to keep in mind. The reviewer was particularly concerned about the comment that ethanol was used because it did not show the coking problem of other fuels.

\section{Reviewer 5:}

The reviewer said the project needs an OEM to be involved to provide feedback regarding an implementation pathway.

Question 4: Proposed future research-the degree to which the project has effectively planned its future work in a logical manner by incorporating appropriate decision points, considering barriers to the realization of the proposed technology and, when sensible, mitigating risk by providing alternate development pathways. 


\section{Reviewer 1:}

The reviewer said that this is an interesting and fun thermodynamic exercise. The reviewer was concerned that there may not be enough enthalpy in the exhaust gas to achieved steam reforming, which appears to show the highest potential for efficiency improvement.

\section{Reviewer 2:}

The reviewer said that the future work will focus on catalytic reforming, which is appropriate given that incylinder reforming was found have some issues. The reviewer added that that the researcher plans on doing engine experiments using several catalysts with the assistance of Umicore's pre-commercial Rh-based catalyst. The reviewer observed that additionally, bench flow reactor studies will be performed to evaluate catalyst durability, and that these activities seem reasonable. The reviewer commented that missing among them seems to be linking with an OEM to provide more credibility to the approach; namely, that the OEMs can envision EGR loopreforming as a viable technology. The reviewer said that this project has been pursued for six years now, and it seems reasonable that a jumping-off point be developed where an OEM will pursue commercialization of the idea.

\section{Reviewer 3:}

The reviewer said the researchers need to expand the study with the catalyst reforming beyond a single speed load.

\section{Reviewer 4:}

The reviewer said that the work may want to step back and assess the potential of their present approach of high dilution and thermochemical recuperation in light of other approaches pursued commercially, such as Rankine cycle and turbocompounding, both from an efficiency and cost-effectiveness standpoint.

\section{Reviewer 5:}

The reviewer stated that catalyst performance will be the key to success for the EGR loop reforming. Catalyst deactivation mechanisms need to be thoroughly identified and explored. The reviewer commented that for the 3D CFD work, the reviewer would like to see how well the $\mathrm{H}_{2}$ production, and its impact on combustion, can be modeled. The reviewer remarked that it would also be good to do a quantitative comparison between the best found on EGR loop-reforming and Southwest Research Institute's dedicated EGR concept, ideally on the same engine.

\section{Question 5: Does this project support the overall DOE objectives of petroleum displacement? Why or why not?}

\section{Reviewer 1:}

The reviewer stated that considering the modest budget of $\$ 300,000$, a lot has been done. The reviewer commented that this is the kind of stretch efficiency combustion work DOE should be sponsoring.

\section{Reviewer 2:}

The reviewer said that if the catalyzed reforming approach works, the system can be leveraged to improve dilution tolerance and increase engine efficiency.

\section{Reviewer 3:}

The reviewer said that this project is relevant from a broad perspective, but greater relevance would be established if an engine OEM were part of the team, along with the interest toward commercialization of the proposed concept that such a partner would bring to the project.

\section{Reviewer 4:}

The reviewer said that this work has the potential to quantify the opportunities and challenges of optimizing the thermochemical processes of fuel energy conversion with practical engine-imposed constraints.

\section{Reviewer 5:}

The reviewer said there must be active research in the high-risk and high-reward space. The reviewer also said that the benefits are being assessed in a proper and relevant way.

Question 6: Resources: How sufficient are the resources for the project to achieve the stated milestones in a timely fashion? 


\section{Reviewer 1:}

The reviewer stated that resources seem adequate although ultimate judgement would have to come from a cost/ benefit analysis based on DOE's investment relative to the commercialization potential. 


\section{High-Efficiency Clean Combustion in Multi-Cylinder Light-Duty Engines: Scott Curran (Oak Ridge National Laboratory) - ace016}

\section{Presenter}

Scott Curran, Oak Ridge National Laboratory

\section{Reviewer Sample Size}

A total of four reviewers evaluated this project.

\section{Question 1: Approach to performing the work-the degree to which technical barriers are addressed, the project is well-designed, feasible, and integrated with other efforts.}

\section{Reviewer 1:}

The reviewer said the project took an excellent experimental approach, combining $1 \mathrm{D}$ and $3 \mathrm{D}$, multi-cylinder, and transient dyno hardware-inthe-loop capability. The reviewer commented that this is a very good and practical approach to measuring virtual in-vehicle fuel economy of advanced combustion concepts.

\section{Reviewer 2:}

The reviewer said that it would be good to show the results of the simulation on top of the experimental results that were shown. The reviewer questioned if the simulation is being used for suggesting optimal operating conditions with the many variables they must control, and if not, it should be. The reviewer said this could be helpful in developing transient control strategies.

\section{Reviewer 3:}

The reviewer said the approach is overall sound in that emissions aftertreatment is included in the overall scope of ORNL projects. However, more than one organization has investigated reactivity-controlled compression ignition (RCCI) combustion for several years. The reviewer observed that its benefits are high efficiency, low engine-out $\mathrm{NO}_{\mathrm{x}}$ and PM. Its challenges are high engine-out $\mathrm{HC}$ and $\mathrm{CO}$ emissions. The reviewer suggested that rather than continue to push the efficiency benefits higher, it is time to focus on minimizing $\mathrm{HC}$ and $\mathrm{CO}$ emissions while retaining efficiency. The reviewer said the researcher should attack key barriers first.

Question 2: Technical accomplishments and progress toward overall project and DOE goals-the degree to which progress has been made, measured against performance indicators and demonstrated progress towards DOE goals. 


\section{Reviewer 1:}

The reviewer said the project showed good, solid work and that understanding transients will be a key to the researchers' success. The reviewer stated that knowing the transient behavior of the entire engine system, including air handling, EGR, and boosting system, and matching those conditions with the optimal RCCI control algorithm will be a large technical challenge.

\section{Reviewer 2:}

The reviewer commented that the project did a very thorough job showing the benefit, the combustion phasing control, and how it compares to other combustion approaches. However, the reviewer said the project needs to show more on combustion noise with increasing load as the engine switches between the combustion modes.

\section{Reviewer 3:}

The reviewer questioned what benefit a combustion system development and mapping guideline is when the combustion system, especially with regards to $\mathrm{CO}$ and $\mathrm{HC}$ emissions, is not meeting engine-out requirements. The reviewer said that conducting a fundamental investigation of the discrepancies between measured and modeled efficiencies to look for areas of efficiency loss is a sound scientific approach. The reviewer commented that contributions at this level will be more valuable to industry than optimizing a given set of hardware. The reviewer added that the project needs to look at five-cycle comparisons. Also, the researchers should make fuel economy comparisons to a relevant downsized, boosted baseline. The reviewer concluded that the fact that the RCCI engine does not meet $\mathrm{CO}$ and $\mathrm{HC}$ emissions standards must somehow be taken into account.

\section{Question 3: Collaboration and coordination with other institutions.}

\section{Reviewer 1:}

The reviewer stated that collaboration with multiple stakeholders is very good and that, as pointed out during the questions and answers, close collaboration with industry will be critical in moving this project forward. The reviewer added that the fundamental issues they are exploring are closely coupled with what will be practical constraints to actually getting this concept out of the laboratory.

Question 4: Proposed future research-the degree to which the project has effectively planned its future work in a logical manner by incorporating appropriate decision points, considering barriers to the realization of the proposed technology and, when sensible, mitigating risk by providing alternate development pathways.

\section{Reviewer 1:}

The reviewer said that the research team has a good understanding of what the issues are.

\section{Reviewer 2:}

The reviewer remarked that quantifying the benefit of the approach over a transient cycle will be key. Also, the researchers need to look at more heavily-loaded cycles (e.g., US06), to see if the aftertreatment system cost and complexity can really be reduced. If conventional diesel combustion is still used at higher loads, the lower light load engine-out $\mathrm{NO}_{\mathrm{x}}$ may have no impact on the aftertreatment system. The reviewer said the researchers also need to make improving combustion efficiency a priority. The reviewer commented that getting the rest of the fuel to burn would be a huge win, and that maybe this is an area to focus the 3D CFD efforts.

\section{Reviewer 3:}

The reviewer remarked that the focus should be on addressing high $\mathrm{HC}$ and $\mathrm{CO}$ emissions during both steady-state and transient operation.

\section{Question 5: Does this project support the overall DOE objectives of petroleum displacement? Why or why not?}

\section{Reviewer 1:}

The reviewer said that anything that has the potential to reduce emissions and improve efficiency is relevant.

\section{Reviewer 2:}

The reviewer said that RCCI has the advantage of having a very powerful additional control lever (i.e., a second 
fuel) for controlling LTC processes. It still has many challenges that need to be understood and overcome if it is to successfully make it from the laboratory to the market. The reviewer expressed that this effort addresses these issues.

\section{Question 6: Resources: How sufficient are the resources for the project to achieve the} stated milestones in a timely fashion?

\section{Reviewer 1:}

The reviewer would like to see this work expedited. The reviewer questioned if within the next year the team can assess the benefit and address the barriers. The sooner this can be done, the sooner it will move to market or the team can refocus on a different technology. 


\section{Accelerating Predictive Simulation of IC Engines with High Performance Computing: Kevin Edwards (Oak Ridge National Laboratory) - ace017}

\section{Presenter}

Kevin Edwards, Oak Ridge National Laboratory

\section{Reviewer Sample Size}

A total of seven reviewers evaluated this project.

\section{Question 1: Approach to performing the work-the degree to which technical barriers are addressed, the project is well-designed, feasible, and integrated with other efforts.}

\section{Reviewer 1:}

The reviewer said that the project appears to be well-designed, with the overall goal to provide innovative simulation tools for improved predictive modeling. The work is compartmentalized in several projects with strong teams represented. The reviewer commented that the project may need to clarify the need or appropriateness of extending the work to on-board diagnostics (OBD). The reviewer added that OBD flags major faults on emission-related equipment; while intricate in many instances, the approach applied here seems to be rather distant from the detailed modeling here.

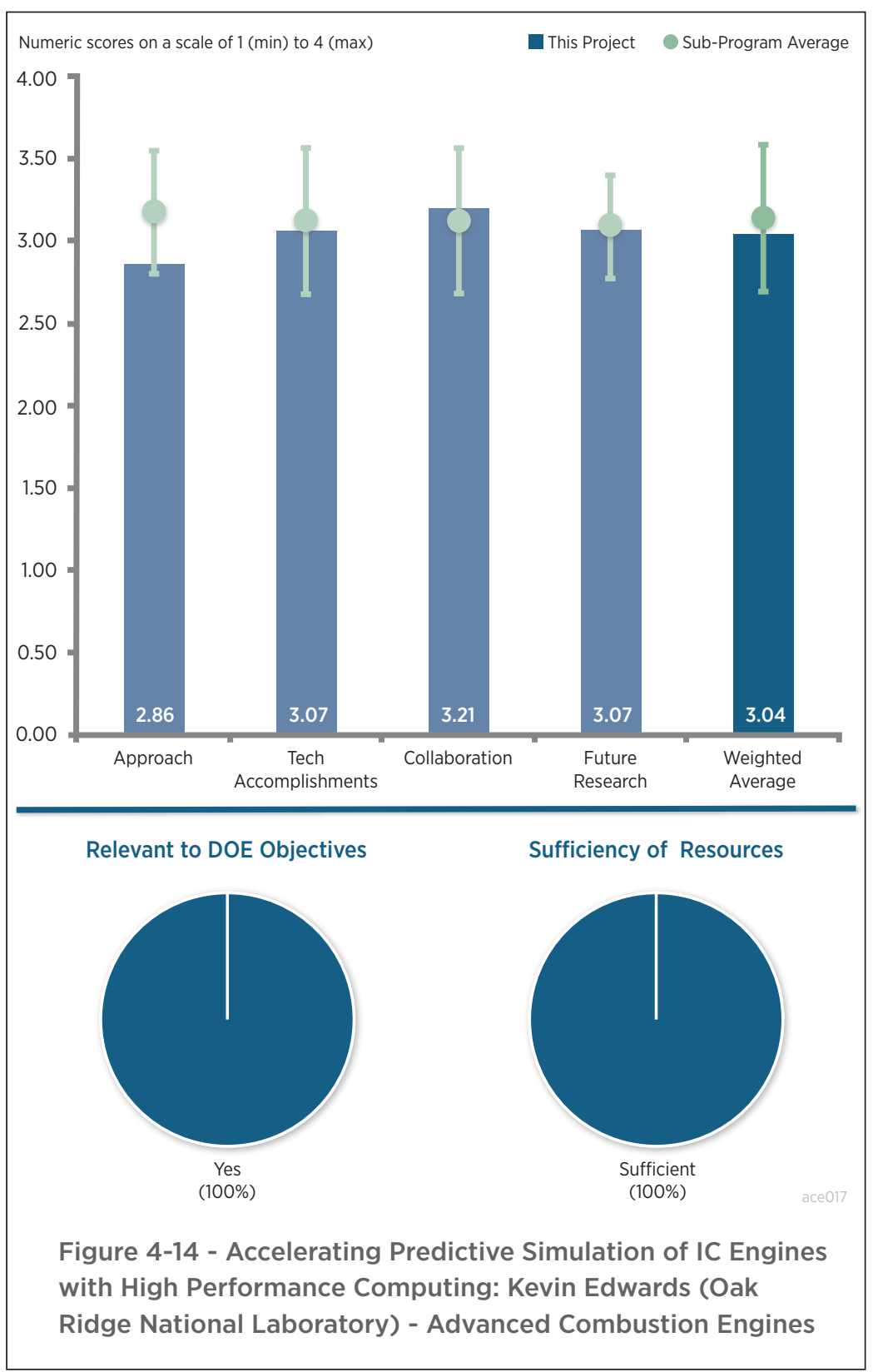

\section{Reviewer 2:}

The reviewer noted that the approach is to utilize the HPC capabilities available at ORNL to examine various approaches to speed up, expand, or otherwise promote large-scale computing for engine design purposes. In this, the team is doing well.

\section{Reviewer 3:}

The reviewer commented that the project was a good use of the national laboratories' supercomputing resources.

\section{Reviewer 4:}

The reviewer said that leveraging large computational resources to solve complex engine problems is very important, but questioned how to know if all the simulations that are being run are correct.

\section{Reviewer 5:}

The reviewer said that this project is developed to enable virtual design of an engine, which is a worthwhile venture. The reviewer added that it includes tasks such as virtual engine design through GPU solvers and detailed 
kinetics, refining a metamodel approach with LES turbulence modeling, and simulating a GM GDI engine, among other things.

The reviewer stated that the relevance and approach were presented at such a high level as to make it difficult to evaluate technical details of the project. This approach did not support effective communication of the results obtained and their rationale. The reviewer commented that under technical accomplishments, the PI notes that "virtual design has potential to significantly accelerate and expand exploration of the design space." However, the reviewer said that this statement does not belong as a technical accomplishment. Rather, it is an observation that belongs in an introductory slide. The reviewer commented that the code used was unclear, and questioned if it was CONVERGE running on Titan. The reviewer said that if so, this should be clearly stated.

The reviewer remarked that the approach of this project may be logical; however, what the presenter provided did not permit a deeper dive into the methodology to allow an evaluation of it. The reviewer said that the PI should not assume that the audience is familiar with all aspects of the project.

\section{Reviewer 6:}

The reviewer commented that Slide 7 recognizes that fast and accurate predictions are two "among the many barriers." The emphasized vision of virtual engine design will not be fulfilled in industry without a commensurate investment in expert, knowledge-based systems that drastically reduce the expertise required by non-specialist engineers to select, assemble, and verify simulation models. The reviewer added that analogous comments apply to reduced models for OBD-based control. Both areas are good candidates for being downstream and outside of this project. The reviewer referred to prior comments under Question three. The reviewer said that pursuing those aggressively now would appear somewhat premature before large discrepancies such as flash boiling and CO are better resolved. The reviewer said that, as another reviewer commented live during the session, "solve accuracy first, and then speed."

\section{Reviewer 7:}

The reviewer commented that goals should be to produce a ranked list of control factors. The reviewer asked which factors can be used to control variability and which to move the mean.

\section{Question 2: Technical accomplishments and progress toward overall project and DOE goals-the degree to which progress has been made, measured against performance indicators and demonstrated progress towards DOE goals.}

\section{Reviewer 1:}

The reviewer stated that accomplishments noted were associated with simulating an atomizer configuration that revealed flash boiling conditions, which was interesting. The reviewer observed that the presenter did not specify the code used in the simulation or the criteria for flash boiling and that also listed in this category was a process for virtual design. However, this is not an accomplishment; it is an observation of a process that belongs elsewhere in the presentation. The reviewer added that the presenter noted that GPU scalability has a significant benefit over CPU. Various results using CONVERGE were shown using heptane. However, the reviewer said that a multicomponent surrogate should be used in the simulations with its associated chemistry, which appears to be a task for future work.

The reviewer observed that there was no discussion of the rational for the 74 reaction kinetics for heptane. The reviewer stated that some simulations of LES realizations were shown. The Oefelein group is developing an LES capability for engine performance. The connection with this effort and the Oefelein group should be noted.

The reviewer said that some of the accomplishments were presented in a rather cryptic form with reference to conference papers where apparently more details would be found. However, this is not conventional. The reviewer said, for example, that the study of the RANS simulation that examines the effect of boundary conditions was hard to follow. The reviewer remarked that the presenter showed a series of figures showing comparisons between measured and predicted crank angles for a kinetic scheme for heptane of 80 species and 450 reactions. However, there was no discussion of the kinetics, model, code, or geometry. The reviewer said, presumably, that this information is found in the SAE publication; however, the presentation should do more than list tasks and include a 
figure and citation or two about them. The reviewer suggested that, otherwise, the presenters could just send papers to the reviewers and ask them to evaluate the work; however, that is not the intent of the AMR.

\section{Reviewer 2:}

The reviewer said the project was well-balanced in execution and in the AMR summary presentation. The reviewer commented that it would be helpful to present more clearly what specific code and model enhancements or calibrations and tunings came directly out of this work.

\section{Reviewer 3:}

The reviewer said the report documents very well the efforts on five separate projects. Work on the injector design is illustrated by detail mapping of flash transition across a wide range of boundary conditions. The reviewer said the two-fluid model is a good improvement from last year. The reviewer commented that in the second project, the work on engine design and calibration highlights some anomalies, such as the CO composition history. The reviewer added that this is of interest, but the presenter did not demonstrate the overall work on effective improved calibration approaches. The work highlights emissions versus computation. The reviewer asked if this should be augmented to performance benchmarks.

The reviewer said the cyclic variability in dilute combustion focused on pressure, temperature, and fuel. The report may explain the justification for the ranges chosen, particularly the large fuel variations that reasonably overshadow the variability. The reviewer asked if other issues would be at play and of interest, such as heat transfer and soot deposits.

\section{Reviewer 4:}

The reviewer said that the presenter reports progress on a variety of joint projects such as virtual injector and engine design and cyclic variability. The reviewer commented that approaches all utilize the computing capacity available at ORNL to extend what groups engaged in CFD-based design do already.

\section{Reviewer 5:}

The reviewer said it looks like injector spray simulations need some improvements and asked what the approach to improve it is. The reviewer said there was good progress on accelerating CONVERGE solution time. The reviewer expressed that cycle-to-cycle variability using LES is interesting, but needs to be sped up to be useful to industry. It is not clear how these tools and approaches can be rolled out to industry.

\section{Reviewer 6:}

The reviewer said the researchers need to continue to pursue accuracy and experimental confirmation of the model.

\section{Reviewer 7:}

The reviewer said that researchers have made reasonable progress with regards to simulating flash boiling gasoline sprays. However, researchers need to make more progress in predicting $\mathrm{CO}$ and $\mathrm{HC}$ emissions. The reviewer said the sensitivity of the code to the random number generator is concerning.

\section{Question 3: Collaboration and coordination with other institutions.}

\section{Reviewer 1:}

The reviewer commented that this was an excellent team. The reviewer asked if the authors could elaborate on how they contribute to the projects.

\section{Reviewer 2:}

The reviewer commented that the correct partners are involved to enable success.

\section{Reviewer 3:}

The reviewer said that the team works with a wide range of industrial partners and other government laboratories.

\section{Reviewer 4:}

The reviewer said that although collaboration is robust, the project appears to extend into some areas that are mature or routine enough to hand off to others such as: simulation-based workflows for virtual engine design; demonstration of CFD scalability to $\mathrm{O}(100)$ cores; generic GPU enablement of a CFD solver; more generally, 
adaption of software to the characteristics of HPC environments; and sparse-grid sampling to generate design-ofexperiments metamodels.

The reviewer said that VTO should consider an industry-led competitive funding opportunity announcement as an alternative mechanism for those parts of the work in order to do the following: conserve expert DOE resources for the core R\&D challenges; ensure more sustainable software commercialization; and help promote a healthy competition and ultimately greater diversity among industrial software tools than the sole CFD partner and platform utilized in this research.

The reviewer suggested that as another alternative to the above suggestion, and following on from last year's review comments and the presented responses on this issue, the research team could adopt a deliberate multi-code approach in a few of the areas where conclusions or calibrations are historically proven to be influenced, to varying degrees, by the peculiar limitations, methods, or assumptions of an individual simulation tool. The reviewer commented that in these situations, notwithstanding the OEM partner's choice of a CFD platform and despite the added cost, code-redundancy can add value by not only avoiding such distortions, but also exposing otherwisehidden lessons and uncertainty sources.

\section{Reviewer 5:}

The reviewer observed that collaborators include GM, General Electric, Ford, LLNL, Cummins, and others; however, it was not clear precisely what these collaborators provided to the project. For example, the reviewer said that under injector design, somewhat curiously, the Powell group at ANL is not listed, yet their project seeks to develop an X-ray capability for improving nozzle design. This person also noted that all collaborators are listed under injector design.

\section{Question 4: Proposed future research-the degree to which the project has effectively planned its future work in a logical manner by incorporating appropriate decision points, considering barriers to the realization of the proposed technology and, when sensible, mitigating risk by providing alternate development pathways.}

\section{Reviewer 1:}

The reviewer said that efforts to understand cycle-variation is particularly important, valuable, and appropriate for continuing work.

\section{Reviewer 2:}

The reviewer said that the authors may want to tie the upcoming work closer to demonstrable targets that translate to improved engine and hardware design. For example, the reviewer suggested applying the flash boiling modeling predictions to optimize the combustion system on engine with resulting improvements on efficiency, emissions, or overall control. The reviewer commented that, to this effect, collaborating with OEMs on specific designs or engine programs may prove to be useful.

\section{Reviewer 3:}

The reviewer remarked that work with larger chemistry mechanisms using LLNL's sparse chemistry solver technology and additional cyclic variability studies, including some LES utilization, is projected. The reviewer said these are incremental steps in the right direction of exercising the capabilities possible with large-scale computing.

\section{Reviewer 4:}

The reviewer said that proposed work on gaining insights into the SNL's partial fuel stratification combustion concept will be interesting.

\section{Reviewer 5:}

The reviewer stated that future work is to include more simulations that include larger chemistry mechanisms, but questioned if it was for heptane or a surrogate. The future work will also include efforts to assess grid size, assess computational time for CONVERGE, and LES simulations on Titan that will investigate nozzle wobble. The reviewer asked if the Oefelein group will be involved with the LES simulations on Titan, and asked for confirmation that assessment of computational time was for CONVERGE. The reviewer said that the researcher listed tasks but they were a bit vague and the rationale for them was not evident. For example, a future task such 
as "assess potential of our approach to improve accuracy and reduce wall-time for virtual engine calibration" does not offer much about precisely how this assessment is to be carried out and what engine calibration means. Finally, the reviewer said that the project lists a lot of presentations. The reviewer encouraged the PI to transition the conference presentations into archival journal articles.

\section{Question 5: Does this project support the overall DOE objectives of petroleum displacement? Why or why not?}

\section{Reviewer 1:}

The reviewer said the project scope is relevant.

\section{Reviewer 2:}

The reviewer stated that speeding up the design process and expanding the design space via HPC will lead to better, more efficient engines that burn less petroleum.

\section{Reviewer 3:}

The reviewer commented that if the tools are developed and provided to industry, then they can potentially be used to improve engine efficiency in the future.

\section{Reviewer 4:}

The reviewer said, yes, from a broad perspective, but the presentation was presented at such a high level that it was difficult to understand how the various tasks combined to meet broad project objectives. The reviewer commented that the PI might consider in future presentations to focus on just one element and provide in-depth information about that. The PI can list all tasks as well, but focus on one. The reviewer said that doing so will allow a capability to assess the quality of the work pursued and its relevance to overall project goals.

\section{Question 6: Resources: How sufficient are the resources for the project to achieve the stated milestones in a timely fashion?}

\section{Reviewer 1:}

The reviewer commented that resources seem adequate for the program plan described.

\section{Reviewer 2:}

The reviewer expressed that the project was a good leverage of advanced scientific computing research (ASCR) resources for HPC.

\section{Reviewer 3:}

The reviewer stated that resources seem adequate although ultimate judgement would have to come from a cost/ benefit analysis based on DOE's investment relative to the commercialization potential. 
Joint Development and Coordination of Emissions Control Data and Models (CLEERS Analysis and Coordination): Stuart Daw (Oak Ridge National Laboratory) - ace022

\section{Presenter}

Josh Pihl, Oak Ridge National

Laboratory

\section{Reviewer Sample Size}

A total of four reviewers evaluated this project.

Question 1: Approach to performing the work-the degree to which technical barriers are addressed, the project is well-designed, feasible, and integrated with other efforts.

\section{Reviewer 1:}

The reviewer said that the combination of approaches used by ORNL and Pacific Northwest National Laboratory (PNNL) are very useful in knowing how new work may contribute. The reviewer said that the priority survey results are very interesting and unique for seeing how technologies mature and grow to ask for more work.

\section{Reviewer 2:}

The reviewer said that the approach to address needed research is wellgrounded in Cross-Cut Lean Exhaust Emissions Reduction Simulations (CLEERS) surveys conducted with OEMs and the catalyst community. The reviewer stated that the shift in research emphasis to passive $\mathrm{NO}_{\mathrm{x}}$ adsorber (PNAs) is consistent with the needs of aftertreatment groups to final solutions for low-temperature operating conditions. Also, continuing to characterize selective catalytic reduction (SCR) ammonia $\left(\mathrm{NH}_{3}\right)$ inventory and other utilization metrics is very desirable for optimizing the use of reductants in lean exhaust aftertreatment systems. However, the reviewer said that real-world aging conditions should be considered in more detail to provide accurate models.

\section{Reviewer 3:}

The reviewer stated that CLEERS audios and workshops are invaluable opportunities for sharing technical work in the aftertreatment community. The reviewer said that CLEERS priority surveys are also extremely helpful and useful to understand industry trends and where research money is being spent.

\section{Reviewer 4:}

The reviewer said there was a good blend of experimental work and modeling. The reviewer said the annual

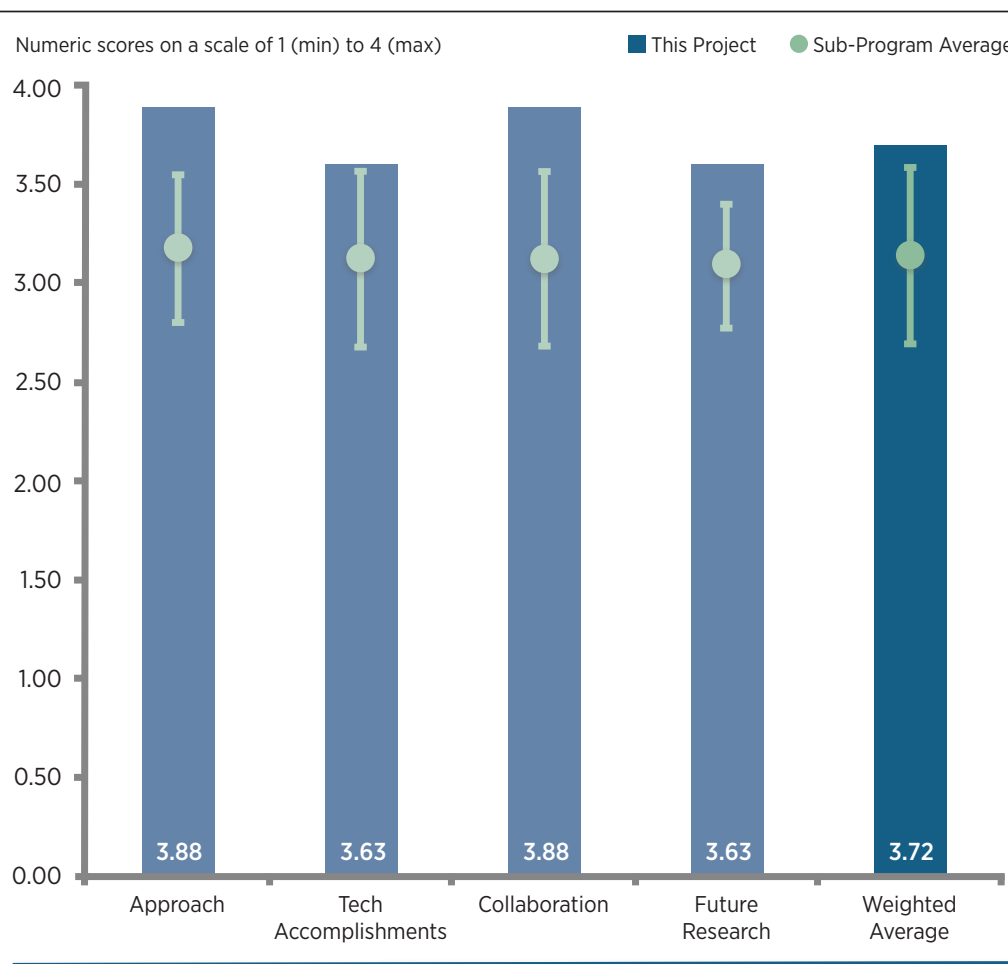

Relevant to DOE Objectives

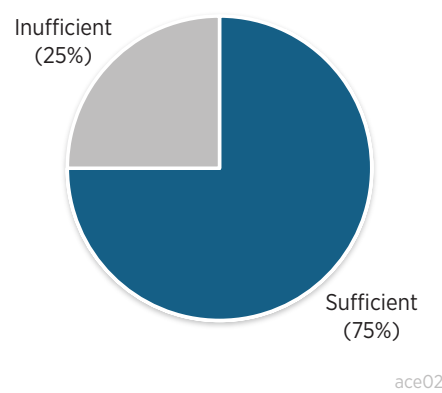

Figure 4-15 - Joint Development and Coordination of Emissions Control Data and Models (CLEERS Analysis and Coordination): Stuart Daw (Oak Ridge National Laboratory) Advanced Combustion Engines 
CLEERS workshop/meeting should definitely be continued, with perhaps a move to the late summer or fall so it does not occur right after SAE, especially if the DEER conferences are not going to continue. The reviewer said the monthly audios also should be continued as well as the annual priorities survey, as these provide guidance on what technologies need to be developed.

\section{Question 2: Technical accomplishments and progress toward overall project and DOE goals-the degree to which progress has been made, measured against performance indicators and demonstrated progress towards DOE goals.}

\section{Reviewer 1:}

The reviewer said that the measurement of steady-state isotherms and their analysis for the $\mathrm{NH}_{3}$ adsorption on SCR catalysts and shows the necessity for two different sites to explain the $\mathrm{NH}_{3}$ inventory results. The reviewer said this excellent work is very useful in modeling along with showing some impact of water and aging on one or both of the sites.

\section{Reviewer 2:}

The reviewer commented that the SCR $\mathrm{NH}_{3}$ inventory and catalyst characterization protocol development are critical activities for ensuring that appropriate catalyst technologies are optimally utilized and advanced to meet current and future low temperature aftertreatment (LTAT) solutions. The reviewer stated that developing models to predict the $\mathrm{NH}_{3}$ storage capacity of SCR catalysts and the storage sites is critical to understanding how best to react $\mathrm{NO}_{\mathrm{x}}$ under lean conditions. The reviewer commented that this type of research effort, which is also supported by CLEERS, is best provided by a national laboratory. However, the reviewer said that shifting focus to include the study of PNAs brings into question the availability to conduct the necessary research given the available resources.

\section{Reviewer 3:}

The reviewer said the project showed nice work on fundamental $\mathrm{NH}_{3}$ adsorption behavior on relevant SCR catalysts. The reviewer said the models are probably more detailed than can be used on a vehicle, but they do shed light on catalyst behavior that could be useful for understanding their operation.

\section{Reviewer 4:}

The reviewer said good work on the $\mathrm{NH}_{3}$ storage modeling. The project needs to start working more intently on PNAs for cold-start $\mathrm{NO}_{\mathrm{x}}$ control, which is the highest rated in the most recent CLEERS survey; HC traps; and low-temperature catalysts, which are needed for the $150^{\circ} \mathrm{C}$ challenge and to provide emission control on the more fuel-efficient engines of the future for both gasoline and diesel.

\section{Question 3: Collaboration and coordination with other institutions.}

\section{Reviewer 1:}

The reviewer noted that there was good collaboration with PNNL on the $\mathrm{NH}_{3}$ storage modeling. The reviewer commented that the researchers did a great job in running the CLEERS conference and the CLEERS audios, which promote pre-competitive collaboration among OEMs, suppliers, universities, and national laboratories. The reviewer added that the CLEERS website is a good place to store the catalyst testing protocols, pre-competitive data, models, and reaction mechanisms so that others can have access to them.

\section{Reviewer 2:}

The reviewer suggested that, with respect to the SCR, more interaction with OEMs and catalyst suppliers in identifying and addressing the emissions and OBD needs of the industry. The reviewer stated that with respect to the protocol development, there is very good interaction and communication with the OEMs to address their needs and those of the catalyst community to provide useful information and technologies.

\section{Reviewer 3:}

The reviewer stated that there was an inherently collaborative set of supporters of this project. The reviewer said that the survey requires a lot of interest in developing a consensus, and the conference calls usually provide an early view of topics of broad general interest. 


\section{Reviewer 4:}

The reviewer remarked that the project included numerous collaborations with industry, national laboratories, and universities. That is how CLEERS operates.

\section{Question 4: Proposed future research-the degree to which the project has effectively planned its future work in a logical manner by incorporating appropriate decision points, considering barriers to the realization of the proposed technology and, when sensible, mitigating risk by providing alternate development pathways.}

\section{Reviewer 1:}

The reviewer said that shifting focus to include PNAs is very appropriate for low-temperature catalyst solutions. The reviewer added that continuing to define aging effects on the performance of SCR catalysts is also of value for the industry.

\section{Reviewer 2:}

The reviewer said that although the future work was not strongly emphasized in the presentation, continuing the work of CLEERS and related efforts is very important.

\section{Reviewer 3:}

The reviewer said there were quick response to CLEERS survey priorities. The reviewer said that focus on lowtemperature catalysis and cold adsorbers for $\mathrm{HC}$ and $\mathrm{NO}_{\mathrm{x}}$ is very relevant going forward. A more detailed look on copper $(\mathrm{Cu})$ and chazabite (CHA) materials with Raj Gounder sounds interesting, but it will not be enough to simply identify $\mathrm{NH}_{3}$ storage sites. The reviewer said the project also needs to know how the sites change over catalyst aging and poisoning and how to eliminate sites that do not contribute to $\mathrm{NO}_{\mathrm{x}}$ conversion to give faster response. The reviewer added that the large buffer of $\mathrm{NH}_{3}$ storage at low temperatures is undesirable from a controls standpoint.

\section{Reviewer 4:}

The reviewer commented that the project needs to start emphasizing PNAs, HC traps, and low-temperature catalysts. The reviewer added that work can, and should, continue on catalysts for lean applications, but there should be some simultaneous research on stoichiometric catalysts as well. The reviewer stated that due to the challenges of providing 99.5\% $\mathrm{NO}_{\mathrm{x}}$ conversion over the Federal Test Procedure (FTP) cycle as well as other test cycles, stoichiometric engines are probably going to be here for a long time. The reviewer said that, in addition, even lean gasoline engines will run at stoichiometry at high loads, where the exhaust temperatures are too high for lean $\mathrm{NO}_{\mathrm{x}}$ catalysts to provide the high $\mathrm{NO}_{\mathrm{x}}$ conversions necessary to satisfy stringent emission standards.

\section{Question 5: Does this project support the overall DOE objectives of petroleum displacement? Why or why not?}

\section{Reviewer 1:}

The reviewer said the project directly addresses the need of the automotive OEMs to meet future emissions standards by utilizing lean and LTAT technologies in a timely manner.

\section{Reviewer 2:}

The reviewer said this work allows the use of efficient engines that lead to effective aftertreatment in lowtemperature exhaust.

\section{Reviewer 3:}

The reviewer commented that low-temperature catalysis and cold adsorbers will allow for more fuel-efficient powertrains.

\section{Reviewer 4:}

The reviewer remarked that the good experimental data being generated and the resulting models will help engineers design efficient and cost-effective emission control systems for the more fuel-efficient engines of the future. The reviewer said that this will reduce national fuel consumption as well as satisfy the clean air requirements. 


\section{Question 6: tResources: How sufficient are the resources for the project to achieve the} stated milestones in a timely fashion?

\section{Reviewer 1:}

The reviewer is slightly cautious with regard to resources. The reviewer said that given the funding levels and the increasing breadth of apparent projects, resources may be somewhat strained.

\section{Reviewer 2:}

The reviewer commented that resources may not be sufficient to continue the $\mathrm{NH}_{3}$ storage modeling work on several new SCR catalysts while adding in a lot of new work on PNAs, HC traps, and low-temperature catalysts.

Reviewer 3:

The reviewer said that resources seem sufficient to support the current level of effort. 
CLEERS: Aftertreatment Modeling and Analysis: Yong Wang (Pacific Northwest National Laboratory) - ace023

\section{Presenter}

Yong Wang, Pacific Northwest

National Laboratory

\section{Reviewer Sample Size}

A total of four reviewers evaluated this project.

\section{Question 1: Approach to performing the work-the degree to which technical barriers are addressed, the project is well-designed, feasible, and integrated with other efforts.}

\section{Reviewer 1:}

The reviewer remarked that there is a wide range of active projects that appear to address very different areas of catalysis and catalyst development. The reviewer said this leads to the concern that there is not an overriding focus of the research to address a central topic, but rather unrelated areas of catalyst development. The reviewer remarked that, having said that, the quality of work and the researchers is high and the information is useful to the industry.

\section{Reviewer 2:}

The reviewer commented that the projects dovetail very nicely with CLEERS modeling efforts and that the coordination is very effective. The reviewer said that the industrial input is key to working on changing needs. However, the reviewer was critical of the large amount of work done on older problems. The reviewer said that, for example, contemporary direction is on $\mathrm{Cu}$ zeolites, not iron (Fe) zeolites, and on gasoline particulate filter (GPF) and SCR filters, not diesel particulate filters (DPF). However, the shift to adsorbers is ahead of the game. The reviewer remarked that, having stated this, the use of modern tools and analyses is exceptional. Identifying the active Fe sites and using this cutting edge information to explain and improve performance is very impressive. The reviewer added that the approaches to developing test protocols for adsorbers and catalysts was also well thought-out and coordinated. Differences appear to be as high as $30^{\circ} \mathrm{C}$, which can be significant.

\section{Reviewer 3:}

The reviewer said that the PNNL has many nice instruments to analyze and characterize catalyst materials and behavior. The knowledge of how to make active $\mathrm{Cu}$ and $\mathrm{Fe} / \mathrm{CHA}$ is extremely useful. The reviewer stated that there seems to be a disparate number of technologies included in the program and that there is not much modeling 
included, although the project is associated with CLEERS. The reviewer could not give more than a good rating due to the mismatch between the content and the title.

\section{Reviewer 4:}

The reviewer commented that the project was excellent and there were copious amounts of experimental data. However, the reviewer did not see much modeling and said that the last letter in CLEERS stands for Simulations. The reviewer said that other than helping ORNL model the $\mathrm{NH}_{3}$ storage capacity of the SCR catalyst, there is not a lot of modeling here. This project seems to be several different catalyst projects-PNA, DPF, SCR, and lowtemperature catalysis - under one umbrella project, with an emphasis on gathering experimental data rather than modeling or simulations. The reviewer asked if the presenter planned to start modeling the data generated at some point.

\section{Question 2: Technical accomplishments and progress toward overall project and DOE goals-the degree to which progress has been made, measured against performance indicators and demonstrated progress towards DOE goals.}

\section{Reviewer 1:}

The reviewer said that the results are impressive and contributing to the advancement of the field; the Cu-SSZ13 contribution is a good example. However, the results on Fe zeolites, although thorough and new, are perhaps of little interest. The reviewer said that emphasis today is on $\mathrm{Cu}$ zeolites and that perhaps the learnings on structure versus performance can be transferred into more meaningful systems. The reviewer added that the DPF results are a little confusing. Wafers were used with one surface being as-received and the other surface being machined, changing the surface porosity. The reviewer asked if the wafer was representative of actual DPF walls. The reviewer expressed that perhaps the presenter needs to run the wafer experiments with two machined surfaces rather than one. Finally, the reviewer said that the PNA work is impressive, but that not much new from what was reported by Ford in August 2014. Nonetheless, the methods and results seem transferrable to other materials.

\section{Reviewer 2:}

The reviewer said that the SCR characterization and optimization activities are well regarded and appropriate for providing LTAT solutions for the automotive industry. The reviewer commented that this activity coupled with the engagement of PNNL with catalyst protocol development to advance useful catalyst technologies is critical to meeting future exhaust emissions standards. The reviewer remarked that this work should continue; however, when developing catalyst technologies such as SCR, the presenter should address aging conditions and give consideration to poisoning and deactivation mechanisms at an earlier stage.

\section{Reviewer 3:}

The reviewer said that there was a good understanding of the deactivation of the Cu/SSZ13 SCR catalyst. Also, the reviewer said good job on identifying Fe monomers as primarily responsible for the low-temperature $\mathrm{NO}_{\mathrm{x}}$ conversion of $\mathrm{Fe} / \mathrm{SSZ}$ catalysts, while Fe dimers are primarily responsible for the $\mathrm{NO}_{\mathrm{x}}$ conversion at high temperatures. The reviewer commented that the presentation demonstrated great efforts on the oxidation protocol and adsorber protocol development. The reviewer said kudos to Ken Rappe and also said good work on the DPF investigations. The reviewer commented that the researchers need to accelerate efforts on PNA development, which ended up as the highest priority item on the annual CLEERS survey, with an emphasis on the sulfur (S) tolerance and thermal durability of the PNA. The reviewer stated that the response to last year's comment that more emphasis needs to be placed on the $\mathrm{S}$ tolerance of catalysts was not very satisfying; in other words, this work is occurring in a cooperative R\&D agreement (CRADA) with Cummins because data generated in a CRADA are typically only available to the participants of that CRADA and not available to the general community.

\section{Reviewer 4:}

The reviewer could not tell what the transportable emissions measurement system image on Slide 8 was supposed to show. The reviewer commented that there was a nice explanation of Fe/CHA aging results. There is not much chance to use a three-site $\mathrm{NH}_{3}$ storage model on a vehicle because it is too complicated. The reviewer added that it may be useful to explain catalyst behavior in the lab. The reviewer commented that there was nice insight into other devices (i.e., Ce PNA, SCR-coated DPF [SDPF]). 


\section{Question 3: Collaboration and coordination with other institutions.}

\section{Reviewer 1:}

The reviewer said that the projects related to protocol development and the work to characterize and optimize catalyst solutions for lean applications is well supported with interaction with either OEMs and/or catalyst suppliers.

\section{Reviewer 2:}

The reviewer remarked that it is good to validate reactors through ACEC protocols with the round robin catalyst. The reviewer noted several collaborations with ORNL, universities, and industry.

\section{Reviewer 3:}

The reviewer said that there was good collaboration with ORNL on the $\mathrm{NH}_{3}$ storage modeling and with the ACEC Technical Team of U.S. DRIVE.

\section{Reviewer 4:}

The reviewer said the researcher has a broad team with much depth and that perhaps it is okay to have a few partners doing the work with others to provide guidance. The reviewer said that the exception is the round robin work on LTAT protocol. The reviewer added that the researcher ought to consider more collaboration with zeolite and/or catalyst companies, at least from an advisory point of view.

Question 4: Proposed future research-the degree to which the project has effectively planned its future work in a logical manner by incorporating appropriate decision points, considering barriers to the realization of the proposed technology and, when sensible, mitigating risk by providing alternate development pathways.

\section{Reviewer 1:}

The reviewer said that inclusion of PNA development into the research portfolio is very appropriate as is the continuing development of protocols for down-selecting advanced catalyst technologies for LTAT. The reviewer said, however, that the resources available to support the wide range of projects does not appear to match funding and manpower.

\section{Reviewer 2:}

The reviewer was very comfortable with future direction. The reviewer said that using the zeolite structure data for SCR improvement is clearly the next step. The reviewer was very optimistic the team will make significant progress and said that no changes are recommended in this portion of the program.

The reviewer said that on LTAT, certainly methane $\left(\mathrm{CH}_{4}\right)$ oxidation is important for natural gas engines. However, the reviewer did not see much market penetration of natural gas (NG) vehicles (greater than $10 \%$ ), even if oil prices increase. The reviewer said that, further, recent work reported satisfactory $\mathrm{CH}_{4}$ light-off of around $240^{\circ}$ Celsius $(\mathrm{C})$ (A.I. Osman et al./Applied Catalysis B: Environmental 187 (2016) 408-418). The reviewer suggested focusing on HCs from LTC of diesel and/or gasoline-based systems.

The reviewer suggested that the presenter proceed with SCR filter work. The reviewer said that the tools are phenomenal and recommended looking at different SCR coatings such as membrane on inlet and outlet walls or in-wall coatings. Also, the reviewer suggested that the presenter should consider looking at GPF.

\section{Reviewer 3:}

The reviewer said that a move away from cerium (Ce) and towards zeolitic low-temperature nitric oxide (NO) traps is a good one. The reviewer would like to see more modeling in the program per the title. The reviewer commented that other topics of high interest would be nitrous oxide $\left(\mathrm{N}_{2} \mathrm{O}\right)$ minimization and formaldehyde trapping and oxidation. The reviewer added that PNNL would seem well-suited to tackle those topics.

\section{Reviewer 4:}

The reviewer said that the presenter needed to emphasize low-temperature catalysts for both diesel (lean) and gasoline (stoichiometric) applications. The reviewer commented that the focus should be on the low-temperature reduction of $\mathrm{NO}_{\mathrm{x}}$ in addition to the oxidation of $\mathrm{CH}_{4}$ and other hydrocarbons because the future emission standards 
will require very high conversions of both species. The reviewer said that, again, the assessment of S tolerance and desulfation capability needs to be investigated for any catalyst that is developed.

\section{Question 5: Does this project support the overall DOE objectives of petroleum displacement? Why or why not?}

\section{Reviewer 1:}

The reviewer said that all catalyst projects support the current and future catalyst knowledge base desired by the automotive OEMs.

\section{Reviewer 2:}

The reviewer said that knowledge of lean, LTAT devices promotes more widespread use of fuel-efficient powertrains and development of new, improved fuel-efficient powertrains.

\section{Reviewer 3:}

The reviewer stated that all of the technologies being developed will be needed to allow more fuel-efficient engines to satisfy stringent emission standards.

\section{Question 6: Resources: How sufficient are the resources for the project to achieve the stated milestones in a timely fashion?}

\section{Reviewer 1:}

The reviewer said that there appears to be a wide range of different projects underway. The reviewer added that known funding restraints and the scope do not seem to align as well as they should.

\section{Reviewer 2:}

The reviewer commented that it seems like a lot of money to cover the approaches. The reviewer added that most money seems to be spent on SCR activity and PNA seems to be just starting. The reviewer added that lowtemperature oxidation was limited to round robin as presented and that SDPF work was limited in scope.

\section{Reviewer 3:}

The reviewer stated that resources seem sufficient for the current level of effort. 


\section{Ash-Durable Catalyzed Filters for Gasoline Direct Injection (GDI) Engines: Hee Je Seong (Argonne National Laboratory) - ace024}

\section{Presenter}

Hee Je Seong, Argonne National Laboratory

\section{Reviewer Sample Size}

A total of five reviewers evaluated this project.

\section{Question 1: Approach to performing the work-the degree to which technical barriers are addressed, the project is well-designed, feasible, and integrated with other efforts.}

\section{Reviewer 1:}

The reviewer said that the approach for studying a three-way catalyst (TWC) plus GPF systems appears well-designed.

\section{Reviewer 2:}

The reviewer stated that there was a clear goal description as well as approach.

\section{Reviewer 3:}

The reviewer said that this activity supports the need for research in the area of filter technology to meet future emissions and $\mathrm{PM} /$ particle number $(\mathrm{PN})$ requirements globally. The reviewer commented that focusing research on the combination of the TWC plus GPFs is also very appropriate from an OEM's point of view for performance and packaging. The reviewer added that understanding the mechanisms of TWC plus GPF catalysts is important R\&D for OEMs trying to adapt to future PM standards and diagnosing catalyst functionality and health.

\section{Reviewer 4:}

The reviewer commented that the described approach to investigating ash-durable catalyzed filters is focused on integral studies using a bench-scale flow reactor. Use of the Advanced Photon Source (APS) and other substrate characterization techniques really only tells you about the physical effects on the substrate or blocking of channels due to ash. The reviewer said that you might be able to see where the ash is, but you are not measuring chemical changes in the washcoat or even the substrate. The reviewer added that the focus on engine scale or bench scale devices complicates the understanding of the effective phenomena seen. The reviewer noted the incredible integrity of the presenter, Dr. Song, who was very honest about how his laboratory results differed from results seen previously in the literature (Slide 15). The reviewer said that Dr. Song may not yet be able to explain why he sees differences, but he is continuing to investigate.

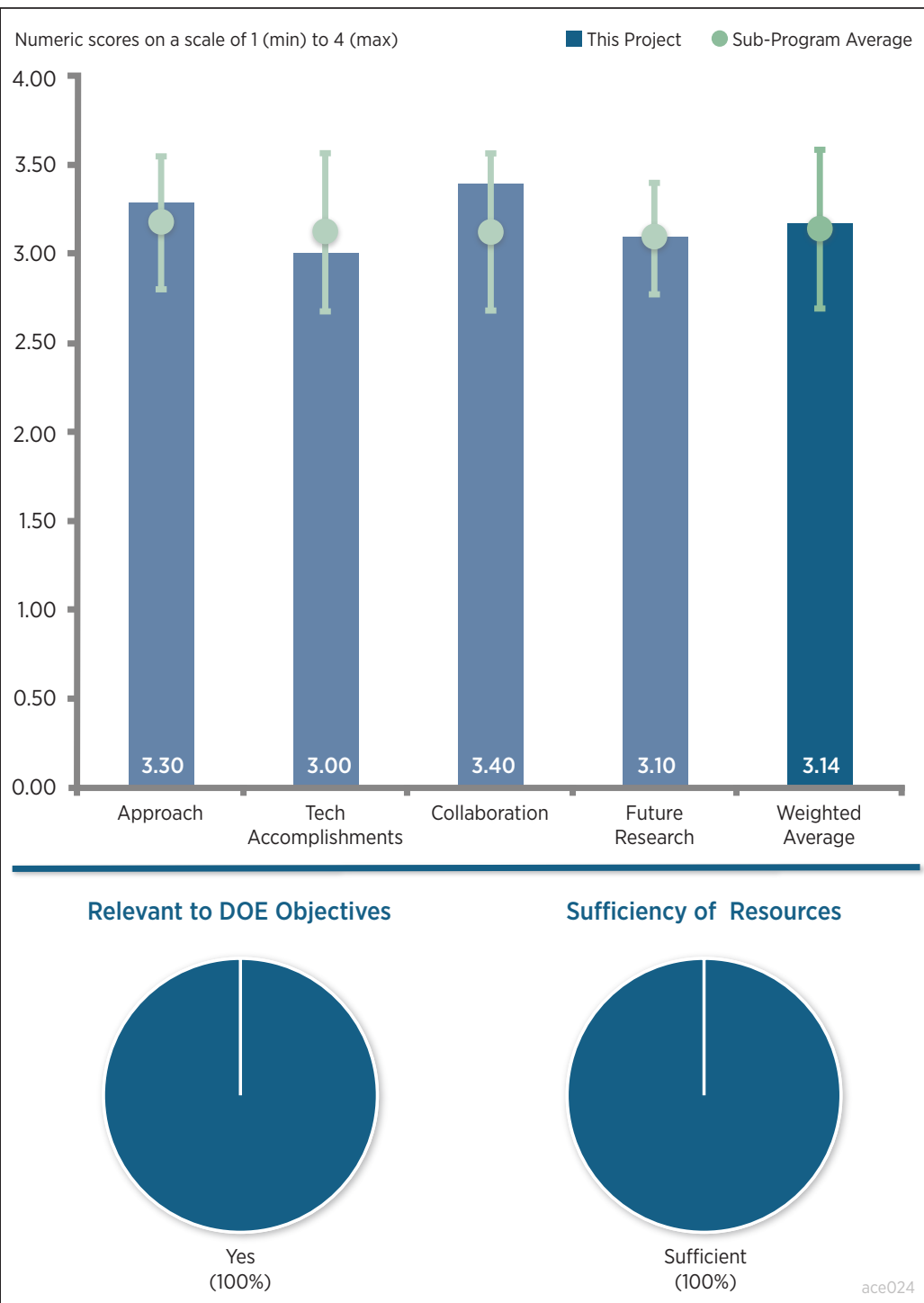

Figure 4-17 - Ash-Durable Catalyzed Filters for Gasoline Direct Injection (GDI) Engines: Hee Je Seong (Argonne National Laboratory) - Advanced Combustion Engines 


\section{Reviewer 5:}

The reviewer said that the presenter needs to emphasize field-aged GPFs. The reviewer suspected that the ash developed from injecting oil into the fuel and rapidly aging the GPF is not the same as normal ash from a gasoline engine driven over tens of thousands of miles. The reviewer said that researchers are exposing the GPF to much less fuel and therefore much less $\mathrm{S}$ with this procedure, which might account for the lack of calcium sulfate $\left(\mathrm{CaSO}_{4}\right)$ in the ash. The reviewer said that some of the test conditions were not apparent in the presentation, like how many miles the field-aged GPF had been driven and under what conditions like time, temperature, and flow rates. Also, the conditions used during the rapid aging were not apparent, such as the exhaust temperature, flow rate, and the duration of aging. The reviewer did not know why the presenter did not observe ash deposits along the walls with the laboratory-aged filters, when published literature indicates that there is ash along the wall with field-aged GPFs. The reviewer said it is not primarily in the end plugs as the presenter suggests.

\section{Question 2: Technical accomplishments and progress toward overall project and DOE goals-the degree to which progress has been made, measured against performance indicators and demonstrated progress towards DOE goals.}

\section{Reviewer 1:}

The reviewer said that the project shows interesting results, especially on ash, although it seems there could be more data considering the effort level.

\section{Reviewer 2:}

The reviewer observed that there was a systematic approach and very thorough analysis. The reviewer added that the presenter needs to correlate more to real-life conditions and filter exposure as well as different oil type exposure.

\section{Reviewer 3:}

The reviewer said that determining the effects of ash and ash components on the performance of TWC and GPF catalysts is very important to understanding how to diagnose the state of the catalyst as well as what to expect from an activity point of view. The reviewer said that establishing where the ash components are located within the GPF helps predict how the catalyst will perform. Similarly, understanding the impact of the calcium $(\mathrm{Ca})$ and phosphorous $(\mathrm{P})$ on reactions is also critical for predicting the performance for the required 150,000-mile life of the aftertreatment system. The reviewer said, however, that correlation with field-aged catalysts is needed to better predict aging mechanisms and performance of in-use catalysts. The reviewer said that artificial means of loading ash-loading catalysts may be significantly different. Also, identifying where the ash components reside in the GPF and why that distribution occurs would be important information for OEMs. The reviewer said, finally, understanding how washcoat load effects light-off must be understood better. The reviewer added that why higher washcoat load increases the light-off temperature requires more understanding.

\section{Reviewer 4:}

The reviewer said that technical achievements are appropriate to a new project. This project only started in October 2015 so results to date are mostly preliminary, which may explain the deviation from what is shown in the literature. The reviewer said that it would be nice to see experiments done to isolate the independent phenomena instead of convoluting the mass transport and kinetic limitations.

\section{Reviewer 5:}

The reviewer expressed a desire to understand why the results are not consistent with published literature in regards to ash layer deposition and the presence of $\mathrm{CaSO}_{4}$ in the ash. The reviewer said that if the presenter's comments are based on the rapid aging results, the presenter might need to rethink the procedure. The reviewer said that if the presenters' comments are based on field-aged GPFs, the presenter needs to reveal how many miles the vehicles were driven and the conditions during the mileage accumulation. The reviewer said that it was not apparent on Slide 17 what the designations LL, LH, HL, and HH mean. Also, the test conditions are missing, such as temperature and oxygen $\left(\mathrm{O}_{2}\right)$ level.

\section{Question 3: Collaboration and coordination with other institutions.}




\section{Reviewer 1:}

The reviewer observed contributions from academia and industry.

\section{Reviewer 2:}

The reviewer said that the inclusion of an automotive OEM that has direct injection (DI) engines in the marketplace along with substrates and catalyst suppliers and facilities that provide analytical characterization services enhances the value of the information and work.

\section{Reviewer 3:}

The reviewer noted that there was good collaboration with Hyundai and Corning.

\section{Reviewer 4:}

The reviewer commented that there was an excellent set of collaborators; however, where they are having impact is not conveyed for all of them.

\section{Reviewer 5:}

The reviewer said that additional laboratory or university partners for chemical characterization studies would be useful. The reviewer added that more focus on the fundamentals would help the overall project.

Question 4: Proposed future research-the degree to which the project has effectively planned its future work in a logical manner by incorporating appropriate decision points, considering barriers to the realization of the proposed technology and, when sensible, mitigating risk by providing alternate development pathways.

\section{Reviewer 1:}

The reviewer said that further establishing the effects of oil derived poisons on the performance of the washcoat and filter is important. However, the presenter must give consideration to the interaction with the upstream catalyst that will also be present in these aftertreatment systems.

\section{Reviewer 2:}

The reviewer said that there are many choices to consider for focused efforts.

\section{Reviewer 3:}

The reviewer suggested that the presenter consider real-life conditions for field performance of filters and oil samples as would be seen in the real world.

\section{Reviewer 4:}

The reviewer said that proposed future work is appropriate and that there was strong interest in the kinetic studies and ash chemistry.

\section{Reviewer 5:}

The reviewer said that the presenter needed to emphasize field-aged filters instead of laboratory-aged filters.

\section{Question 5: Does this project support the overall DOE objectives of petroleum displacement? Why or why not?}

\section{Reviewer 1:}

The reviewer said that global emissions requirements will include PM/PN management to meet standards. The combination of catalyst technologies to achieve these standards while minimizing the effect on engine performance and packaging is critical to designing acceptable aftertreatment solutions.

\section{Reviewer 2:}

The reviewer said it was key for proper engine performance.

\section{Reviewer 3:}

The reviewer suggested that the presenter could enable the use of more lean gasoline engine systems, meeting DOE's goals. 
Reviewer 4:

The reviewer said that this is definitely relevant to DOE interests. The reviewer added that ash can be a very strong, negative influence on the life of aftertreatment catalysts.

Reviewer 5:

The reviewer said the GPFs will be necessary to allow more fuel-efficient gasoline turbocharged DI engines to meet future particulate standards.

Questions 6: Resources: How sufficient are the resources for the project to achieve the stated milestones in a timely fashion?

Reviewer 1:

The reviewer said this project is appropriately funded and staffed.

Reviewer 2:

The reviewer said the resources seem sufficient at the time. 


\section{Enhanced High- and Low- Temperature Performance of $\mathrm{NO}_{x}$ Reduction Materials: Feng Gao (Pacific Northwest National Laboratory) - ace026}

\section{Presenter}

Feng Gao, Pacific Northwest National Laboratory

\section{Reviewer Sample Size}

A total of four reviewers evaluated this project.

\section{Question 1: Approach to performing the work-the degree to which technical barriers are addressed, the project is well-designed, feasible, and integrated with other efforts.}

\section{Reviewer 1:}

The reviewer said the focus of this work to improve both the low- and high-temperature performance of SCR catalysts is very consistent with the needs of the automotive OEMs. The reviewer added that both ends of the operating window must be addressed to have a viable solution for $\mathrm{NO}_{\mathrm{x}}$ control in lean systems. The reviewer commented that using fully formulated catalysts that incorporate alkali earth co-cations into the formulation to achieve better performance on the low-temperature operating end of the window is somewhat novel and unexpected due to the poisoning nature of sodium $(\mathrm{Na})$ in vehicle applications. The reviewer said that explaining why this is the case would also be of interest.

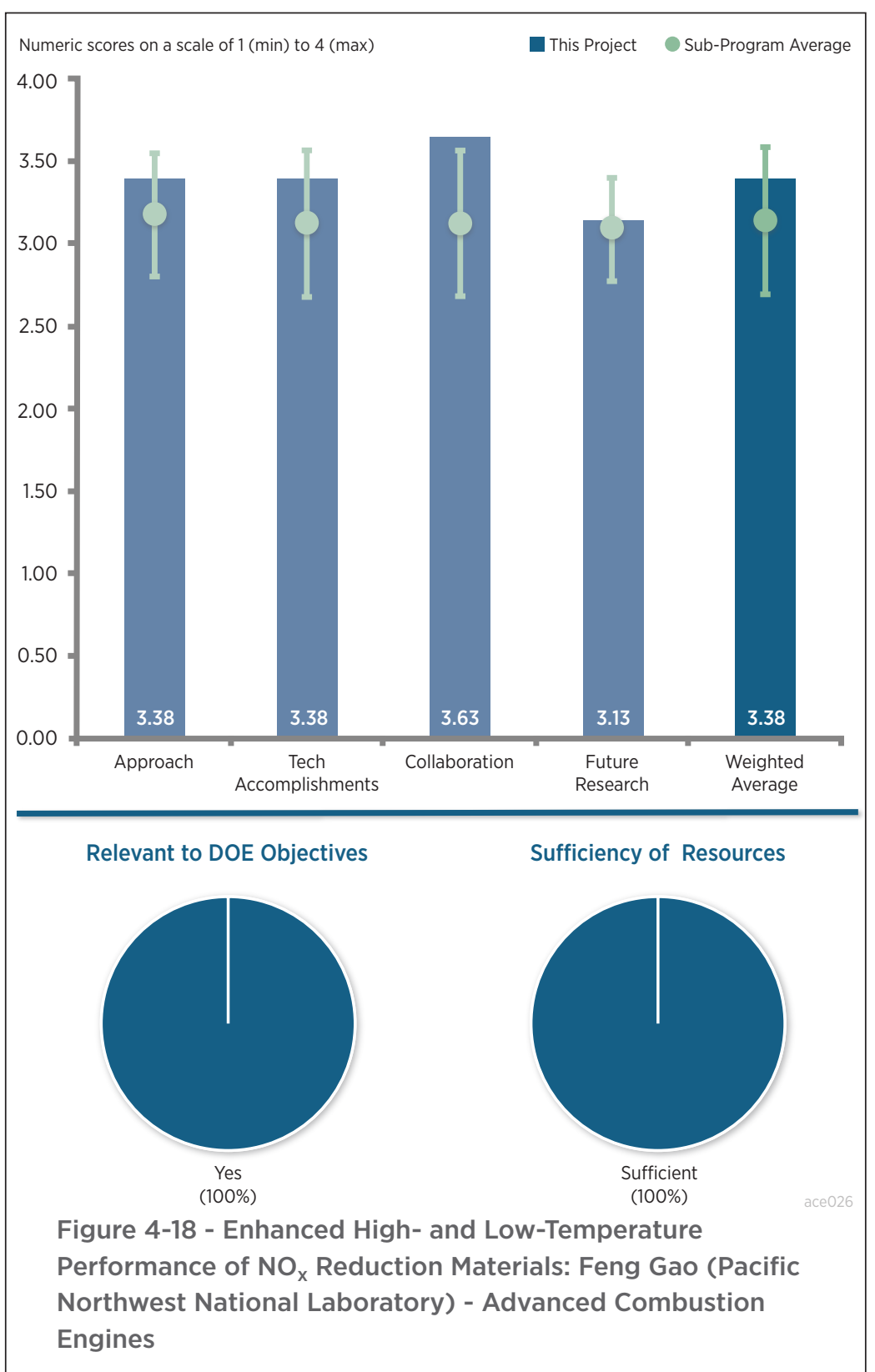

\section{Reviewer 2:}

The reviewer commented that characterizing real-world materials in various aging states using the most advanced techniques is delivering exceptional results. Getting guidance on direction from an OEM keeps the direction pertinent. The reviewer added that the method of isolating $\mathrm{Cu}$ sites to determine which are significant is impressive. The reviewer commented that emphasis on low-temperature performance is also critical.

\section{Reviewer 3:}

The reviewer said that SCR catalysts are relevant for use in lean, fuel-efficient powertrains. The reviewer remarked that there were model catalysts used to represent proprietary catalysts that could not be discussed in public. The reviewer expressed that there is no interest to use beta zeolite in SCR catalysts and that the HC adsorption is catastrophic. The reviewer said that the presenter did not show any work on adding $\mathrm{Fe}$ to $\mathrm{Cu} / \mathrm{CHA}$. This would have been interesting. 


\section{Reviewer 4:}

The reviewer said the presenter needed to include $\mathrm{S}$ poisoning assessments of the catalysts being developed. The reviewer added that $\mathrm{S}$ can be particularly detrimental to the low-temperature activity of SCR catalysts.

\section{Question 2: Technical accomplishments and progress toward overall project and DOE goals-the degree to which progress has been made, measured against performance indicators and demonstrated progress towards DOE goals.}

\section{Reviewer 1:}

The reviewer commented that the presenters did great work in probing the $\mathrm{Cu}$ sites that give good activity and selectivity at both low and high temperatures. Again, the reviewer said, the project needed to assess $\mathrm{S}$ poisoning.

\section{Reviewer 2:}

The reviewer said that the results on the two different $\mathrm{Cu}$ sites are very impressive and valuable. It is this kind of fundamental understanding that drives catalyst compositional work. The reviewer added that the recommendations on zeolite formulation is valuable and impressive. The reviewer commented that it was interesting work on Fe$\mathrm{Cu}$ beta zeolite and with $\mathrm{Na}$. The reviewer suggested that it would be valuable to see recommendations and then verifying.

\section{Reviewer 3:}

The reviewer said that the technical approach to lower the light-off temperature for $\mathrm{NO}_{\mathrm{x}}$ reduction by using alkali metals is novel. The reviewer commented that a significant amount of characterization work has been done to substantiate the activity of the catalyst and the mechanism that is driving the observed behavior; this is very appropriate work for PNNL to perform. The reviewer commented that, going forward, more attention has to be directed at understanding aging mechanism that include HCs and S poisoning. The reviewer remarked that optimizing catalyst formulations for either standard or fast $\mathrm{NO}_{\mathrm{x}}$ reactions is also appropriate. Because different lean applications will produce different ratios of $\mathrm{NO}$ /nitrogen dioxide $\left(\mathrm{NO}_{2}\right)$, catalyst formulations should be optimized to take advantage of the available species. The reviewer added that with regard to combining $\mathrm{Cu}$ and $\mathrm{Fe}$ catalysts, some of this work has been performed by others and should not be emphasized in this project.

\section{Reviewer 4:}

The reviewer commented that the fundamental attribute study of $\mathrm{Cu} / \mathrm{SSZ} 13$ seems contradictory to patent literature on desirable silicon $(\mathrm{Si}) /$ aluminum $(\mathrm{Al})$ ratio. The reviewer said that the use of $\mathrm{Na}+$ to neutralize Bronsted sites seems novel, and improved, high-temperature $\mathrm{NO}_{\mathrm{x}}$ conversion was an unexpected result. The reviewer said that it would be good if it could be shown that adding $\mathrm{Na}$ also improves the SCR activity response with $\mathrm{NH}_{3}$ load on the catalyst.

\section{Question 3: Collaboration and coordination with other institutions.}

\section{Reviewer 1:}

The reviewer said that the inclusion of Cummins and Johnson Matthey Catalysts (JM) in this work should help achieve results that are meaningful and applicable to lean $\mathrm{NO}_{\mathrm{x}}$ aftertreatment solutions.

\section{Reviewer 2:}

The reviewer commented that the team is very strong with key players. The reviewer added that state-of-the-art equipment with industry samples and guidance made results pertinent and will likely lead to faster implementation.

\section{Reviewer 3:}

The reviewer remarked that there was good collaboration with Cummins and JM.

\section{Reviewer 4:}

The reviewer noted that partners were Cummins and JM.

Question 4: Proposed future research-the degree to which the project has effectively planned its future work in a logical manner by incorporating appropriate decision points, considering barriers to the realization of the proposed technology and, when sensible, mitigating risk by providing alternate development pathways. 


\section{Reviewer 1:}

The reviewer said that future research should be primarily focused on extending the operating range of the SCR formulations to low temperatures and secondarily toward extending the upper temperature limit. The reviewer added that preserving activity after appropriate aging is performed will be critical to determining if the technologies under development will survive in vehicle aftertreatment systems.

\section{Reviewer 2:}

The reviewer said that realizing the project is closed, the reviewer is still craving recommendations for future work for other research groups.

\section{Reviewer 3:}

The reviewer noted that the project was completed. The reviewer said that the presenter mentioned transferring remaining work under CLEERS, but the reviewer said that it was not clear exactly what this means.

\section{Reviewer 4:}

The reviewer noted that the project is completed.

\section{Question 5: Does this project support the overall DOE objectives of petroleum displacement? Why or why not?}

\section{Reviewer 1:}

The reviewer commented that there was very relevant R\&D and it was consistent with the call for LTAT solutions for highly efficient lean combustion engines. The reviewer said that the United States Council for Automotive Research (USCAR) has stated this area of research is needed going forward and would strongly support this work.

\section{Reviewer 2:}

The reviewer said that high lean $\mathrm{NO}_{\mathrm{x}}$ conversion across a wide temperature window is an enabler for more fuelefficient powertrains.

\section{Reviewer 3:}

The reviewer commented that we will need SCR catalysts with better $\mathrm{NO}_{\mathrm{x}}$ conversion at both low temperatures and high temperatures as well as better thermal durability for future emission standards and future fuel economy standards. The reviewer said, of course, that the low-temperature activity will depend on whether $\mathrm{NH}_{3}$ is available at those low temperatures.

\section{Question 6: Resources: How sufficient are the resources for the project to achieve the stated milestones in a timely fashion?}

\section{Reviewer 1:}

The reviewer said there was no issue with resources or funding.

\section{Reviewer 2:}

The reviewer noted that it was a co-funded CRADA.

\section{Reviewer 3:}

The reviewer noted that the project is completed. 


\section{Next Generation SCR-Dosing System Investigation: Abhijeet Karkamkar (Pacific Northwest National Laboratory) - ace027}

\section{Presenter}

Abhijeet Karkamkar, Pacific Northwest National Laboratory

\section{Reviewer Sample Size}

A total of six reviewers evaluated this project.

\section{Question 1: Approach to performing the work-the degree to which technical barriers are addressed, the project is well-designed, feasible, and integrated with other efforts.}

\section{Reviewer 1:}

The reviewer remarked that the approach to most of the work is very good and has even focused on making new materials, such as the double salts. The reviewer said there are still other materials that can provide $\mathrm{NH}_{3}$ upon decomposition, especially some liquids, which have not been included for evaluation based on the literature. The reviewer commented that a number of these have been looked at by the Paul Scherer Institute.

\section{Reviewer 2:}

The reviewer remarked that the investigator seemed to understand the technical gaps well and the real need for the project. The reviewer was curious to know how the SCRs make the engine more efficient. The reviewer said that was listed on the slide, but the reviewer was not clear how that is accomplished. Also, it was not clear how the system would work to drive the $\mathrm{NH}_{3}$ into the SCR. The reviewer said the project description says that it is about a system, but the data seem like the project is more of a study on $\mathrm{NH}_{3}$ storage.

\section{Reviewer 3:}

The reviewer said that the project goal is to develop alternative $\mathrm{NH}_{3}$ carriers for $\mathrm{NH}_{3}$ dosing in low-temperature exhaust. The reviewer observed that several materials were studied for specific properties, including volumetric expansion, $\mathrm{NH}_{3}$ storage capacity, $\mathrm{NH}_{3}$ decomposition temperature, material stability, and safety.

\section{Reviewer 4:}

The reviewer said that the researchers should investigate urea alternatives to determine if they are more appropriate for the low-temperature exhaust conditions that will exist in global driving cycles and with the emergence of everincreasing engine efficiency leaving less energy in the exhaust for catalysis. The reviewer said that this activity 
supports an important function of establishing OEM specifications and targets for urea alternatives. Additionally, the reviewer commented that higher density $\mathrm{NH}_{3}$ storage materials that release $\mathrm{NH}_{3}$ at the appropriate temperature will be needed to obtain the level of $\mathrm{NO}_{\mathrm{x}}$ control required for both $\mathrm{LD}$ and $\mathrm{HD}$ applications while minimizing the need for replacing the $\mathrm{NH}_{3}$ source.

\section{Reviewer 5:}

The reviewer said that the research follows clear direction from USCAR CRADA to focus on material selection and development for vapor $\mathrm{NH}_{3}$ delivery for SCR systems. The reviewer commented that technical barriers identified by the research plan, such as improved $\mathrm{NH}_{3}$ storage and low-temperature delivery, as well as reviewers' concerns from the prior year, namely, mitigating hydrochloric acid $(\mathrm{HCl})$ are being addressed methodically through base material and structural material considerations. The reviewer said that although it was not in the scope of the project, it would improve the score to present any available system-level requirements and status for a vapor $\mathrm{NH}_{3}$ delivery system. The reviewer said the researchers claimed that no injector would be needed to deliver vapor $\mathrm{NH}_{3}$ for SCR; however, precise amounts of $\mathrm{NH}_{3}$ would be required for a proper SCR reaction, and it is not clear how this would be achieved. The reviewer added that additional system considerations include the packaging of solid $\mathrm{NH}_{3}$ delivery systems.

\section{Reviewer 6:}

The reviewer said that it is good that the presenter recognized the big issue with $\mathrm{HCl}$ production and worked to minimize it. The reviewer remarked that a steady dose of 600 parts per million $\mathrm{HCl}$ that was observed with magnesium chloride would not be good for the exhaust system, the paint around the exhaust pipe, or the environment.

\section{Question 2: Technical accomplishments and progress toward overall project and DOE goals - the degree to which progress has been made, measured against performance indicators and demonstrated progress towards DOE goals.}

\section{Reviewer 1:}

The reviewer said there was very good progress towards stated goals with the synthesis and study of eutectics. The reviewer suggested quantifying and minimizing volume expansion and said that the project took an innovative look at micro-encapsulation with porous supports.

\section{Reviewer 2:}

The reviewer expressed that there was a very good review of the materials and the researcher used those studies to determine next steps with new materials. The reviewer would like to see a system-level demonstration of the dosing system and how it works effectively on the vehicle.

\section{Reviewer 3:}

The reviewer said that excellent technical progress has been made in the areas chosen for study. While statements from USCAR members state that the deposits from carbamate make it a poor choice, such deposits can form in any system that has carbon dioxide $\left(\mathrm{CO}_{2}\right)$ and $\mathrm{NH}_{3}$ together in the gas phase. The reviewer said this issue could be explored and taken as a challenge because many patents and some papers have been written to address keeping important areas of a system above $70^{\circ} \mathrm{C}$.

\section{Reviewer 4:}

The reviewer commented that the presenter did a good job on minimizing the $\mathrm{HCl}$ production with the composites and on minimizing the volume expansion. The reviewer added that it was a clever method to evaluate volume expansions with the syringes.

\section{Reviewer 5:}

The reviewer said that technical accomplishments for this year are solid, though the reviewer would have liked to see more progress on the $\mathrm{HCl}$ as this is a potentially very serious issue that needs to be addressed. The reviewer said, additionally, that the impact of $\mathrm{CO}_{2} /$ water $\left(\mathrm{H}_{2} \mathrm{O}\right)$ on material performance seems like a show-stopping issue. The reviewer asked if there are risk mitigation plans for either of these. 


\section{Reviewer 6:}

The reviewer said the exclusion of potentially viable alternatives to urea, such as carbamate, has not been adequately explained. The reviewer said the decomposition products are essentially $\mathrm{CO}_{2}$ and $\mathrm{NH}_{3}$. With respect to project goals, a better definition of target release temperatures and maximum recovery of $\mathrm{NH}_{3}$ is needed. The reviewer said that volumetric increases under $\mathrm{NH}_{3}$ loading is important, but not necessary for the primary metric. The reviewer added that as a national laboratory project, the researcher must present more understanding of the kinetics involved.

\section{Question 3: Collaboration and coordination with other institutions.}

\section{Reviewer 1:}

The reviewer questioned how many meetings with USCAR are occurring because the presenters have increased the communications. The reviewer asked if those meetings have aided in the relevance of the project by getting feedback. The reviewer also asked if there are USCAR participants who are willing to partner on a demonstration. The reviewer said that is a good opportunity to work those details and next steps.

\section{Reviewer 2:}

The reviewer said the inclusion of the USCAR OEM members in this work is essential because the OEMs will use this information to write specifications for dosing systems. However, the use of a consultant chemist in this field, with thorough knowledge of reactions involving these $\mathrm{NH}_{3}$ compounds, would provide good feedback on the metrics used in this work. The reviewer added that frequent group meetings to discuss the project progress keep this project focused.

\section{Reviewer 3:}

The reviewer said that while USCAR is certainly a broadly-based group of relevant collaborators, this group could be expanded to include other OEMs interested in SCR.

\section{Reviewer 4:}

The reviewer said that there was good communications with the USCAR SCR team.

\section{Reviewer 5:}

The reviewer noted that there was good collaboration within tightly defined CRADA. The reviewer added that possible improvement would be interactive dialogue for system-level requirements and possibilities as they relate to this subsystem work.

\section{Reviewer 6:}

The reviewer said that the partnership with USCAR is good, but asked if a university or laboratory that could help with analysis. The reviewer commented that there are material evaluation and characterization studies and mechanistic studies that would help this project considerably, much more so than the volumetric expansion study.

\section{Question 4: Proposed future research-the degree to which the project has effectively planned its future work in a logical manner by incorporating appropriate decision points, considering barriers to the realization of the proposed technology and, when sensible, mitigating risk by providing alternate development pathways.}

\section{Reviewer 1:}

The reviewer said these are good next steps on the project. The reviewer would like to see a system demonstration if it can be accomplished. The reviewer did not understand how the use of SCR makes engines more efficient and said that it would be helpful to quantify how the use of $\mathrm{NH}_{3}$ will help the process directly and improve efficiency.

\section{Reviewer 2:}

The reviewer observed that there was a good focus on eutectics and double salts, but commented that the researchers could throw an even wider net. The reviewer suggested that a look at materials, with a greater focus on how they would actually be used in a system, would also be useful. 


\section{Reviewer 3:}

The reviewer expressed that it was a good follow-on for composites and eutectic double salts to continue to improve potential applicability with additives to improve form retention.

\section{Reviewer 4:}

The reviewer was not clear how to mitigate chloride $(\mathrm{Cl})$ ion effects in the exhaust. The reviewer said that as this work progresses, the researchers should develop more appropriate targets and goals.

\section{Reviewer 5:}

The reviewer noted that there were concerns raised by another reviewer on the stability of the air conditioning (AC) materials if they are exposed to air or the exhaust gas, which would be likely in an on-vehicle system. The reviewer said that future work should include additional analysis on how delivery conditions in a vehicle system would impact the material stability. The reviewer said that this is currently not included in the future plans and that the future work plan is vague.

\section{Reviewer 6:}

The reviewer said the researcher needs to develop materials that will release large amounts of $\mathrm{NH}_{3}$ at temperatures between $150^{\circ} \mathrm{C}$ and $180^{\circ} \mathrm{C}$ in order to show an advantage over the aqueous urea system. The reviewer added that it looks like some of the double salts do not release all of the $\mathrm{NH}_{3}$ until about $300^{\circ} \mathrm{C}$, although some is released at lower temperatures. The reviewer said that the presenter might propose how the $\mathrm{NH}_{3}$ storage materials would be recharged, for example, during oil changes. The reviewer asked how long it would take.

Also, the reviewer asked how the gaseous $\mathrm{NH}_{3}$ that is released from the solid source would be stored. The reviewer questioned if there would be a ballast that could store the $\mathrm{NH}_{3}$ because the presenter cannot count on the rate of $\mathrm{NH}_{3}$ release from the solid source to match the $\mathrm{NO}_{\mathrm{x}}$ flux emitted from the engine. Finally, the reviewer asked how much energy would be needed to heat the solid source in order to release the $\mathrm{NH}_{3}$.

\section{Question 5: Does this project support the overall DOE objectives of petroleum displacement? Why or why not?}

\section{Reviewer 1:}

The reviewer commented that the development of a solid source of $\mathrm{NH}_{3}$ that releases $\mathrm{NH}_{3}$ below $180^{\circ} \mathrm{C}$ will allow the use of SCR catalysts that can reduce $\mathrm{NO}_{\mathrm{x}}$ at lower temperatures. The reviewer said that this allows the minimization of the fuel used to pre-heat the exhaust during the cold start, which will save fuel. The reviewer added that it will also allow effective emission control on more fuel-efficient diesel engines that produce lower exhaust temperatures.

\section{Reviewer 2:}

The reviewer said that the urea alternatives that provide high density $\mathrm{NH}_{3}$ storage are required by OEMs in lean aftertreatment systems to meet more stringent emissions standards and the need for LTAT. The reviewer commented that minimizing the need for reductant refills is important from a customer satisfaction perspective and for packing in vehicles.

\section{Reviewer 3:}

The reviewer suggested enabling lower temperature aftertreatment, especially for $\mathrm{NO}_{\mathrm{x}}$.

\section{Reviewer 4:}

The reviewer commented that low-temperature delivery of $\mathrm{NH}_{3}$ can enable efficient engine operation through a wider ambient and engine operating map by providing an aftertreatment solution that operates more broadly and requires less energy penalty, in other words, heating DPF or heating exhaust for DPF to be functional. The reviewer said that solid delivery methods of $\mathrm{NH}_{3}$, furthermore, offer potential for longer service intervals and simplify transportation.

\section{Reviewer 5:}

The reviewer did not understand how the use of SCR makes engines more efficient. The reviewer said that it would be helpful to quantify how the use of $\mathrm{NH}_{3}$ will help the process directly and improve efficiency. 


\section{Reviewer 6:}

The reviewer remarked that the need for new materials is significant, but this project seems to be moving further from its true mission and into materials that are unlikely to be relevant for DOE petroleum displacement objectives.

\section{Question 6: Resources: How sufficient are the resources for the project to achieve the stated milestones in a timely fashion?}

\section{Reviewer 1:}

The reviewer stated that the resources were sufficient for component-level effort. The reviewer added that if USCAR sees a value proposition for vapor-based $\mathrm{NH}_{3}$ delivery, additional system-level considerations should be included, and this would require additional resources.

\section{Reviewer 2:}

The reviewer commented that it would be good to have or see a system demonstration with this equipment. The reviewer did not see this as part of the project, but it would be good to add this on the project for some kind of either engine demonstration or vehicle demonstration to show how it will work.

\section{Reviewer 3:}

The reviewer said that there was no issue with funding or staffing.

Reviewer 4:

The reviewer said that the resources seem sufficient. 


\section{Cummins-ORNL \FEERC Emissions CRADA: $\mathrm{NO}_{\mathrm{x}}$ Control and Measurement Technology for Heavy- Duty Diesel Engines, Self- Diagnosing Smart Catalyst Systems: Bill Partridge (Oak Ridge National Laboratory) - ace032}

\section{Presenter}

Bill Partridge, Oak Ridge National Laboratory

\section{Reviewer Sample Size}

A total of five reviewers evaluated this project.

\section{Question 1: Approach to performing the work-the degree to which technical barriers are addressed, the project is well-designed, feasible, and integrated with other efforts.}

\section{Reviewer 1:}

The reviewer found the work to be an excellent approach that utilizes a fundamental understanding of $\mathrm{NH}_{3}$ adsorption, analyses using spatially resolved capillary inlet, testing methods, and acquisition and analyses of field-aged samples on an applesto-apples basis. The approach has a high probability of achieving desired results. The only improvement may be in expanding into more contemporary zeolite systems, just to check if results are transferable. Non-transferability could be a major risk to the program.

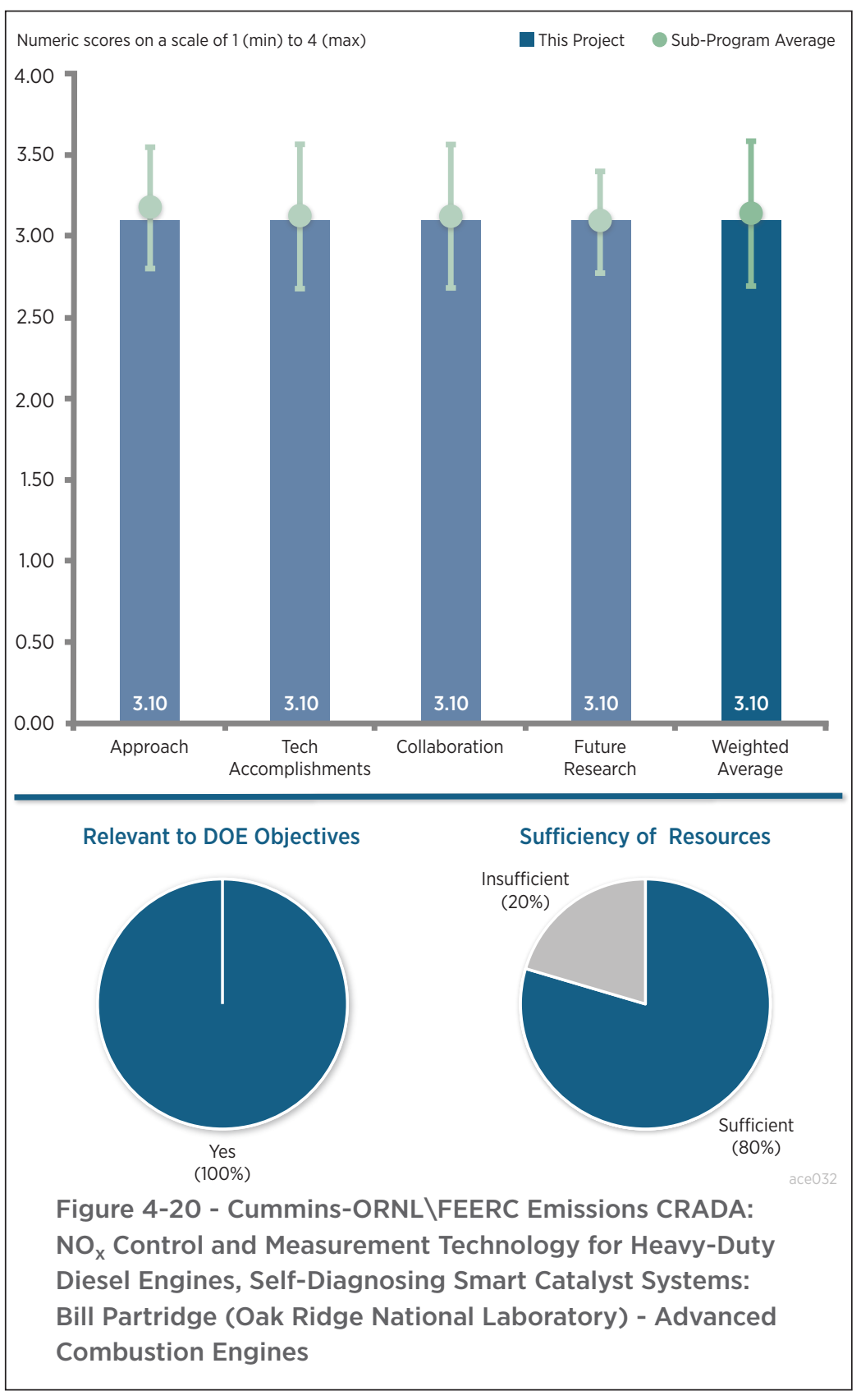

\section{Reviewer 2:}

The reviewer thought the project had a very good approach, especially to use field-aged samples to help develop and verify the model. However, the reviewer found the units and type of field-aging process needed to be explained. The reviewer wanted to know if the process was expressed in miles, cycle, or fuel; whether everything was prepared the same way; and whether the two samples were repeated or intended to be different to provide some range to the model. The reviewer also questioned whether the use of actual field fuel and good quality fuel were part of the model and how much S was in the fuel.

\section{Reviewer 3:}

The reviewer found the project to be tightly focused, and it was targeting key challenges in a stepwise manner. 


\section{Reviewer 4:}

The reviewer commented that the ultimate goal needs to be clearly defined. The reviewer also wanted the objectives of the project to be correlated with the title of the CRADA.

\section{Reviewer 5:}

The reviewer noted that general VTO barriers (cost-effective emission control, modeling for emission control, emission control durability) translated well into specific project objectives, which follow: enable and improve predictive catalyst-performance models, based on controlling physics and chemistry and independent of specific application platform (truck, bus, boat, power); characterize spatiotemporally distributed catalyst performance; investigate aging impacts (performance at different catalyst states); validate and improve models, and mine data and insights for OBD and control methodologies; and develop methods for real-time catalyst-state assessment.

The reviewer commented that the implemented approach could be improved by considering a larger sample of field-aged catalysts for characterization and direct mapping of field-aged samples to physical phenomena (temperature aging, flow-based aging, reaction-based [i.e., oxidation] aging, and poisoning). By correlating/ modeling catalyst aging and performance assessment techniques with a very limited set of field- aged samples, which were reported to have been subjected to a typical but uncharacterized duty cycle, the reviewer noted that current research and subsequent models have a high risk of being application/platform dependent and of less general usefulness.

The reviewer proposed that an improved approach would employ a multi-application, field characterization study using advanced spatiotemporal characterization and assessments to properly characterize failure mechanisms across applications with known field-aging schedules to insure that the catalyst-capacity measure is the appropriate model.

\section{Question 2: Technical accomplishments and progress toward overall project and DOE goals-the degree to which progress has been made, measured against performance indicators and demonstrated progress towards DOE goals.}

\section{Reviewer 1:}

The reviewer commented that this project seemed to provide some significant technical accomplishments with the catalyst modeling. The reviewer said that it will be interesting to actually perform a trial of the model versus the actual performance to determine the model performance and find out if the trial is truly self-diagnosing and able to predict within some $\mathrm{X} \%$ of efficiency.

\section{Reviewer 2:}

The reviewer found good progress in extending the confidence in the measurements, modeling, and real-world behavior through additional catalyst measurements. The reviewer also stated that the interpretation of the operando data to confirm how the sites are degrading in real catalysts is very useful in understanding bench- versus field-aging.

\section{Reviewer 3:}

The reviewer found the evaluation led to some very promising diagnosis methods. The use of unused $\mathrm{NH}_{3}$ capacity (UC) is novel, and the correlation of pulsed response to UC is valuable. Models are not perfect, and more work is needed to improve them, but they are impressive. However, the reviewer questioned why the first field-aged sample (FA-1) is different from the FA-2 (e.g., poisoned, thermal, etc.). Deterioration can be caused by many things, and the reviewer advised knowing what effect the project team is modeling.

\section{Reviewer 4:}

The reviewer noted that the work was a good systematic approach using field-aged catalysts, but in order to have better correlation, the reviewer needed to understand what those catalysts were exposed to.

\section{Reviewer 5:}

The reviewer proposed that a reasonable laboratory approach to assess capacity, once additional field samples are confirmed, may offer possibilities for diagnostics if control steps can be effectively integrated into the SCR control system as active control and not intrusive control. The reviewer stated that the claim that the project has 
"demonstrated a practical method for catalyst state assessment" is premature without relating the aging impacts/ echanism to the state assessment, considering a larger sample of catalyst applications, and understanding the mechanisms involved.

In 2015 the reviewers indicated that more thorough characterization methods for field-aged parts were necessary, and this continues to be an unaddressed issue in 2016. The budget may be constraining the performance of catalyst field aging along with data acquisition; however, the reviewer stated that a larger sample of field-aged samples could potentially be correlated to rapid aging test (RAT) results and the controlled RAT tests used to characterize the physics.

\section{Question 3: Collaboration and coordination with other institutions.}

\section{Reviewer 1:}

The reviewer praised the collaboration with Cummins as clearly excellent. Even though this is a CRADA, the reviewer would like to see more direct paths to distributing the results to other industry members beyond the coordination with the CLEERS organization. The reviewer wondered if this could evolve into a memorandum of understanding.

\section{Reviewer 2:}

The reviewer stated that the collaboration seemed sufficient.

\section{Reviewer 3:}

The reviewer noted a good variety of collaborators in industry and academia and suggested that it may make sense to include a catalyst supplier for additional input into the project.

\section{Reviewer 4:}

The reviewer stated that the close collaboration with the CRADA is obvious and obviously working well. The reviewer noted, however, that it is not clear how the others have helped.

\section{Reviewer 5:}

The reviewer found that the CRADA somewhat constrains participants and limits the sharing of detailed results.

Question 4: Proposed future research-the degree to which the project has effectively planned its future work in a logical manner by incorporating appropriate decision points, considering barriers to the realization of the technology and, when sensible, mitigating risk by providing alternate development pathways.

\section{Reviewer 1:}

The reviewer stated that future plans are well-focused on practical OBD-type developments. On the other hand, the reviewer would like to see some additional work/focus on how to integrate the work with sensor developments-it is mentioned, but could be more definitive.

\section{Reviewer 2:}

The reviewer commented that future work seems logical and noted that it will be interesting to actually perform a trial of the model versus the actual performance to determine the model performance. The reviewer asked if the model is truly self-diagnosing and predicting within some $\mathrm{X} \%$ of efficiency. Also, the reviewer wondered about any impact of fuel and fuel impurities on SCR performance that would need to be incorporated into the aging conditions, or whether that would be an issue for SCRs.

\section{Reviewer 3:}

The reviewer would like the project to focus on transient behavior and OBD work for real-life comparison/ applicability.

\section{Reviewer 4:}

The reviewer noticed one gap in the future approaches that relates to determining the cause of deterioration. The reviewer believed that the model might only be related to thermal or poisoning deterioration and suggested that the project determine the nature of the deterioration being modeled. The reviewer found another gap dealing with 
transferring these models to other zeolite systems and suggested that the model only need to get a peak in the box. The reviewer commented that the project team would not want to be here in two years with a working model that is only pertinent to a catalyst that is two generations old.

\section{Reviewer 5:}

The reviewer commented that future work was generally described but could provide more detail. The reviewer cautioned not to expect a robust solution for a catalyst aging model, which has been developed and fit by data from only two field-aged catalysts with little known about aging history.

The reviewer suggested that the researchers' approach in the future work plan, "Evaluation of alternate aging conditions, sample locations and catalyst types," could provide a bit more detail on the next priority for aging conditions and catalyst types. An improved approach would be to confirm the catalyst state assessment model across a range of field-aged and RAT-aged catalysts with some association to mechanisms of performance change.

Another suggestion from the reviewer was to possibly investigate productive control schemes that are approaching the duty cycle - the intrusive $\mathrm{NH}_{3}$ step method — but which actually are part of SCR dosing control to make a more robust onboard solution. (The reviewer pointed out that the Air Resources Board generally does not accept intrusive OBD tests.) Providing data to quantify tailpipe criteria emissions during the $\mathrm{NH}_{3}$ step and $\mathrm{NH}_{3}$ slip in the analysis would also improve the future work.

\section{Question 5: Does this project support the overall DOE objectives of petroleum displacement? Why or why not?}

\section{Reviewer 1:}

The reviewer commented that the development of practical OBD methods for lean aftertreatment is critical for continuing to develop lean combustion systems.

\section{Reviewer 2:}

The reviewer commented that proper aftertreatment technology allows best optimization of engine thermodynamics.

\section{Reviewer 3:}

The reviewer stated that modeling of catalyst aging is relevant to minimize safety factors (overdesign) in applied catalysts and controls by saving money and reducing fuel consumption in SCR systems.

\section{Reviewer 4:}

The reviewer stated that SCR can improve fuel economy, but it is not clear how the modeling process could assist in the improvement of fuel economy, except for modeling when the SCR has reached a low-efficiency point and would need to be regenerated or replaced.

\section{Question 6: Resources: How sufficient are the resources for the project to achieve the stated milestones in a timely fashion?}

\section{Reviewer 1:}

To properly perform catalyst aging characterization, modeling, and controls development, the reviewer believed there needs to be significantly more funding than has been allocated for this project. Additional funding or funding of a similar project could enable improvements in the field-aging component as well as a more appropriate assessment of the modeling effort for catalyst state and possibly more highly vetted suggestions for control and OBD algorithms.

\section{Reviewer 2:}

The reviewer commented that it seems like there is a lot being accomplished for the project budget. While the reviewer was not sure about the specific headcount and hardware, the outcome could be significant for the HD and LD markets with a catalyst modeling approach. 
Reviewer 3:

The reviewer stated that progress appears to be at a good pace for the size of the project.

Reviewer 4:

The reviewer found no indication that resources were not sufficient nor was there any negative impact on the work. 


\section{Emissions Control for Lean Gasoline Engines: Jim Parks (Oak Ridge National Laboratory) - ace033}

\section{Presenter}

Jim Parks, Oak Ridge National Laboratory

\section{Reviewer Sample Size}

A total of three reviewers evaluated this project.

\section{Question 1: Approach to performing the work-the degree to which technical barriers are addressed, the project is well-designed, feasible, and integrated with other efforts.}

\section{Reviewer 1:}

The reviewer stated that the approach is excellent, particularly the iterative experiments using modeling, bench, and vehicle work. Running rich/lean to generate $\mathrm{NH}_{3}$ for a downstream SCR, and quantifying the fuel penalty, seems feasible. Ford (Theis) substantiated the approach, favoring a TWC or a lean $\mathrm{NO}_{\mathrm{x}}$ trap due to $\mathrm{S}$ poisoning.

The reviewer expressed concerns about this being an older lean burn engine approach. More recent concepts are more diluted, either with air or EGR, to give lower $\mathrm{NO}_{\mathrm{x}}$ and temperatures, perhaps more like diesel. The reviewer suggested that the project team look at GCI work, or a Toyota paper (SAE 2015-01-1896). Given the FC advantages, even versus stoichiometric high-EGR approaches ( $2 \%$ versus EGR to $15 \%$ versus traditional stoichiometry), there is money to play with (e.g., \$200-\$250 in the 2020-2025 timeframe at 2\% benefit). This might pay for a urea system (\$250) if the cost of platinum group metals (PGM) can come down. The reviewer suggested that the project team explore lower $\mathrm{NO}_{\mathrm{x}}$, lower temperature, and urea-SCR supplementation.

\section{Reviewer 2:}

The reviewer found this project to be a nice blend of lab work and engine work. The reviewer commented about the investigation of the effect of the TWC formulation on the light-off performance on Slide 18. The reviewer asked whether the project team had assessed the different formulations for $\mathrm{NH}_{3}$ selectivity and $\mathrm{HC}$ conversion during rich operation. It is critical that the TWC provide essentially $100 \%$ steam reforming capability during the rich periods to satisfy the very stringent $\mathrm{HC}$ standards. This means there has to be some ceria in the TWC because it is needed for steam reforming, but the resulting $\mathrm{O}_{2}$ storage capacity will delay the production of $\mathrm{NH}_{3}$ after a lean/ rich transition. So the amount of ceria needs to be optimized to maximize the $\mathrm{NH}_{3}$ generation while still providing $100 \%$ steam reforming capability. The reviewer recommended that the project team initiate some controls 
development and investigate multi-step purges in order to reduce the CO slip and fuel economy penalty associated with the rich periods.

\section{Reviewer 3:}

The reviewer commented that the focus of this presentation is on technical aspect of the technology. The reviewer believed the project needs to also address the commercial aspect of the approach, benefits versus cost/complexity, and impact on fuel economy of running rich/lean versus SCR costs.

\section{Question 2: Technical accomplishments and progress toward overall project and DOE goals-the degree to which progress has been made, measured against performance indicators and demonstrated progress towards DOE goals.}

\section{Reviewer 1:}

The technical results are excellent to outstanding. The reviewer commented that the project team had quantified most or all major characteristics of the system under steady-state conditions, and the reviewer thinks that the approach looks feasible. Light-off seems good enough. The $\mathrm{NH}_{3}$ and $\mathrm{N}_{2} \mathrm{O}$ trade-off in rich-lean and light-off is quantified and reasonable. Cycle time, $\mathrm{NH}_{3}: \mathrm{NO}_{\mathrm{x}}$, and SCR loading on engine are impressive and seem reasonable. Poisoning and aging impacts are not stoppers, although desulfation can be a problem at $650^{\circ} \mathrm{C}$ if advanced lowtemperature lean burn concepts are used. Although not shown here, the reviewer presumed that the PI has evaluated the above as a function of temperature; if not, this will be needed.

\section{Reviewer 2:}

The reviewer found the project to be an interesting investigation into PGM sintering versus aging time. The reviewer noted the good work in evaluating the effects of $\mathrm{S}$ on $\mathrm{NH}_{3}$ production, $\mathrm{HC}$ conversion during rich operation, and the ability to recover the performance with a rich desulfation. Because the $\mathrm{NH}_{3}$ production and $\mathrm{HC}$ conversion of the TWC both decreased significantly after S poisoning, it will be important to keep the TWC purged of S by periodically exposing it to high temperatures and slightly rich operation. In the close-coupled location, this should happen passively. But, a desulfation procedure will need to be available for the case of extended lowtemperature operation.

$\mathrm{HC}$ slip will be the biggest challenge during rich operation. It will require that the TWC operates at a minimum of $400^{\circ} \mathrm{C}$ and preferably $500^{\circ} \mathrm{C}$ to promote steam reforming of the different types of $\mathrm{HC}$ in the exhaust. The higher temperatures will allow for running less rich and still generating more $\mathrm{NH}_{3}$ and less $\mathrm{N}_{2} \mathrm{O}$. The less rich operation will in turn help minimize the CO slip and fuel economy impacts during rich operation. Two-step purges should still need to be investigated (extra-rich initially to reduce the catalyst, then less rich to generate $\mathrm{NH}_{3}$ during the rest of the purge).

\section{Reviewer 3:}

The reviewer believed there was good consideration of reviewers' comments, but suggested that the project team consider different SCR formulations to address $\mathrm{N}_{2} \mathrm{O}$ emissions.

\section{Question 3: Collaboration and coordination with other institutions.}

\section{Reviewer 1:}

The reviewer commented that the project team has all the key parts to the system-OEMs, catalyzer, and aging support. The reviewer really liked the project team's additional collaborators and noted that the project team was well connected with key players.

\section{Reviewer 2:}

The reviewer praised the great combination of academia and industry.

\section{Reviewer 3:}

The reviewer liked the good collaboration with GM and discussions with other groups such as Ford and Fiat Chrysler Automobiles (FCA).

Question 4: Proposed future research-the degree to which the project has effectively planned its future work in a logical manner by incorporating appropriate decision points, 
considering barriers to the realization of the technology and, when sensible, mitigating risk by providing alternate development pathways.

\section{Reviewer 1:}

The future plans appear to address the challenges and barriers, and the reviewer offered several suggestions. As mentioned above in the approaches, the project team needs to look at emerging lean gasoline concepts with lower engine-out $\mathrm{NO}_{\mathrm{x}}$ levels and lower temperatures from Toyota, Delphi, and ANL. Another suggestion was that transient work will be critical to feasibility. The reviewer suggested that the project team consider supplementing the approach with a urea system. Urea consumption could be very low, and perhaps the reduced rich time and/ or PGM can help pay for much of it. Lastly, the reviewer proposed considering SCR filters. A peak in the box is needed, as GPFs are gaining a foothold in the European Union (EU) and China, and may be needed/desired in the United States. This is not as critical as the above considerations.

\section{Reviewer 2:}

The reviewer believed there was a need to really emphasize HC slip during the purges because HC slip will be one of the biggest challenges for any system requiring periodic rich operation. Aging of the SCR catalyst will be interesting as SCR catalysts can be deactivated by high-temperature rich conditions.

\section{Reviewer 3:}

The reviewer suggested considering the possible influences of $\mathrm{H}_{2}$.

\section{Question 5: Does this project support the overall DOE objectives of petroleum displacement? Why or why not?}

\section{Reviewer 1:}

The reviewer commented that less gasoline operation will reduce fuel consumption and satisfy DOE objectives of reduced petroleum use. However, it will require extremely high performing emission control systems to satisfy stringent emission standards such as the Tier 3 standards.

\section{Reviewer 2:}

The reviewer noted that dedicated EGR can be very competitive to lean-burn gasoline. However, CI gasoline is emerging with potential further advantages.

\section{Reviewer 3:}

The reviewer stated that lean burning engines are key for best performance.

\section{Question 6: Resources: How sufficient are the resources for the project to achieve the stated milestones in a timely fashion?}

\section{Reviewer 1:}

The reviewer stated that $\$ 400,000$ is not enough. This ought to be 1.5-2 times higher, given the emerging interest in lean-burn and the challenges and options that need to be evaluated.

\section{Reviewer 2:}

The reviewer encouraged the PI to consider applying more resources to the calibration, including the multi-step purges. Calibration and controls will also be needed to maximize the feed gas $\mathrm{NO}_{\mathrm{x}}$ during rich operation (to generate high levels of $\mathrm{NH}_{3}$ quickly for the SCR catalyst and allow short rich periods) while minimizing the feed gas $\mathrm{NO}_{\mathrm{x}}$ during lean operation (to reduce the consumption of the stored $\mathrm{NH}_{3}$ and allow longer lean periods for improved fuel economy). 
Neutron Imaging of Advanced Transportation Technologies: Todd Toops (Oak Ridge National Laboratory)

- ace052

\section{Presenter}

Todd Toops, Oak Ridge National

Laboratory

\section{Reviewer Sample Size}

A total of five reviewers evaluated this project.

Question 1: Approach to performing the work-the degree to which technical barriers are addressed, the project is well-designed, feasible, and integrated with other efforts.

\section{Reviewer 1:}

The reviewer complimented the project as a very nice effort to develop an advanced spray/injector diagnostic tool. The information gained and models developed could be very useful for industry and academic researchers.

\section{Reviewer 2:}

The reviewer remarked that this is a good approach to complement other injector diagnostic techniques, such as $\mathrm{X}$-rays and visible light spray testing.

\section{Reviewer 3:}

The reviewer stated that the approach develops and uses a novel tool (neutron imaging) available only at the national laboratories to diagnose engine-related problems.

\section{Reviewer 4:}

The reviewer noted that this project is another successful re-tasking of unique DOE resources to aid the engine industry. While some explanation of the differences between this work and the ANL APS work is made here, some more extensive joint report on how the two facilities actually complement each other is needed. Of course, seeing more actual collaboration with complementary results would be even better.

\section{Question 2: Technical accomplishments and progress toward overall project and DOE goals-the degree to which progress has been made, measured against performance indicators and demonstrated progress towards DOE goals.}

\section{Reviewer 1:}

The reviewer commented that fuel injector and particulate filter measurements are reported. Some collaborative, complementary results with the ANL team are included in the injector work, which is noteworthy. 


\section{Reviewer 2:}

The reviewer noted that engaging with the ECN is a very good thing. The reviewer questioned whether it would be possible using other diagnostics, like high-speed movies, to make some sort of assessment of the cycle-by-cycle variability of the injector being studied. This would give some indication of the amount of smoothing contained in the composite images obtained from the neutron imaging.

\section{Reviewer 3:}

The reviewer found very good progress has been made in providing some new insights for gasoline fuel injector behavior using this tool. More results have been provided on flash boiling, sac volume, and dribble on gasoline spray characteristics. Good progress has also been made with GPFs.

\section{Reviewer 4:}

The reviewer commented that resolution of injector and spray measurements so far do not seem to be good enough to be able to draw strong conclusions. GPF measurements are very insightful and provide information that is not available any other way.

\section{Question 3: Collaboration and coordination with other institutions.}

\section{Reviewer 1:}

The reviewer noted collaboration with a number of academic and industrial partners and remarked that more is always better though.

\section{Reviewer 2:}

The reviewer suggested that as the tool becomes more developed, it would be advisable to engage OEMs and other injection equipment suppliers.

\section{Reviewer 3:}

The reviewer stated that it sounds like the amount of beam time available is limiting the amount of collaboration with fuel injector suppliers and preventing looking at more conditions and more injectors. Still, collaboration should be sought with more fuel injector suppliers like Bosch or Delphi as they know the issues needing to be solved in detail and stand to benefit the most, indirectly impacting the OEMs.

\section{Question 4: Proposed future research-the degree to which the project has effectively planned its future work in a logical manner by incorporating appropriate decision points, considering barriers to the realization of the technology and, when sensible, mitigating risk by providing alternate development pathways.}

\section{Reviewer 1:}

The reviewer commented that a fouling study will be interesting. Improved image processing and understanding occlusion effects from heavy hydrocarbons (HHC) are also important for improving diagnostics results to aid modelers, as is improving the geometry description for spray gasoline.

\section{Reviewer 2:}

The reviewer noted that the project had a well laid-out path for further development of the tool.

\section{Reviewer 3:}

The reviewer suggested higher resolution is the correct thing to work on to improve the measurements and make them useful.

\section{Question 5: Does this project support the overall DOE objectives of petroleum displacement? Why or why not?}

\section{Reviewer 1:}

The reviewer remarked that a better understanding of the injection process provided by the diagnostics here will aid in designing more efficient engines that use less petroleum. 


\section{Reviewer 2:}

The reviewer asserted that this is a good example of DOE being able to develop and provide advanced tools and diagnostic capability that could be helpful to industry but which industry could not afford to undertake itself.

\section{Reviewer 3:}

The reviewer noted that the technique could be developed that will provide insight into injector behavior. This is important to understand how injectors interact with the engine combustion system.

\section{Reviewer 4:}

The reviewer stated that neutron imaging has the potential of becoming a useful tool in developing fuel injectors and particulate filters, for example, which are key enablers of high efficiency engines that meet emissions standards.

\section{Question 6: Resources: How sufficient are the resources for the project to achieve the} stated milestones in a timely fashion?

\section{Reviewer 1:}

The reviewer noted that the project needs more resources to accelerate progress.

\section{Reviewer 2:}

The reviewer found that resources seem adequate for the proposed work 


\section{RCM Studies to Enable Gasoline-Relevant Low- Temperature Combustion: Scott Goldsborough (Argonne National Laboratory) - ace054}

\section{Presenter}

Scott Goldsborough, Argonne National Laboratory

\section{Reviewer Sample Size}

A total of five reviewers evaluated this project.

\section{Question 1: Approach to performing the work-the degree to which technical barriers are addressed, the project is well-designed, feasible, and integrated with other efforts.}

\section{Reviewer 1:}

The reviewer remarked that the project is rather narrowly focused on using the RCM to provide combustion properties for developing and evaluating the kinetic chemistries of transportation fuels at a range of pressures and temperatures. A twin piston RMC is used to measure ignition delay time (IDT), which is the main property that is used to compare predicted and measured values using a model of the RCM.

The reviewer noted that the project team refers to the RCM as providing IDT properties under engine relevant conditions and poses the question to the team about how researchers can infer performance under engine, e.g., HCCI, conditions. The reviewer asserted that it is not at all clear that IDT data from an RCM provide such relevant conditions, nor is it evident that there is much in the RCM environment besides temperature and pressure that could be construed as engine relevant conditions. For example, the RCM has no turbulence, swirl, or any liquid within the RCM such as would be present within an engine from sprays and droplets injection, which sets the initial conditions for in-cylinder processes. The condensed phase fuel of an RCM is pre-vaporized so the environment of an RCM may in fact and in reality not be faithful to that of engine. The reviewer recommended that the project team pull back from characterizing the RCM environment as being engine relevant because it is not.

The reviewer noted the mention that engine data are shared with the project; however, the utility of such data is unclear. Currently, in-cylinder conditions cannot be simulated from a first-principles approach with the same high fidelity modeling that the RCM is amenable to, with its single phase and 1D transport environment. As a result, the reviewer stated that it is not clear how engine data will be folded into this project. Some discussion of how engine data are related to the RCM would be relevant. 


\section{Reviewer 2:}

The reviewer stated that the project is very instrumental towards acquiring RCM data to support the improvements in chemical kinetic modeling for relevant fuels for the transportation industry. The work employs a RCM facility with good range capability and is developing novel analysis techniques using UQ and global sensitivity analysis (GSA). The current efforts to improve the RCM machine operational issues should be addressed right away by possibly engaging outside help to eliminate the 2-3 ms delay in the synchronous motion.

\section{Reviewer 3:}

The reviewer noted that fundamental ignition delay data have been collected extensively for gasoline fuel surrogate components and gasoline/ethanol blends in ANL's RCM facility and compared to models yielding reasonable matches with the test data. However, the gasoline surrogate model needs to be improved to capture the lowtemperature, high-pressure region. In order to perform engine high load simulation work, data at higher ambient pressure might be needed.

\section{Reviewer 4:}

The reviewer commented that RCM experiments for ignition delay measurements are very standard, and this project does not go beyond the standard utilization of RCM. The introductory slides indicate the development of new diagnostics, but such results were not shown. While the ignition delay data are important for model validation, it seems that this project merely cranks out more ignition delay data. Thus, it seems to lack novelty in the project approach.

\section{Reviewer 5:}

The reviewer asked how this work compares to, complements, and/or dovetails with kinetic work by Bill Pitz at LLNL.

\section{Question 2: Technical accomplishments and progress toward overall project and DOE goals-the degree to which progress has been made, measured against performance indicators and demonstrated progress towards DOE goals.}

\section{Reviewer 1:}

The reviewer praised the presentation as documenting very well the efforts undertaken in the gasoline surrogate work, with special emphasis on the LTC behavior. For clarity, the reviewer wondered if the project team could include the dilution ratios on the figures related to the five-member ring naphthenes.

The reviewer commented that ignition delay studies of Fuels for Advanced Combustion Engine-F (FACE-F) with Ethanol blends appear to be well correlated with modeling work. The work seems to attempt to correlate the studies with the HCCI engine work from University of California at Berkeley. The reviewer noted that the data presented appears to be unclear and asked if the project team could help by labeling the figures with more informative information and help the readers distinguish the RCM and engine data.

\section{Reviewer 2:}

The reviewer remarked that most of the proposed milestones have been accomplished with others in progress. Results are solid and promising with the collaboration with other institutions, and the first part of this project is well accomplished.

\section{Reviewer 3:}

The reviewer observed that the outcome of this project, ignition delay of various gasoline surrogates, is well within expectation. There are no additional insights into the chemical kinetics of the ignition delay. There have been many similar studies using RCM for gasoline-type fuel chemistry study. The reviewer suggested that it needs to be clear that this project does not repeat what has been done in the literature.

\section{Reviewer 4:}

The reviewer said that a lot of data were obtained in the reporting period, including for gasoline/ethanol blends (E10), principally being IDT with reasonable agreement shown. The reviewer highly recommended that the project team present some information about the sort of modeling of the RCM that is at the heart of comparing IDT data with simulations. 
The reviewer commented about the "New approaches developed in 2016 to demonstrate correlation between RCM measurements and observed engine trends...." by stating that, again, the RCM environment does not appear to be entirely relevant to engine conditions where sprays set the initial conditions for combustion because it pre-vaporizes the fuel. There are certain engines/conditions where ignition can occur within the multiphase domain of the injected spray. Data for this situation are also relevant to developing combustion chemistries. The reviewer stated that the point is researchers do not know how combustion chemistries developed from purely gas phase combustion configurations such as an RCM would compare with chemistries developed from multiphase configurations where the flame/ignition process would occur with the multiphase domain.

\section{Question 3: Collaboration and coordination with other institutions.}

\section{Reviewer 1:}

The reviewer commented that there were very good collaborations with LLNL, University of California at Berkeley, Northeastern University, and international institutions.

\section{Reviewer 2:}

The reviewer said the project team was a very good team. The reviewer suggested that the project team possibly incorporate more engine representation to help validate the findings and provide a way for further migration of models and chemistry to applications.

\section{Reviewer 3:}

The reviewer stated that the collaboration seems to be reasonable. It seems to include the exchange of information only, but not closely working together to interpret the data at a deeper level. The team seems to be focused on RCM experiments. The reviewer said that the presentation indicates that there is collaboration with Northeastern University on UQ, but it is not clear how the interaction would be. The reviewer encourages two-way interaction and more details.

\section{Reviewer 4:}

The reviewer found the collaborations to be well-developed, but it would be useful to provide more discussions of the models used to validate the RCM data as the project has collaborators who provide engine data. Some discussion for precisely how these data are used to develop combustion chemistries and how the RCM fits in with them would be useful.

\section{Question 4: Proposed future research-the degree to which the project has effectively planned its future work in a logical manner by incorporating appropriate decision points, considering barriers to the realization of the technology and, when sensible, mitigating risk by providing alternate development pathways.}

\section{Reviewer 1:}

The reviewer said that the work provides significant information on ignition delays. The reviewer queried whether the current work is looking at other metrics to help improve or validate today's mechanisms (e.g., select speciation).The reviewer also wanted to know how the present work is considering the uncertainties associated with the LTC mechanisms. On the uncertainty front, the reviewer asked what role the absence of chemical intermediaries plays and whether there are any effects present in the measurements due to local perturbations.

\section{Reviewer 2:}

The reviewer stated that, basically, the proposed future work showed a good extension of present work and offered that study at higher ambient pressure might be needed for the future engine (diesel or gasoline) case study.

\section{Reviewer 3:}

The reviewer noted that future work will include more testing of surrogates and collaborations with groups at SNL to further quantify autoignition behavior. The project team mentioned that tests will be conducted on multicomponent surrogates. The reviewer suggested that some discussion of how blending ratios would be selected is appropriate, and an E10 surrogate (RD-587) will be examined. The reviewer remarked that a simple experiment would be to also measure the IDT with the certification fuel and compare results with E10 and asked how this will be done. The plan to collaborate with LLNL to reduce uncertainty in rate controlling reactions and improve model 
predictions was not clear to the reviewer, who asked about how the collaboration will be done. The reviewer also wanted to know the specific role in the RCM in this process as it was vague.

\section{Reviewer 4:}

The reviewer encouraged proposed future work to use gas chromatography (GC) to obtain gas speciation. The project needs to go beyond the standard ignition delay measurements.

\section{Question 5: Does this project support the overall DOE objectives of petroleum displacement? Why or why not?}

\section{Reviewer 1:}

The reviewer commented that understanding combustion chemistry is important in the design of new, more efficient engines that will reduce petroleum usage.

\section{Reviewer 2:}

The reviewer found that this project is relevant from a broad perspective. However, as noted previously, the RCM does not in general provide engine relevant conditions for reasons mentioned previously. It provides but one of a number of combustion properties useful for validating combustion chemistry.

\section{Reviewer 3:}

The reviewer noted that this project is mostly on fossil fuel (gasoline). Although there are results for ethanol/ gasoline blends, ethanol chemistry is very well established. Thus, it is not clear how this project will help improve the understanding of ethanol chemistry to promote the displacement of petroleum fuels.

\section{Question 6: Resources: How sufficient are the resources for the project to achieve the stated milestones in a timely fashion?}

\section{Reviewer 1:}

The reviewer stated that funding appears to be adequate for proposed plan.

\section{Reviewer 2:}

The reviewer noted that resources seem to be reasonable.

\section{Reviewer 3:}

The reviewer stated that resources seem adequate although ultimate judgement would have to come from a cost/ benefit analysis based on DOE's investment relative to the commercialization potential. 


\section{Fuel-Neutral Studies of Particulate Matter Transport Emissions: Mark Stewart (Pacific Northwest National Laboratory) - ace056}

\section{Presenter}

Mark Stewart, Pacific Northwest National Laboratory

\section{Reviewer Sample Size}

A total of four reviewers evaluated this project.

\section{Question 1: Approach to performing the work-the degree to which technical barriers are addressed, the project is well-designed, feasible, and integrated with other efforts.}

\section{Reviewer 1:}

The reviewer expressed that the approach of characterizing PM with a very wide range of tools is excellent.

\section{Reviewer 2:}

The reviewer commented that this is a very important research project as OEMs are looking for data to help support development and implementation of GPFs for vehicles. The reviewer liked the fact that the project team is also trying to determine the particle size for filtration efficiency and regeneration. The reviewer observed that it is very important to understand the fuel properties of both splash and matchblended fuels, so it would be helpful to list that in the data for the project. It would also be helpful to list the tools and methods used to collect the data on the slides.

\section{Reviewer 3:}

The reviewer said that the approach appropriately leverages project team core competencies and facilities, with UW running advanced engine testing with candidate next-generation gasoline engine technologies and PNNL conducting highly detailed PM characterization.

\section{Reviewer 4:}

The reviewer stated that there was an excellent use of advanced tools on a wide range of filters. Wafers are a good idea, and also looking at early loadings is very important. The reviewer noted that particulate characterization is important at this stage of program, but ash is more important than soot for GPF. Both were evaluated.

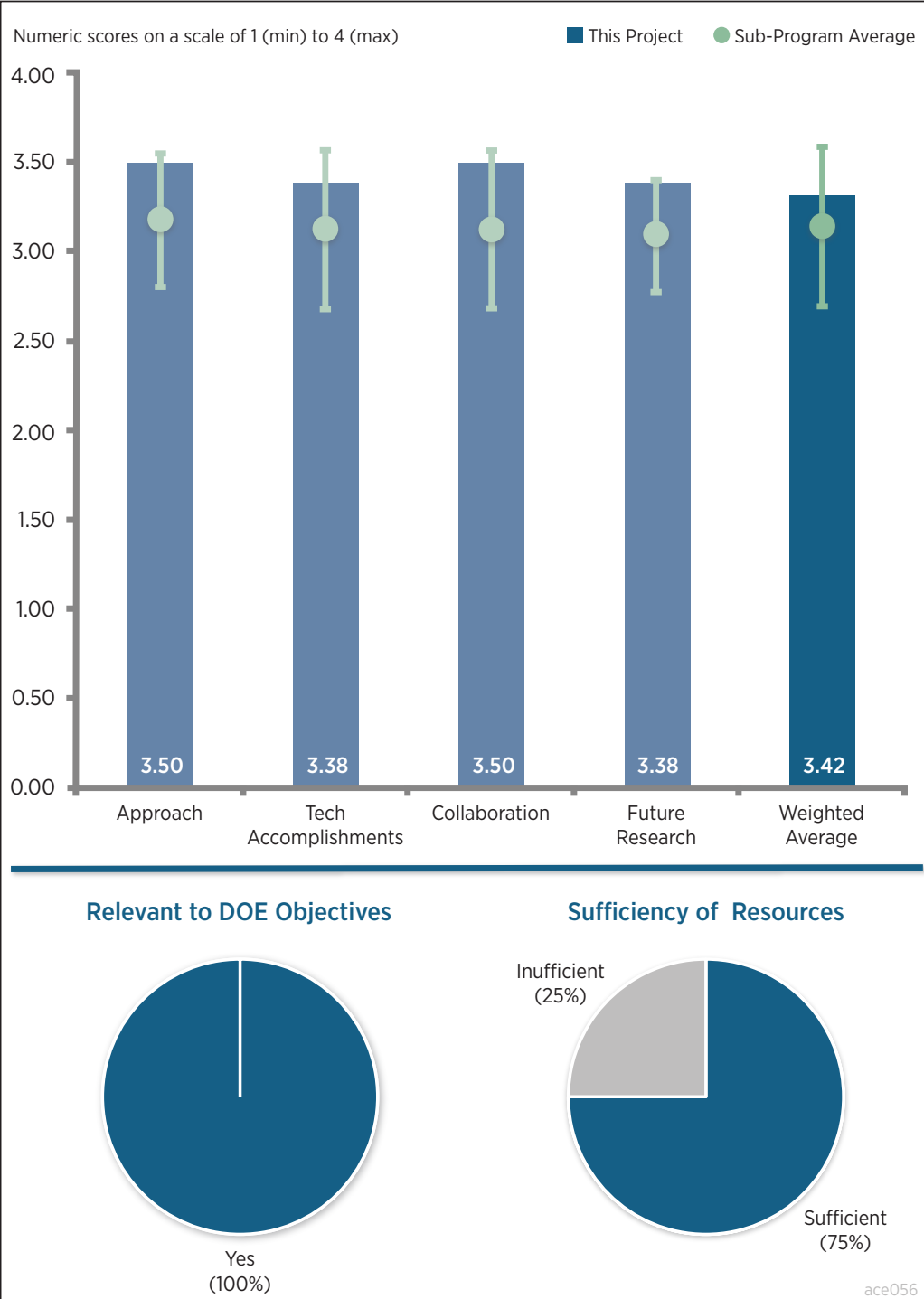

Figure 4-24 - Fuel-Neutral Studies of Particulate Matter Transport Emissions: Mark Stewart (Pacific Northwest National Laboratory) - Advanced Combustion Engines 
Question 2: Technical accomplishments and progress toward overall project and DOE goals-the degree to which progress has been made, measured against performance indicators and demonstrated progress towards DOE goals.

\section{Reviewer 1:}

The reviewer found the overall research accomplishments to be fantastic and very relevant. Again, further clarifying the fuel properties to the particle size and number is important for a complete understanding of the soot loading and GPF efficiency. It would also be helpful to list the tools and methods used to collect the data on the slides. It is hard to make sense of the data on Slide 7 as the reviewer is not sure if this is splash or match- blended fuels. The reviewer asked which tools were used on Slide 8.

\section{Reviewer 2:}

The reviewer observed that technical accomplishments include advances in particulate characterization, device scale modeling, significant amount of new filtration data, and detailed Lattice Boltzmann simulations.

\section{Reviewer 3:}

The reviewer remarked that the data and understanding that have been achieved are impressive, especially the impact of different fuels. Their application into modeling, where appropriate, would be great to see more of. Including ash into the data analysis and modeling would be good also.

\section{Reviewer 4:}

The reviewer found characterization of the soot as a function of fuel and operating conditions to be quite interesting. The operating conditions seem a little broad as well as the fuels, but it is good to see the whole spectrum. The filtration efficiency work versus filter and PM size is also important and valuable. Such data are new for GPF. The Lattice Boltzmann simulations are quite interesting and explain much of the porosity impact on back pressure and filtration efficiency.

\section{Question 3: Collaboration and coordination with other institutions.}

\section{Reviewer 1:}

The reviewer remarked that there was good collaboration with GM and UW on this project. Other collaborators are listed on Slide 21, a very nice list.

\section{Reviewer 2:}

The reviewer commented that this project has a strong project team with good collaboration and partners leveraging their core competencies: GM is the main industry partner providing part of funding, hardware, and expertise; the Engine Research Center (ERC) at UW conducts experiments and assist with analysis and modeling; and four filter and three analysis subcontractors are involved.

\section{Reviewer 3:}

The reviewer asserted that interaction with UW and GM has been very productive.

\section{Reviewer 4:}

The reviewer observed that ERC, GM, and filter suppliers are the key partners, but a catalyzer is missing as the project moves into a TWC plus GPF.

Question 4: Proposed future research-the degree to which the project has effectively planned its future work in a logical manner by incorporating appropriate decision points, considering barriers to the realization of the technology and, when sensible, mitigating risk by providing alternate development pathways.

\section{Reviewer 1:}

The reviewer commented that this is an excellent list of needed future work on this project. The reviewer highly recommended asking for additional funds to overcome any technical challenges or barriers in accomplishing the project (as listed on Slide 22) and to accomplish the goals of Future Work on Slide 23. 


\section{Reviewer 2:}

The reviewer indicated that future research seems appropriate as it involves further expanding the set of tested filter samples, including catalyzed filter substrates to improve filtration models.

\section{Reviewer 3:}

The reviewer remarked that the future work plan is primarily aimed at further refining the models and methods on a wider range of uncoated GPF samples. Although some of the first generation GPFs will be uncoated, the majority of these and all future systems will be catalyzed. The reviewer encouraged the project team to generate future base data to primarily focus on catalyzed samples and recommended shifting to characterizing cordierite only. In gasoline application, high thermal mass materials like AT and silicon carbide ( $\mathrm{SiC})$ have little use. They are valuable for characterizing porosity, as the project team has shown.

Also, a key risk and a general unknown is whether polycyclic aromatic hydrocarbon (PAH) emissions are higher from a GDI engine than an MPI engine. The hypothesis is that PAHs are integrated into immature soot, and this passes through the TWC. Given the toxicity of PAHs on small solid particles, this understanding is perhaps the most critical at this time. This might be beyond the scope of this project, but the project team could take the GDI results and easily slap on a TWC and vary the filter temperature to see PAH response for maybe $10 \%$ ethanol blend with gasoline (E10) to $20 \%$ ethanol blend with gasoline (E20). It might be easy to get similar results on MPI PM characterization. This reviewer noted that China is seriously looking at MPI GPFs, as well as the EU OEMs.)

\section{Reviewer 4:}

The reviewer commented that much is still left to do.

\section{Question 5: Does this project support the overall DOE objectives of petroleum displacement? Why or why not?}

\section{Reviewer 1:}

The reviewer commented that understanding how to manage the filtration efficiency and regeneration of a GPF will help to minimize the back pressure on the engine and thus allow it to operate at the maximum efficiency.

\section{Reviewer 2:}

The reviewer stated that the project allows for use of new combustion technologies.

\section{Reviewer 3:}

The reviewer noted that this project addressed the barriers for enabling high-efficiency engine technology that would result in direct petroleum savings. Specifically, the following are addressed from the VTO multi-year program plan: lack of cost-effective emission control, lack of modeling capability for combustion and emission control, and lack of actual emissions data on pre-commercial and future combustion engines

\section{Reviewer 4:}

The reviewer said that although U.S regulations, even in California $(1 \mathrm{mg} / \mathrm{mi})$, will not require a GPF, some of the emerging high-efficiency concepts may have high PM/particle number (PN) emissions. OEMs are also interested in utilizing GPFs to minimize public relations risk.

\section{Question 6: Resources: How sufficient are the resources for the project to achieve the stated milestones in a timely fashion?}

\section{Reviewer 1:}

The reviewer asserted that it seems that, based on the list of the challenges and barriers listed on Slide 22, the project might need additional funding to complete. The data for a closed coupled GPF and catalyzed filters are a significant hurdle for the industry so these kinds of data are very relevant for the coming years. It would be helpful to support the project with additional funds if the project team deems it necessary to overcome the challenges and barriers in accomplishing the goals and future work.

\section{Reviewer 2:}

The reviewer commented that funding has been $\$ 200,000$ and $\$ 250,000$ for the past two fiscal years, respectively, which appears adequate for the scope of this project. 


\section{SuperTruck - Development and Demonstration of a Fuel-Efficient Class 8 Tractor and Trailer, Engine Systems: Russ Zukouski (Navistar International Corporation) - ace059}

\section{Presenter}

Russ Zukouski, Navistar International Corporation

\section{Reviewer Sample Size}

A total of six reviewers evaluated this project.

\section{Question 1: Approach to performing the work-the degree to which technical barriers are addressed, the project is well-designed, feasible, and integrated with other efforts.}

\section{Reviewer 1:}

The reviewer remarked that the approach is comprehensive and balanced. It hits the major opportunities for efficiencydownspeeding, pumping, combustion, and parasitics. The reviewer liked that Navistar is using many of the technologies it had to develop to get low engine-out $\mathrm{NO}_{\mathrm{x}}$ and that WHR is not needed to demonstrate 50\% BTE. The reviewer commented that it seems that all these 50\% BTE strategies are incremental or practical and can be readily employed, delivering immediate societal benefit.

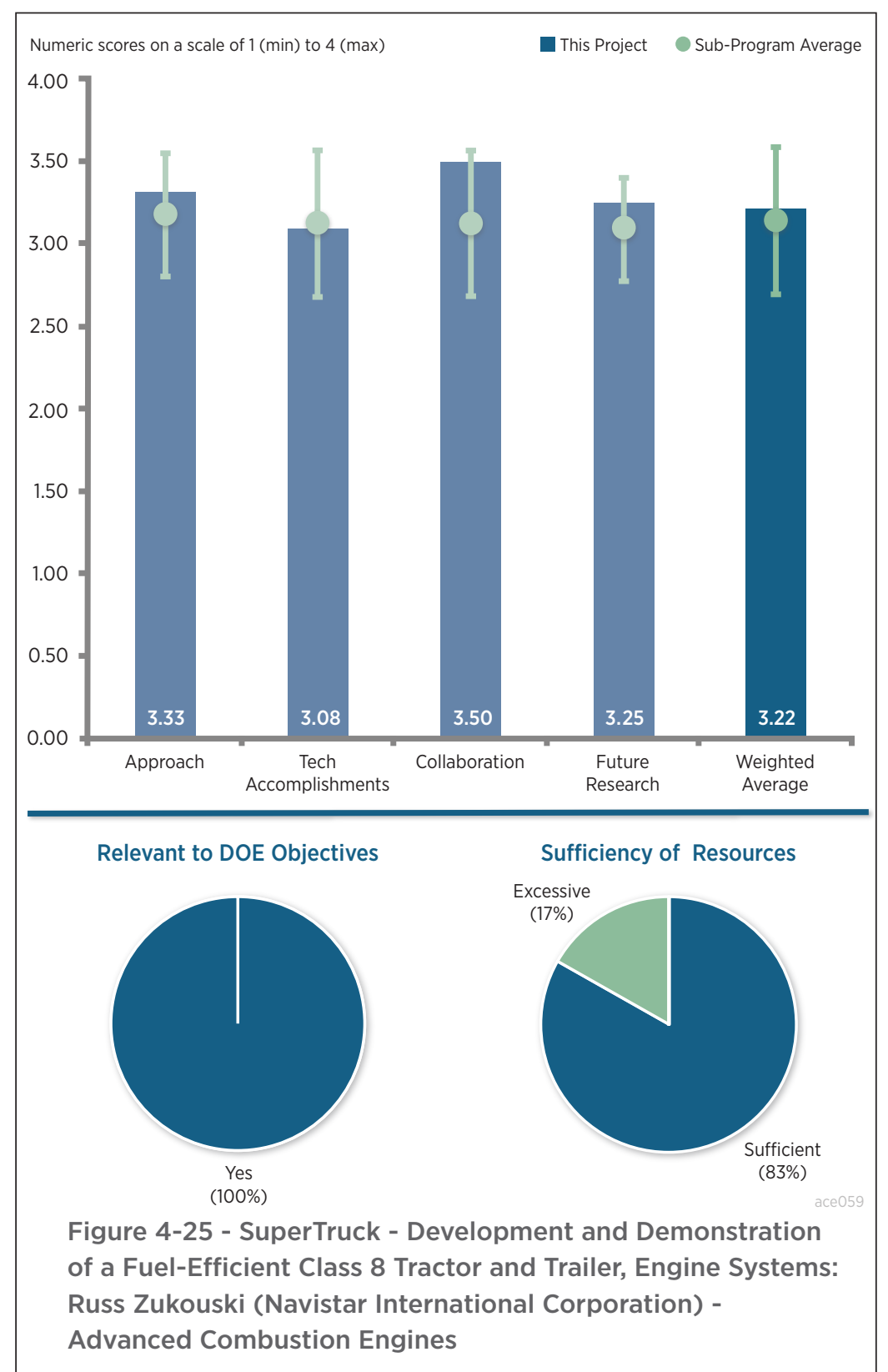

\section{Reviewer 2:}

The reviewer stated that a good set of technologies has been developed and demonstrated. The reviewer found a $50 \%$ BTE to be an impressive demonstration. The approach is systematic and produced good results, and the 55\% path is somewhat more tenuous but not bad.

\section{Reviewer 3:}

The reviewer found an excellent approach with selected technologies, which included a focus on combustion heat retention, friction reduction, alternative fuels, two types of waste heat recovery, and a very good effort at rational electrification of parasitics by considering multi-voltage (12 Volt [V], $24 \mathrm{~V}$, and $48 \mathrm{~V}$ ) architecture to achieve goals. The reviewer commented that it was outstanding to find bandwidth to consider alternative fuels to achieve stretch goals and facilitate parallel work in this area. The plan to leverage industry partner knowledge is excellent.

\section{Reviewer 4:}

The reviewer noted that it is good to see that Navistar finally brought back WHR with Rankine cycle technology, 
which had been criticized last year. There is no way to achieve 50\% BTE goal without WHR. The reviewer said that a lack of technical planning and technical vision in the early stage caused the program to miss the timing and the goal.

\section{Reviewer 5:}

The reviewer commented that this team was caught in an unfortunate situation that was outside their control. Under these difficult situations, the team did the best that they could, and the reviewer commended the team for their perseverance and hard work. The reviewer said that the fundamentals of their approach were sound, the project team just did not get to pursue them to the extent necessary to leave the reviewer with confidence that the end results in September will be very inspiring. Nor did the team have the opportunity to really push on the most challenging longer term technical approaches for improving engine or transport efficiency.

\section{Reviewer 6:}

The reviewer commented that the project objectives were clearly identified, but the path to achieve them is somewhat muddled. While the main path to achieve a 50\% BTE engine is a combination of improved combustion, downspeeding, improved air breathing, and parasitic loss reduction, a number of other technologies appear to have been tried that yielded no promising results. eTurbo and thermal barrier coatings are some, to name a few. The technical pathway identified towards a 55\% BTE engine constitutes use of diesel plus natural gas (NG) or diesel plus ethanol, which are far removed from practical use. The reviewer commented that though the technology mix towards an integrated truck with a 50\% freight efficiency improvement was identified through CFD simulations, the reviewer doubts that it will ever be demonstrated.

\section{Question 2: Technical accomplishments and progress toward overall project and DOE goals-the degree to which progress has been made, measured against performance indicators and demonstrated progress towards DOE goals.}

\section{Reviewer 1:}

The reviewer mentioned balanced contributions from downspeeding, pumping, combustion, and parasitics to get $50 \%$ BTE. The variable valve actuation (VVA) work is unique and valuable as is the rocker stop device to eliminate idling. This is especially critical and unique for the vocational vehicle segment. The work on late combustion enhancement is also unique and important to minimize emissions. It appears to have delivered unexpected efficiencies.

Regarding 55\% BTE, the reviewer believed that Navistar appears to be the only holdout now that may pursue the dual fuel strategy, and the company has generated impressive results. Of the four participants, Navistar seems best poised to actually employ NG-diesel LTC in the market, given their vocational vehicle business and proximity to NG fueling stations (local or fleet). In the reviewer's opinion, this is a key consideration in the next step to sponsoring 55\% BTE work. It would be nice to get LTC more developed to see if it is real.

Navistar also contributed by discovering WHR using eTurbo is not the way to go, and this is a mixed blessing. The reviewer commented that the company is late on ORC, but this forced the company to not rely on WHR for $50 \%$ BTE, the only participant to do this, possibly unique worldwide. (Iveco was first to state it was possible.)

\section{Reviewer 2:}

The reviewer found that the descriptions of Navistar's activities were very generic in comparing this presentation with the results/accomplishments presented by the other SuperTruck team at this year's AMR. Navistar has accomplished a $49.6 \%$ BTE with the final target of $50 \%$ to be demonstrated in the next several months so despite their handicap, the team made progress.

In looking at the results presented, the reviewer expressed concern with the disparity between Navistar's system model and the actual dynamometer results shown in the graphs on Slide 13 of the presentation. Likewise, the data for dual fuel testing on Slide 14 and the current performance of the organic Rankine cycle (ORC) system do lend confidence to the viability of achieving the improvement projected for the dual fuel, downsized, and ORC II engine given in the bar chart on Slide 15. The reviewer commented that coupling the above remarks to brief statements made in the presentation indicating that Navistar is still working on aftertreatment performance and combustion modeling (kinetics) gave the reviewer concern as to the level of success the company will be able to claim at the 
end of the program in September 2016. Lack of technical backup slides for the reviewer to probe further into their accomplishments only enhances this feeling. These comments are made strictly based on demonstrated accomplishments. The reviewer said that they have not been weighted with a consideration of the handicap the researchers were handed after the project started.

\section{Reviewer 3:}

Regarding the 50\% BTE engine, the reviewer noted good progress with the technology mix identified. With the development of WHR system, Navistar is optimistic that the current $49.6 \%$ efficiency can be improved beyond $50 \%$. As for the 55\% freight efficiency vehicle demonstration, CFD simulations were performed to identify the technology mix. The reviewer said that it appears unlikely that this milestone will be met before the end of the project. Concerning the 55\% BTE engine, the reviewer commented that details provided are somewhat sketchy and heavily reliant on combustion system improvement and the WHR system.

The reviewer concluded that though the overall performance does not measure up with that of other awardees like Cummins or Volvo, the path taken to achieve the 50\% BTE is more practical. Navistar has relied on a strategy that is a combination of improved gas exchange, improved combustion system, and WHR, which is commendable.

\section{Reviewer 4:}

The reviewer remarked that it was very good work.

\section{Reviewer 5:}

The reviewer said that even without the pause in the middle of program, Navistar should have been able to achieve $50 \%$ BTE goal if the company had WHR in the early stage.

\section{Reviewer 6:}

The reviewer noted the Navistar has achieved the 50\% BTE goal with technologies that have a high potential for production, such as a variable geometry turbo, friction reduction through base engine redesign, and mild electrification. Efficiency is further enabled by closely coupled and integrated high efficiency aftertreatment.

The reviewer commented that the novel packaging for WHR on the rail is an interesting approach for R\&D simplicity and relatively low-risk potential for early adopters to confirm the technology in their fleets. The reviewer remarked that it was great to share data on approaches that did not make the downselect and the logic for future technology improvement/application consideration. The reviewer said that the three voltage architecture for mild electrification for efficiency is outstanding.

\section{Question 3: Collaboration and coordination with other institutions.}

\section{Reviewer 1:}

The reviewer said that Navistar leveraged key players in major aspects of this project. It appears the company delivered unique results in several of these, like VVA (Jacobs), dual fuel (ANL), and friction using crank and cam shafts (Mahle, ANL).

\section{Reviewer 2:}

The reviewer noted that it was an outstanding effort to leverage a large industry partner/supplier knowledge base to achieve successful results. The extensive list of suppliers and national laboratories supporting modeling work and product development facilitates knowledge transfer and accelerates technology to production through the supply chain.

\section{Reviewer 3:}

The reviewer noted that Navistar had many collaborators.

\section{Reviewer 4:}

The reviewer commented that Navistar has worked with various Tier 1 suppliers and two national laboratories in this effort to evaluate various technologies, and the work seems to be well coordinated.

\section{Reviewer 5:}

The reviewer remarked that there was a large number of active partners contributing to the program. 


\section{Reviewer 6:}

The reviewer suggested that working with so many partners, considering the lack of resource internally, would be the best way to go at this stage.

\section{Question 4: Proposed future research-the degree to which the project has effectively planned its future work in a logical manner by incorporating appropriate decision points, considering barriers to the realization of the technology and, when sensible, mitigating risk by providing alternate development pathways.}

\section{Reviewer 1:}

The reviewer commented that the program is coming to an end and Navistar had a good plan to finish this program.

\section{Reviewer 2:}

The reviewer wanted to see at least future DOE 55\% BTE participants pursue a dual fuel LTC approach. In the reviewer's opinion, Navistar seems best poised and most serious to do this. Achieving 55\% BTE is very difficult so the reviewer had expected a winnowing by any participants to common approaches. The reviewer asserted that it is best to spend public money for this on diversification so reviewers can get a peek at a range of technologies.

\section{Reviewer 3:}

The reviewer said that the project is successfully completed and with an excellent result to meet the 50\% BTE goal and modeled the $55 \%$ BTE target with a rational plan to move forward. Success factors, as a result of SuperTruck, are intensely integrated modeling design approaches across the supply chain, an industry improved focus, and a highly upgraded capability to analyze, design, and apply critical efficiency technologies to commercial truck engines.

\section{Reviewer 4:}

The reviewer had no comment, saying comments were not applicable for this project as it is ending.

\section{Reviewer 5:}

The reviewer noted that the pathway to 55\% BTE engine appears to rely on dual-fuel technology, a prospect that is less desirable to most customers.

\section{Reviewer 6:}

The reviewer noted that it is not clear how Navistar can achieve the 55\% BTE goal, even with analytical solution.

\section{Question 5: Does this project support the overall DOE objectives of petroleum displacement? Why or why not?}

\section{Reviewer 1:}

The reviewer commented that if this project results in improved high efficiency truck engines, the achievement could severely reduce our nation's overall fuel consumption. This in turn could lead to reduced $\mathrm{CO}_{2}$ emissions and environmental impact.

\section{Reviewer 2:}

The reviewer noted that there were large petroleum savings.

\section{Reviewer 3:}

The reviewer remarked that $50 \%$ BTE technologies on the path to production will definitely improve petroleum displacement in the high fuel consumption Class $8 \mathrm{HD}$ truck sector. The 55\% pathway modeled provides a road map for industry and DOE to eliminate barriers for production engines to perform at superefficient levels.

\section{Reviewer 4:}

The reviewer said yes.

\section{Question 6: Resources: How sufficient are the resources for the project to achieve the stated milestones in a timely fashion?}




\section{Reviewer 1:}

The reviewer noted that the resources seem to have been appropriate to the effort required.

\section{Reviewer 2:}

The reviewer commented that obviously Navistar lacked resources to keep the project going, but the reviewer thought the company recovered splendidly.

\section{Reviewer 3:}

The reviewer observed that the very large budget coupled with substantial industry commitment has resulted in outstanding results for this project and the entire SuperTruck effort. The momentum is a great start for further acceleration of efficiency and smart, rational electrification going forward.

\section{Reviewer 4:}

The reviewer noted that, with the time running down and lack of internal resources, the project team had to rely more on suppliers. The reviewer thought that with their help, the project team should be able to achieve the goal. However, time is not on the team's side because it is way behind other competitors.

\section{Reviewer 5:}

The reviewer stated that even after accounting for the fact that Navistar has suffered financial difficulties, which seem to seep into the technical progress of this effort, the progress made seems less than that of other companies. Unless Navistar comes through with final outstanding results, the $\$ 40$ million investment from DOE needs to be considered excessive compared to the returns. 


\section{Volvo SuperTruck - Powertrain Technologies for Efficiency Improvement: Pascal Amar (Volvo Trucks) - ace060}

Presenter

John Gibble, Volvo Trucks

\section{Reviewer Sample Size}

A total of seven reviewers evaluated this project.

\section{Question 1: Approach to performing the work-the degree to which technical barriers are addressed, the project is well-designed, feasible, and integrated with other efforts.}

\section{Reviewer 1:}

The reviewer commented that the approach used in the project was outstanding. The team took aggressive approaches including novel combustion strategies to achieve the fuel efficiency gains. Furthermore, the project team used a systems-level approach to maximize efficiency from several components, which also was the key to the team's success.

\section{Reviewer 2:}

The reviewer stated that this was an interesting program. Volvo has developed a unique approach to achieving 55\% BTE. It will be very interesting to see if the company is successful. Personally, the reviewer expressed concern about the increased losses associated with the additional gas exchange processes Volvo is introducing.

\section{Reviewer 3:}

The reviewer commented that Volvo is unique in elucidating its approach: first, evaluate the best technologies for hitting the end point, $55 \%$ BTE, and then apply the best to meeting the immediate goal of 50\% BTE. The expenditures up front might have appeared to not be an efficient engineering approach to meeting the short-term objective, but it appears to have been quite successful.

\section{Reviewer 4:}

The reviewer found the project to be well designed and thought over: the design targets for the 55\% BTE engine were first scoped through simulations, which in turn determined the scope and technology mix for the 50\% BTE engine. The 50\% BTE engine's performance was validated through dynamometer tests. Finally, the 50\% BTE engine along with a mix of vehicle technologies was integrated into a final demonstration vehicle exhibiting $88 \%$

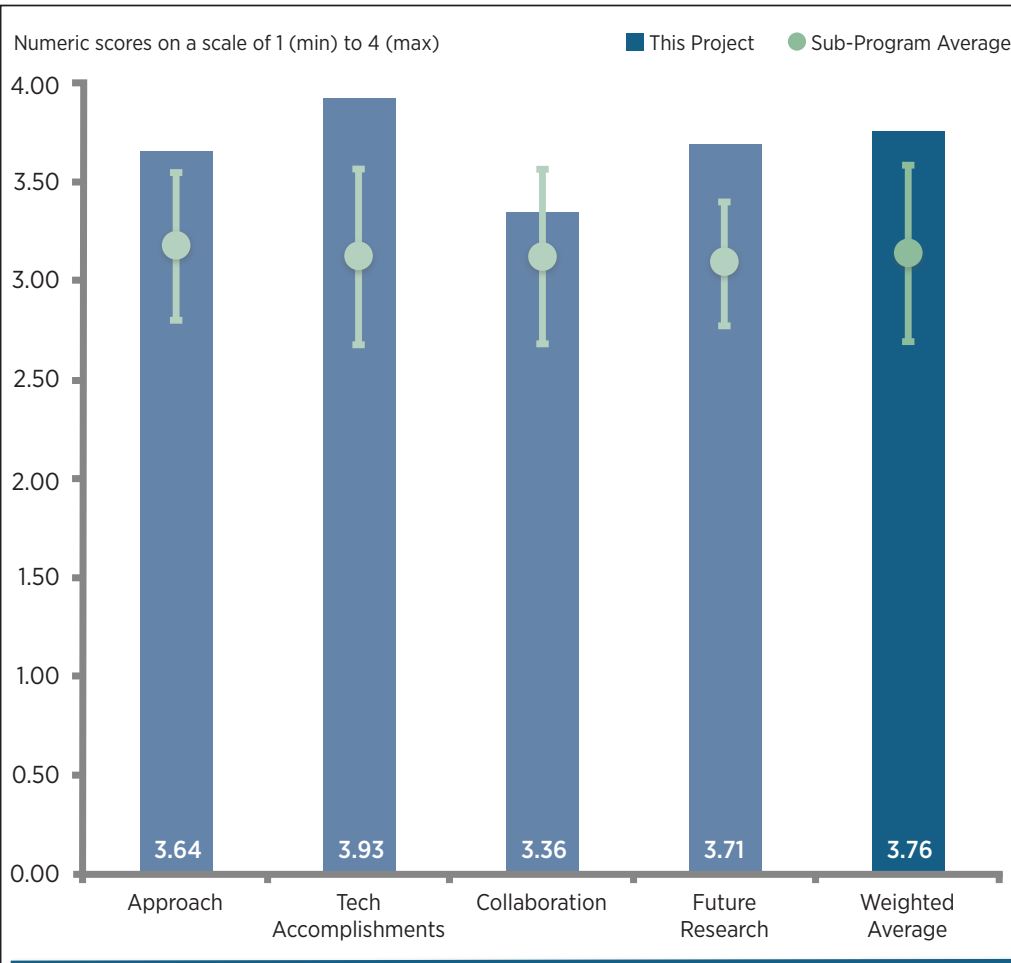

Relevant to DOE Objectives

Sufficiency of Resources
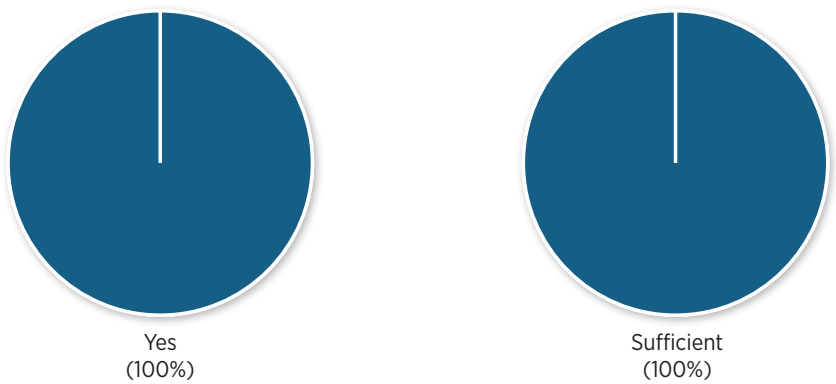

Figure 4-26 - Volvo SuperTruck - Powertrain Technologies for Efficiency Improvement: Pascal Amar (Volvo Trucks) Advanced Combustion Engines 
improvement in vehicle efficiency. Yet, the reviewer stated that the only reason for concern is the high CR concept that uses two cylinders: one for compression and the second one for combustion. Similar concepts are being proposed elsewhere and appear to offer promise. However, considering the fact that the real estate under the hood is limited, the proposed concept might not be viable for transportation purposes.

\section{Reviewer 5:}

The reviewer remarked that Volvo used an excellent modeling approach to achieve 55\% engine BTE using an integrated computational approach (CFD, GT POWER, probability density function [PDF], and chemical kinetics) with confirmation peer review of assumptions and experimental testing where possible, such as in an optical engine.

\section{Reviewer 6:}

The reviewer commented that Volvo had an interesting, alternate approach to achieving the 55\% BTE goal of SuperTruck I. Some clarification as to the real world feasibility of this approach would have been helpful to differentiate this from an academic paper study using 55:1 CR strategy.

\section{Reviewer 7:}

Regarding the 55\% BTE goal, the reviewer was not convinced that a new novel engine concept would be the way to go mainly because of the modeling fidelity. Because this concept has not been tested in a multi-cylinder dynamometer cell, the combustion models cannot be validated. If the 3D and kinetic combustion models are used to calibrate the GT-POWER model, the fidelity of the model would be in question. The 300-bar peak cylinder pressure (PCP) is way too high, which can change the chemistry of the modeling base that we have not dealt with in a conventional engine As a result, the heat release in the GT-POWER model may run too fast, thus predicting unrealistic performance. Furthermore, the temperature can be very high, which can have a big impact on $\mathrm{NO}_{\mathrm{x}}$ emissions. In addition, the pumping loss would be huge, and how the team models the flow loss through valves is unknown. The reviewer is not sure if this engine is 2010 emission compliant.

Regarding 50\% BTE, the reviewer said that it is not clear whether turbocompounding and WHR with Rankine cycle technologies would be used together. If so, there is little or no chance that this type of technology would be accepted by the customer. The reviewer asked for clarification and stated that it would not be a good idea to put all technologies into one presentation, unless it is really used together to demonstrate a $50 \%$ goal.

\section{Question 2: Technical accomplishments and progress toward overall project and DOE goals-the degree to which progress has been made, measured against performance indicators and demonstrated progress towards DOE goals.}

\section{Reviewer 1:}

The reviewer noted that the piston bowl design achieved remarkable results, and it was great to see the amount of SuperTruck developed technology that is making its way into product.

\section{Reviewer 2:}

The reviewer complimented the project team on a very nice job completing the work and was particularly impressed with the team's plans for a 2017 commercial introduction of technologies developed in this program. That is the sign of a very successful program.

\section{Reviewer 3:}

The reviewer commented that the team made excellent gains in fuel efficiency. It was very encouraging to hear the volume of fuel already saved using the introduction of SuperTruck technologies into Volvo trucks.

\section{Reviewer 4:}

The reviewer remarked that reaching the $50 \%$ goal is amazing with one year shorter than their competitors, considering that the project team was two years behind at the beginning and then made up one year. The reviewer enthused well done.

\section{Reviewer 5:}

The reviewer praised the outstanding results overall for 50\% BTE engine demonstration with multiple technologies 
graduating as technology to the customer on production engines (injection system, wave piston, turbocompounding unit, and aftertreatment system). The reviewer noted that results on modeling with a pathway to achieve $56 \%$ engine BTE were published in an SAE paper.

\section{Reviewer 6:}

The reviewer commented that like the others, Volvo developed technologies in the 50\% BTE stage that will make it to market. However, as the company elucidated these technologies more than the others, it appears that more technologies and higher efficiencies will be delivered. The wave bowl design combined with 3,000 bar pressure is valuable, unique (at least at 3,000 bar), and impressive. Turbocompounding has been used a number of years by Volvo and Daimler, and the box design of aftertreatment is coming over from Europe. Nonetheless, the DOE work seems to have helped move these to the next stage. The five-stage WHR steam turbine also is impressive.

The results on 55\% BTE at this stage are very advanced compared to others. This is a direct result of Volvo's longterm approach. If the company can pull off the new combustion strategy that delivers a 55:1 CR, Volvo is uniquely estimating that only a $1 \%$ BTE point is needed from WHR to attain $55 \%$ BTE. If so, the company is a short shot away from eliminating WHR. That is a unique concept.

\section{Reviewer 7:}

The reviewer noted that all the program targets were met or exceeded. However, the outstanding contribution of this effort are the technologies - wave piston, high-pressure fuel injection system, improved aftertreatment system, turbocompounding, etc. - that are likely to make it into the 2017 model year (MY) Volvo vehicles. Very rarely do technologies developed in an R\&D effort make it to a final product. This effort appears to be an exception.

\section{Question 3: Collaboration and coordination with other institutions.}

\section{Reviewer 1:}

The reviewer asserted that there was a good interface with universities.

\section{Reviewer 2:}

Per the slide deck that was provided for developing the 55\% BTE engine, the reviewer noted that CFD simulations were performed in collaboration with Pennsylvania State University (Penn State). Also, single- cylinder engine tests and GT-POWER simulations were carried out at University of Michigan (UM) and Lund University.

\section{Reviewer 3:}

The reviewer effused about the impressive use of advanced modeling methods to derive the 55\% BTE approach. The reviewer suggested that the Lund collaboration in particular likely resulted in the unique $55 \%$ BTE combustion approach.

\section{Reviewer 4:}

The reviewer remarked that this project had a good combination of industrial and academic collaborators, which helped contribute to successfully meeting the program goals.

\section{Reviewer 5:}

The reviewer noted that it was difficult to improve collaboration. Strong universities were well represented and tasked for modeling expertise (i.e., UM, Penn State, and Lund University). The reviewer noted that critical engineering and Tier 1 partners were also incorporated for testing and improving the innovation to market path (Delphi and Ricardo) and consulting for lubricants from the petroleum industry (Mobil).

\section{Reviewer 6:}

The reviewer commented that it is good to see the team work with so many partners.

\section{Reviewer 7:}

The reviewer found that the team had collaborations and a good working relationship among the team members, but collaborations could be improved by broadening the team beyond the suppliers who were the major collaboration partners. 
Question 4: Proposed future research-the degree to which the project has effectively planned its future work in a logical manner by incorporating appropriate decision points, considering barriers to the realization of the technology and, when sensible, mitigating risk by providing alternate development pathways.

\section{Reviewer 1:}

The reviewer noted that this project is over, but hopes that work on Volvo's proposed 55\% BTE concept will continue and be reported in public forums in the future.

\section{Reviewer 2:}

The reviewer stated that Volvo appears to be offering a high-risk, unique approach to meet the 55\% BTE goal. Many have proposed these unique compression cylinder engines. The high CRs, sometimes more than 100:1, offer high efficiency. However, no one has been able to make a practical engine. The reviewer said that this is well-worth DOE funds and a good expenditure of public money. Volvo appears uniquely poised to deliver a 55\% BTE engine with minimal Rankine cycle WHR.

\section{Reviewer 3:}

The reviewer commented that the project has come to an end. Future incorporation of technologies developed in this program into product in the marketplace speak highly of the approach taken by this SuperTruck I team.

\section{Reviewer 4:}

The reviewer remarked that the project is an outstanding success and complete. The lessons learned are DOE funding has enabled parallel engineering efforts in complete engine systems design and vehicle design that facilitated step improvements with an integrated approach. Modeling capabilities to accelerate development and engineering have been significantly improved and more intensely integrated and operationalized into the design process to improve product development going forward. The stretch 55\% BTE goals are a real roadmap for future designs.

\section{Reviewer 5:}

The reviewer said that the project is near its end so there is not much to report on regarding future research.

\section{Reviewer 6:}

The reviewer stated that because the project has ended, this criterion does not apply.

\section{Reviewer 7:}

The reviewer asserted that working with universities on a new engine concept is good. However, the reviewer was not convinced that the new engine concept would be needed, which can diversify the funding source, which may not be a good investment in terms of the future of the product.

\section{Question 5: Does this project support the overall DOE objectives of petroleum displacement? Why or why not?}

\section{Reviewer 1:}

The reviewer commented that the planned commercial introduction of many of the technologies developed in this program is a testimony to its relevance.

\section{Reviewer 2:}

The reviewer stated that the SuperTruck I goals were exceeded, directly supporting DOE objectives.

\section{Reviewer 3:}

The reviewer noted that per Volvo's projections, the 50\% BTE engine concepts to be introduced into its engines are likely to save 120 million gallons of (diesel) fuel consumption spread over the next 5 years.

\section{Reviewer 4:}

The reviewer found the success factor to be clear: multiple engine-efficiency technologies graduate to market at an accelerated pace through the SuperTruck program. 


\section{Reviewer 5:}

The reviewer remarked that the project is highly relevant to DOE objectives for petroleum displacement because truck fuel-efficiency gains are central to the project's goals and deliverables. The project has already realized petroleum displacement.

Reviewer 6:

The reviewer said yes.

\section{Question 6: Resources: How sufficient are the resources for the project to achieve the stated milestones in a timely fashion?}

Reviewer 1:

The reviewer stated that this was a good use of DOE funds, investing in technology development that is making its way into consumers' hands.

Reviewer 2:

The reviewer noted that the project team had a large budget and accomplished large results for a well-managed and delivered project.

Reviewer 3:

The reviewer commented that Volvo had already achieved the program goal. 


\section{Advancements in Fuel Spray and Combustion Modeling with High-Performance Computing Resources: Sibendu Som (Argonne National Laboratory) - ace075}

\section{Presenter}

Sibendu Som, Argonne National Laboratory

\section{Reviewer Sample Size}

A total of three reviewers evaluated this project.

\section{Question 1: Approach to performing the work-the degree to which technical barriers are addressed, the project is well-designed, feasible, and integrated with other efforts.}

\section{Reviewer 1:}

The reviewer said that the work provides a good approach seeking to minimize excessive tuning of models to experimental data and promoting improved predictive simulations with higher fidelity models. The work focuses on detailed chemistry combustion models, finer mesh for grid-convergence, high-fidelity LES turbulence models, and two-phase physics-based fuel spray and nozzleflow models. This is combined with high-performance computing facilities.

\section{Reviewer 2:}

The reviewer commented that the work is moving towards predictive simulation of the ICE with more high fidelity codes by finding ways to make them faster. Now the project team is working on capacity computing, where some fidelity is sacrificed for speed and quantity of cases simulated.

\section{Question 2: Technical accomplishments and progress toward overall project and DOE goals-the degree to which progress has been made, measured against performance indicators and demonstrated progress towards DOE goals.}

\section{Reviewer 1:}

The reviewer noted that the speedup achieved with the tabulated equivalent strain flamelet (TESF) model is good progress in an important direction. The project team seemed to assert that all of the significant observed variation in individual nozzle flow rates is due to upstream flow conditions in the injector. The reviewer is skeptical of this claim and recommends a careful review and/or experimental and/or UQ consideration of other sources of variation, including orifice geometry, etc.

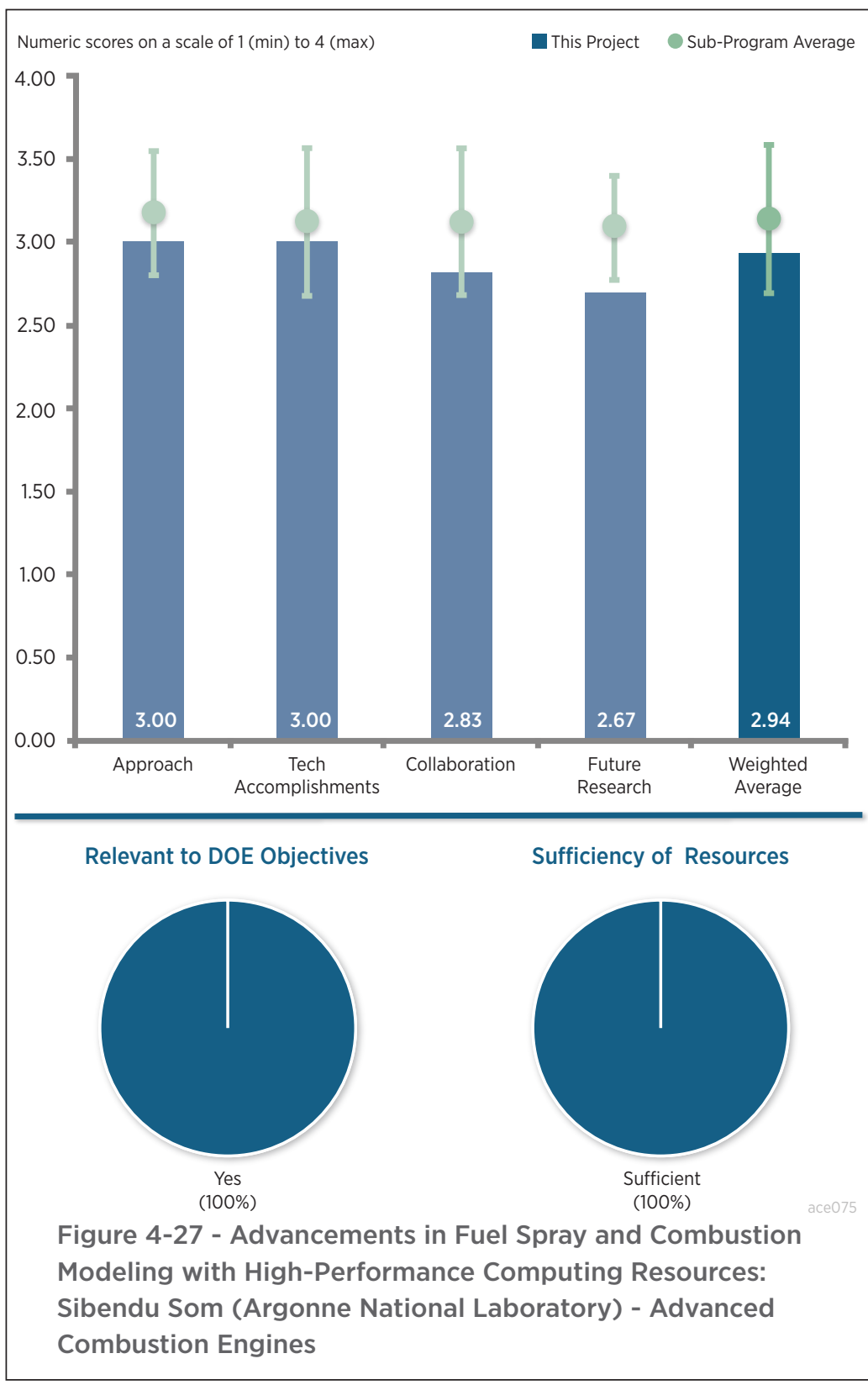




\section{Reviewer 2:}

The reviewer remarked that the work is technically sound. It covers several distinct areas of work. The gasolineinjector modeling features an overall assessment leading to more effective simulations, consideration of flash boiling, and spray work including useful discussions on phase change and effects of backflow. The reviewer asked whether the project team could clarify if this work is being benchmarked with experimental studies and whether the team could explain the novelty of their cavitation work and compare it with the current state-of-the-art.

The reviewer stated that the LES modeling continues to be of great interest, in particular the plume-merging studies. The reviewer questioned if the authors would be able to apply these to selective engine cases to assess and provide what significant improvements may be attained in the context of emissions and fuel economy.

The reviewer would have liked to have seen how the earlier work on wobble, optimized reduced mechanisms for a diesel surrogate, and dribble mass predictions are being utilized. Some of these were indicated as future work in 2015.

\section{Reviewer 3:}

The reviewer stated that progress has been good. The TESF model has been developed that can simulate the turbulent chemistry cost effectively. Collapsing phenomena have been observed for flash boiling conditions as well as high back-pressure conditions. The reviewer remarked that collapsing of sprays at both these conditions is now being predicted, especially with LES. In addition, the details of the entrainment flow measured by SNL are also being predicted.

\section{Question 3: Collaboration and coordination with other institutions.}

\section{Reviewer 1:}

The reviewer found the team to be very good.

\section{Reviewer 2:}

The reviewer complimented the very good collaboration existing among other national laboratories, universities, Convergent Science, and industry.

\section{Reviewer 3:}

The reviewer noted that leveraging ASCR resources for HPC using industrially relevant software and enginedesign use cases by means of collaboration were all good. However, in this mode the reviewer pointed out that it is crucial to avoid (in reality or perception) unfair subsidies for product development for a single chosen partner. The reviewer commented that DOE should ensure this project does not result in unfair subsidies for any specific commercial partner and does not create such an appearance now, or in the future. Source code access for spray and HPC could lead to post-competitive product development if DOE is not careful.

The reviewer stated that mitigation of these issues should be considered in one or both of two ways: removing those activities from the project scope and/or a deliberate multi-code strategy. Because conclusions or calibrations are historically proven to be artificially shaped (to varying degrees) by the peculiar limitations, methods, or assumptions of an individual simulation tool, the latter redundancy approach can add value by not only avoiding such distortions but (frequently, from past experience) also by exposing otherwise-hidden lessons and uncertainty sources.

\section{Question 4: Proposed future research-the degree to which the project has effectively planned its future work in a logical manner by incorporating appropriate decision points, considering barriers to the realization of the technology and, when sensible, mitigating risk by providing alternate development pathways.}

\section{Reviewer 1:}

The reviewer noted that future research is clearly indicated. The authors could establish targeting selective engine cases to assess improvements in the context of emissions and fuel economy. This may be done in closer collaboration and allocating some of the resources with engine OEMs. 


\section{Reviewer 2:}

The reviewer suggested that the model, with its new developments and capacity computing, should be exercised on a small subset of engine design, for example, just spray geometry variables that affect spray characteristics.

The following spray variables can be swept over relevant ranges of interest: L/D; number of plumes; spray angle; nozzle entrance effects; nozzle surface roughness; pre-hole diameter; fuel temperature; and pitch circle.

\section{Question 5: Does this project support the overall DOE objectives of petroleum} displacement? Why or why not?

No comments were received in response to this question.

Question 6: Resources: How sufficient are the resources for the project to achieve the stated milestones in a timely fashion?

No comments were received in response to this question. 


\section{Improved Solvers for Advanced Engine Combustion Simulation: Matthew McNenly (Lawrence Livermore National Laboratory) - ace076}

\section{Presenter}

Matthew McNenly, Lawrence

Livermore National Laboratory

\section{Reviewer Sample Size}

A total of six reviewers evaluated this project.

\section{Question 1: Approach to performing the work-the degree to which technical barriers are addressed, the project is well-designed, feasible, and integrated with other efforts.}

\section{Reviewer 1:}

The reviewer commented that the project team gave a good, clear presentation of its approach, which was tied meaningfully to others' efforts.

\section{Reviewer 2:}

The reviewer remarked that the approach has been reasonably proven and has yielded excellent results.

\section{Reviewer 3:}

The reviewer commented that the broad approach is similar to that used in past years. The project team is creating reactor Jacobians for LLNL's chemistry algorithms. This effort is anticipated to reduce computational time significantly.

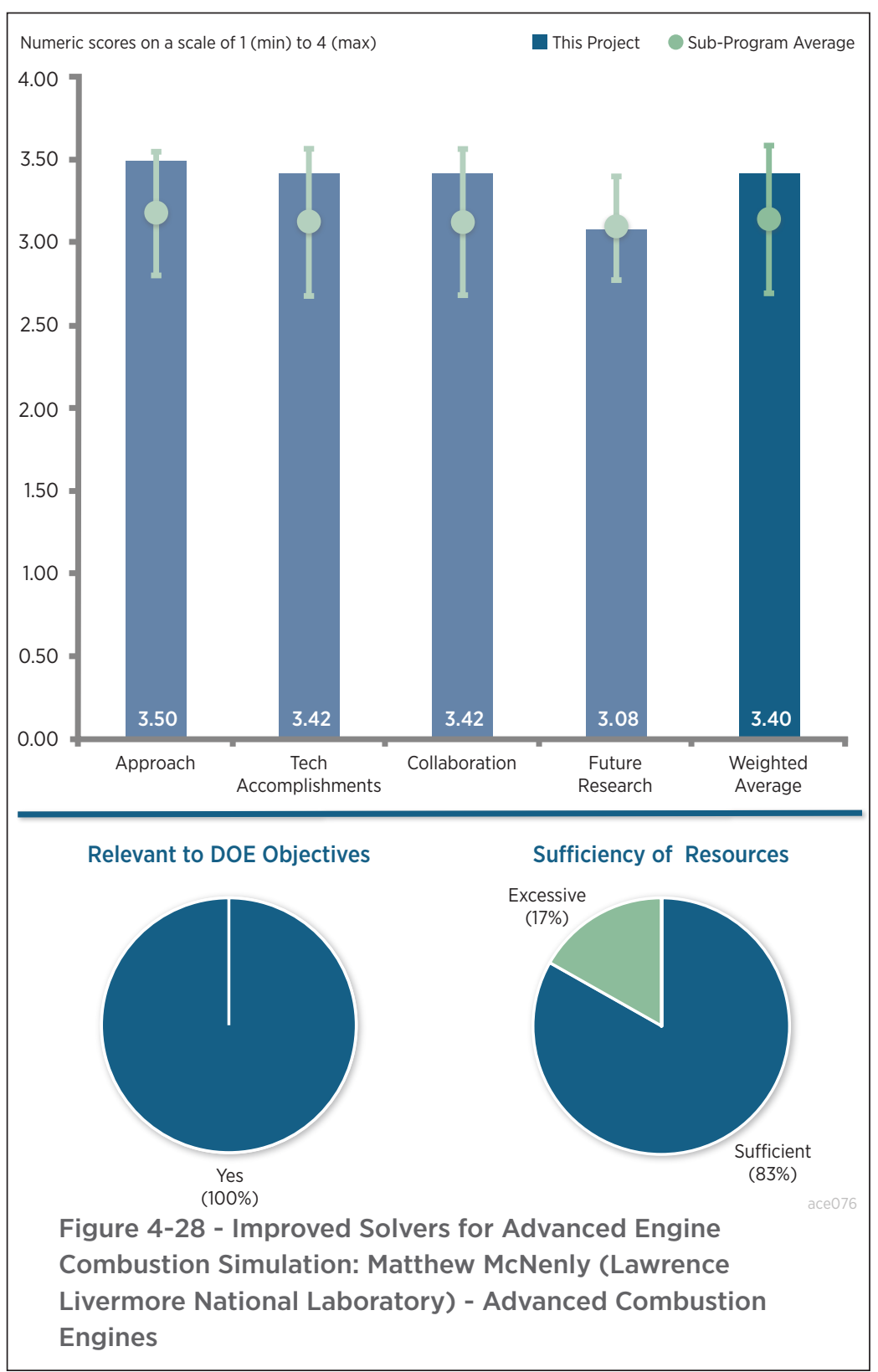

Some simulation results were shown for a homogeneous reactor. The reviewer asked the project team to please provide a rationale for the relevance of this configuration to an engine. The chemistry solver is incorporated with CONVERGE, and results are shown for HCCI simulation. The reviewer commented that the approach of verification/validation of the chemistry solver with a $1 \mathrm{D}$ counterflow flame should be further discussed. This configuration is a bit removed from an engine environment and does not include any multiphase effects such as multicomponent vaporization which is intrinsic to both certain engine conditions and most certainly multicomponent surrogates of the type this project is investigating. The reviewer would like an explanation.

\section{Reviewer 4:}

The reviewer noted that the project team is developing an advanced mathematical method to solve full mechanisms of multicomponent fuels, which has the potential to improve the design tool for industrial engine design and optimization. The demonstrated computational efficiency for multicomponent fuels (more than 2,000 species) 
makes it feasible for full 3D CFD simulations with full mechanisms for chemistry in an affordable time although it has to demonstrate its scalability within the 3D CFD solver on an HPC/GPU.

\section{Reviewer 5:}

The reviewer commented about the impressive portfolio of modeling tools and improvements to those tools but found it hard to determine the accuracy of the simulations (i.e., compared to experimental data) from the presentation content. The reviewer suggested that perhaps the project team should include some detail on how the simulations results are confirmed in future reviews.

\section{Question 2: Technical accomplishments and progress toward overall project and DOE goals-the degree to which progress has been made, measured against performance indicators and demonstrated progress towards DOE goals.}

\section{Reviewer 1:}

The reviewer remarked that being able to significantly reduce the computer time while obtaining the same solution is outstanding.

\section{Reviewer 2:}

The reviewer commented that better treatments to support soot prediction are a welcome and valuable direction of progress. The research on heuristic logic for automated mechanism debugging and timestep improvement is innovative and broadly valuable. The reviewer enthusiastically looked forward to seeing the planned report and user guide.

\section{Reviewer 3:}

The reviewer congratulated the project team for earning a 2015 R\&D 100 award. The solver has demonstrated good speed-up on a homogenous reactor. The reviewer found some issues that needed improvement or clarification. For example, the reviewer observed that the accuracy of the faster solver was not well validated. The reviewer questioned how the solver would perform in a heterogeneous reactor, e.g., in-cylinder combustion. Lastly, the reviewer asked whether this solver/algorithm is robust/effective for different multicomponent fuels other than the demonstrated fuels.

\section{Reviewer 4:}

The reviewer noted that $\mathrm{C}_{20} \mathrm{H}_{42}$ is listed as a biodiesel component, and that this needs some further elaboration. As a general rule, it will not be a biodiesel component. The reviewer remarked that it depends highly on the biofeedstock. For example, algae will yield different dominant species from rapeseed, which in turn will be very different from a fatty acid methyl ethers (FAME) fuel.

The reviewer commented that there is confusion about the fuel systems being investigated. The project team notes a 9-component surrogate last year, then a 12-component surrogate this year, and yet further a 13-component surrogate this year. The reviewer noted that an improved cetane mechanism is being incorporated into the model development and stated that this is confusing. The reviewer asked that the project team please try to bring some clarity and rationale to the choice of surrogate components.

The reviewer noted that some IDT data for methyl decanoate (MD) are included, but asked about the relevance of MD and whether it covered the performance for a FAME (biodiesel) fuel. The reviewer wanted further discussion of this issue. For the surrogates investigated, the reviewer questioned why they have to contain tens of thousands of reactions as it seemed a bit of overkill. LLNL has capabilities for mechanism reduction so this concern needs greater clarity.

The reviewer commented that the Zero-RK award is impressive and offered congratulations. Among the things that are listed as important to add to the Zero-RK model are a pressure-dependent reaction rate table and large molecules. The reviewer asked if Zero-RK could be incorporated into CFD code that addresses multiphase sprayinjection effects.

The reviewer asked about how the soot is handled. Presumably gaseous precursors can be computed (e.g., polycyclic aromatic hydrocarbons [PAHs], acetylene, etc.). It would be instructive to compute a flow configuration 
and predict the distribution of soot precursors, which presumably can be done right now. Then, when a soot model is incorporated, it would be useful to see how the distribution of soot aggregates track with the predictions of the precursors.

\section{Question 3: Collaboration and coordination with other institutions.}

\section{Reviewer 1:}

The reviewer noted that the project has collaborations with academia, industry, and national laboratories to have solver development/validation, make the solver accessible to industry, and closely work with industry on the applications of the solver.

\section{Reviewer 2:}

The reviewer stated that the collaborators on this project are adequate and span the range from academia, industry, and national laboratories. The project includes several industries that market or use computer codes (Convergent Sciences, Cummins, GM, and NVIDIA). The reviewer would like an explanation as to why King Abdullah University of Science and Technology is not listed among the academic collaborators, while the university is providing some data.

\section{Reviewer 3:}

The reviewer observed that Co-Optima has clearly increased the depth of collaboration.

\section{Reviewer 4:}

The reviewer said that the team has collaborated with other institutions, including academics and industry.

\section{Reviewer 5:}

The reviewer noted that GM is evaluating tools in CONVERGE and said to continue to pursue collaborations with OEMs.

\section{Question 4: Proposed future research-the degree to which the project has effectively planned its future work in a logical manner by incorporating appropriate decision points, considering barriers to the realization of the technology and, when sensible, mitigating risk by providing alternate development pathways.}

\section{Reviewer 1:}

The reviewer commented that a range of challenges for future work is listed, one of which is soot. The project team may be able to get some insights about soot with the team's current capabilities by simply tracking predicted soot precursor distributions and use this information to compare with measured PM emissions from a suitable combustion configuration. The reviewer suggested that an engine may be too complex to use as an experimental configuration for this purpose, but a combustion fluid (CF) flame might work. The reviewer noticed that spray dynamics are listed as remaining challenges and asked whether this include multicomponent evaporation processes. The reviewer wanted to know the meaning of nonlinear fuel component interactions.

\section{Reviewer 2:}

The reviewer remarked that turbulence/chemistry interaction closure is an appropriate focus and may change some of the present conclusions relative to mechanism optimization and computational approaches. This future work may necessitate closer collaboration with the CFD solvers that utilize Zero-RK, and the reviewer recommended a deliberate multi-code strategy to ensure the greatest impact on the industry.

\section{Reviewer 3:}

The reviewer commented that the proposed research will extend the solver with new soot model algorithms and chemistry-turbulence interaction, which would potentially improve soot prediction and solution of chemistry in a turbulent flow using considering transport effects. However, it does not provide feasible approaches and potential barriers/alternatives.

\section{Reviewer 4:}

The reviewer encouraged future work in simulating practical engine combustion using CFD. Soot modeling is also necessary at the next step. However, the reviewer did not encourage tackling the problem of spray dynamics 
because the spray model uses a parcel approach and does not truly represent the fuel drops and spray dynamics are heavily influenced by turbulence. The reviewer noted that this project is mainly focused on the chemistry solver; thus, it does not seem logical to tackle the problem of turbulence. Nonetheless, the reviewer encouraged the project team to pursue the problem of turbulence-chemistry interactions.

\section{Question 5: Does this project support the overall DOE objectives of petroleum displacement? Why or why not?}

\section{Reviewer 1:}

The reviewer commented that better, faster chemistry will aid in the development of better, more efficient engines that burn less petroleum.

\section{Reviewer 2:}

The reviewer noted that this work can be applicable to biofuel combustion simulation.

Reviewer 3:

The reviewer responded, yes, from a broad perspective.

\section{Question 6: Resources: How sufficient are the resources for the project to achieve the} stated milestones in a timely fashion?

\section{Reviewer 1:}

The reviewer found the resources to be adequate for the planned activity.

\section{Reviewer 2:}

The reviewer said that the resources are appropriate.

\section{Reviewer 3:}

The reviewer stated that resources seem adequate although ultimate judgement would have to come from a cost/ benefit analysis based on DOE's investment relative to the commercialization potential. 


\section{Cummins-ORNL\FEERC Combustion CRADA: Characterization and Reduction of Combustion Variations: Bill Partridge (Oak Ridge National Laboratory) - ace077}

\section{Presenter}

Bill Partridge, Oak Ridge National Laboratory

\section{Reviewer Sample Size}

A total of four reviewers evaluated this project.

\section{Question 1: Approach to performing the work-the degree to which technical barriers are addressed, the project is well-designed, feasible, and integrated with other efforts.}

\section{Reviewer 1:}

The reviewer stated that the project seeks to assess fluctuations in cylinder charge and to apply remedies in hardware and control strategies. The results will be improved combustion uniformity and implementation of advanced combustion strategies.

\section{Reviewer 2:}

The reviewer commented that the project team used a good approach relative to developing the new diagnostic capabilities. The reviewer understood that this is a CRADA, but stated it would be good to see what insights are learned given the capability of modern CFD tools.

The reviewer asked if tools are able to accurately match (i.e., predict) the measurement. If required, the reviewer suggested that maybe this can be done independently of the CRADA partner, if that is what is required to make the information public.

\section{Reviewer 3:}

The reviewer noted that this project addresses the need for accurate and efficient diagnostics for in-cylinder processes in engines. The project team has developed a range of diagnostics for engines, including the development of an EGR probe that has proven to be quite effective that the team continues to improve, a wavelength modulation spectroscopy method that improves the signal-to-noise ratio (SNR), a method to measure cycle-to-cycle variations in oxygen concentration, and a diagnostic to measure exhaust transients.

The reviewer observed that the overall importance of diagnostics to evaluate engine performance cannot be overstated. In that regard, the project is relevant. In developing the project team's approach, the reviewer remarked that it may be beneficial for the team, and indeed all the national laboratories, to consider developing some sort 
of a diagnostics center spread across the laboratories that catalogues capabilities. This effort could begin with making a list of all laboratory capabilities, who does what, identifying what needs to be measured that cannot now be measured, and then coordinating activities. In this project, as an example, the EGR probe is interesting, and the reviewer asked whether other laboratories have a need for it. The reviewer presumed the answer to be yes.

In the development and application of the diagnostics mentioned, the reviewer commented that it would be relevant to compare capabilities against competing technologies. Though this may have been done in prior presentations, the reviewer stated that it would be useful to make the comparison part of the presentation of experimental results.

\section{Question 2: Technical accomplishments and progress toward overall project and DOE goals-the degree to which progress has been made, measured against performance indicators and demonstrated progress towards DOE goals.}

\section{Reviewer 1:}

The reviewer thought it was impressive that ORNL was able to improve the signal and reduce the noise for the new probe design. The reviewer commented that it would have been good to have the on-engine results performed sooner so they could have been included in the presentation.

\section{Reviewer 2:}

The reviewer found the work presented to be practical and valuable and an example of a well-run CRADA. The work studied back-flow measurements using a multi-color EGR probe. The probe is effective in estimating the exhaust gases moving upstream of the intake port during the engine valve overlap. This year's work has focused on analysis of the cylinder charge. The probe has been reworked to improve the data quality and ability to capture fast transients. Some questions arise regarding the uncertainty evaluation of the measurements. There is no formal treatment of uncertainty (e.g., benchmarking the optical technique with gas analyzers).

\section{Reviewer 3:}

The project team reported EGR probe measurements of $\mathrm{CO}_{2}$ and $\mathrm{H}_{2} \mathrm{O}$ in-cylinder measurements, developed a new probe design with improved SNR, and developed a new diagnostic for measurement of exhaust $\mathrm{O}_{2}, \mathrm{H}_{2} \mathrm{O}$, temperature, and pressure. The effectiveness of these diagnostics was demonstrated with some experimental results. The reviewer thought it would be useful for the project team to consider ways to make the EGR more robust such that it could be used in the sooting region of engines.

\section{Question 3: Collaboration and coordination with other institutions.}

\section{Reviewer 1:}

The reviewer remarked that it was a very impressive team, which has been very successful in providing good performance results.

\section{Reviewer 2:}

he reviewer said that there was good collaboration with the CRADA partner.

\section{Reviewer 3:}

The reviewer pointed out that the project team has a CRADA with Cummins that has provided valuable input to the project. Academic partners are also included that have facilitated improved performance of the EGR probe. The reviewer wanted to have better clarity on what each of the partners - from academia and industry - provides to the project and their relevance as doing so would establish the need for the partnership. The reviewer also stated that it would be useful to provide some data from the partners to show how they are folded into the project.

\section{Question 4: Proposed future research-the degree to which the project has effectively planned its future work in a logical manner by incorporating appropriate decision points, considering barriers to the realization of the technology and, when sensible, mitigating risk by providing alternate development pathways.}

\section{Reviewer 1:}

The reviewer commented that future work is indicated as it would be important to develop approaches on how to 
use the information provided to limit the variability on flow. It will be important to understand how this variability influences engine efficiency or causes it to deteriorate.

\section{Reviewer 2:}

The reviewer thought it would be good to see this new diagnostic evaluated on other projects and to see how well current simulation tools are able to predict.

\section{Reviewer 3:}

The reviewer observed that future work will include improving the EGR probe with a new design for collimated fiber output, measuring high temperature exhaust, adding a CO-measurement capability, and developing new measurements for parameters. The reviewer stated that these tasks are relevant though are a bit vague. More specificity would be advantageous to the understanding of the research going forward. Presumably, at some point the probe development effort will be completed. The reviewer asked when the EGR probe design is expected to become a mature diagnostic.

\section{Question 5: Does this project support the overall DOE objectives of petroleum displacement? Why or why not?}

\section{Reviewer 1:}

The reviewer agreed that reducing variations is a good thing and asked if the CRADA partner can quantify the potential benefit. It would help to gauge the relevance (and budget) of this as compared to other projects.

\section{Reviewer 2:}

From a broad perspective, the reviewer remarked that this project is relevant by its ability to provide new and more accurate measurements of engine performance, though it is somewhat narrowly focused. As noted above, it would be beneficial to combine the efforts here with those from other national laboratories to develop a national consortium of engine diagnostics (e.g., a catalog of engine measurement capabilities). Such an effort could both alert the community at large of diagnostic capabilities and thereby draw attention to the unique capabilities and perhaps also better focus the development and instrument efforts of existing diagnostics (e.g., such as is included as part of this project).

\section{Question 6: Resources: How sufficient are the resources for the project to achieve the stated milestones in a timely fashion?}

\section{Reviewer 1:}

The reviewer noted that resources are sufficient, but again it would be good to see this new diagnostic evaluated on other projects.

\section{Reviewer 2:}

The reviewer stated that resources seem adequate although ultimate judgement would have to come from a cost/ benefit analysis based on DOE's investment relative to the commercialization potential. 
Thermally Stable Ultra-Low Temperature Oxidation Catalysts: Janos Szanyi (Pacific Northwest National Laboratory) - ace078

\section{Presenter}

Janos Szanyi, Pacific Northwest

National Laboratory

\section{Reviewer Sample Size}

A total of four reviewers evaluated this project.

\section{Question 1: Approach to performing the work-the degree to which technical barriers are addressed, the project is well-designed, feasible, and integrated with other efforts.}

\section{Reviewer 1:}

The reviewer found a good blend of experimental work and characterization data. The reviewer commented that there was a nice balanced division of effort between GM and PNNL that harnessed the strengths of both organizations (GM for reactor testing and PNNL for catalyst characterization).

\section{Reviewer 2:}

The reviewer noted that the approach with various characterization tools was strong. The reviewer commented that not knowing the compositions of the excellent catalysts GM brought to the project is a deficiency. The temperature of hydrothermal aging was only $750^{\circ} \mathrm{C}$.

Higher temperatures are important to mimic real aging conditions, in the reviewer's opinion.

\section{Reviewer 3:}

The reviewer stated that this work supports the need for LTAT requested by USCAR and the automotive OEMs. Additionally, this activity also supports the OEM's need for reduced PGM usage and cost by using Cu-based catalysts in place of both platinum ( $\mathrm{Pt}$ ) and palladium (Pd) for CO oxidation. In the past, the use of Cu-based catalysts did not hold up well after hydrothermal aging. In contrast, as this work shows, the reviewer acknowledged that selecting the correct praseodymium ( $\mathrm{Pr}$ ) and $\mathrm{Zr}$ support material can greatly enhance the survivability of the catalyst after hydrothermal aging. A second challenge that was addressed in this work was the resistance to $\mathrm{S}$ poisoning of the $\mathrm{CO}$ oxidation reaction or the ability to regain that function after regeneration. The reviewer noted that using these challenges as part of a benchmarking process for various $\mathrm{Cu}$ formulations easily discriminated the $\mathrm{CO}$ activity of the catalysts tested.

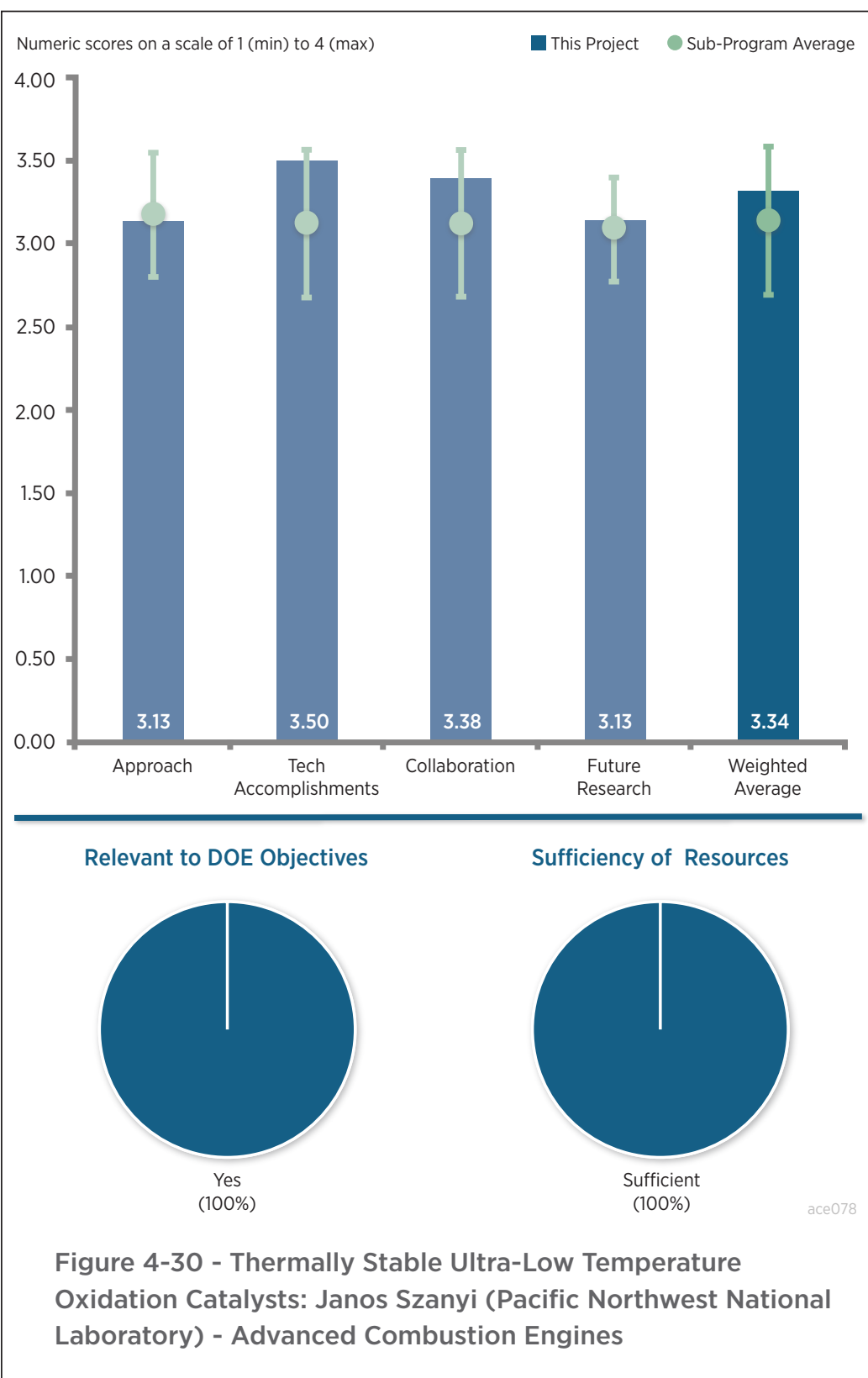




\section{Reviewer 4:}

The reviewer stated that the focus on $\mathrm{C}_{3}$ hydrocarbons is a good start, and moving to longer chains and aromatics would be beneficial. The reviewer remarked that including $\mathrm{S}$ studies in base-metal catalysts is critical, as well as hydrothermal aging. The use of theoretical density functional theory (DFT) in addition to experiments is very nice. PNNL has many instruments to fully analyze and characterize catalyst materials and their behavior. The reviewer commented that the project team should have included $\mathrm{CO}_{2}$ in feedgas. Another reviewer comment was that it would have been nice to look at $\mathrm{HC}$ and $\mathrm{NO}_{\mathrm{x}}$ interactions and the potential to reduce $\mathrm{N}_{2} \mathrm{O}$ formation versus $\mathrm{Pt} / \mathrm{Pd}$ diesel oxidation catalysts (DOCs).

\section{Question 2: Technical accomplishments and progress toward overall project and DOE goals-the degree to which progress has been made, measured against performance indicators and demonstrated progress towards DOE goals.}

\section{Reviewer 1:}

The reviewer commented that the results presented used appropriate gas feeds, fuel poisons, and aging and showed that $\mathrm{Cu}$ formulations incorporating $\mathrm{Pr}$ and $\mathrm{Zr}$ can act as effective $\mathrm{CO}$ oxidation catalysts at low temperatures. Although not resistant to $\mathrm{S}$ poisoning, the catalyst does show the ability to be regenerated at higher temperatures. The characterization work was able to directly link the deactivation of other $\mathrm{Cu}$ formulations to either the loss of surface area or the formation of surface carbonates. The reviewer commented that what was not explained well was why the hydrothermally aged $\mathrm{Cu} / \mathrm{Pr} / \mathrm{Zr}(\mathrm{Cu} / \mathrm{GMR} 6)$ formulation showed a lower light-off temperature than the fresh. This is not expected because the fresh catalyst should have high surface area and should be relatively free of carbonates. With respect to $\mathrm{S}$, requiring $750^{\circ} \mathrm{C}$ for effective regeneration may have a detrimental effect on the long-term activity of the catalyst and limit where in the aftertreatment system the catalyst can be located. Finally, the reviewer commented that formation of $\mathrm{N}_{2} \mathrm{O}$ under the reaction conditions presented in this study must be investigated due to GHG accounting.

\section{Reviewer 2:}

The reviewer commented that the GMR5 and GMR6 catalysts are good, and the GMR6 catalyst even gets better because it shows that lanthanum (La) is a structural stabilizer and Pr increases oxygen mobility. The reviewer noted the interesting result where carbonate formation in the absence of S leads to loss of activity at $175^{\circ} \mathrm{C}$ as well as the interesting isotopic results shown related to oxygen mobility. This reviewer also reported the following: HC and NO light-off impact; $\mathrm{S}$ does not make $\mathrm{Cu}$ sulfate; and just the support. In conclusion, the reviewer observed very interesting results.

\section{Reviewer 3:}

The reviewer said that the work on catalyst fundamentals was excellent, but the reviewer had just some issues with the approach as noted above.

\section{Reviewer 4:}

The reviewer stated that the project team needed to include $\mathrm{CO}_{2}$ during the activity measurements. It will always be present in the exhaust as long as hydrocarbons are combusted, and if carbonate formation caused the deactivation at $175^{\circ} \mathrm{C}$ without $\mathrm{CO}_{2}$ in the feedgas (as shown on Slide 12), the presence of $\mathrm{CO}_{2}$ will only make it worse. The thermal aging assessments (72 hours of high-temperature hydrothermal aging $[\mathrm{HTA}]$ at $750^{\circ} \mathrm{C}$ ) is much more realistic than what was used for the $\mathrm{NO}$ oxidation work last year $\left(1\right.$ hour at $\left.700^{\circ} \mathrm{C}\right)$.

The S poisoning work on Slide 15 demonstrates the well-known S sensitivity of base-metal catalysts as they have to operate at $600^{\circ} \mathrm{C}$ or more to keep them purged of S and to maintain high activity (see SAE 922251). The project team indicated that it assessed S regenerations at $500^{\circ} \mathrm{C}$ and $750^{\circ} \mathrm{C}$, but the reviewer does not recall seeing the performance after the $750^{\circ} \mathrm{C}$ desulfation. The reviewer asked if it was more effective than the $500^{\circ} \mathrm{C}$ desulfation.

The reviewer was not sure what the project team meant in the summary when it said "modest $\mathrm{C}_{3}$ hydrocarbon oxidation activities (...but not $\mathrm{Co}_{2} \mathrm{O}_{3}$ and $\mathrm{Mn}_{2} \mathrm{O}_{3}$ )." The reviewer wanted to know what exhibited more $\mathrm{C}_{3}$ activity than the $\mathrm{Cu} / \mathrm{CeO}_{2}$ catalyst. 


\section{Question 3: Collaboration and coordination with other institutions.}

\section{Reviewer 1:}

The reviewer observed that combining the synthesis and characterization capability of PNNL with GM formulation and testing is a good match for this work. Perhaps including a catalyst supplier to supply additional preparation expertise would benefit the formulation aspect.

\section{Reviewer 2:}

The reviewer pronounced the collaboration between the groups involved to be good.

\section{Reviewer 3:}

The reviewer commented that the GM partner directed the work.

\section{Reviewer 4:}

The reviewer liked the good collaboration with GM and mentioned the commendable division of labor.

Question 4: Proposed future research-the degree to which the project has effectively planned its future work in a logical manner by incorporating appropriate decision points, considering barriers to the realization of the technology and, when sensible, mitigating risk by providing alternate development pathways.

\section{Reviewer 1:}

The reviewer suggested that future work to address any remaining characterization using long chain HCs is appropriate for the intended application.

\section{Reviewer 2:}

The reviewer said that the project ends this year, but it has raised a number of questions that will hopefully be more fully explored.

\section{Reviewer 3:}

The reviewer stated that the project was completed, and final reports and publications will be completed but no new research. The reviewer picked a middle rating here because "not applicable" was not a choice.

\section{Reviewer 4:}

The reviewer remarked that the project is complete and there are no future plans.

\section{Question 5: Does this project support the overall DOE objectives of petroleum displacement? Why or why not?}

\section{Reviewer 1:}

The reviewer stated that this project supports USCAR - U.S. DRIVE initiatives to address the need for effective lean aftertreatment systems and technologies at low temperatures.

\section{Reviewer 2:}

The reviewer commented that catalysts like this are enablers of using more efficient combustion methods that give lower exhaust temperatures.

\section{Reviewer 3:}

The reviewer mentioned that low-temperature DOCs are important to improve fuel efficiency of lean powertrains.

\section{Reviewer 4:}

The reviewer said that low-temperature catalysts will be a requirement for future engines with improved fuel economy that reduce our dependence on foreign oil, as the higher efficiency engines will generate lower exhaust temperatures. 
Question 6: Resources: How sufficient are the resources for the project to achieve the stated milestones in a timely fashion?

Reviewer 1:

The reviewer found no issue with funding or resources in general.

Reviewer 2:

The reviewer stated that a good amount of funding from GM shows industry interest.

Reviewer 3:

The reviewer said that the project is complete. 


\section{High-Efficiency GDI Engine Research, with Emphasis on Ignition Systems: Thomas Wallner (Argonne National Laboratory) - ace084}

\section{Presenter}

Riccardo Scarcelli, Argonne National Laboratory

\section{Reviewer Sample Size}

A total of one reviewer evaluated this project.

\section{Question 1: Approach to performing the work-the degree to which technical barriers are addressed, the project is well-designed, feasible, and integrated with other efforts.}

\section{Reviewer 1:}

The reviewer stated that this project addresses a near-term technology of increasing dilution tolerance with advanced ignition systems. While the improvement in efficiency in a given of impacting the whole LD fleet and therefore having a large impact on petroleum reduction.

Question 2: Technical accomplishments and progress toward overall project and DOE goals-the degree to which progress has been made, measured against performance indicators and demonstrated progress towards DOE goals. engine will be small, it has the potential

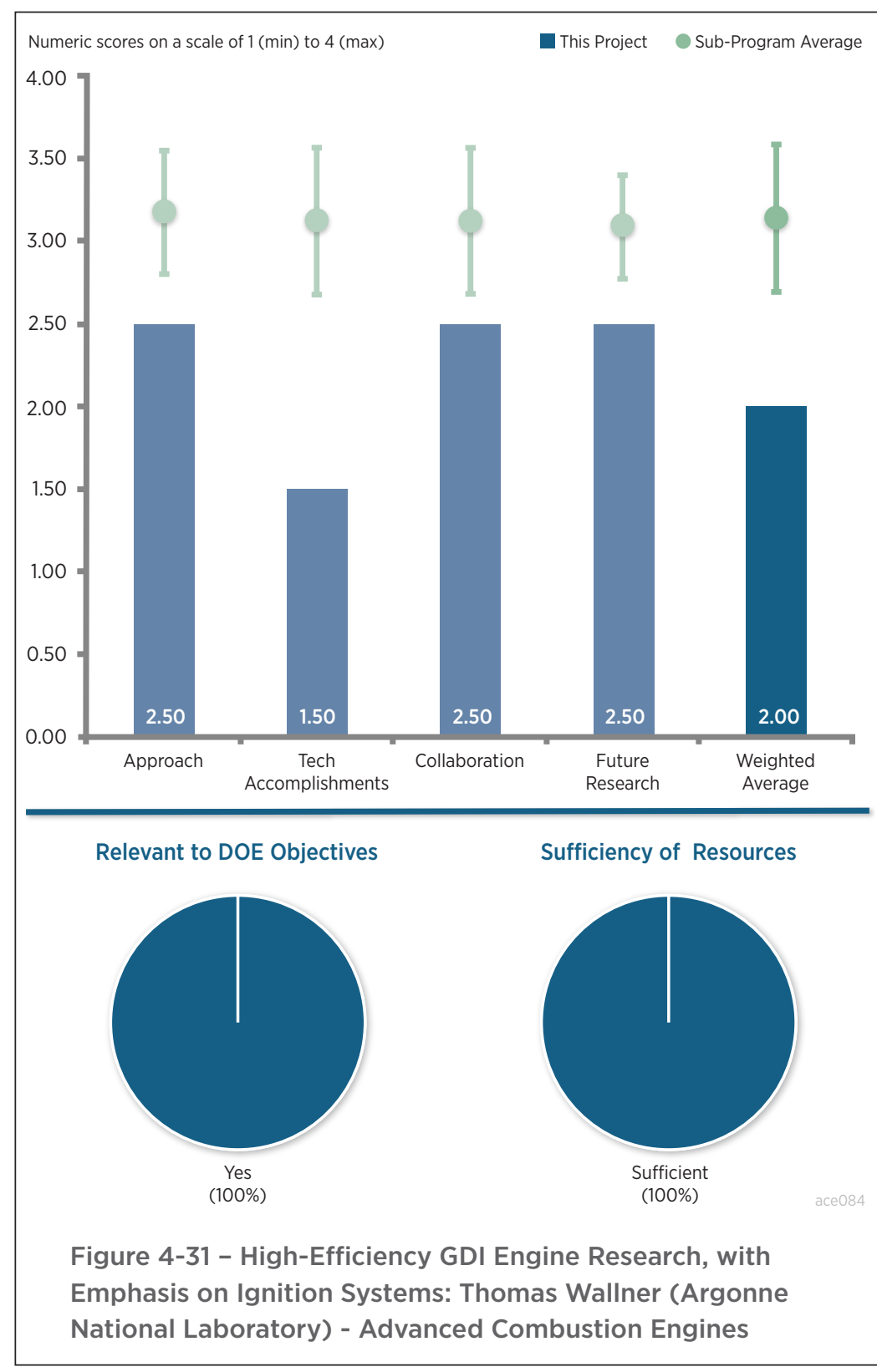

Reviewer 1:

In the reviewer's opinion, progress has been poor this year. The modeling progress is good but adds minimally to the knowledge base. The modeling work supports the main experimental evaluation but cannot justify the project itself.

The stretch goal of 20\% over a stoichiometric GDI engine with production spark was adopted in June of 2015. The reviewer questioned where this goal is coming from and stated that this is an incorrect goal as the baseline should be a stoichiometric GDI engine with production spark and EGR. Thus, the reviewer would anticipate increases of the order of 1\%-5\% with advanced ignition systems. According to the reviewer, the data in Slide 15 show this point very clearly.

The reviewer suggested that the focus should be largely on an experimental evaluation of various advanced ignition concepts like transient plasmas, corona ignition, and laser ignition. Over the past three years much of this 
experimental work has been done and reported. Small gains in engine efficiency and combustion stability have been reported. Thus, the reviewed noted that the big picture conclusion and information of the worth and current state-of-the-art of advanced ignition systems have been accomplished. The reviewer questioned what more the project could hope to contribute.

\section{Question 3: Collaboration and coordination with other institutions.}

Reviewer 1:

The reviewer remarked that better guidance from OEMs is needed to keep this project focused on the main thing. Collaboration should not occur for the sake of collaboration.

Question 4: Proposed future research-the degree to which the project has effectively planned its future work in a logical manner by incorporating appropriate decision points, considering barriers to the realization of the technology and, when sensible, mitigating risk by providing alternate development pathways.

Reviewer 1:

The reviewer commented that the focus should be on understanding the mechanism of ignition with Transient Plasma Systems, Inc. (TPS), Corona, or laser ignition, and improving the process to gain dilution tolerance. More optical engine experiments should be conducted.

\section{Question 5: Does this project support the overall DOE objectives of petroleum} displacement? Why or why not?

No comments were received in response to this question.

Question 6: Resources: How sufficient are the resources for the project to achieve the stated milestones in a timely fashion?

No comments were received in response to this question. 


\section{Low-Temperature \\ Emission Control to Enable \\ Fuel-Efficient Engine \\ Commercialization: Todd \\ Toops (Oak Ridge National \\ Laboratory) - ace085}

Presenter

Todd Toops, Oak Ridge National

Laboratory

\section{Reviewer Sample Size}

A total of five reviewers evaluated this project.

Question 1: Approach to performing the work-the degree to which technical barriers are addressed, the project is well-designed, feasible, and integrated with other efforts.

\section{Reviewer 1:}

The reviewer praised the range of methods used in analyzing the catalytic materials in the project as excellent. Also, the reviewer commented that the goals of the characterization include mimicking realistic conditions as well as exploring the impact of S.

\section{Reviewer 2:}

The reviewer stated that the evaluation techniques were great. Regarding proposing to compare the low-temperature combustion diesel (LTC-D) fuel to real-life fuels, the reviewer suggested considering the worst case fuel, which contains $\mathrm{HC}$ chains and aromatics.

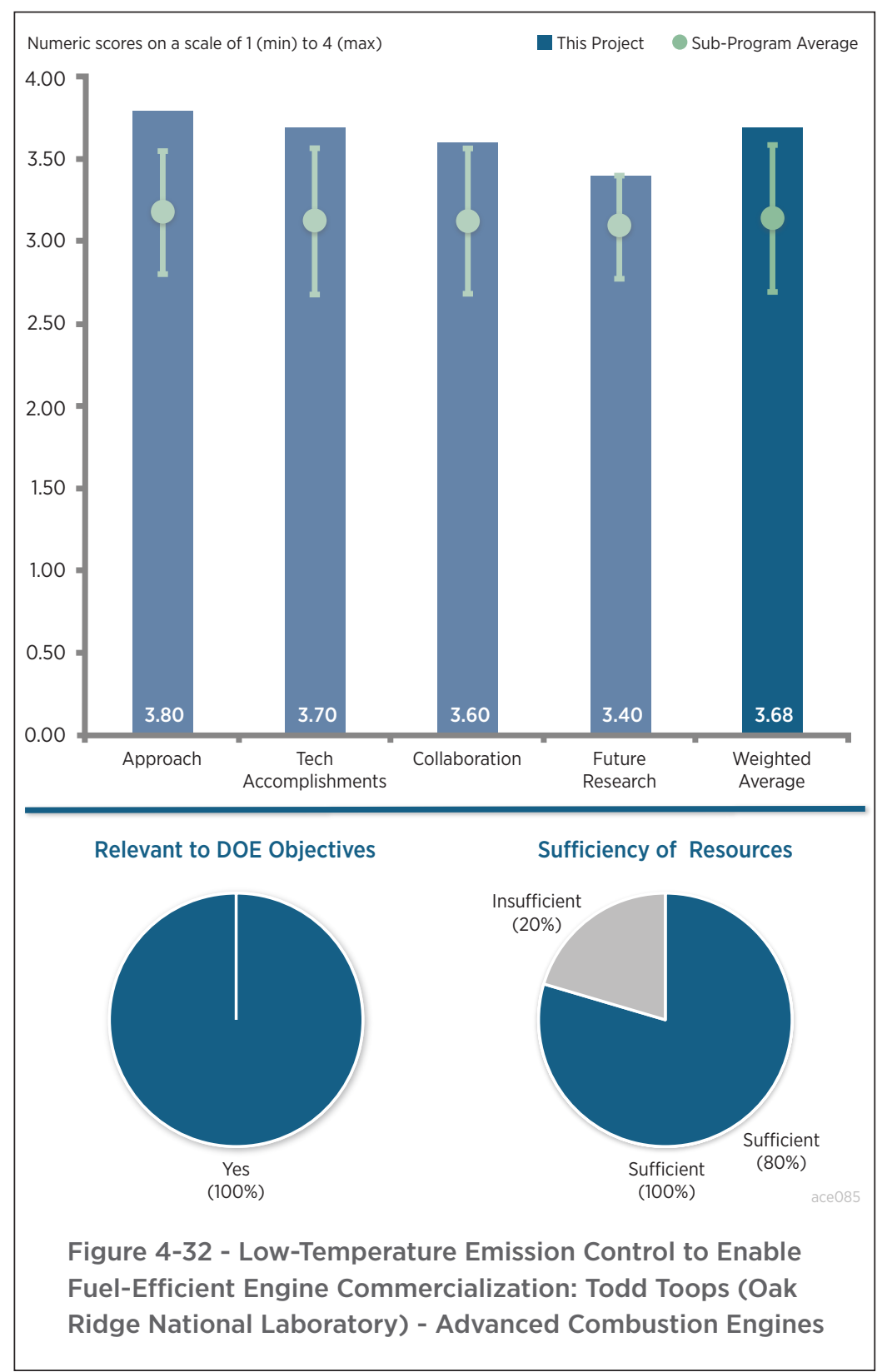

\section{Reviewer 3:}

The reviewer found a clear approach, developed with strong relevance to the CLEERS priorities, the U.S. DRIVE workshop report, and the U.S. DRIVE ACEC Technical Team Roadmap. ORNL is developing and employing the protocols to evaluate novel catalysts. In addition, the laboratory is leading round-robin testing and identifying new materials through collaborations.

\section{Reviewer 4:}

The reviewer stated that the project was a nice detailed look at several different types of catalysts (oxidation catalysts, PNAs, and $\mathrm{HC}$ traps). The reviewer commented that Slide 6 (comparing $\mathrm{SnO}_{2}-\mathrm{MnO}_{\mathrm{x}}-\mathrm{CeO}_{2}$ with simple conditions and full protocol) shows that it is very important to use the protocols recommended by the ACEC Technical Team. For some reason, the NO curve is missing from the graph on the right side. 


\section{Reviewer 5:}

The reviewer commented that the breadth of work occurring at ORNL under the central theme of carbon monoxide/ hydrocarbon $(\mathrm{CO} / \mathrm{HC})$ oxidation and remediation appears well coordinated and very appropriate. Exploring catalyst solutions for low-temperature oxidation of $\mathrm{CO}$ and $\mathrm{HC}$ species is strongly supported by USCAR engine and aftertreatment objectives. The inception stage exploration of multiple pathways to achieve high $\mathrm{CO}$ and $\mathrm{HC}$ oxidation performance is critical to finding viable solutions employing different catalyst technologies in a timely manner. However, the reviewer commented that a better understanding of how poisons, such as S, alter the activity of the catalysts under development is needed to provide a thorough characterization of the technologies.

\section{Question 2: Technical accomplishments and progress toward overall project and DOE goals-the degree to which progress has been made, measured against performance indicators and demonstrated progress towards DOE goals.}

\section{Reviewer 1:}

The reviewer commented that the findings of this project are very interesting and potentially useful. The work on zirconia as the shell of a catalyst was very interesting and should be continued. Also, the silver (Ag)-zeolite system for $\mathrm{HC}$ storage is very interesting.

\section{Reviewer 2:}

The reviewer said that the evaluation techniques were great. There is a need to fully understand observed phenomena, such as why PGM inclusion improves $\mathrm{S}$ tolerance and removal. The reviewer also asked why $\mathrm{CuO}_{x^{-}}$ $\mathrm{CoO}_{\mathrm{y}}-\mathrm{CeO}_{2}(\mathrm{CCC})$ shows the ability to store and release $\mathrm{NO}_{\mathrm{x}}$ with peaks at certain temps and whether that that peak can be influenced.

\section{Reviewer 3:}

The reviewer stated that there were several strong technical accomplishments achieved this year: identified mixed metal oxides that improve $\mathrm{HC}$ conversion (need for additional catalyst for low-temperature activity); measured S tolerance of CCC (showed need for PGM, specifically Pt); explored S mitigation strategies for PGM with CCC, which improves $\mathrm{S}$ tolerance; developed a new core/shell technique that improved zirconium dioxide $\left(\mathrm{ZrO}_{2}\right)$ surface area, which led to improved $\mathrm{HC}$ conversion; implemented nano Pd dispersion on $\mathrm{Ce}-\mathrm{Zr}$ nanoparticle dispersed on aluminate and approaching target activities; determined that the key attribute of a Ag/Al HC trap is deep ion exchange and low Si:Al ratio; and demonstrated the NO adsorption on the ZSM-5 zeolite and the impact of the pretreatment temperature.

\section{Reviewer 4:}

The review commended the project team for considering both thermal aging and S poisoning effects. Slide 13 shows the harsh impact of $\mathrm{SO}_{2}$ on the $\mathrm{CCC}$ catalyst (with and without $\mathrm{Pt}$ ), although having a front zone of $\mathrm{Pt}$ / $\mathrm{Al}_{2} \mathrm{O}_{3}$ appeared to mitigate the effect of $\mathrm{S}$ on the CCC catalyst. Unfortunately, the reviewer noted that the $\mathrm{C}_{3} \mathrm{H}_{6}$ performance on Slide 12 was better with $\mathrm{CCC}$ in front of the $\mathrm{Pt} / \mathrm{Al}_{2} \mathrm{O}_{3}$ catalyst, presumably by oxidizing the $\mathrm{CO}$ before the $\mathrm{Pt} / \mathrm{Al}_{2} \mathrm{O}_{3}$ catalyst and removing the $\mathrm{CO}$ inhibition. The reviewer asked if the project team had tried a $\mathrm{PA}+\mathrm{CCC}$ split-bed system during the light-off tests.

The reviewer also wanted to know why the $\mathrm{Pt} / \mathrm{CCC}$ catalyst looks so much worse than the CCC catalyst after poisoning on Slide 14 and whether the Pt catalyzes the oxidation of $\mathrm{SO}_{2}$ to $\mathrm{SO}_{3}$. The reviewer complimented the nice work on the $\mathrm{Zr}$-on-Si core-shell catalyst and the very nice micrographs. The reviewer noted that $\mathrm{CO}_{2}$ needed to be included in the feedgas (Slide 12) as it can promote carbonate formation. If the project team wants to see $\mathrm{CO}_{2}$ formation, maybe the team could run tests with and without the feedgas $\mathrm{CO}_{2}$.

The reviewer remarked that the researchers involved in this work have developed novel approaches to achieve higher $\mathrm{CO}$ and $\mathrm{HC}$ oxidation activity at low temperatures. The use of these techniques of dispersing the precious metal and/or combining PGMs with non- PGM catalysts to enhance oxidation activity is of significant interest in the catalyst community and applicable to aftertreatment systems in use today. Both the formulation and characterization assets of the national laboratory are well suited for this work. However, the reviewer pointed out that understanding the $\mathrm{S}$ poisoning mechanism for the Pt plus carbon composite catalyst $(\mathrm{Pt}+\mathrm{CCC})$ and using $\mathrm{HCs}$ other than propylene would benefit the research and provide a clearer picture of the performance of the catalysts. In 
addition, work at Ford has shown that Rh, without Pd, can perform as a more effective CO light-off catalyst on the right support. This work should including Rh-based catalysts as a comparison of activities. The HC trap work also supports the need for $\mathrm{HC}$ remediation, but requires additional testing that includes aged samples and appropriate feed conditions.

\section{Question 3: Collaboration and coordination with other institutions.}

\section{Reviewer 1:}

The reviewer commented that interaction with the automotive OEMs through USCAR and catalyst formulators increases the value of the research performed here. Also, this work takes advantage of the many assets at ORNL.

\section{Reviewer 2:}

The reviewer remarked that there is a broad group of collaborators, both commercial and university-based.

\section{Reviewer 3:}

The reviewer stated that there was a good combination of industry and academia.

\section{Reviewer 4:}

The reviewer found the addition of more partners, especially catalyst suppliers, to be excellent.

\section{Reviewer 5:}

The reviewer indicated that it was good to have a catalyst supplier as a partner, and an OEM would also be a helpful partner.

\section{Question 4: Proposed future research-the degree to which the project has effectively planned its future work in a logical manner by incorporating appropriate decision points, considering barriers to the realization of the technology and, when sensible, mitigating risk by providing alternate development pathways.}

\section{Reviewer 1:}

The reviewer noted that given the breath of research and the limited funding available, it is not certain that all the work can be addressed satisfactorily. Given that, the future work discussed is appropriate and has the potential to significantly add to the activity of the catalysts and their characterization.

\section{Reviewer 2:}

The reviewer found the future work proposed to be very appropriate. The areas of trapping and enhanced activity by maintaining dispersion are very important. It is hard to tell if the trapping will receive the effort it deserves because of the importance of preventing emissions at low temperatures.

\section{Reviewer 3:}

The reviewer stated that performance analysis under laboratory conditions was okay, and there is a need to consider real-life conditions as well.

\section{Reviewer 4:}

The reviewer noted strong future directions to investigate $\mathrm{S}$ interactions and support modifications but plans for trapping materials could be better described.

\section{Reviewer 5:}

The reviewer suggested that the project team should plan to include other hydrocarbons in its PNA and HC trap work, such as $\mathrm{C}_{2} \mathrm{H}_{4}$ as it is more abundant in diesel exhaust than $\mathrm{C}_{3} \mathrm{H}_{6}$.

\section{Question 5: Does this project support the overall DOE objectives of petroleum displacement? Why or why not?}

\section{Reviewer 1:}

The reviewer stated that this is highly relevant inception stage research and consistent with the call for LTAT by USCAR and the needs of the OEMs in general. 


\section{Reviewer 2:}

The reviewer noted that, as for most projects in this category, by enabling the use of more efficient combustion, these systems support the move to improving overall fuel economy.

\section{Reviewer 3:}

The reviewer stated that aftertreatment performance is the key for best efficiency of engine.

\section{Reviewer 4:}

The reviewer stressed that low-temperature catalysis is the key barrier to high efficiency combustion strategies.

\section{Reviewer 5:}

The reviewer said that low-temperature catalysts will be required for emission control on future engines with higher fuel efficiency and therefore lower exhaust temperatures.

\section{Question 6: Resources: How sufficient are the resources for the project to achieve the stated milestones in a timely fashion?}

\section{Reviewer 1:}

The reviewer stated that these experiments involving synthesis and bench studies are costly as more resources are necessary to meet project and program goals, especially for the $\mathrm{NH}_{3} \mathrm{SCR}$.

\section{Reviewer 2:}

The reviewer found funding and resources to be borderline sufficient.

\section{Reviewer 3:}

Despite comments about insufficient resources last year, the reviewer noted that the goals of the project have been modified slightly to move forward.

\section{Reviewer 4:}

The reviewer said that resources seem sufficient. 


\section{High-Dilution Stoichiometric Gasoline Direct-Injection (SGDI) Combustion Control Development: Brian Kaul (Oak Ridge National Laboratory) - ace090}

\section{Presenter}

Brian Kaul, Oak Ridge National Laboratory

\section{Reviewer Sample Size}

A total of five reviewers evaluated this project.

\section{Question 1: Approach to performing the work-the degree to which technical barriers are addressed, the project is well-designed, feasible, and integrated with other efforts.}

\section{Reviewer 1:}

The reviewer stated that the research team is good and has previously demonstrated high competence in identifying deterministic coupling of causes and effects. It has leveraged the Cummins-ORNL CRADA, which is pursuing this question from a diagnostic development approach. The team is moving toward developing models of the phenomena of interest, and its approach to the problem appears technically sound.

\section{Reviewer 2:}

The reviewer noted that this project uses a fast EGR probe to measure the external EGR cycle-by-cycle variation in order to understand the physics behind the effect of EGR on high dilution gasoline combustion. The results shown are encouraging and with good repeatability. Based on the feedback from the EGR measurement, engine cycle-to-cycle and next- cycle control strategies have been developed. However, further improvement is needed for implementation in engines. HPC modeling has also been used to assist the development of the control strategy.

\section{Reviewer 3:}

The reviewer suggested that it might be worth developing a P-diagram for combustion stability and identifying real world sources of noise. Some could have significant impact and would need to be addressed (i.e., injector deposits). OEM input could be very helpful here.

\section{Reviewer 4:}

The reviewer commented that engine control development to reduce cycle-to-cycle variability is an important topic, but it is not clear how simulation is going to impact the project.

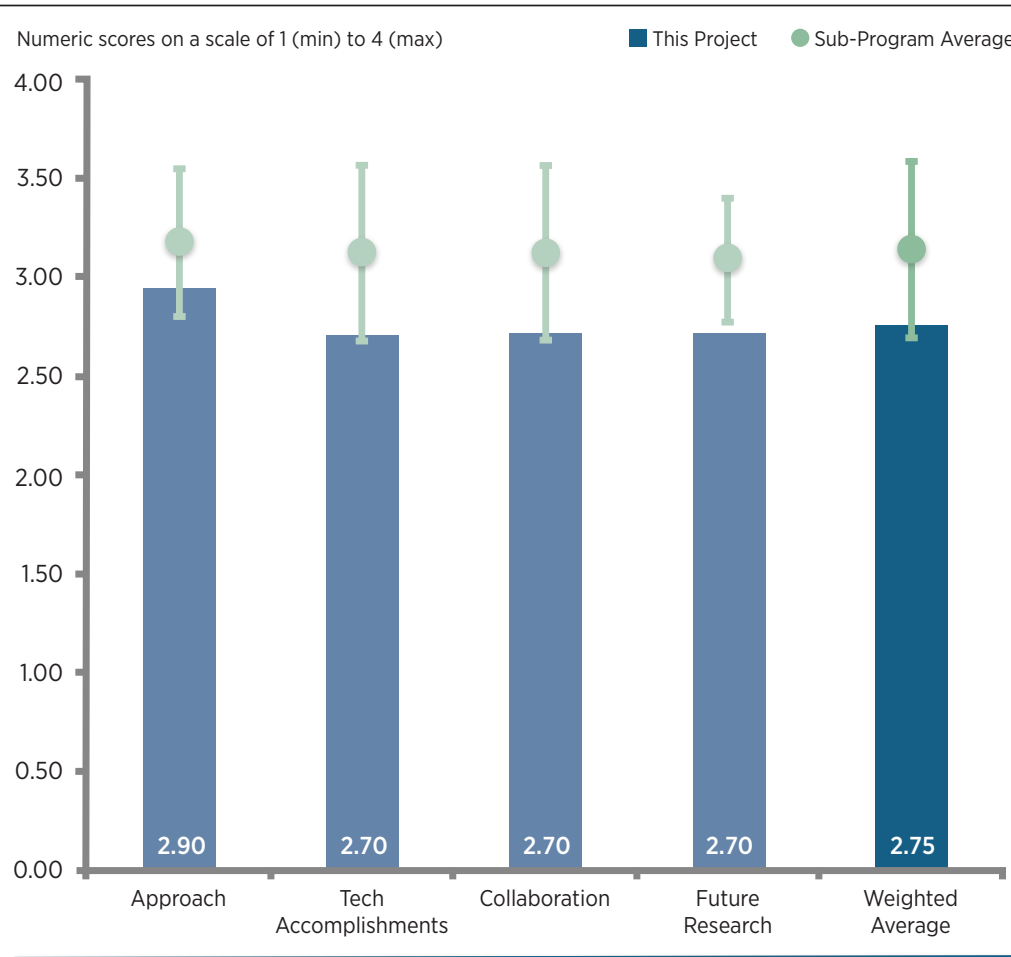

Relevant to DOE Objectives

Sufficiency of Resources
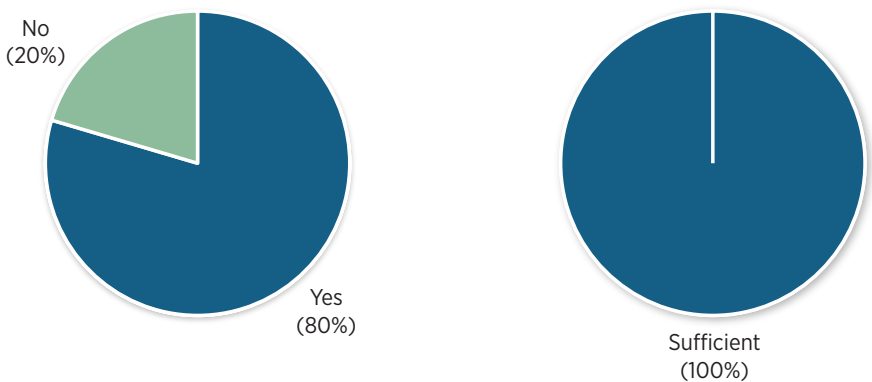

Figure 4-33 - High-Dilution Stoichiometric Gasoline Direct-Injection (SGDI) Combustion Control Development: Brian Kaul (Oak Ridge National Laboratory) - Advanced Combustion Engines 


\section{Reviewer 5:}

The reviewer stated that this project is not focused on key barriers related to improving engine efficiency in a significant way to reduce petroleum usage. An attempt should be made to quantify benefits of reducing cyclic variability to permit operation at the edge of stability to determine if it is worthwhile, especially in light of the fact that OEMs already have model-based controls to control a variety of engines, each with its own idiosyncratic longloop EGR dynamic composition behavior. Much of this work falls in the domain of OEM controls engineering, and given their vast resources and knowledge of real-world, hardware-specific behavior, this project will not have much, if any, impact on future controls direction.

\section{Question 2: Technical accomplishments and progress toward overall project and DOE goals-the degree to which progress has been made, measured against performance indicators and demonstrated progress towards DOE goals.}

\section{Reviewer 1:}

The reviewer commented that good progress has been made against the objectives of the program. The techniques used lead this program to the right direction. However, more work is needed to develop better control models. CFD modeling will definitely help in finding the way to improve the control models by providing more insight of what is happening inside the cylinder.

\section{Reviewer 2:}

The reviewer remarked that this is a very challenging problem. It is still not clear the exact extent to which overcoming this problem will benefit engine performance. However, from the perspective that every little bit helps, it is important to understand. It would be helpful to quantify the potential benefit. The reviewer thought that a prediction of the potential benefits could be made.

\section{Reviewer 3:}

The reviewer commented that the use of a fast EGR sensor is an interesting technique to diagnose engine operation. Only minor progress has been demonstrated with the controls development. It is not relevant to reduce the coefficient of variation (COV) by $2 \%$ when the starting point is near $20 \% \mathrm{COV}$.

\section{Reviewer 4:}

The reviewer stated that it is absolutely worthwhile to go after $1 \%$ efficiency gains by reducing cyclic variations, but given the vast number of engineers working at the OEMs on reducing cyclic variations due to a variety of causes, the reviewer asked how this project will make an impact. None of the results presented this year are any closer to showing to this reviewer that all of this is going to be worthwhile at the big picture level and that there is a pathway for industry to capture the findings of this study.

\section{Question 3: Collaboration and coordination with other institutions.}

\section{Reviewer 1:}

The reviewer remarked that there is very good collaborations with Cummins, National Instruments, ANL, and Bosch. Broader collaboration could be sought.

\section{Reviewer 2:}

The reviewer suggested that if the project team could engage with OEM control engineers, it might be constructive. The reviewer also wanted to know how the approach the team is taking melds with what industry is doing.

\section{Reviewer 3:}

The reviewer stated that the project team needs to include an OEM controls team to understand how techniques in this project might be implemented in a production controls environment.

\section{Reviewer 4:}

The reviewer stated that it is imperative that the project should be guided by a controls team at an OEM. Otherwise, there is a risk that this project can end up doing a lot of good work, but be largely irrelevant or a duplication.

Question 4: Proposed future research-the degree to which the project has effectively planned its future work in a logical manner by incorporating appropriate decision points, 


\section{considering barriers to the realization of the technology and, when sensible, mitigating risk by providing alternate development pathways.}

\section{Reviewer 1:}

The reviewer noted that, basically, the proposed future work showed a good extension of present work.

\section{Reviewer 2:}

The reviewer suggested that the team should make an estimate of the potential benefit.

\section{Reviewer 3:}

The reviewer found that it is not clear what model-based control means for this project. Future work needs to include a practical assessment of the potential engine efficiency improvement that is available based on this work.

\section{Reviewer 4:}

The reviewer commented that it is risky to apply learnings from a lean combustion project. In that case, engine torque (or indicated mean effective pressure [IMEP]) is largely proportional to fueling level alone. Hence, using fueling to control the next cycle heat release to reduce COV is appropriate. Using such a fueling scheme to control a stoichiometric combustion engine, where torque is proportional to fuel plus air, may have some unforeseen challenges.

\section{Question 5: Does this project support the overall DOE objectives of petroleum displacement? Why or why not?}

\section{Reviewer 1:}

The reviewer indicated that better controls can lead to more efficient engines that reduce fuel consumption.

\section{Reviewer 2:}

The reviewer asserted that further understanding the causes of cyclic variations, and their relative weights, will be useful information.

\section{Reviewer 3:}

The reviewer remarked that improved controls to allow increased dilution tolerance can be leveraged to increase engine efficiency.

\section{Reviewer 4:}

The reviewer commented that the chances of having a significant impact on petroleum displacement are minimal.

Question 6: Resources: How sufficient are the resources for the project to achieve the stated milestones in a timely fashion?

\section{Reviewer 1:}

The reviewer stated that resources seem sufficient for the proposed work. 


\section{High-Efficiency VCR Engine with Variable Valve Actuation and New Supercharging Technology: Charles Mendler (Envera LLC) - ace092}

Presenter

Charles Mendler, Enerva LLC

\section{Reviewer Sample Size}

A total of eight reviewers evaluated this project.

\section{Question 1: Approach to performing the work-the degree to which technical barriers are addressed, the project is well-designed, feasible, and integrated with other efforts.}

\section{Reviewer 1:}

The reviewer suspected poor combustion performance with the high CR because typical pent roof combustion chamber geometries are not conducive to CRs much beyond 12.5-13:1. The reviewer suggested that some CFD or fixed CR experiments would be valuable prior to completing the build of the variable CR (VCR) engine to define the correct maximum CR.

Internal EGR is necessary for good combustion stability at low loads, but at a high $\mathrm{CR}$, there will be no provision for the required valve overlap to generate the internal EGR.

The reviewer noted that the project team claims improved time to torque due to low CR operation. There is a need for at least GT-POWER modeling to demonstrate that this is feasible with the turbo required for the brake mean effective pressure (BMEP) needed for the application.

The claimed CR increase over several seconds means that VCR will not be useful on typical vehicle transients, which are quite fast relative to a several second switching time. Fast switching is needed for high-to-low CR transients, and slow switching is acceptable for low-to-high transients.

\section{Reviewer 2:}

The reviewer thought that friction (the work associated with changing the CR) will be a big challenge and that there may not be a way to ameliorate this if it turns out to be the problem the reviewer thought it will be. To achieve such a significant improvement in engine efficiency, no potential improvement should be left on the table. In light of the likelihood of higher friction, the reviewer asked why not try to recoup some of that loss by pursuing lean combustion.

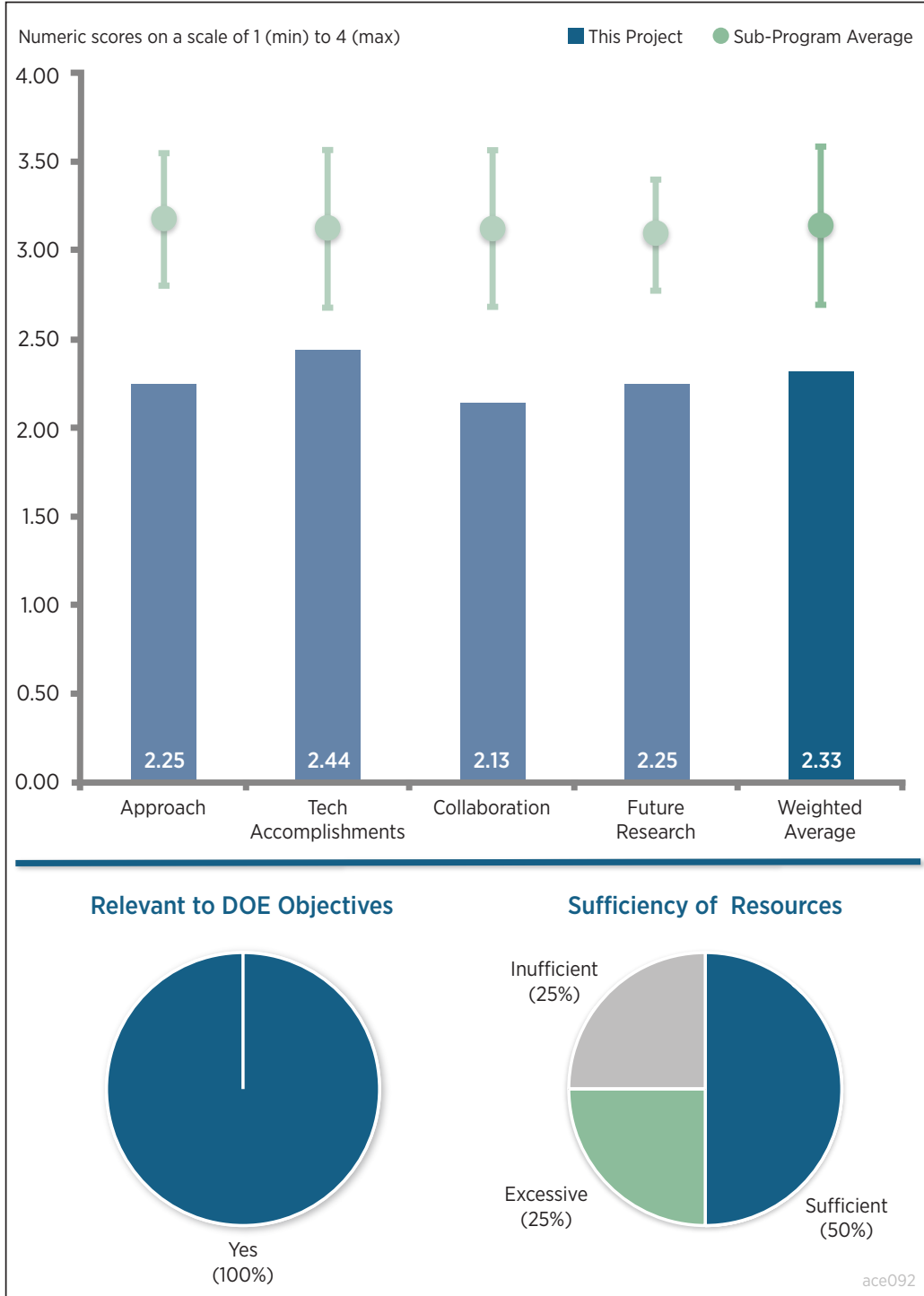

Figure 4-34 - High-Efficiency VCR Engine with Variable Valve Actuation and New Supercharging Technology: Charles Mendler (Envera LLC) - Advanced Combustion Engines 


\section{Reviewer 3:}

The reviewer stated that the project appears to be addressing the issues of VCR basic function, but is not yet addressing some of the logistical issues that have been created. For example, power requirement for moving the cylinder liners should be estimated/calculated and accounted for. Because this is targeted for light duty, operation during a realistic drive cycle needs to be considered to fully quantify the potential benefits of this system. The comparison to Chrysler and Ford BSFC and power is not clear, and the reviewer questioned whether this is really an apples-to-apples comparison.

\section{Reviewer 4:}

The reviewer remarked that the approach to performing the work has strengths of generally following industry accepted approach (establish baselines and metrics [example: V8 pickup $/ 40 \%$ fuel economy improvement, similar power], modeling of potential benefits [GT-POWER modeling], hardware development, dyno testing, and controls). The reviewer was concerned that the overhead valve (OHV) V8 baseline powertrain selected for comparison may need reconsideration and that single-cylinder work to confirm GT-POWER results and optimize injector system/combustion chamber is not in scope. The reviewer noted that an unoptimized combustion system may not perform well when moving directly to a multi-cylinder engine.

\section{Reviewer 5:}

The reviewer acknowledged that the appeal of a VCR engine is not new and many approaches have been suggested and studied over the years. The approach taken here is perhaps more complicated and risky than some previous avenues pursued so the question (yet to be answered) is can it be made to work effectively.

\section{Reviewer 6:}

The reviewer stated that the technical solution proposed (i.e., VCR, Atkinson, and valve lift profile switching) is quite reasonable, but the approach to execute the project is quite poor. At a minimum, there should have been some level of engine testing (e.g., single cylinder) to confirm the combustion performance and to inform the GT-POWER model. To simply say "it is lambda $=1$ " does not allow the project team to avoid the requisite analyses.

\section{Reviewer 7:}

The reviewer commented that it seems there should have been much more thermodynamic analysis done up front. For the two years of review, there has only been one point from GT-POWER simulation discussed that shows a good BSFC, not great, but just good. Beyond that, it seems a single-cylinder version of this mechanism and cylinder arrangement should be the first logical step. Going from one point on GT-POWER to a full four-cylinder engine seems a big investment and big step without much validation.

\section{Reviewer 8:}

The reviewer stated that the VCR 2.0 is an improvement from the previous iterations from a design standpoint. Using GT-POWER to do the efficiency calculations does not consider the changes to the actual combustion event. So, this project would benefit from CFD modeling to provide insight into the effect of changes to the combustion chamber as CR changes. This will feed into changes in the heat release rate. If the heat release rate is not correct from a trend-wise standpoint, then the estimates of friction, heat release, and ultimately friction will be erroneous. This project would also benefit from additional focus on engine thermodynamic tradeoffs (i.e., higher indicated efficiency versus higher friction losses with a higher CR).

\section{Question 2: Technical accomplishments and progress toward overall project and DOE goals - the degree to which progress has been made, measured against performance indicators and demonstrated progress towards DOE goals.}

\section{Reviewer 1:}

The reviewer found reasonable progress with the main focus on developing hardware prototypes for a multicylinder engine.

\section{Reviewer 2:}

The reviewer commented that the project team has made good progress in moving towards a testable configuration. That is where the fun and challenges will start. 


\section{Reviewer 3:}

The reviewer stated that the progress on building a working prototype of VCR 2.0 is looking good.

\section{Reviewer 4:}

The reviewer said that progress on hardware development is at a good pace, and the evolution from the 1.0 to 2.0 VCR mechanism is good. But, there was significant reference to GT-POWER results but none were shown so it is impossible to evaluate the technical progress from a combustion/performance/efficiency process. The reviewer said that before making hardware, a detailed modeling study should show the expected full-map performance and efficiency to determine if the VCR system is appropriate.

\section{Reviewer 5:}

The reviewer stated that the engine design is progressing nicely. However, the amount of learning associated with this project could be increased by presenting tradeoffs with different valve strategies, different CRs, etc.

\section{Reviewer 6:}

The reviewer asserted that there are no data available yet or shown on efficiency improvement and performance. Testing with new hardware is planned. It will be good to have some numerical analysis prior to engine testing to make estimates of performance and provide guidance.

\section{Reviewer 7:}

The reviewer remarked that the project has a design and parts are being procured, but there is little confidence that the goal of $40 \% \mathrm{FE}$ improvement will be achieved. No analysis was shown to show how the engine is predicted to compare to $40 \%$. There was no comprehension on the part of the project team how quickly changing CR (during a drive cycle) directly relates to fuel economy; CR being increased over seconds shows a lack of understanding. The reviewer said that, similarly, saying gas loads will be used to lower CR as a way to keep friction low to minimize losses is flawed. Using gas pressure to move the structure is a loss. The statement that no valve pockets are needed because no valve overlap is needed at high CR is also flawed. The reviewer asked about internal EGR at light- to mid-loads. The reviewer contended that by not doing the upfront combustion development on a single-cylinder engine, the project runs the risk of condemning a good idea.

\section{Reviewer 8:}

The reviewer observed that the project lacks added work on the simulation of the mechanical systems to consider parasitic losses.

\section{Question 3: Collaboration and coordination with other institutions.}

\section{Reviewer 1:}

The reviewer remarked that collaboration with Eaton on valve train technology is good, but there is a substantial lack of combustion researchers, OEM's who can give input on the design, or other support on the various aspects of the engine that need to be considered.

\section{Reviewer 2:}

The reviewer commented that Eaton is a good partner. The reviewer would like to see more active engagement of OEMs other than sighing the activity.

\section{Reviewer 3:}

The reviewer said that compared to the other 15 projects being reviewed by this reviewer, this has the fewest collaborators although the coordination is good with the ones the project team has.

\section{Reviewer 4:}

The reviewer stated that Eaton and Envera are working well together. For the VCR device, collaboration would be greatly improved if an OEM or Tier 1 were co-developing. The reviewer noted that the production pathway will require a significant base engine design change with high level commercialization issues that must be resolved relating to combustion design for DI potential durability risks (base engine block and head change) and existing manufacturing considerations (new engine line tooling may be needed). 


\section{Reviewer 5:}

The reviewer noted that Envera is the lead and Eaton is a subcontractor, but the roles seem to be separate and not collaborative. This project could really benefit from university solid modeling and CFD modeling to provide additional understanding of the structure of the engine architecture, vibration dynamics, and the effect on the combustion process. The reviewer said that it will help to identify where and when losses occur and provide insight to paths forward after the completion of the project.

\section{Reviewer 6:}

The reviewer noted that there is still a need for an engine testing partner.

\section{Reviewer 7:}

The reviewer offered that for this project to be successful and generate any useful information, it needs to engage an OEM or an experienced engineering service provider.

\section{Reviewer 8:}

The reviewer commented that a small fraction of the funding going to a university to build a full map GT-POWER model would have been money well spent and would have lent validity to the project.

\section{Question 4: Proposed future research-the degree to which the project has effectively planned its future work in a logical manner by incorporating appropriate decision points, considering barriers to the realization of the technology and, when sensible, mitigating risk by providing alternate development pathways.}

\section{Reviewer 1:}

The reviewer found the test plans to be good. It seems that modeling could play a much bigger role in this project. The reviewer suggested that the project team may need an outside partner to help with this and should consider this in the team's future plans.

\section{Reviewer 2:}

The reviewer said that the plan to run the multi-cylinder engine and obtain results is part of the base plan. It is critical to measure power, performance, and engine-out emissions and relate them clearly to the baseline. Additional OEM or Tier 1 collaboration for confirmation of modeling, combustion system, and value proposition would improve the future research plan.

\section{Reviewer 3:}

The reviewer remarked that there is not much to see other than a schedule in the presentation. More details of work to be performed would be needed to properly assess the engine test plan, etc.

\section{Reviewer 4:}

The reviewer commented that the actual answer to the question about future research needs will be determined once testing has started and problems need to be addressed.

\section{Reviewer 5:}

The reviewer stated that the project team gave far too little discussion of how the engine performance will be validated. The reviewer continued that not only steady-state points, but transient demonstrations, simulated Federal Test Procedure/New European Driving Cycle/US06/World Harmonized Test Cycle (FTP/NEDC/US06/WHTC) operation, and so on are required to evaluate the systems. Other reviewers noted significant work was needed to validate the hardware design of the VCR system.

\section{Reviewer 6:}

The reviewer commented that steady-state results will show the benefit of the combustion approach. Given the chosen VCR approach, steady-state results are almost meaningless for quantifying the benefit of VCR. The future work must show the transient response capability of the hardware (i.e., how fast from the CR change).

\section{Reviewer 7:}

The reviewer said that the experiments that are proposed appear to be a demonstration. It is not clear that the amount of data collected and analysis associated with the project will provide insight into the thermodynamic 
tradeoffs. For instance, if the BTE does not increase as expected, the reviewer questioned whether the data will provide insight into whether this is a friction problem, a heat transfer problem, or a combustion chamber design problem. These learnings are ultimately how DOE and the community benefit from the project.

\section{Reviewer 8:}

The reviewer stated that the approach lacks a fundamental plan to demonstrate the thermodynamic and mechanical measures (like a friction measurement).

\section{Question 5: Does this project support the overall DOE objectives of petroleum displacement? Why or why not?}

\section{Reviewer 1:}

The reviewer noted that a successful VCR engine will contribute to additional efficiency gains beyond business as usual and support DOE objectives.

\section{Reviewer 2:}

The reviewer said this was an interesting idea, and important information could be generated.

\section{Reviewer 3:}

The reviewer found VCR to be a very intriguing technology. It has the potential to increase engine efficiency, but also has the potential to make engines a lot more compatible with a wider range of fuel composition in the marketplace. It is a worthwhile technology to pursue in the DOE portfolio. The value of the project will be maximized by focusing on the thermodynamic tradeoffs associated with changing $\mathrm{CR}$.

\section{Reviewer 4:}

The reviewer remarked that if a production-viable VCR system could be developed, it has been shown that useful efficiency improvements could be realized.

\section{Reviewer 5:}

The reviewer stated that the approach would appear to be a possible path to higher efficiency engines that will reduce petroleum usage.

\section{Reviewer 6:}

The reviewer suggested that if the approach were successful, it has potential. The barriers should include cost versus competitive technologies.

\section{Reviewer 7:}

The reviewer remarked that VCR technology and variable valve train technology have been clearly identified as enablers to support DOE petroleum reduction objectives. The value proposition and pathway to production for a VCR device could be clearer to insure potential is achieved the marketplace in the near term.

\section{Reviewer 8:}

The reviewer commented that the technologies being explored are relevant, but they are not being explored in a robust and relevant way.

\section{Question 6: Resources: How sufficient are the resources for the project to achieve the stated milestones in a timely fashion?}

\section{Reviewer 1:}

The reviewed stated that the resources look okay.

\section{Reviewer 2:}

The reviewer said that the resources are sufficient for the project as scoped. Additional support for more advanced GT-POWER modeling and single cylinder work could improve the score.

\section{Reviewer 3:}

The reviewer remarked that it seems as there is a lack of thermodynamic analysis and expertise in this project. 
Reviewer 4:

The reviewer commented that there is a very high project amount for making hardware that may or may not achieve the necessary performance.

Reviewer 5:

The reviewer commented that there are some key elements missing from the project (e.g., 1D and 3D simulations and understanding the impact of friction and control) and the project team does not seem willing to address them. Without these, the project can easily come to the wrong conclusion. As such, the funding is excessive given that the reviewer will not have confidence in the result.

Reviewer 6:

The reviewer would like to see a higher contractor share for work of this magnitude. 


\section{Lean Miller Cycle System Development for Light-Duty Vehicles: David Sczomak (General Motors) - ace093}

\section{Presenter}

David Sczomak, General Motors

\section{Reviewer Sample Size}

A total of five reviewers evaluated this project.

\section{Question 1: Approach to performing the work-the degree to which technical barriers are addressed, the project is well-designed, feasible, and integrated with other efforts.}

\section{Reviewer 1:}

The reviewer remarked that an integrated, comprehensive strategy was presented in very nice detail. This appears to be a well thought-out plan. The use of the modeling tools is contributing to the fuel spray and combustion chamber geometry design.

\section{Reviewer 2:}

The reviewer stated that the authors addressed the technical barriers in a systematic and organized way, at least in terms of concepts, by adopting 1D and 3D CFD analysis for engine and for aftertreatment design. If successful, the project is relevant to DOE objectives. Regarding the 35\% targeted efficiency improvement, perhaps a more detailed explanation should be provided of how exactly such a high portion of $18 \%$ efficiency gain is expected to come just from lean combustion strategies and from Miller cycle implementation. It is known that the Miller cycle early intake valve closing (EIVC) strategy has $\mathrm{NO}_{\mathrm{x}}$ reduction benefits while the late intake valve closing (LIVC) shows thermal efficiency improvement if carefully tuned. The project team does not mention which one (of the two) Miller cycle strategies it has chosen.

\section{Reviewer 3:}

The reviewer stated that the lean Miller cycle approach is an excellent approach to achieve fuel efficiency gains. Included as well in the approach is a systems-level approach including downsizing to achieve the fuel efficiency gains. Both approaches are needed to meet the aggressive targets. It was good to see challenging approaches being pursued (DOE funding is well suited for pushing the boundaries).

\section{Reviewer 4:}

The reviewer commented that the approach on this five-year project includes annual go/no-go milestones for DOE review with a projected completion in December 2019 with a final vehicle demonstration. The technical 
approach includes integration of all key engine components and systems (air handling, fuel injection, heat transfer, friction, aftertreatment, and $12 \mathrm{~V}$ system hybridization). Most of the targeted $35 \%$ efficiency improvements are expected from advanced combustion (18\%) followed by advanced integration (10\%), downsizing $(8 \%)$, and lean aftertreatment system $(-1 \%)$.

\section{Reviewer 5:}

The reviewer indicated that the schedule and approach follow a generally accepted R\&D plan: modeling, singlecylinder, multi-cylinder dynamometer, and vehicle. Technologies identified have the potential for production implementation.

Efforts to downsize similar to the current approach to downsize a $3.5 \mathrm{~L}$ port fuel injection (PFI) to a $2.5 \mathrm{~L}$ GDI application for fuel savings are already appearing in the marketplace and may not represent a substantial R\&D benefit. U.S. Environmental Protection Agency (EPA) studies have shown that GDI downsizing has not reaped the targeted $8 \%$ potential targeted for this project and has been more like $1 \%-3 \%$. Considering marketplace available GDI applications, the schedule of single-cylinder work could be more aggressive and modeling work more detailed to indicate potential for progress.

Data metrics for go/no-go should be presented (i.e., key 12 FTP/Highway Fuel Economy [HWFE] speed load points modeled to $25 \%$ efficiency and peak usable power/torque match $3.5 \mathrm{~L}$ PFI baseline engine).

\section{Question 2: Technical accomplishments and progress toward overall project and DOE goals - the degree to which progress has been made, measured against performance indicators and demonstrated progress towards DOE goals.}

\section{Reviewer 1:}

The reviewer remarked that the baseline single-cylinder engine testing and CFD spray modeling have been performed, helping in understanding the spray, the mixing, and the combustion behavior, but also revealing aspects that should be avoided, i.e., spray collapsing. Major engine components are being redesigned, while thermal management 3D analysis is being performed to ensure if the flows, temperatures, and stresses in the engine are within acceptable limits. The air handling system is also being analyzed under several boosting options (super-charger, turbocharger and their combinations) along with their advantages and disadvantages. Among the challenges, it is not clear yet if the lean combustion regimes at low temperatures will ensure sufficient exhaust temperatures for aftertreatment light-off and proper operation. If thermal efficiency individual percentage improvement methods prove successful, i.e., lean combustion and Miller cycle, the project will support DOE's goal of reducing petroleum dependence significantly, given its large number of gasoline engines applications.

\section{Reviewer 2:}

The reviewer stated that all year one milestones (single-cylinder work) have been met and year two (multi-cylinder effort) are underway.

\section{Reviewer 3:}

The reviewer commented that limited results are available after 1.5 years out of 2 years of single-cylinder development, and very limited data were presented on lean Miller cycle results with go/no-go gate approaching in 4 months (October 2016).

With a downsized engine, data for the potential to meet power requirements and actual engine-out emissions should be presented to confirm that the end result will directly relate to DOE metrics. Proof of concept must include the capability to match power performance, capability to meet efficiency targets, and engine-out emissions, which have the potential to be managed with aftertreatment systems potentially available for production in the near term.

\section{Reviewer 4:}

The reviewer stated that there is still a lot to do until the decision gate in late 2016, but the progress has been good. The reviewer asserted that the models for the injection process were good.

\section{Question 3: Collaboration and coordination with other institutions.}




\section{Reviewer 1:}

The reviewer remarked that the collaborators and the technical partners are representative for this research work. AVL single-cylinder testing baseline testing has been completed. It is not mentioned if the transient reactor capability study for aftertreatment development is being performed at an internal GM facility or at a partner/ collaborator's external facility.

\section{Reviewer 2:}

The reviewer complimented the very good collaboration with GM as the project lead supported by the following key suppliers-AVL, Bosch, NGK, Delphi, Eaton, and Umicore - all leveraging their core capabilities.

\section{Reviewer 3:}

The reviewer pronounced it good that top Tier 1 suppliers have been named to fill in the technology roadmap (Bosch, Delphi, NGK, and Umicore). More details on each supplier's role, work plan, and data would have improved the score. The reviewer listed injection system strategy, ignition strategy, aftertreatment configuration, and noted a very limited indication of progress.

\section{Reviewer 4:}

The reviewer commented that the strategic partners were identified; however, it was not clear whether they were strictly supplying what GM asks for or whether their expertise is directly contributing to the project.

\section{Reviewer 5:}

The reviewer found the collaborations to be limited to suppliers and one subcontract (AVL). However, the coordination among the team is good. The project would benefit from expanding the collaborations beyond suppliers.

\section{Question 4: Proposed future research-the degree to which the project has effectively planned its future work in a logical manner by incorporating appropriate decision points, considering barriers to the realization of the technology and, when sensible, mitigating risk by providing alternate development pathways.}

\section{Reviewer 1:}

The reviewer stated that the project milestones appear relevant to meeting objectives: optimizing a stratified charge engine (SCE) using piston, sprays, ports, injection, and dilution strategies; designing a multi-cylinder engine with new boost and new aftertreatment; and incorporating appropriate go/no-go gates in October 2016 followed by procurement of hardware, building a multi-cylinder engine, and demonstrating the fuel efficiency targets.

\section{Reviewer 2:}

The reviewer said that future FY 2016 tasks of optimizing single-cylinder engines (piston, sprays, ports, injection, and dilution strategies), optimizing Miller cycle strategies (LIVC, EIVC) and designing a multi-cylinder engine with new boost and aftertreatment seem very appropriate. FY 2017 tasks include hardware procurement for multicylinder engine builds, optimization of multi-cylinder engine on a dynamometer, and demonstrating fuel efficiency targets.

\section{Reviewer 3:}

The reviewer stated that the plan looks good and comprehensive.

\section{Reviewer 4:}

The reviewer stated that the project is on track and the next steps are consistent with the project's goals leading up to the late 2016 gate review. After that gate review, a closer examination of next steps in research will be more relevant.

\section{Reviewer 5:}

The reviewer indicated that the future research follows an R\&D plan that could have had more aggressive timing. The proof of concept for single-cylinder work (progress demonstrating potential to meet $25 \%$ fuel efficiency while matching power to 3.5 L PFI and reasonable engine-out emissions) should be demonstrated before a significant effort is made on a multi-cylinder engine. 


\section{Question 5: Does this project support the overall DOE objectives of petroleum displacement? Why or why not?}

\section{Reviewer 1:}

The reviewer said that higher efficiency engines such as being developed here will reduce petroleum usage.

\section{Reviewer 2:}

The reviewer remarked that this project entails a very comprehensive approach to reaching the $35 \%$ fuel efficiency target compared to the 2010 baseline. Increased engine efficiency directly contributes to petroleum displacement.

\section{Reviewer 3:}

The reviewer stated there is a 35\% improvement in baseline fuel economy, and the stretch goals shown on Slide 3 are directly relevant to DOE's objectives.

\section{Reviewer 4:}

The reviewer noted that this project directly enables petroleum displacement by fuel efficiency improvements in gasoline engines, which dominate the U.S. passenger car and light truck fleet.

\section{Reviewer 5:}

The reviewer commented that, generally, the $25 \%$ fuel savings effort support DOE objectives. The value of downsized GDI application development should be revisited.

\section{Question 6: Resources: How sufficient are the resources for the project to achieve the stated milestones in a timely fashion?}

\section{Reviewer 1:}

The reviewer stated that the good combination of DOE and corporate funds should be adequate to meet goals.

\section{Reviewer 2:}

The reviewer noted that this is a relatively large project at $\$ 20$ million ( $\$ 8.2$ million DOE share) spanning 5 years but very appropriate given the high level goal that is very challenging.

\section{Reviewer 3:}

The reviewer said that DOE and cost share resources combined appear appropriate for this research.

\section{Reviewer 4:}

The reviewer commented that resources are sufficient. OEM commitment is substantial and indicates commitment to the technology. 


\section{Ultra-Efficient Light-Duty Powertrain with Gasoline Low-Temperature Combustion: Keith Confer (Delphi Advanced Powertrain) - ace094}

\section{Presenter}

Keith Confer, Delphi Advanced Powertrain

\section{Reviewer Sample Size}

A total of three reviewers evaluated this project.

\section{Question 1: Approach to performing the work-the degree to which technical barriers are addressed, the project is well-designed, feasible, and integrated with other efforts.}

\section{Reviewer 1:}

The reviewer enthused that this is an excellent approach progressing through a single-cylinder combustion chamber and controls activities, steady-state dynamometer, and onto the FTP with a keen eye on emissions and cost considerations. The project approach calls for an outstanding three generations of engines and a realistic plan for systematic drivability and emissions development. The approach should consider torque/power targets and time to torque.

\section{Reviewer 2:}

The reviewer stated that the project proposes to utilize a unique LTC regime, GDI CI (GDCI), to achieve the targeted 35\% fuel economy improvement. While promising very high engine efficiency, GDCI requires an aftertreatment system approach that works with the low-temperature challenges of a highly efficient engine that is also planned. FY 2015 milestones are complete, and significant progress has already been made towards many FY 2016 milestones.

\section{Reviewer 3:}

The reviewer said that this is a traditional, proven approach to work on single-cylinder, multi-cylinder, then generational improvements on identified problems. The reviewer saw no mention of modeling except for emissions approaches, but assumed it is integral to the engine design and approach. The reviewer believed that simulations had been done on fuel consumption, etc., several years ago.

Question 2: Technical Accomplishments and Progress toward overall project and DOE goals-the degree to which progress has been made, measured against performance indicators and demonstrated progress towards DOE goals. 


\section{Reviewer 1:}

The reviewer found the continuous progress to be impressive as $210-215 \mathrm{~g} / \mathrm{kWhr}$ over the range of loads is close to world-class for engines in this stage of development. Identification of emissions issues with some early performance data and several possible solutions is a big step. Achieving and characterizing transient operation on a vehicle, with Gen 3 designed, is real progress and reduces one of the larger risk factors. The reviewer had a much better understanding of the potential for this system based on these results.

\section{Reviewer 2:}

The reviewer listed numerous technical accomplishments: engine controls and calibration, evaluation of fuel efficiency on test cycles, test vehicle using Gen 1.0 and Gen 1.8 GDCI hardware, development of Gen 2, and design of Gen 3 engine and aftertreatment for the GDCI multi-cylinder engine.

\section{Reviewer 3:}

The reviewer said excellent progress. Full engine and vehicle FTP results shown for GDCI LTC are on path and producing $32 \%$ of the target $35 \%$ fuel economy improvement over baseline for a vehicle.

The reviewer noted good progress on the level of data provided to the development community for the Gen 1 engine-out criteria emission and temperatures for aftertreatment considerations. FTP data presented for the Gen 1 engine show engine-out emissions comparable to current spark ignition engines with significantly lower $\mathrm{NO}_{\mathrm{x}}$ and some $\mathrm{HC}$ and $\mathrm{CO}$ penalty.

Substantially lower exhaust temperatures of $200^{\circ} \mathrm{C}-300^{\circ} \mathrm{C}$ versus $450^{\circ} \mathrm{C}-700^{\circ} \mathrm{C}$ have been characterized on the FTP for potential novel LTC aftertreatment. The challenge has been identified for lower temperature exhaust.

The Gen 2 engine has data with about 11\% improved BSFC over the Gen 1 engine, indicating a potential to meet the $35 \%$ target. A realistic consideration for $\mathrm{NO}_{\mathrm{x}} / \mathrm{BSFC}$ tradeoff has been presented.

The reviewer found clear plans and accomplishments for aftertreatment approaches presented for temperature considerations, such as insulated exhaust and high power intake air heater, expanded exhaust re-breathing, close coupled catalyst, HC trap, SCR, and low-temperature catalysts.

It was indicated during the presentation that peak power and torque tests were run successfully on the Gen 1 engine, implying that targets were met. It would improve accomplishments to present data on torque/power targets and time to torque.

The reviewer proposed that characterization of a full range of temperatures/constituents in the exhaust (peak temperatures, temperature histograms, levels of and $\mathrm{CO}$ ) would be helpful to the aftertreatment development community to develop or apply solutions.

\section{Question 3: Collaboration and coordination with other institutions.}

\section{Reviewer 1:}

The reviewer commented that Delphi is the project lead and is currently in negotiations with an OEM partner. UW, ORNL, and Umicore round out the team, all contributing their key expertise, which is much needed to set up this project for success. The lack of an automotive OEM partner is a big concern as the originally proposed OEM declined to participate in the project and feedback from other OEMs has generally been that they are currently pursuing other technical directions and cannot take another project on.

\section{Reviewer 2:}

The reviewer remarked that this program continues to be mostly a Delphi program, with a significant contribution on emissions by Umicore. Some apparently minor collaboration on injectors is being done at UW and on emissions analyses by ORNL. ANL is doing significant work on GCI combustion fundamentals, and the reviewer wondered why Delphi is not making use of this. This reviewer certainly hoped that their public reports were not being solely relied upon. The reviewer stated that the top priority needs to be on securing an OEM partner. This establishes credibility, and, without this, skepticism will prevail and the project team will lose significant inputs on issues. 


\section{Reviewer 3:}

The reviewer found very good collaboration with institutions to resolve critical barriers relating to LTAT (Umicore). There is a good level of data sharing for the development community considering the level of investment in cost share, and a good level of OEM involvement until 2016. The reviewer expressed concern with the apparent dropout of OEM partner Hyundai (Hyundai America Test Center Inc.) as engine technology is highly linked to this OEM. The score can be improved with the addition of an OEM partner and the effort to engage the broader aftertreatment community with high level requirements for this LTC. For example, if exhaust temperatures are consistently low, traditionally underused technologies for light duty such as $\mathrm{HC} / \mathrm{NO}_{\mathrm{x}}$ traps may be a potential solution.

\section{Question 4: Proposed future research-the degree to which the project has effectively planned its future work in a logical manner by incorporating appropriate decision points, considering barriers to the realization of the technology and, when sensible, mitigating risk by providing alternate development pathways.}

\section{Reviewer 1:}

The reviewer remarked that excellent progress has been made so far. Future work very appropriately includes further refinement of controls and calibration (transient emphasis), Gen 3 GDCI engine design/build/test with next generation hardware including fuel injectors and aftertreatment architecture, and low-temperature exhaust aftertreatment system.

\section{Reviewer 2:}

The reviewer commended the work as outstanding because it has third-generation engine hardware incorporating lessons learned. As a low-temperature exhaust aftertreatment system is one of the highest priority remaining GDCI technical challenges, it is excellent to leverage ORNL expertise in low-temperature catalysts as well as Umicore. If possible, some general requirements for this LTC aftertreatment should be made available to a broader community as it may accelerate solutions.

Transient results planned should include consideration for maximum power/torque, power density (kW/l), time to torque/power relative to baselines (for acceleration considerations), and some indication of capabilities in the real world, such as cold start drivability down to $-10^{\circ} \mathrm{C}$. With lower temperatures, traditionally underused (due to durability issues) $\mathrm{HC} / \mathrm{NO}_{\mathrm{x}}$ traps may be a robust consideration.

\section{Reviewer 3:}

The reviewer commented that the future work is boiler plate: build and test Gen 3 and work on aftertreatment and calibration. The future tasks generally address the barriers, but the details are missing. $\mathrm{NO}_{\mathrm{x}}$ control seems a major gap, with no specific mention. The reviewer noted gasoline oxidation catalyst (GOC), exotherm temperatures, and durability of the catalyst against flaking and damaging the turbo, and asked how the project team will test and address this before the turbo is wrecked.

A major milestone will be benchmarking with alternative approaches. There are several systems at various stages of development that look quite competitive on GHG emissions. An honest assessment is needed. Securing an OEM partner will validate any advantages.

Various versions of $48 \mathrm{~V}$ hybridization will be the norm if/when this architecture goes into production. Even a P2 hybrid electric vehicle (HEV) can significantly help on numerous transient trouble spots. The reviewer encouraged the project team to look at opportunities to take advantage of this as a way of maybe relieving emissions issues, delivering incremental fuel consumption benefits, or relieving demanding transient demand, etc. The project team should not be spending many resources on trying to solve these kinds of problems. This belongs in more fundamental work such as at ANL. The project team needs to find engineering solutions like this, and perhaps some simulation time on this would be very valuable in this regard.

\section{Question 5: Does this project support the overall DOE objectives of petroleum displacement? Why or why not?}




\section{Reviewer 1:}

The reviewer remarked that this project supports the overall DOE objective of petroleum displacement by improving engine efficiency (targeting 35\%). Specifically, this project supports VTO's goal to improve the efficiency of LD engines for passenger vehicles through advanced combustion and minimization of thermal and parasitic losses.

\section{Reviewer 2:}

The reviewer commented that a $35 \%$ reduction in LD fuel consumption is clearly in line with DOE goals for petroleum displacement.

\section{Question 6: Resources: How sufficient are the resources for the project to achieve the stated milestones in a timely fashion?}

\section{Reviewer 1:}

The reviewer said that this is a relatively large project at \$25 million (nearly \$10 million DOE share) spanning 4 years, but the funding level is very appropriate given the broad scope encompassing optimization of all engine systems to reach the very challenging goal of 35\% engine efficiency improvement over the 2010 baseline.

\section{Reviewer 2:}

The reviewer found a generally sufficient level of resources as excellent progress is being achieved. With additional proof of concept demonstration for efficiency, engine-out emissions, and transient performance, more resources could be justified to accelerate LTC aftertreatment solutions to enable production use of the technology.

\section{Reviewer 3:}

The reviewer warned that the project is in serious jeopardy without a contributing OEM partner. 


\section{Metal Oxide Nano-Array \\ Catalysts for Low- \\ Temperature Diesel Oxidation: \\ Pu-Xian Gao (University of \\ Connecticut) - ace095}

Presenter

$\mathrm{Pu}-\mathrm{Xian}$ Gao, University of

Connecticut

\section{Reviewer Sample Size}

A total of four reviewers evaluated this project.

\section{Question 1: Approach to performing the work-the degree to which technical barriers are addressed, the project is well-designed, feasible, and integrated with other efforts.}

\section{Reviewer 1:}

The reviewer commented that one aspect of this project that is very unique is the growth of nano-array catalysts in an existing honeycomb support. This is still a key area that needs to be demonstrated to work under a wide range of conditions, but this remains an interesting concept. With ORNL and Umicore involved, it should be handled in an appropriate way.

\section{Reviewer 2:}

The reviewer stated that the use of rare earth and base metals combined with unique support in place of traditionally supported PGM catalysts is a novel, inception stage approach for achieving low-temperature $\mathrm{CO}$ oxidation. However, the project team continues to employ conditions in much of the characterization work that do not reflect the actual exhaust environment the catalyst will experience. Minor testing under these more realistic conditions showed considerably less performance than with idealized flow conditions. In order to downselect appropriate materials to advance to the next level, realistic test conditions and aging treatments must be employed sooner. Otherwise, considerable time is wasted on catalysts that will not be utilized in aftertreatment systems. Specifically, feed components and aging protocols consistent with USCAR initiatives must be used to lend credibility to results.

\section{Reviewer 3:}

The reviewer noted that the in-situ growth of nano-array catalysts on monoliths is very interesting, although the reviewer wondered how relevant this would be commercially. The reviewer wanted to know, for example, how possible is the scale-up of this coating method, how durable are the nano-arrays, and are they more susceptible to thermal stresses, sintering, and aging.

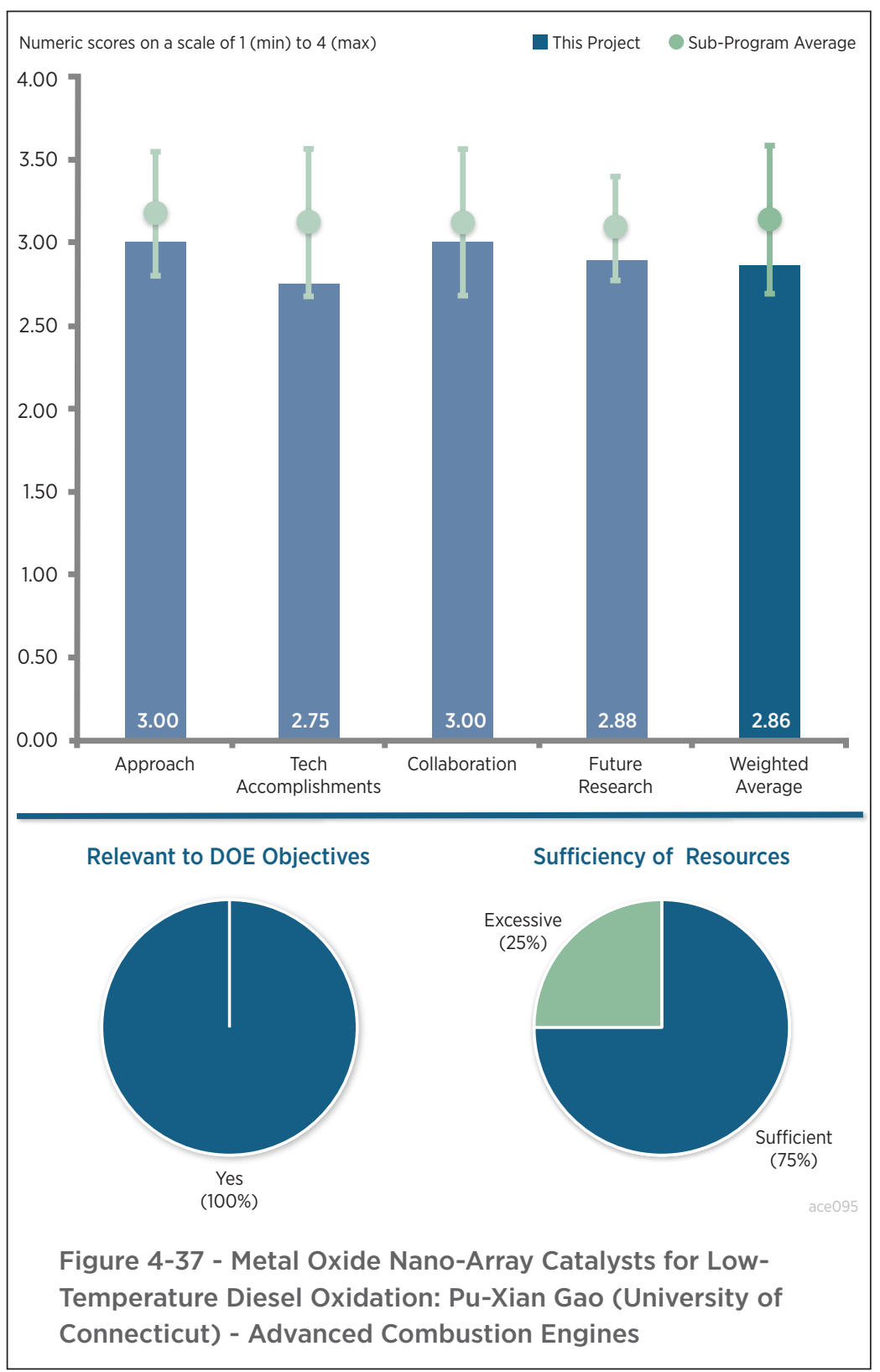




\section{Reviewer 4:}

The reviewer pointed out the need to have assessed $\mathrm{S}$ poisoning effects by now (based on comments from last year). There is no need to present any more data collected under unrealistic conditions (e.g., no $\mathrm{H}_{2} \mathrm{O}$, no $\mathrm{CO}_{2}$, and no $\mathrm{NO}_{\mathrm{x}}$ ). The reviewer only wanted to see data that have been collected with the full catalyst testing protocol specified by the USCAR ACEC Technical Team in the future.

\section{Question 2: Technical accomplishments and progress toward overall project and DOE goals-the degree to which progress has been made, measured against performance indicators and demonstrated progress towards DOE goals.}

\section{Reviewer 1:}

The reviewer noted that minor progress had been achieved in the characterization of multiple catalyst formulations using more realistic feed conditions and aging treatments. The $\mathrm{HC}$ species used were appropriate and represented challenging molecules to convert at low temperatures. However, poisoning effects were not addressed and comparison to a reference, traditional PGM catalyst was not done as a benchmark. The reviewer noted that from a manufacturing perspective, using a growth technique to deposit an active catalyst material on a substrate may preclude the adoption of this technology. Manufacturability is a critical element to both OEMs and catalyst manufacturers. The reviewer inquired as to whether any progress has been made to reduce this challenge.

\section{Reviewer 2:}

The reviewer commented that progress forward in a project with so many pieces and types of catalysts is very challenging. Interesting results have been obtained, but some aspect of the data is difficult to understand, particularly the very abrupt propane light-offs for some catalyst systems, the doped cobalt oxides, and the Pt-titania $\left(\mathrm{TiO}_{2}\right)$ catalysts. These data, and CO-oxidation, are very interesting; however, in the last year more work needs to have been done using the ACEC protocol compositions with HTA, which they have begun. A truer idea of the merit of these catalysts can then be made.

\section{Reviewer 3:}

The reviewer stated that technical accomplishments in the past year include both PGM-free and Pt nano-arrays grown in-situ. One concern is that the Pt size increased due to HTA, which again brings up the question as to the nano-array stability. Current testing methods do not allow for separation of kinetic and mass transport properties, and this is very important in understanding the mechanism.

The reviewer commented that the to-do list on this project remains very long and it looks like things are a bit behind schedule. Hopefully, the collaborations with ORNL and Umicore will allow for pathways to make some quicker progress and keep the project focused.

\section{Reviewer 4:}

The reviewer noted that transition metal results on Slide 12 are of limited value because they were collected under totally unrealistic conditions. Similar considerations hold for other data, such as the perovskite catalysts on Slide 15 and the $\mathrm{Pt} / \mathrm{TiO}_{2}$ data on Slides 19 and 21. Slide 23 demonstrates the huge difference in catalyst performance between the simple conditions and the full protocol conditions. The $\mathrm{CO}$ and $\mathrm{C}_{3} \mathrm{H}_{6}$ data with the full protocol are encouraging.

The reviewer commended the project team on its thermal aging conditions (50 hours at $800^{\circ} \mathrm{C} \mathrm{HTA} \mathrm{aging),} \mathrm{but}$ recommended that the team must incorporate realistic evaluation conditions in its work.

\section{Question 3: Collaboration and coordination with other institutions.}

\section{Reviewer 1:}

The reviewer commented that there was good collaboration with ORNL and Umicore.

\section{Reviewer 2:}

The reviewer remarked that this project has improved some since last year with the inclusion of additional universities and hoped that the planned Umicore work will help keep the evaluations realistic and the work focused and on track. 


\section{Reviewer 3:}

The reviewer stated that including or consulting with an OEM should be part of this project as this would provide a reality check on the work and helpful suggestions for testing.

\section{Reviewer 4:}

The reviewer asserted that while there are excellent collaborators on this project, their contributions are hard to pull out from the discussion of the results.

\section{Question 4: Proposed future research-the degree to which the project has effectively planned its future work in a logical manner by incorporating appropriate decision points, considering barriers to the realization of the technology and, when sensible, mitigating risk by providing alternate development pathways.}

\section{Reviewer 1:}

The reviewer noted that lots of work remains and it may be necessary to focus on the most promising one or maybe two of the catalyst systems to get as complete a set of analyses as possible.

\section{Reviewer 2:}

The reviewer said that there is still a very large to-do list with rather vague plans presented in this presentation. Efforts to mitigate sintering, water, and S are still necessary. The reviewer was not sure that this project will progress to engine testing, even in the final year, but would much rather see the efforts focused and relevant than moving on to engine testing just for the sake of the demonstration. Some effort should be spent on understanding the role of kinetics versus mass transport.

\section{Reviewer 3:}

The reviewer stated that the project team must include S poisoning assessments in the future work. Also, the team must utilize full exhaust mix on all experiments.

\section{Reviewer 4:}

The reviewer stated that the project team indicated last year that more progress would be made testing under realistic conditions and aging methods; that has not materialized as expected. There reviewer expressed concern that this will be appropriately addressed going forward.

\section{Question 5: Does this project support the overall DOE objectives of petroleum displacement? Why or why not?}

\section{Reviewer 1:}

The reviewer found this project to be consistent with the industry need for low-temperature catalyst solutions to meet future emissions standards while minimizing cost. However, unless the concept catalysts are characterized under more appropriate conditions in a timely manner, the technology and usefulness of the data are questionable.

\section{Reviewer 2:}

The reviewer said that developing low-temperature activity is offered by this project, especially in non-PGM catalysts, so the studies with aging and $\mathrm{S}$ are very pertinent to assess these catalysts.

\section{Reviewer 3:}

The reviewer stated that LTAT will be necessary for more efficient engines in the future.

\section{Reviewer 4:}

The reviewer commented that, theoretically, yes, this meets DOE's petroleum displacement objectives. The further this project progresses, the less likely this is becoming, not because it was a bad idea, but just because the research community is learning that there were unforeseen complications with these materials. 


\section{Question 6: Resources: How sufficient are the resources for the project to achieve the}

stated milestones in a timely fashion?

Reviewer 1:

The reviewer found funding and staffing to be appropriate.

\section{Reviewer 2:}

The reviewer remarked that with the development of catalyst studies using more realistic conditions, the resources should be sufficient. The main issue may be based more on focusing on the most effective catalysts.

\section{Reviewer 3:}

The reviewer noted that the budget on this project is quite high considering the accomplishments to date and the likelihood that it will not get to engine testing. 


\section{Micro-Jet Enhanced Ignition with a Variable Orifice Fuel Injector for High-Efficiency Lean-burn Combustion: Chia- Fon Lee (University of Illinois) - ace096}

\section{Presenter}

Chia-Fon Lee, University of Illinois

\section{Reviewer Sample Size}

A total of four reviewers evaluated this project.

\section{Question 1: Approach to performing the work-the degree to which technical barriers are addressed, the project is well-designed, feasible, and integrated with other efforts.}

\section{Reviewer 1:}

The reviewer found this project difficult to assess. Because of the constraints on information disclosure associated with an incubator project, the presentation could only talk in generalities. It seems that the approaches being pursued are fundamentally sound and the evaluation being done is comprehensive. The proof will be in the data, which are yet to come.

\section{Reviewer 2:}

The reviewer commented that the barrier to address is stated to be "Lack of cost-effective emissions control," but stratified charge engines are known to produce high $\mathrm{NO}_{\mathrm{x}}$ due to near stoichiometric combustion and high particulates due to inadequate mixing. The G equation in KIVA is an inadequate and insufficient tool to predict emissions in a stratified charge engine.

\section{Reviewer 3:}

The reviewer stated that the development approach is reasonable with some preliminary simulations followed by single-cylinder evaluation and optical measurements. Not knowing more details about the technology approach, it is hard to say if the preliminary simulations were adequate.

\section{Question 2: Technical accomplishments and progress toward overall project and DOE goals-the degree to which progress has been made, measured against performance indicators and demonstrated progress towards DOE goals.}

\section{Reviewer 1}

The reviewer noted that it seems that a significant technical work has been accomplished. How good it is will not

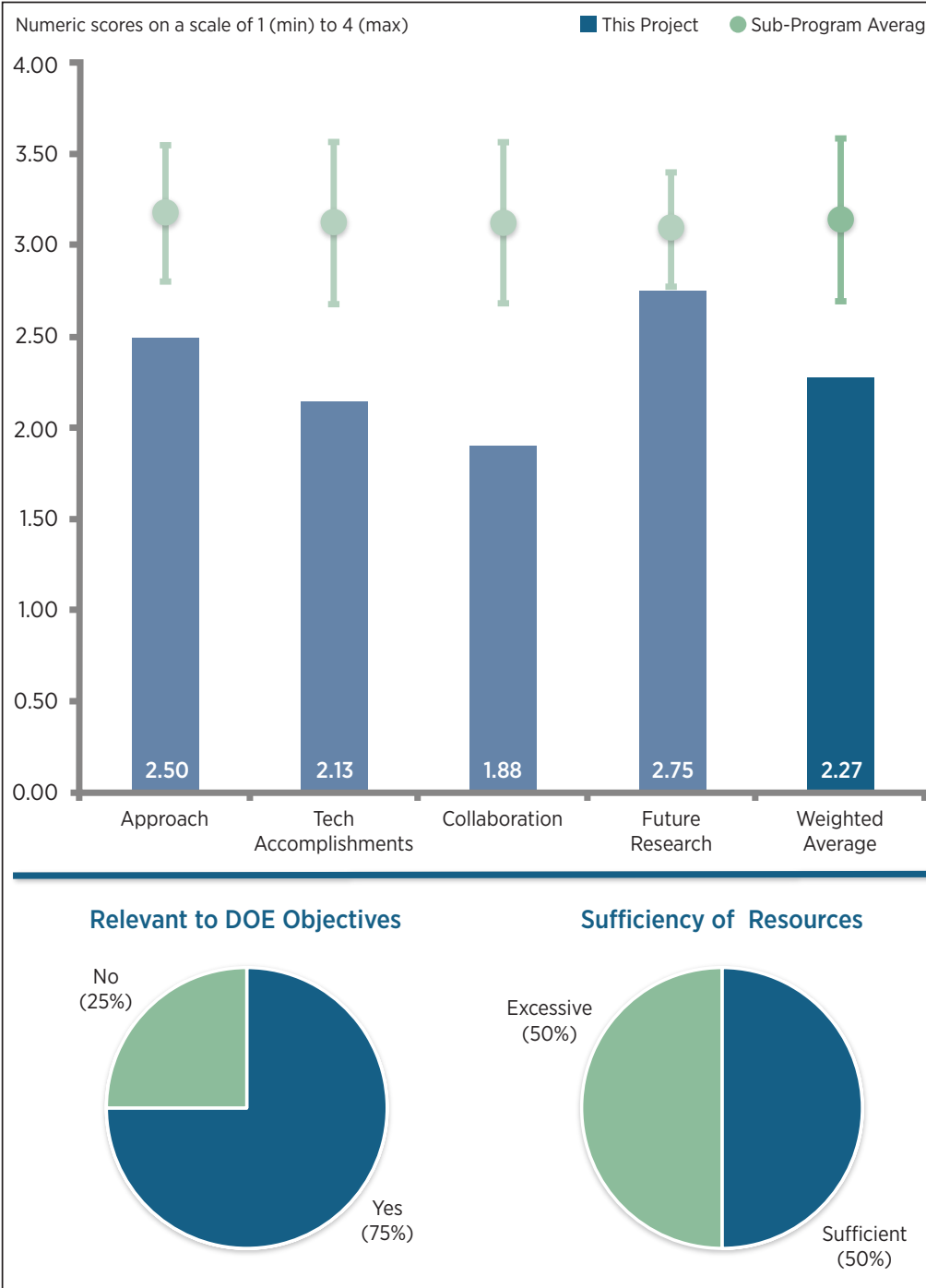

Relevant to DOE Objectives
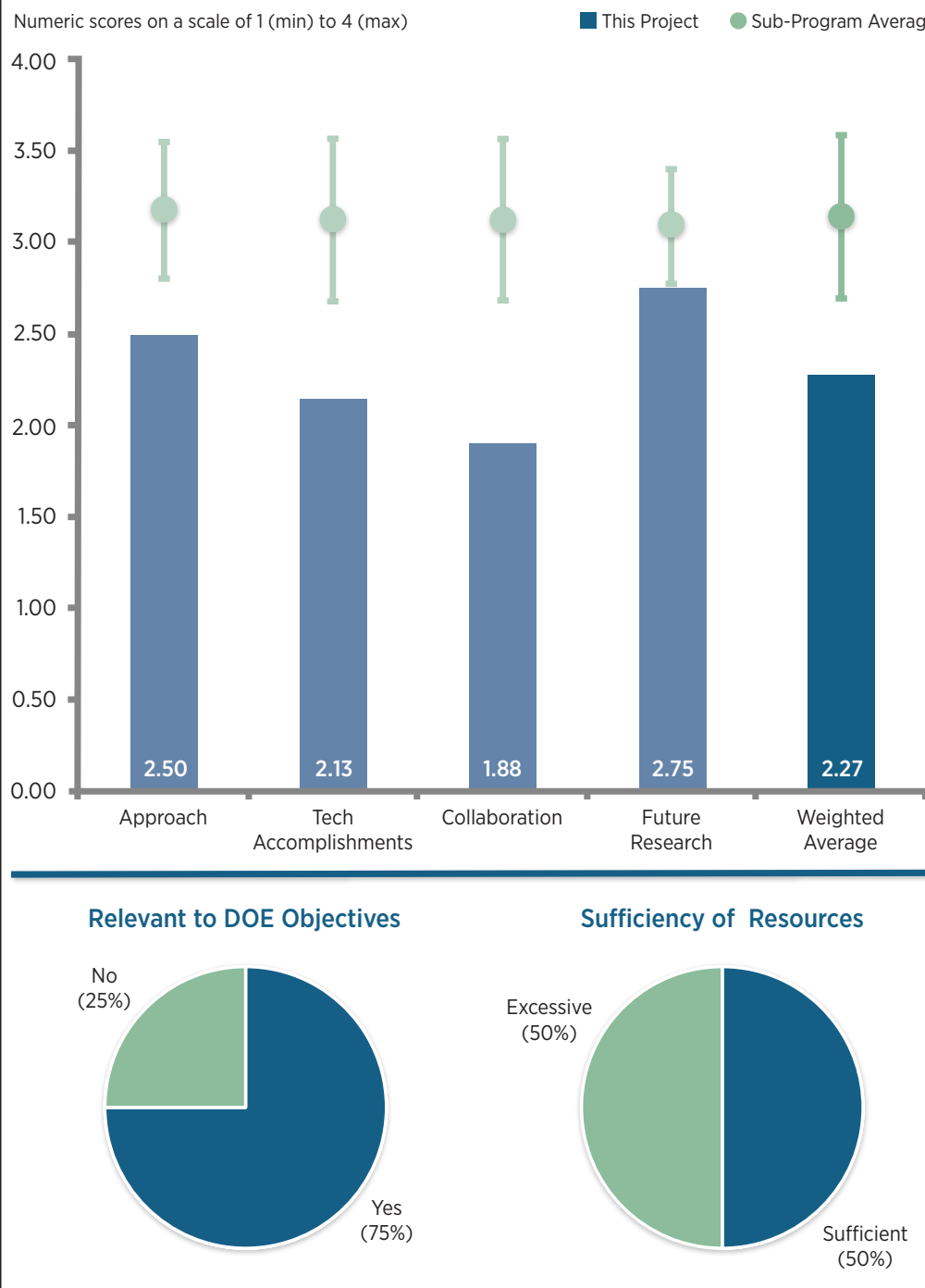

Figure 4-38 - Micro-Jet Enhanced Ignition with a Variable Orifice Fuel Injector for High-Efficiency Leanburn Combustion: Chia-Fon Lee (University of Illinois) Advanced Combustion Engines

ace096 
be known until the data have been obtained. This reviewer also asserted that the PI is very good and his work is credible.

\section{Reviewer 2:}

The reviewer remarked that progress is impossible to assess because no results were presented.

\section{Reviewer 3:}

The reviewer observed that, again, this is hard to rate given the lack of details. There are several easy criticisms/ questions to offer. There was a focus of not putting fuel on the liner, but nothing was said about the piston. The $150-\mathrm{mm}$ liquid length seems excessive. Depending on the approach, this could produce a noncommercial amount of soot off the piston top.

The reviewer asked for the combustion efficiency (e.g., will the lean regions near the walls burn). The reviewer asked what the approach is to deal with the high $\mathrm{NO}_{x}$, and how that impacts engine efficiency. The reviewer would like to know where the spark plug is located and also what happens at high load. The reviewer also inquired about the power density, if still stratified. Finally, if lambda is one, the reviewer asked what the knock limit is given the newly styled piston and combustion chamber.

\section{Question 3: Collaboration and coordination with other institutions.}

\section{Reviewer 1:}

The reviewer wished there was a not applicable review category to check. As an incubator project, collaboration does not seem appropriate for this project

\section{Reviewer 2:}

The reviewer stated that there is no collaboration.

\section{Reviewer 3:}

The reviewer suggested that this project would benefit from the involvement of someone with combustion system development experience (e.g., an OEM or an engineering service provider). Without this involvement, the reviewer was skeptical that the project will deliver any meaningful results.

\section{Question 4: Proposed future research-the degree to which the project has effectively planned its future work in a logical manner by incorporating appropriate decision points, considering barriers to the realization of the technology and, when sensible, mitigating risk by providing alternate development pathways.}

\section{Reviewer 1:}

The reviewer looked forward to results.

\section{Reviewer 2:}

The reviewer commented that it seems that a viable plan is in place to get data and evaluate the concept.

\section{Reviewer 3:}

The reviewer stated that getting measured results on a running single-cylinder engine is a good next step. This should inform the models and allow them to be correlated. The big unknown is will the technology deliver the expected results on the single-cylinder engine, and if not, can it ever be made to work.

\section{Question 5: Does this project support the overall DOE objectives of petroleum displacement? Why or why not?}

\section{Reviewer 1:}

The reviewer was not familiar with the details of the incubator program, but it seemed to the reviewer that if this project is successful, it could contribute to improved lean burn engine development. 


\section{Reviewer 2:}

The reviewer stated agreement with any technology that addresses emissions and efficiency is relevant, but it is unclear how relevant this project truly will be, given the minimal details shared.

\section{Reviewer 3:}

The reviewer remarked that the concept is unlikely to work.

Question 6: Resources: How sufficient are the resources for the project to achieve the stated milestones in a timely fashion?

No comments were received in response to this question. 
Affordable Rankine Cycle (ARC) Waste Heat Recovery for Heavy-Duty Trucks: Swami Subramanian (Eaton Corporation) - ace097

\section{Presenter}

Swami Subramanian, Eaton

Corporation

\section{Reviewer Sample Size}

A total of seven reviewers evaluated this project.

Question 1: Approach to performing the work-the degree to which technical barriers are addressed, the project is well-designed, feasible, and integrated with other efforts.

\section{Reviewer 1:}

The reviewer stated that most of the work is still in progress. The steps presented on the Approach/Strategy slide seem to be in the right direction. Perhaps additional plots and visual representations other than words would help the reader understand the project team's intent faster.

The reviewer remarked that the use of the existing coolant as the working fluid eliminates the driver's burden to buy additional fluids - a feasibility study is in progress. The evaluation of different WHR architectures should include at least a basic schematic/drawing of the main WHR components: pump, boiler, expander, condenser, etc.

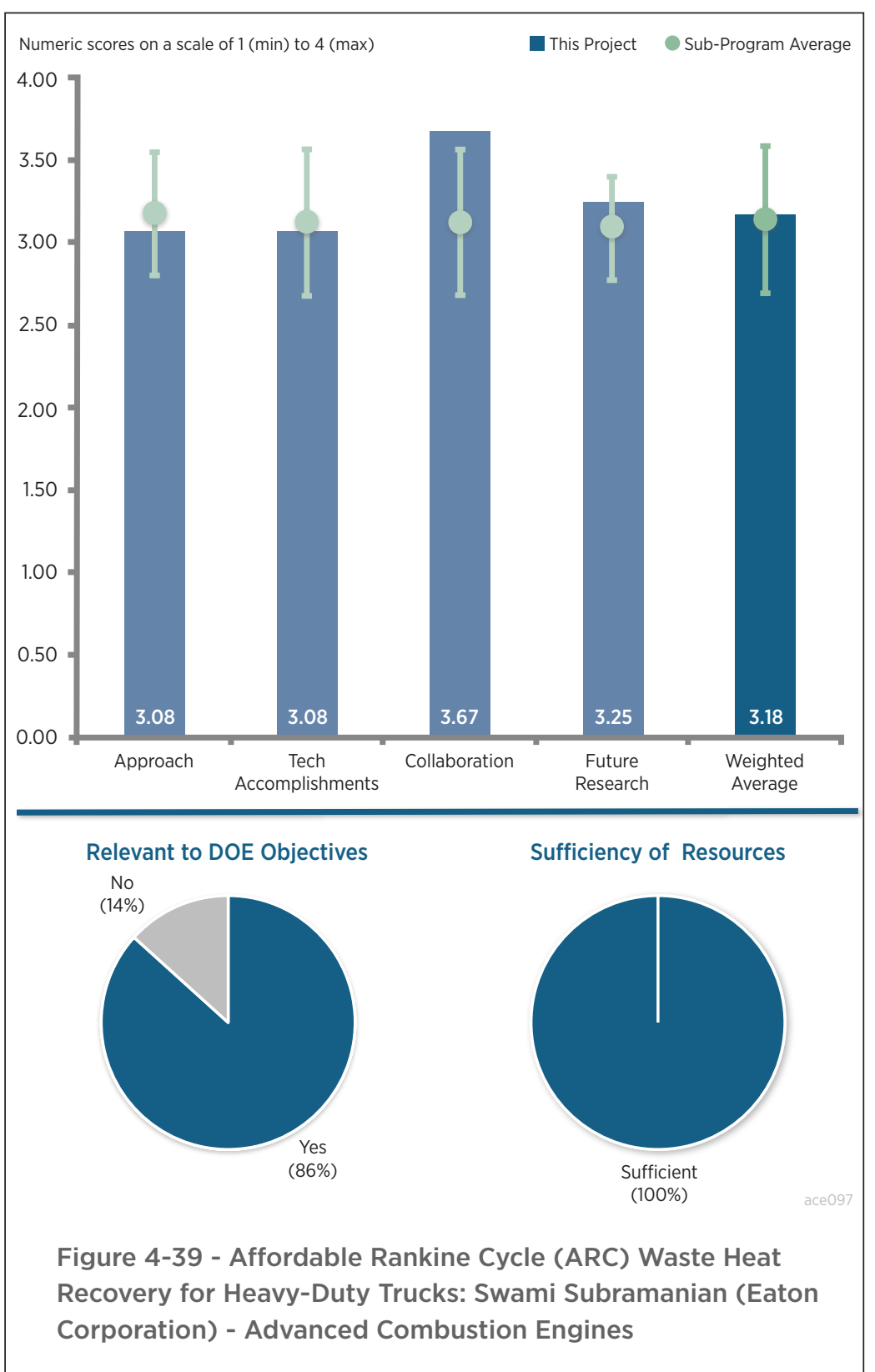

\section{Reviewer 2:}

The reviewer noted that very early in the program, the plan looks fair. It would go a long way to have had a first-order analysis that would show what it takes to get a 5\% fuel economy (FE) improvement — how much heat is needed, what efficiencies are needed, etc.

\section{Reviewer 3:}

The reviewer stated that, in the literature, the working fluid for WHR is typically ethanol, and the expected fuel economy benefit in real world driving is around 3\%-5\%. This project chooses to use the engine coolant as the working fluid with a target 5\% FE improvement. If successful, it would represent a significant advance in WHR technology.

The project is well-designed and covers all the tasks related to the WHR system development. A major challenge with WHR is the systems integration/optimization. The reviewer commented that this task should start from the 
very beginning instead of near the end of the project. The reviewer recommended that increasing the fan power requirement should also be considered along with the charge air cooler design.

\section{Reviewer 4:}

The reviewer noted that this project is at an early stage and the project team is pursuing an interesting strategy. The reviewer had concerns regarding the 5\% FE increase with WHR systems. There were no thermodynamic data supporting this number presented in the presentation, and it seems like an aggressive goal. The reviewer said that it would be good to know the assumptions that go into the projected 5\% FE benefit from this project.

\section{Reviewer 5:}

The reviewer stated that although it is early in the project, the approach seems appropriate. However, the working fluid composition study is the basis of the viability of the concept. The reviewer had concerns about some boundary conditions that need attention. The first is that the ratio of glycol to water directly impacts the heat capacity of the system. The reviewer asked how the integrity of the working fluid will be maintained and how dissolved gases will be addressed. Second, the reviewer referred the project team to concerns about the fact that the heat transfer into the working fluid may include nucleate boiling. The reviewer wanted to know what steps will be taken to minimize the effects of insulating air bubbles inhibiting heat transfer (i.e., abrasive slurry extrusion through coolant passages to increase smoothness and the addition of surfactant to the working fluid to help disperse vapor bubbles that may form).

\section{Reviewer 6:}

The reviewer commented that using coolant as a working fluid is an excellent idea; however, the performance would be challenging to meet the target due to high-temperature decomposition of the coolant. It is not clear how $5 \% \mathrm{FE}$ is defined. The reviewer wanted to know if it would be for a single point at $65 \mathrm{mph}$ cruise speed or over the 13 mode composite Supplemental Emission Test point.

\section{Question 2: Technical accomplishments and progress toward overall project and DOE goals-the degree to which progress has been made, measured against performance indicators and demonstrated progress towards DOE goals.}

\section{Reviewer 1:}

The reviewer noted that this project has just started and good progress has been made in identifying the working fluid.

\section{Reviewer 2:}

The reviewer commented that the project team has been meeting the schedule since the start in February.

\section{Reviewer 3:}

The reviewer remarked that the project is at a very early stage. The work accomplished to date is appropriate for the stage of the project.

\section{Reviewer 4:}

The reviewer observed that the progress is organized, and the results from the Roots expander and its drive design will be critical in establishing the viability of this system.

\section{Reviewer 5:}

The reviewer did not observe much, other than performing the 13-point engine testing baseline. The project team has laid out a plan at least regarding its intentions. The plot in Slide 13 could use additional explanatory notes. Regarding the CFD analysis, no details are provided regarding what the simulation conditions are; how the calibration, if any, will be performed and assessed for accuracy; what will be achieved; and what exactly is to be modeled. At this stage, it is too early to make further comments in the absence of some intermediate results.

\section{Reviewer 6:}

The reviewer found not too much progress that can be evaluated because the program just started not too long ago.

\section{Question 3: Collaboration and coordination with other institutions.}




\section{Reviewer 1:}

The reviewed found the collaboration and coordination with industry partners, universities, and/or subcontractors to be excellent.

\section{Reviewer 2:}

The reviewer stated that there was a good cross section of academia and industry.

\section{Reviewer 3:}

The reviewer remarked that this project includes a large number of collaborators with distinct roles. Each brings a unique expertise to the project.

\section{Reviewer 4:}

The reviewer praised working with PACCAR as an excellent starting point, specifically with PACCAR's MX-13 engine. Also, a list of partners seems to be very clearly defined for their roles.

\section{Reviewer 5:}

The reviewer said that the list of collaborators contains appropriate industry leaders. The success of this project is dependent on the integration of the Roots expander with the PACCAR engine/control systems.

\section{Reviewer 6:}

The reviewer commented that the Collaborations and Coordination information seems to be more explicit, revealing which task each partner should address. The partners seem to be well coordinated by the project team. Perhaps more explanations (i.e., what models or testing facilities and what laboratories each of the collaborators use) would help the reviewer to understand the team's activities better.

\section{Question 4: Proposed future research-the degree to which the project has effectively planned its future work in a logical manner by incorporating appropriate decision points, considering barriers to the realization of the technology and, when sensible, mitigating risk by providing alternate development pathways.}

\section{Reviewer 1:}

The reviewer commented that it will be interesting to see the project moving forward.

\section{Reviewer 2:}

The reviewer commented that all plans are in place to deliver the results. The reviewer looked forward to hearing from the progress next year.

\section{Reviewer 3:}

The reviewer noted that the project is logical and well planned in general. It seems to lack an alternative in term of working fluid if the current choice fails to meet the target.

\section{Reviewer 4:}

The reviewer commented that the future research includes most of the necessary steps, but has not been performed yet by the project team. The project milestones are relevant to DOE objectives according to this reviewer, who listed the following: multi-component CFD analysis; two-phase heat transfer correlation development; Affordable Rankine Cycle (ARC) analytical model development; and WHR components (expander, working fluid, and heat exchangers) design finalized with a go/no-go review in December 2016.

\section{Reviewer 5:}

The reviewer stated that the Critical Assumptions and Issues slides list a number of risks that have a potentially high impact on the project. As a result, the go/no-go decision at the end of FY 2016 is very important. Given the early stage of the project, this go/no-go decision point may be too early in the project timeline. If these critical assumptions and risks cannot be satisfactorily answered at this time, the go/no-go decision point should be postponed to an appropriate time.

\section{Reviewer 6:}

The reviewer pointed out that, while understanding that the project is in its earliest stages, there are some areas that 
need attention. The first is the sensitivity of the WHR system to coolant mixture regarding efficiency and engine heat transfer. The second is the failure mode analyses of the WHR and expander systems to address the effects on the control system when departures from expected Roots expander output occur. The third is the criteria emissions output failure mode effect management (FMEM) when the Roots expander underachieves with power addition.

\section{Question 5: Does this project support the overall DOE objectives of petroleum displacement? Why or why not?}

\section{Reviewer 1:}

The reviewer noted that effective WHR will increase overall engine efficiency and hence reduce petroleum usage.

\section{Reviewer 2:}

The reviewer mentioned that WHR would be likely seen in the Phase 2 of EPA HD GHG rules. WHR is one of the most effective technologies to achieve high performance in FE.

\section{Reviewer 3:}

The reviewer commented that low-cost WHR is in direct alignment with DOE's objectives and supports petroleum reduction.

\section{Reviewer 4:}

The reviewer commented that, if successful, the project would be relevant to a significant fuel consumption reduction.

\section{Reviewer 5:}

The reviewer remarked that, if successful, the technology would results in fuel savings that would support DOE objectives of petroleum displacement.

\section{Reviewer 6:}

The reviewer stated that so much opportunity exists to have dramatic reductions in transport fuel consumption, given the poor fuel economy exhibited by over-the-road freight and other commercial vehicles. If successful, the objectives have the potential to reduce both the GHG impact of transport and the cost as well.

\section{Question 6: Resources: How sufficient are the resources for the project to achieve the stated milestones in a timely fashion?}

\section{Reviewer 1:}

The reviewer commented that the project resources of $\$ 4$ million may just be sufficient to meet the stated milestones in a timely fashion, given the multitude of collaborations under such a tight schedule.

\section{Reviewer 2:}

The reviewer stated that funding seems to be adequate for the remaining tasks.

\section{Reviewer 3:}

The reviewer remarked that given the early stage of the project, the project resources appear to be sufficient.

\section{Reviewer 4:}

The reviewer commented that, so far, it is still too early to tell, but it seems that the project is on schedule. 
Cummins 55\% BTE Project: Lyle Kocher (Cummins)

- ace098

Presenter

Lyle Kocher, Cummins

\section{Reviewer Sample Size}

A total of eight reviewers evaluated this project.

Question 1: Approach to performing the work-the degree to which technical barriers are addressed, the project is well-designed, feasible, and integrated with other efforts.

\section{Reviewer 1:}

The reviewer stated that the Cummins team has laid out an aggressive, yet viable, path to achieving 55\% BTE.

\section{Reviewer 2:}

The reviewer stated that the approach looks good and noted the project team's attack on thermal heat losses and heat recovery barriers to enable a $55 \% \mathrm{BTE}$ demonstration.

\section{Reviewer 3:}

The reviewer commented that the project is using a traditional, fully comprehensive approach that utilizes Cummins' strengths, particularly in fuel injected engines (FIE), turbocharging, and WHR. Some technologies offer big gains, like WHR (4\%-4.5\% BTE) and combustion and FIE (1.3\% BTE each), while others offer smaller increments.

The integrated approach and estimated BTE improvements of various approaches gets the project team to its goal.

\section{Reviewer 4:}

The reviewer found the approach to be reasonable and systematic to meet a stretch 55\% BTE goal by targeting high-pressure injection, multiple EGR loops, and a higher CR. The reviewer mentioned that it was excellent to set interim real goals to have initial 50\% BTE on an engine dynamometer running very soon without WHR. Major risks have been identified. It is a very nice approach to manage moisture level in the low-temperature EGR loop with the WHR system.

To improve the approach, the reviewer said that more detail on risk mitigation and contingency plans for known challenges could improve the score. The reviewer wanted to know, for example, what specific plans are in this work to overcome known high risk issues with the planned approach. For thermal coatings, the reviewer referenced the statement, "previous work with insulated combustion systems have been challenged to demonstrate improved efficiencies," and opined that more detail on what novel approach not attempted with thermal coatings or contingency to make up efficiency would improve the approach. Also a risk is that engine-out $\mathrm{NO}_{\mathrm{x}}$ will be 
higher due to higher temperature combustion. The reviewer commented that in this case, the upper limit to engineout $\mathrm{NO}_{\mathrm{x}}$ should be reported as a metric as it is likely well understood by aftertreatment system designers and manufacturers.

\section{Reviewer 5:}

The reviewer commented that the project entails a systems-level approach towards the challenging BTE goal. A systems-level approach is required to achieve success for such an aggressive goal. Emissions are included in the approach including impacts on fuel efficiency. WHR is also included.

\section{Reviewer 6:}

The reviewer stated that the approach for this project is very good. There is a recognition that, to push to the maximum efficiency, every component on the engine system needs to be evaluated. There is no single silver bullet.

\section{Reviewer 7:}

Based on other presentations the reviewer had seen on advanced combustion regimes, the key to progress is a robust engine control system and strategy. In this project, fuel and air handling system control define the combustion and effectiveness of the aftertreatment system. The reviewer commented that whether the hardware/ software pairing for 50\% BTE (engine only) is sufficient or appropriate for a production vehicle is unknown, but the controls system effectiveness will dictate the overall performance of the whole engine system.

\section{Reviewer 8:}

The reviewer noted that it would be a good R\&D project, but was not convinced that the proposed approach has any potential path to become a commercially viable approach. Two noticeable issues are high engine-out $\mathrm{NO}_{\mathrm{x}}$ and condensation issues for the LP EGR system. The reviewer asked if the engine can meet the 2010 emission standards, specifically on the cold FTP cycle with such high engine-out $\mathrm{NO}_{\mathrm{x}}$. Condensation can bring up a big warranty issue if the LP EGR cooler fails. The reviewer did not see any good solution for that.

Also, it seemed to the reviewer that the Cummins ISX engine would be used for this program. This engine has limitations on peak cylinder pressure. It is not going to be a good engine platform to achieve the program goal because this engine is old and it will be at the end of life when the program is completed.

\section{Question 2: Technical accomplishments and progress toward overall project and DOE goals-the degree to which progress has been made, measured against performance indicators and demonstrated progress towards DOE goals.}

\section{Reviewer 1:}

The reviewer stated that the project has just started, but the accomplishments to date are impressive. The project team has a detailed technical road map that has been developed through verified simulation and laboratory testing. The project team is engaging in the fundamental activities necessary to overcome the barriers it has identified.

\section{Reviewer 2:}

The reviewer commented that there has been an impressive delivery of results in such a short period. Cavitation modeling and piston crown thermal barriers are cutting edge. The decision on SCR filters and dual-loop EGR, which is borrowed from Cummins' and others' light-duty programs, is promising. As a result of this, the reviewer observed $0.4 \%$ BTE points from unique aspects of this and noted exhaust manifold design and turbocharging optimization. The reviewer said that the high EGR rates allow much leverage in $\mathrm{NO}_{\mathrm{x}}$ and efficient combustion. The next-generation WHR is significantly adding to the knowledge base in the industry. The reviewer thanked the project team for publicly reporting the amount of data and results

\section{Reviewer 3:}

The reviewer noted that the project has just started, and the accomplishments are appropriate for the short timeframe.

\section{Reviewer 4:}

The reviewer remarked that the results were excellent for a newly started project with leverage from prior SuperTruck engine work. The piston design data indicate that targeted incremental efficiency improvements have 
been comfortably exceeded. The reviewer said that injector prototypes, engine friction reduction prototypes, and WHR prototypes have been tested with good results and plans for further confirmation/optimization.

\section{Reviewer 5:}

The reviewer commented that the technical accomplishments being made in this project are outstanding. From an academic standpoint, the reviewer would like to encourage Cummins to make as much information as possible public through technical publications. The reviewer understood that there are issues with proprietary information, but the thermodynamic and structural analyses that were conducted would be very informative.

\section{Reviewer 6:}

The reviewer stated that the project team had advised the reviewer that the engine will be run on certification diesel, but various fuel compositions need to be evaluated. The reviewer had two items of concern: the first is that at some point key parameters of those fuels will affect items related to technical barriers, such as $\mathrm{NO}_{\mathrm{x}}$ performance (cetane and FAME content) and PM emissions (aromatic content). The other is that BTE is also dependent on the catalyst efficiency at the feedgas temperature and equivalence ratio. The additional fuel required to maintain catalyst light off and/or DPF effectiveness will reduce BTE.

\section{Reviewer 7:}

The reviewer said that this project is just beginning so there is not much to report on at this stage.

\section{Reviewer 8:}

The reviewer remarked that it is too early to tell, with a big question mark.

\section{Question 3: Collaboration and coordination with other institutions.}

\section{Reviewer 1:}

The reviewer stated that the work is basically an internal Cummins program; however, the company is engaging with the national laboratories doing relevant work in CRADAs and spray diagnostic and modeling development.

\section{Reviewer 2:}

The reviewer said that there are no collaborations on this project external to Cummins. The reviewer was not sure any are necessary though. Including a university partner to perform thermodynamic analyses could be useful to add academic depth, and increasing the amount of public information could be useful.

\section{Reviewer 3:}

The reviewer noted that this is all internal work to Cummins. The reviewer would have preferred to see project benefit, and knowledge, spread to numerous parties. However, given the tight timeframe, external collaborations may have been difficult. The reviewer commented that proper project management could have pulled this off though. There is work being done with the supply chain partners.

\section{Reviewer 4:}

The reviewer commented that the project appears to be all internal Cummins, without external collaborators. Although Cummins has excellent capabilities in the fuels systems and turbo areas, the team should consider additional institutions to provide alternate viewpoints on the approaches.

\section{Reviewer 5:}

The reviewer found this work to be a very Cummins specific project with limited need for external collaboration. Work products are well supported by analysis and data are presented that are excellent. The reviewer commented that including an aftertreatment partner, and potentially a third-party engine laboratory for confirmation testing, would improve the score. The expertise of unnamed suppliers is clearly needed to complete development and their recognition/inclusion for major work could improve the score.

\section{Reviewer 6:}

The reviewer remarked that it appears that all of the work is being done at Cummins with no outside partners on the project. Working with different divisions of the same company is not really collaborating. The project needs to improve collaborations by bringing in outside partners to the project. 


\section{Reviewer 7:}

The reviewer acknowledged not knowing the entire history of this project, but the only participants appear to be Cummins companies. The reviewer was unsure how to rate this because other projects had outside vendor/ university/government laboratory participation.

\section{Reviewer 8:}

The reviewer was unsure that Cummins Turbo Technologies and Cummins Fuel Systems can be viewed as partners because they all belong to Cummins. The reviewer opined that this would not be the best use of funds to support a single company.

\section{Question 4: Proposed future research-the degree to which the project has effectively planned its future work in a logical manner by incorporating appropriate decision points, considering barriers to the realization of the technology and, when sensible, mitigating risk by providing alternate development pathways.}

\section{Reviewer 1:}

The reviewer was looking forward to additional development from this project—excellent work.

\section{Reviewer 2:}

The reviewer stated that Cummins has an excellent plan for this project. The reviewer looked forward to the results to come.

\section{Reviewer 3:}

The reviewer said that the project is just in the beginning stages. The planned research is in good shape for now (as proposed).

\section{Reviewer 4:}

The reviewer stated that it seems that SCR filters are a key technology, and Cummins states higher $\mathrm{NO}_{\mathrm{x}}$ will likely be needed to deliver the goals. However, there are no plans stated here to pursue advance de- $\mathrm{NO}_{\mathrm{x}}$. This would especially be critical to implementation, given the likelihood of a nationwide low- $\mathrm{NO}_{\mathrm{x}}$ standard. The reviewer noted that other barriers have been identified, and steps appear in place to chase them down.

\section{Reviewer 5:}

The reviewer noted that a very good plan was presented and the approach is being followed in future plans. As indicated, more information on risk mitigation and contingency (what other pathways are possible if one of the high risk approaches does not achieve targets) would improve the score.

\section{Reviewer 6:}

The reviewer was surprised that some form of port injected water is not under investigation for $\mathrm{NO}_{\mathrm{x}}$ mitigation coincident with efforts to reduce combustion duration for more efficient heat release characteristics. The reviewer noted that, historically, efficiency comes at the expense of higher engine-out $\mathrm{NO}_{\mathrm{x}}$ and lower $\mathrm{NO}_{\mathrm{x}}$ comes at the expense of torque and/or efficiency.

\section{Reviewer 7:}

The reviewer said that the future work seems to be comprehensive, including all pieces that are needed for the demonstration.

\section{Question 5: Does this project support the overall DOE objectives of petroleum displacement? Why or why not?}

\section{Reviewer 1:}

The reviewer stated that 55\% BTE enabling technologies contribute directly to DOE objectives of petroleum displacement through efficiency gains.

\section{Reviewer 2:}

The reviewer noted that work to achieve a 55\% BTE target performed by engine manufacturers with a high level of cost share clearly supports DOE objectives for petroleum displacement. 


\section{Reviewer 3:}

The reviewer suggested that this project can lead directly to petroleum displacement by improving the fuel efficiency of diesel engines, which dominate the transportation industry in the United States.

\section{Reviewer 4:}

The reviewer found this project to be highly relevant because there is a direct link to reduced petroleum consumption.

\section{Reviewer 5:}

The reviewer commented that development of any efficient technologies can always support overall DOE goal.

\section{Reviewer 6:}

The reviewer stated that it is assumed that achieving a 55\% BTE for a given operating point implies a broad area of in-cycle efficiency gains. Modeling of many more operating points should be performed to ensure there is indeed a reasonable BTE gain translating into real fuel economy gains.

\section{Question 6: Resources: How sufficient are the resources for the project to achieve the stated milestones in a timely fashion?}

\section{Reviewer 1:}

The reviewer said that reaching 55\% BTE is a tough goal, and resources for this project look to be on target for the difficulty to achieve this.

\section{Reviewer 2:}

The reviewer remarked that the budget is good considering this is follow-on work from SuperTruck. It is difficult to obtain this level of activity at the project's current funding level without leverage of the prior work. The reviewer noted that if prior SuperTruck work had not been done, then the budget would be very lean.

\section{Reviewer 3:}

The reviewer commented that it seems that it is okay at this early stage.

\section{Reviewer 4:}

The reviewer noted that Cummins stands to gain quite a bit of expertise (and potentially a competitive advantage) with this research. The reviewer was surprised that there are no other participants to both fund and participate in this project. 


\section{Improved Fuel Efficiency through Adaptive Radio Frequency Controls and Diagnostics for Advanced Catalyst Systems: Alexander Sappok (Filter Sensing Technologies, Inc.) - ace099}

\section{Presenter}

Alexander Sappok, Filter Sensing Technologies, Inc.

\section{Reviewer Sample Size}

A total of seven reviewers evaluated this project.

\section{Question 1: Approach to performing the work-the degree to which technical barriers are addressed, the project is well-designed, feasible, and integrated with other efforts.}

\section{Reviewer 1:}

The reviewer applauded the approach to the project as excellent. The research teaming is strong, with partners having well-developed roles (Slide 13 is great). Year 1 is focused on development and refinement of the sensor and screening tests. The reviewer remarked that two quarters into the project (started in October 2015), the project team is on track to meet the goals.

\section{Reviewer 2:}

The reviewer noted that the approach of using live RF sensors to measure catalyst state during vehicle operation appears to be feasible based on researchers' previous work and, of course, would be highly desirable. The planned research appears to cover the bases in terms of developing and testing the technology.

\section{Reviewer 3:}

The reviewer remarked that a nice approach was laid out.

\section{Reviewer 4:}

The reviewer observed that the project was an excellent extension of the technology. The methodology to sort through the challenges looks solid, and the project team is working with a great cross section of the industry.

\section{Reviewer 5:}

The reviewer noted that the RF sensor project for soot-loading measurement was quite successful. An RF sensor provides more information than the pressure drop across the DPF. This information helps to reduce the frequency of trap regeneration, thus improving fuel economy. It is not obvious, however, that fuel efficiency could be improved when the same technology is being used for SCR and/or a TWC. The feasibility study should include if 
additional information obtained by the radio frequency (RF) sensor (i.e., $\mathrm{NH}_{3}$ or oxygen storage) could actually improve fuel efficiency. The reviewer said that this task should be performed at an early stage of the project. Other than this missing link, the rest of the project is well-designed.

\section{Reviewer 6:}

The reviewer stated that improved sensing supports OBD requirements and can enable some improvements in closed-loop control. It is not clear from the presented material how much better RF sensing is than other competing technologies

\section{Question 2: Technical accomplishments and progress toward overall project and DOE goals - the degree to which progress has been made, measured against performance indicators and demonstrated progress towards DOE goals.}

\section{Reviewer 1:}

The reviewer stated that this is a new project so there are no major accomplishments to review.

\section{Reviewer 2:}

The reviewer remarked that this is a new project in its first year so only the first six months' work has been completed. However, the sensors have been developed and testing is underway so progress is good for the limited time the project has been underway.

\section{Reviewer 3:}

The reviewer mostly graded the project high for the prior work, but there is no reason to think the background shown is not considered as great understanding of the technology.

\section{Reviewer 4:}

The reviewer commented that the success of the DPF sensing was good background to have, but it was a little confusing differentiating that work from this project's accomplishments.

\section{Reviewer 5:}

The reviewer commented that there has been good progress in the first two quarters of the project based on solid prior work on DPF sensor project. Already in this new project, there has been significant work on the RF cavity, both in development and simulations. Additionally, work has begun in regard to catalyst selection and a bench reactor has been commissioned to do the testing. The reviewer noted that preliminary results show excellent promise for $\mathrm{NH}_{3}$ storage.

\section{Reviewer 6:}

The reviewer noted that there has been good progress in term of sensor-related development, testing, and planning. The $\mathrm{RF}$ sensor response to $\mathrm{NH}_{3}$ storage on the SCR catalyst is very promising. The reviewer found the demonstration of fuel savings (the DOE goal) is not very convincing if the project is under the assumption that added information on catalyst state would naturally lead to improvement in fuel efficiency.

\section{Question 3: Collaboration and coordination with other institutions.}

\section{Reviewer 1:}

The reviewer lauded the truly outstanding research team and collaborator list, which has well-defined roles and regular, in-person meetings (which the reviewer thought was excellent).

\section{Reviewer 2:}

The reviewer stated that collaboration and coordination with industry partners, national laboratory, city fleet, and/or subcontractors seems to be in place.

\section{Reviewer 3:}

The reviewer said that there were good partnerships for hardware, testing, and carry-through to implementation. 


\section{Reviewer 4:}

The reviewer noted that a very good, comprehensive team has been assembled, which should contribute to a successful project.

\section{Reviewer 5:}

The reviewer found an excellent level of collaboration, with involvement from the national laboratories, OEMs, and Tier 1 suppliers.

\section{Reviewer 6:}

The reviewer praised working with partners that know what is needed for the industry and understand all the application challenges.

\section{Reviewer 7:}

The reviewer stated that a pretty broad team has been assembled including DOE national laboratories, several engine makers, and fleet operators. The reviewer commented that adding a couple of universities would make the project perfect.

\section{Question 4: Proposed future research-the degree to which the project has effectively planned its future work in a logical manner by incorporating appropriate decision points, considering barriers to the realization of the technology and, when sensible, mitigating risk by providing alternate development pathways.}

\section{Reviewer 1:}

The reviewer stated that this program is probably the best planned seen this year and it was again working on relevant issues with a directly applicable solution.

\section{Reviewer 2:}

The reviewer said that the R\&D plan looks satisfactory for achieving the project goals.

\section{Reviewer 3:}

This project has an excellent trajectory, and the reviewer looked forward to watching its progress.

\section{Reviewer 4:}

The reviewer said that the plan looks good.

\section{Reviewer 5:}

The reviewer stated that this was a good plan going forward.

\section{Reviewer 6:}

The reviewer commented that the proposed future work is logical and well planned in terms of sensor-related development. Again, a major void in the project is the pathway from the sensor development to the actual vehicle fuel efficiency improvement. The reviewer said that the knowledge of the SCR catalyst state seems to be more useful for diesel emissions fluid dosing control than engine control itself. The reviewer questioned how the engine would operate in a more efficient way given the knowledge of the SCR catalyst state and suggested that this question be addressed as soon as possible and used as a decision point.

\section{Question 5: Does this project support the overall DOE objectives of petroleum displacement? Why or why not?}

\section{Reviewer 1:}

The reviewer stated that maximizing aftertreatment system performance will aid in reducing fuel consumption, thus reducing petroleum usage.

\section{Reviewer 2:}

The reviewer said that reducing the uncertainty in aftertreatment effectiveness/maintenance will lead to improved efficiency, thus supporting the DOE objectives of petroleum displacement. 


\section{Reviewer 3:}

The reviewer stated the project allows the engine to run at more efficient conditions and still control the catalyst to very high effectiveness and be capable for OBD. The project should be a very cost-effective solution towards running such conditions.

\section{Reviewer 4:}

The reviewer commented that use of the sensor and avoidance of unnecessary regeneration events would positively impact fuel economy and the durability of aftertreatment devices.

\section{Reviewer 5:}

The reviewer stated that, if successful, the technology would results in fuel savings, which would support DOE objectives of petroleum displacement.

\section{Reviewer 6:}

The reviewer remarked that there is some potential to increase vehicle efficiency through improved sensing technology. It is not the strongest knob though.

\section{Question 6: Resources: How sufficient are the resources for the project to achieve the stated milestones in a timely fashion?}

\section{Reviewer 1:}

The reviewer said that budget and spend rate seem to be in line and sufficient for progress.

\section{Reviewer 2:}

The reviewer stated the resources look appropriate.

\section{Reviewer 3:}

The reviewer remarked that the funding levels seem reasonable for this work.

\section{Reviewer 4:}

The reviewer indicated that funding seems to be adequate for the remaining tasks.

\section{Reviewer 5:}

The reviewer noted that the resources are sufficient given the scope of project. The reviewer would always like to see contractors match the DOE input, but that can be difficult for small companies. 


\section{Acronyms and Abbreviations}

1D

$3 \mathrm{D}$

$\mathrm{AC}$

ACE

ACEC

$\mathrm{Al}$

AMR

ANL

APS

ARC

ASCR

BMEP

BSFC

BTE

${ }^{\circ} \mathrm{C}$

$\mathrm{Ca}$

$\mathrm{CaSO}_{4}$

CAE

$\mathrm{CCC}$

$\mathrm{Ce}$

CF

CFD

$\mathrm{CH}_{4}$

CHA

CI
One dimensional

Three dimensional

Air Conditioning

Advanced combustion engine

Advanced Combustion and Emissions Control

Aluminum

Annual Merit Review

Argonne National Laboratory

Advanced Photon Source

Affordable Rankine Cycle

Advanced Scientific Computing Research

Brake Mean Effective Pressure

Brake-specific fuel consumption

Brake Thermal Efficiency

Degrees Celsius

Calcium

Calcium Sulfate

Computer-Aided Engineering

Co-precipitated $\mathrm{CuOx}, \mathrm{CoOy}$, and $\mathrm{CeO} 2$ catalyst

Cerium

Combustion Fluid

Computational Fluid Dynamics

Methane

Chabazite

Compression Ignition 


\begin{tabular}{|c|c|}
\hline $\mathrm{Cl}$ & Chloride \\
\hline CLEERS & Cross-Cut Lean Exhaust Emissions Reduction Simulations \\
\hline $\mathrm{CO}$ & Carbon Monoxide \\
\hline $\mathrm{CO}_{2}$ & Carbon Dioxide \\
\hline $\mathrm{COV}$ & Coefficient of variance \\
\hline CPU & Central processing unit \\
\hline CR & Compression Ratio \\
\hline CRADA & Cooperative Research and Development Agreement \\
\hline $\mathrm{Cu}$ & Copper \\
\hline DEF & Diesel Emissions Fluid \\
\hline DFT & Density Functional Theory \\
\hline DI & Direct Injection \\
\hline DOC & Diesel oxidation catalyst \\
\hline DOE & U.S. Department of Energy \\
\hline DPF & Diesel particulate filter \\
\hline E10 & $10 \%$ ethanol blend with gasoline \\
\hline E20 & $20 \%$ ethanol blend with gasoline \\
\hline $\mathrm{ECN}$ & Engine Collaboration Network \\
\hline EGR & Exhaust Gas Recirculation \\
\hline EIVC & Early Intake Valve Closing \\
\hline EPA & U.S. Environmental Protection Agency \\
\hline ERC & Engine Research Center \\
\hline EU & European Union \\
\hline FA & Field Aged \\
\hline FACE & Fuels for Advanced Combustion Engines \\
\hline FAME & Fatty Acid Methyl Ethers \\
\hline
\end{tabular}


FCA Fiat Chrysler Automobiles

$\mathrm{Fe} \quad$ Iron

FE $\quad$ Fuel Economy

FEM Finite Element Model

FIE Fuel Injected Engines

FRESCO Fast and Reliable Engine Simulation Code

FTP Federal Test Procedure

FY Fiscal year

GC Gas Chromatography

GDI Gasoline Direct-injected

GDCI Gasoline Direct Compression Engine

GHG Greenhouse gas

GM General Motors Corporation

GOC Gasoline Oxidation Catalyst

GPF Gasoline Particulate Filter

GPU Graphics Processing Unit

GSA Global sensitivity analysis

$\mathrm{H}_{2} \quad$ Hydrogen

$\mathrm{H}_{2} \mathrm{O} \quad$ Water

HC Hydrocarbon

HCCI Homogeneous Charge Compression Ignition

$\mathrm{HCl} \quad$ Hydrochloric Acid

HD Heavy-Duty

HEV Hybrid Electric Vehicle

HHC Heavy Hydrocarbons

HPC High Performance Computing 


\begin{tabular}{|c|c|}
\hline HTA & High-temperature hydrothermal aging \\
\hline HWFE & Highway Fuel Economy \\
\hline ICE & Internal Combustion Engine \\
\hline IDT & Ignition delay time \\
\hline IMEP & Indicated Mean Effective Pressure \\
\hline IR & Infrared \\
\hline ISFC & Indicated Specific Fuel Consumption \\
\hline IVC & Intake Valve Closing \\
\hline JM & Johnson Matthey Catalysts \\
\hline $\mathrm{L}$ & Liter \\
\hline $\mathrm{La}$ & lanthanum \\
\hline LANL & Los Alamos National Laboratory \\
\hline LD & Light-Duty \\
\hline LES & Large Eddy Simulation \\
\hline LIVC & Late Intake Valve Closing \\
\hline LLNL & Lawrence Livermore National Laboratory \\
\hline LP & Low-pressure \\
\hline LTAT & Low temperature Aftertreatment \\
\hline LTC & Low-Temperature Combustion \\
\hline LTGC & Low-Temperature Gasoline Combustion \\
\hline MD & Methyl Decanoate \\
\hline $\mathrm{mm}$ & Millimeter \\
\hline MON & Motor Octane Number \\
\hline MPI & Multi-Point Injection \\
\hline $\mathrm{ms}$ & Milliseconds \\
\hline MY & Model Year \\
\hline
\end{tabular}




\begin{tabular}{|c|c|}
\hline $\mathrm{N}_{2} \mathrm{O}$ & Nitrous Oxide \\
\hline $\mathrm{Na}$ & Sodium \\
\hline NG & Natural gas \\
\hline $\mathrm{NH}_{3}$ & Ammonia \\
\hline NI & National Instruments \\
\hline $\mathrm{NO}$ & Nitric Oxide \\
\hline $\mathrm{NO}_{x}$ & Oxides of Nitrogen \\
\hline $\mathrm{NO}_{2}$ & Nitrogen Dioxide \\
\hline NVO & Negative Valve Overlap \\
\hline $\mathrm{O}_{2}$ & Oxygen \\
\hline OBD & On-Board Diagnostics \\
\hline OEM & Original Equipment Manufacturer \\
\hline ORAU & Oak Ridge Associated Universities \\
\hline $\mathrm{ORC}$ & Organic Rankine Cycle \\
\hline ORNL & Oak Ridge National Laboratory \\
\hline PAH & Polycyclic aromatic hydrocarbon \\
\hline PCP & Peak Cylinder Pressure \\
\hline $\mathrm{Pd}$ & Palladium \\
\hline PDF & Probability Density Function \\
\hline Penn State & Pennsylvania State University \\
\hline PFI & Port Fuel Injection \\
\hline PGM & Platinum group metals \\
\hline $\mathrm{PI}$ & Principal Investigator \\
\hline PIV & Particle image velocimetry \\
\hline PM & Particulate matter \\
\hline $\mathrm{PN}$ & Particulate number \\
\hline
\end{tabular}




\begin{tabular}{|c|c|}
\hline PNA & Passive $\mathrm{NO}_{\mathrm{x}}$ adsorber \\
\hline PNNL & Pacific Northwest National Laboratory \\
\hline $\operatorname{Pr}$ & Praseodymium \\
\hline PR & Pressure Rise \\
\hline $\mathrm{Pt}$ & Platinum \\
\hline $\mathrm{R} \& \mathrm{D}$ & Research and development \\
\hline RANS & Reynolds-Averaged Navier Strokes \\
\hline RAT & Rapid Aging Test \\
\hline RCCI & Reactivity Controlled Compression Ignition \\
\hline $\mathrm{RCM}$ & Rapid compression machines \\
\hline $\mathrm{RF}$ & Radio-Frequency \\
\hline RFI & Radio frequency interference \\
\hline $\mathrm{Rh}$ & Rhodium \\
\hline RK & Reaction kinetics \\
\hline $\mathrm{RON}$ & Research octane number \\
\hline $\mathrm{S}$ & Sulfur \\
\hline SAE & Society of Automotive Engineers \\
\hline SCE & Stratified Charge Engine \\
\hline SCR & Selective Catalytic Reduction \\
\hline SDPF & SCR-Coated DPF \\
\hline $\mathrm{Si}$ & Silicon \\
\hline $\mathrm{SiC}$ & Silicon Carbide \\
\hline SNL & Sandia National Laboratories \\
\hline SNR & Signal to Noise Ratio \\
\hline SOI & Start of Ignition \\
\hline TESF & Tabulated Equivalent Strain Flamelet \\
\hline
\end{tabular}


$\mathrm{TiO}_{2} \quad$ Titanium Dioxide

TWC Three-Way Catalyst

UC Unused Capacity

UM University of Michigan

UQ Uncertainty quantification

USCAR U.S. Council for Automotive Research

U.S. DRIVE U.S. Driving Research and Innovation for Vehicle efficiency and Energy sustainability

UW University of Wisconsin

VCR Variable compression ratio

VTO Vehicle Technologies Office

VVA Variable Valve Actuation

WHR Waste Heat Recovery

Zr Zirconium

$\mathrm{ZrO}_{2} \quad$ Zirconium Dioxide 


\section{Fuel and Lubricant Technologies}

More than $90 \%$ of transportation relies on petroleum-based fuels: gasoline and diesel. While alternative fuels and plug-in electric vehicles offer great promise to reduce America's petroleum consumption, petroleum-based fuels are likely to play a substantial role for years to come. However, the sources of these petroleum-based fuels are changing, with more fuels than ever from unconventional sources. Canada, which is the United States' largest foreign supplier of crude oil, is currently getting more than half of its petroleum from oil sands. In addition, new advanced combustion engines are particularly sensitive to variations in fuel composition.

The Vehicle Technologies Office (VTO) supports research into fuels to enable more efficient engines and more effective emissions control systems that improve fuel economy and reduce emissions. Much of this research can also provide insight into how biofuels' unique properties can affect these engines and systems.

Currently, VTO is supporting the Co-Optimization of Fuels and Engines Initiative (Co-Optima). Over the course of this multi-year initiative, VTO and the Bioenergy Technologies Office are supporting research and development (R\&D) to concurrently accelerate the introduction of affordable, scalable, and sustainable biofuels along with high-efficiency, low-emission vehicle engines. This coordinated R\&D effort brings together nine U.S. Department of Energy (DOE) national laboratories and numerous industry and academic partners to integrate the research areas of biofuels, combustion, and analysis. The Co-Optimization Initiative has three concurrent phases of R\&D:

- Optimizing spark ignition (SI) fuels and engines for near-term effect;

- Developing fuels and engines to enable advanced compression ignition (CI) technologies needed for revolutionary long-term solutions; and

- Developing and applying analysis tools to assess the economic and environmental impact of the proposed technologies.

Investigating technologies such as lubricants that will improve the efficiency of today's vehicles is essential, as most vehicles are on the road for more than 15 years before they are retired. VTO supports R\&D on lubricants that can improve the efficiency of vehicles with internal combustion engines. Because $11.5 \%$ of fuel energy is consumed by engine friction, improving lubricants enough to result in a $1 \%$ fuel savings in the existing vehicle fleet could save more than 90 thousand barrels of oil a day.

Research that the VTO supports includes:

- Developing better base oils and oil additives that have the potential to improve the mechanical efficiency of internal combustion engines by $10 \%$ without causing increased wear, emissions, or damage to emission aftertreatment systems. In particular, using ionic liquids (IL), which are salts in a liquid state, as lubricants or lubricant additives may result in $30 \%$ less friction than comparable lubricants.

- Working to improve the understanding of the relationship between benchtop and engine tests when studying friction and wear performance data. This work will help improve standards and the accuracy of future research.

- Developing and optimizing tribochemical films (the protective layer that forms on metal surfaces when using oil additives) to reduce friction, reduce wear, and improve fuel economy.

- Developing additives to lubricants that are compatible with the use of higher levels of biofuels that will enable the adoption of low-carbon fuels. 


\section{Subprogram Feedback}

The U.S. Department of Energy (DOE) received feedback on the overall technical subprogram areas presented during the 2016 Annual Merit Review (AMR). Each subprogram technical session was introduced with a presentation that provided an overview of subprogram goals and recent progress, followed by a series of detailed topic area project presentations.

The reviewers for a given subprogram area responded to a series of specific questions regarding the breadth, depth, and appropriateness of that DOE VTO subprogram's activities. The subprogram overview questions are listed below, and it should be noted that no scoring metrics were applied. These questions were used for all VTO subprogram overviews.

Question 1: Was the program area, including overall strategy, adequately covered?

Question 2: Is there an appropriate balance between near- mid- and long-term research and development?

Question 3: Were important issues and challenges identified?

Question 4: Are plans identified for addressing issues and challenges?

Question 5: Was progress clearly benchmarked against the previous year?

Question 6: Are the projects in this technology area addressing the broad problems and barriers that the Vehicle Technologies Office (VTO) is trying to solve?

Question 7: Does the program area appear to be focused, well-managed, and effective in addressing VTO's needs?

Question 8: What are the key strengths and weaknesses of the projects in this program area? Do any of the projects stand out on either end of the spectrum?

Question 9: Do these projects represent novel and/or innovative ways to approach these barriers as appropriate?

Question 10: Has the program area engaged appropriate partners?

Question 11: Is the program area collaborating with them effectively?

Question 12: Are there any gaps in the portfolio for this technology area?

Question 13: Are there topics that are not being adequately addressed?

Question 14: Are there other areas that this program area should consider funding to meet overall programmatic goals?

Question 15: Can you recommend new ways to approach the barriers addressed by this program area? 
Question 16: Are there any other suggestions to improve the effectiveness of this program area?

Responses to the subprogram overview questions are summarized in the following pages. Individual reviewer comments for each question are identified under the heading Reviewer 1, Reviewer 2, etc. Note that reviewer comments may be ordered differently; for example, for each specific subprogram overview presentation, the reviewer identified as Reviewer 1 in the first question may not be Reviewer 1 in the second question, etc. 


\title{
Overview of the VTO Fuel and Lubricant Technologies R\&D: Kevin Stork (U.S. Department of Energy) - ft000
}

\author{
Question 1: Was the program area, including overall strategy, adequately covered? \\ Reviewer 1: \\ The reviewer stated that the overall strategy of the Fuel and Lubricant Technologies program was very well \\ described: directly displace petroleum and enhance combustion using lubricants to retrofit existing technology to \\ provide a $4 \%$ fuel economy improvement.
}

\section{Reviewer 2:}

The reviewer replied yes, the program was covered in adequate detail.

\section{Reviewer 3:}

The reviewer said the presentation gave a nice, broad overview of this large program, which has two very separate activities (fuels and lubricants). However, the reviewer remarked that the presentation lacked details about selection of the fuels and status and criteria of that selection. The reviewer stated that it was not possible to get a real idea of the programs until after attending individual merit review talks.

\section{Question 2: Is there an appropriate balance between near- mid- and long-term research and development?}

\section{Reviewer 1:}

The reviewer stated that there appears to be an appropriate balance between practical goals and long term research goals. The reviewer further stated that there also appears to be an appropriate balance between technology development and development of new methods for assessing technologies.

\section{Reviewer 2:}

The reviewer asserted that the program area adequately discussed near-, mid-, and long-term R\&D. The work in this area has strategies to help address the 2017 to 2025 U.S. Environmental Protection Agency (EPA) Tier 3 emissions regulations, the fuel economy standard, and the Renewable Fuels Standard in 2022 and 2025. This reviewer also noted that the co-optimization of fuels and engines looks at long-term fuel economy gains in the 2040 timeframe.

\section{Reviewer 3:}

The reviewer replied yes, the Co-Optima program is an excellent example of looking at 5-year and 20-year programs.

\section{Question 3: Were important issues and challenges identified?}

\section{Reviewer 1:}

The reviewer asserted that the three automotive challenges of meeting fuel economy standards, renewable fuels standards, and EPA emissions standards were discussed.

\section{Reviewer 2:}

The reviewer said yes, the presentation gave an overview about octane and advanced combustion fuels.

\section{Reviewer 3:}

The reviewer indicated that the presentation was mainly focused on SI engines and octane effects. This person observed very little detail about diesel/CI engines and fuels, apparently because they are mainly being covered in Phase 2. The reviewer was not sure why diesel was assigned to second place, unless this was due to budget considerations and pressure from advisor groups. Although there was no mention of fuel effects on low-speed preignition, the reviewer later learned that this criterion is part of the Co-Optima screening criteria. 


\section{Question 4: Are plans identified for addressing issues and challenges?}

\section{Reviewer 1:}

The reviewer agreed yes, there were clear plans and projects identified to meet the challenges, especially through the Co-Optimization of Fuel and Engines projects.

\section{Reviewer 2:}

The reviewer asserted that the program discussed the lubricants challenges with good detail.

\section{Reviewer 3:}

This reviewer replied yes, but really got the sense of identified plans for addressing issues and challenges from following the review talks, as this presentation was a broad overview. The reviewer elaborated that the development of a new fuel and corresponding engine types are enormous challenges and this project could be important for laying the groundwork for further work. The reviewer concluded that, to a large extent, the issues and challenges will be identified as the project progresses, rather than at the front end.

\section{Question 5: Was progress clearly benchmarked against the previous year?}

\section{Reviewer 1:}

The reviewer agreed that yes, the benchmarks and progress were clearly shown.

\section{Reviewer 2:}

The reviewer stated that some detail was provided about accomplishments including the novel lubricant formulation scheme for $2 \%$ fuel efficiency improvement and power cylinder friction reduction through coating and surface finish.

\section{Reviewer 3:}

The reviewer indicated that, on Slide 3, the goals and accomplishments for fuels research were not aligned for direct comparison. In addition, the reviewer remarked that for the funnel slide describing fuel selection and screening, it would be useful to know real numbers. This reviewer inquired about how many fuels and components are going to be screened, and how many have already advanced to more rigorous evaluation.

\section{Question 6: Are the projects in this technology area addressing the broad problems and barriers that the Vehicle Technologies Office (VTO) is trying to solve?}

\section{Reviewer 1:}

The reviewer replied yes and characterized this program as supporting the broad program goals of improved fuel efficiency, lower greenhouse gas (GHG) emissions, and increased use of low-carbon fuels. The reviewer also stated that the program is well aligned with the biofuels program and with the Advanced Combustion Engine program, as it should be.

\section{Reviewer 2:}

The reviewer observed that fuels and lubricants are one area that directly affect DOE's goals for reducing GHGs and petroleum dependence.

\section{Reviewer 3:}

The reviewer indicated that there was not much information provided regarding many of the projects in the Fuel and Lubricant Technologies program. Therefore, it is difficult to address whether it is addressing the VTO barriers. However, the reviewer added that through the discussion of the plans of the program, it appears the barriers would be adequately addressed.

Question 7: Does the program area appear to be focused, well-managed, and effective in addressing VTO's needs? 


\section{Reviewer 1:}

The reviewer declared that the Technology Manager is doing an excellent job of managing the program and the recent addition of program staff is likely to make the program stronger.

\section{Reviewer 2:}

The reviewer stated yes, the program is well-managed and should be effective in addressing VTO's needs.

\section{Reviewer 3:}

The reviewer commented that the presentation did not give sufficient detail to assess effectiveness of management and focus, elaborating that while the program certainly has all the right elements and work is progressing, the reviewer could not decide if things were adequately managed or if the program could benefit from stronger management.

\section{Question 8: What are the key strengths and weaknesses of the projects in this program area? Do any of the projects stand out on either end of the spectrum?}

\section{Reviewer 1:}

The reviewer remarked that the new effort of Co-Optima should develop into a strength of the program. This person also observed that it is essential to work on fuel and engines as a pair to make sure the goals can be attained.

\section{Reviewer 2:}

The reviewer commented that the lubricants portion of the program has obvious advantages for legacy vehicles that the rest of the VTO program cannot help and that the only weakness is the lack of funding for lubricants. Fuels appears to be on a good track now with the Co-Optima program, and the reviewer added that it would be nice if the Advanced Combustion Engine program would try to integrate more fuels research into its program.

\section{Reviewer 3:}

The reviewer said that Co-Optima is a very important thrust and could be considered a key strength, while recognizing that the program has limited resources to achieve rapid and real benefits and that such a large, diverse group will be hard to manage and coordinate. Regarding the lubricants area, the reviewer acknowledged not really being qualified to assess the detailed technologies being studied, and thinks it is important to maintain industry involvement to ensure that DOE is working on relevant topics.

\section{Question 9: Do these projects represent novel and/or innovative ways to approach these barriers as appropriate?}

\section{Reviewer 1:}

The reviewer said that the program has a good balance of practical, traditional approaches focusing on octane, ignition delay, and low-carbon fuels combined with engine performance research. The program also addresses the need for new methods or metrics for screening new technologies as they are developed.

\section{Reviewer 2:}

This reviewer indicated that the Co-Optima project represents a very good approach to creating mechanisms to reach the goals and eliminate program barriers.

\section{Reviewer 3:}

The reviewer replied yes to this question.

\section{Question 10: Has the program area engaged appropriate partners?}

\section{Reviewer 1:}

The reviewer said the collaboration with the United States Driving Research and Innovation for Vehicle efficiency and Energy sustainability (U.S. DRIVE) Fuels Working Group (FWG), and the Coordinating Research Council (CRC) on the fuels side, is excellent and a very good way to engage fuel and engine manufacturing in a manner that could lead to real change in future fuels and engines design. The reviewer characterized collaboration with lubricant and additive manufacturers and engine and vehicle designers as critical to ensuring that lubricants work is properly focused to yield both short- and long-term results. 


\section{Reviewer 2:}

The reviewer observed that the program works with all of the appropriate partners including many national laboratories and members of the FWG in U.S. DRIVE.

\section{Reviewer 3:}

The reviewer replied yes, the lubricants partners are all excellent. The reviewer suggested that the fuels program could use more energy companies, but speculated that most of these companies do not want to be involved in government work.

\section{Question 11: Is the program area collaborating with them effectively?}

\section{Reviewer 1:}

The reviewer stated that the program seems to have a very good relationship with the program partners and there is great collaboration and coordination with them.

\section{Reviewer 2:}

The reviewer remarked that the current partners are working together well.

\section{Reviewer 3:}

The reviewer stated that the collaborations appear to be effective, but suggested collaborations might be a little slow moving because of collaborators that are mainly consensus-driven groups and organizations. As more practical results are achieved, the reviewer opined that maybe things can be accelerated.

\section{Question 12: Are there any gaps in the portfolio for this technology area?}

\section{Reviewer 1:}

The reviewer stated that with the addition of the Co-Optima projects, there do not appear to be any gaps in this program area.

\section{Reviewer 2:}

The reviewer commented that more work needs to be done on how to introduce new fuels to the market.

\section{Reviewer 3:}

The reviewer pointed out that there was not any discussion of fuel properties beyond octane, ignition delay, and bio equivalence. Based upon follow-on talks, the reviewer learned that secondary fuel properties such as flame velocity, detailed chemistry, and molecular weight range, among others, are being studied. The reviewer stated that long term, there has to be a step taken beyond research octane number (RON) and cetane number $(\mathrm{CN})$.

The reviewer offered that more details about fuel chemistry are probably best studied using a combination of detailed kinetic modeling, surrogate fuels, and refinery-based blended fuels. The reviewer also suggested that new lubricants and additives can enable new lighter weight or lower friction engine designs through a long term iterative process. It was not clear to this reviewer whether there is much focus on engine design.

\section{Question 13: Are there topics that are not being adequately addressed?}

\section{Reviewer 1:}

The reviewer commented that projects in the program area adequately cover the necessary topics to have the program meets its goals.

\section{Reviewer 2:}

The reviewer stated that it was not clear from the presentation if the following important areas had been addressed by the program: fuel properties beyond octane and cetane; kinetic modeling of fuel chemistry effects; and coevolution of lubricants and engine design. The review acknowledged being more comfortable about the program's progress after hearing the follow-on talks.

\section{Reviewer 3:}

The reviewer stated that more work needs to be done on how to introduce new fuels to the market. 


\section{Question 14: Are there other areas that this program area should consider funding to meet} overall programmatic goals?

Reviewer 1:

The reviewer commented that the program should continue funding the project areas in its portfolio.

\section{Reviewer 2:}

The reviewer replied no, but added that increased or accelerated funding might be appropriate.

\section{Reviewer 3:}

The reviewer offered that some work looking at stoichiometric compression ignition (SCI) with lower octane fuels would be a welcome addition.

\section{Question 15: Can you recommend new ways to approach the barriers addressed by this program area?}

\section{Reviewer 1:}

The reviewer replied that the program seems to be adequately addressing the barriers.

\section{Reviewer 2:}

The reviewer stated that the start of the Co-Optima project is a very good way to approach the barriers and was glad to see it was implemented.

Question 16: Are there any other suggestions to improve the effectiveness of this program area?

\section{Reviewer 1:}

The reviewer replied no, adding that the program appears to be pursuing appropriate projects and is funded adequately.

\section{Reviewer 2:}

The reviewer suggested the program continue to communicate results in a timely manner so that feedback from interested groups can be obtained. 


\section{Project Feedback}

In this merit review activity, each reviewer was asked to respond to a series of questions, involving multiple-choice responses, expository responses where text comments were requested, and numeric score responses (on a scale of 1.0 to 4.0). In the pages that follow, the reviewer responses to each question for each project will be summarized: the multiple choice and numeric score questions will be presented in graph form for each project, and the expository text responses will be summarized in paragraph form for each question. A table presenting the average numeric score for each question for each project is presented below.

\section{Table 5-1 - Project Feedback}

\begin{tabular}{|c|c|c|c|c|c|c|c|}
\hline Presentation Title & $\begin{array}{c}\text { Principal } \\
\text { Investigator } \\
\text { and } \\
\text { Organization }\end{array}$ & $\begin{array}{l}\text { Page } \\
\text { Number }\end{array}$ & Approach & $\begin{array}{c}\text { Technical } \\
\text { Accomplishments }\end{array}$ & Collaborations & $\begin{array}{c}\text { Future } \\
\text { Research }\end{array}$ & $\begin{array}{c}\text { Weighted } \\
\text { Average }\end{array}$ \\
\hline $\begin{array}{l}\text { Engine Friction } \\
\text { Reduction } \\
\text { Technologies }\end{array}$ & $\begin{array}{c}\text { Fenske, } \\
\text { George (ANL) }\end{array}$ & 5-12 & 3.50 & 3.50 & 3.63 & 3.25 & 3.48 \\
\hline $\begin{array}{l}\text { Ionic Liquids as } \\
\text { Engine Lubricant } \\
\text { Additives, Impact } \\
\text { on Emission } \\
\text { Control Catalysts, } \\
\text { and Compatibility } \\
\text { with Coatings }\end{array}$ & $\begin{array}{l}\text { Qu, Jun } \\
\text { (ORNL) }\end{array}$ & $5-15$ & 3.67 & 3.50 & 3.83 & 3.50 & 3.58 \\
\hline $\begin{array}{l}\text { Integrated } \\
\text { Friction Reduction } \\
\text { Technology to } \\
\text { Improve Fuel } \\
\text { Economy without } \\
\text { Sacrificing } \\
\text { Durability }\end{array}$ & $\begin{array}{l}\text { Hsu, Stephen } \\
\text { (George } \\
\text { Washington } \\
\text { University) }\end{array}$ & $5-18$ & 2.67 & 3.00 & 3.50 & 3.00 & 2.98 \\
\hline $\begin{array}{l}\text { Hybrid lonic- } \\
\text { Nano-Additives } \\
\text { for Engine } \\
\text { Lubrication to } \\
\text { Improve Fuel } \\
\text { Efficiency }\end{array}$ & $\begin{array}{l}\text { Zhao, Bin } \\
\text { (University of } \\
\text { Tennessee) }\end{array}$ & $5-21$ & 2.67 & 2.83 & 2.83 & 2.83 & 2.79 \\
\hline $\begin{array}{l}\text { Hyperbranched } \\
\text { Alkanes for Lubes }\end{array}$ & $\begin{array}{l}\text { Cosimbescu, } \\
\text { Lelia (PNNL) }\end{array}$ & $5-24$ & 3.33 & 3.17 & 3.00 & 3.33 & 3.21 \\
\hline
\end{tabular}




\begin{tabular}{|c|c|c|c|c|c|c|c|}
\hline Presentation Title & $\begin{array}{c}\text { Principal } \\
\text { Investigator } \\
\text { and } \\
\text { Organization }\end{array}$ & $\begin{array}{l}\text { Page } \\
\text { Number }\end{array}$ & Approach & $\begin{array}{c}\text { Technical } \\
\text { Accomplishments }\end{array}$ & Collaborations & $\begin{array}{l}\text { Future } \\
\text { Research }\end{array}$ & $\begin{array}{l}\text { Weighted } \\
\text { Average }\end{array}$ \\
\hline $\begin{array}{l}\text { Lubricant Effects } \\
\text { on Combustion, } \\
\text { Emissions, and } \\
\text { Efficiency }\end{array}$ & $\begin{array}{c}\text { Wagner, } \\
\text { Robert (ORNL) }\end{array}$ & $5-27$ & 3.50 & 3.67 & 3.83 & 3.33 & 3.60 \\
\hline $\begin{array}{l}\text { Co-Optimization } \\
\text { of Fuels and } \\
\text { Engines Overview }\end{array}$ & $\begin{array}{l}\text { Farrell, John } \\
\text { (NREL) }\end{array}$ & $5-30$ & 2.88 & 2.88 & 3.13 & 2.88 & 2.91 \\
\hline $\begin{array}{c}\text { Co-Optimization } \\
\text { of Fuels and } \\
\text { Engines (Co- } \\
\text { Optima) -- Fuel } \\
\text { Properties and } \\
\text { Thrust I Engine } \\
\text { Research }\end{array}$ & $\begin{array}{l}\text { Szybist, Jim } \\
\text { (SNL) }\end{array}$ & $5-35$ & 3.13 & 3.13 & 3.50 & 3.13 & 3.17 \\
\hline $\begin{array}{l}\text { Co-Optimization } \\
\text { of Fuels and } \\
\text { Engines (Co- } \\
\text { Optima) -- } \\
\text { Thrust II Engine } \\
\text { Research, Sprays } \\
\text { Research, and } \\
\text { Emissions Control } \\
\text { Research }\end{array}$ & $\begin{array}{l}\text { Miles, Paul } \\
\text { (ORNL) }\end{array}$ & $5-41$ & 2.63 & 2.88 & 2.75 & 2.63 & 2.77 \\
\hline $\begin{array}{l}\text { Co-Optimization } \\
\text { of Fuels and } \\
\text { Engines (Co- } \\
\text { Optima) -- } \\
\text { Simulation Toolkit } \\
\text { Team }\end{array}$ & $\begin{array}{l}\text { McNenly, Matt } \\
\text { (LLNL) }\end{array}$ & $5-45$ & 2.88 & 3.00 & 3.25 & 2.75 & 2.97 \\
\hline $\begin{array}{c}\text { Utilizing } \\
\text { Alternative } \\
\text { Fuel Ignition } \\
\text { Properties to } \\
\text { Improve Spark- } \\
\text { Ignited and } \\
\text { Compression- } \\
\text { Ignited Engine } \\
\text { Efficiency }\end{array}$ & $\begin{array}{l}\text { Wooldridge, } \\
\text { Margaret } \\
\text { (University of } \\
\text { Michigan) }\end{array}$ & $5-51$ & 2.40 & 2.50 & 3.20 & 2.60 & 2.58 \\
\hline
\end{tabular}




\begin{tabular}{|c|c|c|c|c|c|c|c|}
\hline Presentation Title & $\begin{array}{c}\text { Principal } \\
\text { Investigator } \\
\text { and } \\
\text { Organization }\end{array}$ & $\begin{array}{l}\text { Page } \\
\text { Number }\end{array}$ & Approach & $\begin{array}{c}\text { Technical } \\
\text { Accomplishments }\end{array}$ & Collaborations & $\begin{array}{l}\text { Future } \\
\text { Research }\end{array}$ & $\begin{array}{l}\text { Weighted } \\
\text { Average }\end{array}$ \\
\hline $\begin{array}{c}\text { E85/Diesel } \\
\text { Premixed } \\
\text { Compression } \\
\text { Ignition }\end{array}$ & $\begin{array}{l}\text { Kocher, Lyle } \\
\text { (Cummins) }\end{array}$ & $5-56$ & 3.64 & 3.64 & 3.07 & 3.43 & 3.54 \\
\hline $\begin{array}{c}\text { GEFORCE: } \\
\text { Gasoline Engine } \\
\text { and Fuels Offering } \\
\text { Reduced Fuel } \\
\text { Consumption and } \\
\text { Emissions }\end{array}$ & $\begin{array}{l}\text { Sluder, Scott } \\
\text { (ORNL) }\end{array}$ & $5-60$ & 3.50 & 3.50 & 3.20 & 3.30 & 3.44 \\
\hline GDI Metrics & $\begin{array}{l}\text { Goldsborough, } \\
\text { Scott (ANL) }\end{array}$ & $5-64$ & 3.17 & 3.17 & 3.17 & 3.17 & 3.17 \\
\hline $\begin{array}{c}\text { Efficiency- } \\
\text { Optimized Dual } \\
\text { Fuel Engine } \\
\text { with In-Cylinder } \\
\text { Gasoline/CNG } \\
\text { Blending }\end{array}$ & $\begin{array}{c}\text { Wallner, } \\
\text { Thomas (ANL) }\end{array}$ & $5-68$ & 3.00 & 3.30 & 3.10 & 3.20 & 3.19 \\
\hline Overall Average & & & 3.10 & 3.18 & 3.27 & 3.09 & 3.16 \\
\hline
\end{tabular}




\section{Engine Friction Reduction Technologies: George Fenske (Argonne National Laboratory) - ft012}

\section{Presenter}

George Fenske, Argonne National Laboratory

\section{Reviewer Sample Size}

A total of four reviewers evaluated this project.

\section{Question 1: Approach to performing the work-the degree to which technical barriers are addressed, the project is well-designed, feasible, and integrated with other efforts.}

\section{Reviewer 1:}

The reviewer praised the approaches identified to address the barriers in this project and stated that they are very good. The reviewer remarked that the use of multiple approaches of using base fluids, additives, and coatings to reduce engine friction will lead to a high likelihood of success of the project. The reviewer further commented that the approach to develop and assess lab protocols to replicate tribological environments in engines and drivetrains will provide for innovative lab-engine correlation.

\section{Reviewer 2:}

The reviewer remarked that the modification to the reciprocating rig that includes the tilt angle impact on scuffing is a good improvement. The reviewer questioned what the fundamental mechanism is that could be looked at to understand why this has an impact instead of just testing it on the rig.

\section{Reviewer 3:}

The reviewer commented that the project takes a very broad approach to developing new lubricant related technologies, including base fluids, additives, and coatings. The reviewer noted that the project includes boundary, mixed, and hydrodynamic regimes, screening methods, and surface science related measurements.

\section{Reviewer 4:}

The reviewer remarked that the project is producing vital information regarding major issues. The reviewer would have liked to hear more in-depth information on the project. However, due to presentation time constraints and the wide breadth of the program, it is hard to tell in-depth information on any given project. This is not the fault of the presenter, but this reviewer would like to see a deeper dive in one or two projects, instead of a general overview of several. Any given project within the program could easily be the topic of a presentation. 
Question 2: Technical accomplishments and progress toward overall project and DOE goals-the degree to which progress has been made, measured against performance indicators and demonstrated progress towards DOE goals.

\section{Reviewer 1:}

The reviewer said the technical accomplishments this year have been excellent. The reviewer said that the project accomplishments including development and validation of scuffing protocols and the identification of factors that affect scuffing including temperature, speed, and load have provided information to successfully address the barriers of the project.

\section{Reviewer 2:}

The reviewer praised the good progress in developing standardized scuffing test. The reviewer further remarked on the good progress with catalytic coatings, i.e., narrowed down focus to a single representative coating so that performance and underlying science can be studied in greater detail.

\section{Reviewer 3:}

The reviewer remarked that the focus on base fluids and additives appears synergistic towards the goals, and the coating work is also progressing well.

\section{Reviewer 4:}

The reviewer commented that the project spans several huge issues in the tribology world. In particular, scuffing predictability, tribofilm generation mechanisms, and creating a benchtop test that has the ability to predict engine performance are all longstanding endeavors that have not been well understood even though they have been researched for decades.

\section{Question 3: Collaboration and coordination with other institutions.}

\section{Reviewer 1:}

The reviewer remarked that the coordination and collaboration in this project is excellent. The reviewer commented that the project works with industry consortia, vehicle and engine original equipment manufacturers (OEMs), additive and lubricant OEMs, engineering companies, and suppliers. Through the interaction with all of these entities, the project is able to address barriers that are important to industry so that the results of this effort will be useful.

\section{Reviewer 2:}

The reviewer remarked on the good mix of collaborative partners and opportunities for disseminating information and gaining feedback. The reviewer further noted the good involvement with interested groups.

\section{Reviewer 3:}

The reviewer remarked that the Massachusetts Institute of Technology consortium is necessary to stay close to the industry and keep the program focused. The reviewer asked if there are any programs that Argonne National Laboratory (ANL) is working on that have plans for production from the collaborations.

\section{Reviewer 4:}

The reviewer commented that this is an immense project involving several major collaborators.

Question 4: Proposed future research-the degree to which the project has effectively planned its future work in a logical manner by incorporating appropriate decision points, considering barriers to the realization of the proposed technology and, when sensible, mitigating risk by providing alternate development pathways. 


\section{Reviewer 1:}

The reviewer commented that the program will continue to support DOE goals through developing new technologies and methods.

\section{Reviewer 2:}

The reviewer said future direction of lab-engine correlation, basefluid development, and catalytic coatings will continue to provide advancements in this project's work to develop engine friction reduction technologies.

\section{Reviewer 3:}

The reviewer said that it seems all of the components within the project are continuing adequately and that the future directions were addressed for each and seem appropriate, but due to presentation time constraints, the reviewer would have liked a higher level of project detail.

\section{Reviewer 4:}

The reviewer noted that there should be more work on non-ferrous materials and compatibility with advanced lubricants because the trend is towards more lightweight, non-ferrous materials. The reviewer said many conventional lubricant additives have trouble with non-ferrous materials.

\section{Question 5: Does this project support the overall DOE objectives of petroleum displacement? Why or why not?}

\section{Reviewer 1:}

The reviewer noted that by developing lubricants to reduce frictional losses and improve fuel economy, this project helps to address the DOE objective of petroleum displacement.

\section{Reviewer 2:}

The reviewer said lubricants were one of the few spots in the VTO program that can affect both future and legacy vehicles.

\section{Reviewer 3:}

The reviewer remarked that the project is aimed at both the development of new lubricant science and new lubricant methods to support industry in the advancement of fuel economy and downsizing goals.

\section{Reviewer 4:}

The reviewer said this project addressed many of the major questions of the tribology research realm. However, achieving the level of understanding necessary to elucidate these questions is no easy task.

\section{Question 6: Resources: How sufficient are the resources for the project to achieve the stated milestones in a timely fashion?}

\section{Reviewer 1:}

The reviewer remarked that the addition of an experienced formulator was a welcome addition a few years ago. The current team is excellent but could benefit from another experienced industry hire.

\section{Reviewer 2:}

The reviewer said the resources appear to be adequate to keep progress and goals on track.

\section{Reviewer 3:}

The reviewer commented that funding appears to be adequate for this project to meet its goals and objectives.

\section{Reviewer 4:}

The reviewer said due to the wide scope of the program, the funding level seems adequate. 
Ionic Liquids as Engine

Lubricant Additives, Impact on Emission Control Catalysts, and Compatibility with Coatings: Jun Qu (Oak Ridge National Laboratory)

- ft014

\section{Presenter}

Jun Qu, Oak Ridge National

Laboratory.

\section{Reviewer Sample Size}

A total of three reviewers evaluated this project.

\section{Question 1: Approach to} performing the work-the degree to which technical barriers are addressed, the project is well-designed, feasible, and integrated with other efforts.

\section{Reviewer 1:}

The reviewer praised the approach taken by the project team and said it has been well thought out and provides excellent insight into how ILs, as well as zinc dialkyldithiophosphates (ZDDP), function. The investigator's efforts to understand the antagonistic behavior between ILs and ZDDPs, as well as diamond-like carbon (DLC) coatings was fruitful.

\section{Reviewer 2:}

The reviewer said the approach of this project was well designed and described for this project and that each of the tasks including using ILs as a lubricant to enhance engine efficiency, lubricant effects on emission control technologies, and compatibility of lubricant additives with hard coatings were described fully. The reviewer remarked that it was clear the project team would provide a path to addressing the barriers of this project.

\section{Reviewer 3:}

The reviewer said the approach to look at the tribofilm is excellent and said the project team is doing a great job to fundamentally explain why the ZDDP and ILs have a synergistic effect. The reviewer noted that the project needs to conduct more full-engine tests to look at the compatibility with some of the nonferrous materials under real operating conditions.

Question 2: Technical accomplishments and progress toward overall project and DOE goals-the degree to which progress has been made, measured against performance indicators and demonstrated progress towards DOE goals. 


\section{Reviewer 1:}

The reviewer remarked that the technical accomplishments in this project throughout this three-year program have been excellent and that all of the required milestones in this project have been met. The reviewer further noted that the accomplishments to complete the project in fiscal years (FY) 2015 and 2016 (i.e., completing dynamometer tests to demonstrate fuel economy improvement when using a prototype oil and also the studies of the impact of additives on three-way catalysts which showed less impact of the IL additive) specifically addressed the barriers of this project.

\section{Reviewer 2:}

The reviewer praised the technical progress to date as excellent and very promising. The reviewer also noted that additional work on materials compatibility with non-ferrous materials, such as copper, is required to prove the potential corrosion issues are manageable.

\section{Reviewer 3:}

The reviewer commented that the investigators were able to make significant headway against the barriers. The reviewer noted that the effects of ILs on three-way catalyst (TWC) seems to be clearly understood and that the investigation into ILs compatibility with non-metallic coatings is well underway with significant findings related to the charge of the phosphate ion and its impact on wear of DLCs.

\section{Question 3: Collaboration and coordination with other institutions.}

\section{Reviewer 1:}

The reviewer praised the project team as one of the best examples of a superstar team with members from DOE, the world's largest lubricant supplier, one of the largest automotive OEMs, a large additive company, and a national laboratory.

\section{Reviewer 2:}

The reviewer commented that the team assembled for this project, which included energy companies, additive manufacturers, laboratories, and academia, brought together all of the entities that are needed to be involved in developing new lubricant additives to ensure acceptance of the results for potential future use in vehicles.

\section{Reviewer 3:}

The reviewer remarked that the project seems to have had good cooperation between the collaborators.

\section{Question 4: Proposed future research-the degree to which the project has effectively planned its future work in a logical manner by incorporating appropriate decision points, considering barriers to the realization of the proposed technology and, when sensible, mitigating risk by providing alternate development pathways.}

\section{Reviewer 1:}

The reviewer commented that the project team has identified and understands the important barriers that remain. The reviewer remarked that the barriers that have been investigated during this project to date are relevant and important for the successful transition of this technology to the commercial sector.

\section{Reviewer 2:}

The reviewer said this current project is $100 \%$ complete. The reviewer also noted that there is, however, a new joint funding opportunity announcement (FOA) project with General Motors that has tasks identified that will continue to address fuel economy improvement through the development of lubricant technology for engine lubrication. The reviewer remarked that the project will also investigate the impact of these lubricants on TWCs, which will help eliminate the identified barriers. 


\section{Reviewer 3:}

The reviewer said that the future research should include identifying how low viscosity can go with advanced additives that decrease boundary friction and wear. The reviewer said some testing to push the limits on viscosity would be interesting if funding allows.

\section{Question 5: Does this project support the overall DOE objectives of petroleum displacement? Why or why not?}

\section{Reviewer 1:}

The reviewer said the project supports the DOE goal of oil displacement because the development of engine oil additives will improve the efficiency of internal combustion engines.

\section{Reviewer 2:}

The reviewer said lubricants are one of the few research areas in VTO that can affect both future and legacy vehicles.

\section{Reviewer 3:}

The reviewer said this project will result in a better understanding of the chemical mechanisms underpinning the development of tribofilms that will enable the use of lower viscosity, energy efficient lubricants, and a reduction in overall petroleum use.

\section{Question 6: Resources: How sufficient are the resources for the project to achieve the stated milestones in a timely fashion?}

\section{Reviewer 1:}

The reviewer remarked that additional funds are needed to complete additional materials compatibility work. The reviewer said materials compatibility is a major hurdle hindering production.

\section{Reviewer 2:}

The reviewer said the resources appear to be sufficient to meet the goals of the project, with the assumption that the technology being developed will be licensed or otherwise taken up by a commercial champion at some point and then further developed into a commercial product.

\section{Reviewer 3:}

The reviewer commented that the funding is adequate to complete the project. 


\section{Integrated Friction \\ Reduction Technology to Improve Fuel Economy without Sacrificing Durability: Stephen Hsu (George Washington University) - ft033}

\section{Presenter}

Stephen Hsu, George Washington University.

\section{Reviewer Sample Size}

A total of three reviewers evaluated this project.

\section{Question 1: Approach to performing the work-the degree to which technical barriers are addressed, the project is well-designed, feasible, and integrated with other efforts.}

\section{Reviewer 1:}

The reviewer remarked that the project's approach is sound. The reviewer said the project looked at the base oil composition, additive components, and conducted some friction and wear bench testing leading up to fuel economy testing in an industry standardized engine test. The reviewer noted that the project team did not supply any statistical information concerning the variability of the bench test results, which made it difficult assess the results. The reviewer further remarked that no detail was provided about the composition of the test oils other than a lube code and said that this lack of detail resulted in difficulty in understanding the significance of some testing.

\section{Reviewer 2:}

The reviewer said attempts were made to formulate futuristic American Petroleum Institute (API) automotive specifications for passenger car motor oils (GF-6A and GF-6B) with low viscosity passenger car crankcase oils using available base oils and additives. The reviewer noted that the project considered novel microencapsulation techniques to protect ZDDP and the reviewer noted that friction modifier was carried out. The reviewer said the project involved Edisonian screening of numerous additive and base oil technologies and the reviewer noted that this may not be the most effective way to address existing challenges. The reviewer commented that more direct focus on additives microencapsulation techniques should be pursued and suggested this focus may provide potential for technical breakthroughs.

\section{Reviewer 3:}

The reviewer said the VTO program appears to rely heavily on a formulation similar to current production

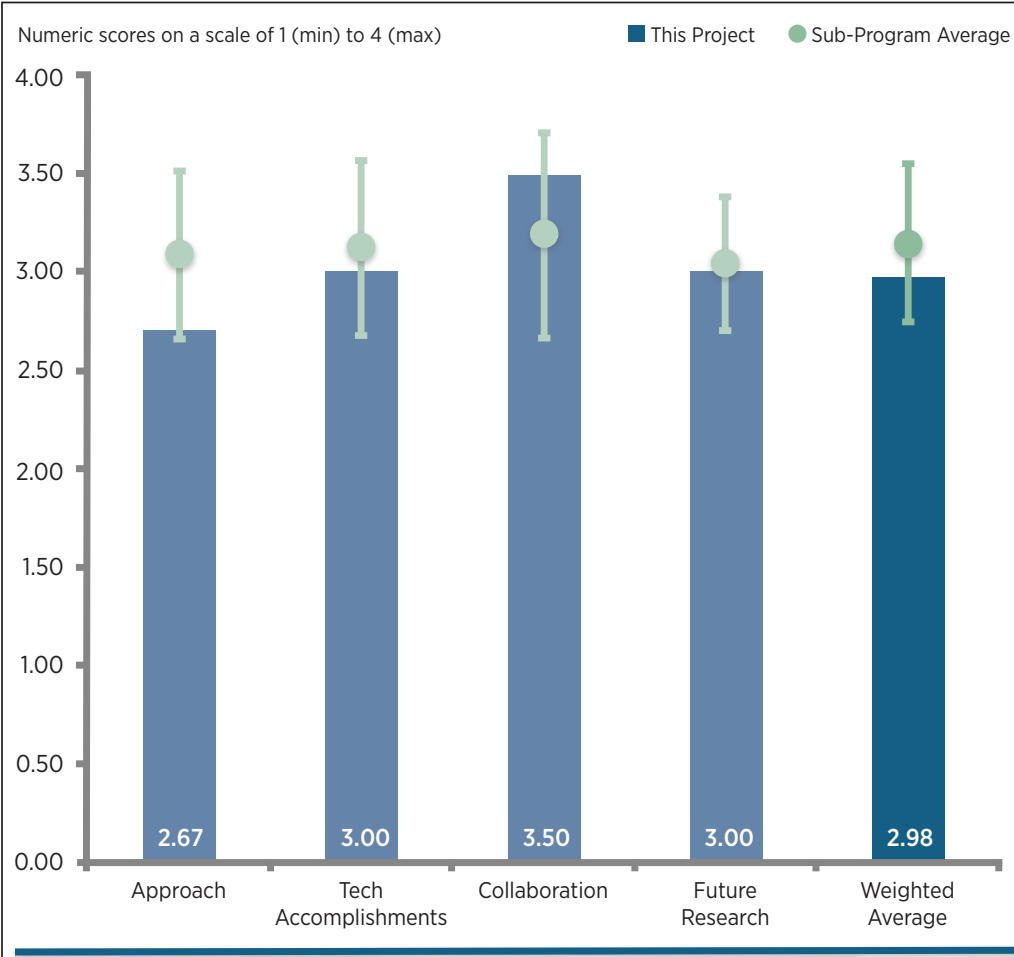

Relevant to DOE Objectives
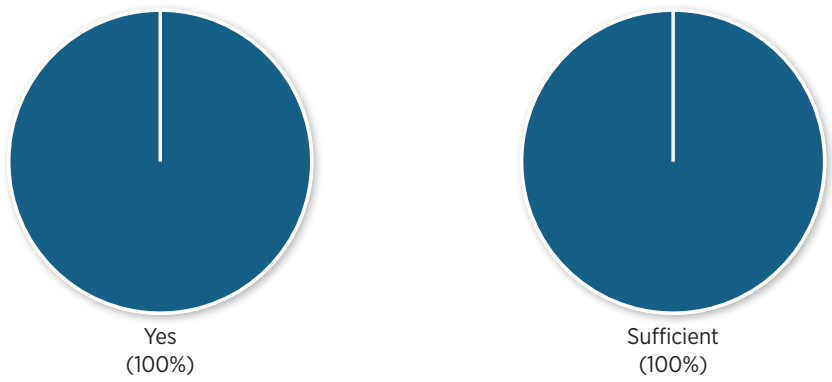

Figure 5-3 - Integrated Friction Reduction Technology to Improve Fuel Economy without Sacrificing Durability: Stephen Hsu (George Washington University) - Fuel and Lubricant Technologies

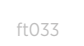


formulations. The reviewer suggested that it would be advantageous to be more aggressive and look at more aggressive changes to viscosity.

\section{Question 2: Technical accomplishments and progress toward overall project and DOE goals-the degree to which progress has been made, measured against performance indicators and demonstrated progress towards DOE goals.}

\section{Reviewer 1:}

The reviewer noted that the project addressed numerous base oils and additives that were examined in screen tests focusing on friction and wear measurements of fresh oils including Plint tester, high frequency reciprocating rig (HFRR) with temperatures up to $130^{\circ}$ Celsius $\left({ }^{\circ} \mathrm{C}\right)$, four ball at room temperature only, and tribological experiments using a mini traction machine (MTM). The reviewer further noted the team introduced a novel thermal gravimetric analysis (TGA) test run on neat base oils using argon rather than nitrogen. The reviewer said the project addressed "Sequence variable interest entity (VIE)" tests run before final test conditions are officially approved and further commented that the Sequence VIE tests showed potential for fuel consumption benefit. The reviewer said no information was offered on the type of base oil or novel additive chemistry in generic terms that was used to formulate candidate GF-6A oil. The reviewer said that because no statistical assessments with bars representing confidence level of reported bench tests data were provided, it was difficult to judge the actual level of improvements in frictional and wear performance observed in reported screen tests. The reviewer further commented that no hypothesis was put forward to address why microencapsulated ZDDP or friction modifier should offer improved wear or frictional performance. The reviewer remarked that no used oil analysis was reported from oxidation or low-temperature and high-temperature deposits screen bench tests. The reviewer questioned if encapsulated additives impact upper piston deposits formation. The reviewer indicated that no storage stability, corrosion, or seals results were reported.

\section{Reviewer 2:}

The reviewer said the project was able to formulate an oil which when tested in an industry standard engine fuel economy test showed significant improvement over a relevant baseline engine oil. The reviewer stated these baselines were $0.89 \%$ for new oil and $1.51 \%$ for aged oil. The reviewer claimed the method of summing the new and aged fuel economy improvement is only relevant for the American Society for Testing and Materials (ASTM) and International Lubricants Standardization and Approval Committee ILSAC test limits. The reviewer further stated that a driver will not experience a summing of these two improvements. The reviewer said that a driver can expect to get some combination or average of the new or aged fuel economy benefit but not the sum. The reviewer remarked that it will be interesting to see if the encapsulation of additives will provide benefits for these formulations.

\section{Reviewer 3:}

The reviewer noted that the VTO program is on track for the objectives.

\section{Question 3: Collaboration and coordination with other institutions.}

\section{Reviewer 1:}

The reviewer said the team is excellent and has all of the necessary components for success.

\section{Reviewer 2:}

The reviewer noted that the project includes representatives of key OEM and oil additive suppliers.

\section{Reviewer 3:}

The reviewer commented that the project worked closely with collaborators in the oil industry to develop its current $0 \mathrm{~W}-20$ lubricant.

Question 4: Proposed future research-the degree to which the project has effectively planned its future work in a logical manner by incorporating appropriate decision points, 


\section{considering barriers to the realization of the proposed technology and, when sensible, mitigating risk by providing alternate development pathways.}

\section{Reviewer 1:}

The reviewer remarked that the proposed future work includes durability testing of the lower viscosity $0 \mathrm{~W}-16$. The reviewer noted that the project also plans to continue its work on surface textures and investigate the use of microencapsulated friction modifiers to prolong friction reduction. The reviewer concluded that these are logical extensions of the current work.

\section{Reviewer 2:}

The reviewer noted that the project made a list of several relevant challenges that were described in a relatively general fashion. The reviewer remarked that no timeline or listing of specific tasks assigned to key project collaborators, specifically their labs, was given. The reviewer stated that no analysis of metal contact areas to explain mechanisms of lubricating film formation or chemistry was planned or proposed.

\section{Reviewer 3:}

The reviewer stated that work looking at coating every surface in an engine is of limited value and said that not all moving surfaces may benefit from coatings or surface texturing. The reviewer offered that it would be more interesting to look at coating or texturing select components and then testing them one by one instead of as a whole system.

\section{Question 5: Does this project support the overall DOE objectives of petroleum displacement? Why or why not?}

\section{Reviewer 1:}

The reviewer said lubricants are one of the few research areas in VTO that can affect both future and legacy vehicles.

\section{Reviewer 2:}

The reviewer pointed out that the project goal is to improve the fuel economy of vehicles which will reduce the use of fuel.

\section{Reviewer 3:}

The reviewer stated that forthcoming API GF-6A and GF-6B oils will offer significant fuel economy improvements. The reviewer remarked that no specific focus to provide vital fundamental knowledge regarding utilization and potential barriers of novel microencapsulated additives technologies is described. The reviewer said there was no description of how the future technology discovery steps and intellectual property (IP) will be handled.

\section{Question 6: Resources: How sufficient are the resources for the project to achieve the stated milestones in a timely fashion?}

\section{Reviewer 1:}

The reviewer remarked that the project has not commented on current or future funds available. The reviewer cautioned that the project may run short of funds specifically when multiple sets of API GF-6A and GF-6B engine tests need to be carried out.

\section{Reviewer 2:}

The reviewer said according to the overview the funding received for FY 2016 is $\$ 751,000$. The reviewer stated that this funding amount appears to be sufficient for the project to complete new formulations, bench testing, and engine testing. 


\section{Hybrid Ionic-Nano-Additives for Engine Lubrication to Improve Fuel Efficiency: Bin Zhao (University of Tennessee) - ft034}

\section{Presenter}

Bin Zhao, University of Tennessee

\section{Reviewer Sample Size}

A total of three reviewers evaluated this project.

\section{Question 1: Approach to performing the work-the degree to which technical barriers are addressed, the project is well-designed, feasible, and integrated with other efforts.}

\section{Reviewer 1:}

The reviewer remarked that the project seems like a well thought out nanoparticle (NP) study. The reviewer expressed some concern on the stability and dispersion side. The reviewer stated that there was little mention of how stable and dispersed these formulations are over time. The reviewer assumed particle size data over time by way of a technique such as dynamic light scattering was conducted by the project, but not shown due to presentation time constraints. The reviewer specified that zeta potential of the particles over time would also be a very good metric to quantify stability. The reviewer further noted that stability and dispersion state can vary greatly over many parameters, such as concentration, agitation state, temperature, interface materials, and others.

The reviewer cautioned that it is imperative to be certain the nanofluid subjected to tribological testing is the true intended monodispersed nanofluid, not a system of agglomerates. The reviewer further remarked that if this cannot be proven, the performance could vary greatly and thus the validity of the data could be brought into question.

\section{Reviewer 2:}

The reviewer said the approach was satisfactory but it would be useful to have a more detailed explanation of how the project is going to be implemented.

\section{Reviewer 3:}

The reviewer said that there does not appear to be any baselining against current technology, that is to say, current motor oil or against current dispersants. The reviewer warned that the use of plain Polyalphaolefin (PAO) 4 as a baseline is not really realistic and said that probably almost anything added to it will improve friction and wear.

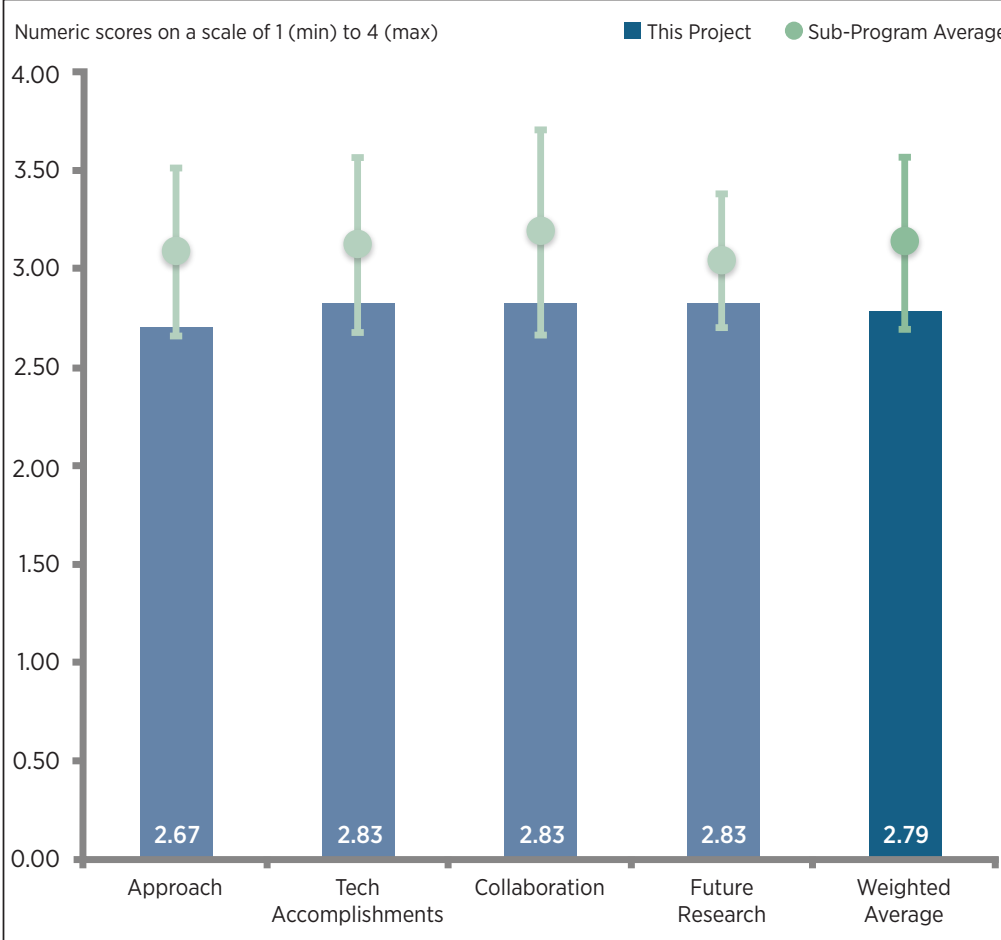

Relevant to DOE Objectives
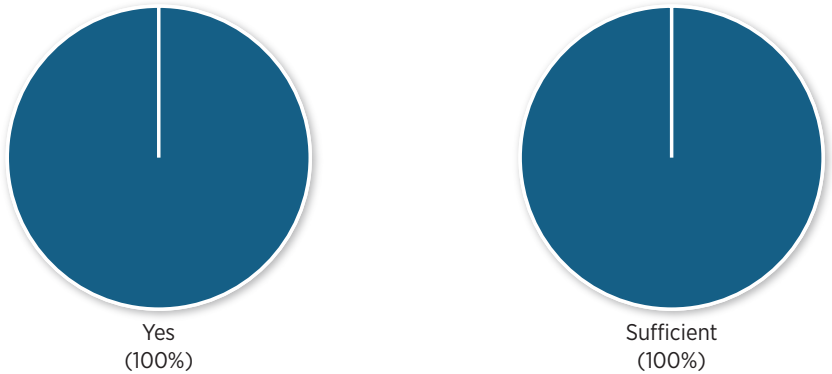

Figure 5-4 - Hybrid Ionic-Nano-Additives for Engine Lubrication to Improve Fuel Efficiency: Bin Zhao (University of Tennessee) - Fuel and Lubricant Technologies 
The reviewer remarked that the project needs a better way to assess suspension stability beyond taking photographs to show transparency. The reviewer said that there was no detail or discussion about engine dyno testing.

\section{Question 2: Technical accomplishments and progress toward overall project and DOE goals-the degree to which progress has been made, measured against performance indicators and demonstrated progress towards DOE goals.}

\section{Reviewer 1:}

The reviewer remarked that several technical accomplishments have shown friction reduction of as much as $50 \%$. The reviewer said that milestones have been met or are on track, which will contribute to overcoming the barriers of this effort.

\section{Reviewer 2:}

The reviewer noted that the project conveyed the good potential in the novel nanofluid formulations. The reviewer specified that the room temperature performance of the ionic liquid nanoparticles (IL-NPs), albeit beneficial, is not very important and indicated that this is because it lacks practicality to the real world application. The reviewer said scuffing issues at higher temperature are a major concern that must be immediately addressed. The reviewer claimed that at higher temperatures, the oil will thin out, and the smaller fluid film thickness could lead to NP build up on the leading edge and oil starvation in the contact. The reviewer stated that this problem could be exacerbated if the solution has agglomerates and suggested that a more detailed dispersion study may provide information regarding the scuffing issue.

\section{Reviewer 3:}

Program appears to be a little behind schedule based on $30 \%$ completion number, but it does appear that milestones are being met.

The reviewer said the selection of NPs is not described and therefore it is hard to assess how novel they are. The reviewer warned that failure of IL-NP lubrication at $100^{\circ} \mathrm{C}$ is troubling and warrants much more study. The reviewer suggested that maybe the NPs desorb from the metal surface or the polymers desorb from the NPs.

\section{Question 3: Collaboration and coordination with other institutions.}

\section{Reviewer 1:}

The reviewer said the national laboratory and university support seems adequate. The reviewer noted that there is a strong influence from the national laboratory regarding the IL-NP dispersion science and said that this is vital for program success. The reviewer remarked that there is no influence from industry and said that this is not a major concern at this stage of the project, but also suggested that the project team may want to seek this in the future. The reviewer would like to see more influence from additive companies. The reviewer commented that the performance may be vastly different once added to a fully formulated oil and that an additive company could provide important guidance on how to overcome these barriers.

\section{Reviewer 2:}

The reviewers remarked on the useful partnerships with Oak Ridge National Laboratory (ORNL) for ILs and testing. The reviewer said there was a useful partnership with the University of California-Merced for modeling. The reviewer pointed out that there does not appear to be a relationship with a lubricant manufacturer to deal with formulation issues and that this was a project weakness. The reviewer said there also does not appear to be a collaboration with engine, vehicle, or component manufacturers to indicate industrial interest in new technology.

\section{Reviewer 3:}

The reviewer said collaboration between ORNL and the University of California-Merced is good. The reviewer commented that it may be useful to also have interactions with industry partners such as OEMs or engine lubrication companies.

Question 4: Proposed future research-the degree to which the project has effectively planned its future work in a logical manner by incorporating appropriate decision points, 
considering barriers to the realization of the proposed technology and, when sensible,
mitigating risk by providing alternate development pathways.

\section{Reviewer 1:}

The reviewer said the proposed future work is very good and that it will address the challenges and barriers that remain in the project.

\section{Reviewer 2:}

The reviewer would like to see more stability-over-time data addressing the previously stated concerns.

\section{Reviewer 3:}

The reviewer remarked that there was no discussion about addressing $100^{\circ} \mathrm{C}$ performance issues, other than developing new formulations. The reviewer asked if there was a systematic plan for developing new formulations, along with selection criteria, or if the project was conducting more Edisonian type research. The reviewer said that it sounds like there could be a lot of combinations for NPs, ILs, polymers, and polymer molecular weights (MWs). The reviewer indicated that the matrix was not defined in the talk, relative to the total number of combinations and screening tests being used. The reviewer said there was no discussion about engine dyno testing plans or about baselining against current oil and additive technologies.

\section{Question 5: Does this project support the overall DOE objectives of petroleum displacement? Why or why not?}

\section{Reviewer 1:}

The reviewer said the main objective of the project is to improve engine efficiency by developing additives to be used in engine lubrication and that this definitely supports the DOE objective of petroleum displacement.

\section{Reviewer 2:}

The reviewer said that this project illustrated the potential to reduce both friction and wear, which could ultimately lead to fuel efficiency gains.

\section{Reviewer 3:}

The reviewer affirmed by saying yes, the development of new or novel additives can result in friction and wear reduction to enable more efficient engines. The reviewer noted that it is also possible that some of these additives could be used in the existing vehicle fleet with lower viscosity base stocks.

\section{Question 6: Resources: How sufficient are the resources for the project to achieve the stated milestones in a timely fashion?}

\section{Reviewer 1:}

The reviewer said that the level of control over the NP surface chemistry demands an appropriate budget and strong partners, which this project has.

\section{Reviewer 2:}

The reviewer said that there appears to be sufficient funds to accomplish the project objectives and milestones.

\section{Reviewer 3:}

The reviewer commented that the resources appear to be adequate to complete the project and said the current progress does not warrant an increase in effort. 


\section{Hyperbranched Alkanes for Lubes: Lelia Cosimbescu (Pacific Northwest National Laboratory) - ft035}

\section{Presenter}

Lelia Cosimbescu, Pacific Northwest National Laboratory.

\section{Reviewer Sample Size}

A total of three reviewers evaluated this project.

\section{Question 1: Approach to performing the work-the degree to which technical barriers are addressed, the project is well-designed, feasible, and integrated with other efforts.}

\section{Reviewer 1:}

The reviewer remarked that the project approach was divided into two components: design, synthesis, and screening of molecular structures with unique hyperbranched architectures for proof of concept experiments; and engine testing on the developed additives that pass the screening criteria, which has proven to be an excellent method.

\section{Reviewer 2:}

The reviewer said the project's approach was thorough and included synthesis of new viscosity modifiers (VMs), simulation and bench testing, and also engine testing.

\section{Reviewer 3:}

The reviewer said the approach for the go/no go points was well designed. The reviewer stated that the project team had a nice design to focus on the synthesis and testing of the additives before spending much effort in full engine tests.

\section{Question 2: Technical accomplishments and progress toward overall project and DOE goals-the degree to which progress has been made, measured against performance indicators and demonstrated progress towards DOE goals.}

\section{Reviewer 1:}

The reviewer said that the technical accomplishments in this project provided data and information to meet the milestones that were identified. The reviewer noted that engine testing demonstrated the feasibility of the Pacific Northwest National Lab (PNNL) polymer with commercial packages and also showed fuel economy increases that met the objective of $2 \%$ fuel economy increase.

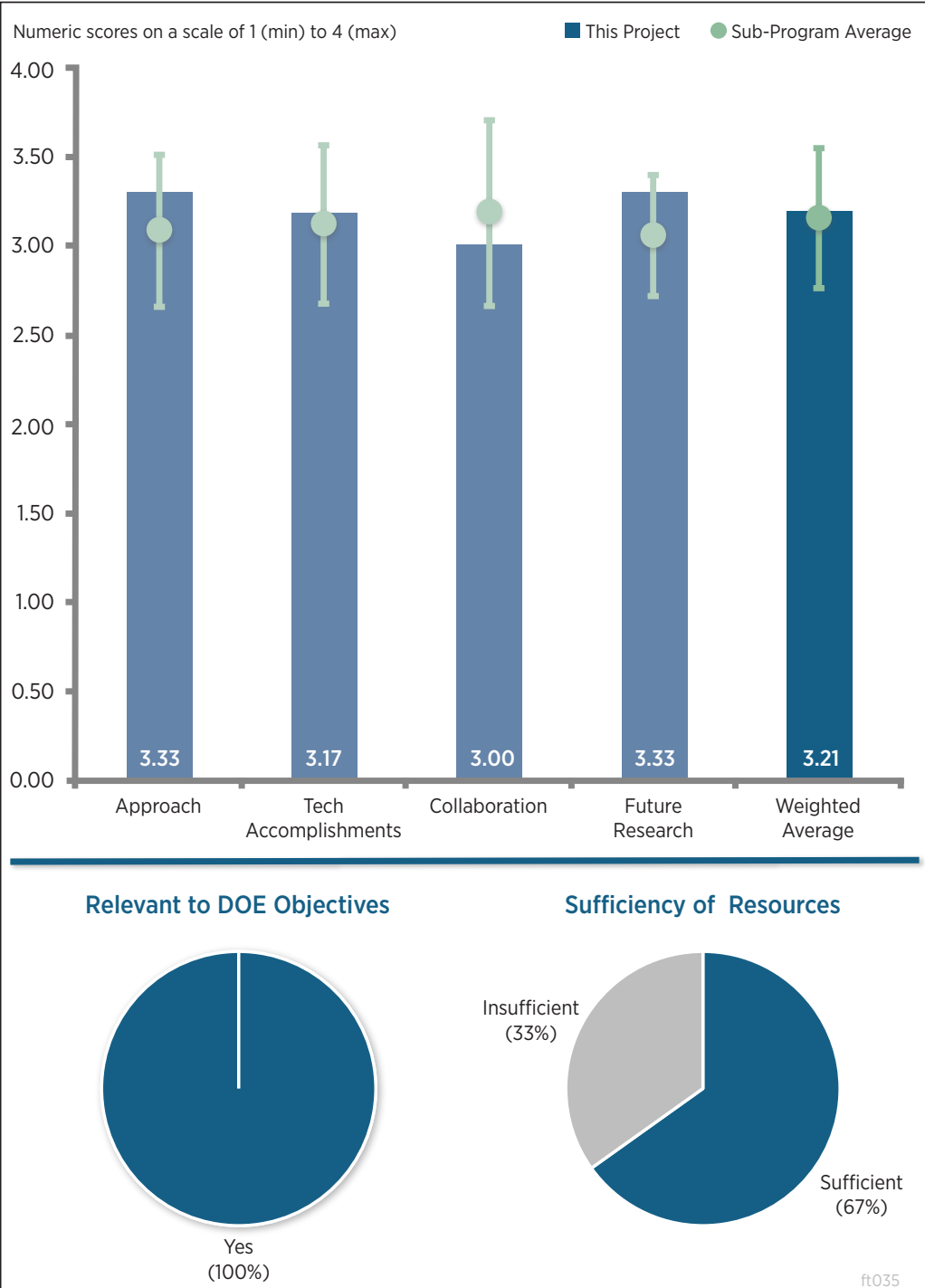

Figure 5-5 - Hyperbranched Alkanes for Lubes: Lelia Cosimbescu (Pacific Northwest National Laboratory) - Fuel and Lubricant Technologies 


\section{Reviewer 2:}

The reviewer stated that the project is essentially finished so the progress is good. The reviewer said that the demonstration of $2 \%$ fuel economy could have been improved with different baseline fluids but that this is a moot point now.

\section{Reviewer 3:}

The reviewer remarked that although the project did achieve a fuel economy benefit over the reference oil, the reference oil is not a modern fuel efficient formulation. The reviewer pointed out that additional optimization of the formulation could lead to improvements. The reviewer said that other significant concerns include shear stability of the new VMs and the potential for increased wear.

\section{Question 3: Collaboration and coordination with other institutions.}

\section{Reviewer 1:}

There reviewer remarked that there is very good collaboration and coordination in this project with other national laboratories, academia, and industry partners, which have provided guidance and advice on the project to make it a success.

\section{Reviewer 2:}

The reviewer said the project team brings excellent experience, but a partner familiar with full engine formulation would have been useful.

\section{Reviewer 3:}

The reviewer said the project included adequate collaboration between the project team and an industry additive marketer. The reviewer noted that the bench and engine testing were also completed by collaborators.

\section{Question 4: Proposed future research-the degree to which the project has effectively planned its future work in a logical manner by incorporating appropriate decision points, considering barriers to the realization of the proposed technology and, when sensible, mitigating risk by providing alternate development pathways.}

\section{Reviewer 1:}

The reviewer said the project team identified important barriers and issues that still need to be addressed prior to this product becoming commercialized.

\section{Reviewer 2:}

The reviewer remarked that the proposed future work to continue the development of hyperbranched structures and to develop and strengthen industrial relationships will help to address the remaining challenges and barriers identified for this project.

\section{Reviewer 3:}

The reviewer mentioned that the pressure and viscosity effect and shear stability were not evaluated and should be considered if the project continues. The reviewer indicated that the project needs another reference oil for a baseline if the project continues.

\section{Question 5: Does this project support the overall DOE objectives of petroleum displacement? Why or why not?}

\section{Reviewer 1:}

The reviewer stated that the project is relevant to the overall DOE objective of petroleum displacement through the development of novel lubricant formulations that are expected to improve fuel efficiency of vehicles.

\section{Reviewer 2:}

The reviewer said lubricants are one of the few research areas in VTO that can affect both future and legacy vehicles. 


\section{Reviewer 3:}

The reviewer said the project goals were to reduce fuel consumption through the development of novel friction and viscosity modifiers combined on the same molecule. The reviewer indicated that a successful project would lead to reduced use of petroleum.

\section{Question 6: Resources: How sufficient are the resources for the project to achieve the stated milestones in a timely fashion?}

Reviewer 1:

The reviewer said the project could use some additional funding to continue some necessary work.

\section{Reviewer 2:}

The reviewer said that the resources were sufficient for the envisioned project, but further work will be needed and noted that it is unclear if resources are available.

Reviewer 3:

The reviewer said that the resources are sufficient to complete the project. 


\section{Lubricant Effects on Combustion, Emissions, and Efficiency: Robert Wagner (Oak Ridge National Laboratory) - ft036}

Presenter

Brian West, Oak Ridge National

Laboratory.

\section{Reviewer Sample Size}

A total of three reviewers evaluated this project.

\section{Question 1: Approach to performing the work-the degree to which technical barriers are addressed, the project is well-designed, feasible, and integrated with other efforts.}

\section{Reviewer 1:}

The reviewer said the investigative techniques used to test the various hypothesis are excellent and unique.

\section{Reviewer 2:}

The reviewer said that the approach of targeted engine, vehicle, and flow reactor studies with in-depth characterization of combustion, emission control devices, and fuel economy to better understand fuel and lubricant effects is very good and provides for a plan to adequately address the barriers

\section{Reviewer 3:}

The reviewer said that the fuel and lubricant interface is a critical area, but is also immensely complex and dynamic. The reviewer remarked that the mixture of fuel, lube, soot, and others may not be adequately investigated with gas chromatography-mass spectrometry (GC-MS) analysis alone. The reviewer suggested that the project consider testing the rheology and lubricity of the mixture.

\section{Question 2: Technical accomplishments and progress toward overall project and DOE goals-the degree to which progress has been made, measured against performance indicators and demonstrated progress towards DOE goals.}

\section{Reviewer 1:}

The reviewer said the accomplishments have been adequate thus far. The reviewer stated that the proof of concept apparatus seems viable and that it will be interesting to see further research with this approach.

\section{Reviewer 2:}

The reviewer said the technical accomplishments for this project-including quantification of fuel and lubricant impacts on gasoline direct injection (GDI) particulate matter (PM) and the work to establish vehicle-based methods 
to measure fuel economy improvement from lubricants-have provided excellent results to address the barriers of this project.

\section{Reviewer 3:}

The reviewer remarked that the progress to date was excellent.

\section{Question 3: Collaboration and coordination with other institutions.}

\section{Reviewer 1:}

The reviewer remarked that collaboration and coordination with other organizations was excellent. The reviewer said that working with industry, academia, and other national laboratories provides for an excellent set of partners, which will lead to the success of the project.

\section{Reviewer 2:}

The reviewer said, as usual, Oak Ridge is collaborating with excellent, world-class partners.

\section{Reviewer 3:}

The reviewer said due to the applied nature of this project that it was appropriate to have a solid influence from industry collaborators, which this project has.

\section{Question 4: Proposed future research-the degree to which the project has effectively planned its future work in a logical manner by incorporating appropriate decision points, considering barriers to the realization of the proposed technology and, when sensible, mitigating risk by providing alternate development pathways.}

\section{Reviewer 1:}

The reviewer stated that the future direction of the project has identified very good plans that will help to address the remaining barriers.

\section{Reviewer 2:}

The reviewer said that it seems like the direction of the project is well thought out and clearly defined. The reviewer suggested that if the project team can determine what the fuel, lubricant, or soot mixture is composed of then that could lead to many interesting tribological studies. The reviewer said an interesting starting point would be to test the mixture lubricity in a Plint TE 77 with segmented ring and liner.

\section{Reviewer 3:}

The reviewer indicated that low- and high-temperature fuel economy would be very high impact tests for future research. The reviewer said that demonstrating how a high viscosity index gives more real world benefit would help the scientific community.

\section{Question 5: Does this project support the overall DOE objectives of petroleum displacement? Why or why not?}

\section{Reviewer 1:}

The reviewer stated that determining the effects of lubricants on combustion, emissions, and efficiency is very relevant to the DOE objective of petroleum displacement.

\section{Reviewer 2:}

The reviewer said the area where the lubricant and fuel meet is not well understood. The reviewer remarked that this project addresses some of those concerns and associated phenomena. The reviewer said, if successful, the project could elucidate some observed technical issues.

\section{Reviewer 3:}

The reviewer said lubricants are one of the few research areas in VTO that can affect both future and legacy vehicles. 
Question 6: Resources: How sufficient are the resources for the project to achieve the stated milestones in a timely fashion?

Reviewer 1:

The reviewer said the resources appear to be sufficient to achieve the work identified in this project.

Reviewer 2:

The reviewer said that the program needs to expand and do fuel economy tests at more temperatures and with more vehicles on multiple drive cycles.

Reviewer 3:

The reviewer said the amount of funding seems adequate. The reviewer remarked that it was not clear to what level the collaborators are contributing, and noted in-kind contributions, hardware/component, testing, consultation, etc. 


\section{Co-Optimization of Fuels and Engines Overview: John Farrell (National Renewable Energy Laboratory) - ft037}

\section{Presenter}

John Farrell, National Renewable Energy Laboratory.

\section{Reviewer Sample Size}

A total of four reviewers evaluated this project.

\section{Question 1: Approach to performing the work-the degree to which technical barriers are addressed, the project is well-designed, feasible, and integrated with other efforts.}

\section{Reviewer 1:}

The reviewer remarked that overall the project team appears to be doing an excellent job approaching a very difficult task. The reviewer provided praise by saying that this is one of the highest impact programs to come from VTO or the DOE Bioenergy Technologies Office (BETO) since the E15 program in 2008-2011. The reviewer said there is a buzz in the industry that resonates outside of the typical fuels and engines community and that the VTO program is influencing industry research programs in the energy industry as far away as China and Saudi Arabia.

The reviewer said the split between Thrust I and Thrust II is critical compression ignition, including low-temperature combustion (LTC), too quickly. The reviewer also remarked that adding a decision point to extend the Thrust I program beyond 2019 would be beneficial. The reviewer cautioned that switching the project's full R\&D effort to Thrust II too quickly would put any gains from Thrust I at risk. For example, if a high-octane and increased ethanol content fuel is chosen, it will likely have trouble with market introduction. The reviewer pointed out that some extension of Thrust I to make a more market friendly fuel with the majority of the chosen fuel benefits might be the better path forward. The reviewer suggested that, perhaps, a higher-octane refinery fuel without the ideal properties would be the better path.

The reviewer said the distillate fuel volumes are projected to dominate the U.S. fuel pool in the coming decades. The reviewer remarked that optimizing light-duty vehicles (LDVs) is a noble goal but heavy-duty vehicles (HDVs) might be the more critical need. The reviewer said change of fuels would also be simpler for on-highway heavyduty (HD) engines than light-duty (LD) simply because the vehicle parc is smaller and the refueling system is not as large as gasoline. The reviewer also noted that Thrust II should consider splitting the HD and LD activities. 
The reviewer further remarked that engine size does make a difference; that is to say, the large bore engines have different chemical requirements due to operating range and torque requirements.

\section{Reviewer 2:}

The reviewer remarked that the work involved with the Thrust I projects is very interesting to OEMs and will support the industry's progress towards further improvements in fuel economy. The focus on improvement in a fuel for these near-term needs is imperative. However, as shown with a timeline decision point in 18 months on the Thrust I fuel and given the overall length of the project, it seemed to this reviewer that very little focus is being given to these near-term needs. The reviewer said that seems extremely ambitious given some of the existing challenges with current market fuels and current technologies that would be addressed with a new fuel going forward for the Thrust I technologies. The reviewer said it seems that more focus should be spent on the Thrust I portion of the project to really understand the fuel aspects of technologies, which would then apply to understanding the challenges of other technologies.

In reference to The Approach Strategy slides, the reviewer commented that it is not clear what the metrics are to know that the project team has success in these areas. The reviewer wanted to know if there is a set of metrics being developed (e.g., goal/outcome; ASTM specification/proposal, etc.). The reviewer questioned if Thrust I and Thrust II should really just be combined.

\section{Reviewer 3:}

The reviewer said that from a technical perspective, this project seems well thought out. The reviewer expressed concern that there is an undue focus on using biomass for a fuel source. The reviewer noted that with the abundance of natural gas (NG) and the problems associated with diverting crops from food to fuel that it seems to make better sense converting NG into fuels. The reviewer also mentioned that the other problem with this is that there seems to be little to no cooperation with universities, fuel manufacturers, and vehicle manufacturers. The reviewer was uncomfortable because the entire program seems to be run using only the national laboratories.

\section{Reviewer 4:}

Considering that introducing a new fuel into the market is a monumental task, this reviewer suggested that it would behoove the Co-Optima panel to perform a thorough investigation to assess the use of the same fuel for both Thrust I and Thrust II engine concepts. The reviewer warned that if the Thrust II fuel is dramatically different than the Thrust I fuel, then the challenges posed by introduction of a second new fuel and new powertrains that require use of the new fuel may pose insurmountable challenges.

The reviewer indicated that development of the modeling toolkit is extremely valuable work and should be given as much support as possible. The reviewer suggested that care should be taken to ensure that appropriate experimental hardware is used for validating the models being developed. For instance, the optical single cylinder engine at Sandia National Laboratories (SNL) is an excellent tool for helping with the development and validation of computational models but that engine is not representative of the Thrust I production engine technologies. The reviewer said that the use of the optical engine to validate computational models may lead to validation of combustion regimes, which would not be encountered in Thrust I production technology.

The reviewer remarked that as part of the proposed research, extensive data will be generated on the physical and chemical properties of fuels and their impact on engine combustion. The reviewer suggested that this may be a good opportunity to develop and define a new knock resistance metric that is more representative of combustion in modern engines compared to the research octane number (RON) and motor octane number (MON) tests.

\section{Question 2: Technical accomplishments and progress toward overall project and DOE goals - the degree to which progress has been made, measured against performance indicators and demonstrated progress towards DOE goals.}

\section{Reviewer 1:}

The reviewer said the project only recently began but that it has made excellent progress. The reviewer noted that the screening criteria led by the project team are excellent and a needed tool for the industry. The reviewer remarked that, while some individuals will disagree with the criteria, the development of a tool fills a critical need. 
The reviewer also said the market transformation reports are needed and appear to be on-track for completion by the end of 2016.

\section{Reviewer 2:}

The reviewer said that it looks like the project is just getting started and therefore there is not much progress yet on the project. The reviewer noted that the project team make some important notes on the critical assumptions and issues of the project. The reviewer asked how those issues are being addressed so that the project will be successful. The reviewer said those are very insightful and show the great leadership on the project.

\section{Reviewer 3:}

The reviewer said that while the targets and milestones for the Co-Optima program are clearly spelled out, the metrics for assessing the success and completion of the program are not well defined. The reviewer stated that it would be helpful if the deliverables and targets for the overall program are well defined and quantifiable along with the metrics to be used for assessing the completion and success of the project. The reviewer also noted that good progress has been made on the down-selection of biomass-based fuel components with the ultimate goal of identifying components for engine testing (Tier 3 candidate evaluation). The reviewer specified that as part of the Analysis of Sustainability, Scale, Economics, Risk, and Trade (ASSERT) tasks on Slide 13, it was not clear how the economy wide benefits have been quantified when the fuels and engine technologies have not been finalized.

\section{Reviewer 4:}

The reviewer said it seems like ethanol fuels are a pre-determined winner here. The reviewer acknowledged that other fuels definitely will be tested, but opined that ethanol seems to be the winner already. As was stated, the project purpose is to see what can compete with ethanol. The reviewer expressed uncertainty about how to make sufficient ethanol to supply the need without significantly impacting the food supply.

\section{Question 3: Collaboration and coordination with other institutions.}

\section{Reviewer 1:}

The reviewer said the span of partners is very impressive and the collaboration effort is the largest since the E15 effort. The reviewer also remarked that the team has made a great effort to engage external stakeholders and organize an advisory board. The reviewer noted that the current board is well covered by government, engine OEMs, energy companies, and the biofuel industry. In relation to the stakeholder groups, the reviewer asked about off-road manufacturers (e.g., marine, lawn equipment, and recreational equipment). The reviewer noted that these entities do not use much fuel but will be impacted and should be considered for a place on the external advisory board. The reviewer remarked that the emissions equipment manufacturer should also be considered for a position on the external advisory board simply because emissions will be critical for future success and transformation. The reviewer stated that the United States Council for Automotive Research (USCAR) should not be relied upon to represent them. The reviewer noted that the nine national laboratories appear to have well defined splits in the work to take full advantage of their individual specialties.

\section{Reviewer 2:}

The reviewer said that it seems that there is good collaboration on the project. The reviewer also pointed out that the project seems low on the stakeholders that would be involved in implementation of any new market fuels, whether there is one or more new fuels for various engine technologies. The reviewer noted that the project team indicated such stakeholders were suggested as a barrier and challenge to the project and suggested it would be helpful to open the lines of communication and add these kinds of stakeholders to the discussion. The reviewer said a project on new and improved fuels and engine technologies would be a terrible waste of money if it could not be implemented in the market for the reduction of petroleum consumption and benefit of all stakeholders.

\section{Reviewer 3:}

The reviewer stated that it is encouraging to see the close collaboration between the various national laboratories and the concerted effort to maximize the synergies between the participating teams. The reviewer emphasized that considering the scale of the Co-Optima program and the significance of the scope of work, stakeholder feedback and engagement are critical for the ultimate success of the project. The reviewer said stakeholder conference calls 
and listening days are steps in the right direction for soliciting feedback from stakeholders. The reviewer pointed out that it is not clear how the stakeholder feedback is being incorporated into planning Co-Optima activities.

\section{Reviewer 4:}

The reviewer asked why universities or fuel manufacturers are not a part of this work. The reviewer said that universities have already done a lot of this work, so it could be that researchers are recreating the wheel. The reviewer also said that fuel manufacturers need to be involved to make sure that whatever fuel is chosen can be manufactured, stored, and distributed. The reviewer further asked if researchers know that the fuel can be run in the vehicle without first developing a new fuel system.

\section{Question 4: Proposed future research-the degree to which the project has effectively planned its future work in a logical manner by incorporating appropriate decision points, considering barriers to the realization of the proposed technology and, when sensible, mitigating risk by providing alternate development pathways.}

\section{Reviewer 1:}

The reviewer remarked that the Co-Optima program has aggressive timelines and the desired targets for the 18 month decision point are well specified. The reviewer said developing a good understanding of the various market transformation scenarios is essential for the successful adoption of the new fuels and engine technologies that are expected to be developed as part of the program. The reviewer expressed that it would behoove the project team to ensure that the aggressive timeline does not compromise the research and analysis required for market adoption and growth of Thrust I technologies.

\section{Reviewer 2:}

The reviewer commented that the team seems to be making good progress towards their 18 month decision point. The reviewer remarked that the ASSERT team should include the impact on petroleum refinery efficiency, GHGs, and economics if they are not planning for it. The reviewer said impacts of biorefineries and biofuel production is critical but the petroleum refineries should not be trivialized. The small changes in the fuel octane rating or distillation changes from the refinery can impact the refinery efficiency by several percent. The reviewer said that, while several percent on the well-to-tank is often trivialized by people considering the entire lifecycle, the economic impact is more severe. The reviewer remarked that the margins from refineries are typically miniscule and small changes can make or break the refinery.

\section{Reviewer 3:}

The reviewer remarked that this is a very interesting and challenging project that appears to have just gotten started and therefore, much of the work ahead is in the future. The reviewer commented that reviewers were shown a set of very high level tasks for the different integrated teams, but not all tasks, and that it was unclear on the timeline when the project would to accomplish these tasks. The reviewer said that due to this it was not really clear how aggressive the project is in completing the Thrust I goals for the 18 month decision point. The reviewer said that the Thrust I projects are very important to the OEMs for introduction of the new downsized boosted engines and therefore the timeline should be worked out and communicated to stakeholders immediately. The reviewer stated that the hardest part of the project will be the implementation of the fuel in the market and that the project needs to show greater focus and emphasis in this area. The reviewer concluded that most of the work is needed in demonstrating a clear plan in the near-term for Thrust I.

\section{Reviewer 4:}

The reviewer did not like the fact that the first milestone is time driven and not event driven. The reviewer said that this time constraint could leave new developments out of the picture.

\section{Question 5: Does this project support the overall DOE objectives of petroleum displacement? Why or why not?}

Reviewer 1:

The reviewer applauded this effort as one of the most critical VTO programs for the DOE goals of reducing 
petroleum dependence and GHGs. The reviewer said the project actually has a chance to drastically change the fuels landscape and have a larger impact than most of the electric vehicle and materials programs.

\section{Reviewer 2:}

The reviewer remarked that improvement of existing engine combustion technologies, identification of desirable fuel properties, and development of new biofuels are all expected to contribute to DOE's goal of petroleum displacement.

\section{Reviewer 3:}

The reviewer said that there are certainly aspects of the Thrust I portion of the project that will enable displacement of petroleum through improvement in fuel efficiency. The reviewer explained that it has been shown in the literature and it is well known that higher octane and higher sensitivity fuels with downsized boosted engines will provide fuel economy improvements. The reviewer remarked that the Thrust I project is interesting and will benefit the auto industry goal of furthering the downsized boosted engines. The reviewer also said that the Thrust II projects are not clear if they will provide petroleum displacement through improved efficiency.

\section{Question 6: Resources: How sufficient are the resources for the project to achieve the stated milestones in a timely fashion?}

\section{Reviewer 1:}

The reviewer said that it is not clear how the money will be divided between the projects but highly encouraged the project team to move more of the funds to the near-term Thrust I projects rather than the Thrust II projects. The reviewer remarked that the timeline appears as if most of the research will focus on Thrust II projects. The reviewer pointed out that the Thrush II projects are not the prime time technologies that OEMs are working on because these are technologies that are more than 15 years away from market introductions. The reviewer further commented that the timeline shows very little focus on the Thrust I projects and said that is surprising given that the technologies are the main line technologies for current OEMs.

\section{Reviewer 2:}

The reviewer remarked that, while the individual subprojects have budgets associated with them, it would also be helpful if the overview presentation included a slide showing the breakdown of the overall budget and how the resources are allocated to the different activities.

\section{Reviewer 3:}

The reviewer said the level of funding appears sufficient for the current goals. The reviewer mentioned that expansion of funding may increase in the coming years depending on the initial results. The reviewer said additional funds at the current time would not significantly contribute to the scope or success of the project and might detract from other critical activities in the VTO Fuel and Lubricant Technologies program. The reviewer said if additional funding from the Fuel and Lubricant Technologies program is required, it should be taken from any gaseous fuel activities. Direct-injection propane is a waste of money. NG should be used in power plants to displace coal first; further effort in engine development is not very useful. 


\section{Co-Optimization of Fuels and Engines (Co-Optima) - Fuel Properties and Thrust I Engine Research: Jim Szybist (Oak Ridge National Laboratory) - ft038}

\section{Presenter}

Jim Szybist, Oak Ridge National

Laboratory.

\section{Reviewer Sample Size}

A total of four reviewers evaluated this project.

\section{Question 1: Approach to} performing the work-the degree to which technical barriers are addressed, the project is well-designed, feasible, and integrated with other efforts.

\section{Reviewer 1:}

The reviewer remarked that the general approaches identified for the overall Co-Optima program and for the 13 individual projects discussed during the presentations were excellent and should be effective in addressing the barriers identified.

\section{Reviewer 2:}

The reviewer said that the approach follows a logical progression for defining key fuel properties and parameters, screening candidate fuels according to those properties as well as some other key characteristics, and testing performance of the identified fuels in laboratory combustion experiments, along with kinetic modeling followed by engine testing. The reviewer noted that in order to do this, new test methods such as heat of vaporization and auto-ignition quality from small samples are to be developed. The reviewer said promising approaches to those have been described.

\section{Reviewer 3:}

The reviewer remarked this project had a good approach to a very complex set of problems. The reviewer did not see oil company involvement. The reviewer acknowledged not being aware of efforts to involve industry or universities from the outset. The reviewer expressed skepticism about the ultimate importance of LTC-based engines. The data on cold starting and full load range capability remain missing. The reviewer noted that with this absence, it was unclear if there was a real benefit. The reviewer said some work suggests aftertreatment requirements have largely eliminated the originally expected fuel economy benefits. The reviewer cautioned that given this, the major emphasis on fuels for such engines may be misdirected.

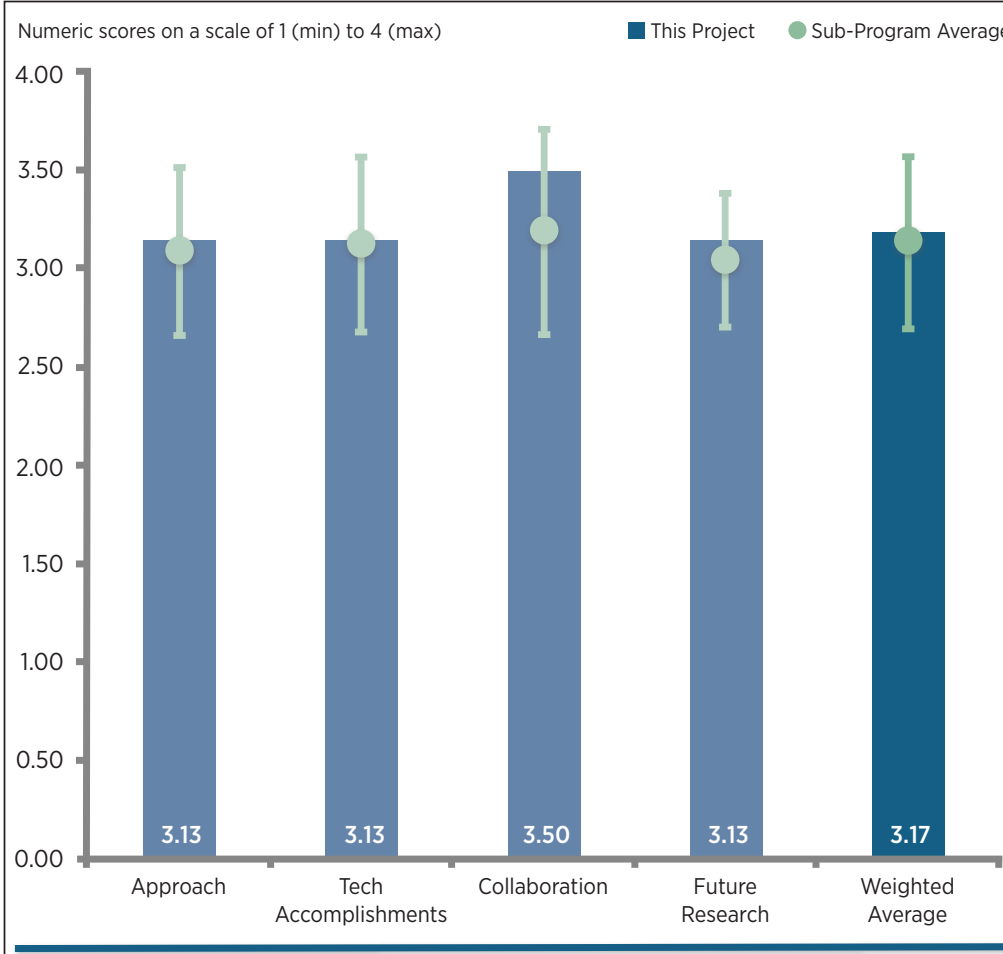

Relevant to DOE Objectives
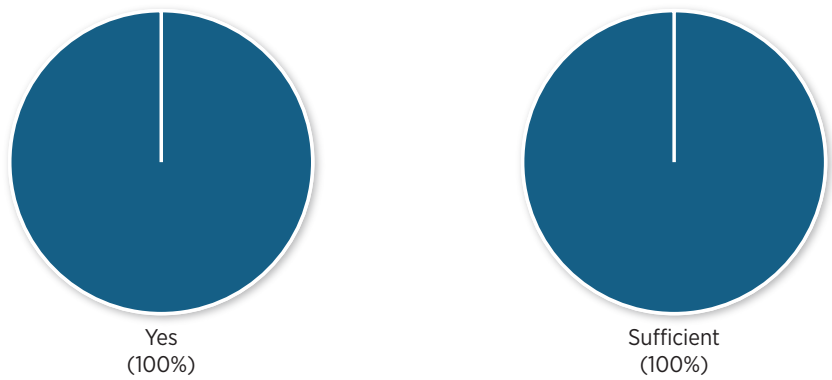

Figure 5-8 - Co-Optimization of Fuels and Engines (CoOptima) -- Fuel Properties and Thrust I Engine Research: Jim Szybist (Oak Ridge National Laboratory) - Fuel and Lubricant Technologies 


\section{Reviewer 4:}

The reviewer said that the following Co-Optima projects have been reviewed as one and provided one set of comments for all four presentations: FT037, Co-Optima Overview (John Farrell, et al); FT038, Co-Optima Fuel Property and Advanced Engine Development Team, Part 1-Fuel Properties and Chemical Kinetics (Robert McCormick and Jim Szybist, et. al.) and Part 2-Thrust I engine projects (Jim Szybist, et. al.); ft039, Co-Optima of Fuels and Engines and Advanced Engine Development Team, Part 1-Thrust II engine projects (Paul Miles and Matt Ratcliff, et. al.); and ft040, Co-Optima Simulation Toolkit Team (Matt McNenly and Sibendu Som, et. al.). The reviewer provided 11 Thrust I comments that follow below.

Beginning the first Thrust I group of comments, the reviewer remarked that Co-Optima and Thrust I should be sharply focused on the overall Co-Optima goal vision of "Better fuels and better vehicles sooner." The reviewer noted that a lot of effort has gone into collaborating and coordinating between the various national laboratories and that these efforts and results are commended. The reviewer pointed out that collaboration and coordination is obviously needed and beneficial. For example, bringing to bear all the simulation work going on at the national laboratories on fuel property and engine response work, this work is bound to have high impact even if later than sooner. The reviewer cautioned that, with all the emphasis on collaboration between the various national laboratories and the coordination of the various teams with their diverse skills and tasks, it is easy for the program to become narrowly focused and lose sight of the forest for the trees. The reviewer also noted that the Co-Optima program has quickly become bureaucratic, with sub-teams providing reports to other teams within itself and to DOE. The reviewer said that while the project is admittedly a non-trivial challenge, in order to gain the trust of its stakeholders as being a legitimate effort to bring about significant GHG reduction, Co-Optima should continuously strive to be seamless across DOE, VTO, BETO, and the individual national laboratories and to keep the focus on the customer, vision, and the stakeholders.

For the second Thrust I group of comments, the reviewer remarked that Co-Optima has correctly concluded that the major barrier and challenge to realizing better fuels and better vehicles sooner in the marketplace is related to the large number of stakeholders with competing value propositions. The reviewer also said that because this is the major barrier, the full strength and weight of the Co-Optima program should be brought to bear squarely on removing this major barrier and not simply end up majoring on the minors.

Initiating the third Thrust I group of comments, this reviewer asked what was driving the 18-month decision point for Thrust I and noted that it gives the perception of Co-Optima being eager to hastily disengage from Thrust I issues and challenges and desiring to move on to Thrust II. The reviewer said that the one thing this implies, perhaps inadvertently, is that Thrust II engines, which are yet to be identified, and Thrust II fuels, which are also yet to be identified, will be more impactful in reaching Co-Optima goals than Thrust I engines and fuels. The reviewer questioned, if given the well-acknowledged risks associated with Thrust II engine concepts, whether there is overwhelming evidence that the only barrier remaining for Thrust II engines is the lack of a co-optimized Thrust II fuel. On the other hand, Thrust I engines and fuels are largely already identified by overwhelming industry and OEM practice and literature evidence, both recent and historical. Therefore, it seems prudent and a more reasonable strategy for Co-Optima to first tackle co-optimizing Thrust I engines and fuels, focusing sharply on the major barrier for Thrust I, which this reviewer referenced in the second Thrust I group of comments.

Starting the fourth Thrust I group of comments, the reviewer recommended that Co-Optima should refrain from proposing timelines for commercialization targets because Co-Optima can only provide research information, motivation, and incentive for commercialization, and perhaps play the very valuable role of bringing all stakeholders to the table. Those timelines can probably only be set by the stakeholders themselves because stakeholders typically have to follow free enterprise principles for commercial success.

For the fifth Thrust I group of comments, this reviewer remarked that Co-Optima should include a R\&D milestone titled "Can Thrust II fuel be the same as Thrust I fuel?" Having the same fuel for both Thrust I downsized boosted SI engines as well as Thrust II advanced compression ignition (ACI) engines has obvious benefits. The reviewer referenced the second Thrust I group of comments, and then remarked that the major barrier for any new fuel, Thrust I or Thrust II, has already been acknowledged to be the large number of stakeholders with competing value propositions. The reviewer asked if there is an opportunity to bust this major roadblock once, why choose a path that would need this roadblock busted twice. Having Thrust I and Thrust II fuels be the same is an incredible opportunity to further the main goal of Co-Optima vision of "Better fuels and better vehicles sooner." The reviewer also noted a related sub-milestone comment under Thrust II. 
Opening the sixth Thrust I group of comments, the reviewer remarked that the Co-Optima program should verify that high RON and high sensitivity fuels do indeed substantially increase engine and vehicle fuel efficiency. The reviewer commented that this should be done quickly and with minimal research, as this ground has been plowed numerous times by many studies, and the answer is generally well-known and accepted.

Beginning the seventh Thrust I group of comments, this person stated that the Co-Optima program should focus major resources and effort on identifying promising low-GHG fuel compositions and blendstocks that are sustainable, affordable, scalable, and with attractive infrastructure and retail attributes. The reviewer said that the understanding is that Co-Optima has already started the life-cycle and techno-economic analysis on the 20 Thrust I fuel blendstocks.

Introducing the eighth Thrust I group of comments, the reviewer said that it is anticipated, that of the six teams that Co-Optima has put in place, the following hold the key to overcoming the main barrier already discussed: the Low Greenhouse Gas Fuels (LGGF) team, which is focused on low-GHG blendstock properties and pathway attributes; the ASSERT team, which is focused on environmental impacts, cost, scalability, and feed logistics; and the Market Transformation (MT) team, which is focused on infrastructure and legacy fleet compatibility. The reviewer is of the opinion that these three teams will need to be primarily engaged in assisting stakeholders going forward. The reviewer also warned that, if Co-Optima declares Thrust I complete and disengages at FY 2019, it may fall short of its goal and will have at most a flash-in-the-pan effect.

Starting the ninth Thrust I group of comments, this reviewer said that the Thrust I engine projects currently being carried out on the 1.6-L Ford Ecoboost engine at ORNL and the 2.0-LTG General Motors (GM) engine at the National Renewable Energy Laboratory (NREL) are scoped reasonably well. The reviewer remarked that several appropriate changes have been made to the base engines to make it more relevant for evaluating high octane fuel performance. The reviewer also noted that appropriate changes in the engine compression ratio (CR) are planned, along with an appropriate selection of relevant fuels. The reviewer also remarked that appropriate issues like low-speed pre-ignition (LSPI) are being studied. The reviewer also commented that the project has the potential of informing the community about the tradeoffs that need to be dealt with when designing downsized boosted engines with high octane fuel to realize high fuel efficiency.

For the $10^{\text {th }}$ Thrust I group of comments, the reviewer opined that the single cylinder head engine choice at SNL may not be representative of the state-of-the-art Thrust I engine that industry currently has and continues to evolve. The referenced engine at SNL has good optical access and also has port and other information needed for computational fluid dynamics (CFD) simulation provided by an OEM. However, the typical downsized, boosted SI engine today utilizes high-tumble ports, special combustion chamber and piston topologies, and Miller cycle valve strategies, of which none are present in that cylinder head. The reviewer explained that work on knock-limited performance on this cylinder head will fall way short of understanding real low- and high-speed wide open throttle (WOT) knock-limited behavior.

Presenting the $11^{\text {th }}$ Thrust I group of comments, this person stated that engine downsizing causes the load-factor of an engine to increase. Therefore, the current work on the effect of laminar flame speed increasing dilution tolerance at light loads should be extended to mid-loads also where knock typically compromises the optimum location of CA50.

The reviewer provided five Thrust II comments that follow below.

Commencing the first Thrust II group of comments, this person remarked that verifying whether Thrust I fuel and Thrust II fuel can be the same should be the first, and foremost, Thrust II goal. The reviewer noted that in this regard, the use of an Octane Index (OI) as a means to evaluate fuel properties simultaneously suitable for both Thrust I and Thrust II engines is encouraged.

For the second Thrust II comment, the reviewer asserted that Co-Optima should pursue the path of discovering a new Thrust II fuel and by implication, a new Thrust II engine, only after it has been convincingly proved that these two fuels cannot be the same.

Presenting the third Thrust II group of comments, this reviewer commented that the literature on Thrust II engine concepts suggests more than a handful of recipes for ACI combustion. The reviewer inquired about what would be the anticipated Co-Optima fuel requirements for each recipe. The reviewer provided that choices could be as follows: exactly the same; slightly different; or significantly different. The reviewer noted if the answer turns out to 
be "exactly the same," then the path forward is simplified. If it also stands that Thrust II fuel equals Thrust I fuel, then the path forward is incredibly faster and beneficial for all stakeholders, as previously noted.

Starting the fourth Thrust II group of comments while also referencing the third Thrust II group of comments, the reviewer questioned whether the path forward would be fraught with huge challenges if the answer turns out to be "significantly different," or even "slightly different." The reviewer expressed interest in knowing who decides the winning Thrust II engine-plus-fuel combination. This person further inquired whether Co-Optima could really be the one who picks the winner, or if each stakeholder would have a differing opinion on the winning combination. The reviewer also asked about the role of free market enterprise for selecting the winning combination.

Concluding with the fifth Thrust II group of comments and referencing the fifth Thrust I group of comments, this reviewer remarked that Co-Optima could include a sub-milestone under another milestone. Recognizing that in the context of the current discussion, perhaps the suggestion that Thrust II fuel and Thrust I fuel can be the same may hold true only for LD applications because Thrust I engines largely imply SI, gasoline, LD engines. The reviewer offered that, on the other hand, Thrust II fuel can be targeted to any or all applications including LD, medium-duty (MD), or HD. For example, if it turns out that new Thrust II fuels can be profitably optimized with Thrust II engine recipes for MD or HD applications, perhaps then a case could be made that Thrust II fuel should be different than Thrust I fuel for these applications.

\section{Question 2: Technical accomplishments and progress toward overall project and DOE goals-the degree to which progress has been made, measured against performance indicators and demonstrated progress towards DOE goals.}

\section{Reviewer 1:}

The reviewer commented that there was a great deal of progress in both the fuel property team and the Thrust I engine projects, especially because the projects were only started recently. The reviewer said progress and accomplishments identified in the presentations from both teams appear to be addressing the barriers adequately and milestones in the project have been met or are on track. The reviewer noted that there were seven individual projects discussed in the Fuels and Engines Fuel Properties Team and six individual projects discussed in the Advanced Engine Development team. The reviewer suggested that in the future, it may be necessary to devote more time for these individual projects to allow a more in-depth review of each project.

\section{Reviewer 2:}

The reviewer commented that there is a mix, with some new projects and some older projects. The reviewer remarked that, overall, there was good progress and useful data. The reviewer assumed that there will be work to collate the various results and understand results on the many different engines and test rigs. The reviewer wanted to know if there would be any work to correlate different labs and pointed out that very small details of test methods can have large effects on results. The reviewer said that cold start conditions are critical for the ability to run lean, for hydrocarbon (HC) speciation for catalyst light off, and for the ability to operate heavily retarded to generate heat for catalyst light off. The reviewer said experimental plans and models should consider this because $90 \%$ of emissions occur in the first 60 seconds of engine operation.

\section{Reviewer 3:}

The reviewer remarked that the project was in the early stages and therefore most of the technical accomplishments are yet to come. The reviewer said that the presentation was necessarily abbreviated considering the wide scope of project activities. The reviewer pointed out that while a fair amount of relevant data were presented within the presentations, it was not clear how much of this was actually from project results and how much was pre-existing data. The reviewer remarked that notwithstanding this, the data presented, along with the well planned and explained work plan, indicate a more than satisfactory level of progress.

\section{Question 3: Collaboration and coordination with other institutions.}

\section{Reviewer 1:}

The reviewer said the project includes six national laboratories and that the collaboration between them shows every indication of being ample and productive. 


\section{Reviewer 2:}

The reviewer said that the Co-Optima project has a very extensive set of researchers collaborating and coordinating the research. The reviewer commented that the nine national laboratories bring together some of the best talent in the country to help solve the fuel and lube barriers identified. The reviewer expressed a concern that, with so many entities participating, there is a possibility that so much time could be spent on coordination meetings and it will take away from the time available to do the research.

\section{Reviewer 3:}

The reviewer suggested that more collaboration with industry and universities would be helpful in the long run. The reviewer expressed some concern that the large number of players means a lot of time will be spent on meeting, coordination, and reporting. The reviewer asked what the overhead cost of project management was versus actual research. The reviewer noted that DOE had a policy 20 years ago of putting certain kinds of research in specific labs. The reviewer said that SNL's combustion lab did optical engines, ORNL did full scale engines, and Lawrence Livermore National Laboratory (LLNL) did kinetics, etc. The reviewer remarked that this is now diffused so that there is a large overhead of engines at multiple labs, with large mission overlap. The reviewer said that this requires a lot of coordination, and the reviewer suspected there will also be a big management cost [DOE Program Clarification: DOE plans to increase industry engagement over the life of this project, and still strives to not duplicate efforts at the national laboratories.].

Question 4: Proposed future research-the degree to which the project has effectively planned its future work in a logical manner by incorporating appropriate decision points, considering barriers to the realization of the proposed technology and, when sensible, mitigating risk by providing alternate development pathways.

\section{Reviewer 1:}

The reviewer said that the proposed work of both the fuels properties project team and the Thrust I engine project team to include the incorporation of biofuels, with a wide range of chemical compositions, into the experiments will provide information to continue to address the goals and objectives of the project.

\section{Reviewer 2:}

The reviewer observed a good plan towards important goals.

\section{Reviewer 3:}

The reviewer remarked that the directions of future research described are logical and potentially very useful, particularly in getting better understanding of fuel property impacts including octane sensitivity, heat of vaporization (HOV), exhaust gas recirculation (EGR) dilution tolerance, and others on combustion flame, autoignition, and ultimately efficiency of high CR engines. The reviewer commented that the 18 month make-or-break decision point, apparently dictated to the program office and project team exogenously, is very unfortunate and probably unrealistic [DOE Program Clarification: The decision point is only to decide what compounds can reasonably be made at scale by the year 2025. It will not be the end of investigations of fuels for spark-ignition engines, and therefore, should not be viewed as a "make-or-break" decision.].

\section{Question 5: Does this project support the overall DOE objectives of petroleum displacement? Why or why not?}

\section{Reviewer 1:}

The reviewer said that both the fuel properties and chemical kinetics, as well as the Thrust I engine projects, are very relevant to helping to meet the DOE objectives of petroleum displacement because a major goal is to develop a robust and quantitative understanding on how efficiency is impacted by fuel properties.

\section{Reviewer 2:}

The reviewer noted a core of work on future engine systems. 


\section{Reviewer 3:}

The reviewer said the project is potentially very relevant in that it would enable future development of much more efficient SI engines, as well as use with mid-level biofuels blends. The reviewer noted an issue with the relevance was, however, that fuel candidates are being selected without substantial grading by production cost or difficulty. The reviewer said while this is very difficult to assess at this point or even conterminously with the project progress, it is the ultimate determinant of the relevance of the project. The reviewer commented that while it is known that many different compounds can be produced from various biofuels processes, the reality is that attempts to commercially produce biofuels other than corn ethanol and heavily subsidized biodiesel have been unsuccessful to date despite seemingly viable pathways for cellulosic ethanol having been identified for decades.

\section{Question 6: Resources: How sufficient are the resources for the project to achieve the stated milestones in a timely fashion?}

\section{Reviewer 1:}

The reviewer said the funding appears to be sufficient for the tasks to be completed in this project.

\section{Reviewer 2:}

The reviewer acknowledged not having a basis for evaluating the sufficiency of the resources provided other than that the work plans appear to be well-tailored based on those resources.

\section{Reviewer 3:}

The reviewer said the project is a well-funded large effort. The reviewer said that it seems misleading to call this a \$21 million program because most of the work is a continuation of earlier efforts and rebranded as Co-Optima. The reviewer remarked that, still, the overall funding seems about right [DOE Program Clarification: Although much of the work is building on earlier efforts, the novel aspect of Co-Optima is the explicit coordination of the programs.]. 


\section{Co-Optimization of Fuels and Engines (Co-Optima) - Thrust II Engine Research, Sprays Research, and Emissions Control Research: Paul Miles (Sandia National Laboratories) - ft039}

\section{Presenter}

Paul Miles, Sandia National Laboratories

\section{Reviewer Sample Size}

A total of four reviewers evaluated this project.

\section{Question 1: Approach to performing the work-the degree to which technical barriers are addressed, the project is well-designed, feasible, and integrated with other efforts.}

\section{Reviewer 1:}

The reviewer remarked that the advanced combustion regimes appear to be focused on making the combustion actually possible, which is critical. The reviewer said that it would be beneficial to also consider emissions early in the program and noted that it appears to not even be a part of the combustion program. The reviewer commented that the only emissions work seems to be with current engines. The reviewer expressed that emissions problems will prevent certain combustion regimes from ever entering the market, barring miraculous efforts on the aftertreatment. The reviewer offered that getting an early look at the engineout emissions would help downselect candidate fuels and regimes. The reviewer commented that the spray work feeding into the modeling team is a critical effort that should be continued and noted that the modeling efforts are of very limited value without the empirical data to support them. The reviewer remarked that the emissions work looking at fuel impurities (i.e., Toops/Pihl at ANL) is critical to get an early look at unintended consequences with biofuels. The reviewer stated that the unexpected impact of molecules such as potassium should be considered early and not after a fuel is picked. The reviewer said that this work should continue.

\section{Reviewer 2:}

The reviewer wanted to know what efforts will be included in Thrust II that can address all of the barriers to implementation of these new advanced combustion modes. The reviewer said that these barriers include, but are not limited to, transient response, tailpipe emissions over a drive cycle, cold start performance, variations in market fuel, robustness to ambient conditions, and robustness to production-build tolerances and engine aging affects. The reviewer referenced Slide 3 of the presentation and pointed out that the Thrust I milestone is March 2017 and that the testing of Thrust I fuels in gasoline compression ignition (GCI) has a target date of September 30, 2016. 
The reviewer wanted to clarify if this implied that all five Tier 3 fuels are to be tested by September 30, 2016, even though the fuels have not been down-selected as of June 2016. The reviewer commented that understanding the impact of new fuel components on the engine emissions is critical for evaluating the feasibility of the fuels and engine concepts being evaluated. The reviewer remarked that it is encouraging to see that emissions assessment is being considered from the start of the project.

\section{Reviewer 3:}

The reviewer said that although this is a very admirable project, the Thrust II objectives are too aggressive for market implementation even after the technology might be well developed. The reviewer remarked that the timeline development for the project seems rather novice, as many of the issues that OEMs will have with the technology on controls and meeting emissions regulations seem to be an oversight. The reviewer said that as the presenter was going over the projects, it was difficult to determine if the team was talking about something that was for a diesel engine or a gasoline engine or both. The reviewer also said that it seems like there are many combustion modes and processes and on-going work, but no clear identification process for techniques or technologies so a down-selection can be made based on metrics. The reviewer stated that it seemed like a laundry list of projects without a clear method to identify the winners and losers. The reviewer suggested that it would be best to combine both the Thrust I and Thrust II aspects to better help in the development and definition of fuels and the technology winners and losers. With regard to Thrust I and Thrust II fuels, the reviewer noted that much of the framework and metrics will be the same, so it seems like a duplication of effort to separate them.

\section{Reviewer 4:}

The reviewer expressed concern that there is no apparent input from industry or academia on this work. The reviewer further questioned if researchers are re-inventing the wheel on certain subtasks. The reviewer's final point of concern was that no thought seems to have been made regarding the final fuel cost and said that this will directly affect market acceptance.

The reviewer noted that, later in the presentation, the project team stated that a cooperative research and development agreement (CRADA) is currently being drafted with Ford Motors and Caterpillar. The reviewer questioned why this was not done up front. The reviewer also commented that there were too many undefined acronyms in the presentation.

\section{Question 2: Technical accomplishments and progress toward overall project and DOE goals-the degree to which progress has been made, measured against performance indicators and demonstrated progress towards DOE goals.}

\section{Reviewer 1:}

The reviewer remarked that the technical accomplishments to date are impressive.

\section{Reviewer 2:}

The reviewer remarked that there are a lot of projects and on-going work being accomplished, but it is unclear how this is moving towards meeting the goals of the Thrust II projects. The reviewer said that those goals were not clearly explained here, just a very brief list of objectives and milestones. The reviewer asked what the goal of Thrust II was. The reviewer also wanted to know how the project team will know that they made it or not. The reviewer further questioned how the technologies are being sorted for good or bad progress towards the goals. The reviewer commented that it all seems very disjointed, by the look of the slides and the summary of the progress. The reviewer remarked that the projects on the spray and emission control research were much better. The reviewer said it seems like these pertain to the Thrust I and Thrust II areas, so will support both projects.

\section{Reviewer 3:}

The reviewer remarked that it is critical to test the compatibility of Thrust I fuels with Thrust II engine concepts. The reviewer said that thus, it is encouraging to see that this activity is a priority for the Thrust II engine program. The reviewer said that most of the emissions research outlined in the presentation is focused on Thrust I engine technology. The reviewer noted that while this research is very pertinent and needs to continue, it is not clear how the planned emissions activities will address aftertreatment for LTC or ACI engine concepts. The reviewer offered that similar to the testing of Thrust I fuels on Thrust II engines, the aftertreatment efforts should also include some 
work on Thrust II technologies with the goal of early identification of challenges that may influence the feasibility of the advanced combustion concepts. The reviewer questioned if particulate matter index (PMI) translated from Thrust I to Thrust II.

\section{Reviewer 4:}

The reviewer got the sense that E30 is the presumptive winner. The reviewer asserted that both FEV and AVL have done substantial work on E30 and wanted to know why these groups were not part of this effort. The reviewer also questioned why fuel bulk modulus was not taken into account. The reviewer stated that depending on the fuel system, this property can have a great effect on ignition.

\section{Question 3: Collaboration and coordination with other institutions.}

\section{Reviewer 1:}

The reviewer remarked that it is encouraging to see the close collaboration between the various national laboratories and the concerted effort to maximize the synergies between the participating teams.

\section{Reviewer 2:}

The reviewer said that the collaborative work between the labs is impressive. The reviewer remarked that they appear to actually be leveraging their individual strengths instead of competing against each other. The reviewer noted that collaborations with external stakeholders and companies is either not presented or at a low level. The reviewer suggested that some additional interaction with non-government stakeholders would ensure the programs are working towards relevant problems.

\section{Reviewer 3:}

The reviewer said that this looked like it was mostly collaborations between the national laboratories, but not so much on other collaborations. The reviewer noted that it would be helpful to include OEMs and industry in this process.

\section{Reviewer 4:}

The reviewer said that again, the partner list is very good but it only includes national laboratories. The reviewer asked where the industrial partners and academics were.

\section{Question 4: Proposed future research-the degree to which the project has effectively planned its future work in a logical manner by incorporating appropriate decision points, considering barriers to the realization of the proposed technology and, when sensible, mitigating risk by providing alternate development pathways.}

\section{Reviewer 1:}

The reviewer remarked that the focus on the evaluation of Thrust I fuels in Thrust II advanced combustion concepts is encouraging. The reviewer said that the spray and emissions research outlined for future work is very relevant. The reviewer noted that, however, additional work should be included to identify and address aftertreatment challenges for advanced combustion concepts.

\section{Reviewer 2:}

The reviewer commented that it would be beneficial to expand the work to look at unintended consequences.

\section{Reviewer 3:}

The reviewer said, again, it seems that the proposed projects and work is scattered with no clear plan to determine winners and losers. The reviewer commented that it was not clear how the technologies will be completely evaluated and down-selected to focus on more promising areas. The reviewer remarked, as is stated on the Thrust II slide, the project focus is to focus on projects across the labs rather than an approach to develop the technologies into usable solutions for industry. The reviewer said it is not clear how one would know that the Thrust II is completed and successful without metrics. The reviewer noted that, also, it is not clear that the future work is helping to move the project forward to achieve the challenges and barriers. The reviewer stated that more work needs to be done at a high level to determine what the goal to be achieved really is. 


\section{Question 5: Does this project support the overall DOE objectives of petroleum displacement? Why or why not?}

\section{Reviewer 1:}

The reviewer said that, while long-term, the advanced combustion concepts are expected to help achieve DOE's goal of petroleum displacement. In addition, the emissions research is expected to contribute to achieving improved engine efficiency along with lower emissions.

\section{Reviewer 2:}

The reviewer said that fuels and lubricants are one of the few research areas in VTO that can affect both future and legacy vehicles.

\section{Reviewer 3:}

The reviewer asked if petroleum displacement is being looked at too hard. The reviewer wanted to know where ethanol will come from if it wins this and further inquired if it will come from food production.

\section{Reviewer 4:}

The reviewer remarked that it is not clear that the Thrust II technologies will prove to be more fuel efficient and displace petroleum. The reviewer questioned if those data are available and in the literature. The reviewer was unsure what this technology will help support because the project team did not cover that. With regard to the question about data in the literature, the reviewer commented that it would be good to establish that first and to determine if this technology will afford a winning solution for the future as compared to current OEMs technology paths.

\section{Question 6: Resources: How sufficient are the resources for the project to achieve the stated milestones in a timely fashion?}

\section{Reviewer 1:}

The reviewer said that the project needs resources to look at toxics and other unintended consequences.

\section{Reviewer 2:}

The reviewer remarked that it seems like the Thrust II projects should be evaluated on an engine that is relevant for what current OEMs are working on for the market. The reviewer also said that it seems that the project could be evaluated in just one engine for the different combustion modes. The reviewer noted that the Thrust II does not have a clear focus and seems a bit excessive, especially given that this technology is more than 15 years away and not the current focus of OEMs. 


\section{Co-Optimization of Fuels and Engines (Co-Optima) - Simulation Toolkit Team: Matt McNenly (Lawrence Livermore National Laboratory) - ft040}

\section{Presenter}

Matthew McNenly, Lawrence Livermore National Laboratory

\section{Reviewer Sample Size}

A total of four reviewers evaluated this project.

\section{Question 1: Approach to performing the work-the degree to which technical barriers are addressed, the project is well-designed, feasible, and integrated with other efforts.}

\section{Reviewer 1:}

The reviewer remarked that the overall approach of the Simulation Toolkit Team to leverage existing VTO software and expertise and to build a shared community around computing and data to accelerate research is very good. In addition, for each of the subtasks in this project, a detailed goal, approach, and Co-Optima impact were identified that will contribute towards meeting the milestones and barriers of the effort.

\section{Reviewer 2:}

The reviewer praised that this is a very good approach to develop a highly skilled team and certainly world leading technology. The reviewer said that it would be more effective if all of the team were co-located, but that is probably impossible. The reviewer commented that the wide geographical spread necessarily means some overhead cost in organization and management. The reviewer remarked that the best collaboration is over a water cooler, not a telephone.

\section{Reviewer 3:}

The reviewer said that the approach as laid out in the overview and on Slide 33 appears reasonable and potentially beneficial. The reviewer commented that specific task descriptions, however, are very difficult to understand, both individually, and in terms of the relationships between them, particularly the relationships between different kinds of data being input in the simulations. The reviewer remarked that it is not even clear if this project includes generation of much of the data identified and depicted in the photos of testing equipment, etc., or if this project team is simply processing data received from other Co-Optima teams. The reviewer pointed out that no indication is given of a logical progression being followed but rather of certain types of data being generated, or otherwise obtained, on certain surrogate fuels without clear direction based on analysis of what is needed and what steps should lead to what others.

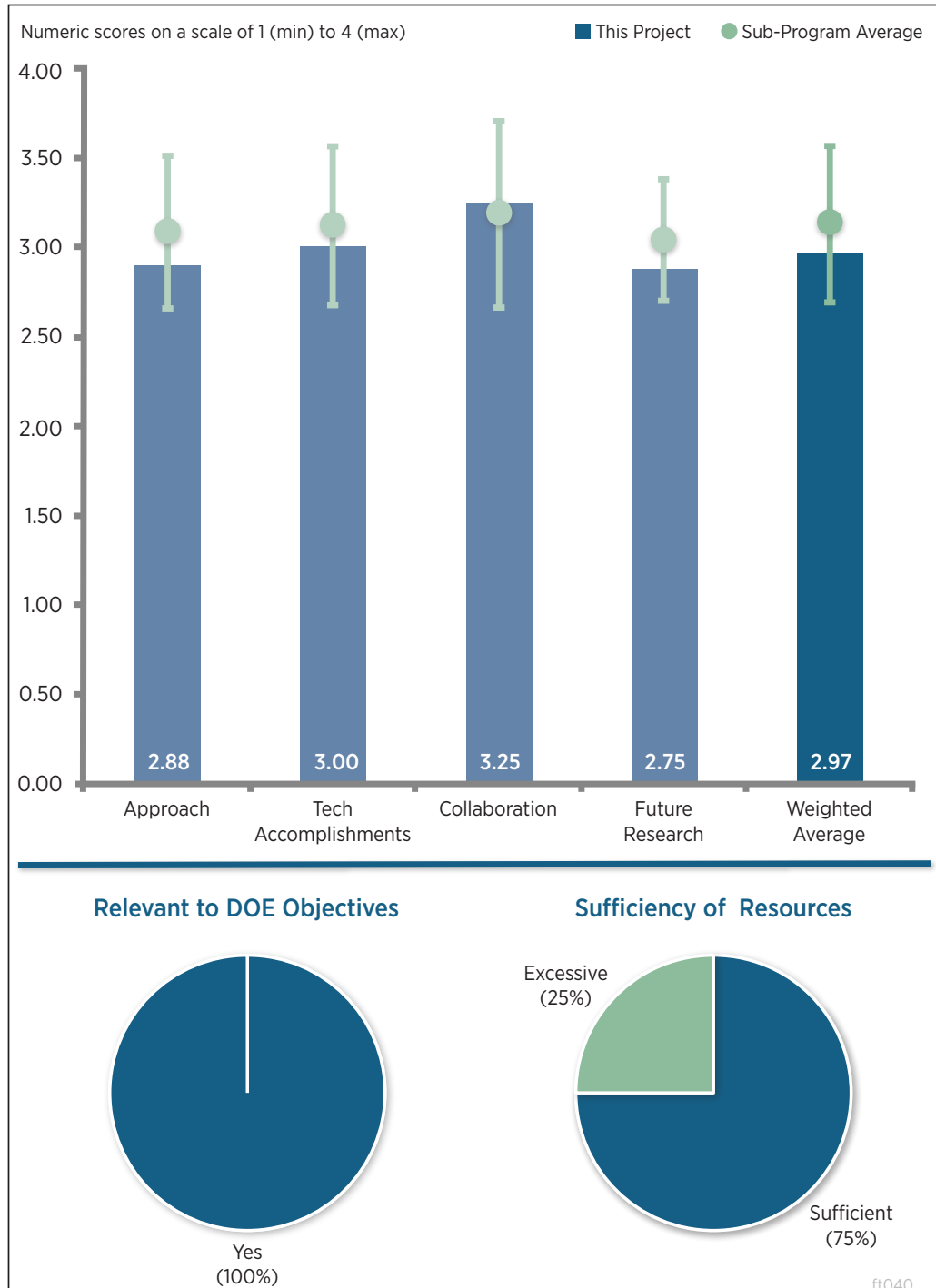

Figure 5-10 - Co-Optimization of Fuels and Engines (CoOptima) -- Simulation Toolkit Team: Matt McNenly (Lawrence Livermore National Laboratory) - Fuel and Lubricant Technologies 


\section{Reviewer 4:}

The reviewer said that the following Co-Optima projects have been reviewed as one and provided one set of comments for all four presentations: FT037, Co-Optima Overview (John Farrell, et al); FT038, Co-Optima Fuel Property and Advanced Engine Development Team, Part 1-Fuel Properties and Chemical Kinetics (Robert McCormick and Jim Szybist, et. al.) and Part 2-Thrust I engine projects (Jim Szybist, et. al.); ft039, Co-Optima of Fuels and Engines and Advanced Engine Development Team, Part 1-Thrust II engine projects (Paul Miles and Matt Ratcliff, et. al.); and ft040, Co-Optima Simulation Toolkit Team (Matt McNenly and Sibendu Som, et. al.). The reviewer provided 11 Thrust I comments that follow below.

Beginning the first Thrust I group of comments, the reviewer remarked that Co-Optima and Thrust I should be sharply focused on the overall Co-Optima goal vision of "Better fuels and better vehicles sooner." The reviewer noted that a lot of effort has gone into collaborating and coordinating between the various national laboratories and that these efforts and results are commended. The reviewer pointed out that collaboration and coordination is obviously needed and beneficial. For example, bringing to bear all the simulation work going on at the national laboratories on fuel property and engine response work, this work is bound to have high impact even if later than sooner. The reviewer cautioned that, with all the emphasis on collaboration between the various national laboratories and the coordination of the various teams with their diverse skills and tasks, it is easy for the program to become narrowly focused and lose sight of the forest for the trees. The reviewer also noted that the Co-Optima program has quickly become bureaucratic, with sub-teams providing reports to other teams within itself and to DOE. The reviewer said that while the project is admittedly a non-trivial challenge, in order to gain the trust of its stakeholders as being a legitimate effort to bring about significant GHG reduction, Co-Optima should continuously strive to be seamless across DOE, VTO, BETO, and the individual national laboratories and to keep the focus on the customer, vision, and the stakeholders.

For the second Thrust I group of comments, the reviewer remarked that Co-Optima has correctly concluded that the major barrier and challenge to realizing better fuels and better vehicles sooner in the marketplace is related to the large number of stakeholders with competing value propositions. The reviewer also said that because this is the major barrier, the full strength and weight of the Co-Optima program should be brought to bear squarely on removing this major barrier and not simply end up majoring on the minors.

Initiating the third Thrust I group of comments, this reviewer asked what was driving the 18-month decision point for Thrust I and noted that it gives the perception of Co-Optima being eager to hastily disengage from Thrust I issues and challenges and desiring to move on to Thrust II. The reviewer said that the one thing this implies, perhaps inadvertently, is that Thrust II engines, which are yet to be identified, and Thrust II fuels, which are also yet to be identified, will be more impactful in reaching Co-Optima goals than Thrust I engines and fuels. The reviewer questioned, if given the well-acknowledged risks associated with Thrust II engine concepts, whether there is overwhelming evidence that the only barrier remaining for Thrust II engines is the lack of a co-optimized Thrust II fuel. On the other hand, Thrust I engines and fuels are largely already identified by overwhelming industry and OEM practice and literature evidence, both recent and historical. Therefore, it seems prudent and a more reasonable strategy for Co-Optima to first tackle co-optimizing Thrust I engines and fuels, focusing sharply on the major barrier for Thrust I, which this reviewer referenced in the second Thrust I group of comments.

Starting the fourth Thrust I group of comments, the reviewer recommended that Co-Optima should refrain from proposing timelines for commercialization targets because Co-Optima can only provide research information, motivation, and incentive for commercialization, and perhaps play the very valuable role of bringing all stakeholders to the table. Those timelines can probably only be set by the stakeholders themselves because stakeholders typically have to follow free enterprise principles for commercial success.

For the fifth Thrust I group of comments, this reviewer remarked that Co-Optima should include a R\&D milestone titled "Can Thrust II fuel be the same as Thrust I fuel?" Having the same fuel for both Thrust I downsized boosted SI engines as well as Thrust II ACI engines has obvious benefits. The reviewer referenced the second Thrust I group of comments, and then remarked that the major barrier for any new fuel, Thrust I or Thrust II, has already been acknowledged to be the large number of stakeholders with competing value propositions. The reviewer asked if there is an opportunity to bust this major roadblock once, why choose a path that would need this roadblock 
busted twice. Having Thrust I and Thrust II fuels be the same is an incredible opportunity to further the main goal of Co-Optima vision of "Better fuels and better vehicles sooner." The reviewer also noted a related sub-milestone comment under Thrust II.

Opening the sixth Thrust I group of comments, the reviewer remarked that the Co-Optima program should verify that high RON and high sensitivity fuels do indeed substantially increase engine and vehicle fuel efficiency. The reviewer commented that this should be done quickly and with minimal research, as this ground has been plowed numerous times by many studies, and the answer is generally well-known and accepted.

Beginning the seventh Thrust I group of comments, this person stated that the Co-Optima program should focus major resources and effort on identifying promising low-GHG fuel compositions and blendstocks that are sustainable, affordable, scalable, and with attractive infrastructure and retail attributes. The reviewer said that the understanding is that Co-Optima has already started the life-cycle and techno-economic analysis on the 20 Thrust I fuel blendstocks.

Introducing the eighth Thrust I group of comments, the reviewer said that it is anticipated, that of the six teams that Co-Optima has put in place, the following hold the key to overcoming the main barrier already discussed: the LGGF team, which is focused on low-GHG blendstock properties and pathway attributes; the ASSERT team, which is focused on environmental impacts, cost, scalability, and feed logistics; and the MT team, which is focused on infrastructure and legacy fleet compatibility. The reviewer is of the opinion that these three teams will need to be primarily engaged in assisting stakeholders going forward. The reviewer also warned that, if Co-Optima declares Thrust I complete and disengages at FY 2019, it may fall short of its goal and will have at most a flash-in-the-pan effect.

Starting the ninth Thrust I group of comments, this reviewer said that the Thrust I engine projects currently being carried out on the 1.6-L Ford Ecoboost engine at ORNL and the 2.0-LTG GM engine at NREL are scoped reasonably well. The reviewer remarked that several appropriate changes have been made to the base engines to make it more relevant for evaluating high octane fuel performance. The reviewer also noted that appropriate changes in the engine CR are planned, along with an appropriate selection of relevant fuels. The reviewer also remarked that appropriate issues like LSPI are being studied. The reviewer also commented that the project has the potential of informing the community about the tradeoffs that need to be dealt with when designing downsized boosted engines with high octane fuel to realize high fuel efficiency.

For the $10^{\text {th }}$ Thrust I group of comments, the reviewer opined that the single cylinder head engine choice at SNL may not be representative of the state-of-the-art Thrust I engine that industry currently has and continues to evolve. The referenced engine at SNL has good optical access and also has port and other information needed for CFD simulation provided by an OEM. However, the typical downsized, boosted SI engine today utilizes high-tumble ports, special combustion chamber and piston topologies, and Miller cycle valve strategies, of which none are present in that cylinder head. The reviewer explained that work on knock-limited performance on this cylinder head will fall way short of understanding real low- and high-speed WOT knock-limited behavior.

Presenting the 11th Thrust I group of comments, this person stated that engine downsizing causes the load-factor of an engine to increase. Therefore, the current work on the effect of laminar flame speed increasing dilution tolerance at light loads should be extended to mid-loads also where knock typically compromises the optimum location of CA50.

The reviewer provided five Thrust II comments that follow below.

Commencing the first Thrust II group of comments, this person remarked that verifying whether Thrust I fuel and Thrust II fuel can be the same should be the first, and foremost, Thrust II goal. The reviewer noted that in this regard, the use of an OI as a means to evaluate fuel properties simultaneously suitable for both Thrust I and Thrust II engines is encouraged.

For the second Thrust II comment, the reviewer asserted that Co-Optima should pursue the path of discovering a new Thrust II fuel and by implication, a new Thrust II engine, only after it has been convincingly proved that these 
two fuels cannot be the same.

Presenting the third Thrust II group of comments, this reviewer commented that the literature on Thrust II engine concepts suggests more than a handful of recipes for ACI combustion. The reviewer inquired about what would be the anticipated Co-Optima fuel requirements for each recipe. The reviewer provided that choices could be as follows: exactly the same; slightly different; or significantly different. The reviewer noted if the answer turns out to be "exactly the same," then the path forward is simplified. If it also stands that Thrust II fuel equals Thrust I fuel, then the path forward is incredibly faster and beneficial for all stakeholders, as previously noted.

Starting the fourth Thrust II group of comments while also referencing the third Thrust II group of comments, the reviewer questioned whether the path forward would be fraught with huge challenges if the answer turns out to be "significantly different," or even "slightly different." The reviewer expressed interest in knowing who decides the winning Thrust II engine-plus-fuel combination. This person further inquired whether Co-Optima could really be the one who picks the winner, or if each stakeholder would have a differing opinion on the winning combination. The reviewer also asked about the role of free market enterprise for selecting the winning combination.

Concluding with the fifth Thrust II group of comments and referencing the fifth Thrust I group of comments, this reviewer remarked that Co-Optima could include a sub-milestone under another milestone. Recognizing that in the context of the current discussion, perhaps the suggestion that Thrust II fuel and Thrust I fuel can be the same may hold true only for LD applications because Thrust I engines largely imply SI, gasoline, LD engines. The reviewer offered that, on the other hand, Thrust II fuel can be targeted to any or all applications including LD, MD, or HD. For example, if it turns out that new Thrust II fuels can be profitably optimized with Thrust II engine recipes for MD or HD applications, perhaps then a case could be made that Thrust II fuel should be different than Thrust I fuel for these applications.

\section{Question 2: Technical accomplishments and progress toward overall project and DOE goals-the degree to which progress has been made, measured against performance indicators and demonstrated progress towards DOE goals.}

\section{Reviewer 1:}

The reviewer said that project team has made a lot of progress for only starting the project a short while ago. The reviewer remarked that each of the tasks and subtasks have identified several accomplishments that will provide the necessary tools to help meet the projects milestone and overcome the barriers. The reviewer noted that the milestones identified in this project are on schedule to be completed, and said because there are so many projects in the simulation toolkit team, it may be necessary to have each subtask reviewed independently during the AMR.

\section{Reviewer 2:}

The reviewer noted lots of good progress. However, researchers need to ensure that the needs of boosted, highoutput engines that will be the main part of the market are being addressed. The reviewer said that there are a lot of tools and it seems like there is some overlap that needs to be controlled.

\section{Reviewer 3:}

The reviewer stated that the accomplishments and progress in the presentation appeared to be largely a data dump, with only cursory references to the significance of the relationships shown and almost no explanation given of any relationships between them. The reviewer concluded that, therefore, it is unclear to what extent the enormous computing power utilized has been needed, effectively utilized, or what progress has actually been made.

\section{Question 3: Collaboration and coordination with other institutions.}

\section{Reviewer 1:}

The reviewer commented that this was an amazing job of focusing research into one organized program.

\section{Reviewer 2:}

The reviewer remarked that the overall team assembled of national laboratories and an external advisory board have brought together a very high caliber of expertise that will be able to implement the program to meet the 
required milestones and eliminate barriers. The reviewer's only concern was that the team is so large and so many tasks are being worked on, there may be too much time spent on coordination and outside meetings that may take away from the research.

\section{Reviewer 3:}

The reviewer said that the project apparently involves collaboration and cooperation between six national laboratories. The reviewer said some of the slides showed data that identify the source as one or more of the specific lab partners, but most do not. In reference to the data displayed in the presentation, the reviewer said that some show testing equipment identified as at institutions that are not project partners. The reviewer said that thus, overall, the presentation is unclear as to how much actual collaboration and coordination is occurring between the partners beyond what the project team stated in an answer to a question that there is a phone call between the principal investigators (PIs) every month.

\section{Question 4: Proposed future research-the degree to which the project has effectively planned its future work in a logical manner by incorporating appropriate decision points, considering barriers to the realization of the proposed technology and, when sensible, mitigating risk by providing alternate development pathways.}

\section{Reviewer 1:}

The reviewer remarked that the following future work planned are all appropriate and that these tasks should continue helping to overcome the barriers: Task G.1.2, Support Small Volume Fuel Testing; Task G.2.1, Extreme Mechanism Reduction for Direct Injection Spark Ignition (DISI) (which will include a focus on Thrust 1 ethanol gasoline blends and bio-diesel); and Task G-3, Blendstock-to-Efficiency Application (single and multi-cylinder engine modeling).

\section{Reviewer 2:}

The reviewer commented that the project team needs engine data for high brake mean effective pressure (BMEP), boosted, and Miller Cycle operation. The reviewer said that the bulk seems aimed at LTC, homogeneous charge compression ignition (HCCI), and reactivity controlled compression ignition (RCCI) and expressed doubt that those will ever be large volume products.

\section{Reviewer 3:}

The reviewer said that most of the future research slides are stated in general terms which appear to project very useful models linking efficiency to fuel properties and engine parameters for both gasoline spark ignition (GSI) and gasoline compression ignition (GCI) engines, based on fuel maps as well as external data on vehicle miles traveled (VMT), market penetration, etc. It was not clear how any (if at all), let alone all, of the prior work is to be integrated into this model. Moreover, a question was raised as to the appropriateness of using the optical engine at SNL for the initial part of this work, and the reviewer said that the PIs did not provide a particularly good answer to that question.

\section{Question 5: Does this project support the overall DOE objectives of petroleum displacement? Why or why not?}

\section{Reviewer 1:}

The reviewer said that the work of the Simulation Toolkit Team is very relevant to the DOE goal of petroleum displacement. The reviewer remarked that by developing models to show the impact of new fuels on engine performance, the project team will provide the necessary information to aid in developing optimum fuels to be used in advanced engines to reduce fuel consumption.

\section{Reviewer 2:}

The reviewer noted a large impact on future fuel efficiency.

\section{Reviewer 3:}

The reviewer remarked that the project would support the objectives of petroleum displacement if it achieves its objectives of better understanding the relationships between fuel properties, engine design, and efficiency. The 
reviewer commented that the presentation, however, did not establish the likelihood of those objectives being realized.

\section{Question 6: Resources: How sufficient are the resources for the project to achieve the stated milestones in a timely fashion?}

\section{Reviewer 1:}

The reviewer said that the funding for this Co-Optima Simulation Toolkit Team is adequate to achieve the milestones in the project.

\section{Reviewer 2:}

The reviewer said that it seems to be a good use for skilled personnel and big computers.

\section{Reviewer 3:}

The reviewer said that the presentation did not provide any basis for this reviewer to make judgments as to the sufficiency in terms of achieving the objectives in a well-planned and organized progression. The reviewer remarked that the overall impression given is that lots of activities are being undertaken and data generated without a logical progression and decision tree. 


\section{Utilizing Alternative Fuel Ignition Properties to Improve Spark-Ignited and Compression-Ignited Engine Efficiency: Margaret Wooldridge (University of Michigan) - ft042}

\section{Presenter}

Andre Boehman, University of Michigan

\section{Reviewer Sample Size}

A total of five reviewers evaluated this project.

\section{Question 1: Approach to performing the work-the degree to which technical barriers are addressed, the project is well-designed, feasible, and integrated with other efforts.}

\section{Reviewer 1:}

The reviewer remarked that this project is very well planned and executed. The reviewer stated that aside from not meeting the $\mathrm{CI}$ target and requiring a no-cost time extension, that the project team were meeting all objectives of the project.

\section{Reviewer 2:}

The reviewer noted that overall, the various research tasks individually seem to be interesting and useful. However, together as a program things seem a bit unfocused. The reviewer said that it was not clear how exactly the project plans to move towards the goal of achieving $40 \%$ brake thermal efficiency (BTE).

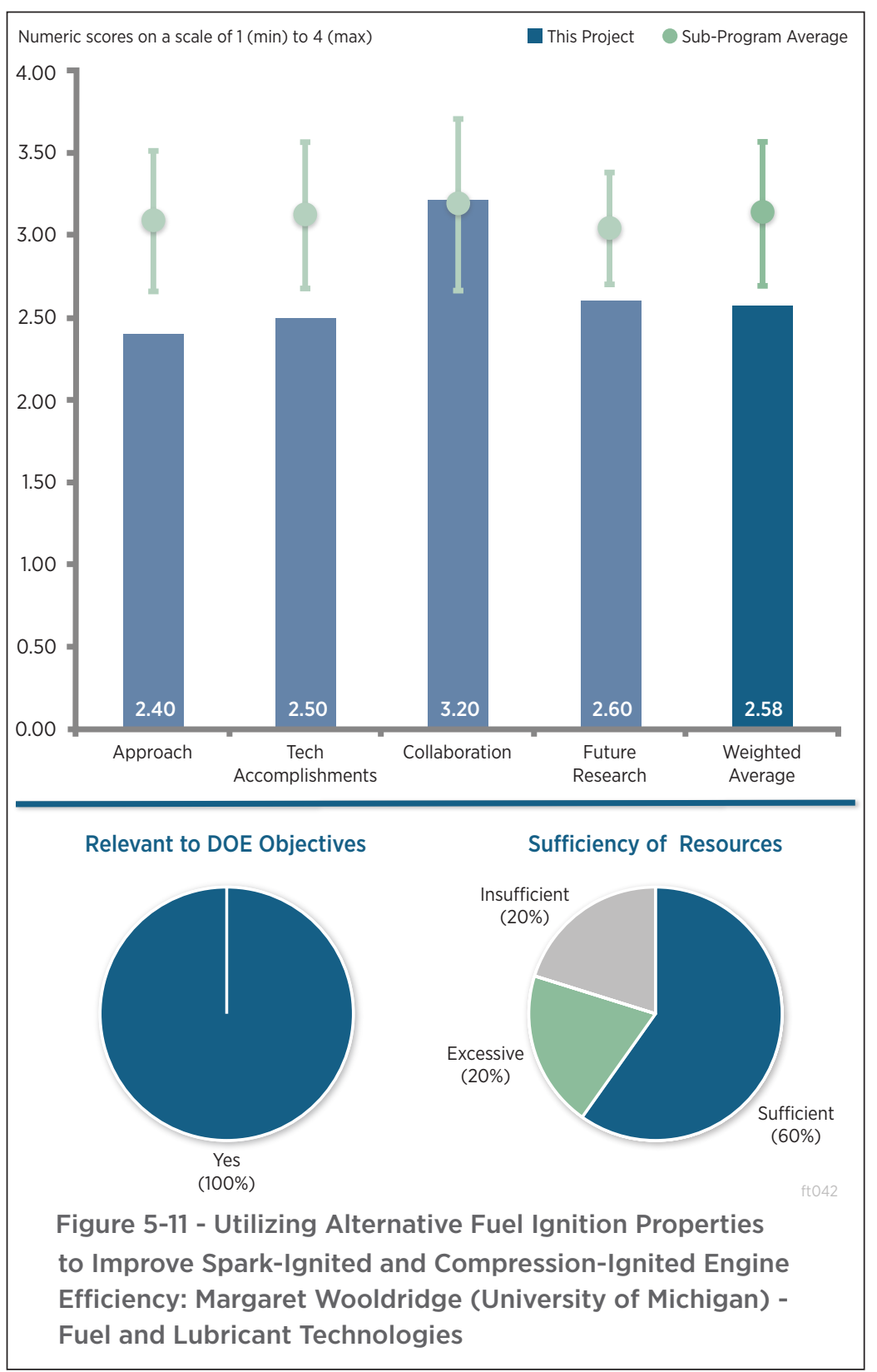

The reviewer stated that the project team has, as presenter put it, lots of knobs to turn to improve efficiency, but that there does not appear to be a systematic approach. The reviewer commented that the project team wants to use rapid compression machine (RCM), ignition quality tester (IQT), and CI demonstration studies to learn about autoignition and single-cylinder engines to learn about the various sensitivities to the knobs that the project team can turn. The reviewer stated that this was vague and does not map out a clear plan. Furthermore, by the project team's own admission it is not assured that the project team will be able to take advantage of what they learn in the simpler platforms including RCM, IQT, and single-cylinder engine when the project team goes to the multicylinder engine. The reviewer stated that the project team indicated that they very well might not be able to achieve the commanded conditions.

\section{Reviewer 3:}

The reviewer remarked that the approach including progression from simulations, ignition quality tester, RCM, single cylinder engine, multicylinder engine, and others was a logical way of examining the potential for increased FE with high-level ethanol blends in SI engines by varying engine conditions such as intake pressure, EGR 
dilution, spark timing, and others, and as well as benefitting from charge cooling. The reviewer said that the project appears on track to meet objectives. The reviewer commented that the investigation of CI dimethyl-ether (DME) and propane blends applicability was less clear and the objectives determined were not attainable. The reviewer remarked that this investigation appears to have been prompted largely by another study that the project team now has determined was flawed.

\section{Reviewer 4:}

The reviewer stated that the deliverables and scope of the project are not well defined. The reviewer remarked that one of the key deliverables of the project has been defined as achieving 40\% BTE on an SI engine. The reviewer said that the presentation, as well as the slide deck, made available for review did not elaborate on any qualifying criteria for the $40 \%$ BTE. The reviewer said that for instance, if the goal of the project is to demonstrate a peak BTE of $40 \%$ on an SI engine, then that has already been demonstrated. The reviewer indicated that prior DOE funded projects have demonstrated peak BTEs well in excess of $40 \%$. The reviewer also pointed out that current Atkinson cycle production engines from Toyota and Hyundai have achieved a peak BTE of $40 \%$. The reviewer noted that it was also not clear if the peak 40\% BTE target is to be met while retaining the power and torque output of the engine. The reviewer said that if the efficiency needs to be increased while retaining the specific output of the engine, then the project may need to include development activities such as turbocharger matching, EGR system modifications, and ignition system upgrades.

The reviewer said that the reference or baseline condition was not well defined. The reviewer claimed that the peak BTE of the stock Daimler M274 engine is in excess of 37\% and that thus, achieving a peak BTE of $40 \%$ is not a very difficult task. For instance, increasing the CR from a stock value of one equal to 9.8 to one equal to 12 might be sufficient to achieve an increase of $3 \%$ in part load BTE. The reviewer concluded that thus, in the absence of the baseline or reference condition being well defined, achieving $40 \%$ BTE was not a very challenging target.

The reviewer said that extensive data already exists in the literature on the impact of ethanol and exhaust gas recirculation (EGR) on combustion separately. The reviewer claimed that data also exist on the combined impact of ethanol and EGR. The reviewer noted that one recent example of a study investigating ethanol and EGR effects is SAE 2016-01-0715. The reviewer suggested that the project leads may want to clearly define the new technical information that this study expects to contribute to the existing body of knowledge.

The reviewer remarked that Task 1.2 refers to the use of simulations for evaluating the impact on knock and flame limits of alternate fuels and combustion strategies on engine efficiency. The reviewer expressed that it was not clear if that task is referring to one-dimensional or three-dimensional simulations, or both. The reviewer cautioned that one-dimensional analysis alone was not sufficient to assess the impact on knock and flame limits of fuel properties such as octane number and heat of vaporization. The reviewer said that also, as alluded by Task 1.2, one-dimensional analysis is probably not the most effective and accurate tool for assessing the impact of fuel spray on combustion.

\section{Reviewer 5:}

The reviewer remarked that it was disappointing to see DOE fund such poorly scoped and funded projects. The reviewer stated that the project seemed to be scoped poorly to begin with. The reviewer remarked that the goal of $40 \%$ BTE for SI and 50\% for CI was a worthy goal, but that the resources and the pathway to achieve it seems to fall way short. The reviewer commented that the original time of one year as well as the funding are way below what would be needed for a project of this magnitude. The reviewer said no wonder a no-cost extension has been requested and no wonder the diesel portion of the goal has been abandoned. The reviewer said that fundamentally, operating an SI engine with ethanol and ethanol-gasoline blends, which are higher in RON, and increasing the CR to gain efficiency is a well-known and studied strategy. The reviewer said that if that was all this project was going to demonstrate for an SI engine, then it is a waste of money. The reviewer wanted to know what new efficiencyimproving proposals were going to be studied in this project.

\section{Question 2: Technical accomplishments and progress toward overall project and DOE goals-the degree to which progress has been made, measured against performance indicators and demonstrated progress towards DOE goals.}




\section{Reviewer 1:}

The reviewer remarked that one technical objective was not met, but the presenter promised a continued effort as part of another program.

\section{Reviewer 2:}

The reviewer said that the overall progress and accomplishments are generally on target but that the score is slightly downgraded due to lack of success on CI engine work.

\section{Reviewer 3:}

The reviewer referenced prior comments, and said that useful data were collected on SI combustion with gasoline and ethanol fuels at various pressures and EGR rates. The reviewer remarked that the test engine calibration and modelling was completed. The reviewer also stated that data on CI was useful mainly in dispelling interest in pursuing indicated strategies.

\section{Reviewer 4:}

The reviewer said that considering the ignition delay testing, development of a GT-Power model, and the testing on the Ford Motors single cylinder engine, that it appears that reasonable amount of work has been performed as part of the project. However, it is not clear how the work performed thus far has contributed to the existing body of knowledge on combustion of ethanol-gasoline blends. The reviewer remarked that with discontinuation of the dualfuel CI work stream, this project is essentially an investigation on combustion of gasoline-ethanol blends, which is a topic that has been extensively investigated. The reviewer said that the project leads may want to identify and define deliverables that are expected to contribute new information and in the process help achieve DOE's goals.

\section{Reviewer 5:}

The reviewer said that the project was made up of what seems like loosely connected sub-projects, with no clear indication of how each sub-project contributes to reaching the main goal of $40 \%$ BTE. The reviewer provided as an example that single-cylinder engine studies have been conducted and that these studies show an indicated thermal efficiency of about $38 \%$ with E100. The reviewer would like to know what the plan was to get to $40 \%$ thermal efficiency on a brake basis on the multi-cylinder. The reviewer said that GT-Power simulations can only offer analytical insight. Furthermore, the single cylinder engine is a Ford Fox engine, but that the multi-cylinder is a Daimler M274 2.0-L engine. The reviewer said that the single cylinder engine probably has a multi-hole fuel spray while the multi-cylinder engine has a piezo spray. The reviewer asked what the plan was to make sure that the single cylinder engine learnings translate to the multi-cylinder. The reviewer questioned what the hope was that E100 will be the fuel of the future.

The reviewer also questioned why a PM emission study was included in this project. The reviewer asked if it has that significant a bearing on demonstrating the BTE goals of the project. The reviewer said that more details would be appreciated, otherwise, it is left to the interpretation of the reviewer.

The reviewer asked how conducting Engine Combustion Network (ECN) Spray G experiments contributes to demonstrating the BTE goals of the project. The reviewer also wanted information on how these measurements were going to be related to the different sprays in the single- and multi-cylinder engines. The reviewer said that more details would be appreciated, otherwise, it is left to the interpretation of the reviewer.

The reviewer remarked that the go/no-go decision on the diesel part of the project was commended. The reviewer offered that it was a wise decision to not proceed and waste money, time, and resources in trying to execute a poorly scoped and unplanned project.

\section{Question 3: Collaboration and coordination with other institutions.}

\section{Reviewer 1:}

The reviewer remarked that the involvement of a major OEM and a Tier 1 supplier, along with research institutions such as NREL and SNL, suggests a reasonable level of collaboration between project participants and collaborators. The reviewer said that based on the presentation, it was clear that as the project partner Bosch was providing a lot of hardware support. The reviewer said that if Bosch's role extends beyond hardware and monetary support, that the project leads may want to comment on that as well. 


\section{Reviewer 2:}

The reviewer said that there appears to be extensive and fruitful collaboration ongoing between the two national laboratory partners, University of Michigan, and the three industry partners, and that these collaborations involved both sharing of responsibilities and equipment and consultation on implications of results. The reviewer said that the results of all partners should ultimately be combined to show total efficiency gains possible from optimization of engines on the selected ethanol blends.

\section{Reviewer 3:}

The reviewer remarked that the project team suggested that they were collaborating and coordinating with several partners, but some of these relationships are unclear or seemingly irrelevant. The reviewer provided an example that the project team claimed that the SNL ECN was a collaborator, but the project team did not make a credible case that the Spray G imaging work is relevant to this program. The reviewer said that in fact, the project team barely paid lip service to that work. The reviewer offered that a second example was Horiba and said that it appears that the extent of that collaboration was a discount that offered to the project team on their equipment. The reviewer would not agree that this constitutes a collaboration.

\section{Reviewer 4:}

The reviewer said that the collaboration with the various organizations listed is fine and that they are mainly for the purpose of procuring hardware or information. The reviewer said that Bosch is playing a critical role in providing and possibly running the Daimler multi-cylinder engine tests. The reviewer remarked that the bulk of the responsibility of successfully demonstrating the goals of this project are upon the shoulders of the University of Michigan. The reviewer expressed the concern that the project team has bitten off more than it can chew.

\section{Question 4: Proposed future research-the degree to which the project has effectively planned its future work in a logical manner by incorporating appropriate decision points, considering barriers to the realization of the proposed technology and, when sensible, mitigating risk by providing alternate development pathways.}

\section{Reviewer 1:}

The reviewer remarked that the project has access to excellent facilities and investigative tools. However, as described in the presentation, the project scope is perhaps too wide, which may be driving work streams that are not necessarily the most effective use of the facilities. The reviewer stated that the project leads may want to identify research activities that are expected to contribute new information and to focus on those instead of trying to perform too many tasks. For instance, the impact of gasoline-ethanol blends on fuel spray and soot formation could be a work stream worthy of an in-depth study.

\section{Reviewer 2:}

The reviewer said that the future research identified was the extension of the work as planned into multi-cylinder engine testing, as well as other testing being extended with different CRs and spray strategies.

\section{Reviewer 3:}

The reviewer said that by the presenter's own admission, the single-cylinder engine studies (and spray and ignition studies) may very well point the project team in directions that it cannot necessarily go with the multi-cylinder engine. The reviewer remarked that the best approach would be to either focus on aspects that that the project team knows it can control/change in the multi-cylinder engine when doing the project's fundamental studies (but that work appears to be mostly complete), or to ensure that the project's multi-cylinder engine system can accomplish the conditions that the project team anticipates needing to achieve.

\section{Reviewer 4:}

The reviewer remarked that except for the time delay, this program will finally achieve all targets, within or without of this project.

\section{Reviewer 5:}

The reviewer said that the task list for future work was exhaustive and it probably will not be completed in the extended time requested. The reviewer commented that very little useful information or knowledge can be salvaged from the work done so far or from the future work. 


\section{Question 5: Does this project support the overall DOE objectives of petroleum displacement? Why or why not?}

\section{Reviewer 1:}

The reviewer said that clearly, achievement of significantly higher BTE would support the primary objective of petroleum displacement.

\section{Reviewer 2:}

The reviewer remarked that the project has access to appropriate tools and facilities that can be used for making valuable contributions towards meeting DOE's objectives. The reviewer stated that however, the scope of the project and deliverables need to be defined such that they are better aligned with DOE's goals.

\section{Reviewer 3:}

The reviewer remarked that this project could ultimately help identify optimal ways of using ethanol supply in U.S. gasoline to maximize FE and equalize or improve miles per gallon (MPG) in contrast to gasoline, increasing overall FE and allowing for greater usage of ethanol than currently, as constrained by mileage penalty, cost per mile, frequency of refueling, etc.

\section{Question 6: Resources: How sufficient are the resources for the project to achieve the stated milestones in a timely fashion?}

\section{Reviewer 1:}

The reviewer said that, as usual, the University of Michigan was well suited to complete the task, including both people and equipment.

\section{Reviewer 2:}

The reviewer said that the resources and time provided have been grossly underestimated.

\section{Reviewer 3:}

The reviewer said that resources appear sufficient based on work being performed. The reviewer noted that the budget for FY 2017 was reduced from previous years, apparently as an adjustment for discontinuation of the CI work on DME and propane.

\section{Reviewer 4:}

The reviewer said that the University of Michigan has significant resources and that therefore this is not expected to be an issue.

\section{Reviewer 5:}

The reviewer observed that the current budget is excessive in view of the existing work streams. However, this ranking may be changed to "Sufficient" if the project scope and deliverables are aligned better with DOE's goals. 


\section{E85/Diesel Premixed Compression Ignition: Lyle Kocher (Cummins) - ft043}

\section{Presenter}

Lyle Kocher, Cummins

\section{Reviewer Sample Size}

A total of seven reviewers evaluated this project.

\section{Question 1: Approach to performing the work-the degree to which technical barriers are addressed, the project is well-designed, feasible, and integrated with other efforts.}

\section{Reviewer 1:}

The reviewer remarked that this was an outstanding example of a well thought out project with aggressive objective, correct technical content, and the right resources to make it happen.

\section{Reviewer 2:}

The reviewer remarked that the project was scoped well and said that this may be the first RCCI attempt by an industry OEM. The reviewer further noted that because the team uses E85 as one of the fuels, it has potential for significant petroleum reduction.

\section{Reviewer 3:}

The reviewer remarked that the approach to design a combustion and engine system to operate over the entire speed and torque map, to design the integration of a secondary fuel system, and the development of closed loop control during transient operation have been excellent. The reviewer commented that the final step of the approach will be to demonstrate the engine in a vehicle on a developed calibration. The reviewer said this final step was an excellent way to show that this technology will truly have an impact on petroleum reduction.

\section{Reviewer 4:}

The reviewer said that the project was well structured and the approach was very clear. The reviewer voiced that the project team was taking a very logical approach to addressing the project goals of reducing petroleum use by $50 \%$ while considering other emissions.

\section{Reviewer 5:}

The reviewer said that the approach was excellent and logical, as expected from a successful engine OEM. The reviewer commented that the team was properly considering hydrocarbon (HC) and PM emissions as a constraint

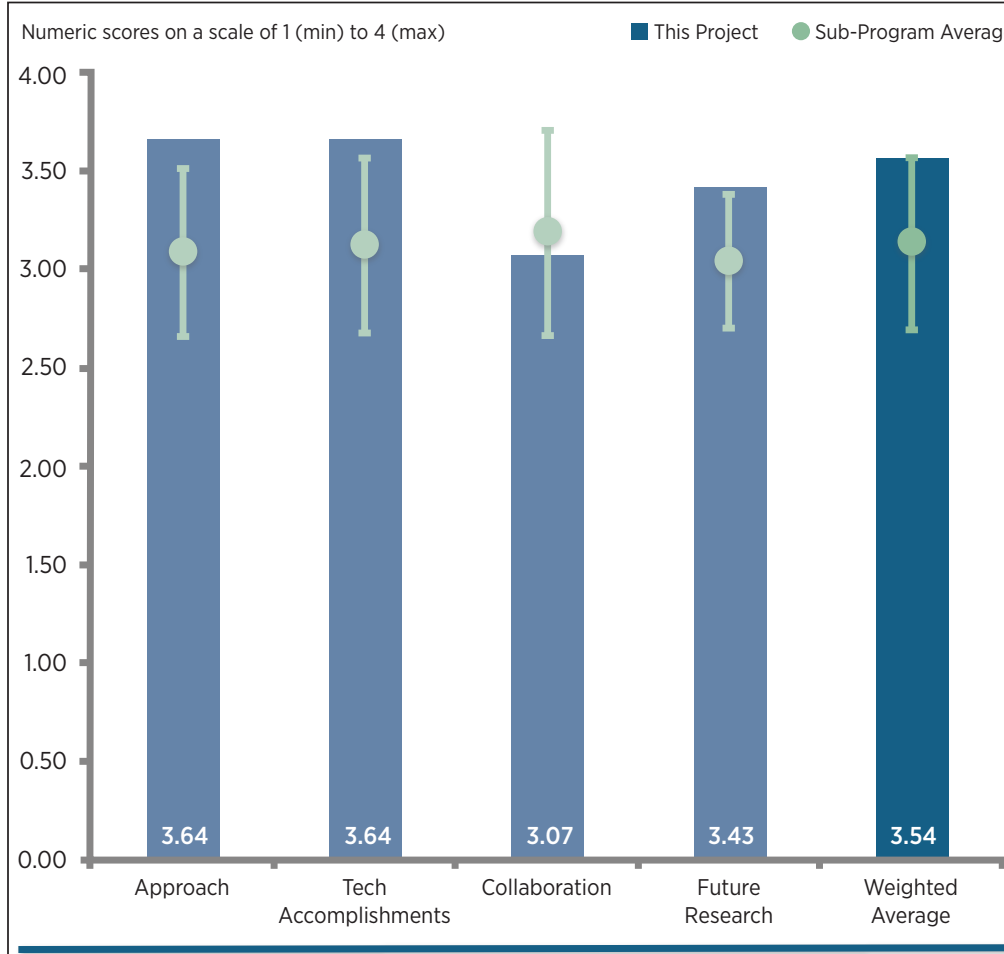

Relevant to DOE Objectives
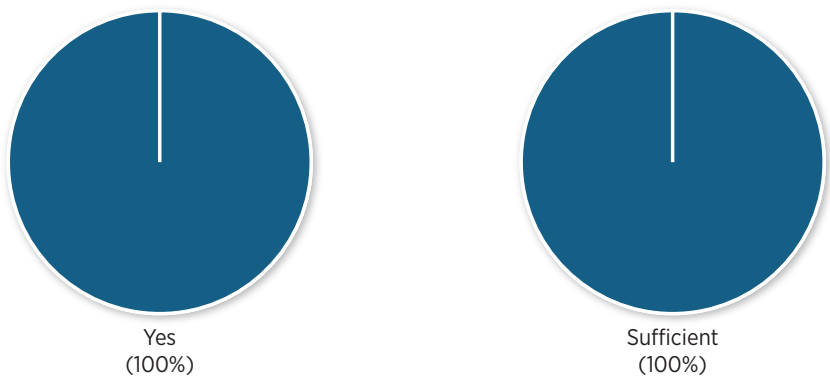

Figure 5-12 - E85/Diesel Premixed Compression Ignition: Lyle Kocher (Cummins) - Fuel and Lubricant Technologies 
which was an improvement over many R\&D projects that focus only on efficiency. The reviewer remarked that reducing $\mathrm{HC}$ with the low exhaust temps will be a problem that needs some aftertreatment consideration. The reviewer suggested that further work will be needed with varying ethanol levels, diesel cetane levels, and colder temperatures. The reviewer suggested that some demonstration that the system would still be viable with a glowplug to start or robust with the pressure feedback would be beneficial.

\section{Reviewer 6:}

The reviewer said that the project team has a great approach to the project. The reviewer commented that the only things missing were DI of E85 and a deeper dive into the variability of E85 between the summer and winter, which the reviewer noted were beyond the scope of this project.

\section{Question 2: Technical accomplishments and progress toward overall project and DOE goals-the degree to which progress has been made, measured against performance indicators and demonstrated progress towards DOE goals.}

\section{Reviewer 1:}

The reviewer praised that the project has demonstrated excellent progress according to plan. The reviewer said that results to date were clearly articulated and the remaining challenges appropriately quantified. The reviewer commented that additional plans to address the remaining challenges were clearly articulated.

\section{Reviewer 2:}

The reviewer said that the objectives seem to have been fully met, with the remaining work well in hand.

\section{Reviewer 3}

The reviewer said that the project appears to progressing nicely. The reviewer commented that the hardware design was quite clever and that it seems production ready.

\section{Reviewer 4:}

The reviewer said that the project's technical accomplishments have been excellent and have met all of the required milestones to date. The reviewer remarked that Cummins has successfully designed and demonstrated a duelfuel engine for a Class 8 heavy truck. The reviewer noted that Cummins has demonstrated over 50\% petroleum reduction over a 13 mode and FTP and have also shown engine-out nitrogen oxides $\left(\mathrm{NO}_{\mathrm{x}}\right)$ and $\mathrm{PM}$ reductions over its diesel counterpart.

\section{Reviewer 5:}

The reviewer remarked that the results for petroleum reduction have been reported, which are very good. The reviewer noted that it would have been informative to know how the absolute BTE with this two-fuel, E85 plus diesel, concept compared to the base engine operating on one fuel, diesel.

\section{Reviewer 6:}

The only area that this reviewer could find fault with was $\mathrm{HC}$ and carbon monoxide $(\mathrm{CO})$ emissions from the end gasses, which is a common problem with this approach.

\section{Question 3: Collaboration and coordination with other institutions.}

\section{Reviewer 1:}

The reviewer remarked that the project does not have collaborators or coordination outside of Cummins but because of the way the project was designed that it did not appear necessary to have outside collaboration. The reviewer said that there was collaborative work with Cummins Fuel Systems to provide an advanced direct injection fuel system.

The reviewer remarked that collaborations were mainly internal and that few other companies have such complete internal capabilities. The reviewer commented that because Cummins has the tools that this seems to be an appropriate level of collaboration. The reviewer said that much of this work was clearly informed by previous research at national laboratories and contractors. 


\section{Reviewer 2:}

The reviewer said that there were no external partners but that external partners are not necessary for this project. The reviewer remarked that Cummins has the resources to complete the project.

\section{Reviewer 3:}

The reviewer stated that this project was internal to Cummins and not a team with academia or national laboratory participation.

\section{Reviewer 4:}

The reviewer commented that this project relies primarily on internal collaboration within the organization. The reviewer said that while the project was well thought out and the progress is strong that perhaps deeper interactions with the labs, universities, and other performers beyond attendance at the program meetings could generate additional ideas to tackle remaining challenges.

\section{Question 4: Proposed future research-the degree to which the project has effectively planned its future work in a logical manner by incorporating appropriate decision points, considering barriers to the realization of the proposed technology and, when sensible, mitigating risk by providing alternate development pathways.}

\section{Reviewer 1:}

The reviewer remarked that the proposed future work of developing transient calibration and controls and also to build and test the vehicle, including emission validation testing, will complete the project and eliminate the barriers outlined in the project.

\section{Reviewer 2:}

The reviewer said the project path forward seems to systematically address the stated objectives. The reviewer commented that the project team has clearly thought through the project and carefully planned how to manage the hydrocarbon emissions challenge.

\section{Reviewer 3:}

The reviewer noted a strong plan.

\section{Reviewer 4:}

The reviewer said that the project team understood the deficiencies of this approach and suggested several areas and approaches, such as DI and low-pressure EGR, to overcome their problems.

\section{Reviewer 5:}

The reviewer remarked that the acknowledgement of the challenge and focus on $\mathrm{HC}$ emissions is good. The reviewer said that there could be more work on how such a fuel strategy could actually be implemented.

\section{Reviewer 6:}

Pending successful development of transient engine operation, HC emissions, and cold-start and warm-up calibration, the reviewer asked what some of the other barriers are that would prevent this concept from being commercialized. The reviewer asked what the issues are related to the use of E85 by Class 8 truck manufacturers.

\section{Question 5: Does this project support the overall DOE objectives of petroleum displacement? Why or why not?}

\section{Reviewer 1:}

The reviewer said that the project definitely supports the overall DOE objective of petroleum displacement, because the goal of the project was to meet 50\% petroleum reduction in an E85 diesel premixed CI engine.

\section{Reviewer 2:}

The reviewer said large petroleum reduction on a relevant product platform.

\section{Reviewer 3:}

The reviewer said that the project was explicitly focused on displacing petroleum fuel by $50 \%$ by using E85. 


\section{Reviewer 4:}

The reviewer said that there was a significant portion of petroleum displaced by E85.

\section{Reviewer 5:}

The reviewer said that the project was meeting the goal of 50\% petroleum reduction and noted that it was therefore relevant. The reviewer said that with current E85 and diesel fuel prices, there does not appear to be a business case for anyone to pursue this currently. The reviewer commented that future price swings could change the case.

Question 6: Resources: How sufficient are the resources for the project to achieve the stated milestones in a timely fashion?

\section{Reviewer 1:}

The reviewer said that the funding seems to be adequate to complete the project and meet the milestones.

\section{Reviewer 2:}

The reviewer commented that the project was adequately funded when considering the cost share from Cummins.

\section{Reviewer 3:}

The reviewer said the funding seems to be about right. 


\section{GEFORCE: Gasoline Engine and Fuels Offering Reduced Fuel Consumption and Emissions: Scott Sluder (Oak Ridge National Laboratory) - ft044}

\section{Presenter}

Scott Sluder, Oak Ridge National Laboratory.

\section{Reviewer Sample Size}

A total of five reviewers evaluated this project.

\section{Question 1: Approach to performing the work-the degree to which technical barriers are addressed, the project is well-designed, feasible, and integrated with other efforts.}

\section{Reviewer 1:}

The reviewer remarked that the project was scoped very well. The reviewer said that several appropriate changes have been made to the base engine to make it more relevant for evaluating high octane fuel performance. The reviewer commented that appropriate changes in the engine $\mathrm{CR}$ are planned, along with an appropriate selection of relevant fuels. The reviewer stated that the project has the potential of informing the community about the tradeoffs that need to be dealt with when designing engines for high octane fuel to realize high fuel efficiency.

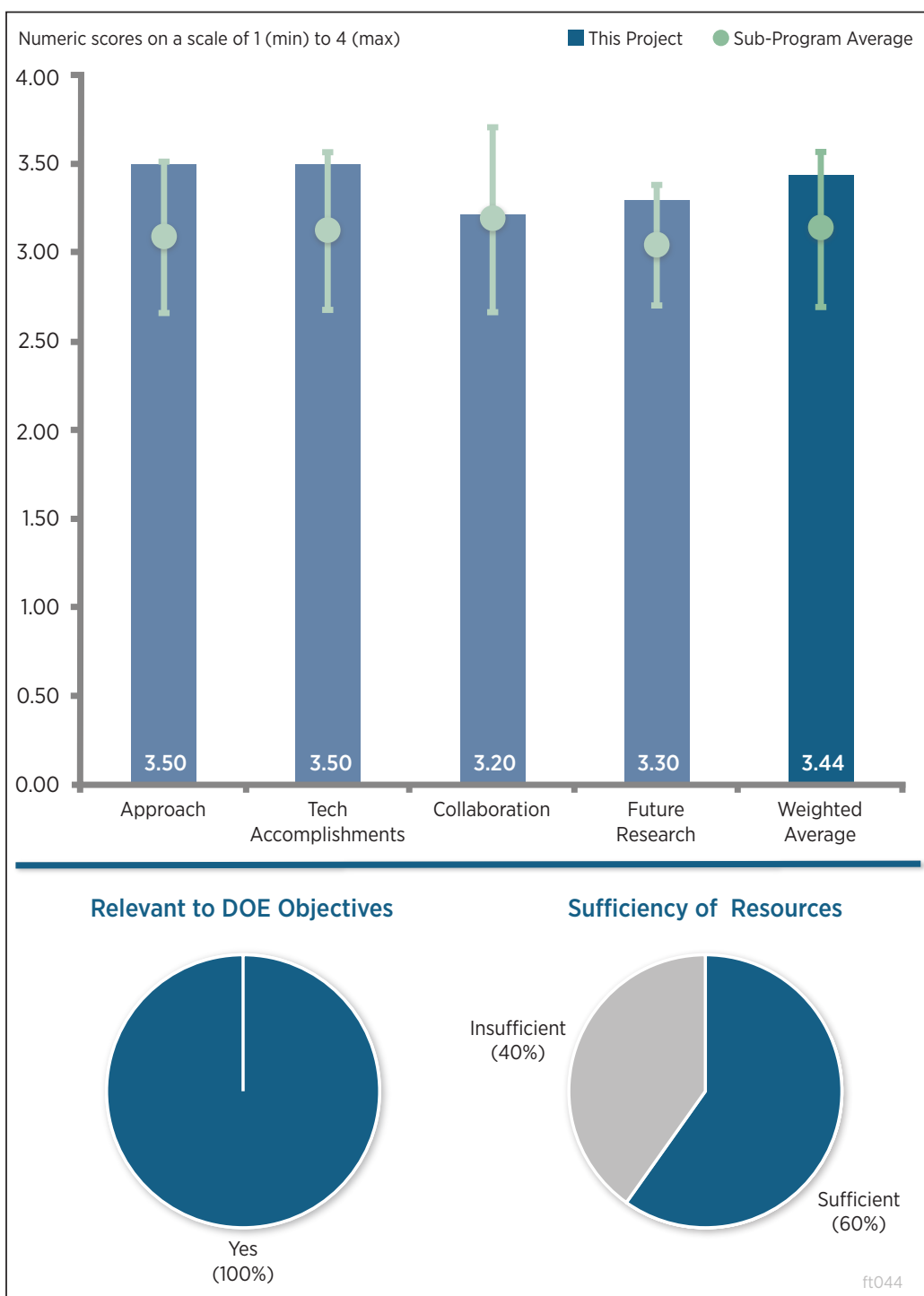

Figure 5-13 - GEFORCE: Gasoline Engine and Fuels Offering Reduced Fuel Consumption and Emissions: Scott Sluder (Oak Ridge National Laboratory) - Fuel and Lubricant Technologies

\section{Reviewer 2:}

The reviewer remarked that the approach was useful in anticipating engine designs for the 15 to 20 year horizon and in determining optimal fuel properties for efficiency and low emissions as used in such engines. The reviewer commented that various fuel mixtures in matrix of RON, ethanol, and final boiling point were to be tested at various operating conditions including, power densities and gear ratios initially, to find the optimum conditions for each fuel. The reviewer said that it provides the first step for future engine and fuel co-development.

\section{Reviewer 3:}

The reviewer remarked that this CRADA was well thought out. The reviewer noted that it was set up to systematically evaluate potential future gasoline in terms of its potential to increase the efficiency of gasoline engines.

\section{Reviewer 4:}

The reviewer said that the approach used to determine what the dyno coefficients would be for a representative midsized car was clever. The reviewer commented that running simulations for an actual vehicle would also be 
useful to compare the simulation results to the real world results. The reviewer said this would allow for additional vehicle testing if this project should receive additional funds.

The reviewer remarked that the project seemed to rely heavily on the experimental GM engine and cautioned that this limits the ability to test advanced technologies beyond those GM provides. The reviewer commented that it might have been better to start with a current state of the art engine so that the baseline data would be very reliable. The reviewer said that measuring the effect of the fuels on something like a 40\% BTE Prius engine, or Mazda Skyactive, would eliminate engine unknowns. The reviewer said that if a boosted engine was really necessary, perhaps that one of the Coordinating Research Council (CRC) members could support with an engine and controller.

\section{Reviewer 5:}

The reviewer remarked that overall the approach was sound and quite interesting, and that it seemed highly appropriate when considering the context of many of the discussions that were taking place at this Peer Review meeting. The reviewer said that the only complaint was that the approach was slightly vague, in that it seemed as though the project team was going to try a bunch of stuff and see what happens. The reviewer stated that there does not seem to be a clear hypothesis, other than the idea that you will find ways to improve engine efficiency. The reviewer noted that this was only a minor complaint considering how useful this work is likely to be.

\section{Question 2: Technical accomplishments and progress toward overall project and DOE goals-the degree to which progress has been made, measured against performance indicators and demonstrated progress towards DOE goals.}

\section{Reviewer 1:}

The reviewer said that progress was on track with program objectives.

\section{Reviewer 2:}

The reviewer remarked that very significant progress has been made in the area of procuring relevant engine hardware and fuel. In addition, the base engine has been calibrated. The reviewer noted that the OEM partner's ability to free up resources to support this project at ORNL was limiting further progress, but patience should be exercised as the payoff has the potential of having significant impact.

\section{Reviewer 3:}

The reviewer noted that the project team was still setting up to do the experiments. The reviewer stated that the progress appears to be on track with the program schedule. The reviewer said that an appropriately representative modern engine was being used, which is critically important.

\section{Reviewer 4:}

The reviewer said that the project was in the early stages and that legal agreements took longer than anticipated. The reviewer noted that despite this, an advanced research engine was near operational and a baseline engine was operational. The reviewer stated that other key data have been obtained as a basis for initiating this research project.

\section{Reviewer 5:}

The reviewer said that the lengthy contracting process seems to have delayed the project quite a bit. The reviewer said that hopefully this coming year will be productive. The reviewer remarked that the fuel matrix and fuel formulations are done so one major hurdle has been overcome.

\section{Question 3: Collaboration and coordination with other institutions.}

\section{Reviewer 1:}

The reviewer said that the collaboration with GM appears to be excellent. The reviewer remarked that GM seemed heavily integrated into the project and that they are an excellent partner. The reviewer stated that the CRC allows access to more partners but that they do not appear to be heavily involved at this time. 


\section{Reviewer 2:}

The reviewer stated that the fact that this project was partly CRC funded was a huge plus. The reviewer said that with the auto and oil company personnel working under the same umbrella, that the chances of success will be greater.

\section{Reviewer 3:}

The reviewer stated that the nature of a CRADA is strong collaboration.

\section{Reviewer 4:}

The reviewer stated that the project was a collaboration between ORNL and the CRC, and that key CRC individuals from GM and Chevron were identified as co-investigators. The reviewer noted that problems with legal agreements with each collaborator have occurred but that they were apparently resolved. The reviewer said that not much basis for evaluating extent of collaboration has been possible thus far.

\section{Reviewer 5:}

The reviewer indicated a slight downgrade on score, but only because it was not entirely clear what the role of $\mathrm{CRC}$ was. The reviewer noted that the $\mathrm{CRC}$ was the source of all collaboration on this project. The reviewer commented that the project team made it clear that CRC has provided money and resources. The reviewer expressed a lack of understanding about how this project specifically fits into CRC's plans and objectives.

\section{Question 4: Proposed future research-the degree to which the project has effectively planned its future work in a logical manner by incorporating appropriate decision points, considering barriers to the realization of the proposed technology and, when sensible, mitigating risk by providing alternate development pathways.}

\section{Reviewer 1:}

The reviewer stated that the hardware, fuels, and test plans seem to be in place. The reviewer said that it seemed like the main part of the work will begin in the middle of 2016 with support from the OEM partner.

\section{Reviewer 2:}

The reviewer remarked that the project team were setting up for fundamental thermodynamic analysis of the engine performance. The reviewer commented that this project seems like it would also be an excellent opportunity to interface with the Co-Optima program relative to fuel characteristics and associated engine performance. The reviewer asked if the project team would be able to obtain the generic composition of the chemical-molecular class distributions of the fuels being used and if that information could be incorporated into the database being developed by Co-Optima. The reviewer questioned if the octane index $\mathrm{K}$ factor could be determined for this engine, and if the OI could be determined for the range of fuels used. The reviewer asked if this could contribute to a baseline for the Co-Optima program. Then the reviewer stated that this could contribute to Thrust 1 activities.

\section{Reviewer 3:}

The reviewer remarked that comments on the proposed future research were essentially the same as comments on the project's approach because real experimental work has not yet been initiated.

\section{Reviewer 4:}

The reviewer commented that engine calibration work seems to be a bit nebulous. The reviewer stated that developing a more defined plan for what engine map the project team will run should be part of the plan before running too many tests. The reviewer also noted that the limited funds will likely limit future work to less than desired, and referenced prior comments regarding resources.

\section{Reviewer 5:}

The reviewer stated that the future work plans were not specifically outlined, but rather left mostly general. The reviewer remarked that the project team may very well have a clear plan for your future work, but that it was not communicated clearly.

\section{Question 5: Does this project support the overall DOE objectives of petroleum displacement? Why or why not?}




\section{Reviewer 1:}

The reviewer remarked that this project has the potential of being the most relevant project in the DOE portfolio for some time now. The reviewer said that it is not a far-reaching research project that promises large hard-to-realize benefits, and yet it is not a project that is addressing insignificant efficiency. Instead, it is going after the issue of high-octane fuel that has been severely limiting the potential of SI internal-combustion engines for two or three decades now.

\section{Reviewer 2:}

The reviewer remarked that the project meets the DOE goals and creates some necessary data for the influence of fuel properties with future engine technology.

\section{Reviewer 3:}

The reviewer remarked that the project clearly supports a primary objective of petroleum displacement.

\section{Reviewer 4:}

The reviewer stated that the project could provide a guide to auto and fuel industries regarding future engine fuel combinations for greater efficiency, hence, petroleum reduction. The reviewer remarked that the project could serve as a prelude to Co-Optima work. Focusing on fuel combinations (e.g., ethanol and gasoline) that are already available at commercial scales and supplies could be ramped up to progressively to meet demands of the future engines designed for these fuels as market penetration proceeds.

\section{Question 6: Resources: How sufficient are the resources for the project to achieve the stated milestones in a timely fashion?}

\section{Reviewer 1:}

The reviewer expressed the opinion that given the scope of the project, the funds for this project fall way short. The reviewer remarked that efforts should be made to increase the funding of this project.

\section{Reviewer 2:}

The reviewer stated that the calibration activity alone may use up most of the money. The reviewer stated that a $50 \%$ increase in funds seems more reasonable if CRC will come up with the additional cost share.

\section{Reviewer 3:}

The reviewer remarked that the resources were adequate for the initial phase of the work, but that it could be anticipated that additional work varying engine parameters further might be desirable with additional funding.

\section{Reviewer 4:}

The reviewer remarked that manpower seems appropriate and that the project was receiving reasonable direct and indirect support from CRC. The reviewer said that in addition, as the project team pointed out, associating with CRC enables broader indirect resources. 


\section{GDI Metrics: Scott Goldsborough (Argonne National Laboratory) - ft045}

\section{Presenter}

Scott Goldsborough, Argonne National Laboratory.

\section{Reviewer Sample Size}

A total of six reviewers evaluated this project.

\section{Question 1: Approach to performing the work-the degree to which technical barriers are addressed, the project is well-designed, feasible, and integrated with other efforts.}

\section{Reviewer 1:}

The reviewer stated that the approach to use ANL's RCM to acquire fundamental autoignition data, understand effects of fuel composition on LTC trends, and to formulate correlation based on data should prove to be very successful in addressing the barriers of this project.

\section{Reviewer 2:}

The reviewer remarked that the project was clearly addressing the need to have quantitative fuel quality metrics to characterize fuels across LTC operating modes. The reviewer noted that the approach was sound, using two complementary devices, RCM and GCI, to characterize the fuel blends and systematically vary the conditions.

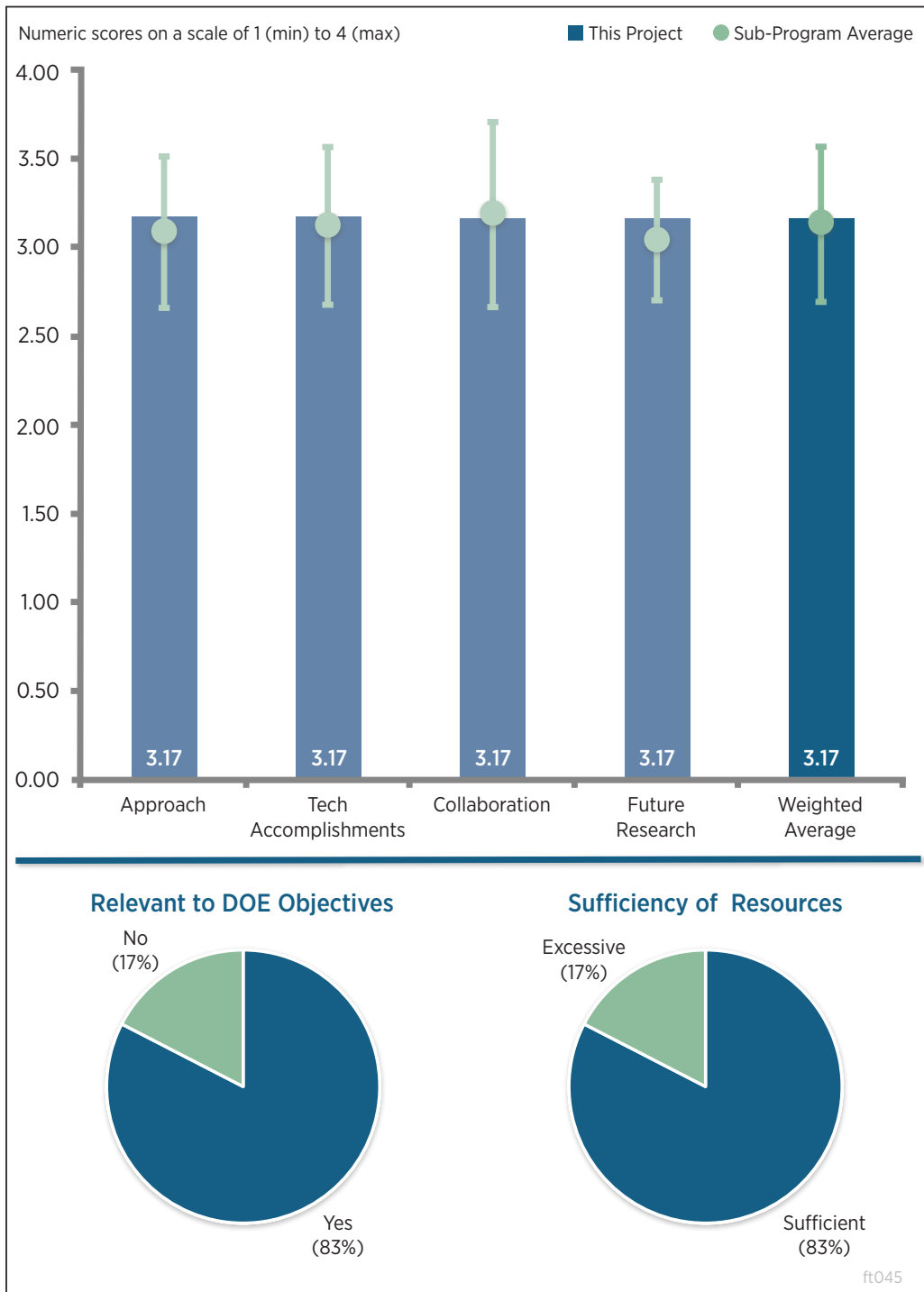

Figure 5-14 - GDI Metrics: Scott Goldsborough (Argonne National Laboratory) - Fuel and Lubricant Technologies

\section{Reviewer 3:}

The reviewer stated that the project has a good range of autoignition characteristics as well as fuel properties. The reviewer remarked that defining the low-temperature heat release (LTHR) and intermediate temperature heat release (ITHR) as a fraction of lower heating value (LHV) was a nice metric to evaluate LTHR and ITHR.

\section{Reviewer 4:}

The reviewer said good idea. The reviewer expressed that it was somewhat unclear exactly how the two data streams fit together.

\section{Reviewer 5:}

The reviewer remarked that the approach as listed on Slide 5 seems very short sighted. The reviewer said that much emphasis was given to understanding the fuel quality metrics for a LTC fuel, but that there seems to be little emphasis on how the project will employ a GCI engine to run on a variety of fuels, given that there was not much in the project about an engine calibration or development of an engine to run a transient cycle. The reviewer stated that quantifying the fuel performance on key engine points was only one part of the process in bringing the technology to market. The reviewer remarked that the project seems to overstate the planned accomplishments on 
the Approach slide and in the title, but then clearly articulates the planned accomplishments on the Future Work slide.

\section{Question 2: Technical accomplishments and progress toward overall project and DOE goals-the degree to which progress has been made, measured against performance indicators and demonstrated progress towards DOE goals.}

\section{Reviewer 1:}

The reviewer commented that the project was on course and the data were extremely detailed and complete. The reviewer stated that the HCCI autoignition has a wide range of conditions that are hard to mimic outside of an engine. The reviewer said that the work linking the autoignition characteristics between the two was impressive.

\section{Reviewer 2:}

The reviewer commented that the characterization and testing of engines and fuels in FY 2015 has provided data for successfully meeting the project milestones. The reviewer remarked that there were several technical accomplishments this year: the project has shown excellent correlation between RCM and GCI engine data under quasi-HCCI conditions for baseline gasoline; fuels with a range of reactivity and molecular structure blended with physical-chemical properties were successfully characterized; and GCI engine experiments were used to quantify fuel influences over a range of operating conditions to validate fuel quality metrics.

\section{Reviewer 3:}

The reviewer remarked on the nice initial progress and said that it seemed like there was something here to be learned.

\section{Reviewer 4:}

The reviewer stated that the project team presented a suite of test data and seemed to be on a solid path forward to identifying an approach for characterizing fuels with a quantitative quality metric. The reviewer remarked that the project team identified some interesting trends regarding intermediate heat release rates and reactivity trends that may inform the metric.

\section{Reviewer 5:}

The reviewer said that the technical accomplishments were just getting started with Phase II of this project, which included looking at fuel properties and the fuel quality metrics. The reviewer remarked that this is at the heart of the project. The reviewer stated that it was unclear why a proposed $20 \%$ vol./vol. would help support the objectives of the project, and that those are not listed on the project milestone slide. The reviewer stated that it was unclear why the chemicals were chosen for the $10 \%$ vol./vol. volume and what had been learned for the metrics, and that therefore it was also hard to understand the need for a second set of fuels.

\section{Question 3: Collaboration and coordination with other institutions.}

\section{Reviewer 1:}

The reviewer commented that this project has great coordination between national laboratories and industry.

\section{Reviewer 2:}

The reviewer stated that the coordination and collaboration between ANL and Chevron was very good and that it will help to ensure a successful project completion.

\section{Reviewer 3:}

The reviewer commented that the primary partners on this project are ANL and Chevron. The reviewer remarked that the roles and responsibilities were clear and that the team seems well-coordinated in its efforts.

\section{Reviewer 4:}

The reviewer said it was good that the project team had a fuel supplier involved. 


\section{Reviewer 5:}

The reviewer said that it seems that it would be helpful to have more collaborators for such a significant portion of DOE budget on this project.

\section{Question 4: Proposed future research-the degree to which the project has effectively planned its future work in a logical manner by incorporating appropriate decision points, considering barriers to the realization of the proposed technology and, when sensible, mitigating risk by providing alternate development pathways.}

\section{Reviewer 1:}

The reviewer remarked that the proposed future work will continue to move the project towards meeting the milestones and overcoming the barriers of this project. The reviewer commented that it was very good that the future work will include a Phase III, which will include a $20 \%$ blended fuel.

\section{Reviewer 2:}

The reviewer stated that the key challenge associated with the project was whether a robust, quantitative metric or correlation could be obtained. The reviewer stated that the proposed work seems to provide a logical path forward.

\section{Reviewer 3:}

The reviewer stated that proposed future work seems like a good path. High-risk but potentially high-reward.

\section{Reviewer 4:}

The reviewer commented that isolating Phi sensitivity and EGR effects, as well as HOV, will make this work extremely useful in GCI and LTC fuel evaluation.

\section{Reviewer 5:}

The reviewer stated that the proposed future work follows the planned milestones for the project. The reviewer stated that it was still unclear why the Phase III was proposed $20 \%$ vol./vol. and how that will help support the objectives of the project, and pointed out those objectives were not listed on the Project Milestone slide. It was unclear why this is needed and how it will support the outcomes of the project. The reviewer said that it seemed too early to be planning for a Phase III, without the data for both Phase I and Phase II being completed. The reviewer remarked that the technical questions from the initial sets of data would be helpful to direct the Phase III.

\section{Question 5: Does this project support the overall DOE objectives of petroleum displacement? Why or why not?}

\section{Reviewer 1:}

The reviewer stated that yes, this project supports the DOE goal of petroleum displacement. The reviewer said the work in this project to develop new fuel quality metrics could be utilized to overcome barriers that inhibit the specification of fuels for low-temperature combustion engines in vehicle fleets, which will enable gains in engine efficiency to help achieve petroleum displacement.

\section{Reviewer 2:}

The reviewer stated that having quantitative metrics to characterize fuels across LTC operating conditions was critical for characterizing these advanced combustion regimes. The reviewer remarked that characterizing fuels was critical to understanding the effectiveness of alternative fuels that can displace petroleum-based fuels.

\section{Reviewer 3:}

The reviewer remarked that this type of work leads to enhancing the basic understanding of LTC and autoignition. The reviewer commented that this will lead to better control of LTC engines and higher efficiency, hence lower petroleum consumption.

\section{Reviewer 4:}

The reviewer commented that a good ignition quality metric was needed for future fuel specifications. 


\section{Reviewer 5:}

The reviewer remarked that it was not clearly explained how the project can aid in the objective of petroleum displacement. The reviewer asked if there are data showing that this technology can enable improvements and suggested that, if the data exist, it would be helpful to highlight these in this project as the impetus for the research.

\section{Question 6: Resources: How sufficient are the resources for the project to achieve the stated milestones in a timely fashion?}

\section{Reviewer 1:}

The reviewer said that the funding for this project seemed to be sufficient to complete the project.

\section{Reviewer 2:}

The reviewer noted that the funding seemed about right. The reviewer said it would be hard to contain the experiments with a smaller budget.

\section{Reviewer 3:}

The reviewer said that there were significant funds provided in this project, but the results seem rather light for the project goals. The reviewer stated that it was unclear how the project supported the goal of fuel economy improvements, and that no data were listed for expected results or improvements. The reviewer remarked that it was unclear how this project will be quantified. 


\section{Efficiency-Optimized Dual Fuel Engine with In-Cylinder Gasoline/CNG Blending: Thomas Wallner (Argonne National Laboratory) - ft046}

\section{Presenter}

Thomas Wallner, Argonne National Laboratory.

\section{Reviewer Sample Size}

A total of five reviewers evaluated this project.

\section{Question 1: Approach to performing the work-the degree to which technical barriers are addressed, the project is well-designed, feasible, and integrated with other efforts.}

\section{Reviewer 1 :}

The reviewer remarked that this was a well-defined project with some challenging goals. The reviewer commented that the project was moving quite well with some interesting learnings. The reviewer said that there were significant remaining challenges and barriers, noting Slide 20, but Slide 22 suggested that metrics were being met. The reviewer commented that it was not clear that the learnings will provide accurate vehicle level fuel economy data from single cylinder engine results. The reviewer said that this is usually a first step at an OEM for comparison of technologies and that it is not always accurate. The reviewer provided a few additional questions for the project team to add to the technical approach explanation: if a transient response is being comprehended somewhere, or only steady state points of an engine map; if an octane equivalent of the blends can be calculated; and if the aftertreatment needs to be changed as a result of methane use, and if that will be comprehended into the cost assessment.

\section{Reviewer 2:}

The reviewer remarked that overall the approach was sound and appropriate. The reviewer commented that the score was slightly downgraded because the project does not address the major barrier of infrastructure. The reviewer pointed out that admittedly, this was not something the research can address readily, but that the project team listed this as a major barrier, and thus put the project on the hook to address it.

\section{Reviewer 3:}

The reviewer said that the balance of simulation and experimental activities makes for a comprehensive approach 
to the project. The reviewer stated that some aftertreatment studies of methane slip past the catalyst would be beneficial.

\section{Reviewer 4:}

The reviewer remarked that the approach is sound in terms of the operating parameters, flame patterns, pressures, efficiencies, etc., that are being studied for various mixtures of a $10 \%$ ethanol blend with gasoline (E10) and NG using different direct injection (DI) NG injection types and placements to optimize engine efficiency utilizing the high anti-knock properties of the NGl, rather than running the vehicle on as much NG as possible and then switching to all-gasoline. The reviewer stated that some results presented were not clear as to whether a $25 \% \mathrm{NG}$ mixture or $50 \%$ NG mixture was being used. The reviewer said it might have been better if the approach started with an examination of other constraints on the level of NG to be used. The reviewer said that efficiency gains are to be realized through increasing $\mathrm{CR}$ and otherwise exploiting the anti-knock properties of NG. The reviewer stated that this means that running on $100 \%$ gasoline, for example E10, will have to be avoided, apart from limiting the application of such engines to those not requiring fast refueling, such as long-distance. The reviewer remarked that in light of this constraint, available space for conforming tanks might determine limits on volume of natural gas to be utilized somewhere short of $50 \%$.

\section{Reviewer 5:}

The reviewer said that as stated in this project's presentation, one of the barriers for NG was limited fueling infrastructure and refueling station availability. The reviewer asked what this project was doing to address this barrier. The reviewer asked why the project team would work on improving engine designs to run on NG when the limiting factor was the NG fueling infrastructure for LD engines. The reviewer noted that another barrier to the wider use of NG in internal combustion engines is the methane catalysis, and wondered if this barrier is recognized in this project. If this barrier is acknowledged, this reviewer asked why there is work on improving engine designs to run on NG when the limiting factor could be meeting emissions standards due to aftertreatment limitations. The reviewer commented that this presentation's assessment of the Advanced Research Projects Agency - Energy (ARPA-E) Methane Opportunities for Vehicular Enhancement (MOVE) program seems dated. The reviewer said that DOE's ARPA-E MOVE program started in 2012 and is almost completed. The reviewer asked if the conclusions of that program are suggesting that the infrastructure barrier for LD application of NG can be overcome.

\section{Question 2: Technical accomplishments and progress toward overall project and DOE goals-the degree to which progress has been made, measured against performance indicators and demonstrated progress towards DOE goals.}

\section{Reviewer 1:}

The reviewer remarked that the project seemed to be on track with the listed objectives and that an impressive amount of work has been completed so far.

\section{Reviewer 2:}

The reviewer said that the progress to date was impressive, including collection of data on key operating parameters with various mixtures, injection strategies, injector locations, etc. The reviewer said that the goals of $10 \%$ efficiency improvement have been exceeded but noted that it was not clear from the project team's presentation if that was with $25 \%$ or $50 \% \mathrm{NG}$. The reviewer also noted that some acronyms were not explained.

\section{Reviewer 3:}

The reviewer stated that the project was on time to according to plan. The reviewer expressed that this was encouraging because the project was almost $50 \%$ complete and the experimental work was well underway.

\section{Reviewer 4:}

The reviewer said that the technical approach on this project was good. The reviewer expressed that it was unclear how some of the items on Slide 20 of the project's presentation will be accomplished. The reviewer asked if there was an octane model being developed for NG and an E10 fuel such as 87 anti-knock index (AKI). 
The reviewer provided a few additional questions to add to the technical approach explanation. The reviewer asked if transient response was being comprehended somewhere, or only for steady state points of an engine map. The reviewer asked if an octane equivalent of the blends can be calculated. The reviewer also asked if the aftertreatment needs to be changed as a result of methane use, and if that will be comprehended into the cost assessment.

\section{Reviewer 5:}

The reviewer asked what the reasoning was behind blending compressed natural gas $(\mathrm{CNG})$ and gasoline. The reviewer asked if it was because that blending was the status quo of limited on-board CNG supply constraints, or if it was because that approach provided the highest efficiency, or if there was some other reason. If it is already recognized that bi-fuel vehicles (e.g., due to a compromised CR) leave the overall fuel consumption potential of CNG untapped, the reviewer asked what or how this project will get around that barrier.

The reviewer said that the very large WOT efficiency and performance benefits of NG, due to more optimum phasing of the combustion process, that have been demonstrated is not surprising and it is well known. The reviewer remarked that at light load, when knock is not a problem, the data show that the blending strategy has very little benefit, approximately a $0.5 \%$ ITE benefit. The reviewer further pointed out that at heavy load, when the engine is knock limited with E10, the data shows that the blending approach leaves a significant amount of efficiency untapped.

\section{Question 3: Collaboration and coordination with other institutions.}

\section{Reviewer 1:}

The reviewer remarked that it seemed like there was good collaboration between the members of the project based on the schedule of actions and project coordination meetings.

\section{Reviewer 2:}

The reviewer said that the collaboration with Fiat Chrysler Automobiles (FCA) and Ford makes for an excellent team. The reviewer remarked that a catalyst partner would be beneficial to address methane slip and the effect on net GHG.

\section{Reviewer 3:}

The reviewer remarked that work was done as collaboration between ANL, Ford, and FCA, with automakers providing injection hardware and technical guidance. The reviewer commented that the nature and extent of technical guidance, mechanisms of consultation, etc., were not explained.

\section{Reviewer 4:}

The reviewer commented that overall, collaborations are good and appropriate, though not very broad. The reviewer said that there seemed to be some missed opportunities. For one, the reviewer thought that collaborations with academia would be possible (e.g., West Virginia University, University of Alabama, or Mississippi State University), as well as with the CNG industry.

Question 4: Proposed future research-the degree to which the project has effectively planned its future work in a logical manner by incorporating appropriate decision points, considering barriers to the realization of the proposed technology and, when sensible, mitigating risk by providing alternate development pathways.

\section{Reviewer 1:}

The reviewer commented that there was discussion of a vehicle level control strategy in the future work. The reviewer believed that this is to be able to comprehend a vehicle-level analysis of the project. The reviewer remarked that it will be interesting to see how a potential transmission shift schedule will accommodate an optimized fuel economy map between the fuels. The reviewer asked if the vehicle level analysis will assume a Federal Test Procedure and US06 cycle and be modeled. The reviewer is excited to see the outcome of this project. 


\section{Reviewer 2:}

The reviewer remarked that the future work plan was appropriate to demonstrate the Funding Opportunity Announcement goals.

\section{Reviewer 3:}

The reviewer remarked that the plan looked fine. The reviewer expressed that the only comment was that it seemed like much work remained for a relatively short amount of time.

\section{Reviewer 4:}

The reviewer said that follow-up work would complete the engine optimization experiments and design a control strategy on actual vehicle. The reviewer stated that this could be an opportunity to integrate optimization with tank and refueling constraints, although that opportunity was not explicit in the presentation.

\section{Reviewer 5:}

The reviewer commented that the planned increase in CR and increase in tumble ratio were in the correct direction to maximize engine efficiency and performance potential with CNG. The reviewer expressed uncertainty why outwardly opening injectors were going to be investigated for side injection and inwardly opening for central injection. The reviewer asked if it would not be the other way around.

\section{Question 5: Does this project support the overall DOE objectives of petroleum displacement? Why or why not?}

\section{Reviewer 1:}

The reviewer remarked that the project clearly supports primary objective of petroleum displacement.

\section{Reviewer 2:}

The reviewer said, yes, the use of NG would aid in the displacement of liquid petroleum. The reviewer commented that using the high octane of NG has potential to aid in the efficiency improvement across the engine map of some advanced technologies.

\section{Reviewer 3:}

The reviewer said the project supports petroleum displacement by both increasing fuel economy and substituting NG for gasoline. The reviewer said but the relevance will ultimately be determined by how broadly it can be applied in terms of driving and refueling patterns.

\section{Reviewer 4:}

The reviewer stated that the project meets the DOE goals for petroleum reduction but meeting the goal for GHG reduction was questionable. The reviewer remarked that the CNG does not really help GHGs and methane slip may actually make the well-to-wheels greenhouse gases worse.

\section{Question 6: Resources: How sufficient are the resources for the project to achieve the stated milestones in a timely fashion?}

\section{Reviewer 1:}

The reviewer said that the resources are appropriate for current and proposed future work.

\section{Reviewer 2:}

The reviewer remarked that overall funding of \$1 million appears adequate and the progress to date conforms well to the indicated timelines and annual budget levels provided.

\section{Reviewer 3:}

The reviewer stated that the current resources are sufficient and do not need to be increased. 


\section{Reviewer 4:}

The reviewer said that it was difficult to determine with the information provided if the funds are for hardware or headcount (or both) for the project, and what the collaborators are providing directly for the project. 


\section{Acronyms and Abbreviations}

ACI

AKI

AMR

ANL

API

ARPA-E

ASSERT

ASTM

BETO

BMEP

BTE

${ }^{\circ} \mathrm{C}$

CA50

CFD

CI

$\mathrm{CN}$

$\mathrm{CO}$

$\mathrm{CNG}$

CR

CRADA

CRC

DI

DISI

DLC

DME
Advanced compression ignition

Anti-knock index

Annual Merit Review

Argonne National Laboratory

American Petroleum Institute

Advanced Research Projects Agency - Energy

Analysis of Sustainability, Scale, Economics, Risk, and Trade

American Society for Testing and Materials

Bioenergy Technologies Office

Brake mean effective pressure

Brake thermal efficiency

Degrees Celsius (Centigrade)

Crank angle position at which $50 \%$ of heat is released

Computational fluid dynamics

Compression ignition

Cetane number

Carbon monoxide

Compressed natural gas

Compression ratio

Cooperative research and development agreement

Coordinating Research Council

Direct injection

Direct injection spark ignition

Diamond-like carbon

Dimethyl-ether 
DOE

E10

E15

E30

E85

E100

ECN

EGR

EPA

EV

FCA

FE

FOA

FWG

FY

GCI

GC-MS

GDI

GHG

GSI

$\mathrm{HC}$

HCCI

HD

HDV

HOV

HFRR

\section{U.S. Department of Energy}

$10 \%$ ethanol blend with gasoline

$15 \%$ ethanol blend with gasoline

$30 \%$ ethanol blend with gasoline

$85 \%$ ethanol blend with gasoline

$100 \%$ ethanol

Engine combustion network

Exhaust gas recirculation

U.S. Environmental Protection Agency

Electric vehicle

Fiat Chrysler Automobiles

Fuel economy

Funding opportunity announcement

Fuels Working Group

Fiscal year

Gasoline compression ignition

Gas chromatography- mass spectrometry

Gasoline direct injection

Greenhouse gas

Gasoline spark ignition

Hydrocarbon

Homogeneous charge compression ignition

Heavy-duty

Heavy-duty vehicle

Heat of vaporization

High frequency reciprocating rig 


\begin{tabular}{|c|c|}
\hline IL & Ionic liquid \\
\hline IL-NP & Ionic liquid nanoparticle \\
\hline ILSAC & International Lubricants Standardization and Approval Committee \\
\hline IP & Intellectual property \\
\hline IQT & Ignition quality tester \\
\hline ITHR & Intermediate temperature heat release \\
\hline LD & Light-duty \\
\hline LDV & Light-duty vehicle \\
\hline LGGF & Low Greenhouse Gas Fuels team \\
\hline LTHR & Low-temperature heat release \\
\hline LLNL & Lawrence Livermore National Laboratory \\
\hline LSPI & Low-speed pre-ignition \\
\hline LTC & Low-temperature combustion \\
\hline MIT & Massachusetts Institute of Technology \\
\hline MON & Motor octane number \\
\hline MOVE & Methane Opportunities for Vehicular Enhancement \\
\hline MPG & Miles per gallon \\
\hline MT & Market transformation \\
\hline MTM & Mini traction machine \\
\hline MW & Molecular weight \\
\hline NG & Natural gas \\
\hline $\mathrm{NO}_{\mathrm{x}}$ & Oxides of nitrogen \\
\hline NP & Nanoparticle \\
\hline NREL & National Renewable Energy Laboratory \\
\hline OBD & On-board diagnostics \\
\hline OEM & Original equipment manufacturer \\
\hline
\end{tabular}


OI Octane index

ORNL Oak Ridge National Laboratory

PAO Polyalphaolefin

PI Principal Investigator

PM Particulate matter

PMI Particulate matter index

PNNL Pacific Northwest National Laboratory

R\&D Research and development

RCCI Reactivity controlled compression ignition

RCM Rapid compression machine

RON Research octane number

SCI Stoichiometric compression ignition

SI Spark ignition

SNL Sandia National Laboratories

TGA Thermal gravimetric analysis

TWC Three-way catalyst

USCAR United States Council for Automotive Research

U.S. DRIVE United States Driving Research and Innovation for Vehicle efficiency and Energy sustainability

VIE Variable interest entity

VM Viscosity modifier

VMT Vehicle miles traveled

VTO Vehicle Technologies Office

WOT Wide open throttle

ZDDP Zinc dialkyldithiophosphate 


\section{Lightweight Materials}

Advanced materials are essential for boosting the fuel economy of modern automobiles while maintaining safety and performance. Because it takes less energy to accelerate a lighter object than a heavier one, lightweight materials offer great potential for increasing vehicle efficiency. A 10\% reduction in vehicle weight can result in a 6\%-8\% fuel economy improvement. Replacing cast iron and traditional steel components with lightweight materials such as high-strength steel, magnesium (Mg) alloys, aluminum (Al) alloys, carbon fiber (CF), and polymer composites can directly reduce the weight of a vehicle's body and chassis by up to $50 \%$ and therefore reduce a vehicle's fuel consumption. Using lightweight components and high-efficiency engines enabled by advanced materials in one quarter of the U.S. fleet could save more than 5 billion gallons of fuel annually by 2030.

By using lightweight structural materials, cars can carry additional advanced emission control systems, safety devices, and integrated electronic systems without increasing the overall weight of the vehicle. While any vehicle can use lightweight materials, they are especially important for hybrid electric, plug-in hybrid electric, and electric vehicles. Using lightweight materials in these vehicles can offset the weight of power systems such as batteries and electric motors, improving the efficiency and increasing their all-electric range. Alternatively, the use of lightweight materials could result in needing a smaller and lower cost battery while keeping the all-electric range of plug-in vehicles constant.

Research and development into lightweight materials is essential for lowering their cost, increasing their ability to be recycled, enabling their integration into vehicles, and maximizing their fuel economy benefits.

The Vehicle Technologies Office (VTO) works to improve these materials in four ways:

- Increasing understanding of the materials themselves through modeling and computational materials science.

- Improving their properties (such as strength, stiffness, and ductility).

- Improving their manufacturing (material cost, production rate, or yield).

- Developing alloys of advanced materials.

In the short term, replacing heavy steel components with materials such as high-strength steel, Al, or glass fiberreinforced polymer composites can decrease component weight by $10-60 \%$. Scientists already understand the properties of these materials and the associated manufacturing processes. Researchers are working to lower their cost and improve the processes for joining, modeling, and recycling these materials.

In the longer term, advanced materials such as $\mathrm{Mg}$ and $\mathrm{CF}$ reinforced composites could reduce the weight of some components by $50-75 \%$. The Office is working to increase our knowledge of these materials' chemical and physical properties and reduce their cost.

\section{Subprogram Feedback}

The U.S. Department of Energy (DOE) received feedback on the overall technical subprogram areas presented during the 2016 Annual Merit Review (AMR). Each subprogram technical session was introduced with a presentation that provided an overview of subprogram goals and recent progress, followed by a series of detailed topic area project presentations.

The reviewers for a given subprogram area responded to a series of specific questions regarding the breadth, depth, and appropriateness of that DOE VTO subprogram's activities. The subprogram overview questions are listed below, and it should be noted that no scoring metrics were applied. These questions were used for all VTO subprogram overviews. 
Question 1: Was the program area, including overall strategy, adequately covered?

Question 2: Is there an appropriate balance between near- mid- and long-term research and development?

Question 3: Were important issues and challenges identified?

Question 4: Are plans identified for addressing issues and challenges?

Question 5: Was progress clearly benchmarked against the previous year?

Question 6: Are the projects in this technology area addressing the broad problems and barriers that the Vehicle Technologies Office (VTO) is trying to solve?

Question 7: Does the program area appear to be focused, well-managed, and effective in addressing VTO's needs?

Question 8: What are the key strengths and weaknesses of the projects in this program area? Do any of the projects stand out on either end of the spectrum?

Question 9: Do these projects represent novel and/or innovative ways to approach these barriers as appropriate?

Question 10: Has the program area engaged appropriate partners?

Question 11: Is the program area collaborating with them effectively?

Question 12: Are there any gaps in the portfolio for this technology area?

Question 13: Are there topics that are not being adequately addressed?

Question 14: Are there other areas that this program area should consider funding to meet overall programmatic goals?

Question 15: Can you recommend new ways to approach the barriers addressed by this program area?

Question 16: Are there any other suggestions to improve the effectiveness of this program area?

Responses to the subprogram overview questions are summarized in the following pages. Individual reviewer comments for each question are identified under the heading Reviewer 1, Reviewer 2, etc. Note that reviewer comments may be ordered differently; for example, for each specific subprogram overview presentation, the reviewer identified as Reviewer 1 in the first question may not be Reviewer 1 in the second question, etc. 


\section{Subprogram Overview Comments: Felix Wu (U.S. Department of Energy) - Im000}

\section{Question 1: Was the program area, including overall strategy, adequately covered?}

\section{Reviewer 1:}

The reviewer observed that the program area was well covered, including the establishment of performance metrics, justification for the focus on materials, identifying the portfolio of lightweight materials, road map exercise and progress to date. The reviewer suggested that some clarification is needed between performance metrics (body, chassis, and interior) when the following slide - for example, "Material Lightweighting: Broad Application"-does not highlight interiors as a focus area. The reviewer added that the "Increasing Focus" slide needs to be clarified as to whether this is a DOE focus or an industry focus, or both.

\section{Reviewer 2:}

The reviewer remarked that the sole sub-program goal presented (Slide three) does not appear to link with the Propulsion Materials part of the portfolio. Despite sound strategy materials subsequently presented for each part, this raised the following questions in this reviewer's mind about the overall strategy. The reviewer would like to know why these two categories, what other relevant material classes exist but are not being targeted (the reviewer added that the hallmark of a complete strategy is to say what you are not going to do), and how was the balance of funding/efforts determined.

\section{Reviewer 3:}

The reviewer answered no, the presentation did not include a strategy to achieve objective and noted that the focus of the presentation was instead on 2015 accomplishments. Near-, mid- and long-term strategy need be developed for each material system. The reviewer also emphasized that Slide eight needs to be updated, and asked whether glazing, metal matrix composite, and titanium really are the future focus. Finally, the reviewer stated that baseline, near-, mid-, and long-term mass reduction goals associated vehicle subsystem need be established and updated annually.

\section{Question 2: Is there an appropriate balance between near- mid- and long-term research and development?}

\section{Reviewer 1:}

The reviewer replied yes, there appears to be a balance between near-, mid-, and long-term research, although DOE did not describe a timescale in the Overview document. The reviewer observed that automotive experience would dictate that structural, safety related systems would require more long-term research in order to design, develop, and test these applications. The lightweight materials research appears to cover the spectrum of critical challenges described, including items such as predictive modeling, cost, recycling, and improving properties, among others.

\section{Reviewer 2:}

The reviewer stated that except for one slide (Slide 15), it was difficult to deduce the targeted timescales from the presented material and thus it is difficult to answer this question. The reviewer suggested more explicit treatment of major targets and time ranges.

\section{Reviewer 3:}

The reviewer replied no, remarking that the predominate focus was on past accomplishments. The reviewer recommended that future presentations include more information as to gaps that will be addressed in mid-term and long-term research and development (R\&D).

\section{Question 3: Were important issues and challenges identified?}

\section{Reviewer 1:}

The reviewer answered yes, critical challenges were outlined for each of the material categories and elaborated that challenges described as critical — regardless of severity of the challenge - are still critical, meaning that 
they all require some level of research in order to solve those challenges for the technologies to be successful in the marketplace. The reviewer offered as an example that for carbon fiber composites (CFCs), what are needed are nondestructive evaluation (NDE) methods, as well as predictive modeling and low-cost fibers. The reviewer concluded that all of these challenges are required to be solved in order for the materials technology to be successfully implemented into the industry.

\section{Reviewer 2:}

The reviewer replied yes, important challenges were identified, but added that they were lost in the clutter of Slide eight.

\section{Reviewer 3:}

To reviewer answered to some degree, characterizing Slide eight in particular as a useful catalogue of materials and ranking of challenges. However, the reviewer added that in some cases (e.g., perhaps manufacturing), greater specificity about the identified issues would be helpful.

\section{Question 4: Are plans identified for addressing issues and challenges?}

\section{Reviewer 1:}

The reviewer replied yes, and elaborated that DOE outlined plans for addressing these issues and challenges based on feedback from, and development of, light- and heavy-duty vehicle roadmaps with plans outlined in three areas. These include properties and manufacturing, which looks to reduce cost of raw materials and processing and improve performance and manufacturing; multi-material enabling, which looks to join dissimilar materials, prevent corrosion, and develop NDE techniques; and modeling and simulation, which looks to develop tools for modeling and accurately predicting behavior.

Similarly, the reviewer observed that DOE outlined plans for the Propulsion Materials Program that also include three areas of focus, namely, Engine Materials, Exhaust System Materials, and Integrated Computational Materials Engineering. The reviewer stated that in both the Lightweight Materials Program and the Propulsion Material Program, demonstration, validation, and analysis are keys to success for rapid acceleration into the marketplace.

\section{Reviewer 2:}

The reviewer replied in some cases, yes, but added that the magnitude and breadth of challenges is so broad that it seemed to overshadow the progress of the several worthy 2015 accomplishments. The reviewer suggested that perhaps this is partly a matter of emphasis in assembling the presentation material.

\section{Reviewer 3:}

The reviewer judged that the plan to address issues and challenge was not presented.

\section{Question 5: Was progress clearly benchmarked against the previous year?}

\section{Reviewer 1:}

The reviewer replied yes, and recounted that DOE provided five examples of progress made in 2015, including: plasma oxidation technology for rapid throughput of CFCs with reduced energy usage; laser assisted adhesive joining of CF-reinforced polymer (CFRP) to $\mathrm{Al}$; $\mathrm{Mg}$ intensive demonstration structure (shock tower); high-strength steel with increased yield; and completed characterization of $\mathrm{Mg}$ alloys.

\section{Reviewer 2:}

The reviewer answered no, benchmark data was not provided as it need be developed. The reviewer recommended that VTO set mass reduction goals for key vehicle subsystems based on a 2013 model year (MY) baseline highvolume C-segment vehicle to demonstrate the pathway to achieve $30 \%$ full vehicle mass reduction as follows: 


\begin{tabular}{|l|l|l|l|l|}
\hline & Baseline $(\mathrm{kg})$ & Demonstrated & $\begin{array}{l}\text { Goal } \\
(\mathrm{kg})\end{array}$ & \\
\hline BIW & 326 & 231 & 162 & $50 \%$ \\
\hline Closures & 98 & 57 & 43 & $56 \%$ \\
\hline Chassis & 57 & 54 & 31 & $46 \%$ \\
\hline Bumpers & 37 & 11 & 11 & $70 \%$ \\
\hline Total & 518 & 353 & 247 & $52 \%$ \\
\hline
\end{tabular}

The reviewer replied no, stating that that there was a considerable amount of recycling of last year's material but in such a way that the reviewer did not get a good sense of continuity or incremental benchmarking. The reviewer clarified that specific accomplishments are naturally highly focused, but to a newcomer, the year-to-year continuity and an overall integrative approach appear to be weaker aspects.

\section{Question 6: Are the projects in this technology area addressing the broad problems and barriers that the Vehicle Technologies Office (VTO) is trying to solve?}

\section{Reviewer 1:}

The reviewer observed that VTO is focused on energy security and reduced dependence on foreign oil, and that by focusing on lightweight materials development and usage in the automotive industry, car companies will have solutions in their tool kit to enable lightweight (and safe) vehicles to be produced that will also use less fuel and emit fewer greenhouse gases.

\section{Reviewer 2:}

The reviewer replied yes to this question.

\section{Reviewer 3:}

The reviewer answered yes, but cautioned that projects need be focused on developing technologies to demonstrate the mass reduction goal for each subsystem.

Question 7: Does the program area appear to be focused, well-managed, and effective in addressing VTO's needs?

\section{Reviewer 1:}

The reviewer replied yes, adding that the program appears focused, well-managed, and effective in addressing VTO's needs.

\section{Reviewer 2:}

This reviewer could obtain little insight into the number of current projects, the portfolio balance (other than by financial figures for lightweight versus. propulsion), the strategy relative to time horizons (or alternatively, technology readiness levels [TRL]), or the rationale for the selection or emphasis among individual projects.

\section{Reviewer 3:}

The reviewer stated no, the program lacks a clear vision for the future, indicating the need for direction. The reviewer said a budget reduction of $25 \%$ is a signal of clarity and a lack of a defined plan to achieve the objective.

\section{Question 8: What are the key strengths and weaknesses of the projects in this program area? Do any of the projects stand out on either end of the spectrum?}

\section{Reviewer 1:}

The reviewer was unable to provide meaningful comments, partly for the reason cited in the reply to Question 7. 


\section{Reviewer 2:}

The reviewer stated that the projects are not focused to achieve a common goal of $30 \%$ full vehicle mass reduction, adding that if the goal is $30 \%$ full vehicle mass reduction by 2020, a roadmap is needed for each subsystem.

\section{Question 9: Do these projects represent novel and/or innovative ways to approach these barriers as appropriate?}

\section{Reviewer 1:}

The reviewer responded yes, characterizing these projects as representing new and innovative approaches to address these barriers. The reviewer declared that gone are the days when a single organization has the capability to fully innovative across the supply chain. The reviewer further remarked that collaboration in the industry has become critically important and helps drive new material development, combined with new processing methods, and combined with new tooling methods, concluding that DOE-funded projects help facilitate these collaborate activities.

\section{Reviewer 2:}

The reviewer replied yes to this question.

\section{Reviewer 3:}

The reviewer stated that many of the projects are novel and innovative, but are not coordinated to achieve a commercialization objective by 2020 , adding that the R\&D projects need to be aligned with the needs of the demonstration/validation projects. The reviewer said that several projects specify a vehicle subsystem and a target mass reduction and incremental cost per pound saved are focused and add to the commercialization objective. The reviewer also specified that materials development and joining projects need to specify clear measurable objectives and target mass reduction potential specific to a vehicle subsystem.

\section{Question 10: Has the program area engaged appropriate partners?}

\section{Reviewer 1:}

The reviewer said yes, the program actively engages national laboratories, universities, original equipment manufacturer (OEM) companies, tier suppliers, material suppliers, and other research institutes.

\section{Reviewer 2:}

The reviewer replied yes, the program seems to have a good mixture of academic, industry, and government research groups.

\section{Reviewer 3:}

The reviewer replied yes, but added that there is too much emphasis on partners and instead recommended focusing on aligning and coordinating partners to deliver the commercialized result. The reviewer cited as a good example friction stir scribe technology. The reviewer said the project team includes a hand-off from FRDL to industrial partners to supply commercial application equipment to the OEM/tier community.

\section{Question 11: Is the program area collaborating with them effectively?}

\section{Reviewer 1:}

The reviewer answered yes, the program teams are collaborating well with their partners.

\section{Reviewer 2:}

The reviewer replied yes to this question.

\section{Reviewer 3:}

The reviewer was unable to say conclusively from material presented, noting that while many other organizations and initiatives were referenced (e.g., MGI, LightMat, the Energy Materials Network, the United States Automotive Materials Partnership [USAMP]), the program's connections to them were not clearly explained for the benefit of outsiders.

Question 12: Are there any gaps in the portfolio for this technology area? 


\section{Reviewer 1:}

The reviewer stated that there do not appear to be gaps at this time, adding that the program is taking a balanced approach to solve technical challenges facing the materials industry. The reviewer observed that DOE is funding research in many areas, including reducing costs for raw materials and processing, improving performance and manufacturability, evaluating joining methods for dissimilar materials, developing nondestructive testing methods, and developing tools for modeling and simulation. Continuing this approach across materials solutions provides for a level playing field for suppliers and a broader portfolio of solutions for the automotive industry.

\section{Reviewer 2:}

The reviewer remarked that the five 2015 accomplishments in Slides 18 through 22, while technically impressive, appeared to remain some distance from implementation in manufactured, in-service vehicles. The reviewer suggested that because the sub-program has been running for a longer time, it would be valuable for it to track the progressive industry adoption and deployment of previous years' progress, and take some credit for them (as certain other sub-programs appear to do) to avoid any misperception of gaps or any disconnect with commercial relevance. The reviewer was unable to provide further comments, partly for the reason cited in the answer to question seven above.

\section{Reviewer 3:}

The reviewer said the gap is the lack of a defined goal, documentation relative to baseline and progress of lightweighting vehicle subsystems. The reviewer offered that there has been significant progress that has not been documented which has led to the reduction in funding.

\section{Question 13: Are there topics that are not being adequately addressed?}

\section{Reviewer 1:}

The reviewer replied that technical gaps and challenges identified in industry roadmap sessions appear to be well covered.

\section{Reviewer 2:}

The reviewer stated that gaps are being addressed but progress has not been documented.

\section{Reviewer 3:}

The reviewer observed that the increased safety challenges associated with lightweighting appear (at least from this overview presentation) to be receiving little attention, and suggested it would be beneficial to have a stronger recognition of that tradeoff (alongside cost, comfort, etc.), than simply having a brief mention on the Summary slide.

\section{Question 14: Are there other areas that this program area should consider funding to meet overall programmatic goals?}

\section{Reviewer 1:}

The reviewer recommended that the program should continue with its balanced approach to solving technical issues across the various material solutions.

\section{Reviewer 2:}

The reviewer suggested that given the emphasis being placed on integrated computational materials engineering (ICME) in both this program area and some others, the program might consider pre-competitive funding for better software, following the Computer-Aided Engineering for Electric-Drive Vehicle Batteries (CAEBAT) project approach, so that the "expanded ICME capabilities" referenced on Slide 14 can be deployed more broadly, sustainably, and in a way that directly impacts vehicle manufacturing. The reviewer further noted that presently, the gap in usability between simulating true molecular-scale fundamentals and real processes/vehicle systems is so large that general references to ICME (e.g., Slide 17 title, say) can appear aspirational and insufficiently supported by tangible results. 


\section{Reviewer 3:}

The reviewer replied yes, noting that reduction of manufacturing cost and life-cycle assessment (LCA) associated with fiber-reinforced polymer (FRP) are not being addressed. The reviewer elaborated that the effort to reduce the cost of carbon has realized the objective but the incremental cost of FRP remains $\$ 10$ per pound ( $\$ 10 / 1 \mathrm{~b}$.) mass saved and the carbon footprint is high, prohibiting commercial application. For commercial use, cost per pound saved needs to be under $\$ 2.50 / \mathrm{lb}$. The reviewer specified that a benchmark needs to be published for cost per pound saved (e.g., \$10/lb.) and LCA along with a plan as to where we are going, adding that if we do not have a plan to realize the commercialization barriers, we need to reallocate funding.

\section{Question 15: Can you recommend new ways to approach the barriers addressed by this program area?}

\section{Reviewer 1:}

The reviewer replied no to this question.

\section{Reviewer 2:}

The reviewer recommended that DOE should continue to work with individual companies, industry associations, research organizations, and universities to understand technologies in the pipeline, as well as work closely with the car companies and regulators to understand upcoming needs. The reviewer also suggested that DOE should continue to evaluate short-, medium- and long-term technology solutions, and should do so across the broad portfolio of lightweight material solutions.

\section{Question 16: Are there any other suggestions to improve the effectiveness of this program area?}

\section{Reviewer 1:}

The reviewer had no other further suggestions at this time, and thanked the program for its efforts and providing a publicly-accessible Annual Merit Review.

\section{Reviewer 2:}

The reviewer said improve focus by downselecting carefully from the myriad challenges, which includes everything from materials data to NDE to missing basic material data to manufacturing processes to ICME. The reviewer cautioned that this area seems too broad for maximum effectiveness with the limited available funding and the diversity of vehicle materials. The reviewer offered that focusing on fewer topics might provide greater leadership and progress in those areas and suggested use of a rigorous metric or scorecard to determine what is the low-hanging fruit that gains maximum benefit from this government-led collaboration, adding that perhaps that already exists, but that it was not evident from this presentation. 


\section{Project Feedback}

In this merit review activity, each reviewer was asked to respond to a series of questions, involving multiple-choice responses, expository responses where text comments were requested, and numeric score responses (on a scale of 1.0 to 4.0). In the pages that follow, the reviewer responses to each question for each project will be summarized: the multiple choice and numeric score questions will be presented in graph form for each project, and the expository text responses will be summarized in paragraph form for each question. A table presenting the average numeric score for each question for each project is presented below.

\section{Table 6-1 - Project Feedback}

\begin{tabular}{|c|c|c|c|c|c|c|c|}
\hline Presentation Title & $\begin{array}{c}\text { Principal } \\
\text { Investigator } \\
\text { and } \\
\text { Organization }\end{array}$ & $\begin{array}{l}\text { Page } \\
\text { Number }\end{array}$ & Approach & $\begin{array}{c}\text { Technical } \\
\text { Accomplishments }\end{array}$ & Collaborations & $\begin{array}{c}\text { Future } \\
\text { Research }\end{array}$ & $\begin{array}{c}\text { Weighted } \\
\text { Average }\end{array}$ \\
\hline $\begin{array}{l}\text { Scale-Up of } \\
\text { Magnesium } \\
\text { Production by } \\
\text { Fully Stabilized } \\
\text { Zirconia } \\
\text { Electrolysis }\end{array}$ & $\begin{array}{l}\text { Powell, Adam } \\
\text { (INFINIUM, } \\
\text { Inc.) }\end{array}$ & $6-13$ & 3.33 & 3.50 & 3.83 & 3.75 & 3.53 \\
\hline $\begin{array}{c}\text { Integrated } \\
\text { Computational } \\
\text { Materials } \\
\text { Engineering } \\
\text { Approach to } \\
\text { Development } \\
\text { of Lightweight } \\
\text { 3GAHSS Vehicle } \\
\text { Assembly }\end{array}$ & $\begin{array}{l}\text { Hector, Lou } \\
\text { (USAMP) }\end{array}$ & $6-16$ & 3.13 & 3.50 & 3.63 & 3.00 & 3.36 \\
\hline $\begin{array}{l}\text { Validation of } \\
\text { Material Models } \\
\text { for Crash } \\
\text { Simulation of } \\
\text { Automotive } \\
\text { Carbon Fiber } \\
\text { Composite } \\
\text { Structures (VMM) }\end{array}$ & $\begin{array}{l}\text { Berger, Libby } \\
\text { (GM) }\end{array}$ & $6-19$ & 3.33 & 3.33 & 3.75 & 3.17 & 3.36 \\
\hline $\begin{array}{l}\text { Collision Welding } \\
\text { of Dissimilar } \\
\text { Materials by } \\
\text { Vaporizing Foil } \\
\text { Actuator: A } \\
\text { Breakthrough } \\
\text { Technology } \\
\text { for Dissimilar } \\
\text { Materials Joining }\end{array}$ & $\begin{array}{l}\text { Daehn, Glenn } \\
\text { (Ohio State } \\
\text { University) }\end{array}$ & $6-23$ & 3.00 & 2.88 & 3.25 & 3.00 & 2.97 \\
\hline $\begin{array}{l}\text { Active, Tailorable } \\
\text { Adhesives for } \\
\text { Dissimilar Material } \\
\text { Bonding, Repair } \\
\text { and Assembly }\end{array}$ & $\begin{array}{l}\text { Haq, Mahmood } \\
\text { (Michigan } \\
\text { State } \\
\text { University) }\end{array}$ & $6-26$ & 3.38 & 3.50 & 3.13 & 3.38 & 3.41 \\
\hline
\end{tabular}




\begin{tabular}{|c|c|c|c|c|c|c|c|}
\hline Presentation Title & $\begin{array}{c}\text { Principal } \\
\text { Investigator } \\
\text { and } \\
\text { Organization }\end{array}$ & $\begin{array}{c}\text { Page } \\
\text { Number }\end{array}$ & Approach & $\begin{array}{c}\text { Technical } \\
\text { Accomplishments }\end{array}$ & Collaborations & $\begin{array}{c}\text { Future } \\
\text { Research }\end{array}$ & $\begin{array}{c}\text { Weighted } \\
\text { Average }\end{array}$ \\
\hline $\begin{array}{l}\text { High-Strength } \\
\text { Electroformed } \\
\text { Nanostructured } \\
\text { Aluminum for } \\
\text { Lightweight } \\
\text { Automotive } \\
\text { Applications }\end{array}$ & $\begin{array}{l}\text { Hilty, Robert } \\
\text { (Xtalic } \\
\text { Corporation) }\end{array}$ & $6-29$ & 3.00 & 3.13 & 2.88 & 3.00 & 3.05 \\
\hline $\begin{array}{c}\text { Vehicle } \\
\text { Lightweighting: } \\
\text { Mass Reduction } \\
\text { Spectrum Analysis } \\
\text { and Process Cost } \\
\text { Modeling }\end{array}$ & $\begin{array}{l}\text { Mascarin, } \\
\text { Tony (IBIS } \\
\text { Associates) }\end{array}$ & $6-32$ & 2.58 & 2.50 & 2.42 & 2.90 & 2.56 \\
\hline $\begin{array}{l}\text { Laser-Assisted } \\
\text { Joining Process } \\
\text { of Aluminum and } \\
\text { Carbon Fiber } \\
\text { Components }\end{array}$ & $\begin{array}{l}\text { Sabau, Adrian } \\
\text { (ORNL) }\end{array}$ & $6-36$ & 3.38 & 3.00 & 3.38 & 3.13 & 3.16 \\
\hline $\begin{array}{l}\text { Brazing Dissimilar } \\
\text { Metals with a } \\
\text { Novel Composite } \\
\text { Foil }\end{array}$ & $\begin{array}{l}\text { Weihs, Tim } \\
\text { (John Hopkins } \\
\text { University) }\end{array}$ & $6-39$ & 2.90 & 2.90 & 2.30 & 2.80 & 2.81 \\
\hline $\begin{array}{l}\text { High-Strength, } \\
\text { Dissimilar Alloy } \\
\text { Aluminum Tailor- } \\
\text { Welded Blanks }\end{array}$ & $\begin{array}{l}\text { Hovanski, Yuri } \\
\quad \text { (PNNL) }\end{array}$ & $6-43$ & 3.75 & 3.63 & 3.75 & 3.50 & 3.66 \\
\hline $\begin{array}{c}\text { Upset Protrusion } \\
\text { Joining } \\
\text { Techniques For } \\
\text { Joining Dissimilar } \\
\text { Metals }\end{array}$ & $\begin{array}{l}\text { Logan, Steve } \\
\text { (Fiat Chrysler } \\
\text { Automobiles } \\
\text { US LLC) }\end{array}$ & $6-46$ & 3.60 & 3.30 & 3.20 & 2.80 & 3.30 \\
\hline $\begin{array}{c}\text { Integrated } \\
\text { Computational } \\
\text { Materials } \\
\text { Engineering } \\
\text { (ICME) } \\
\text { Development } \\
\text { of Carbon Fiber } \\
\text { Composites for } \\
\text { Lightweight } \\
\text { Vehicles }\end{array}$ & $\begin{array}{l}\text { Su, Xuming } \\
\text { (Ford) }\end{array}$ & $6-49$ & 3.40 & 3.50 & 3.50 & 3.40 & 3.46 \\
\hline
\end{tabular}




\begin{tabular}{|c|c|c|c|c|c|c|c|}
\hline Presentation Title & $\begin{array}{c}\text { Principal } \\
\text { Investigator } \\
\text { and } \\
\text { Organization }\end{array}$ & $\begin{array}{l}\text { Page } \\
\text { Number }\end{array}$ & Approach & $\begin{array}{c}\text { Technical } \\
\text { Accomplishments }\end{array}$ & Collaborations & $\begin{array}{l}\text { Future } \\
\text { Research }\end{array}$ & $\begin{array}{l}\text { Weighted } \\
\text { Average }\end{array}$ \\
\hline $\begin{array}{l}\text { Predictive Models } \\
\text { for Integrated } \\
\text { Manufacturing } \\
\text { and Structural } \\
\text { Performance of } \\
\text { Carbon Fiber } \\
\text { Composites for } \\
\text { Automotive } \\
\text { Applications }\end{array}$ & $\begin{array}{l}\text { Aitharaju, } \\
\text { Venkat (GM) }\end{array}$ & $6-52$ & 3.50 & 3.50 & 3.50 & 3.63 & 3.52 \\
\hline $\begin{array}{l}\text { E. Coli Derived } \\
\text { Spider Silk } \\
\text { MaSp1 and } \\
\text { MaSp2 Proteins } \\
\text { as Carbon Fiber } \\
\text { Precursors }\end{array}$ & $\begin{array}{l}\text { Lewis, Randy } \\
\text { (Utah State } \\
\text { University) }\end{array}$ & $6-55$ & 3.50 & 3.43 & 3.50 & 3.14 & 3.42 \\
\hline $\begin{array}{l}\text { Solid-State Body- } \\
\text { in-White Spot } \\
\text { Joining of Al to } \\
\text { AHSS at Prototype } \\
\text { Scale }\end{array}$ & $\begin{array}{l}\text { Feng, Zhili } \\
\text { (ORNL) }\end{array}$ & $6-58$ & 3.25 & 3.38 & 3.25 & 3.13 & 3.30 \\
\hline $\begin{array}{c}\text { Friction Stir Scribe } \\
\text { Joining of Al to } \\
\text { Steel }\end{array}$ & $\begin{array}{l}\text { Hovanski, Yuri } \\
\text { (PNNL) }\end{array}$ & $6-60$ & 3.60 & 3.60 & 3.60 & 3.50 & 3.59 \\
\hline $\begin{array}{c}\text { Enhanced } \\
\text { Sheared Edge } \\
\text { Stretchability of } \\
\text { AHSS/UHSS }\end{array}$ & $\begin{array}{l}\text { Sun, Xin } \\
\text { (PNNL) }\end{array}$ & $6-63$ & 3.50 & 3.50 & 3.67 & 3.50 & 3.52 \\
\hline $\begin{array}{l}\text { Optimizing } \\
\text { Heat Treatment } \\
\text { Parameters for } \\
\text { 3rd Generation } \\
\text { AHSS Using } \\
\text { an Integrated } \\
\text { Experimental- } \\
\text { Computational } \\
\text { Framework }\end{array}$ & $\begin{array}{l}\text { Sun, Xin } \\
\text { (PNNL) }\end{array}$ & $6-65$ & 3.00 & 3.00 & 2.83 & 2.50 & 2.92 \\
\hline $\begin{array}{l}\text { Development } \\
\text { of Low-Cost, } \\
\text { High-Strength } \\
\text { Automotive } \\
\text { Aluminum Sheet }\end{array}$ & $\begin{array}{l}\text { Long, Russell } \\
\text { (ALCOA) }\end{array}$ & $6-68$ & 3.33 & 3.33 & 3.33 & 3.33 & 3.33 \\
\hline
\end{tabular}




\begin{tabular}{|l|c|c|c|c|c|c|c|}
\hline Presentation Title & $\begin{array}{c}\text { Principal } \\
\text { Investigator } \\
\text { and } \\
\text { Organization }\end{array}$ & $\begin{array}{c}\text { Page } \\
\text { Number }\end{array}$ & Approach & $\begin{array}{c}\text { Technical } \\
\text { Accomplishments }\end{array}$ & Collaborations & $\begin{array}{c}\text { Future } \\
\text { Research }\end{array}$ & $\begin{array}{c}\text { Weighted } \\
\text { Average }\end{array}$ \\
\hline Overall Average & & & 3.29 & 3.28 & 3.30 & 3.19 \\
\hline
\end{tabular}


Scale-Up of Magnesium Production by Fully Stabilized Zirconia Electrolysis: Adam Powell (INFINIUM, Inc.) - Im035

\section{Presenter}

Steve Derezinski, Infinium, Inc.

\section{Reviewer Sample Size}

A total of three reviewers evaluated this project.

\section{Question 1: Approach to performing the work-the degree to which technical barriers are addressed, the project is well-designed, feasible, and integrated with other efforts.}

\section{Reviewer 1:}

The reviewer applauded this as a great project, and clarified that creating master Mg-neodymium (Nd) alloys is a step in the right direction if combining the two parent metal oxides and reducing them is cheaper in the long run. The reviewer remarked that the $\mathrm{Mg}$ suppliers could further alloy them into conventional AE42-type alloys, hopefully at less cost penalty.

\section{Reviewer 2:}

The reviewer found that the project has shifted from production of a primary metal to alloy seeding, and opined that this was a wise move. The reviewer commented that it is certainly strategic for the U.S./North America market and should result in stronger prospects for $\mathrm{Mg}$ deployment in vehicle components.

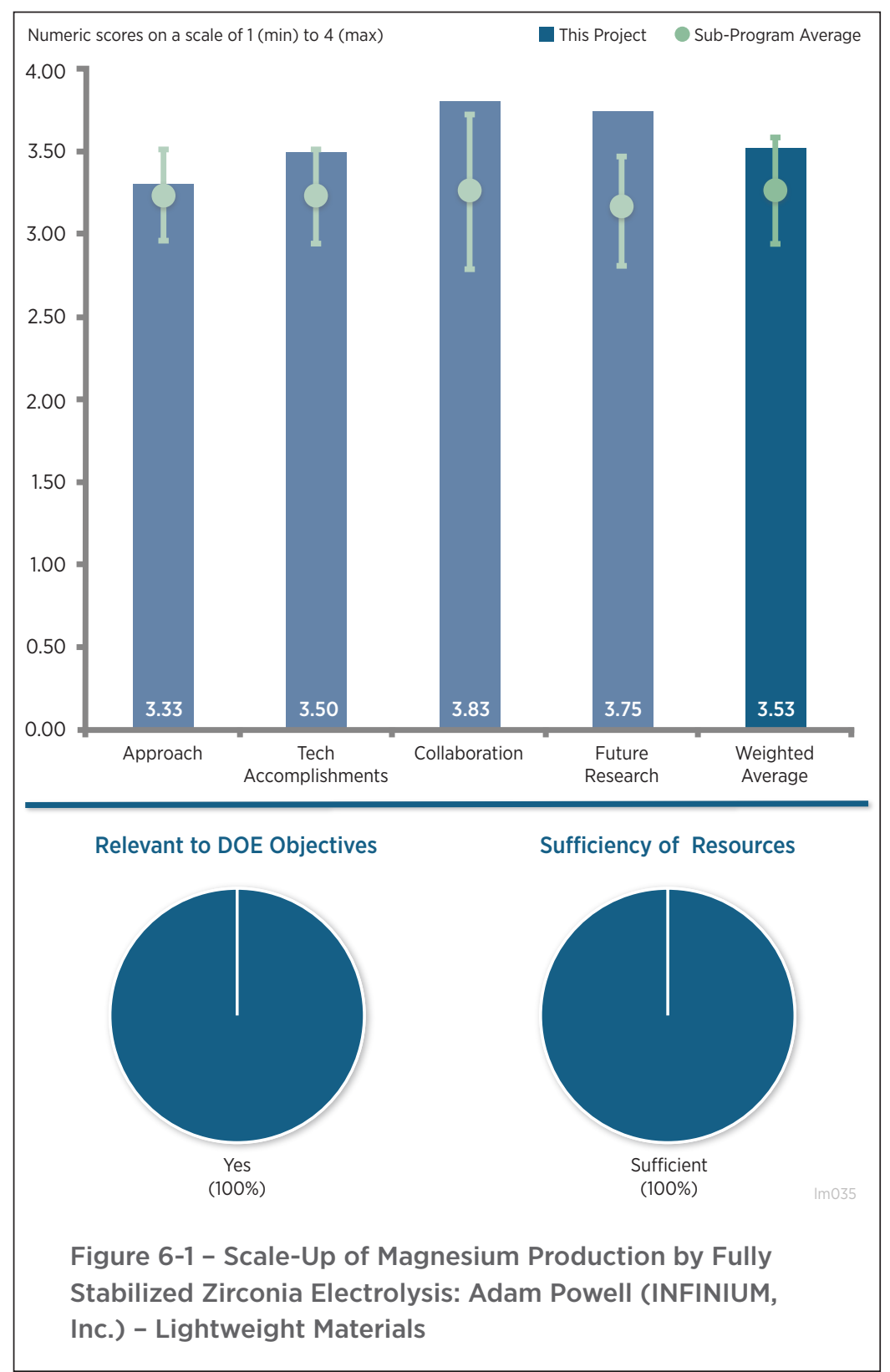

\section{Reviewer 3:}

The reviewer pointed out that the overall goal is to provide an inexpensive and clean domestic source of $\mathrm{Mg}$, yet the program changed to supply a Mg-Nd master alloy. The reviewer was not sure where the change in approach originated from, but it appears to be needed to assist in reaching possible production rates needed for mainstream production. The reviewer was concerned that the rare earth metal availability could be an issue in the future as production ramps up. The reviewer observed that it would be nice to see production rate availability of the rareearth metal coming from other countries, and evaluate if this supply would accommodate the U.S. demand once production begins.

Question 2: Technical accomplishments and progress toward overall project and DOE goals-the degree to which progress has been made, measured against performance indicators and demonstrated progress towards DOE goals. 


\section{Reviewer 1:}

The reviewer commented that there is significant innovation in moving from a primary magnesium oxide $(\mathrm{MgO})$ to a complex $\mathrm{MgO} / \mathrm{Nd}_{2} \mathrm{O}_{3}$ system as a reduction technology to drive the cost out of specialized $\mathrm{Mg}$ alloys like AE42, etc.

\section{Reviewer 2:}

The reviewer observed great improvements in efficiency in such a short period of time. The reviewer said that compared to current manufacturing processes, it is amazing to see such a high efficiency, and looks very promising going forward. Production rate increases of 10 times from Delta 1 to Delta 1.1 are also very promising to see. The reviewer said that once the gamma cell is running, it will be interesting to see if the efficiencies and production rates meet project goals. The reviewer observed that from an energy consumption standpoint, improvements over current manufacturing processes are very impressive and will assist in process adoption once production rates are up. Environmental impacts just from the reduction of by-products are also amazing, and will enable ISO 14001-compliant companies to further improve the manufacturing impact on the environment. The reviewer cautioned that Slide 11 does not show the efficiency expected from Gamma production; if the efficiency falls off from the newer larger unit, production rates might not be achievable as anticipated.

\section{Reviewer 3:}

The reviewer remarked that the shift in project focus/methodology will, understandably, have slowed progress against milestones as new targets are developed. Overall, this person commented that good forward progress appears to have been made. The reviewer concluded that the enhancements to cell efficiency and process safety and robustness (eight hours unattended operation) is very encouraging.

\section{Question 3: Collaboration and coordination with other institutions.}

\section{Reviewer 1:}

The reviewer applauded that this small start-up firm has made collaboration an integral part of their project, and this strategy has worked very well.

\section{Reviewer 2:}

The reviewer commented that collaboration efforts have the correct institutions selected, and the collaborators are performing what they do best. The reviewer applauded a great use of resources to focus on each entities' expertise to accomplish a common goal.

\section{Reviewer 3:}

The reviewer is hoping to see, during the life of the project, the master $50 / 50 \mathrm{Mg} / \mathrm{Nd}$ soon making an AER42 alloy at a primary $\mathrm{Mg}$ supplier.

Question 4: Proposed future research-the degree to which the project has effectively planned its future work in a logical manner by incorporating appropriate decision points, considering barriers to the realization of the proposed technology and, when sensible, mitigating risk by providing alternate development pathways.

\section{Reviewer 1:}

The reviewer remarked that the plan looks very good going forward.

\section{Reviewer 2:}

The reviewer suggested that as in past years, the project team consider expanding into other rare-earth additional, such as yttrium, erbium, and others.

\section{Question 5: Does this project support the overall DOE objectives of petroleum} displacement? Why or why not? 


\section{Reviewer 1:}

The reviewer emphasized yes, and opined that the project also helps to secure the supply chain for this strategic material (i.e., $\mathrm{Mg}$ ) in the United States, which is important given the potential difficulties associated with the present supply chain that is based in Asia.

\section{Reviewer 2:}

The reviewer commented that $\mathrm{Mg}$ is still viewed as an enabling lightweight material for the transportation industry, so this project is well aligned with DOE's objectives.

\section{Reviewer 3:}

The reviewer said that with just a pure material swap, $\mathrm{Mg}$ has the potential weight savings of over $30 \%$. If successfully able to ramp up production, this effort will enable high-strength castable $\mathrm{Mg}$ to be used for automotive purposes.

\section{Question 6: Resources: How sufficient are the resources for the project to achieve the stated milestones in a timely fashion?}

\section{Reviewer 1:}

The reviewer remarked that resources appear to be adequate.

\section{Reviewer 2:}

The reviewer commented that the program is wrapping up and should have sufficient resources available to overcome the few remaining barriers and reach the milestones.

\section{Reviewer 3:}

The reviewer said that funding is appropriate. 


\section{Integrated Computational \\ Materials Engineering \\ Approach to Development of Lightweight 3GAHSS Vehicle Assembly: Lou Hector (United States Automotive Materials Partnership LLC) - Im080}

\section{Presenter}

Lou Hector, USAMP

\section{Reviewer Sample Size}

A total of four reviewers evaluated this project.

\section{Question 1: Approach to performing the work-the degree to which technical barriers are addressed, the project is well-designed, feasible, and integrated with other efforts.}

\section{Reviewer 1:}

The reviewer remarked excellent approach.

\section{Reviewer 2:}

The reviewer said that the approach is leading in the right direction to achieve the goals set forth in the program. Objectives are laid out nicely and a defined path with milestones is in place. According to the reviewer, following what is laid out, if successful, will enable the barriers for third-generation advanced high-strength steels (3GAHHS) to be overcome.

\section{Reviewer 3:}

The reviewer observed that this is a very large, complex and challenging project with a lot of moving parts, but it is well-designed, and focused very clearly on the goal of commercializing the technologies being developed. The reviewer stated that the scope and execution are impressive.

\section{Reviewer 4:}

The reviewer commented that the mass saving objective was not met. The reviewer commented that an increase in strength will not result in mass reduction in a stiffness driven application, and that no plan to address this topic was presented, significantly reducing the relevance.

Question 2: Technical accomplishments and progress toward overall project and DOE goals-the degree to which progress has been made, measured against performance indicators and demonstrated progress towards DOE goals.

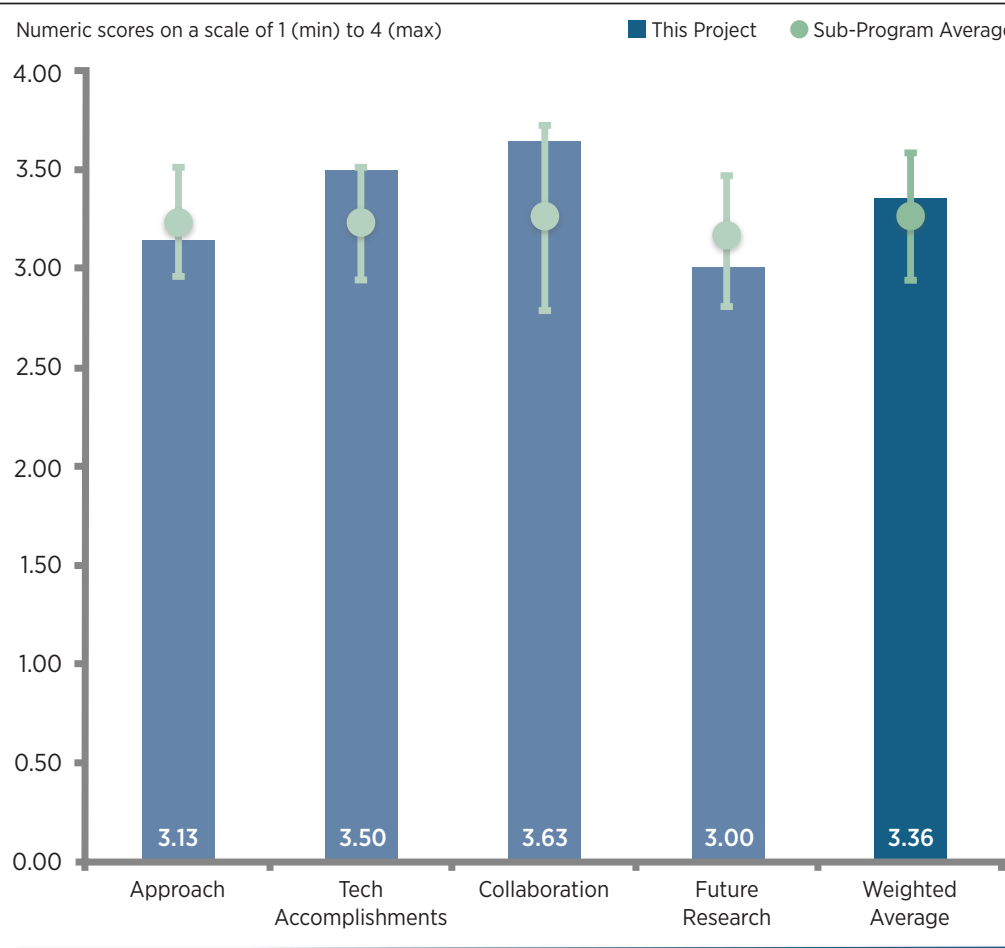

Relevant to DOE Objectives

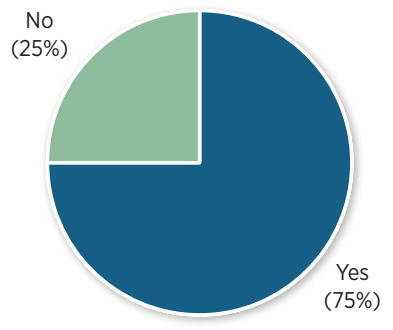

Sufficiency of Resources

Figure 6-2 - Integrated Computational Materials Engineering Approach to Development of Lightweight 3GAHSS Vehicle Assembly: Lou Hector (United States Automotive Materials Partnership LLC) - Lightweight Materials 


\section{Reviewer 1:}

The reviewer was impressed with the progress and accomplishments to date.

\section{Reviewer 2:}

The reviewer said once again, accomplishments and progress toward the goals are impressive. Nonetheless, a lot of work remains.

\section{Reviewer 3:}

The reviewer commented that the stated meso-scale model was in agreement with experimental results, but the presentation never identified how closely it meets the objective of validation within $15 \%$ of experiments. The new procedure to measure retained austenite would be very beneficial for production purposes. The reviewer remarked that more details about this would be interesting to show the relation between testing and model validation.

The reviewer pointed out that the forming simulation and validation of Task 3 never stated how closely the models were. The project appears to be able to produce the components needed for validation, just need the results and how closely it achieves the goals. The reviewer commented that the design optimization results are very promising going forward and should be able to achieve the stiffness requirements before program ends.

\section{Reviewer 4:}

The reviewer said that progress relative to alloy development is very good, but cautioned that commercial application to realize 35\% mass reduction may not be achievable. We can replace DP980 with a higher-strength material with increased formability with minimal mass reduction potential.

\section{Question 3: Collaboration and coordination with other institutions.}

\section{Reviewer 1:}

The reviewer said that collaboration within this project is extensive and appears to be harmonious, productive and worthwhile in the achievement of project goals.

\section{Reviewer 2:}

The reviewer applauded great usage of leading experts within the industry to form cross-functional teams. Utilizing cross-functional teams provides a checks and balances for each task and allows each entity to bring their expertise into the project to overcome barriers and reach goals. The reviewer said great usage of collaboration.

\section{Reviewer 3:}

The reviewer remarked that the collaboration on coordination is excellent.

Question 4: Proposed future research-the degree to which the project has effectively planned its future work in a logical manner by incorporating appropriate decision points, considering barriers to the realization of the proposed technology and, when sensible, mitigating risk by providing alternate development pathways.

\section{Reviewer 1:}

The reviewer said that time did not allow for a full discussion of future work, but it did appear that a good plan has been developed going forward.

\section{Reviewer 2:}

The reviewer commented that this is obviously an industry-driven effort, and it shows in the planning. The reviewer expressed concern that this material might be used for conformal hydrogen $\left(\mathrm{H}_{2}\right)$ storage on the vehicle. A definitive declaration from the developers on the compatibility with $\mathrm{H}_{2}$ and natural gas would be useful to avoid a compatibility incident.

\section{Reviewer 3:}

The reviewer said that the tasks laid out for the future work will lead to a valid solution and hopefully achieve 
goals set forth for the project. The reviewer said that very little information was provided about how or what tasks are involved in the model calibration. The reviewer said that this - meeting $15 \%$ validation - is a very important aspect of the program, and very little evidence was presented that this goal will or can be met.

\section{Reviewer 4:}

The reviewer commented that the plan needs be revised to address the mass reduction potential.

\section{Question 5: Does this project support the overall DOE objectives of petroleum displacement? Why or why not?}

\section{Reviewer 1:}

The reviewer commented that this activity will help reduce vehicle operating cost and increase safety.

\section{Reviewer 2:}

The reviewer said yes, this project is very well integrated with DOE's program goals.

\section{Reviewer 3:}

The reviewer said that 3 GAHHS will enable DOE goals to be met if this project is successful. Two new materials are already developed that are very close to meeting all DOE goals.

\section{Reviewer 4:}

The reviewer said that no mass is saved, and requires heat treatment.

\section{Question 6: Resources: How sufficient are the resources for the project to achieve the stated milestones in a timely fashion?}

\section{Reviewer 1:}

The reviewer asserted that resources amazingly appear to be adequate given how big this actually has become.

\section{Reviewer 2:}

The reviewer said that the team put together should be able to achieve the milestones laid out. Model calibration could be an issue as newer materials are developed. 
Validation of Material Models for Crash Simulation of Automotive Carbon Fiber Composite Structures (VMM): Libby Berger (General Motors) - Im084

Presenter

Omar Faruque, Ford Motor Company

\section{Reviewer Sample Size}

A total of six reviewers evaluated this project.

\section{Question 1: Approach to performing the work-the degree to which technical barriers are addressed, the project is well-designed, feasible, and integrated with other efforts.}

\section{Reviewer 1:}

The reviewer remarked that the approach to this work is outstanding. The program team benchmarked a design (steel bumper system) from a current vehicle, developed a new design using composites, manufactured and assembled the new design, tested the bumper system, tested the system using nondestructive evaluation methods, and plans to compare to analytic predictions.

\section{Reviewer 2:}

The reviewer said that the approach is good. This person observed that various fabrication and assembly methods to manufacture an automotive component are being addressed and tested as a vehicle subsystem.

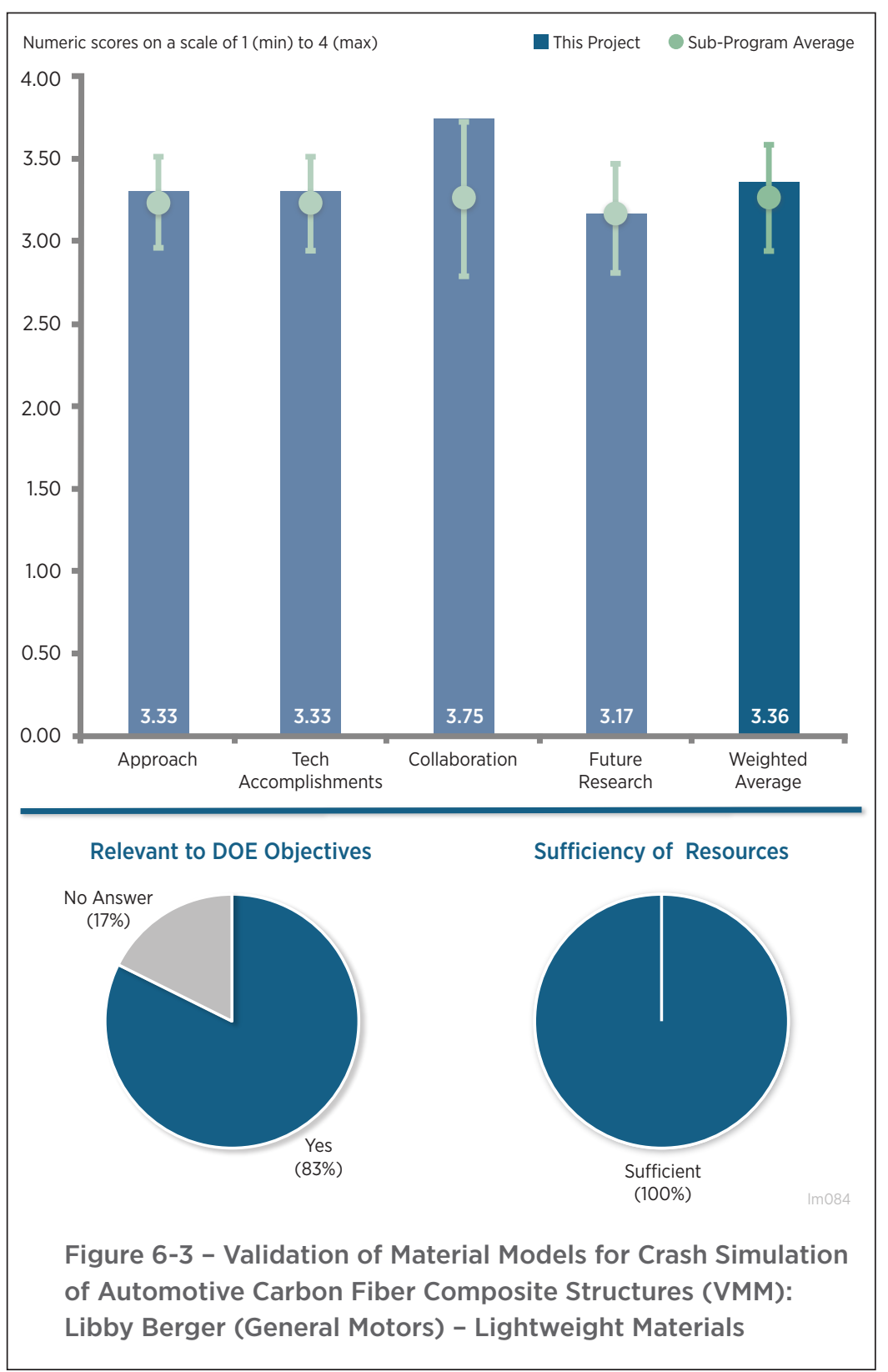

\section{Reviewer 3:}

The reviewer found that overall, the approach is excellent. It would be good to include manufacturing process simulations in the validation models, not only at coupon level but also at component level.

\section{Reviewer 4:}

The reviewer exclaimed that the experimental approach is excellent. The project established the metal values and/or set goals for composites, test composites, and comparison to predictive models. The reviewer noted that the CF processing must be suitable for 100,000/year. The reviewer asked how well results will translate to other geometries and account for other composite manufacturing methods. This person also inquired about the CF tow that was used. The reviewer assumed 12,000, and queried how CF tow size impacts predictive models. The reviewer asked what the ability is to predict 12-layer versus 24 layers. The reviewer observed that parts used were not made with production processes - translation of model validation to production-ready produced components instead of prototypes. The reviewer suggested extending material investigation to include thermoplastic non-woven as an alternative to sheet molding compound (SMC). 


\section{Reviewer 5:}

The reviewer remarked that the authors have done a comprehensive job in developing a work plan that serves the important purpose of validating dynamic crash simulations against experimental results. The use of multiple manufacturing methodologies combined with integrated bonded assemblies is an important step towards a reliable predictive capability.

The reviewer said that interrogating several different theoretical models and calibrating these tools demonstrates a comprehensive understanding of the range of technologies available and improves the usefulness of this work. The reviewer understood that this cannot be all-encompassing, but commented that the use of quasi-isotropic laminates limits the usefulness of the validation effort. Failure modes have a significant effect on energy absorption and the predictive models must include the physics to capture these effects. The reviewer remarked that the work would be strengthened by acknowledging this and either including experimental and analytical work, or recommending and proposing follow-on efforts to validate predictive models for a range of laminate architectures and failure modes.

\section{Reviewer 6:}

The reviewer needed to know a lot more about the joining and highlighted that it was barely mentioned. The reviewer understood that there are intellectual property (IP) issues there, but explained that there are a lot of elements of non-competitive nature and that these should be openly shared.

\section{Question 2: Technical accomplishments and progress toward overall project and DOE goals-the degree to which progress has been made, measured against performance indicators and demonstrated progress towards DOE goals.}

\section{Reviewer 1:}

The reviewer said that the technical accomplishments of this project are excellent and make significant progress towards DOE goals. The reviewer summarized the following accomplishments: correlation analysis of baseline steel to predictions; calibration of material models for thermoset materials; calibration of PAM-CRASH MAT131 material model; calibration of Northwestern University microplane material model; University of Michigan drop tower testing for model calibration and joining configuration; ESI predicted NCAP load case for composite bumper system; manufacturable design of composite bumper system; fabrication of composite bumper system; and NDE of adhesive bonding and joints.

\section{Reviewer 2:}

The reviewer remarked that the extensive level of work accomplished is impressive. The authors discussed the importance of including the effects of manufacturing variance, but little appears in the work accomplished that assesses the ability to capture the effects of variance in manufacturing within predictive models.

\section{Reviewer 3:}

The reviewer said that it appears the project is proceeding as planned.

\section{Reviewer 4:}

The reviewer commented that the progress is pretty good, and added that it would be good to include the NDE results in the model validation.

\section{Reviewer 5:}

The reviewer listed C-channel; continuous fiber and SMC ribs; and non-primary structure. The reviewer also noted crush cans. The reviewer said that the project contributes to understanding the current codes, but the new project is to validate production components that are cost effective relative to metal options. According to the reviewer, key questions include whether the technology is production ready with predictable results, and whether it can be afforded.

\section{Reviewer 6:}

The reviewer observed excellent progress, but expressed skepticism regarding whether the project team will finish as stated. To this reviewer, work remained to be done before the official end of the project. 


\section{Question 3: Collaboration and coordination with other institutions.}

\section{Reviewer 1:}

The reviewer said that collaboration and coordination are well-balanced and coordinated across the supply chain, including with government, industry, and academia. The reviewer pointed out that 14 different companies and organizations were involved with the project. Material suppliers, tier suppliers, and OEMs are involved and contribute to characterization, modeling, testing of the materials, and applications.

\section{Reviewer 2:}

The reviewer observed outstanding outreach to partners and other contributing suppliers.

\section{Reviewer 3:}

The reviewer said that the project leverages the respective strengths from a variety of competent stakeholders, including OEMs, academic institutions, software developers (to support predictive modeling), Tier 1 suppliers to provide manufacturing support, and material suppliers. The reviewer commented that this was very well done.

\section{Reviewer 4:}

The reviewer observed excellent collaboration with university groups. The University of Michigan, Northwestern, and Wayne State were each responsible for unique tasks.

\section{Reviewer 5:}

The reviewer would have liked to see more variety among the companies. The reviewer understood that IP issues may be a problem, but at some points, there are a lot of elements that are pre-competitive, and those elements have to be shared throughout the industry.

\section{Reviewer 6:}

The reviewer said none.

Question 4: Proposed future research-the degree to which the project has effectively planned its future work in a logical manner by incorporating appropriate decision points, considering barriers to the realization of the proposed technology and, when sensible, mitigating risk by providing alternate development pathways.

\section{Reviewer 1:}

The reviewer detailed that the proposed future work is very specifically laid out with a completion date targeted for later in 2016 and report writing in 2017. The reviewer noted that completing the bumper system testing, comparing results to predictions, and evaluating thermoplastic solutions and NDE methods are planned for the rest of 2016.

\section{Reviewer 2:}

The reviewer thought that the proposed future research was very good, but would have liked to see a future work proposal that demonstrates if the computer-aided engineering (CAE) results apply to other high-volume production processes that have attractive business case relative. The technology will not be used if the other technologies are more attractive. The reviewer said that future work needs to include validation of predictive codes on parts that can be produced at high volume and with attractive business cases.

\section{Reviewer 3:}

The reviewer said that the proposed work is responsive to the overall project objectives and is sufficient to meet those goals. As the reviewer noted previously, the overall strength of the project would be improved by evaluating a range of fiber architectures (e.g., fiber dominated and matrix dominated) and shedding light on the ability of predictive models to capture the difference in performance as a function of changing failure modes. The reviewer said that shedding more light on the role of NDE methodologies on predictive analysis and effects of manufacturing variance would further strengthen the value of the proposed future research. 


\section{Reviewer 4:}

The reviewer said that future work includes only reporting test results. It appeared to this person that there is no follow-on interest. Early stage application like the bumper application are key steps to achieve commercialization, the objective.

\section{Reviewer 5:}

The reviewer emphasized that because the project is almost over, it is difficult to assess whether the remainder is good, or better. In the case of this particular project, this reviewer suggested that there should be another option (e.g., Does Not Apply) describing that the project has ended.

\section{Reviewer 6:}

The reviewer referenced prior comments.

\section{Question 5: Does this project support the overall DOE objectives of petroleum displacement? Why or why not?}

\section{Reviewer 1:}

The reviewer commented that the work done on this project is directly applicable to DOE objectives and can be translated to other application areas on the vehicle that can benefit from the strength, stiffness, impact, and lightweighting benefits of thermoplastic and thermoset composites.

\section{Reviewer 2:}

The reviewer said that mass reduction is relevant.

\section{Reviewer 3:}

The reviewer pointed out that any weight-saving is going a long way toward the DOE goals.

\section{Reviewer 4:}

The reviewer said that validating predictive methods, particularly in the simulation of crash events for automotive design, is a prerequisite of incorporating high specific property materials like CF reinforced polymers into transportation systems. Without a high level of confidence in the fidelity of these methods, CF reinforced polymers will not make it onto future platforms. Thus, the reviewer concluded that the overall DOE goals of reducing petroleum based fuel use is predicated on success of this program and others like it.

\section{Question 6: Resources: How sufficient are the resources for the project to achieve the stated milestones in a timely fashion?}

\section{Reviewer 1:}

The reviewer said that the resources applied towards this program are sufficient to complete the goals and objectives outlined in the program. The project work should be completed in 2016 with report writing scheduled for 2017.

\section{Reviewer 2:}

The reviewer observed a good number of resources that appear well coordinated.

\section{Reviewer 3:}

The reviewer commented that the level of effort, resources expended, and results obtained are consistent. Resourcing appears entirely appropriate for this ambitious and important work.

\section{Reviewer 4:}

The reviewer reiterated that when a project is toward the end, there should be a statement of whether there are enough funds to finish the project (how much in dollars). 
Collision Welding of Dissimilar Materials by Vaporizing Foil

Actuator: A Breakthrough Technology for Dissimilar Materials Joining: Glenn Daehn (Ohio State University) - Im086

Presenter

Glenn Daehn, Ohio State University

\section{Reviewer Sample Size}

A total of four reviewers evaluated this project.

Question 1: Approach to performing the work-the degree to which technical barriers are addressed, the project is well-designed, feasible, and integrated with other efforts.

\section{Reviewer 1:}

The reviewer observed a good approach at a coupon level to demonstrate feasibility.

\section{Reviewer 2:}

The reviewer said that the project shows promise, and a good deal of basic science work has been done, but additional detail on deployment with commercial partners would have been useful.

\section{Reviewer 3:}

The reviewer noted an interesting joining technique with very limited applicability. This person opined that there are possible aerospace applications, and expressed certainty that there are no high or intermediate applications.

\section{Question 2: Technical accomplishments and progress toward overall project and DOE} goals-the degree to which progress has been made, measured against performance indicators and demonstrated progress towards DOE goals.

\section{Reviewer 1:}

The reviewer observed good progress relative to timeline.

\section{Reviewer 2:}

The reviewer remarked that the group appears to have made significant progress toward the project goals and this is commendable. The reviewer questioned how realistic it is to see this process as nearly ready for commercialization. The reviewer thought that a great deal of work on fatigue, corrosion, and joint design will be required before this interesting technology could be used on an actual vehicle. For example, no curved part/joint geometries have been investigated and there are a range of workplace issues such as noise, evolved gas and soot that will require a good 
deal of work prior to deployment. Nonetheless, according to the reviewer, this is an interesting and potentially quite useful technology that does warrant ongoing investigation.

\section{Reviewer 3:}

The reviewer said that materials systems seem to be randomly chosen with no apparent final application in mind. The reviewer commented that technical accomplishments and progress are good for an R\&D project, but observed no real immediate applications.

\section{Reviewer 4:}

The reviewer said that it is still not clear how to maintain a one millimeter gap next to welded spots.

\section{Question 3: Collaboration and coordination with other institutions.}

\section{Reviewer 1:}

The reviewer said that collaboration efforts are good, a pathway to commercialization with an equipment provider and OEM are recognized.

\section{Reviewer 2:}

The reviewer said that the issue of interactions was a key concern in the 2015 review, and the group appears to have addressed this and engaged more effectively.

\section{Reviewer 3:}

The reviewer observed a good mix of external partners, and suggested a few more from the transportation industry.

Question 4: Proposed future research-the degree to which the project has effectively planned its future work in a logical manner by incorporating appropriate decision points, considering barriers to the realization of the proposed technology and, when sensible, mitigating risk by providing alternate development pathways.

\section{Reviewer 1:}

The reviewer said that the plan is to continue towards commercial application, and the future is bright for this technology.

\section{Reviewer 2:}

The reviewer observed a good plan to continue on the path to a commercially viable process, although the issue of joint geometry needs additional attention.

\section{Reviewer 3:}

The reviewer suggested more focus on a targeted minimum joint strength rather than just reporting random and/or various mixed metal combinations. The reviewer reiterated pulling in some industry partners to point the project team to mix materials systems of interest.

\section{Question 5: Does this project support the overall DOE objectives of petroleum displacement? Why or why not?}

\section{Reviewer 1:}

The reviewer remarked that joining dissimilar materials is a core enabling technology for lightweighting vehicle structures, and asserted that the project is aligned with DOE program goals.

\section{Reviewer 2:}

The reviewer commented that this technology is an enabler to lightweighting, specifically metamaterial joining. 


\section{Reviewer 3:}

The reviewer noted the project team's path is relevant as related to mixed metal; albeit high level and loose, it may result in a potential future application.

Question 6: Resources: How sufficient are the resources for the project to achieve the stated milestones in a timely fashion?

\section{Reviewer 1:}

The reviewer said that resources appear to be adequate, including additional investment from the state of Ohio and increased involvement from industrial sponsors (Honda, Coldwater Machine, and Magna International).

\section{Reviewer 2:}

The reviewer said that the project is appropriately funded. 


\section{Active, Tailorable Adhesives for Dissimilar Material Bonding, Repair and Assembly: Mahmood Haq (Michigan State University) - Im087}

\section{Presenter}

Mahmood Haq, Michigan State

University

\section{Reviewer Sample Size}

A total of four reviewers evaluated this project.

\section{Question 1: Approach to performing the work-the degree to which technical barriers are addressed, the project is well-designed, feasible, and integrated with other efforts.}

\section{Reviewer 1:}

The reviewer said that the overall approach is excellent. This is exciting fundamental work that will have far reaching impact on the composites re-engineering. The reviewer suggested studying the temperature inside the microwave with and without graphene and accordingly make a work plan to engineer the chemistry. The reviewer also suggested investigating the time dependency of melting the adhesive with and without graphene so as to learn the impact of graphene on the bondability of the synthesized adhesive.

\section{Reviewer 2:}

The reviewer said that although not specifically identified as an approach, the Summary of Progress on Slide five shows an approach of material development and optimization for a process using thermoplastic and graphene nanoparticles to produce active adhesive pellets and films followed by laboratory evaluations and design tools and database development that can be applied to four different substrates and four different thermoplastic adhesives. The reviewer said that the uniqueness of the approach is in the use of graphene nanoparticles to overcome some of the technical barriers typically associated with bonding metals to composite materials. The milestone descriptions support this approach. The reviewer commented that the results of this research integrate well with other efforts that are currently ongoing to address the technology gaps identified in the EERE VTO Workshop Report titled, "Light-Duty Vehicles Technical Requirements and Gaps for Lightweight and Propulsion Materials February 2013."

\section{Reviewer 3:}

The work being done at Michigan State University (MSU) on reversible bonded joints is very important for the automotive industry. The approach can be better defined in order to improve the understanding of the overall approach. 
Question 2: Technical accomplishments and progress toward overall project and DOE goals - the degree to which progress has been made, measured against performance indicators and demonstrated progress towards DOE goals.

\section{Reviewer 1:}

The reviewer said that a significant technical accomplishment presented was that the lap-shear strength of a specific functionalized adhesive was improved by more than $30 \%$ over a pristine adhesive with only a 3 weight percent addition of graphene nanoparticles. Also, microwave activated joints showed better performance over joints formed with conventional ovens. This resulted in three types of tailorable metal-composite joints that can be bonded, disbonded, and re-assembled. The reviewer remarked that this is very significant if the process can be commercialized. The reviewer commented that another significant achievement was the experimental validation of a nano-, meso-, and macro-scale model to predict the structural behavior beyond the experimental results of the project.

\section{Reviewer 2:}

The reviewer remarked that excellent progress has been made on this project: addition of nano-graphene particles into thermoplastic substrates and the development of adhesive films; production of various test coupons and joints; evaluation of the results; nano-graphene functionalization has been shown to improve properties; and microwave bonding improves performance versus conventional thermal method.

\section{Reviewer 3:}

The reviewer said that excellent progress has been made so far and referenced prior comments.

\section{Question 3: Collaboration and coordination with other institutions.}

\section{Reviewer 1:}

The reviewer stated that the list of collaborators includes two research and development organizations and an Army activity that is reviewing the project's progress and providing guidance on relevant materials for automotive applications. This reviewer observed no collaboration with supply chain activities where bonding is needed for their products or automotive original equipment manufacturers who would be the technology transfer entities. Although the presenter stated that their input is being incorporated in this project and future work will directly involve them, the project only has one year left and that is very little time to get an automobile manufacture or their suppliers directly involved where they will accept the technology and process being developed.

\section{Reviewer 2:}

The reviewer said that MSU collaborates formally with Eaton on this program, but has also directly worked with at least four different automobile companies, many different composite and material suppliers, government agencies, and industry associations.

\section{Reviewer 3:}

The reviewer said none.

Question 4: Proposed future research-the degree to which the project has effectively planned its future work in a logical manner by incorporating appropriate decision points, considering barriers to the realization of the proposed technology and, when sensible, mitigating risk by providing alternate development pathways.

\section{Reviewer 1:}

This reviewer explained that the plan for future work includes corrosion studies, optimization work on processing, thermal testing, re-assembly and repair work, and efforts in NDE. The reviewer commented that additional evaluation on commercialization methods for large automotive applications would be beneficial for this type of joining method. 


\section{Reviewer 2:}

The reviewer said that the only proposed future research presented was for the remainder of the current research period. The future work described is more upcoming tasks and how they will be accomplished rather than future research needed. This reviewer opined that milestones efforts show that the upcoming work is effectively planned in a logical manner by incorporating appropriate decision points, but do not necessarily coincide with challenges and barriers pointed out later in the presentation. The reviewer said that some significant challenges are addressed, such as processing problems with thermoplastics and equipment needed to handle larger sample sizes as would be the case with industrial applications.

\section{Reviewer 3:}

The reviewer referenced prior comments.

\section{Question 5: Does this project support the overall DOE objectives of petroleum displacement? Why or why not?}

\section{Reviewer 1:}

The reviewer said that the work being done at MSU on reversible bonded joints is directly applicable to DOE's goal of building lightweight vehicles and enables the use of multi-materials by automotive companies.

\section{Reviewer 2:}

The reviewer observed that Slide four of the presentation showed eight key technical gaps for light-duty vehicles systems that this project addresses directly. These include lack of technology and fast, robust, and reliable processes for joining dissimilar materials; lack of modeling, simulation, and predictive engineering design and modeling tools; lack of high-volume manufacturing capacity; and design knowledge and databases, which are contained in the Office of Energy Efficiency and Renewable Energy (EERE) VTO Workshop Report titled, "LightDuty Vehicles Technical Requirements and Gaps for Lightweight and Propulsion Materials February 2013."

\section{Reviewer 3:}

The reviewer said absolutely, because composites repair is a growing need for a futuristic transportation sector that has started to advocate composites in the mainstream.

\section{Question 6: Resources: How sufficient are the resources for the project to achieve the stated milestones in a timely fashion?}

\section{Reviewer 1:}

The reviewer remarked that this is a three-year research project to improve a bonding technique for $\$ 600,000$ (\$200,000 per year average). All previous milestones and go-no go decision points have been successfully met on schedule with the funding provided with only three milestones to meet in the final year of the project. The reviewer commented that the project is well managed and, when received, funding (approximately $\$ 263,000$ ) will be sufficient to achieve the stated milestones.

\section{Reviewer 2:}

The reviewer said that the resource levels for this work appear appropriate for this project. Additional work and a future project(s) could branch from this work. 


\section{High-Strength Electroformed Nanostructured Aluminum for Lightweight Automotive Applications: Robert Hilty (Xtalic Corporation) - Im089}

\section{Presenter}

Robert Hilty, Xtalic Corporation

\section{Reviewer Sample Size}

A total of four reviewers evaluated this project.

\section{Question 1: Approach to performing the work-the degree to which technical barriers are addressed, the project is well-designed, feasible, and integrated with other efforts.}

\section{Reviewer 1:}

The reviewer said that the approach is good, and emphasized that the change to clad onto traditional sheet is key to the cost/benefit relationship. This person further noted that the true application is thin layer, but the funding opportunity announcement topic forces to thick layer.

\section{Reviewer 2:}

The reviewer said that this looks like a really interesting project on an important issue, but the reviewer admitted that the feasibility of the plating process to build up a structural element is new. The reviewer suspected that a lot of testing will need to be done to establish this technology.

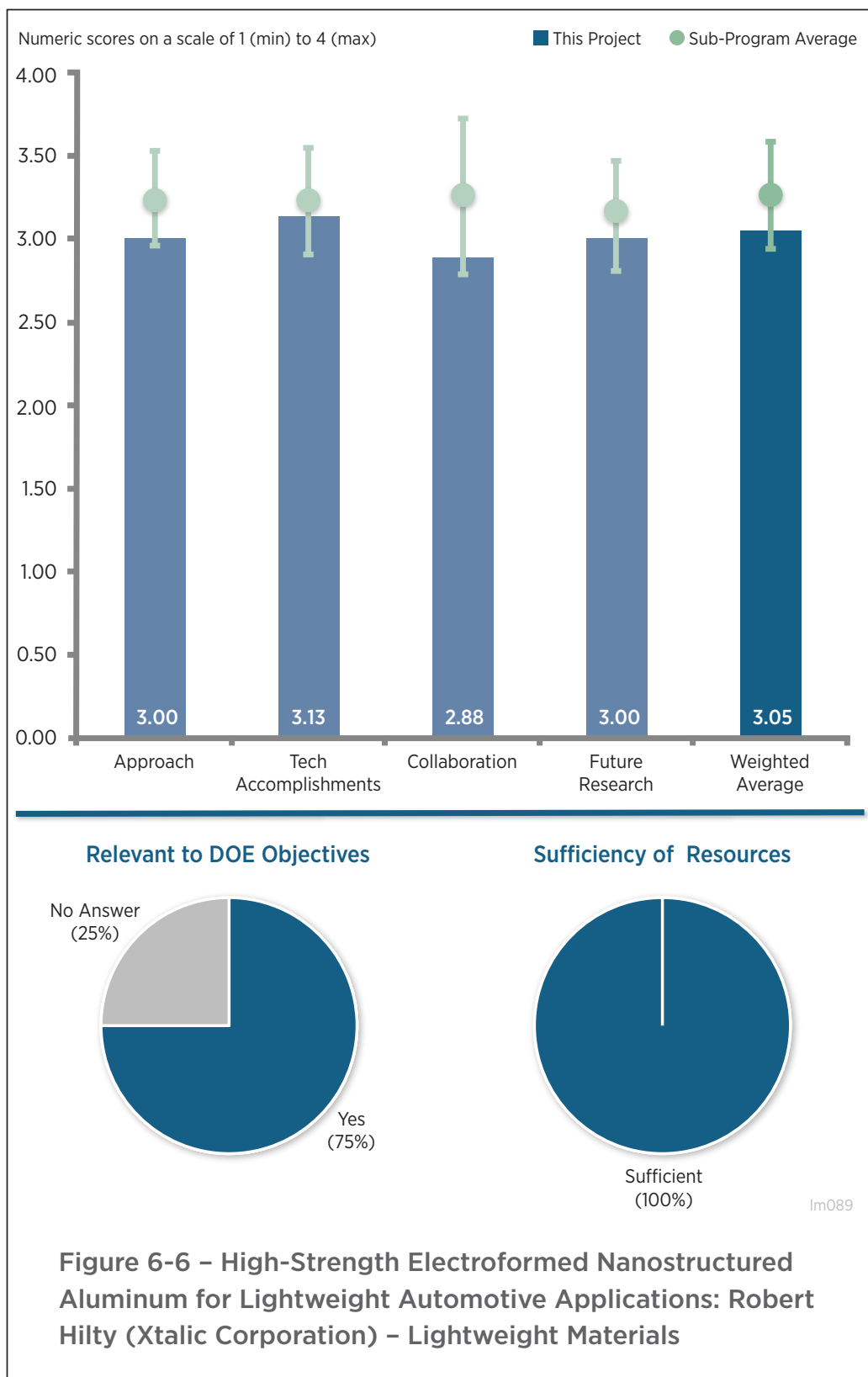

\section{Reviewer 3:}

The reviewer commented that manufacturing advanced materials is said to be a barrier, yet the approach starts with a current material and makes it better to meet an end goal. While this approach should meet final project goals, this is not a new material, only a surface treatment. Cost is also a barrier defined by the project, yet starting with a thin rolled product caused this reviewer to think the process is inherently more expensive because the starting material would be more expensive. Every reduction in thickness of a rolled product increases product cost. This just seems to contradict general manufacturing rules as the proposer states a layered structure improves cost without sacrificing performance. The reviewer added that the use of go/no-go is acceptable.

\section{Reviewer 4:}

The reviewer said that the approach is good for this stage. However, the reviewer wanted to see more information on potential methods to scale up this technology to large volume production. The reviewer said that the efforts on the sheet/plating work appears solid for this project. Further, this reviewer indicated that the manufacturing time sounds good. 


\section{Question 2: Technical accomplishments and progress toward overall project and DOE goals-the degree to which progress has been made, measured against performance indicators and demonstrated progress towards DOE goals.}

\section{Reviewer 1:}

The reviewer commented on-time with progress.

\section{Reviewer 2:}

The reviewer explained that it appeared that good progress has been made against a number of key goals, but it appeared that a good deal of work remains, including cost modeling, thermal stability in coatings processes, and perhaps some work in design variables that will be required for modeling of actual vehicle parts.

\section{Reviewer 3:}

The reviewer observed great progress on plating development for deposition layers and finding possible additives to overcome the dendrite formation and increased layer thickness. The reviewer expressed some concern about moving from rods to sheet and still meeting the project goals. The reviewer asked whether the machine places the final thickness in one pass through the bath, multiple passes are required to increase thickness, or the process just needs to slow down through the bath to increase thickness.

The reviewer commented that Slide five shows a stress/strain curve that appears to meet DOE goals with an AA6061 inner layer, but Slide 12 shows the test ran on AA3104. There needs to be a defined path for what material/materials are the substrate and what materials will meet or exceed goals.

The reviewer said that process control development is coming along nicely, though there is concern on the nondestructive testing (NDT) method presented. The current NDT method allows for $100 \mu \mathrm{m}$ thickness to be measured, yet the end product will have a thickness layer greater than 400 micrometers. The reviewer said that the presentation showcased a continuous electroforming system to develop six-inch wide samples. The reviewer remarked having a hard time believing a six-inch wide sample will have much usage in the automotive world other than for a few possible applications. The reviewer said that only a rear door side impact beam was shown as a possibility, and this seems like a lot of funding and development for just one part. The reviewer suggested possibly identifying multiple possibilities and parts where the technology can be used.

The reviewer reported that the cost model has been built and will be used to identify best opportunities to reduce manufacturing costs. However, the models were not available for review, which led the reviewer to question the process expense and how close goals are to being achieved.

\section{Reviewer 4:}

The reviewer described the strength as great, and expressed hope that the project team can get the ductility to where it is wanted for high formability. The plating results are great and are cautiously optimistic. This reviewer asked if there are any corrosion concerns.

\section{Question 3: Collaboration and coordination with other institutions.}

\section{Reviewer 1:}

The reviewer said that collaboration appears to be good.

\section{Reviewer 2:}

The reviewer observed great collaborators, solid division of assignments, and clear roles and responsibilities.

\section{Reviewer 3:}

The reviewer commented that partners meet the minimum needed to accomplish goals. The team will be able to meet the goals as they are laid out.

\section{Reviewer 4:}

The reviewer said that the project does not require significant collaboration. 
Question 4: Proposed future research-the degree to which the project has effectively planned its future work in a logical manner by incorporating appropriate decision points, considering barriers to the realization of the proposed technology and, when sensible, mitigating risk by providing alternate development pathways.

\section{Reviewer 1:}

The reviewer said that future plans going forward appear to be realistic and focused on the goal.

\section{Reviewer 2:}

The reviewer said that future work is to demonstrate feasibility, and that starting with sheet substrate is a very positive change in approach. The reviewer recommended that a lifecycle analysis needs be conducted. The side door beam may not be the target application, but due to roll width constraints and acceptable proof of principle.

\section{Reviewer 3:}

The reviewer wanted to see more details on the next steps, as well as how the cost model and cost estimates will be done and hopefully influence future plans. The reviewer commented that the efforts on the wider sheet will be a good addition.

\section{Question 5: Does this project support the overall DOE objectives of petroleum displacement? Why or why not?}

\section{Reviewer 1:}

The reviewer asserted that high strength, high ductility Al enables lightweight vehicles.

\section{Reviewer 2:}

The reviewer indicated that $\mathrm{Al}$ is clearly an important material at present and, if cost, strength, and predictability constraints can be addressed, it has a very promising future for wider application in lightweighting vehicles. Thus, according to the reviewer, the project is definitely aligned with DOE program goals.

\section{Reviewer 3:}

The reviewer commented that the real application is $\mathrm{Al}$ deposition on $\mathrm{Mg}$ substrate.

\section{Question 6: Resources: How sufficient are the resources for the project to achieve the stated milestones in a timely fashion?}

\section{Reviewer 1:}

The reviewer observed that resources look to be okay. 


\section{Vehicle Lightweighting: Mass Reduction Spectrum Analysis and Process Cost Modeling: Tony Mascarin (IBIS Associates) - Im090}

\section{Presenter}

Tony Mascarin, IBIS Associates

\section{Reviewer Sample Size}

A total of six reviewers evaluated this project.

\section{Question 1: Approach to performing the work-the degree to which technical barriers are addressed, the project is well-designed, feasible, and integrated with other efforts.}

\section{Reviewer 1:}

The reviewer said that this project clearly outlined the tasks and overall approach to reviewing, identifying, and prioritizing weight reduction path scenarios; collecting data and updating models to develop model scenarios; and refining and analyzing the models in order to present results.

\section{Reviewer 2:}

The reviewer did not believe the approach taken will provide meaningful output relative to project objectives. Categorization of the barriers and extracting the cost data provided in the previous literature studies in not optimum for generating useful information.

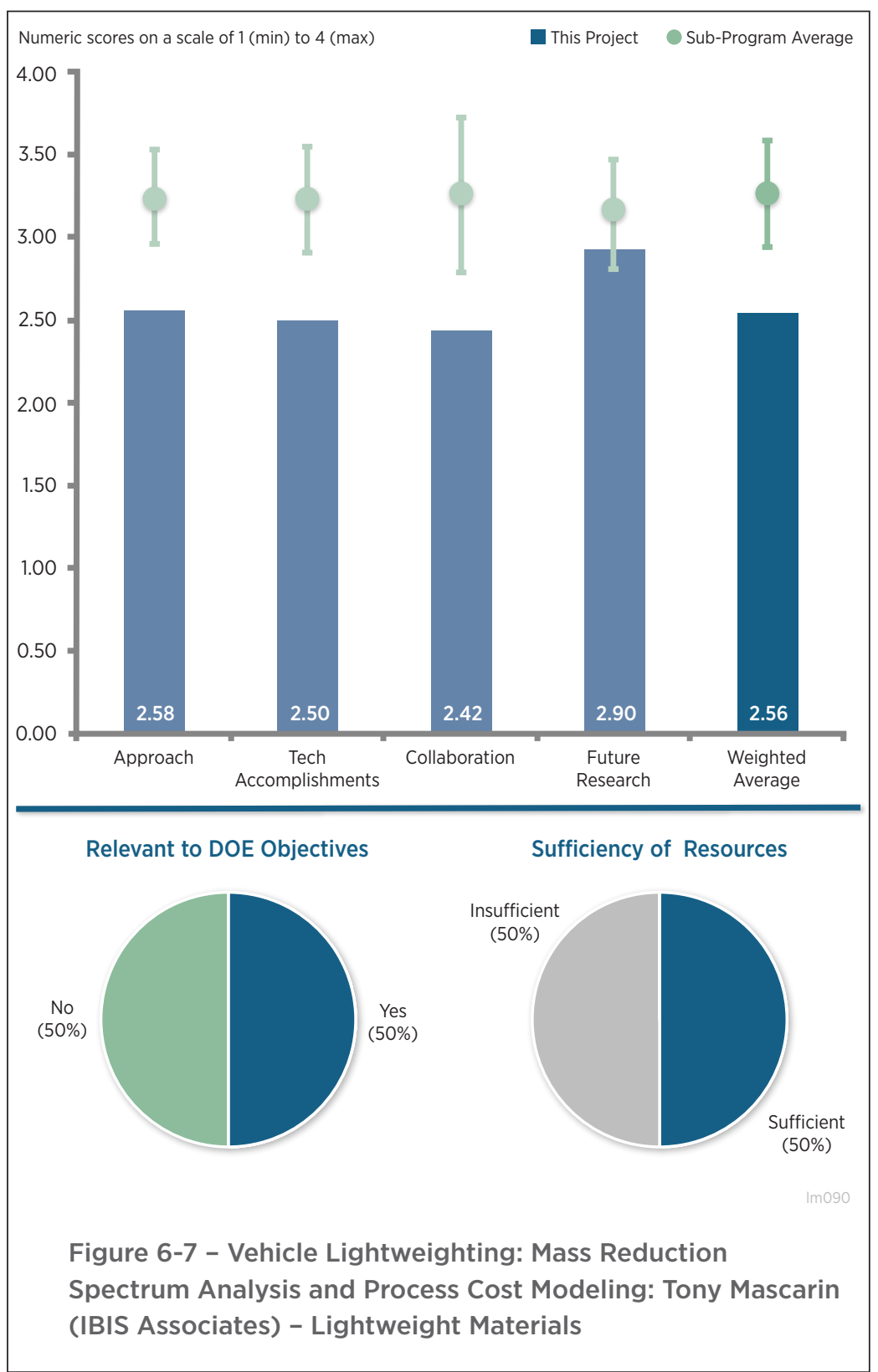

\section{Reviewer 3:}

The reviewer said that the assembly of data from previous vehicle lightweighting initiatives is a useful approach to evaluate their effectiveness and establish a comparative cost basis for each material technology, forming technology, and assembly technology. According to the reviewer, it is unfortunate additional projections of cost based on hybrid composite technologies and the potential opportunities of reducing total part count through part integration has not been considered.

\section{Reviewer 4:}

The reviewer did not think that the project really was additive to the question on lightweighting costs. Essentially, the project team took all the lightweight evaluation projects and tried to homologate the outputs and provide a sensible, holistic view on attractiveness. The reviewer did not think it worked. 
The reviewer remarked that all of the projects used for this study had some basic flaws in the assumptions, which were increased by trying to map them into a unified report. It seemed like an overly good idea, but the results did not seem realistic, as noted by the animated reviewer response.

\section{Reviewer 5:}

The reviewer expressed concern that interpretation of a DOE-funded cost study based on a literature review associated with various studies of different baseline vehicles and assumptions is not a cost modeling effort.

\section{Question 2: Technical accomplishments and progress toward overall project and DOE goals-the degree to which progress has been made, measured against performance indicators and demonstrated progress towards DOE goals.}

\section{Reviewer 1:}

The reviewer said that the program did a fine job of assembling and analyzing available program data. The project team drew insightful conclusions and established an important set of metrics to support or justify particular strategies for vehicle lightweighting.

\section{Reviewer 2:}

The reviewer detailed that strategies for vehicle lightweighting were characterized into low-, medium-, and high risk. The costs for each strategy were obtained, either from previous work or the team's work, and then presented in the report. The debate regarding cost model accuracy and ease of implementation into vehicles will continue between organizations wishing to position these technologies and those organizations responsible for producing the end product.

\section{Reviewer 3:}

The reviewer said that technical accomplishments were not articulated well during the delivery of the presentation. Responses to reviewer questions also did not provide additional clarity on the technical content presented.

\section{Reviewer 4:}

The reviewer remarked the team did not seem to have enough technical expertise to sort through the subject matter to make a sound analysis.

\section{Reviewer 5:}

The reviewer said that the technical accomplishment is negative in value, and providing misleading information will result in legislation that has a negative impact to industry. The thought that the cost of lightweighting up to $6 \%$ is free, and from $6 \%$ to $35 \%$ can be realized at $\$ 1.25 / \mathrm{lbs}$. weight saved, was described as ludicrous by this reviewer.

\section{Question 3: Collaboration and coordination with other institutions.}

\section{Reviewer 1:}

The reviewer said that the level of resources available required a balance of collaborating institutions. The experience of Ibis and the inclusion of Energetics and the national laboratory is admirable.

\section{Reviewer 2:}

The reviewer remarked that collaboration appears satisfactory to accomplish this task, although direct interaction with OEMs, tiers, and materials suppliers would be required to more accurately capture cost and risk information.

\section{Reviewer 3:}

The reviewer commented that collaboration with the authors of various studies took place.

\section{Reviewer 4:}

The reviewer believed that this is one area that more could have been done, and cited as an example collaboration with the Ford/Cosma program on the Multi-Material Lightweight Vehicle (MMLV) program results. 


\section{Reviewer 5:}

The reviewer said that it would have been good to have included industry composites experts to help with data assessment.

Question 4: Proposed future research-the degree to which the project has effectively planned its future work in a logical manner by incorporating appropriate decision points, considering barriers to the realization of the proposed technology and, when sensible, mitigating risk by providing alternate development pathways.

\section{Reviewer 1:}

The reviewer said that this work is complete.

\section{Reviewer 2:}

The reviewer remarked that work is completed.

\section{Reviewer 3:}

The reviewer emphatically commented that no proposed future research is good.

\section{Question 5: Does this project support the overall DOE objectives of petroleum displacement? Why or why not?}

\section{Reviewer 1:}

The reviewer said that cost information is needed to make educated decisions for vehicle production, as well as in making regulatory policy. Cost is only one dimension, of course. The reviewer noted that safety implications, globalization, and consumer demand are but a few other considerations.

\section{Reviewer 2:}

The reviewer said that a comprehensive evaluation of lightweighting strategies and the incremental cost associated with their implementation is essential for providing a roadmap for future designs to follow in wringing out the last kilogram of mass possible and the lowest incremental cost. The result will be a reduction of petroleum fuel consumed to meet the nation's transportation needs.

\section{Reviewer 3:}

The reviewer said that it was not very obvious how the program is linked broadly to DOE's objectives. The presented categorization of cost/complexity were not articulated well and that is perhaps why this reviewer did not see the relevance.

\section{Reviewer 4:}

The reviewer did not believe the output of this report is useful or representative of costs and implementation of technologies.

\section{Reviewer 5:}

The reviewer said that the subject is not related to petroleum displacement.

\section{Question 6: Resources: How sufficient are the resources for the project to achieve the stated milestones in a timely fashion?}

\section{Reviewer 1:}

The reviewer said that the resources available were commensurate with the work performed. The reviewer noted that the level of resources were equivalent to approximately three-fourths of a man year's effort and this reflects the level of work accomplished by the program. 


\section{Reviewer 2:}

The reviewer said that the cost model cannot be conducted by a third party, and that only the manufacturer can conduct such. The reviewer recommended that Ford and Magna be contracted to conduct an incremental cost analysis of the 2013 Fusion, Mach I and Mach II (Body in White [BIW], Chassis \& Closures). There is interest by both parties to clarify the incremental cost by subsystem for Mach I.

\section{Reviewer 3:}

The reviewer said that this project is complete. 


\section{Laser-Assisted Joining Process of Aluminum and Carbon Fiber Components: Adrian Sabau (Oak Ridge National Laboratory) - Im097}

\section{Presenter}

David Warren, Oak Ridge National Laboratory

\section{Reviewer Sample Size}

A total of four reviewers evaluated this project.

\section{Question 1: Approach to performing the work-the degree to which technical barriers are addressed, the project is well-designed, feasible, and integrated with other efforts.}

\section{Reviewer 1:}

The reviewer noted a great approach to investigate a laser method to improve adhesive bond robustness between $\mathrm{CFCs}$ and sheet Al.

\section{Reviewer 2:}

The reviewer said that the laser assisted surface preparation approach used to obtain higher bonding strength is innovative, and could potentially be adopted in high volume automotive manufacturing in the future.

\section{Reviewer 3:}

The reviewer pointed out that it would have been great if the current state of the art in the automotive industry would have been described to fully articulate the defects and need for improvements. The BMW I3 program utilizes two different bonding technologies for their BIW construction. The BMW 7 series also utilizes a number of adhesive joining techniques to join $\mathrm{Al}$ to $\mathrm{CFC}$, and CFC to steel. The reviewer inquired about the following: how the selected joining techniques differ; what steps in the surface preparation or joining are eliminated as a result of using the suggested joining techniques; and whether it is possible to claim that the best possible adhesive is selected only based on lap-shear evaluations. The reviewer said that the project team should consider alternative joints such as cross-tension or KS2 style specimens to fully evaluate the efficiencies with the proposed work.

\section{Reviewer 4:}

The reviewer said that it would be good to have a schematic of the process and suggested either a picture, animation, or video.

Question 2: Technical accomplishments and progress toward overall project and DOE goals-the degree to which progress has been made, measured against performance

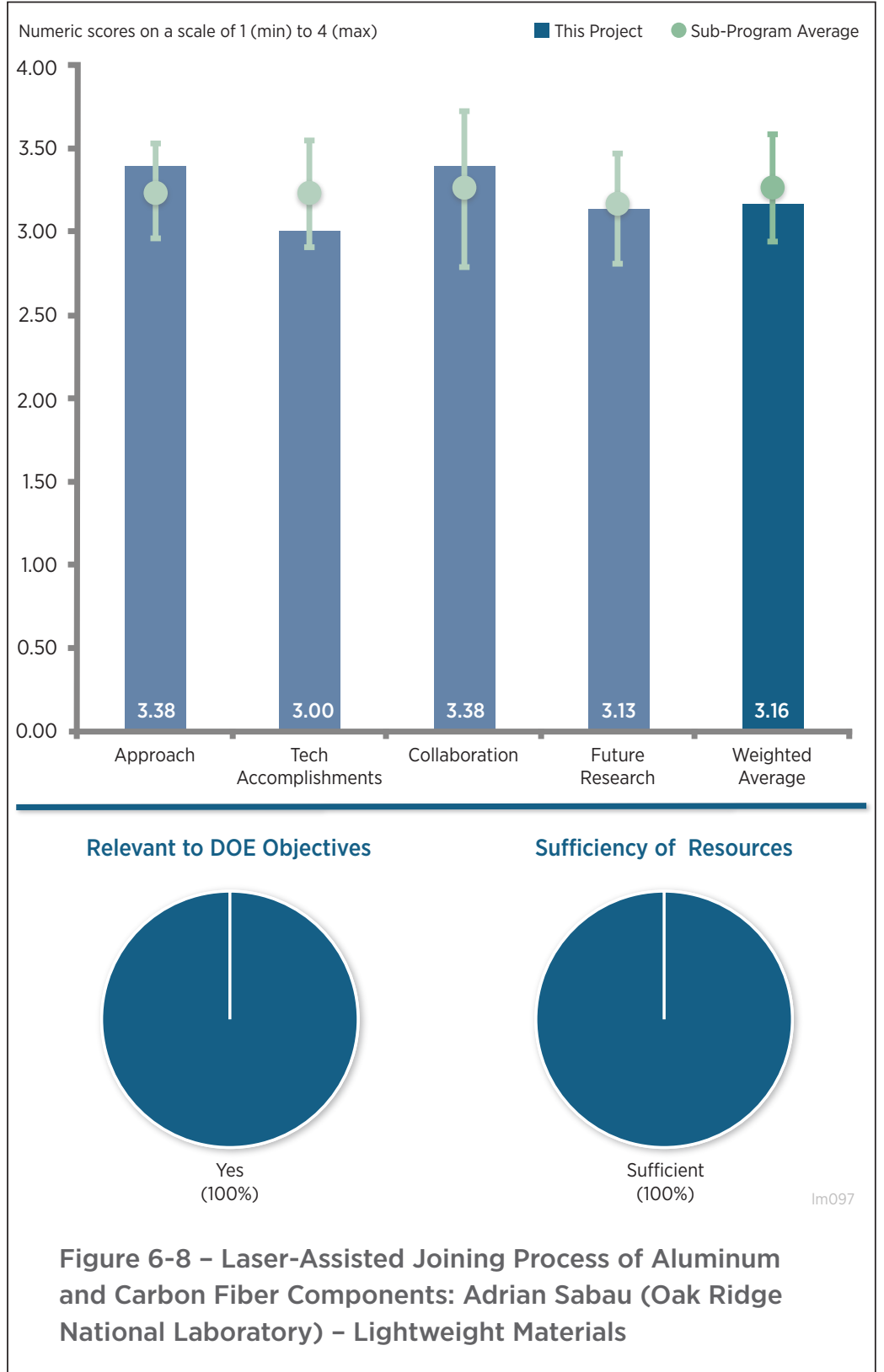




\section{indicators and demonstrated progress towards DOE goals.}

\section{Reviewer 1:}

The reviewer said that the project successfully completed DOE's and surface investigations to quantify the improvements and surface conditions.

\section{Reviewer 2:}

The reviewer remarked that different joint configurations could have been studied as the samples made for the lap joint are already available. This could have helped to understand the benefits in the modes other than shear studied in this project.

\section{Reviewer 3:}

The reviewer suggested incorporating alternative joint geometries. The reviewer also suggested that the team consider adding wedge impact peel, very simple geometry, very simple test setup, and data analysis to better understand the benefit of suggested joining on dynamic impact problems.

\section{Reviewer 4:}

The reviewer said that although the presented results are satisfactory, it would be good to make the following change so as to look at the problem more accurately. The reviewer said that the bondline thickness measurement should be substituted by contact area which is more in the laser-rastered sample than non-laser sample and then re-analyze the results to see if the same conclusions are reached. The reviewer said that technically, an electrically insulating layer is not provided because the carbon within CFRP is exposed using laser etching.

\section{Question 3: Collaboration and coordination with other institutions.}

\section{Reviewer 1:}

The reviewer noted wonderful cooperation to get the samples made, conditioned, and tested.

\section{Reviewer 2:}

The reviewer said none.

Question 4: Proposed future research-the degree to which the project has effectively planned its future work in a logical manner by incorporating appropriate decision points, considering barriers to the realization of the proposed technology and, when sensible, mitigating risk by providing alternate development pathways.

\section{Reviewer 1:}

The reviewer observed solid proposals for scale-up to volume production.

\section{Reviewer 2:}

The reviewer said that the team has a good plan in place to scale the developed process to a production intended process. Also, the tests planned for assessing the joint strength are necessary to understand the impact of laser assisted joining process in other configurations.

\section{Reviewer 3:}

The reviewer referenced prior comments.

\section{Question 5: Does this project support the overall DOE objectives of petroleum displacement? Why or why not?}

\section{Reviewer 1:}

The reviewer said that the technology developed in this project directly helps the future multi-material material designs adopted by the automotive industry for lightweighting.

\section{Reviewer 2:}

The reviewer remarked that joining will remain the number one barrier in usage of all lightweight materials. 
Reviewer 3:

The reviewer said that this is a very critical problem to be solved in the current context of multi-material implementation within the automotive sector.

\section{Reviewer 4:}

The reviewer pointed out that bonding $\mathrm{CFC}$ to $\mathrm{Al}$ is a critical joining technology for lightweight vehicles of the future.

Question 6: Resources: How sufficient are the resources for the project to achieve the stated milestones in a timely fashion?

No comments were received in response to this question. 


\section{Brazing Dissimilar Metals with a Novel Composite Foil: Tim Weihs (Johns Hopkins University) - Im098}

\section{Presenter}

Tim Weihs, Johns Hopkins University

\section{Reviewer Sample Size}

A total of five reviewers evaluated this project.

\section{Question 1: Approach to performing the work-the degree to which technical barriers are addressed, the project is well-designed, feasible, and integrated with other efforts.}

\section{Reviewer 1:}

The reviewer observed a good approach at solving issues with reactive metal oxides. Good analysis of processes between diluent and reactive zones. The approach evaluates four metal oxide compounds used in redox foils fabricated by consolidating, swaging, and rolling micron sized powders and the effects of quenching and dilution at the microstructure level. The reviewer commented that the experimental approach resulted in the selection of $\mathrm{Al}$ : copper (I) oxide: copper ( $\left.\mathrm{Al}: \mathrm{Cu}_{2} \mathrm{O}: \mathrm{Cu}\right)$ as the best candidate to use in the next level of experimentation.

\section{Reviewer 2:}

The reviewer said that this is a worthwhile project because the future vehicle will require a range of different materials to achieve greenhouse gas (GHG) and crashworthiness targets at a realistic cost. Joining these materials is therefore a core enabling technology in this reviewer's view. The approach being taken by this group appears to be realistic and systematic as the project team endeavors to find just the right formulation for its brazing foils. It appeared to this reviewer that the project team is making progress and has made progress on addressing the comments of earlier reviews. Acknowledging that it is beyond the scope of the present work, the reviewer wondered how repairs might ever be accomplished on an actual vehicle in, for example, a commercial auto body repair shop. The reviewer emphasized that this remark is not intended in any way as a criticism of the present project.

\section{Reviewer 3:}

The reviewer commented that this is a wild research/stretch approach to see the applicability of using foils for joining dissimilar metals. However, the approach lacks robustness for other performance issues such as corrosion, paint ability, surface finish, etc. The reviewer said that this is okay for such an early research project, and asked if cost or joint processing parameters were part of the project scope.

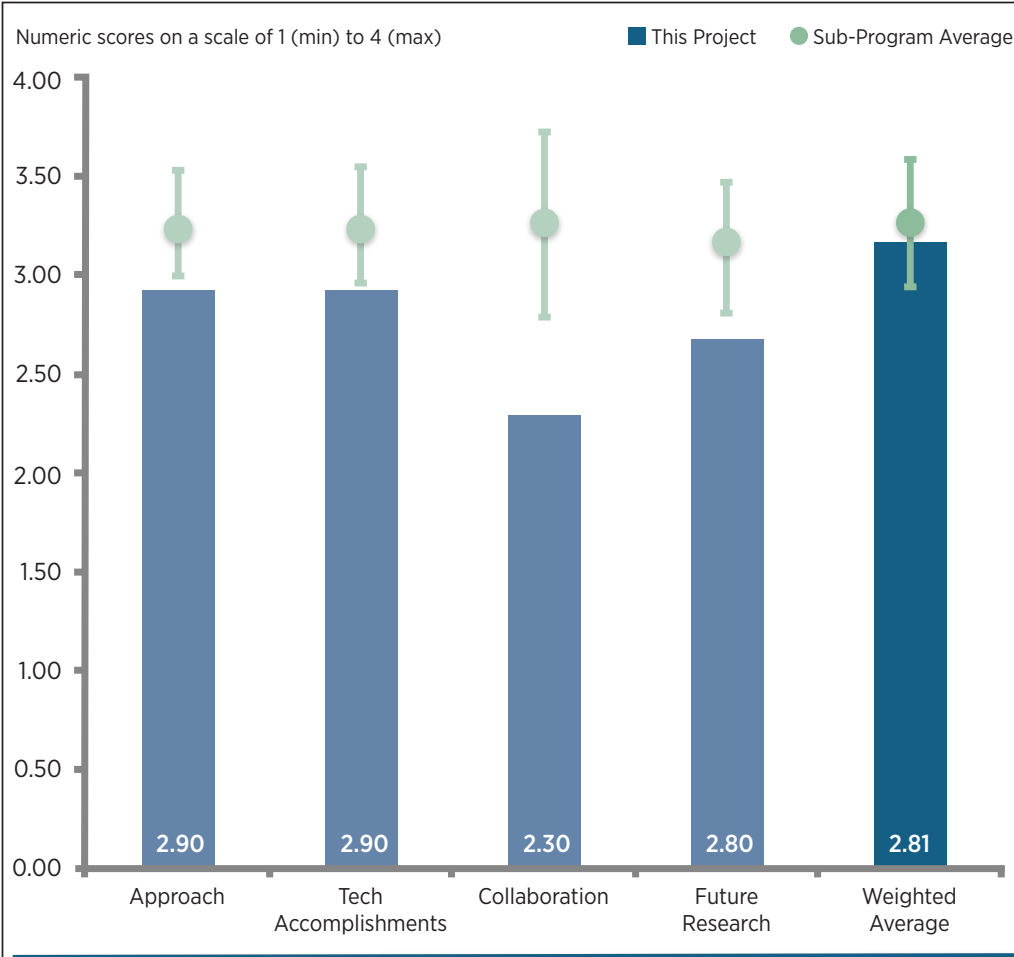

Figure 6-9 - Brazing Dissimilar Metals with a Novel Composite Foil: Tim Weihs (Johns Hopkins University) Lightweight Materials 


\section{Reviewer 4:}

The reviewer said that the approach is without potential application to transportation vehicles, and commented that it should be funded by Advanced Research Projects Agency - Energy (ARPA-E) or the National Institute of Standards and Technology (NIST).

\section{Question 2: Technical accomplishments and progress toward overall project and DOE goals-the degree to which progress has been made, measured against performance indicators and demonstrated progress towards DOE goals.}

\section{Reviewer 1:}

The reviewer commented that it appears that the group has identified key concerns in the work and appears to have a plan for addressing each one.

\section{Reviewer 2:}

The reviewer remarked that the project is progressing on track against its plan. The developments and investigations in the diluent and reactive components appear to be going on track, but the chemistry is still apparently elusive, and the lack of cost discussion in this project is disappointing. The reviewer said that the chemistry evaluations appear to guide you to the best solutions, and the additional costs need to be included in the investigations. The reviewer asked how the strengths can be increased.

\section{Reviewer 3:}

The reviewer said that the presenter did not address DOE goals; however, the technical accomplishments and progress have addressed the major technical issues for the materials being considered. The research is technically sound, but the relationship to DOE goals is weak. The reviewer remarked that experimental results have shown ways of increasing the bond strength, minimizing excessive melting, and decreasing porosity from excessive gas generation during bond formation. Investigations into ball milling resulted in increased homogeneity and reduction of heat diffusion distances, which are very good results. The reviewer said that the heat diffusion modeling efforts are beneficial to improving the technology. All of these contribute to the degree of progress to date as measured against performance indicators found in the project milestones.

\section{Reviewer 4:}

The reviewer remarked that 10 megapascals (MPa) lap shear strength is not applicable to vehicle technologies.

\section{Question 3: Collaboration and coordination with other institutions.}

\section{Reviewer 1:}

The reviewer observed only satisfactory cooperation, and that the roles are not as clearly defined as the reviewer wanted to see.

\section{Reviewer 2:}

The reviewer commented that the presenter only listed two sources for collaboration and coordination; one is a fellow researcher and post-doctoral employee, and the second is a supplier of material to be tested. There is no collaboration with potential sources in the supply chain or with manufacturers who would be interested in the technology, if the research is successful. The reviewer observed minimal collaboration and no OEMs.

\section{Reviewer 3:}

It appeared to this reviewer that the major work is being done at Johns Hopkins University with some involvement from a former student who is based in Germany. The only industrial sponsor identified is Severstal, which supplies the materials. The reviewer would have appreciated knowing that an actual body structure manufacturer is interested in this work because without an actual application or potential target customer, it is challenging to see how viable an investment this represents for the DOE program.

\section{Reviewer 4:}

The reviewer said that collaboration with a former post doc is not collaboration. 
Question 4: Proposed future research-the degree to which the project has effectively planned its future work in a logical manner by incorporating appropriate decision points, considering barriers to the realization of the proposed technology and, when sensible, mitigating risk by providing alternate development pathways.

\section{Reviewer 1:}

The reviewer said that the plans are okay for this level of research project as measured against the stated project proposals.

\section{Reviewer 2:}

The reviewer remarked that overall, the future research proposed seems to address potential problems with the technology.

\section{Reviewer 3:}

The reviewer commented that the plan lacks direction, and that there is no plan to address corrosion issues.

\section{Reviewer 4:}

The reviewer indicated that the proposed future work only supports half of the remaining challenges and barriers and quoted "Mass ejection and porosity in bonds" and "Molten braze from Redox reaction wets poorly." The reviewer pointed out that no future work is proposed to address issues with brazing. Instead, two other efforts are proposed: "Create statistically signification datasets for shear strengths of bonds and determine the modes of failure in the joint;" and "Analyze the braze and base metal interface for any changes in mechanical properties of base metal due to heating from the reaction of the Redox Foil." The reviewer said that nothing is mentioned regarding the four months for each effort that addresses bond strength, failure modes, corrosion behavior, and component degradation shown in the milestone chart on Slide four.

\section{Question 5: Does this project support the overall DOE objectives of petroleum displacement? Why or why not?}

\section{Reviewer 1:}

The reviewer said that all new ideas for joining dissimilar materials need to be explored to enable lightweight vehicle construction.

\section{Reviewer 2:}

The reviewer said it potentially supports DOE goals, but is essential to identify a potential target customer soon to ensure that there will be a return on the investment.

\section{Reviewer 3:}

The reviewer said that although the objective stated that the effort is to develop and characterize novel reactive foils for use in bonding dissimilar materials, there is nothing in the written or oral presentation that explains how this research will be used in lightweighting applications that will directly support the overall DOE objectives to make lightweight vehicles that will displace or reduce the use of petroleum. The relevance discussed applies to determining the best chemistry and increasing quantity of braze in the foils from $65 \%$ to $74 \%$, which is relevant to research goals, not DOE goals.

\section{Reviewer 4:}

The reviewer remarked that there is no tangible means as to how reduction oxidation can reduce vehicle mass and associated fuel reduction.

\section{Question 6: Resources: How sufficient are the resources for the project to achieve the stated milestones in a timely fashion?}

\section{Reviewer 1:}

The reviewer said that this is a four-year research project to improve a bonding technique for $\$ 640,000$ ( $\$ 160,000$ per year average). All previous milestones have been met with the funding provided and all future milestones are reported as on track for the remaining amount of funds. The reviewer remarked that resources are sufficient to achieve the stated milestones. 
Reviewer 2:

The reviewer said that resources look okay.

Reviewer 3:

The reviewer strongly recommended that this project be cancelled. 
High-Strength, Dissimilar Alloy Aluminum Tailor-Welded Blanks: Yuri Hovanski (Pacific Northwest National Laboratory) - Im099

\section{Presenter}

Yuri Hovanski, Pacific Northwest

National Laboratory

\section{Reviewer Sample Size}

A total of five reviewers evaluated this project.

\section{Question 1: Approach to performing the work-the degree to which technical barriers are addressed, the project is well-designed, feasible, and integrated with other efforts.}

\section{Reviewer 1:}

The reviewer said that this is one of the best funded projects in the current DOE portfolio, well scoped out and executed.

\section{Reviewer 2:}

The reviewer observed an outstanding approach to developing and progressing the state-of-the-art for friction-stir welding (FSW) of $\mathrm{Al}$ tailor blanks.

\section{Reviewer 3:}

The reviewer noted a vertical supply chain with a tangible product application, which provides mass reduction benefit.

\section{Reviewer 4:}

The reviewer said that this project appears to be highly focused, well organized, and is on-track to achieve its goals. A key thing is that the project team has engaged the entire supply chain, right through to an eventual technology deployment client (General Motors).

\section{Question 2: Technical accomplishments and progress toward overall project and DOE goals-the degree to which progress has been made, measured against performance indicators and demonstrated progress towards DOE goals.}

\section{Reviewer 1:}

The reviewer said that the project is reportedly ahead of schedule and somewhat underspent, despite the fact that the team is considering a very wide array of variables and all of the necessary potential negative factors in the process. The learnings and progress toward a viable process are all impressive.

\section{Reviewer 2:}

The reviewer observed good results to date.

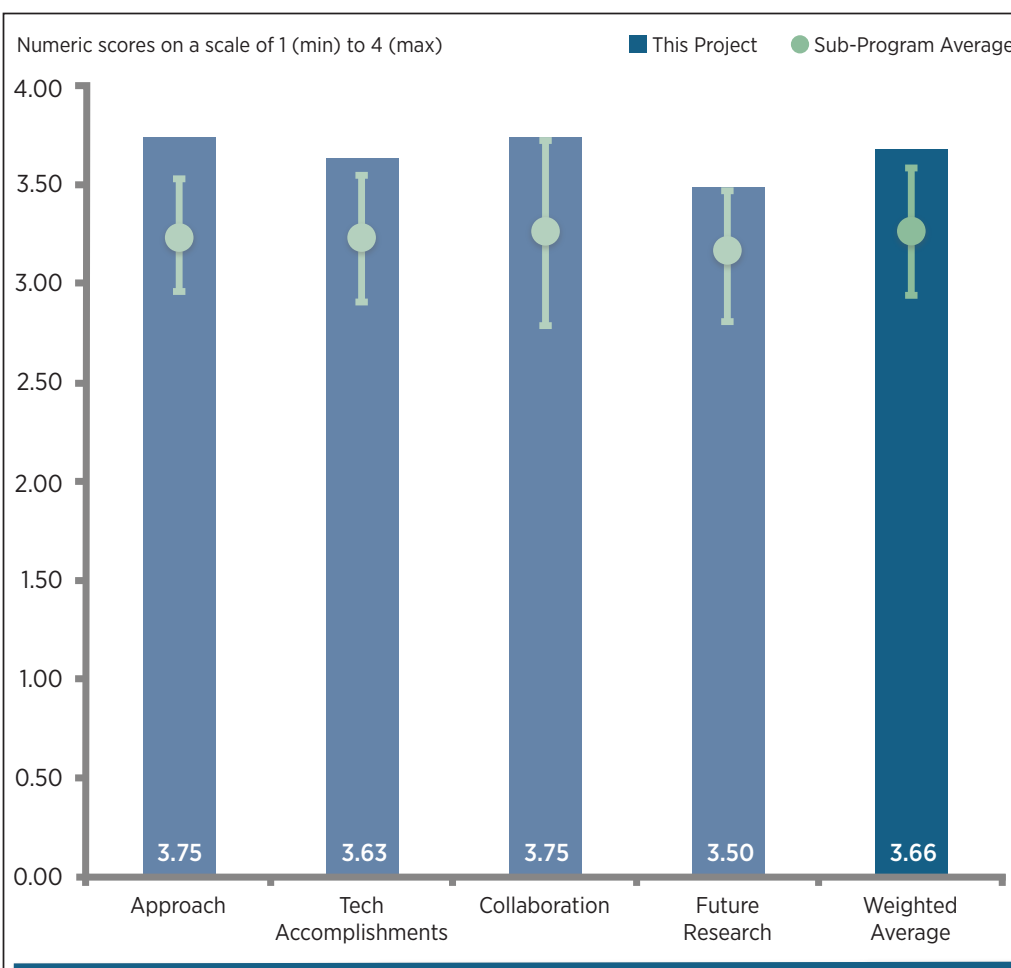

Relevant to DOE Objectives

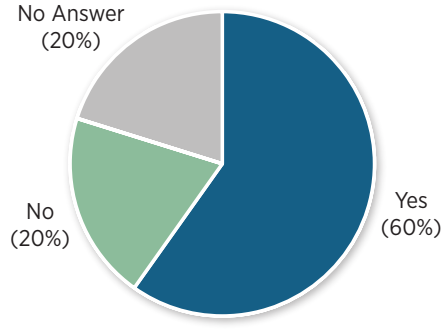

Figure 6-10 - High-Strength, Dissimilar Alloy Aluminum Tailor-Welded Blanks: Yuri Hovanski (Pacific Northwest National Laboratory) - Lightweight Materials

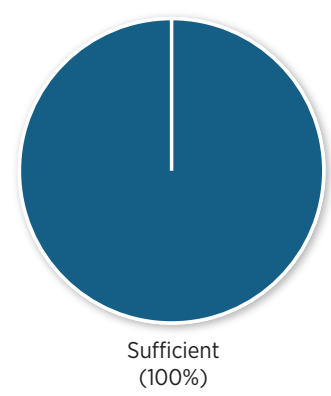

Im099 


\section{Reviewer 3:}

The reviewer said that work has focused on the barriers and has solid experimental foundations.

\section{Reviewer 4:}

The reviewer commented that results look excellent. The reviewer would like to see actual stamping trials in the last phase to show performance of the FSW blanks under truly high strain rate forming, and taking it beyond limiting dome height (LDH) testing.

\section{Question 3: Collaboration and coordination with other institutions.}

\section{Reviewer 1:}

The reviewer observed great teamwork.

\section{Reviewer 2:}

The reviewer said that it would appear that the team is properly constituted, working effectively together, and sharing results in a positive fashion.

\section{Reviewer 3:}

The reviewer stressed that collaboration is the key.

\section{Reviewer 4:}

The reviewer described a perfect mix of collaborators that include an OEM, national laboratory, and academia.

Question 4: Proposed future research-the degree to which the project has effectively planned its future work in a logical manner by incorporating appropriate decision points, considering barriers to the realization of the proposed technology and, when sensible, mitigating risk by providing alternate development pathways.

\section{Reviewer 1:}

The reviewer said that the plan to integrate FSW of $7 \mathrm{xxx} \mathrm{Al}$ is a great stretch target.

\section{Reviewer 2:}

The reviewer said that the project looks like a great plan for the next phase of work-comprehensive, realistic, and goal focused.

\section{Reviewer 3:}

The reviewer said that the project has solid proposed work to address remaining tasks, and there are good ideas for future commercialization through partners.

\section{Reviewer 4:}

The reviewer suggested trying to include the high-strain rate testing via conventional stamping.

\section{Question 5: Does this project support the overall DOE objectives of petroleum displacement? Why or why not?}

\section{Reviewer 1:}

The reviewer asserted that this project is definitely and firmly aligned with the program goals. The reviewer also pointed out that the project team seems to be very conscious of final product cost, which is also a crucial aspect of success for a new technology.

\section{Reviewer 2:}

The reviewer said that tailor-welded blanks (TWB) are a proven weight-saving opportunity, and that we need this technology for $\mathrm{Al}$ sheets. 
Reviewer 3:

The reviewer remarked that this project is definitely focused on next-generation joining and vehicle lightweighting.

Reviewer 4:

The reviewer declared that mass is not saved, and that the process saves cost by improving scrap utilization.

Question 6: Resources: How sufficient are the resources for the project to achieve the stated milestones in a timely fashion?

Reviewer 1:

The reviewer said that yes, this experienced team appears to be adequately resourced.

Reviewer 2:

The reviewer said that the project is appropriately funded. 


\section{Upset Protrusion Joining Techniques For Joining Dissimilar Metals: Steve Logan (Fiat Chrysler Automobiles US LLC) $-\operatorname{Im} 100$}

\section{Presenter}

Steve Logan, Fiat Chrysler Automobiles USA LLC

\section{Reviewer Sample Size}

A total of five reviewers evaluated this project.

\section{Question 1: Approach to performing the work-the degree to which technical barriers are addressed, the project is well-designed, feasible, and integrated with other efforts.}

\section{Reviewer 1:}

The reviewer observed a great test matrix.

\section{Reviewer 2:}

The reviewer noted an outstanding approach and testing plan with great statistical analysis, and added that this will supply a solid data set to the industry.

\section{Reviewer 3:}

The reviewer said that this is an excellent project, well scoped-out, and related to the needs for more mixed materials joining solutions.

\section{Reviewer 4:}

The reviewer said that the program covers the important aspects of applying this technology to production.

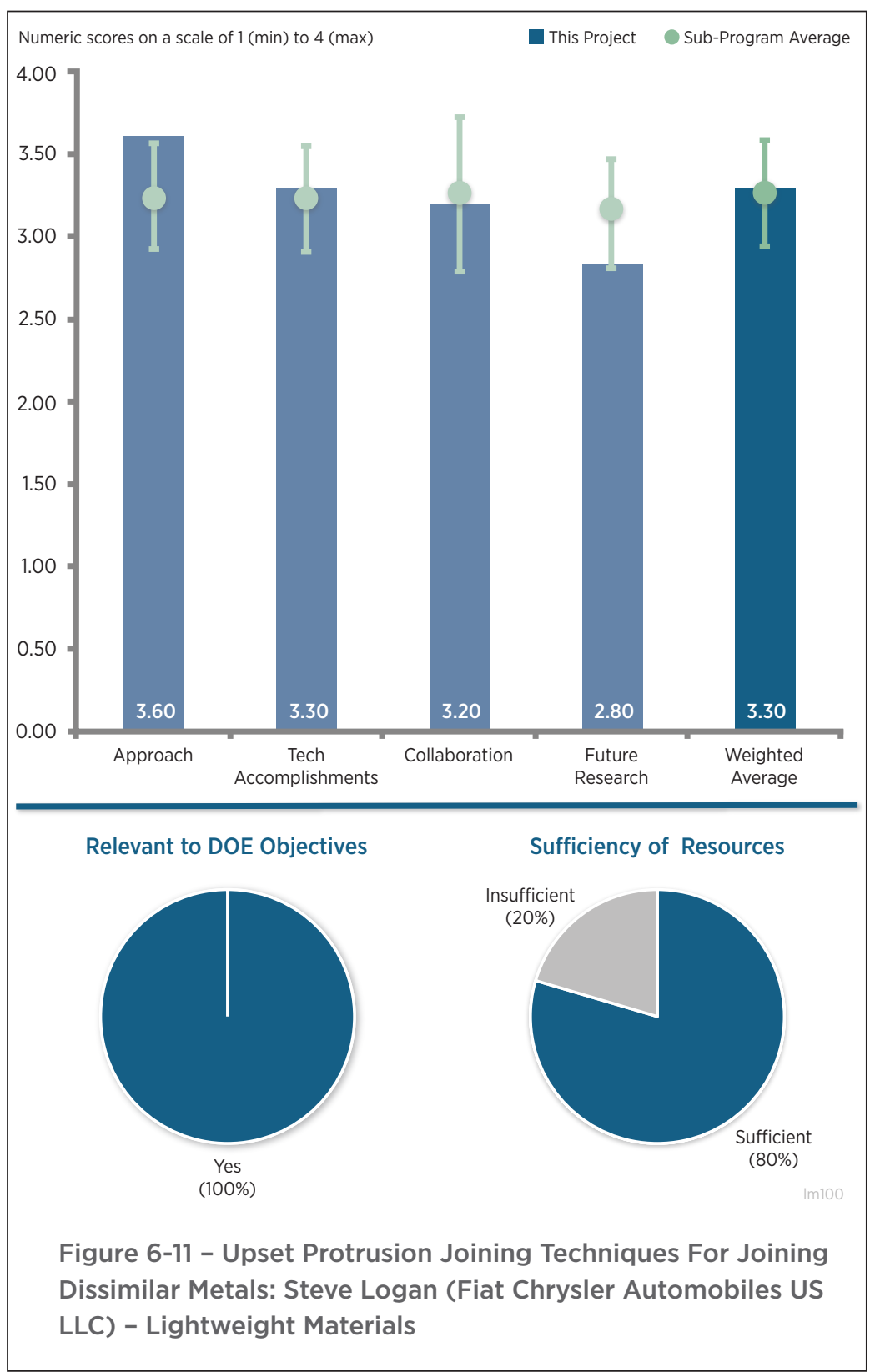

\section{Question 2: Technical accomplishments and progress toward overall project and DOE goals-the degree to which progress has been made, measured against performance indicators and demonstrated progress towards DOE goals.}

\section{Reviewer 1:}

The reviewer remarked great progress and interesting results, and the reviewer hoped the last phase might include a run at rate study including repeatability studies, mechanical properties, and a roughed-out business case.

\section{Reviewer 2:}

The reviewer noted excellent accomplishments and timely data, and that the accomplishments are keeping the project on track. 


\section{Reviewer 3:}

The reviewer said that the project seems to have developed the information needed for a business case decision to be made on applying this technology to production, and a sound base of information on which to develop a similar case for other material systems.

\section{Reviewer 4:}

The reviewer said that results associated with the test plan revealed difficult challenges and the limitations of the process.

\section{Reviewer 5:}

The reviewer said that the project is very ambitious and there is too much to do to satisfy DOE requirements. As a consequence, the number of tests per material combination is too small and the reviewer questioned the reliability aspect of the project.

\section{Question 3: Collaboration and coordination with other institutions.}

\section{Reviewer 1:}

The reviewer observed a great mix of OEM, national laboratory, and academic involvement.

\section{Reviewer 2:}

The reviewer described utilization of outside resources to conduct casting and corrosion testing as good.

\section{Reviewer 3:}

The reviewer observed a good team of collaborators with clear roles and responsibilities.

\section{Reviewer 4:}

The reviewer said that collaboration was good, but too restricted.

\section{Reviewer 5:}

The reviewer said that the interconnection of efforts was not as readily apparent as with other projects.

Question 4: Proposed future research-the degree to which the project has effectively planned its future work in a logical manner by incorporating appropriate decision points, considering barriers to the realization of the proposed technology and, when sensible, mitigating risk by providing alternate development pathways.

\section{Reviewer 1:}

The reviewer commented that the proposed future work will address what questions and tasks remain in this project.

\section{Reviewer 2:}

The reviewer remarked that the project is close to completion and does not respond to the question of the process reliability. The principal investigator (PI) commented that Fiat Chrysler Automobiles is doing other work on the subject.

\section{Reviewer 3:}

Looking for more component level assemblies with post corrosion testing of mechanical joint strength was reported by this reviewer.

\section{Reviewer 4:}

The reviewer did not observe many insights on how to build on this work, and highlighted that only corrosion testing is being finished. 


\section{Question 5: Does this project support the overall DOE objectives of petroleum displacement? Why or why not?}

Reviewer 1:

The reviewer emphasized that dissimilar material joining is basically the issue for multi-material lightweight auto bodies, and this technology is a valuable addition to the slate of possibilities.

\section{Reviewer 2:}

The reviewer said that joining of dissimilar materials is challenging, and that the project illustrated the proper project methodology and demonstration of plan execution.

\section{Reviewer 3:}

The reviewer commented that joining to $\mathrm{Mg}$ is one of the key enablers for lightweight mixed material structures.

\section{Reviewer 4:}

The reviewer said that the project fulfills a need for more innovative and effective mix metal joining technology.

Question 6: Resources: How sufficient are the resources for the project to achieve the stated milestones in a timely fashion?

\section{Reviewer 1:}

The reviewer observed an appropriate amount of funding for the project.

\section{Reviewer 2:}

The reviewer remarked about the fact that the number of tests per material combination is too small. 


\section{Integrated Computational Materials Engineering (ICME) Development of Carbon Fiber Composites for Lightweight Vehicles: Xuming Su (Ford Motor Company) - Im101}

\section{Presenter}

Xuming Su, Ford Motor Company

\section{Reviewer Sample Size}

A total of five reviewers evaluated this project.

\section{Question 1: Approach to performing the work-the degree to which technical barriers are addressed, the project is well-designed, feasible, and integrated with other efforts.}

\section{Reviewer 1:}

The reviewer remarked that the ICME approach developed in this project for predicting the manufacturing and structural performance will exponentially speed up the lightweighting efforts of automobiles using CFCs. The technology being developed in this project has the potential to meet DOE targets; namely, $25 \%$ weight reduction and cost increase less than $\$ 4.27 / \mathrm{lbs}$. compared to current steel assemblies.

\section{Reviewer 2:}

The reviewer said that the plan seems quite realistic, but additional detail on how the technology will eventually be deployed would be welcome.

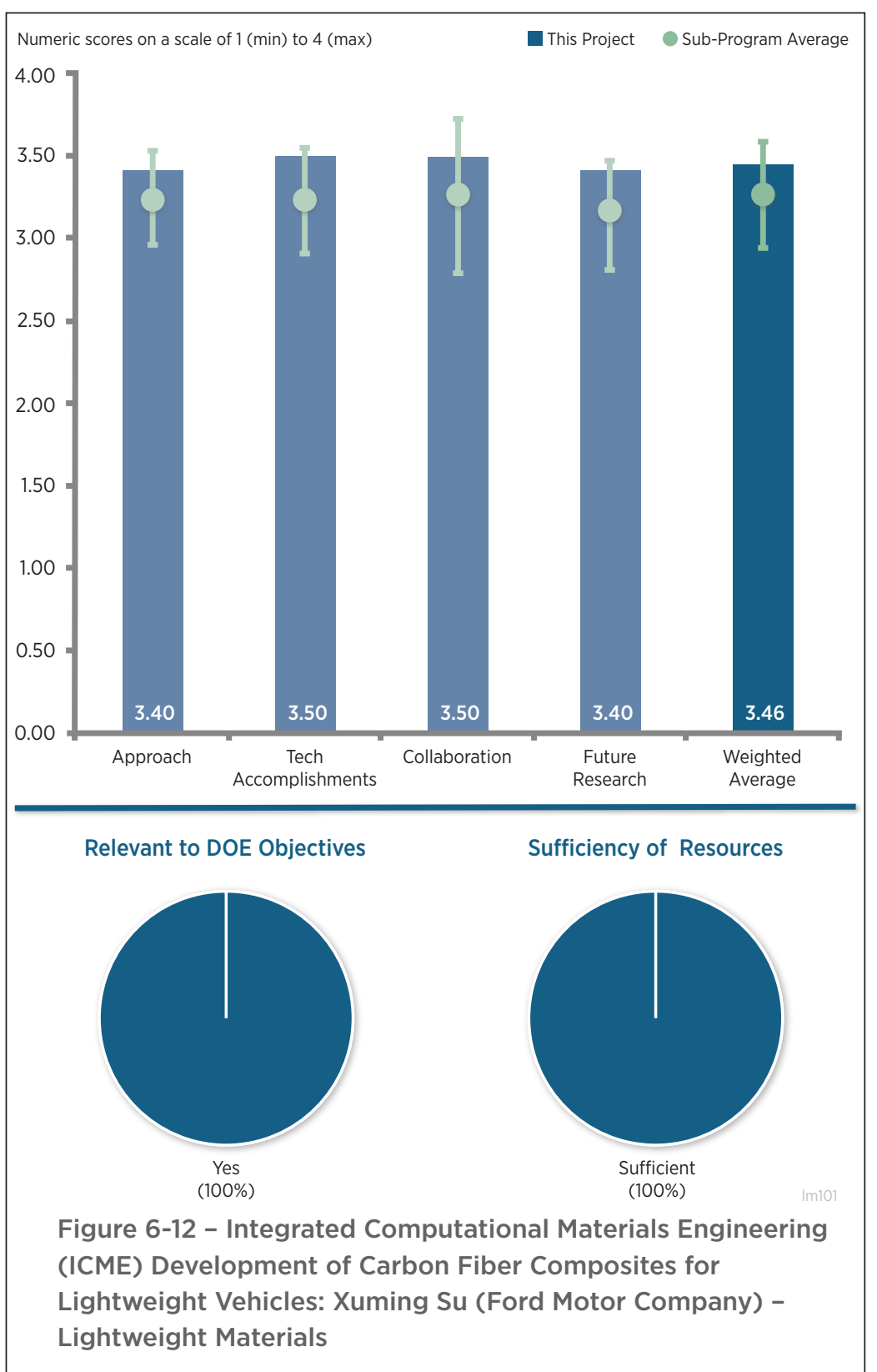

\section{Reviewer 3:}

The reviewer said that the approach taken to developing the ICME models, modules, and processes is very well conceived. There is little doubt that the project will lead to capabilities that accomplish the project's technical goals. The reviewer said that the only element that might improve the work is consideration of alternative continuous fiber material forms. The reviewer said that using woven carbon reinforcements makes the overall cost targets (i.e., less than $\$ 4.27 / \mathrm{lbs}$. weight saved) close to unattainable.

\section{Reviewer 4:}

The reviewer asked what the impact is on data with large-tow CF, and what the impact is of using same-tow CF but different sizes of CF. The reviewer suggested adding thermoplastic (polyamide [PA]/polyphthalamide [PPA] or high performance) non-woven material to evaluation materials as a lower cost alternative to thermoset.

\section{Reviewer 5:}

The reviewer asked how the team intends to do the cost analysis to meet cost limitations. Meeting cost targets is a 
big challenge and the team should consider cost targets well in advance of the project by looking at different fiber forms and tow sizes rather than dwelling too deep into existing fiber forms.

\section{Question 2: Technical accomplishments and progress toward overall project and DOE goals-the degree to which progress has been made, measured against performance indicators and demonstrated progress towards DOE goals.}

\section{Reviewer 1:}

The reviewer said that the team appears to be making good progress against the objectives.

\section{Reviewer 2:}

The reviewer reported excellent progress in the last year: selecting the material system; representative volume element (RVE) unidirectional (UD) development; performing simulations; molding simulation of compression molding; and process integration, including Moldflow, LS-DYNA, NASTRAN, and nCode. The reviewer asked if molecular dynamic analysis (MDA) tools are integrated with the developed ICME tools or whether they work alone. The reviewer also asked what the plans are to link uncertainty quantification models with the deterministic ICME models.

\section{Reviewer 3:}

The reviewer said that the work on MDA is particularly impressive with a strong correlation of predicted mechanical and thermal properties. The work completed on fatigue analysis is also very well done. The balance of the work accomplished is commensurate with the technical approach and expectations of the program. The reviewer said that the project needs to address cost factors. The progress on technical modeling is outstanding. However, this reviewer opined that it will not see the light of day without similar attention paid to economics of the processes studied and development of integrated cost models that provide a similar level of fidelity regarding the cost of structures fabricated.

\section{Reviewer 4:}

The reviewer commented that deformation of the fiber mat during processing should be considered while deriving the material properties to be used in finite element analysis (FEA).

\section{Question 3: Collaboration and coordination with other institutions.}

\section{Reviewer 1:}

The reviewer noted excellent collaboration among the project team members.

\section{Reviewer 2:}

The reviewer commented that the inclusion of strong academic institutions (i.e., Northwestern and Maryland) to support development of analytical tools and material characterization, a leading OEM and material supplier, along with important commercial software developers, makes this a winning team.

\section{Reviewer 3:}

The reviewer said that collaboration looks okay.

\section{Reviewer 4:}

The reviewer suggested investigating thermoplastic options.

\section{Reviewer 5:}

The reviewer said none.

Question 4: Proposed future research-the degree to which the project has effectively planned its future work in a logical manner by incorporating appropriate decision points, considering barriers to the realization of the proposed technology and, when sensible, mitigating risk by providing alternate development pathways. 


\section{Reviewer 1:}

The reviewer said that proposed future research addresses some of the major challenging facing the industry in large-scale implementation of composite materials in automobiles. Based on the strong performance last year, the reviewer expects the project to accomplish all the remaining challenges.

\section{Reviewer 2:}

The reviewer said that the forward plan appears to be well focused and the project is likely to continue resulting in good progress.

\section{Reviewer 3:}

The reviewer said that the proposed work for the coming period is well tailored to meet the technical objectives to demonstrate application of a strong ICME environment for automotive component design for CFRP. The reviewer said that the overall program will be strengthened if more consideration is placed on the economic modeling. Providing a strong cost model as part of the integrated design environment is an essential part of ensuring cost targets that result in viable commercial components are achieved.

\section{Reviewer 4:}

The reviewer referenced prior comments.

\section{Question 5: Does this project support the overall DOE objectives of petroleum displacement? Why or why not?}

\section{Reviewer 1:}

The reviewer said that the ICME technology being developed in this project has the potential to achieve the overall DOE objective of $25 \%$ lighter assemblies in automobiles using CFCs. This will directly improve the fuel economy and reduce emissions. The reviewer also pointed out that using this technology, significant reduction in manufacturing costs of components with improved quality can be obtained and while eliminating costly trial and error.

\section{Reviewer 2:}

The reviewer asserted that the project does appear to be aligned with DOE's program goals.

\section{Reviewer 3:}

The reviewer said that this program will ensure that future designers have the tools needed and the methodologies established that will result in reliable automotive composite designs that exploit the use of high specific proprietary materials in transportation systems reducing wait and thereby reducing fuel consumption. The reviewer said that it remains important to consider the trade of incremental cost for each pound of weight saved. More work should be done to provide an economic basis for that trade-off.

\section{Reviewer 4:}

The reviewer said the project is very much needed in the current context of extensive use of composites within transportation sector because ICME development will lead to not only rapid advancements in materials but also virtual investigations prior to fabrication and testing. Thereby, millions of dollars in cost would be saved.

\section{Question 6: Resources: How sufficient are the resources for the project to achieve the stated milestones in a timely fashion?}

\section{Reviewer 1:}

The reviewer said that resources look okay.

\section{Reviewer 2:}

Given the scope of work identified, the reviewer found that the goals stated and approaches proposed are commensurate with the budget established for this work. The resources available should be sufficient to support the vast amount of data generated and analysis tools developed along with the design work required to complete this effort. 


\section{Predictive Models for Integrated Manufacturing and Structural Performance of Carbon Fiber Composites for Automotive Applications: Venkat Aitharaju (General Motors) - Im102}

\section{Presenter}

Venkat Aitharaju, General Motors

\section{Reviewer Sample Size}

A total of five reviewers evaluated this project.

\section{Question 1: Approach to} performing the work-the degree to which technical barriers are addressed, the project is welldesigned, feasible, and integrated with other efforts.

\section{Reviewer 1:}

The reviewer noted that the performance of CFRP is strongly dependent on manufacturing processes and varies from location to location. The project takes an integrated analysis approach, considering manufacturing and local variability, and is exactly needed for the problem.

\section{Reviewer 2:}

The reviewer said that the approach is a bit vague and the overall scope is overly optimistic. Characterizing material models, and evaluating process simulation and structural performance for a full suite of thermosets, thermoplastics, chopped-, uni-, and woven composites, were described as lofty goals by this reviewer. The reviewer added that the project seems too ambitious to be completed by 2019 , particularly with one goal being to account for uncertainty across scales. The reviewer commented that the process flow of tool development needs to be refined to more clearly show the process steps.

\section{Reviewer 3:}

The reviewer suggested that the team look into other distributions (than uniform well in-advance of the work plan), which might result in different process development flow and uncertainty modeling approaches.

\section{Question 2: Technical accomplishments and progress toward overall project and DOE goals-the degree to which progress has been made, measured against performance indicators and demonstrated progress towards DOE goals.}

\section{Reviewer 1:}

The reviewer said that great progress has been made, and a lot of data has been collected for the short period.

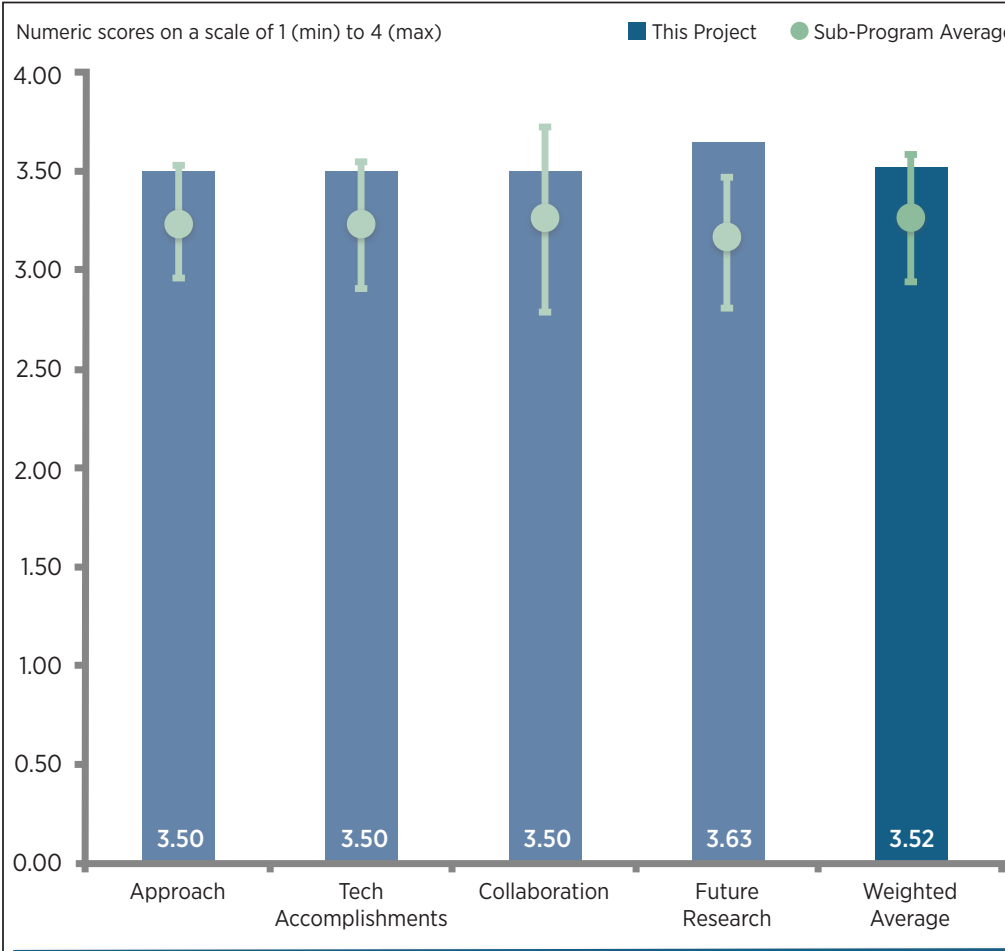

Relevant to DOE Objectives

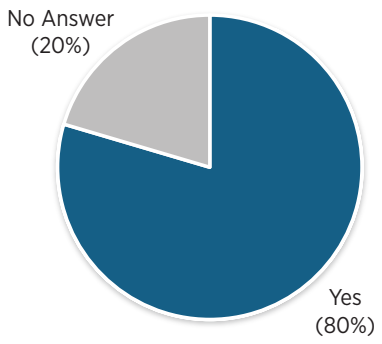

Figure 6-13 - Predictive Models for Integrated Manufacturing and Structural Performance of Carbon Fiber Composites for Automotive Applications: Venkat Aitharaju (General Motors) Lightweight Materials) 


\section{Reviewer 2:}

The reviewer observed good progress so far.

\section{Reviewer 3:}

The reviewer noted that the project was initiated in May 2015 and it is still early in the project. The reviewer listed the following: analysis method was developed; manufacturing methods were identified; material systems were selected and tested; material characterization for tension, three-point bending, and crush was done; and crush testing was completed. This reviewer would like to see more specific results presented in future reviews.

\section{Question 3: Collaboration and coordination with other institutions.}

\section{Reviewer 1:}

The reviewer commented that major relevant elements of a successful project have been assembled.

\section{Reviewer 2:}

The reviewer said that a good balance of collaborators exist, with one OEM, a Tier 2 supplier, modeling companies, and a university, but suggested that the project should consider material suppliers. This reviewer is interested in the pre-competitive research that will be generated in order to benefit the industry.

\section{Reviewer 3:}

The reviewer would like to see the project include chopped CF thermoplastic prepreg or three-dimensional (3D) preform materials. This is a lower cost approach than resin transfer molding (RTM)/thermoset. In overview, the reviewer would like to see more thermoplastic in the project based on recycling, cycle time, and more simplistic chemistry than thermosets.

\section{Reviewer 4:}

The reviewer said there is more to do for the common good than what is presented.

\section{Reviewer 5:}

The reviewer remarked none.

Question 4: Proposed future research-the degree to which the project has effectively planned its future work in a logical manner by incorporating appropriate decision points, considering barriers to the realization of the proposed technology and, when sensible, mitigating risk by providing alternate development pathways.

\section{Reviewer 1:}

The reviewer said that the proposed future work seems appropriate. Project plans for 2016 include completing data collection for manufacturing and structural performance simulation tools, completing the stochastic manufacturing simulation tool, and completing the stochastic performance simulation tool for three material systems.

\section{Reviewer 2:}

The reviewer said that the proposed future works emphasizes uncertainty characterization. As predictive tools, modeling of uncertainty transfer from one scale to another would be important and interesting.

\section{Reviewer 3:}

The reviewer referenced prior comments.

Question 5: Does this project support the overall DOE objectives of petroleum displacement? Why or why not?

\section{Reviewer 1:}

The reviewer asserted that the work outlined in this project supports the overall DOE objectives of helping to develop and support lightweight automotive applications to reduce fuel and GHG emissions. 


\section{Reviewer 2:}

The reviewer said that predictive modeling of composites is a challenging issue to solve in the current context of composites modeling efforts. If successful, the project will help to reduce the overall system costs by advance probability estimates of failures.

\section{Reviewer 3:}

The reviewer said yes, but RTM with thermoset chemistry has not been demonstrated as a cost-effective high volume process. The European OEMs who typically lead this type of advanced technology development seemed to have dropped this as a prime path. The reviewer suggested including a high-temperature thermoplastic, such as PPA.

\section{Question 6: Resources: How sufficient are the resources for the project to achieve the stated milestones in a timely fashion?}

\section{Reviewer 1:}

The reviewer said that the resource funding for this project seems appropriate for the amount of work and results expected. This reviewer would like to better understand the precompetitive work that will benefit the industry.

\section{Reviewer 2:}

A perfect set of resources was observed by this reviewer. 


\section{E. Coli Derived Spider Silk MaSp1 and MaSp2 Proteins as Carbon Fiber Precursors: Randy Lewis (Utah State University) - Im103}

Presenter

Randy Lewis, Utah State University

\section{Reviewer Sample Size}

A total of seven reviewers evaluated this project.

\section{Question 1: Approach to performing the work-the degree to which technical barriers are addressed, the project is well-designed, feasible, and integrated with other efforts.}

\section{Reviewer 1:}

The reviewer noted an outstanding approach to solving the challenges of lightweight composites in the automotive industry using spider silk as CF precursors. Simplified, the approach is as follows: first, produce fibers; second, convert to CF; and third, complete economic analysis.

\section{Reviewer 2:}

The reviewer pointed out that the biomaterial is green and would be abundant.

\section{Reviewer 3:}

The reviewer observed a good plan to evaluate non-traditional material source.

\section{Reviewer 4:}

The reviewer said that the problem being worked on (producing low-cost CF) is potentially a very important component of future lightweight vehicle technology. The overall approach is intriguing and appears to be successful at producing actual CF, but whether this can be done at a commercially feasible cost in commercially relevant quantities remains to be seen. The reviewer further explained that key problems that have come up appear to have been addressed in the project plan. Subsequently, the overall approach is, in this reviewer's view, very good. The reviewer looked forward to further results from this project team as the team continues with its work on this very interesting project.

\section{Reviewer 5:}

Referencing the presentation (slide deck), the approach could be better defined for the reviewer. The reviewer asked that notes be added in the slide deck next year. The project approach is a bit confusing without the presenter. The reviewer did understand the project approach much better after the presentation. From a research perspective, the scientific approach is interesting and innovative. The reviewer looked forward to seeing the project results next year. The reviewer did not believe the team has enough time in the project to allow for Oak Ridge National Laboratory (ORNL) to optimize the fiber properties to show the true potential.

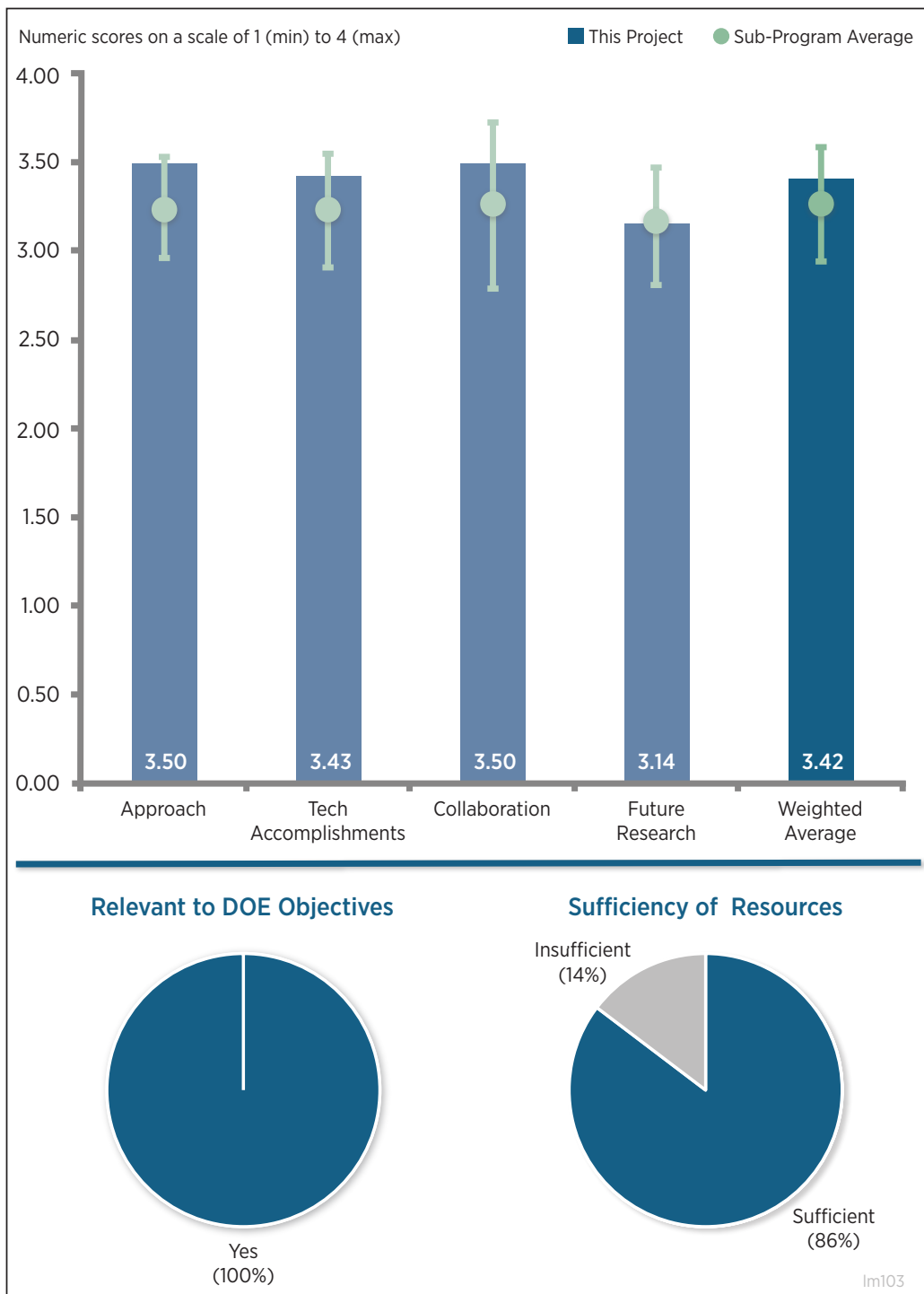

Figure 6-14 - E. Coli Derived Spider Silk MaSp1 and MaSp2 Proteins as Carbon Fiber Precursors: Randy Lewis (Utah State University) - Lightweight Materials 
Question 2: Technical accomplishments and progress toward overall project and DOE goals-the degree to which progress has been made, measured against performance indicators and demonstrated progress towards DOE goals.

Reviewer 1:

The reviewer observed good progress.

Reviewer 2:

The reviewer said that accomplishments look good, lots of issues remain, and to keep going.

\section{Reviewer 3:}

The reviewer said that the technical accomplishments are good and can help meet DOE goals for producing lightweight vehicles; however, many challenges are still outstanding, including producing fibers with sufficient strength and a reasonable cost. The learnings from this project may lead to continued research in this area.

\section{Question 3: Collaboration and coordination with other institutions.}

\section{Reviewer 1:}

The reviewer said that this is a complex initiative, and it appears that the right people are involved and working together effectively. The blend of engineering, biotechnology, entomology (insects), and manufacturing is really rather unique.

\section{Reviewer 2:}

The reviewer said that the project team seems to have the right partners and are working well together.

\section{Reviewer 3:}

The reviewer detailed that Utah State University is working with two other universities (i.e., University of California and Arizona State University) and two national laboratories. The reviewer said it was mentioned in the Question and Answer Session that collaboration was occurring with an automotive composites supplier and an aircraft composites supplier. This is good science and may lead to more research. Thus, sharing the knowledge via publication could be important.

\section{Reviewer 4:}

The reviewer noted limited collaboration due to nature of project, and that collaboration with ORNL was efficient.

Question 4: Proposed future research-the degree to which the project has effectively planned its future work in a logical manner by incorporating appropriate decision points, considering barriers to the realization of the proposed technology and, when sensible, mitigating risk by providing alternate development pathways.

\section{Reviewer 1:}

The reviewer said that it looks good but it is likely too early to say for sure. Overall, the presentation was realistic but optimistic, and this reviewer is in the same mindset with respect to this project. In this reviewer's view, it is worth a try.

\section{Reviewer 2:}

The reviewer said good, and elaborated that the proposed future work describes several activities to improve the fiber properties, including optimizing the oxidation process, using crosslinking agents, improving processing conditions, etc. The reviewer's confidence is low that goals and objectives will be met before the end of the defined program, and future research in this area may be beneficial.

\section{Reviewer 3:}

The reviewer would like to see go/no go decisions incorporated into the plan, and an additional emphasis on cost 
comparison and impacts on carbonization process. The reviewer also noted demonstration of benefits at small scale component.

\section{Reviewer 4:}

The reviewer would like to see efforts in commercializing the research before completion of the project, and would like to see a CFC made with fibers from spider silk.

\section{Reviewer 5:}

The reviewer does not believe the team has allocated enough time to do the proper oxidation, test cross linking, and test different spider silk proteins. The reviewer hoped that the team obtains some promising results and can continue with another grant and more time to develop.

\section{Question 5: Does this project support the overall DOE objectives of petroleum} displacement? Why or why not?

\section{Reviewer 1:}

The reviewer commented that it certainly does meet DOE's objectives, if this all works.

\section{Reviewer 2:}

The reviewer asserted that this project supports the overall DOE objective of developing lightweight automotive applications. As an incubator project, it is still early in the development phase, yet the approach seems solid. There is some good work established, but this reviewer indicated that there is a long way to go to meet the objectives.

\section{Reviewer 3:}

The reviewer responded yes, but only if the research shows promise to meet cost and performance of current CF. The minimum properties target physical properties need to be as good as Toray T700.

\section{Question 6: Resources: How sufficient are the resources for the project to achieve the stated milestones in a timely fashion?}

\section{Reviewer 1:}

The reviewer said that the resources will not be sufficient to meet the stated goals and objectives outlined in the documentation. The project is three-quarters of the way completed with a target end date of October 2016, yet the program has a long way to go. The reviewer said that the science is good, and more work needs to be done.

\section{Reviewer 2:}

The reviewer commented that the team appears to be well-resourced. 


\section{Solid-State Body-in-White Spot Joining of Al to AHSS at Prototype Scale: Zhili Feng (Oak Ridge National Laboratory) - Im104}

\section{Presenter}

Zhili Feng, Oak Ridge National Laboratory

\section{Reviewer Sample Size}

A total of four reviewers evaluated this project.

\section{Question 1: Approach to performing the work-the degree to which technical barriers are addressed, the project is well-designed, feasible, and integrated with other efforts.}

\section{Reviewer 1:}

The reviewer said that the project appears to be well-organized and sensibly designed to work toward actual onvehicle deployment.

\section{Reviewer 2:}

The reviewer said that the chemistry of adhesive used should be presented. An understanding of the temperature at the joint interface should also be presented so as to investigate adhesive degradability. The reviewer remarked that any aging study should be planned because if there are issues at the adhesive interphase then they will get magnified due to aging.

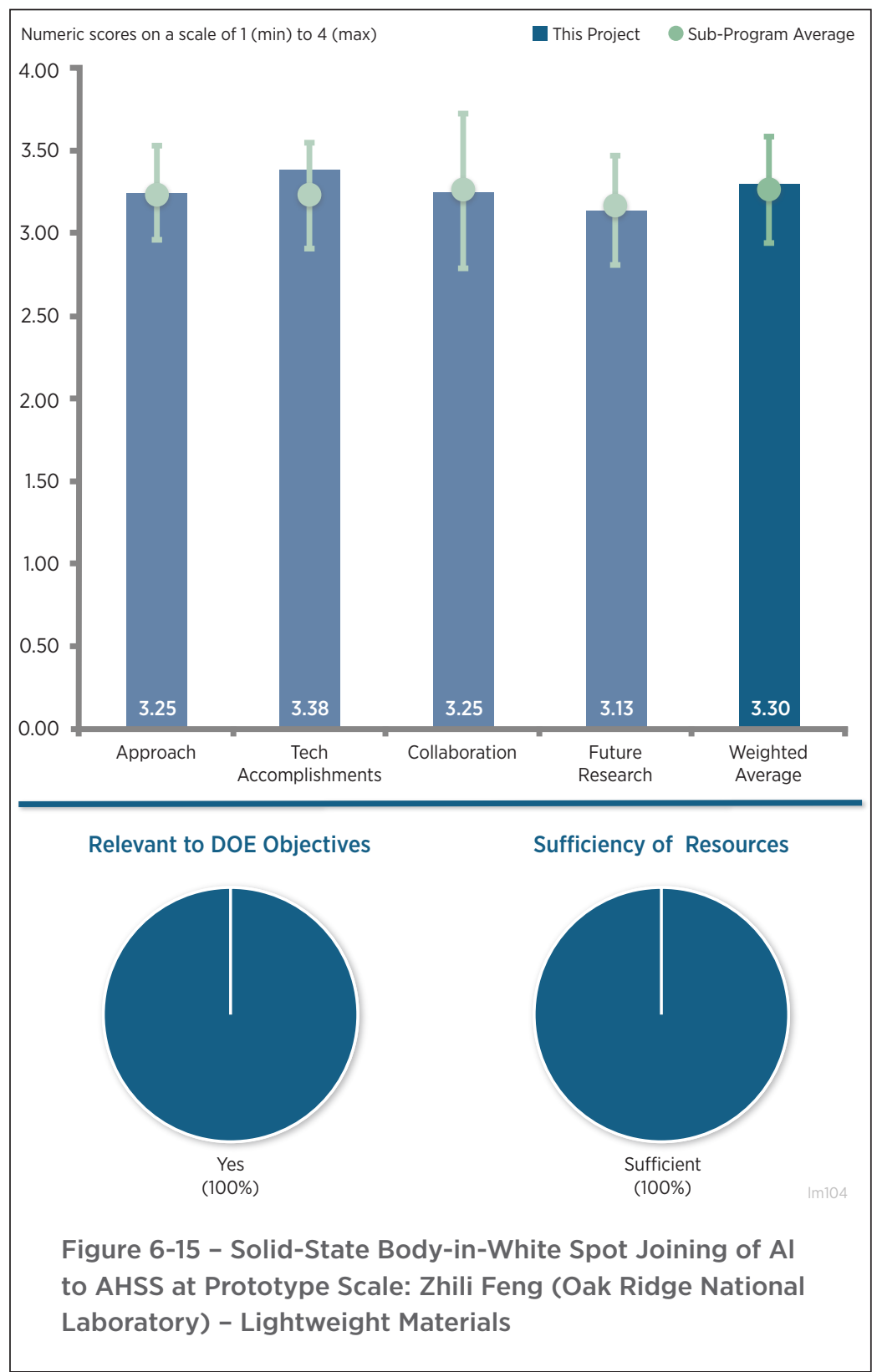

\section{Reviewer 3:}

The reviewer said that the details on the modeling effort to predict microstructures is not well described in the presentation. The reviewer would like to know how this effort will integrate into the process. This reviewer also inquired about the following: the processing variables from either process that are being correlated to good resulting microstructures; how the modeling is being informed by experiment; and the existence of any generic predictive capability, or the need to be completely re-trained on each new material system pair.

\section{Question 2: Technical accomplishments and progress toward overall project and DOE goals-the degree to which progress has been made, measured against performance indicators and demonstrated progress towards DOE goals.}

\section{Reviewer 1:}

The reviewer commented that the mechanical testing seems to be moving along well, but the modeling was not presented beyond thermal distortion FEA predictions. The assertion that the friction stir spot welding process squeezes out the adhesive at the point of welding was made with little backup evidence. The reviewer asked 
whether the degraded adhesive changes the local chemistry at the weld, affecting either corrosion performance or strength.

Reviewer 2:

The reviewer said that progress toward project goals appears to be on track, although additional information on the role and type of the adhesive would be welcome as this is a unique aspect of this project versus other dissimilar materials joining projects.

\section{Reviewer 3:}

The reviewer referenced prior comments.

\section{Question 3: Collaboration and coordination with other institutions.}

\section{Reviewer 1:}

The reviewer said that there seems to be a nice tie in between the joining experts and the industrial end users. The reviewer said that the modelers and how they tie in and interact with the team was not completely fleshed out in the presentation. The reviewer inquired about who owns the friction bit joining IP.

\section{Reviewer 2:}

The reviewer said that collaboration among the various participants in the project appears to be good, although relatively little was said about the specific contributions and roles of each partner.

\section{Reviewer 3:}

The reviewer said none.

Question 4: Proposed future research-the degree to which the project has effectively planned its future work in a logical manner by incorporating appropriate decision points, considering barriers to the realization of the proposed technology and, when sensible, mitigating risk by providing alternate development pathways.

\section{Reviewer 1:}

The reviewer said that the group appears to have identified the major concerns and issues going forward, but that little was said about the details. Thus, a real evaluation of the forward plan is difficult.

\section{Reviewer 2:}

The reviewer referenced prior comments.

Question 5: Does this project support the overall DOE objectives of petroleum displacement? Why or why not?

\section{Reviewer 1:}

The reviewer said that dissimilar joining technologies are critical to the implementation of a multi-material lightweight vehicle body, and understanding these processes will be well worth the effort

\section{Reviewer 2:}

The reviewer declared that this one is well aligned with the goals of the DOE program, as is the case with all dissimilar materials joining projects.

Question 6: Resources: How sufficient are the resources for the project to achieve the stated milestones in a timely fashion?

\section{Reviewer 1:}

The reviewer said that resources appear to be okay. 


\section{Friction Stir Scribe Joining of Al to Steel: Yuri Hovanski (Pacific Northwest National Laboratory) - Im105}

\section{Presenter}

Yuri Hovanski, Pacific Northwest National Laboratory

\section{Reviewer Sample Size}

A total of five reviewers evaluated this project.

\section{Question 1: Approach to performing the work-the degree to which technical barriers are addressed, the project is well-designed, feasible, and integrated with other efforts.}

\section{Reviewer 1 :}

The reviewer said as the need for more mixed metal joining techniques arise for multi-material vehicles, this project is perfectly aligned, well scoped, and executed. The reviewer said to keep up the great work.

\section{Reviewer 2:}

The reviewer commented that the approach of solid state joining of materials with different melting points considered in this project is very innovative and has potential to address some of the critical challenges faced by the automotive industry working on multi-materials. The reviewer commented that it is great to see the project also addresses optimizing joining parameters for each of the OEMs interested in material system and finally technology transfer to all OEMs.

\section{Reviewer 3:}

The reviewer said that it is a good place to be because the cost of project outcome is below DOE's target. The reviewer also reported repeatability tested.

\section{Reviewer 4:}

The reviewer commented that it would be illustrative to see an estimate of the time required to develop the information needed to join two $\mathrm{Al}$ alloys chosen at random, and wondered if the timeframe is days, weeks, months, or years.

Question 2: Technical accomplishments and progress toward overall project and DOE goals-the degree to which progress has been made, measured against performance indicators and demonstrated progress towards DOE goals.

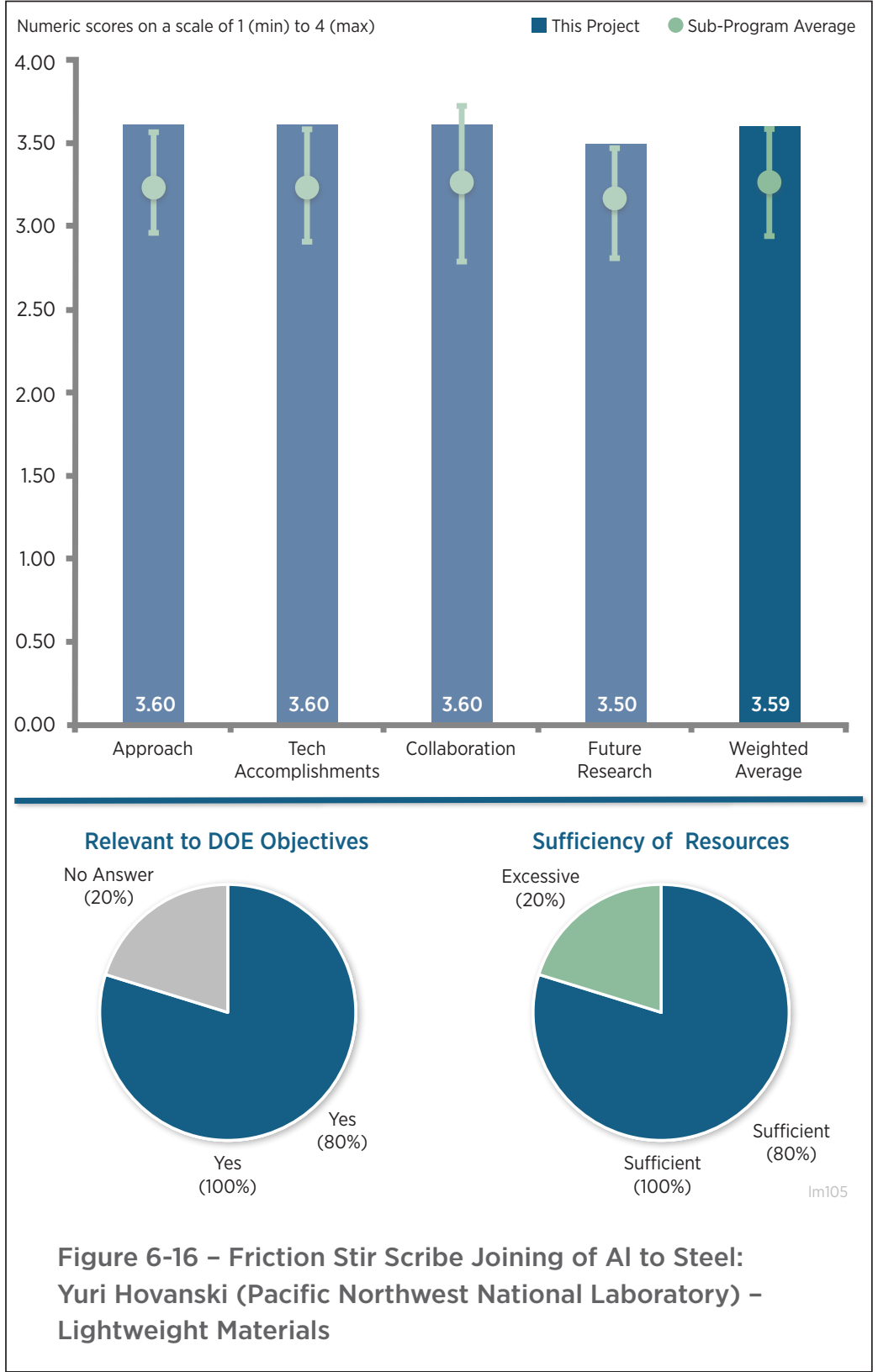

Figure 6-16 - Friction Stir Scribe Joining of Al to Steel: Yuri Hovanski (Pacific Northwest National Laboratory) Lightweight Materials 


\section{Reviewer 1:}

The reviewer noted impressive achievement. To this reviewer, accomplishments appear to be too good. The reviewer pointed out that grain refinement in the weld at low temperature is very good.

\section{Reviewer 2:}

The reviewer commented excellent progress, and said that it will be interesting to consider joint configurations other than the lap joint (peel, etc.), and that it will be interesting to see whether the weld parameters optimized in the laboratory work well after scaling to high volume manufacturing.

\section{Reviewer 3:}

The reviewer observed a very nice mix of different Al to steel combinations, and noted good directional results showing the potential for the process. The reviewer suggested adding some microstructural characterization to the study and that this would then be truly outstanding, and that results to date look very promising.

\section{Question 3: Collaboration and coordination with other institutions.}

\section{Reviewer 1:}

The reviewer noted excellent collaboration that is even reaching out to OEMs.

\section{Reviewer 2:}

The reviewer said collaboration seems good.

\section{Reviewer 3:}

The reviewer said that collaboration is excellent, but for this reviewer the team is a bit small. It is unclear to this reviewer what is available for the common good.

\section{Reviewer 4:}

The reviewer commented on an excellent mix of OEM participation and national laboratory involvement. Honda invested a lot of money in developing the FSW mixed metal process for the Accord subframe. This reviewer further explained that it looks like this project is a repeat, hopefully to make their joint more robust.

Question 4: Proposed future research-the degree to which the project has effectively planned its future work in a logical manner by incorporating appropriate decision points, considering barriers to the realization of the proposed technology and, when sensible, mitigating risk by providing alternate development pathways.

\section{Reviewer 1:}

The reviewer said that computational tool development considered in the next stage of this project is very crucial. The developed tool will help in minimizing the costs for joining with improved quality and eliminate costly trial and error.

\section{Reviewer 2:}

The reviewer observed an excellent work plan and objectives for subsequent years of funding, and said to keep up the great deliverables. As mentioned previously, the reviewer would welcome including more interfacial material characterization and some specific info on tool wear/life as a function of linear friction stir scribe length.

\section{Reviewer 3:}

The reviewer inquired if there is a need to evaluate corrosion performance, and whether it is possible to incorporate adhesive joining with the proposed process. The reviewer asked if adding adhesive joining provides any additional benefits, and whether the benefits can be demonstrated on crush members. 


\section{Reviewer 4:}

This reviewer liked what was understood from the PI, though it appeared that proposed future outreach is too ambitious.

\section{Question 5: Does this project support the overall DOE objectives of petroleum displacement? Why or why not?}

\section{Reviewer 1:}

The reviewer commented that using metals of appropriate strength, thickness, and formability in a TWB is an excellent method of lightweighting. Also, combining different $\mathrm{Al}$ alloys with varying characteristics such as these without degradation of properties at the joint would be an extremely valuable technology to have available.

\section{Reviewer 2:}

The reviewer said that the project addresses the major barriers faced by automakers in joining multi-materials. Using multi-materials, automakers can deliver lightweight solutions for automobiles, which will in turn help improve fuel economy, reduce dependence on foreign oil and reduce emissions.

\section{Reviewer 3:}

The reviewer said that mixed metal vehicles will be a near-term lightweighting enabler for the next 5-7 years and DOE's support for this project clearly shows a well thought-out and aligned portfolio of funded research.

\section{Reviewer 4:}

The reviewer remarked that joining is always a key consideration for use of lightweight materials.

\section{Question 6: Resources: How sufficient are the resources for the project to achieve the stated milestones in a timely fashion?}

\section{Reviewer 1:}

The project looks to be appropriately funded.

\section{Reviewer 2:}

The reviewer clarified that it is because others, namely GM, TWB Company, and Alcoa, are so committed to be successful. 


\section{Enhanced Sheared Edge Stretchability of AHSS/UHSS: Xin Sun (Pacific Northwest National Laboratory) - Im106}

\section{Presenter}

Xin Sun, Pacific Northwest National Laboratory

\section{Reviewer Sample Size}

A total of three reviewers evaluated this project.

\section{Question 1: Approach to performing the work-the degree to which technical barriers are addressed, the project is well-designed, feasible, and integrated with other efforts.}

\section{Reviewer 1:}

The reviewer said that formability is an important parameter for formed sheet stock. Tools to predict the formability are important for the selection and adoption of new materials.

\section{Reviewer 2:}

The reviewer said that the project is a new start and the direction to develop a model directionally is a big need for shear edge trimming predictions or quality parts.

\section{Reviewer 3:}

This reviewer commented that the project will study the edge stretchability of advanced high-strength steels (AHSS)/ ultra-high-strength steels (UHSS) to increase application of AHSS/UHSS into vehicle structures for weight reduction and crash performance. The reviewer was pleased to see some numerical and physics studies other than experimental testing and characterization. The link between the material microstructure and edge stretchability is critical to guide the design and development of future generation of AHSS/UHSS.

\section{Question 2: Technical accomplishments and progress toward overall project and DOE goals-the degree to which progress has been made, measured against performance indicators and demonstrated progress towards DOE goals.}

\section{Reviewer 1:}

The reviewer said that progress and accomplishments are in keeping with industry expectations.

\section{Reviewer 2:}

The reviewer said that accomplishments so far are very good, considering the project was just started in fiscal year (FY) 2015 and equipment purchase is needed before testing.

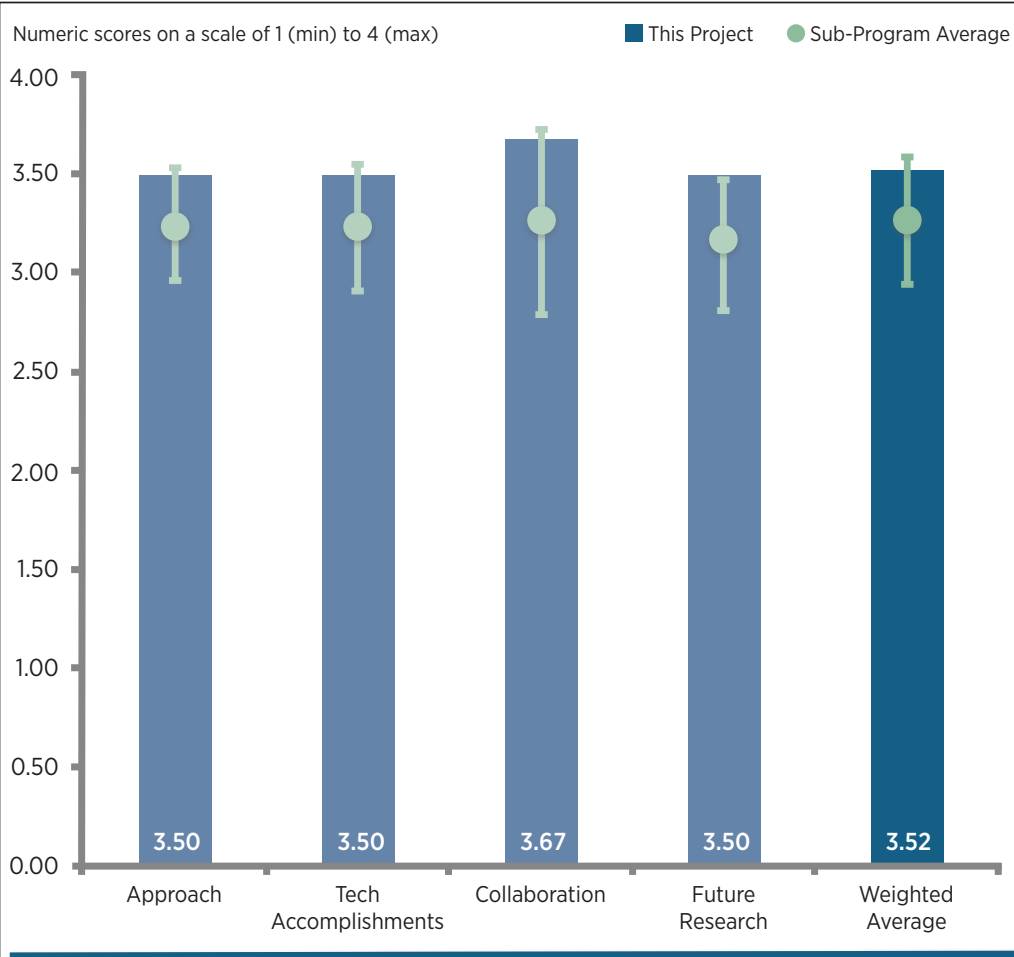

Relevant to DOE Objectives

Sufficiency of Resources
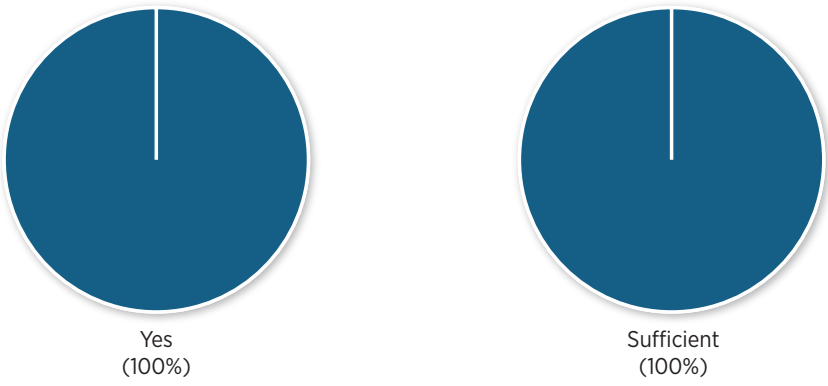

Im106

Figure 6-17 - Enhanced Sheared Edge Stretchability of AHSS/UHSS: Xin Sun (Pacific Northwest National Laboratory) - Lightweight Materials 


\section{Reviewer 3:}

The reviewer said that constitutive equations are sound and directionally correct, and that the project needs to deliver process models as part of the deliverables. The reviewer anticipates these results will be provided based on the strength of the team.

\section{Question 3: Collaboration and coordination with other institutions.}

\section{Reviewer 1:}

The reviewer said that the selection of collaborators appears to be appropriate.

\section{Reviewer 2:}

The reviewer noted an excellent mix of industry (OEM), academia, and national laboratory participation.

\section{Reviewer 3:}

The reviewer stated that the project is well organized and distributed efficiently to different collaborators.

Question 4: Proposed future research-the degree to which the project has effectively planned its future work in a logical manner by incorporating appropriate decision points, considering barriers to the realization of the proposed technology and, when sensible, mitigating risk by providing alternate development pathways.

\section{Reviewer 1:}

The reviewer observed a great start, reported that the project is on track, and recommended no changes in the technical direction.

\section{Reviewer 2:}

The reviewer said that future work is well planned.

\section{Reviewer 3:}

The reviewer said that proposed future work appears to be suitable to assist in the adoption of lighter-weight structural materials.

\section{Question 5: Does this project support the overall DOE objectives of petroleum displacement? Why or why not?}

\section{Reviewer 1:}

The reviewer said that this will assist with the adoption of lighter-weight structural materials.

\section{Reviewer 2:}

The reviewer noted that lightweighting is not only important to traditional gasoline vehicles, but also to electric vehicles/hybrid electric vehicles for extending driving range.

\section{Reviewer 3:}

The reviewer said that AHSS and UHSS are lightweighting solutions and yet still pose challenges, and this project is an enabler for high quality parts.

\section{Question 6: Resources: How sufficient are the resources for the project to achieve the stated milestones in a timely fashion?}

\section{Reviewer 1:}

The reviewer said that the project is appropriately funded.

\section{Reviewer 2:}

The reviewer said that the budget is sufficient for the project. 
Optimizing Heat Treatment Parameters for $3^{\text {rd }}$ Generation AHSS Using an Integrated Experimental-Computational Framework: Xin Sun (Pacific Northwest National Laboratory) - Im107

\section{Presenter}

Xin Sun, Pacific Northwest National Laboratory

\section{Reviewer Sample Size}

A total of three reviewers evaluated this project.

\section{Question 1: Approach to performing the work-the degree to which technical barriers are addressed, the project is well-designed, feasible, and integrated with other efforts.}

\section{Reviewer 1:}

The reviewer said that this project appears to be primarily a literature study on heat treating high manganese steels. The reviewer commented that validation data would be helpful.

\section{Reviewer 2:}

The reviewer remarked that the approach and strategy look good. It seems the team has a sound plan to approach the technical barriers.

\section{Reviewer 3:}

The reviewer said that the approach seems satisfactory. The presentation does not give clear details on the how the improvements will be found. There is good details on the testing methods. The reviewer noted that picking judicious methods in Thrust 4 is not defined. The reviewer commented that this plan for improvements seems too vague for a robust approach, and that the project team is using too many acronyms that are not generally well known. The reviewer noted that HEXRD, RA, ASPPRC, APS, IA, TRIP, TOF-SIM, and CCE are only known to material scientists, and asked that the project team please define these the first time they are used in slides or reports.

Question 2: Technical accomplishments and progress toward overall project and DOE goals-the degree to which progress has been made, measured against performance indicators and demonstrated progress towards DOE goals.

\section{Reviewer 1:}

The reviewer said that the project was started early this year and many comprehensive results have been shown. The reviewer said well done.

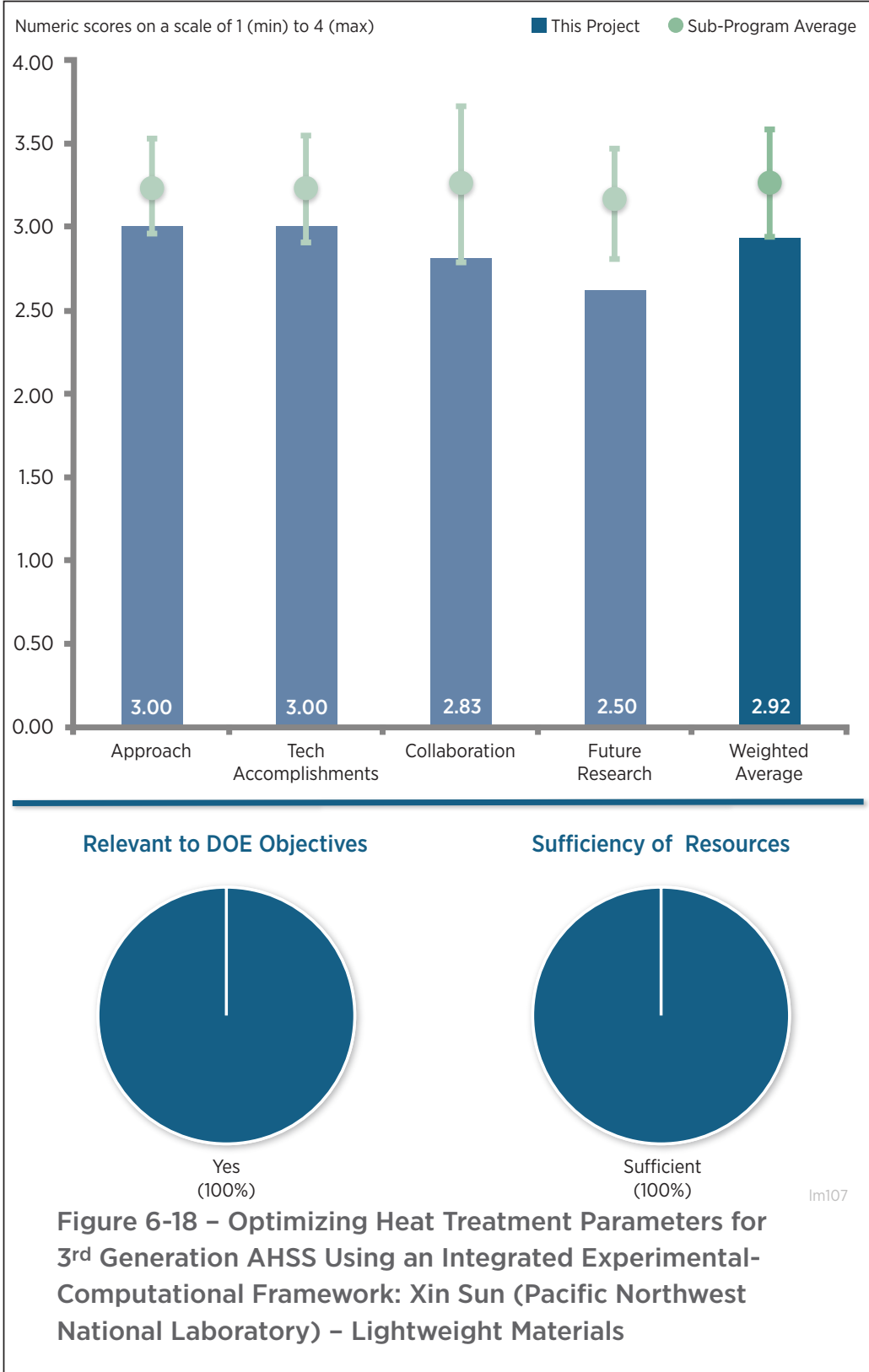




\section{Reviewer 2:}

The reviewer said that the progress to date appears to be primarily generating models, and that more experimental results would be helpful.

\section{Reviewer 3:}

The reviewer said that this project has just started and there are few accomplishments at this time. How the experimental results will be used in the ICME models is unclear to this reviewer and has not been defined in the presentation.

\section{Question 3: Collaboration and coordination with other institutions.}

\section{Reviewer 1:}

The reviewer said that the collaboration and partnership appears to be appropriate.

\section{Reviewer 2:}

The reviewer noted national laboratory, university, and industry involvement, and that different parties are responsible for different tasks.

\section{Reviewer 3:}

The reviewer emphasized that there is not a clear division of labor or clear roles and responsibilities. There is an assembled team, but the project would be improved if the assignments to each member were more clear and distinct. The reviewer commented that where the experiments will be done and who will be doing the math modeling should be clearer.

Question 4: Proposed future research-the degree to which the project has effectively planned its future work in a logical manner by incorporating appropriate decision points, considering barriers to the realization of the proposed technology and, when sensible, mitigating risk by providing alternate development pathways.

\section{Reviewer 1:}

The reviewer said that proposed future research is appropriate.

\section{Reviewer 2:}

The reviewer said that the presentation does not have a specific slide to show the future work, but it can be seen from the tasks listed in Slide 5. The reviewer said that it is good to have the future work that will be done before the next Annual Merit Review meeting listed in a separate slide.

\section{Reviewer 3:}

The reviewer said that there is no definition of the next steps in the developments. The reviewer understands there will be testing, but the reviewer saw no details on how the specimens will be developed, or what will be done with the test results.

\section{Question 5: Does this project support the overall DOE objectives of petroleum displacement? Why or why not?}

\section{Reviewer 1:}

The reviewer said that this supports the acceptance on new steel alloys.

\section{Reviewer 2:}

The reviewer commented that high-strength steels might reduce the weight of future cars and trucks. 
Reviewer 3:

The reviewer said that, similar to the presenter's other project, the development of lightweight material is important for energy saving and electric vehicle driving range extension.

Question 6: Resources: How sufficient are the resources for the project to achieve the stated milestones in a timely fashion?

Reviewer 1:

The reviewer said that the budget is sufficient for the project. 


\section{Development of Low-Cost, High-Strength Automotive Aluminum Sheet: Russell Long (Alcoa) - Im108}

\section{Presenter}

Russell Long, Alcoa

\section{Reviewer Sample Size}

A total of three reviewers evaluated this project.

\section{Question 1: Approach to performing the work-the degree to which technical barriers are addressed, the project is well-designed, feasible, and integrated with other efforts.}

\section{Reviewer 1:}

The reviewer said that this is a large and comprehensive project being carried out by a well-qualified team and the project plan as presented was clear and goal-focused.

\section{Reviewer 2:}

The reviewer observed a good approach and project plan. The reviewer said that a weak link is demonstrating in a production plant environment with alternate objectives.

\section{Reviewer 3:}

The reviewer said that the approach to improve Al performance is well-directed. The reviewer questioned why any efforts are invested in the FSW TWB, and whether the Pacific Northwest National Laboratory (PNNL) successes can be used directly. The reviewer suggested a smaller study on the FSW blanks to confirm and repeat the PNNL learnings. Producing the full scale coils for the parts will be great. The reviewer recommended consideration of keeping or tightening the radii at the closed box ends of the project team's parts to confirm the CAE stamping/warm forming predictions all the way to splits.

\section{Question 2: Technical accomplishments and progress toward overall project and DOE goals-the degree to which progress has been made, measured against performance indicators and demonstrated progress towards DOE goals.}

\section{Reviewer 1 :}

The reviewer noted excellent accomplishments in this first year, and suggested that the team consider using TWB Company and PNNL for the TWB development.

\section{Reviewer 2:}

The reviewer said that accomplishments are on plan.

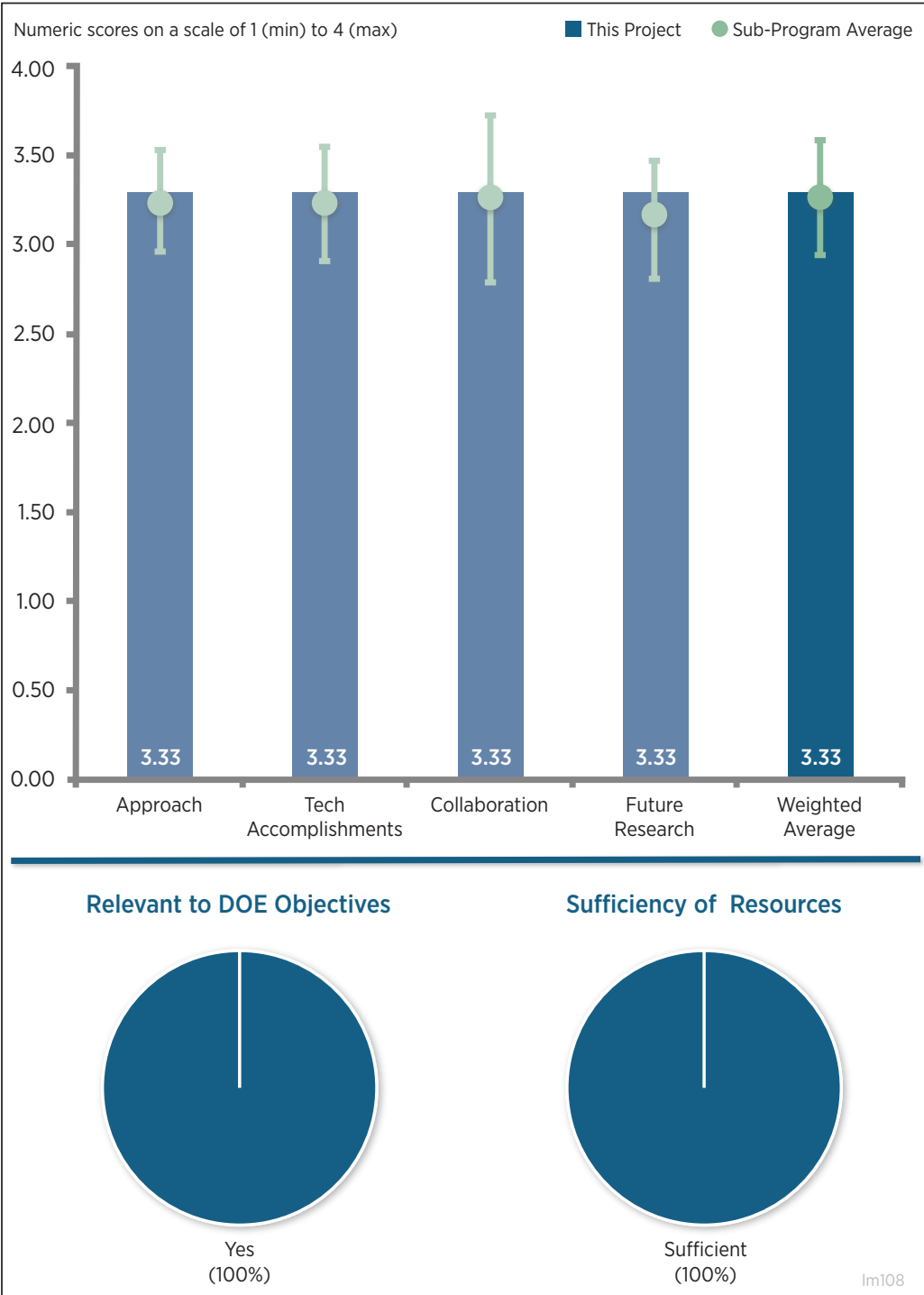

Figure 6-19 - Development of Low-Cost, High-Strength Automotive Aluminum Sheet: Russell Long (Alcoa) Lightweight Materials 


\section{Reviewer 3:}

The reviewer said that the presentation showed that goals are being achieved and that the project appears to be on-track toward its targets. The reviewer noted that significantly more progress is anticipated in the next phases as process equipment becomes available.

\section{Question 3: Collaboration and coordination with other institutions.}

\section{Reviewer 1:}

The reviewer said that collaboration appears to be productive, collegial, and successful. The plan clearly shows that each partner is taking an important role and one that makes sense for the total project.

\section{Reviewer 2:}

The reviewer noticed good collaboration with a vertical partnership OEM, material supplier, and Tier 1.

\section{Reviewer 3:}

The reviewer observed clear divisions of duties among the participants, and great collaboration to achieve overall project success. The reviewer suggested investigating whether the team can use the PNNL FSW for the TWB, and borrowing the lessons from PNNL tailor welded FSW blanks.

Question 4: Proposed future research-the degree to which the project has effectively planned its future work in a logical manner by incorporating appropriate decision points, considering barriers to the realization of the proposed technology and, when sensible, mitigating risk by providing alternate development pathways.

\section{Reviewer 1:}

The reviewer observed clear definition of the next steps to get to the target. The reviewer also wanted to see more clarity on the corrosion investigations.

\section{Reviewer 2:}

The reviewer pointed out that the portion of the presentation that dealt with future challenges was a bit brief, but showed that the team appreciates the potential barriers and that the team appears to have a plan to meet these challenges. The reviewer suspected that springback may prove to be a bigger problem than expected, however.

Question 5: Does this project support the overall DOE objectives of petroleum displacement? Why or why not?

\section{Reviewer 1:}

The reviewer said that the project is definitely aligned with DOE's goals.

\section{Reviewer 2:}

The reviewer said that high-strength lightweight $\mathrm{Al}$ is needed for future lightweight multi-material vehicles.

\section{Reviewer 3:}

The reviewer remarked that the high-strength Al will enable reduced weight of vehicles.

Question 6: Resources: How sufficient are the resources for the project to achieve the stated milestones in a timely fashion?

\section{Reviewer 1:}

The reviewer said that resources look to be quite adequate. 


\section{Acronyms and Abbreviations}

$3 \mathrm{D}$

3GAHHS Third-generation advanced high-strength steels

AHHS

$\mathrm{Al}$

AMR

ARPA-E

BIW

CAEBAT

$\mathrm{CF}$

CFC

CFRP

$\mathrm{Cu}$

$\mathrm{Cu}_{2} \mathrm{O}$

DOE

EERE

FEA

FRP

FSW

FY

GHG

$\mathrm{H}_{2}$

ICME

IP

ISO

lb.

LCA

LDH

LLC

MDA

$\mathrm{Mg}$
Advanced high-strength steels

Aluminum

Annual Merit Review

Advanced Research Projects Agency - Energy

Body in white

Computer-Aided Engineering for Electric-Drive Vehicle Batteries

Carbon fiber

Carbon fiber composite

Carbon fiber-reinforced polymer

Copper

Copper (I) oxide

U.S. Department of Energy

Office of Energy Efficiency and Renewable Energy

Finite element analysis

Fiber-reinforced polymer (fiber-reinforced plastic)

Friction-stir welding

Fiscal year

Greenhouse gas

Hydrogen

Integrated computational materials engineering

Intellectual property

International Organization for Standardization

Pound

Life-cycle assessment (life-cycle analysis)

Limiting dome height

Limited liability company

Molecular dynamic analysis

Magnesium 


\begin{tabular}{|c|c|}
\hline $\mathrm{MgO}$ & Magnesium oxide \\
\hline MMLV & Multi-Material Lightweight Vehicle \\
\hline $\mathrm{MPa}$ & Megapascal \\
\hline MSU & Michigan State University \\
\hline MY & Model year \\
\hline $\mathrm{Nd}$ & Neodymium \\
\hline $\mathrm{Nd}_{2} \mathrm{O}_{3}$ & Neodymium (III) oxide \\
\hline NDE & Nondestructive evaluation \\
\hline NDT & Nondestructive testing \\
\hline NIST & National Institute of Standards and Technology \\
\hline OEM & Original equipment manufacturer \\
\hline ORNL & Oak Ridge National Laboratory \\
\hline PA & Polyamide \\
\hline PI & Principal Investigator \\
\hline PNNL & Pacific Northwest National Laboratory \\
\hline PPA & Polyphthalamide \\
\hline $\mathrm{R} \& \mathrm{D}$ & Research and development \\
\hline RTM & Resin transfer molding \\
\hline RVE & Representative volume element \\
\hline SMC & Sheet molding compound \\
\hline TRL & Technology readiness levels \\
\hline TWB & Tailor-welded blanks \\
\hline UD & Unidirectional \\
\hline UHSS & Ultra-high strength steels \\
\hline U.S. & United States \\
\hline USAMP & United States Automotive Materials Partnership \\
\hline VTO & Vehicle Technologies Office \\
\hline
\end{tabular}




\section{Propulsion Materials}

\section{Advanced materials are essential for boosting the fuel economy of modern automobiles while}

maintaining safety and performance.

Propulsion materials enable higher efficiencies in propulsion systems of all types. For example, many combustion engine components require advanced propulsion materials so they can withstand the high pressures and temperatures of high-efficiency combustion regimes. Similarly, novel propulsion materials may be able to replace the current expensive materials in electric motors and drivetrain components, thus lowering the cost of electric-drive vehicles.

Using lightweight components and high-efficiency engines enabled by advanced materials in one quarter of the U.S. fleet could save more than 5 billion gallons of fuel annually by 2030 .

The U.S. Department of Energy (DOE) Vehicle Technologies Office (VTO) collaborates with industry to improve materials that will increase vehicle efficiency while meeting consumer and industry expectations. It does this through work on both Lightweight Materials and Propulsion Materials. In the case of Propulsion Materials, VTO works closely with other VTO subprogram technology areas to identify and meet requirements for materials needed to develop cost-effective, highly efficient, and environmentally friendly next-generation heavy and light duty powertrains.

Research and development (R\&D) is done in collaboration with industry, national laboratories, and universities. The VTO contributes to the Materials Genome Initiative, a federal interagency effort to support Integrated Computational Materials Engineering. It also works through government/industry partnerships:

- The United States Driving Research and Innovation for Vehicle Efficiency and Energy sustainability (U.S. DRIVE) Partnership focusing on light-duty vehicles;

- The $21^{\text {st }}$ Century Truck Partnership, focusing on heavy-duty (HD) vehicles; and

- The U.S. Automotive Materials Partnership (USAMP).

The Propulsion Materials (PM) subprogram's major R\&D goal is to develop high performance cost-effective materials that solve key challenges that currently limit the performance of propulsion systems (high-efficiency engines and electric drive, and compatibility with alternative fuels).

\section{Subprogram Feedback}

The U.S. Department of Energy (DOE) received feedback on the overall technical subprogram areas presented during the 2016 Annual Merit Review (AMR). Each subprogram technical session was introduced with a presentation that provided an overview of subprogram goals and recent progress, followed by a series of detailed topic area project presentations.

The reviewers for a given subprogram area responded to a series of specific questions regarding the breadth, depth, and appropriateness of that DOE VTO subprogram's activities. The subprogram overview questions are listed below, and it should be noted that no scoring metrics were applied. These questions were used for all VTO subprogram overviews.

Question 1: Was the program area, including overall strategy, adequately covered? Question 2: Is there an appropriate balance between near- mid- and long-term research and development?

\section{Question 3: Were important issues and challenges identified?}


Question 4: Are plans identified for addressing issues and challenges?

Question 5: Was progress clearly benchmarked against the previous year?

Question 6: Are the projects in this technology area addressing the broad problems and barriers that the Vehicle Technologies Office (VTO) is trying to solve?

Question 7: Does the program area appear to be focused, well-managed, and effective in addressing VTO's needs?

Question 8: What are the key strengths and weaknesses of the projects in this program area? Do any of the projects stand out on either end of the spectrum?

Question 9: Do these projects represent novel and/or innovative ways to approach these barriers as appropriate?

Question 10: Has the program area engaged appropriate partners?

Question 11: Is the program area collaborating with them effectively?

Question 12: Are there any gaps in the portfolio for this technology area?

Question 13: Are there topics that are not being adequately addressed?

Question 14: Are there other areas that this program area should consider funding to meet overall programmatic goals?

Question 15: Can you recommend new ways to approach the barriers addressed by this program area?

Question 16: Are there any other suggestions to improve the effectiveness of this program area?

Responses to the subprogram overview questions are summarized in the following pages. Individual reviewer comments for each question are identified under the heading Reviewer 1, Reviewer 2, etc. Note that reviewer comments may be ordered differently; for example, for each specific subprogram overview presentation, the reviewer identified as Reviewer 1 in the first question may not be Reviewer 1 in the second question, etc. 


\section{Overview of VTO Material Technologies: Jerry Gibbs (U.S. Department of Energy) - pmo00}

Question 1: Was the program area, including overall strategy, adequately covered?

\section{Reviewer 1:}

The reviewer replied yes, stating there was a well-rounded portfolio addressing many areas of materials research focused on expediting the process of moving innovative materials to the marketplace. The reviewer stated that the combination of improving lightweight materials that increase the tolerance of the engine to higher temperatures and pressures and combustion strategies to achieve greater efficiency and the resulting need of low-temperature aftertreatment is very appropriate.

\section{Reviewer 2:}

The reviewer answered yes, remarking it was very clearly structured and the content is accurately conveyed.

\section{Reviewer 3:}

The reviewer replied yes, that a nice overview was given, with both high-level points and some detail on each area and why it was included. The reviewer concluded that there is an overall strategy uniting the program elements together.

\section{Reviewer 4:}

The reviewer replied yes to this question.

\section{Reviewer 5:}

The reviewer stated that the program area and overall strategy was sufficiently covered. This included the roadmap and materials requirements for powertrain components, as well as both current limitations and anticipated properties out until 2050. The reviewer commented that one of the innovative claims for this program is that advances in materials for current internal combustion engines, transmission and exhaust components will improve the materials available for other propulsion systems (such as electric) because of the extreme environments and approaches to material design.

\section{Reviewer 6:}

The reviewer replied yes, the scope was clearly presented. However, this reviewer does not understand the decrease in funding that the program is seeing given the obvious need for increased materials capabilities to support efficiency requirements for internal combustion engines going forward. The reviewer claimed the reality is that the hydrogen economy is not the future, and for the foreseeable future (say to 2030), the answer will remain the internal combustion engine. The reviewer also observed that increasing efficiency of internal combustion using currently available materials has been optimized, and little changes are available within that space. The reviewer concluded that the obvious answer is that the properties of the materials associated with the combustion event must be changed (temperatures and strength limits) to create a new space for increased efficiency.

\section{Question 2: Is there an appropriate balance between near- mid- and long-term research and development?}

\section{Reviewer 1:}

The reviewer offered that in a normal situation, the proper materials research balance requires significantly more long-term research as it is at least a second tier to a product. However, the reviewer stated that the program leader has successfully used materials processing to fill in the shorter term research and is doing a great job of continuing to create wins for the program. Praising this is as a novel and creative way to continue to create commercial wins for the program, this reviewer is impressed with this insight.

\section{Reviewer 2:}

The reviewer remarked that the balance between near-, mid-, and long-term elements is sufficient, adding that the program has some immediate goals for materials to push the boundary of current material properties as well as to evaluate the knowledge gaps in computational material programs. The reviewer observed that these results enable the mid-term programs to collaborate with the development of solutions to fill these gaps. The reviewer 
concluded that the long-term goal of achieving the materials with properties will be enabled by both the near-term investigation as well as the future tools in the mid-term efforts.

\section{Reviewer 3:}

The reviewer replied yes to this question.

\section{Reviewer 4:}

The reviewer replied yes to this question.

\section{Reviewer 5:}

The reviewer noted that the idea is to speed up the long-term research timeline from greater than 40 years to about 5 years, and as a result, the long-term research becomes near and mid-term.

\section{Reviewer 6:}

The reviewer stated that there appears to be, adding that the overarching theme of trying to move innovations to market faster through computational methods is a good way to achieve the desired result for materials. However, the reviewer cautioned, with respect to the aftertreatment projects, more funding is necessary to investigate the wide range of technologies needed to support advanced engine development.

\section{Question 3: Were important issues and challenges identified?}

\section{Reviewer 1:}

The reviewer said yes, these are very clearly identified.

\section{Reviewer 2:}

The reviewer replied yes to this question.

\section{Reviewer 3:}

The reviewer answered yes, elaborating that speeding up long-term research is very challenging and requires intense collaborations between industry partners including original equipment manufacturers (OEMs) and suppliers, national laboratories, and academia. The reviewer added that most of the challenges are simply in communication between partners and access by the partners to the right analytical tools, and characterized DOE as well-positioned to facilitate these interactions.

\section{Reviewer 4:}

The reviewer described as sufficient the identification of important issues and challenges with accompanying metrics to identify progress in resolving these, adding that the goals to reduce weight, increase power density, and engine metrics are admirable goals that must be solved in parallel with material improvements. The reviewer concluded the tools of using computational materials and national laboratories equipment to measure results on the scale that they are predicted are viable for solving near- and mid-term challenges.

\section{Reviewer 5:}

The reviewer replied yes, both in the area of lightweight materials and engine efficiency/aftertreatment. However, from the data provided, the reviewer suggested that the program more generously fund projects under the materials umbrella, characterizing the breadth and scope of potential projects that could impact advancement in this area as not being at an acceptable level. The reviewer warned that the trend points to even less funding going forward and urged that this must be reversed to effectively support the activities of the major vehicle OEMs to meet efficiency targets for emissions and fuel economy. The reviewer remarked that given the size of the fleets that use internal combustion engines, even small advancements will have a significant impact on the nation's energy independence.

\section{Reviewer 6:}

The reviewer said yes, the workshop data projecting 2050 was presented and was a great overview. However, the reviewer noted that the data were from 2011 and said it would be great to see this renewed and see where we are in 2016. The reviewer also said it would be nice to see what else is available to do but did not make the cut because of the available budget. The reviewer commented that the challenges around holes in the models as they are combined is very important, and it is disappointing that this work was not funded to fill those holes. 


\section{Question 4: Are plans identified for addressing issues and challenges?}

\section{Reviewer 1:}

The reviewer answered yes, noting that plans were clearly laid out and clearly-leveled targets stated.

\section{Reviewer 2:}

The reviewer replied yes to this question.

\section{Reviewer 3:}

The reviewer characterized plans identified for addressing issues and challenges as sufficient and remarked that the portfolio of projects reviewed in this program look comprehensive in the mechanical property requirements for future applications. The reviewer referenced the question and answer session, and added that tools feedback in the form of a meeting at The Minerals, Metals \& Materials Society (TMS) 2017 to discuss gaps in integrated computational materials engineering (ICME) is helpful. Further structuring of these programs with specific deliverables toward these ICME gaps would be helpful as well.

\section{Reviewer 4:}

The reviewer replied yes, observing that the incorporation of very fast computing can directly speed up development. The reviewer further remarked that solicitations containing multiple partner-type requirements, including national laboratories with fast computing and sophisticated analytical tools, also help speed up materials development.

\section{Reviewer 5:}

The reviewer said yes, the talk covered issues with the trend of increasing peak cylinder pressures and increased engine out exhaust temperatures, and it also identified the lowering of catalyst inlet temperatures due to turbocharging and waste heat recovery. However, the reviewer added, that the presenters do not intend to fix the modeling issues they identified, and that is a major missed opportunity. The reviewer realized this is due to funding, but would be remiss if it was not identified in this review.

\section{Reviewer 6:}

The reviewer answered not with respect to funding, and that this must be addressed.

\section{Question 5: Was progress clearly benchmarked against the previous year?}

\section{Reviewer 1:}

The reviewer answered yes, adding it was also benchmarked against the time to market using non-computational methods to speed the movement of technology to market.

\section{Reviewer 2:}

The reviewer replied yes to this question.

\section{Reviewer 3:}

The reviewer replied yes to this question.

\section{Reviewer 4:}

The reviewer said yes, remarking that the program continues to create wins with decreasing resources and then commenting that this is either a strong testament to the program manager's capability to identify key technologies for impact, or to the fact that the field is so wrought with opportunity that it becomes a challenge to select a lowimpact project.

\section{Reviewer 5:}

The reviewer stated that including the chart looking at the technology implementation timeline was a useful metric to demonstrate the accomplishments of this program against the stated goals and also noted that the stated goals for the projects for achieving materials properties were accomplished. The reviewer remarked that a final technology product would be nice to highlight although difficult by the proprietary nature of working directly with an OEM or first tier suppliers. 


\section{Reviewer 6:}

The reviewer indicated that the overview did not include much in terms of progress details, observing that a lot of slides had the same words as last year. The reviewer pointed out that there was a crankshaft project noted as an example of faster progress than normal materials development and another item noted was access gained to the Titan supercomputing system at Oak Ridge National Laboratory (ORNL) for greatly increased computational speed for metal alloy modeling. The reviewer commented that the program is divesting itself of hybrid electric vehicle (HEV) materials in favor of the SuperTruck II project.

\section{Question 6: Are the projects in this technology area addressing the broad problems and barriers that the Vehicle Technologies Office (VTO) is trying to solve?}

\section{Reviewer 1:}

The reviewer praised the work that is being done as spot on with the intent and well aligned with the scope. The level of work in this area is well beyond any other presentations this reviewer attended at the Annual Merit Review. The reviewer added that it is very telling that so much of the U.S. Council for Automotive Research (USCAR) identified needs were materials issues.

\section{Reviewer 2:}

The reviewer replied yes to this question.

\section{Reviewer 3:}

The review replied yes to this question.

\section{Reviewer 4:}

The reviewer stated that addressing the broad problems and barriers in the VTO is sufficient, in particular, the targets of high efficiency internal combustion engines and reduction of emissions through high performance materials is applicable. The reviewer added the parallel activities of properties and components is essential to the success of the VTO program.

\section{Reviewer 5:}

The reviewer remarked that the goal is higher engine efficiency and the projects included in this area are enablers for meeting that overall goal.

\section{Reviewer 6:}

The reviewer responded not adequately, characterizing the funding as a major roadblock with respect to the breadth of technologies that must be addressed.

\section{Question 7: Does the program area appear to be focused, well-managed, and effective in addressing VTO's needs?}

\section{Reviewer 1:}

The reviewer declared absolutely, characterizing it as an extremely efficiently managed program, well aligned with scope, and in step with the industry needs to maintain the competitive position of U.S. companies.

\section{Reviewer 2:}

The reviewer replied yes, the interaction of the program managers through yearly face to face meetings and multiple program updates throughout the year appears to be an effective means to keep projects focused and adaptive to changing project needs.

\section{Reviewer 3:}

The reviewer stated that the program appears to be well-managed, adding that this is good because on the surface it appears to be a very disparate set of technology areas. The reviewer remarked, however, the program manager appears to have both managerial skills and deep technical understanding that can help bring everything together.

\section{Reviewer 4:}

The reviewer replied yes to this question. 


\section{Reviewer 5:}

The reviewer replied yes to this question.

\section{Reviewer 6:}

The reviewer characterized the focus of this program on materials properties and use of OEMs to develop materials and create applications as sufficient to address VTO's needs. The reviewer cautioned that whether this approach is effective depends on the extent to which the OEM partners and principals implement and publish results.

\section{Question 8: What are the key strengths and weaknesses of the projects in this program area? Do any of the projects stand out on either end of the spectrum?}

\section{Reviewer 1:}

The reviewer characterized as a key strength the linkage of materials properties back to combustion modeling through optimizing thermal efficiency without materials constraints and then deducing what needs to be changed in materials technology to support this.

\section{Reviewer 2:}

This reviewer liked the program involving ICME and Materials for Hybrid and Electric Drive Systems. The former would significantly reduce the materials $R \& D$ time and the latter is very important for vehicles using renewable energy.

\section{Reviewer 3:}

The reviewer remarked that the computational approaches to advancing innovative materials appears to be working well and appears funded adequately, but that the engine efficiency and aftertreatment programs must be funded at a higher level to improve the ability to address all the needed areas of research.

\section{Reviewer 4:}

The reviewer described the key strengths of the projects in this program areas as including the following: Using a computational materials approach is a strength to this program to deliver a gap assessment as well as identify collaborative programs for further tools development; and many of the programs have OEM leads (e.g., Ford, GM, and Caterpillar) that have the benefit of both knowing materials requirements and using materials to design the latest components in propulsion materials. A key weakness observed by the reviewer is the reduction of funding year over year in a critical tool development environment.

\section{Reviewer 5:}

The reviewer noted for a few areas, budget seems to have been cut, which might impact continuity and incremental technological improvements in those areas.

\section{Reviewer 6:}

The reviewer stated one potential problem is that improving engine materials to withstand higher temperatures and allow for higher exhaust gas temperatures could jeopardize progress made in low-temperature catalysts. The reviewer elaborated that typically, there is a tradeoff to be made in catalyst activity at low temperatures and the high-temperature stability. The reviewer noted that the current catalyst technologies for stoichiometric operation can withstand $1,000^{\circ}$ Celsius (C), but future materials might be only able to withstand $900^{\circ} \mathrm{C}$ or even $800^{\circ} \mathrm{C}$ peak temperatures. Thus, it is important to pay attention to the system impacts of changing components.

\section{Question 9: Do these projects represent novel and/or innovative ways to approach these barriers as appropriate?}

\section{Reviewer 1:}

This reviewer strongly agreed that the projects comprising part of the materials portfolio represent novel and/or innovative technologies and approaches to help overcome the barriers and challenges that the industry is facing in a number of powertrain areas.

\section{Reviewer 2:}

The reviewer declared that exploiting the physical limits of the materials will enable new concepts in component design that incorporate the very best design approaches and will yield benefits beyond the immediate program 
success. The reviewer also applauded the use of national laboratory equipment in new ways to validate computational materials approaches at the modeling length scale.

\section{Reviewer 3:}

This reviewer affirmed seeing many capable, good reputable OEMs, institutes, and universities involved in appropriate areas in which they have expertise.

\section{Reviewer 4:}

The reviewer replied yes, for example, the reverse use of combustion modeling is great.

\section{Reviewer 5:}

The reviewer replied yes to this question.

\section{Reviewer 6:}

The reviewer remarked that this was an overview and there were not enough details given on the projects to judge novelty. The reviewer also noted that no intellectual property (IP) was mentioned.

\section{Question 10: Has the program area engaged appropriate partners?}

\section{Reviewer 1:}

The reviewer remarked that from the presentation, it appears that the lightweight materials portion of the portfolio is well engaged. Speaking directly for the propulsion materials projects, the reviewer described all the projects as supported with the necessary partners to help ensure successful outcomes.

\section{Reviewer 2:}

The reviewer characterized the program has having engaged appropriate partners from OEMs, national laboratories, and academic institutions and suggested that further partnerships with computational materials companies will be useful in the future, adding that some of this engagement is dependent on the relationship of the OEM to the computational materials companies.

\section{Reviewer 3:}

The reviewer said yes, the major concerns in the U.S. were clearly engaged.

\section{Reviewer 4:}

The reviewer replied yes to this question.

\section{Reviewer 5:}

The reviewer replied yes to this question.

\section{Reviewer 6:}

The reviewer noted that the projects include numerous industrial OEMs, suppliers, and national laboratories as partners.

\section{Question 11: Is the program area collaborating with them effectively?}

\section{Reviewer 1:}

The reviewer affirmed yes, they were obviously well connected, and their views well represented.

\section{Reviewer 2:}

The reviewer stated that meetings, experiments, and publications demonstrate effective collaboration between all partners.

\section{Reviewer 3:}

The reviewer answered yes, noting multiple project meetings with the interested parties each year and at least one face-to-face meeting between DOE, national laboratories involved, and the other members.

\section{Reviewer 4:}

The reviewer replied yes to this question. 


\section{Reviewer 5:}

The reviewer replied yes to this question.

\section{Reviewer 6:}

The reviewer stated that it appears that the program area is collaborating but cautioned how effective it is will be shown in a few years when approaching commercialization opportunities for the materials developed in the program.

\section{Question 12: Are there any gaps in the portfolio for this technology area?}

\section{Reviewer 1:}

Not as far as this reviewer is aware.

\section{Reviewer 2:}

The reviewer stated that, in general, there is good coverage with just one issue around the needed sensing technology which the reviewer thinks will come along with low-temperature operations very soon.

\section{Reviewer 3:}

The reviewer commented that adding low-temperature aftertreatment closes a previously existing gap, but that engine changes should be treated as part of the system, along with aftertreatment changes.

\section{Reviewer 4:}

The reviewer noted that gaps in the computational materials area have been discussed and will be reviewed with the broader community during a session of TMS 2017. This was discussed in the question and answer after the presentation.

\section{Reviewer 5:}

The reviewer asserted that resources are a gap in the portfolio. This person further explained that this is a major area for opportunity and noted that the resources are dwindling.

\section{Reviewer 6:}

The reviewer specified one gap that has emerged, apparently as a result of reduced funding, is the area of lowtemperature aftertreatment research projects. The reviewer pointed out that this was an area of innovation that was strongly stressed by the automotive OEMs through USCAR and the associated workshops and technology roadmaps. The reviewer also remarked that the current level of funding does not appear to be able to support this activity at the level required to help the OEMs find new technologies to meet future emissions challenges.

\section{Question 13: Are there topics that are not being adequately addressed?}

\section{Reviewer 1:}

The reviewer replied no to this question.

\section{Reviewer 2:}

The reviewer replied not really to this question.

\section{Reviewer 3:}

The reviewer referenced prior comments to question 12 and stated low-temperature aftertreatment.

\section{Reviewer 4:}

The reviewer answered clearly so, but added that given the program's resource constraints, it is doing amazing things.

\section{Reviewer 5:}

The reviewer said that component demonstration of advanced materials is pending but identified this as a difficult task, and that this would be a good area of focus, adding that nondestructive testing and examination of these new materials is missing in the approaches discussed. 


\section{Reviewer 6:}

The reviewer remarked it needs to be acknowledged that improving high-temperature stability of engine materials could result in a lack of low-temperature aftertreatment materials that can withstand the potential higher peak exhaust temperatures. The reviewer suggested that this tradeoff should be emphasized more.

\section{Question 14: Are there other areas that this program area should consider funding to meet overall programmatic goals?}

\section{Reviewer 1:}

The reviewer replied no, the program is very comprehensive.

\section{Reviewer 2:}

The reviewer remarked that given the current budget, it would be difficult to include additional areas that would benefit from additional $\mathrm{R} \& \mathrm{D}$ resources.

\section{Reviewer 3:}

This reviewer does not see how this program area can take on anything else given the limited budget.

\section{Reviewer 4:}

The reviewer replied yes, and added they are too numerous to list. The reviewer declared this is an amazing area of opportunity and the presentation did a great job of relating intent and scope.

\section{Reviewer 5:}

The reviewer noted sensing technology and materials to prevent heat loss from the engine skin/piping system.

\section{Reviewer 6:}

The reviewer offered that broader demonstrations of computational materials tools validated with research from this program area would follow well from the component demonstration mentioned above. The reviewer added that quality assurance and testing limits would be a helpful area to include in the future for these advanced materials.

\section{Question 15: Can you recommend new ways to approach the barriers addressed by this program area?}

\section{Reviewer 1:}

The reviewer remarked that the program is going well and making progress continuously. This reviewer does not have any new ways to recommend.

\section{Reviewer 2:}

The reviewer commented that the current approach seems to be good at this moment.

\section{Reviewer 3:}

The reviewer replied none at this time.

\section{Reviewer 4:}

The reviewer stated that this is an underfunded area that shows a lot of opportunity.

\section{Reviewer 5:}

The reviewer remarked that for barriers to be addressed in an effective manner, adequate funding of joint DOE and OEM projects must be in place and that powertrain technologies based on combustion engines will be the primary mode of propulsion in the near- to medium-term as least. The reviewer concluded that not supporting activities that support these powertrains, where incremental improvements can have a large effect on fleet miles per gallon (MPG) and emissions, in preference to battery-based alternatives, would not be the best course of action.

\section{Reviewer 6:}

The reviewer warned that with half the funding going to SuperTruck II, and a lack of real information about what that will include because it is in the competitive phase, it is hard to offer up new approaches. 
Question 16: Are there any other suggestions to improve the effectiveness of this program area?

Reviewer 1:

The reviewer had no specific suggestions and instead said to keep the current good working relationships with involved partners.

Reviewer 2:

The reviewer replied no to this question.

Reviewer 3:

The reviewer said none at this time.

Reviewer 4:

The reviewer recommended a reverse to the trend of funding cuts.

\section{Reviewer 5:}

The reviewer said more budget and claimed that the use of half the budget on SuperTruck II will jeopardize opportunities to fund other projects in the future. The reviewer suggested that spinning off the HEV component would help somewhat.

\section{Reviewer 6:}

The reviewer exclaimed increase resources, stating that this work is pivotal both to have the United States maintain the lead in transportation technology as well as in increasing efficiency and thus lowering energy usage rate.

The reviewer commented that U.S. companies routinely compete against foreign concerns that are not under capitalist profitability expectations, and that the only way to maintain or grow U.S. market share is via technology improvements. The reviewer explained that if the future is not in unique materials, then we are building the future engines today, and we are defeated.

The reviewer remarked that the lead time for materials research puts it into a space where companies cannot afford to invest in it due to the distance in economic return. This person added that stakeholders do not have the patience to wait 15 to 20 years before a return on investment is seen. The reviewer concluded that work in this program effectively decreases that horizon time to a point where it is palatable and allows U.S. companies to maintain technology leadership. 


\section{Project Feedback}

In this merit review activity, each reviewer was asked to respond to a series of questions, involving multiple-choice responses, expository responses where text comments were requested, and numeric score responses (on a scale of 1.0 to 4.0). In the pages that follow, the reviewer responses to each question for each project will be summarized: the multiple choice and numeric score questions will be presented in graph form for each project, and the expository text responses will be summarized in paragraph form for each question. A table presenting the average numeric score for each question for each project is presented below.

Table 7-1 - Project Feedback

\begin{tabular}{|c|c|c|c|c|c|c|c|}
\hline Presentation Title & $\begin{array}{c}\text { Principal } \\
\text { Investigator } \\
\text { and } \\
\text { Organization }\end{array}$ & $\begin{array}{l}\text { Page } \\
\text { Number }\end{array}$ & Approach & $\begin{array}{c}\text { Technical } \\
\text { Accomplishments }\end{array}$ & Collaborations & $\begin{array}{l}\text { Future } \\
\text { Research }\end{array}$ & $\begin{array}{l}\text { Weighted } \\
\text { Average }\end{array}$ \\
\hline $\begin{array}{c}\text { Novel } \\
\text { Manufacturing } \\
\text { Technologies } \\
\text { for High-Power } \\
\text { Induction and } \\
\text { Permanent } \\
\text { Magnet Electric } \\
\text { Motors }\end{array}$ & $\begin{array}{l}\text { Grant, Glenn } \\
\text { (PNNL) }\end{array}$ & 7-15 & 3.45 & 3.45 & 3.45 & 3.10 & 3.41 \\
\hline $\begin{array}{c}\text { Materials Issues } \\
\text { Associated with } \\
\text { EGR Systems }\end{array}$ & $\begin{array}{l}\text { Lance, Michael } \\
\text { (ORNL) }\end{array}$ & $7-22$ & 3.38 & 3.13 & 3.50 & 3.38 & 3.27 \\
\hline $\begin{array}{l}\text { High-Temperature } \\
\text { Materials for High- } \\
\text { Efficiency Engines }\end{array}$ & $\begin{array}{l}\text { Muralidharan, } \\
\text { Govindarajan } \\
\text { (ORNL) }\end{array}$ & $7-26$ & 3.17 & 3.17 & 3.00 & 3.00 & 3.13 \\
\hline $\begin{array}{c}\text { Enabling } \\
\text { Materials for } \\
\text { High-Temperature } \\
\text { Power Electronics }\end{array}$ & $\begin{array}{l}\text { Wereszczak, } \\
\text { Andrew } \\
\text { (ORNL) }\end{array}$ & $7-28$ & 3.33 & 3.33 & 3.33 & 3.17 & 3.31 \\
\hline $\begin{array}{c}\text { Biofuel Impacts } \\
\text { on Aftertreatment } \\
\text { Devices }\end{array}$ & $\begin{array}{l}\text { Lance, Michael } \\
\text { (ORNL) }\end{array}$ & 7-31 & 3.33 & 3.25 & 3.08 & 3.17 & 3.24 \\
\hline $\begin{array}{l}\text { Applied } \\
\text { Computational } \\
\text { Methods for } \\
\text { New Propulsion } \\
\text { Materials }\end{array}$ & $\begin{array}{l}\text { Finney, Charles } \\
\text { (ORNL) }\end{array}$ & $7-34$ & 3.39 & 3.50 & 3.39 & 3.33 & 3.44 \\
\hline
\end{tabular}




\begin{tabular}{|c|c|c|c|c|c|c|c|}
\hline Presentation Title & $\begin{array}{c}\text { Principal } \\
\text { Investigator } \\
\text { and } \\
\text { Organization }\end{array}$ & $\begin{array}{l}\text { Page } \\
\text { Number }\end{array}$ & Approach & $\begin{array}{c}\text { Technical } \\
\text { Accomplishments }\end{array}$ & Collaborations & $\begin{array}{l}\text { Future } \\
\text { Research }\end{array}$ & $\begin{array}{l}\text { Weighted } \\
\text { Average }\end{array}$ \\
\hline $\begin{array}{l}\text { Development of } \\
\text { Advanced High- } \\
\text { Strength Cast } \\
\text { Alloys for Heavy- } \\
\text { Duty Engines }\end{array}$ & $\begin{array}{l}\text { Huff, Rich } \\
\text { (Caterpillar) }\end{array}$ & $7-39$ & 3.39 & 3.50 & 3.39 & 3.33 & 3.44 \\
\hline $\begin{array}{c}\text { Integrated } \\
\text { Computational } \\
\text { Materials } \\
\text { Engineering } \\
\text { Guided } \\
\text { Development of } \\
\text { Advanced Cast } \\
\text { Aluminum Alloys } \\
\text { For Automotive } \\
\text { Engine } \\
\text { Applications }\end{array}$ & Li, Mei (Ford) & 7-44 & 3.46 & 3.33 & 3.42 & 3.33 & 3.38 \\
\hline $\begin{array}{l}\text { Computational } \\
\text { Design and } \\
\text { Development of a } \\
\text { New, Lightweight } \\
\text { Cast Alloy } \\
\text { for Advanced } \\
\text { Cylinder Heads in } \\
\text { High-Efficiency, } \\
\text { Light-Duty } \\
\text { Engines }\end{array}$ & $\begin{array}{l}\text { Walker, Mike } \\
\text { (General } \\
\text { Motors) }\end{array}$ & 7-51 & 3.13 & 2.88 & 3.31 & 3.00 & 3.01 \\
\hline $\begin{array}{l}\text { High-Performance } \\
\text { Cast Aluminum } \\
\text { Alloys for Next } \\
\text { Generation } \\
\text { Passenger Vehicle } \\
\text { Engines }\end{array}$ & $\begin{array}{l}\text { Shyam, Amit } \\
\text { (ORNL) }\end{array}$ & $7-57$ & 3.40 & 3.50 & 3.50 & 3.40 & 3.46 \\
\hline $\begin{array}{l}\text { Development of } \\
\text { High-Performance } \\
\text { Cast Crankshafts }\end{array}$ & $\begin{array}{l}\text { Huff, Rich } \\
\text { (Caterpillar) }\end{array}$ & $7-61$ & 3.38 & 3.13 & 3.50 & 3.38 & 3.27 \\
\hline $\begin{array}{l}\text { Innovative SCR } \\
\text { Materials and } \\
\text { Systems for } \\
\text { Low-Temperature } \\
\text { Aftertreatment }\end{array}$ & $\begin{array}{l}\text { Wang, Yong } \\
\text { (PNNL) }\end{array}$ & $7-64$ & 3.25 & 3.50 & 3.17 & 3.17 & 3.35 \\
\hline
\end{tabular}




\begin{tabular}{|c|c|c|c|c|c|c|c|}
\hline Presentation Title & $\begin{array}{c}\text { Principal } \\
\text { Investigator } \\
\text { and } \\
\text { Organization }\end{array}$ & $\begin{array}{l}\text { Page } \\
\text { Number }\end{array}$ & Approach & $\begin{array}{c}\text { Technical } \\
\text { Accomplishments }\end{array}$ & Collaborations & $\begin{array}{c}\text { Future } \\
\text { Research }\end{array}$ & $\begin{array}{c}\text { Weighted } \\
\text { Average }\end{array}$ \\
\hline $\begin{array}{c}\text { Next Generation } \\
\text { Three-Way } \\
\text { Catalysts for } \\
\text { Future, Highly } \\
\text { Efficient Gasoline } \\
\text { Engines }\end{array}$ & $\begin{array}{l}\text { Lambert, } \\
\text { Christine } \\
\text { (Ford) }\end{array}$ & $7-68$ & 3.50 & 3.50 & 3.30 & 3.30 & 3.45 \\
\hline $\begin{array}{l}\text { Sustained Low- } \\
\text { Temperature } \\
\text { NOx Reduction } \\
\text { (SLTNR) }\end{array}$ & $\begin{array}{l}\text { Zha, Yuhui } \\
\text { (Cummins) }\end{array}$ & $7-73$ & 3.50 & 3.63 & 3.50 & 3.38 & 3.55 \\
\hline Overall Average & & & 3.36 & 3.33 & 3.37 & 3.22 & 3.33 \\
\hline
\end{tabular}




\section{Novel Manufacturing \\ Technologies for High-Power Induction and Permanent Magnet Electric Motors: Glenn Grant (Pacific Northwest National Laboratory) - pm004}

\section{Presenter}

Glenn Grant, Pacific Northwest National Laboratory

\section{Reviewer Sample Size}

A total of 10 reviewers evaluated this project

\section{Question 1: Approach to performing the work-the degree to which technical barriers are addressed, the project is well-designed, feasible, and integrated with other efforts.}

\section{Reviewer 1:}

The reviewer observed that this project is near completion with no new funding for fiscal year (FY) 2016 and noted that Dr. Grant provided an excellent overview of the project and its accomplishments. In addition, the reviewer highlighted that the approach to developing a solid state weld procedure that will ultimately lead to lower cost manufacturing of copper $(\mathrm{Cu})$ rotors was well stated and included systematically developing solutions to controlling temperature, experimental determination of weld protocols, tool design and working with the industrial partner to characterize welds and iteratively improve the overall process.

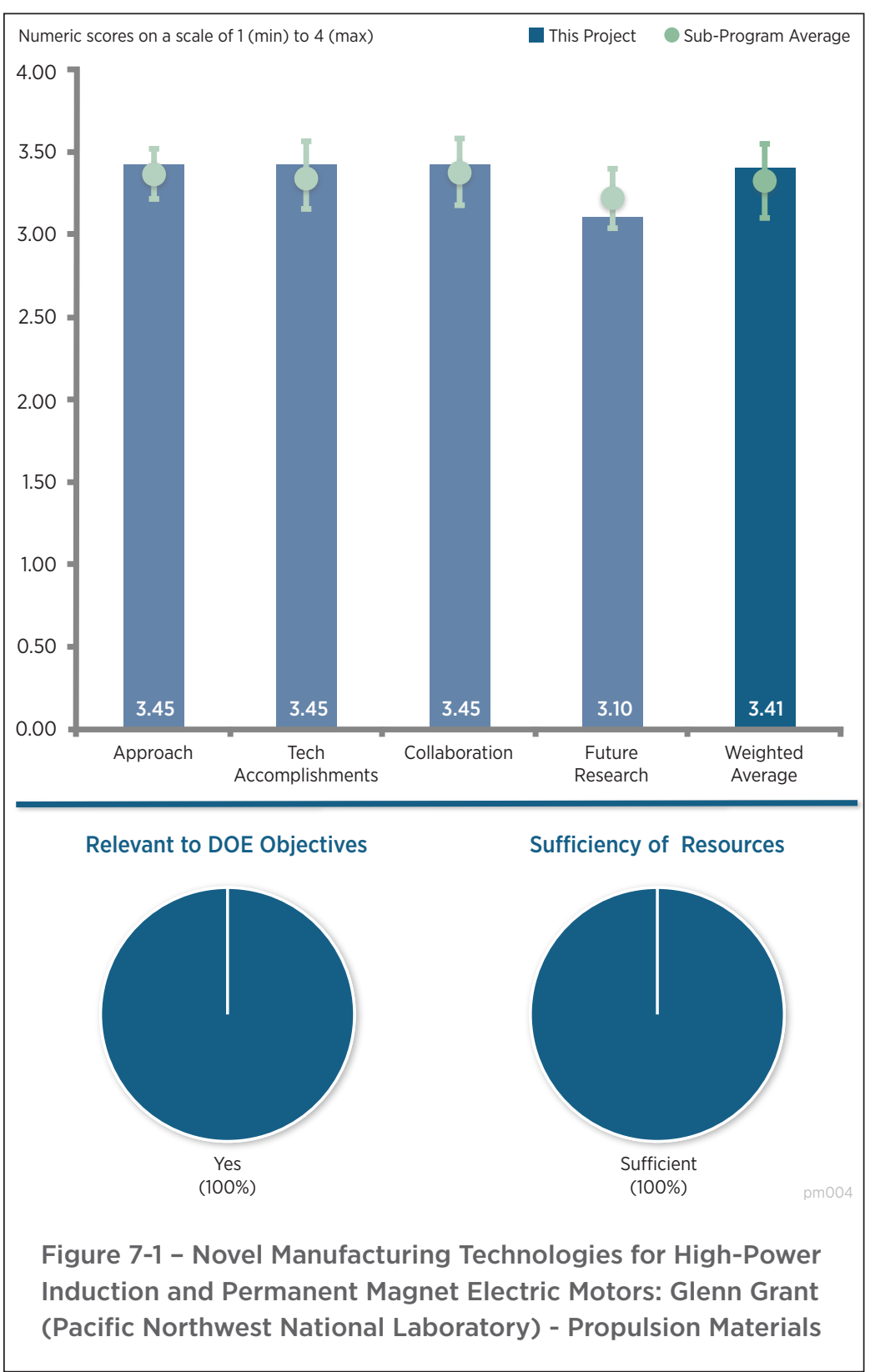

\section{Reviewer 2:}

The reviewer indicated the approach is addressing improvements to induction motors that have the opportunity for lower cost and removal of critical materials (rare earths), which is very important and that the project addresses a key efficiency loss area for induction motors (joining of rotor bars to end caps). In addition, the reviewer acknowledged the approach applies a previously developed DOE VTO materials technology process (friction stir welding [FSW]) for a new problem, which is an excellent use of DOE funds to extend previous success to a new area. This project has a good balance of technology exploration, scientific research, and practical manufacturing considerations. The process for the work is appropriate for the technology readiness levels (TRL) being addressed.

\section{Reviewer 3:}

The reviewer concluded the project has excellent manufacturing process development with FSW and an automotive OEM. 


\section{Reviewer 4:}

The reviewer stated the project has an excellent approach and that the technique can be applied to other applications.

\section{Reviewer 5:}

The reviewer described looking at things like the buy to drive ratio and other production concerns as great work. The project team has a clear understanding of what is necessary for commercial success of the process.

\section{Reviewer 6:}

The reviewer explained the project is specifically focused on improving manufacturing techniques associated with producing higher performance and lower cost motors. In addition, the focus is on moving from aluminum (Al) to $\mathrm{Cu}$ to take advantage of greater conductivity leading to greater energy density. The reviewer noted the project is being performed under a cooperative research and development agreement (CRADA) and at the end of the project the process technology will be transferred to the industrial partner.

\section{Reviewer 7:}

The reviewer reported the approach looks satisfactory and that welds proved good and viable manufacturing route.

\section{Reviewer 8:}

The reviewer said the project is a TRL 4 to 7 project and not traditional research. In addition, there is less fundamentals characterization with more focus on manufacturing process development. The focus is on improving performance and lowering the cost through better use of materials and simpler manufacturing method of Cu-based induction motors as an alternative to rare-earth based interior permanent magnet motors. The reviewer stated that while there is OEM support for this project by General Motors (GM), besides Tesla Motors using them in their vehicles, there does not seem to be significant interest from other OEMs. Most OEMs have taken the pathway to minimize or completely eliminate dysprosium (Dy), due to relatively very high cost, along with reducing other rare-earth materials neodymium $(\mathrm{Nd})$. In addition, the reviewer explained there have been several successes in reducing rare-earth magnet content significantly (and therefore cost and potential future volatility associated with rare-earth prices) in integrated permanent magnet (IPM) motors without impacting the performance.

\section{Reviewer 9:}

The reviewer explained the project is designed to move the technology from research (TRL 4) to nearer to production-ready (TRL 6) to hand off to GM to commercialize. The reviewer agreed the approach seems to be well-designed for solving the technical issues of developing the friction-stir welding process for $\mathrm{Cu}$. The reviewer said that the justification for this as an alternative over would-be expensive $\mathrm{Cu}$ was stated, so the approach seems valid. The stated barriers are decreased motor cost, decreased weight, increased durability, and increased efficiency. The application baseline is an induction motor, so a motor produced by the method developed in the project would increase efficiency and decrease weight (two of the barriers). The reviewer cautioned improved durability is only stated on a summary slide with no technical backup. The total motor cost impact is not stated. The project objective was to develop a low-cost FSW method, but the cost relative to the baseline was not stated. In addition, the overall motor cost (conventional versus the developed motor) was not stated, so unable to rate progress at the motor level.

\section{Question 2: Technical accomplishments and progress toward overall project and DOE goals-the degree to which progress has been made, measured against performance indicators and demonstrated progress towards DOE goals.}

\section{Reviewer 1:}

The reviewer pointed out that the defect free rotor welds are very impressive. It shows a lot of work went into this accomplishment. The complex control system algorithm necessary to improve temperature control is also impressive work. This controls approach shows very good control of temperature in the weld, which is critical for avoiding distortion of the welded parts. The reviewer agreed that thorough examination of the weld end problem gives a simple and manufacturing-appropriate solution and indicated that runoff tabs are an appropriate solution. The reviewer commented that it is very important that the rotors have passed all the rigorous OEM tests for durability and performance, as this demonstrates both the quality of this research product and the OEM's interest in the technology. 


\section{Reviewer 2:}

The reviewer applauded that the project is completing on budget and is extending the period of performance without increasing cost. The reviewer reported that the technology is ready for commercial application. In addition, the control of torque plus temperature has improved quality of weld. This reviewer noted that the exit hole solution is a runoff tab. The reviewer stated lower scrap metal is the easiest approach to complete weld.

\section{Reviewer 3:}

The reviewer explained the project is nearly complete and with the exception of the forthcoming technology transfer to the industrial partner and uncertainty associated with production costs, the project achieved its goals and fully addressed the barriers. In addition, the reviewer reported that $\mathrm{Cu}$ rotors have passed all electrical and performance tests at GM. The reviewer indicated a CRADA is the mechanism for transferring technology to industry.

\section{Reviewer 4:}

The reviewer concluded the project appears to be close to providing a product, and the project team is now working closely with production engineers at this point. The reviewer agrees the project team appears to have learned a great deal about manufacturing issues and developed several solutions along the way. The project team also appears to have developed welding methods that provide defect-free units by developing temperature-controlled welding methods. In addition, the project team now has identified what the team believes to be the best approach for minimizing complexity and waste by using a runoff tab. The reviewer remarked overall progress on the project has been a bit slower than anticipated throughout the project, although progress has clearly been made. There was one milestone delayed from fourth quarter 2014 to 2016.

\section{Reviewer 5:}

The reviewer stated several technical accomplishments including temperature control algorithm effectively addressed the rising temperature issue related to rotor welds which is $+/-2^{\circ} \mathrm{C}$ temperature control. The amount of $\mathrm{Cu}$ removed has been minimized in exit holes of shorting bars in the rotor, successfully. The reviewer noted the project team completed destructive, balance, resistance and electrical continuity and homogeneity of welds tests. The reviewer suggested because this project is relevant to the DOE VTO Electric Drive Technologies (EDT) subprogram, progress should be coordinated with EDT and also be measured against its targets (electric motor costs, specific power, power density, and efficiency) to better gauge significance of the potential impact of this project.

\section{Reviewer 6:}

The reviewer commented that feedback control on temperature of the welds is an innovative step forward for the FSW process.

\section{Reviewer 7:}

The reviewer observed that it was nice work changing the friction stir to constant torque to make the process more consistent. Controlling temperature is critical to more than this project and the reviewer mentioned the technology will find other applications.

\section{Reviewer 8:}

The reviewer cautioned several slides and accomplishments were repeated in 2015 and 2016, so the presentation does not only show last year's progress. The temperature control effect on final weld is clear in the picture and data. It is unclear what accomplishments happened in FY 2015, which is the period under review. The results shown indicate that the runoff tab is a successful exit hole solution that appears to be the best option for GM to implement. Full rotor testing has been done by GM and had good results of same or similar resistance and induction performances compared to based cast rotors and spin and balance tests. The presentation was focused on the FSW process. The reviewer said that the project's performance for meeting DOE's overall motor cost, weight, durability, and efficiency at the motor level were not described. The project has been delayed some, but appears to be in the final stage where the process is being readied to transfer to GM. The Stationary Shoulder Tool development plan was shown in the 2015 and 2016 presentations. The reviewer was concerned that finding a workable solution appears to be an issue. Because the presenter briefly mentioned this and stated that the project team has a working 
solution, the reviewer opined that this implies it is an issue. The reviewer indicated it is unclear whether the issue is really solved, or if this approach was abandoned.

\section{Question 3: Collaboration and coordination with other institutions.}

\section{Reviewer 1:}

The reviewer commented that the collaboration shows a good distribution of responsibilities based on expertise between Pacific Northwest National Laboratory (PNNL) and GM. The project team has had close collaboration with GM, including final testing of completed rotors and use of this testing to improve the PNNL FSW process. The reviewer stated the team's goal of passing the complete process to GM is excellent, as it will enable the OEM to begin using the process to manufacture electric motor components for themselves.

\section{Reviewer 2:}

The reviewer indicated the project team is very well connected with GM, their industrial partner. In addition, the reviewer stated that the testing GM has performed was important to provide commercial expectations.

\section{Reviewer 3:}

The reviewer acknowledged the project is closely coordinated with an ultimate user, GM (under a CRADA). At the end of this fiscal year, the plan is to transition the project to GM, reported the reviewer.

\section{Reviewer 4:}

The reviewer stated there were very close collaborations and interactions with GM.

\section{Reviewer 5:}

The reviewer pointed out that GM, as the largest domestic OEM, is a very appropriate partner and has been engaged throughout the project.

\section{Reviewer 6:}

The reviewer stated that the project team is in continual iterative interaction (sharing parts, test data, and results) with GM, which is the sole commercial partner due to CRADA arrangement. The reviewer indicated that because this is a CRADA, no other collaborations were expected.

\section{Reviewer 7:}

The reviewer stated GM is heavily involved and is transitioning this process to commercial production.

\section{Reviewer 8:}

The reviewer mentioned the cost share and technical input provided by the industrial partner contributed substantially to the success of this project. Given that the results of this project were closer to the implementation phase (TRL 4 to TRL 6), the focus on process control, manufacturing technique and cooperation and feedback from the partner was instrumental.

\section{Reviewer 9:}

The reviewer said collaboration was explained during the presentation and GM seems interested in continuing to manufacture rotors like the one discussed in the future.

\section{Question 4: Proposed future research-the degree to which the project has effectively planned its future work in a logical manner by incorporating appropriate decision points, considering barriers to the realization of the proposed technology and, when sensible, mitigating risk by providing alternate development pathways.}

\section{Reviewer 1:}

The reviewer indicated the extent of the possibilities of this work is not nearly completed, and the funding is ending. The reviewer concluded this project deserves a second look as the commercialization opportunities are evident and close to a deployment stage. 


\section{Reviewer 2:}

The reviewer stated the project is scheduled to end in September 2016. The Principle Investigator (PI) clearly identified several remaining challenges and barriers to be addressed through the end of FY 2016, but appeared to feel these activities would be completed on time.

\section{Reviewer 3:}

The reviewer stated the project is ending this year.

\section{Reviewer 4:}

The reviewer said the goal is to transfer technology to GM and by the end of the project in FY 2016, four full size optimized, fully defect free and weld temperature controlled rotors will be delivered to GM for testing. The project is also aiming to develop and demonstrate shouldered tool assembly for further minimizing material wastage and part deformation by the end of the project at the end of FY 2016.

\section{Reviewer 5:}

The reviewer stated that the work is very appropriate and to wrap up this project will transfer the FSW process to GM. The reviewer suggested it would be good to transfer some future work to the EDT program in VTO which will continue the great progress and address broader and larger scale motor designs.

\section{Reviewer 6:}

The reviewer indicated the use of $\mathrm{Cu}$ with a higher conductivity is proposed to lighten the weight of the rotor assembly and questioned if this process is viable at lighter weight designs.

\section{Reviewer 7:}

The reviewer said the project is in its last phase and PNNL will have the fixturing problem and solution developed by the end of the project. The reviewer reported that transferring the process to GM is the last step and will be done next year.

\section{Reviewer 8:}

The reviewer indicated technology has been developed for FSW process and the project team is looking at other applications in the $60-80 \mathrm{~kW}$ motor range. The reviewer observed continued effort to determine GM's interest regarding how to move forward, however, GM is interested. The reviewer noted no plan forward with other applications.

\section{Reviewer 9:}

The reviewer observed the project is near completion and the only task remaining in FY 2016 is completing the fixturing system

\section{Question 5: Does this project support the overall DOE objectives of petroleum displacement? Why or why not?}

\section{Reviewer 1:}

The reviewer stated the processing was fast enough to support a commercial application on a common opportunity that has many obvious applications in propulsion materials. The novel concept opens a lot of new doors for related opportunities as well, suggested the reviewer.

\section{Reviewer 2:}

The reviewer observed the project is focused on improving electric motor performance and cost for electric motors through improved manufacturing techniques. The reviewer suggested this is highly relevant for electrified vehicle applications. These motors are also used in non-electrified vehicle applications that are aimed at efficiency improvements, such as cylinder deactivation. The reviewer said by moving to $\mathrm{Cu}$-based motors, there can be reductions in weight of $23 \%$ and size of $30 \%$. The reviewer stated that this work is highly relevant to DOE and VTO overall objectives.

\section{Reviewer 3:}

The reviewer stated this activity enables more rapid and cost effective manufacturing of a key electric motor component. 


\section{Reviewer 4:}

The reviewer indicated electric drive vehicles are one of the key technologies for petroleum reduction, which is the main DOE VTO goal. Current commercial electric drive vehicles are primarily depended on IPMs. IPMs use almost exclusively neodymium magnets with Dy used for protection from demagnetization at higher operating temperatures. Because Nd and Dy are heavy rare-earth magnets and are identified as critical materials by the DOE, improving induction motors, which is a competing technology to IPM, is a good alternative for protecting vehicle manufacturers from rare-earth magnet price volatility, which occurred in 2011 due to a significant spike in price due to limited supply. The reviewer said that improvements to induction motors that have been around for nearly 100 years have been very hard to come by.

\section{Reviewer 5:}

The reviewer stated this project addresses the material needs of the EDT team by developing lower cost and higher efficiency motors that will ultimately reduce petroleum use.

\section{Reviewer 6:}

The reviewer mentioned there is a good opportunity to use a more efficient material which has difficulties to pour due to higher melting temperatures and that weight and power efficiency improvements are expected.

\section{Reviewer 7:}

The reviewer stated induction motors are less expensive than permanent magnet motors because there are no expensive rare-earth metals, therefore, there is support for market adoption of plug-in electric vehicles. The reviewer indicated the specific motor involved in the project is a low-power mild-hybrid motor which the market appears to be moving to as the next step (e.g., 48 vehicle dynamic control mild-hybrids) for fuel economy improvements. The reviewer mentioned the project uses $\mathrm{Cu}$ shorting bars which replace $\mathrm{Al}$ bars. This provides higher power density because of Cu's higher conductivity. The result is a motor that is $23 \%$ lighter and $30 \%$ smaller than an Al-intensive machine. This is vital for electric vehicles (EVs) and for meeting DOE mass reduction goals. The reviewer explained it appears induction motors are the standard for the mild-hybrid application and indicated the presenter stated that the project is to develop a low-cost method to join the bars to the end caps using FSW. The reviewer said this is good for improving the power density. The reviewer commented the cost comparison between conventional manufacturing (die-cast) and this approach was not described and the presenter did not mention the cost impact of the developed approach (including the materials cost), especially when compared to current low-cost die casting for all-Al motors.

\section{Reviewer 8:}

The reviewer stated this project provides the low cost process to get the induction motor cost competitive and in production.

\section{Reviewer 9:}

The reviewer said the project contributes to improved process control and manufacturing techniques and greater efficiency is a key vehicle component.

\section{Reviewer 10:}

The reviewer indicated the development of cost-efficient manufacturing technologies of motors is beneficial to lowering the price of EVs and HEVs.

\section{Question 6: Resources: How sufficient are the resources for the project to achieve the stated milestones in a timely fashion?}

\section{Reviewer 1:}

The reviewer stated the project team has achieved their milestones within their budget constraints.

\section{Reviewer 2:}

The reviewer reported that work during FY 2016 was under a no-cost extension, and that the PI thinks the project is fine. The reviewer suggested after FY 2016, when the project will be transitioned to GM, there could be some future support through the EDT program. 


\section{Reviewer 3:}

The reviewer said the resources allocated for the project are adequate to complete the proposed work. The reviewer indicated there was no FY 2016 funding as the project is continuing on carryover funds from FY 2015.

\section{Reviewer 4:}

The reviewer stated the project team is doing a very good job with the resources appropriated, and the resources have been sufficient to complete the work.

\section{Reviewer 5:}

The reviewer reported this is the last year of their project and the tasks seem to focus on contributions at GM for implementation.

\section{Reviewer 6:}

The reviewer stated the project is developing a friction stir welded manufacturing process for joining $\mathrm{Cu}$ stator bars to $\mathrm{Cu}$ end caps. The project is developing the process to the stage it can be transferred to industry which at this time is only to GM because of the work being done under a CRADA. The reviewer commented that the overall motor design seems to have been done outside of this project and asked if it was done by GM. The reviewer indicated it is unclear whether developing this manufacturing process could have been done for less than $\$ 2.8$ million total. It seems that the industry cost-share should have been higher to reduce DOE funding for this work because the CRADA was only with GM, who will be the only beneficiary. Resources were rated as sufficient because the reviewer may have missed some details.

\section{Reviewer 7:}

The reviewer noted the project is fully funded and is coming to a conclusion this year.

\section{Reviewer 8:}

The reviewer stated the money was well spent in this project. 


\section{Materials Issues Associated with EGR Systems: Michael Lance (Oak Ridge National Laboratory) - pm009}

\section{Presenter}

Michael Lance, Oak Ridge National Laboratory

\section{Reviewer Sample Size}

A total of four reviewers evaluated this project.

\section{Question 1: Approach to performing the work-the degree to which technical barriers are addressed, the project is well-designed, feasible, and integrated with other efforts.}

\section{Reviewer 1:}

The reviewer stated the project appears to have a properly-focused and reasonably complete approach and is addressing exhaust gas recirculation (EGR) cooler fouling from a materials perspective. The reviewer indicated that to start the project, ORNL went to all the U.S. diesel engine manufacturers to find out what the biggest issue was facing EGR systems. The reviewer reported all of the engine manufacturers said EGR cooler fouling was the biggest issue. The reviewer mentioned the project team then obtained in-use EGR coolers from industrial partners and worked to address fouling through both active and passive (geometry/materials) control.

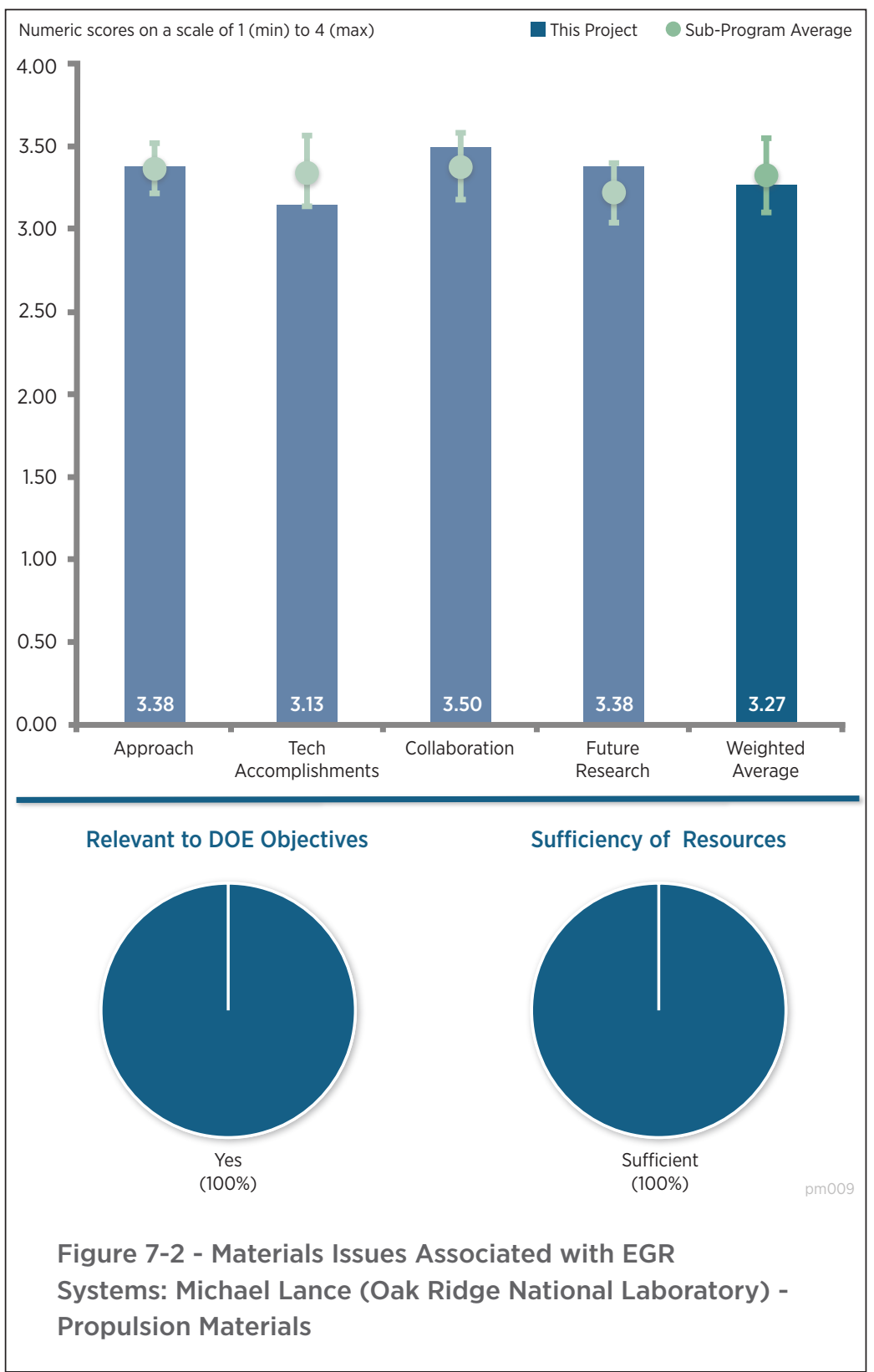

\section{Reviewer 2:}

The reviewer said the use of EGR for increased oxides of nitrogen $\left(\mathrm{NO}_{\mathrm{x}}\right)$ emission performance and engine efficiency suffer from loss of thermal efficiency due to hydrocarbon (HC) fouling. The reviewer mentioned the developed techniques used in this work to characterize HC fouling mechanisms of EGR has been useful, as an initial step, to compare and contrast actual deposition with that predicted from models. The reviewer noted this provides a way to further validate predictions from computational fluid dynamics (CFD) models to capture the necessary components such as shear forces to predict the deposition process. The techniques used to determine the deposit thickness and distribution are very appropriate and utilize the resources available at the national laboratories, said the reviewer.

\section{Reviewer 3:}

The reviewer stated the project is aimed at getting a better understanding of EGR cooler fouling mechanisms to affect more efficient cooler designs for supporting the confluence of future more restrictive $\mathrm{NO}_{\mathrm{x}}$ limits and greater use of advanced combustion techniques like low-temperature combustion. In addition, the project is using a systematic approach involving initial characterization of deposit formation and aging, and investigating EGR 
cooler designs and geometries for mitigating deposit formation. The reviewer mentioned FY 2016's five factor, three-level design methodology is a good example of this systematic approach to determine main influencers on deposit formation and mitigation. Also, two new techniques for measuring deposit thickness were developed as part of the research. The reviewer explained that the project team did look at new coatings and alloys early in the project to determine their impacts. The reviewer agreed the survey to elicit industry input on the research problem and industry participation is good.

Reviewer 4:

The reviewer stated the project team did a nice job tying the change being sought to an improved brake thermal efficiency.

\section{Question 2: Technical accomplishments and progress toward overall project and DOE goals-the degree to which progress has been made, measured against performance indicators and demonstrated progress towards DOE goals.}

\section{Reviewer 1:}

The reviewer explained the work showing the temperature variation in the cooler effecting the location of the deposit was stellar. The reviewer indicated this was a nice find and great work. The reviewer said that data also indicate that a good portion of the EGR cooler is not removing heat, indicating they can remove mass and not effect efficiency. The reviewer indicated the shear force hypothesis is important as well.

\section{Reviewer 2:}

The reviewer mentioned good progress has been made to characterize the actual deposit depth and how that varies with temperature, EGR geometries, and shear forces present. The reviewer explained that using this information to correlate with a CFD model will help predict the expected behavior under realistic conditions.

\section{Reviewer 3:}

The reviewer observed that the project found that heavily fouled coolers were not really impacted by geometry design, but low-hydrocarbon deposit coolers were. ORNL had John Deere foul coolers so they could look at the impact of geometry. The reviewer noted the result of this effort was that the project team found that the center is often significantly hotter than the outside of the cooler and that lower EGR inlet temperatures led to greater deposition, as did hydrocarbon content. The reviewer explained that the project team also developed a real-time method for generating three-dimensional (3D) images to see inside operating cooler and that actual deposit thickness measurements contradicted modeling results. The reviewer said the project team indicated that modeling did not adequately account for shear forces on the upstream side of the cooler fin and that the project team has started working with the Georgia Institute of Technology (GT) to improve the model.

\section{Reviewer 4:}

The reviewer indicated the total project timeframe seems like an inordinate amount of time to obtain significant results; however, the DOE Program Manager explained in the question and answer session that the project initially took some time to get traction while waiting for OEMs to provide information and support. The reviewer concluded that given the extended project timeframe, the continued industry participation in the project is critical to ensure the project stays relevant to industry research and needs in this area of $\mathrm{NO}_{\mathrm{x}}$ control. The reviewer pointed out that a major accomplishments achieved this year included two new techniques for measuring deposit thickness and determination of two primary factors impacting deposit formation, which are EGR inlet temperature and $\mathrm{HC}$ concentration. The reviewer indicated the new optical profilometer technique deposit measurements indicated different results than the CFD modeling conducted earlier in the project in terms of deposit locations. The researcher believed that this was due to the interaction of exhaust gas shear forces and cooler geometry which is currently not incorporated in the CFD model. The reviewer concluded this offers an opportunity for model validation and updating, which will be the focus of next year's research under the project.

\section{Question 3: Collaboration and coordination with other institutions.}

\section{Reviewer 1:}

The reviewer indicated the inclusion of HD engine and vehicle manufacturers along with ORNL and university 
assets will benefit this project. This will provide a platform to provide appropriately prepared samples and analysis, the reviewer said.

\section{Reviewer 2:}

The reviewer mentioned the project is collaborating with all U.S. diesel engine manufacturers, plus Modine and GT, and indicated it took some time to get all manufacturers on board, but that was seen as critical to the completeness of the project. The reviewer pointed out it was perhaps a bit surprising that there was unanimity in industry's response on the largest issue facing EGR systems, which was EGR cooler fouling. The reviewer noted that from the presentation it was somewhat unclear how engaged many of the industrial partners, specifically the manufacturers, are in the project. Of the manufacturers, John Deere and GM seem the most heavily involved, the reviewer reported.

\section{Reviewer 3:}

The reviewer stated the project team has maintained good collaborative efforts by involving an industry advisory team comprised of nine industry partners: Caterpillar, Cummins, Detroit Diesel, Ford, GM, John Deere, Navistar, PACCAR, and Volvo/Mack. The reviewer indicated the industry team has provided significant input and participation in the project. The reviewer mentioned that in order to address the CFD model inconsistencies identified through deposit measurement observations in the work, the project team has enlisted GM and GT for collaboration on CFD model updates next year.

\section{Reviewer 4:}

The reviewer noted the project team has nine industrial collaborators, including parts and data.

\section{Question 4: Proposed future research-the degree to which the project has effectively planned its future work in a logical manner by incorporating appropriate decision points, considering barriers to the realization of the proposed technology and, when sensible, mitigating risk by providing alternate development pathways.}

\section{Reviewer 1:}

The reviewer stated future research activities seem appropriate, leveraging off past work and recent findings on CFD model inconsistencies with deposit formation to first update the model to incorporate shear force influences and focus on cooler designs for deposit formation mitigation and removal. The reviewer observed future work will also review fouled coolers provided by industry to measure deposits and assessing varying geometries against the sinusoidal geometry already evaluated and indicated this work should provide additional information on optimal cooler designs for mitigating deposit formation.

\section{Reviewer 2:}

The reviewer observed the future work planned to address remaining questions and fill the knowledge gaps is appropriate.

\section{Reviewer 3:}

The reviewer emphasized creating the model with GM and GT will be a great asset and should be quickly deployed to the industry.

\section{Reviewer 4:}

The reviewer pointed out the presentation indicated future efforts to revise models and revisit additional EGR designs geometries. In particular, collaboration with GM and GT was highlighted that it intended to result in recommendations for improved EGR cooler design. The reviewer indicated the project will also revisit coolers donated by industry partners for use in the geometry analysis.

\section{Question 5: Does this project support the overall DOE objectives of petroleum displacement? Why or why not?}

\section{Reviewer 1:}

The reviewer stated this work is relevant and supports overall DOE objectives by addressing EGR cooler fouling and its deleterious impacts on $\mathrm{NO}_{\mathrm{x}}$ emission control efficiency. The reviewer suggested the problem of EGR cooler 
fouling may be exacerbated by future advanced combustion approaches, such as low-temperature combustion and efficiency enabling technologies such as waste heat recovery.

\section{Reviewer 2:}

The reviewer said the project, which is aimed at reducing $\mathrm{NO}_{\mathrm{x}}$ emissions with no fuel economy penalty, clearly is in line with DOE/VTO objectives. The reviewer indicated current technology tends to have a $1 \%$ efficiency loss now. The reviewer concluded as advanced engine technologies expand, there will be more demanding EGR operating environments, including lower temperature combustion and greater waste heat recovery and both of these will be hindered by EGR cooler fouling.

\section{Reviewer 3:}

The reviewer reported technologies focused on increasing engine efficiency while lower $\mathrm{NO}_{\mathrm{x}}$ emissions is critical to achieving the required performance targets of HD lean engine applications.

\section{Reviewer 4:}

The reviewer noted if the cooler becomes more efficient, engine efficiency increases as data in the presentation indicated showing a greater than $1 \%$ increase in thermal efficiency.

\section{Question 6: Resources: How sufficient are the resources for the project to achieve the stated milestones in a timely fashion?}

\section{Reviewer 1:}

The reviewer said annual project funding has been fairly consistent for the last four years and indicated the project team estimates that about 3\% progress had been accomplished in FY 2016, and estimates total project progress is now at $75 \%$. The reviewer commented project completion is anticipated in FY 2018, leaving two years to complete $25 \%$ of the research work. The reviewer mentioned the research team is adding additional resources including GT and GM, for supporting the CFD modeling initiative next year.

\section{Reviewer 2:}

The reviewer commented there has been good progress and project seems to be on track.

\section{Reviewer 3:}

The reviewer indicated this project is appropriately funded and staffed.

\section{Reviewer 4:}

The reviewer said no indication was made that the funding is not sufficient. 


\section{High-Temperature Materials for High-Efficiency Engines: Govindarajan Muralidharan (Oak Ridge National Laboratory) - pm053}

\section{Presenter}

Govindarajan Muralidharan, Oak Ridge National Laboratory

\section{Reviewer Sample Size}

A total of three reviewers evaluated this project.

\section{Question 1: Approach to performing the work-the degree to which technical barriers are addressed, the project is well-designed, feasible, and integrated with other efforts.}

\section{Reviewer 1:}

The reviewer observed this project seeks to develop new alloys that have combined improved strength, oxidation resistance and lifetime when operating at high temperature, up to $950^{\circ} \mathrm{C}$. The approach combines computational thermodynamics including the ICME component of the research to support identification and characterization of higher strength alloys with experimental efforts to validate the modeling and to characterize the oxidation resistance of these alloys. The reviewer stated the project uses a combination of predictive materials science and trial and error.

\section{Reviewer 2:}

The reviewer was still confused at the progression of alloys studied in this work. The reviewer stated that Slide 8 in the presentation shows the third-generation alloy developed at ORNL but questions what the comparison on Slide 9 shows. The reviewer pointed out that the alloy designations are confusing.

\section{Question 2: Technical accomplishments and progress toward overall project and DOE goals-the degree to which progress has been made, measured against performance indicators and demonstrated progress towards DOE goals.}

\section{Reviewer 1:}

The reviewer commented that the oxidation resistance is explained as better or worse many times during the presentation. The justification seems to have both characteristics present. The reviewer asked to please include a consistent metric for comparison of all results for determining the order of oxidation resistance.

\section{Reviewer 2:}

The reviewer noted that establishing a computational approach for predicting the yield strength of materials at temperature from average particle size distributions provided experimentally is a positive accomplishment though 
it requires additional investigation to quantify the disagreement between measurement and prediction of yield strengths. The reviewer stated establishing a weight percent minimum for nickel (Ni) plus cobalt to improve yield strength of new material versus commercial alloys and increasing $\mathrm{Ni}$ content to improve oxidation resistance is also notable. Overall progress on this project appears to be slow and behind schedule, reported the reviewer.

\section{Question 3: Collaboration and coordination with other institutions.}

\section{Reviewer 1:}

The reviewer pointed out the relationship between ORNL and Carpenter seems productive especially seeing the processing window determination.

\section{Reviewer 2:}

The reviewer said collaborations appear to be limited to use of Carpenter Technologies for material supply and some characterization and to Argonne National Laboratory (ANL) for additional characterization. The reviewer explained that based on the presentation material these relationships appear more like contractual agreements for support.

\section{Question 4: Proposed future research-the degree to which the project has effectively planned its future work in a logical manner by incorporating appropriate decision points, considering barriers to the realization of the proposed technology and, when sensible, mitigating risk by providing alternate development pathways.}

\section{Reviewer 1:}

The reviewer commented that there does not appear to be proposed work beyond the project scope and that the project is behind schedule and still has substantial work to complete prior to dissemination of final results.

\section{Reviewer 2:}

The reviewer described proposed future research as sufficient for the present project.

\section{Question 5: Does this project support the overall DOE objectives of petroleum displacement? Why or why not?}

\section{Reviewer 1:}

The reviewer indicated the data for this material should have a positive influence on decisions to create a higher temperature and more efficient combustion cycle.

\section{Reviewer 2:}

The reviewer noted the project's intent was to develop alloys capable of supporting more efficient engines (valves) at higher temperatures.

\section{Reviewer 3:}

The reviewer stated the development of high-temperature material is to increase the engine efficiency for fuel savings.

\section{Question 6: Resources: How sufficient are the resources for the project to achieve the stated milestones in a timely fashion?}

\section{Reviewer 1:}

The reviewer warned it is not clear that there are sufficient remaining resources to complete the tasks outlined as future work for the remainder of 2016. Future work includes adding reactive elements, fabrication of downselected alloy, testing, and completion of characterization and preparation of final report. This person said given that the project is already beyond its intended schedule, completion of all tasks with the remaining budget will be challenging. The September 2015 milestone was scaled back from down-selecting two alloys to one. The reviewer suggested that overall, it appears that the initial goals of the project were too ambitious for the level of funding provided or that there were unanticipated costs at some point. 


\section{Enabling Materials for High- Temperature Power Electronics: Andrew Wereszczak (Oak Ridge National Laboratory) - pm054}

\section{Presenter}

Andrew Wereszczak, Oak Ridge National Laboratory

\section{Reviewer Sample Size}

A total of three reviewers evaluated this project.

\section{Question 1: Approach to performing the work-the degree to which technical barriers are addressed, the project is well-designed, feasible, and integrated with other efforts.}

\section{Reviewer 1:}

The reviewer reported a fairly thorough discussion of the issues, approach and results associated with high-temperature power electronics was provided. Substantial attention was given to the understanding of the potential manufacturing issues and the implementation challenges associated with sintered silver. In addition, a comprehensive research plan has been conceived, developed and executed. The reviewer commented for the funding provided, significant progress has been made but additional work is required to determine if this material is viable. The reviewer suggested that because this is the end of this project, the researchers should be encouraged to report results in a journal publication for reference in future efforts to continue development. The reviewer indicated the discussion on electric motors was minimal. Thermal conductivity was characterized through several methods and knowledge was gained on the anisotropy of the material said the reviewer. Because this task was apparently slated for only $20 \%$ effort, the results are useful but leave much work to be done. The reviewer commented that hopefully a publication either already exists or will follow that details proposed additional efforts.

\section{Reviewer 2:}

The reviewer said the team has effectively focused on two key material challenges in high-temperature power electronics; the interconnect material and high-temperature, high-thermal conductivity potting compounds. Other high-temperature material challenges were recognized but appropriately ranked at lower priorities. The reviewer indicated this effort will generate data that will be effectively integrated with other efforts, particularly National Renewable Energy Laboratory's (NREL) efforts on measuring and modeling heat transfer.

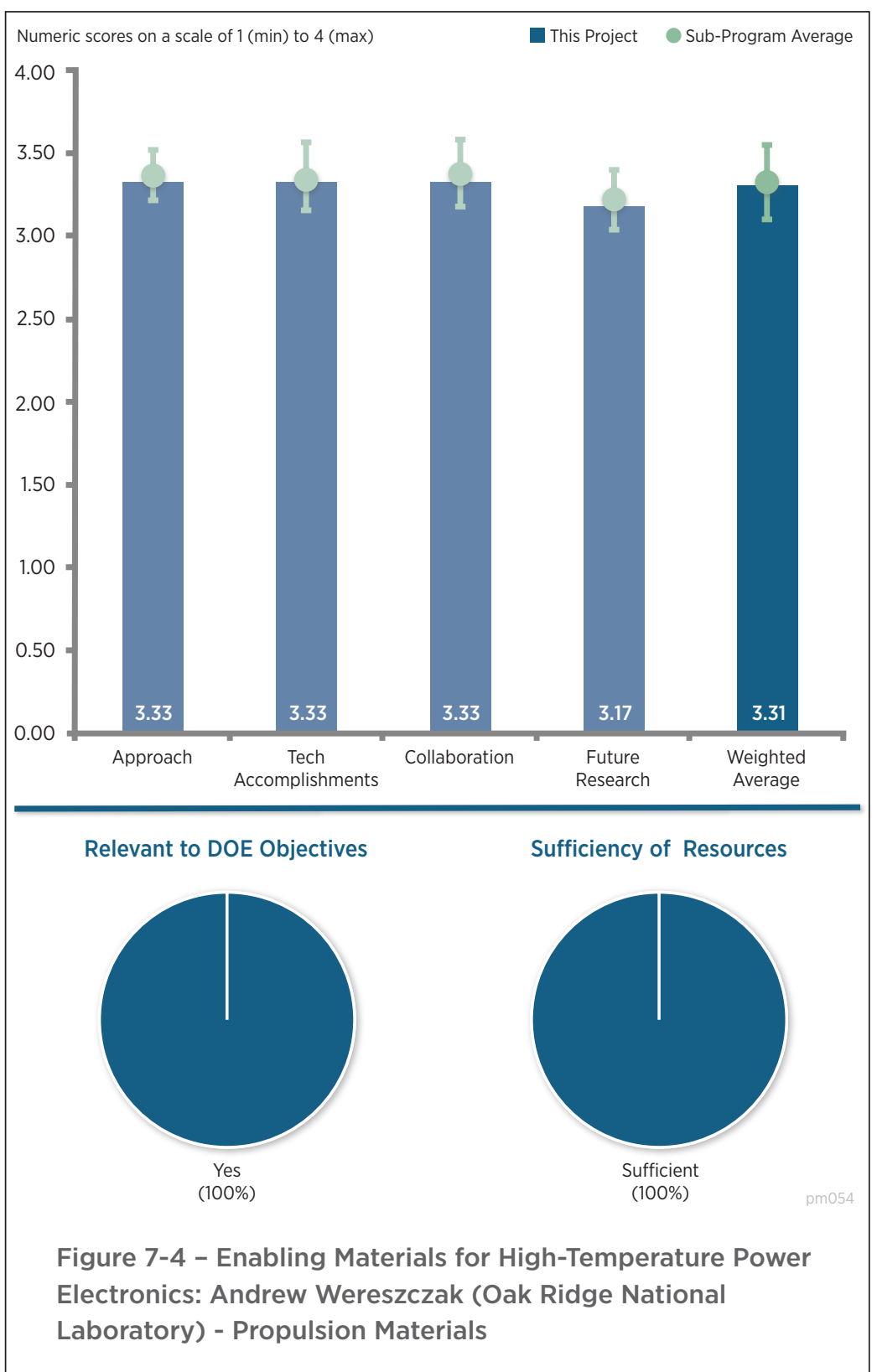




\section{Reviewer 3:}

The reviewer explained in addition to the measurement of the anisotropic thermal conduction of this system, a model showing the limits or just system behavior is warranted.

\section{Question 2: Technical accomplishments and progress toward overall project and DOE goals-the degree to which progress has been made, measured against performance indicators and demonstrated progress towards DOE goals.}

\section{Reviewer 1:}

The reviewer praised the project team for making outstanding progress on developing sintered silver interconnects as an alternative interconnect material. The processing challenges of surface condition and plating requirements were well understood and tested. The important performance metric of failure stress was identified and measured, and specification limits were determined from those measurements. The reviewer said the various fracture and fatigue studies that were undertaken will provide valuable data for assessing the reliability and lifetime of systems manufactured with this technology. The reviewer pointed out the measurements of the thermally conductive potting compounds used in electric motor compounds seemed to be at an earlier stage of completion. The test matrix to be developed was not apparent but presumably will be in the paper that is in preparation the reviewer reported.

\section{Reviewer 2:}

The efforts to understand the challenges associated with the use of sintered silver in high-temperature power electronics cover material development, understanding material properties, manufacturing and mechanical testing, these tasks all provided substantially useful information leading to a greater understanding of the viability of sintered silver for this application. Several mechanisms associated with the potential loss of integrity of electronic structures were identified and characterized.

\section{Reviewer 3:}

The reviewer questioned if a modeling approach was incorporated into this project such that the data and knowledge can be leveraged in the design of a new system.

\section{Question 3: Collaboration and coordination with other institutions.}

\section{Reviewer 1:}

The reviewer said the team seems to have excellent interaction with ORNL and NREL in electric motor winding sample preparation and measurement. In addition, the team also seems to have had fruitful interaction with ORNL in assessing the fracture mechanics of the sintered silver interconnects.

\section{Reviewer 2:}

The reviewer affirmed good collaboration is seen for both sample creation and testing.

\section{Reviewer 3:}

The reviewer indicated the teaming effort was solid.

Question 4: Proposed future research-the degree to which the project has effectively planned its future work in a logical manner by incorporating appropriate decision points, considering barriers to the realization of the proposed technology and, when sensible, mitigating risk by providing alternate development pathways.

\section{Reviewer 1:}

The reviewer stated the remaining tasks focus on report writing, which seems appropriate given that the project is nearing the end of its period of performance.

\section{Reviewer 2:}

The reviewer reported that because the project is near completion, articles should be submitted for publication prior to end of the funding. 


\section{Reviewer 3:}

The reviewer noted that sintered silver seemed to be favored, but the reasoning behind this choice was not clear. The reviewer asked whether this was CTE, strength driven, or something else.

\section{Question 5: Does this project support the overall DOE objectives of petroleum displacement? Why or why not?}

\section{Reviewer 1:}

The reviewer observed these projects indirectly supports the overall goal of petroleum displacement by enabling the development of more efficient power electronics and electric machines.

\section{Reviewer 2:}

The reviewer commented reducing the cost and reliability of electric drive systems.

\section{Reviewer 3:}

The reviewer stated that if an argument for addressing the DOE objectives of petroleum displacement was made, it was missed. Improved performance in power electronics and electric motors will lead to greater efficiencies and longer life. The reviewer concluded that if that is sufficient for supporting DOE's objective, then a case can be made for this project supporting the objective.

\section{Question 6: Resources: How sufficient are the resources for the project to achieve the stated milestones in a timely fashion?}

\section{Reviewer 1:}

The reviewer reported given the limited funding and the challenges of getting work done at national laboratories, the tasks accomplished within schedule and budget are notable.

\section{Reviewer 2:}

The reviewer stated the remaining resources seem sufficient to carry the project through its scheduled endpoint at the end of 2016.

\section{Reviewer 3:}

The reviewer said the project is ending shortly with additional tests and data. 
Biofuel Impacts on

Aftertreatment Devices: Michael

Lance (Oak Ridge National

Laboratory) - pm055

Presenter

Michael Lance, Oak Ridge National

Laboratory

\section{Reviewer Sample Size}

A total of three reviewers evaluated this project.

Question 1: Approach to performing the work-the degree to which technical barriers are addressed, the project is well-designed, feasible, and integrated with other efforts.

Reviewer 1:

The reviewer observed nice applied work looking at a potential real world issue of biodiesel contaminant effects on exhaust gas catalyst system. The reviewer explained it is important to understand the underlying fundamentals of this issue, and noted that the barriers being addressed do not seem to match those in the propulsion materials area.

\section{Reviewer 2:}

The reviewer explained that the validation of the American Society of Testing and Materials (ASTM) standard is a great measurement tool for the effect of biofuel. The reviewer pointed out it is a nice approach to look at both running engines as well as forensic methods.

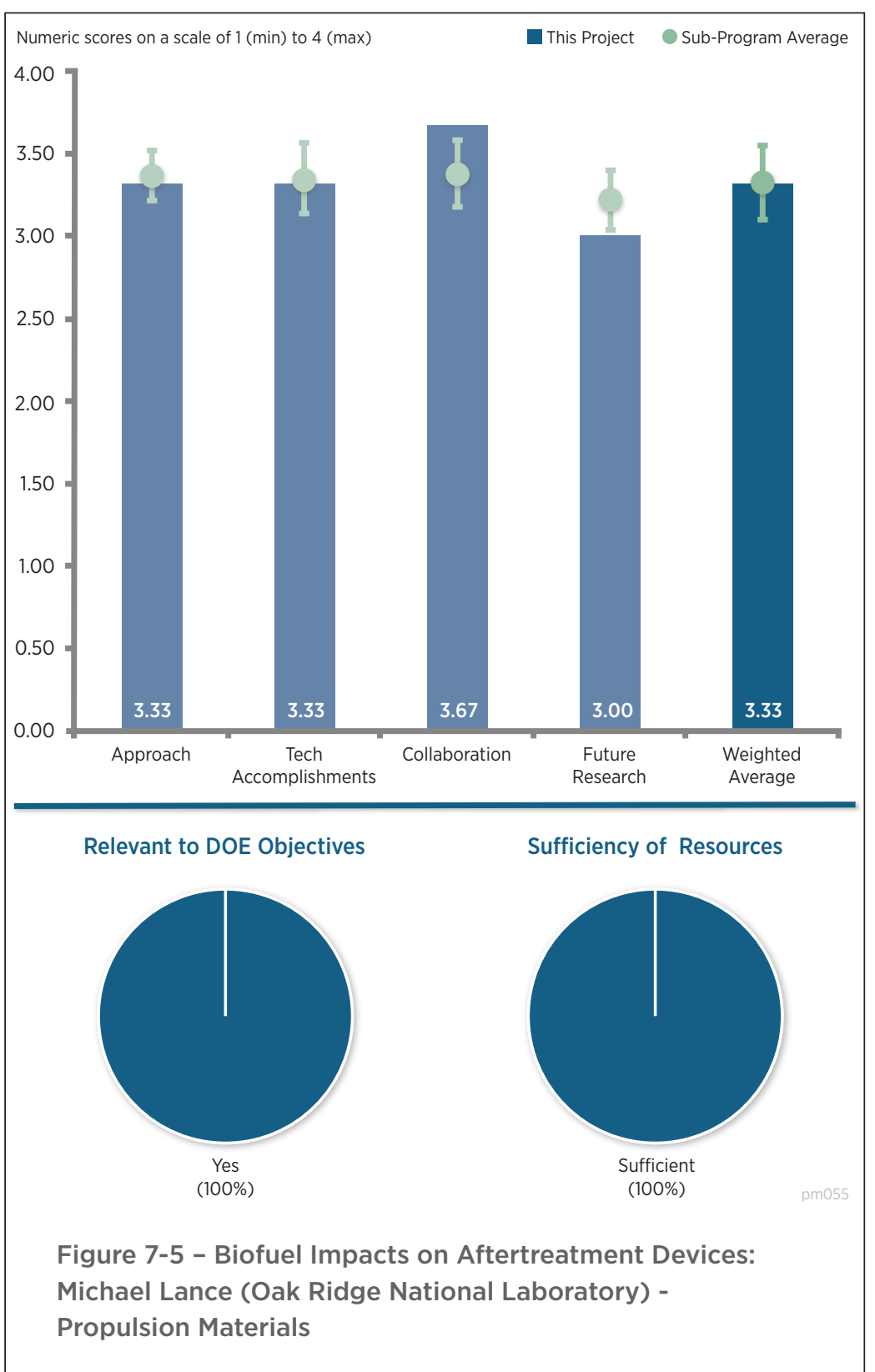

\section{Reviewer 3:}

The reviewer reported this work seeks to understand how components of biodiesel, remaining from the fuel synthesis process, effect the performance of the catalysts used in the aftertreatment systems used for diesel engines. Also included in the study is the effect of phosphorous, from engine oil, effects the performance of the aftertreatment catalysts. With respect to the contaminants in the biodiesel, those species studied here, sodium (Na) and potassium $(\mathrm{K})$, result from first generation homogeneous fuel processing of biodiesel. Second-generation heterogeneous biofuel processes use catalysts that are composed of different elements. Therefore, the reviewer concluded one should expect the presence of $\mathrm{Na}$ and $\mathrm{K}$ to be much lower and less of a concern as a source of catalyst deactivation. In fact, the concentration of these species in the limited fuel survey may support this change in fuel source. The reviewer suggested an expanded fuel study, to determine if other species are present in higher concentrations, should be invested as a risk to catalyst performance. The actual testing of the effects of contaminants is reasonable reported the reviewer. 
Question 2: Technical accomplishments and progress toward overall project and DOE goals-the degree to which progress has been made, measured against performance indicators and demonstrated progress towards DOE goals.

\section{Reviewer 1:}

The reviewer stated it was nice protocol to remove single elements and identify changes. In addition, the reviewer commented it was great work covering the lack of change in mechanical properties of the diesel particulate filter (DPF). The reviewer suggested tying the Na back to the ash content is very good and was nice to extrapolate the effect on real products.

\section{Reviewer 2:}

The reviewer noted nice progress towards detailed understanding biodiesel effects on each component in diesel exhaust system. However, the reviewer suggested because mostly steady state accelerated aging is only partially realistic, the stepwise approach to discover the fundamental issue of platinum $(\mathrm{Pt})$ poisoning on direct oxidation catalyst (DOC) activity was interesting and novel.

\section{Reviewer 3:}

The reviewer stated this work used a thought-out method to assign the loss of catalyst functionality to a particular contaminant in the exhaust environment. The use of analytical tools and contaminant removal processes worked well and showed the effect of the contaminants on the activity of the various components of the exhaust aftertreatment. However, the reviewer suggested the evidence is less convincing for assigning Pt contamination as the cause of selective catalytic reduction (SCR) selectivity reduction. The inability to see the Pt in the SCR catalyst and where it might be concentrated, coupled with the placement of the SCR after the DPF, is not convincing evidence for this failure mechanism.

\section{Question 3: Collaboration and coordination with other institutions.}

\section{Reviewer 1:}

The reviewer explained the Manufacturer of Emission Controls Association, NREL and Engine Manufacturers Association participation increase the quality of this project and it is great to have them on board.

\section{Reviewer 2:}

The reviewer pointed out there is strong interactions with industrial partners.

\section{Reviewer 3:}

The reviewer said the partners involved in this work are appropriate.

\section{Question 4: Proposed future research-the degree to which the project has effectively planned its future work in a logical manner by incorporating appropriate decision points, considering barriers to the realization of the proposed technology and, when sensible, mitigating risk by providing alternate development pathways.}

\section{Reviewer 1:}

The reviewer observed the generator work going forward is a good addition and is a very cost effective way to get the data and create samples. The reviewer concluded this may offer an opportunity to look at the variations associated with changing biodiesel feed stocks.

\section{Reviewer 2:}

The reviewer suggested the actual composition of biodiesel should be investigated first to assure the presence of $\mathrm{Na}$ and $\mathrm{K}$ are still present at levels that are significant to the aftertreatment system.

\section{Reviewer 3:}

The reviewer suggested ORNL may want to look at the effect of exhaust condensate on DPF ash. The reviewer indicated it was mentioned that the presence of $\mathrm{Na}$ increases the total ash mass, but this is most likely mitigated by cold start conditions when liquid water is present in the exhaust system. 


\section{Question 5: Does this project support the overall DOE objectives of petroleum}

displacement? Why or why not?

\section{Reviewer 1:}

The reviewer said understanding deactivation mechanisms related to contaminants in biodiesel fuel are of importance, because the use of biodiesel is increasing in the fuel marketplace along with green diesel.

\section{Reviewer 2:}

The reviewer reported if the degradation is not identified then a larger system would be required for bio compatibility.

\section{Reviewer 3:}

The reviewer indicated understanding fuel and oil effects on aftertreatment devices can lead to more active emission control systems that enable more fuel efficient powertrains.

Question 6: Resources: How sufficient are the resources for the project to achieve the stated milestones in a timely fashion?

\section{Reviewer 1:}

The reviewer stated this project is appropriately funded and staffed.

\section{Reviewer 2:}

The reviewer indicated the project team is on track and have a lot of data and analysis to support their conclusions.

\section{Reviewer 3:}

The reviewer said resources appeared adequate to cover the work. 


\section{Applied Computational Methods for New Propulsion Materials: Charles Finney (Oak Ridge National Laboratory) - pm057}

\section{Presenter}

Charles Finney, Oak Ridge National Laboratory

\section{Reviewer Sample Size}

A total of six reviewers evaluated this project.

\section{Question 1: Approach to performing the work-the degree to which technical barriers are addressed, the project is well-designed, feasible, and integrated with other efforts.}

\section{Reviewer 1 :}

The reviewer praised the technical approach that combines CFD, FEM and FE-SAFE to evaluate the material properties and said it is a systematic and reasonable method.

\section{Reviewer 2:}

The reviewer said this activity is successfully developing a combined CFD and finite element modeling (FEM) approach to predict where and how high-temperature thermal exposure affects engine components. There is good coupling with engine efficiency targets that require higher temperature materials to reach the efficiency levels needed going forward. The reviewer suggested understanding where hightemperature events are impacting the cylinder components and how the material reacts is very important to the survivability of the engine. Therefore, the reviewer concluded this work is highly encouraged.

\section{Reviewer 3:}

The reviewer acknowledged the project's approach appears rational and well-developed, based upon extensive experience in the technical area. Overall, the project is aimed at bringing together modeling and experimental validation to improve HD vehicle engine designs as well as to eventually extend successful techniques to lightduty (LD) engines. The reviewer noted that specifically, the project is focused upon using CFD to identify the overall operating environment within engines at higher peak cylinder pressures. Then, FEM is used to identify the impacts upon various engine components, thus estimating the performance needs for the materials used for these components. In addition, the reviewer stated this effort supports all SuperTruck activities. The reviewer mentioned that it should be noted that this review appears limited to only Task 4 of the five project tasks.

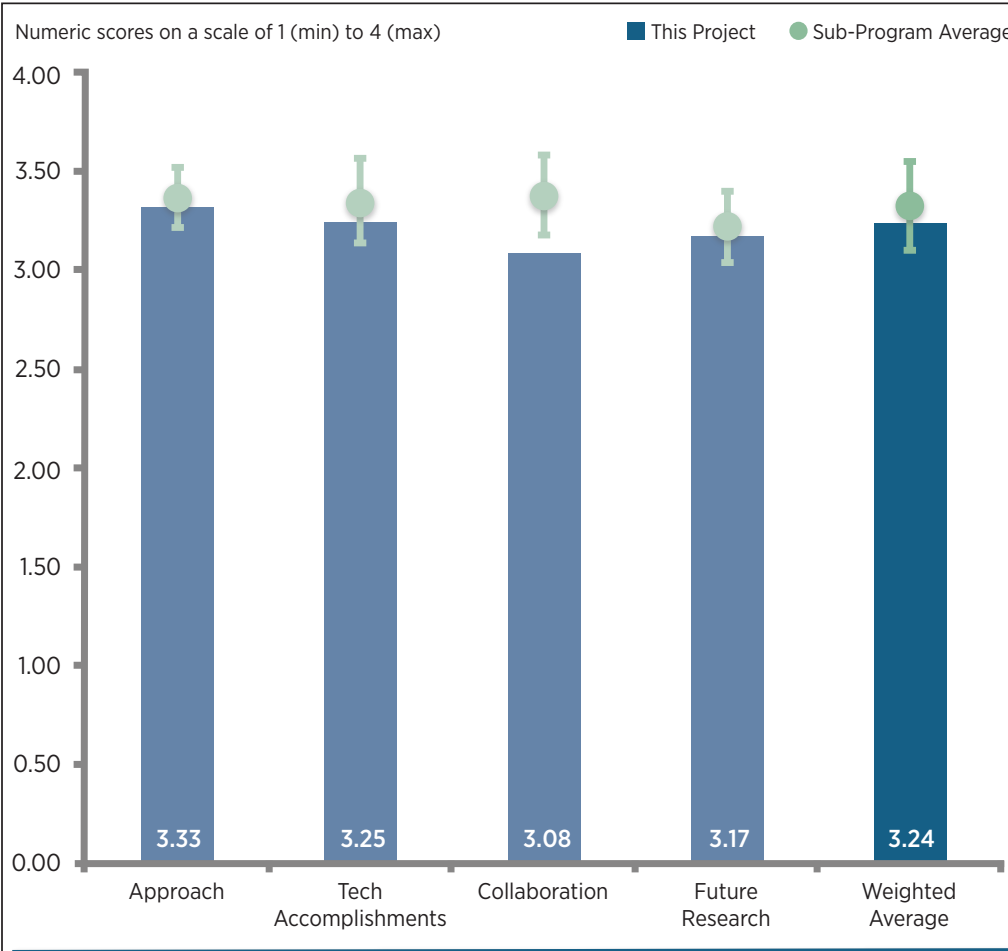

Relevant to DOE Objectives

Sufficiency of Resources
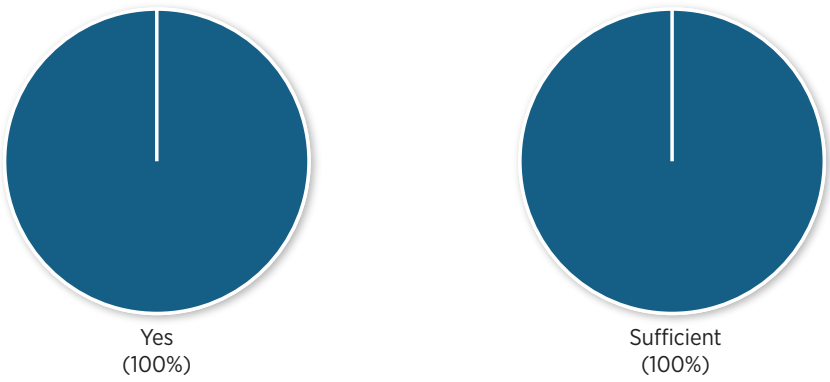

Figure 7-6 - Applied Computational Methods for New Propulsion Materials: Charles Finney (Oak Ridge National Laboratory) - Propulsion Materials 


\section{Reviewer 4:}

The reviewer explained that the review covered mostly Task 4, HD Engine Materials Requirement, like last year in 2015 but did also mention Task 5, Materials Characterization and Evolution, which was added from last year's review. The compacted graphite iron (CGI) characterization helped link Task 4 and 5 together. The reviewer pointed out that overall, the work is interesting and seems feasible in developing an improved understanding on the material property needs for both HD and LD engines.

The reviewer reported that because modeling is such a large component of this project, it would be appropriate to acknowledge the computational time and/or power needed for the CFD with conjugate heat transfer (CHT) coupled into the FEM. The reviewer indicated this would provide a better idea on the scalability of the CFD-FEM approach from the two material test cases to assessing a large number of candidate materials for new engine materials as part of the ICME effort.

\section{Reviewer 5:}

The reviewer pointed out that this approach seems like it should be common in the engine design community. The reviewer questioned if there are current cases that could be used as a baseline for the initial CHT simulations. In addition, the reviewer asked if these simulations will be validated at some point.

\section{Reviewer 6:}

The reviewer explained that the approach combines feeding relevant experimentally-derived material properties for compacted graphite iron ( $\mathrm{Fe}$ ) into the modeling stream that includes CFD that are integrated into finite element codes and conjugate heat transfer to explore various effects at higher temperature and pressures. The reviewer indicated that the results of these studies ultimately provide the stress and temperature parameters of engine components at peak cylinder pressures and drive fatigue models.

\section{Question 2: Technical accomplishments and progress toward overall project and DOE goals - the degree to which progress has been made, measured against performance indicators and demonstrated progress towards DOE goals.}

\section{Reviewer 1:}

The reviewer agreed combining computation methods to better assign hot spots and how material will respond to temperature and pressure is a very appropriate. The work appears well thought out and focused. The predicted results are overall very consistent with the experiment. The reviewer suggested continued validation of the modeling is required to improve confidence, but current work appears to be driving material changes at the manufacturing level.

\section{Reviewer 2:}

The reviewer emphasized that during FY 2016 the project has nearly completed all milestones and focused on measuring properties for compacted graphite iron at higher engine temperatures. The only effort remaining for compacted graphite iron appears to be testing of short-term creep (which is currently underway). The reviewer indicated additional efforts were also completed in combustion modeling, and that CHT simulations are in progress.

\section{Reviewer 3:}

The reviewer said that the researcher provided a thorough discussion of the HD engine materials task within this project. The reviewer indicated this appears to be the only remaining task and is independent of tasks addressed at previous review meetings, which included piezoelectric, non-rare-earth and low-temperature catalyst materials tasks. The reviewer pointed out that possibly some of the methodology carried over from the other tasks. The reviewer commented that accomplishments in this task are on target and that the co-simulation of combustion and thermal properties is notable.

\section{Reviewer 4:}

The reviewer said the experimental part of the project is almost done and CFD simulation was started.

\section{Reviewer 5:}

The reviewer noted that Task 4 makes up a large component of this project and seems to be progressing well. The 
new conjugate heat transfer model implemented will provide better temperature distributions at the peak cylinder pressures of interest, in which the finite element method should simulate more accurate peak stresses of actual working engines. The reviewer stated that the results of the experimentally measured properties of compacted graphite iron are not only useful for this project, but also for the engine modeling community. The reviewer concluded that at this time, there is insufficient information on how the models will be evaluated for determining the guidance on the limits for targeted material properties, which will hopefully be present in the next review.

\section{Reviewer 6:}

The reviewer pointed out that variation is shown for the peak cylinder pressures, and questions how this will be compared to current practice to ensure that these predictions are in the right range and are showing the right behavior.

\section{Question 3: Collaboration and coordination with other institutions.}

\section{Reviewer 1:}

The reviewer stated there is good partnering with a leading HD engine manufacturer and that the project would also benefit from a LD engine partner because this work can carry over to that segment as well.

\section{Reviewer 2:}

The reviewer noted that ORNL is collaborating with Cummins and Convergent Science. Cummins provided everything it had on the ISX engine to the project, and has outfitted test engine units to identify real engine operating environment requirements. Cummins indicated that it has learned a great deal already that will impact their future engine designs. The reviewer indicated that most of this information on collaboration was gained from the PI and the Cummins representative in the review room, rather than from the presentation. The reviewer said in future planned research the PI identified a desire to move toward adding LD engines, which will require an addition of a LD engine partner. The reviewer commented that the PI indicated the team is working on that, and have identified a common spark-ignition (SI) engine and partner for when those efforts occur.

\section{Reviewer 3:}

The reviewer explained that the contributions of the two partners listed were mentioned during the review and the collaboration effort briefly mentioned on the summary slide. The reviewer stated that while more discussion of the collaboration efforts would always be appreciated, it is acknowledged that partners may not want their roles to be described as pointed out during the presentation.

\section{Reviewer 4:}

The reviewer was not able to gain a clear picture of the collaborative relationships although it would appear likely that Convergent Science provided CFD support and Cummins would provide materials and operational input.

\section{Question 4: Proposed future research-the degree to which the project has effectively planned its future work in a logical manner by incorporating appropriate decision points, considering barriers to the realization of the proposed technology and, when sensible, mitigating risk by providing alternate development pathways.}

\section{Reviewer 1:}

The reviewer indicated the proposed future research is well planned out and on track to addressing the remaining subtasks within Task 4.

\section{Reviewer 2:}

The reviewer observed that models must be continuously updated to more accurately reflect the in-use experience both in the HD and LD markets.

\section{Reviewer 3:}

The reviewer stated future research is well planned for the remainder of FY 2016 and FY 2017 and that longer term opportunities for extension of methods to LD vehicles appear viable. The reviewer indicated a fully coupled CFDFEM approach could be challenging. 


\section{Reviewer 4:}

The reviewer acknowledged the PI clearly laid out the plan for future research, for FY 2016, FY 2017, and the future. The elements identified appear to make sense, and it is interesting to see that there has been thought given to how the techniques developed here would be applied to LD engines including the changes in concerns and challenges that sector would bring. The reviewer was concerned that the future portion of the list (beyond FY 2017) appears to show the full implementation of tools, which might have been hoped for before the project ended in FY 2017.

\section{Reviewer 5:}

The reviewer questioned if the baseline results will be published or how will this information be communicated to the broader audience.

\section{Question 5: Does this project support the overall DOE objectives of petroleum displacement? Why or why not?}

\section{Reviewer 1:}

The reviewer pointed out the project is aimed at identifying future engine materials needs to improve performance and thus efficiency, particularly through increased peak cylinder pressures and temperatures. Additional objectives are to identify materials with domestic supplies, reduce materials commercialization lead-times, and reduce engine weight. These are all extremely relevant to DOE and VTO objectives, the reviewer said.

\section{Reviewer 2:}

The reviewer remarked the project is well aligned with the DOE objectives of petroleum displacement by accelerating the discovery and development of new materials used in future engines for improved fuel efficiency.

\section{Reviewer 3:}

The reviewer indicated the project is to develop a faster and cheaper method of material development to enable advanced engine and powertrain systems for propulsion applications. The final goal is to increase vehicle efficiency for fuel saving.

\section{Reviewer 4:}

The reviewer noted the project directly addresses the needs for improvement in vehicle materials capabilities to enable operations at higher temperatures and pressures.

\section{Reviewer 5:}

The reviewer observed the establishment of material property targets for a baseline internal combustion engine is a good achievement. Further efficiency improvement may be more realistically set based on results such as this, the reviewer concluded.

\section{Reviewer 6:}

The reviewer indicated that improving survivability of materials under more demanding conditions due to increased engine efficiency is critical for manufacturers to ensure performance over engine life.

\section{Question 6: Resources: How sufficient are the resources for the project to achieve the stated milestones in a timely fashion?}

\section{Reviewer 1:}

The reviewer stated this project is appropriately funded and staffed.

\section{Reviewer 2:}

The reviewer reported that no indication was made by the PI concerning the sufficiency of the funds, although the FY 2016 level appears to be a bit of a ramp-down from earlier years. The reviewer concluded this may simply represent the stage of the project, but it is unclear. Given overall funding tightness within PM in future years, it would be anticipated that any FY 2017 funding would likely be lower, suggested the reviewer.

\section{Reviewer 3:}

The reviewer said the resources appear to be sufficient to complete the remaining tasks. 


\section{Reviewer 4:}

The reviewer commented that to the extent it can be evaluated based on information provided in the presentation, the resources appear reasonable. It would be interesting to see where the project could go with additional funds dedicated towards it, the reviewer reported. 


\section{Development of Advanced High-Strength Cast Alloys for Heavy-Duty Engines: Rich Huff (Caterpillar) - pm059}

\section{Presenter}

Rich Huff, Caterpillar

\section{Reviewer Sample Size}

A total of nine reviewers evaluated this project.

\section{Question 1: Approach to performing the work-the degree to which technical barriers are addressed, the project is well-designed, feasible, and integrated with other efforts.}

\section{Reviewer 1:}

The reviewer commented that the approach is logical and the PI has described the steps quite well in the presentation. The process of starting with ICME and moving to prototype alloys and verification is appropriate. The reviewer said that the inclusion of cost modeling to understand how these alloys will impact the cost of components and systems is an important step for moving this research work into actual production. Especially notable is the use of advanced laboratory capabilities (e.g., the ANL advanced photon source [APS]) to understand the materials science behind these lightweight materials, the reviewer stated.

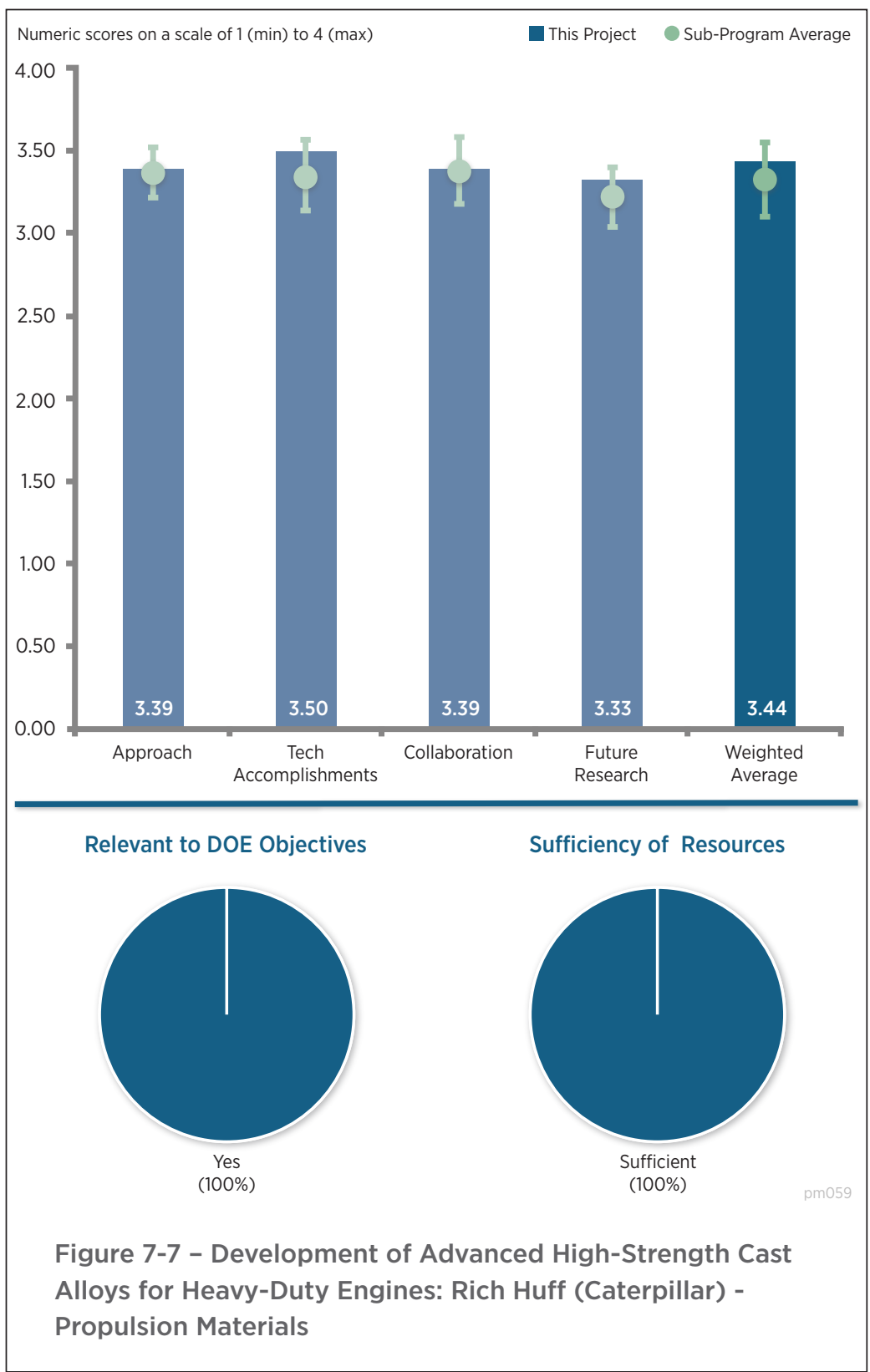

\section{Reviewer 2:}

The reviewer pointed out the project is an outstanding implementation of what now is becoming a standard approach to new materials development, which is computationally designing new material sets based on prior expert knowledge, developing prototype melts, characterizing microstructural and mechanical properties, developing test castings of final alloy, and comparing results with modeling and predicted results.

\section{Reviewer 3:}

The reviewer indicated the thoroughness of the experimental approaches were impressive and seemed appropriate, but the materials system (cast iron) is extremely challenging due to barriers in modeling and predicting the structure of the Fe-graphite system. The reviewer said the approach considered refining primary austenite dendrites, refining eutectic cell size via inoculation, and improving the strength of the matrix by alloying or nano-precipitates, but the structure of these materials is extremely sensitive to cooling rates, which is a difficult boundary condition. The directional solidification studies to evaluate effects of thermal gradients and cooling rates added some interesting scientific understanding, the reviewer noted. 


\section{Reviewer 4:}

The reviewer indicated the approach (interrupted solidification, visualization techniques, etc.) appears to be thorough, well-designed, and well-executed for understanding the formation mechanisms and composition the project is targeting to understand. The reviewer highlighted the following: the efficiency target is to achieve 214 Megapascal (MPa) endurance limit to enable higher cylinder pressure and temperature combustion regimes; the power density target is to achieve $25 \%$ increase in strength over A 842 compacted graphite Fe; and various approaches for quantifying and improving strength were described. However, the reviewer noted the presentation lacked quantification of the endurance limit and tensile strength performance of the investigated alloys (including relative to the baseline). Also, there is no described effort yet regarding validating cost target (less than or equal to $110 \%$ of production A 48 gray Fe cast units). This reviewer stated that cost models will be used this year to quantify. The reviewer mentioned it seems like cost analyses would have been included throughout project to ensure that the research path was cost-effective.

\section{Reviewer 5:}

The reviewer stated due to the need of higher strength materials that will survive the increased pressure and temperature requirements of more efficient engines, advanced modeling techniques are needed to predict performance. In addition, the pace of innovation in this area will benefit significantly from models that are capable of directionally predicting how materials will respond to harsher environments. The reviewer pointed out that the work in this project directly addresses these needs through ICME. However, setting benchmark targets for cost of material must be well grounded in feedback from manufacturers. In addition, the reviewer suggested that heat rejection and conductivity must be an integral component of a model that predicts material behavior and suitability for an application.

\section{Reviewer 6:}

The reviewer said it is good to see some modeling work of the microstructure to study the physics behind it.

\section{Reviewer 7:}

The reviewer mentioned it is nice that the project is addressing the issue with modeling graphite influence in the Fe. The reviewer noted it is surprising that the project team is only looking at varying the nucleation akin to CGI instead of something with a higher return possibility.

\section{Reviewer 8:}

The reviewer commented the project team is using advanced experimental methods and characterizations to achieve the material properties needed, and ICME is being used to help with this.

\section{Question 2: Technical accomplishments and progress toward overall project and DOE goals-the degree to which progress has been made, measured against performance indicators and demonstrated progress towards DOE goals.}

\section{Reviewer 1:}

The reviewer mentioned the project team has conducted extensive and systematic work to create and test a number of potential alloys, and conduct the required down-select based on microstructure and properties. In addition, the project team is doing a careful study of microstructures and their connection to material properties. The reviewer noted the interrupted solidification work appears to have been very successful, and provided considerable additional understanding of microstructure evaluation. The project team has made good use of the ANL APS for in situ solidification experiments, which provides a very interesting look at the evolution of the microstructure over time as the alloy solidifies. Thee reviewer concluded that this provides the team with extensive information on how to get the desired microstructures.

\section{Reviewer 2:}

The reviewer said the researchers have implemented their plans well and have made substantial progress this year, particularly with respect to casting materials, characterizing structural and mechanical properties, and multiple solidification studies.

\section{Reviewer 3:}

The reviewer reported there has been good progress and a lot of work has been done. 


\section{Reviewer 4:}

The reviewer stated the project team identified 30 high potential alloys, and developed strength models. The reviewer indicated solidification experiments with APS at ANL illustrated the issues of graphite formation, moving toward non-graphite (white) options.

\section{Reviewer 5:}

The reviewer reported good progress has been made identifying potential materials and characterizing those materials appropriately. The reviewer suggested more work must be performed to account for additional functional requirements of the materials such as heat rejection and the tradeoff involved in the casting process

\section{Reviewer 6:}

The reviewer reported that an impressive array of results (interrupted solidification, visualization techniques, etc.) was presented. The project team provided a detailed understanding of the alloy production process. This reviewer opined that these will be useful for Caterpillar and industry, possibly via DOE shared data, to develop commercialized metals. The reviewer noted that only FY 2015 accomplishments are shown, which makes it clear what was done in the period being reviewed. The reviewer indicated the milestone dates (completed, ongoing, and future) for many milestones changed between the 2015 and 2016 presentations, which created uncertainty as to whether or when the milestones were achieved and this is especially true for completed milestones.

\section{Reviewer 7:}

The reviewer said it was clear that improved properties have been achieved, but it was not clear in the presentation or the summary if the DOE materials properties targets are anticipated to be transferable to the cooling rates of actual cast components. The reviewer reported the DOE targets were $25 \%$ increase in strength over A842 CGI, and $214 \mathrm{MPa}$ endurance limit at no more than $120 \%$ of cast of A48 gray Fe. The reviewer questioned at what temperature this occurred. Unfortunately, the presentation did not clearly identify the baseline strength of A842 CGI, so it was difficult to gauge progress, the reviewer observed. The reviewer pointed out the solidification, characterization and modeling work was impressive. The clear conclusion that satisfactory ICME tools are not available for fundamental modeling and design of cast irons is a valuable outcome, and can help shape both future reliance and investment in computational methods for cast irons.

\section{Reviewer 8:}

The reviewer noted the presentation did not clearly identify where the team is in relation to the targets with the down-selected alloys they are developing. The application of the solidification rate changes is important and they were addressed, they are keeping the option of post processing, such as heat treatment. The reviewer said the project has shown the complexity of grey $\mathrm{Fe}$, and the need for increased modeling capability

\section{Question 3: Collaboration and coordination with other institutions.}

\section{Reviewer 1:}

The reviewer said there appeared to be excellent collaboration with other organizations, and the roles of Questek, University of Alabama at Birmingham (UAB), and ANL were all clearly described.

\section{Reviewer 2:}

The reviewer stated there is excellent balance of partners, academic and national laboratory capabilities which are integrated into the project.

\section{Reviewer 3:}

The reviewer reported the collaborative team appears well suited to achieve the project goals. Automotive OEMs should be involved as well to provide addressable needs for the LD market as well.

\section{Reviewer 4:}

The reviewer mentioned the project team is clearly working between several institutions as necessary.

\section{Reviewer 5:}

The reviewer noted the team has a good balance of industry, national laboratory, materials, and academic partners with the necessary capabilities to complete the work successfully. 


\section{Reviewer 6:}

The reviewer said that there is a clear distribution of tasks among the collaborators that enables work to be performed in an efficient and timely manner.

\section{Reviewer 7:}

The reviewer mentioned the collaboration map is nice and clear.

\section{Reviewer 8:}

The reviewer explained that the project team is collaborating on the project with QuesTek Innovations (ICME), UAB (experimental testing), and ANL (APS facility for measurements and visualization). The reviewer mentioned the Northwestern University role was not clear.

\section{Question 4: Proposed future research-the degree to which the project has effectively planned its future work in a logical manner by incorporating appropriate decision points, considering barriers to the realization of the proposed technology and, when sensible, mitigating risk by providing alternate development pathways.}

\section{Reviewer 1:}

The reviewer said the project team provided a clear explanation of the remaining barriers that demonstrates their understanding of the challenges that remain. The project team is working on component designs and scale up of casting processes, both of which will be very useful for eventual commercialization.

\section{Reviewer 2:}

The reviewer agrees with future activities.

\section{Reviewer 3:}

The reviewer commented that the future steps were clearly described and were in alignment with project goals.

\section{Reviewer 4:}

The reviewer commented that the plan for scale-up trials (1,000 lbs. and prototype parts using the developed ICME approach) are good steps to validate model and material performance. The reviewer pointed out that the budget for the project is $70 \%$ complete, and that Slide 26 shows a long list of challenges, barriers, future steps, and six milestones that remain. The reviewer questioned if there is enough time and budget to complete them all, and if so, whether the project will be completed on time.

\section{Reviewer 5:}

The reviewer reported that the project is one that is well planned and executed, and is leading to the development of alloys that can be integrate into Caterpillar's product line. One concern the reviewer noted is that Caterpillar has moved away from the on-highway market. Application of these improved materials may not obtain significant fuel economy improvement in off-road vehicles. The reviewer suggested the results from this project could be used by many engine manufacturers for on-road applications.

The reviewer said future research focusing on the selection of the best material option should continue. Also, the reviewer suggested the project team should investigate novel (phases) approaches for precipitate strengthening and heat treating should be investigated to enhance the properties.

\section{Reviewer 6:}

The reviewer noted plans for research over the coming year are ambitious and include modeling, experimental validation and casting. Several new developments are planned that include developing experimental process for increasing the solidification rate of step block castings using a new supplier, developing new models, and demonstrating component design. The reviewer questioned if there are there sufficient resources to complete all tasks.

\section{Reviewer 7:}

The reviewer indicated the project team has lots of work ahead of them. 


\section{Question 5: Does this project support the overall DOE objectives of petroleum displacement? Why or why not?}

\section{Reviewer 1:}

The reviewer explained that increased cylinder pressures and reduced cost for engines and engine materials do support the DOE petroleum reduction goals (as the work will increase engine efficiency and push implementation into production). Power density/package size will be a major benefit of this work for the HD engine applications (475 horsepower [hp] from 9 liter [L] instead of $15 \mathrm{~L}$ ).

\section{Reviewer 2:}

The reviewer reported that the project is developing higher strength Fe metals to enable higher temperature, pressure and efficiency of combustion. Once commercialized, this directly results in reduced petroleum consumption.

\section{Reviewer 3:}

The reviewer stated this project's focus on the development of higher power density engines can lead to a reduction in engine size and a more efficient engine.

\section{Reviewer 4:}

The reviewer indicated that the project is geared towards developing higher efficiency engines with improvement in component strength using conventional casting techniques.

\section{Reviewer 5:}

The reviewer commented that both the pace of innovation and the materials developed using this approach will benefit the manufacturers in addressing their requirements for high-strength/temperature materials going forward.

\section{Reviewer 6:}

The reviewer observed decreasing mass via higher strength and allowing higher peak cylinder pressure.

\section{Reviewer 7:}

The reviewer noted the following quote: "Advanced materials that are lighter and/or stronger are essential for boosting the fuel economy and reducing emissions of modern vehicles while maintaining performance and safety."

\section{Question 6: Resources: How sufficient are the resources for the project to achieve the stated milestones in a timely fashion?}

\section{Reviewer 1:}

The reviewer commented that this project is appropriately funded and staffed.

\section{Reviewer 2:}

The reviewer indicated the project team is on track, but there are a lot of deliverables on the horizon for the project team.

\section{Reviewer 3:}

The reviewer stated that the he resources appear to be sufficient to complete the work described on the timetable outlined.

\section{Reviewer 4:}

The reviewer said that funding appears to be sufficient and did not note that funding was lacking.

\section{Reviewer 5:}

The reviewer noted the project is sufficiently funded and well balanced between industry cost share and government funding.

\section{Reviewer 6:}

The reviewer indicated that sufficient information on distribution of funds was not provided, but it appears that the resources have been adequately allocated to enable the completion of tasks. 


\section{ICME Guided Development of Advanced Cast Aluminum Alloys for Automotive Engine Applications: Mei Li (Ford Motor Company) - pm060}

Presenter

Mei Li, Ford Motor Company

Reviewer Sample Size

A total of 12 reviewers evaluated this project

\section{Question 1: Approach to} performing the work-the degree to which technical barriers are addressed, the project is welldesigned, feasible, and integrated with other efforts.

\section{Reviewer 1:}

The reviewer emphasized that the presentation was impressive. The alloy design approach was effective, driven by the awareness of the need for both a stable precipitate strategy (via slow diffusers) and adequate volume fraction of strengthening precipitate(s) at the target temperatures. A higher $\mathrm{Cu}$ alloy was chosen to achieve adequate volume fraction of strengthening phases. The reviewer reported the dual strengthening precipitate strategy was impressive and interesting. The two stage heat treatments seem necessary, but will add cost, the reviewer commented.

\section{Reviewer 2:}

The reviewer commented that the reputation of both Mei and the project team are well established. In addition, the approach to the alloy development is reasonably well thought out and appears to be ideal for the focus, which is an incremental increase in critical cast Al properties for a specific Ford engine block. The reviewer praised the computational approach presented early on that illustrates the desired magnesium $(\mathrm{Mg})$ and $\mathrm{Cu}$ levels for strength optimization.

\section{Reviewer 3:}

The reviewer pointed out that the project team did good work on identifying and quantifying the baseline compositions and the roll of precipitates. The reviewer suggested it would add to the presentation if the impact of a successful completion was quantified in impact on an engine. The reviewer questioned how much lighter is the engine when this technology is deployed and how does that weight change effect overall efficiency. The reviewer commented that the $\mathrm{Cu}$ and $\mathrm{Mg}$ diffusivity work is impressive and thought there was a nice presentation of the requirements and expectations.

\section{Reviewer 4:}

The reviewer said the approach includes a focus on multiple components to improving $\mathrm{Al}$ alloy performance

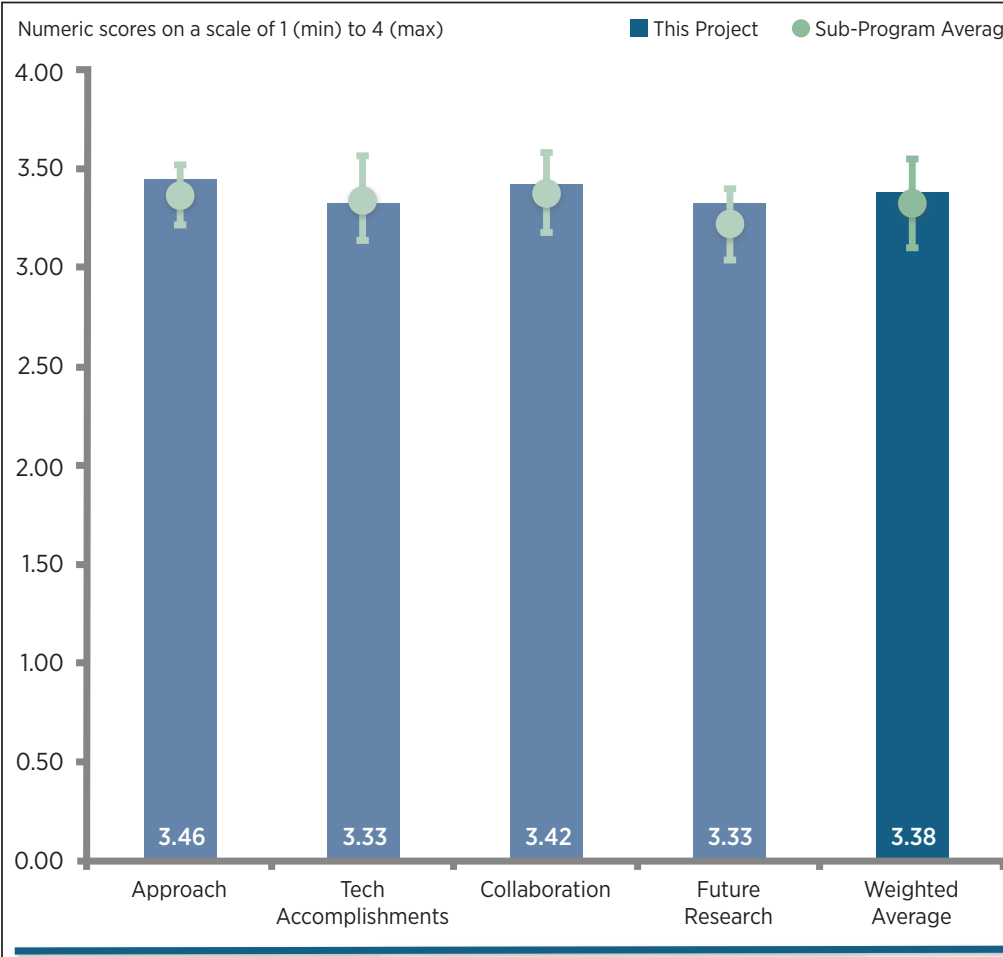

Relevant to DOE Objectives

Sufficiency of Resources

Figure 7-8 - ICME Guided Development of Advanced Cast Aluminum Alloys for Automotive Engine Applications: Mei Li (Ford Motor Company) - Propulsion Materials 
including precipitation strengthening, grain boundary strengthening, and solute strengthening. The reviewer mentioned the approach appears clearly based upon previous developments in the technology area, specifically aimed at achieving target properties and that the project also includes clear plans for application of project results to next-generation products.

\section{Reviewer 5:}

The reviewer indicated the approach seems appropriate to meet the objective to develop a new class of advanced, cost competitive $\mathrm{Al}$ casting alloys with 25\% greater strength compared to A319 or A356 alloys for highperformance engine applications. In addition, the approach includes ICME tools for accelerating the development of new materials and processing techniques, as well as to identify the gaps in ICME capabilities.

\section{Reviewer 6:}

The reviewer noted the project team's approach to the Al alloy design is logical, uses sound metallurgical and scientific processes, and addresses the key material properties necessary to increase engine thermal efficiency. The project team has a clear grasp of the science behind improving the properties of these lightweight alloys. The reviewer reported that the team is conducting extensive development of proposed alloys to optimize their composition and resulting properties.

\section{Reviewer 7:}

The reviewer concluded the approach seems effective in selecting an alloy and the current castability assessment activities seem sufficient.

\section{Reviewer 8:}

Overall, this reviewer noted that the project appears to be well-designed to accomplish the goal of developing stronger alloys at room temperature and high temperatures that exceed DOE yield strength targets. The presented work appears to be experimental with analysis of experimental results. The reviewer cautioned that the role of how ICME was used to guide the approach is not clearly stated or clear. It appears that ICME was used to develop the alloying and heat treatment process, but was not specifically described. Slide 32, just before the summary, showed how ICME will be used to develop a commercial process using the project results with a $15-25 \%$ development time savings estimate. Otherwise, the project presents a comprehensive study of how to design (structure, heat treatment, cooling rate control) and quantify alloys to meet the DOE targets (tensile strength, fatigue strength, etc.). The reviewer indicated that cost analysis to ensure an incremental increase within $10 \%$ was mentioned, but apparently with no work in this past year. This reviewer further explained that the cost model was implied to have been developed before and will be refined next year.

\section{Reviewer 9:}

The reviewer noted that ICME allows for cost effective design of materials with big computing resources now available to the industry. For example, many variables for precipitates can be modeled to accelerate the selection of alloy components. The reviewer concluded this type of approach is much improved versus the trial and error methods of the past.

\section{Reviewer 10:}

The reviewer mentioned a common approach of employing ICME techniques to select initial alloy compositions is employed. Initial alloy compositions include varying contributions from silicon, $\mathrm{Cu}$, and $\mathrm{Mg}$ (later $\mathrm{X}$ and Y). The reviewer noted new heat treatments are then employed to enhance precipitation hardening followed by microstructural characterization and mechanical testing.

\section{Question 2: Technical accomplishments and progress toward overall project and DOE goals-the degree to which progress has been made, measured against performance indicators and demonstrated progress towards DOE goals.}

\section{Reviewer 1:}

The reviewer noted overall, the accomplishments are very good for this project. The project team's heat treatment and aging process results in increased strength with thermally stable precipitates, which is a good accomplishment. In addition, the project team has seen good results in achieving the microstructure desired for the necessary 
mechanical properties. The team has achieved the DOE target for basic properties of tensile strength at room temperature and high temperature, and is working on thermomechanical fatigue. This person said the project team has increased thermomechanical fatigue performance over baseline alloys and has seen good improvement in fatigue strength.

\section{Reviewer 2:}

The reviewer pointed out the project has already met and/or exceeded DOE targets for performance of yield strength and ultimate tensile strength for several alloys, so efforts now are targeting further improvements. This was achieved through a combination of compositional changes as well as processing, particularly heat treating, improvements. This person indicated that the project also completed the plans for the block bulkhead and the gasoline turbocharged direct injection (GTDI) cylinder head, tying together a future application for the results of this project. The reviewer said that the only concern on accomplishments is related to timing. At last year's AMR, the project was reported as $70 \%$ complete compared to $80 \%$ complete reported this year. The project was scheduled for completion by February 2016, which has now been extended to November 2016.

\section{Reviewer 3:}

The reviewer reported the project objectives include developing a new class of advanced Al casting alloys and demonstrating the power of ICME for accelerating materials and processing development. This person indicated that the project is making very good progress on both of these objectives. Yield, tensile and fatigue strengths are above target values. The reviewer said modeling of casting and heat treatments feed predictive results for material properties and lifetimes have served to optimize the development process and reduced development times.

\section{Reviewer 4:}

The reviewer said that the room temperature and $300^{\circ} \mathrm{C}$ high-temperature properties were met in the as annealed materials, which was an impressive accomplishment. However, the averaged $300^{\circ} \mathrm{C}$ ultimate tensile strength (UTS) data after 100 hour exposure appeared to be lower than the DOE targets. Also, the $300^{\circ} \mathrm{C}$ exposures were shown after only 100 hours, whereas the other two cast Al projects in the same session showed data after longer exposures of 200 hours. The reviewer noted that leads to the question of how stable are the alloy properties at $300^{\circ} \mathrm{C}$. This person said it would be useful to see properties after longer-term exposure in the 2017 meeting. The reviewer pointed out that the improvements in $120^{\circ} \mathrm{C}$ fatigue strength for the new alloys were very promising. The reviewer stated that overall this is very nice work.

\section{Reviewer 5:}

The reviewer indicated the creation of the alloy and the required heat treatment within a single project is a lofty goal, and the project team is doing this as well as looking at the influence of cooling rates of the casting. This person noted it is nice that the project team is covering all of the issues associated with market deployment.

\section{Reviewer 6:}

The reviewer noted the project finished the design of new cast Al alloys (H16 and H17) that met DOE requirements for high temperature and fatigue strength. The new alloys with the optimum heat treatment process achieved greater than $20 \mathrm{MPa}$ fatigue testing above the baseline at $120^{\circ} \mathrm{C}$. This person reported that out-phase thermomechanical properties demonstrated thermal stability of the new alloys at low strain range and long life regime.

\section{Reviewer 7:}

The reviewer remarked that the alloy selection seems complete and data generated for designers seems sufficient.

\section{Reviewer 8:}

The reviewer indicated the project is leading toward the development of materials that can be used in the engine block bulkhead and GTDI cylinder head. This person noted the design of alloys was completed, alloys meet DOE Targets and there is a need to demonstrate performance in the target application.

\section{Reviewer 9:}

The reviewer noted the project is approaching its deadline but is only $30 \%$ completed.

\section{Reviewer 10:}

The reviewer reported that the presentation shows that it is $30 \%$ complete, which is the same as 2015 . The reviewer 
concluded that this was just a typographical error based on the accomplishments and the fact that the project ends in November 2016. The reviewer noted that funding numbers were not accurate or updated between 2015 and 2016 presentations, which made it unclear where spending was last year to compare to progress. The presented results describe the process and results to better understand the structure, heat treatment, and cooling rate control of the developed alloys. The reviewer observed that the project determined aging needs for particle distribution and stability. This person stated that progress towards meeting DOE cost targets was not described and it is unclear how ICME was used throughout project and how or what ICME gaps were identified.

\section{Reviewer 11:}

The reviewer said the presentation itself provided little information such that the reviewer must have missed a key point made orally during the presentation. This reviewer re-evaluated the slides, but the slides did not provide insight. The reviewer questioned why the program is approaching its completion date and is only $30 \%$ complete. The reviewer indicated there may well have been a sound reason for this, but did not recall what it was. This person noted the presentation itself was long on introductory matter and general, if nuanced, commentary on accomplishments and future work that was to validate models and identify gaps. The reviewer concluded that presentations with this level of detail could be generated long before a program even begins. More project-specific information on what is actually being pursued would be very welcome, even when acknowledging that the project is having success in the stated goals. The reviewer pointed out this project team is too talented not to provide seminal commentary on cutting-edge ICME approaches.

\section{Question 3: Collaboration and coordination with other institutions.}

\section{Reviewer 1:}

The reviewer noted he team has the right combination of collaborating skill sets. This is as good a team as one might find in this topic area.

\section{Reviewer 2:}

This person commented that clearly Ford and Alcoa are communicating well and working in a synergistic manner.

\section{Reviewer 3}

The reviewer pointed out that Alcoa (materials expertise), Nemak (Ford engine supplier), Magma (alloys) and University of Michigan (UM) (simulation and experimentation support) are project partners and their roles in the project seem very appropriate leveraging their respective core competencies.

\section{Reviewer 4:}

The reviewer said that the team appears to have an appropriate balance of suppliers and academia in combination with the OEM prime contractor. A slide explicitly describing the collaborations would be helpful for reviewers to fully judge the quality of the collaborations. This reviewer commented that Ford is collaborating internally to move this alloy to a 2020 release for production, which is extremely important.

\section{Reviewer 5:}

The reviewer stated the project has a highly qualified team with substantial pre-existing resources and capabilities.

\section{Reviewer 6:}

The reviewer stated the project has an impressive list of collaborators, all of whom were brought on for their potential involvement in the next steps (deployment and use), their capabilities, and/or their facilities. The collaborators include Alcoa, Nemak, MAGMA Foundry, and UM. The reviewer noted the collaborators roles were explained in previous year's presentations, but not as clearly in this year's.

\section{Reviewer 7:}

The reviewer observed that there seemed to be good collaboration with UM for transmission electron microscope (TEM) and atom probe analysis. This person noted the roles of Alcoa, Nemak and Magma were not clear.

\section{Reviewer 8:}

The reviewer indicated that collaborators Alcoa, Nemak, MAGMA Foundry Technologies, and UM, were 
mentioned but their roles were briefly described. This person suggested that the roles of the collaborators can also be logically-inferred. The fact that the project has made a lot of progress implies the partners are collaborating well.

\section{Reviewer 9:}

The reviewer said team members include several industry members and one academic institution. This person suggested the addition of a national laboratory should be considered to gain access to their computing capability.

\section{Question 4: Proposed future research-the degree to which the project has effectively planned its future work in a logical manner by incorporating appropriate decision points, considering barriers to the realization of the proposed technology and, when sensible, mitigating risk by providing alternate development pathways.}

\section{Reviewer 1:}

The reviewer noted that the modeling of phase transformation kinetics is important and exciting.

This person stated that the project team is committed to identifying the gaps in the microstructure and property models, but the team does not have a loop to address them.

\section{Reviewer 2:}

The reviewer reported the project is scheduled to be completed in November 2016. The plan is to demonstrate inclusion of successful alloys in block bulkheads. The alloys will also be used under another program (i.e., Ford's GTDI Engine) under a separate DOE contract. Ford's plans appear to focus on inclusion of these advanced alloys into available products in 2020, with incorporation into the GTDI engine likely first. The reviewer voiced a concern that there are a number of activities remaining to be completed by the end of the project in November 2016, so time may be tight.

\section{Reviewer 3:}

The reviewer commented that the stated goal of a possible application of the new alloy for the 2020 model year was intriguing. The plan to demonstrate a new alloy on a new GTDI I4 architecture was impressive, and indicates a serious interest in bringing the new alloy(s) to market eventually. This person said it will be interesting to see the final cost analysis with the added heat treatment step.

\section{Reviewer 4:}

The reviewer indicated that future research includes demonstrating the performance of new alloys in block bulkhead and Ford GTDI cylinder head and that bench testing has been scheduled.

This person stated that the project will appropriately assess the cost of the new alloy compared to the baseline alloys (A319 and A356).

\section{Reviewer 5:}

The reviewer reported that the project team has a logical list of future work activities to complete the project this year. The team will demonstrate casting of actual engine components in this future work, which is very appropriate and useful for eventual commercialization.

\section{Reviewer 6:}

The reviewer explained that the plans for completion of project including demonstration of developed alloys in cast components (cylinder heads) appear to be on track.

\section{Reviewer 7:}

The reviewer indicated the future plans appear to address most of the remaining challenges. However, it is unclear how the project contributes to determining ICME gap analysis. This reviewer indicated that the plan for refining the cost model and how the cost impact will be determined was not described. Especially because the project ends in November 2016, the reviewer questioned the remaining effort and cost based on inaccuracies of stated percent progress and budget. 


\section{Reviewer 8:}

The reviewer commented that the gaps in the ICME tools are not clear from the presentation. This person said these remain on the future work slides but one would suspect that these areas of improvement have been discovered along the way.

\section{Reviewer 9:}

The reviewer indicated the project demonstrated performance of alloys and the target is in 2020 for commercialization into product line. This reviewer also observed a significantly accelerated approach versus conventional material development timelines. The reviewer noted that focus on commercializing lighter weight high-pressure diecast (HPDC) block bulkhead and Ford GTDI cylinder head appears to be a good application of this material.

\section{Reviewer 10:}

The reviewer referenced prior comments and indicated that the integration of wide-ranging ICME models is not readily identifiable from the work that was presented. This person suggested that for a program that is so heavily leveraged by DOE funding, the discovery and application of ICME tools should be a key focus area, allowing that Ford has intellectual property that it will want to protect. A program like this is most beneficial to DOE goals when some element of shareable discovery is made public. From a program-specific standpoint, the results for different processing approaches to the H16 and H17 alloys are not overly compelling. The overlap in the limited data points indicates that some statistical significance exists that would allow the program to conclude that the claimed improvements are real, but a more strict presentation of the data that outline this significance would help immensely. The reviewer stated that additionally, for a program at this stage it is difficult to believe that no previous reviews provided critiques that were worthy of addressing in the presentation and that no such information was provided.

\section{Question 5: Does this project support the overall DOE objectives of petroleum displacement? Why or why not?}

\section{Reviewer 1:}

The reviewer agreed that advanced cast alloys are certainly germane to DOE objectives vis-à-vis the transportation sector. Increasing both strength and stability of lightweight cast alloys, which this program is accomplishing, is a definite step forward.

\section{Reviewer 2:}

The reviewer stated that the increase in strength will allow decreased mass in the casting at a similar temperature and pressure during service.

\section{Reviewer 3:}

The reviewer said the project is aimed at improving the strength of Al alloys, demonstrating ICME tools for materials processing, developing cost models, and conducting technology transfer and commercialization planning for deployment of the new alloys. The result of this project should be higher performance $\mathrm{Al}$ alloys allowing for higher efficiency, clearly of relevance to the DOE objectives.

\section{Reviewer 4:}

The reviewer commented that the development of an alloy that meets the challenging DOE goals enables design of much higher efficiency engines in the future.

\section{Reviewer 5:}

The reviewer indicated that high-temperature and pressure metal alloys enable high-efficiency internal combustion engines and therefore, enable petroleum displacement.

\section{Reviewer 6:}

This person reported that new high-temperature alloy work for lighter weight and increased efficiency engines is directly relevant to DOE petroleum reduction goals. 


\section{Reviewer 7:}

The reviewer reported the alloy has been improved with use in the design of a new cylinder head and efficiency improvement is expected.

\section{Reviewer 8:}

The reviewer pointed out that the project is developing higher strength $\mathrm{Al}$ alloys to enable higher temperature, pressure, and efficiency during combustion. Once commercialized, this directly results in reduced petroleum consumption.

\section{Reviewer 9:}

The reviewer acknowledged that lighter weight and higher performance propulsion materials being developed lead to more efficient and lighter vehicles.

\section{Reviewer 10:}

This person observed that overall cost of materials and component development may increase but the new alloys should lead to lighter weight vehicles and higher efficiency.

\section{Question 6: Resources: How sufficient are the resources for the project to achieve the stated milestones in a timely fashion?}

\section{Reviewer 1:}

The reviewer commented that the project is on track for completion at the stated time, and the amount of work completed seems to correlate with their schedule.

\section{Reviewer 2:}

The reviewer said no indication was made that the resources are not sufficient, and the project team appears to have made significant progress under existing funding.

\section{Reviewer 3:}

The reviewer indicated that the budget seems appropriate for the presented project scope. FY 2016 budget is $\$ 1.3$ million to focus in on demonstrations and total DOE project funding is $\$ 3.24$ million with project team contributing $\$ 1.39$ million in cost share.

\section{Reviewer 4:}

The reviewer said the project appears to be appropriately funded, based on the technical achievements displayed.

\section{Reviewer 5:}

The reviewer reported funding appears to be sufficient and did not note funding was lacking.

\section{Reviewer 6:}

The reviewer said that the project remains on schedule and funding should continue on the originally planned timeline to ensure commercialized product arrives in the market in 2020.

\section{Reviewer 7:}

The reviewer noted the project spending was not addressed; however, it appears that resources are sufficient.

\section{Reviewer 8:}

The reviewer noted the question of timeliness still remains. The program is considerably lagging a straight-line use of funds if it is scheduled to end in five months. 


\section{Computational Design and Development of a New, Lightweight Cast Alloy for Advanced Cylinder Heads in High-Efficiency, Light-Duty Engines: Mike Walker (General Motors) - pm061}

\section{Presenter}

Qigui Wang, General Motors

\section{Reviewer Sample Size}

A total of eight reviewers evaluated this project

\section{Question 1: Approach to performing the work-the degree to which technical barriers are addressed, the project is well-designed, feasible, and integrated with other efforts.}

\section{Reviewer 1:}

The reviewer stated the approach is logical and appropriately thought out. The project team is focusing on high-temperature strength and fatigue characteristics of these lightweight alloys, and both characteristics are critical for engine durability. This person pointed out that the examination of higher strength lightweight alloys is important to achieve higher peak cylinder pressures and engine efficiency. The project team is considering the effects of alloy processing and microstructure on the key material properties of relevance to engine structures, which is quite appropriate, commented the reviewer.

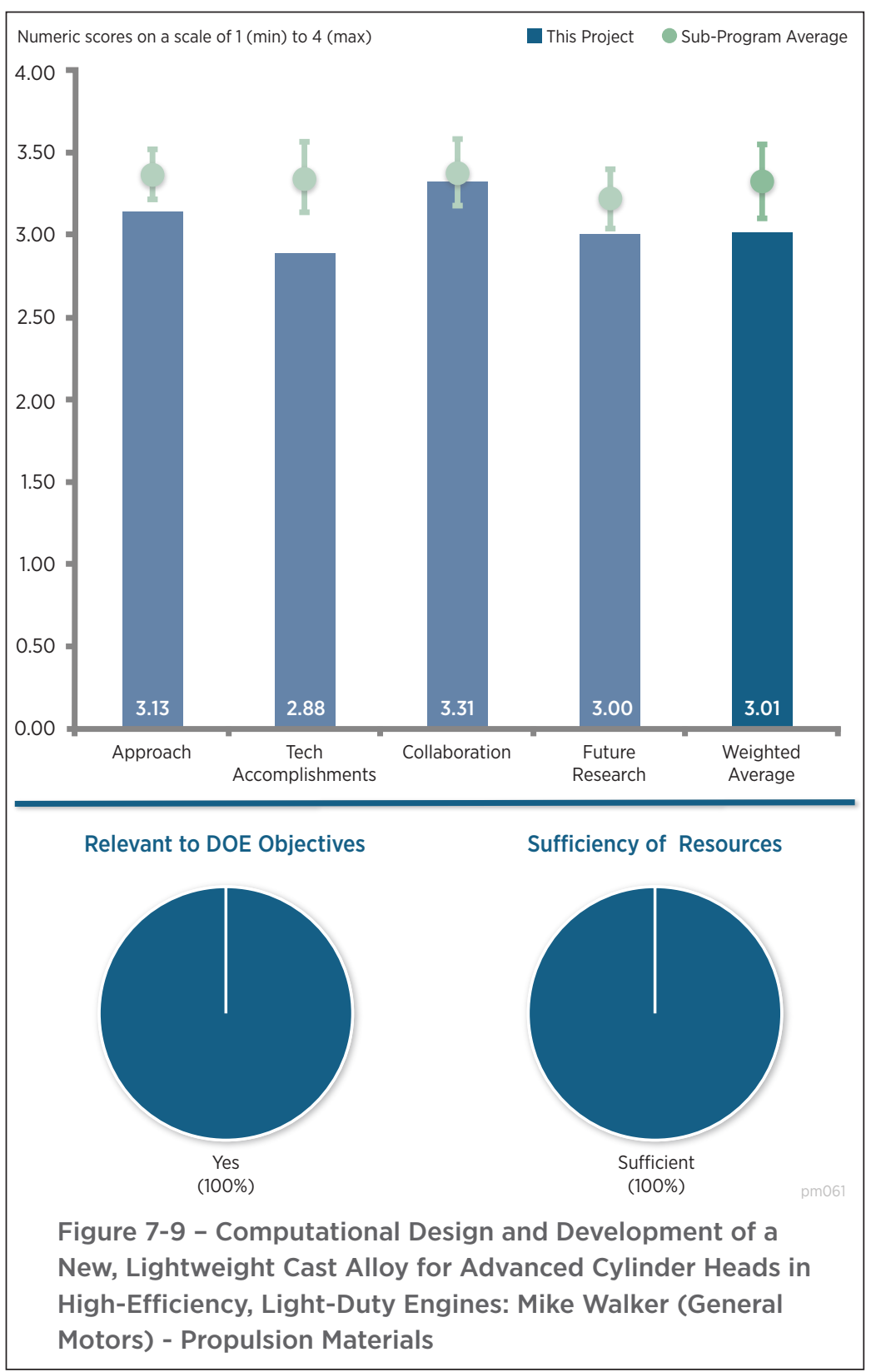

\section{Reviewer 2:}

The reviewer observed that the approach of using density functional theory (DFT) models for alloy concept development and alloy selection, button castings for structural and physical properties is solid. This person stated that the modification to the approach outlined in the critical assumptions and issues slide is a reasonable adaptation to the original approach.

\section{Reviewer 3:}

The reviewer stated that the focus for the approach has been improving high-temperature strength to improve engine performance. The key element of the approach for 2015-2016 was clearly identified as analysis of two new alloys. This includes a careful look at cast defects because they have significant impacts on ultimate fatigue. The reviewer noted that the approach, as presented, included a matrix-type approach across processing, structure, and properties, in an attempt to provide the level of in-depth analysis needed. 


\section{Reviewer 4:}

The reviewer commented that was a nice graphic in the presentation to explain the approach and strategy on Slide 5. In addition, this person said there was a well explained scope and an alignment with the goals. The reviewer suggested there could be an increase in merit by surveying a wider view of the material options.

\section{Reviewer 5:}

The reviewer commented that the down selection approach and evaluations to date seem appropriate for the goal, but nothing overly transformational is being shown. This person said just a sound approach to alloy improvement through relatively minor precipitate structures was shown. The program is progressing with regard to ongoing evaluations, but despite the detailed analyses there is not yet an overly compelling reason to think that the Q alloy will be entirely successful. The reviewer suggested it would appear that the Rio Tinto alloy is more promising despite the low ductility. Regardless, the foundation is being established for continuing alloy development efforts that begin with atomistic/DFT simulations and end with component trials. The reviewer stated that the results from the dispersoid element trials will be of great interest with regard to the stated property targets and cost modeling.

\section{Reviewer 6:}

The reviewer reported that the computational approach was very broad, but narrowed to a strategy centered on the $Q$ phase. In addition, focusing on casting defects and fatigue properties was of value, commented the reviewer. This person suggested that tit would have been helpful to have a slide explaining why the Q phase was selected for a $300^{\circ} \mathrm{C}$ strengthening strategy.

\section{Reviewer 7:}

The reviewer mentioned it would be interesting to evaluate the stability of the elevated temperature property response at 200 hours. This person questioned how stable are the properties for the Q alloy.

\section{Reviewer 8:}

The reviewer suggested that the presenter better define the additive selection process to clarify the additives needed to improve material performance. The project may actually achieve the end results but there are better methods to identify these alloys such as ICME. The reviewer pointed out that this project used ICME earlier, and connecting these results to the previous ICME research would help clarify the selection process. This person commented that the virtual casting approach to reduce the weight of the cylinder head is innovative.

\section{Question 2: Technical accomplishments and progress toward overall project and DOE goals-the degree to which progress has been made, measured against performance indicators and demonstrated progress towards DOE goals.}

\section{Reviewer 1:}

The reviewer commented that it appears all go/no-go points to date have been successfully passed. The project has appeared to down-select from six alloys to one, which is a Q phase alloy. This person pointed out that the Rio Tinto Alcan (RTA) alloy met all DOE high-temperature requirements, but ductility was an issue. Ultimately, new alloy concepts were generated. The reviewer indicated there were some setbacks, but the project appears to have retrenched and, based upon what the team had learned so far, moved ahead with the new concepts.

\section{Reviewer 2:}

The reviewer noticed that the status of any significant breakthroughs is not coming across in the presentation, although to be fair this type of effort is not at all straightforward. This person said that the project team is to be commended for identifying approaches that simply did not pan out along with successes, as there are bound to be both in alloy development that draws upon multi-scale approaches. The reviewer commented that the reasoning behind the alloy selection process is sound.

\section{Reviewer 3:}

The reviewer observed that it is impressive that the project team has already created 100 cylinder heads with the new material. This is very fast progress on design to market timing. However, the reviewer noted the project seems more iterative than transformative. The reviewer suggested it would add merit if the project team made some estimation of the actual impact on the change in weight if this project is successful. 


\section{Reviewer 4:}

The reviewer stated that the project has established a viable approach for selection of new materials to meet DOE targets. In executing the work the project has generated a substantial amount of data and knowledge but has yet to identify materials with an acceptable combination of strength and ductility. The shift to exploring dispersoids may yield better results. This person commented that project milestones have been met.

\section{Reviewer 5:}

The reviewer pointed out that in past work, the team has conducted a systematic exploration of prospective alloys with differing precipitate structures, and down-selected to the $\mathrm{Q}$ phase precipitate. The team has now validated the basic characteristics and microstructure models of the precipitates, which appears to be good science-based work. This person commented that this has led to the down-selection of a candidate precipitate for further development. The reviewer noted that all of these are good accomplishments.

\section{Reviewer 6:}

The reviewer observed that an alloy has been identified which meets the targets in high-temperature mechanical properties. In addition, strength model prediction and optimization looks promising for the future.

\section{Reviewer 7:}

The reviewer commented still no final alloy configuration has been identified. This person suggested that some of this information may be corporate confidential. However, it is difficult to determine if the project is leading to success without this information.

\section{Reviewer 8:}

The reviewer reported that it appears that a high volume of thoughtful computational and experimental work has been conducted, and improvements in alloy properties have been achieved. However, the results indicate that that the primary approaches to creating a stable, higher strength alloy at the target temperature has not been successful. The reviewer indicated that the mechanical property targets for $300^{\circ} \mathrm{C}$ have not yet been achieved, nor were there strong indications in the presentation that the present alloy design approach will eventually achieve such targets.

In addition, the introduction of the RTA alloys seems to confirm that the $\mathrm{Q}$ or Theta $\mathrm{Q}$ alloys will not achieve targets. However, the RTA alloys do not have sufficient ductility, as was pointed out by the presenter. The reviewer said that the definitions of coarse and fine structure were unclear. The reviewer mentioned it was also unclear why other strengthening strategies were not evaluated when the Q phase emphasis did not prove adequate for $300^{\circ} \mathrm{C}$ properties. The reviewer observed it was stated that hundreds of thousands of crystal structures were analyzed by high-throughput DFT. This person suggested it would be interesting to know how those analyses along with the calculations of elemental segregation and interfacial strengthening predicted success with the Q phase at $300^{\circ} \mathrm{C}$. It will be of value to the ICME community to see what properties or advantages were predicted for the Q-phase through computational approaches, and then compare to actual measured properties or improvements in properties versus baseline materials.

\section{Question 3: Collaboration and coordination with other institutions.}

\section{Reviewer 1:}

The reviewer indicated that the project includes a long list of collaborators: Questek, Northwestern, American Foundry Society, Fred Major, Camaneo Associates, and Massachusetts Institute of Technology (MIT). This person pointed out all have clearly identified roles under the project.

\section{Reviewer 2:}

The reviewer noted that the team has a good balance of collaborators including OEMs, a material supplier, academics, and ICME experts that provide the right expertise. The team is taking advantage of this expertise through the distribution of activities under GM's leadership.

\section{Reviewer 3:}

The reviewer stated that the project is comprised of a strong set of collaborators. 


\section{Reviewer 4:}

The reviewer indicated that all collaborators appear to be working together.

\section{Reviewer 5:}

The reviewer commented that including MIT was a good addition with the academic community. This person pointed out the project could still use a national laboratory to improve the team's expertise and capabilities. National laboratory expertise could add computational capabilities to the team.

\section{Reviewer 6:}

The reviewer observed that the project team is both diverse and talented in a number of specific areas that are a benefit to the proposed effort. This person suggested that specific roles and responsibilities are not coming across in the presentation, and the amount of work being carried out by Questek, for instance, is not afforded an appropriate level of significance. A great deal of the work being done was not identified until the response to the reviewer comments section.

\section{Reviewer 7:}

The reviewer said that there are clear ties to GM and well-grounded in what is necessary to commercialize. This person pointed out it is surprising that GM did not have a clear test procedure for material aging, or that it was not shared. The reviewer commented there was no clear tie between aging time and product requirements for durability and useful life.

\section{Reviewer 8:}

The reviewer commented that there appear to be very strong collaborations with Questek for the computational activity; however, the roles of the other partners were not quite clear.

\section{Question 4: Proposed future research-the degree to which the project has effectively planned its future work in a logical manner by incorporating appropriate decision points, considering barriers to the realization of the proposed technology and, when sensible, mitigating risk by providing alternate development pathways.}

\section{Reviewer 1:}

The reviewer indicated that the presentation included a number of specific activities planned for the rest of 2016 and for 2017. The list makes reasonable sense, assuming that at least one alloy can be developed to move all the way through the test and development process. This person pointed out that should there be a setback; the project team will need to stay flexible, as they showed in 2015-2016.

\section{Reviewer 2:}

The reviewer commented that the future work plan looks reasonable and appropriate, with casting trials of the down-selected alloys and associated testing/analysis. This person said that the work plan tasks are appropriate to complete the work by the end of FY 2017.

\section{Reviewer 3:}

The reviewer stated the future work slide details the planned work for 2016 and 2017 and this is a reasonable approach for achieving project goals.

\section{Reviewer 4:}

The reviewer commented that some level of confidence that the $\mathrm{Q}$ alloy will be successful in meeting all of the targets is still in question, but the project team is pushing forward with planning for full component trials. This person indicated that the project team is making interesting and scientifically significant progress, but commented that were a major GM stakeholder would want to see more concrete results that the project team is on the absolute correct path prior to moving from evaluations to the practical component test phase.

\section{Reviewer 5:}

The reviewer suggested that comments regarding the utility of high-throughput DFT calculations, the Open Quantum Materials Database (OQMD), would be useful. 


\section{Reviewer 6:}

The reviewer indicated that the future work was not well presented and passed quickly. This person noted that in reviewing the presentation the project team has a path forward but it is still narrowly focused.

\section{Reviewer 7:}

The reviewer observed that the future effort could be clearer if the specifics were provided. This person stated with the given information, it is not clear that this project will achieve the end result with the remaining funding and the established approach.

\section{Question 5: Does this project support the overall DOE objectives of petroleum displacement? Why or why not?}

\section{Reviewer 1:}

The reviewer noted that this project addresses the material issues facing LD engine designers as they push the limits of efficiency, with resulting achievement of DOE petroleum reduction goals. Increasing engine materials capability (peak cylinder pressure and temperature) are critical considerations for raising engine brake thermal efficiency that can often be overlooked.

\section{Reviewer 2:}

The reviewer stated that alloys found in the study will lead to efficiency and weight reduction improvements in internal combustion engines.

\section{Reviewer 3:}

The reviewer commented that the project is specifically relevant to DOE and VTO objectives, as it is aimed at moving toward DOE materials targets to allow for increased performance and efficiency for light-duty spark ignition engines.

\section{Reviewer 4:}

The reviewer mentioned that developing improved alloys will allow more aggressive combustion strategies to enable higher engine efficiencies.

\section{Reviewer 5:}

The reviewer said that the target material will provide the capability to increase engine efficiency.

\section{Reviewer 6:}

The reviewer pointed out that the project strives to achieve DOE goals for lightweight materials that tolerate higher pressures and temperatures in light-duty vehicles.

\section{Reviewer 7:}

The reviewer indicated that the program follows the stated targets of cast lightweight alloys and those metrics are being used to evaluate progress.

\section{Reviewer 8:}

This reviewer commented that efficiency increases would push higher mass materials if this option was not available. The reviewer further explained that the casting can be minimized using these improved materials properties, which would be lighter as well.

\section{Question 6: Resources: How sufficient are the resources for the project to achieve the stated milestones in a timely fashion?}

\section{Reviewer 1:}

The reviewer commented that funds appear sufficient, at least at this point. It is unclear how much of the overall DOE funding has been received, but with the shrinking overall Propulsion Materials funding, there could be issues if significant funding is required in FY 2017.

\section{Reviewer 2:}

The reviewer observed that the team has moved through the down-selection phase, and although there is 
considerable work to be done the team appears to be progressing well against the proposed timeline with completion in September of 2017.

\section{Reviewer 3:}

The person stated that the project team is on target and meeting milestones.

Reviewer 4:

The resources appear to be sufficient to complete the work as described over the timeframe of the project.

\section{Reviewer 5:}

The reviewer noted funding seems appropriate to execute this approach, given the previous ICME work. This person pointed out that it appears that the remaining funding will mostly be used for durability testing.

\section{Reviewer 6:}

The reviewer observed that sufficient information on the use of project resources was not provided. This person suggested the project appears to be adequately funded. 


\section{High-Performance Cast Aluminum Alloys for Next Generation Passenger Vehicle Engines: Amit Shyam (Oak Ridge National Laboratory) - pm062}

\section{Presenter}

Amit Shyam, Oak Ridge National Laboratory

\section{Reviewer Sample Size}

A total of five reviewers evaluated this project.

\section{Question 1: Approach to performing the work-the degree to which technical barriers are addressed, the project is well-designed, feasible, and integrated with other efforts.}

\section{Reviewer 1:}

The reviewer reported that the results of this alloy design study are impressive.

\section{Reviewer 2:}

The reviewer noted that the approach seems to be appropriate and focused on accomplishing project objectives. The project is aimed at reducing the cost of improving the performance of engine materials through ICME. The reviewer observed that in particular, project efforts focus on balancing the need to satisfy several properties simultaneously.

\section{Reviewer 3:}

The reviewer commented that there are very interesting results thus far, and the program appears to be proceeding successfully. With a focus on the microstructural stability, however, more emphasis on the practical response of the alloys following extended aging would be very beneficial. This person said all targets are met, but questioned what the effect is on precipitate size and aspect ratio following times that are more reflective of engine lifetimes rather than minimum qualification targets. The reviewer asked if the results that are being seen by the project team still valid. The reviewer noted that the microstructures and schematic of Slide 8 do not really close the argument.

\section{Reviewer 4:}

The reviewer observed that the project has two goals; the first is to determine an appropriate high-temperature Al-alloy with the target properties through an ICME approach, and the second to determine the gaps in the ICME approach to discovering a new appropriate alloy in this specific case. The reviewer stated that overall the approach is reasonable, but ultimately, as the project team suggested, does not work well due to the current lack of knowledge and models for the microstructure evolution. This person suggested that the technical accomplishments shown in the presentation indicate that the necessary knowledge can be achieved by atomistic simulations. 
The reviewer mentioned that presumably, the ICME approach will still be used to evaluate the performance versus cost trade-off of the different candidate Al-alloys, which is equally as important as the discovery of new alloys.

\section{Reviewer 5:}

The reviewer stated that the researchers have successfully implemented an iterative ICME approach consisting of modeling to suggest material composition, casting and mechanical testing, component casting and testing. The focus of the results is on elevated temperature mechanical properties and castability and hot tear resistance. This person indicated the project team has recognized the limitations of this approach, including functionality in cylinder heads and unknowns including thermal conductivity, corrosion resistance, residual stress and cost.

\section{Question 2: Technical accomplishments and progress toward overall project and DOE goals-the degree to which progress has been made, measured against performance indicators and demonstrated progress towards DOE goals.}

\section{Reviewer 1:}

The reviewer noted that the technical accomplishments are substantial. These include developing computational capability to predict useful elemental combinations, generation of high-temperature alloys that exceed tensile strength targets at $300^{\circ} \mathrm{C}$, confirmation of hot tear resistance, and proof of microstructural stability.

\section{Reviewer 2:}

The reviewer indicated that the team has utilized some powerful tools and practical approaches to alloy casting to provide broad test matrices for evaluation. This person observed that this is a real plus of the program's presented results to date.

\section{Reviewer 3:}

The reviewer pointed out that the project has identified improved alloys, and is now casting components. To date, the high-temperature alloy, at $300^{\circ} \mathrm{C}$, exceeds the technical target by more than two times. It also has exceptionally stable microstructure, which was confirmed through testing at Brookhaven National Laboratory. This person observed the project then took what it learned to develop computational capabilities to predict microstructure results for other material combinations, which can be used for future stabilization efforts. This approach has identified which elements provide good high-temperature stability, and which ones do not. The reviewer stated that to date, the project has cast about 30 alloy compositions using the project's concepts and that most of the hightemperature alloys appear to have very good hot tear resistance.

\section{Reviewer 4:}

The reviewer noted a strong understanding on improving high-temperature strength in $\mathrm{Al}$ alloys was shown in the presentation. This is achieved through control of the mobility of the semi-coherent interfaces of the precipitates, thus controlling the precipitate size at elevated temperature. The reviewer noticed that from the modeling effort, it seems that several potential candidate $\mathrm{Al}$ alloys have been determined and the project is moving along well towards its objectives.

\section{Reviewer 5:}

The reviewer indicated that it was interesting use of DFT models to predict stable precipitates.

\section{Question 3: Collaboration and coordination with other institutions.}

\section{Reviewer 1:}

The reviewer stated that the project appears highly collaborative, with regular calls and face-to-face meetings. Partners (under a CRADA) include Fiat Chrysler Automobiles (FCA) and Nemak, plus collaboration with Granta MI, ESI North America, Flow Science, Magma Foundry, and Minco. All have specifically-identified roles covering the project's needs, and there is significant cost-share. The reviewer noted that FCA has already indicated it will soon (within two years) incorporate an alloy from this project into an engine component for a higher efficiency engine. 


\section{Reviewer 2:}

The reviewer indicated that researchers have engaged partners from automotive and manufacturing industries and collaborations appear to be working well.

\section{Reviewer 3:}

The reviewer pointed out that the actual contribution of team members was questioned in a past review, as shown in the responses to reviewer comments section, and the team responded that all members were contributing significantly. The reviewer reported that there is no reason not to believe this, but little concrete evidence was specifically addressed in the body of the presentation to put this concern to rest. There is certainly a group of highly regarded names from ORNL being acknowledged, but whether there is equally significant contributions coming from outside of ORNL must be taken on the statement of the presenter. That being said, the cost share provided to supplement the DOE share was considerably higher in this program than for other similar programs, so there is a clear collaborator presence, this person commented.

\section{Reviewer 4:}

The reviewer reported that collaboration with CRADA partners seem well established and organized. The largescale castings by the CRADA partners will provide a nice comparison to the current results. This person observed some mention of other partners was made, though the amount of collaboration effort between those partners is unclear.

\section{Question 4: Proposed future research-the degree to which the project has effectively planned its future work in a logical manner by incorporating appropriate decision points, considering barriers to the realization of the proposed technology and, when sensible, mitigating risk by providing alternate development pathways.}

\section{Reviewer 1:}

The reviewer enthusiastically looked forward to the results of the larger heats and component trials.

\section{Reviewer 2:}

The reviewer reported that a number of specific activities have been identified for the remainder of 2016 and 2017. In particular, the project is looking at optimizing alloys for both technical and cost targets. The reviewer pointed out that additional elements need characterization, such as thermal conductivity, corrosion resistance, machinability, and residual stress. There will also be additional ICME modeling work to fill gaps in current capabilities. The reviewer observed that future efforts appear to culminate in preparation for large scale evaluation on an engine platform and development of a cost analysis and commercialization plan.

\section{Reviewer 3:}

The reviewer stated that the future plans are well thought out and were presented adequately at the review. The plans follow a logical progression to completion and include additional mechanical testing, attempting to understand those properties not addressed thus far including thermal conductivity, corrosion resistance and residual stress. The reviewer commented that also included are plans to refine the ICME approach, produce a gap analysis and a cost and commercialization plan.

\section{Reviewer 4:}

The reviewer indicated that the future work seems on-track with the proposed milestones in the project and the immediate work plans are well outlined. The microstructure stability characterization coupled with the casting evaluation will provide useful information between the structure and property relationships. This person has a slight concern in the down-selection of the new alloy composition, which was originally proposed as one of the future work plans to be done in the 2015 review. The ICME approach will give several alloys each with their pros and cons for the target key properties, but the reviewer predicts that the cost analysis will ultimately help reduce this to one. The reviewer suggests that the team put some more effort into the cost analysis coupled with the ICME approach sooner rather than later.

\section{Reviewer 5:}

The reviewer noted a good gap assessment in the ICME tools. This person questioned if any nondestructive testing improvements will be necessary in the final demonstration. 


\section{Question 5: Does this project support the overall DOE objectives of petroleum displacement? Why or why not?}

\section{Reviewer 1:}

The reviewer stated that the project is focused on improving alloys allowing for increased engine efficiency. Specifically, the project's goals include improved castability, high-temperature strength, and fatigue performance. Overall, the new alloy cylinder heads will have more than a $25 \%$ improvement in strength at a cost of less than $10 \%$ more. The reviewer pointed out these project objectives are all in line with the overall DOE VTO objectives.

\section{Reviewer 2:}

The reviewer noted that this project is aligned with the DOE objectives of petroleum displacement through the discovery and development of new $\mathrm{Al}$ alloys with high-temperature strength and fatigue properties. This person indicated this will enable the lightweighting of engine materials and consequently improved fuel economy.

\section{Reviewer 3:}

The reviewer observed that success of these alloys will lead to efficiency improvements in the internal combustion engine.

\section{Reviewer 4:}

The reviewer said the project directly addresses objectives set forth by DOE associated with material properties of tensile strength at high temperature.

\section{Reviewer 5:}

The reviewer indicated that the specific DOE targets for cast alloys are both stated and used to gauge progress.

\section{Question 6: Resources: How sufficient are the resources for the project to achieve the stated milestones in a timely fashion?}

\section{Reviewer 1:}

The reviewer indicated that the program is on target based on the progress to date, and the accomplishments as presented indicate that the next phase of the program that moves beyond alloy development and analysis is ready to commerce.

\section{Reviewer 2:}

The reviewer remarked no indication was made concerning the sufficiency of the resources, so they were assumed to be appropriate. This person said it should be noted that the presentation did not include any estimated DOE funding for 2017.

\section{Reviewer 3:}

The reviewer noted resources appear sufficient for the completion of this project. The largest cost seems to be in computational time or power, which is covered by the facilities at ORNL.

\section{Reviewer 4:}

The reviewer indicated that funding appears sufficient, although little information is provided regarding project costs. 


\section{Development of High- Performance Cast Crankshafts: Rich Huff (Caterpillar) - pm065}

\section{Presenter}

Rich Huff, Caterpillar

\section{Reviewer Sample Size}

A total of four reviewers evaluated this project.

\section{Question 1: Approach to performing the work-the degree to which technical barriers are addressed, the project is well-designed, feasible, and integrated with other efforts.}

\section{Reviewer 1:}

The reviewer said the work has a clear direction, is well documented with a nice flow chart of the selection and development process.

\section{Reviewer 2 :}

The reviewer commented that proper targets were clearly defined and described for reviewers and indicated the approach appears to be sound. The reviewer pointed out that it would have been useful to see a simple castability study for some of the selected steels versus nodular iron, in order to give reviewers a sense of the relative castability of these steels. Casting and defect analysis of crankshafts, and comparison to the MAGMA software, was valuable.

\section{Reviewer 3:}

The reviewer indicated this is interesting work and has good progress. The reviewer referenced Slide 7 and requested an explanation regarding the allowable forecast design given the current alloy choice. The reviewer questioned if these are only composition dependent, or whether more is intended by this statement.

\section{Reviewer 4:}

The reviewer pointed out that using ICME allows faster development and lower cost alloy development. The reviewer said the project is also using APS at ANL to measure phase evolutions. The reviewer pointed out that use of Accelerated Insertion of Materials with ICME permits an accelerated iteration of material composition.

\section{Question 2: Technical accomplishments and progress toward overall project and DOE goals-the degree to which progress has been made, measured against performance indicators and demonstrated progress towards DOE goals.}

\section{Reviewer 1:}

The reviewer observed that good progress has been made in the first $30 \%$ of the project, but it seems that there are 
substantial processing hurdles to consistently cast clean steel cranks of complex geometry, particularly if hollow cranks are eventually cast to reduce mass. This person pointed out that casting defect modeling seemed to be very effective in predicting defect-prone areas. The reviewer noted that validation and calibration of ICME tools in subsequent years will be valuable. The vacuum assisted counter gravity (VACG) casting process is interesting, and cleanliness comparisons with other casting methods will be valuable, commented the reviewer.

\section{Reviewer 2:}

The reviewer said that progress toward the desired crankshaft is reasonable. In addition, alloy selection appears complete and sample crankshafts have been produced.

\section{Reviewer 3:}

The reviewer explained that 20 alloys were assessed but the yield strength target was not met. The reviewer pointed out the project casted a prototype steel crankshaft that used one of the highest potential alloys to determine how close to target it was and established a baseline. This person indicated crankshaft results were even better than the test bars and a counter gravity chamber was designed to eliminate quality issues.

\section{Reviewer 4:}

The reviewer reported the yield was low and this was addressed by the presenter. However, there was not a strong path presented if the project team needs to increase this beyond what it has seen other than just some tuning.

\section{Question 3: Collaboration and coordination with other institutions.}

\section{Reviewer 1:}

The reviewer reported there is good collaboration between ANL, GM and Caterpillar.

\section{Reviewer 2:}

The reviewer said that there appear to be strong collaborations with GM, University of Iowa and ANL.

\section{Reviewer 3:}

The reviewer stated the project is teamed with GM, ANL, Iowa and Northwestern, and are also working with two foundries.

\section{Question 4: Proposed future research-the degree to which the project has effectively planned its future work in a logical manner by incorporating appropriate decision points, considering barriers to the realization of the proposed technology and, when sensible, mitigating risk by providing alternate development pathways.}

\section{Reviewer 1:}

The reviewer said that the proposed future research plan appears to be sound. A castability comparison to nodular cast iron would be of value in identifying the risk factors associated with steel casting of complex geometries the reviewer commented.

\section{Reviewer 2:}

The reviewer stated that many available options going forward were discussed and that there is a lot of space for options ahead and for post processing.

\section{Reviewer 3:}

The reviewer noted fatigue life testing seems critical to the next phase of the project.

\section{Reviewer 4:}

The reviewer observed that crankshafts are built for infinite miles and lifetime at Caterpillar. This person indicated that there is a need to find a material that can meet Caterpillar's and DOE's need. The reviewer concluded the project is getting close, but not there yet.

Question 5: Does this project support the overall DOE objectives of petroleum displacement? Why or why not? 


\section{Reviewer 1:}

The reviewer noted that more accessible cast steel crankshafts will increase internal combustion engine efficiency.

\section{Reviewer 2:}

The reviewer stated that lighter weight crankshaft leads to a lighter, more efficient vehicle.

\section{Reviewer 3:}

The reviewer pointed out that it is not clear how a cast crankshaft improves fuel efficiency from the current forged component. The project is trying to approach forged steel strengths and this would not exceed those strengths. Given that the mass is the same, there is no material decrease (no loss of weight) available, and because strength will be less, there is no gain available from decreasing the journal diameters and therefore reducing the amount of oil sheared during rotation.

\section{Question 6: Resources: How sufficient are the resources for the project to achieve the stated milestones in a timely fashion?}

\section{Reviewer 1:}

The reviewer observed that it appears that the funding has been significantly reduced and is unsure why this happened. The reviewer pointed out the reduction in the Propulsion Materials budget forced the cut in funding in this project. This person indicated it appears that the funding needed to complete this project may not be allocated, which is very concerning.

\section{Reviewer 2:}

The reviewer reported that for a project that requires both alloy development and casting process development, both the time and funding appear to be inadequate.

\section{Reviewer 3:}

The reviewer stated the project team is on target to down-select from its 14 initial alloys. 


\section{Innovative SCR Materials and Systems for Low-Temperature Aftertreatment: Yong Wang (Pacific Northwest National Laboratory) - pm066}

\section{Presenter}

Yong Wang, Pacific Northwest National Laboratory

\section{Reviewer Sample Size}

A total of six reviewers evaluated this project

\section{Question 1: Approach to performing the work-the degree to which technical barriers are addressed, the project is well-designed, feasible, and integrated with other efforts.}

\section{Reviewer 1 :}

The reviewer noted an excellent job defining suspect elements and concentrations, as evidenced by the improvements in the first generation part. This person suggested the need to add the effect of sulfur on the new formulations.

\section{Reviewer 2:}

The reviewer commented that this project team's CRADA approach is logical and takes advantage of the expertise areas of the various partners. The project team has a logical progression of tasks that should achieve the required goals to adapt new SCR materials, develop SCR catalysts, and verify catalyst performance to meet relevant LD emission standards. The reviewer said that the approach includes work to examine costs and system impacts, which will be very important.

\section{Reviewer 3:}

The reviewer indicated that the project is aimed at cost-effective, durable, and low-temperature emissions control systems. The approach seems relatively straight-forward, focusing on development of appropriate catalyst material and then demonstrating its performance, resulting in an SCR cost model at the end. This person observed that while not included in the presentation, according to the PI, the project also plans to look at the impact of sulfur on catalyst performance.

\section{Reviewer 4:}

The reviewer pointed out that the project is focused on developing new enabling SCR catalysts with conversion efficiency necessary to meet regulations that are necessary for commercializing high-efficiency engine technologies.

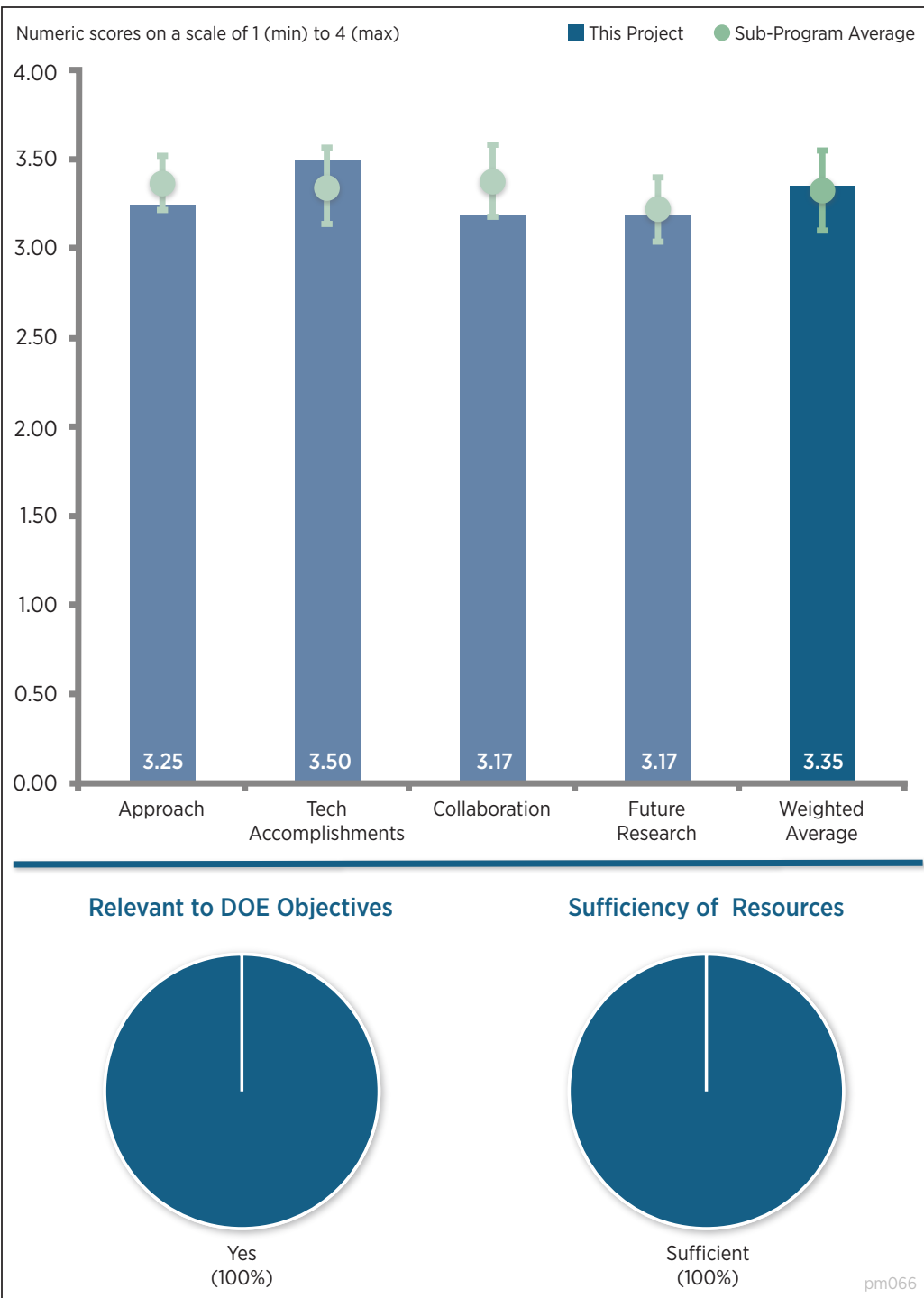

Figure 7-12 - Innovative SCR Materials and Systems for LowTemperature Aftertreatment: Yong Wang (Pacific Northwest National Laboratory) - Propulsion Materials 


\section{Reviewer 5:}

The reviewer stated the project is assessing the viability of developing a second generation SCR catalyst system. This person mentioned that low-temperature $\mathrm{NO}_{\mathrm{x}}$ conversion performance improvement can be obtained by a twostage process.

\section{Reviewer 6:}

The reviewer indicated that the barriers being approached do not match those in the PM area. The reviewer said that the barriers listed are from Advanced Combustion and Emission Control area and this is a PM project.

\section{Question 2: Technical accomplishments and progress toward overall project and DOE goals - the degree to which progress has been made, measured against performance indicators and demonstrated progress towards DOE goals.}

\section{Reviewer 1:}

The reviewer commented that the team has had a number of successes in its first year or so of work. The project team has demonstrated a first generation catalyst that achieves the required $\mathrm{NO}_{\mathrm{x}}$ conversion efficiency at a temperature approaching the $150^{\circ} \mathrm{C}$ goal, which is a good initial result. This person indicated that the project team has conducted good basic science to explore ways to improve SCR catalyst activity at low temperatures. The reviewer stated that the team has demonstrated reproducible catalyst performance with multiple low-volume production batches, which is a valuable contribution to understanding how this catalyst can move to production.

\section{Reviewer 2:}

The reviewer mentioned that it is very encouraging that the project team has already decreased activation temperature significantly.

\section{Reviewer 3:}

The reviewer reported that all project milestones for late 2015 to early 2016 were achieved. So far, for the first year of the project, the project demonstrated greater than $90 \%$ conversion efficiency at $175^{\circ} \mathrm{C}$, much better than current commercial systems. This person also indicated the project delivered a large batch of the first generation catalyst. From there the reviewer pointed out that the project focused on identifying a pathway to get to a second generation system. It appears that improved crystallization can improve catalyst performance. The reviewer said that the project has also down-selected a possible (ammonia $\left.\left[\mathrm{NH}_{3}\right]\right)$ generation strategy.

\section{Reviewer 4:}

The reviewer observed improved current generation SCR catalysts, laying the baseline for generation two catalyst design. This person noted adding calcium ( $\mathrm{Ca}$ ) improves performance for some instances; however; $\mathrm{Cu}$ additions neutralize these gains, but improve lower temperature conversion. The reviewer commented that $\mathrm{Cu}$ additions from $1-3 \%$ are only needed to improve the $\mathrm{NO}_{\mathrm{x}}$ conversion from $50-66 \%$ at $150^{\circ} \mathrm{C}$. There was a good use of capabilities at PNNL, the reviewer reported.

\section{Reviewer 5:}

The reviewer noted that the technical accomplishments include research direction for catalyst efficiency improvement, synthesis of a large batch of first generation catalyst to identify key issues for future studies, and down-selection of a possible $\mathrm{NH}_{3}$ generation strategy.

\section{Reviewer 6:}

The reviewer observed that the large batch of $\mathrm{Cu}$ /chabazite (CHA) catalyst was really a lot of combined small batches. This person questioned why a major catalyst supplier is not involved. The reviewer said that $\mathrm{NH}_{3}$ generation catalyst details were not described.

\section{Question 3: Collaboration and coordination with other institutions.}

\section{Reviewer 1:}

The reviewer stated that the project is a collaboration between FCA and PNNL, with assistance from the University of Houston (UH). FCA (with the help of $\mathrm{UH}$ ) will focus on passive $\mathrm{NH}_{3}$ generation and system integration, along with supplemental $\mathrm{NO}_{\mathrm{x}}$ control. PNNL will focus primarily on low-temperature SCR development, along 
with system integration. The reviewer pointed out that this is a relatively small team, but appears to have the key members needed. The reviewer noted that there is a strong degree of communication among the members, including monthly conference calls along with face-to-face meetings every six months.

\section{Reviewer 2:}

The reviewer said that the project team includes a good combination of OEM, laboratory, and university collaboration with good communication and noted this team has a good balance of technical expertise relevant to the work to be completed.

\section{Reviewer 3:}

The reviewer indicated there is good collaboration and includes the right team.

\section{Reviewer 4:}

The reviewer acknowledged that there is appropriate collaboration among project partners leveraging their core competencies and include: PNNL (catalyst development and advanced characterizations), FCA (system integration and operational parameters), and UH (catalyst characterization, degradation, and modeling).

\section{Reviewer 5:}

The reviewer commented that the partners included an OEM, national laboratory, and university, and that collaboration level seems adequate.

\section{Reviewer 6:}

The reviewer noted that national laboratory, industry and academia are all participating in this project. This person suggested that the project could use an aftertreatment firm that specializes in SCR systems to complement the team and that a HD vehicle manufacturer could also add some insight into issues and possible solutions.

\section{Question 4: Proposed future research-the degree to which the project has effectively planned its future work in a logical manner by incorporating appropriate decision points, considering barriers to the realization of the proposed technology and, when sensible, mitigating risk by providing alternate development pathways.}

\section{Reviewer 1:}

The reviewer said the project has appropriate future work focusing on development of second-generation catalysts to improve efficiency, stability and aging.

\section{Reviewer 2:}

The reviewer indicated that the future work is appropriate and is logical for achieving the goals of the project.

\section{Reviewer 3:}

The reviewer noted a good path forward to continue the gains that they have already realized.

\section{Reviewer 4:}

The reviewer observed that the project includes several very specific plans for future research, and include development of the second generation catalysts and verifying their performance stability, designing the $\mathrm{NH}_{3}$ generation system, and conducting system component aging.

\section{Reviewer 5:}

The reviewer pointed out future work will include development of a second generation SCR catalyst to meet the conversion efficiency target, which is a primary goal; verifying sufficient hydrothermal stability of second generation SCR catalysts; designing an $\mathrm{NH}_{3}$ generation strategy; and performing system component aging. This person indicated that all of these activities will be needed to resolve the issues currently preventing the development of a low-temperature $\mathrm{NO}_{\mathrm{x}}$ conversion system.

\section{Reviewer 6:}

The reviewer stated that washcoat loading of SCR catalysts is very important but was not mentioned in future work slide. In addition, this person pointed out that it was not clear if the $\mathrm{NH}_{3}$ generation catalyst would also be scaled up to a core level. 


\section{Question 5: Does this project support the overall DOE objectives of petroleum displacement? Why or why not?}

\section{Reviewer 1:}

The reviewer observed that this project supports the DOE petroleum displacement objective by developing emissions aftertreatment solution to enable high efficiency engines. Specifically, the project is addressing the $150^{\circ} \mathrm{C}$ Challenge identified in the 2012 USCAR workshop.

\section{Reviewer 2:}

The reviewer said yes, lower temperature aftertreatment capability supports DOE's goals of higher fuel efficiency.

\section{Reviewer 3:}

The reviewer stated yes, the ability to limit or remove thermal management would create a significant improvement in fuel economy.

\section{Reviewer 4:}

The reviewer indicated that the project is specifically aimed at addressing the $150^{\circ} \mathrm{C}$ Challenge identified at the 2012 USCAR workshop. As such, this project is trying to ensure high $(90 \%)$ conversion efficiency SCR catalyst systems at a low temperature $\left(150^{\circ} \mathrm{C}\right)$, which are anticipated to be needed by advanced technology powertrains.

\section{Reviewer 5:}

The reviewer noted that improved SCR materials can enable meeting stringent emission standards with more efficient catalysts and facilitate new more efficient powertrains. This in turn creates the opportunity for petroleum reduction.

\section{Reviewer 6:}

The reviewer indicated that higher SCR conversion efficiency will reduce fuel and aftertreatment fluid use.

\section{Question 6: Resources: How sufficient are the resources for the project to achieve the stated milestones in a timely fashion?}

\section{Reviewer 1:}

The reviewer said that the resources are sufficient to complete the work as described in the presentation.

\section{Reviewer 2:}

This person indicated that funding appears appropriate for this project.

\section{Reviewer 3:}

The reviewer stated that no indication was made that the funding was insufficient.

\section{Reviewer 4:}

The reviewer pointed out that the project funding of $\$ 500,000$ per year over a three-year period seems appropriate given the project scope.

\section{Reviewer 5:}

The reviewer noted the project team seems to be on track and meeting their milestones.

\section{Reviewer 6:}

The reviewer observed that it was not clear how much funding PNNL and UH were each receiving. 


\section{Next Generation Three-Way Catalysts for Future, Highly Efficient Gasoline Engines: Christine Lambert (Ford Motor Company) - pm067}

\section{Presenter}

Christine Lambert, Ford

\section{Reviewer Sample Size}

A total of five reviewers evaluated this project.

\section{Question 1: Approach to performing the work-the degree to which technical barriers are addressed, the project is well-designed, feasible, and integrated with other efforts.}

\section{Reviewer 1:}

The reviewer observed this is an excellent approach to investigating new catalyst materials and options including identifying and characterizing new materials and predicting performance and costs. In addition, the project will identify and capitalize on synergies between various catalyst materials within and between partners, increase surface area but not increasing the content of metals, investigate catalyst architectures containing a core shell conformation composed of a metal nanoparticle surrounded by a metal oxide shell, and where appropriate, use ICME to help with material development activities.

\section{Reviewer 2:}

The reviewer noted that the project's approach appears straightforward, and there is a rational path of activities to accomplish its objectives. The project is looking at new ways of characterizing innovative materials and catalyst designs, rather than simply increasing the content of precious metals. The reviewer further explained that this would drive up cost, although some comparisons were run by increasing these materials. These include oxide overlayers and improved mixing. A specific component of the approach is also to leverage the partners' analytical capabilities. The reviewer observed that at this time, the testing is being done using Iso-octane, not 10\% ethanol blend with gasoline (E10), which represents probably $95 \%$ of the commonly-available gasoline at refueling stations. Upon questioning, the PI indicated future efforts may consider this. The reviewer commented the specific fuel formulation could impact catalyst design.

\section{Reviewer 3:}

The reviewer stated that the technical approach is logical, and will push three-way catalyst technology forward by looking at catalyst fundamentals. The team is exploring novel approaches for catalyst materials to improve catalyst activity. It is good to see Ford's focus on cost estimation for these new materials. The reviewer mentioned that this 
is very important, particularly for a relatively mature technology like the three-way catalyst that is produced at high volume and low cost.

\section{Reviewer 4:}

The reviewer said predictive capabilities are being utilized in tandem with characterization techniques. The reviewer suggested that the multiple approaches presented offer a better chance of success and it is good that the project can run these in parallel.

\section{Reviewer 5:}

The reviewer noted that this work directly addresses the request for expanded efforts of low-temperature aftertreatment resulting from USCAR engine and aftertreatment workshops and roadmaps. As such and in recognition of global drive cycles experiencing temperatures in the $150^{\circ} \mathrm{C}$ range, developing catalyst solutions for low-temperature oxidation of carbon monoxide (CO) and $\mathrm{HC}$ species for three-way catalyst (TWC) applications is very appropriate and supported research. The inception stage exploration of multiple pathways to achieve high $\mathrm{CO}$ and $\mathrm{HC}$ oxidation performance and reduced metal cost at low temperature through maintaining highly dispersed Pt- group metal (PGM) is a viable strategy to achieve the desired performance of catalysts. However, maintaining this functionality after experiencing real aging conditions (thermal and poisons) is essential to the adoption of any of these technologies. The reviewer suggested that a better understanding of how poisons, such as sulfur, alter the activity of the catalysts under development is needed to provide a thorough characterization of the technologies.

\section{Question 2: Technical accomplishments and progress toward overall project and DOE goals-the degree to which progress has been made, measured against performance indicators and demonstrated progress towards DOE goals.}

\section{Reviewer 1:}

The reviewer agreed that beginning a study by baseline reactors that will be used to judge the performance of the various catalysts is the proper way to begin. Using the protocols developed by USCAR adds an additional layer of confidence to the results. This person noted that, the light-off performance of the palladium (Pd) versus rhodium $(\mathrm{Rh})$ formulations is somewhat unexpected, but interesting. The fresh CO light-off performance of the fresh $\mathrm{Rh}$ formulation is unexpected. The reviewer suggested that because most catalyst formulation use a combination of $\mathrm{Pd}$ and $\mathrm{Rh}$, it would be of interest to determine how the resulting light-off performance would illuminate the interaction between the metals. This person also asked if $\mathrm{Rh}$ will also be compared on the titanium dioxide $\left(\mathrm{TiO}_{2}\right)$ support. The reviewer explained that Pd suffers more from sulfur poisoning and that light-off data may also change the ranking of the different formulations. The reviewer noted that with respect to the silicon dioxide $\left(\mathrm{SiO}_{2}\right)$ core catalyst, spectroscopically comparing the fresh to the aged sample would be of interest. In general, the effect of sulfur at low temperature on the light-off performance of the well dispersed Pd catalysts is needed. In all cases, determining the selectivity of the $\mathrm{NO}_{\mathrm{x}}$ species toward nitrous oxide $\left(\mathrm{N}_{2} \mathrm{O}\right)$ formation should be assessed for greenhouse gas (GHG) inventory estimates. However, the reviewer observed overall the data support encouraging oxidation performance at temperatures approaching the goal of $150^{\circ} \mathrm{C}$.

\section{Reviewer 2:}

The reviewer indicated that the project team's systematic work starts with the basics of laboratory reactor performance confirmation, which is an important step. The team has done quite a bit of good scientific work on catalyst materials development in the first half of this project. The reviewer observed that the project team has not yet quite gotten to the $150^{\circ} \mathrm{C}$ temperature goal, but has shown some promising catalyst materials that are moving in that direction. The core shell catalyst material and its associated synthesis process are interesting, said the reviewer. The team is focusing on making its own materials, versus sourcing black box materials from suppliers, to understand how they work and can be used is a good approach. This person mentioned that this project has shown great early results and it appears there are more to come.

\section{Reviewer 3:}

The reviewer observed that the round robin for commercial catalysts is complete, but took longer than anticipated. There was good agreement on results among the three research laboratories including Ford, ORNL and the UM. The reviewer pointed out that the project team learned a great deal about performance of various catalyst formulations as well as the impact of aging. 


\section{Reviewer 4:}

The reviewer reported that round robin testing is completed and investigated production catalysts first, the $\mathrm{Rh}$ contribution gets close to $150^{\circ} \mathrm{C}$ after $950^{\circ} \mathrm{C}$, lean aging, and that titanium worked well. In addition, this person pointed out the $\mathrm{Rh}$ in four mode aging at $960^{\circ} \mathrm{C}$ was best performer and that $\mathrm{Rh}$ has good promise as an addition to the catalyst material. The reviewer said that $4 \% \mathrm{Pd}$ also looks like a good potential element to add from tests. Multiple catalysts to address each pollutant could be an option, ternary base metal oxides has significant $\mathrm{CO}$ reduction and can store $\mathrm{NO}_{\mathrm{x}}$ at low temperatures; however, it has little effect on the others. The reviewer also noted that dispersed Pd improved catalyst light-off.

\section{Reviewer 5:}

The reviewer noted that low-temperature light-off changes are promising for improved efficiency, and less thermal management.

\section{Question 3: Collaboration and coordination with other institutions.}

\section{Reviewer 1:}

The reviewer stated that the partnering in this project is appropriate for the scope of the project and the background of the groups involved.

\section{Reviewer 2:}

Three participants, Ford, ORNL, and UM, were characterized as equal contributors to the project by the presenter. This person pointed out that adding a coater would be a positive. It would be disappointing if the project team developed a material that could not be applied in a consistent way in production.

\section{Reviewer 3:}

The reviewer stated that the project includes Ford, ONRL, and UM. All three are considered as contributing equally under the project, with specifically identified tasking. This person pointed out that monthly calls are held among the partners, along with in-person meetings once or twice per year. The reviewer noted that the project will partner with a catalyst supplier in the third year of the project.

\section{Reviewer 4:}

The reviewer remarked that Ford and ORNL are partners and are leveraging their core capabilities for this work. The team has a good mix of OEM, laboratory, and university partners. The team is looking to add a catalyst supplier in the third year, which will be a critical step. The reviewer observed that is good to develop and understand your own materials, but a catalyst supplier can provide additional insight.

\section{Reviewer 5:}

The reviewer acknowledged that academic, industry and national laboratory partnerships are in place, and that adding a Tier 1 powder supplier would help. In addition, the year three addition of catalyst manufacturer will also improve team capabilities.

\section{Question 4: Proposed future research-the degree to which the project has effectively planned its future work in a logical manner by incorporating appropriate decision points, considering barriers to the realization of the proposed technology and, when sensible, mitigating risk by providing alternate development pathways.}

\section{Reviewer 1:}

The reviewer said that the team has good plans to address the remaining barriers, particularly lead times and costs for materials. The system-level work should be interesting and add to the community's knowledge, noted the reviewer.

\section{Reviewer 2:}

The reviewer noted that the future work to address remaining questions and fill the knowledge gaps is appropriate.

\section{Reviewer 3:}

The reviewer observed that several specific future activities were proposed. In particular, the third year of the project calls for combining novel materials into a complete catalyst and then partnering with a major automotive 
catalyst supplier. Other future efforts include activities focused on shortening materials commercialization leadtimes and reducing costs, culminating in identifying systems solutions and estimating vehicle performance and cost, the reviewer reported.

\section{Reviewer 4:}

The reviewer suggested that the novel materials should be a parallel path rather than waiting until the third year to add them to the project because if the project team has issues, there are not sufficient resources left to recover and meet deliverables.

\section{Reviewer 5:}

This reviewer provided the following comments: long lead time in materials commercialization; add new materials to base to understand catalyst materials; investigate base metals as a PGM substitute to reduce cost; capitalize on catalyst material synergies to develop an emission solution/system; develop cost estimate of new system; and demonstrate full aging and performance of coated monolith cores. The reviewer suggested that a good additional activity would be developing a functional, prototype catalyst system using the most promising materials and configuration.

\section{Question 5: Does this project support the overall DOE objectives of petroleum displacement? Why or why not?}

\section{Reviewer 1:}

The reviewer pointed out that the project is focused on developing improved catalysts for high-efficiency engines, including addressing long lead times for materials commercialization and cost. Because next generation engines are anticipated to have lower exhaust temperatures, the project is aiming at improving catalyst performance at lower temperatures through materials selection. This person stated that this is highly relevant to DOE VTO objectives.

\section{Reviewer 2:}

The reviewer commented that this project supports USCAR and U.S. Driving Research and Innovation for Vehicle Efficiency and Energy sustainability (U.S. DRIVE) initiatives to address the need for effective low-temperature aftertreatment solutions for highly efficient engines. This work also supports the need for cost effective emissions control.

\section{Reviewer 3:}

The reviewer said that removal of thermal management is a big win and it would be good to add a calculation relating the impact of meeting the goals on the effect of petroleum displacement.

\section{Reviewer 4:}

The reviewer indicated that low-temperature catalysis is important for future advanced combustion engine technologies to reduce fuel consumption. More thermally efficient engines will have lower exhaust temperatures, but still be required to meet criteria emission standards. Lower temperature catalysts are thus critical enablers for these efficient engines, the reviewer remarked.

\section{Reviewer 5:}

The reviewer mentioned that higher efficiency conversion of emissions can allow more efficient, lower temperature combustion techniques.

\section{Question 6: Resources: How sufficient are the resources for the project to achieve the stated milestones in a timely fashion?}

\section{Reviewer 1:}

The reviewer observed that with the anticipated near-term commercialization of advanced lower temperature combustion engines, this critical need will require a solution to be developed to meet current and future emission regulations. This person suggested an increase in funding would accelerate completion of the project milestones.

\section{Reviewer 2:}

The reviewer explained that no indication was made that the funds were insufficient, so the reviewer were assumed 
to be fine. It should be noted that there is only a $20 \%$ cost-share on this project. The reviewer observed that given the higher levels of cost-share on related projects within PM, this appears a bit unusual.

\section{Reviewer 3:}

The reviewer stated that this project is appropriately funded and staffed.

Reviewer 4:

The reviewer said the project is on track and meeting milestones.

Reviewer 5:

The reviewer indicated that resources appear to be sufficient to address the work described. 


\section{Sustained Low-Temperature $\mathrm{NO}_{x}$ Reduction (SLTNR): Yuhui Zha (Cummins) - pm068}

\section{Presenter}

Yuhui Zha, Cummins

\section{Reviewer Sample Size}

A total of four reviewers evaluated this project.

\section{Question 1: Approach to performing the work-the degree to which technical barriers are addressed, the project is well-designed, feasible, and integrated with other efforts.}

\section{Reviewer 1:}

The reviewer pointed out that the approach as outlined is logical and addresses the key technical points for low-temperature $\mathrm{NO}_{\mathrm{x}}$ reduction with a systems approach. The approach addresses the commercial viability of the system, which is very useful to move this to implementation. This person stated that the on-engine test verification of the catalyst system is even more important for implementation. Including the reductant system is essential, as there are well-known challenges with low-temperature deposit formation with current reductant dosing systems. The reviewer reported that the project team is making good use of partner core capabilities through the extensive catalyst expertise at PNNL.

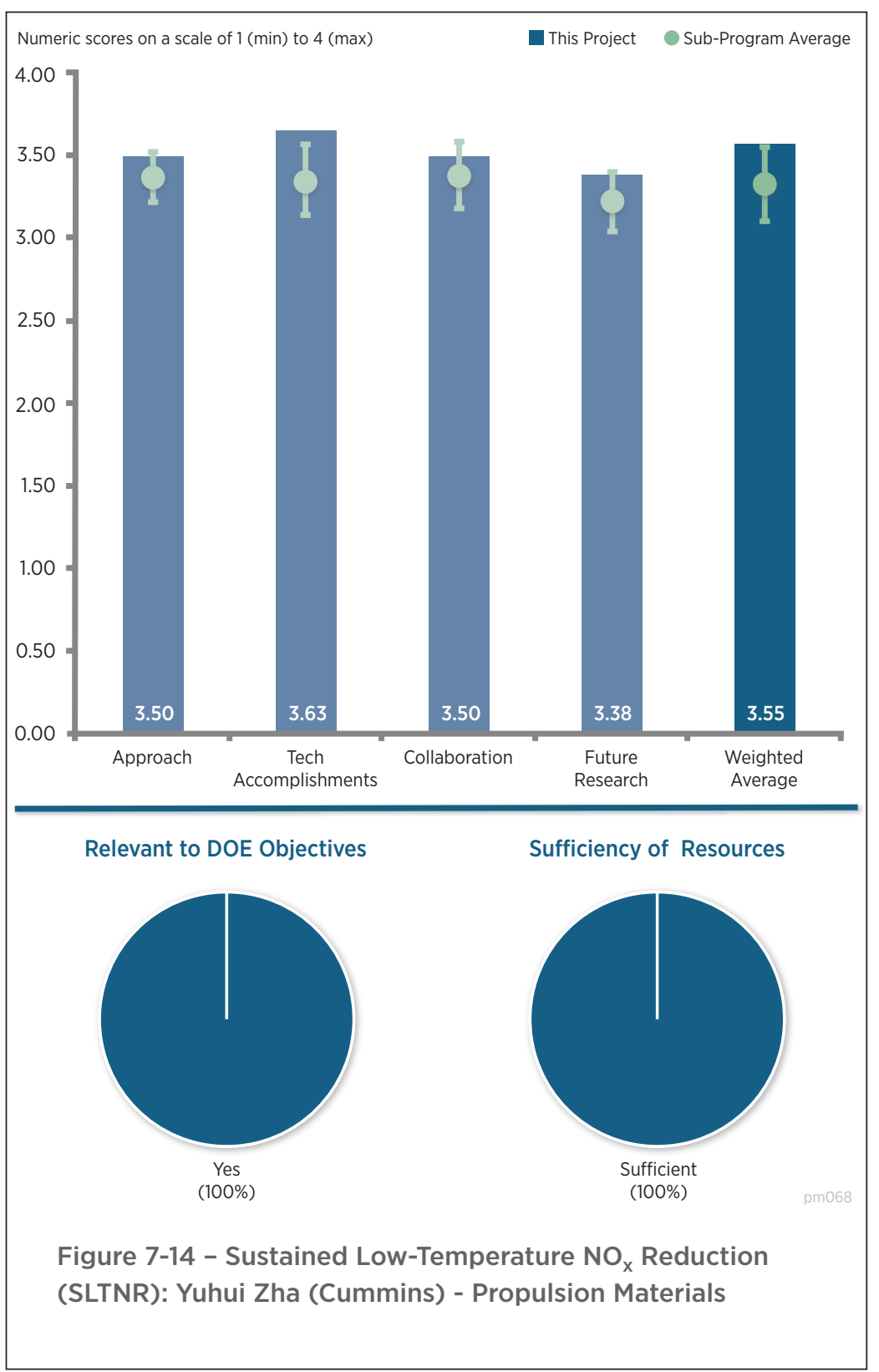

\section{Reviewer 2:}

The reviewer stated this is a nice balanced approach between system development and modeling approach to enhance catalyst performance. The PI is using a creative, outside-the-box approach to emission control by considering alternative positions and locations for emission control systems. This solution will require a redesign of the emission control system. This person suggested that opportunities exist to utilize the pre-turbo exhaust temperatures of $230^{\circ} \mathrm{C}$ to improve pollutant conversion. However, there is limited space in/near the engine for a conventional solution. The reviewer mentioned that a vaporizer is being tested to delivery diesel exhaust fluid to the exhaust stream in this area.

\section{Reviewer 3:}

The reviewer observed that the project's approach seems to be reasonable for accomplishing the identified objectives of developing an appropriate catalyst system, conducting on-engine testing to verify performance, and conducting a commercial viability assessment. A number of discrete steps (milestones) were identified for the project, which assists greatly in tracking project progress, the reviewer said. 


\section{Reviewer 4:}

The reviewer indicated this is a sound approach focusing on DOC, SCR, and reductant delivery systems as well as modeling to size catalysts and optimize design.

\section{Question 2: Technical accomplishments and progress toward overall project and DOE goals-the degree to which progress has been made, measured against performance indicators and demonstrated progress towards DOE goals.}

\section{Reviewer 1:}

The reviewer reported that the team has made very good progress on completing its technical milestones. PNNL's work in identifying the critical low-temperature SCR characteristics and developing promising catalysts to take advantage of this work represents a significant accomplishment. The proposed pre-turbo catalyst design is interesting, but ensures access to heat for proper catalyst operation. This person observed that the project team has completed a good comprehensive modeling study of concept designs to look at catalyst location and sizing. The vaporizer for SCR dosing is also interesting, particularly as it takes advantage of something that appeared unrelated to SCR dosing (a commercial humidifier). The reviewer pointed out that challenges remain for this system, but initial results are promising. The reviewer said that the team is also making good progress on commercial viability with the initial framework and key technical and market considerations.

\section{Reviewer 2:}

The reviewer noted that all calendar year 2015 milestones were completed. Initial results are that ammonium nitrate formation is a key inhibitor, so its decomposition must be promoted catalytically through material changes and pore structure design. Adding iron/zeolite helps conversion performance. In addition, $\mathrm{HC}$ also inhibit low-temperature nitric oxide oxidation, and both $\mathrm{HC}$ and nitrogen dioxide $\left(\mathrm{NO}_{2}\right)$ are dependent upon exhaust gas recirculation (EGR) fraction, which tends to be set to maximize diesel oxidation catalyst performance. The reviewer observed that it appears if the EGR fraction is 0.4 , the team can reach the desired $\mathrm{NO}_{\mathrm{x}}$ conversion at $210^{\circ}-230^{\circ} \mathrm{C}$ (which is a pre-turbo temperature, but somewhat higher than the goal of $150^{\circ} \mathrm{C}$ ). PNNL has already developed a catalyst formulation that appears to achieve greater than $90 \% \mathrm{NO}_{\mathrm{x}}$ conversion at $150^{\circ} \mathrm{C}$ under laboratory conditions, and Johnson Matthey has successfully scaled this material up to a monolith sample.

\section{Reviewer 3:}

The reviewer stated that accomplishments include SCR development and design, integrated high $\mathrm{NO}_{2}$ strategy, as well as modeling and vaporizer design. This person noted that commercial viability assessment was also started.

\section{Reviewer 4:}

The reviewer indicated that all milestones are on schedule. The Fe/zeolite formulation can achieve $90 \% \mathrm{NO}_{\mathrm{x}}$ conversion at $150^{\circ} \mathrm{C}$, said the reviewer. This person pointed out there is continuing modeling analysis with $\mathrm{Cu} /$ Zeolite to determine optimized system sizing. In addition, the $\mathrm{Fe}$ plus $\mathrm{Cu}$ components together reduces sensitivities to $\mathrm{NO}_{2}$ fractions and $\mathrm{Cu}$ needs to be seven to eight times greater than the Fe component. The reviewer commented that two good options were identified up to $230^{\circ} \mathrm{C}$ (i.e., low- to mid-cost, and mid- to low-size). Proof of concept illustrated that there is limited space for catalyst pre- or post-turbo. The reviewer noted that the vaporizer creates 10 times smaller size particles than dosers, and that the project is using off-the-shelf components in vaporizer design, except for housings. The project completed a prototype in June 2016. The reviewer suggested assessing risk factors to determine commercial viability.

\section{Question 3: Collaboration and coordination with other institutions.}

\section{Reviewer 1:}

The reviewer indicated that the project is a collaboration between Cummins, PNNL, and Johnson Matthey. Each appears to have critical roles under the project. While it is perhaps a small team, the partners appear to cover the project's needs at this time and are working together well.

\section{Reviewer 2:}

The reviewer stated that it seems like very close collaboration among Cummins, PNNL and Johnson Matthey leveraging their aftertreatment system development, modeling, and catalyst design expertise, respectively. 


\section{Reviewer 3:}

The reviewer acknowledged that the team has an appropriate mix of partners, with an engine OEM, a catalyst supplier, and a national laboratory. The team has balanced the work among the partners in ways appropriate to each partner's core competencies.

\section{Reviewer 4:}

The reviewer noted that industry, emission control Tier 1 supplier, and a national laboratory are partnering on this project. The reviewer suggested an academic institution should be considered as an addition to the team.

\section{Question 4: Proposed future research-the degree to which the project has effectively planned its future work in a logical manner by incorporating appropriate decision points, considering barriers to the realization of the proposed technology and, when sensible, mitigating risk by providing alternate development pathways.}

\section{Reviewer 1:}

The reviewer commented that the future work plan appears to be logical and appropriate for finalizing and testing the low-temperature $\mathrm{NO}_{\mathrm{x}}$ catalysts and reductant system. It was not mentioned in the presentation, but it is presumed that the engine testing of the full SCR system will be in 2017, which appears appropriate.

\section{Reviewer 2:}

The reviewer reported that a number of future activities were identified, but the key ones were to test catalyst durability and robustness and develop and integrate the urea vaporizer system. In addition, next steps were identified as fabrication and insulation, and then engine testing.

\section{Reviewer 3:}

The reviewer indicated that future tasks include optimizing the SCR system, completing the integrated high $\mathrm{NO}_{2}$ strategy, and designing, building, and testing the reductant delivery system.

\section{Reviewer 4:}

The reviewer explained that durability, robustness of performance in operating conditions still needs to be shown and the project will develop an integrated high $\mathrm{NO}_{2}$ strategy. Three SCR designs are to be assessed in 2016. The reviewer noted there is a need to complete a proof a concept design for reductant delivery system and to complete CFD analysis, bench testing, system integration and engine tests, which are all planned for 2016.

\section{Question 5: Does this project support the overall DOE objectives of petroleum displacement? Why or why not?}

\section{Reviewer 1:}

The reviewer observed that the project is focused on ensuring highly-efficient $\mathrm{NO}_{\mathrm{x}}$ conversion at the low temperatures that may be more likely to characterize advanced higher efficiency engines. Thus, this is entirely relevant to DOE/VTO objectives.

\section{Reviewer 2:}

The reviewer emphasized that this project supports the overall DOE objectives of petroleum displacement by enabling high-efficiency medium and HD diesel engines that meet emissions requirements. More efficient engines have lower exhaust temperatures which create very challenging conditions for exhaust aftertreatment. This person concluded that this project directly addresses the low exhaust temperature performance of the SCR system.

\section{Reviewer 3:}

The reviewer explained that low-temperature $\mathrm{NO}_{\mathrm{x}}$ reduction will be important for future HD efficiency applications. In addition, higher efficiency will mean lower exhaust temperatures, and manufacturers will need to meet criteria emission regulations at these conditions. This person pointed out that more efficient emission controls at the conditions presented by future high brake thermal efficiency engines are a necessary enabler for deployment of these engines and resulting petroleum displacement. 


\section{Reviewer 4:}

The reviewer stated that high efficiency emission control can reduce the need for frequent DPF regeneration, which uses a significant amount of fuel.

\section{Question 6: Resources: How sufficient are the resources for the project to achieve the stated milestones in a timely fashion?}

\section{Reviewer 1:}

The reviewer observed that no indication was made that the funding was either excessive or insufficient, so it was assumed to be appropriate. Overall funding appears to include a $40 \%$ cost-share from Cummins.

\section{Reviewer 2:}

The reviewer said that the approximate $\$ 3$ million total budget (\$2 million DOE share) seems appropriate for a three-year project scope.

\section{Reviewer 3:}

The reviewer indicated that the resources appear to be sufficient for the work described, over the timeframe proposed.

\section{Reviewer 4:}

The reviewer indicated the review was considered as a phase one project, and as such the funding appears to be sufficient. This person suggested that risk factors need to be assessed to determine follow on work towards a commercial solution, especially regarding the vaporizer. 


\section{Acronyms and Abbreviations}

$3 \mathrm{D}$

Al

AMR

ANL

APS

ASTM

${ }^{\circ} \mathrm{C}$

$\mathrm{Ca}$

CFD

CGI

CHA

CHT

$\mathrm{CO}$

CRADA Cooperative research and development agreement

$\mathrm{Cu}$

DFT

DOC

DOE

DPF

Dy

E10

EDT

EGR

EV

FCA

$\mathrm{Fe}$

FEM

FSW

FY

GHG
Three-dimensional

Aluminum

Annual Merit Review

Argonne National Laboratory

Advanced photon source

American Society of Testing and Materials

Degrees Celsius (Centigrade)

Calcium

Computational fluid dynamics

Compacted graphite iron

Chabazite

Conjugate heat transfer

Carbon Monoxide

Copper

Density functional theory

Diesel oxidation catalyst

U.S. Department of Energy

Diesel particulate filter

Dysprosium

$10 \%$ Ethanol Blended with Gasoline

Electric Drive Technologies

Exhaust gas recirculation

Electric vehicle

Fiat Chrysler Automobiles

Iron

Finite element modeling

Friction-stir welding

Fiscal year

Greenhouse gas 


\begin{tabular}{|c|c|}
\hline GM & General Motors \\
\hline GT & Georgia Tech University \\
\hline GTDI & Gasoline turbocharged direct injection \\
\hline $\mathrm{HC}$ & Hydrocarbon \\
\hline HD & Heavy-duty \\
\hline HDV & Heavy-duty vehicle \\
\hline HPDC & High-pressure diecast \\
\hline $\mathrm{HEV}$ & Hybrid electric vehicle \\
\hline $\mathrm{Hp}$ & Horsepower \\
\hline ICME & Integrated computational materials engineering \\
\hline IP & Intellectual property \\
\hline IPM & Interior permanent magnet \\
\hline $\mathrm{K}$ & Potassium \\
\hline $\mathrm{L}$ & Liter \\
\hline LD & Light-duty \\
\hline $\mathrm{Mg}$ & Magnesium \\
\hline MGI & Materials Genome Initiative \\
\hline MPG & Miles per gallon \\
\hline MIT & Massachusetts Institute of Technology \\
\hline $\mathrm{MPa}$ & Megapascal \\
\hline $\mathrm{N}_{2} \mathrm{O}$ & Nitrous Oxide \\
\hline $\mathrm{Na}$ & Sodium \\
\hline $\mathrm{Nd}$ & Neodymium \\
\hline $\mathrm{NH}_{3}$ & Ammonia \\
\hline $\mathrm{Ni}$ & Nickel \\
\hline $\mathrm{N}_{2} \mathrm{O}$ & Nitrous oxide \\
\hline $\mathrm{NO}_{2}$ & Nitrogen Dioxide \\
\hline $\mathrm{NO}_{\mathrm{x}}$ & Oxides of nitrogen \\
\hline NREL & National Renewable Energy Laboratory \\
\hline OEM & Original equipment manufacturer \\
\hline OQMD & Open Quantum Materials Database \\
\hline
\end{tabular}




\begin{tabular}{|c|c|}
\hline ORNL & Oak Ridge National Laboratory \\
\hline $\mathrm{Pd}$ & Palladium \\
\hline PGM & Platinum group metal \\
\hline PI & Principal Investigator \\
\hline PM & Propulsion Materials \\
\hline PNNL & Pacific Northwest National Laboratory \\
\hline $\mathrm{Pt}$ & Platinum \\
\hline $\mathrm{R} \& \mathrm{D}$ & Research and development \\
\hline $\mathrm{Rh}$ & Rhodium \\
\hline RTA & Rio Tinto Alcan \\
\hline SCR & Selective catalytic reduction \\
\hline SI & Spark ignition \\
\hline $\mathrm{SiO}_{2}$ & Silicon dioxide \\
\hline TEM & Transmission electron microscope \\
\hline $\mathrm{TiO}_{2}$ & Titanium dioxide \\
\hline TMS & The Materials, Metals and Minerals Society \\
\hline TRL & Technology readiness levels \\
\hline TWC & Three-way catalyst \\
\hline USAMP & United States Automotive Materials Partnership \\
\hline U.S. DRIVE & U.S. Driving Research and Innovation for Vehicle efficiency and Energy sustainability \\
\hline UAB & University of Alabama at Birmingham \\
\hline $\mathrm{UH}$ & University of Houston \\
\hline UM & University of Michigan \\
\hline USCAR & United States Council for Automotive Research \\
\hline UTS & Ultimate tensile strength \\
\hline VACG & Vacuum assisted counter gravity \\
\hline VTO & Vehicle Technologies Office \\
\hline
\end{tabular}




\section{Technology Integration}

Our nation's energy security depends on the efficiency of our transportation system and on which fuels we use. Transportation in the United States already consumes much more oil than we produce here at home and the situation is getting worse. The U.S. Department of Energy's (DOE's) Vehicle Technologies Office (VTO) supports research and development (R\&D) that will lead to new technologies that reduce our nation's dependence on imported oil, further decrease vehicle emissions, and serve as a bridge from today's conventional powertrains and fuels to tomorrow's hydrogen-powered hybrid fuel cell vehicles. VTO also supports implementation programs that help to transition alternative fuels and vehicles into the marketplace, as well as collegiate educational activities to help encourage engineering and science students to pursue careers in the transportation sector. Following are some of the activities that complement the VTO's mission.

Energy Policy Act of 1992

The Office of Energy Efficiency and Renewable Energy (EERE) manages several programs designed to fulfill the requirements of the original and amended versions of the Energy Policy Act of 1992 (EPAct) that regulate and guide specific types of fleets with the goal of reducing the United States' petroleum consumption.

EERE's VTO implements the EPAct Alternative Fuel Transportation Program. This compliance program works with covered fleets - which include certain state and alternative fuel provider fleets (e.g., utilities) - to reduce petroleum consumption and increase the use of alternative fuels.

\section{Clean Cities}

DOE's Clean Cities program advances the nation's economic, environmental, and energy security by supporting local actions to cut petroleum use in transportation. Part of DOE's VTO, Clean Cities has saved more than 7.5 billion gallons of petroleum since its inception in 1993.

Nearly 100 local coalitions serve as the foundation of the Clean Cities program by working to cut petroleum use in communities across the country. Clean Cities coalitions are comprised of businesses, fuel providers, vehicle fleets, state and local government agencies, and community organizations. Each coalition is led by an on-theground Clean Cities coordinator, who tailors projects and activities to capitalize on the unique opportunities in their communities. Nationwide, nearly 15,000 stakeholders participate in Clean Cities coalitions, and through their collective efforts they are transforming local and regional transportation markets and contributing to Clean Cities' goals and accomplishments.

At the national level, the program develops and promotes partnerships, publications, tools, and other unique resources. At the local level, coalitions leverage these resources to create networks of local stakeholders and provide technical assistance to fleets implementing alternative and renewable fuels, idle-reduction measures, fuel economy improvements, and emerging transportation technologies.

Clean Cities efforts support reduced dependence on petroleum at the local, state, and national levels. Clean Cities activities include:

- Building partnerships with local coalitions of public- and private-sector transportation stakeholders;

- Developing unbiased and objective information resources about alternative fuels, advanced vehicles, and other strategies to cut petroleum use;

- Advancing interactive, data-driven online tools to help stakeholders evaluate options and achieve goals;

- Collecting and sharing best practices, data, and lessons learned to inform choices and build a strong national network; 
- Providing technical assistance to help fleets deploy alternative fuels, advanced vehicles, and idle-reduction measures;

- Working with industry partners and fleets to identify and address technology barriers;

- Empowering local decision makers to successfully implement the best petroleum reduction strategy for their circumstance; and

- Seeding local alternative fuels markets through projects that deploy vehicles and fueling infrastructure.

Clean Cities dates back to the Alternative Motor Fuels Act of 1988 and the Clean Air Act Amendments of 1990. These laws, which encouraged the production and use of AFVs and the reduction of vehicle emissions, led to the creation of the Alternative Fuels Data Center (AFDC) in 1991. The AFDC's mission was to collect, analyze, and distribute data used to evaluate alternative fuels and vehicles.

In 1992, the enactment of EPAct required certain vehicle fleets to acquire AFVs. Subsequently, DOE created Clean Cities in 1993 to provide informational, technical, and financial resources to EPAct-regulated fleets and voluntary adopters of alternative fuels and vehicles.

The AFDC became and continues to be the clearinghouse for these resources. Its sister website, FuelEconomy. gov, provides consumers with information on fuel economy, emissions, and energy impact of light-duty vehicles, based on vehicle data from the U.S. Environmental Protection Agency (EPA). The site also provides tips for drivers on maximizing fuel efficiency. FuelEconomy.gov was created in response to DOE's requirement under the 1975 Energy Policy and Conservation Act to publish and distribute an annual fuel economy guide for consumers.

\section{Educational Activities}

VTO offers a variety of resources and opportunities for students, university researchers and professionals. It also provides information for consumers through FuelEconomy.gov and the AFDC.

VTO's educational efforts focus on higher education and public outreach, but its parent EERE offers resources for K-12 energy education. VTO has hosted student competitions in advanced vehicle technologies for more than 25 years to educate the next generation of automotive engineers and accelerate the development of vehicle technologies.

VTO's graduate education program supports efforts at top universities to train a future workforce of automotive engineering professionals in developing and commercializing advanced automotive technologies. These universities' multidisciplinary curriculums and unique laboratory facilities will prepare students to overcome technology barriers preventing the development and production of cost-effective, high-efficiency vehicles for the U.S. market.

\section{Subprogram Feedback}

The U.S. Department of Energy (DOE) received feedback on the overall technical subprogram areas presented during the 2016 Annual Merit Review (AMR). Each subprogram technical session was introduced with a presentation that provided an overview of subprogram goals and recent progress, followed by a series of detailed topic area project presentations.

The reviewers for a given subprogram area responded to a series of specific questions regarding the breadth, depth, and appropriateness of that DOE VTO subprogram's activities. The subprogram overview questions are listed below, and it should be noted that no scoring metrics were applied. These questions were used for all VTO subprogram overviews.

\section{Question 1: Was the program area, including overall strategy, adequately covered? \\ Question 2: Is there an appropriate balance between near- mid- and long-term research and development?}


Question 3: Were important issues and challenges identified?

Question 4: Are plans identified for addressing issues and challenges?

Question 5: Was progress clearly benchmarked against the previous year?

Question 6: Are the projects in this technology area addressing the broad problems and barriers that the Vehicle Technologies Office (VTO) is trying to solve?

Question 7: Does the program area appear to be focused, well-managed, and effective in addressing VTO's needs?

Question 8: What are the key strengths and weaknesses of the projects in this program area? Do any of the projects stand out on either end of the spectrum?

Question 9: Do these projects represent novel and/or innovative ways to approach these barriers as appropriate?

Question 10: Has the program area engaged appropriate partners?

Question 11: Is the program area collaborating with them effectively?

Question 12: Are there any gaps in the portfolio for this technology area?

Question 13: Are there topics that are not being adequately addressed?

Question 14: Are there other areas that this program area should consider funding to meet overall programmatic goals?

Question 15: Can you recommend new ways to approach the barriers addressed by this program area?

Question 16: Are there any other suggestions to improve the effectiveness of this program area?

Responses to the subprogram overview questions are summarized in the following pages. Individual reviewer comments for each question are identified under the heading Reviewer 1, Reviewer 2, etc. Note that reviewer comments may be ordered differently; for example, for each specific subprogram overview presentation, the reviewer identified as Reviewer 1 in the first question may not be Reviewer 1 in the second question, etc. 


\section{Subprogram Overview Comments: Linda Bluestein (U.S. Department of Energy) - tiooo}

\section{Question 1: Was the program area, including overall strategy, adequately covered?}

\section{Reviewer 1:}

The reviewer commented that the deployment technology portfolio activities, which support replacement, elimination, or reduction of petroleum use, were very adequately discussed. In addition, the reviewer affirmed that the deployment activities also align extremely well with the EERE strategic plan to increase the use of higher efficiency advanced technology vehicles, to improve overall efficiency of the transportation systems, and to reduce greenhouse gas (GHG) emissions in the future.

\section{Reviewer 2:}

The reviewer stated that there was a good explanation of how DOE vehicle technologies deployment efforts work to address overall transportation system efficiency and directly support national GHG emissions reduction goals. The reviewer added that the program portfolio is based on a well-balanced, three-legged approach for petroleum use reduction (replace, reduce, and eliminate).

\section{Reviewer 3:}

The reviewer observed that the presentation appeared to cover the entire Technology Integration (TI) program including Clean Cities, Legislative and Regulatory, and Student Competitions. This program, being a bit different than the R\&D portion of VTO, requires a different presentation structure.

\section{Question 2: Is there an appropriate balance between near- mid- and long-term research and development?}

\section{Reviewer 1:}

The reviewer summarized that the program area addresses near-, mid- and long-term goals by supporting the national goals to reduce emissions by $17 \%$ by $2020,26-28 \%$ by 2025 , and $83 \%$ by 2050 .

\section{Reviewer 2:}

The reviewer stated that this appears to be an area of emerging focus for the program, noting that recent program planning has been strategically assessing next priority opportunities for fuels and technologies, and how to develop deployment efforts supporting these in the mid-longer term.

\section{Reviewer 3:}

The reviewer answered yes, clarifying that because of the nature of TI activities as related to deployment, most of the emphasis is specifically (and appropriately) upon near-term efforts. The reviewer noted that the one longer-term focused effort perhaps is the education of the next generation of engineers under EcoCAR and other educational activities.

\section{Question 3: Were important issues and challenges identified?}

\section{Reviewer 1:}

The reviewer replied yes, describing the TI Program as focused upon deploying (and also providing the information necessary for deploying) new and advanced technologies, and elaborating that these technologies are aimed at reducing emissions (particularly GHGs) and petroleum use, which are the specific issues/challenges facing VTO. Within these overall issues/challenges, the reviewer noted that TI efforts are specifically focused on increasing the deployment of technologies that can be solutions, by providing critical technical, outreach, and other assistance necessary for adopters of these technologies.

\section{Reviewer 2:}

The reviewer remarked that the challenge to replace, eliminate, or reduce the use of petroleum was identified and discussed thoroughly. 


\section{Reviewer 3:}

The reviewer observed that the presentation focused more on strategies and recent/current solutions - as well as areas of opportunity — rather than specific challenges.

\section{Question 4: Are plans identified for addressing issues and challenges?}

\section{Reviewer 1:}

The reviewer replied yes, elaborating that in addition to a well-proven approach to moving technologies into use (over 20 years of Clean Cities and regulatory fleet experience), there is a new effort to work with the U.S. Department of Transportation (DOT) on the Systems and Modeling for Accelerated Research in Transportation (SMART) Mobility implementation. As for educating the next generation of engineers on these technologies, this reviewer affirmed that there is also a similar several-decade proven track record of success that continues to expand and innovate, moving the bar higher and enhancing this experience for students.

\section{Reviewer 2:}

The reviewer noted that a new area of emphasis was discussed, seeking the opportunity to incorporate existing alternative and advanced vehicle technology programs and efforts with Smart Cities/smart mobility technologies (connected vehicles and infrastructure, automation, transport planning, etc.), and that additional program plans are being developed.

\section{Reviewer 3:}

The reviewer stated yes, the program area identified the use of low carbon fuels, idle reduction, fuel economy improvements, and increasing the use of hybrid vehicles to help reduce petroleum consumption.

\section{Question 5: Was progress clearly benchmarked against the previous year?}

\section{Reviewer 1:}

This reviewer confirmed yes, pointing out that cumulative contributions from GHG emissions and petroleum reductions from Clean Cities were shown, dating back to the beginning of Clean Cities activities in the mid-1990s. The reviewer also recounted that regulatory program developments (compliance) were shown going back over a decade, emphasizing the virtually $100 \%$ compliance level, while educational programs listed the numbers of universities and students involved and detailed recent accomplishments.

\section{Reviewer 2:}

The reviewer declared that progress of GHG reduction and petroleum use reduction in the deployment activity continues to be one of the best success stories in VTO.

\section{Reviewer 3:}

The reviewer affirmed that program progress - in terms of petroleum displaced — has been tracked annually since 1993, showing an excellent record of progress (currently at 7.5 billion GGEs saved).

\section{Question 6: Are the projects in this technology area addressing the broad problems and barriers that the Vehicle Technologies Office (VTO) is trying to solve?}

\section{Reviewer 1:}

The reviewer replied yes and elaborated that the projects are specifically addressing reducing emissions and petroleum use through ensuring that technologies move into use successfully. The reviewer explained that this not only requires setting up a programmatic structure to focus on introduction of use, but ensuring that the necessary information gets into the hands of users and potential users. This is important for helping to make decisions to adopt these technologies, and to ensure their success once in place (particularly by making sure that users' expectations are managed and met).

The reviewer also observed that additional deployments are also occurring through the regulated fleets effort, a program with a nearly-20-year history of virtually $100 \%$ compliance. The reviewer added that this is virtually unheard of in the regulatory arena, and it is largely a testament to the high level of fleet outreach conducted by the project. 
The reviewer concluded that as time moves on, both government and industry will have a continuing need for specially-trained engineers who understand these advanced technologies, and pointed out that this is the specific focus of the student competitions.

\section{Reviewer 2:}

The reviewer stated that the program is executing a broad array of activities to foster alternative fuel and advanced vehicle technology deployment and adoption, developing critical market pull.

\section{Reviewer 3:}

The reviewer characterized all of the projects in the TI Program as continuing to address the issues the VTO is trying to solve.

\section{Question 7: Does the program area appear to be focused, well-managed, and effective in addressing VTO's needs?}

\section{Reviewer 1:}

The reviewer praised the overall program, including deployment, legislative and rulemaking, and advanced vehicle competitions as extremely well managed and effective.

\section{Reviewer 2:}

The reviewer replied yes, elaborating that TI's role is to focus on deploying new and advanced technologies, particularly as these emerge from VTO's R\&D efforts, and remarking that this link is important and must be continued. The reviewer observed that as programs such as VTO are being pushed to "do more with less," increasing the overall impact of VTO will require an even greater tie between deployment and R\&D activities, since it is only through deployment that any of these technologies reduce emissions or petroleum use.

\section{Reviewer 3:}

The reviewer noted that Clean Cities Program staff are regionally assigned, which allows for area-specific focus on fuels, technologies, and partners most prevalent and important to each region.

\section{Question 8: What are the key strengths and weaknesses of the projects in this program area? Do any of the projects stand out on either end of the spectrum?}

\section{Reviewer 1:}

The reviewer characterized all five major deployment activities of the program as very important. That said, of all of them, the reviewer suggested the consumer information outreach and education activities may have the greatest degree of reach, while training and stakeholder development and competitive funding are perhaps the next most important program activities.

\section{Reviewer 2:}

The reviewer remarked that the strength of the program is the real world reduction of petroleum use that is quantified each year through the deployment accomplishments. The reviewer highlighted the Clean Cities Program as the one that continues to stand out in the TI Program, adding that it continues to be a true success story.

\section{Reviewer 3:}

The reviewer stated that the key strengths really include the knowledge base built within the TI Program (in DOE, the national laboratories, the field, and with key consultants) and the relationships developed with thousands of stakeholders (other agencies, industry, associations, states, etc.), as well as involvement of knowledgeable users within the Clean Cities Coalitions. The reviewer remarked that the national network of coalitions is truly the strength of the program, and noted in addition that the program places significant emphasis on data collection from the coalitions to ensure programmatic metrics are being met.

Similarly, the reviewer stated, the regulated fleets program has built relationships with hundreds of state and utility fleets to ensure high levels of deployment, while the student competitions have created amazing relationships with numerous universities and with industry. 


\section{Question 9: Do these projects represent novel and/or innovative ways to approach these barriers as appropriate?}

\section{Reviewer 1:}

The reviewer observed that while the Clean Cities network structure is over 20 years old, it still works and allows for continued innovative developments. In addition, the reviewer stated that the Clean Cities Program is adept at listening to users and developing information, tools, and other items based upon needs identified by these users. The program has specific events gauged to gather this input from its thousands of stakeholders.

The reviewer praised Fueleconomy.gov as still a highly novel approach for delivering efficiency (and also alternative fuel) information to users, continuing to be one of the Federal Government's most-used sites. The reviewer remarked that the Legislative and Regulatory program is always looking for new ways to engage fleets in adopting new technologies at rates that go far above simple compliance. The reviewer specified that with each new student competition series, the innovation required by the teams grows, by design, and that this process thus allows for a way to continue developing better and better competitions.

\section{Reviewer 2:}

The reviewer commented that the Clean Cities Program and EcoCAR3 both are considered to be novel approaches to helping to address the barriers of the program, adding that they both are able to leverage government funds and provide a vast number of groups to collaborate with to reduce petroleum use.

\section{Reviewer 3:}

The reviewer noted that the Alternative Fuels Data Center (AFDC) website has expanded to include a substantial number of novel tools, and the program's recent competitive funding topics have sought and funded a wide range of innovative projects.

\section{Question 10: Has the program area engaged appropriate partners?}

\section{Reviewer 1:}

The reviewer remarked that partnerships are really the key to the TI efforts, observing that in Clean Cities, there are thousands of stakeholders ranging from local fleets to manufacturers to other government agencies. The reviewer offered that there are probably few other government programs that focus this heavily upon developing and maintaining the relationships necessary for success. The reviewer noted that regulated fleets efforts coordinate not only with hundreds of covered fleets, but also additional state organizations, fuels associations, and others, such as Clean Cities, federal fleets, and fuel suppliers (such as a joint workshop series a few years ago). The reviewer stated that under the educational program (particularly EcoCAR), there is specific coordination with not only universities, but also industry partners for the competitions. The reviewer concluded that all efforts under TI also include strong involvement of the national laboratories and key consultants, each of whom has developed expertise related to TI Program needs.

\section{Reviewer 2:}

The reviewer characterized the number of entities that the TI Program engages as extremely impressive. The reviewer elaborated that partners in the National Clean Fleets, Clean Cities, national laboratories, and colleges and universities in the EcoCAR3 program make the overall TI Program very effective.

\section{Reviewer 3:}

The reviewer remarked that the program has developed strong partnerships - with industry, corporate fleets, states, municipalities and many other stakeholders. The reviewer observed that Clean Cities Coalitions are completely built on partnerships and driven by independent stakeholders. The reviewer stated that the National Clean Fleets Partners Program is a good way for large national fleets to partner with the program; many of these fleets serve as pace-setters. The reviewer noted that the program has developed strong partnerships with the National Park Service (NPS).

\section{Question 11: Is the program area collaborating with them effectively?}




\section{Reviewer 1:}

The reviewer remarked that, as evidenced by the success and results that the TI Program has experienced over the years, the collaboration with partners has been extremely effective.

\section{Reviewer 2:}

The reviewer noted that several of the Clean Cities Program's main activity areas are organized around and support partner collaboration, especially technical and problem solving assistance, training and stakeholder coordination, field metrics tracking, and funded projects.

\section{Reviewer 3:}

The reviewer replied yes, as indicated above, the TI Program has been designed to explicitly work with all these partners, day in and day out. The reviewer elaborated that in Clean Cities, this involves stakeholder gatherings, funding opportunity announcements (FOAs), and participation in numerous outreach events. The legislative and regulatory activities also emphasize outreach to covered fleets, which has been key to ensuring the 100\% compliance level within the fleets' area. Finally, the reviewer added, in the educational area, DOE has very strong relationships with a number of universities, which has resulted in thousands of trained engineers, many of whom have moved into jobs related to transportation technologies. These educational competitions are specifically made possible through a collaboration with auto manufacturers and other component manufacturers.

Over the new few years, the reviewer observed, there may be an even greater need for coordination with other federal agencies (perhaps begun with the recent SMART Mobility Memorandum of Understanding [MOU] with U.S. DOT), as well as states, cities, industrial partners, and local technology implementers.

\section{Question 12: Are there any gaps in the portfolio for this technology area?}

\section{Reviewer 1:}

The reviewer replied no, the overall program does a very good job of addressing the issues that need to be resolved to help reduce petroleum use.

\section{Reviewer 2:}

The reviewer noted that recent focus has appeared to be on electric technologies, but Clean Cities Coalitions appear to also remain interested in gaseous fuel and biofuel technologies, as well as efficiency and idle reduction, so it appears there is still a need for efforts that provide better balance among technologies.

\section{Reviewer 3:}

The reviewer offered that hydrogen (H2) may be seen as lacking program focus, so perhaps the program could identify and articulate minimum thresholds in terms of practical vehicle price point and vehicle/fueling availability, to clarify why the program is not doing more in this area.

\section{Question 13: Are there topics that are not being adequately addressed?}

\section{Reviewer 1:}

The reviewer stated that topics in the TI Program all appear to be adequately addressed.

\section{Reviewer 2:}

The reviewer suggested that perhaps the TI Program could be more coordinated with the Bioenergy Technologies Office (BETO), particularly on biofuel infrastructure deployment and early information sharing on the Co-Optima initiative (i.e., what would deployment trends need to look like to best encourage/enable co-optimized internal combustion engine (ICE) vehicle development and deployment? The reviewer wondered what consumer and fleet issues would need to be addressed and how Clean Cities could assist.

\section{Reviewer 3:}

The reviewer stated that it was unclear from the presentation what the real goals of the National Clean Fleets Partnership are, how it is being implemented, what the real successes are from it, and how the needs for this activity are changing/increasing over time. The reviewer observed that it seems like it has the potential to achieve a great deal, but it is unclear how that is going. 
The reviewer also noted that there is a great demand for more and better outreach in TI Program areas and that these efforts tend to be largely limited by available funding. The reviewer commented that the joint workshops (regulated fleets, Clean Cities, and federal fleets) from a few years ago seemed like a very effective mechanism, and could be continued/expanded. The reviewer pointed out that none have been held in over two years, and that with changes in infrastructure development status and vehicle technology options (most were before plugin electric vehicles [PEVs] were significantly available), this argues for a need to resume the workshops. In addition, the reviewer said, with DOE interests in reaching beyond fleets to consumers, there is a need for perhaps expanding/adapting this model to more consumer-oriented stakeholders, such as automobile dealers.

\section{Question 14: Are there other areas that this program area should consider funding to meet overall programmatic goals?}

\section{Reviewer 1:}

The reviewer characterized funding in the TI Program as being allocated appropriately. The reviewer does not think there are other program areas that need to be funded to meet the goals.

\section{Reviewer 2:}

The reviewer offered that staying aware and on top of what other agencies are doing — and co-investing/leveraging those activities where it makes sense-is valuable for the program. The reviewer suggested coordination with U.S. DOT, EPA, the U.S. Department of Agriculture (USDA), and others on how those agencies are approaching efforts to support transportation system efficiency and energy diversity (i.e., DOT and SMART Mobility, USDA and bioeconomy development, EPA and its latest GHG analyses and priority reduction strategies).

\section{Reviewer 3:}

The reviewer observed that it seems like the funding approach in Clean Cities has moved from deployment to community readiness, but that it appears as though a mix of these two areas is still needed, particularly in the area of infrastructure. In addition, the reviewer remarked, more of the focus the past few years has appeared to be on electric technologies, but contributions in the program to date have appeared to come more from gaseous fuels and biofuels. So, again, as far as vehicle types, balance is important. Other suggestions from the reviewer include resuming workshops as described in question 13 above.

\section{Question 15: Can you recommend new ways to approach the barriers addressed by this program area?}

\section{Reviewer 1:}

The reviewer stated that over the years this program area has been and continues to be very effective in addressing the barriers to allow for petroleum displacement.

\section{Reviewer 2:}

The reviewer suggested that since Clean Cities is such a strong public and industry-facing effort, perhaps other VTO programs (hydrogen, fuels and lubricants, advanced vehicle technology, etc.) could utilize Clean Cities more as a way to demonstrate their funded technologies in the field, and collect feedback/information from valuable stakeholder groups. The reviewer also recommended that perhaps the Clean Cities Program could be positioned to serve as an outreach arm (of sorts) for the other VTO areas (e.g., if there is a lubricants technology that fleets should know about, can Clean Cities help communicate on that topic?).

\section{Reviewer 3:}

The reviewer's key recommendation is to continue to expand and innovate in the critical area of outreach, elaborating that this can mean more targeted multidisciplinary workshops (as described in question 13 above) or the use of new tools such as fleet data collection technologies or social media. The real key in TI, the reviewer remarked, is making sure users have the information necessary to make decisions and support the operation of the vehicles once in place (including through training technicians and others). The reviewer concluded that the best ways to do these things continue to change, so, the program, while having established highly effective methods for conducting this outreach, will need to continue to innovate and improve the delivery of these services. 


\section{Question 16: Are there any other suggestions to improve the effectiveness of this program area?}

\section{Reviewer 1:}

The reviewer replied no, the program area as currently implemented is extremely effective.

\section{Reviewer 2:}

The reviewer suggested perhaps some better alignment between EcoCAR activities and the other VTO research areas, offering, though, that this may already be happening. The reviewer also stated that university and vehicle education activities are very good, but that they do seem a bit disjointed from the rest of the portfolio. The reviewer clarified that it is fine; all program activities do not need to be clearly and directly linked, and this is just an observation.

\section{Reviewer 3:}

The reviewer said to remember how all of these TI project areas have been built and how successful they are, and use these attributes and structures when moving forward. The reviewer observed that a new technology or even integration approach is unlikely to be needed to change these bedrocks of the program, but new opportunities will require additional efforts (and funding) to address the new issues and challenges that come along with them. 


\section{Project Feedback}

In this merit review activity, each reviewer was asked to respond to a series of questions, involving multiple-choice responses, expository responses where text comments were requested, and numeric score responses (on a scale of 1.0 to 4.0). In the pages that follow, the reviewer responses to each question for each project will be summarized: the multiple choice and numeric score questions will be presented in graph form for each project, and the expository text responses will be summarized in paragraph form for each question. A table presenting the average numeric score for each question for each project is presented below.

\section{Table 8-1 - Project Feedback}

\begin{tabular}{|c|c|c|c|c|c|c|c|c|}
\hline $\begin{array}{l}\text { Presentation } \\
\text { Title }\end{array}$ & $\begin{array}{c}\text { Principal } \\
\text { Investigator } \\
\text { and } \\
\text { Organization }\end{array}$ & $\begin{array}{l}\text { Page } \\
\text { Number }\end{array}$ & Objectives & Approach & $\begin{array}{l}\text { Accomplishments } \\
\text { and Progress }\end{array}$ & Collaboration & $\begin{array}{l}\text { Market } \\
\text { Impact }\end{array}$ & $\begin{array}{c}\text { Weighted } \\
\text { Average }\end{array}$ \\
\hline $\begin{array}{l}\text { Plug-In Hybrid } \\
\text { Electric } \\
\text { Vehicle } \\
\text { Demonstration } \\
\text { Program and } \\
\text { Social Media } \\
\text { Campaign }\end{array}$ & $\begin{array}{l}\text { Ardisana, } \\
\text { Lizabeth } \\
\text { (ASG } \\
\text { Renaissance) }\end{array}$ & 8-13 & 2.63 & 2.50 & 2.63 & 2.75 & 2.13 & 2.56 \\
\hline $\begin{array}{c}\text { Drive } \\
\text { Electric } \\
\text { Orlando }\end{array}$ & $\begin{array}{c}\text { Combs, April } \\
\text { (Florida } \\
\text { Department } \\
\text { of Agriculture } \\
\text { and } \\
\text { Consumer } \\
\text { Services/ } \\
\text { Office of } \\
\text { Energy) }\end{array}$ & 8-17 & 3.38 & 3.38 & 3.00 & 3.38 & 3.25 & 3.21 \\
\hline $\begin{array}{l}\text { Alternative } \\
\text { Fuel Vehicle } \\
\text { Curriculum } \\
\text { Development } \\
\text { and Outreach } \\
\text { Initiative }\end{array}$ & $\begin{array}{l}\text { Moore, } \\
\text { Judy (West } \\
\text { Virginia } \\
\text { University } \\
\text { Research } \\
\text { Corporation) }\end{array}$ & $8-21$ & 3.50 & 3.10 & 3.20 & 2.80 & 2.70 & 3.15 \\
\hline $\begin{array}{c}\text { Nationwide } \\
\text { AFV } \\
\text { Emergency } \\
\text { Responder, } \\
\text { Recovery, Re- } \\
\text { Construction } \\
\text { and } \\
\text { Investigation } \\
\text { Training }\end{array}$ & $\begin{array}{c}\text { Klock, } \\
\text { Andrew } \\
\text { (National Fire } \\
\text { Protection } \\
\text { Association) }\end{array}$ & $8-25$ & 3.40 & 3.40 & 3.30 & 3.10 & 3.00 & 3.29 \\
\hline
\end{tabular}




\begin{tabular}{|c|c|c|c|c|c|c|c|c|}
\hline $\begin{array}{l}\text { Presentation } \\
\text { Title }\end{array}$ & $\begin{array}{c}\text { Principal } \\
\text { Investigator } \\
\text { and } \\
\text { Organization }\end{array}$ & $\begin{array}{c}\text { Page } \\
\text { Number }\end{array}$ & Objectives & Approach & $\begin{array}{l}\text { Accomplishments } \\
\text { and Progress }\end{array}$ & Collaboration & $\begin{array}{l}\text { Market } \\
\text { Impact }\end{array}$ & $\begin{array}{l}\text { Weighted } \\
\text { Average }\end{array}$ \\
\hline $\begin{array}{l}\text { Safe } \\
\text { Alternative } \\
\text { Fuels } \\
\text { Deployment in } \\
\text { Mid-America } \\
\text { (The SAF-D } \\
\text { Project) }\end{array}$ & $\begin{array}{c}\text { Gilbert, Kelly } \\
\text { (Metropolitan } \\
\text { Energy } \\
\text { Center, Inc.) }\end{array}$ & $8-29$ & 2.80 & 2.70 & 2.90 & 3.00 & 2.80 & 2.84 \\
\hline $\begin{array}{l}\text { Initiative for } \\
\text { Resiliency } \\
\text { in Energy } \\
\text { through } \\
\text { Vehicles } \\
\text { (IREV) }\end{array}$ & $\begin{array}{l}\text { Powers, } \\
\text { Cassie } \\
\text { (National } \\
\text { Association } \\
\text { of State } \\
\text { Energy } \\
\text { Officials) }\end{array}$ & $8-33$ & 3.40 & 3.10 & 3.30 & 3.10 & 3.00 & 3.23 \\
\hline EcoCAR 3 & $\begin{array}{l}\text { Wahl, Kristen } \\
\text { (ANL) }\end{array}$ & $8-38$ & 3.60 & 3.60 & 3.50 & 3.80 & 3.60 & 3.58 \\
\hline $\begin{array}{l}\text { Overall } \\
\text { Average }\end{array}$ & & & 3.24 & 3.11 & 3.12 & 3.13 & 2.93 & 3.12 \\
\hline
\end{tabular}




\section{Plug-In Hybrid Electric Vehicle Demonstration Program and Social Media Campaign: Lizabeth Ardisana (ASG Renaissance) - ti064}

\section{Presenter}

Brenda Prebo, ASG Renaissance

\section{Reviewer Sample Size}

A total of four reviewers evaluated this project.

Question 1: Project objectives-the degree to which the project objectives support the DOE/VTO objectives of reducing reliance on petroleum based fuels and reducing emissions. This includes the impact the project has on addressing the technical barriers from the Vehicle Technologies Office (VTO) Multi-Year Program Plan.

\section{Reviewer 1:}

The reviewer stated that the project objective and overview slides described the project's specific objectives, as well as how the project addressed specific barriers in the VTO Multi-Year Program Plan 2011-2015. Furthermore, the project objectives appeared to be generally effective.

\section{Reviewer 2:}

The reviewer found that the project had innovative outreach objectives; however, the extent to which these project objectives would translate into deployment (i.e., electric vehicle [EV] sales) was not entirely clear. This lack of clarity was, in part, due to limited original equipment manufacturer (OEM) involvement and/or specific messaging driving web audiences to auto dealerships.

\section{Reviewer 3:}

The reviewer stated that the objective of reaching potential plug-in hybrid electric vehicle (PHEV) buyers to influence their decisions and steer them towards PHEVs was fundamentally sound. Given the pervasive reach of social media in today's culture and the likely predisposition of tech-savvy consumers to be inclined toward a plugin vehicle, the use of online EV Ambassadors sounds like a good idea. The reviewer was not convinced that this type of campaign will have an immediate, significant, or even measurable impact on PHEV sales, however. Thus, the reviewer did not score this project highly in terms of achieving DOE/VTO objectives of reducing petroleum use or reducing emissions.

\section{Reviewer 4: \\ Reviewer 4:}

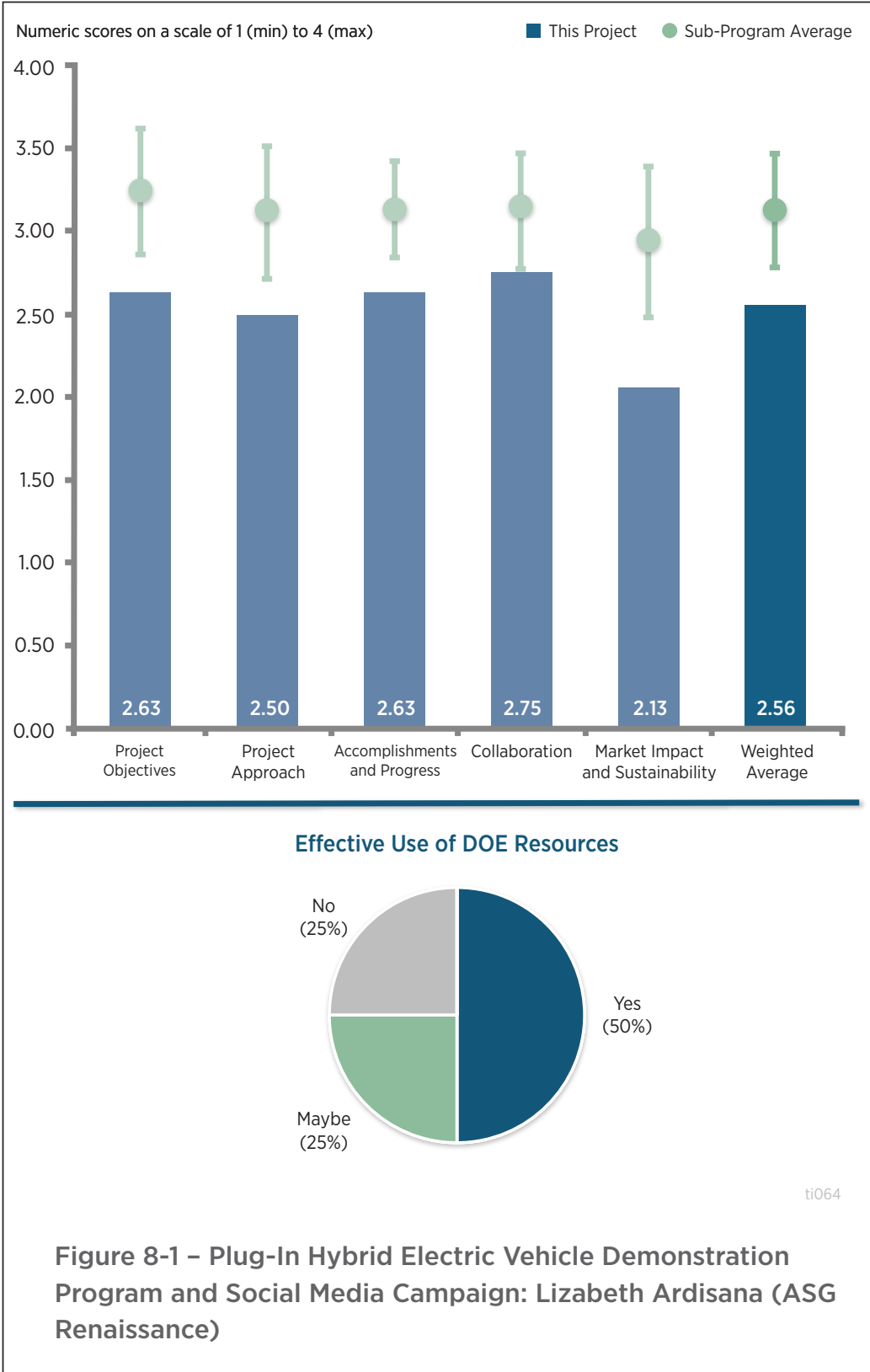


The reviewer did not agree that increasing awareness is commensurate with increasing acceptance of AFVs. The reviewer commented that social media is a very extemporaneous, transient, and fleeting — really superficial — way of increasing awareness. The reviewer stated that the impression people get on social media is momentary but not long-lasting, and the purpose of social media seems to be instant gratification and sharing instant gratification.

Regarding progress and goal tracking, the reviewer noted that there was no tracking or measurement of how one-month free trials led to actual AFV purchases. Furthermore, the project manager was not able to provide an example of where social media was instrumental in major purchases (greater than $\$ 20,000$ ). Thus, the reviewer determined that DOE/VTO objectives of reducing emissions and reliance on petroleum based fuels are too serious to be achieved by the sharing of extemporaneous, transient, and fleeting impressions.

\section{Question 2: Project approach to supporting deployment of petroleum reduction technologies and practices, alternative fuel vehicles, infrastructure, emissions reductions and related efforts-the degree to which the project is well-designed, feasible, and integrated with other efforts.}

\section{Reviewer 1:}

The reviewer noted that the project approach section provided a generally effective methodology to accomplish the project objectives. Adequate detail was provided on the approach and milestone slides with regards to the planned tasks and activities.

\section{Reviewer 2:}

Although the reviewer found the project approach somewhat risky, it was substantively innovative in its targeting of social media marketing. The project ensured a thorough amount of time was allocated for each influencer test drive (one month). However, the reviewer suggested that the project may have benefited from targeting higherprofile, up-and-coming YouTube stars, whose videos and blogs are more widely viewed than smaller bloggers with a far more limited reach. Lastly, the reviewer suggested that a greater emphasis on making video and blog content visible to general internet/Google searches (strong focus on making posts searchable) would broaden exposure far beyond just followers of the Influencers/EV Ambassadors.

\section{Reviewer 3:}

The reviewer asked how EV Ambassadors are selected, and if their age/income was in line with existing PHEV owners or intenders. The reviewer asked if the project team's initial research indicated that PHEV intenders are also active on social media. The reviewer noted that reaching a large number of people is great, but it is critical to know who is being reached, and if they can afford to buy PHEVs.

The reviewer acknowledged the value in using social media to tout the benefits of PHEVs, because it is a popular source of cultural information in today's society. However, the reviewer found the presentation was vague on how the influencers were chosen and wanted more analysis of the results. Finally, the reviewer commented that the project grossly overestimated the expected rate of engagement, and the reviewer expected a better understanding of the fundamentals of social media before undertaking a project like this. Thus the reviewer was a little dubious on the effectiveness of this approach.

\section{Reviewer 4:}

The reviewer believed that social media is best suited for low-cost, spur-of-the-moment purchases, such as recommending a restaurant after dining, a movie after going to the theatre, a night club after drinking, or avoiding the Metro subway because of an incident, etc. Larger purchases and investments, such as purchase of an AFV, are better suited to personal contact and word-of-mouth communication. The reviewer reiterated that sharing of good impressions does not necessarily lead to a conscious effort to make a purchase of, or rent, an AFV for the purpose of reducing emissions and use of petroleum-based fuels.

\footnotetext{
Question 3: Project accomplishments and progress toward overall project and DOE goals - the degree to which progress/significant accomplishments have been achieved, measured against performance indicators and demonstrated progress toward project and DOE goals.
} 


\section{Reviewer 1:}

The reviewer stated that good progress had been made towards achieving Phase One project goals for fiscal year (FY) 2015-2016. All initiatives and activities appeared to be on track for successful completion. Activities related to the program design, program website, selection of EV ambassadors, and first wave of Phase One demonstrations resulted in the majority of project metrics/targets being achieved with regards to program reach, views and engagement. The reviewer noted that no concerns had been identified.

\section{Reviewer 2:}

The reviewer noted that to date the project had recruited about 50 Influencers/EV Ambassadors, which was a strong number. The number of published content pieces and program views thus far was also strong. However, the reviewer stated that the degree of web audience engagement was far under project objectives. The Principal Investigator (PI) explained that this was due to a (rather grossly) misjudged expectation, and that the actual percent engagement was closer to industry standards. Still, the reviewer pointed out that the large difference between expected total program engagement $(100,000)$ and engagement at the time of the presentation $(13,600)$ raised the question of whether the applicant would have designed a substantively different type of project had the project team had previous knowledge about web marketing industry standards and what could reasonably be expected for web engagement.

\section{Reviewer 3:}

The reviewer said that the project's accomplishments and progress to date were underwhelming. Some project goals were exceeded, others were not even close. The reviewer commented that 44 people producing, on average $14 \mathrm{blogs}$, tweets and/or posts over the course of a month was hardly a feverish pace. The reviewer expressed concern regarding the inability to analyze the impact of the influencers, and questioned whether or not any PHEVs were sold as a result of the campaign.

\section{Reviewer 4:}

The reviewer observed that the PI showed a slide indicating that as of April 28, 2016, half of the target goals were met and half were not. The reviewer noted that the PI's claim of meeting or exceeding total program goals was true only by asserting that some of the original total program goals were set unrealistically high.

\section{Question 4: Collaboration and coordination among project team-the degree to which the appropriate team members and partners are involved in the project work and the effectiveness of the collaboration between and among partners.}

\section{Reviewer 1:}

The reviewer stated that an effective project team was assembled to carry out this project, as industry and Clean Cities Coalition partners provided an appropriate mix of expertise among team members. Roles of project team were defined and collaboration/communication among project partners appeared to be appropriate for the project of this scope. The reviewer noted that during the oral presentation, the presenter commented that Ford had not been very engaged in the program to date, besides providing the vehicles, but it appeared that they are more interested in Phase 2 involvement. The reviewer stated that it is important that the project's vehicle OEM is onboard with the direction and data associated with this project, to ensure the activities being carried out and data collected are valuable to them and will result in increased vehicle awareness/sales.

\section{Reviewer 2:}

The reviewer stated that the project would have benefited quite a bit from more OEM/Ford involvement, including from dealers.

\section{Reviewer 3:}

The reviewer stated that the team and partnership appeared to be fairly well-organized, and the project chose the kinds of influencers they were targeting. However, glitches like the insurance issue, which forced the team to hire the EV Ambassadors, showed a lack of thorough planning, or ineffective project management. Also, the reviewer questioned whether the shift to fewer influencers with larger followings for Phase 2 was planned from the beginning, or if it was in response to the results of Phase 1. 
Question 5: Market impact and sustainability-the degree to which the project has already contributed, as well as the potential to continue to contribute in the future, to a sustainable alternative fuel vehicle market, alternative fuel market expansion, and reduced petroleum dependence/greenhouse gas emissions in the transportation sector. This would include the potential to reduce barriers to large scale alternative fuel vehicle market penetration, making information about alternative fuels and petroleum reduction opportunities widely available to target audiences, and ability for the project to be replicated in other geographic areas or with other technologies.

\section{Reviewer 1:}

The reviewer noted that the project may contribute to reduced petroleum dependence, GHG emissions reduction and AFV market sustainability goals through raising consumer awareness of the availability and benefits of PEVs in several key Northeastern markets, through the use of social media influencers. However, the reviewer stated that a weakness identified in this section of the presentation involved the lack of providing a methodology to define/ demonstrate how the project metrics of reach, views, and engagement directly or indirectly translated into vehicle sales.

\section{Reviewer 2:}

The reviewer commented that there appeared to be no future for the program once the car loans are done, and no way of measuring the results in terms of how many PHEVs were sold as a result of this program. The reviewer suggested augmenting the influencer loans with some shorter test drives directly with EV-intenders, and still tying it in with social media. For instance, set up in an Ikea parking lot on a Saturday morning, give short test drives, and gather data from an exit poll.

\section{Reviewer 3:}

The reviewer stated that there was no tracking or tracing of the impact of social media impressions on purchases or rental of AFVs. Furthermore, there was no measure of how long good memories of the experiences with AFVs were retained.

Question 6: Use of resources-are DOE funds being used wisely? Should DOE fund similar efforts in the future? If not, what would be a better use of DOE resources to achieve alternative fuel vehicle and infrastructure expansion to support the broader goal of petroleum displacement and greenhouse gas reductions?

\section{Reviewer 1:}

The reviewer stated that in lieu of funding for hardware (i.e., vehicles and fueling sites), the use of DOE funding to inform the public about the availability of PEVs was critically important to advance the market of these vehicles. As newer marketing methods are implemented, such as this project's use of social media, it is critical to understand how the industry calculates the benefits of these activities and how they translate into vehicle sales and market development.

\section{Reviewer 2:}

The reviewer stated that this project focused on an innovative and modern marketing approach, which is really the future of consumer outreach. The reviewer noted that more OEM involvement and greater state-of-the-art web marketing program design (e.g., targeting YouTube celebrities, realistic engagement targets, and searchable posts) would have improved the project.

\section{Reviewer 3:}

The reviewer stated that that the return on investment was lower than expected based on projected and actual media value. Spending money, time, and effort to develop and generate awareness for a website that adds little to the available online PHEV knowledge base has limited value beyond the life of the project. The reviewer noted that there are existing and well-established websites that give this information.

\section{Reviewer 4:}

The reviewer referenced prior comments regarding project objectives and approach. 
Drive Electric Orlando: April Combs (Florida Department of Agriculture and Consumer Services/Office of Energy) - ti065

\section{Presenter}

April Combs, Florida Department of Agriculture and Consumer Services

\section{Reviewer Sample Size}

A total of four reviewers evaluated this project.

\section{Question 1: Project} objectives-the degree to which the project objectives support the DOE/VTO objectives of reducing reliance on petroleum based fuels and reducing emissions. This includes the impact the project has on addressing the technical barriers from the Vehicle Technologies Office (VTO) Multi-Year Program Plan.

\section{Reviewer 1:}

The reviewer stated that this was an excellent project with excellent objectives at the right time and right place.

\section{Reviewer 2:}

The reviewer found that the wellresearched objectives showed a clear understanding and analysis of the demographics of the target market area, such as the knowledge that most rental buyers make short trips. The reviewer stated that putting drivers behind the wheel of an EV for an extended test drive in real-world conditions was a great way to engage and influence their purchasing decision.

\section{Reviewer 3:}

The reviewer stated that the project objective and overview slides described the project's specific objectives, as well as how the project addresses specific barriers in the VTO's Multi-Year Program Plan 2011-2015. The reviewer added that project objectives appeared to be generally effective.

\section{Reviewer 4:}

The reviewer stated that putting consumers into a moderate-term rental experience was an excellent idea and project. However, the total number of rental experiences targeted during the project period was not clear (or the reviewer may have missed this figure).

Question 2: Project approach to supporting deployment of petroleum reduction technologies and practices, alternative fuel vehicles, infrastructure, emissions reductions 
and related efforts-the degree to which the project is well-designed, feasible, and integrated with other efforts.

\section{Reviewer 1:}

The reviewer said that the strategy to attract EV rental customers with perks (free charging, free drinks, admission, free Clear membership, etc.) are smart and effective. Furthermore, ensuring that the cost of a Volt rental was the same as a regular mid-size sedan was a strong measure to ensure optimum participation.

\section{Reviewer 2:}

The reviewer stated that the project approach section provided a detailed and effective methodology to accomplish the project objectives. Appropriate detail was provided on the approach and milestone slides regarding the planned tasks and activities.

\section{Reviewer 3:}

The reviewer stated that this was a solid approach with strong partners onboard from the start. The project planning seemed to have covered all bases in terms of which partners to seek, identifying a target-rich environment of rental customers, gathering driver feedback, and analyzing the results.

\section{Reviewer 4:}

The reviewer found the approach to be excellent, but better quality control over the training of the rental car agents was absolutely needed. The reviewer would like to have seen all drivers be informed about, and given an opportunity, to rent an electric car, if available, even if the customer wanted a conventional car.

\section{Question 3: Project accomplishments and progress toward overall project and DOE} goals - the degree to which progress/significant accomplishments have been achieved, measured against performance indicators and demonstrated progress toward project and DOE goals.

\section{Reviewer 1:}

The reviewer stated that good progress had been made towards achieving Year 1 project goals. Activities related to project engagement with rental car, theme park, hotel, and travel partners resulted in initial progress towards engaging these entities and rental car customers to provide vehicles, charging, and incentives. All initiatives and activities appeared to be on track for successful completion and no concerns had been identified.

\section{Reviewer 2:}

The reviewer noted that, to date, only one rental car company partner was involved, despite Orlando being the largest rental car market in the world. The reviewer stated that the project team had done a very good job attracting other various stakeholder partners.

\section{Reviewer 3:}

The reviewer noted that progress was good, and the project had proactively worked to attract more partners. However, the reviewer thought more attention could have been paid to actively marketing the availability of EV rentals; it appeared that the EV rentals were happening more by chance or by whim of the consumer who saw that they were available.

\section{Reviewer 4:}

The reviewer said that no target performance metrics were given for Year 1.

\section{Question 4: Collaboration and coordination among project team-the degree to which the appropriate team members and partners are involved in the project work and the effectiveness of the collaboration between and among partners.}

\section{Reviewer 1:}

The reviewer stated that the project team appeared well-coordinated. The project demonstrated numerous broad strategic stakeholder partnerships. 


\section{Reviewer 2:}

The reviewer stated that an effective project team was assembled to carry out this project, with government, EV industry trade association, and Clean Cities Coalition partners involved, which provided an excellent mix of expertise among team members. Project team roles were defined and collaboration/communication among project partners appeared to be appropriate for a project of this scope. Additionally, the project interacted with a good mix of local stakeholders, which included city governments, infrastructure providers, utilities, automakers and other firms, service providers, and regulators, as well as Orlando-based rental car companies, theme parks, and hotels.

\section{Reviewer 3:}

The reviewer was impressed, not only that the project leaders were able to engage as many hotels as they did, but that the team also went the extra mile to secure prime parking locations for re-charging the electric cars. The reviewer thought one potential improvement was the signage telling drivers where those electric charging spots are, particularly at parking lots that are so huge that it is hard to find those spots.

\section{Reviewer 4:}

The reviewer was concerned that employee education among the partner companies was an issue, as these types of companies can have a high turnover rate, making it difficult to keep momentum going; however, the reviewer thought it sounded like the coalition was actively engaged in continuing education among the partners and was seeking ways to expand and enhance the program.

Question 5: Market impact and sustainability-the degree to which the project has already contributed, as well as the potential to continue to contribute in the future, to a sustainable alternative fuel vehicle market, alternative fuel market expansion, and reduced petroleum dependence/greenhouse gas emissions in the transportation sector. This would include the potential to reduce barriers to large scale alternative fuel vehicle market penetration, making information about alternative fuels and petroleum reduction opportunities widely available to target audiences, and ability for the project to be replicated in other geographic areas or with other technologies.

\section{Reviewer 1:}

The reviewer said that the project offered an excellent way to expose a broad consumer base to electric vehicles and technology. As a pilot project (involving only $30 \mathrm{EVs}$ in a massive rental car market), the market impact of the individual project was rather small; however, it did create a replicable template that can and should be broadened across Orlando and in other large rental car markets.

\section{Reviewer 2:}

The reviewer noted that the EV infrastructure will remain in place so the potential is there for sustaining the program into the future. The program recognized and is continuing to address the sustainability challenges, such as employee training at the partner companies. The reviewer thought that continuing to expand the charging network within the tourism corridor is great. The program had been both well-conceived and well-executed.

\section{Reviewer 3:}

The reviewer stated that there needed to be tracking or tracing of a customer's experience with renting an electric car to the customer purchasing an electric car or even renting one elsewhere or later. It was not possible to determine the near-term and longer-term impact of Drive Electric Orlando on customer purchase or rental of electric cars.

\section{Reviewer 4:}

The reviewer stated that the project may contribute to reduced petroleum dependence, GHG reduction, and AFV market sustainability goals by leveraging America's top tourism destination and rental car market to expose millions of visitors to EVs with the goal of, ultimately, turning renters into buyers. The reviewer identified as a weakness in this section of the presentation the lack of providing a methodology to define/demonstrate how the project rental metrics, directly or indirectly, translate into vehicle sales. As the project progresses into Year two, the customer feedback surveys should provide important data related to the customer experience and potential for EV purchases. 
Question 6: Use of resources-are DOE funds being used wisely? Should DOE fund similar efforts in the future? If not, what would be a better use of DOE resources to achieve alternative fuel vehicle and infrastructure expansion to support the broader goal of petroleum displacement and greenhouse gas reductions?

\section{Reviewer 1:}

The reviewer stated that the coalition had leveraged its resources very effectively, attracting a strong network of volunteer partners. The reviewer considered this a good use of funds.

\section{Reviewer 2:}

The reviewer stated that in lieu of funding for hardware (i.e., vehicles and fueling sites), the use of DOE funding to inform the public on the availability of EVs was critically important to advance the market of these vehicles. While the industry's "butts in seats" programs/activities are deployed across target markets, it is critical to understand how the industry calculates the benefits of these activities and how they translate into vehicle sales and market development.

\section{Reviewer 3:}

The reviewer stated that the project made a solid effort to expose EVs in a significant way to a very large potential consumer base. The success of the current project should be followed up by an effort where Enterprise can share highlights of its EV rental program with other rental companies in Orlando. An information exchange workshop among rental car companies could be a good next step to keep the momentum going, encourage other rental companies to join in, and help spur more charging infrastructure.

\section{Reviewer 4:}

The reviewer wished that the visitor arriving in Orlando could also experience electric buses and see electric trucks supporting the backbone of the area. The presence of electric cars should be extended to all vehicles — buses, trucks, vans, etc. 


\section{Alternative Fuel Vehicle Curriculum Development and Outreach Initiative: Judy Moore (West Virginia University Research Corporation) - ti066}

\section{Presenter}

Judy Moore, West Virginia University

\section{Reviewer Sample Size}

A total of five reviewers evaluated this project.

Question 1: Project objectives-the degree to which the project objectives support the DOE/VTO objectives of reducing reliance on petroleum based fuels and reducing emissions. This includes the impact the project has on addressing the technical barriers from the Vehicle Technologies Office (VTO) Multi-Year Program Plan.

\section{Reviewer 1:}

The reviewer stated that training underserved audiences (tow and salvage, collision, fueling and maintenance, etc.) was an excellent objective.

\section{Reviewer 2:}

The reviewer thought the project had solid objectives. Thinking to reach these underserved audiences and having the knowledge to recognize all of the ancillary people and positions who touch and have an effect on the life cycle of these vehicles showed the strength of this partnership.

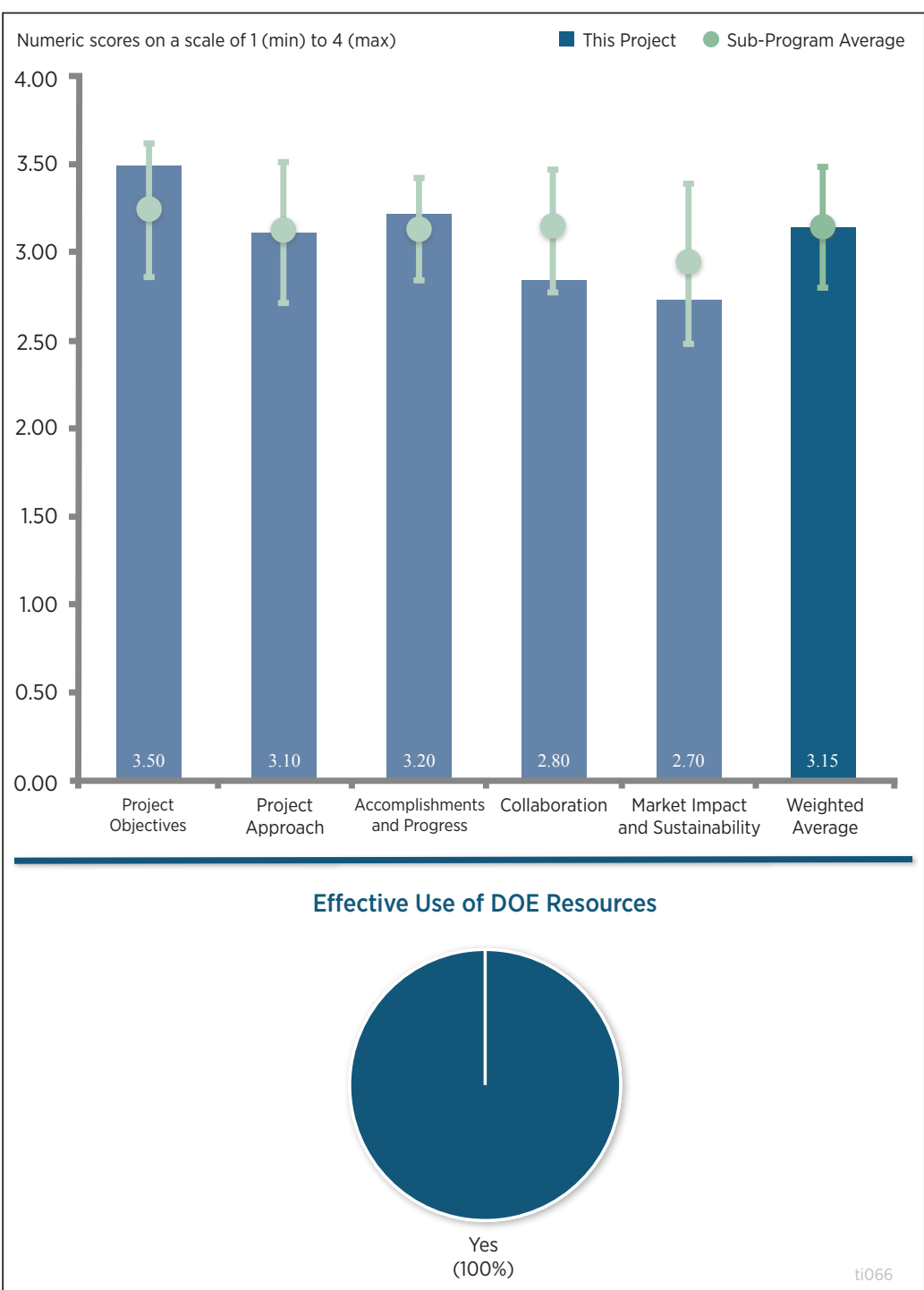

Figure 8-3 - Alternative Fuel Vehicle Curriculum Development and Outreach Initiative: Judy Moore (West Virginia University Research Corporation)

\section{Reviewer 3:}

The reviewer stated that the project objective and overview slides described the project's specific objectives/ deliverables, as well as how the project addressed specific barriers in the VTO's Multi-Year Program Plan 20112015. Project objectives appeared to be generally effective.

\section{Reviewer 4:}

The reviewer stated that the instructional designers seemed to have taken into account targeting the lowest common denominator for the educational level of the audience - in particular, the 8th grade level of reading/comprehension.

\section{Reviewer 5}

The reviewer thought that overall, the project addressed barriers in outreach to unique stakeholders, but was in the very early stages of deploying the material. 
Question 2: Project approach to supporting deployment of petroleum reduction technologies and practices, alternative fuel vehicles, infrastructure, emissions reductions and related efforts-the degree to which the project is well-designed, feasible, and integrated with other efforts.

\section{Reviewer 1:}

The reviewer thought the project approach seemed to be effective and contributed to achieving the majority of project objectives.

\section{Reviewer 2:}

The reviewer thought that this partnership had a strong background in education and had developed an allencompassing approach to get the word out to the right people. The inclusion of online, in-person, and event-based training covered all the bases, and this curriculum integrated well with existing training programs from the same group.

\section{Reviewer 3:}

The reviewer stated that the project's approach followed a well-established National Alternative Fuels Training Consortium (NAFTC) curriculum development format. The Odyssey Day activity was not well described in terms of how the products of this project would be specifically marketed or distributed at those events.

\section{Reviewer 4:}

The reviewer stated that the project approach (relevance) section provided an effective methodology to accomplishing the project objectives. Despite the fact that the revised presentation template for 2016 was not followed, appropriate detail was provided on the approach, impact and milestone slides regarding the planned tasks and activities. The reviewer identified the lack of a table with fiscal year (FY) two Milestones as one weakness.

\section{Reviewer 5:}

The reviewer would like to have seen a state department of transportation traffic operations and state department of transportation highway incident response organization as a consultant. The project team did not seem to take into full account potential bridge, tunnel, and highway restrictions on AFVs or towing of AFVs.

\section{Question 3: Project accomplishments and progress toward overall project and DOE goals - the degree to which progress/significant accomplishments have been achieved, measured against performance indicators and demonstrated progress toward project and DOE goals.}

\section{Reviewer 1:}

The reviewer stated that the program appeared to be on schedule and had set attainable goals for deployment. Milestones were clearly identified and progress was well-documented.

\section{Reviewer 2:}

The reviewer found that good progress has been made towards achieving Year one project goals. Activities related to curriculum development, marketing, and outreach were all underway and appeared to be on track for successful completion. The reviewer had no identified concerns.

\section{Reviewer 3:}

The reviewer noted that the project schedule appeared to be generally on track.

\section{Reviewer 4:}

The reviewer stated that at the current stage of the project, the accomplishments and progress were satisfactory.

Question 4: Collaboration and coordination among project team-the degree to which the appropriate team members and partners are involved in the project work and the effectiveness of the collaboration between and among partners. 


\section{Reviewer 1:}

The reviewer thought that the project made good use of a Clean Cities Coalition advisory committee, as well as other fleet stakeholders, to inform curriculum development.

\section{Reviewer 2:}

The reviewer stated that an effective project team was assembled to carry out this project, with industry stakeholders and multiple Clean Cities Coalition partners involved, which provided an excellent mix of expertise among team members. The roles of the project team were defined and collaboration/communication among project partners appeared to be appropriate for a project of this scope.

\section{Reviewer 3:}

The reviewer was delighted that the project sought out and included as stakeholders/partners the Automotive Recyclers Association (ARA). However, the reviewer did not see the Towing and Recovery Association of America (TRAA), North American Towing Academy (NATA), or the International Institute of Towing and Recovery (IITR).

\section{Reviewer 4:}

The reviewer stated that this was a strong team with deep experience in curriculum development and knowledge of AFVs, along with a well-established nationwide network of schools and trainers. The reviewer would like to have seen more collaboration/sharing with other grantees who have created similar curriculum programs to maximize the effect of both. There were some areas of overlap in the intended audience and delivery methods, as well as areas that were unique to this program.

\section{Reviewer 5:}

The reviewer commented that it was unfortunate the project was not leveraging with other similar projects within the VTO portfolio, regardless of how the information is disseminated. The reviewer thought that this could also be extended to the project's hydrogen modules, which did not seem to match up with current hydrogen deployment activities. It was also unclear who was participating on the advisory committee. The reviewer suggested that one stakeholder of interest could be the insurance industry. Finally, the reviewer asked how international activities could be best leveraged.

Question 5: Market impact and sustainability-the degree to which the project has already contributed, as well as the potential to continue to contribute in the future, to a sustainable alternative fuel vehicle market, alternative fuel market expansion, and reduced petroleum dependence/greenhouse gas emissions in the transportation sector. This would include the potential to reduce barriers to large scale alternative fuel vehicle market penetration, making information about alternative fuels and petroleum reduction opportunities widely available to target audiences, and ability for the project to be replicated in other geographic areas or with other technologies.

\section{Reviewer 1:}

The reviewer stated that this team had a proven track record of excellent training programs, and has built in the ability to update the curriculum as technology evolves and new vehicles enter the market.

\section{Reviewer 2:}

The reviewer stated that the project can have tremendous effectiveness and contribute to overcoming most barriers and informing appropriate audiences to the AFV market expansion. The reviewer noted that it was still early to determine the degree of impact.

\section{Reviewer 3:}

The reviewer commented that by creating brand-new, standing NAFTC courses, the products of this project were positioned to have an enduring shelf-life. However, it was not entirely clear how the new courses will be marketed to the various new underserved audiences that are targeted.

\section{Reviewer 4:}

The reviewer saw a need for providing emergency responder training on AFVs, but said it was a very long stretch that such training would significantly improve the market for AFVs and sustainability. The reviewer had not seen 
the survey data, but had never heard a consumer ask if emergency responders were prepared to deal with incidents involving AFVs before buying one.

\section{Reviewer 5:}

The reviewer thought that the project may contribute to reduced petroleum dependence, GHG emissions reduction, and AFV market sustainability goals by developing and delivering four new training courses and the associated marketing and outreach materials to support them, which will provide multiple new, underserved audiences with technical experience working with AFVs and advanced technology vehicles. The reviewer identified as a weakness that because the 2016 presentation format template was not used, the presentation did not specifically address market impact and sustainability with any level of detail.

\section{Question 6: Use of resources-are DOE funds being used wisely? Should DOE fund similar efforts in the future? If not, what would be a better use of DOE resources to achieve alternative fuel vehicle and infrastructure expansion to support the broader goal of petroleum displacement and greenhouse gas reductions?}

\section{Reviewer 1:}

The reviewer found that this team was judicious in use of funds and achieved maximum impact for every dollar spent.

\section{Reviewer 2:}

The reviewer wondered whether the Advanced Technology Vehicle Training for Towing and Roadside Assistance course could also be targeted to first responders, as it will contain content very relevant to that audience as well.

\section{Reviewer 3:}

The reviewer commented that that Federal funds were being used wisely in support of this project. However, the reviewer wondered why the DOT-National Highway Traffic Safety Administration (NHTSA) was not funding this project instead of DOE (possibly due to DOT funding constraints).

\section{Reviewer 4:}

The reviewer stated that the use of DOE funding to develop/deliver training and associated outreach/marketing for these underserved audiences is critically important and necessary. The project will provide these audiences with technical experience working with AFVs and advanced technology vehicles, thus filling a critical gap in educational opportunities, reducing apprehension and resistance to supporting these new fuels/technologies, and breaking down barriers. There appears to be some overlap between this project and the National Fire Protection Association (NFPA) project, and DOE should work with the project teams to minimize duplication [DOE Program Clarification: Each training project has a different target audience and focuses on different geographic regions in the country. So, duplication and overlap of training initiatives are minimized. DOE project officers with oversight for this work also coordinate to further ensure that information and resources are broadly leveraged while each project remains separate and distinct.]. 


\section{Nationwide AFV Emergency \\ Responder, Recovery, \\ Reconstruction and \\ Investigation Training: Andrew \\ Klock (National Fire Protection \\ Association) - ti067}

\section{Presenter}

Andrew Klock, National Fire Protection

Association

\section{Reviewer Sample Size}

A total of five reviewers evaluated this project.

Question 1: Project

objectives-the degree to

which the project objectives

support the DOE/VTO

objectives of reducing reliance

on petroleum based fuels

and reducing emissions. This

includes the impact the project

has on addressing the technical

barriers from the Vehicle

Technologies Office (VTO)

Multi-Year Program Plan.

\section{Reviewer 1:}

The reviewer found the development and execution of free Train-the-Trainer (T-t-T), classroom, and online AFV safety training for fire professionals and responders was a highly valuable activity. The objective of creating stronger relationships between NFPA and OEMs to bolster safety data sharing was critical.

\section{Reviewer 2:}

The reviewer agreed that there is definitely a need for training in the emergency medical services (EMS), fire, and salvage communities. This partnership, anchored by the NFPA, had the right partners and depth of relevant experience to produce this kind of specialized training.

\section{Reviewer 3:}

The reviewer stated that the project objective and overview slides described the project's specific objectives, which appeared to be generally effective.

\section{Reviewer 4:}

The reviewer was surprised but satisfied with the inclusion of biodiesel in the scope of alternative fuels covered. The reviewer was impressed with the comprehensiveness of the passenger car makes/models covered.

\section{Reviewer 5:}

The reviewer found that overall, the project addressed barriers in outreach to unique stakeholders, but was in the very early stages of deploying the material.

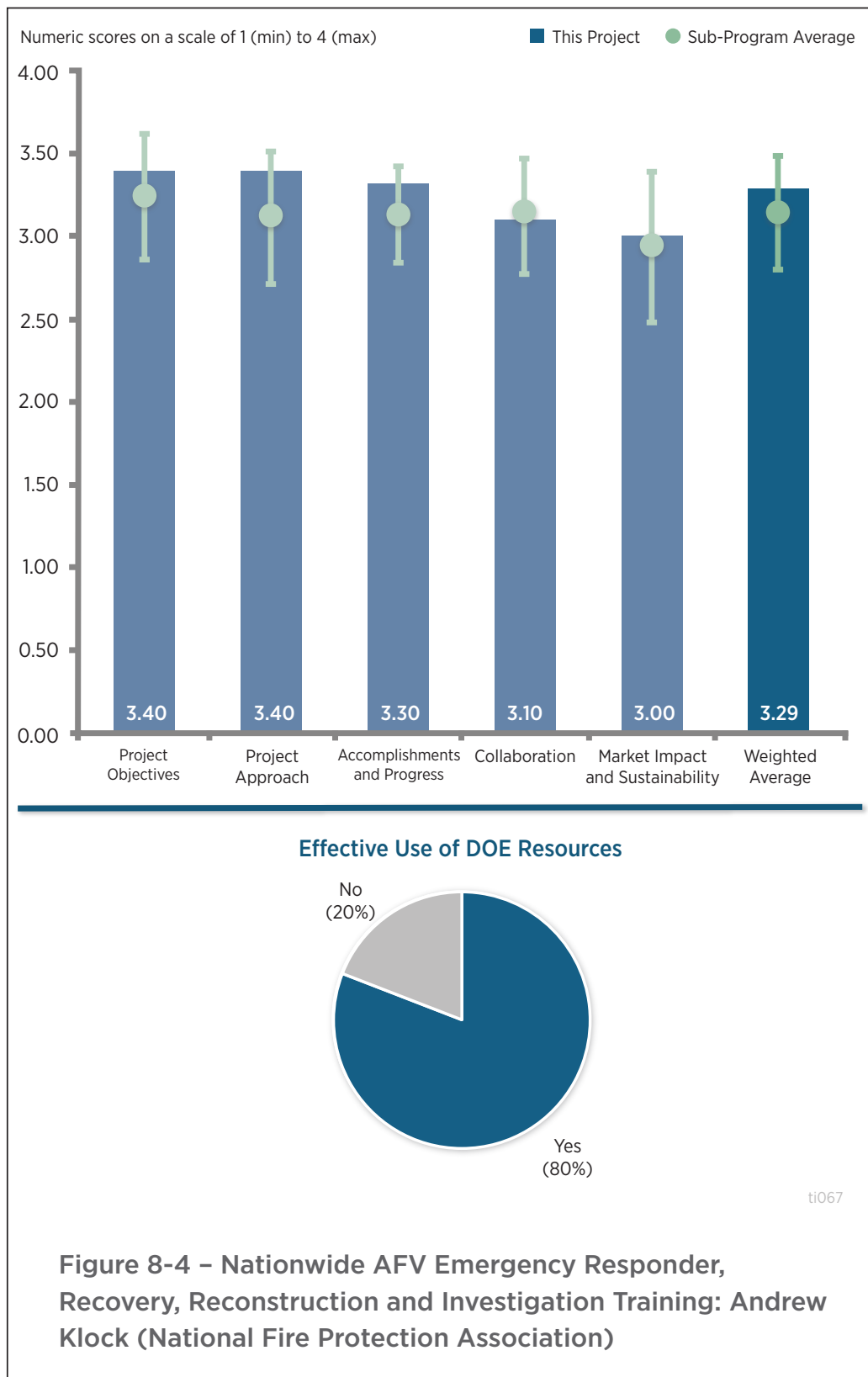


Question 2: Project approach to supporting deployment of petroleum reduction technologies and practices, alternative fuel vehicles, infrastructure, emissions reductions and related efforts-the degree to which the project is well-designed, feasible, and integrated with other efforts.

\section{Reviewer 1:}

The reviewer found that the project included a comprehensive approach and thorough strategy for implementation.

\section{Reviewer 2:}

The reviewer stated that the project approach seemed to be effective and contributed to achieving the majority of project objectives.

\section{Reviewer 3:}

The reviewer thought the project approach/relevance section provided a detailed and effective methodology to accomplish the project objectives. Significant detail was provided on the approach and milestone slides with regards to the planned tasks and activities, and risks/barrier analysis.

\section{Reviewer 4:}

The reviewer particularly liked the fact that the project was making the instructional materials online and free of charge. The reviewer stated that safety is everyone's business regardless of whether it is the public or private sector; there should be no charge for safety advice. The reviewer would have liked to have seen a state department of transportation traffic operations and state department of transportation highway incident response organization as consultants. The project team did not seem to take into full account potential bridge, tunnel, and highway restrictions on AFVs or towing of AFVs.

\section{Reviewer 5:}

The reviewer stated that this program seemed to take a fuel-specific approach, as opposed to a vehicle-specific approach. Although a vehicle-specific guide was available from the anchor organization, it was not highlighted in the presentation. The reviewer thought that the scope and breadth of partnerships with industry trade groups, like salvage and towing associations, could have been expanded to help address their stated barrier of disseminating information. The project team obviously had a strong presence and credibility within the fire and EMS community.

\section{Question 3: Project accomplishments and progress toward overall project and DOE goals - the degree to which progress/significant accomplishments have been achieved, measured against performance indicators and demonstrated progress toward project and DOE goals.}

\section{Reviewer 1:}

The reviewer found that the progress to date and the numbers were impressive.

\section{Reviewer 2:}

The reviewer stated that the project was on pace with stated goals, and video components were especially well crafted.

\section{Reviewer 3:}

The reviewer commented that effective progress had been made towards achieving Year 1 project goals. Activities related to the formation of the Technical Advisory Panel, OEM partnership development, fire service and investigation classroom module development, and video development were completed. The reviewer added that all initiatives and activities appeared to be on track for successful completion. The reviewer had no concerns identified.

\section{Reviewer 4:}

The reviewer stated that at the current stage of the project, the accomplishments and progress were satisfactory. 


\section{Reviewer 5:}

The reviewer commented that the developed curriculum materials were very high quality (especially video modules). However, the reviewer noted that the project was $50 \%$ complete, and no classroom trainings had yet been held at time of AMR review presentation submission (though they have begun in June).

Question 4: Collaboration and coordination among project team-the degree to which the appropriate team members and partners are involved in the project work and the effectiveness of the collaboration between and among partners.

\section{Reviewer 1:}

The reviewer noted that the project made good progress in establishing AFV OEM partners to participate in curriculum development.

\section{Reviewer 2:}

The reviewer noted that an effective project team was assembled to carry out this project, with government, industry, codes and safety organizations, and Clean Cities Coalition partners involved, which provided an excellent mix of expertise among team members. Project team roles were defined and collaboration/communication among project partners appeared to be appropriate for a project of this scope.

\section{Reviewer 3:}

The reviewer had similar comments to the project out of West Virginia University. The reviewer was delighted that the project sought out and included as stakeholders/partners the ARA. However, the reviewer did not see the TRAA, NATA, or the IITR.

\section{Reviewer 4:}

The reviewer found it unfortunate that the project was not leveraging with other similar projects within the VTO portfolio, regardless of how the information is disseminated. The reviewer thought it was nice to see the project leveraging the right folks on hydrogen, the Pacific Northwest National Laboratory (PNNL), and the California Fuel Cell Partnership (CaFCP). The reviewer added that one stakeholder of interest could be the insurance industry, and asked how international activities could be best leveraged.

\section{Reviewer 5:}

The reviewer would have liked to see more collaboration/sharing with other grantees who created similar curriculum programs, to maximize the effectiveness and reach of both. There were areas of overlap between the two projects in terms of subject matter and intended audience, and each brought a unique approach or angle to their projects. Better collaboration between grantees could have served the end-user better and more fully advanced DOE's objectives.

Question 5: Market impact and sustainability-the degree to which the project has already contributed, as well as the potential to continue to contribute in the future, to a sustainable alternative fuel vehicle market, alternative fuel market expansion, and reduced petroleum dependence/greenhouse gas emissions in the transportation sector. This would include the potential to reduce barriers to large scale alternative fuel vehicle market penetration, making information about alternative fuels and petroleum reduction opportunities widely available to target audiences, and ability for the project to be replicated in other geographic areas or with other technologies.

\section{Reviewer 1:}

The reviewer thought that the project had good potential to contribute to reduced petroleum dependence, GHG emissions reduction, and AFV market sustainability goals by determining best practices and disseminating knowledge from OEMs, subject matter experts (SMEs), and national laboratories to emergency responders/ investigators.

\section{Reviewer 2:}

The reviewer stated that the NFPA is a well-established organization with deep ties to the fire/EMS community and a full slate of training and curriculum projects, with which this project will integrate nicely. 


\section{Reviewer 3:}

The reviewer stated that the T-t-T model and other online training tools provided a reasonable strategy for promoting curricula sustainability and replication. Other emergency responder audiences - besides fire professionals - are allowed to participate in classroom trainings; however, no specific strategy for recruiting their attendance was presented by the PI.

\section{Reviewer 4:}

The reviewer commented that the project could have tremendous effectiveness and contribute to overcoming most barriers and informing appropriate audiences about the AFV market expansion. The reviewer noted that it was still early to determine the degree of impact.

\section{Reviewer 5:}

The reviewer did see a need for providing emergency responder training on AFVs, but said it was a very long stretch that such training would significantly improve the market for AFVs and sustainability. The reviewer acknowledged perhaps not seeing the survey data, but the reviewer had never heard a consumer ask if emergency responders were prepared to deal with incidents involving AFVs before buying one.

\section{Question 6: Use of resources-are DOE funds being used wisely? Should DOE fund similar efforts in the future? If not, what would be a better use of DOE resources to achieve alternative fuel vehicle and infrastructure expansion to support the broader goal of petroleum displacement and greenhouse gas reductions?}

\section{Reviewer 1:}

The reviewer stated that this was a solid partnership, a well-crafted project, and a great use of resources.

\section{Reviewer 2:}

The reviewer stated that the use of DOE funding to educate the nationwide safety communities will help keep responders and the public safe, promote AFV acceptance, and avoid serious high profile events, which is critically important and necessary. There appeared to be some overlap between this project and the West Virginia University project and DOE should have ensured that these two projects had complimentary versus duplicative efforts.

\section{Reviewer 3:}

The reviewer said that the NFPA and West Virginia University projects appear to be very similar, and that DOE should work with project managers to minimize overlap and duplication of work being supported with Federal funds. Second, the reviewer wondered why the DOT-National Highway Traffic Safety Administration (NHTSA) was not funding this project instead of DOE (possibly due to DOT funding constraints) [DOE Program Clarification: Each training project has a different target audience and focuses on different geographic regions in the country. So, duplication and overlap of training initiatives are minimized. DOE project officers with oversight for this work also coordinate to further ensure that information and resources are broadly leveraged while each project remains separate and distinct.]. 
Safe Alternative Fuels Deployment in Mid-America (The SAF-D Project): Kelly Gilbert (Metropolitan Energy Center, Inc.) - ti068

Presenter

Kelly Gilbert, Metropolitan Energy

Center, Inc.

\section{Reviewer Sample Size}

A total of five reviewers evaluated this project.

\section{Question 1: Project} objectives-the degree to which the project objectives support the DOE/VTO objectives of reducing reliance on petroleum based fuels and reducing emissions. This includes the impact the project has on addressing the technical barriers from the Vehicle Technologies Office (VTO) Multi-Year Program Plan.

\section{Reviewer 1:}

The reviewer stated that the project had a strong objective of removing financial and logistical barriers to deploying AFV safety training to fire and rescue/ responders in Kansas and Missouri.

\section{Reviewer 2:}

The reviewer stated that, overall, the project addressed barriers in outreach to unique stakeholders, but was in the very early stages of deploying the material.

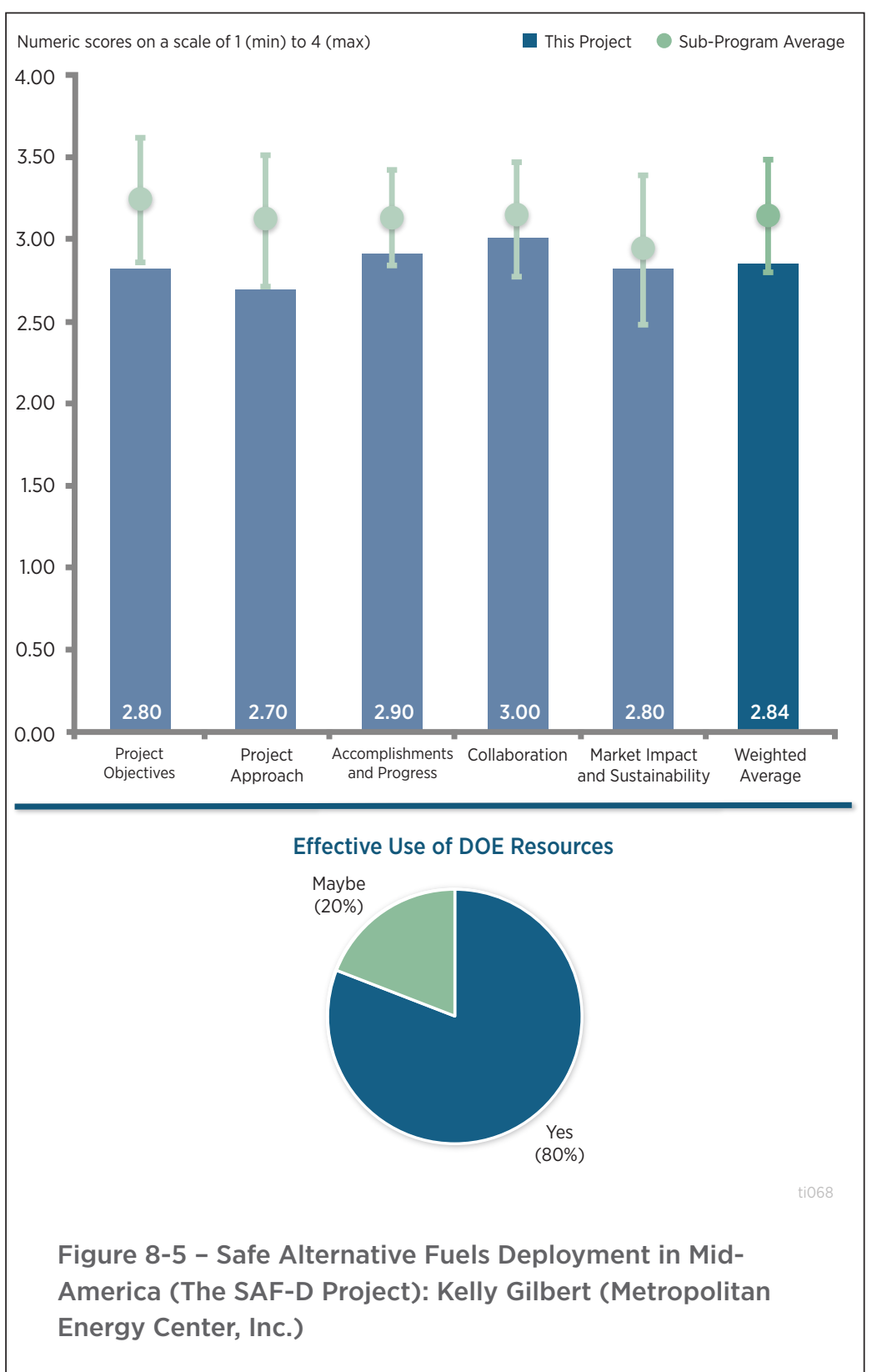

\section{Reviewer 3:}

The reviewer stated that the project objective and overview slides described the project's specific objectives, as well as how the project addressed specific barriers in the VTO's Multi-Year Program Plan 2011-2015. The reviewer noted that the project objectives appeared to be generally effective.

\section{Reviewer 4:}

The reviewer commented that the project objectives were certainly valid and fit within DOE's stated area of need. The reviewer was not convinced that the regional nature and limited scope of the project justified the need to spend resources to develop new training that exists or is in development on the national level.

\section{Reviewer 5:}

The reviewer said that the project did not include an assessment of the needs in the mid-America area or a business case analysis. The reviewer said that an assessment should have been performed to determine how many different types of AFVs there are in Kansas and Missouri, how many vehicles there are of each type, and where they are primarily concentrated (i.e., urban, suburban or rural and, if urban, which cities). Second, the reviewer thought it was presumptuous to say that Kansas and Missouri needed emergency response training on AFV incidents. An 
assessment should have been performed to determine where there are AFVs, and which agencies in the areas lack the training to deal with incidents involving such vehicles. The reviewer also asked if there are enough AFVs in Kansas and Missouri to justify such training. For example, if an area has only one or two such vehicles, the reviewer asked if hazmat could deal with such incidents.

\section{Question 2: Project approach to supporting deployment of petroleum reduction technologies and practices, alternative fuel vehicles, infrastructure, emissions reductions and related efforts-the degree to which the project is well-designed, feasible, and integrated with other efforts.}

\section{Reviewer 1:}

The reviewer noted that the project approach was smart by leveraging existing training infrastructure that is already widely established and used to dispense/disseminate training to fire professionals and first responders.

\section{Reviewer 2:}

The reviewer stated that the project approach seemed to be effective and contributed to achieving the majority of project objectives.

\section{Reviewer 3:}

The reviewer stated that the project approach section provided an effective methodology to accomplish the project objectives. The project team provided appropriate detail on the approach and milestone slides with regards to the planned tasks and activities.

\section{Reviewer 4:}

The reviewer thought that the need for this type of training is undeniable, but added that effective programs already existed or were being developed by other grantees with stronger backgrounds in this specific area. Some questions remained on exactly how the online portion will be made interactive, despite the interactive part being touted heavily in the presentation as a differentiator between this project and others.

\section{Reviewer 5:}

The reviewer expressed concern that the Safe Alternative Fuels Deployment in Mid-America (SAF-D) Project appears to duplicate work being done by NFPA and West Virginia University under their projects. The SAF-D Project should have been aware of the other two curricula and worked to avoid duplication and/or pointed out how its curriculum differs from the other two [DOE Program Clarification: Each training project has a different target audience and focuses on different geographic regions in the country. So, duplication and overlap of training initiatives are minimized. DOE project officers with oversight for this work also coordinate to further ensure that information and resources are broadly leveraged while each project remains separate and distinct.].

The reviewer stated that contrary to what was indicated on Slide 7, the SAF-D Project did not leverage previous DOE investments. In addition, the reviewer thought that Slides 7 and 15 needed to be corrected: Intellectual property (patented) developed with U.S. Government funds may be allowed royalty-free use by the U.S. government as so designated. Documents and instructional materials developed with U.S. government funds may not be copyrighted and are in the public domain.

\section{Question 3: Project accomplishments and progress toward overall project and DOE goals - the degree to which progress/significant accomplishments have been achieved, measured against performance indicators and demonstrated progress toward project and DOE goals.}

\section{Reviewer 1:}

The reviewer stated that the project was working through clear milestones, though a substantial amount of work remained to be conducted in the remaining project period.

\section{Reviewer 2:}

The reviewer thought that at the current stage of the project, the accomplishments and progress were satisfactory. 


\section{Reviewer 3:}

The reviewer stated that good progress had been made towards achieving Year 1 project goals. Activities related to establishing teaming arrangements and classroom curriculum development were underway and appear to be on track for successful completion. The reviewer identified no concerns.

\section{Reviewer 4:}

The reviewer stated that the project had set attainable goals for itself, but the reviewer got the sense that the project team was lagging behind in development of the curriculum, particularly the online portion.

\section{Question 4: Collaboration and coordination among project team-the degree to which the appropriate team members and partners are involved in the project work and the effectiveness of the collaboration between and among partners.}

\section{Reviewer 1:}

The reviewer stated that the project had a strong core of partners with relevant experience in the subject matter, especially the North American Fire Training Directors (NAFTD) and state training organizations.

\section{Reviewer 2:}

The reviewer stated that an effective project team was assembled to carry out this project, with State Fire and Rescue Training Institutes in Missouri and Kansas, and the Kansas City Clean Cities Coalition involved, which provided an appropriate mix of expertise among team members. Project team roles were defined and collaboration/ communication among project partners appeared to be appropriate for a project of this scope.

\section{Reviewer 3:}

The reviewer stated that the project's interdisciplinary project team appeared well coordinated to execute the project. Some further information on the project's curriculum development partner (capacities, experience, and role) would have been helpful for reviewer understanding.

\section{Reviewer 4:}

The reviewer thought it is unfortunate that the project was not leveraging other similar projects within the VTO portfolio, regardless of how the information is disseminated.

\section{Reviewer 5:}

The reviewer commented that the only partner that made the SAF-D different or unique from the similar efforts of NFPA and West Virginia University was NAFTD. However, it was not clear if the responsibility for national dissemination of the training was going to fall wholly on NAFTD or what NAFTD was going to do. The reviewer stated that this definitely has to be clarified.

Question 5: Market impact and sustainability-the degree to which the project has already contributed, as well as the potential to continue to contribute in the future, to a sustainable alternative fuel vehicle market, alternative fuel market expansion, and reduced petroleum dependence/greenhouse gas emissions in the transportation sector. This would include the potential to reduce barriers to large scale alternative fuel vehicle market penetration, making information about alternative fuels and petroleum reduction opportunities widely available to target audiences, and ability for the project to be replicated in other geographic areas or with other technologies.

\section{Reviewer 1:}

The reviewer thought that the project did a good job targeting rural fire departments and responder professionals. The project incorporated a T-t-T component that was intended to replicate and advance training material dissemination beyond the project period.

\section{Reviewer 2:}

The reviewer stated that the project may have contributed to reduced petroleum dependence, GHG emissions reduction, and AFV market sustainability goals by developing and delivering training courses for fire and public safety personnel to train on alternative fuel safety. 


\section{Reviewer 3:}

The reviewer stated that the project could have tremendous effectiveness and contribute to overcoming most barriers and informing appropriate audiences to the AFV market expansion. It is still early to determine the degree of impact.

\section{Reviewer 4:}

The reviewer stated that integrating this curriculum into existing training programs and including T-t-T components would ensure that this curriculum has a shelf-life well beyond the project period. The reviewer was also concerned that not having the depth of experience or the inventory of related training programs of other grantees may limit the long-term effectiveness of this project, as technology evolves and new models enter the market.

\section{Reviewer 5:}

The reviewer did see a need for providing emergency responder training on AFVs, but said it was a very long stretch that such training would significantly improve the market for AFVs and sustainability. Perhaps the reviewer had not seen the survey data, but the reviewer had never heard a consumer ask if emergency responders were prepared to deal with incidents involving AFVs before buying one.

\section{Question 6: Use of resources-are DOE funds being used wisely? Should DOE fund similar efforts in the future? If not, what would be a better use of DOE resources to achieve alternative fuel vehicle and infrastructure expansion to support the broader goal of petroleum displacement and greenhouse gas reductions?}

\section{Reviewer 1:}

The reviewer stated that this program was disseminating valuable information into a targeted market, and would definitely help further the DOE's stated objectives.

\section{Reviewer 2:}

The reviewer stated that the use of DOE funding to develop/deliver training for fire and safety officials was critically important and necessary. Once the training programs are incorporated into state fire training centers, adoptions of gaseous fueled vehicles will accelerate due to fewer objections from well-prepared critical emergency and mechanical services personnel.

\section{Reviewer 3:}

The reviewer noted that another reviewer commented about Kansas University Fire retaining all copyright to developed course materials. Although Kansas University is expected to grant free use licenses to Clean Cities and fire safety trainers, a valid question was raised as to whether or not the government should actually be free to distribute or deliver any developer materials as it chooses to beyond the project period.

\section{Reviewer 4:}

The reviewer questioned why three projects are being funded for the same purpose. The reviewer also asked how this project is sufficiently different from the other two to justify the funding [DOE Program Clarification: Each training project has a different target audience and focuses on different geographic regions in the country. So, duplication and overlap of training initiatives are minimized. DOE project officers with oversight for this work also coordinate to further ensure that information and resources are broadly leveraged while each project remains separate and distinct.]. 
Initiative for Resiliency in Energy through Vehicles (IREV): Cassie Powers (National Association of State Energy Officials) - ti069

\section{Presenter}

Cassie Powers, National Association of State Energy Officials

\section{Reviewer Sample Size}

A total of five reviewers evaluated this project.

\section{Question 1: Project}

objectives-the degree to which

the project objectives support the DOE/VTO objectives of reducing reliance on petroleum based fuels and reducing emissions. This includes the impact the project has on addressing the technical barriers from the Vehicle Technologies Office (VTO) Multi-Year Program Plan.

\section{Reviewer 1:}

The reviewer stated that the project had a strong central objective to educate and coordinate local/state/ regional emergency response and planning entities on the incorporation of alternative fuels into their long-term plans.

\section{Reviewer 2:}

The reviewer stated that this project targeted an under-served audience, and addressed a crucial need to coordinate strategies among regional and national organizations. The reviewer thought it provided an innovative approach to increasing the use of AFVs on a large scale.

\section{Reviewer 3:}

The reviewer believed that the project objectives were focused on supporting the VTO mission and the project had a direct and substantial impact on addressing technical barriers.

\section{Reviewer 4:}

The reviewer stated that the project objective and overview slides described the project's specific objectives, as well as how the project addressed specific barriers in the VTO's Multi-Year Program Plan 2011-2015. The reviewer noted that the project objectives appeared to be generally effective.

\section{Reviewer 5:}

The reviewer found it difficult to evaluate how much impact results from the use of AFVs during an emergency when those emergencies are infrequent. This uncertainty makes it in turn difficult to evaluate how much reduction

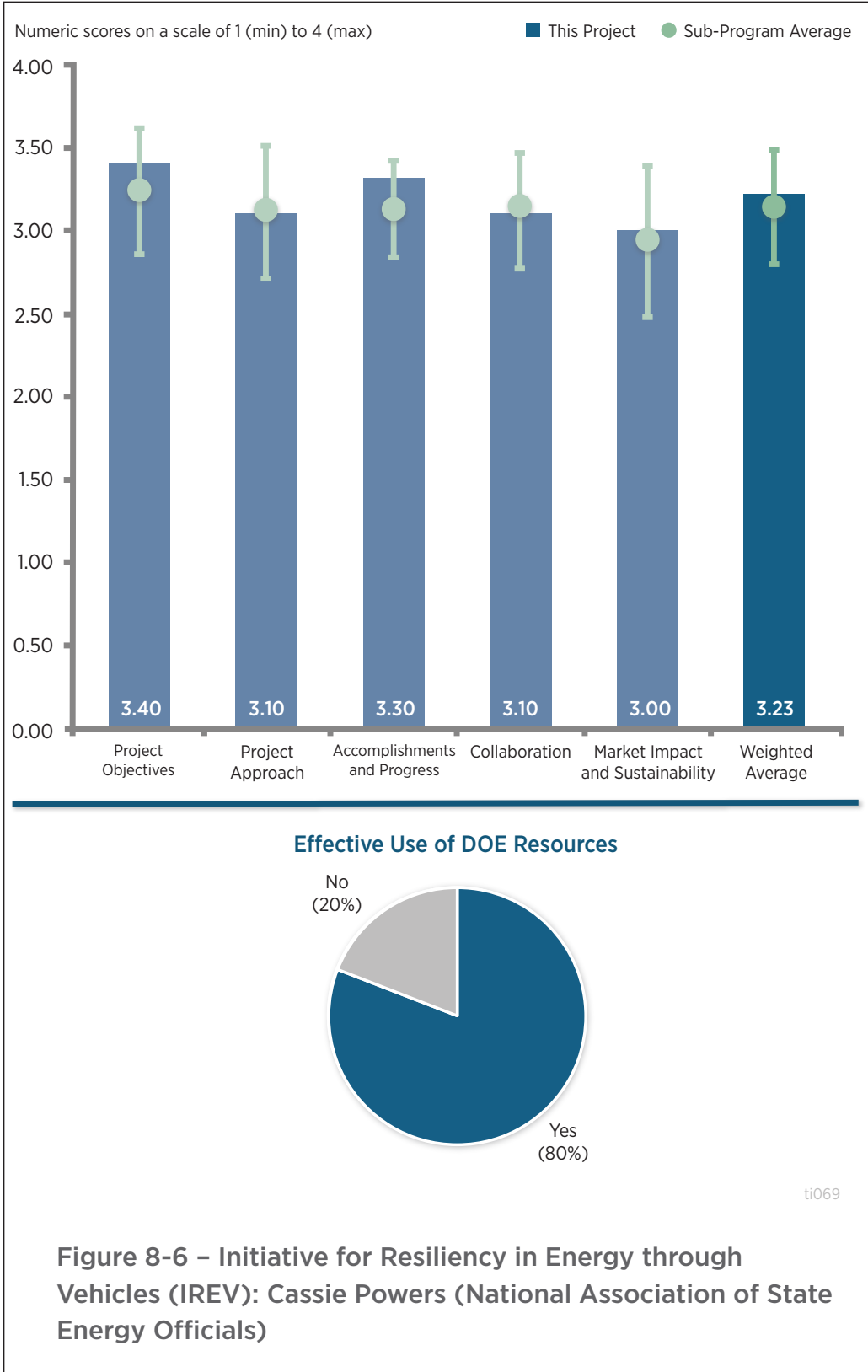

Figure 8-6 - Initiative for Resiliency in Energy through Energy Officials) 
in emissions, and in the use of petroleum-based fuels resulted from the use of AFVs during emergencies. This type of evaluation was not undertaken by this particular project. The reviewer opined that because emergencies are so infrequent, the use of AFVs during emergencies would have hardly any appreciable effect on reduction of emissions and use of petroleum-based fuels.

\section{Question 2: Project approach to supporting deployment of petroleum reduction technologies and practices, alternative fuel vehicles, infrastructure, emissions reductions and related efforts-the degree to which the project is well-designed, feasible, and integrated with other efforts.}

\section{Reviewer 1:}

The reviewer stated that compiling critical fleet data and organizing existing information in new ways to help emergency preparedness organizations and officials integrate AFV into their long-term planning was a solid approach. The project identified a unique need, appeared to be well-researched, and had a solid plan to disseminate this information to key people. Finally, the reviewer believed the Initiative for Resiliency in Energy through Vehicles tool to be simple to use and easy to update.

\section{Reviewer 2:}

The reviewer stated that the project approach seemed to be effective and contributed to achieving the majority of the project's objectives.

\section{Reviewer 3:}

The reviewer found that the project approach section provided an effective methodology to accomplishing the project objectives. Appropriate detail was provided on the approach and milestone slides with regards to the planned tasks and activities.

\section{Reviewer 4:}

The reviewer stated that co-locating AFV safety workshops with existing national events for emergency planners was a smart approach. The project involved a comprehensive approach, assessed existing emergency plans among 20 largest U.S. municipalities, created tools and resources, educated stakeholders, and laid the groundwork for incorporating alternative fuels into emergency plans nationwide. The reviewer noted that criteria or methodology for selecting cities and regions (for workshops and pilots) were not entirely clear.

\section{Reviewer 5:}

The reviewer did not find the approach to this project to be well thought out and strategized. At the time of the presentation, the project seemed to target all 50 states across the nation, even when it may not have been appropriate to do so in some places. The reviewer commented that the project could be much better focused starting on geographic regions of the country that both encounter emergencies (e.g., hurricanes, tornados, floods, earthquakes, etc.) and have AFVs and fueling infrastructure already in place. Furthermore, the reviewer stated that the scope seemed to be narrowed to alternative fuel automobiles. The reviewer found absolutely no reason why alternative fuel vans, trucks, and buses should have been ignored or excluded. The reviewer referenced more detailed comments in the Collaboration and Coordination section.

\section{Question 3: Project accomplishments and progress toward overall project and DOE goals - the degree to which progress/significant accomplishments have been achieved, measured against performance indicators and demonstrated progress toward project and DOE goals.}

\section{Reviewer 1:}

The reviewer stated that the case studies and reports on how AFVs have been incorporated into existing emergency plans were very valuable. The project appeared to be making steady progress towards achieving objectives.

\section{Reviewer 2:}

The reviewer found that significant progress had been made towards achieving Year 1 project goals. Activities related to establishing the project steering committee and tools/resource development were underway, and appeared to be on track for successful completion. The reviewer identified no concerns. 


\section{Reviewer 3:}

The reviewer stated that at the current stage of the project, the accomplishments and progress were satisfactory.

\section{Reviewer 4:}

The reviewer stated that the project established attainable goals and appeared to be on schedule. The reviewer worried that coordinating with a large number of emergency planning groups and convincing them to get on board with the inclusion of AFVs in their master plans will not be an easy task.

\section{Reviewer 5:}

The reviewer noted that no target performance goals were given for Year 1 .

Question 4: Collaboration and coordination among project team-the degree to which the appropriate team members and partners are involved in the project work and the effectiveness of the collaboration between and among partners.

\section{Reviewer 1:}

The reviewer found the project was based on a highly cooperative structure among a broad range of states, emergency managers, and alternative fuel communities.

\section{Reviewer 2:}

The reviewer stated that the core partners in this project had strong ties and credibility within the emergency planning community. It appeared that the team was enjoying excellent collaboration and coordination among its members.

\section{Reviewer 3:}

The reviewer commented that an effective project team was assembled to carry out this project, with International Association of Emergency Managers (IAEM), National Governors Association (NGA), alternative fuel industry trade organizations, and seven Clean Cities Coalitions involved, which provided an excellent mix of expertise among team members. The roles of the project team were defined and collaboration/communication among project partners appeared to be appropriate for a project of this scope.

\section{Reviewer 4:}

The reviewer stated that the project seemed to have effective collaboration with industry to continue to build on what has been started.

\section{Reviewer 5:}

The reviewer strongly recommended the inclusion of additional stakeholders and stronger collaboration and coordination among project partners. In being resilient to an emergency, public utilities, such as electric, gas, water supply, sewage, telephone, internet, and communications need to be included. Alternative fuel trucks of public utility companies can play a critical role in recovery and restoration after a widespread emergency.

The reviewer also commented that state trucking associations needed to be involved. Trucks bring necessary provisions such as fuel, food, and other staple items for people, as well as much needed supplies and equipment for recovery and restoration after a widespread emergency. Thus, the reviewer thought alternative fuel trucks needed to be considered.

Finally, the reviewer said that buses needed to be involved, as they are needed for evacuation of residents and bringing in relief workers. Furthermore, the reviewer said that the transit bus fleets are the most aggressive adopters of alternative fuel (besides the refuse industry), so transit agencies needed to be involved as stakeholders and partners, and the American Public Transportation Association (APTA) should be made a national stakeholder and partner.

Question 5: Market impact and sustainability-the degree to which the project has already contributed, as well as the potential to continue to contribute in the future, to a sustainable alternative fuel vehicle market, alternative fuel market expansion, and reduced petroleum dependence/greenhouse gas emissions in the transportation sector. This would include the potential to reduce barriers to large scale alternative fuel vehicle 
market penetration, making information about alternative fuels and petroleum reduction opportunities widely available to target audiences, and ability for the project to be replicated in other geographic areas or with other technologies.

\section{Reviewer 1:}

The reviewer thought that the PI highlighted several great examples of how communities were able to leverage alternative fuels in emergency management (Florida Power and Light's use of biodiesel; Pacific Gas and Electric conducting power-takeoff with bucket trucks; New Jersey shore communities using heavy-duty natural gas vehicles $[\mathrm{NGVs}]$ during post-hurricane power disruptions, etc.). The reviewer indicated that the project had good potential to help communities/states share information on these successful cases and examples.

\section{Reviewer 2:}

The reviewer noted that the project could have tremendous effectiveness, contributing to overcoming most barriers, and informing appropriate audiences about the AFV market expansion. The reviewer noted that it was still early to determine the degree of impact.

\section{Reviewer 3:}

The reviewer stated that the project had potential to contribute to reduced petroleum dependence, GHG emissions reduction, and AFV market sustainability goals. This would be accomplished by creating customized tools and providing information for emergency management decision-makers to examine the potential costs, benefits, and trade-offs of incorporating alternative fuels into their plans.

\section{Reviewer 4:}

The reviewer commented that disaster plans are not revised frequently, so any inclusion of AFVs in these plans will remain for a long time. The reviewer trusted that NASEO will continue to monitor AFV fleets and provide updated information to relevant parties as needed.

\section{Reviewer 5:}

The reviewer referenced prior comments from Question 1. The reviewer did not think this project will have any appreciable impact on the market for AFVs and on sustainability because of the infrequency of emergencies.

\section{Question 6: Use of resources-are DOE funds being used wisely? Should DOE fund similar efforts in the future? If not, what would be a better use of DOE resources to achieve alternative fuel vehicle and infrastructure expansion to support the broader goal of petroleum displacement and greenhouse gas reductions?}

\section{Reviewer 1:}

The reviewer found the project to be a great idea, and was well-planned and executed.

\section{Reviewer 2:}

The reviewer stated that there were a lot of expanding activities among the larger transportation planning community focusing on climate resiliency. This project represented a good start towards integrating alternative fuels and AFVs into this emerging topic area.

\section{Reviewer 3:}

The reviewer found that the use of DOE funding to develop customized resources and tools for emergency management officials, to increase the prevalence of alternative fuels in existing and future state and local emergency response and planning operations, was appropriate. The reviewer also found that DOE funding was necessary to reach new audiences that may not otherwise realize the role alternative fuels could play in their emergency response plans.

\section{Reviewer 4:}

The reviewer did not think that this project makes the best use of DOE funds. The reviewer thought that if DOE wanted to cover the union of emergencies and AFVs, it should have looked at getting fire departments, rescue services, ambulances, police departments, and other first responders to deploy more AFVs. The reason is that first of all, the kinds of emergencies that these first responders handle are more regular, and thus, would have greater 
impact on emissions reduction and petroleum-based fuel use than irregular emergencies that fall under the province of emergency managers. Secondly, the reviewer pointed out that there is a lot of idling that needs to be reduced in the conventional vehicles used by these first responders. Therefore, replacing them with AFVs would significantly reduce emissions. The reviewer suggested a program to incentivize natural gas fire trucks. 


\section{EcoCAR 3: Kristen Wahl (Argonne National Laboratory) - ti070}

\section{Presenter}

Kristen Wahl, Argonne National Laboratory

\section{Reviewer Sample Size}

A total of five reviewers evaluated this project

\section{Question 1: Project objectives- the degree to which the project objectives support the DOE/ VTO objectives of reducing reliance on petroleum based fuels and reducing emissions. This includes the impact the project has on addressing the technical barriers from the Vehicle Technologies Office (VTO) Multi-Year Program Plan.}

\section{Reviewer 1:}

The reviewer stated that the project represented a strong ongoing advanced technology vehicle workforce development activity.

\section{Reviewer 2 :}

The reviewer stated that the objectives of workforce development, demonstration of energy-efficient powertrains, alternative fuels and innovative technologies, and their impact on reducing environmental impact, supported the overall VTO goal of reducing dependence on petroleum-based fuels.

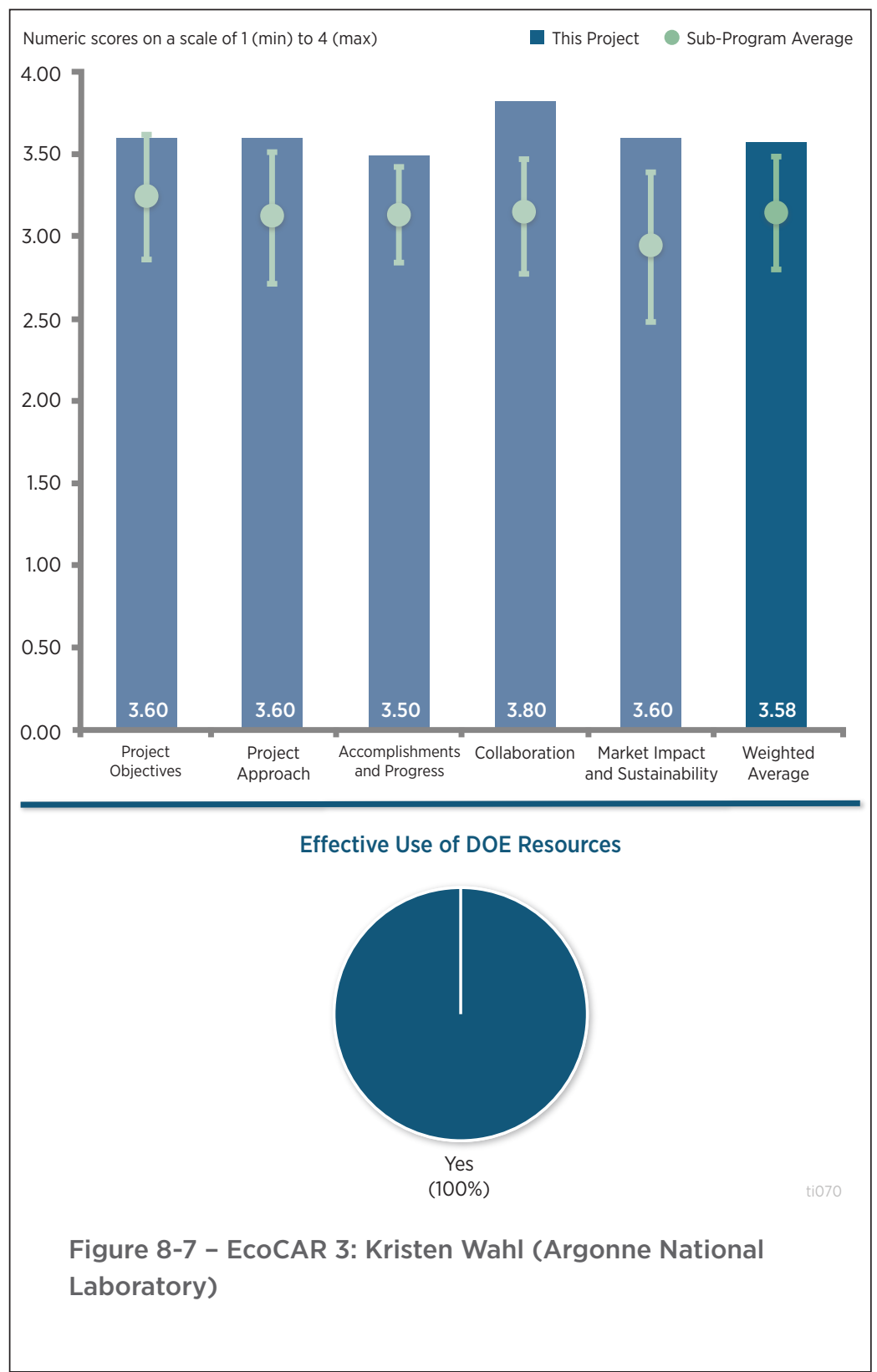

\section{Reviewer 3:}

The reviewer commented that this project clearly supports VTO's objectives, particularly in addressing the lack of trained engineers and scientists and the lack of advanced vehicle technology curricula. The project has a strong collaboration with industry, government, and universities. It is very impressive that since 1988, more than 17,000 graduates of Advanced Vehicle Technology Competitions (AVTC) have worked in the automotive industry and that more than $80 \%$ of AVTC graduates work in the automotive industry. This competition provides a strong impact by training students to become ready for work in industry. The reviewer remarked that for Slide 3, it is hard to gauge how good the number is of 53\% of AVTC alumni that have credit for producing intellectual property. Please show what the number is for non-AVTC alumni.

\section{Reviewer 4:}

The reviewer noted that the project had a number of objectives that clearly addressed barriers to the VTO and DOE goals. The EcoCAR3 competition activities addressed workforce development directly, through the students involved in the training and hands-on experience during the competition, as well as less directly by developing curricula that is made openly available to other academic institutions. 
Though the presentation reported on a number of quantifiable outcomes, such as the number of Science, Technology, Engineering, and Mathematics (STEM) events and the number of program graduates since the beginning of the AVTC program, it was not clear if there were project targets or objectives for these outcomes on an annual/competition cycle basis. The reviewer commented that these could be useful in understanding the yearto-year objectives and how much progress is being made towards them.

\section{Reviewer 5:}

The reviewer noted that the leading university-level engineering competition project was a Workforce Development Program, so it did not develop or demonstrate commercial-intent petroleum and emissions reducing technologies. That said, this program squarely addresses a crucial U.S. automotive industry (OEMs and Tier system manufacturers) need to develop the next generation of automotive engineers and related necessary disciplines (e.g., marketing, business, communications, project management, etc.) in the United States. This directly addressed the program's barriers of a lack of trainer engineers and scientists, and a lack of advanced vehicle technology (AVT) curricula.

\section{Question 2: Project approach to supporting deployment of petroleum reduction technologies and practices, alternative fuel vehicles, infrastructure, emissions reductions and related efforts-the degree to which the project is well-designed, feasible, and integrated with other efforts.}

\section{Reviewer 1:}

The reviewer stated that the multi-year vehicle development process modeled after the auto industry was an excellent way to prepare future engineers to be able to walk into their first position with an OEM and would allow them to provide near immediate contributions to the OEM.

\section{Reviewer 2:}

The reviewer commented that the student curriculum was well designed, and followed a four-year development timeframe, mirroring the typical vehicle development timescale occurring in the auto industry. The reviewer noted that the project's curriculum was no longer just a mechanical engineering activity, but it invoked a much wider range of other technical and business/professional skills that are key in the auto industry.

\section{Reviewer 3:}

The reviewer stated that the EcoCAR3 approach of using a collegiate competition to train future AVT project teams, and also to develop curricula and demonstrate innovative technologies, supported both the project objectives and the DOE/VTO objectives of enabling AVTs by addressing issues related to workforce development, education, and training.

The reviewer found that the competition's plan of modeling the development process of the real-world auto industry, by following the multi-year Vehicle Development Process, and including not just engineers but also project and communication managers as part of the teams, and developing a multi-disciplinary curriculum, should make for an effective approach to addressing broader workforce development barriers.

\section{Reviewer 4:}

The reviewer said that the competition focuses on reducing petroleum use, total energy usage, and emissions on a variety of vehicle platforms. The project has a multifaceted approach on working both the technical aspects of the vehicles and the communications efforts of reaching out to the local communities. The only area that this reviewer sees that could be improved is that only 16 universities are selected. While this is understandable due to the huge scope of this competition, there could be a way to expand the scope of EcoCAR by having universities work together. The reviewer suggested that maybe there could be a lead university that has the car, but several other universities that support the effort by taking the curricula and doing activities that can be done offsite, for example computer design and additional communication efforts. The project could become even more interdisciplinary if this was done.

\section{Reviewer 5:}

The reviewer stated that as a workforce development program, the program does not deploy petroleum-reducing and other sustainable vehicle technologies. However, the program's student participants' outreach and public 
interactions support deployment of these technologies during the project. The students' impact while at work in industry post-project that the program enabled will be much greater because of the program. This reviewer explained that the combined group of vehicles use most fuels, powertrain architectures, options, etc., which allows for outreach to a wide range of audiences. The program's design squarely addressed a crucial U.S. automotive industry (OEMs and Tier system manufacturers) need to develop the next generation of automotive engineers and related necessary disciplines (e.g., marketing, business, communications, project management, etc.) in the United States. The program evolved to mimic the automotive design process (timing, steps, computational tools, experimental tools, etc.), which will allow students to transition into industry and contribute from the start. The reviewer stated that the interaction between industry (vehicle OEMs, suppliers, computational tool developers, etc.) was very good and helpful for improving the project and students' education/training. The technologies and vehicles were developed with a performance goal, but not a prescribed solution path. The reviewer commented that this was good and gave the teams freedom to explore more broadly than OEMs, which developed creativity and unique solutions. The reviewer found that the Advanced Driver Assistance Systems (ADAS) activity (new in 2016) was a nice addition, given the market interest and development of connected/autonomous vehicles. The reviewer noted that being a four-year program has created challenges for information and experience retention, and student motivation, as students cycle out of and into the program based on when they start college. This was not mentioned as a limitation/issue, but this reviewer expressed interest in knowing how the program (and universities) address this.

\section{Question 3: Project accomplishments and progress toward overall project and DOE goals - the degree to which progress/significant accomplishments have been achieved, measured against performance indicators and demonstrated progress toward project and DOE goals.}

\section{Reviewer 1:}

The reviewer found that the progress in this project was excellent in year two of the program, including vehicle integration, new progress management, communications programs, as well as media and public relations activities.

\section{Reviewer 2:}

The reviewer stated that the project's revised safety focus (improved structural modification waiver process, inspections, and systems safety activity) was well considered and added substantial value to the EcoCAR curricula.

\section{Reviewer 3:}

The reviewer remarked that the project has a broad reach and has many accomplishments. In addition, it appears that the project team is continually trying to improve the project, with the reviewer citing the new ADAS work as an example.

\section{Reviewer 4:}

The reviewer evaluated based on the impact on developing students into highly-skilled employees for the automotive industry, not on direct petroleum/emissions reduction. The reviewer noted that this was the $11^{\text {th }}$ generation of DOE-sponsored engineering design/business competitions (since 1988). Over this period, the program claimed over 17,000 students who have participated in the program. The presenter commented that this large number underestimated the impact because it only includes the core group of dedicated students on each team. Teams always have a much larger number of people who play a smaller role, but are also impacted by the project. The result was a much larger number of impacted students (over 100,000). The reviewer noted that the program outreach (both by the Argonne National Laboratory [ANL]-led EcoCAR3 headquarters (national) and by teams (in their local markets)) increased the awareness of, and interest in, the program and the sustainable vehicle technologies used by the teams to the broader market. This included the general population (consumers and students, to get them excited about working in the industry when they are older). The Model-Based Design Curriculum and Applied Automotive Engineering Curriculum were a good way to allow students at universities who were not in the competition to learn some of the same skills (for \$0).

\section{Reviewer 5:}

The reviewer found that substantial accomplishments and progress were reported, and the competition was running on-time based on the provided schedule. In addition to the activities directly related to the competition stages, other 
supporting accomplishments were reported, such as the addition of an ADAS activity and an innovation initiative developed in partnership with the National Science Foundation (NSF).

The focus on STEM outreach will help broaden the impacts of the work, particularly with the emphasis on diverse, underserved audiences. The reviewer stated that because the milestones reported were primarily timeline based, it was difficult to evaluate how the accomplishments measure against performance measures.

The reviewer commented that there were some areas where it would be useful to include more quantitative outcomes for the current year, or for the competition to date. For example, though the project reported having over 17,000 graduates since 1988, the total number of core competitors for this competition (either this year or for this competition cycle) was not reported.

\section{Question 4: Collaboration and coordination among project team-the degree to which the appropriate team members and partners are involved in the project work and the effectiveness of the collaboration between and among partners.}

\section{Reviewer 1:}

The reviewer said that the collaborations for EcoCAR are truly outstanding. It is very impressive to have the support of so many key industrial companies and universities. In addition, this project links to the Clean Cities Program, providing over 100 interns per year. The reviewer commented that the amount of additional cost-share that is provided for this project ( $\$ 915$ million) is staggering.

\section{Reviewer 2:}

The reviewer thought that the collaboration and coordination in this project was outstanding. The 16 university teams were able to work with a variety of OEMs and suppliers that helped to increase their knowledge base and provided an excellent source of contacts as the students move into the work force.

\section{Reviewer 3:}

The reviewer stated that student vehicle teams were 100 people in size and ranged across a breadth of disciplines, which taught students very important collaboration and team building skills. General Motors (GM) and OEM involvement with the project appeared to be very strong.

\section{Reviewer 4:}

The reviewer noted that the project had a very large number of partners (30) in addition to the competing university teams, and this broad level of collaboration was focused on, and important for, meeting the project objectives. Based on the reported outcomes, the collaboration of all these partners appeared to be very well managed and coordinated.

The reviewer commented that given the limited time for the presentation and the large number of partners involved, it was understandable that only a few partners' specific roles were discussed, but an indication of what organizations were involved in which activities (e.g., mentoring, hardware supplies) would be of interest.

\section{Reviewer 5:}

The reviewer stated that there was strong collaboration between DOE, GM, 16 universities, and more than 30 government and industry sponsors, and noted that the coordination work to accomplish this was impressive. The program's success and track record likely made it an easier sell generation. The reviewer stated that the presenter was clearly leading a team at ANL for this scale of project, but it was unclear how big the team is. The ANL/ DOE team and the teams interacted on a regular schedule (workshops, planning sessions, and competition). The presenter did not indicate that there were any coordination issues. The reviewer said that it would be good to know why the vehicle OEM has been GM since 1999 (maybe earlier). The reviewer questioned if the other domestic OEMs (Ford and Fiat Chrysler Automobiles) were not interested.

Question 5: Market impact and sustainability-the degree to which the project has already contributed, as well as the potential to continue to contribute in the future, to a sustainable alternative fuel vehicle market, alternative fuel market expansion, and reduced petroleum dependence/greenhouse gas emissions in the transportation sector. 
This would include the potential to reduce barriers to large scale alternative fuel vehicle market penetration, making information about alternative fuels and petroleum reduction opportunities widely available to target audiences, and ability for the project to be replicated in other geographic areas or with other technologies.

\section{Reviewer 1:}

The reviewer said that the market impact is strong. It is hard to fully quantify the results, as so many students have developed skills and passions for sustainability in this program and have gone on to work for the automotive industry. The reviewer noted that there are over 17,000 lives that have been impacted by this project. It is so important to ignite a flame in a student, and competitions like this one can do it. The reviewer remarked that it would be interesting to hear some personal stories of how this project impacted their lives and their careers.

\section{Reviewer 2:}

The reviewer stated that the project continued to build on its long history of advancing both advanced and AFV technologies, and developing the workforce needed to support and grow the industry. The integration of ADAS and connected and automated vehicle (CAV) technologies into the EcoCAR curricula was timely and very valuable.

\section{Reviewer 3:}

The reviewer stated that this project, as well as previous student competitions, definitely contributed to alternative fuel market expansion and reduced petroleum dependence. This was achieved by developing curricula and graduating knowledgeable engineers to work in the AFV industry.

\section{Reviewer 4:}

The reviewer stated that the market impact and sustainability was strong, but indirect. The program's most direct market impact was on developing students into highly-skilled employees for the automotive industry, not on direct petroleum/emissions reduction. The program claimed over 17,000 core students who have participated in the program since 1988 (this number increases to over 100,000, a large number, when non-core students are included). The students developed through the program enter industry and work to develop the next generation of sustainable vehicle technologies that DOE targets and consumers need.

\section{Reviewer 5:}

The reviewer commented that this project supported the sustainability goals by focusing the competition on energy efficient and alternative fuel technologies. Furthermore, the program used environmental impact as part of the competition criteria, as listed in the back-up slides. Reduction in energy consumption, petroleum usage, and criteria and GHG emissions were some of the things evaluated in the competition. The reviewer added that although the project did not directly impact the market, it addressed important barriers to market expansion by supporting the future workforce for advanced vehicle technologies and addressing education issues.

\section{Question 6: Use of resources-are DOE funds being used wisely? Should DOE fund similar efforts in the future? If not, what would be a better use of DOE resources to achieve alternative fuel vehicle and infrastructure expansion to support the broader goal of petroleum displacement and greenhouse gas reductions?}

\section{Reviewer 1:}

The reviewer commented that this project is truly a win-win-win for universities, companies, and government. This is an excellent use of DOE funds, and the reviewer said that it would be great to see competitions like this in other areas where DOE has not established them.

\section{Reviewer 2:}

The reviewer commented that the funds used for EcoCAR3 were definitely being used for a wonderful program. The DOE should continue to fund these types of projects.

\section{Reviewer 3:}

The reviewer stated that this project had provided substantial results for the DOE resources provided. The reviewer added that this project was able to significantly leverage the funding from DOE. In addition to the direct funding by DOE, this project had sponsors that, since FY 2012, have funded about a third to half of the budget. On top of 
that, in-kind support to the competition, cash, and in-kind support to the competing universities totaled over $\$ 900$ million.

\section{Reviewer 4:}

The reviewer stated that DOE funds were being well-used and heavily-leveraged with industry funding. The presenter noted that the actual industry cost-sharing was much higher if all of the donated industry time (mentoring and other interactions), software tools, etc., were accounted for. The reviewer suggested fully-accounting for all of this somehow to strengthen the impression of how well industry funding has been leveraged.

The reviewer suggested that the EcoCAR program consider leveraging some of the curricula that have been developed through other university vehicle competitions that are focused on connected and automated vehicles, as well with DOT-sponsored University Transportation Centers. 


\section{Acronyms and Abbreviations}

ADAS Advanced Driver Assistance Systems

AFDC Alternative Fuels Data Center

AFV Alternative fuel vehicle

AMR Annual Merit Review

ANL Argonne National Laboratory

APTA American Public Transportation Association

ARA Automotive Recyclers Association

AVT Advanced vehicle technology

AVTC Advanced Vehicle Technology Competitions

BETO Bioenergy Technologies Office

CaFCP California Fuel Cell Partnership

CAV Connected and automated vehicle

DOE U.S. Department of Energy

DOT U.S. Department of Transportation

EERE Office of Energy Efficiency and Renewable Energy

EMS Emergency medical services

EPA U.S. Environmental Protection Agency

EPAct Energy Policy Act of 1992

EV Electric vehicle

FOA Funding opportunity announcement

FY Fiscal year

GGE Gasoline Gallon equivalents

GHG Greenhouse gas

GM General Motors

$\mathrm{H}_{2} \quad$ Hydrogen

IAEM International Association of Energy Managers

ICE Internal combustion engine

IITR International Institute of Towing and Recovery

IREV Initiative for Resiliency in Energy through Vehicles

MOU Memorandum of Understanding 
NAFTC National Alternative Fuels Training Consortium

NAFTD North American Fire Training Directors

NATA North American Towing Academy

NFPA National Fire Protection Association

NGA National Governors Association

NGV Natural gas vehicle

NHTSA National Highway Traffic Safety Administration

NPS National Park Service

NSF National Science Foundation

OEM Original equipment manufacturer

PEV Plug-in electric vehicle

PHEV Plug-in hybrid-electric vehicle

PI Principal Investigator

PNNL Pacific Northwest National Laboratory

R\&D Research and development

SAF-D Safe Alternative Fuels Deployment in Mid-America

SMART Systems and Modeling for Accelerated Research in Transportation

SME Subject matter expert

STEM Science, Technology, Engineering, and Mathematics

TI Technology Integration

TRAA Towing and Recovery Association of America

T-t-T Train-the-Trainer

USDA U.S. Department of Agriculture

VTO Vehicle Technologies Office 


\section{Vehicle Analysis}

The portfolio of the Vehicle Technologies Office's Analysis Subprogram broadly comprises data, modeling, and applied analysis. A subset of the portfolio is presented and reviewed at the VTO Annual Merit Review. The presentations or posters are available in the Annual Merit Review Database and described here in the Annual Merit Review outcome report.

The VTO works with its national laboratories to collect and analyze data on the transportation sector to help better understand the sector's needs and guide the Office's research investments. VTO publishes the Transportation Energy Data Book annually with Oak Ridge National Laboratory, which compiles information on petroleum consumption, vehicle use, environmental impacts, household vehicle characteristics, and fleet characteristics. It also collaborates with Oak Ridge to publish the Vehicle Technologies Market Report, which describes major trends in light and heavy-duty markets. VTO also publishes the Fact of the Week, a fact with an accompanying graph or chart, to draw attention to particularly useful statistics.

The Vehicle Technologies Office (VTO) has supported the development of a number of software packages and online tools to model individual vehicles and the overall transportation system. Most of these tools are available for free or a nominal charge. Modeling tools that simulate entire vehicles and components allow researchers to create and test entire "virtual vehicles." Integration and validation tools help researchers test how multiple components interact.

With participation from the energy, electric utility, and automobile industries, VTO and the Fuel Cells Technology Office conducted a cradle-to-grave analysis activity that encompasses resource extraction (cradle), transformation of resources into fuels and vehicles, vehicle operation, and vehicle end-of-life disposal and recycling (grave). VTO also works with Argonne National Laboratory to publish performance reports under the Government Performance and Results Act (GPRA).

\section{Subprogram Feedback}

The U.S. Department of Energy (DOE) received feedback on the overall technical subprogram areas presented during the 2016 Annual Merit Review (AMR). Each subprogram technical session was introduced with a presentation that provided an overview of subprogram goals and recent progress, followed by a series of detailed topic area project presentations.

The reviewers for a given subprogram area responded to a series of specific questions regarding the breadth, depth, and appropriateness of that DOE VTO subprogram's activities. The subprogram overview questions are listed below, and it should be noted that no scoring metrics were applied. These questions were used for all VTO subprogram overviews.

Question 1: Was the program area, including overall strategy, adequately covered?

Question 2: Is there an appropriate balance between near- mid- and long-term research and development?

Question 3: Were important issues and challenges identified?

Question 4: Are plans identified for addressing issues and challenges?

Question 5: Was progress clearly benchmarked against the previous year?

Question 6: Are the projects in this technology area addressing the broad problems and barriers that the Vehicle Technologies Office (VTO) is trying to solve?

Question 7: Does the program area appear to be focused, well-managed, and effective in addressing VTO's needs? 
Question 8: What are the key strengths and weaknesses of the projects in this program area? Do any of the projects stand out on either end of the spectrum?

Question 9: Do these projects represent novel and/or innovative ways to approach these barriers as appropriate?

Question 10: Has the program area engaged appropriate partners?

Question 11: Is the program area collaborating with them effectively?

Question 12: Are there any gaps in the portfolio for this technology area?

Question 13: Are there topics that are not being adequately addressed?

Question 14: Are there other areas that this program area should consider funding to meet overall programmatic goals?

Question 15: Can you recommend new ways to approach the barriers addressed by this program area?

Question 16: Are there any other suggestions to improve the effectiveness of this program area?

Responses to the subprogram overview questions are summarized in the following pages. Individual reviewer comments for each question are identified under the heading Reviewer 1, Reviewer 2, etc. Note that reviewer comments may be ordered differently; for example, for each specific subprogram overview presentation, the reviewer identified as Reviewer 1 in the first question may not be Reviewer 1 in the second question, etc. 
Subprogram Overview Comments: Jake Ward (U.S. Department of Energy) - van999

Question 1: Was the program area, including overall strategy, adequately covered?

\section{Reviewer 1:}

The reviewer replied yes, the program's goal, objective, and strategy were all defined and/or covered, adding that funding and model/tool definition and integration were provided to demonstrate strategy execution.

\section{Reviewer 2:}

The reviewer stated that the program area and strategy were well covered and linkages between the various components are clear, although the link between the new initiative in Systems and Modeling for Accelerated Research in Transportation (SMART) Mobility could be made more explicit.

\section{Reviewer 3:}

The reviewer said the program area was adequately covered. However, the reviewer specified areas to consider for improvement, namely, the objective can be read as vague and does not effectively speak to the relevance or usefulness of the program for various users of the output: government, industry, non-governmental organizations (NGOs). The reviewer also cautioned that "speaks for itself" is very subjective, and that it may be beneficial to reword this even if it is duller but measureable.

\section{Reviewer 4:}

The reviewer remarked that the linkage between the pyramid levels could have been made clearer with an example showing how information flows from one level to the next.

\section{Question 2: Is there an appropriate balance between near- mid- and long-term research and development?}

\section{Reviewer 1:}

The reviewer stated that the balance seems appropriate.

\section{Reviewer 2:}

The reviewer expressed that efforts appear to be appropriately balanced.

\section{Reviewer 3:}

The reviewer remarked that this is subjective, but the work seems to be weaker in early applied (long-term) research, and that this seems to be the result of higher level strategic direction at DOE versus a decision issue at the program management level.

\section{Reviewer 4:}

The reviewer commented that it was not clear from the presentation that there were near-, mid-, and long-term research and development (R\&D) goals, and that most of the "future work" seemed like near-term goals.

\section{Question 3: Were important issues and challenges identified?}

\section{Reviewer 1:}

The reviewer stated that the speaker clearly articulated the issues and challenges that the analysis program is addressing.

\section{Reviewer 2:}

The reviewer replied yes, elaborating that modeling and simulation improvements were presented and described including data updates, coding/software revisions, testing, calibration, integration, and review. It is an extensive set of analytical tools requiring a lot of care and maintenance. The reviewer suggested that there might be some opportunities for greater consolidated effort, but added that given the specificity of model function, integration with other tools, etc., desired analytical capability may preclude a scaling effort over multiple platforms. 


\section{Reviewer 3:}

The reviewer remarked that there were not many issues and challenges identified, and that the presentation could have benefited from detailing the "why" or need for each of the highlights or accomplishments.

\section{Reviewer 4:}

The reviewer stated that these were not addressed in the presentation.

\section{Question 4: Are plans identified for addressing issues and challenges?}

\section{Reviewer 1:}

The reviewer replied yes, core analytical model and tool program issues and successes were identified with plans provided for future activities.

\section{Reviewer 2:}

The reviewer stated that the speaker highlighted activities planned for the coming year and provided the motivation for the topical areas.

\section{Reviewer 3:}

The reviewer stated that these were not addressed in the presentation.

\section{Reviewer 4:}

The reviewer responded N/A and referenced prior comments.

\section{Question 5: Was progress clearly benchmarked against the previous year?}

\section{Reviewer 1:}

The reviewer replied yes to this question.

\section{Reviewer 2:}

The reviewer commented that the focus of the program has shifted somewhat from last year, especially with the addition of the SMART Mobility initiative, and that highlights of accomplishments indicated continued progress over the previous year in all major program areas.

\section{Reviewer 3:}

The reviewer observed that the prior year presentation was provided, which was helpful in benchmarking year-toyear progress, but that the 2016 slide deck did not have much benchmarking data.

\section{Reviewer 4:}

The reviewer stated that this was not addressed in the presentation.

\section{Question 6: Are the projects in this technology area addressing the broad problems and barriers that the Vehicle Technologies Office (VTO) is trying to solve?}

\section{Reviewer 1:}

The reviewer praised the projects in this program as providing foundational data, models, and analyses that provide insight into the value of transitioning to alternative vehicles and fuels supported by VTO. The reviewer applauded the program as solid and well-structured to provide context for the broad problems and barriers VTO is trying to address.

\section{Reviewer 2:}

The reviewer replied yes to this question.

\section{Reviewer 3:}

The reviewer stated that they seem to be closely aligned with VTO-identified problems and barriers.

\section{Reviewer 4:}

The reviewer replied yes, but warned that there are some acute concerns and not all projects are as appropriate or as effective in addressing the broad problems and barriers. The reviewer also noted that some areas are better covered 
by the projects in terms of multiple contributors, while others are more limited, and remarked that this remains a chronic challenge of the program. The reviewer also stated that acute feedback is provided for the individual presentations.

\section{Question 7: Does the program area appear to be focused, well-managed, and effective in addressing VTO's needs?}

\section{Reviewer 1:}

The reviewer described the program as well-organized and structured, adding that the suite of activities provides information, capabilities, and studies that highlight the potential and challenges of transitioning to alternative fuels and vehicles. The reviewer affirmed that program management seems strong, with clear linkages between the various complementary efforts, and that shifts in the program are responsive to VTO needs.

\section{Reviewer 2:}

The reviewer replied yes to this question.

\section{Reviewer 3:}

The reviewer said yes but, as stated above (in question 1), an example of information/data flows between the pyramid levels would help solidify the program area.

\section{Reviewer 4:}

The reviewer stated that the program is generally well managed and that the program manager has a confident vision. The reviewer remarked that it is difficult to discern where the root cause of shortcomings derives from: DOE leadership and direction, or program decision making. The reviewer concluded that it appears to be more the former.

\section{Question 8: What are the key strengths and weaknesses of the projects in this program area? Do any of the projects stand out on either end of the spectrum?}

\section{Reviewer 1:}

The reviewer stated that a key strength is the complementary structure and synergy across the project portfolio, and that all projects presented seem solid.

\section{Reviewer 2:}

The reviewer observed that there are a broad set of tools being used to analyze a broad set of issues, and remarked that many of these tools represent significant contribution to the analytical community. The reviewer offered that there are redundancies in scope, but nuanced capability provides added insight on critical issues.

\section{Reviewer 3:}

The reviewer commented that a key strength seemed to be the engagement/collaboration with other federal laboratories, industry, academia, and area specific experts, while the perceived weakness was how all the information between the projects ties together.

\section{Reviewer 4:}

The reviewer judged that work performed on the Greenhouse gases, Regulated Emissions, and Energy use in Transportation (GREET) model stands out on the weak end along with the VTO program benefits analysis (see individual review). The reviewer stated that the Autonomie system simulation tool is stronger.

\section{Question 9: Do these projects represent novel and/or innovative ways to approach these barriers as appropriate?}

\section{Reviewer 1:}

The reviewer observed that many of the projects are longstanding ones within the portfolio and remarked that they provide continuity, capability, and information that the analysis community has come to rely upon. The reviewer added that the addition of new projects over the past few years has provided new and innovative modeling capabilities. 


\section{Reviewer 2:}

The reviewer replied yes, adding that underlying assumptions should be clearly stated.

\section{Reviewer 3:}

The reviewer commented that it is hard to judge this based on the level of detail in this presentation.

\section{Reviewer 4:}

The reviewer said no, they do not, and added that Autonomie is one of the better executed projects by DOE's national laboratories this year.

\section{Question 10: Has the program area engaged appropriate partners?}

\section{Reviewer 1:}

The reviewer commented that the program area is engaged across multiple government, laboratory, university, and industry partners, and that these partnerships link to key organizations and thought leaders that provide relevant input and feedback, as well as consumers of the analysis and data.

\section{Reviewer 2:}

The reviewer replied yes to this question.

\section{Reviewer 3:}

The reviewer stated that it seems so, based on Slide 21.

\section{Reviewer 4:}

The reviewer remarked that this was not covered in the overview presentation but that the program manager explained how the different components of the program engage each other.

\section{Question 11: Is the program area collaborating with them effectively?}

\section{Reviewer 1:}

The reviewer affirmed that program management has clearly made a concerted effort to engage a broad set of relevant stakeholders and partners, which informs the issues addressed and strengthens the resulting analysis products.

\section{Reviewer 2:}

The reviewer replied yes to this question.

\section{Reviewer 3:}

The reviewer was unable to determine.

\section{Reviewer 4:}

The reviewer stated that there is not sufficient information to answer this question.

\section{Question 12: Are there any gaps in the portfolio for this technology area?}

\section{Reviewer 1:}

The reviewer replied no to this question.

\section{Reviewer 2:}

The reviewer stated none that come to mind.

\section{Reviewer 3:}

The reviewer answered that there are no obvious gaps at this time.

\section{Reviewer 4:}

The reviewer replied that, consistent with the comments provided above, there are two high-level weaknesses to consider: First, the greenhouse gas (GHG) emissions measurement is dependent on one model, which the reviewer cautioned greatly narrows the input and fails to recognize the critique and limitations established in the literature 
or capture broader measurement techniques and perspectives that are hallmarks of good analysis. Second, all of the projects, and it appears that almost all of the funding, are supporting DOE laboratories. The reviewer offered that there was not a good rationale provided for why there is not more engagement with academia or other independent actors who bring different approaches, skills, and insights to the research (the reviewer said to see prior comment in this section as an example).

\section{Question 13: Are there topics that are not being adequately addressed?}

\section{Reviewer 1:}

The reviewer replied no to this question.

\section{Reviewer 2:}

The reviewer replied no to this question.

\section{Reviewer 3:}

The reviewer offered that including SMART Mobility is a much-needed enhancement to the program, and added that the program will need to create new capabilities to effectively address key challenges with new technologies that go beyond the powertrains and fuels that have been the focus of the program.

\section{Reviewer 4:}

The reviewer stated that GHG impacts/measurements are not being adequately addressed.

\section{Question 14: Are there other areas that this program area should consider funding to meet overall programmatic goals?}

\section{Reviewer 1:}

The reviewer replied no to this question.

\section{Reviewer 2:}

The reviewer replied no to this question.

\section{Reviewer 3:}

The reviewer suggested that further sensitivity studies to help identify and bound uncertainties in the modeling and analyses could provide further insight into the impact of technology, policy, and consumer choice.

\section{Reviewer 4:}

The reviewer suspected that some might suggest SMART mobility, which was added to the portfolio. The reviewer warned that while SMART mobility will affect energy utilization of the transportation system, this is a subject area that is likely better handled by DOT and others, as it is fundamentally a question of transportation system operation, regulation, and development/funding policy, even if VTO can generate an argument to show that energy is somehow tied in. The reviewer added that the material presented suggests large amounts of scope and mission creep despite the argument presented to the contrary, and that moving into this space needs to be very carefully considered.

\section{Question 15: Can you recommend new ways to approach the barriers addressed by this program area?}

\section{Reviewer 1:}

The reviewer replied no to this question.

\section{Reviewer 2:}

The reviewer stated not at this time.

\section{Reviewer 3:}

This reviewer's understanding is that the program has historically convened and also has planned workshops for stakeholder engagement, but observed that this was not covered in the presentation. The reviewer offered that 
highlighting how this outreach is conducted and the lessons learned would provide even better context for the program focus areas.

\section{Reviewer 4:}

The reviewer remarked that this program continues to sustain funding for the same projects that tend to produce the same results from the same perspective. The reviewer offered that a simple new approach would be to allow or instruct the program manager to roll over the projects and bring in a new set of researchers and modelers/analysts to bring a different and complementary perspective to the work. The reviewer claimed that an exorbitant amount of money is being spent to produce fundamentally the same results (with the same limitations and embedded errors and uncertainty) that have persisted for years, and added that the return on investment (ROI) from a different perspective is probably going to be higher.

\section{Question 16: Are there any other suggestions to improve the effectiveness of this program area?}

\section{Reviewer 1:}

The reviewer replied no to this question.

\section{Reviewer 2:}

The reviewer replied no to this question.

\section{Reviewer 3:}

The reviewer stated that the reviews for GREET, Autonomie, and VISION/NEAT need to be separated, elaborating that while individual comments can be provided in technical sections, the ratings for each category do not apply to the individual projects. The reviewer pointed out that a stronger project receives a lower grade and the weak(er) projects are over-graded. The reviewer commented that this is a poor way to make project funding and continuation decisions and is a disservice to the higher performing individuals. 


\section{Project Feedback}

In this merit review activity, each reviewer was asked to respond to a series of questions, involving multiple-choice responses, expository responses where text comments were requested, and numeric score responses (on a scale of 1.0 to 4.0). In the pages that follow, the reviewer responses to each question for each project will be summarized: the multiple choice and numeric score questions will be presented in graph form for each project, and the expository text responses will be summarized in paragraph form for each question. A table presenting the average numeric score for each question for each project is presented below.

\section{Table 9-1 - Project Feedback}

\begin{tabular}{|c|c|c|c|c|c|c|c|}
\hline Presentation Title & $\begin{array}{c}\text { Principal } \\
\text { Investigator } \\
\text { and } \\
\text { Organization }\end{array}$ & $\begin{array}{l}\text { Page } \\
\text { Number }\end{array}$ & Approach & $\begin{array}{c}\text { Technical } \\
\text { Accomplishments }\end{array}$ & Collaborations & $\begin{array}{c}\text { Future } \\
\text { Research }\end{array}$ & $\begin{array}{c}\text { Weighted } \\
\text { Average }\end{array}$ \\
\hline $\begin{array}{c}\text { Transportation } \\
\text { Data Program: } \\
\text { A Multi-Lab } \\
\text { Coordinated } \\
\text { Project }\end{array}$ & $\begin{array}{l}\text { Davis, Stacy } \\
\text { (ORNL) }\end{array}$ & $9-10$ & 3.08 & 3.42 & 3.50 & 3.00 & 3.29 \\
\hline $\begin{array}{c}\text { ANL Vehicle } \\
\text { Technologies } \\
\text { Analysis Modeling } \\
\text { Program }\end{array}$ & $\begin{array}{l}\text { Wang, Michael } \\
\text { (ANL) }\end{array}$ & $9-14$ & 3.25 & 3.17 & 3.58 & 3.25 & 3.25 \\
\hline $\begin{array}{l}\text { VTO Program } \\
\text { Benefits Analysis }\end{array}$ & $\begin{array}{l}\text { Stephens, Tom } \\
\text { (ANL) }\end{array}$ & $9-18$ & 2.50 & 2.67 & 2.75 & 3.00 & 2.68 \\
\hline $\begin{array}{l}\text { Assessing Energy } \\
\text { and Cost Impact } \\
\text { of Advanced } \\
\text { Technologies } \\
\text { through Model } \\
\text { Based Design }\end{array}$ & $\begin{array}{l}\text { Rousseau, } \\
\text { Aymeric } \\
\text { (ANL) }\end{array}$ & $9-22$ & 3.58 & 3.67 & 3.67 & 3.50 & 3.63 \\
\hline Overall Average & & & 3.10 & 3.23 & 3.38 & 3.19 & 3.21 \\
\hline
\end{tabular}


Transportation Data Program:

A Multi-Lab Coordinated

Project: Stacy Davis (Oak Ridge National Laboratory) - van016

Presenter

Stacy Davis, Oak Ridge National

Laboratory

\section{Reviewer Sample Size}

A total of six reviewers evaluated this project.

\section{Question 1: Approach to performing the work-the degree to which technical barriers are addressed, the project is well-designed, feasible, and integrated with other efforts.}

\section{Reviewer 1:}

The reviewer said that all three projects seem well-designed, feasible, and integrated with other DOE efforts. Principal investigators (PIs) are trying to address technical barriers. The reviewer commented that for the TEDB, which informs the DOE and external models, the biggest barrier seems to continue to be inconsistency across the time series due to changes in data inputs. For the consumer survey on plug-in electric vehicles (PEVs), the most significant barrier seems to be the limitations of stated preference data (as opposed to revealed preference data). However, the reviewer remarked that the presenter aptly noted that some data are better than no data. The reviewer said that for questions assessing PEV awareness, a consumer survey is the right tool, and this will be a particularly valuable year-by-year data set. The presentation stated that the National Renewable Energy Laboratory (NREL) is also seeking opportunities to contextualize study results with external data sets, which seems useful. The reviewer remarked that for the electric drive E-Drive project, monthly PEV sales data are published on Argonne National Laboratory's (ANL) website. This is a valuable resource for researchers and stakeholders for tracking the early PEV market. If international sales data and U.S. regional breakdowns are also being regularly collected, the reviewer suggested making these public as well (on the same or linked ANL website) rather than just providing to VTO.

\section{Reviewer 2:}

The reviewer said the project team has a strong approach that enables successful multi-lab coordination. The reviewer remarked that tasks and roles are clearly defined and that the focus on publicly accessible material enables transparency in the data reported.

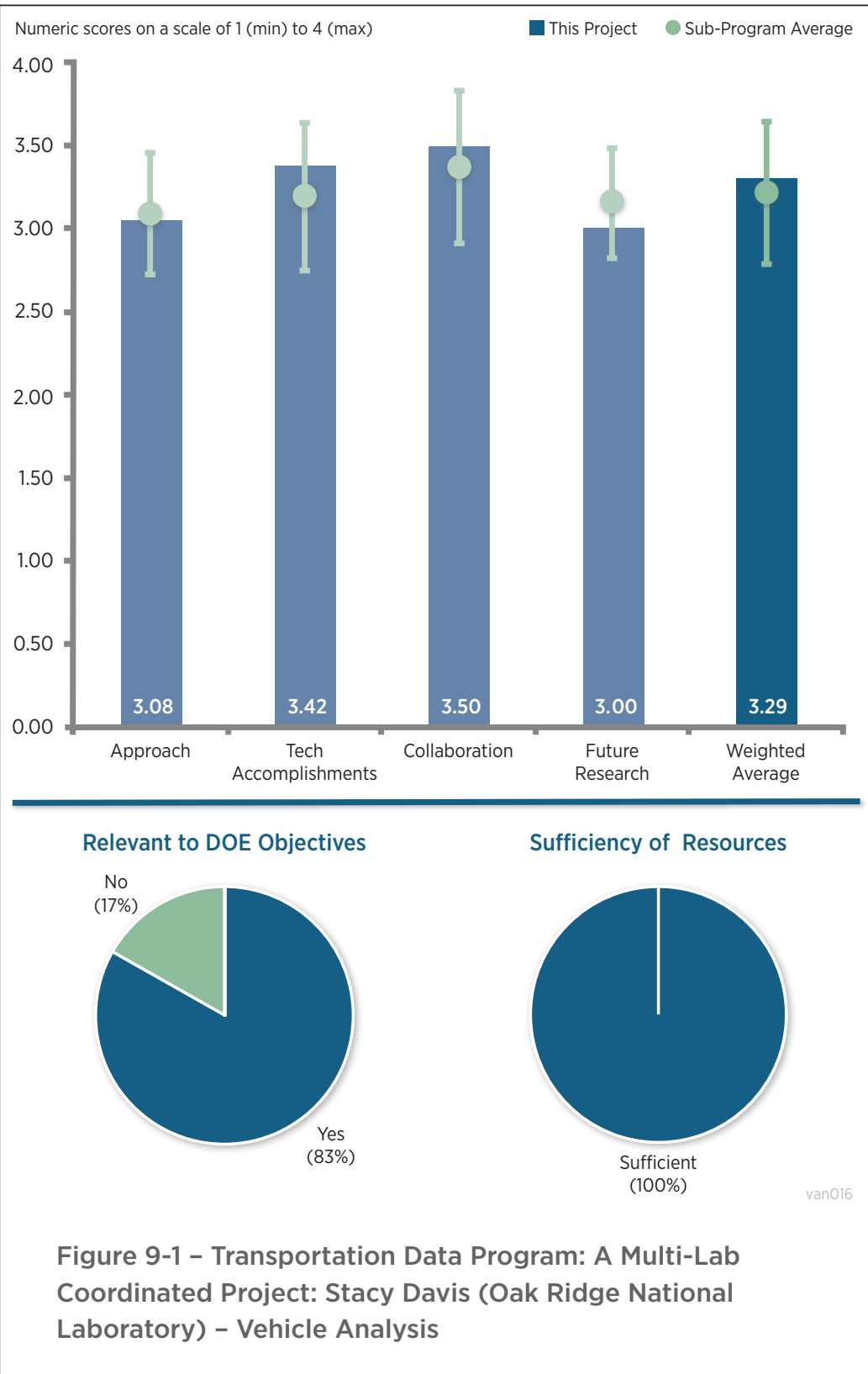




\section{Reviewer 3:}

The reviewer stated that the approach is logical and provides foundational information relevant for a community of researchers. The team has had long-term engagement in the project and provided thoughtful attention to details that have come to be expected by the community of users. The reviewer said that the addition of E-Drive data are particularly relevant given the growing interest in vehicle electrification.

\section{Reviewer 4:}

The reviewer said that the project appears to be designed to specifically address data, market and analytical needs of the VTO. The reviewer commented that the design, feasibility, and integration support the VTO programs and involve multiple stakeholders.

\section{Reviewer 5:}

The reviewer said the work is straightforward and satisfactory, and that there are no major issues or challenges. The reviewer found it unclear that preference analysis is very robust or better than competing alternatives, which is why it is not used versus other sources for the analysis that the reviewer saw being performed or utilized; however, the reviewer said it seems valuable as is to other users. The reviewer had some concern that the data are focused on only nine companies.

\section{Reviewer 6:}

The reviewer commented that the presentation lacked detail on how the data were collected. It was not clear how the data and information from this project are used by the other projects.

\section{Question 2: Technical accomplishments and progress toward overall project and DOE goals-the degree to which progress has been made, measured against performance indicators and demonstrated progress towards DOE goals.}

\section{Reviewer 1:}

The reviewer noted that milestones have been met or are on track for all project components. The reviewer was particularly impressed by the outreach efforts for the TEDB and Vehicle Technologies Market Report: monthly website visits increased substantially from Fiscal Year (FY) 2015 to FY 2016 on both projects and topped 11,000 for TEDB. The reviewer observed that even granting that part of the increase for TEDB was a shift from hard copies to the web, the impressive usage numbers are indicative of the importance of this data to external researchers and transportation stakeholders. The reviewer noted that both the Consumer Benchmark Report and E-Drive project will provide important data to help understand and track early PEV adoption and identify challenges and barriers. The reviewer commented that the slides on the consumer survey state, "In an early adoption market, it is helpful to identify where further investigation is warranted." To that end, researchers might consider adding a few state- or region-specific questions in early adoption markets to explore how consumer views differ in these areas and to explore the impact of state incentives, visible public charging stations, or (if applicable) region-specific advertising and consumer education campaigns. If that is not possible, the reviewer suggested that an alternative may be to increase the total number of consumers surveyed such that the sample size is large enough to analyze responses both nationally and regionally. The reviewer recognized this is outside the current scope of the project and that while national benchmarking is very valuable on its own, regional data would be an additional, valuable resource.

\section{Reviewer 2:}

The reviewer remarked that the project continues to make steady progress and publish the market report, data book, and facts of the week. A significant increase in hits to the website for the TEDB indicates the value of this resource, and the reviewer suspected that this is attributed to the value of the data beyond the reduction in hard copy distribution mentioned during the presentation. The reviewer suggested that the team track what information is accessed to focus and prioritize which areas may either merit expansion or perhaps less frequent updates. It would also be helpful to understand more clearly how the data are used by the other parts of the VTO analysis portfolio.

\section{Reviewer 3:}

The reviewer stated that the team has shown good progress and is on track to meet all its milestones, and in some cases is even ahead of schedule. For instance, the market report was published early this year. 


\section{Reviewer 4:}

The reviewer noted that this project has met defined accomplishments and progress toward meeting desired DOE goals.

\section{Reviewer 5:}

The reviewer commented, again, that the project was straightforward.

\section{Question 3: Collaboration and coordination with other institutions.}

\section{Reviewer 1:}

The reviewer commented that PIs are working with an impressive cross-section of government agencies, industry, and academia and have plans to expand outreach. The reviewer suggested the presenters might consider also reaching out to Idaho National Laboratory (INL). Early adopter data for PEV usage related to driving and charging could inform future questions on consumer surveys. The reviewer said that one of the slides on E-Drive mentioned the limitations of using the National Household Travel Survey data to model the behavior of PEV drivers and that perhaps INL data or other early adopter data sets could also be helpful here.

\section{Reviewer 2:}

The reviewer said that this multi-lab project shows strong coordination across the three partners - ORNL, NREL, and ANL. The team also has significant cross-sector collaboration involving industry, academia, and other government agencies, including DOT and EPA.

\section{Reviewer 3:}

The reviewer noted that the project team continues to collaborate with various providers and users of data. The project has clearly sought out feedback and has judiciously responded to input.

\section{Reviewer 4:}

The reviewer commented that there is a broad spectrum of stakeholder involvement including other government agencies, private sector, national laboratories, and academia.

\section{Reviewer 5:}

The reviewer said that the collaboration comes across as forced farming of the work in order to split the effort across the laboratories. The reviewer stated that the results are fine, but it is not clear how this is necessary or value added. The nature of the collaboration with the broader list was not explained.

\section{Question 4: Proposed future research-the degree to which the project has effectively planned its future work in a logical manner by incorporating appropriate decision points, considering barriers to the realization of the proposed technology and, when sensible, mitigating risk by providing alternate development pathways.}

\section{Reviewer 1:}

The reviewer remarked that the project has a sustained history of delivering a solid set of data for the community and clearly plans to continue on this path forward.

\section{Reviewer 2:}

The reviewer said that all projects have feasible plans for future work that are consistent with DOE goals. For the consumer survey, the reviewer suggested considering expanding the scope to allow regional evaluations in early adopter markets. For E-Drive, the reviewer suggested expanding outreach and making data sets publicly available, for example, by adding a global sales bar graph to ANL's monthly sales website.

\section{Reviewer 3:}

The reviewer stated that proposed future work is well planned with weekly, monthly, and annual milestones identified.

\section{Reviewer 4:}

The reviewer commented that future plans should include identifying more recent data for inclusion in the presenters' reports. The reviewer strongly recommended that the presenters look into updating some data more 
often than annually to make it available for use faster, as the presenter suggested could be a possibility. The reviewer stated that it would be interesting to see the team investigate why they found that in colder climates there is a reduced battery electric vehicle (BEV) share of the market. The reviewer commented that BEVs have been very successful in Scandinavia and questioned why they could not also be successful in the northern United States.

\section{Reviewer 5:}

The reviewer commented that proposed future work was not explained well, and that the slide was too high level.

\section{Question 5: Does this project support the overall DOE objectives of petroleum displacement? Why or why not?}

\section{Reviewer 1:}

The reviewer stated that the projects provide valuable information to DOE, other policymakers, researchers, and the public on key transportation data. The National Benchmark Report will be particularly helpful in understanding how consumer awareness of PEVs changes or does not change over time, and it may also be helpful in assessing how certain incentives, infrastructure, and other factors impact that awareness. The reviewer said that this knowledge can be used to help reduce barriers to adoption. E-Drive sales data likewise provides important information for tracking and understanding early PEV market penetration.

\section{Reviewer 2:}

The reviewer remarked that the data collected by the team directly informs the VTO's Multi-Year Research, Development, and Demonstration (MYRD\&D) Plan and is critical to ensuring relevance of program activities. The reviewer said that the data point from FY 2014 that stated there were 1,200 users of the reviewer book is a clear indicator of the relevance of the work.

\section{Reviewer 3:}

The reviewer said the project provides foundational data and important highlights that are used extensively by the modeling and analysis community.

\section{Reviewer 4:}

The reviewer expressed that the project supports data and analytic needs.

\section{Reviewer 5:}

The reviewer questioned who was using the data and how it was being used. The reviewer said it would be beneficial to add some content and provide examples of the data usage.

\section{Reviewer 6:}

The reviewer stated that good data are foundational for good modeling.

\section{Question 6: Resources: How sufficient are the resources for the project to achieve the stated milestones in a timely fashion?}

\section{Reviewer 1:}

The reviewer said the resources seem sufficient for the current project milestones, but that if the scope of the projects expands, for example, by adding region-based consumer surveys, then additional funds may be needed.

\section{Reviewer 2:}

The reviewer stated that no gaps were identified that would warrant additional resources. 


\section{ANL Vehicle Technologies Analysis Modeling Program: Michael Wang (Argonne National Laboratory) - van017}

\section{Presenter}

Michael Wang, Argonne National Laboratory

\section{Reviewer Sample Size}

A total of six reviewers evaluated this project.

\section{Question 1: Approach to performing the work-the degree to which technical barriers are addressed, the project is well-designed, feasible, and integrated with other efforts.}

\section{Reviewer 1:}

The reviewer said the project uses an excellent approach that enables comparison of differing technologies in a consistent way. There is clear integration of this effort with other VTO-funded analysis.

\section{Reviewer 2:}

The reviewer stated that there were strong technical achievements on all four models discussed and that plans for continued development seem welldesigned and feasible. The reviewer expressed that there seemed to be particularly strong technical progress in Autonomie and the GREET model, for example, water consumption.

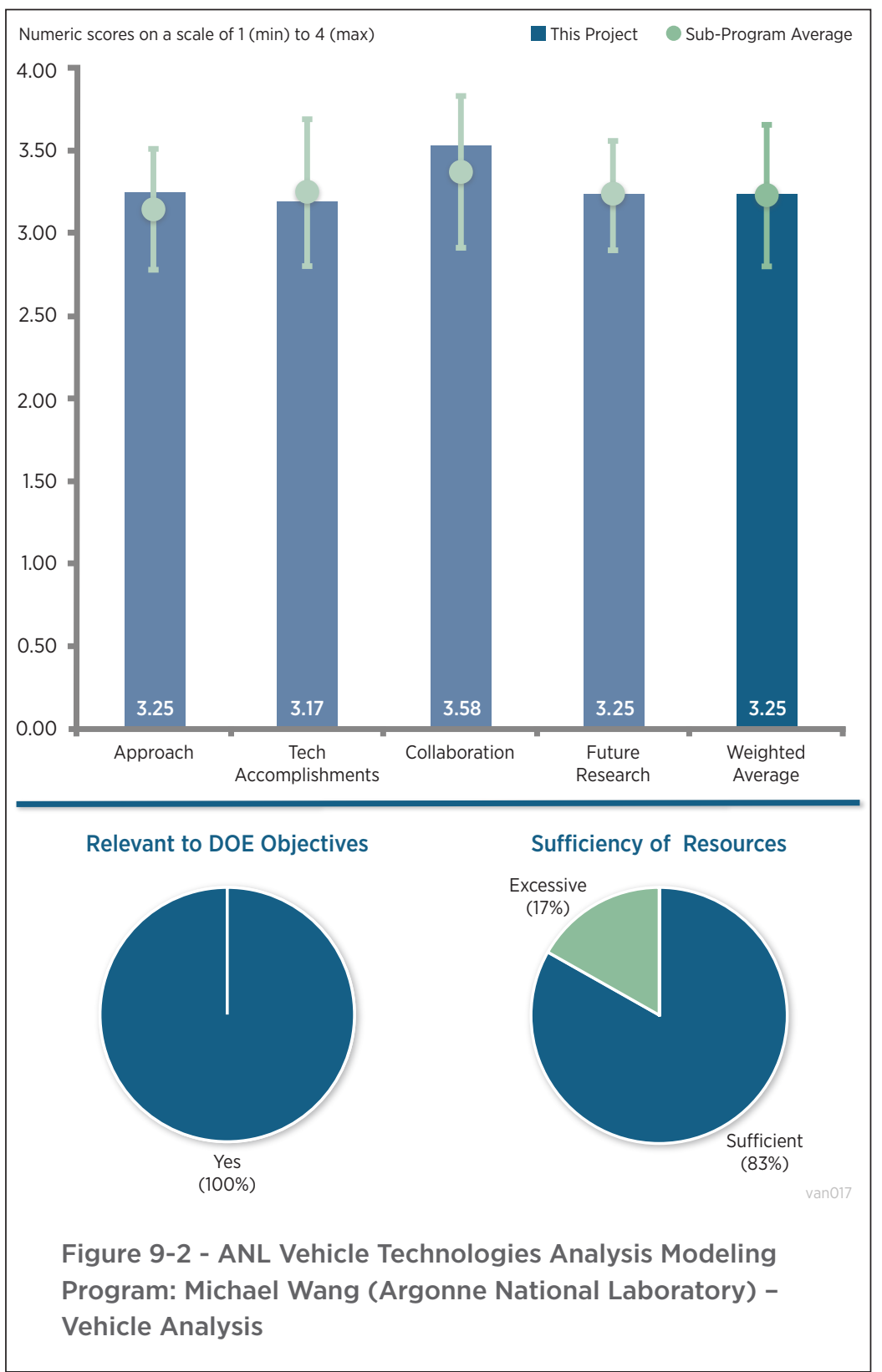

The reviewer said the technical

challenges seem highest for the household vehicle ownership model due to data limitations. The reviewer commented that it would have been helpful to see more detail on which datasets are being used for calibration and how different powertrain technologies are being handled; for example, a household's decision to buy a PEV. The reviewer understood this was difficult given how many topics needed to be covered in 20 minutes and suggested that in future merit reviews, it may be helpful for this to be a standalone topic. The models seem well-integrated with other DOE efforts.

\section{Reviewer 3:}

The reviewer commented that the suite of models developed under this project is useful for a broad user community. The continued model development, refinement, and use are important for supporting assessments made across the user base. The reviewer said the team seems to take a logical approach to balancing across gathering information, building the models, and performing analysis. The expansion of features, including access in the ".net" platform, is logical and reflects capabilities that are being increasingly demanded by the analysis community. 


\section{Reviewer 4:}

The reviewer commented that updates and integration of Autonomie, GREET, and VISION/ NEAT are welldeveloped and defined; however, vehicle market dynamics are not well-defined or developed. The reviewer questioned if this portion of the project will be the primary focus toward the end of the project's timeline, and the reviewer said that, if so, the presenter should be clear about that in the presentation.

\section{Reviewer 5:}

The reviewer said it would have been helpful if the presentation better addressed the first objective of overcoming inconsistent data, assumptions, and guidelines.

\section{Reviewer 6:}

The reviewer said the modeling approach is not the best choice or particularly well-suited for evaluating environmental sustainability. This, in part, derives from limitations on how the boundary and factors considered in GREET are determined. The reviewer stated that comparing across technologies, which inherently encompass systems that have different components and input flows, is very challenging. However, GREET continues to present their results as having more usefulness and accuracy than is warranted for many of these applications, and that can be misinforming the decision-making process. The reviewer said the approach of Autonomie, however, is good.

The reviewer also said that the approach of VISION/NEAT, and the reliance on the exogenous inputs, limits its usefulness. It is unclear if there is a mechanism to ensure that the inputs are self-consistent. As such, the model functions more as a deterministic calculator for which the output may or not be realistic or relevant for informing decision making by DOE.

\section{Question 2: Technical accomplishments and progress toward overall project and DOE goals - the degree to which progress has been made, measured against performance indicators and demonstrated progress towards DOE goals.}

\section{Reviewer 1:}

The reviewer said that both Autonomie and GREET are robust, well-vetted models, with an extensive list of technology pathways and fuel pathways that are widely used by outside stakeholders. The reviewer was particularly impressed by reported GREET updates and said that the development of regional platforms will be especially important for evaluating impacts of electric vehicles (EVs) due to wide variations in the grid. The reviewer was also glad to see the new video tutorial.

The reviewer said that the ability to model long-range scenarios (2050 or 2100) in VISION/NEAT seems limited by a lack of advanced technology powertrain options in the freight sector. The reviewer suggested that the presenter consider the addition of electrification; for example, plug-in hybrid electric vehicles (PHEV) or fuel cell vehicles (FCV), for medium-duty vehicles (MDVs) and heavy-duty vehicles (HDVs). While the reviewer recognized that data were limited and that many simplifying assumptions and caveats may need to be made, the reviewer said that projections to 2100 that do not allow for any freight electrification limit the models' usefulness and may inadvertently imply that these are not viable technologies.

The reviewer also commented that for the household vehicle ownership model, it was not clear from the presentation what data are being used to calibrate PEV model components. The reviewer commented that the presenter might consider evaluating early adopter data on PEV usage from INL or other PEV-specific data sets as they become available given how different travel behavior and needs may be for owners of these vehicles relative to internal combustion engine (ICE) vehicles.

\section{Reviewer 2:}

The reviewer said that the publication of the cradle-to-grave report on June 1st was a significant accomplishment from four years of efforts. Integrating all of the modeling analysis and results is extremely valuable to the clean transportation community. 


\section{Reviewer 3:}

The reviewer commented that the project continues to make good progress on developing capabilities that have become widely used by the academic, policy, and industry communities.

\section{Reviewer 4:}

The reviewer remarked that when evaluating fuel and vehicle emissions at a state level, electric power should reflect utility generation mix of consumption, not state production. VISION model calibration techniques should be clearly described and tested to indicate the impact calibration has on projected values.

\section{Reviewer 5:}

The reviewer said that the technical accomplishments vary. Inputs used to show results in GREET may be vetted by experts, but they are also refuted by experts. The reviewer said that the intransigence in which the researchers have failed to consider legitimate critique and the limits it implies on how the model should and should not be used continue to linger as problems. The reviewer commented, however, that the Autonomie results are valuable and well-received by the range of stakeholders.

\section{Question 3: Collaboration and coordination with other institutions.}

\section{Reviewer 1:}

The reviewer stated that the project had excellent collaboration with other laboratories, researchers and original equipment manufacturers (OEMs). The reviewer commented that there was good coordination with the BETO WATER 2.0 model ensuring integration and consistency of assumptions.

\section{Reviewer 2:}

The reviewer said that the team obviously has a vast network of collaborators and partners that inform and shape the models and analyses. Sustained investment over multiple years has enabled this capability to flourish.

\section{Reviewer 3:}

The reviewer commented that the PIs are working with appropriate government agencies, industry, and academia.

\section{Reviewer 4:}

The reviewer said that Autonomie should continue to collaborate with industry and others to continue to seek good empirical input and review.

\section{Question 4: Proposed future research-the degree to which the project has effectively planned its future work in a logical manner by incorporating appropriate decision points, considering barriers to the realization of the proposed technology and, when sensible, mitigating risk by providing alternate development pathways.}

\section{Reviewer 1:}

The reviewer said that the project had very well-defined milestones extending to FY 2018 for Autonomie, GREET, and VISION/NEAT. There was less detail provided on plans for the household vehicle ownership model. The reviewer suggested that the presenter consider additional powertrain and technology options for MDVs and HDVs in VISION/NEAT.

\section{Reviewer 2:}

The reviewer said that the planned future work is relevant and valuable. The team should include the proposed updates to Autonomie, GREET, and VISION/NEAT as outlined in the presentation.

\section{Reviewer 3:}

The reviewer remarked that incorporating new components and powertrain technologies is a very logical expansion. The addition of quality assurance (QA) / quality control (QC), uncertainty analyses, and web processing tools also seems like it would be useful for the broader user community. The reviewer commented that water consumption accounting should consider all feasible technology options just as the GHG emissions considers all options in order to enable consistent comparisons. The reviewer also stated that the household vehicle ownership description was not clear and that more background is needed to understand how this is going to be used. 


\section{Reviewer 4:}

The reviewer said that Autonomie was on point and likely to be accomplished. However, the reviewer commented that the GREET model continues to take a Band-Aid approach instead of addressing fundamental problems. The reviewer also said that the VISION/NEAT model was adequate.

\section{Question 5: Does this project support the overall DOE objectives of petroleum displacement? Why or why not?}

\section{Reviewer 1:}

The reviewer remarked that the project is extremely relevant as it shows the impact of VTO technology developments and provides pathway analysis to show the sustainability and economic viability of current and future vehicle technology pathways.

\section{Reviewer 2:}

The reviewer said that the linking of the three tools seems to provide the DOE the capability to project benefits from VTO-funded R\&D activities.

\section{Reviewer 3:}

The reviewer said that the suite of models in this project has become a standard set of tools used by the analysis community and is directly relevant to DOE objectives.

\section{Reviewer 4:}

The reviewer said that modeling is important for assessing the potential for advanced vehicle technologies to reduce petroleum and for identifying barriers to the adoption of these technologies.

\section{Reviewer 5:}

The reviewer opined that, overall, DOE petroleum displacement objectives are supported by this project, and noted better support from some parts than others. The reviewer expressed that providing misinformation, even if the answer is aligned with the objective, is probably more damaging than not providing information at all, but the reviewer said that is hard to measure with the yes or no question.

\section{Question 6: Resources: How sufficient are the resources for the project to achieve the stated milestones in a timely fashion?}

\section{Reviewer 1:}

The reviewer stated that the resources seem relevant to continue steady progress.

\section{Reviewer 2:}

The reviewer said that it is not clear how the large resources allocated to GREET are making it a fundamentally more useful tool or improving the usefulness enough to justify the investment. The reviewer also said that the resources for Autonomie are sufficient. The reviewer said that the three models covered in this project should be reviewed individually. 
VTO Program Benefits Analysis:

Tom Stephens (Argonne National Laboratory) - van018

Presenter

Tom Stephens, Argonne National

Laboratory

\section{Reviewer Sample Size}

A total of six reviewers evaluated this project.

\section{Question 1: Approach to performing the work-the degree to which technical barriers are addressed, the project is well-designed, feasible, and integrated with other efforts.}

\section{Reviewer 1:}

The reviewer stated that the approach to the work enables an unbiased comparison of the impact of the VTO investments. It is a very valuable way to show the impact of the VTO funding. The reviewer recommended that other offices adopt the same approach.

\section{Reviewer 2:}

The reviewer said that, in general, the project seems well-designed and the PI has feasible plans for making the analysis more robust, for example, by refining retail PEV costs, incorporating more cost components, and doing sensitivities around fuel prices. The reviewer commented that understanding which benefits are attributable to the VTO program, as opposed to other policies and market effects, remains a significant challenge. The reviewer suggested incorporating the light-duty GHG standards into the base case and doing additional sensitivities to account for other non-VTO potential drivers for technology improvements.

The reviewer observed that there are four consumer choice models used to help address uncertainty in light-duty vehicle (LDV) sales share. While this seems like a good approach, the reviewer stated that it would have been helpful to know what technology penetration rates each model found. The reviewer said that if the results are similar, this could indicate that additional sensitivities need to be performed by altering the inputs to the consumer choice models; in other words, high adoption cases where tipping point for PEVs is reached, rather than just using different models.

\section{Reviewer 3:}

The reviewer said that the current analysis approach assigns all fossil energy improvements to VTO-funded R\&D and ignores corporate average fuel economy (CAFE)/GHG standards through 2025. This seems to double count the benefits that are due to CAFE/GHG standards. The reviewer said that VTO benefits should be relative to CAFE/ 
GHG standards (baseline) and show the improvement of fuel economy above and beyond CAFE/GHG or show how VTO-funded R\&D lowers the cost of complying with CAFE/GHG standards.

\section{Reviewer 4:}

The reviewer said that the project goal and approach are very clearly focused on program evaluation. The reviewer commented that the researchers are careful in capturing the appropriate attributes of the program relative to the no program case. The presenters are providing a logical methodology for evaluating the environmental, cost, and petroleum consumption effects of the program.

\section{Reviewer 5:}

The reviewer stated that not enough work was completed, and that some are behind schedule. The reviewer said that this happens, but it speaks to the design and feasibility as outlined.

The reviewer said that the baseline is highly suspect and is predicated on an internal belief and understanding within the DOE of technology improvement, without considering private market, university, and other driven technology development. This black and white approach to technology development is problematic, particularly because a lot of DOE investments empirically demonstrated over several decades are duplicative and/or lag behind privately generated technology advancement. The reviewer also stated that the project lead indicated that the nonVTO case did not comply with CAFE standards, and this is functionally a non-starter for accepting the research as valid or useful.

\section{Question 2: Technical accomplishments and progress toward overall project and DOE goals-the degree to which progress has been made, measured against performance indicators and demonstrated progress towards DOE goals.}

\section{Reviewer 1:}

The reviewer said that this is an ambitious project with significant potential to inform future policy by quantifying benefits of VTO technology performance goals. It can also help inform future advanced technology research by addressing challenges associated with such a complex modeling undertaking, for example, addressing discrepancies in assumptions and handling uncertainty across multiple models such as Autonomie, VISION, Advanced Vehicle Cost and Energy-Use Model (AVCEM), GREET, and the Plug-In Electric Vehicle Infrastructure model (PEVI).

The reviewer commented that good progress has been made. The reviewer was especially impressed by the integration of the agent-based charging infrastructure model with the Multi-Agent Transport Simulation (MATSim) framework. The reviewer suggested that multiple data sets, including both early PEV adopter data and more general household vehicle travel data, be used to calibrate the model and perform sensitivities around charging behavior.

\section{Reviewer 2:}

The reviewer stated that the project is consistent and has made steady progress. The presenters are focused on methodology and quantitative metrics for assessing program goals. The reviewer said that breaking down the levelized cost of driving for different components for a variety of powertrain technologies helps provide insight on where costs can be cut, both at the vehicle level and for the overall vehicle parc. The reviewer observed that the team has incorporated all of the LDV consumer choice models to get a sense of the uncertainty in the models as well as the uncertainty in the impact of the overall program. It would be worthwhile to explore what the underlying causes are for the differences in the models.

The reviewer commented that the infrastructure to grid interaction analysis is also a significant addition to the project. More information on the model, assumptions, and insight that the project team expects to learn should be made more explicit. The reviewer said that, similarly, information on the AVCEM model and expected insight should be more explicit.

\section{Reviewer 3:}

The reviewer said that the team has made significant progress and continues to update its analysis assumptions; however, some of the basis for the cost data are unclear. For example, the reviewer wonders what the hydrogen 
$\left(\mathrm{H}_{2}\right)$ cost is in 2025 and what the basis is. The reviewer questioned if this is assuming a high volume market and what the delivery pressure is. The reviewer observed that this does not seem consistent with the latest records from the Fuel Cell Technologies Office (FCTO), and that the same applies to the fuel cell cost.

\section{Reviewer 4:}

The reviewer commented that the baseline issue mentioned above makes it difficult to comment on the progress that has been made. The reviewer said that the retail cost markup research should be coordinated with DOT and EPA.

\section{Reviewer 5:}

The reviewer said that, overall, the project seems to be behind, but there are some positive outcomes. The project appears to have at least one foundational flaw that makes the output highly suspect. Regarding petroleum savings, the reviewer also stated that it is difficult to accept, given the approach concerns. On the topic of PEV to grid interactions, the reviewer stated that it is hard to evaluate as work is still in progress. Finally, regarding AVCEM, the reviewer remarked that it is hard to evaluate as work is still in progress.

\section{Question 3: Collaboration and coordination with other institutions.}

\section{Reviewer 1:}

The reviewer said that collaboration with the other laboratories involved in the work is strong; however, the work would benefit from greater collaboration outside of the project team. Detailed peer review of the work, with more depth than can be achieved in a 20-minute AMR presentation, could provide valuable input. The reviewer commented that OEMs, fuel providers, and other technology offices should be involved in reviewing the cost estimates and assumptions.

\section{Reviewer 2:}

The reviewer commented that collaboration across the program partners is clear. This activity brings together the analysis portfolio team. The reviewer remarked that reviewing results with additional stakeholders could benefit this analysis.

\section{Reviewer 3:}

The reviewer stated that the PI is working with appropriate government agencies and academia. The reviewer suggested also reaching out to INL regarding early PEV adopter data, which could be used to help inform modeling components on consumer behavior and charging usage.

\section{Reviewer 4:}

The reviewer stated that based on the omission of CAFE/GHG standards, it does not seem that there is enough coordination with other agencies.

\section{Question 4: Proposed future research-the degree to which the project has effectively planned its future work in a logical manner by incorporating appropriate decision points, considering barriers to the realization of the proposed technology and, when sensible, mitigating risk by providing alternate development pathways.}

\section{Reviewer 1:}

The reviewer stated that there were well-designed, feasible plans to make analysis more robust and incorporate more components. The reviewer suggested incorporating existing policies such as LDV GHG standards into the base case.

\section{Reviewer 2:}

The reviewer said that, overall, the updates proposed are good and will improve the analysis. The reviewer stated that it is not clear what the side cases are and how they will address the previous years' reviewer comment about the uncertainty around fuel prices. 


\section{Reviewer 3:}

The reviewer commented that the proposed future work is logical. There is a long list of factors to address, but it is not clear how these will be prioritized and which have the potential to have most impact.

\section{Reviewer 4:}

The reviewer said that the proposed work focused more on completing expected work versus addressing post-work assessment that identifies new needs and weaknesses that were revealed. The reviewer remarked that a review of the feedback provided from the prior year indicates that the project is not aligned or has chosen not to address important recommendations.

\section{Question 5: Does this project support the overall DOE objectives of petroleum displacement? Why or why not?}

\section{Reviewer 1:}

The reviewer expressed that this analysis directly provides insight into the impact of the VTO program and its objectives of petroleum displacement and GHG emissions.

\section{Reviewer 2:}

The reviewer expressed that the project will inform the DOE on which technologies and technology performance goals can have the biggest impact on petroleum reduction, GHGs, and other social benefits.

\section{Reviewer 3:}

The reviewer remarked that the project helps to identify the areas in which the VTO can have the most impact.

\section{Reviewer 4:}

The reviewer said that understanding and comparing the cost to consumers and society is an important project.

\section{Reviewer 5:}

The reviewer said that the program benefits analysis effort should explicitly state what assumptions are made regarding federal- and state-level vehicle emissions and fuel economy and fuel efficiency standards when evaluating the impacts of VTO-supported technologies. The reviewer remarked that not including federal and state minimum requirements could lead to considerable over-estimation of projected benefits.

\section{Reviewer 6:}

The reviewer said that this is a difficult choice to select no, and perhaps the preferred response would be maybe. The reviewer expressed concern that the research is less informative and more focused on reaching an answer of yes, in effect, providing the sponsor with the answer they want. The reviewer commented that, phrased differently, the analysis shows that the VTO work could achieve the goals; however, it does not provide good insight into where the risks are for it falling short or where the key value of efforts is, and this appeared to be part of the project objective.

The reviewer stated that the results are meaningless if CAFE compliance and other market-driven improvements are not part of a baseline. Comments last year raised this concern, and this major flaw has persisted.

\section{Question 6: Resources: How sufficient are the resources for the project to achieve the stated milestones in a timely fashion?}

\section{Reviewer 1:}

The reviewer said that to complete all the goals listed, the funding level seems low.

\section{Reviewer 2:}

The reviewer stated that the resources are sufficient; however, that is independent on if they are being used effectively. The resources should be enough to achieve the project requirements. 


\section{Assessing Energy and Cost Impact of Advanced Technologies through Model Based Design: Aymeric Rousseau (Argonne National Laboratory) - van023}

\section{Presenter}

Aymeric Rousseau, Argonne National Laboratory

\section{Reviewer Sample Size}

A total of six reviewers evaluated this project.

\section{Question 1: Approach to performing the work-the degree to which technical barriers are addressed, the project is well-designed, feasible, and integrated with other efforts.}

\section{Reviewer 1:}

The reviewer stated that the work is well-designed with clear milestones through FY 2018. The PI is addressing usability concerns identified by prior year reviewers and users through new plug and play features and improved workflow of Autonomie 2.0.

\section{Reviewer 2:}

The reviewer said that the project is very clear with a good approach. This is excellent work that is highly utilized by the industry. The reviewer remarked that the team had good responses to reviewer questions from last year. The approach taken to have two versions of Autonomie depending on the user, a simple version and an in-depth version, is excellent. The reviewer commented that it will improve greatly the use rate of the technology and the flexibility for advanced users.

\section{Reviewer 3:}

The reviewer said the feedback from the user community really seems to have improved the Autonomie tool.

\section{Reviewer 4:}

The reviewer said this capability has been developed over multiple years and has expanded its reach. Having full vehicle models for a wide range of powertrains is an important objective. The reviewer commented that making the tool available to a broad community is significant and that gathering community consensus to prioritize model inputs and assessments is a solid approach.

\section{Reviewer 5:}

The reviewer commented that the project was, analytically and scientifically, well-grounded and rigorous. Research and model improvements continue to add value and advance the state of the art.

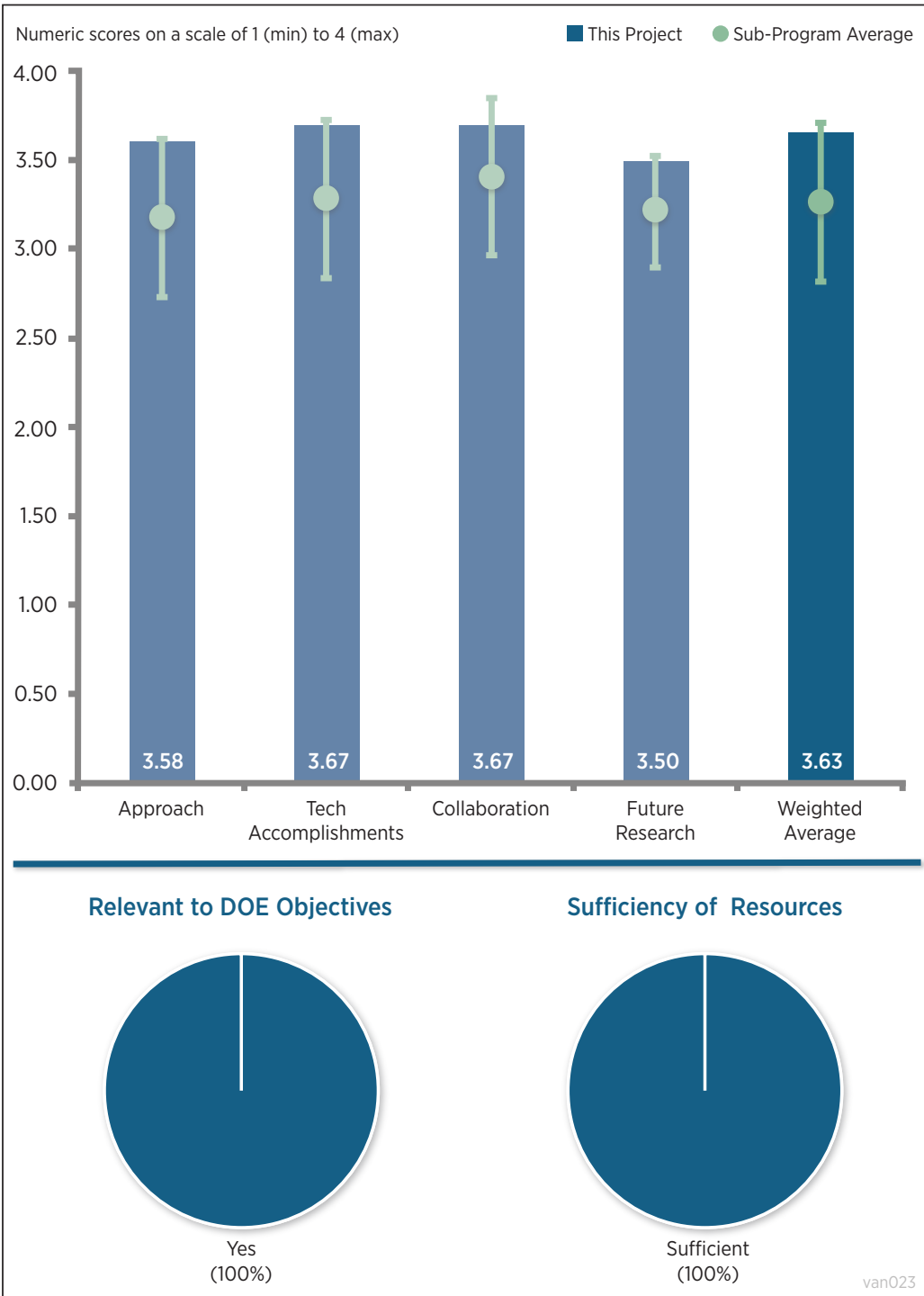

Figure 9-4 - Assessing Energy and Cost Impact of Advanced Technologies through Model Based Design: Aymeric Rousseau (Argonne National Laboratory) - Vehicle Analysis 


\section{Question 2: Technical accomplishments and progress toward overall project and DOE goals - the degree to which progress has been made, measured against performance indicators and demonstrated progress towards DOE goals.}

\section{Reviewer 1:}

The reviewer said that Autonomie provides robust system modeling capability of advanced technologies. There was significant technical progress including 100 new turn-key vehicles, new platform, and vehicle thermal validation. The reviewer was particularly impressed by the progress in building capacity related to smart mobility. The reviewer commented that, in particular, expanding modeling capabilities for autonomous vehicles and work on Autonomie EcoSystem are likely to be great assets to researchers and policymakers going forward.

\section{Reviewer 2:}

The reviewer said that the emphasis on validation is important and should continue. The accomplishments reflect a solid quality execution of research. The reviewer commented that accomplishments reflect a well thought-out process.

\section{Reviewer 3:}

The reviewer said the team has made excellent progress. This is highly valuable work that informs projects across VTO and FCTO and is also used for education in other agencies.

\section{Reviewer 4:}

The reviewer said that the team has focused on improving the usability of the tool. Adding application programming interface (APIs) and the ability to compare a wider set of simulations has enabled expanded assessments by the user community. The reviewer commented that the team has included an expanded set of turn-key vehicles to represent existing and future technologies. This, combined with the ability to look at individual component technologies, has allowed further exploration by the users. The reviewer remarked that the new workflow that enables the different access by user versus developer needs can expand the accessibility of the models.

\section{Question 3: Collaboration and coordination with other institutions.}

\section{Reviewer 1:}

The reviewer observed that very strong collaborations are apparent with industry, academia, and other federal agencies. Also, the team shows good international collaboration. The reviewer remarked that the collaboration with the software developers is very valuable to the work as well.

\section{Reviewer 2:}

The reviewer said that the project seems to engage in extensive coordination with labs, OEMs, and other stakeholders on data inputs and vehicle testing.

\section{Reviewer 3:}

The reviewer observed that the team collaborates across the program as well, with a broad network of external companies, users, and researchers who provide insight into the technologies being evaluated.

\section{Reviewer 4:}

The reviewer said that the collaborations are important, well-linked directionally with Autonomie, and are valuable. However, it was not clear how they are all linked to each other.

\section{Reviewer 5:}

The reviewer stated that the interaction with industry and the user community seems vital for the further refinements of the tool.

Question 4: Proposed future research-the degree to which the project has effectively planned its future work in a logical manner by incorporating appropriate decision points, considering barriers to the realization of the proposed technology and, when sensible, mitigating risk by providing alternate development pathways. 


\section{Reviewer 1:}

The reviewer said that the future plans to continue to try to integrate traffic flow data into the model are very interesting and are a good focus. The plans to also expand and integrate other analysis tools into the model make excellent use of investments already in place to expand upon the capabilities. The reviewer said the development of BMW's i3 extended range electric vehicle, the i3-EREV, is a very interesting case study bridging the BEV and fuel cell electric vehicle (FCEV) technologies.

\section{Reviewer 2:}

The reviewer said that detailed plans for future work seem well-designed and feasible.

\section{Reviewer 3:}

The reviewer remarked that, overall, the proposed future research is good and continues enhancements of the project. One significant new direction proposed is modeling of autonomous vehicles. However, the reviewer said that there were few details provided on the approach the project team will take to go from vehicle level to system level analysis.

\section{Reviewer 4:}

The reviewer said that the project recognizes the importance of engaging industry and continuing to improve the acceptance by industry and others. The project deserves credit for honestly considering the critical feedback provided and having clear ideas on how to address them in concrete ways. The reviewer said there are some concerns with how the work will support smart mobility. It will be necessary to disaggregate individual vehicle performance improvements and total system improvements or systemic impacts imparted on an individual vehicle when energy consumption and efficiency are being measured. The reviewer commented that the discussion provided by the project lead is cognizant of this challenge, but may benefit from deeper clarification beyond the discussion that occurred during the review.

\section{Question 5: Does this project support the overall DOE objectives of petroleum displacement? Why or why not?}

\section{Reviewer 1:}

The reviewer remarked that this work is extremely relevant, not only to the VTO objectives, but also to those of the sustainable transportation office providing a consistent platform across which to measure vehicle performance.

\section{Reviewer 2:}

The reviewer stated that the project provides a unique capability that is a resource to a very extensive analysis community. It allows for vehicle-level assessments of fuel consumption and costs of various technologies that provide insight, and is also a foundational input to other models.

\section{Reviewer 3:}

The reviewer said that the Autonomie tool enables the evaluation of technologies that are currently not available for on-road or dynamometer testing.

\section{Reviewer 4:}

The reviewer commented that the work very clearly links to outputs in evaluating the impact of the individual technologies the VTO is working with.

\section{Reviewer 5:}

The reviewer said that providing system modeling capability of advanced technologies can support OEMs bringing vehicles to market and policymakers trying to understand and address barriers to adoption.

\section{Question 6: Resources: How sufficient are the resources for the project to achieve the stated milestones in a timely fashion?}

\section{Reviewer 1:}

The reviewer said the resources are sufficient but on the low side. 
Reviewer 2:

The reviewer said that the resources appear to be producing a high ROI. Future resources appear to be aligned with clear use and project improvement and work. 


\section{Acronyms and Abbreviations}

AFDC Alternative Fuels Data Center

AMR Annual Merit Review

ANL Argonne National Laboratory

API Application programming interface

AVCEM Advanced Vehicle Cost and Energy-Use Model

BEV Battery electric vehicle

CAFE Corporate average fuel economy

DOE U.S. Department of Energy

DOT U.S. Department of Transportation

EIA Energy Information Administration

EPA U.S. Environmental Protection Agency

EREV Extended range electric vehicle

EV Electric vehicle

FCEV Fuel cell electric vehicle

FCTO Fuel Cell Technologies Office

FCV Fuel cell vehicle

FHWA Federal Highway Administration

FY Fiscal year

GHG Greenhouse gas

GREET Greenhouse gas, Regulated Emissions, and Energy use in Transportation

$\mathrm{H}_{2} \quad$ Hydrogen

HDV Heavy-duty vehicle

ICE Internal combustion engine

INL Idaho National Laboratory

LDV Light-duty vehicle

MATSim Multi-Agent Transport Simulation

MDV Medium-duty vehicle

MYRD\&D Multi-Year Research, Development, and Demonstration

NGO Non-governmental organization

NREL National Renewable Energy Laboratory 
OEM Original equipment manufacturer

ORNL Oak Ridge National Laboratory

OTAQ Office of Transportation and Air Quality

PEV Plug-in electric vehicle

PEVI Plug-in electric vehicle infrastructure

PHEV Plug-in hybrid electric vehicle

PI Principal investigator

QA Quality assurance

QC Quality control

R\&D Research and development

ROI Return on investment

SMART Systems and Modeling for Accelerated Research in Transportation

TEDB Transportation Energy Data Book

VTO Vehicle Technologies Office 


\section{Acronyms}

${ }^{\circ} \mathrm{C}$

$\mu \mathrm{m}$

1D

3D

3GAHHS

A

$\mathrm{A} / \mathrm{C}$

ABR

$\mathrm{AC}$

ACE

ACEC

ACI

ADAS

ADP

AEC

AEC

AEV

AFDC

AFV

Ag

Ah

AHHS

AIM

AKI

$\mathrm{Al}$

ALD

$\mathrm{AlF}_{3}$

AlNiCo

AMR

AMT

AMT

ANL

APEEM

API

API

APS

APTA

ARA

ARC

ARK

ARL
Degrees Celsius (Centigrade)

Micrometer (micron)

One dimensional

Three dimensional

Third-generation advanced high-strength steels

Ampere

Air conditioning

Advanced Battery Research

Alternating current

Advanced Combustion Engines (VTO program)

Advanced Combustion and Emissions Control

Advanced compression ignition

Advanced Driver Assistance Systems

Advanced drying process

Advanced engine combustion

Automotive Electronics Council

All-electric vehicle

Alternative Fuels Data Center

Alternative fuel vehicle

Silver

Ampere-hour

Advanced high-strength steels

Accelerated insertion of materials

Anti-knock index

Aluminum

Atomic layer deposition

Aluminum fluoride

Aluminum-nickel-cobalt

Annual Merit Review

Air maintenance technology

Automated manual transmission

Argonne National Laboratory

Advanced Power Electronics and Electric Motors

American Petroleum Institute

Application programming interface

Advanced photon source

American Public Transportation Association

Automotive Recyclers Association

Affordable Rankine cycle

Abuse reaction kinetics

Army Research Laboratory 


\begin{tabular}{|c|c|}
\hline ARPA-E & Advanced Research Projects Agency - Energy \\
\hline ASCR & Advanced scientific computing research \\
\hline ASI & Area specific impedance \\
\hline ASSERT & Analysis of Sustainability, Scale, Economics, Risk, and Trade \\
\hline ASTM & American Society for Testing and Materials \\
\hline ATF & Automatic transmission fluid \\
\hline ATR & Attenuated total reflectance \\
\hline AV & Advanced vehicle \\
\hline AVCEM & Advanced Vehicle Cost and Energy-Use Model \\
\hline AVT & Advanced vehicle technology \\
\hline AVTA & Advanced Vehicle Testing Activity \\
\hline AVTC & Advanced Vehicle Technology Competitions \\
\hline B & Magnetic flux density \\
\hline BATO & Bridgestone Americas Tire Operations \\
\hline BATT & Batteries for Advanced Transportation Technologies \\
\hline BCMS & Battery/capacitor management system \\
\hline BES & Office of Basic Energy Sciences \\
\hline BET & Battery electric truck \\
\hline BETO & Bioenergy Technologies Office \\
\hline $\mathrm{BEV}$ & Battery electric vehicle \\
\hline $\mathrm{B}-\mathrm{H}$ & Magnetic hysteresis curve (magnetic flux density versus magnetic field strength) \\
\hline BIW & Body in white \\
\hline BMEP & Brake mean effective pressure \\
\hline BMR & Battery Materials Research \\
\hline BMS & Battery management system \\
\hline $\mathrm{BNL}$ & Brookhaven National Laboratory \\
\hline BOM & Bill of materials \\
\hline $\mathrm{B}_{\mathrm{r}}$ & Magnetic remanence \\
\hline BSFC & Brake-specific fuel consumption \\
\hline BTE & Brake thermal efficiency \\
\hline $\mathrm{C}$ & Carbon \\
\hline C2ES & Center for Climate and Energy Solutions \\
\hline $\mathrm{Ca}$ & Calcium \\
\hline CA50 & Crank angle position at which $50 \%$ of heat is released \\
\hline CAD & Computer-aided design \\
\hline CAE & Computer-aided engineering \\
\hline CAEBAT & Computer-Aided Engineering for Electric-Drive Vehicle Batteries \\
\hline $\mathrm{CaFCP}$ & California Fuel Cell Partnership \\
\hline CAFE & Corporate average fuel economy \\
\hline CAISO & California Independent System Operator \\
\hline CAM & Cathode Active Material \\
\hline CAM-7 & Proprietary catholic material for lithium-ion batteries \\
\hline CAMP & Cell analysis, modeling, and prototyping \\
\hline
\end{tabular}




\begin{tabular}{|c|c|}
\hline CAN & Controller area network \\
\hline $\mathrm{CaSO}_{4}$ & Calcium sulfate \\
\hline CAV & Clean air vehicle \\
\hline CAV & Connected and automated vehicle \\
\hline $\mathrm{CC}$ & Constant current \\
\hline $\mathrm{CC} / \mathrm{S}$ & Carbon sulfur composite \\
\hline $\mathrm{CCC}$ & Co-precipitated $\mathrm{CuO}_{\mathrm{X}}, \mathrm{CoO}_{\mathrm{y}}$, and $\mathrm{CeO}_{2}$ catalyst \\
\hline $\mathrm{Cd}$ & Drag coefficient \\
\hline $\mathrm{Ce}$ & Cerium \\
\hline $\mathrm{CE}$ & Coulombic efficiency \\
\hline $\mathrm{Ce}$ & Cerium \\
\hline CEC & California Energy Commission \\
\hline CEMI & Clean Energy Manufacturing Initiative \\
\hline $\mathrm{CF}$ & Carbon fiber \\
\hline $\mathrm{CF}$ & Combustion fluid \\
\hline $\mathrm{CFC}$ & Carbon fiber composite \\
\hline CFD & Computational fluid dynamics \\
\hline CFRP & Carbon fiber-reinforced polymer \\
\hline CGI & Compacted graphite iron \\
\hline $\mathrm{CH}_{4}$ & Methane \\
\hline $\mathrm{CHA}$ & Chabazite \\
\hline $\mathrm{CHT}$ & Conjugate heat transfer \\
\hline $\mathrm{CI}$ & Compression ignition \\
\hline $\mathrm{Cl}$ & Chlorine \\
\hline CLEERS & Cross-Cut Lean Exhaust Emissions Reduction Simulations \\
\hline $\mathrm{CN}$ & Cetane number \\
\hline $\mathrm{CNG}$ & Compressed natural gas \\
\hline $\mathrm{CO}$ & Carbon monoxide \\
\hline Co & Cobalt \\
\hline $\mathrm{CO}_{2}$ & Carbon dioxide \\
\hline $\mathrm{CoS}_{2}$ & Cobalt-sulfide cattierite \\
\hline $\mathrm{COV}$ & Coefficient of variance \\
\hline CPES & Center for Power Electronics Systems \\
\hline $\mathrm{CPU}$ & Central processing unit \\
\hline $\mathrm{Cr}$ & Chromium \\
\hline $\mathrm{CR}$ & Compression ratio \\
\hline CRADA & Cooperative Research and Development Agreement \\
\hline $\mathrm{CRC}$ & Coordinating Research Council \\
\hline $\mathrm{Cu}$ & Copper \\
\hline $\mathrm{Cu}_{2} \mathrm{O}$ & Copper (I) oxide \\
\hline $\mathrm{CuF}_{2}$ & Copper (II) fluoride \\
\hline $\mathrm{CV}$ & Cyclic voltammetry \\
\hline $\mathrm{CY}$ & Calendar year \\
\hline
\end{tabular}




\begin{tabular}{|c|c|}
\hline $\mathrm{DC}$ & Direct current \\
\hline DEF & Diesel exhaust fluid \\
\hline DEF & Diesel Emissions Fluid \\
\hline $\mathrm{DF}$ & Dissipation factor \\
\hline DFT & Density functional theory \\
\hline $\mathrm{DI}$ & Direct injection \\
\hline DISI & Direct injection spark ignition \\
\hline DLC & Diamond-like carbon \\
\hline DME & Dimethyl ether \\
\hline DME & Dimethoxyethane \\
\hline DOC & Diesel oxidation catalyst \\
\hline DOD & Depth of discharge \\
\hline DOE & U.S. Department of Energy \\
\hline DOD & U.S. Department of Defense \\
\hline DOT & U.S. Department of Transportation \\
\hline DPF & Diesel particulate filter \\
\hline DPP & Dynamic particle-packing \\
\hline DSC & Differential scanning calorimetry \\
\hline DSRC & Dedicated short range communications \\
\hline DST & Dynamic stress test \\
\hline Dy & Dysprosium \\
\hline E0 & $0 \%$ ethanol blend with gasoline \\
\hline E10 & $10 \%$ ethanol blend with gasoline \\
\hline E100 & $100 \%$ ethanol blend with gasoline \\
\hline E15 & $15 \%$ ethanol blend with gasoline \\
\hline E20 & $20 \%$ ethanol blend with gasoline \\
\hline E30 & $30 \%$ ethanol blend with gasoline \\
\hline E85 & $85 \%$ ethanol blend with gasoline \\
\hline EAVS & Electrically assisted variable speed \\
\hline $\mathrm{EC}$ & Ethylene carbonate \\
\hline $\mathrm{ECN}$ & Engine Combustion Network \\
\hline EDT & Electric Drive Technologies (VTO program) \\
\hline EDV & Electric drive vehicle \\
\hline EERE & Office of Energy Efficiency and Renewable Energy \\
\hline EETT & Electrical and Electronics Technical Team \\
\hline EGR & Exhaust gas recirculation \\
\hline EIA & Energy Information Administration \\
\hline EIS & Electrochemical impedance spectroscopy \\
\hline EIVC & Early intake valve closing \\
\hline EMC & Electromagnetic compatibility \\
\hline EMI & Electromagnetic interference \\
\hline EMS & Emergency medical services \\
\hline EOL & End-of-life \\
\hline
\end{tabular}




\begin{tabular}{|c|c|}
\hline EPA & U.S. Environmental Protection Agency \\
\hline EPAct & Energy Policy Act of 1992 \\
\hline EPD & Electrophoretic deposition \\
\hline ERC & Engine Research Center \\
\hline EREV & Extended range electric vehicle \\
\hline ES & Electrochemical Energy Storage (VTO program) \\
\hline ESR & Equivalent series resistance \\
\hline EU & European Union \\
\hline EUCAR & European Council for Automotive Research and Development \\
\hline EV & Electric vehicle \\
\hline EVAS & Electrically-assisted variable speed \\
\hline eVMT & Electric vehicle miles traveled \\
\hline EVSE & Electric vehicle supply equipment \\
\hline EVSP & Electric vehicle service provider \\
\hline $\mathrm{F}$ & Fluorine \\
\hline FA & Field aged \\
\hline FACE & Fuels for Advanced Combustion Engines \\
\hline FAME & Fatty acid methyl ester \\
\hline FASTSim & Future Automotive Systems Technology Simulator \\
\hline $\mathrm{FC}$ & Fuel cell \\
\hline FCA & Fiat Chrysler Automobiles \\
\hline $\mathrm{FCC}$ & First Cycle Capacity \\
\hline FCE & First Cycle Efficiency \\
\hline FCEV & Fuel cell electric vehicle \\
\hline FCTO & Fuel Cell Technologies Office \\
\hline FCV & Fuel cell vehicle \\
\hline $\mathrm{Fe}$ & Iron \\
\hline FE & Fuel economy \\
\hline FEA & Finite element analysis \\
\hline $\mathrm{Fe}-\mathrm{Co}$ & Iron-cobalt \\
\hline $\mathrm{FeF}_{3}$ & Iron fluoride \\
\hline FEM & Finite element modeling \\
\hline $\mathrm{FeS}_{2}$ & Iron sulfide \\
\hline FGC & Fuel gradient cathode \\
\hline FHWA & Federal Highway Administration \\
\hline FIE & Fuel injected engines \\
\hline FMEA & Failure mode and effects analysis \\
\hline FMVSS & Federal Motor Vehicle Safety Standards \\
\hline FOA & Funding opportunity announcement \\
\hline FRESCO & Fast and Reliable Engine Simulation Code \\
\hline FRP & Fiber-reinforced polymer (fiber-reinforced plastic) \\
\hline FSW & Friction-stir welding \\
\hline FT & Fuel and Lubricant Technologies (VTO program) \\
\hline
\end{tabular}




\begin{tabular}{|c|c|}
\hline FTP & Federal Test Procedure \\
\hline FWG & Fuels Working Group \\
\hline FY & Fiscal year \\
\hline $\mathrm{g}$ & Gram \\
\hline $\mathrm{GaN}$ & Gallium nitride \\
\hline $\mathrm{GC}$ & Gas chromatography \\
\hline GCI & Gasoline compression ignition \\
\hline GC-MS & Gas chromatography - mass spectrometry \\
\hline GDCI & Gasoline direct compression engine \\
\hline GDI & Gasoline direct injection \\
\hline GE & General Electric \\
\hline GGE & Gallons gasoline equivalent \\
\hline GHG & Greenhouse gas \\
\hline GITT & Grid Integration Technical Team \\
\hline GM & General Motors \\
\hline GOC & Gasoline oxidation catalyst \\
\hline GPF & Gasoline particulate filter \\
\hline GPU & Graphics processing unit \\
\hline $\mathrm{Gr} / \mathrm{S}$ & Graphite Sulfur \\
\hline GREET & Greenhouse gas, Regulated Emissions, and Energy use in Transportation \\
\hline GSA & Global sensitivity analysis \\
\hline GSF2 & Generic speed form 2 \\
\hline GSI & Gasoline spark ignition \\
\hline GT & Georgia Tech University \\
\hline GTDI & Gasoline turbocharged direct injection \\
\hline GTR & Global Technical Regulation \\
\hline $\mathrm{H}$ & Henry (unit of electrical inductance) \\
\hline $\mathrm{H}$ & Magnetic field strength \\
\hline $\mathrm{H}_{2}$ & Hydrogen \\
\hline $\mathrm{H}_{2} \mathrm{O}$ & Water \\
\hline HA & High-active \\
\hline $\mathrm{HC}$ & Hydrocarbon \\
\hline $\mathrm{HCCI}$ & Homogeneous charge compression ignition \\
\hline Hci & Intrinsic coercivity \\
\hline $\mathrm{HCl}$ & Hydrochloric Acid \\
\hline HCMR & High-capacity manganese rich \\
\hline HD & Heavy-duty \\
\hline HDV & Heavy-duty vehicle \\
\hline HEV & Hybrid electric vehicle \\
\hline HFRR & High frequency reciprocating rig \\
\hline $\mathrm{HHC}$ & Heavy hydrocarbon \\
\hline HIL & Hardware-in-the-loop \\
\hline $\mathrm{HOV}$ & Heat of vaporization \\
\hline
\end{tabular}




\begin{tabular}{|c|c|}
\hline hp & Horsepower \\
\hline HPC & High-performance computing \\
\hline HPDC & High-pressure diecast \\
\hline hr. & Hour \\
\hline HTA & High-temperature hydrothermal aging \\
\hline HV & High-voltage \\
\hline HVAC & Heating, ventilating, and air-conditioning (heat, ventilation, and air-conditioning) \\
\hline HWFE & Highway fuel economy \\
\hline IAEM & International Association of Energy Managers \\
\hline IAPG & Interagency Advanced Power Group \\
\hline $\mathrm{IC}$ & Integrated circuit \\
\hline ICE & Internal combustion engine \\
\hline ICME & Integrated computational materials engineering \\
\hline IDT & Ignition delay time \\
\hline IGBT & Insulated-gate bipolar transistors \\
\hline IITR & International Institute of Towing and Recovery \\
\hline IL & Ionic liquid \\
\hline IL-NP & Ionic liquid nanoparticle \\
\hline ILSAC & International Lubricants Standardization and Approval Committee \\
\hline IMEP & Indicated mean effective pressure \\
\hline INL & Idaho National Laboratory \\
\hline IoT & Internet of things \\
\hline IP & Intellectual property \\
\hline IPM & Integrated permanent magnet \\
\hline IPM & Interior permanent magnet \\
\hline IQT & Ignition quality tester \\
\hline IR & Infrared \\
\hline IREV & Initiative for Resiliency in Energy through Vehicles \\
\hline $\mathrm{IrO}_{2}$ & Iridium oxide \\
\hline ISFC & Indicated specific fuel consumption \\
\hline ISO & International Organization for Standardization \\
\hline ITHR & Intermediate temperature heat release \\
\hline IVC & Intake valve closing \\
\hline $\mathrm{J}$ & Joule \\
\hline JM & Johnson Matthey catalyst \\
\hline K & Potassium \\
\hline $\mathrm{Kg}$ & Kilogram \\
\hline $\mathrm{kHz}$ & Kilohertz \\
\hline $\mathrm{kV}$ & Kilovolt \\
\hline $\mathrm{kW}$ & Kilowatt \\
\hline $\mathrm{kWh}$ & Kilowatt-hour \\
\hline $\mathrm{L}$ & Liter \\
\hline $\mathrm{La}$ & Lanthanum \\
\hline
\end{tabular}




\begin{tabular}{|c|c|}
\hline LANL & Los Alamos National Laboratory \\
\hline lb. & Pound \\
\hline LBNL & Lawrence Berkeley National Laboratory \\
\hline LCA & Life-cycle assessment (life-cycle analysis) \\
\hline LCO & Lithium cobalt oxide \\
\hline LCP & Lithium Cobalt Phosphate \\
\hline LD & Light-duty \\
\hline $\mathrm{LDH}$ & Limiting dome height \\
\hline LDV & Light-duty vehicle \\
\hline LES & Large eddy simulation \\
\hline LFO & Lithium iron oxide \\
\hline LFP & Lithium iron phosphate \\
\hline LGGF & Low Greenhouse Gas Fuels team \\
\hline $\mathrm{Li}$ & Lithium \\
\hline $\mathrm{Li}_{2} \mathrm{CO}_{3}$ & Lithium carbonate \\
\hline $\mathrm{Li}_{2} \mathrm{O}$ & Lithium oxide \\
\hline $\mathrm{Li}_{2} \mathrm{~S}$ & Lithium sulfide \\
\hline LIB & Lithium ion battery \\
\hline LiBOB & Lithium bis(oxalato)borate \\
\hline LIC & Lithium ion capacitor \\
\hline $\mathrm{LiCoO}_{2}$ & Lithium cobalt oxide \\
\hline $\mathrm{LiFePO}_{4}$ & Lithium iron phosphate \\
\hline LiFSI & Lithium bis(fluorosulfonyl)imide \\
\hline Li-ion & Lithium ion \\
\hline $\mathrm{LiO}_{2}$ & Lithium superoxide \\
\hline $\mathrm{LiPF}_{6}$ & Effective electrolyte salt for lithium-ion battery \\
\hline Li-S & Lithium sulfur \\
\hline LiTFSI & Lithium bis(trifluoromethanesulfonyl)imide \\
\hline LIVC & Large intake valve closing \\
\hline LLC & Limited liability company \\
\hline LLNL & Lawrence Livermore National Laboratory \\
\hline LM & Lightweight Materials (VTO program) \\
\hline LMNO & Lithium-manganese nickel oxide \\
\hline $\mathrm{LMO}$ & Lithium manganese oxide \\
\hline LMR & Lithium manganese rich \\
\hline LNMO & Lithium nickel manganese oxide \\
\hline LP & Low-pressure \\
\hline LS-DYNA & Non-linear finite element analysis software program \\
\hline LSPI & Low-speed pre-ignition \\
\hline LTAT & Low-temperature aftertreatment \\
\hline LTC & Low-temperature combustion \\
\hline LTGC & Low-temperature gasoline combustion \\
\hline LTHR & Low-temperature heat release \\
\hline
\end{tabular}




\begin{tabular}{|c|c|}
\hline LTO & Lithium titanium oxide \\
\hline $\mathrm{M} \& \mathrm{~S}$ & Modeling and Simulation \\
\hline MD & Medium-duty \\
\hline MD & Methyl decanoate \\
\hline MDA & Molecular dynamic analysis \\
\hline MDV & Medium-duty vehicle \\
\hline MECA & Manufacturers of Emission Controls Association \\
\hline MERF & Materials Engineering Research Facility \\
\hline MFCA & Multi-functional cathode additive \\
\hline MFx & Metal fluoride \\
\hline $\mathrm{Mg}$ & Magnesium \\
\hline MGI & Materials Genome Initiative \\
\hline $\mathrm{MgO}$ & Magnesium oxide \\
\hline MGOe & Megagauss-oersteds \\
\hline mi. & Mile \\
\hline MIT & Massachusetts Institute of Technology \\
\hline $\mathrm{mJ}$ & Millijoule \\
\hline $\mathrm{mm}$ & Millimeter \\
\hline MMLV & Multi-material lightweight vehicle \\
\hline $\mathrm{Mn}$ & Manganese \\
\hline $\mathrm{Mo}_{2} \mathrm{C}$ & Molybdenum carbide \\
\hline MON & Motor octane number \\
\hline MOS & Metal-oxide-semiconductor \\
\hline MOSFET & Metal-oxide-semiconductor field-effect transistor \\
\hline MOU & Memorandum of Understanding \\
\hline MOVE & Methane Opportunities for Vehicular Enhancement \\
\hline $\mathrm{MPa}$ & Megapascal \\
\hline MPG & Miles per gallon \\
\hline MPGe & Miles per gallon gasoline equivalent \\
\hline MPI & Multi-point injection \\
\hline $\mathrm{ms}$ & Millisecond \\
\hline MSMD & Multi-scale multi-domain \\
\hline MSR & Multi-speed range \\
\hline MSU & Michigan State University \\
\hline MT & Market transformation \\
\hline MTC & Michigan Mobility Transformation Center \\
\hline MTM & Mini traction machine \\
\hline MW & Molecular weight \\
\hline MY & Model year \\
\hline MYRD\&D & Multi-Year Research, Development, and Demonstration \\
\hline $\mathrm{N}_{2}$ & Nitrogen \\
\hline $\mathrm{N}_{2} \mathrm{O}$ & Nitrous oxide \\
\hline NA & North American \\
\hline
\end{tabular}




\begin{tabular}{|c|c|}
\hline $\mathrm{Na}$ & Sodium \\
\hline NAFTC & National Alternative Fuels Training Consortium \\
\hline NAFTD & North American Fire Training Directors \\
\hline $\mathrm{NaOH}$ & Sodium hydroxide \\
\hline NATA & North American Towing Association \\
\hline NBB & National Biodiesel Board \\
\hline NCA & Nickel cobalt aluminum oxide \\
\hline $\mathrm{NCM}$ & Nickel cobalt manganese \\
\hline ND & Neutron diffraction \\
\hline $\mathrm{Nd}$ & Neodymium \\
\hline $\mathrm{Nd}_{2} \mathrm{O}_{3}$ & Neodymium (III) oxide \\
\hline NDE & Non-destructive evaluation \\
\hline $\mathrm{NdFeB}$ & Neodymium magnet \\
\hline NDT & Non-destructive testing \\
\hline NFPA & National Fire Protection Association \\
\hline NG & Natural gas \\
\hline NGA & National Governors Association \\
\hline NGO & Non-governmental organization \\
\hline NGV & Natural gas vehicle \\
\hline $\mathrm{nH}$ & Nanohenry \\
\hline $\mathrm{NH}_{3}$ & Ammonia \\
\hline $\mathrm{NH}_{4} \mathrm{NO}_{3}$ & Ammonium nitrate \\
\hline NHTSA & National Highway Traffic Safety Administration \\
\hline $\mathrm{Ni}$ & Nickel \\
\hline NI & National Instruments \\
\hline NIST & National Institute of Standards and Technology \\
\hline $\mathrm{nm}$ & Nanometer \\
\hline NMC & Nickel manganese cobalt oxide \\
\hline NMO & Nickel Manganese Oxide \\
\hline NMP & N-Methylpyrrolidone \\
\hline NMR & Nuclear magnetic resonance \\
\hline NNMI & National Network for Manufacturing Innovation \\
\hline NO & Nitric oxide (nitrogen oxide, nitrogen monoxide) \\
\hline $\mathrm{NO}_{2}$ & Nitrogen dioxide \\
\hline $\mathrm{NO}_{\mathrm{x}}$ & Oxides of nitrogen \\
\hline NP & Nanoparticle \\
\hline NPS & National Park Service \\
\hline NRE & Non-rare earth \\
\hline NREL & National Renewable Energy Laboratory \\
\hline NSF & National Science Foundation \\
\hline NSWC & Naval Surface Warfare Center \\
\hline NVO & Negative valve overlap \\
\hline $\mathrm{O}_{2}$ & Oxygen \\
\hline
\end{tabular}




\begin{tabular}{|c|c|}
\hline OAS & Open architecture software \\
\hline $\mathrm{OBC}$ & On-board charger \\
\hline OBD & On-board diagnostics \\
\hline Oe & Oersteds \\
\hline $\mathrm{OE}$ & Office of Electricity Delivery and Energy Reliability \\
\hline OEM & Original equipment manufacturer \\
\hline $\mathrm{OH}$ & Hydroxide \\
\hline OI & Octane index \\
\hline OQMD & Open Quantum Materials Database \\
\hline ORAU & Oak Ridge Associated Universities \\
\hline ORC & Organic Rankine Cycle \\
\hline ORNL & Oak Ridge National Laboratory \\
\hline ORR & Oxygen reduction reaction \\
\hline OS & Organosilicon \\
\hline OTAQ & Office of Transportation and Air Quality \\
\hline $\mathrm{P}$ & Phosphorous \\
\hline $\mathrm{Pa}$ & Pascal \\
\hline PA & Polyamide \\
\hline PAA & Poly(acrylic acid) \\
\hline PAH & Polycyclic aromatic hydrocarbon \\
\hline $\mathrm{PAO}$ & Polyalphaolefin \\
\hline PBA & Planar-Bond-All \\
\hline PCM & Phase change material \\
\hline PCP & Peak cylinder pressure \\
\hline $\mathrm{Pd}$ & Palladium \\
\hline PDF & Probability density function \\
\hline $\mathrm{PE}$ & Polyethylene \\
\hline PE & Power electronics \\
\hline PEI & Polyetherimide \\
\hline Penn State & Pennsylvania State University \\
\hline PEV & Plug-in electric vehicle \\
\hline PEVI & Plug-in electric vehicle infrastructure \\
\hline PFI & Port fuel injection \\
\hline PFM & Poly(9,9-dioctylfluorene-co-9-fluorenone-co-methyl benzoic ester \\
\hline PGM & Platinum group metal \\
\hline PHET & Plug-in hybrid electric truck \\
\hline PHEV & Plug-in hybrid electric vehicle \\
\hline PI & Principal Investigator \\
\hline PIV & Particle image velocimetry \\
\hline PM & Particulate matter \\
\hline PM & Permanent magnet \\
\hline PM & Propulsion Materials (VTO program) \\
\hline PMI & Particulate matter index \\
\hline
\end{tabular}




\begin{tabular}{|c|c|}
\hline PML & Polymer-multi-layer \\
\hline $\mathrm{PN}$ & Particulate number \\
\hline PNA & Passive $\mathrm{NO}_{\mathrm{x}}$ adsorber \\
\hline PNNL & Pacific Northwest National Laboratory \\
\hline $\mathrm{PP}$ & Polypropylene \\
\hline ppm & Part per million \\
\hline PPy & Polypyrrole \\
\hline $\operatorname{Pr}$ & Praseodymium \\
\hline PR & Pressure rise \\
\hline PS & Polysulfide \\
\hline psi & Pounds per square inch \\
\hline $\mathrm{Pt}$ & Platinum \\
\hline PTO & Power Takeoff \\
\hline PVDF & Polyvinylidene difluoride \\
\hline Q\&A & Question and answer \\
\hline QA & Quality assurance \\
\hline $\mathrm{R} \& \mathrm{D}$ & Research and development \\
\hline RANS & Reynolds-averaged Navier-Stokes equations \\
\hline RASIC & Responsible, Approving, Supporting, Informed, and Consulted \\
\hline RAT & Rapid Aging Test \\
\hline $\mathrm{RCCI}$ & Reactivity controlled compression ignition \\
\hline $\mathrm{RCM}$ & Rapid compression machine \\
\hline RE & Rare earth \\
\hline RF & Radio frequency \\
\hline RFI & Radio frequency interference \\
\hline RFS & Renewable Fuel Standard \\
\hline $\mathrm{Rh}$ & Rhodium \\
\hline RK & Reaction kinetics \\
\hline ROI & Return on investment \\
\hline RON & Research octane number \\
\hline RTA & Rio Tinto Alcan \\
\hline RTM & Resin transfer molding \\
\hline $\mathrm{Ru}$ & Ruthenium \\
\hline RVE & Representative volume element \\
\hline $\mathrm{S}$ & Sulfur \\
\hline SAE & Society of Automotive Engineers \\
\hline SAF-D & Safe Alternative Fuels Deployment in Mid-America \\
\hline $\mathrm{Sb}$ & Antimony \\
\hline SBIR & Small Business Innovation Research \\
\hline SCE & Stratified charge engine \\
\hline $\mathrm{SCI}$ & Stoichiometric compression ignition \\
\hline SCR & Selective catalytic reduction \\
\hline SDPF & SCR-Coated DPF \\
\hline
\end{tabular}




\begin{tabular}{|c|c|}
\hline $\mathrm{Se}$ & Selenium \\
\hline SEI & Solid electrolyte interface \\
\hline SEM & Scanning electron microscope \\
\hline SEO & Symmetric polystyrene-block-poly (ethylene oxide) \\
\hline $\mathrm{Si}$ & Silicon \\
\hline SI & Spark ignition \\
\hline $\mathrm{SiC}$ & Silicon carbide \\
\hline SIMS & Secondary ion mass spectrometry \\
\hline $\mathrm{SiO}_{2}$ & Silicon dioxide \\
\hline SLA & Sealed lead acid \\
\hline SMART & Systems and Modeling for Accelerated Research in Transportation \\
\hline SMC & Sheet molding compound \\
\hline SME & Subject matter expert \\
\hline $\mathrm{Sn}$ & Tin \\
\hline SNL & Sandia National Laboratories \\
\hline SNR & Signal-to-noise ratio \\
\hline SOA & Semiconductor optical amplifier \\
\hline $\mathrm{SOC}$ & State of charge \\
\hline SOI & Start of ignition \\
\hline SPaT & Signal phase and timing \\
\hline SS & Start/stop \\
\hline SSE & Solid state electrolyte \\
\hline STEM & Science, Technology, Engineering, and Mathematics \\
\hline SUS & Stainless steel \\
\hline SWCNT & Single wall carbon nanotube \\
\hline SwRI & Southwest Research Institute \\
\hline TARDEC & U.S. Army Tank and Automotive Research, Development and Engineering Center \\
\hline TEDB & Transportation Energy Data Book \\
\hline TEM & Transmission electron microscope \\
\hline TESF & Tabulated equivalent strain flamelet \\
\hline TGA & Thermal gravimetric ignition \\
\hline $\mathrm{TI}$ & Technology Integration (VTO program) \\
\hline $\mathrm{Ti}$ & Titanium \\
\hline $\mathrm{TiO}_{2}$ & Titanium dioxide \\
\hline TIR & Technical Information Report \\
\hline $\mathrm{TiS}_{2}$ & Titanium disulfide \\
\hline TMS & The Materials, Metals and Minerals Society \\
\hline TOF & Time of flight \\
\hline TRAA & Towing and Recovery Association of America \\
\hline TRL & Technology readiness levels \\
\hline TSDC & Transportation Secure Data Center \\
\hline T-t-T & Train-the-Trainer \\
\hline TWB & Tailor-welded blanks \\
\hline
\end{tabular}




\begin{tabular}{|c|c|}
\hline TWB Co. & TWB Company \\
\hline TWC & Three-way catalyst \\
\hline U.S. & United States \\
\hline U.S. DRIVE & $\begin{array}{l}\text { United States Driving Research and Innovation for Vehicle efficiency and Energy } \\
\text { sustainability }\end{array}$ \\
\hline UAB & University of Alabama at Birmingham \\
\hline $\mathrm{UC}$ & Unused capacity \\
\hline UD & Unidirectional \\
\hline UH & University of Houston \\
\hline UHSS & Ultra-high strength steels \\
\hline $\mathrm{UM}$ & University of Michigan \\
\hline UMTRI & University of Michigan Transportation Research Institute \\
\hline UN & United Nations \\
\hline UnCoVerCPS & Unifying Control and Verification of Cyber-Physical Systems \\
\hline UQ & Uncertainty quantification \\
\hline USABC & United States Advanced Battery Consortium \\
\hline USAMP & United States Automotive Materials Partnership \\
\hline USCAR & United States Council for Automotive Research \\
\hline USDA & U.S. Department of Agriculture \\
\hline UTEMPRA & Unitary Thermal Energy Management for Propulsion Range Augmentation \\
\hline UTK & University of Tennessee, Knoxville \\
\hline UTS & Ultimate tensile strength \\
\hline UV & Ultraviolet \\
\hline $\mathrm{V}$ & Vanadium \\
\hline $\mathrm{V}$ & Volt \\
\hline V2G & Vehicle-to-grid \\
\hline V2I & Vehicle-to-infrastructure \\
\hline $\mathrm{V} 2 \mathrm{~V}$ & Vehicle-to-vehicle \\
\hline $\mathrm{V} 2 \mathrm{X}$ & Vehicle-to-grid, infrastructure, and/or vehicle (vehicle-to-everything) \\
\hline VAC & Volt alternating current \\
\hline VACG & Vacuum assisted counter gravity \\
\hline VAN & Vehicle Technologies Analysis (VTO program) \\
\hline VCR & Variable compression ratio \\
\hline VDC & Vehicle dynamic control \\
\hline VFM & Variable frequency microwave \\
\hline VIBE & Virtual integrated battery environment \\
\hline VIE & Variable interest entity \\
\hline VM & Viscosity modifier \\
\hline VMT & Vehicle miles traveled \\
\hline VS & Vehicle Systems (VTO program) \\
\hline VSATT & Vehicle Systems Analysis Technical Team \\
\hline VT & Vehicle Technologies \\
\hline Vth & Threshold voltage \\
\hline VTO & Vehicle Technologies Office \\
\hline
\end{tabular}




$\begin{array}{ll}\text { VVA } & \text { Variable valve actuation } \\ \mathrm{W} & \text { Watt } \\ \mathrm{WBG} & \text { Wide bandgap } \\ \mathrm{Wh} & \text { Watt-hour } \\ \mathrm{Wh} / \mathrm{mi} & \text { Watt-hour per mile } \\ \mathrm{WHR} & \text { Waste heat recovery } \\ \mathrm{W}-\mathrm{hr} . & \text { Watt-hour } \\ \text { WOT } & \text { Wide open throttle } \\ \text { WPT } & \text { Wireless power transfer } \\ \mathrm{XAS} & \text { X-ray absorption spectroscopy } \\ \mathrm{xEV} & \text { Generic term for any hybrid, plug-in, or electric vehicle } \\ \mathrm{ZDDP} & \text { Zinc dialkyldithiophosphates } \\ \mathrm{Zn} & \text { Zinc } \\ \mathrm{Zr} & \text { Zirconium } \\ \mathrm{ZrO} & \text { Zirconium dioxide }\end{array}$




\section{Appendix A: 2016 Annual Merit Review Attendees}

\begin{tabular}{|c|c|}
\hline Name & Affiliation \\
\hline $\begin{array}{l}\text { Mohamad } \\
\text { Abdul-Hak }\end{array}$ & $\begin{array}{c}\text { Mercedes Benz Research \& } \\
\text { Development }\end{array}$ \\
\hline Daniel Abraham & $\begin{array}{l}\text { Argonne National } \\
\text { Laboratory }\end{array}$ \\
\hline Judi Abraham & CMA, Inc./ATS \\
\hline Salvador Aceves & LLNL \\
\hline Jesse Adams & U.S. Department of Energy \\
\hline Radoslav Adzic & $\begin{array}{c}\text { Brookhaven National } \\
\text { Laboratory }\end{array}$ \\
\hline Kareem Afzal & PDC Machines \\
\hline Anant Agarwal & EERE/DOE \\
\hline Nick Agathocleous & Hanon Systems \\
\hline $\begin{array}{l}\text { Eduardo } \\
\text { Aguilera-Gomez }\end{array}$ & University of Guanajuato \\
\hline Rajesh Ahluwalia & $\begin{array}{l}\text { Argonne National } \\
\text { Laboratory }\end{array}$ \\
\hline Iftikhar Ahmad & Lambda Technologies \\
\hline Alauddin Ahmed & University of Michigan \\
\hline Shabbir Ahmed & $\begin{array}{l}\text { Argonne National } \\
\text { Laboratory }\end{array}$ \\
\hline Channing Ahn & Caltech \\
\hline $\begin{array}{l}\text { Christopher } \\
\text { Ainscough }\end{array}$ & NREL \\
\hline Venkat Aitharaju & General Motors \\
\hline Yoshio Akimune & $\begin{array}{l}\text { Innovative Structural } \\
\text { Materials Association }\end{array}$ \\
\hline Mohamed Alamgir & LG Chem Power \\
\hline Shaun Alia & $\begin{array}{l}\text { National Renewable } \\
\text { Energy Laboratory }\end{array}$ \\
\hline
\end{tabular}

\begin{tabular}{|c|c|}
\hline Name & Affiliation \\
\hline James Alkire & U.S. Department of Energy \\
\hline Jan Allen & Army Research Laboratory \\
\hline Mark Allendorf & $\begin{array}{l}\text { Sandia National } \\
\text { Laboratories }\end{array}$ \\
\hline Laura Allerston & $\begin{array}{l}\text { The University of } \\
\text { Birmingham }\end{array}$ \\
\hline Charles Alsup & U.S. Department of Energy \\
\hline Pascal Amar & Volvo Group North America \\
\hline Joseph Ambrosio & $\begin{array}{l}\text { Unique Electric } \\
\text { Solutions LLC }\end{array}$ \\
\hline Khalil Amine & $\begin{array}{l}\text { Argonne National } \\
\text { Laboratory }\end{array}$ \\
\hline Ramin Amin-Sanayei & Arkema \\
\hline Seong Jin An & $\begin{array}{c}\text { Oak Ridge National } \\
\text { Laboratory }\end{array}$ \\
\hline David Anderson & $\begin{array}{l}\text { DOE/Vehicle Technologies } \\
\text { Office }\end{array}$ \\
\hline Michele Anderson & Office of Naval Research \\
\hline Norm Anderson & Goodyear Tire \& Rubber Co \\
\hline Robert Angel & ORAU \\
\hline Donald Anton & $\begin{array}{c}\text { Savannah River National } \\
\text { Laboratory }\end{array}$ \\
\hline Shane Ardo & $\begin{array}{l}\text { University of California } \\
\text { Irvine }\end{array}$ \\
\hline Muhammad Arif & NIST \\
\hline Brett Aristegui & U.S. Department of Energy \\
\hline John Arnold & Miltec UV International \\
\hline Christopher Arntsen & $\begin{array}{l}\text { University of Chicago, } \\
\text { Department of Chemistry }\end{array}$ \\
\hline
\end{tabular}




\begin{tabular}{|c|c|}
\hline Name & Affiliation \\
\hline Rajendran Arunagiri & $\begin{array}{c}\text { Eastman Chemical } \\
\text { Company }\end{array}$ \\
\hline Koichiro Asazawa & Daihatsu Motor \\
\hline Alex Askari & $\begin{array}{c}\text { Naval Surface Warfare } \\
\text { Center }\end{array}$ \\
\hline Hikaru Aso & Toyota Technical Center \\
\hline Plamen Atanassov & University of New Mexico \\
\hline Lance Atkins & $\begin{array}{c}\text { Nissan Technical Center } \\
\text { North America }\end{array}$ \\
\hline Debra Audus & Not Applicable \\
\hline Marc Auger & Magna \\
\hline Tom Autrey & $\begin{array}{l}\text { Pacific Northwest } \\
\text { National Laboratory }\end{array}$ \\
\hline Scott Averitt & Robert Bosch LLC \\
\hline Michelle Avillanoza & $\begin{array}{c}\text { Allegheny Science \& } \\
\text { Technology }\end{array}$ \\
\hline Katherine Ayers & Proton OnSite \\
\hline Susan Babinec & ARPA-E \\
\hline Chulsung Bae & $\begin{array}{l}\text { Rensselaer Polytechnic } \\
\text { Institute }\end{array}$ \\
\hline Bamdad Bahar & Xergy Inc. \\
\hline Hua Bai & Kettering University \\
\hline Jianming Bai & $\begin{array}{c}\text { Brookhaven National } \\
\text { Laboratory }\end{array}$ \\
\hline Qiang Bai & University of Maryland \\
\hline Seongmin Bak & $\begin{array}{c}\text { Brookhaven National } \\
\text { Laboratory }\end{array}$ \\
\hline David Baker & U.S. Army Research Labs \\
\hline Warren Baker & $\begin{array}{c}\text { Naval Surface Warfare } \\
\text { Center }\end{array}$ \\
\hline
\end{tabular}

\begin{tabular}{|c|c|}
\hline Name & Affiliation \\
\hline Balu Balachandran & $\begin{array}{l}\text { Argonne National } \\
\text { Laboratory }\end{array}$ \\
\hline Perla Balbuena & Texas A\&M University \\
\hline Nina Balke & $\begin{array}{c}\text { Oak Ridge National } \\
\text { Laboratory }\end{array}$ \\
\hline Chunmei Ban & $\begin{array}{l}\text { National Renewable } \\
\text { Energy Laboratory }\end{array}$ \\
\hline James Banas & JSR Micro Inc/JM Energy \\
\hline Jai Bansal & $\begin{array}{l}\text { Argonne National } \\
\text { Laboratory }\end{array}$ \\
\hline Dan Barba & US EPA \\
\hline Julie Barber & ORAU \\
\hline Ewa Bardasz & Energetics Inc \\
\hline Javier Bareno & $\begin{array}{l}\text { Argonne National } \\
\text { Laboratory }\end{array}$ \\
\hline Nick Barilo & $\begin{array}{c}\text { Pacific Northwest National } \\
\text { Laboratory }\end{array}$ \\
\hline James Barnes & Barnes Tech Advising \\
\hline Ted Barnes & $\begin{array}{c}\text { Gas Technology Institute } \\
\text { (GTI) }\end{array}$ \\
\hline Brian Barnett & CAMX Power \\
\hline Vincent Battaglia & LBNL \\
\hline Olga Baturina & $\begin{array}{l}\text { Naval Research } \\
\text { Laboratory }\end{array}$ \\
\hline Alexandre Bayen & LBNL and UCB \\
\hline Armin Bayer & Greenerity GmbH \\
\hline Joseph Bayer & $\begin{array}{c}\text { Marine Corps Systems } \\
\text { Command }\end{array}$ \\
\hline John Bays & $\begin{array}{c}\text { Pacific Northwest National } \\
\text { Laboratory }\end{array}$ \\
\hline Christian Beauvais & Rowan University \\
\hline
\end{tabular}




\begin{tabular}{|c|c|}
\hline Name & Affiliation \\
\hline Michael Beckman & Linde \\
\hline Noriko Behling & Kyushu University \\
\hline Guido Bender & $\begin{array}{l}\text { National Renewable } \\
\text { Energy Laboratory }\end{array}$ \\
\hline Leonid Bendersky & NIST \\
\hline Tom Benjamin & $\begin{array}{l}\text { Argonne National } \\
\text { Laboratory }\end{array}$ \\
\hline Jeffrey Bennett & $\begin{array}{c}\text { Southwest Research } \\
\text { Institute }\end{array}$ \\
\hline Kevin Bennion & $\begin{array}{l}\text { National Renewable } \\
\text { Energy Laboratory }\end{array}$ \\
\hline Bryan Benson & Solvay \\
\hline Gene Berdichevsky & Sila Nanotechnologies \\
\hline Naveen Berry & $\begin{array}{c}\text { South Coast Air Quality } \\
\text { Management District }\end{array}$ \\
\hline Susan Bershad & NFPA \\
\hline Erwan Bertin & NIST \\
\hline Dmitri Bessarabov & HySA Center at NWU \\
\hline Johan Beyer & PowerCell Sweden AB \\
\hline Connie Bezanson & $\begin{array}{c}\text { DOE Vehicle Technologies } \\
\text { Office }\end{array}$ \\
\hline Erik Bigelow & CTE \\
\hline Matthias Binder & Greenerity GmbH \\
\hline Alicia Birky & Energetics Incorporated \\
\hline Bryan Blackburn & Redox Power Systems \\
\hline Mauricio Blanco & Ballard Power Systems \\
\hline Myra Blaylock & Sandia National Labs \\
\hline
\end{tabular}

\begin{tabular}{|c|c|}
\hline Name & Affiliation \\
\hline Nicholas Bleich & $\begin{array}{c}\text { DOE/Vehicle Technologies } \\
\text { Office }\end{array}$ \\
\hline David Blekhman & Cal State LA \\
\hline George Blomgren & $\begin{array}{c}\text { Blomgren Consulting } \\
\text { Services }\end{array}$ \\
\hline Ira Bloom & $\begin{array}{l}\text { Argonne National } \\
\text { Laboratory }\end{array}$ \\
\hline James Blount & Hydrogen fuelcell \\
\hline Cary Bloyd & $\begin{array}{c}\text { Pacific Northwest National } \\
\text { Laboratory }\end{array}$ \\
\hline Linda Bluestein & $\begin{array}{c}\text { DOE/Vehicle Technologies } \\
\text { Office }\end{array}$ \\
\hline Andre Boehman & University of Michigan \\
\hline Eric Boettcher & Honda R\&D Americas, Inc. \\
\hline Richard Bogacz & $\begin{array}{l}\text { Allegheny Science \& } \\
\text { Technology }\end{array}$ \\
\hline Brian Bolton & Nissan Technical Center \\
\hline Rod Borup & Los Alamos National Lab \\
\hline $\begin{array}{l}\text { Remy Botalla } \\
\text { Gambetta }\end{array}$ & Embassy of France \\
\hline Jessey Bouchard & Aramco Services Company \\
\hline Ellen Bourbon & $\begin{array}{c}\text { Allegheny Science \& } \\
\text { Technology }\end{array}$ \\
\hline Nico Bouwkamp & $\begin{array}{l}\text { California Fuel Cell } \\
\text { Partnership/BKI }\end{array}$ \\
\hline Mark Bowden & $\begin{array}{c}\text { Pacific Northwest National } \\
\text { Laboratory }\end{array}$ \\
\hline Robert Bowman & $\begin{array}{c}\text { Oak Ridge National } \\
\text { Laboratory }\end{array}$ \\
\hline Steven Boyd & $\begin{array}{c}\text { DOE Vehicle Technologies } \\
\text { Office }\end{array}$ \\
\hline Brian Bratvold & John Deere \\
\hline Leo Breton & $\begin{array}{c}\text { DOE/Vehicle Technologies } \\
\text { Office }\end{array}$ \\
\hline
\end{tabular}




\begin{tabular}{|c|c|}
\hline Name & Affiliation \\
\hline Thomas Briggs & $\begin{array}{l}\text { Southwest Research } \\
\text { Institute }\end{array}$ \\
\hline Robert Brinker & DENSO \\
\hline Michael Britt & UPS \\
\hline Eugene Broerman & $\begin{array}{l}\text { Southwest Research } \\
\text { Institute }\end{array}$ \\
\hline Paul Brooker & $\begin{array}{l}\text { University of } \\
\text { Central Florida }\end{array}$ \\
\hline Kriston Brooks & $\begin{array}{c}\text { Pacific Northwest National } \\
\text { Laboratory }\end{array}$ \\
\hline Claire Brown & Lucite International, Inc \\
\hline Craig Brown & $\begin{array}{l}\text { National Insititue of } \\
\text { Standards and Technology }\end{array}$ \\
\hline Daryl Brown & PNNL \\
\hline Tobias Brunner & HYNERGY GmbH \\
\hline Judy Brunson & Mercedes Benz R\&D, N.A. \\
\hline Norman Bucknor & General Motors \\
\hline Ratnakumar Bugga & $\begin{array}{l}\text { Jet Propulsion Laboratory, } \\
\text { Caltech }\end{array}$ \\
\hline Emilio Bunel & $\begin{array}{l}\text { Argonne National } \\
\text { Laboratory }\end{array}$ \\
\hline Eric Bunnelle & ExxonMobil \\
\hline Bruce Bunting & Energetics, Inc. \\
\hline Robert Burgess & $\begin{array}{l}\text { National Renewable } \\
\text { Energy Laboratory }\end{array}$ \\
\hline Harry Burns & $\begin{array}{c}\text { Directed Vapor Technologies } \\
\text { International }\end{array}$ \\
\hline Kenneth Burtt & Dexmet Corporation \\
\hline Stephen Busch & $\begin{array}{l}\text { Sandia National } \\
\text { Laboratories }\end{array}$ \\
\hline Brian Bush & $\begin{array}{l}\text { National Renewable } \\
\text { Energy Laboratory }\end{array}$ \\
\hline
\end{tabular}

\begin{tabular}{|c|c|}
\hline Name & Affiliation \\
\hline Colleen Butcher & US DOE / NETL \\
\hline William Buttner & $\begin{array}{l}\text { National Renewable } \\
\text { Energy Laboratory }\end{array}$ \\
\hline Stephanie Byham & CSRA International \\
\hline Russ Campbell & CSRA/ DOE VTO \\
\hline Christopher Capuano & Proton OnSite \\
\hline Marco Nicola Carcassi & University of Pisa \\
\hline Barney Carlson & Idaho National Laboratory \\
\hline Steve Carlson & Optodot Corporation \\
\hline Anna Carlsson & MyfC \\
\hline Everett Carpenter & Nanofoundry, LLC \\
\hline David Carrington & $\begin{array}{l}\text { Los Alamos National } \\
\text { Laboratory }\end{array}$ \\
\hline Mark Carroll & Idaho National Laboratory \\
\hline Andrea Casalegno & Politecnico di Milano \\
\hline Jonathan Castellano & $\begin{array}{c}\text { Allegheny Science \& } \\
\text { Technology }\end{array}$ \\
\hline Gerbrand Ceder & $\begin{array}{c}\text { Lawrence Berkeley National } \\
\text { Laboratory }\end{array}$ \\
\hline Kevin Centeck & U.S. Army TARDEC \\
\hline Dhanesh Chandra & $\begin{array}{l}\text { University of Nevada, } \\
\text { Reno }\end{array}$ \\
\hline Sung-Chun Chang & $\begin{array}{l}\text { Industrial Technology } \\
\text { Research Institute }\end{array}$ \\
\hline Zhihong Chang & Vanderbilt University \\
\hline Bryan Chapman & ExxonMobil \\
\hline Elana Chapman & $\begin{array}{l}\text { General Motors- Global } \\
\text { Propulsion Systems }\end{array}$ \\
\hline
\end{tabular}




\begin{tabular}{|c|c|}
\hline Name & Affiliation \\
\hline Mangesh Chaudhari & Sandia National Labs \\
\hline Bulent Chavdar & Eaton \\
\hline Nay Chehab & $\begin{array}{c}\text { Allegheny Science \& } \\
\text { Technology }\end{array}$ \\
\hline Chaoji Chen & UMD \\
\hline Dejun Chen & Georgetown University \\
\hline Guoying Chen & LBNL \\
\hline Hai-Ying Chen & Johnson Matthey \\
\hline Yanan Chen & University of Maryland \\
\hline Yuche Chen & $\begin{array}{l}\text { National Renewable } \\
\text { Energy Laboratory }\end{array}$ \\
\hline Zonghai Chen & $\begin{array}{l}\text { Argonne National } \\
\text { Laboratory }\end{array}$ \\
\hline Jiangtao Cheng & Virginia Tech \\
\hline Mark Cheng & Wayne State University \\
\hline Yang-Tse Cheng & University of Kentucky \\
\hline William Chernicoff & Toyota Motor North America \\
\hline Miaofang Chi & $\begin{array}{l}\text { Oak Ridge National } \\
\text { Laboratory }\end{array}$ \\
\hline Sean Chigusa & $\begin{array}{c}\text { Kobelco Compressors } \\
\text { America, Inc }\end{array}$ \\
\hline Anthony Childress & Clemson University \\
\hline Inhyuk Cho & Samsung SDI \\
\hline Jinhee Cho & KETEP \\
\hline Nam Hyo Cho & John Deere \\
\hline Sungjin Cho & $\begin{array}{c}\text { North Carolina A\&T State } \\
\text { University }\end{array}$ \\
\hline
\end{tabular}

\begin{tabular}{|c|c|}
\hline Name & Affiliation \\
\hline Brian Choe & SCAQMD \\
\hline Yoong-Kee Choe & $\begin{array}{c}\text { National Institute of } \\
\text { Advaced Industrial Science } \\
\text { \& Technology }\end{array}$ \\
\hline Baeck Choi & University of Florida \\
\hline Sun Hee Choi & $\begin{array}{c}\text { Korea Institute of Science } \\
\text { and Technology }\end{array}$ \\
\hline Biswajit Choudhury & DuPont \\
\hline Sourav Chowdhury & MAHLE \\
\hline lek-Heng Chu & $\begin{array}{l}\text { University of California, } \\
\text { San Diego }\end{array}$ \\
\hline Steven Chuang & The University of Akron \\
\hline Jaesik Chung & PCTEST \\
\hline Stephen Ciatti & $\begin{array}{l}\text { Argonne National } \\
\text { Laboratory }\end{array}$ \\
\hline James Ciszewski & $\begin{array}{l}\text { Argonne National } \\
\text { Laboratory }\end{array}$ \\
\hline Chris Claxton & $\begin{array}{l}\text { Argonne National } \\
\text { Laboratory }\end{array}$ \\
\hline Corie Cobb & PARC, a Xerox Company \\
\hline Yehonathan Cohen & Amprius \\
\hline Lewis Collins & ANSYS, Inc. \\
\hline $\begin{array}{c}\text { Hector } \\
\text { Colon-Mercado }\end{array}$ & SRNL \\
\hline Allen Comfort & US Army TARDEC \\
\hline Eric Condemine & IFP Energies nouvelles \\
\hline Keith Confer & Delphi \\
\hline Vincent Contini & Battelle \\
\hline Christy Cooper & $\begin{array}{l}\text { DOE Vehicle Technologies } \\
\text { Office }\end{array}$ \\
\hline
\end{tabular}




\begin{tabular}{|c|c|}
\hline Name & Affiliation \\
\hline Claudio Corgnale & Savannah River Consulting \\
\hline Liviu Cosacescu & GWM Limited \\
\hline Lelia Cosimbescu & $\begin{array}{c}\text { Pacific Northwest National } \\
\text { Laboratory }\end{array}$ \\
\hline Philip Cox & $\begin{array}{c}\text { Mainstream Engineering } \\
\text { Corporation }\end{array}$ \\
\hline Timothy Craig & MAHLE \\
\hline Stephen Creager & Clemson University \\
\hline Steven Crouch-Baker & SRI International \\
\hline Jason Croy & $\begin{array}{l}\text { Argonne National } \\
\text { Laboratory }\end{array}$ \\
\hline Jun Cui & Ames Laboratory \\
\hline Yi Cui & Stanford University \\
\hline David Cullen & $\begin{array}{c}\text { Oak Ridge National } \\
\text { Laboratory }\end{array}$ \\
\hline Brian Cunningham & $\begin{array}{c}\text { DOE/Vehicle Technologies } \\
\text { Office }\end{array}$ \\
\hline Scott Curran & $\begin{array}{c}\text { Oak Ridge National } \\
\text { Laboratory }\end{array}$ \\
\hline Sandra Curtin & FCHEA \\
\hline Tracie Curtright & ORAU \\
\hline Anthony Da Costa & Independant \\
\hline Philippe Degeilh & IFPEN \\
\hline Glenn Daehn & The Ohio State University \\
\hline Wendy Dafoe & $\begin{array}{l}\text { National Renewable } \\
\text { Energy Laboratory }\end{array}$ \\
\hline Jiaqi Dai & University of Maryland \\
\hline Qiang Dai & $\begin{array}{l}\text { Argonne National } \\
\text { Laboratory }\end{array}$ \\
\hline
\end{tabular}

\begin{tabular}{|c|c|}
\hline Name & Affiliation \\
\hline Sheng Dai & $\begin{array}{c}\text { Oak Ridge National } \\
\text { Laboratory }\end{array}$ \\
\hline Nilesh Dale & $\begin{array}{c}\text { Nissan Technical Center } \\
\text { North America }\end{array}$ \\
\hline Arrelaine Dameron & $\begin{array}{l}\text { National Renewable } \\
\text { Energy Laboratory }\end{array}$ \\
\hline Nick D'Amico & DOE / NETL \\
\hline Claus Daniel & $\begin{array}{c}\text { Oak Ridge National } \\
\text { Laboratory }\end{array}$ \\
\hline Nemanja Danilovic & Proton OnSite \\
\hline Anirban Das & Wayne State University \\
\hline Papiya Das & Not Applicable \\
\hline Moni Datta & University of Pittsburgh \\
\hline Rachel Davenport & Alliance Technical Services \\
\hline Lamuel David & $\begin{array}{c}\text { Oak Ridge National } \\
\text { Laboratory }\end{array}$ \\
\hline Bill Davis & $\begin{array}{l}\text { WVU/National Alternative } \\
\text { Fuels Training Consortium }\end{array}$ \\
\hline Patrick Davis & SMI \\
\hline Stacy Davis & $\begin{array}{c}\text { Oak Ridge National } \\
\text { Laboratory }\end{array}$ \\
\hline Emory De Castro & Advent Technologies, Inc. \\
\hline Katherine Deason & WorleyParsons \\
\hline John Dec & Sandia National Laboratories \\
\hline Andrew DeCandis & $\begin{array}{c}\text { Houston-Galveston Area } \\
\text { Council }\end{array}$ \\
\hline Gerald DeCuollo & $\begin{array}{l}\text { TreadStone Technologies, } \\
\text { Inc. }\end{array}$ \\
\hline Dennis Dees & $\begin{array}{l}\text { Argonne National } \\
\text { Laboratory }\end{array}$ \\
\hline Oscar Delgado & ICCT \\
\hline
\end{tabular}




\begin{tabular}{|c|c|}
\hline Name & Affiliation \\
\hline Samuel Delp & $\begin{array}{l}\text { Army Research } \\
\text { Laboratory }\end{array}$ \\
\hline Jack Deppe & Deppe Consulting \\
\hline Daniel DeSantis & Strategic Analysis Inc. \\
\hline Dean Deter & ORNL \\
\hline John Deur & Cummins \\
\hline Todd Deutsch & $\begin{array}{c}\text { National Renewable } \\
\text { Energy Laboratory }\end{array}$ \\
\hline Pete Devlin & U.S. Department of Energy \\
\hline Jeremy Diez & Intertek \\
\hline Sara Dillich & Retired \\
\hline Shen Dillon & University of Illinois \\
\hline Craig DiMaggio & FCA US LLC \\
\hline Mirjana Dimitrievska & $\begin{array}{c}\text { National Renewable } \\
\text { Energy Laboratory }\end{array}$ \\
\hline Yi Ding & RDECOM-TARDEC \\
\hline Huyen Dinh & NREL \\
\hline Eric Dirschka & $\begin{array}{c}\text { NASA/ Kennedy Space } \\
\text { Center }\end{array}$ \\
\hline Charles Dismukes & Rutgers University \\
\hline Tabbetha Dobbins & Rowan University \\
\hline Marca Doeff & Berkeley Lab \\
\hline Connor Dolan & FCHEA \\
\hline Byron Dorgan & U.S. Senate (retired) \\
\hline $\begin{array}{c}\text { Lucas } \\
\text { Dos Santos Freire }\end{array}$ & PPG \\
\hline
\end{tabular}

\begin{tabular}{|c|c|}
\hline Name & Affiliation \\
\hline PJ Dougherty & SMI Inc. \\
\hline James Drakes & Aerojet Rocketdyne \\
\hline Lawrence Drzal & Michigan State University \\
\hline Nandika DSouza & University of North Texas \\
\hline Bingchen Du & DataRevive \\
\hline Peng Du & Silatronix, Inc \\
\hline Zhijia Du & $\begin{array}{c}\text { Oak Ridge National } \\
\text { Laboratory }\end{array}$ \\
\hline Nancy Dudney & $\begin{array}{c}\text { Oak Ridge National } \\
\text { Laboratory }\end{array}$ \\
\hline Gopalakrishnan Duleep & H-D Systems \\
\hline Alison Dunlop & $\begin{array}{l}\text { Argonne National } \\
\text { Laboratory }\end{array}$ \\
\hline Catherine Dunwoody & $\begin{array}{c}\text { California Air Resources } \\
\text { Board }\end{array}$ \\
\hline Tien Duong & $\begin{array}{c}\text { DOE/Vehicle Technologies } \\
\text { Office }\end{array}$ \\
\hline Edward Duranty & $\begin{array}{c}\text { Pacific Northwest National } \\
\text { Laboratory }\end{array}$ \\
\hline Michael Dwyer & Energetics Incorporated \\
\hline Trevor Dzwiniel & $\begin{array}{l}\text { Argonne National } \\
\text { Laboratory }\end{array}$ \\
\hline Jonas Edman & FS Dynamics Sweden AB \\
\hline David Edwards & Air Liquide \\
\hline Glenn Eisman & $\begin{array}{l}\text { Eisman Technology } \\
\text { Consultants, LLC }\end{array}$ \\
\hline Isaac Ekoto & $\begin{array}{c}\text { Sandia National } \\
\text { Laboratories }\end{array}$ \\
\hline S. Elangovan & Ceramatec, Inc. \\
\hline Jaafar El-Awady & John Hopkins University \\
\hline
\end{tabular}




\begin{tabular}{|c|c|}
\hline Name & Affiliation \\
\hline Ronald Elder & FCA \\
\hline Amgad Elgowainy & $\begin{array}{l}\text { Argonne National } \\
\text { Laboratory }\end{array}$ \\
\hline Ayman El-Refaie & GE Global Research \\
\hline Bill Elrick & $\begin{array}{l}\text { California Fuel Cell } \\
\text { Partnership }\end{array}$ \\
\hline Hadi El-Shayeb & University of Waterloo \\
\hline Mark Elvington & $\begin{array}{c}\text { Savannah River Consulting, } \\
\text { L.L.C. }\end{array}$ \\
\hline Roger England & Cummins, Inc. \\
\hline Kathi Epping Martin & Alliance Technical Services \\
\hline Robert Erickson & University of Colorado \\
\hline Jonah Erlebacher & Johns Hopkins University \\
\hline Ivan Ermanoski & $\begin{array}{l}\text { Sandia National } \\
\text { Laboratory }\end{array}$ \\
\hline Corey Ershow & EV Everywhere \\
\hline Leslie Eudy & $\begin{array}{l}\text { National Renewable } \\
\text { Energy Laboratory }\end{array}$ \\
\hline Mitch Ewan & $\begin{array}{l}\text { Hawaii Natural Energy } \\
\text { Institute }\end{array}$ \\
\hline Laura Fabeny & $\begin{array}{c}\text { Allegheny Science \& } \\
\text { Technology }\end{array}$ \\
\hline Tibor Fabian & Ardica Technologies \\
\hline Hassan Fagehi & $\begin{array}{l}\text { Western Michigan } \\
\text { University }\end{array}$ \\
\hline Peter Faguy & $\begin{array}{l}\text { DOE Vehicle Technologies } \\
\text { Office }\end{array}$ \\
\hline Xiulin Fan & $\begin{array}{l}\text { University of Maryland, } \\
\text { College Park }\end{array}$ \\
\hline Siamak Farhad & University of Akron \\
\hline Richard Farmer & U.S. Department of Energy \\
\hline
\end{tabular}

\begin{tabular}{|c|c|}
\hline Name & Affiliation \\
\hline John Farrell & $\begin{array}{l}\text { National Renewable } \\
\text { Energy Laboratory }\end{array}$ \\
\hline Rob Farrington & $\begin{array}{l}\text { National Renewable } \\
\text { Energy Laboratory }\end{array}$ \\
\hline Mohammed Faruque & Ford Motor Co \\
\hline Christian Fau & Not Applicable \\
\hline Xuhui Feng & $\begin{array}{c}\text { National Renewable Energy } \\
\text { Laboratory }\end{array}$ \\
\hline Kyle Fenton & Sandia National Laboratories \\
\hline Magali Ferrandon & Argonne National Laboratory \\
\hline Leo Fifield & $\begin{array}{c}\text { Pacific Northwest National } \\
\text { Laboratory }\end{array}$ \\
\hline Zoran Filipi & Clemson University \\
\hline Allison Fisher & Florida Solar Energy Center \\
\hline Brian Fisher & Naval Research Laboratory \\
\hline Galen Fisher & University of Michigan \\
\hline Leah Fisher & CSRA \\
\hline Tanya Flemons & REDHORSE \\
\hline Ronald Flowers & $\begin{array}{l}\text { Greater Washington Region } \\
\text { Clean Cities Coalition }\end{array}$ \\
\hline Stephen Forbes & Air Liquide \\
\hline Matthew Fortini & Eaton Corporation \\
\hline David Foster & $\begin{array}{c}\text { Engine Research Center - } \\
\text { UW-Madison }\end{array}$ \\
\hline Melissa Fox & $\begin{array}{l}\text { Los Alamos National } \\
\text { Laboratory }\end{array}$ \\
\hline James Francfort & Idaho National Laboratory \\
\hline Julieta Francis & $\begin{array}{c}\text { Allegheny Science \& } \\
\text { Technology }\end{array}$ \\
\hline
\end{tabular}




\begin{tabular}{|c|c|}
\hline Name & Affiliation \\
\hline Jeremiah Freeman & U.S. Department of Energy \\
\hline Alexander Freitag & Robert Bosch LLC \\
\hline Douglas Freitag & $\begin{array}{l}\text { Bayside Materials } \\
\text { Technology }\end{array}$ \\
\hline David Friedman & DOE/EERE \\
\hline Matt Fronk & Kodak \\
\hline Kun Fu & University of Maryland \\
\hline Song Fu & University of North Texas \\
\hline Yoshiya Fujiwara & Honda R\&D Co., Ltd. \\
\hline Patrick Fullenkamp & GLWN \\
\hline Brent Fultz & $\begin{array}{l}\text { California Institute of } \\
\text { Technology }\end{array}$ \\
\hline Stuart Funk & LMI \\
\hline Ryosuke Furuhashi & NGK Sparkplug Co., Ltd. \\
\hline Joe Gagliano & $\begin{array}{l}\text { California Fuel Cell } \\
\text { Partnership }\end{array}$ \\
\hline Nicolas Gaillard & University of Hawaii \\
\hline Kevin Gallagher & $\begin{array}{l}\text { Argonne National } \\
\text { Laboratory }\end{array}$ \\
\hline Hong Gan & $\begin{array}{l}\text { Brookhaven National } \\
\text { Laboratory }\end{array}$ \\
\hline Umesh Gandhi & Toyota Research Inst NA \\
\hline Prabhu Ganesan & $\begin{array}{c}\text { Savannah River Consultants } \\
\text { LLC }\end{array}$ \\
\hline Jennifer Gangi & $\begin{array}{l}\text { Fuel Cell and Hydrogen } \\
\text { Energy Association }\end{array}$ \\
\hline John Gangloff & $\begin{array}{l}\text { U.S. Department of Energy- } \\
\text { Fuel Cell Technologies Office }\end{array}$ \\
\hline Feng Gao & PNNL \\
\hline
\end{tabular}

\begin{tabular}{|c|c|}
\hline Name & Affiliation \\
\hline Pu-Xian Gao & University of Connecticut \\
\hline Tao Gao & $\begin{array}{c}\text { University of } \\
\text { Maryland,College Park }\end{array}$ \\
\hline Yong Gao & Southern Illinois University \\
\hline Dick Garard & Lambda Technologies \\
\hline John Garbak & $\begin{array}{l}\text { Allegheny Science \& } \\
\text { Technology }\end{array}$ \\
\hline Alberto Garcia & FCH JU \\
\hline Thomas Garetson & Electric Applications Inc. \\
\hline Richard Garfinkel & Advanced Carbon Products \\
\hline Nancy Garland & U.S. Department of Energy \\
\hline Sarah Garman & U.S. Department of Energy \\
\hline Ryan Garwood & Rowan University \\
\hline Fernando Garzon & University of New Mexico \\
\hline Haiwen Ge & FCA US LLC \\
\hline Chris Gearhart & $\begin{array}{l}\text { National Renewable } \\
\text { Energy Laboratory }\end{array}$ \\
\hline Laura Geiman & W.L. Gore \& Associates, Inc. \\
\hline Michael Geller & $\begin{array}{l}\text { Manufacturers of Emission } \\
\text { Controls Association }\end{array}$ \\
\hline David Gelman & $\begin{array}{l}\text { Sustainable Energy } \\
\text { Strategies, Inc. }\end{array}$ \\
\hline Thomas Gennett & $\begin{array}{l}\text { National Renewable } \\
\text { Energy Laboratory }\end{array}$ \\
\hline Paul George & Battelle \\
\hline Jeff Gerbec & Mitsubishi Chemical \\
\hline Saman Gheytani & University of Houston \\
\hline
\end{tabular}




\begin{tabular}{|c|c|}
\hline Name & Affiliation \\
\hline Sujit Ghosh & $\begin{array}{l}\text { U.S. Maritime } \\
\text { Administration }\end{array}$ \\
\hline John Gibble & Volvo Group \\
\hline Jerry Gibbs & $\begin{array}{l}\text { DOE/Office of Vehicle } \\
\text { Technologies }\end{array}$ \\
\hline James Gilbert & $\begin{array}{l}\text { Argonne National } \\
\text { Laboratory }\end{array}$ \\
\hline Kelly Gilbert & Metropolitan Energy Center \\
\hline Eleanor Gillette & $\begin{array}{l}\text { National Institute of } \\
\text { Standards and Technology }\end{array}$ \\
\hline Vincent Giordani & Liox Power \\
\hline Charles Girard & $\begin{array}{l}\text { Quebec Office in } \\
\text { Washington }\end{array}$ \\
\hline Francois Girard & $\begin{array}{l}\text { National Research Council } \\
\text { Canada }\end{array}$ \\
\hline Craig Gittleman & General Motors \\
\hline Tobias Glossmann & $\begin{array}{c}\text { Mercedes-Benz Research \& } \\
\text { Development North America, } \\
\text { Inc. }\end{array}$ \\
\hline Stephen Goguen & $\begin{array}{l}\text { DOE/Vehicle Technologies } \\
\text { Office }\end{array}$ \\
\hline David Gohlke & U.S. Department of Energy \\
\hline Scott Goldsborough & $\begin{array}{l}\text { Argonne National } \\
\text { Laboratory }\end{array}$ \\
\hline Brian Goldstein & Energy Independence Now \\
\hline Alan Goliaszewski & Ashland \\
\hline Carlos Gomez & FCTO-EERE/DOE \\
\hline Jeff Gonder & $\begin{array}{l}\text { National Renewable } \\
\text { Energy Laboratory }\end{array}$ \\
\hline Yunhui Gong & University of Maryland \\
\hline Marc Goodman & Eastern Research Corp. \\
\hline Anand Gopal & $\begin{array}{l}\text { Lawrence Berkeley } \\
\text { National Laboratory }\end{array}$ \\
\hline
\end{tabular}

\begin{tabular}{|c|c|}
\hline Name & Affiliation \\
\hline Phil Gorney & U.S. DOT/NHTSA \\
\hline Alison Gotkin & UTRC \\
\hline Risei Goto & $\begin{array}{c}\text { Sumitomo Corporation of } \\
\text { Americas }\end{array}$ \\
\hline David Gotthold & PNNL \\
\hline Andrew Goudy & Delaware State University \\
\hline Charles Gough & General Motors \\
\hline S. William Gouse & SAE International \\
\hline Lars Grabow & University of Houston \\
\hline Robert Graham & $\begin{array}{c}\text { DOE Vehicle Technologies } \\
\text { Office }\end{array}$ \\
\hline Glenn Grant & $\begin{array}{c}\text { Pacific Northwest National } \\
\text { Laboratory }\end{array}$ \\
\hline Greg Grant & Delphi Electronics \& Safety \\
\hline Leo Grassilli & ONR \\
\hline Steve Gravante & Ricardo, Inc. \\
\hline Roland Gravel & $\begin{array}{l}\text { DOE/Vehicle Technologies } \\
\text { Office }\end{array}$ \\
\hline Scott Greenway & Greenway Energy LLC \\
\hline Scott Greenway & Savannah River Consulting \\
\hline Clare Grey & University of Cambridge \\
\hline Markus GroB & BMW \\
\hline April Groover Combs & $\begin{array}{l}\text { Florida Department of } \\
\text { Agriculture and Consumer } \\
\text { Services, Office of Energy }\end{array}$ \\
\hline Thomas Gross & Electricore, Inc. \\
\hline Stephen Grot & Ion Power, Inc \\
\hline
\end{tabular}




\begin{tabular}{|c|c|}
\hline Name & Affiliation \\
\hline Katrina Groth & $\begin{array}{l}\text { Sandia National } \\
\text { Laboratories }\end{array}$ \\
\hline Ray Grout & $\begin{array}{l}\text { National Renewable } \\
\text { Energy Laboratory }\end{array}$ \\
\hline Erika Gupta & DOE EERE FCTO \\
\hline Ram Gupta & $\begin{array}{c}\text { Virginia Commonwealth } \\
\text { Univ }\end{array}$ \\
\hline Shalabh Gupta & Ames Laboratory \\
\hline Sreenath Gupta & $\begin{array}{l}\text { Argonne National } \\
\text { Laboratory }\end{array}$ \\
\hline Barry Guthrie & $\begin{array}{l}\text { Prime Mover International, } \\
\text { LLC }\end{array}$ \\
\hline Lisa Guthrie & $\begin{array}{l}\text { Prime Mover International, } \\
\text { LLC }\end{array}$ \\
\hline Ray Gwin & Earth Day Texas \\
\hline Dr. Gerold Hubner & Volkswagen AG \\
\hline Nico Haak & SGL Carbon GmbH \\
\hline Bahman Habibzadeh & U.S. Department of Energy \\
\hline Andrea Haight & $\begin{array}{l}\text { Composite Technology } \\
\text { Development, Inc. }\end{array}$ \\
\hline Barr Halevi & Pajarito Powder, LLC \\
\hline Trev Hall & NETL \\
\hline Andrew Halonen & NanoAI, LLC \\
\hline Masato Hamano & Toyota Motor Corporation \\
\hline Jennifer Hamilton & Ca Fuel Cell Partnership \\
\hline Paul Hamilton & Bridgestone Americas \\
\hline Fei Han & Department of Agriculture \\
\hline Fudong Han & University of Maryland \\
\hline
\end{tabular}

\begin{tabular}{|c|c|}
\hline Name & Affiliation \\
\hline Hui-Ling Han & Lawrence Berkeley Lab \\
\hline Jeongwoo Han & Argonne National Laboratory \\
\hline Taehee Han & Nissan \\
\hline Jason Hanlin & CTE \\
\hline Kiruba Haran & University of Illinois \\
\hline Jonathan Hardis & NIST \\
\hline Ken Hardman & FCA US LLC \\
\hline Bruce Hardy & $\begin{array}{c}\text { Savannah River National } \\
\text { Laboratory }\end{array}$ \\
\hline William Haris & TARDEC \\
\hline James Harkins & $\begin{array}{l}\text { DENSO International } \\
\text { America }\end{array}$ \\
\hline Alleyn Harned & Virginia Clean Cities \\
\hline Rondle Harp & U.S. Department of Energy \\
\hline Ryan Harrington & U.S. DOT - Volpe Center \\
\hline Alexander Harris & $\begin{array}{c}\text { Brookhaven National } \\
\text { Laboratory }\end{array}$ \\
\hline Stephen Harris & $\begin{array}{l}\text { Lawrence Berkeley } \\
\text { National Laboratory }\end{array}$ \\
\hline Tequila Harris & Georgia Tech \\
\hline Kevin Harrison & $\begin{array}{l}\text { National Renewable } \\
\text { Energy Laboratory }\end{array}$ \\
\hline William Harrison & NanoSonic, Inc. \\
\hline John Hart & Dexmet \\
\hline Terence Hart & PPG \\
\hline Brent Hartman & CSA Group \\
\hline
\end{tabular}




\begin{tabular}{|c|c|}
\hline Name & Affiliation \\
\hline Yuichi Hasegawa & Denso \\
\hline Gunther Hasse & $\begin{array}{c}\text { VDI Technologiezentrum } \\
\text { GmbH }\end{array}$ \\
\hline Tatsuya Hatanaka & $\begin{array}{l}\text { Toyota Central R\&D } \\
\text { Labs. Inc. }\end{array}$ \\
\hline David Hauber & Automated Dynamics \\
\hline Andrew Haug & $3 M$ \\
\hline Sheree Haus & Alcoa Inc. \\
\hline Cary Hayner & SiNode Systems \\
\hline Allen Haynes & $\begin{array}{l}\text { Oak Ridge National } \\
\text { Laboratory }\end{array}$ \\
\hline Charles Hays & Texas A\&M University \\
\hline Xiangming $\mathrm{He}$ & Tsinghua University \\
\hline Xin He & Aramco Services Company \\
\hline Xingfeng $\mathrm{He}$ & $\begin{array}{l}\text { University of Maryland, } \\
\text { College Park }\end{array}$ \\
\hline Ethan Hecht & $\begin{array}{l}\text { Sandia National } \\
\text { Laboratories }\end{array}$ \\
\hline Matt Hedgecock & Umicore Inc. \\
\hline Eric Heim & USABC \\
\hline Rupert Heirs & Consultants \\
\hline David Held & DENSO \\
\hline Stuart Hellring & PPG Industries, Inc. \\
\hline Thomas Hellstern & Stanford University \\
\hline Christopher Hendricks & $\begin{array}{l}\text { Naval Surface Warfare } \\
\text { Center Carderock Division }\end{array}$ \\
\hline Su Jeong Heo & University of Connecticut \\
\hline
\end{tabular}

\begin{tabular}{|c|c|}
\hline Name & Affiliation \\
\hline Tae Wook Heo & $\begin{array}{l}\text { Lawrence Livermore } \\
\text { National Laboratory }\end{array}$ \\
\hline Elaine Herbon & FCA US LLC \\
\hline Darrell Herling & $\begin{array}{l}\text { Pacific Northwest } \\
\text { National Laboratory }\end{array}$ \\
\hline Andrew Herring & Colorado School of Mines \\
\hline Robert Hershey & Robert L. Hershey, P.E. \\
\hline Clemens Heske & UNLV \\
\hline Howard Hess & Johnson Matthey \\
\hline Karsten Hess & German Embassy \\
\hline Joseph Heuvers & US Army \\
\hline Peter Heywood & $\begin{array}{c}\text { Allegheny Science \& } \\
\text { Technology }\end{array}$ \\
\hline Michael Hickner & Penn State University \\
\hline Michael Hicks & H2PowerTech \\
\hline Laura Hill & U.S. Department of Energy \\
\hline Abdelkader Hilmi & FuelCell Energy, Inc. \\
\hline Robert Hilty & Xtalic \\
\hline Shinichi Hirano & Ford Motor Company \\
\hline Donna Ho & U.S. Department of Energy \\
\hline Janet Ho & $\begin{array}{l}\text { U.S. Army Research } \\
\text { Laboratory }\end{array}$ \\
\hline John Hoard & University of Michigan \\
\hline Karsten Hofmann & Continental Corporation \\
\hline Chris Hohmann & Sigma Technologies \\
\hline
\end{tabular}




\begin{tabular}{|c|c|}
\hline Name & Affiliation \\
\hline Wendolyn Holland & Holland Consulting, LLC \\
\hline Markus Holzmann & Greenerity GmbH \\
\hline Arthur Homa & ASH Consulting \\
\hline Philip Horacek & Powertech Labs \\
\hline Kaoru Horie & American Honda Motor \\
\hline Deyang Hou & QuantLogic Corp. \\
\hline Yuri Hovanski & $\begin{array}{l}\text { Pacific Northwest } \\
\text { National Laboratory }\end{array}$ \\
\hline Ken Howden & $\begin{array}{c}\text { DOE/Vehicle Technologies } \\
\text { Office }\end{array}$ \\
\hline David Howell & $\begin{array}{c}\text { DOE/Vehicle Technologies } \\
\text { Office }\end{array}$ \\
\hline John Howes & Redland Energy Group \\
\hline Stephen Hsu & $\begin{array}{c}\text { George Washington } \\
\text { University }\end{array}$ \\
\hline Liangbing $\mathrm{Hu}$ & $\begin{array}{c}\text { University of Maryland, } \\
\text { College park }\end{array}$ \\
\hline Xuebu Hu & University of Maryland \\
\hline Yan-Yan Hu & Florida State University \\
\hline Thanh Hua & $\begin{array}{l}\text { Argonne National } \\
\text { Laboratory }\end{array}$ \\
\hline Kevin Huang & $\begin{array}{l}\text { University of } \\
\text { South Carolina }\end{array}$ \\
\hline Qisen Huang & $\begin{array}{l}\text { Contempary Amperex } \\
\text { Technoloty Co., Limited }\end{array}$ \\
\hline Tao Huang & DuPont \\
\hline Zeric Hulvey & $\begin{array}{c}\text { Fuel Cell Technologies } \\
\text { Office, DOE }\end{array}$ \\
\hline Daniel Hussey & NIST \\
\hline Jennie Huya-Kouadio & Strategic Analysis \\
\hline
\end{tabular}

\begin{tabular}{|c|c|}
\hline Name & Affiliation \\
\hline Robert Hwang & $\begin{array}{l}\text { Sandia National } \\
\text { Laboratories }\end{array}$ \\
\hline William Hynes & Allison Transmission \\
\hline Grady Icard & Teledyne Energy Systems \\
\hline Andrew Ickes & $\begin{array}{l}\text { Argonne National } \\
\text { Laboratory }\end{array}$ \\
\hline Bill Ickes & Hyundai Motor Group \\
\hline Hiroshi Igarashi & N.E. Chemcat Corporation \\
\hline Akihiro Ilyama & University of Yamanashi \\
\hline Tetsufumi Ikeda & HySUT \\
\hline William (Bill) Imoehl & $\begin{array}{l}\text { Continental Automotive } \\
\text { Systems, Inc. }\end{array}$ \\
\hline Joseph Impullitti & SCAQMD \\
\hline Louis Infante & Kee Energy LLC \\
\hline Brian Ingram & $\begin{array}{l}\text { Argonne National } \\
\text { Laboratory }\end{array}$ \\
\hline Asim lqbal & FCA US LLC \\
\hline Dan Ireland & $\begin{array}{c}\text { Solvay Specialty Polymers } \\
\text { LLC }\end{array}$ \\
\hline Lawrence Irvine & Plug Power, Inc. \\
\hline Chiaki Ishii & Technova Inc. \\
\hline Hiroya Ishikawa & $\begin{array}{l}\text { NGK SPARK PLUGS } \\
\text { (USA),INC }\end{array}$ \\
\hline Kane Ivory & DSTG \\
\hline Bernadette Jackson & $\begin{array}{c}\text { DOE Vehicle Technologies } \\
\text { Office }\end{array}$ \\
\hline David Jacobson & $\begin{array}{c}\text { National Institute of } \\
\text { Standards and Technology }\end{array}$ \\
\hline Fred Jahnke & FuelCell Energy \\
\hline
\end{tabular}




\begin{tabular}{|c|c|}
\hline Name & Affiliation \\
\hline Justin Jahnke & $\begin{array}{l}\text { Oak Ridge Affliated } \\
\text { Universities }\end{array}$ \\
\hline Ian Jakupca & NASA \\
\hline Radha Jalan & ElectroChem, Inc. \\
\hline Brian James & Strategic Analysis Inc. \\
\hline Rachel James & HCATT \\
\hline Will James & $\begin{array}{l}\text { U.S. DOE Fuel Cell } \\
\text { Technologies Office }\end{array}$ \\
\hline Alexandra Jamis & ICF International \\
\hline $\begin{array}{l}\text { Prashanth Jampani } \\
\text { Hanumantha }\end{array}$ & University of Pittsburgh \\
\hline Raphael Janot & LRCS - CNRS \\
\hline Andrew Jansen & $\begin{array}{l}\text { Argonne National } \\
\text { Laboratory }\end{array}$ \\
\hline Thomas Jaramillo & Stanford University \\
\hline William Jarvis & FLEXcon \\
\hline Casey Jaworsk & $\begin{array}{c}\text { Tanaka Kikinzoku } \\
\text { International (America), Inc. }\end{array}$ \\
\hline Matthew Jeffers & $\begin{array}{l}\text { National Renewable } \\
\text { Energy Laboratory }\end{array}$ \\
\hline Forrest Jehlik & ANL \\
\hline Deborah Jelen & Electricore, Inc. \\
\hline Craig Jensen & University of Hawaii \\
\hline Lisa Jerram & Navigant Research \\
\hline Gerald Jeske & Umicore \\
\hline Hongfei Jia & $\begin{array}{l}\text { Toyota Research Institute } \\
\text { of North America }\end{array}$ \\
\hline Xiaoning Jiang & NC State University \\
\hline
\end{tabular}

\begin{tabular}{|c|c|}
\hline Name & Affiliation \\
\hline Feng Jiao & University of Delaware \\
\hline Safia Jilani & Georgetown University \\
\hline Jonathan Jin & U.S. Department of Energy \\
\hline Christopher Johnson & $\begin{array}{c}\text { Argonne National } \\
\text { Laboratory }\end{array}$ \\
\hline Francis Johnson & GE Global Research \\
\hline John Johnson & $\begin{array}{c}\text { Michigan Technological } \\
\text { University }\end{array}$ \\
\hline Kenneth Johnson & $\begin{array}{l}\text { Pacific Northwest } \\
\text { National Laboratory }\end{array}$ \\
\hline Terry Johnson & $\begin{array}{l}\text { Sandia National } \\
\text { Laboratories }\end{array}$ \\
\hline Timothy Johnson & Corning Incorporated \\
\hline William Joost & $\begin{array}{l}\text { DOE Vehicle Technologies } \\
\text { Office }\end{array}$ \\
\hline Fred Joseck & U.S. Department of Energy \\
\hline Ajey Joshi & Applied Materials \\
\hline Ameya Joshi & Corning Incorporated \\
\hline T. Richard Jow & $\begin{array}{l}\text { U.S. Army Research } \\
\text { Laboratory }\end{array}$ \\
\hline Nidhi Juthani & University of Waterloo \\
\hline Takuya Kadohira & $\begin{array}{l}\text { National Institute for } \\
\text { Materials Science }\end{array}$ \\
\hline Masataka Kadowaki & $\begin{array}{l}\text { NEDO (New Energy and } \\
\text { Industrial Technology } \\
\text { Development Organization) }\end{array}$ \\
\hline Keith Kahl & $\begin{array}{l}\text { Oak Ridge National } \\
\text { Laboratory }\end{array}$ \\
\hline Kahvecioglu Feridun & $\begin{array}{l}\text { Argonne National } \\
\text { Laboratory }\end{array}$ \\
\hline $\begin{array}{c}\text { Mohammad Rejaul } \\
\text { Ozgenur Kaiser }\end{array}$ & University of Maryland \\
\hline Sergiy Kalnaus & $\begin{array}{l}\text { Oak Ridge National } \\
\text { Laboratory }\end{array}$ \\
\hline
\end{tabular}




\begin{tabular}{|c|c|}
\hline Name & Affiliation \\
\hline Motohisa Kamijo & Nissan Motor Co., Ltd. \\
\hline Yasushi Kaneko & Panasonic \\
\hline Hiroyuki Kanesaka & FC-Cubic TRA \\
\hline Argyro Karathanou & FCLAB \\
\hline Abhi Karkamkar & PNNL \\
\hline Donald Karnerr & $\begin{array}{l}\text { Electric Applications } \\
\text { Incorporated }\end{array}$ \\
\hline James Kast & U.S. Department of Energy \\
\hline Hideki Kato & $\begin{array}{l}\text { Hino Motors Manufacturing } \\
\text { USA, Inc }\end{array}$ \\
\hline Brian Kaul & $\begin{array}{l}\text { Oak Ridge National } \\
\text { Laboratory }\end{array}$ \\
\hline Steven Kays & 3M Company \\
\hline Glenn Keller & $\begin{array}{l}\text { Argonne National } \\
\text { Laboratory }\end{array}$ \\
\hline Jay Keller & $\begin{array}{c}\text { Zero Carbon Energy } \\
\text { Solutions }\end{array}$ \\
\hline Patrick Kelly & Path-Two \\
\hline Timothy Keon & NHTSA \\
\hline Keith Kepler & Farasis Energy, Inc. \\
\hline Justin Kern & Robert Bosch LLC \\
\hline Matthew Keyser & $\begin{array}{l}\text { National Renewable } \\
\text { Energy Laboratory }\end{array}$ \\
\hline Michael Kezirian & Century Fathom \\
\hline Alireza Khaligh & University of Maryland \\
\hline Hamid Kia & General Motors R\&D Center \\
\hline Daniyal Kiani & Georgetown University \\
\hline
\end{tabular}

\begin{tabular}{|c|c|}
\hline Name & Affiliation \\
\hline Haekyoung Kim & Yeungnam University \\
\hline Hyoung-Juhn Kim & $\begin{array}{c}\text { Korea Institute of Science } \\
\text { and Technology }\end{array}$ \\
\hline Jeffrey Kim & Maxwell Technologies \\
\hline JuYong Kim & J\&L Tech \\
\hline Ki-woon Kim & Samsung SDI \\
\hline Kiyoung Kim & ILSUNG \\
\hline Tae-Young Kim & $\begin{array}{l}\text { Korea Institute of Energy } \\
\text { Research (KIER) }\end{array}$ \\
\hline Yongmin Kim & $\begin{array}{l}\text { NPL, Stanford University, } \\
\text { Department of Mechanical } \\
\text { Engineering }\end{array}$ \\
\hline Yu Seung Kim & $\begin{array}{c}\text { Los Alamos National } \\
\text { Laboratory }\end{array}$ \\
\hline Tatsusaburo Kimura & Sumitomo Corporation \\
\hline David Kirschner & U.S. DOE / NETL \\
\hline Neil Kirschner & U.S. DOE / NETL \\
\hline Takeaki Kishimoto & Nisshinbo Holdings Inc. \\
\hline Benjamin Klahr & U.S. Department of Energy \\
\hline Greg Kleen & $\begin{array}{l}\text { U.S. DOE Fuel Cell } \\
\text { Technologies Office }\end{array}$ \\
\hline Douglas Knight & Intelligent Energy \\
\hline Shanna Knights & Ballard Power Systems \\
\hline Katy Knopp & FCHEA \\
\hline Kenji Kobayashi & NGK Spark Plugs \\
\hline Shyam Kocha & $\begin{array}{l}\text { National Renewable } \\
\text { Energy Laboratory }\end{array}$ \\
\hline Lyle Kocher & Cummins Inc. \\
\hline
\end{tabular}




\begin{tabular}{|c|c|}
\hline Name & Affiliation \\
\hline Michael Kocsis & $\begin{array}{c}\text { Southwest Research } \\
\text { Institute }\end{array}$ \\
\hline Prakash Kolli & University of Maryland \\
\hline Siddharth Komini Babu & $\begin{array}{l}\text { Carnegie Mellon } \\
\text { Univeristy }\end{array}$ \\
\hline Hiroaki Komoda & Honda R\&D Co., Ltd. \\
\hline Shinji Kondo & Sumitomo Corporation \\
\hline Song-Charng Kong & $\begin{array}{l}\text { National Science } \\
\text { Foundation }\end{array}$ \\
\hline Anusorn Kongkanand & General Motors \\
\hline John Kopasz & $\begin{array}{l}\text { Argonne National } \\
\text { Laboratory }\end{array}$ \\
\hline Robert Koroshetz & $\begin{array}{c}\text { Allegheny Science \& } \\
\text { Technology }\end{array}$ \\
\hline Robert Kostecki & LBNL \\
\hline Tomohito Kotake & Honda R\&D CO.,Ltd. \\
\hline Alison Kraigsley & NIH/NIAID \\
\hline Matthew Kramer & Ames Laboratory \\
\hline Theodore Krause & $\begin{array}{l}\text { Argonne National } \\
\text { Laboratory }\end{array}$ \\
\hline John Kresse & Cummins \\
\hline Ken Kriha & GTI \\
\hline $\begin{array}{l}\text { Shashank } \\
\text { Krishnamurthy }\end{array}$ & UTRC \\
\hline Joachim Kroemer & Borit NV \\
\hline Ryan Kuehn & KeyLogic \\
\hline Jackie Kulfan & PPG \\
\hline Bijayendra Kumar & Energetics \\
\hline
\end{tabular}

\begin{tabular}{|c|c|}
\hline Name & Affiliation \\
\hline Prashant Kumta & University of Pittsburgh \\
\hline Steven Kung & EPRI \\
\hline Sergey Kunin & Cummins \\
\hline Shashi Kuppa & NHTSA \\
\hline Jennifer Kurtz & $\begin{array}{l}\text { National Renewable } \\
\text { Energy Laboratory }\end{array}$ \\
\hline Ahmet Kusoglu & $\begin{array}{l}\text { Lawrence Berkeley } \\
\text { National Laboratory }\end{array}$ \\
\hline Alan Kwan & U.S. DOE-EERE \\
\hline Quon Kwan & USDOT / FMCSA \\
\hline Steven Lacey & UMD College Park \\
\hline Melissa Laffen & $\begin{array}{l}\text { Alliance Technical Services, } \\
\text { Inc. }\end{array}$ \\
\hline Chris LaFleur & $\begin{array}{l}\text { Sandia National } \\
\text { Laboratories }\end{array}$ \\
\hline $\begin{array}{l}\text { Balasubramanian } \\
\text { Lakshmanan }\end{array}$ & General Motors \\
\hline Jennifer Lalli & NanoSonic, Inc. \\
\hline Jacob LaManna & NIST \\
\hline Joshua Lamb & $\begin{array}{l}\text { Sandia National } \\
\text { Laboratories }\end{array}$ \\
\hline Christine Lambert & Ford Motor Company \\
\hline Robin Lamgaday & $\begin{array}{c}\text { The Goodyear Tire \& } \\
\text { Rubber CO. }\end{array}$ \\
\hline Sara Lamnin & City of Hayward \\
\hline Michael Lanagan & Penn State University \\
\hline Michael Lance & $\begin{array}{l}\text { Oak Ridge National } \\
\text { Laboratory }\end{array}$ \\
\hline Christopher Lang & Physical Sciences Inc. \\
\hline
\end{tabular}




\begin{tabular}{|c|c|}
\hline Name & Affiliation \\
\hline Jay Laskin & Hyenergy Consulting, LLC \\
\hline Suzanne Lauer & Alliance Technical Services \\
\hline Andrea Laughlin & Altergy \\
\hline Michael Laughlin & Energetics Incorporated \\
\hline Larry Laws & General Motors \\
\hline David Lax & API \\
\hline Duy Le & $\begin{array}{l}\text { University of Central } \\
\text { Florida }\end{array}$ \\
\hline Jacob Leachman & $\begin{array}{l}\text { Washington State } \\
\text { University }\end{array}$ \\
\hline David LeBlanc & $\begin{array}{c}\text { University of Michigan } \\
\text { UMTRI }\end{array}$ \\
\hline Elias Ledesma & University of Guanjuato \\
\hline Albert Lee & Navigant Research \\
\hline David Lee & BioSolar, Inc. \\
\hline Doohwan Lee & U.S. Department of Energy \\
\hline Doohwan Lee & University of Seoul \\
\hline D-Y Lee & $\begin{array}{l}\text { Argonne National } \\
\text { Laboratory }\end{array}$ \\
\hline Eungje Lee & $\begin{array}{l}\text { Argonne National } \\
\text { Laboroatry }\end{array}$ \\
\hline Jennifer Lee & $\begin{array}{c}\text { California Air Resources } \\
\text { Board }\end{array}$ \\
\hline Jong Kook Lee & Hyundai Motor Group \\
\hline Sang Bok Lee & University of Maryland \\
\hline Uisung Lee & $\begin{array}{l}\text { Argonne National } \\
\text { Laboratory }\end{array}$ \\
\hline Won-Yong Lee & $\begin{array}{c}\text { Korea Institute of Energy } \\
\text { Research }\end{array}$ \\
\hline
\end{tabular}

\begin{tabular}{|c|c|}
\hline Name & Affiliation \\
\hline Mark Lefebvre & Samsung SDI \\
\hline DeLisa Leighton & Luxfert-GTM \\
\hline Rebecca Levinson & $\begin{array}{l}\text { Sandia National } \\
\text { Laboratories }\end{array}$ \\
\hline Terry Levinson & $\begin{array}{c}\text { Allegheny Science \& } \\
\text { Technology }\end{array}$ \\
\hline Randolph Lewis & Utah State University \\
\hline Josh Ley & UQM Technologies, Inc. \\
\hline Alan Li & $\begin{array}{c}\text { Richard Montgomery } \\
\text { High School }\end{array}$ \\
\hline Gong Liang Li & NGK Sparkplugs \\
\hline Hongqiang Li & $\begin{array}{l}\text { Canadian Nuclear } \\
\text { Laboratories }\end{array}$ \\
\hline Jan-Mou Li & $\begin{array}{l}\text { Oak Ridge National } \\
\text { Laboratory }\end{array}$ \\
\hline Jianlin Li & $\begin{array}{l}\text { Oak Ridge National } \\
\text { Laboratory }\end{array}$ \\
\hline Lin-Feng Li & Bettergy Corp. \\
\hline Linsen Li & $\begin{array}{c}\text { Massachusetts Institute of } \\
\text { Technology }\end{array}$ \\
\hline Wenyue Li & American University \\
\hline Xiaogang Li & University of Maryland \\
\hline Xiaolin Li & $\begin{array}{c}\text { Pacific Northwest National } \\
\text { Laboratory }\end{array}$ \\
\hline Yiju Li & University of Maryland \\
\hline Zhenxian Liang & $\begin{array}{l}\text { Oak Ridge National } \\
\text { Laboratory }\end{array}$ \\
\hline Zhixiu Liang & BNL \\
\hline Boryann Liaw & Idaho National Laboratory \\
\hline Gregory Lilik & ExxonMobil \\
\hline
\end{tabular}




\begin{tabular}{|c|c|}
\hline Name & Affiliation \\
\hline Yong Chae Lim & $\begin{array}{l}\text { Oak Ridge National } \\
\text { Laboratory }\end{array}$ \\
\hline Brian Lin & $\begin{array}{l}\text { National Institute of } \\
\text { Standards and Technoloy }\end{array}$ \\
\hline Chenghsiung Lin & $\begin{array}{c}\text { Goodyear Tire \& Rubber } \\
\text { Company }\end{array}$ \\
\hline Dingchang Lin & Stanford University \\
\hline Kevin Lin & NSWC Carderock \\
\hline Ruoqian Lin & Brookhaven National Lab \\
\hline Zhenhong Lin & $\begin{array}{l}\text { Oak Ridge National } \\
\text { Laboratory }\end{array}$ \\
\hline Suljo Linic & University of Michigan \\
\hline Clovis Linkous & $\begin{array}{l}\text { Youngstown State } \\
\text { University }\end{array}$ \\
\hline Timothy Lipman & UC Berkeley TSRC \\
\hline Shawn Litster & Carnegie Mellon University \\
\hline Andrew Littlefield & $\begin{array}{c}\text { US Army RDECOM-ARDEC } \\
\text { BenÃ } \odot t \text { Labs }\end{array}$ \\
\hline Scott Litzelman & Booz Allen Hamilton \\
\hline Bert Liu & The Ohio State University \\
\hline Boyang Liu & University of Maryland \\
\hline Changzheng Liu & $\begin{array}{l}\text { Oak Ridge National } \\
\text { Laboratory }\end{array}$ \\
\hline Di-Jia Liu & $\begin{array}{l}\text { Argonne National } \\
\text { Laboratory }\end{array}$ \\
\hline Dongxia Liu & University of Maryland \\
\hline Hong Liu & Oregon State University \\
\hline Ping Liu & UC - San Deigo \\
\hline Xingbo Liu & West Virginia University \\
\hline
\end{tabular}

\begin{tabular}{|c|c|}
\hline Name & Affiliation \\
\hline Xiujun Liu & American University \\
\hline Yang Liu & Virginia Tech \\
\hline Yangwei Liu & Georgetown University \\
\hline Yayuan Liu & Stanford University \\
\hline Yihua Liu & $\begin{array}{c}\text { Argonne National } \\
\text { Laboratory }\end{array}$ \\
\hline Ying Liu & Nissan Technical Center N.A. \\
\hline Michael Lloyd & Energetics Incorporated \\
\hline Bruce Logan & Penn State University \\
\hline Stephen Logan & FCA US LLC \\
\hline Jonathan Lombardi & U.S. DOE / EERE \\
\hline Douglas Longman & $\begin{array}{c}\text { Argonne National } \\
\text { Laboratory }\end{array}$ \\
\hline Sergey Lopatin & Applied Materials \\
\hline Herman Lopez & Envia Systems \\
\hline Michelle Love & ORAU \\
\hline Edward Lovelace & XL Hybrids \\
\hline Jorge Lozada & PACCAR \\
\hline Dongping Lu & $\begin{array}{l}\text { Pacific Northwest } \\
\text { National Laboratory }\end{array}$ \\
\hline Guo-Quan Lu & Virginia Tech \\
\hline Wenquan Lu & $\begin{array}{l}\text { Argonne National } \\
\text { Laboratory }\end{array}$ \\
\hline Wesley Luc & University of Delaware \\
\hline Timo Lukkarinen & Horizon Educational \\
\hline
\end{tabular}




\begin{tabular}{|c|c|}
\hline Name & Affiliation \\
\hline Hongmei Luo & $\begin{array}{c}\text { New Mexico State } \\
\text { University }\end{array}$ \\
\hline Jinyong Luo & Cummins Inc \\
\hline Wei Luo & University of Maryland \\
\hline Jason Lustbader & $\begin{array}{l}\text { National Renewable } \\
\text { Energy Laboratory }\end{array}$ \\
\hline Jodie Lutkenhaus & Texas A\&M University \\
\hline Simon Lux & $\begin{array}{l}\text { BMW Group Technology } \\
\text { Office }\end{array}$ \\
\hline Lauren Lynch & $\begin{array}{l}\text { National Renewable } \\
\text { Energy Laboratory }\end{array}$ \\
\hline Gregg Lytle & Solvay Specialty Polymers \\
\hline Max Lyubovksy & U.S. Department of Energy \\
\hline Nader M. Hagh & NEI Corporation \\
\hline Beihai Ma & $\begin{array}{l}\text { Argonne National } \\
\text { Laboratory }\end{array}$ \\
\hline Zhong Ma & $\begin{array}{c}\text { Brookhaven National } \\
\text { Laboratory }\end{array}$ \\
\hline Jim Mac Dougall & $\begin{array}{l}\text { Air Products and } \\
\text { Chemicals, Inc. }\end{array}$ \\
\hline Bill MacLeod & Emerald Advisory LLC \\
\hline Miguel Maes & NASA-WSTF \\
\hline Zeeshan Mahmood & Merck KgaA/EMD \\
\hline Eric Majzoub & $\begin{array}{c}\text { University of Missouri - } \\
\text { St. Louis }\end{array}$ \\
\hline Ljiljana Maksimovic & PPG \\
\hline Andreas Malikopoulos & $\begin{array}{c}\text { Oak Ridge National } \\
\text { Laboratory }\end{array}$ \\
\hline John Maloney & $\begin{array}{c}\text { Goodyear Tire \& Rubber } \\
\text { Company }\end{array}$ \\
\hline Sotirios Mamalis & $\begin{array}{c}\text { SUNY - Stony Brook } \\
\text { University }\end{array}$ \\
\hline
\end{tabular}

\begin{tabular}{|c|c|}
\hline Name & Affiliation \\
\hline Pin-Ching Maness & $\begin{array}{l}\text { National Renewable } \\
\text { Energy Laboratory }\end{array}$ \\
\hline Venkat Manivannan & DON \\
\hline Dawn Manley & Samsung \\
\hline Azzam Mansour & NSWCCD \\
\hline Arumugam Manthiram & $\begin{array}{l}\text { University of Texas } \\
\text { at Austin }\end{array}$ \\
\hline Karthish Manthiram & $\begin{array}{l}\text { California Institute of } \\
\text { Technology }\end{array}$ \\
\hline John Maples & EIA \\
\hline Clay Maranville & Ford Motor Company \\
\hline James Marcicki & Ford Motor Company \\
\hline Radenka Maric & University of Connecticut \\
\hline Tony Markel & $\begin{array}{l}\text { National Renewable } \\
\text { Energy Laboratory }\end{array}$ \\
\hline Morry Markowitz & $\begin{array}{l}\text { Fuel Cell \& Hydrogen Energy } \\
\text { Association }\end{array}$ \\
\hline Matthew Marks & SABIC \\
\hline Laura Marlino & Oak Ridge National Lab \\
\hline Carl Maronde & U.S. Dept of Energy/NETL \\
\hline Azadeh Maroufmashat & University of Waterloo \\
\hline Josh Martin & $\begin{array}{l}\text { National Renewable } \\
\text { Energy Laboratory }\end{array}$ \\
\hline Mike Martin & USCAR \\
\hline Richard Martin & Ardica Technologies \\
\hline Andrew Martinez & $\begin{array}{c}\text { California Air Resources } \\
\text { Board }\end{array}$ \\
\hline Alvaro Masias & Ford Motor Company \\
\hline
\end{tabular}




\begin{tabular}{|c|c|}
\hline Name & Affiliation \\
\hline David Masten & General Motors \\
\hline Larry Masur & Xtalic Corp \\
\hline Yuki Matsuda & Technova Inc. \\
\hline Tomoya Matsunaga & $\begin{array}{c}\text { Toyota Research Institute of } \\
\text { North America }\end{array}$ \\
\hline Toshiro Matsushima & Honda Motor Co.,Ltd. \\
\hline Paul Matter & pH Matter, LLC \\
\hline Adam Matzger & University of Michigan \\
\hline Manos Mavrikakis & $\begin{array}{l}\text { University of Wisconsin - } \\
\text { Madison }\end{array}$ \\
\hline Samir Mayekar & SiNode Systems \\
\hline Ahmad Mayyas & $\begin{array}{l}\text { National Renewable } \\
\text { Energy Laboratory }\end{array}$ \\
\hline Lena Mazeina & Miltec UV \\
\hline Brian Mazzeo & Brigham Young University \\
\hline David McCallum & $\begin{array}{l}\text { Argonne National } \\
\text { Laboratory }\end{array}$ \\
\hline Eric McCarty & USAMP LLC \\
\hline Robert McCormick & $\begin{array}{l}\text { National Renewable } \\
\text { Energy Laboratory }\end{array}$ \\
\hline Britney McCoy & U.S. EPA \\
\hline Dr. Anthony McDaniel & $\begin{array}{l}\text { Sandia National } \\
\text { Laboratories }\end{array}$ \\
\hline Robert McDonald & Energetics Incorporated \\
\hline Emma McElroy & FCHEA \\
\hline Brian McKay & $\begin{array}{l}\text { Continental Automotive } \\
\text { Systems, Inc. }\end{array}$ \\
\hline Daniel McKay & Volta Consulting Group \\
\hline
\end{tabular}

\begin{tabular}{|c|c|}
\hline Name & Affiliation \\
\hline Kyle McKeown & Linde ATZ \\
\hline Cindy McMullen & $\begin{array}{l}\text { U.S. DOE Vehicle } \\
\text { Technologies Office }\end{array}$ \\
\hline Matthew McNenly & LLNL \\
\hline Dennis McOwen & University of Maryland \\
\hline Shawna McQueen & Energetics Incorporated \\
\hline Manish Mehta & M-Tech International LLC \\
\hline Vineet Mehta & Tesla Motors \\
\hline Charles Mendler & ENVERA LLC \\
\hline Hui Meng & $\begin{array}{c}\text { Brookhaven National } \\
\text { Laboratory }\end{array}$ \\
\hline Ying Shirley Meng & $\begin{array}{c}\text { University of California San } \\
\text { Diego }\end{array}$ \\
\hline Rebecca Mercuri, Ph.D. & Notable Software Inc. \\
\hline Catherine Mertes & $\begin{array}{l}\text { RCF Economic \& Financial } \\
\text { Consulting, Incs }\end{array}$ \\
\hline Kristen Meyers & Soulbrain MI \\
\hline $\begin{array}{l}\text { Christopher } \\
\text { Michelbacher }\end{array}$ & Idaho National Laboratory \\
\hline Paul Miles & $\begin{array}{l}\text { Sandia National } \\
\text { Laboratories }\end{array}$ \\
\hline Brenton Miller & $\begin{array}{c}\text { Maryland Technical Review, } \\
\text { LLC }\end{array}$ \\
\hline James Miller & $\begin{array}{l}\text { Argonne National } \\
\text { Laboratory }\end{array}$ \\
\hline John Miller & $\begin{array}{c}\text { J-N-J Miller Design Services } \\
\text { PLLC }\end{array}$ \\
\hline JoAnn Milliken & JMilliken Consulting \\
\hline Michael Mills & U.S. Department of Energy \\
\hline Nguyen Minh & $\begin{array}{c}\text { University of California, } \\
\text { San Diego }\end{array}$ \\
\hline
\end{tabular}




\begin{tabular}{|c|c|}
\hline Name & Affiliation \\
\hline Mansour Mirdamadi & Dow Automotive Systems \\
\hline David Mitlin & Clarkson University \\
\hline Matt Miyasato & $\begin{array}{l}\text { South Coast Air Quality } \\
\text { Management District }\end{array}$ \\
\hline John Mizroch & JFM LLC \\
\hline Yifei Mo & $\begin{array}{c}\text { University of Maryland, } \\
\text { College Park }\end{array}$ \\
\hline Thomas Moffat & NIST \\
\hline Surya Moganty & NOHMs Technologies \\
\hline Ashok Moghe & Cisco Systems \\
\hline Ram Mohan & Singular Ventures LLP \\
\hline Rana Mohtadi & $\begin{array}{c}\text { Toyota Research Institute of } \\
\text { North America }\end{array}$ \\
\hline Peter Moilanen & Ford Motor Company \\
\hline Jongsoo Mok & Hyundai Motor Company \\
\hline Judy Moore & $\begin{array}{l}\text { WVU/National Alternative } \\
\text { Fuels Training Consortium }\end{array}$ \\
\hline Karren More & $\begin{array}{c}\text { Oak Ridge National } \\
\text { LAboratory }\end{array}$ \\
\hline Greg Morelandg & CSRA \\
\hline Pietro Moretto & $\begin{array}{l}\text { European Commission / } \\
\text { Joint ResearchCentre }\end{array}$ \\
\hline Marianne Morgan & BASF Corporation \\
\hline John Morgante & AAM \\
\hline Yu Morimoto & $\begin{array}{l}\text { Toyota Central R\&D Labs., } \\
\text { Inc. }\end{array}$ \\
\hline Melody Morris & University of Delaware \\
\hline Geoff Morrison & Cadmus Group \\
\hline
\end{tabular}

\begin{tabular}{|c|c|}
\hline Name & Affiliation \\
\hline Michael Mosburger & Robert Bosch LLC \\
\hline Andrew Moskalik & US EPA \\
\hline Charles Mueller & $\begin{array}{l}\text { Sandia National } \\
\text { Laboratories }\end{array}$ \\
\hline Sanjeev Mukerjee & Northeastern University \\
\hline Partha Mukherjee & Texas A\&M University \\
\hline Calum Munro & PPG \\
\hline George Muntean & PNNL \\
\hline Matteo Muratori & PNNL \\
\hline KP Murphy & $\begin{array}{l}\text { Alliance Technical Services, } \\
\text { Inc. }\end{array}$ \\
\hline Lilia Murphy & $\begin{array}{l}\text { Alliance Technical Services, } \\
\text { Inc. }\end{array}$ \\
\hline Mark Musculus & $\begin{array}{l}\text { Sandia National } \\
\text { Laboratories }\end{array}$ \\
\hline Kenichi Muto & NEDO \\
\hline Charles Myers & CSRA \\
\hline Kotobu Nagai & $\begin{array}{c}\text { National Institute for } \\
\text { Materials Scinece }\end{array}$ \\
\hline Payam Naghshtabrizi & Eaton Corp \\
\hline Koichiro Nakatani & Toyota Motor Corporation \\
\hline Sang Yong Nam & $\begin{array}{c}\text { Gyeongsang National } \\
\text { University }\end{array}$ \\
\hline Jagjit Nanda & $\begin{array}{c}\text { Oak Ridge National } \\
\text { Laboratory }\end{array}$ \\
\hline Dan Nardozzi & NETL/DOE \\
\hline Sreekant Narumanchi & $\begin{array}{l}\text { National Renewable } \\
\text { Energy Laboratory }\end{array}$ \\
\hline Kristen Nawoj & DOE FCTO \\
\hline
\end{tabular}




\begin{tabular}{|c|c|}
\hline Name & Affiliation \\
\hline Rachael Nealer & $\begin{array}{c}\text { DOE Vehicle Technologies } \\
\text { Office }\end{array}$ \\
\hline Norman Newhouse & Hexagon Lincoln \\
\hline Kenneth Neyerlin & $\begin{array}{l}\text { National Renewable } \\
\text { Energy Laboratory }\end{array}$ \\
\hline Simon $\mathrm{Ng}$ & Wayne State University \\
\hline Nha Nguyen & $\begin{array}{l}\text { U.S. Department of } \\
\text { Transportation }\end{array}$ \\
\hline Ralph Nine & U.S. Department of Energy \\
\hline Shin Nishimura & Kyushu University \\
\hline Yoshiaki Nitta & Nissan Motor Co., Ltd. \\
\hline Yuming Niu & Deere \& Company \\
\hline Stephen Nolet & TPI Composites, Inc. \\
\hline William Notardonato & NASA KSC \\
\hline Christopher O'Brien & Ivys Energy Solutions \\
\hline James O'Brien & Idaho National Laboratory \\
\hline Daniel O'Connell & FCHEA \\
\hline Madeleine Odgaard & IRD Fuel Cells LLC \\
\hline Susan Odom & University of Kentucky \\
\hline Joseph Oefelein & $\begin{array}{l}\text { Sandia National } \\
\text { Laboratories }\end{array}$ \\
\hline Robert Oesterreich & Air Liquide \\
\hline Arnold Offner & Phoenix Contact \\
\hline Tadashi Ogitsu & $\begin{array}{l}\text { Lawrence Livermore } \\
\text { National Laboratory }\end{array}$ \\
\hline Carrie Okma & FCA US LLC (Fiat Chrysler) \\
\hline
\end{tabular}

\begin{tabular}{|c|c|}
\hline Name & Affiliation \\
\hline Takefumi Okumura & Hitachi, Ltd. \\
\hline David Olds & General Motors \\
\hline Natalie Olds & USCAR \\
\hline Kraig Olejniczak & Cree Fayetteville, Inc. \\
\hline Peter Olin & Delphi \\
\hline Gina Oliver & American Chemistry Council \\
\hline Miodrag Oljaca & Cabot Corporation \\
\hline David Ollett & U.S. DOE/NETL \\
\hline Sarah Ollila & $\begin{array}{l}\text { DOE Vehicle Technologies } \\
\text { Office }\end{array}$ \\
\hline Gregory Olson & CSRA International, Inc. \\
\hline Tim Olson & $\begin{array}{l}\text { California Energy } \\
\text { Commission }\end{array}$ \\
\hline Travis Omasta & University of Connecticut \\
\hline Fredrick Omenya & Binghamton University \\
\hline Omer Onar & $\begin{array}{l}\text { Oak Ridge National } \\
\text { Laboratory }\end{array}$ \\
\hline Shaun Onorato & $\begin{array}{c}\text { Allegheny Science \& } \\
\text { Technology - DOE Contractor }\end{array}$ \\
\hline Irving Oppenheim & Carnegie Mellon University \\
\hline Grace Ordaz & US DOE \\
\hline $\begin{array}{l}\text { W. Shannan } \\
\text { O'Shaughnessy }\end{array}$ & GVD Corporation \\
\hline Stan Osserman & $\begin{array}{l}\text { HI Ctr. for Advanced } \\
\text { Transportation Tech. }\end{array}$ \\
\hline Graham Ostrander & Automated Dynamics \\
\hline Naoki Ota & 24M Technologies Inc \\
\hline
\end{tabular}




\begin{tabular}{|c|c|}
\hline Name & Affiliation \\
\hline Kevin Ott & Consultant \\
\hline Runqing Ou & NEI Corporation \\
\hline Shiqi Ou & $\begin{array}{c}\text { Oak Ridge National } \\
\text { Laboratory }\end{array}$ \\
\hline Russell Owens & Eneregtics Incorporated \\
\hline Burak Ozpineci & $\begin{array}{c}\text { Oak Ridge National } \\
\text { Laboratory }\end{array}$ \\
\hline Benjamin Paczkowski & TARDEC \\
\hline Narendra Pal & Bethlehem Hydrogen Inc. \\
\hline Jeffrey Pallitto & United Hydrogen, LLC \\
\hline Brad Palmer & Cummins Corp. R\&T \\
\hline Mu Pan & $\begin{array}{c}\text { Wuhan University of } \\
\text { Technology }\end{array}$ \\
\hline $\begin{array}{c}\text { Dimitrios } \\
\text { Papageorgopoulos }\end{array}$ & U.S. Department of Energy \\
\hline Philip Parilla & $\begin{array}{l}\text { National Renewable } \\
\text { Energy Laboratory }\end{array}$ \\
\hline Brian Park & DOT \\
\hline Hoonmo Park & Hyundai Motor Company \\
\hline Jaehyung Park & KETEP \\
\hline Joong Sun Park & Argonne National Laboratory \\
\hline Seokhee Park & $\begin{array}{c}\text { Korea Institute of Energy } \\
\text { Research }\end{array}$ \\
\hline John Michael Parkan & Providence Entertainment \\
\hline Eric Parker & $\begin{array}{c}\text { Fuel Cell and Hydrogen } \\
\text { Energy Association }\end{array}$ \\
\hline Walter Parker & U.S. DOE - NETL \\
\hline George Parks & FuelScience LLC \\
\hline
\end{tabular}

\begin{tabular}{|c|c|}
\hline Name & Affiliation \\
\hline James Parks & $\begin{array}{c}\text { Oak Ridge National } \\
\text { Laboratory }\end{array}$ \\
\hline Antony Parulian & Arbin Corporation \\
\hline Pinakin Patel & FuelCell Energy \\
\hline Sanjay Patel & DOT/NHTSA \\
\hline Howard Pearlman & $\begin{array}{l}\text { Advanced Cooling } \\
\text { Technologies Inc }\end{array}$ \\
\hline Brian Peaslee & General Motors \\
\hline Charles Peden & U.S. Department of Energy \\
\hline William Peirce & General Motors \\
\hline George Pelton & Allison Transmission \\
\hline Ricardo Pena & $\begin{array}{c}\text { DOE/Vehicle Technologies } \\
\text { Office }\end{array}$ \\
\hline Christopher Penney & Rowan University \\
\hline Daniel Peppin & $3 M$ \\
\hline Thomas Perrot & Energetics Incorporated \\
\hline Mike Perry & $\begin{array}{l}\text { United Technologies } \\
\text { Research Center }\end{array}$ \\
\hline Kristin Persson & $\begin{array}{l}\text { Lawrence Berkeley National } \\
\text { Laboratory }\end{array}$ \\
\hline Ahmad Pesaran & $\begin{array}{c}\text { National Renewable Energy } \\
\text { Lab }\end{array}$ \\
\hline Michael Peters & $\begin{array}{l}\text { National Renewable } \\
\text { Energy Laboratory }\end{array}$ \\
\hline David Peterson & U.S. Department of Energy \\
\hline Doug Peterson & USMC, MARCORSYSCOM \\
\hline Lisa Peterson & U.S. Department of Energy \\
\hline Valeri Petkov & Central Michigan University \\
\hline
\end{tabular}




\begin{tabular}{|c|c|}
\hline Name & Affiliation \\
\hline Randy Petri & FuelCell Energy Inc \\
\hline Mark Petrie & SRI International \\
\hline Juergen Pfeiffer & Wacker Chemie AG \\
\hline Scott Phillippi & UPS \\
\hline Lyle Pickett & $\begin{array}{c}\text { Sandia National } \\
\text { Laboratories }\end{array}$ \\
\hline Jeff Pickles & Linde \\
\hline Michael Pien & ElectroChem \\
\hline Daniel Pierpont & $3 M$ \\
\hline Patrick Pietrasz & Ford Motor Company / AFCC \\
\hline Srikanth Pilla & Clemson University \\
\hline Peter Pintauro & Vanderbilt University \\
\hline William Pitz & $\begin{array}{l}\text { Lawrence Livermore } \\
\text { National Laboratory }\end{array}$ \\
\hline Hector Plascencia & University of Guanajuato \\
\hline Olga Polevaya & Nuvera Fuel Cells \\
\hline Darryl Pollica & Ivys Inc. \\
\hline Georgios Polyzos & ORNL \\
\hline Bryant Polzin & $\begin{array}{l}\text { Argonne National } \\
\text { Laboratory }\end{array}$ \\
\hline Neil Popovich & $\begin{array}{l}\text { National Renewable Energy } \\
\text { Laboratory }\end{array}$ \\
\hline Adam Powell & INFINIUM \\
\hline Christopher Powell & $\begin{array}{l}\text { Argonne National } \\
\text { Laboratory }\end{array}$ \\
\hline Rick Powell & Chevron \\
\hline
\end{tabular}

\begin{tabular}{|c|c|}
\hline Name & Affiliation \\
\hline Cassie Powers & NASEO \\
\hline Amit Prakash & Wiretough Cylinders LLC \\
\hline Ajay Prasad & University of Delaware \\
\hline Ravi Prasher & LBNL \\
\hline Joseph Pratt & $\begin{array}{l}\text { Sandia National } \\
\text { Laboratories }\end{array}$ \\
\hline Rick Pratt & PNNL \\
\hline Brendan Prebo & ASG Renaissance \\
\hline David Prendergast & $\begin{array}{l}\text { Lawrence Berkeley } \\
\text { National Laboratory }\end{array}$ \\
\hline Panos Prezas & $\begin{array}{l}\text { Argonne National } \\
\text { Laboratory }\end{array}$ \\
\hline Rebecca Price & Energetics Incorporated \\
\hline Robert Privette & XG Sciences, Inc. \\
\hline Sean Puckett & Volpe Center/OST-R \\
\hline Vincent Puglia & $\begin{array}{l}\text { Quebec Government Office } \\
\text { in Washington }\end{array}$ \\
\hline Krzysztof Pupek & Argonne National Laboratory \\
\hline Yue Qi & Michigan State University \\
\hline Nan Qin & $\begin{array}{l}\text { University of Central Florida- } \\
\text { Florida Solar Energy Center }\end{array}$ \\
\hline Jun Qu & $\begin{array}{l}\text { Oak Ridge National } \\
\text { Laboratory }\end{array}$ \\
\hline Karen Quackenbush & FCHEA \\
\hline Stephan Roser & $\begin{array}{l}\text { Westfalische-Wilhelms } \\
\text { Universitat Munster }\end{array}$ \\
\hline Dan Radomski & Optimal CAE \\
\hline Adam Ragatz & $\begin{array}{l}\text { National Renewable } \\
\text { Energy Laboratory }\end{array}$ \\
\hline
\end{tabular}




\begin{tabular}{|c|c|}
\hline Name & Affiliation \\
\hline Talat Rahman & $\begin{array}{l}\text { University of } \\
\text { Central Florida }\end{array}$ \\
\hline Manikandan Ramani & Plug Power Inc \\
\hline Anibal Ramirez-Cuesta & $\begin{array}{c}\text { Oak Ridge National } \\
\text { Laboratory }\end{array}$ \\
\hline Amy Randall & Bridgestone \\
\hline Katie Randolph & U.S. Department of Energy \\
\hline Jonathan Ranisau & University of Waterloo \\
\hline Kenneth Rappe & $\begin{array}{c}\text { Pacific Northwest National } \\
\text { Laboratory }\end{array}$ \\
\hline Brian Rasimick & Giner, Inc. \\
\hline Matthew Ratcliff & $\begin{array}{c}\text { NREL / Fuels Performance } \\
\text { Group }\end{array}$ \\
\hline Carole Read & $\begin{array}{l}\text { National Science } \\
\text { Foundation }\end{array}$ \\
\hline Steve Reagan & L\&L Products \\
\hline Krishna Reddi & $\begin{array}{l}\text { Argonne National } \\
\text { Laboratory }\end{array}$ \\
\hline Bradley Reese & Dartmouth College \\
\hline Ronald Reese, II & FCA US LLC \\
\hline Julia Rege & $\begin{array}{c}\text { Association of Global } \\
\text { Automakers }\end{array}$ \\
\hline Kurt Reichelderfer & $\begin{array}{c}\text { Toyota Motor Enginering \& } \\
\text { Mfg. NA }\end{array}$ \\
\hline Susan Rempe & $\begin{array}{l}\text { Sandia National } \\
\text { Laboratories }\end{array}$ \\
\hline Jane Rempel & TIAX LLC. \\
\hline Fei Ren & Temple University \\
\hline Xiaoming Ren & $\begin{array}{l}\text { U.S. Army Research } \\
\text { Laboratory }\end{array}$ \\
\hline Yang Ren & $\begin{array}{c}\text { Argonne National } \\
\text { Laboratory }\end{array}$ \\
\hline
\end{tabular}

\begin{tabular}{|c|c|}
\hline Name & Affiliation \\
\hline Julie Renner & Proton OnSite \\
\hline Tobias Renz & Tobias Renz FAIR \\
\hline Brian Rice & $\begin{array}{l}\text { University of Dayton } \\
\text { Research Institute }\end{array}$ \\
\hline Joel Rinebold & CCAT \\
\hline Mike Rinker & $\begin{array}{c}\text { Pacific Northwest National } \\
\text { Laboratory }\end{array}$ \\
\hline Jason Ritter & Blue Origin \\
\hline Nicole Ritzert & NIST \\
\hline Carl Rivkin & $\begin{array}{l}\text { National Renewable } \\
\text { Energy Laboratory }\end{array}$ \\
\hline Jeff Roberts & LLNL \\
\hline Rick Rocheleau & $\begin{array}{l}\text { Hawaii Natural Energy } \\
\text { Institute, Un }\end{array}$ \\
\hline Aida Rodrigues & General Motors LLC \\
\hline Jose Rodriguez & $\begin{array}{c}\text { Brookhaven National } \\
\text { Laboratory }\end{array}$ \\
\hline Michael Roeth & $\begin{array}{c}\text { North American Council for } \\
\text { Freight Efficiency }\end{array}$ \\
\hline Susan Rogers & $\begin{array}{c}\text { DOE Vehicle Technologies } \\
\text { Office }\end{array}$ \\
\hline Aashish Rohatgi & $\begin{array}{l}\text { Pacific Northwest } \\
\text { National Laboratory }\end{array}$ \\
\hline Pradee Rohatgi & $\begin{array}{l}\text { University of Wisconsin- } \\
\text { Milwaukee }\end{array}$ \\
\hline Joseph Ronevich & $\begin{array}{l}\text { Sandia National } \\
\text { Laboratories }\end{array}$ \\
\hline Tim Roney & Idaho National Laboratory \\
\hline Drew Ronneberg & SMI, Inc \\
\hline Jeff Ronning & AAM \\
\hline Marcy Rood & $\begin{array}{l}\text { Argonne National } \\
\text { Laboratory }\end{array}$ \\
\hline
\end{tabular}




\begin{tabular}{|c|c|}
\hline Name & Affiliation \\
\hline Farshid Roumi & Parthian Energy LLC \\
\hline Mahshid Roumi & Parthian Energy LLC \\
\hline Aymeric Rousseau & $\begin{array}{l}\text { Argonne National } \\
\text { Laboratory }\end{array}$ \\
\hline Ian Rowe & $\begin{array}{l}\text { Department of Energy } \\
\text { Bioenergy Technologies } \\
\text { Office }\end{array}$ \\
\hline Subir Roychoudhury & Precision Combustion, Inc. \\
\hline Hanson Ruan & Blue-O Technology Inc. \\
\hline John Rugh & $\begin{array}{l}\text { National Renewable } \\
\text { Energy Laboratory }\end{array}$ \\
\hline Peter Rupnowski & NREL \\
\hline Selena Russell & $\begin{array}{l}\text { U.S. Army Research } \\
\text { Laboratory }\end{array}$ \\
\hline Erin Russell-Story & U.S. DOE/NETL \\
\hline Neha Rustagi & U.S. Department of Energy \\
\hline Mark Ruth & $\begin{array}{l}\text { National Renewable } \\
\text { Energy Laboratory }\end{array}$ \\
\hline Jaeyune Ryu & $\begin{array}{l}\text { Korea Institute of Science } \\
\text { and Technology }\end{array}$ \\
\hline Adrian Sabau & $\begin{array}{l}\text { Oak Ridge National } \\
\text { Laboratory }\end{array}$ \\
\hline Michael Saft & $\begin{array}{l}\text { MCS Technology } \\
\text { Associates }\end{array}$ \\
\hline Nobuhiro Saito & Honda R\&D Co.,Ltd. \\
\hline Kazuo Sakurahara & $\begin{array}{l}\text { Honda R\&D Co.,Ltd. } \\
\text { Automobile R\&D Center }\end{array}$ \\
\hline Kambiz Salari & $\begin{array}{l}\text { Lawrence Livermore } \\
\text { National Laboratory }\end{array}$ \\
\hline Shawn Salisbury & Idaho National Laboratory \\
\hline Christopher San Marchi & $\begin{array}{l}\text { Sandia National } \\
\text { Laboratories }\end{array}$ \\
\hline Gary Sandrock & SNL/DOE \\
\hline
\end{tabular}

\begin{tabular}{|c|c|}
\hline Name & Affiliation \\
\hline $\begin{array}{c}\text { Shriram } \\
\text { Santhanagopalan }\end{array}$ & $\begin{array}{l}\text { National Renewable } \\
\text { Energy Laboratory }\end{array}$ \\
\hline Alexander Sappok & CTS Corporation \\
\hline Reuben Sarkar & DOE/EERE \\
\hline Tsuyoshi Sasaki & $\begin{array}{l}\text { Toyota Central R\&D Labs., } \\
\text { INC. }\end{array}$ \\
\hline Masaharu Sasakura & $\begin{array}{c}\text { The Institute of Applied } \\
\text { Energy }\end{array}$ \\
\hline James Saulsbury & $\begin{array}{l}\text { Oak Ridge National } \\
\text { Laboratory }\end{array}$ \\
\hline Ashok Saxena & Wiretough LLC \\
\hline Puneet Saxena & Delphi Automotive Systems \\
\hline Samveg Saxena & $\begin{array}{l}\text { Lawrence Berkeley } \\
\text { National Laboratory }\end{array}$ \\
\hline Riccardo Scarcelli & $\begin{array}{l}\text { Argonne National } \\
\text { Laboratory }\end{array}$ \\
\hline Michael Scarpino & US DOT-Volpe Center \\
\hline Bob Schauer & Lambda Technologies, Inc. \\
\hline Peter Schihl & US Army TARDEC \\
\hline Brendan Schloerb & Daikin America \\
\hline Martin Schneider & Hydrogenious Technologies \\
\hline David Schroeder & Volta Energy Technologies \\
\hline Marshall Schroeder & $\begin{array}{l}\text { Army Research Laboratory } \\
\text { (Adelphi) }\end{array}$ \\
\hline Klaus Schuegraf & PathTwo \\
\hline Christian Schumacher & $\begin{array}{c}\text { Naval Undersea Warfare } \\
\text { Center (NUWC) }\end{array}$ \\
\hline Carol Schutte & U.S. Department of Energy \\
\hline Jason Schwanke & Robert Bosch LLC \\
\hline
\end{tabular}




\begin{tabular}{|c|c|}
\hline Name & Affiliation \\
\hline Hart Schwartz & $\begin{array}{l}\text { Clarify Consulting \& } \\
\text { Research, LLC }\end{array}$ \\
\hline Viviane Schwartz & DOE/BES \\
\hline David Scrivener & MotorWeek \\
\hline David Sczomak & General Motors \\
\hline Ted Sears & $\begin{array}{l}\text { National Renewable } \\
\text { Energy Laboratory }\end{array}$ \\
\hline Jorge Seminario & Texas A\&M University \\
\hline Hee Je Seong & Argonne National Laboratory \\
\hline Jeff Serfass & $\begin{array}{c}\text { Technology Transition } \\
\text { Corporation }\end{array}$ \\
\hline Alexey Serov & University of New Mexico \\
\hline Pierre Serre-Combe & CEA \\
\hline Willard Shade & $\mathrm{ACl}$ Services inc. \\
\hline Pinakin Shah & $\begin{array}{l}\text { Teledyne Energy Systems, } \\
\text { Inc. }\end{array}$ \\
\hline $\begin{array}{l}\text { Reza Shahbazian- } \\
\text { Yassar }\end{array}$ & $\begin{array}{l}\text { University of Illinois at } \\
\text { Chicago }\end{array}$ \\
\hline Shane Shamblin & $\begin{array}{c}\text { Concurrent Technologies } \\
\text { Corporation }\end{array}$ \\
\hline Huifang Shao & Afton Chemical Corporation \\
\hline Minhua Shao & $\begin{array}{l}\text { The Hong Kong University of } \\
\text { Science and Technology }\end{array}$ \\
\hline Michael Sharpe & ORAU \\
\hline Stephen Sheargold & BASF \\
\hline Yangping Sheng & $\begin{array}{l}\text { Oak Ridge National } \\
\text { Laboratory }\end{array}$ \\
\hline Nicholas Sherman & U.S. Department of Energy \\
\hline Hiroshi Shimanuki & Honda Motor Co.,Ltd. \\
\hline
\end{tabular}

\begin{tabular}{|c|c|}
\hline Name & Affiliation \\
\hline Takahiro Shimizu & $\begin{array}{l}\text { Japan Automobile Research } \\
\text { Institute }\end{array}$ \\
\hline Masatoshi Shimoda & Hino Motors, Ltd. \\
\hline Akira Shimomura & $\begin{array}{c}\text { Sumitomo Corporation of } \\
\text { Americas }\end{array}$ \\
\hline Dongwon Shin & $\begin{array}{l}\text { Oak Ridge National } \\
\text { Laboratory }\end{array}$ \\
\hline Youngho Shin & $\begin{array}{l}\text { Argonne National } \\
\text { Laboratory }\end{array}$ \\
\hline Matthew Shirk & Idaho National Laboratory \\
\hline Pezhman Shirvanian & Tennessee Tech University \\
\hline Tolou Shokuhfar & $\begin{array}{l}\text { University of Illinois at } \\
\text { Chicago }\end{array}$ \\
\hline Steven Shpiner & Prescient \\
\hline Amit Shyam & $\begin{array}{l}\text { Oak Ridge National } \\
\text { Laboratory }\end{array}$ \\
\hline Donald Siegel & University of Michigan \\
\hline Kay Kimberly Siegel & H2Safe, LLC \\
\hline William Siegel & Consultant \\
\hline Robert Sievers & Teledyne Energy Systems \\
\hline Karen Sikes & CSRA \\
\hline Godfrey Sikha & Tesla Motors \\
\hline Aaron Simon & LLNL \\
\hline Mark Singer & $\begin{array}{l}\text { National Renewable } \\
\text { Energy Laboratory }\end{array}$ \\
\hline Brij Singh & John Deere \\
\hline Dileep Singh & $\begin{array}{l}\text { Argonne National } \\
\text { Laboratory }\end{array}$ \\
\hline Gurpreet Singh & $\begin{array}{l}\text { DOE Vehicle Technologies } \\
\text { Office }\end{array}$ \\
\hline
\end{tabular}




\begin{tabular}{|c|c|}
\hline Name & Affiliation \\
\hline Jagat Singh & 3M Company \\
\hline Ranbir Singh & $\begin{array}{c}\text { GeneSiC } \\
\text { Semiconductor Inc. }\end{array}$ \\
\hline Subhash Singhal & Independent Consultant \\
\hline Gal Sitty & Fuel Freedom Foundation \\
\hline Magnus Sjoberg & $\begin{array}{l}\text { Sandia National } \\
\text { Laboratories }\end{array}$ \\
\hline Ganesh Skandan & NEI Corporation \\
\hline Jon Skelly & $\begin{array}{c}\text { Hyundai Americas Technical } \\
\text { Center, Inc. }\end{array}$ \\
\hline Ed Skolnik & Energetics Incorporated \\
\hline Tim Skszek & Magna International \\
\hline Lee Slezak & $\begin{array}{l}\text { DOE Vehicle Technologies } \\
\text { Office }\end{array}$ \\
\hline Scott Sluder & $\begin{array}{l}\text { Oak Ridge National } \\
\text { Laboratory }\end{array}$ \\
\hline John Smart & Idaho National Laboratory \\
\hline Barton Smith & $\begin{array}{l}\text { Oak Ridge National } \\
\text { Laboratory }\end{array}$ \\
\hline David Smith & $\begin{array}{l}\text { Oak Ridge National } \\
\text { Laboratory }\end{array}$ \\
\hline Dennis Smith & $\begin{array}{l}\text { DOE Vehicle Technologies } \\
\text { Office }\end{array}$ \\
\hline Gregory Smith & $\begin{array}{l}\text { Oak Ridge National } \\
\text { Laboratory }\end{array}$ \\
\hline Margaret Smith & Energetics Incorporated \\
\hline Mark Smith & $\begin{array}{l}\text { DOE Vehicle Technologies } \\
\text { Office }\end{array}$ \\
\hline Owen Smith & $\begin{array}{l}\text { National Renewable } \\
\text { Energy Laboratory }\end{array}$ \\
\hline Patricia Smith & $\begin{array}{l}\text { Naval Surface Warfare } \\
\text { Center-Carderock }\end{array}$ \\
\hline Richard Smith & Not Applicable \\
\hline
\end{tabular}

\begin{tabular}{|c|c|}
\hline Name & Affiliation \\
\hline Dallas Smolarek & $\begin{array}{l}\text { DENSO International } \\
\text { America, Inc. }\end{array}$ \\
\hline Amy Snelling & ICF International \\
\hline Joshua Snyder & Drexel University \\
\hline Kimberly Soaper & BCS, Inc. \\
\hline Petros Sofronis & University of Illinois \\
\hline Tanju Sofu & $\begin{array}{l}\text { Argonne National } \\
\text { Laboratory }\end{array}$ \\
\hline Arun Solomon & GM \\
\hline Grigorii Soloveichik & ARPA-E \\
\hline Sibendu Som & $\begin{array}{l}\text { Argonne National } \\
\text { Laboratory }\end{array}$ \\
\hline Huiyu Song & University of Maryland \\
\hline Jianwei Song & $\begin{array}{l}\text { University of Maryland, } \\
\text { College Park }\end{array}$ \\
\hline John Sonnichsen & Hyundai Motor Group \\
\hline Ryan Sookhoo & Hydrogenics \\
\hline Roxana Sosa & Continental Corporation \\
\hline Fernando Soto & Texas A\&M University \\
\hline Jacob Spendelow & LANL \\
\hline Paul Spitsen & U.S. Department of Energy \\
\hline Kevin Spitzer & $\begin{array}{l}\text { Air Force Research } \\
\text { Laboratory }\end{array}$ \\
\hline Sam Sprik & $\begin{array}{l}\text { National Renewable } \\
\text { Energy Laboratory }\end{array}$ \\
\hline Venkat Srinivasan & $\begin{array}{c}\text { Lawrence Berkeley National } \\
\text { Laboratory }\end{array}$ \\
\hline Vojislav Stamenkovic & $\begin{array}{l}\text { Argonne National } \\
\text { Laboratory }\end{array}$ \\
\hline
\end{tabular}




\begin{tabular}{|c|c|}
\hline Name & Affiliation \\
\hline $\begin{array}{l}\text { Jean-Louis } \\
\text { Staudenmann }\end{array}$ & NIST/AMO \\
\hline Vitalie Stavila & $\begin{array}{c}\text { Sandia National } \\
\text { Laboratories }\end{array}$ \\
\hline Leigh Anna Steele & $\begin{array}{l}\text { Sandia National } \\
\text { Laboratories }\end{array}$ \\
\hline Constantin Stefan & Amprius, Inc. \\
\hline Daron Stein & PEC \\
\hline Andrew Steinbach & 3M Company \\
\hline Dietmar Steiner & Robert Bosch GmbH \\
\hline Thomas Stephens & $\begin{array}{l}\text { Argonne National } \\
\text { Laboratory }\end{array}$ \\
\hline Susan Stephenson & ATS \\
\hline Thomas Stephenson & Pajarito Powder, LLC \\
\hline Ned Stetson & U.S. Department of Energy \\
\hline Paul Stevens & $\begin{array}{c}\text { ExxonMobil Research \& } \\
\text { Eng. Co. }\end{array}$ \\
\hline Darren Stevenson & U.S. DOE, NETL \\
\hline Eric Stewart & $\begin{array}{c}\text { RCF Economic \& Financial } \\
\text { Consulting }\end{array}$ \\
\hline Mark Stewart & PNNL \\
\hline Jessie Stolark & EESI \\
\hline Kevin Stork & $\begin{array}{c}\text { DOE Vehicle Technologies } \\
\text { Office }\end{array}$ \\
\hline Gary Stottler & General Motors LLC \\
\hline Jean St-Pierre & $\begin{array}{l}\text { Hawaii Natural Energy } \\
\text { Institute }\end{array}$ \\
\hline Dee Strand & $\begin{array}{l}\text { Wildcat Discovery } \\
\text { Technologies }\end{array}$ \\
\hline Raimund Stroebel & Dana Corporation \\
\hline
\end{tabular}

\begin{tabular}{|c|c|}
\hline Name & Affiliation \\
\hline Andrea Strzelec & Texas A\&M University \\
\hline Philip Stuckey & $\begin{array}{c}\text { U.S. Patent and Trademark } \\
\text { Office }\end{array}$ \\
\hline Sarah Studer & ORISE \\
\hline Dong Su & Brookhaven National Lab \\
\hline Gui-Jia Su & $\begin{array}{c}\text { Oak Ridge National } \\
\text { Laboratory }\end{array}$ \\
\hline Wencong Su & $\begin{array}{l}\text { University of Michigan- } \\
\text { Dearborn }\end{array}$ \\
\hline Xin Su & $\begin{array}{l}\text { Argonne National } \\
\text { Laboratory }\end{array}$ \\
\hline Xuming Su & Ford Motors Company \\
\hline Ram Subbaraman & Robert Bosch LLC \\
\hline $\begin{array}{l}\text { Swaminathan } \\
\text { Subramanian }\end{array}$ & EATON \\
\hline Jens-Peter Suchsland & Greenerity GmbH \\
\hline Kazuhiro Suda & Honda Motor Co., Ltd. \\
\hline Martin Sulic & Savannah River Consulting \\
\hline William Summers & SRNL \\
\hline Ke Sun & Brookhaven National Lab \\
\hline Ruonan Sun & US EPA \\
\hline Wei Sun & University of Maryland \\
\hline Xiaolei Sun & $\begin{array}{l}\text { Electric Applications } \\
\text { Incorporated }\end{array}$ \\
\hline Xin Sun & $\begin{array}{c}\text { Pacific Northwest National } \\
\text { Laboratory }\end{array}$ \\
\hline Yong Sun & Tenneco Inc \\
\hline Yugang Sun & Temple University \\
\hline
\end{tabular}




\begin{tabular}{|c|c|}
\hline Name & Affiliation \\
\hline Yoo Sung Jong & $\begin{array}{l}\text { Korea Institute of Science } \\
\text { and Technology }\end{array}$ \\
\hline Mahendra Sunkara & University of Louisville \\
\hline Joseph Sunstrom & Daikin America \\
\hline Hideki Suzuki & American Honda Motor \\
\hline Keiji Suzuki & $\begin{array}{l}\text { NGK Spark Plugs (USA), } \\
\text { Inc. }\end{array}$ \\
\hline Shingo Suzuki & $\begin{array}{c}\text { Tanaka Kikinzoku } \\
\text { International (America), Inc. }\end{array}$ \\
\hline Scott Swartz & Nexceris, LLC \\
\hline Janos Szanyi & $\begin{array}{l}\text { Pacific Northwest National } \\
\text { Laboratory }\end{array}$ \\
\hline Klaus Szoucsek & BMW AG \\
\hline Esther Takeuchi & Stony Brook University \\
\hline David Tamburello & $\begin{array}{c}\text { Savannah River National } \\
\text { Laboratory }\end{array}$ \\
\hline Satish Tamhankar & Independent \\
\hline Mohammad Tamimi & Eastern Research Group \\
\hline Daniel Tan & GE Global Research \\
\hline Yukihito Tanaka & $\begin{array}{l}\text { Honda R\&D Co., Ltd. } \\
\text { Automobile R\&D Center }\end{array}$ \\
\hline Lixin Tang & $\begin{array}{l}\text { Oak Ridge National } \\
\text { Laboratory }\end{array}$ \\
\hline Maureen Tang & Drexel University \\
\hline Wan Si Tang & UMD / NIST-NCNR \\
\hline Wei Tang & $\begin{array}{l}\text { Oak Ridge National } \\
\text { Laboratory }\end{array}$ \\
\hline Zhijiang Tang & $\begin{array}{l}\text { Oak Ridge National } \\
\text { Laboratory }\end{array}$ \\
\hline Arman Tanman & $\begin{array}{l}\text { U.S. Environmental } \\
\text { Protection Agency }\end{array}$ \\
\hline
\end{tabular}

\begin{tabular}{|c|c|}
\hline Name & Affiliation \\
\hline Katie Tartaglia & Energetics Inc \\
\hline Jacob Tarver & $\begin{array}{l}\text { National Renewable } \\
\text { Energy Laboratory }\end{array}$ \\
\hline Harshad Tataria & GM/USABC \\
\hline Ralph Taylor & Delphi \\
\hline Yoshiaki Tazaki & Oak-Mitsui Technologies \\
\hline Andrei Tchouvelev & $\begin{array}{l}\text { A.V.Tchouvelev \& Associates } \\
\text { Inc. }\end{array}$ \\
\hline Joseph Teprovich & $\begin{array}{c}\text { Savannah River National } \\
\text { Laboratory }\end{array}$ \\
\hline Danny Terlip & $\begin{array}{l}\text { National Renewable } \\
\text { Energy Laboratory }\end{array}$ \\
\hline David Tew & $\begin{array}{l}\text { United Technologies } \\
\text { Research Center }\end{array}$ \\
\hline Michael Thackeray & ANL \\
\hline C. E. (Sandy) Thomas & Not Applicable \\
\hline Mark Thompson & $\begin{array}{l}\text { United Technologies } \\
\text { Research Center }\end{array}$ \\
\hline Matthew Thorington & Robert BOSCH LLC \\
\hline Matthew Thornton & $\begin{array}{l}\text { National Renewable } \\
\text { Energy Laboratory }\end{array}$ \\
\hline Steven Thrush & U.S. Army TARDEC \\
\hline Huajun Tian & University of Maryland \\
\hline Zhenhua Tian & University of South Carolina \\
\hline Will Tilford & Solvay \\
\hline Thomas A. Timbario & $\begin{array}{c}\text { Alliance Technical Services, } \\
\text { Inc. }\end{array}$ \\
\hline Thomas J. Timbario & $\begin{array}{l}\text { Alliance Technical Services, } \\
\text { Inc. }\end{array}$ \\
\hline Kenneth Tobin & $\begin{array}{l}\text { Oak Ridge National } \\
\text { Laboratory }\end{array}$ \\
\hline
\end{tabular}




\begin{tabular}{|c|c|}
\hline Name & Affiliation \\
\hline Richard Todaro & $\begin{array}{c}\text { Allegheny Science and } \\
\text { Technology }\end{array}$ \\
\hline Sascha Toelle & Umicore AG\&Co. KG \\
\hline Wei Tong & LBNL \\
\hline Laurence Toomey & US Army - TARDEC \\
\hline Todd Toops & $\begin{array}{c}\text { Oak Ridge National } \\
\text { Laboratory }\end{array}$ \\
\hline Sarah Topper & PPG \\
\hline Mark Toughiry & $\begin{array}{l}\text { US Department of } \\
\text { Transportation }\end{array}$ \\
\hline Dennis Townsend & Townsend Capital, LLC \\
\hline Justin Townsend & ORAU \\
\hline Aaron Trainor & Zeton Inc. \\
\hline Dat Tran & $\begin{array}{l}\text { U.S. Army Research } \\
\text { Laboratory }\end{array}$ \\
\hline Thanh Tran & NSWC Carderock \\
\hline Stephen Trask & $\begin{array}{l}\text { Argonne National } \\
\text { Laboratory }\end{array}$ \\
\hline Vanessa Trejos & Department of Energy/ CSRA \\
\hline John Trocciola & SRA International \\
\hline Li-Duan Tsai & $\begin{array}{l}\text { Industrial Technology } \\
\text { Research Institute }\end{array}$ \\
\hline Dionisis Tsimis & FCH JU \\
\hline Vasilios Tsourapas & Eaton \\
\hline Shuntaro Tsutsumi & $\begin{array}{l}\text { Toyota Motor North America, } \\
\text { Inc. }\end{array}$ \\
\hline Dylan Tucker & $\begin{array}{c}\text { Allegheny Science \& } \\
\text { Technology }\end{array}$ \\
\hline Eric Tyo & Johnson Matthey \\
\hline
\end{tabular}

\begin{tabular}{|c|c|}
\hline Name & Affiliation \\
\hline Terrence Udovic & $\begin{array}{c}\text { National Institute of } \\
\text { Standards \& Technology }\end{array}$ \\
\hline Yoshitaka Uehara & $\begin{array}{c}\text { NISSAN TECHNICAL CENTER } \\
\text { N.A. }\end{array}$ \\
\hline Michael Ulsh & $\begin{array}{l}\text { National Renewable } \\
\text { Energy Laboratory }\end{array}$ \\
\hline Andrew Ulvestad & $\begin{array}{l}\text { Argonne National } \\
\text { Laboratory }\end{array}$ \\
\hline Mario Urdaneta & Department of Energy, AMO \\
\hline Alex Vaipan & Hexagon Composites \\
\hline Thomas I. Valdez & Jet Propulsion Laboratory \\
\hline Nicholas Vanderborgh & LANL, retired \\
\hline Walter Varhue & University of Vermont \\
\hline John Vaughey & $\begin{array}{l}\text { Argonne National } \\
\text { Laboratory }\end{array}$ \\
\hline $\begin{array}{c}\text { Surya Narayana Prasad } \\
\text { Vegendla }\end{array}$ & $\begin{array}{l}\text { Argonne National } \\
\text { Laboratory }\end{array}$ \\
\hline Gabriel Veith & $\begin{array}{c}\text { Oak Ridge National } \\
\text { Laboratory }\end{array}$ \\
\hline Adriana Vela & Universidad de Guanajuato \\
\hline $\begin{array}{l}\text { Subramanian } \\
\text { Venkatachalam }\end{array}$ & Envia Systems \\
\hline David Ventola & B\&W MEGTEC \\
\hline $\begin{array}{l}\text { Lakshman } \\
\text { Ventrapragada }\end{array}$ & Clemson University \\
\hline Laura Verduzco & Chevron Corporation \\
\hline Pascal Viala & BMW NA \\
\hline Julio Villafuerte & Centerline Windsor Ltd \\
\hline Anil Virkar & University of Utah \\
\hline Anupam Vivek & The Ohio State University \\
\hline
\end{tabular}




\begin{tabular}{|c|c|}
\hline Name & Affiliation \\
\hline Nhon Vo & NanoAI LLC \\
\hline Gary Voelker & Miltec UV International \\
\hline John Vogel & Combined Energies \\
\hline Cung Vu & Vu Consulting LLC \\
\hline Miomir Vukmirovic & $\begin{array}{c}\text { Brookhaven National } \\
\text { Laboratory }\end{array}$ \\
\hline Steve Wach & SRNL \\
\hline Eric Wachsman & University of Maryland \\
\hline Matthew Wade & Virginia Clean Cities \\
\hline David Wagner & Ford Motor Company \\
\hline Fred Wagner & Energetics Incorporated \\
\hline Frederick Wagner & Self (retired from GM) \\
\hline James Waldecker & Ford \\
\hline Mike Walker & General Motors LLC \\
\hline Gordon Waller & NSWCCD \\
\hline Thomas Wallner & $\begin{array}{l}\text { Argonne National } \\
\text { Laboratory }\end{array}$ \\
\hline Sam Waltzer & USEPA SmartWay \\
\hline Jiayu Wan & $\begin{array}{l}\text { Univerisyt of Maryland, } \\
\text { college park }\end{array}$ \\
\hline Yan Wan & University of North Texas \\
\hline Chengwei Wang & University of Maryland \\
\hline Chongmin Wang & $\begin{array}{l}\text { Pacific Northwest } \\
\text { National Laboratory }\end{array}$ \\
\hline Conghua Wang & $\begin{array}{l}\text { TreadStone Technologies, } \\
\text { Inc. }\end{array}$ \\
\hline
\end{tabular}

\begin{tabular}{|c|c|}
\hline Name & Affiliation \\
\hline Dawei Wang & $\begin{array}{c}\text { Brookhaven National } \\
\text { Laboratory }\end{array}$ \\
\hline Donghai Wang & Penn State University \\
\hline Feng Wang & $\begin{array}{c}\text { Brookhaven National } \\
\text { Laboratory }\end{array}$ \\
\hline Francis Wang & SiNode Systems \\
\hline Fred Wang & University of Tennessee \\
\hline Hailiang Wang & Yale University \\
\hline Li Wang & Tsinghua University \\
\hline Liang Wang & University of Delaware \\
\hline Luning Wang & $\begin{array}{l}\text { University of Maryland, } \\
\text { College Park }\end{array}$ \\
\hline Mengqi Wang & $\begin{array}{l}\text { University of Michigan- } \\
\text { Dearborn }\end{array}$ \\
\hline Michael Wang & $\begin{array}{l}\text { Argonne National } \\
\text { Laboratory }\end{array}$ \\
\hline Mingyu Wang & MAHLE \\
\hline Qigui Wang & General Motors \\
\hline Sha Wang & $\begin{array}{c}\text { University of Maryland } \\
\text { College Park }\end{array}$ \\
\hline Wei Wang & $\begin{array}{c}\text { Pacific Northwest National } \\
\text { Laboratory }\end{array}$ \\
\hline Xiaohua Wang & Argonne National Lab \\
\hline Xiqing wang & $\begin{array}{l}\text { Contemporary Amperex } \\
\text { Technology Co., Limited }\end{array}$ \\
\hline Xiwen Wang & University of Maryland \\
\hline Yan Wang & $\begin{array}{l}\text { Worcester Polytechnic } \\
\text { Institute }\end{array}$ \\
\hline Yanyan Wang & Georgetown University \\
\hline Jacob Ward & $\begin{array}{c}\text { DOE Vehicle Technologies } \\
\text { Office }\end{array}$ \\
\hline
\end{tabular}




\begin{tabular}{|c|c|}
\hline Name & Affiliation \\
\hline Patrick Ward & $\begin{array}{c}\text { Savannah River National } \\
\text { Laboratory }\end{array}$ \\
\hline Bruce Warford & ORAU \\
\hline Ryoji Watanabe & Sumitomo Corporation \\
\hline Matt Watkins & EMRE \\
\hline Eric Weaver & US DOT/FHWA \\
\hline Adam Weber & LBNL \\
\hline Brian Weeks & Gas Technology Institute \\
\hline Max Wei & LBNL \\
\hline Zhehao Wei & Johnson Matthey \\
\hline Craig Weich & Sila Nanotechnologies \\
\hline Tim Weihs & John Hopkins University \\
\hline Steven Weiner & Hydrogen Safety Panel \\
\hline Michael Weismiller & $\begin{array}{c}\text { DOE Vehicle Technologies } \\
\text { Office }\end{array}$ \\
\hline Fei Wen & University of Michigan \\
\hline Baicheng Weng & University of Toledo \\
\hline Andrew Wereszczak & $\begin{array}{c}\text { Oak Ridge National } \\
\text { Laboratory }\end{array}$ \\
\hline Chris Werth & AST/DOE \\
\hline Brian West & $\begin{array}{l}\text { Oak Ridge National } \\
\text { Laboratory }\end{array}$ \\
\hline Todd West & $\begin{array}{l}\text { Sandia National } \\
\text { Laboratories }\end{array}$ \\
\hline Brittany Westlake & EPRI \\
\hline Scott Whalen & $\begin{array}{l}\text { Pacific Northwest } \\
\text { National Laboratory }\end{array}$ \\
\hline
\end{tabular}

\begin{tabular}{|c|c|}
\hline Name & Affiliation \\
\hline Christopher Whaling & Synthesis Partners, LLC \\
\hline Dean Wheeler & Brigham Young University \\
\hline Douglas Wheeler & DJW TECHNOLOGY, LLC \\
\hline Sera White & Idaho National laboratory \\
\hline Steve White & Robert Bosch, LLC \\
\hline Russell Whitesides & LLNL \\
\hline Stanley Whittingham & SUNY \\
\hline Jenna Wies & $\begin{array}{l}\text { Forchungszentrum Julich } \\
\text { GmbH }\end{array}$ \\
\hline Gregory Wilcox & Eastern Research Group \\
\hline Herbert Williams & Hanon Systems USA \\
\hline Mark Williams & aecom \\
\hline Margaret Williamson & ORAU \\
\hline Claude Willis & $\begin{array}{c}\text { Greater Wash. Region Clean } \\
\text { Cities }\end{array}$ \\
\hline Keith Wilson & SAE International \\
\hline Robert Wimmer & Toyota \\
\hline Keith Wipke & $\begin{array}{l}\text { National Renewable } \\
\text { Energy Laboratory }\end{array}$ \\
\hline Martin Wissink & $\begin{array}{c}\text { Oak Ridge National } \\
\text { Laboratory }\end{array}$ \\
\hline Thomas Witte & $\begin{array}{c}\text { Pacific Northwest } \\
\text { National Laboratories }\end{array}$ \\
\hline Michael Wixom & $\begin{array}{c}\text { Navitas Advanced Solutions } \\
\text { Group }\end{array}$ \\
\hline William Woebkenberg & $\begin{array}{c}\text { Aramco Research Center - } \\
\text { Detroit }\end{array}$ \\
\hline Ron Wohl & Nanoscale Components Inc \\
\hline
\end{tabular}




\begin{tabular}{|c|c|}
\hline Name & Affiliation \\
\hline Jeff Wolfenstine & $\begin{array}{l}\text { U.S. Army Research } \\
\text { Laboratory }\end{array}$ \\
\hline Ezana Wondimneh & $\begin{array}{l}\text { U.S. Department of } \\
\text { Transportation }\end{array}$ \\
\hline Dominica Wong & $\begin{array}{c}\text { Eastman Chemical } \\
\text { Company }\end{array}$ \\
\hline Brandon Wood & $\begin{array}{l}\text { Lawrence Livermore } \\
\text { National Laboratory }\end{array}$ \\
\hline David Wood & $\begin{array}{c}\text { Oak Ridge National } \\
\text { Laboratory }\end{array}$ \\
\hline Marissa Wood & $\begin{array}{c}\text { Oak Ridge National } \\
\text { Laboratory }\end{array}$ \\
\hline Weston Wood & ENTEK Membranes \\
\hline William Woodford & 24M Technologies Inc \\
\hline Stephen Woods & Jacobs NASA WSTF \\
\hline Felix Wu & $\begin{array}{c}\text { DOE Vehicle Technologies } \\
\text { Office }\end{array}$ \\
\hline Gefei Wu & Valvoline/Ashland \\
\hline Zili Wu & $\begin{array}{c}\text { Oak Ridge National } \\
\text { Laboratory }\end{array}$ \\
\hline Tian Xia & University of Vermont \\
\hline Jie Xiao & University of Arkansas \\
\hline Xingcheng Xiao & General Motors \\
\hline Jian Xie & $\begin{array}{l}\text { Indiana University Purdue } \\
\text { University Indianapolis }\end{array}$ \\
\hline Huolin Xin & $\begin{array}{c}\text { Brookhaven National } \\
\text { Laboratory }\end{array}$ \\
\hline Yangchuan (Chad) Xing & University of Missouri \\
\hline Ben Xiong & $\begin{array}{l}\text { California Fuel Cell } \\
\text { Partnership }\end{array}$ \\
\hline Bingjun $\mathrm{Xu}$ & University of Delaware \\
\hline Fenghua Xu & University of Toledo \\
\hline
\end{tabular}

\begin{tabular}{|c|c|}
\hline Name & Affiliation \\
\hline Gui-Liang Xu & $\begin{array}{l}\text { Argonne National } \\
\text { Laboratory }\end{array}$ \\
\hline Kang Xu & US Army Research Lab \\
\hline Pinghong $\mathrm{Xu}$ & Gotion Inc. \\
\hline Shaomao Xu & University of Maryland \\
\hline Wu Xu & $\begin{array}{c}\text { Pacific Northwest } \\
\text { National Laboratory }\end{array}$ \\
\hline Allen Xue & Novick, Kim \& Lee, PLLC \\
\hline Jisan Xue & DOt/NHTSA \\
\hline Marina Yakovleva & fmc \\
\hline Takahiro Yamada & $\begin{array}{l}\text { University of Dayton } \\
\text { Research Institute }\end{array}$ \\
\hline Kunimitsu Yamamoto & $\begin{array}{c}\text { Toyota Motor Engineering } \\
\text { \& Manufacturing North } \\
\text { America }\end{array}$ \\
\hline Fuminori Yamanashi & HySUT \\
\hline Yushan Yan & University of Delaware \\
\hline Michael Yandrasits & $3 M$ \\
\hline Chongyin Yang & University of Maryland \\
\hline Chuanbo Yang & $\begin{array}{l}\text { National Renewable } \\
\text { Energy Laboratory }\end{array}$ \\
\hline Jihui Yang & University of Washington \\
\hline Taehyun Yang & KETEP \\
\hline Xiao Guang Yang & Ford Motor Company \\
\hline Yong Yang & $\begin{array}{c}\text { Austin Power Engineering } \\
\text { LLC }\end{array}$ \\
\hline Yuan Yang & Columbia University \\
\hline Zhiwei Yang & $\begin{array}{c}\text { United Technologies } \\
\text { Research Center }\end{array}$ \\
\hline
\end{tabular}




\begin{tabular}{|c|c|}
\hline Name & Affiliation \\
\hline Ami Yang Neyerlin & $\begin{array}{l}\text { NREL/Colorado School of } \\
\text { Mines }\end{array}$ \\
\hline Yonggang Yao & University of Maryland \\
\hline Tomio Yasuda & Technova Inc. \\
\hline Koji Yasutomi & Hino Motors Ltd. \\
\hline Brandon Yauch & Electricore, Inc. \\
\hline Siyu Ye & Ballard Power Systems \\
\hline Chiao-Wen Yeh & Epistar \\
\hline Paul Yelvington & $\begin{array}{c}\text { Mainstream Engineering } \\
\text { Corporation }\end{array}$ \\
\hline Angelo Yializis & Sigma Technologies \\
\hline Aaron Yocum & U.S. Department of Energy \\
\hline Snng Jong Yoo & $\begin{array}{l}\text { Korea Institute of Science } \\
\text { and Technology }\end{array}$ \\
\hline Junichi Yoshida & NEDO \\
\hline Makoto Yoshida & Nissan Motor Co Ltd \\
\hline Toshihiko Yoshida & Tokyo Institute Technology \\
\hline Toshiro Yoshida & Technova Inc. \\
\hline Yuichi Yoshida & NEDO \\
\hline Kazunori Yoshitomi & Hino Motors \\
\hline Harry Youmans & Hanon Systems, USA LLC \\
\hline Ronald Young & General Motors \\
\hline Stanley Young & $\begin{array}{l}\text { National Renewable } \\
\text { Energy Laboratory }\end{array}$ \\
\hline Nadia Yousfi Steiner & LABEX ACTION \\
\hline
\end{tabular}

\begin{tabular}{|c|c|}
\hline Name & Affiliation \\
\hline Raman Yousif & Natural Resources Canada \\
\hline Siyoung Yu & University of Connecticut \\
\hline Jie Yue & $\begin{array}{l}\text { University of Maryland, } \\
\text { College Park }\end{array}$ \\
\hline Chao-Yi Yuh & FuelCell Energy, Inc. \\
\hline Gleb Yushin & $\begin{array}{l}\text { Georgia Institute of } \\
\text { Technology }\end{array}$ \\
\hline Elvin Yuzugullu & CSRA \\
\hline Karim Zaghib & Hydro-Quebec \\
\hline Walter Zalis & Energetics Incorporated \\
\hline Elizabeth Zeitler & $\begin{array}{l}\text { Millennium Challenge } \\
\text { Corporation }\end{array}$ \\
\hline Piotr Zelenay & $\begin{array}{l}\text { Los Alamos National } \\
\text { Laboratory }\end{array}$ \\
\hline Xiangqun Zeng & Oakland University \\
\hline Iryna Zenyuk & Tufts University \\
\hline Yuhui Zha & Cummins, Inc. \\
\hline Cuijuan Zhang & University of South Carolina \\
\hline Feng-Yuan Zhang & University of Tennessee \\
\hline Houshun Zhang & EPA \\
\hline Ji-Guang Zhang & PNNL \\
\hline Julia Zhang & Oregon State University \\
\hline Linghong Zhang & $\begin{array}{l}\text { Argonne National } \\
\text { Laboratory }\end{array}$ \\
\hline Pu Zhang & $\begin{array}{c}\text { Navitas Advanced Solutions } \\
\text { Group }\end{array}$ \\
\hline Shengshui Zhang & $\begin{array}{l}\text { U.S. Army Research } \\
\text { Laboratory }\end{array}$ \\
\hline
\end{tabular}




\begin{tabular}{|c|c|}
\hline Name & \multicolumn{1}{|c|}{ Affiliation } \\
\hline Shuo Zhang & University of Maryland \\
\hline Wei Zhang & BNL \\
\hline Xiaoyu Zhang & Old Dominion University \\
\hline Y-H Percival Zhang & Virginia Tech \\
\hline Ying Zhang & University of Maryland \\
\hline Bin Zhao & University of Tennessee \\
\hline Jianming Zheng & Pacific Northwest National \\
\hline Liangyang Zhou & \\
\hline Long Zaboratory
\end{tabular}

\begin{tabular}{|c|c|}
\hline Name & Affiliation \\
\hline Yongning Zhou & Fudan University \\
\hline Huaping Zhu & $\begin{array}{l}\text { Huazhong University of } \\
\text { Science and Technology }\end{array}$ \\
\hline Kai Zhu & $\begin{array}{c}\text { National Renewable Energy } \\
\text { Lab }\end{array}$ \\
\hline Yu Zhu & University of Akron \\
\hline Brad Zigler & NREL \\
\hline Mark Zima & MAHLE \\
\hline Stephen Zimmer & USCAR \\
\hline Jonathan Zimmerman & Sandia National Laboratories \\
\hline Shouzhong Zou & American University \\
\hline Shouzhong Zou & American University \\
\hline Russell Zukouski & Navistar \\
\hline Lei Zuo & Virginia Tech \\
\hline
\end{tabular}




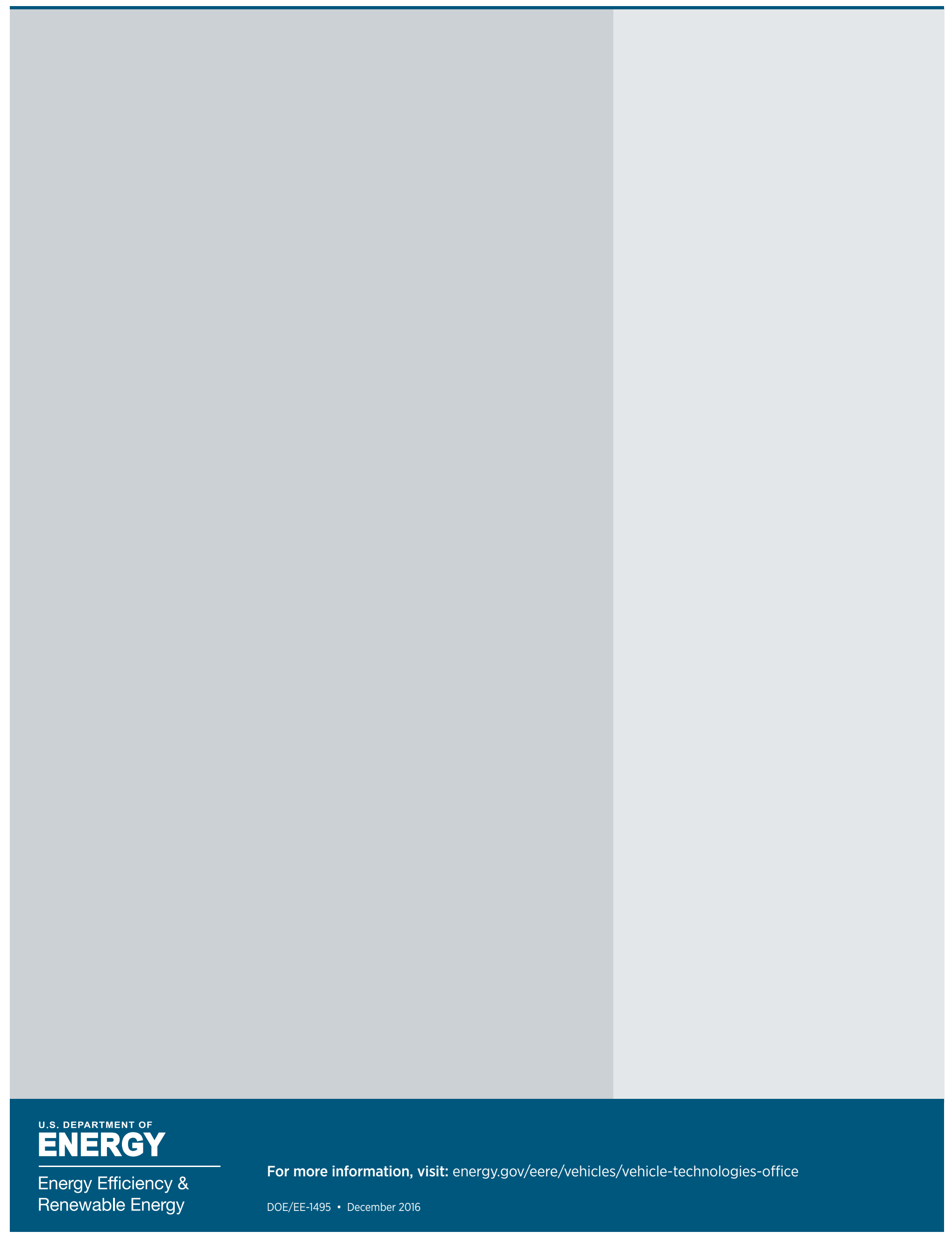

